University of Nebraska - Lincoln

DigitalCommons@University of Nebraska - Lincoln

Geochemical Characterization of Water, Sediment, and Biota Affected by Mercury Contamination and Acidic Drainage from Historical Gold Mining, Greenhorn Creek, Nevada County, California, 1999-2001

\author{
Charles N. Alpers \\ U.S. Geological Survey \\ Michael P. Hunerlach \\ U.S. Geological Survey \\ Jason T. May \\ U.S. Geological Survey \\ Roger L. Hothem \\ U.S. Geological Survey \\ Howard E. Taylor \\ U.S. Geological Survey, hetaylor@usgs.gov \\ See next page for additional authors \\ Follow this and additional works at: https://digitalcommons.unl.edu/usgspubs \\ Part of the Earth Sciences Commons
}

Alpers, Charles N.; Hunerlach, Michael P.; May, Jason T.; Hothem, Roger L.; Taylor, Howard E.; Antweiler, Ronald C.; De Wild, John F.; and Lawler, David A., "Geochemical Characterization of Water, Sediment, and Biota Affected by Mercury Contamination and Acidic Drainage from Historical Gold Mining, Greenhorn Creek, Nevada County, California, 1999-2001" (2004). Publications of the US Geological Survey. 54. https://digitalcommons.unl.edu/usgspubs/54

This Article is brought to you for free and open access by the US Geological Survey at DigitalCommons@University of Nebraska - Lincoln. It has been accepted for inclusion in Publications of the US Geological Survey by an authorized administrator of DigitalCommons@University of Nebraska - Lincoln. 


\section{Authors}

Charles N. Alpers, Michael P. Hunerlach, Jason T. May, Roger L. Hothem, Howard E. Taylor, Ronald C. Antweiler, John F. De Wild, and David A. Lawler 
In cooperation with the Bureau of Land Management, the California State Water Resources Control Board, the Nevada County Resource Conservation District, and the U.S. Department of Agriculture-Forest Service

\section{Geochemical Characterization of Water, Sediment, and Biota Affected by Mercury Contamination and Acidic Drainage from Historical Gold Mining, Greenhorn Creek, Nevada County, California, 1999-2001}

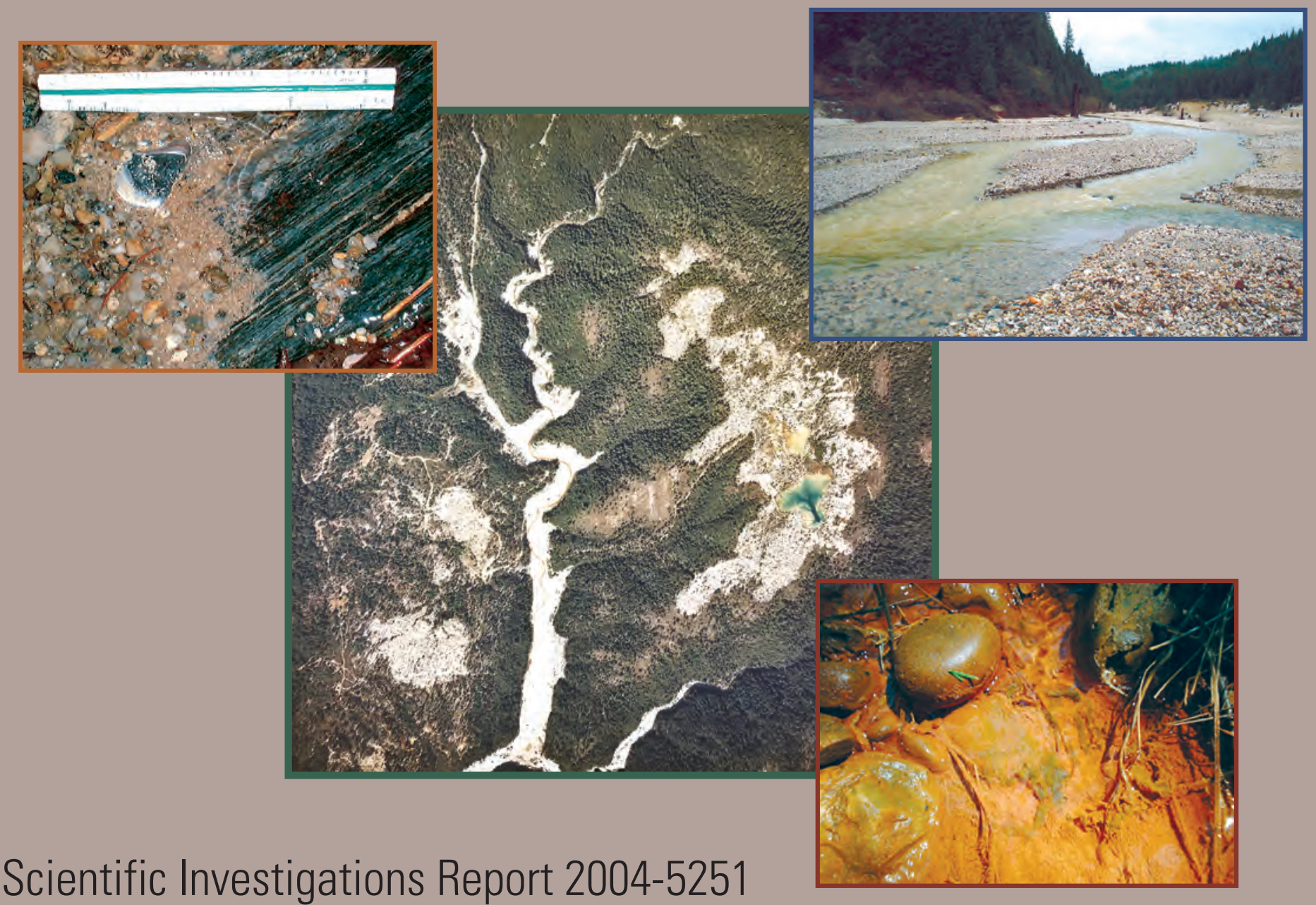

U.S. Department of the Interior U.S. Geological Survey 
Photographs on front cover (clockwise, from upper left):

(upper left) Visible mercury at contact between alluvium and slate bedrock, Sailor Flat Mine, Greenhorn Creek drainage, Nevada County, California; total length of ruler is 4.8 inches. Photograph by M.P. Hunerlach.

(upper right) Confluence of Greenhorn Creek and main drain from Buckeye Flat, looking upstream, Nevada County, California. Photograph by M.P. Hunerlach. (lower right) Iron-rich precipitate in ground sluice at Poore Mine, Greenhorn Creek drainage, Nevada County, California. Photograph by M.P. Hunerlach.

(center) Aerial view of central part of Greenhorn Creek drainage showing Buckeye Flat Mine and pit lake, Nevada County, California. Photograph courtesty of the U.S. Department of Agriculture-Forest Service, Tahoe National Forest. 


\section{Geochemical characterization of water, sediment, and biota affected by mercury contamination and acidic drainage from historical gold mining, Greenhorn Creek, Nevada County, California, 1999-2001}

By Charles N. Alpers, Michael P. Hunerlach, Jason T. May, Roger L. Hothem, Howard E. Taylor, Ronald C. Antweiler, John F. De Wild, and David A. Lawler

Scientific Investigations Report 2004-5251 


\section{U.S. Department of the Interior \\ Gale A. Norton, Secretary \\ U.S. Geological Survey \\ Charles G. Groat, Director}

U.S. Geological Survey, Reston, Virginia: 2005

For sale by U.S. Geological Survey, Information Services
Box 25286, Denver Federal Center
Denver, CO 80225
For more information about the USGS and its products:
Telephone: 1-888-ASK-USGS
World Wide Web: http://www.usgs.gov/

Any use of trade, product, or firm names in this publication is for descriptive purposes only and does not imply endorsement by the U.S. Government.

Although this report is in the public domain, permission must be secured from the individual copyright owners to reproduce any copyrighted materials contained within this report.

Suggested citation:

Alpers, C.N., Hunerlach, M.P., May, J.T., Hothem, R.L., Taylor, H.E., Antweiler, R.C., De Wild, J.F., and Lawler, D.A., 2005, Geochemical characterization of water, sediment, and biota affected by mercury contamination and acidic drainage from historical gold mining, Greenhorn Creek, Nevada County, California, 1999-2001: U.S. Geological Survey Scientific Investigations Report 2004-5251, 278 p. 


\section{Contents}

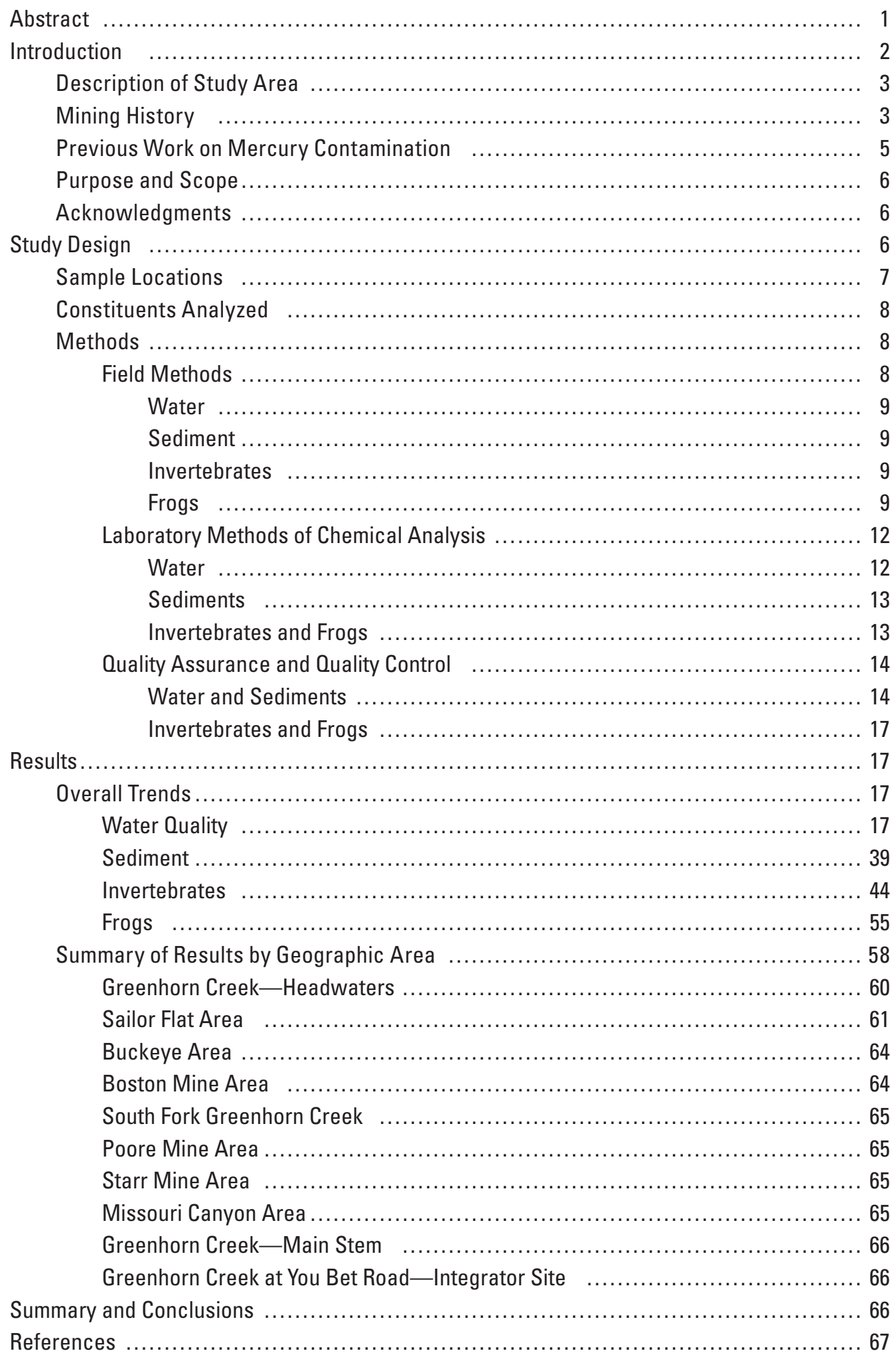


Appendix A. Map of Tunnel Locations and Photographs of Field Sites, Greenhorn Creek Drainage, Nevada County, California

Appendix B. Quality Assurance and Quality Control Plots for Chemical Analyses of Water

Samples from the Greenhorn Creek Drainage, Nevada County, California

Appendix C. Locations and Characteristics of Sampling Stations and Tunnels, Greenhorn

Creek Drainage, Nevada County, California

Appendix D. Quality Assurance and Quality Control Information for Chemical Analyses of Samples from the Greenhorn Creek Drainage, Nevada County, California .......... 255

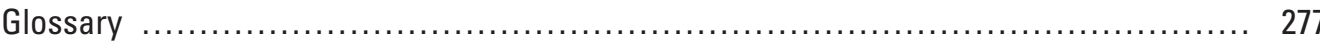

\section{Figures}

Figure 1. Map showing location of Greenhorn Creek drainage in the Bear River watershed, Nevada County, California

Figure 2. Map showing sampling stations in the Greenhorn Creek drainage, Nevada County, California.

Figure 3. Map showing sampling stations in the northern part of Greenhorn Creek drainage, Nevada County, California.

Figure 4. Map showing sampling stations in the central part of Greenhorn Creek drainage, Nevada County, California.

Figure 5. Map showing the "integrator" sampling station in the southern part of Greenhorn Creek drainage, Nevada County, California .

Figure 6. Box plots showing distribution of total mercury concentration and methylmercury concentration in unfiltered and filtered water samples from the Greenhorn Creek drainage, Nevada County, California

Figure 7. Box plots showing distribution of $(A)$ total mercury concentration in unfiltered water, $(B)$ total mercury concentration in filtered water, and $(C)$ methylmercury concentration in unfiltered water, grouped by type of water body, Greenhorn Creek drainage, Nevada County, California.

Figure 8. Box plots showing distribution of the ratio of methylmercury to total mercury in unfiltered water samples grouped by type of water body, Greenhorn Creek drainage, Nevada County, California....

Figure 9. Plot showing relation between total mercury concentration in unfiltered and filtered water samples, Greenhorn Creek drainage, Nevada County, California

Figure 10. Plot showing relation between methylmercury concentration in unfiltered and filtered water samples, Greenhorn Creek drainage, Nevada County, California

Figure 11. Plot showing relation between total mercury and methylmercury concentration in unfiltered water samples, Greenhorn Creek drainage, Nevada County, California

Figure 12. Plot showing relation between total mercury and methylmercury concentration in filtered water samples, Greenhorn Creek drainage, Nevada County, California 35

Figure 13. Plot showing relation between $\mathrm{pH}$ in unfiltered water and sulfate concentration in filtered water, Greenhorn Creek drainage, Nevada County, California 36

Figure 14. Plots showing relation between $\mathrm{pH}$ and $(A)$ total mercury in unfiltered water, $(B)$ total mercury in filtered water, and $(C)$ methylmercury in unfiltered water, Greenhorn Creek drainage, Nevada County, California

Figure 15. Plots showing relation between sulfate concentration in filtered water and

$(A)$ total mercury in unfiltered water, $(B)$ total mercury in filtered water, and

(C) methylmercury in unfiltered water, Greenhorn Creek drainage, Nevada County,

California 
Figure 16. Plot showing relation between total mercury concentration in unfiltered water and total suspended sediment concentration, Greenhorn Creek drainage,

Nevada County, California

Figure 17. Plot showing relation between particulate total mercury concentration and total suspended sediment concentration, Greenhorn Creek drainage, Nevada County, California...

Figure 18. Box plots showing distribution of total mercury and methylmercury concentration in sediment samples from the Greenhorn Creek drainage, Nevada County, California

Figure 19. Plot showing relation between total mercury and methylmercury concentration in sediment samples from the Greenhorn Creek drainage, Nevada County, California...

Figure 20. Box plots showing distribution of methylmercury concentrations in invertebrates and total mercury concentrations in frogs from the Greenhorn Creek drainage, Nevada County, California

Figure 21. Plots showing data for total mercury and methylmercury in predatory insects from the Greenhorn Creek drainage, Nevada County, California

Figure 22. Plot showing relation between methylmercury concentration and average mass of banana slugs from the Greenhorn Creek drainage, Nevada County, California..

Figure 23. Plot showing relation between methylmercury concentration and average mass of dragonflies from the Greenhorn Creek drainage, Nevada County, California .....

Figure 24. Plot showing relation between methylmercury concentration and average mass of dobsonflies from the Greenhorn Creek drainage, Nevada County, California .....

Figure 25. Plot showing relation between methylmercury concentration and average mass of predaceous diving beetles from the Greenhorn Creek drainage, Nevada County, California

Figure 26. Plot showing relation between methylmercury concentration and average mass of predaceous stoneflies from the Greenhorn Creek drainage,

Nevada County, California

Figure 27. Plot showing relation between methylmercury concentration and average mass of water striders from the Greenhorn Creek drainage, Nevada County, California .....

Figure 28. Plot showing relation between total mercury concentration and total or average mass of all frog species from the Greenhorn Creek drainage, Nevada County, California .....

Figure 29. Plot showing relation between total mercury concentration and mass of individual bullfrogs from the Greenhorn Creek drainage, Nevada County, California .....

Figure 30. Plot showing relation between total mercury concentration and mass of individual foothill yellow-legged frogs from the Greenhorn Creek drainage, Nevada County, California...

Figure 31. Plot showing relation between total mercury concentration and total or average mass of Pacific treefrogs from the Greenhorn Creek drainage, Nevada County, California...

Figure 32. Summary of data for mercury and methymercury in water, sediment, and biota, Greenhorn Creek drainage, Nevada County, California 
Figure A1. Map showing locations of mine tunnels, hydraulic pits, and lands managed by federal land management agencies, Greenhorn Creek drainage, Nevada County, California.

Figure A2. Photograph of main drainage gulch at Sailor Flat (station BY105) with visible mercury bead about one inch in diameter, Greenhorn Creek drainage, Nevada County, California ...

Figure A3. Photograph of Sailor Flat main drainage to Greenhorn Creek (station BY106), Nevada County, California.

Figure A4. Photograph of Tom and Jerry Mine tunnel outlet (station BY130), Greenhorn Creek drainage, Nevada County, California 218

Figure A5. Photograph of Buckeye main drain entering Greenhorn Creek (station BY58), Nevada County, California.

Figure A6. Photograph of mercury beads on bedrock at Boston Mine tunnel outlet (station BY21), Greenhorn Creek drainage, Nevada County, California

Figure A7. Historical photograph of Boston Mine (station BY21), Greenhorn Creek drainage, Nevada County, California

Figure A8. Photograph of discharge from Boston Mine tunnel outlet (station BY20), Greenhorn Creek drainage, Nevada County, California 220

Figure A9. Photograph of iron precipitate in ground sluice at Poore Mine (station BY88), Greenhorn Creek drainage, Nevada County, California .

Figure A10. Photograph of Poore Mine pit lake (station BY89), Greenhorn Creek drainage, Nevada County, California.

Figure A11. Photograph of Poore Mine tunnel outlet (station BY91), Greenhorn Creek drainage, Nevada County, California

Figure A12. Photograph of Starr Mine tunnel inlet area (station BY122), Greenhorn Creek drainage, Nevada County, California.

Figure A13. Photograph of midway station in Starr Mine tunnel (station BY123), Greenhorn Creek drainage, Nevada County, California 223

Figure A14. Photograph of Starr Mine tunnel, near outlet (station BY124), Greenhorn Creek drainage, Nevada County, California.

Figure A15. Photograph of Starr Mine pit lake (station BY148), Greenhorn Creek drainage, Nevada County, California. 224

Figure A16. Photograph of Missouri Canyon ground sluice (station BY146), Greenhorn Creek drainage, Nevada County, California

Figure A17. Photograph of Missouri Canyon ground sluice above creek (station BY75), Greenhorn Creek drainage, Nevada County, California .

Figure A18. Photograph of Greenhorn Creek at You Bet Road (station BY59), Nevada County,

California

Figure A19. Photograph of confluence of Little Greenhorn Creek with Greenhorn Creek upstream of You Bet Road (station BY59), Nevada County, California.

Figure A20. Photograph of streambank showing flood incision of gravels, Greenhorn Creek at the Narrows (station BY180), Nevada County, California

Figure A21. Photograph of Greenhorn Creek at the Narrows, looking downstream (station BY180), Nevada County, California

Figure A22. Photograph of Hunts Hill drainage tunnel outlet, near Buckeye Ford in Greenhorn Creek, Nevada County, California

Figure A23. Photograph of confluence of Gas Canyon with Greenhorn Creek (station BY52), 
Figure A24. Photograph of Greenhorn Creek at Buckeye Drain, looking upstream (station BY60), Nevada County, California

Figure A25. Photograph of Greenhorn Creek at Red Dog Road crossing, Nevada County, California

Figure A26. Photograph of pit lake at Buckeye Flat Mine, Greenhorn Creek drainage, Nevada County, California.

Figure B1. Correlation plot of observed values versus reported values for erbium, europium, holmium, lutetium, terbium, thulium, and ytterbium determined in standard reference materials

Figure B2. Correlation plot of observed values versus reported values for cerium, dysprosium, gadolinium, lanthanum, neodymium, praseodymium, samarium, tellurium, and yttrium determined in standard reference materials

Figure B3. Correlation plot of observed values versus reported values for bismuth, rubidium, selenium, and uranium determined in standard reference materials

Figure B4. Correlation plot of observed values versus reported values for arsenic, beryllium, cadmium, cobalt, lead, thallium, and vanadium determined in standard reference materials

Figure B5. Correlation plot of observed values versus reported values for aluminum, antimony, chromium, copper, lithium, nickel, and zinc determined in standard reference materials.

Figure B6. Correlation plot of observed values versus reported values for barium, boron, manganese, molybdenum, and strontium determined in standard reference materials

Figure B7. Correlation plot of observed values versus reported values for mercury determined in standard reference materials.

Figure B8. Bar graph plotting recovery (in percent) for arsenic (As), beryllium $(\mathrm{Be})$, copper (Cu), and zinc $(\mathrm{Zn})$ spiked in blanks for selected analysis dates .

Figure B9. Bar graph plotting recovery (in percent) for mercury $(\mathrm{Hg})$ spiked in blanks for selected analysis dates.

Figure B10. Graph plotting recovery (in percent) for beryllium (Be), cadmium (Cd), copper (Cu), and zinc ( $\mathrm{Zn})$ spiked in samples for selected analysis dates.

Figure B11. Bar graph plotting recovery (in percent) for mercury spiked in samples for selected analysis dates

Figure B12. Correlation plots of field replicate analyses of six elements: arsenic (As), barium $(\mathrm{Ba})$, beryllium $(\mathrm{Be})$, calcium $(\mathrm{Ca})$, cobalt $(\mathrm{Co})$, and copper $(\mathrm{Cu})$

Figure B13. Correlation plots of field replicate analyses of six elements: mercury $(\mathrm{Hg})$, holmium ( $\mathrm{Ho})$, manganese $(\mathrm{Mn})$, neodymium $(\mathrm{Nd})$, lead $(\mathrm{Pb})$, and uranium (U)

Figure B14. Plots of percent relative standard deviation versus concentration for six elements: cadmium $(\mathrm{Cd})$, copper $(\mathrm{Cu})$, gadolinium $(\mathrm{Gd})$, mercury $(\mathrm{Hg})$, magnesium $(\mathrm{Mg})$, and lead $(\mathrm{Pb})$

Figure B15. Plot of total mercury concentrations in unfiltered water showing relation of replicate 1 of 2 and replicate 2 of 2 .

Figure B16. Plot of total mercury concentrations in filtered water showing relation of replicate 1 of 2 and replicate 2 of 2

Figure B17. Plot showing relation of specific conductance with sum of microequivalent concentrations of chloride, sulfate, and bicarbonate alkalinity 


\section{Tables}

Table 1. Data for water-quality parameters measured in the field, Greenhorn Creek drainage, Nevada County, California

Table 2. Concentrations of total mercury in water samples, Greenhorn Creek drainage,

Nevada County, California

Table 3. Concentrations of methylmercury in water samples, Greenhorn Creek drainage, Nevada County, California.

Table 4A. Concentrations of trace metals and selected major elements in unfiltered water samples, Greenhorn Creek drainage, Nevada County, California.

Table 4B. Concentrations of trace metals and selected major elements in filtered water samples, Greenhorn creek drainage, Nevada County, California

Table 5. Concentrations of major anions and alkalinity in filtered water samples, Greenhorn Creek drainage, Nevada County, California .

Table 6. Concentrations of nutrients and organic carbon in water samples, Greenhorn Creek drainage, Nevada County, California

Table 7. Data for suspended sediment in water samples, Greenhorn Creek drainage, Nevada County, California.

Table 8. Data for stable isotopes of sulfur and oxygen in aqueous sulfate and of oxygen and hydrogen in water, Greenhorn Creek drainage, Nevada County, California 26

Table 9. Concentrations of mercury and methylmercury in sediment samples, determined by laboratory methods, Greenhorn Creek drainage, Nevada County, California

Table 10. Concentrations of visible total mercury in sediment samples, determined by field panning methods, Greenhorn Creek drainage, Nevada County, California

Table 11A. Mercury and methylmercury concentration data for invertebrates, Greenhorn Creek drainage, Nevada County, California

Table 11B. Mercury and methylmercury concentration data for invertebrates at baseline site, Nevada County, California

Table 12. Mercury concentration data for frogs, Greenhorn Creek drainage, Nevada County, California

Table C1. Locations, descriptions, and characteristics of sampling stations, Greenhorn Creek drainage, Nevada County, California ...

Table C2. Characteristics of tunnels at hydraulic mines in the Greenhorn Creek drainage, Nevada County, California.

Table C3. Descriptions of photographs of sampling stations, Greenhorn Creek drainage, Nevada County, California.

Table D1. Trace elements analyzed by inductively coupled plasma-mass spectrometry, and corresponding median detection limits.

Table D2. Selected trace elements and regression correlation coefficients $\left(R^{2}\right)$ for correlation plots of observed versus reported values of analyses of standard reference materials

Table D3. Data for total mercury in blanks

Table D4. Data for methylmercury in blanks... 259

Table D5. Data for trace elements in process blanks 260

Table D6. Data for trace elements in source blanks and equipment blanks. 267 


\section{Conversion Factors, Datums, Acronyms, Abbreviations, and Chemical Notation}

\begin{tabular}{lll}
\hline Multiply & \multicolumn{1}{c}{ By } & To obtain \\
\hline cubic foot per second $\left(\mathrm{ft}^{3} / \mathrm{s}\right)$ & 0.02832 & cubic meter per second $\left(\mathrm{m}^{3} / \mathrm{s}\right)$ \\
cubic yard $\left(\mathrm{yd}^{3}\right)$ & 0.7646 & cubic meter $\left(\mathrm{m}^{3}\right)$ \\
foot $(\mathrm{ft})$ & 0.3048 & meter $(\mathrm{m})$ \\
gallon per minute $(\mathrm{gal} / \mathrm{min})$ & 0.002228 & cubic foot per second $\left.\left(\mathrm{ft}^{3} / \mathrm{s}\right)\right)$ \\
gallon per minute $(\mathrm{gal} / \mathrm{min})$ & 0.06309 & liter per second $(\mathrm{L} / \mathrm{s})$ \\
inch $($ in. $)$ & 2.54 & centimeter $(\mathrm{cm})$ \\
mile $(\mathrm{mi})$ & 1.609 & kilometer $(\mathrm{km})$ \\
pound $(\mathrm{lb})$ & 0.4536 & kilogram $(\mathrm{kg})$ \\
\hline
\end{tabular}

Temperature in degrees Celsius $\left({ }^{\circ} \mathrm{C}\right)$ may be converted to degrees Fahrenheit $\left({ }^{\circ} \mathrm{F}\right)$ as follows:

$$
{ }^{\circ} \mathrm{F}=\left(1.8 \times{ }^{\circ} \mathrm{C}\right)+32
$$

\section{Spatial Datums}

Horizontal coordinate information is referenced to the North American Datum of 1927 (NAD 27). Elevation, as used in this report, refers to distance above the National Geodetic Vertical Datum of 1929 (NGVD of 1929).

Specific conductance is given in microsiemens per centimeter at 25 degrees Celsius $(\mu \mathrm{S} / \mathrm{cm}$ at $\left.25^{\circ} \mathrm{C}\right)$.

Concentrations of chemical constituents in water are given in either milligrams per liter (mg/L), micrograms per liter $(\mu \mathrm{g} / \mathrm{L})$, or nanograms per liter $(\mathrm{ng} / \mathrm{L})$.

\section{Acronyms, Abbreviations, and Chemical Notation}

\section{Acronyms}

ASTM, American Society for Testing and Materials

CRV, certified reference value

CVAAS, cold-vapor atomic-absorption spectrometry

CVAFS, cold-vapor atomic-fluorescence spectrometry

DI, deionized (water)

D0, dissolved oxygen

DOC, dissolved organic carbon

EWI, equal width increment

FDA, Food and Drug Administration

GC, gas chromatography

$\mathrm{GI}$, gastrointestinal

IC, ion chromatography

ICP-AES, inductively coupled plasma-atomic emission spectrometry

ICP-MS, inductively coupled plasma-mass spectrometry

MDL, method detection limit 
NCRCD, Nevada County Resource Conservation District

NRCC, National Research Council Canada

NRP, National Research Program

NIST, National Institute of Standards and Technology

PACF, Patuxent Analytical Control Facility

POC, Particulate organic carbon

$\mathrm{QA}$, quality assurance

OC, quality control

$R P D$, relative percentage difference

$R S D$, relative standard deviation

SC, specific conductance

SRM, standard reference material

SRWS, standard reference water sample

SSC, suspended sediment concentration

SSWD, South Sutter Water District

SWRCB, State Water Resources Control Board

TERL, Trace Element Research Laboratory

USDA-FS, U.S. Department of Agriculture-Forest Service

USEPA, United States Environmental Protection Agency

USGS, United States Geological Survey

WDML, Wisconsin District Mercury Laboratory

\author{
Abbreviations \\ $\mathrm{cm}$, centimeter \\ g, gram \\ kg, kilogram \\ $\mathrm{L}$, liter \\ $\mu \mathrm{g}$, microgram \\ $\mathrm{mg}$, milligram \\ $\mathrm{mL}$, milliliter \\ $\mu \mathrm{m}$, micrometer \\ $\mathrm{M} \Omega$-cm, megaohm-centimeter \\ ng, nanogram \\ $\mathrm{ppb}$, part per billion \\ ppm, part per million \\ wt, weight \\ s, second \\ $>$, greater than \\ $<$, less than
}

Chemical Notation

$\mathrm{Al}$, aluminum 
As, arsenic

$B$, boron

$\mathrm{Ba}$, barium

$\mathrm{Be}$, beryllium

Bi, bismuth

B, boron

Ca, calcium

Cd, cadmium

Ce, cerium

$\mathrm{CH}_{2} \mathrm{Cl}_{2}$, methylene chloride

Cl, chloride

Co, cobalt

$\mathrm{Cr}$, chromium

Cs, cesium

Cu, copper

$\mathrm{CuSO}_{4^{\prime}}$, copper sulfate

Dy, dysprosium

Er, erbium

Eu, europium

$\mathrm{Fe}$, iron

$\mathrm{Gd}$, gadolinium

$\mathrm{HCl}$, hydrochloric acid

HF, hydrofluoric acid

$\mathrm{Hg}$, mercury

$\mathrm{Hg}^{0}$, elemental mercury

$\mathrm{Hg}^{2+}$, mercuric ion

$\mathrm{HgCl}_{2}$, mercuric chloride

$\mathrm{HNO}_{3}$, nitric acid

Ho, holmium

In, indium

Ir, iridium

$\mathrm{K}$, potassium

$\mathrm{KBr}$, potassium bromide

La, lanthanum

Li, lithium

Lu, lutetium

$\mathrm{MeHg}$, methylmercury (monomethylmercury)

$\mathrm{Mg}$, magnesium

$\mathrm{Mn}$, manganese

$\mathrm{N}$, nitrogen

$\mathrm{Na}$, sodium

$\mathrm{NaBEt}_{4}$, sodium tetra-ethyl borate 


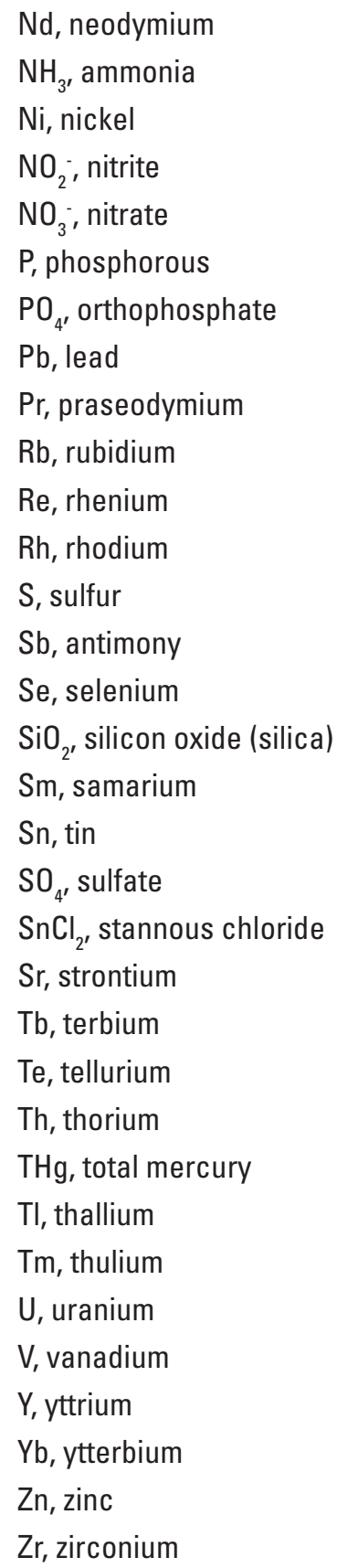




\title{
Geochemical characterization of water, sediment, and biota affected by mercury contamination and acidic drainage from historical gold mining, Greenhorn Creek, Nevada County, California, 1999-2001
}

\author{
By Charles N. Alpers, Michael P. Hunerlach, Jason T. May, Roger L. Hothem, Howard E. Taylor, \\ Ronald C. Antweiler, John F. De Wild, and David A. Lawler
}

\begin{abstract}
In 1999, the U.S. Geological Survey (USGS) initiated studies of mercury and methylmercury occurrence, transformation, and transport in the Bear River and Yuba River watersheds of the northwestern Sierra Nevada. Because these watersheds were affected by large-scale, historical gold extraction using mercury amalgamation beginning in the 1850 s, they were selected for a pilot study of mercury transport by the USGS and other cooperating agencies. This report presents data on methylmercury (MeHg) and total mercury (THg) concentrations in water, bed sediment, invertebrates, and frogs collected at 40 stations during 1999-2001 in the Greenhorn Creek drainage, a major tributary to the Bear River. Results document several mercury contamination "hot spots" that represent potential targets for ongoing and future remediation efforts at abandoned mine sites in the study area.

Water-quality samples were collected one or more times at each of 29 stations. The concentrations of total mercury in 45 unfiltered water samples ranged from 0.80 to 153,000 nanograms per liter (ng/L); the median was $9.6 \mathrm{ng} / \mathrm{L}$. Total mercury concentrations in filtered water (41 samples) ranged from less than 0.3 to $8,000 \mathrm{ng} / \mathrm{L}$; the median was $2.7 \mathrm{ng} / \mathrm{L}$. Concentrations of methylmercury in the unfiltered water (40 samples) ranged from less than 0.04 to $9.1 \mathrm{ng} / \mathrm{L}$; the median was $0.07 \mathrm{ng} / \mathrm{L}$. Methylmercury in filtered water (13 samples) ranged from less than 0.04 to $0.27 \mathrm{ng} / \mathrm{L}$; the median was $0.04 \mathrm{ng} / \mathrm{L}$. Acidic drainage with $\mathrm{pH}$ values as low as 3.4 was encountered in some of the mined areas. Elevated concentrations of aluminum, cadmium, copper, iron, manganese, nickel, and zinc were found at several stations, especially in the more acidic water samples.
\end{abstract}

Total mercury concentrations in sediment were determined by laboratory and field methods. Total mercury concentrations (determined by laboratory methods) in ten samples from eight stations ranged from about 0.0044 to $12 \mu \mathrm{g} / \mathrm{g}$ (microgram per gram, equivalent to part per million). Methylmercury concentrations in these samples ranged from less than 0.00011 to $0.0095 \mu \mathrm{g} / \mathrm{g}$. A field panning method was used to determine the concentration of liquid elemental mercury in 22 samples from 14 stations. Measured quantities of elemental mercury recovered by panning ranged from a trace amount estimated at 100 milligrams per kilogram (equivalent to parts per million) to 45,000 milligrams per kilogram (equivalent to 4.5 per cent, by weight).

In total, 194 invertebrate samples were collected at 31 stations; 78 of the samples were analyzed for concentrations of $\mathrm{THg}$ and $\mathrm{MeHg}$ and used to calculate $\mathrm{MeHg}$ to $\mathrm{THg}$ ratios. In total, 69 frog samples were collected at 19 stations, and all were analyzed only for THg. Ranges of MeHg concentrations $(\mu \mathrm{g} / \mathrm{g}$, wet weight $)$ in invertebrate samples and number of samples (n) were $0.0012-0.048$ for banana slugs (Arionidae, $\mathrm{n}=27$ ), $0.027-0.39$ for dobsonflies (Corydalidae, $\mathrm{n}=$ 14), $0.029-0.50$ for predaceous diving beetles (Dytiscidae, $\mathrm{n}$ $=31$ ), $0.026-0.52$ for predaceous stoneflies (Perlidae, $\mathrm{n}=18$ ), $0.011-1.6$ for dragonflies (Odonata, $\mathrm{n}=46$ ), and $0.061-0.55$ for water striders (Gerridae, $n=56$ ). The ratio of $\mathrm{MeHg}$ to $\mathrm{THg}$ in invertebrates was greater than 50 percent for 74 of 78 samples.

The data from this reconnaissance sampling effort have been used by land-management agencies in selecting abandoned mine sites for remediation. The Forest Service has remediated the Sailor Flat site, and the Bureau of Land Management has initiated plans to remediate the Boston Mine drainage tunnel. 


\section{Introduction}

The Bear River watershed in the northwestern Sierra Nevada (fig. 1) was the site of considerable mining of placer gold deposits using hydraulic methods during the latter half of the 19th century and the first part of the 20th century (Hunerlach and others, 1999; Alpers and Hunerlach, 2000; Hunerlach and Alpers, 2003; Alpers and others, 2005). Gilbert (1917) estimated that between the 1850s and early 1900s hydraulic mining displaced 254 million cubic yards of gravel and sediment in the Bear River watershed. We estimate that 71 million cubic yards of this total were displaced in the Greenhorn Creek drainage, as explained in a later section of this report. The reader is referred to the reports cited above (and references therein) for a description of the history of hydraulic mining in the Sierra Nevada, including documentation of the extensive use of liquid mercury $(\mathrm{Hg})$ to recover gold from placer ores by sluicing methods and amalgamation.

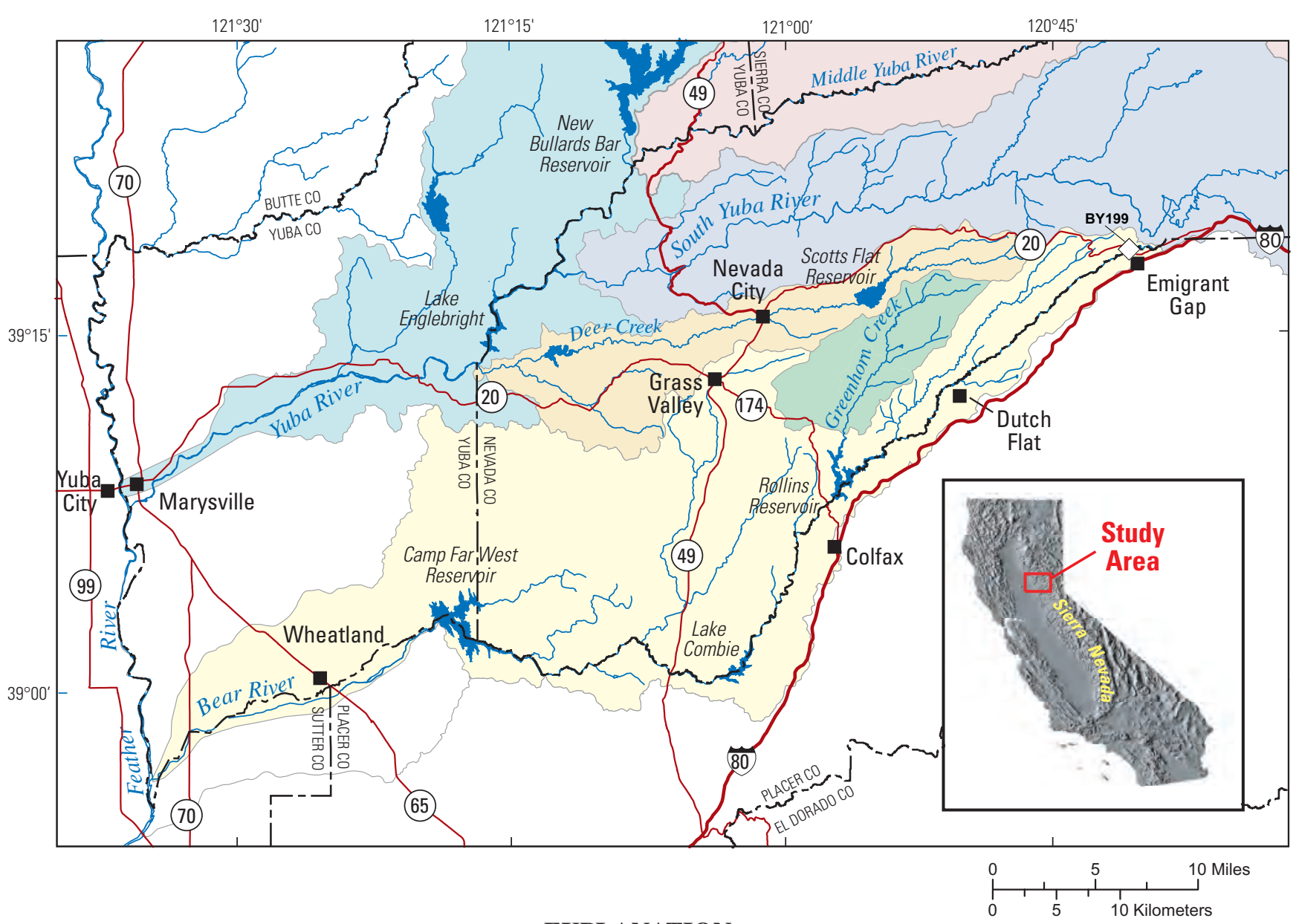

EXPLANATION

Watersheds

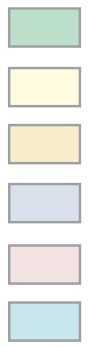

Greenhorn Creek drainage

Bear River

Deer Creek

South Yuba River

Middle Yuba River

Yuba River

Figure 1. Location of Greenhorn Creek drainage in the Bear River watershed, Nevada County, California. 


\section{Description of Study Area}

Greenhorn Creek is a tributary to Rollins Reservoir, one of several man-made impoundments on the Bear River (fig. 1). The Greenhorn Creek drainage is located within Nevada County, in the northwestern Sierra Nevada. The Bear River flows into the lower Feather River, a major tributary to the Sacramento River, which flows into the Sacramento-San Joaquin Delta and San Francisco Bay. The elevation of the Rollins Reservoir spillway is 2,171 feet above sea level. The highest sampling point in the Greenhorn Creek watershed is the Headwaters station (figs. 2, 3) at 4,262 feet above sea level. The area has a Mediterranean climate; precipitation falls primarily between November and April. Annual precipitation in nearby Grass Valley, California (fig. 1), is 53.3 inches per year, including average annual snowfall of about 10 inches per year, based on records from October 1966 through March 2003 (Western Regional Climate Center, 2004).

\section{Mining History}

According to one version of California Gold Rush history, hydraulic mining began during 1852, when Antoine Chabot used a canvas hose to wash loose gravel from a large bench deposit at Buckeye Hill, located on Buckeye Ridge, between the South Fork of Greenhorn Creek and Greenhorn Creek (fig. 4) (May, 1970). In 1853, Edward Matteson applied a steel nozzle to a canvas hose to improve the velocity and force of water delivery at American Hill near Grass Valley, and his success led to the rapid spread of hydraulic mining throughout the vast Tertiary-age river-channel deposits exposed in the drainages of Greenhorn Creek. Large-scale hydraulic mining occurred in the Sierra Nevada from 1855 through the early 1880s (Clark, 1963). Most hydraulic mining stopped in 1884 because of the case of Woodruff versus North Bloomfield, which led to what has become known as the Sawyer Decision (Kelley, 1959). Woodruff was a farmer whose lands were affected by sediments discharged from upstream hydraulic mines, and North Bloomfield was one of the largest mining companies in the Sierra Nevada, active in the Malakoff Diggings area of the South Yuba River watershed (fig. 1).

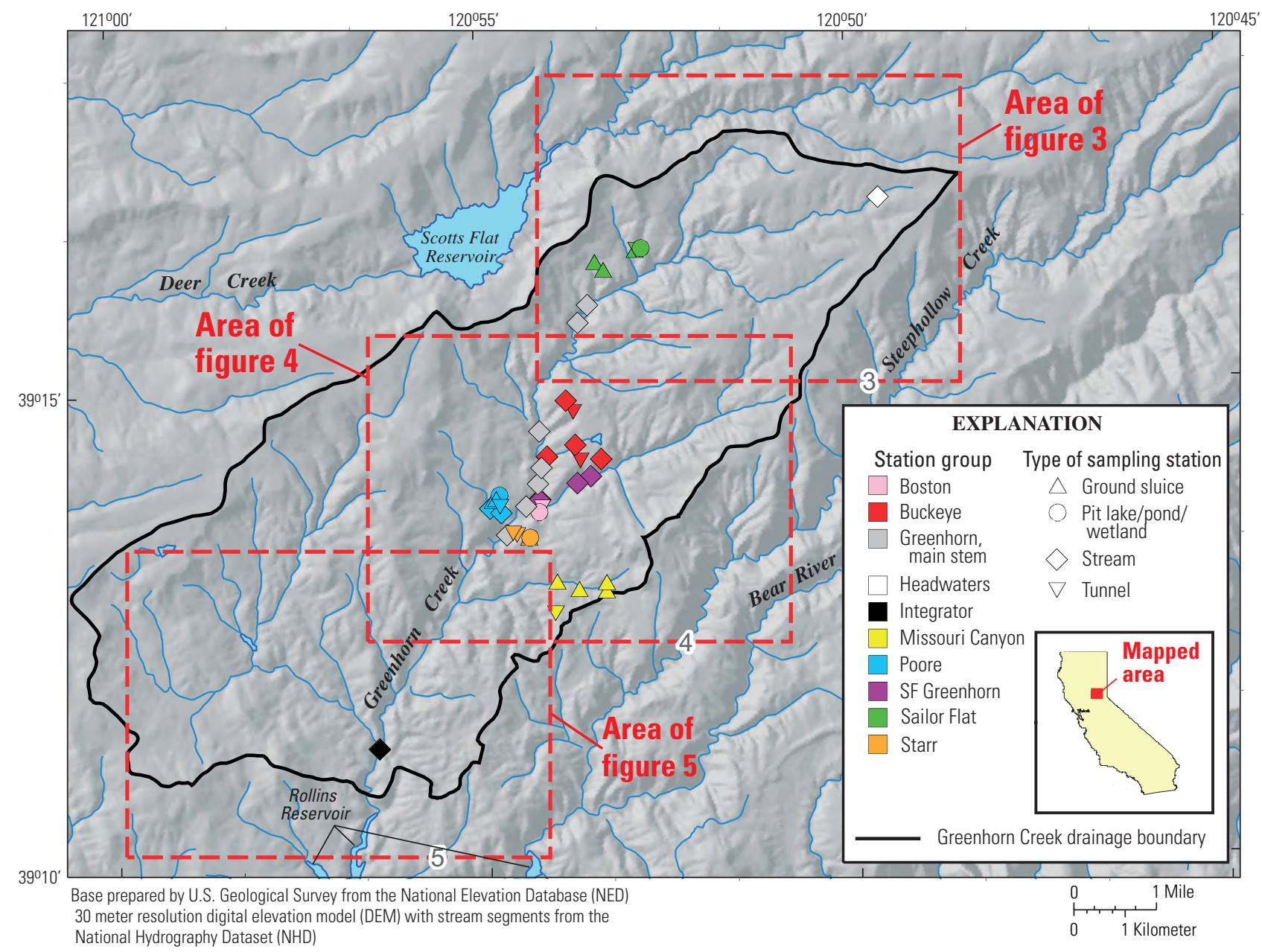

Figure 2. Sampling stations in the Greenhorn Creek drainage, Nevada County, California. Red boxes outline areas of figures $3-5$. 
The rich, gold-bearing gravels in the Greenhorn Creek drainage were part of the ancestral Yuba River system, which flowed from south to north in this area (Lindgren, 1911). The gold-bearing river-channel deposits ranged from one to four miles wide and were up to 600 feet thick near Hunts Hill (formerly known as Gouge Eye) and at Buckeye Hill. Greenhorn Creek and its tributaries drain three principal mining areas-Quaker Hill, Red Dog, and You Bet (fig. Al)—which contributed the bulk of the 71 million cubic yards of production from numerous hydraulic mines. At Quaker Hill, more than 35 million cubic yards of gravel with an average yield of $\$ 0.06$ per cubic yard were processed. (The value of gold during the late 1800s was about $\$ 20$ per ounce; a tenor of $\$ 0.06$ per cubic yard corresponds to 0.003 ounces per cubic yard.) More than 6.7 million cubic yards were processed at Hunts Hill and more than 6 million cubic yards at Buckeye Hill. The You Bet and Red Dog mining areas are in both the
Greenhorn Creek and Steephollow Creek drainages; combined production in these areas was more than 47 million cubic yards, of which about half, some 23.5 million cubic yards with a gold tenor of more than $\$ 0.25$ per yard, probably was discharged into the Greenhorn Creek drainage (Jarmin, 1927), and the remainder was discharged into Steephollow Creek (fig. 2).

The unconsolidated upper gravels in the deposits of the ancestral Yuba River were easily mined by the hydraulic method, whereas the deeper, more cemented gravels were commonly drift mined. Drift mining was done underground through tunnels that commonly followed the deepest portion of the channel parallel to its original course. The wellcemented deeper gravels required stamp mills to break up the ore and liberate of the gold (Hobson and Wiltsee, 1893). At least fifteen stamp mills were operated in the Red Dog and Quaker Hill mining districts during peak production.

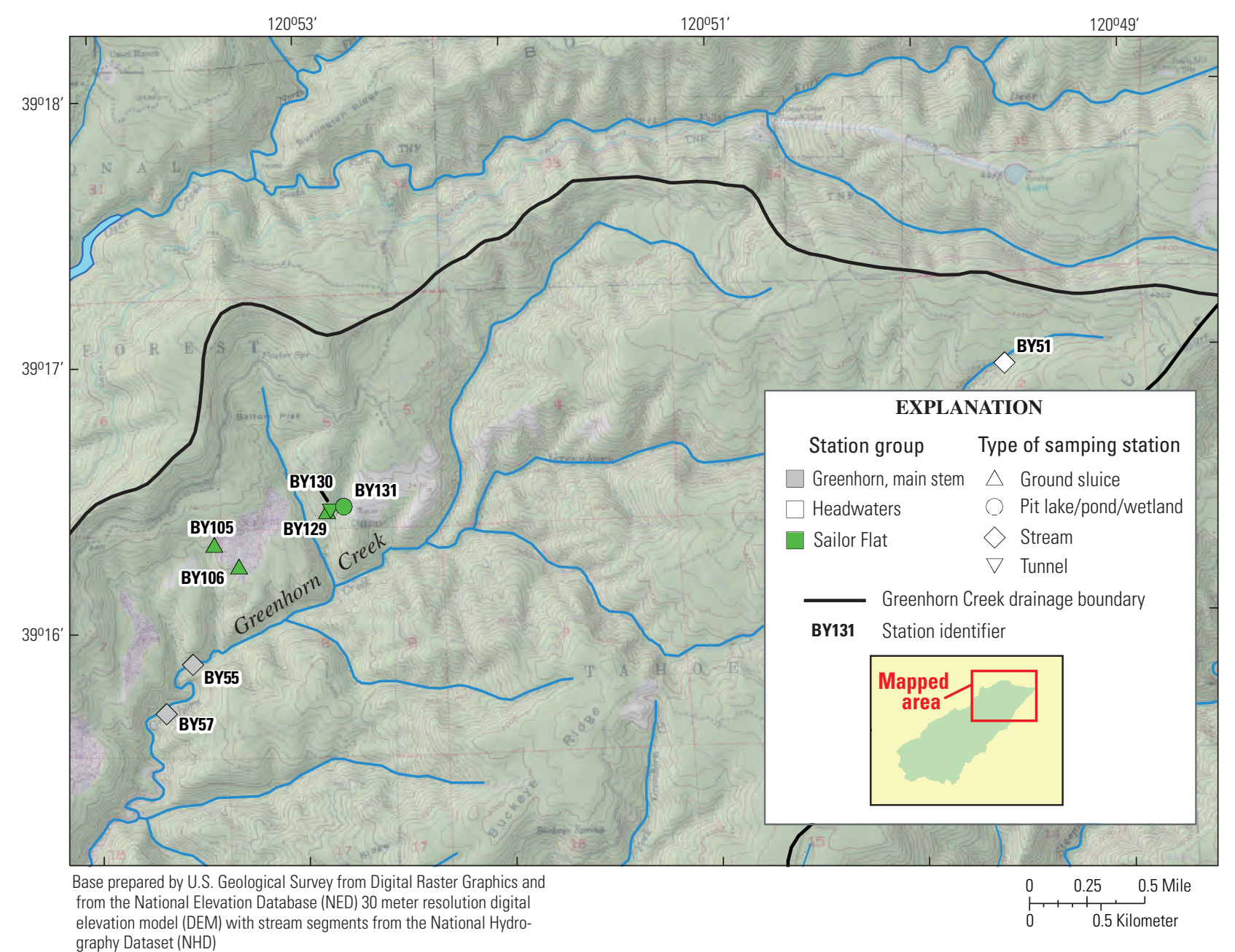

Figure 3. Sampling stations in the northern part of Greenhorn Creek drainage, Nevada County, California. 


\section{Previous Work on Mercury Contamination}

A reconnaissance sampling of mercury in invertebrates and small fish during the mid 1990s indicated that mercury bioaccumulation in the Bear River and South Yuba River watersheds was more severe than in adjacent watersheds in the northern Sierra Nevada, including the North Yuba, Middle Yuba, Feather, and American River watersheds (Slottton and others, 1997). Based in part on the data of Slotton and others (1997), on preliminary studies in the Dutch Flat mining district (Bear River watershed; Hunerlach and others, 1999), and on an analysis of hydraulic mining intensity (Alpers and Hunerlach, 2000), the USGS began a cooperative study with other public agencies in 1999 to address mercury contamination associated with historical gold mining in the Bear River, Deer Creek, and South Yuba River watersheds (hereinafter referred to as the Bear-Yuba study area).
A key component of the multi-agency cooperative study was to assess mercury bioaccumulation in sport fish, the consumption of which is thought to be the primary pathway for mercury to affect human health and ecosystems. The fish-tissue data published by May and others (2000) were the basis for a state-level fish consumption advisory (Klasing and Brodberg, 2003), the first of its kind for the Sierra Nevada. In addition, the fish-tissue data reported by May and others (2000) were used to identify three reservoirs in the Bear River watershed and the segment of the Bear River near Dog Bar Road as impaired water bodies by the State of California under section 303(d) of the Clean Water Act (California Regional Water Quality Control Board, Central Valley Region, 2003). Stakeholders, including federal, state, and local agencies, have expressed interest in attempting to reduce mercury loads to downstream reservoirs by remediating mine sites that may be significant sources of mercury and methylmercury in the upper Bear River watershed.

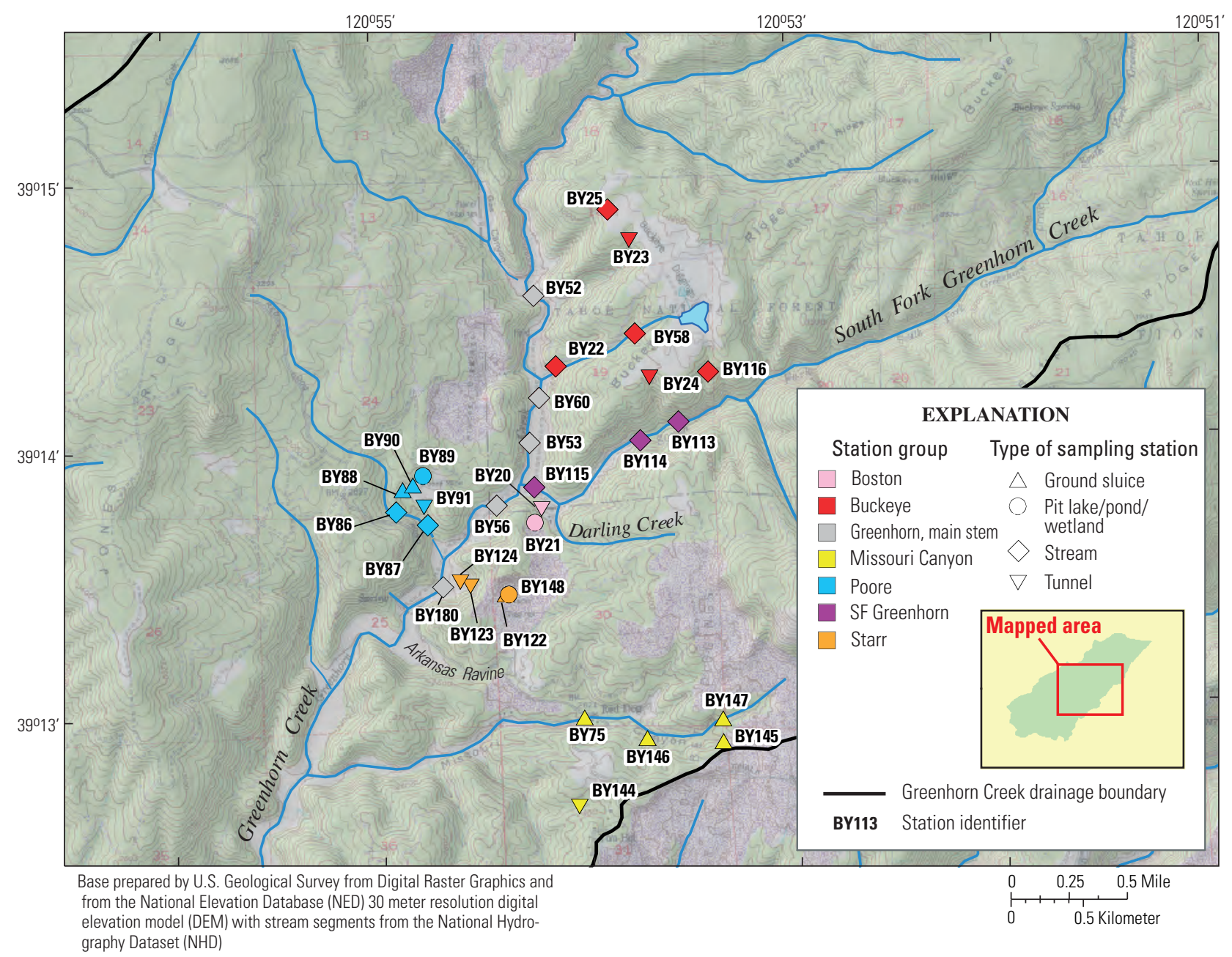

Figure 4. Sampling stations in the central part of Greenhorn Creek drainage, Nevada County, California. 


\section{Purpose and Scope}

A key overall objective of the multi-agency cooperative study is to identify "hot spots" of mercury contamination and bioaccumulation, so that land-management agencies and other stakeholders can design appropriate remediation strategies to protect public health and ecological systems. Preliminary data from about 125 stations sampled for this study in the Bear River, Deer Creek, and South Yuba River watersheds were provided to federal land-management agencies, primarily the Bureau of Land Management (BLM) and the U.S. Department of Agriculture-Forest Service (USDA-FS), so that potential remediation sites could be identified and priorities established for mine-site remediation. Two of the highest priority areas for remediation in the Bear-Yuba watersheds are located within the Greenhorn Creek drainage of the Bear River watershed (fig. 1): the Sailor Flat mine site (USDA-FS lands, Tahoe National Forest; stations shown on figs. 2, 3, Al) and the Boston mine site (primarily on BLM lands; stations shown on figs. 2, 4, Al).

This report documents the results of sampling water, sediment, invertebrates, and frogs for concentrations of methylmercury (MeHg), total mercury ( $\mathrm{THg}$ ), and other constituents at 40 stations in the Greenhorn Creek drainage during 1999-2001. Similar data gathered from other parts of the Bear River watershed and from the Deer Creek and South Yuba River watersheds will be published separately.

The main body of this report consists of this Introduction, followed by a section regarding the study design, which includes subsections on sample locations, constituents analyzed, field and laboratory methods, and quality assurance and quality control; the next section documents the study results, followed by the summary and conclusions. Detailed information on sampling stations in the study area, including photographs and an inventory of mine tunnels, is given in Appendix A (figures) and Appendix $C$ (tables). Details regarding quality assurance and quality control are given in Appendix B (figures) and Appendix D (tables).

\section{Acknowledgments}

The authors are grateful to the Bureau of Land Management (BLM), the California State Water Resources Control Board (SWRCB), the Nevada County Resource Conservation District (NCRCD), and the U.S. Department of Agriculture-Forest Service (USDA-FS) for providing funds and logistical support for the work described in this report. At the BLM, Timothy Carroll (Folsom Field Office) assisted with field work and the development of annual work plans; Richard
Forester and Richard Grabowski (State Office) assisted with administration. Rick Weaver (USDA-FS, Tahoe National Forest) assisted with field work and the development of work plans; Janine Clayton (USDA-FS, Regional Office) assisted with administration and coordination. Rick Humphreys of the SWRCB assisted with field work and contract management. Curtis Davidson, Tamara Gallentine, and Gail Bakker of the NCRCD provided administrative support in conjunction with a Proposition 204 grant managed by Rick Humphreys at the SWRCB. Gail Bakker, Rick Humphreys, and several USGS reviewers provided comments on an earlier draft of this report. Other agencies, including the California Department of Parks and Recreation and the Nevada Irrigation District, have contributed to the study by providing access to stations and available data. In addition, several private landowners allowed sampling on their lands, which is much appreciated. All biological samples were collected under the auspices of scientific collecting permits issued by the California Department of Fish and Game. USGS personnel and employees of the California State University Sacramento Foundation who contributed significantly to this project include Michael Atamian, Stacy Burnett, Michael Casselberry, Michelle Chao, Connie Clapton, Joseph Domagalski, Stephanie Dudash, Mary Elizabeth, Susan Fregien, Steven Gallanthine, Jerry Harmon, Mark Jennings, Mark Johnson, David Kelly, Sandor Kelly, Rebecca Kershnar, Cynthia Kester, Matthew Law, Erick Oshel, Kelly McPherson, Frank Moseanko, Darnella Murphy, Raef Porter, Kelly Rider, and Larry Shelton.

\section{Study Design}

In this report, we document results from sampling water, sediment, invertebrates, and frogs at 40 stations in the Greenhorn Creek drainage during 1999-2001. During the 1999 field season, reconnaissance sampling was done at several mine sites and adjacent areas affected by historical mining activity. On the basis of preliminary data from the initial sampling, follow-up sampling took place during 2000 and 2001 at a number of the locations to confirm anomalously high concentrations of mercury and methylmercury in water, sediment, and biota and to document inter-annual variability; also, some mine sites in the study area were visited for the first time during this period. Results of follow-up sampling that took place during 2002 in the Greenhorn Creek drainage will be published separately, as will results from samples collected during 1999-2002 in the Deer Creek watershed, the South Yuba River watershed, and other parts of the Bear River watershed. 


\section{Sample Locations}

The locations of sampling stations in the Greenhorn Creek drainage are shown in figure 2. A description of each sampling station is given in Appendix C (table C1). More detailed maps of the Greenhorn Creek drainage (figs. 3-5) include map ID numbers (for example BY20), which can be used to track information in the data tables. Stations in Greenhorn Creek itself were divided into three groups: a headwaters station (a reference site), main-stem stations in the central part of the drainage, and an integrator station near the confluence with Rollins Reservoir (fig. 2). The remaining stations were grouped by sub-watershed or mining district. Symbol shapes in figures 2-5 indicate the type of water body or mining feature sampled, including ground sluices; pit lakes, ponds, or wetlands; streams; and tunnels. The same definitions of symbol color and shape are used in many of the plots described in the Results section. Also presented in this report are data for invertebrate samples from a baseline reference sampling station outside of the Greenhorn Creek drainage, in the headwaters of the Bear River watershed, upstream of known mining effects. The location of the baseline reference station (BY199) is shown on figure 1.

Samples were collected from 40 locations in the Greenhorn Creek drainage for this study during 1999-2001. Fortynine water-quality samples were collected at 27 of the sampling stations. Ten sediment samples for laboratory analysis were collected at eight locations. At 5 of these 8 locations, plus 9 others (a total of 14), elemental mercury concentration in sediment was assessed using a field panning method. A total of 194 invertebrate samples were collected at 31 sampling stations in the study area, and 69 frog samples were collected at 19 of the sampling stations. An additional 19 invertebrate samples were collected at the baseline reference station. Banana slugs and six taxa of predaceous aquatic insects were collected as available at each invertebrate sampling station. Three taxa of frogs were collected as available, up to three individuals per taxon per station per year.

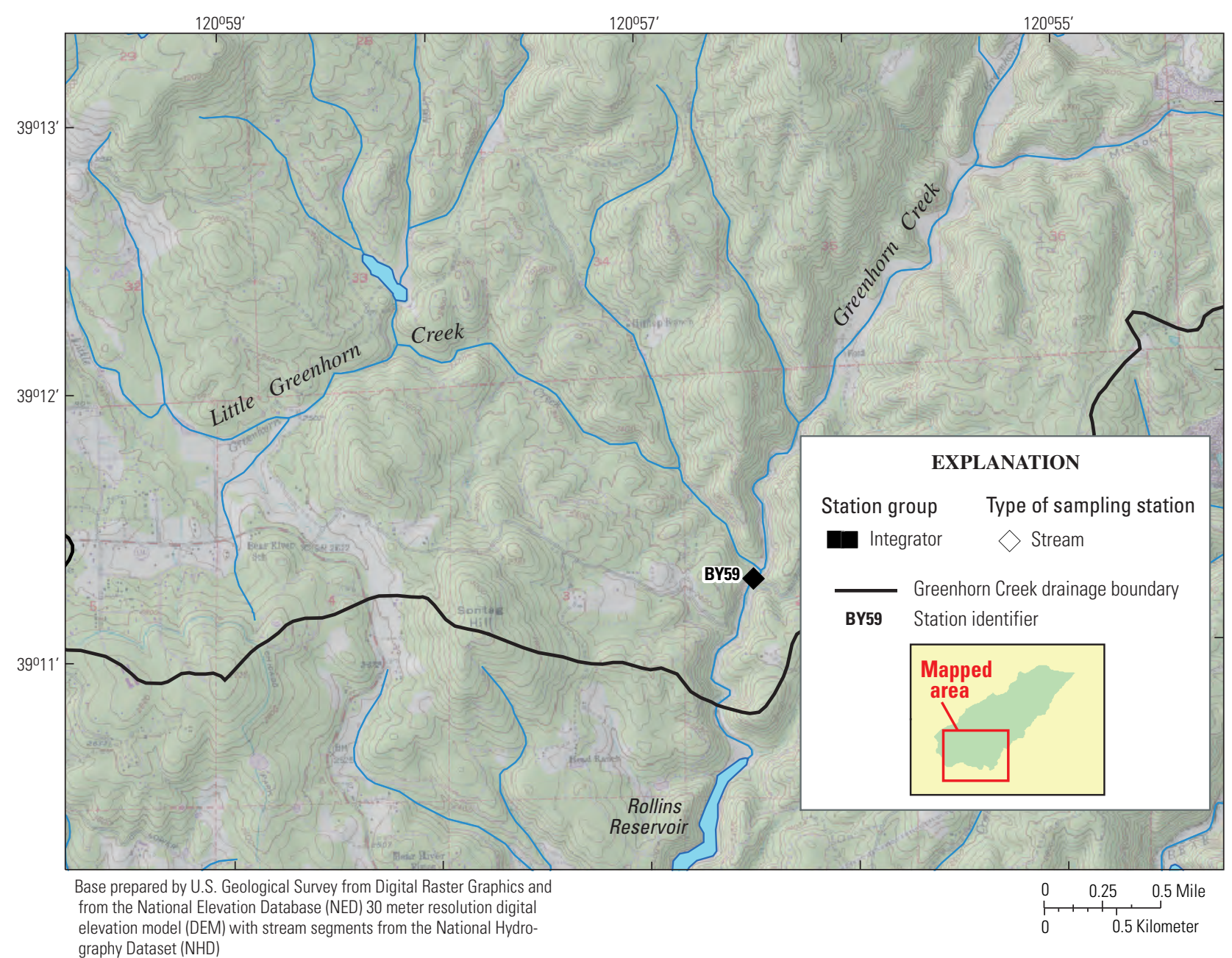

Figure 5. "Integrator" sampling station in the southern part of Greenhorn Creek drainage, Nevada County, California. 


\section{Constituents Analyzed}

Constituents analyzed in water samples included mercury in four categories: (1) total mercury $(\mathrm{THg})$ in unfiltered water, (2) $\mathrm{THg}$ in filtered water, (3) monomethylmercury (MeHg) in unfiltered water, and (4) $\mathrm{MeHg}$ in filtered water. (For the remainder of this report, the term methylmercury [also abbreviated as $\mathrm{MeHg}$ ] is used instead of "monomethylmercury;" note that other methylated forms of mercury such as dimethylmercury may be present but are not included in reported methylmercury analyses.) Total mercury in unfiltered and filtered water and $\mathrm{MeHg}$ in unfiltered water were analyzed in nearly all of the 49 water-quality samples; however, $\mathrm{MeHg}$ in filtered water was analyzed in only 12 samples. All analyses of $\mathrm{THg}$ in water for this study were done by the USGS National Research Program (NRP) laboratory in Boulder, Colorado; analyses of $\mathrm{MeHg}$ in water were done by the USGS Wisconsin District Mercury Laboratory (WDML) in Middleton, Wisconsin.

Water-quality was characterized in the field for unstable constituents and in the laboratory for stable constituents in preserved water samples. Unstable water-quality parameters measured in the field included $\mathrm{pH}$, temperature, specific conductance, and dissolved oxygen. Alkalinity (a proxy for dissolved bicarbonate) was analyzed by titration in the laboratory on a filtered subsample (stored chilled until analysis), usually within 48 hours of collection. Major cations (calcium, iron, magnesium, potassium, silica, and sodium) and more than 40 trace elements were analyzed in filtered and unfiltered samples by the USGS NRP laboratory. Major anions (sulfate and chloride in filtered samples only) also were analyzed by the USGS NRP laboratory. Nutrients in filtered and unfiltered samples were analyzed by the USGS National Water Quality Laboratory (NWQL) in Denver, Colorado. Forms of nitrogen $(\mathrm{N})$ that were analyzed included ammonia plus organic $\mathrm{N}$ in both unfiltered and filtered water, and the following three forms of $\mathrm{N}$ in filtered water only: ammonia, nitrite, and nitrite plus nitrate. Forms of phosphorous $(\mathrm{P})$ that were analyzed included total $\mathrm{P}$ in both unfiltered and filtered water and orthophosphate in filtered water only. Organic carbon in filtered and particulate form was analyzed by the USGS NWQL. Stable isotopes of hydrogen and oxygen were analyzed in unfiltered water by the Geology Department laboratory at the University of California, Davis. Stable isotopes of sulfur and oxygen in dissolved sulfate were analyzed by a USGS laboratory in Denver, Colorado. The USGS laboratory in Marina, California, determined concentration of total suspended sediment in water samples along with the percentage of sand in the suspended sediment, which was used to compute the concentration of suspended silt and clay $(<0.063 \mathrm{~mm}$ diameter $)$.
Sediment samples were analyzed for $\mathrm{MeHg}$ concentration by the USGS WDML. Analyses of THg in sediment were done by the WDML or the USGS NRP laboratory. In nearly all cases, the $\mathrm{MeHg}$ and $\mathrm{THg}$ concentrations are reported on a dry basis. However, the $\mathrm{THg}$ concentration in one sediment sample is reported on a wet basis because the moisture content was not determined. The sediment samples analyzed in the field for visible, elemental mercury by panning methods were analyzed on a wet basis.

The analyzed invertebrates include adult water striders and giant water bugs (order Hemiptera; families Gerridae and Belostomatidae), adult predaceous diving beetles (order Coleoptera; family Dytiscidae), larval stoneflies (order Plecoptera; family Perlidae), larval dobsonflies (order Megaloptera; family Corydalidae), larval dragonflies (order Odonata; families Aeshnidae, Cordulegastridae, Gomphidae, and Libellulidae), and adult banana slugs (order Gastropoda; family Arionidae). Insects were identified using taxonomic keys of Merritt and Cummins (1996) and McCaffrey (1981). All invertebrates were analyzed for $\mathrm{MeHg}$; some of the invertebrate samples were also analyzed for $\mathrm{THg}$, so that the ratio of $\mathrm{MeHg}$ to $\mathrm{THg}$ could be assessed. Previous studies have shown that values of $\mathrm{MeHg} / \mathrm{THg}$ in invertebrates can vary from 20 to 90 percent, depending on functional feeding strategies, with predatory insects generally having higher values (Tremblay and others, 1996; Slotton and others, 1997; Tremblay and Lucotte, 1997; Hall and others, 1998; Mason and others, 2000; Schwarzbach and others, 2002).

Three species of post-metamorphic frogs were collected in this study: bullfrogs (Rana catesbeiana), foothill yellow-legged frogs (Rana boylii), and Pacific treefrogs (Hyla regilla). Frogs were identified using descriptions by Stebbins (1985). Frog samples were analyzed for THg after cleaning and removal of gut contents. Other studies show that the mercury in frog tissue is largely in the form of $\mathrm{MeHg}$ (Roger L. Hothem, written commun., 2002).

\section{Methods}

\section{Field Methods}

Specialized cleaning and sampling techniques were used during all stages of sample collection to prevent sample contamination. Prior to use, all containers and equipment used for water and sediment sampling were cleaned using a dilute liquid soap followed by a 5-percent hydrochloric acid solution and multiple rinses in ultrapure, deionized (DI) water (18 megaohm-centimeter [M $\Omega-\mathrm{cm}]$ ), following standard USGS protocols (Shelton, 1994). Specific sampling and preservation methods are described below. 
Water

Water samples were collected in Teflon or fluorinated plastic containers and then transferred to a Teflon-lined, stainless steel churn for splitting. In small streams discharging less than about $100 \mathrm{gal} / \mathrm{min}$, grab samples typically were collected, because shallow conditions and narrow stream width preclude using integrated sampling techniques. In streams with higher flows, integrated samples were collected using the equal width increment (EWI) method using either 1-L Teflon bottles or D77 samplers. All stations at all times were sampled by wading except for Greenhorn Creek at You Bet Road on December 21, 2001, which was sampled using a D-77 sampler from the You Bet Road bridge.

Water samples collected for analysis of suspended sediment concentration (SSC) were taken from the churn and collected in pre-weighed 1-L Nalgene bottles. In contrast to all other splits, the bottles were not rinsed with the ambient water, to avoid introducing extraneous suspended sediment to the container. The SSC samples were chilled on wet ice or refrigerated at less than $5^{\circ} \mathrm{C}$ until analysis.

Filtration was done in the field using Gelman capsule filters of $0.45-\mu \mathrm{m}$ pore size. Filtered and unfiltered water samples for analysis of THg by the USGS NRP laboratory were stored in acid-washed glass bottles provided by the laboratory and were preserved using a potassium dichromate-nitric acid solution. Filtered and unfiltered water samples for analysis of MeHg were stored in acid-washed Teflon bottles provided by the WDML and were preserved either by freezing on dry ice or by using a 50-percent hydrochloric acid solution provided by the WDML. Filtered and unfiltered samples for analysis of cations and trace elements were preserved using distilled (ultrapure) nitric acid provided by the NRP laboratory.

Filtered samples collected for analysis of anions were chilled on wet ice or refrigerated at less than $5^{\circ} \mathrm{C}$ until analysis. Unfiltered samples collected for analysis of nutrients were preserved using a sulfuric acid solution and then chilled on wet ice followed by refrigeration; filtered nutrient samples were not acidified but were chilled prior to analysis. Samples collected for analysis of stable isotopes of hydrogen and oxygen in water were stored in glass bottles with polyseal caps with minimal headspace to retard evaporation. Aqueous sulfate was precipitated from filtered water samples at $90^{\circ} \mathrm{C}$ using a barium chloride solution after using $\mathrm{HCl}$ to lower the $\mathrm{pH}$ value to about 2; the resulting barium sulfate precipitate was filtered using $0.7-\mu \mathrm{m}$ filters and then transferred to silver-foil trays.

For most water samples, care was taken not to disturb bed sediments before sample collection. Such samples are referred to throughout this report as environmental samples. The amount of suspended sediment in two of the water samples was intentionally increased by human activity. These two samples are referred to throughout this report as disturbed samples.

Unstable water-quality constituents measured in the field included temperature, $\mathrm{pH}$, specific conductance, and dissolved oxygen (table 1). Values of $\mathrm{pH}$ were determined using an Orion 250A or 290A meter with a gel-filled Triode electrode. The $\mathrm{pH}$ meter was calibrated using commercially available $\mathrm{pH}$ 7 and 4 buffers. Water temperatures were measured using a thermometer that was checked against a thermometer certified by the American Society for Testing and Materials (ASTM); temperature data are accurate within $0.5^{\circ} \mathrm{C}$. Specific conductance was determined using a Cole-Parmer meter and probe; a one-point calibration was done with a USGS standard in the approximate concentration range of the unknowns. Dissolved oxygen was determined using a YSI meter and probe, calibrated using standard procedures.

\section{Sediment}

Sediment samples were collected using polycarbonate, Teflon, or stainless steel spoons and knives, and were transferred to Teflon or acid-washed glass containers using acid-cleaned stainless steel spatulas, following the protocols described by Shelton and Capel (1994). Sediment samples collected for analysis of THg were chilled immediately on wet ice and stored at $5^{\circ} \mathrm{C}$ or less until analysis. Sediment samples collected for analysis of $\mathrm{MeHg}$ were stored immediately on dry ice and frozen until analysis.

\section{Invertebrates}

Invertebrates were collected using dip nets and (or) by hand. Captured invertebrates were temporarily stored with native water in zippered plastic bags and were passively depurated over 4-24 hours. Invertebrates typically were combined as composites of multiple individuals to obtain a minimum of one gram of biomass for the analysis. In some cases, however, samples weighing less than one gram were analyzed. Prior to initial processing, samples were held on dry ice or wet ice. Initial processing of invertebrate samples proceeded within 24 hours of collection. Samples were thoroughly rinsed with DI water and patted dry using disposable paper towels. Composites of similar size and (or) age individuals (visually estimated) were placed in chemically cleaned jars (VWR Trace Clean TM jars, certified at $<0.2 \mu \mathrm{g} / \mathrm{L} \mathrm{Hg}$ ). Invertebrate samples were analyzed primarily for $\mathrm{MeHg}$; in some cases, however, samples were analyzed for both $\mathrm{THg}$ and $\mathrm{MeHg}$.

\section{Frogs}

Frogs were held on wet ice for 4-8 hours and then placed on dry ice. The samples were stored frozen up to 3 months before dissecting out the gastrointestinal (GI) tract. After excising the GI tract, frog carcasses were placed in certified, chemically cleaned jars and kept frozen until sent to the analytical laboratory. Usually, individual frog samples were sent for analysis, but, in one case, a composite of immature Pacific treefrogs was sent. Frogs were analyzed only for $\mathrm{THg}$. 


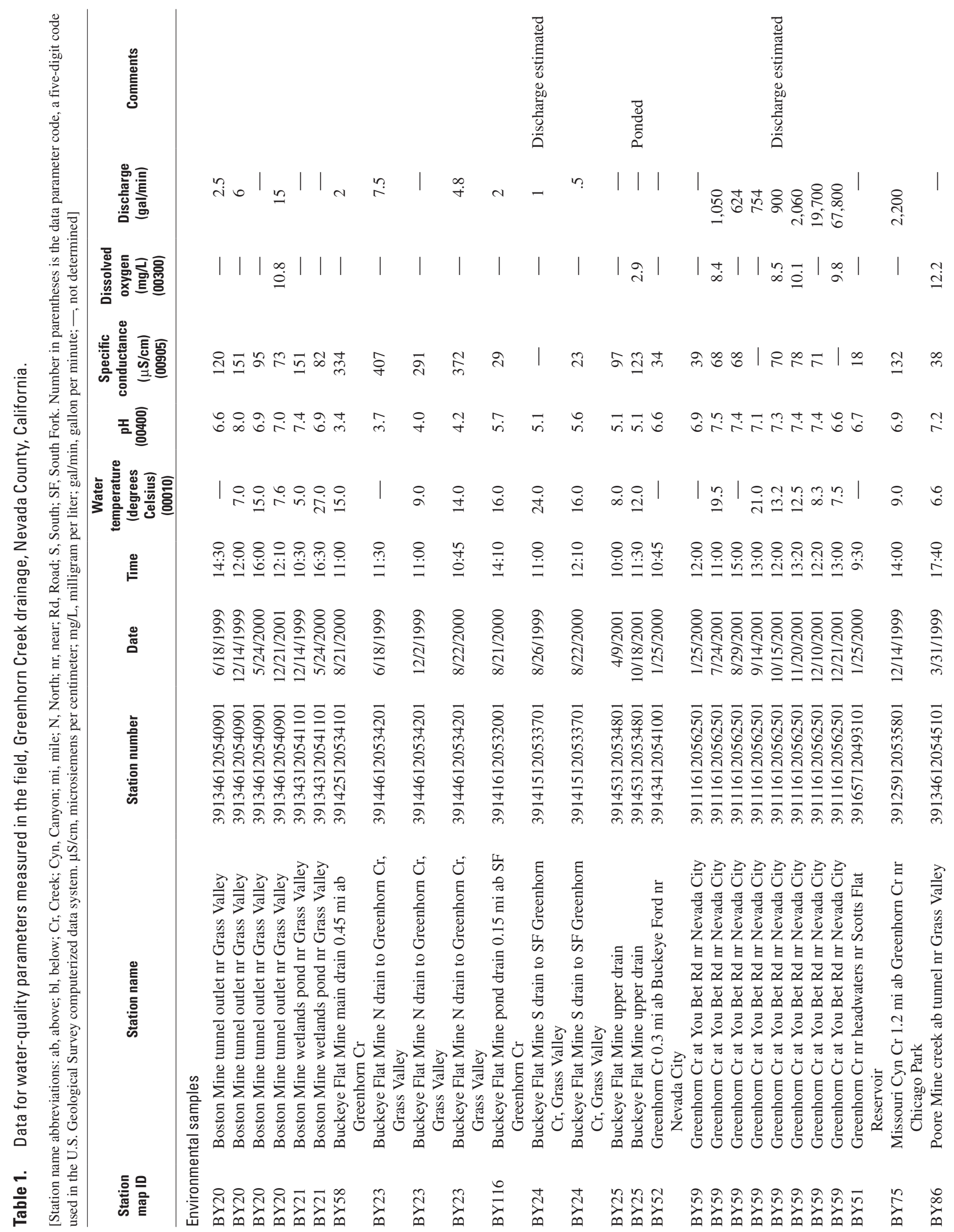




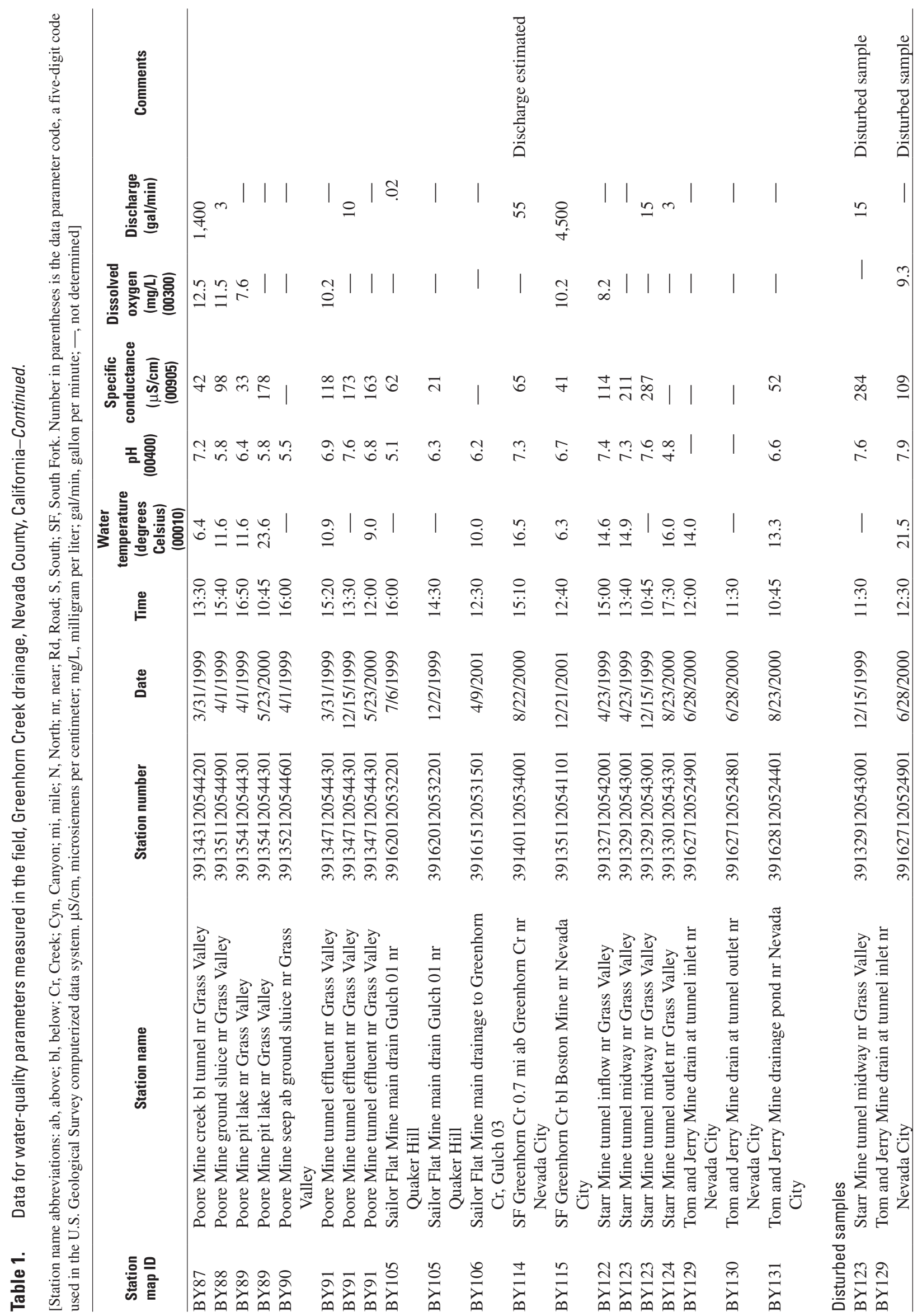




\section{Laboratory Methods of Chemical Analysis}

This section presents information on analytical methods used in this study. Where USGS-approved methods were used by laboratories such as the USGS NWQL, relatively little information is given in this report, and the reader is referred to published sources. More detailed information is given in this section for research methods that are not officially approved by the USGS as "production methods."

\section{Water}

Total mercury $(\mathrm{THg})$ in water was analyzed by the USGS NRP laboratory in Boulder, Colorado, using cold-vapor atomic-fluorescence spectrometry (CVAFS). Mercury stock and standard solutions were made from Puratronic grade (99.9995 percent) mercuric chloride $\left(\mathrm{HgCl}_{2}\right)$ salt (Johnson Matthey Plc) and preserved in a solution of high-purity nitric acid and primary-standard grade potassium dichromate using the same reagents and concentrations as those used to preserve samples. Deionized water (type 1, $18 \mathrm{M} \Omega-\mathrm{cm}$ ) was used for preparing all standards and reagent solutions. A solution of 3 percent hydrochloric acid (volume:volume, hydrochloric acid: type 1, $18 \mathrm{M} \Omega$-cm DI water) was used to prepare a 2-percent stannous chloride $\left(\mathrm{SnCl}_{2}\right.$ ) solution (wt:volume, stannous chloride:3 percent hydrochloric acid), which was used to reduce mercury to its elemental form in the cold vapor reactor. The vapor was transported to the detector with a stream of argon gas. Trace concentration levels of mercury were measured using an automated, cold-vapor atomic-fluorescence spectrometer or CVAFS (PS Analytical) using methods described previously by Roth (1994) and by David A. Roth (U.S. Geological Survey, written commun., 1999). Instrument parameters for the CVAFS mercury analysis were the same as those described by Alpers and others (2000). Peak height intensities of unknown samples were compared to a six-point calibration curve prepared from aqueous standards ranging in concentration from 0 to $50 \mathrm{ng} / \mathrm{L}$.

Methylmercury $(\mathrm{MeHg})$ in water was analyzed at the USGS WDML in Middleton, Wisconsin using ethylationdistillation CVAFS methods with double amalgamation, as described by Olson and De Wild (1999) and by De Wild and others (2002). Analysis of MeHg in water by the WDML was approved by the USGS Branch of Quality Assurance as a production method in August 2002, after the analyses in this report were completed. The method used by the WDML to analyze $\mathrm{MeHg}$ in water samples in this study was identical to the USGS-approved method.

Major cations and trace metals in water were analyzed by inductively coupled plasma (ICP) methods: both atomic emission spectrometry (AES) and mass spectrometry (MS) were used. Major elements including calcium $(\mathrm{Ca})$, iron $(\mathrm{Fe})$, magnesium $(\mathrm{Mg})$, potassium $(\mathrm{K})$, sodium $(\mathrm{Na})$, and silica $\left(\mathrm{SiO}_{2}\right)$ were determined by ICP-AES techniques using a
Perkin-Elmer Optima 3300DV multi-channel emission spectrometer. Use of the dual-view (radial and axial) optical configuration provided optimal sensitivity for various elements regardless of concentration. A description of the analysis conditions and procedures is reported by Garbarino and Taylor (1979). Details of the operational conditions are described by Mitko and Bebek (1999, 2000). Except for mercury, trace-element determinations were done by ICP-MS using a Perkin Elmer Elan Model 6000. Aerosols of acidified aqueous samples were introduced into the spectrometer with a conespray pneumatic nebulizer. Multiple internal standards (indium [In], iridium [Ir], and rhodium [Rh]), which covered the mass range, were used to normalize the system for drift. Details of the specific analysis techniques, procedures, and instrumental settings are described by Garbarino and Taylor (1996) and Taylor (2001). Major anions in filtered water (chloride and sulfate) were analyzed by ion chromatography following procedures described by Fishman and Friedman (1989).

Nutrients in each water sample were analyzed for three forms of phosphorus $(\mathrm{P})$ and five forms of nitrogen $(\mathrm{N})$, as described below. The phosphorus analyses included orthophosphate in filtered water, plus total phosphorus in both unfiltered and filtered water. Orthophosphate was determined using an automated, colorimetric, phosphomolybdate blue procedure with antimony $(\mathrm{Sb})$ added to increase the reduction rate (Patton and Truitt, 1992; Fishman, 1993). Total phosphorus was determined colorimetrically as orthophosphate after Kjeldahl digestion (Patton and Truitt, 1992). Filtered water samples were analyzed for nitrogen in the following forms: (1) nitrite $\left(\mathrm{NO}_{2}^{-}\right)$, (2) nitrite plus nitrate $\left(\mathrm{NO}_{3}^{-}\right)$, (3) ammonia $\left(\mathrm{NH}_{3}\right)$, and (4) ammonia plus organic nitrogen. Ammonia plus organic nitrogen was also determined in raw water samples. The method used to analyze nitrite was diazotization using sulfanilamide and N-1-naphthylethylenediamine under acidic conditions to form a red compound, the absorbance of which was determined colorimetrically using an automated segment flow procedure (Fishman, 1993). The concentration of nitrite plus nitrate was determined by reducing nitrate to nitrite using cadmium metal; the nitrite was then analyzed by diazotization (Fishman, 1993). Ammonia was analyzed using a salicylatehypochlorite method, in the presence of ferricyanide ions, that produces the salicylic acid analog of indophenol blue, which was analyzed colorimetrically using an automated-segmented flow procedure (Fishman, 1993). The concentration of ammonia plus organic nitrogen in raw and filtered samples was determined using the same Kjeldahl digestion as that used for total phosphorus, in which the organic nitrogen is reduced to the ammonium ion, followed by determination of the ammonium ion concentration by the colorimetric salicylatehypochlorite method (Fishman and Friedman, 1989; Patton and Truitt, 1992). 
Concentrations of dissolved organic carbon (DOC) were determined in $100-\mathrm{mL}$ filtered water samples $(0.45-\mu \mathrm{m}$ silver membrane filter). The filtrates were acidified first to remove dissolved and colloidal carbonates and bicarbonates, then the organic carbon was oxidized to carbon dioxide by adding persulfate and exposing the samples to ultraviolet light. The carbon dioxide was then measured by infrared spectrometry using a Dorhmann carbon analyzer (Brenton and Arnett, 1993). Particulate organic carbon (POC) concentrations were determined in the residual material that was collected on the silver membrane filters used to prepare DOC samples. The silver membrane filters were treated with acid to dissolve inorganic forms of carbon, then were reacted with potassium persulfate in glass ampules for 4 hours at $116^{\circ}$ to $130^{\circ} \mathrm{C}$. Then the ampules were broken in the carbon analyzer, releasing carbon dioxide, which was measured by infrared spectrometry using an Oceanography International carbon analyzer (Wershaw and others, 1987).

\section{Sediments}

For most constituents, the analytical methods used for determining concentrations in sediment were similar to those used for water samples. After a small aliquot (usually 50 to $100 \mathrm{mg}$ ) of sediment was selected for analysis and weighed, the constituents of concern were liberated from the sediment by digestion or chemical extraction. After dilution and preservation, the digests were analyzed by methods similar to those used for water samples.

Sediments in subsamples collected for analysis of trace metals and major elements were completely dissolved using a total $\mathrm{HCl}-\mathrm{HNO}_{3}-\mathrm{HF}$ acid microwave digestion procedure (Hayes, 1993). The digested subsamples were diluted at 1:10 (volume:volume, digest:DI water) with $18 \mathrm{M} \Omega$-cm DI water for analysis by ICP methods. The digested sediment subsamples were stabilized with the same reagents used for the preservation of the water samples.

Methylmercury in sediment was analyzed at the USGS WDML in Middleton, Wisconsin, using CVAFS methods with double amalgamation after ethylation of $\mathrm{MeHg}$ by extraction, a method less prone to produce $\mathrm{MeHg}$ artifacts than the distillation method used to analyze $\mathrm{MeHg}$ in water samples. $\mathrm{MeHg}$ can be a difficult constituent to measure in solids because of matrix interferences and the possibility of unintentionally producing MeHg during distillation (Bloom and others, 1997; Hintelmann and others, 1997; Hammerschmidt and Fitzgerald, 2001). Researchers at the WDML have adopted a published technique (Hintelmann, 1999) for extracting MeHg from solids that eliminates formation of $\mathrm{MeHg}$ in samples with high inorganic mercury levels. Solids ( 0.5 to $1.0 \mathrm{~g}$ ) are placed into a centrifuge tube. Potassium bromide $(\mathrm{KBr})$, copper sulfate $\left(\mathrm{CuSO}_{4}\right)$, and methylene chloride $\left(\mathrm{CH}_{2} \mathrm{Cl}_{2}\right)$ are added sequentially. The mixture is allowed to react for an hour and then is shaken for an hour to ensure complete extraction of the
MeHg. Following the shaking, the samples are centrifuged to break any emulsion that has formed. An aliquot of the $\mathrm{CH}_{2} \mathrm{Cl}_{2}$ is cleanly transferred to a vial containing reagent water. These vials are placed in a heating block until all $\mathrm{CH}_{2} \mathrm{Cl}_{2}$ has evaporated and the $\mathrm{MeHg}$ has back-extracted into the reagent water. The $\mathrm{pH}$ of the extractant is adjusted to 4.9 (to maximize ethylation potential) using acetate buffer. The extractant then is ethylated using sodium tetraethyl borate $\left(\mathrm{NaBEt}_{4}\right)$ and allowed to react for 15 minutes. After the reaction, the extractant is purged with nitrogen gas $\left(\mathrm{N}_{2}\right)$ for 20 minutes and the ethylated forms of $\mathrm{Hg}$ are collected on a sample trap containing Carbotrap. These ethylated $\mathrm{Hg}$ species are desorbed thermally from the sample trap, separated using a gas chromatographic (GC) column, reduced using a pyrolytic column, and detected using a CVAFS detector.

\section{Invertebrates and Frogs}

Total mercury and MeHg in biological samples were analyzed at the Trace Element Research Laboratory (TERL) at Texas A\&M University in College Station, Texas. Before samples were analyzed for $\mathrm{THg}$ by cold-vapor atomicabsorption spectrometry (CVAAS), the $\mathrm{Hg}$ was converted to the $\mathrm{Hg}^{2+}$ form. Mercury was digested using a modified version of USEPA methods 245.5 and 245.6. Tissue samples were freeze-dried or wet when analyzed. Freeze-dried tissue samples were homogenized in the original sample containers and then subsampled; wet samples were homogenized in a Tekmar Tissumizer and then subsampled. Samples were digested with a mixture containing nitric acid, sulfuric acid, potassium permanganate, and potassium persulfate in polypropylene tubes in a water bath at $90-95^{\circ} \mathrm{C}$. Before analysis, hydroxylamine hydrochloride was added to reduce excess permanganate, and the samples were brought to full volume using distilled-deionized water.

When the CVAAS procedure for determining $\mathrm{THg}$ in the biological tissue samples is used, divalent mercury $\left(\mathrm{Hg}^{2+}\right)$ in aqueous samples (digested tissue samples) is reduced to the elemental state $\left(\mathrm{Hg}^{0}\right)$ by a strong reducing agent (stannous chloride). Gaseous $\mathrm{Hg}^{0}$ is transported by the sweep gas into an atomic absorption cell, where light produced by a $\mathrm{Hg}$ vapor lamp is absorbed by the free $\mathrm{Hg}$ atoms. The concentration of $\mathrm{Hg}$ in the sample is determined by comparing light absorption of the sample with that of external calibration standards.

The procedure for determining MeHg in biological tissue samples measured the sum of all organo-mercury species extracted by the solvent. Samples were wet or freeze-dried when analyzed. Homogenized aliquots were extracted by an organic solvent with potassium bromide and copper sulfate added to improve extraction efficiency. The organic phase was digested in combusted glass vials, using nitric and sulfuric acids and potassium permanganate, to convert all mercury species to ionic mercury and to remove traces of organic solvent that could affect the measurement. Analysis was based on the CVAAS method. 
Moisture content was determined by weight loss during freeze-drying and is expressed as weight percentage of the original wet sample. Depending upon sample size, either the whole sample or a representative aliquot was frozen and then dried in a vacuum until a constant weight was attained. Samples were prepared and dried using plastic materials to minimize potential contamination artifacts.

\section{Quality Assurance and Quality Control}

A variety of measurements and analyses were used to determine the quality of the data associated with this study. The quality assurance (QA) program consisted of qualitycontrol (QC) measures including field and laboratory blank samples, standard reference materials (SRM) where available, spike recoveries, and replicate samples.

\section{Water and Sediments}

Major and trace elements in water were analyzed by inductively coupled plasma (ICP) methods; each analysis consisted of at least four replicate instrumental measurements. Each filtered and unfiltered sample was collected in duplicate and analyzed in triplicate. The triplicate analyses were analyzed statistically and the analyses were accepted if the standard deviation was within standard tolerances of precision (generally less than 15 percent of the amount present). The error tolerance is increased for analyses close to the detection limit for a given analyte. Median detection limits for trace elements analyzed by ICP-MS are given in table D1.

When each batch of samples was analyzed by ICP for trace element and major cations, several standard reference materials were also analyzed as unknowns as part of the QA program. Plots of reported values in relation to observed values for certified SRM and USGS standard reference water samples (SRWS) are shown in figures $B 1$ through $B 7$. The number of times each standard was analyzed during 1999-2001 is indicated on each figure; the standards typically were run several hundred times during the study period. There is excellent agreement between all reported and observed values for the standards. Regression coefficients were derived from correlation plots of observed and reported values for the analysis of standard reference materials for 21 selected trace elements (table D2); the regression coefficients range from 0.9881 for manganese to 1.000 for beryllium and antimony.

Data for spiked, blank samples for the elements arsenic, beryllium, copper, and zinc analyzed during July 2000 through August 2001 are shown in figure B8. In nearly all cases, the spike recovery was within the control range of 80 to 120 percent of the expected amount. Data for spiked blank samples having an expected $\mathrm{THg}$ concentration of $5 \mathrm{ng} / \mathrm{L}$ are given in figure B9. Nearly all results fall in the control range of 80 to 120 percent recovery. Results for trace-element spikes of unknown samples are shown in figure B10 for four trace elements and in figure B11 for mercury. Spike levels were generally three to ten times the amount present in field samples for each element. Again, nearly all the spike recoveries were in the control range of 80 to 120 percent of the expected value.

Because replicate split samples were routinely analyzed as separate unknowns and each replicate was analyzed in triplicate, plots of replicate 1 versus replicate 2 indicate the analytical variability as well as possible contamination issues regarding bottles and sample-splitting equipment. The plots in figures $B 12$ and $B 13$ for 12 of the elements analyzed by the NRP laboratory during the study period show very few outliers for replicate analyses. Values of relative standard deviation (RSD) for six elements (fig. B14) are generally less than 10 percent at concentrations well above the method detection limit (MDL) for each element, whereas RSD values tend to be higher at concentrations near the MDL, where lower precision is generally indicated by reporting data to fewer significant figures. Results for replicate analyses of specific samples are shown for mercury in unfiltered water (fig. B15) and for mercury in filtered water (fig. B16). One outlier is evident on fig. B15; it was not used to compute averages or median values.

Precision and accuracy criteria for $\mathrm{THg}$ in filtered and unfiltered water samples were evaluated by analyzing results for field and laboratory blank samples, standard reference materials, spike addition recoveries, and replicate samples. All samples were analyzed at least in triplicate. In most cases, each sample was sent to the laboratory in two bottles designated as split replicates " 1 of 2 " and " 2 of 2 ." The MDL for the $\mathrm{THg}$ analyses was 0.3 nanogram per liter (ng/L).

Data for THg in field blanks are given in table D3. Deionized source water from a MilliQ purification system at the USGS laboratory in Sacramento, California, was used for blanks taken in late November 1999. The source water blank had $\mathrm{THg}$ concentrations of 2.1 to $3.3 \mathrm{ng} / \mathrm{L}$. Nine subsequent equipment and process (filtered) blanks taken during January and February 2000 had a median THg concentration of $0.5 \mathrm{ng} / \mathrm{L}$, just above the MDL, and a range from $<0.4 \mathrm{ng} / \mathrm{L}$ to $1.6 \mathrm{ng} / \mathrm{L}$. In comparison, the medians of station-averaged values for unfiltered and filtered $\mathrm{THg}$ in this study were $14.6 \mathrm{ng} / \mathrm{L}$ and $2.8 \mathrm{ng} / \mathrm{L}$, respectively. Several values for filtered $\mathrm{THg}$ in field samples are less than the MDL of $0.3 \mathrm{ng} / \mathrm{L}$ (table 2). Given that some of the blank values for THg are in the same concentration range as the values for some of the filtered samples, these lower-levels values must be interpreted with caution.

Quality-control measures used during analysis of $\mathrm{MeHg}$ in water were described by De Wild and others (2002). Water samples were analyzed in batches of 11 samples plus three laboratory blanks, a matrix spike, and a matrix spike duplicate. The reported values for $\mathrm{MeHg}$ concentrations were corrected for daily blank values, as described by De Wild and others (2002). The MDL for MeHg in water at the USGS Wisconsin District Mercury Laboratory, defined using standard protocol (U.S. Environmental Protection Agency, 1990), was $0.025 \mathrm{ng} / \mathrm{L}$ during 1999-2000 and was $0.04 \mathrm{ng} / \mathrm{L}$ during 2001. A conservative MDL for $\mathrm{MeHg}$ of $0.04 \mathrm{ng} / \mathrm{L}$ is used throughout this report. 


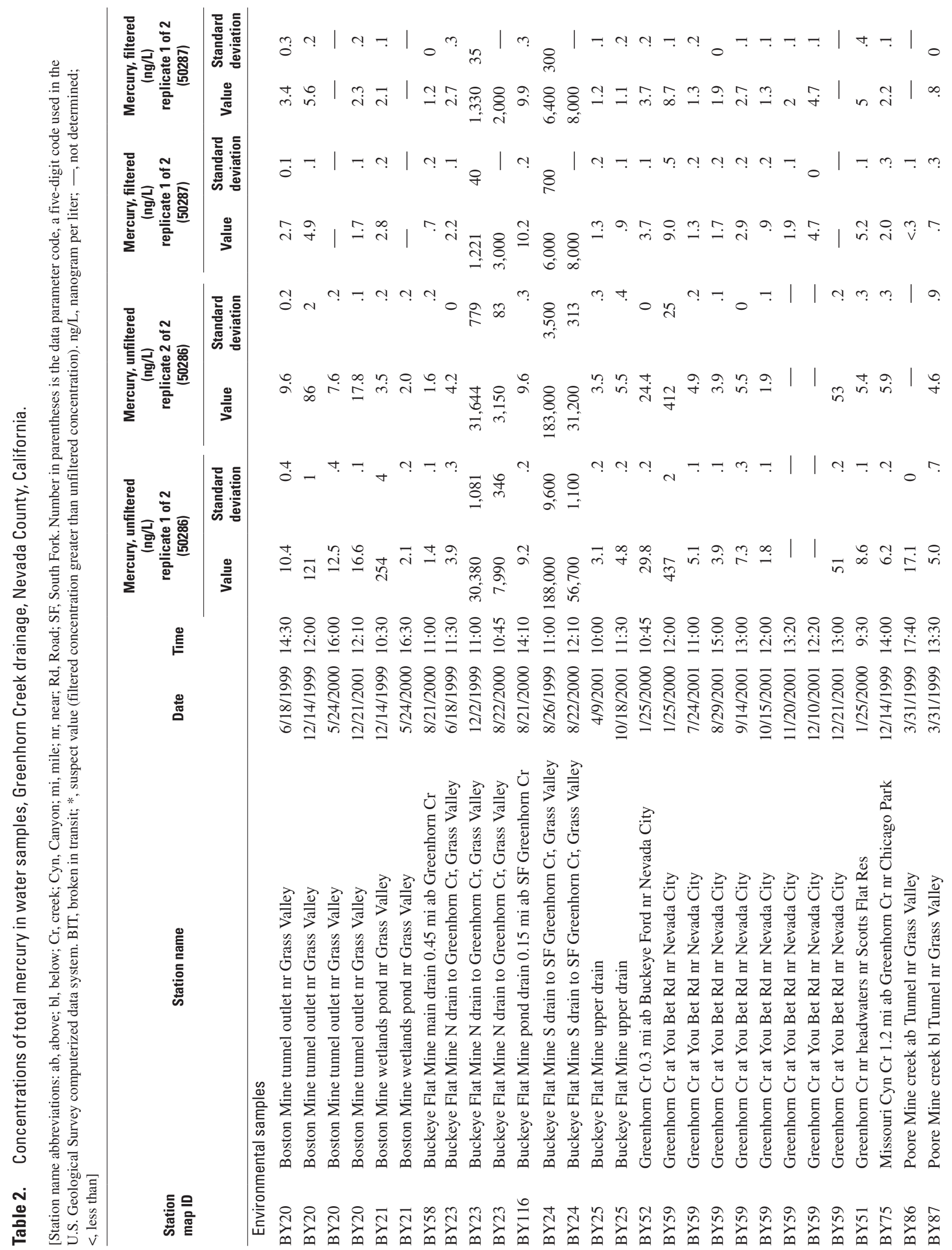




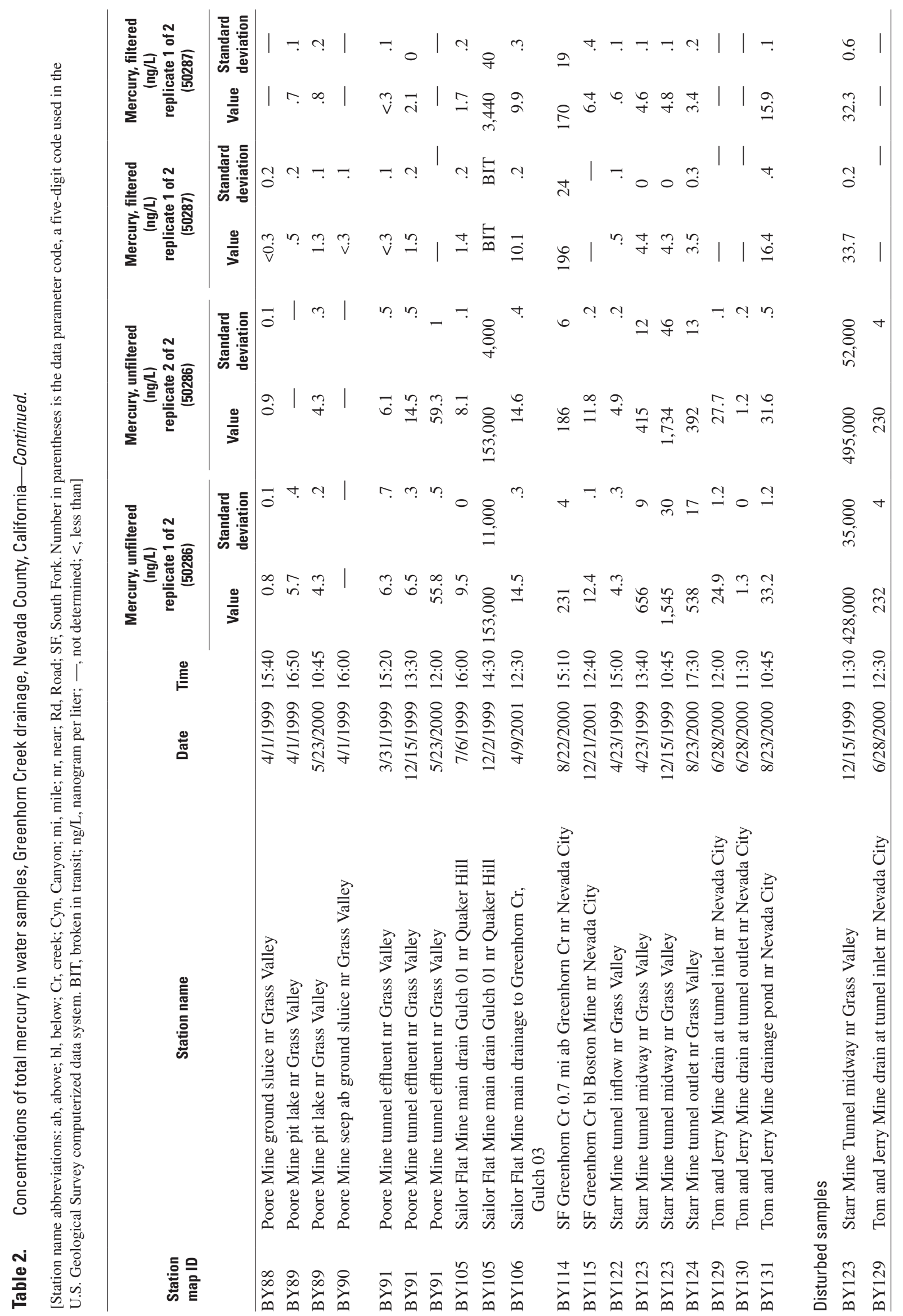


Concentrations of $\mathrm{MeHg}$ in blanks analyzed during this study are given in table D4. Methylmercury was below the MDL of $0.04 \mathrm{ng} / \mathrm{L}$ in all six of the equipment and process blanks taken during the study period. A single process (filtered) blank had a $\mathrm{MeHg}$ value of $0.04 \mathrm{ng} / \mathrm{L}$, right at the detection limit.

Figure B17 shows the relation between specific conductance (SC) and the sum of equivalent change based on analyses of major anions $\left(\mathrm{Cl}^{-}\right.$and $\left.\mathrm{SO}_{4}^{2-}\right)$ plus bicarbonate alkalinity (as $\mathrm{HCO}_{3}^{-}$). Theoretically, the relation in figure B17 should define a straight line. Based on the relation in figure B17, data for SC, anions, and alkalinity for five water samples that plotted as outliers were deemed unreliable and were removed from the data tables.

Concentration values for trace metals and major cations in blank water samples are given in table D5 (filtered and unfiltered process blanks) and table B6 (equipment blanks and source blanks).

\section{Invertebrates and Frogs}

Duplicate samples of invertebrates and frogs were analyzed at a rate of 5 percent of total samples, with at least one duplicate per matrix per analytical run to estimate the precision of the methods. To confirm the accuracy of the methods, procedural blanks, spiked samples, and SRMs were analyzed. To confirm that no analyte was added during the processing of the sample, procedural blanks were analyzed at a rate of 5 percent of the total samples, with at least one per matrix per analytical run. Spiked samples were analyzed at a rate of 5 percent, with at least one spike per matrix per analytical run. Spikes were samples fortified with a known quantity of analyte and analyzed as part of the run. Standard reference materials (dogfish liver and muscle) were analyzed at a rate of 5 percent to ensure that the method worked with naturally incorporated mercury.

The U.S. Fish and Wildlife Service's Patuxent Analytical Control Facility (PACF) was responsible for assuring the quality of the chemical analyses provided by TERL. Their reports indicated that the limits of detection for $\mathrm{THg}$ and $\mathrm{MeHg}$ were all below the maximum acceptable value $(0.20 \mu \mathrm{g} / \mathrm{g}$, dry weight $)$, and analyses of procedural blanks were within normal limits. Spiked sample recoveries averaged 96.7 percent for $\mathrm{THg}(\mathrm{n}=24$ [number of samples]) and ranged between 83.2 and 116 percent. For $\mathrm{MeHg}$, spiked sample recoveries averaged 95.7 percent $(n=21)$ and ranged between 79.3 and 107 percent. For both $\mathrm{THg}$ and $\mathrm{MeHg}$, the spiked sample recoveries were acceptable because they were within 2 standard deviations of the mean in at least 95 percent of the samples. The average percentage recovery (and range) from SRMs for $\mathrm{THg}(\mathrm{n}=26)$ was 96.7 percent $(84.5-110$ percent $)$; for $\mathrm{MeHg}(\mathrm{n}=22)$ the average percentage recovery was 98.8 percent (85.6-112 percent). All percentage recoveries were within acceptable limits. THg was analyzed in duplicate on 25 samples, and the relative percentage difference (RPD) between duplicates ranged from 0.53 to 16.7 percent and averaged 5.2 percent. $\mathrm{MeHg}$ was analyzed in duplicate on 21 samples, and the RPD between duplicates ranged from 0.35 to 24.2 percent and averaged 5.4 percent. All duplicate analyses were within acceptable limits.

\section{Results}

This section is divided into two parts. The first part describes overall trends in the data set, and the second part describes the data by geographic area.

\section{Overall Trends}

In this section, the overall trends in the data are discussed in terms of water quality, sediment, and biota. The relations between mercury and methylmercury concentrations are investigated for water, sediment, and invertebrates. For the water samples, the relations between mercury and methylmercury concentrations and $\mathrm{pH}$ and sulfate are explored.

\section{Water Quality}

Water-quality data for the 49 samples collected in the Greenhorn Creek drainage during 1999-2001 are given in tables $1-8$ (table 4 in back of report) and figures $6-17$. (Note that results less than the MDL, referred to as non-detects, are plotted on all figures using a value equal to one-half the MDL, with an error bar extending to the MDL.)

Field parameters (table 1) include water temperature, $\mathrm{pH}$, specific conductance, and dissolved oxygen. Values of $\mathrm{pH}$ ranged from 3.4 at the Buckeye main drain to 8.0 at the Boston Mine tunnel outlet. Specific conductance values ranged from 18 microsiemens per centimeter $(\mu \mathrm{S} / \mathrm{cm})$ at the Headwaters station (BY51) to $407 \mu \mathrm{S} / \mathrm{cm}$ in the Buckeye Flat Mine north drain (BY23). Dissolved oxygen (DO) was measured in 14 of the 49 water samples. Thirteen of the 14 DO measurements were greater than 7.5 milligrams per liter $(\mathrm{mg} / \mathrm{L})$, indicating near-saturation, typical of flowing surface waters. The lowest recorded value of DO was $2.9 \mathrm{mg} / \mathrm{L}$ from the Buckeye Flat Mine upper drain. Discharge data in table 1 are considered to be accurate within 20 percent of the measured flow rate, except the data marked as estimates, for which the uncertainty may be as high as 50 to 75 percent. 
Table 3. Concentrations of methylmercury in water samples, Greenhorn Creek drainage, Nevada County, California.

[NOTE: Table 4 begins on page 71. Station name abbreviations: ab, above; bl, below; Cr, Creek; Cyn, Canyon; mi, mile; N, North; nr, near; Rd, Road; S, South; SF, South Fork. Number in parentheses is the data parameter code, a five-digit code used in the U.S. Geological Survey computerized data system. The method detection limit for methylmercury was 0.04 nanogram per liter $(\mathrm{ng} / \mathrm{L}) . \ldots$, not determined; <, less than]

\begin{tabular}{|c|c|c|c|c|c|}
\hline Station map ID & Station name & Date & Time & $\begin{array}{c}\text { Methylmercury, } \\
\text { unfiltered } \\
\text { (ng/L) } \\
(\mathbf{5 0 2 8 4 )} \\
\end{array}$ & $\begin{array}{l}\text { Methylmercury, } \\
\text { filtered } \\
\text { (ng/L) } \\
\text { (50285) }\end{array}$ \\
\hline BY20 & Boston Mine tunnel outlet nr Grass Valley & $6 / 18 / 1999$ & $14: 30$ & 0.09 & - \\
\hline BY20 & Boston Mine tunnel outlet nr Grass Valley & $12 / 14 / 1999$ & $12: 00$ & $<.04$ & - \\
\hline BY20 & Boston Mine tunnel outlet nr Grass Valley & $5 / 24 / 2000$ & $16: 00$ & .13 & - \\
\hline BY21 & Boston Mine wetlands pond nr Grass Valley & $5 / 24 / 2000$ & $16: 30$ & .63 & - \\
\hline BY58 & Buckeye Flat Mine main drain $0.45 \mathrm{mi}$ ab Greenhorn $\mathrm{Cr}$ & $8 / 21 / 2000$ & 11:00 & - & - \\
\hline BY23 & Buckeye Flat Mine N drain to Greenhorn Cr, Grass Valley & $6 / 18 / 1999$ & $11: 30$ & $<.04$ & - \\
\hline BY23 & Buckeye Flat Mine N drain to Greenhorn Cr, Grass Valley & $12 / 2 / 1999$ & 11:00 & .30 & - \\
\hline BY23 & Buckeye Flat Mine N drain to Greenhorn Cr, Grass Valley & $8 / 22 / 2000$ & $10: 45$ & - & - \\
\hline BY25 & Buckeye Flat Mine upper drain & $10 / 18 / 2001$ & $11: 30$ & .06 & .05 \\
\hline BY52 & Greenhorn Cr $0.3 \mathrm{mi}$ ab Buckeye Ford nr Nevada City & $1 / 25 / 2000$ & $10: 45$ & $<.04$ & - \\
\hline BY59 & Greenhorn Cr at You Bet Rd nr Nevada City & $1 / 25 / 2000$ & $12: 00$ & .10 & - \\
\hline BY59 & Greenhorn $\mathrm{Cr}$ at You Bet Rd nr Nevada City & $7 / 24 / 2001$ & 11:00 & $<.04$ & - \\
\hline BY59 & Greenhorn Cr at You Bet Rd nr Nevada City & $8 / 29 / 2001$ & $15: 00$ & .11 & .05 \\
\hline BY59 & Greenhorn Cr at You Bet Rd nr Nevada City & $9 / 14 / 2001$ & $13: 00$ & .07 & .04 \\
\hline BY59 & Greenhorn Cr at You Bet Rd nr Nevada City & $10 / 15 / 2001$ & $12: 00$ & .04 & $<.04$ \\
\hline BY59 & Greenhorn Cr at You Bet Rd nr Nevada City & $11 / 20 / 2001$ & $13: 20$ & - & $<.04$ \\
\hline BY59 & Greenhorn Cr at You Bet Rd nr Nevada City & $12 / 10 / 2001$ & $12: 20$ & - & $<.04$ \\
\hline BY59 & Greenhorn Cr at You Bet Rd nr Nevada City & $12 / 21 / 2001$ & 13:00 & .08 & - \\
\hline BY51 & Greenhorn Cr nr headwaters nr Scotts Flat Reservoir & $1 / 25 / 2000$ & $9: 30$ & .06 & - \\
\hline BY91 & Poore Mine tunnel effluent nr Grass Valley & $12 / 15 / 1999$ & $13: 30$ & $<.04$ & - \\
\hline BY91 & Poore Mine tunnel effluent nr Grass Valley & $5 / 23 / 2000$ & $12: 00$ & .88 & - \\
\hline BY105 & Sailor Flat Mine main drain Gulch 01 nr Quaker Hill & 7/6/1999 & $16: 00$ & .30 & - \\
\hline BY105 & Sailor Flat Mine main drain Gulch 01 nr Quaker Hill & $12 / 2 / 1999$ & $14: 30$ & 2.64 & - \\
\hline BY106 & Sailor Flat Mine main drainage to Greenhorn Cr, Gulch 03 & $4 / 9 / 2001$ & $12: 30$ & .10 & .06 \\
\hline BY114 & SF Greenhorn Cr 0.7 mi ab Greenhorn Cr nr Nevada City & $8 / 22 / 2000$ & $15: 10$ & .13 & .27 \\
\hline BY115 & SF Greenhorn Cr bl Boston Mine nr Nevada City & $12 / 21 / 2001$ & $12: 40$ & $<.04$ & $<.04$ \\
\hline BY122 & Starr Mine tunnel inflow nr Grass Valley & 4/23/1999 & $15: 00$ & $<.04$ & - \\
\hline BY123 & Starr Mine tunnel midway nr Grass Valley & $4 / 23 / 1999$ & $13: 40$ & .79 & - \\
\hline BY123 & Starr Mine tunnel midway nr Grass Valley & $12 / 15 / 1999$ & $10: 45$ & .78 & - \\
\hline BY124 & Starr Mine tunnel outlet nr Grass Valley & $8 / 23 / 2000$ & $17: 30$ & .53 & $<.04$ \\
\hline BY129 & Tom and Jerry Mine drain at tunnel inlet nr Nevada City & $6 / 28 / 2000$ & $12: 00$ & .18 & - \\
\hline BY130 & Tom and Jerry Mine drain at tunnel outlet nr Nevada City & $6 / 28 / 2000$ & $11: 30$ & $<.04$ & - \\
\hline BY131 & Tom and Jerry Mine drainage pond nr Nevada City & $8 / 23 / 2000$ & $10: 45$ & $<.04$ & - \\
\hline \multicolumn{6}{|c|}{ Disturbed Samples } \\
\hline BY123 & Starr Mine tunnel midway nr Grass Valley & $12 / 15 / 1999$ & $11: 30$ & 244 & - \\
\hline BY129 & Tom and Jerry Mine drain at tunnel inlet nr Nevada City & $6 / 28 / 2000$ & $12: 30$ & .04 & - \\
\hline
\end{tabular}


Table 5. Concentrations of major anions and alkalinity in filtered water samples, Greenhorn Creek drainage, Nevada County, California.

[Station name abbreviations: ab, above; bl, below; Cr, Creek; Cyn, Canyon; mi, mile; nr, near; Rd, Road; S, south; SF, South Fork. Number in parentheses is the data parameter code, a five-digit code used in the U.S. Geological Survey computerized data system. $\mathrm{CaCO}_{3}$, calcium carbonate; mg/L, milligram per liter; $\leq$ less than; - , not determined]

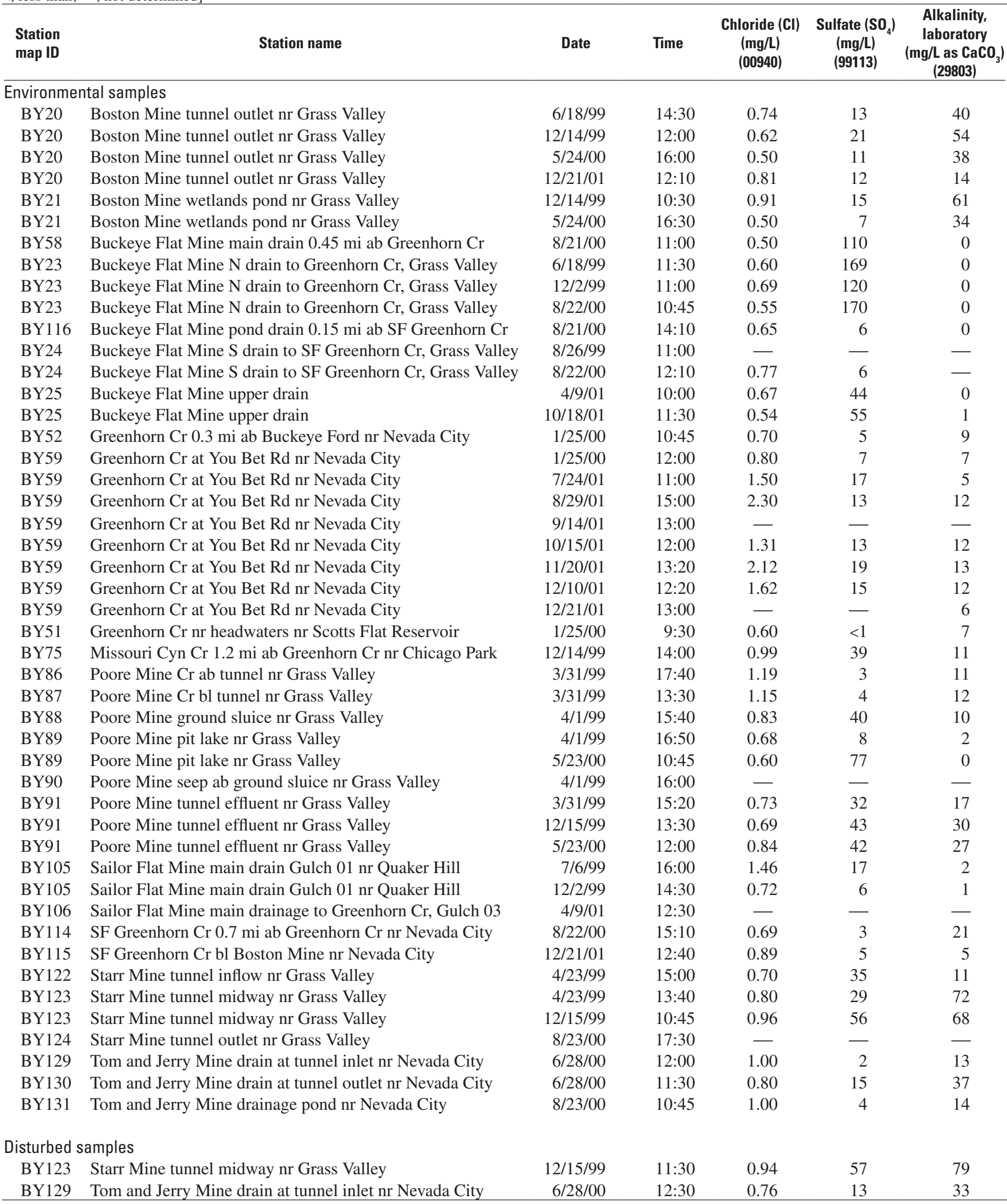




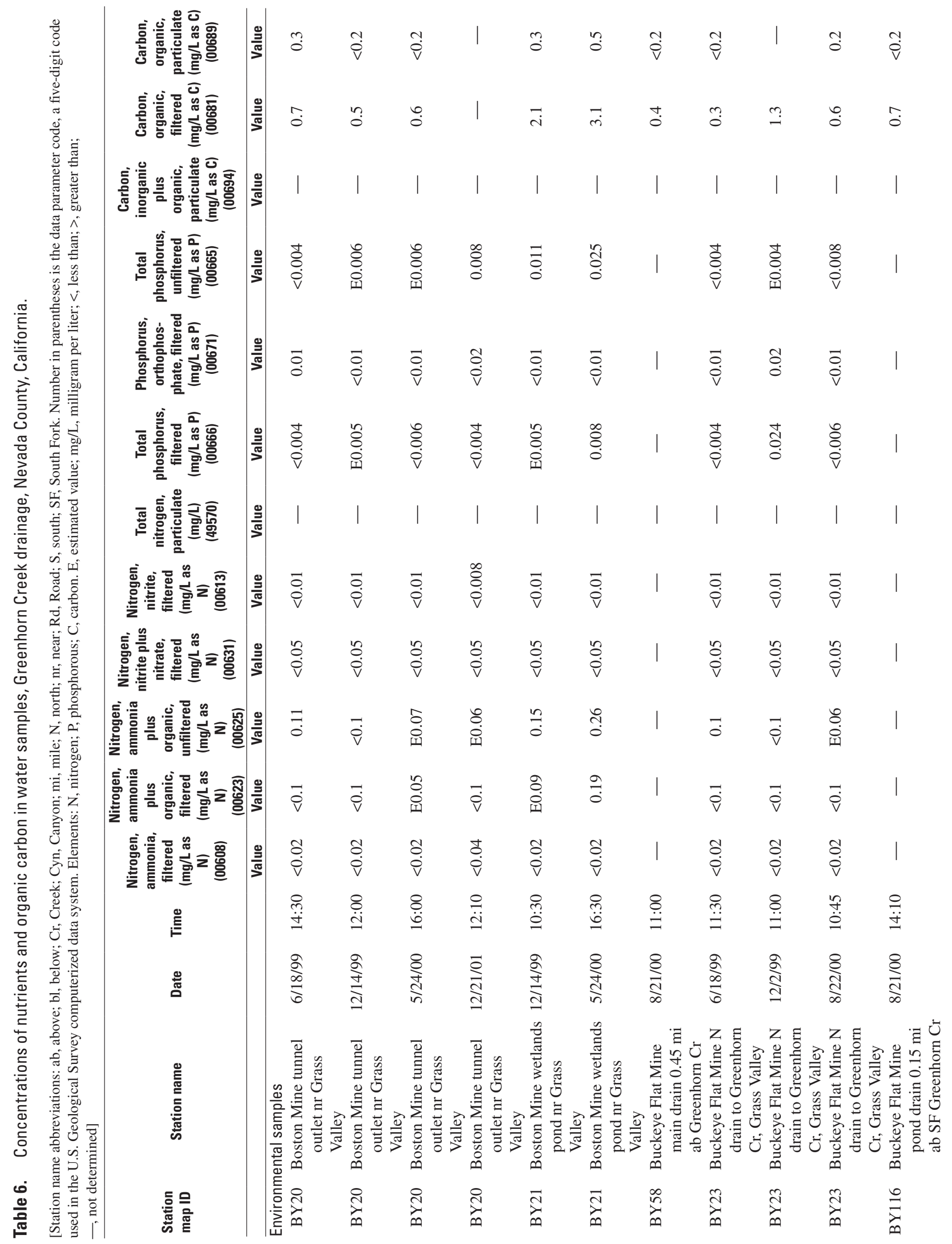




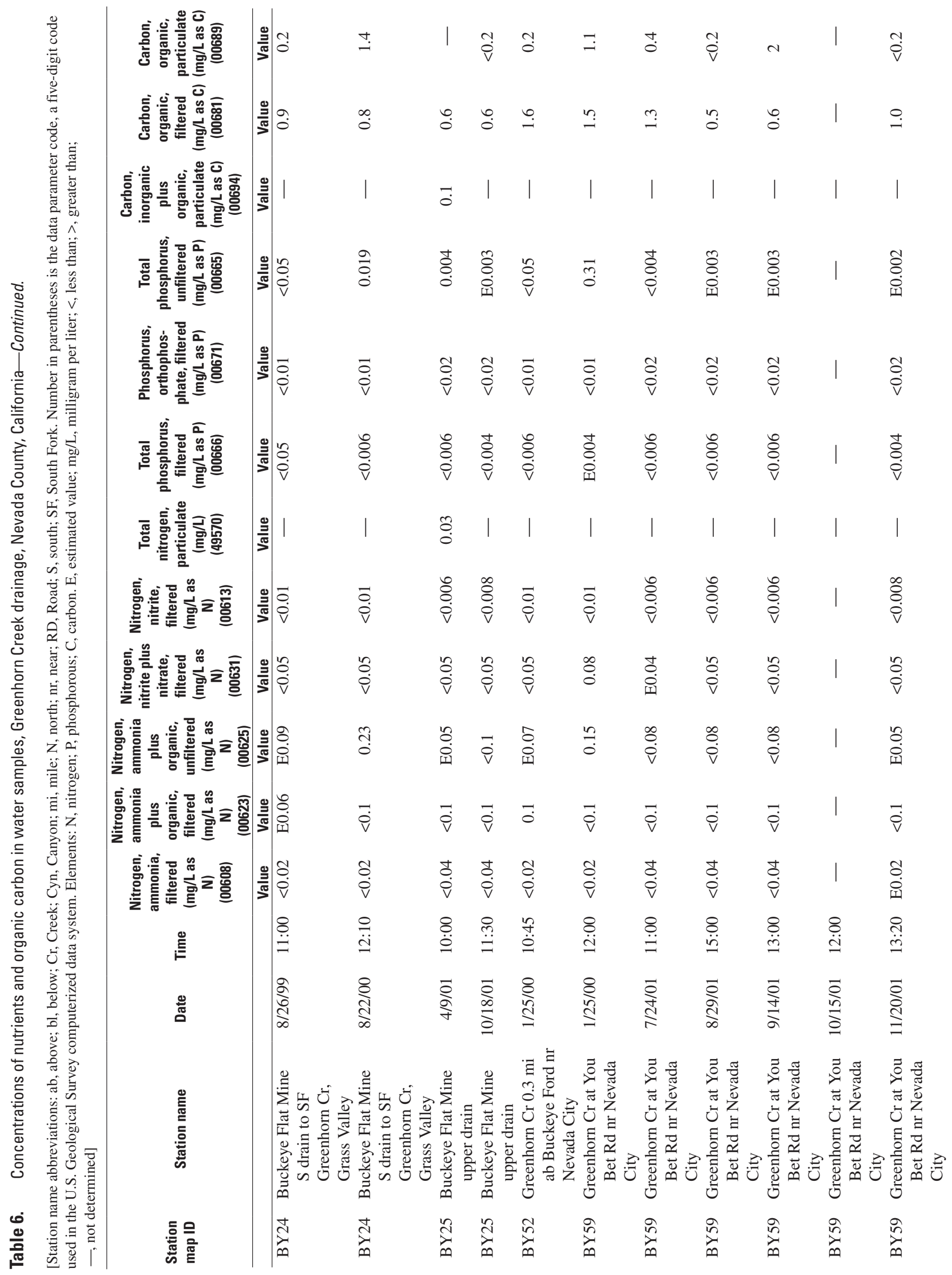




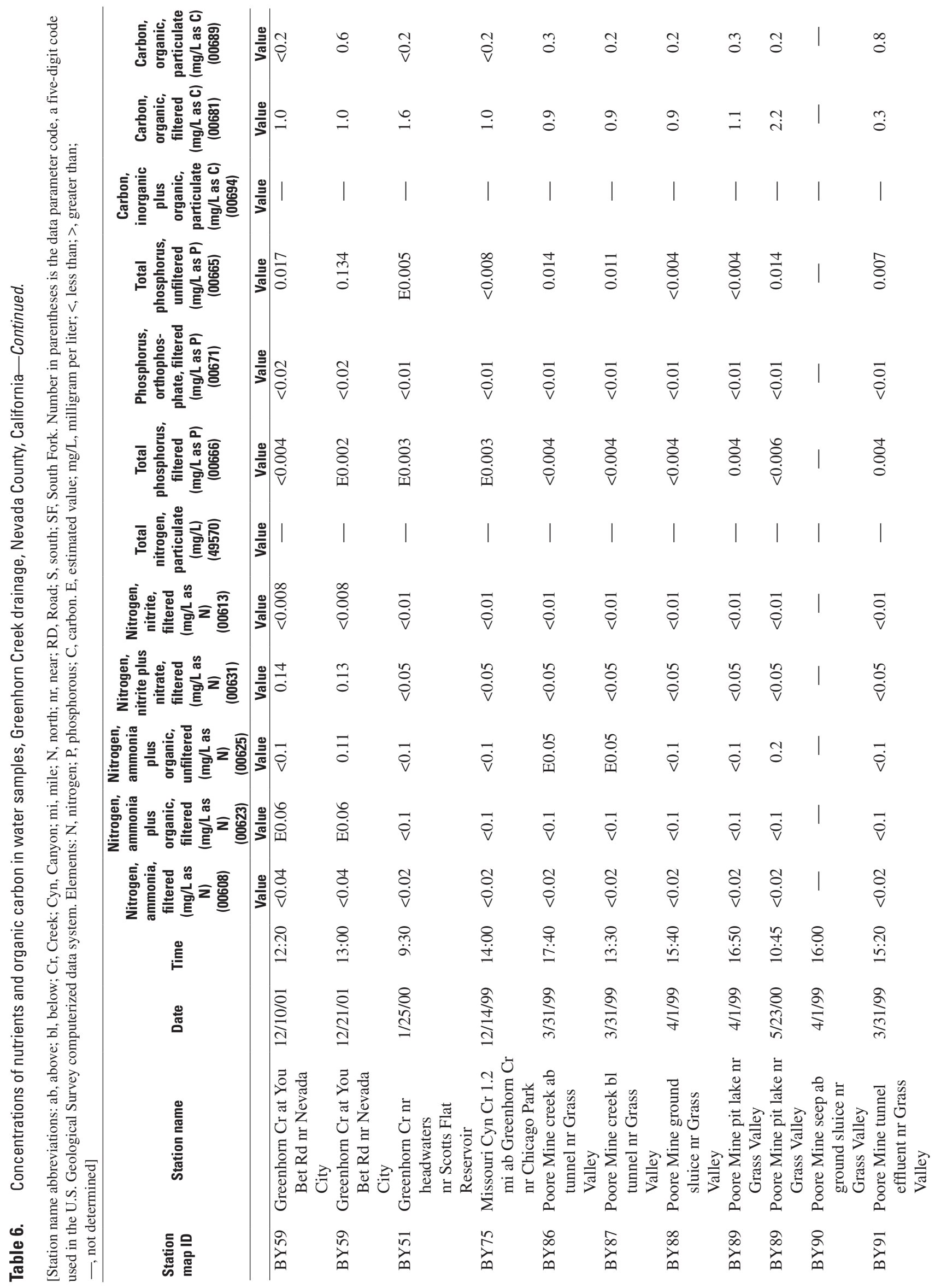




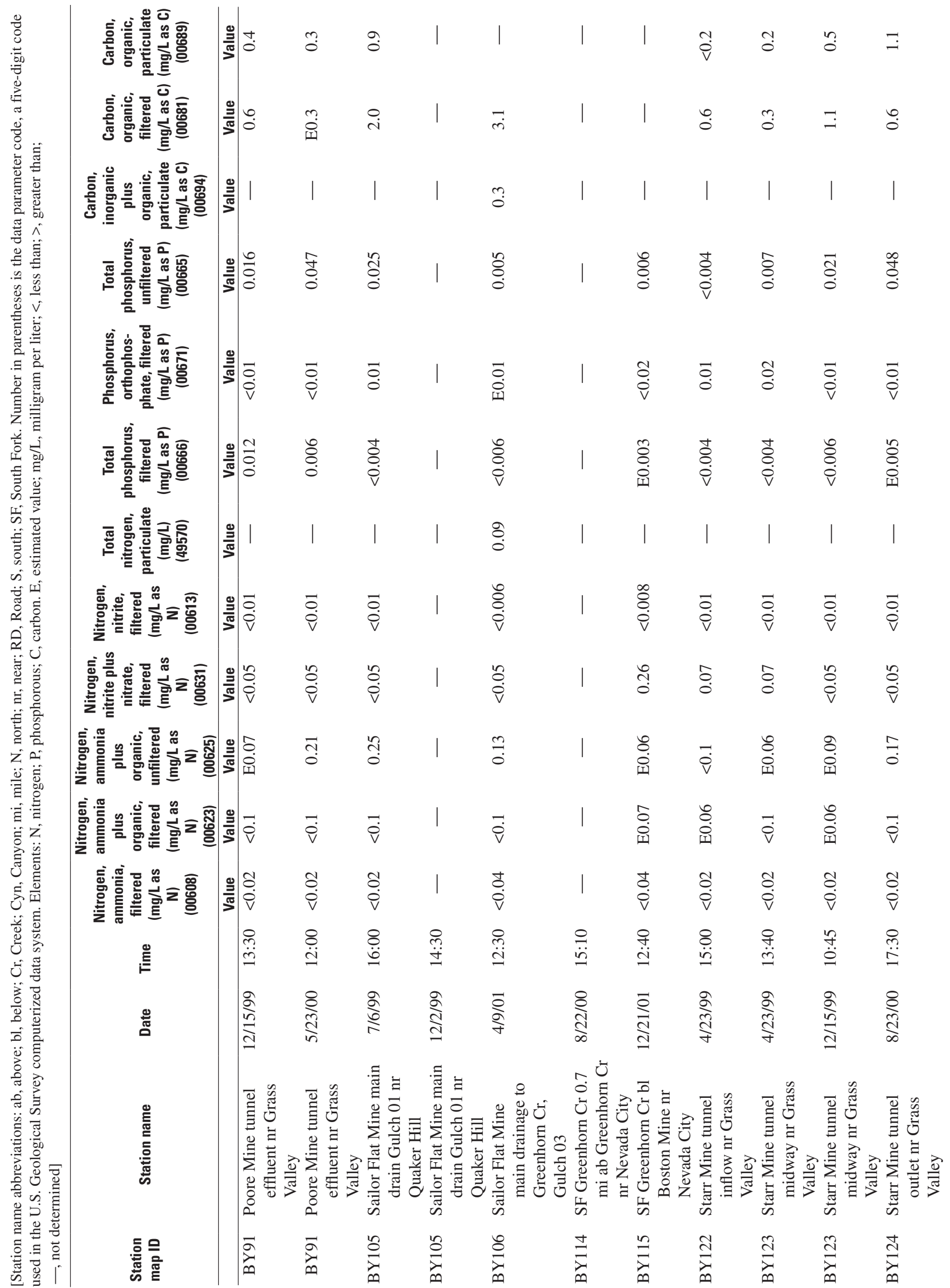




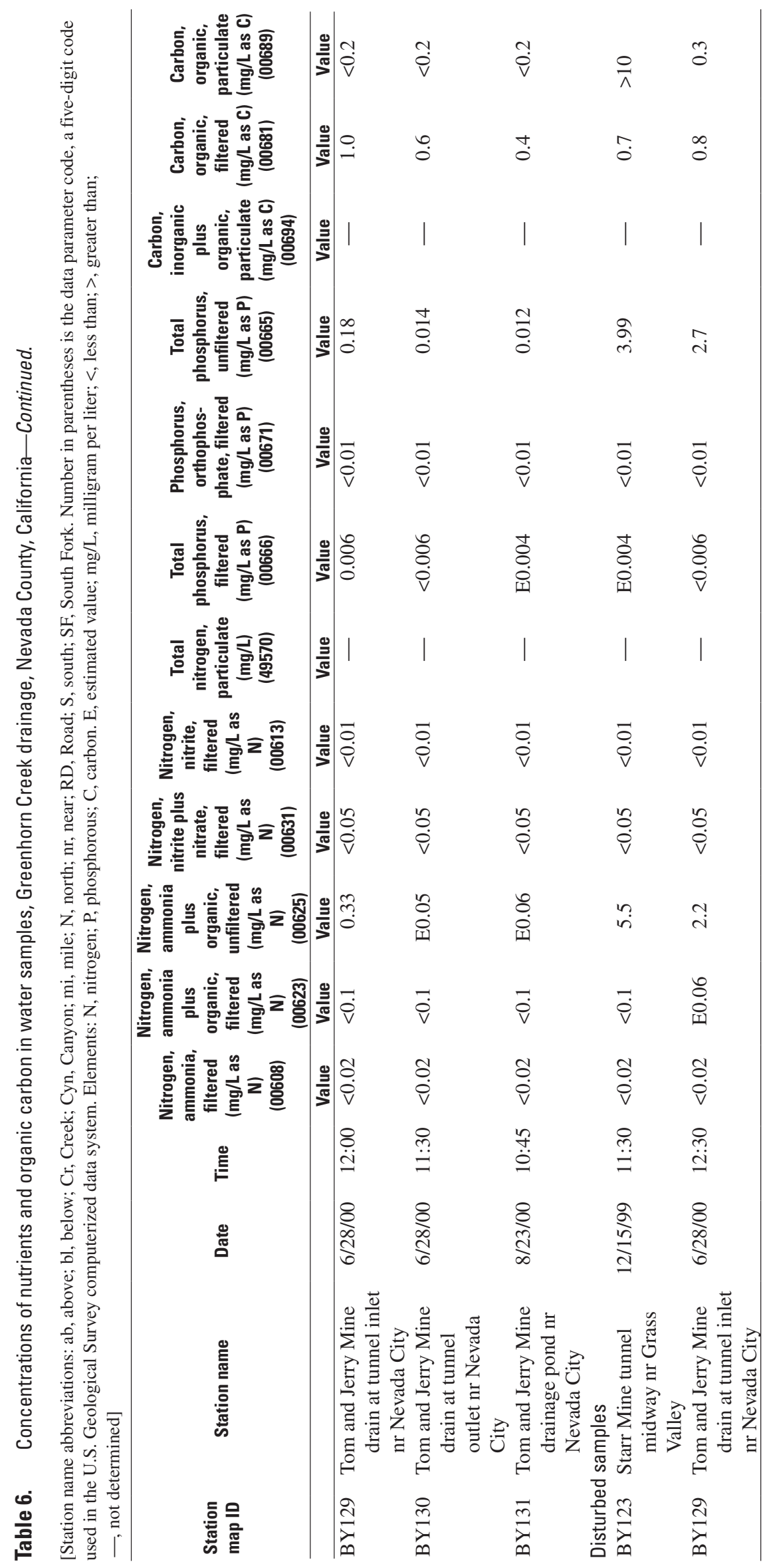


Table 7. Data for suspended sediment in water samples, Greenhorn Creek drainage, Nevada County, California.

[Percentage sand determined gravimetrically using 0.063-millimeter screen. Station name abbreviations: ab, above; bl, below; Cr, Creek; Cyn, Canyon; mi, mile; N, north; nr, near; Rd, Road; S, south; SF, South Fork. mm, millimeter; mg/L milligram per liter; —, not determined]

\begin{tabular}{|c|c|c|c|c|c|}
\hline $\begin{array}{l}\text { Station } \\
\text { map ID }\end{array}$ & Station name & Date & Time & $\begin{array}{c}\text { Suspended } \\
\text { sediment, } \\
\text { percentage finer } \\
\text { than } 0.063 \mathrm{~mm}\end{array}$ & $\begin{array}{c}\text { Suspended } \\
\text { sediment } \\
\text { concentration } \\
\text { (mg/L) }\end{array}$ \\
\hline BY20 & Boston Mine tunnel outlet nr Grass Valley & $6 / 18 / 99$ & $14: 30$ & - & - \\
\hline BY20 & Boston Mine tunnel outlet nr Grass Valley & $12 / 14 / 99$ & $12: 00$ & 40 & 6 \\
\hline BY20 & Boston Mine tunnel outlet nr Grass Valley & $5 / 24 / 00$ & $16: 00$ & - & 7 \\
\hline BY21 & Boston Mine wetlands pond nr Grass Valley & $5 / 24 / 00$ & $16: 30$ & - & 3 \\
\hline BY58 & Buckeye Flat Mine main drain $0.45 \mathrm{mi}$ ab Greenhorn $\mathrm{Cr}$ & $8 / 21 / 00$ & 11:00 & - & 1 \\
\hline BY23 & Buckeye Flat Mine N drain to Greenhorn Cr, Grass Valley & $6 / 18 / 99$ & $11: 30$ & - & - \\
\hline BY23 & Buckeye Flat Mine N drain to Greenhorn Cr, Grass Valley & $12 / 2 / 99$ & $11: 00$ & 72 & 708 \\
\hline BY23 & Buckeye Flat Mine N drain to Greenhorn Cr, Grass Valley & $8 / 22 / 00$ & $10: 45$ & - & 1 \\
\hline BY25 & Buckeye Flat Mine upper drain & $10 / 18 / 01$ & $11: 30$ & 50 & 1 \\
\hline BY52 & Greenhorn Cr 0.3 mi ab Buckeye Ford nr Nevada City & $1 / 25 / 00$ & $10: 45$ & 69 & 32 \\
\hline BY59 & Greenhorn Cr at You Bet Rd nr Nevada City & $1 / 25 / 00$ & $12: 00$ & 64 & 715 \\
\hline BY59 & Greenhorn Cr at You Bet Rd nr Nevada City & $7 / 24 / 01$ & 11:00 & - & 4 \\
\hline BY59 & Greenhorn Cr at You Bet Rd nr Nevada City & $8 / 29 / 01$ & $15: 00$ & 50 & 1 \\
\hline BY59 & Greenhorn Cr at You Bet Rd nr Nevada City & $9 / 14 / 01$ & 13:00 & 67 & 0 \\
\hline BY59 & Greenhorn Cr at You Bet Rd nr Nevada City & $10 / 15 / 01$ & $12: 00$ & 29 & 1 \\
\hline BY59 & Greenhorn Cr at You Bet Rd nr Nevada City & $11 / 20 / 01$ & $13: 20$ & 75 & 3 \\
\hline BY59 & Greenhorn Cr at You Bet Rd nr Nevada City & $12 / 10 / 01$ & $12: 20$ & 52 & 63 \\
\hline BY59 & Greenhorn Cr at You Bet Rd nr Nevada City & $12 / 21 / 01$ & 13:00 & 92 & 266 \\
\hline BY51 & Greenhorn Cr nr headwaters nr Scotts Flat Reservoir & $1 / 25 / 00$ & $9: 30$ & 86 & 1 \\
\hline BY91 & Poore Mine tunnel effluent nr Grass Valley & $12 / 15 / 99$ & $13: 30$ & 43 & 7 \\
\hline BY91 & Poore Mine tunnel effluent nr Grass Valley & $5 / 23 / 00$ & $12: 00$ & - & - \\
\hline BY105 & Sailor Flat Mine main drain Gulch 01 nr Quaker Hill & $7 / 6 / 99$ & $16: 00$ & - & - \\
\hline BY105 & Sailor Flat Mine main drain Gulch 01 nr Quaker Hill & $12 / 2 / 99$ & $14: 30$ & 58 & 1,369 \\
\hline BY106 & Sailor Flat Mine main drainage to Greenhorn $\mathrm{Cr}$, Gulch 03 , & 4/9/01 & $12: 30$ & 87 & 3 \\
\hline BY114 & SF Greenhorn Cr 0.7 mi ab Greenhorn Cr nr Nevada City & $8 / 22 / 00$ & $15: 10$ & - & 1 \\
\hline BY115 & SF Greenhorn Cr bl Boston Mine nr Nevada City & $12 / 21 / 01$ & $12: 40$ & 94 & 7 \\
\hline BY122 & Starr Mine tunnel inflow nr Grass Valley & 4/23/99 & $15: 00$ & - & 3 \\
\hline BY123 & Starr Mine tunnel midway nr Grass Valley & 4/23/99 & $13: 40$ & - & 12 \\
\hline BY123 & Starr Mine tunnel midway nr Grass Valley & $12 / 15 / 99$ & $10: 45$ & 56 & 20 \\
\hline BY124 & Starr Mine tunnel outlet nr Grass Valley & $8 / 23 / 00$ & $17: 30$ & - & - \\
\hline BY129 & Tom and Jerry Mine drain at tunnel inlet nr Nevada City & $6 / 28 / 00$ & $12: 00$ & 83 & 308 \\
\hline BY130 & Tom and Jerry Mine drain at tunnel outlet nr Nevada City & $6 / 28 / 00$ & $11: 30$ & 67 & 6 \\
\hline BY131 & Tom and Jerry Mine drainage pond nr Nevada City & $8 / 23 / 00$ & $10: 45$ & - & 12 \\
\hline \multicolumn{6}{|c|}{ Disturbed samples } \\
\hline BY123 & Starr Mine tunnel midway nr Grass Valley & $12 / 15 / 99$ & $11: 30$ & 60 & 8,781 \\
\hline BY129 & Tom and Jerry Mine drain at tunnel inlet nr Nevada City & $6 / 28 / 00$ & $12: 30$ & 97 & 6,039 \\
\hline
\end{tabular}




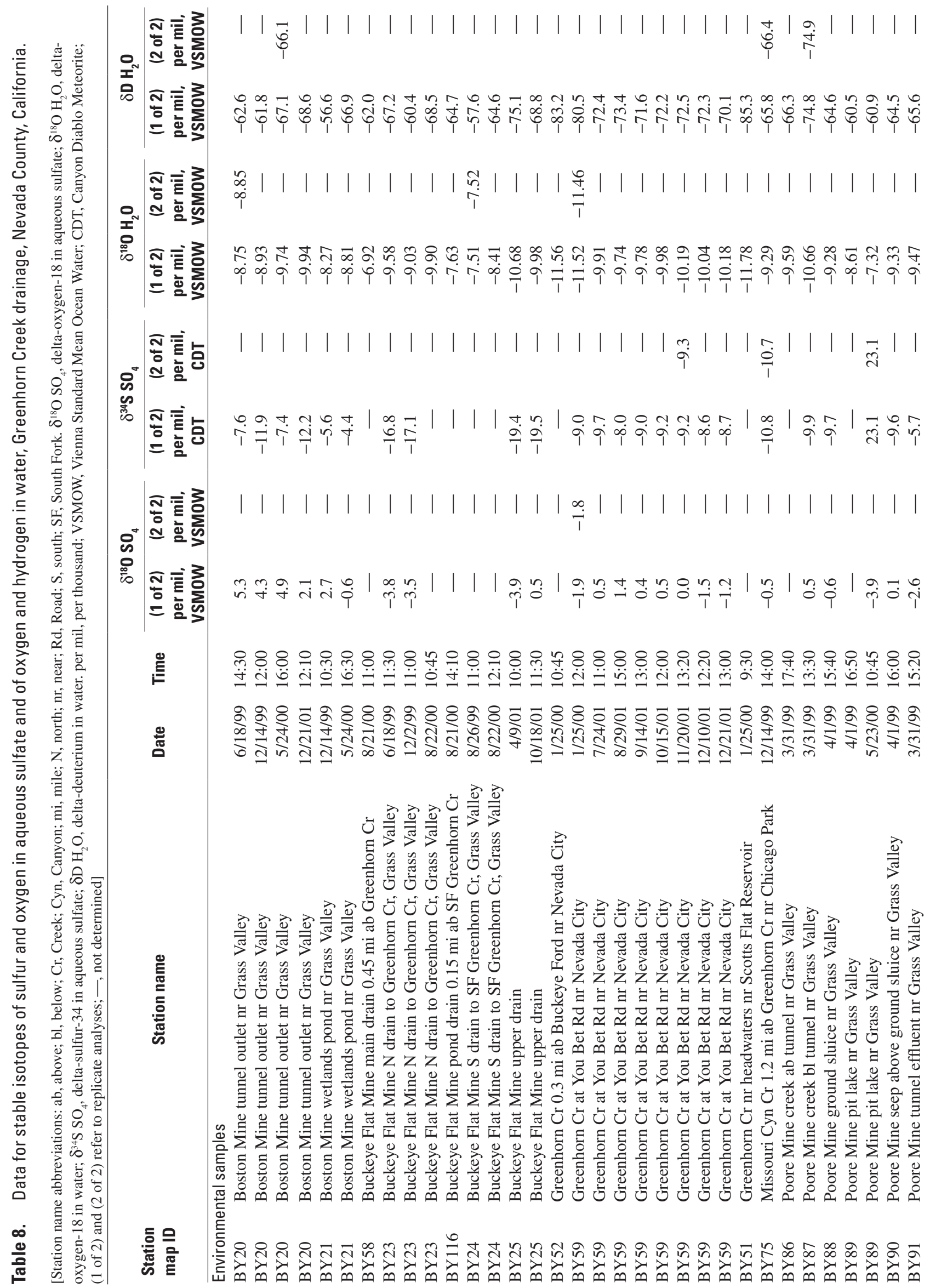




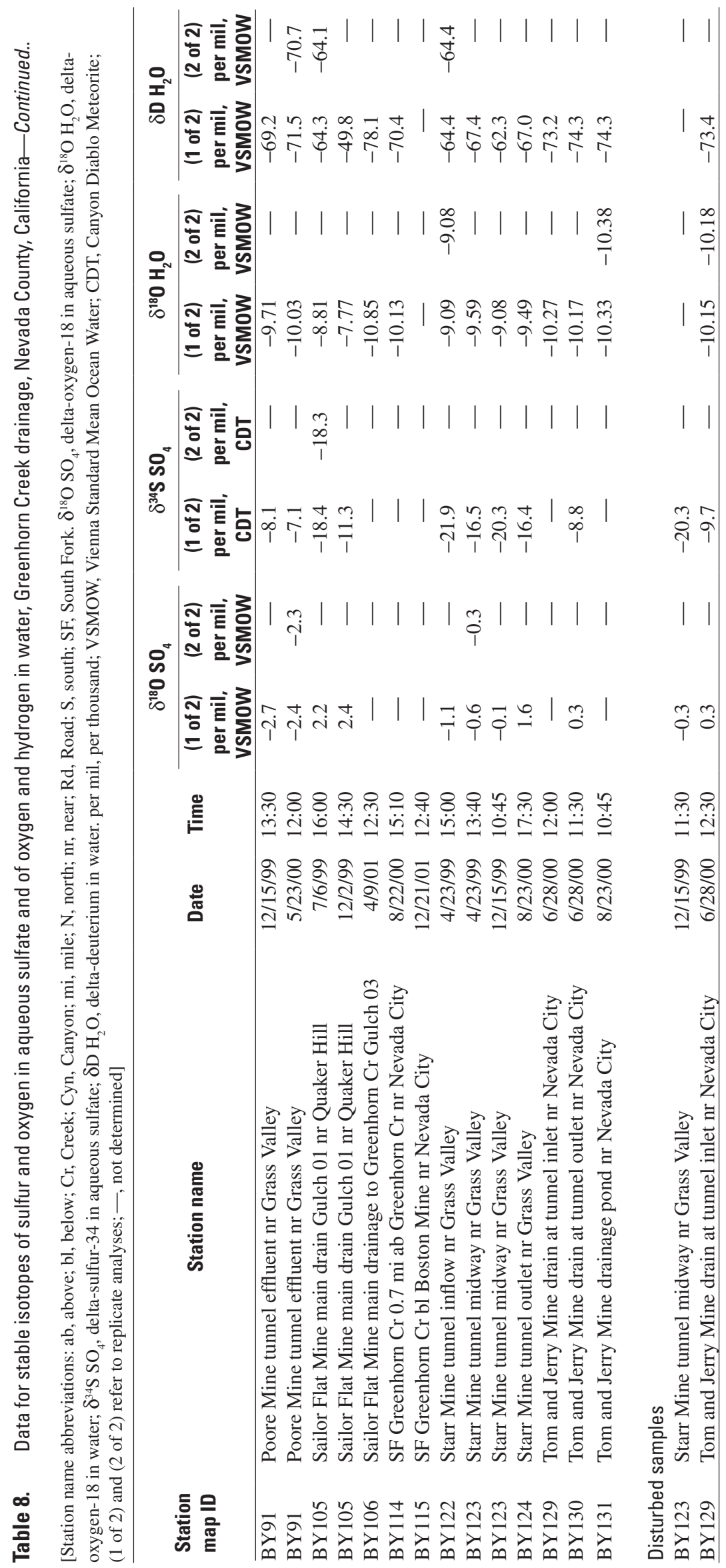


The overall distribution of mercury concentrations in the environmental water samples collected for this study is shown in figure 6 for the following mercury analytes and matrices: $\mathrm{THg}$ in unfiltered water, $\mathrm{THg}$ in filtered water, $\mathrm{MeHg}$ in unfiltered water, and $\mathrm{MeHg}$ in filtered water. Concentrations of $\mathrm{THg}$ in unfiltered water (table 2) are the highest of these four types of mercury samples. The range of concentrations of $\mathrm{THg}$ in the unfiltered, environmental water samples was 0.80 to 153,000 nanograms per liter (ng/L) with a median value of $9.6 \mathrm{ng} / \mathrm{L}$. Total mercury concentrations in filtered water (table 2) ranged from $<0.3$ to $8,000 \mathrm{ng} / \mathrm{L}$ with a median value of $2.7 \mathrm{ng} / \mathrm{L}$. Concentrations of $\mathrm{MeHg}$ in the unfiltered water (table 3) ranged from $<0.04$ to $9.1 \mathrm{ng} / \mathrm{L}$ in 40 undisturbed (environmental) samples, with a median value of $0.07 \mathrm{ng} / \mathrm{L}$. Methylmercury in filtered water (12 samples) ranged from $<0.04$ to $0.27 \mathrm{ng} / \mathrm{L}$ with a median value of $0.04 \mathrm{ng} / \mathrm{L}$.

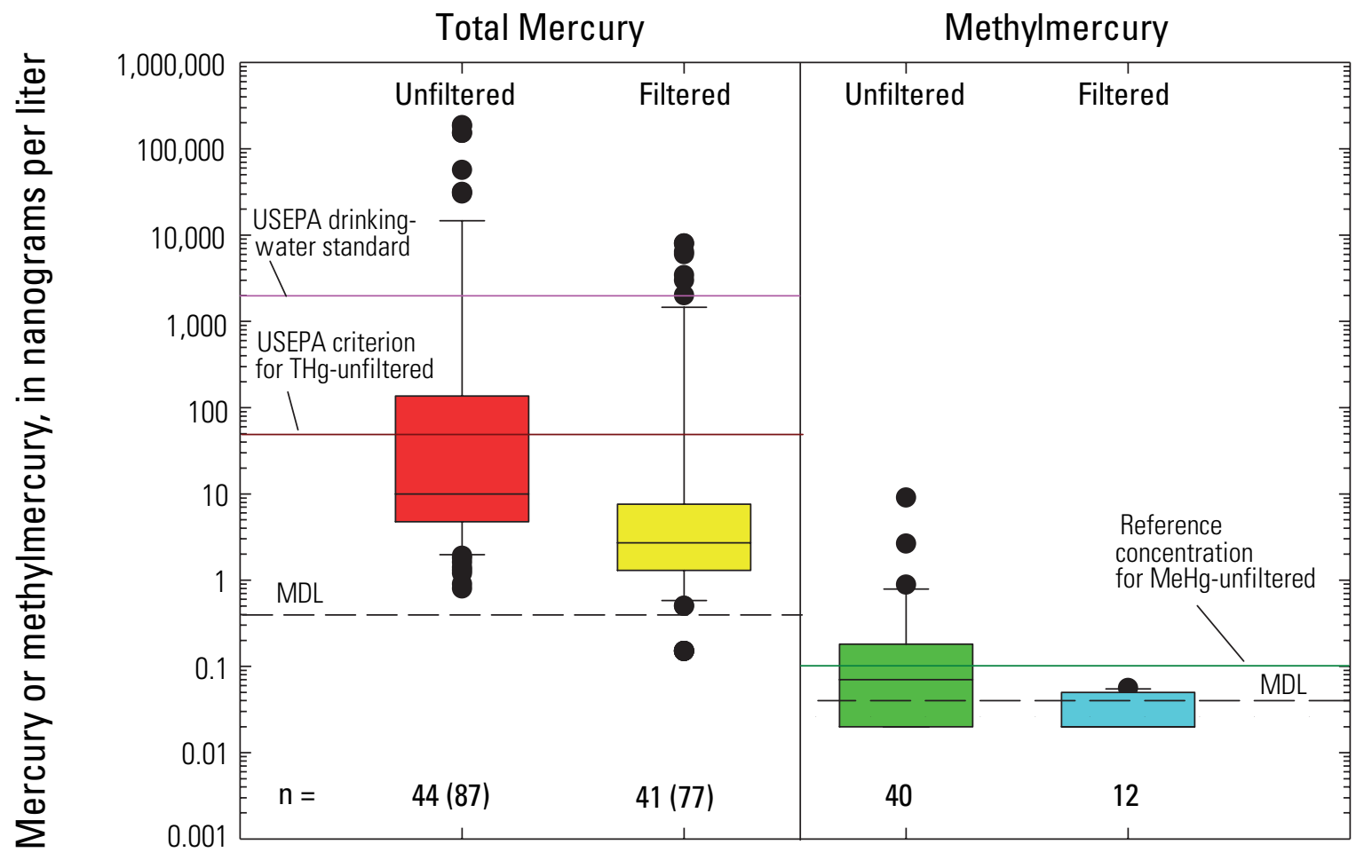

\section{EXPLANATION}

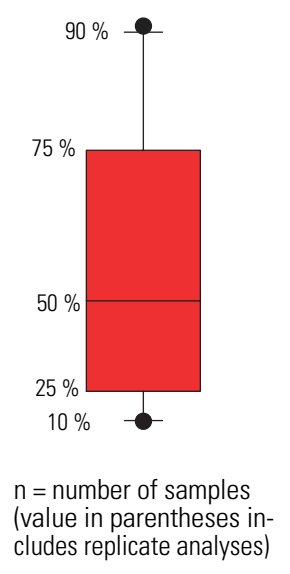

Figure 6. Box plots showing distribution of total mercury concentration and methylmercury concentration in unfiltered and filtered water samples from the Greenhorn Creek drainage, Nevada County, California. Non-detects plotted at 50 percent of method detection limit (MDL). USEPA, U.S. Environmental Protection Agency; THg, total mercury; MeHg, methylmercury. USEPA criterion for THg-unfiltered from U.S. Environmental Protection Agency (1999). USEPA drinking water standard from U.S. Environmental Protection Agency (2003). Reference concentration for MeHg-unfiltered from Rudd (1995). 
The disturbed water sample from the Starr Mine tunnel contained extremely high concentrations of unfiltered $\mathrm{THg}$ (between 400,000 and 500,000 ng/L in two replicate analyses) and unfiltered $\mathrm{MeHg}$ (244 ng/L). Concentrations of this magnitude were related to increased concentrations of suspended sediment associated with human activity and likely persisted only a short time after cessation of the disturbance. Evidence of recent human disturbance was commonly observed in most of the mine tunnels visited for this study. The most likely type of disturbance apparently was related to attempted extraction of residual gold and gold-mercury amalgam in tunnel sluices. Based on observations while working in the tunnels, it is likely that discharge of mercury and $\mathrm{MeHg}$ is greatly increased by the release of suspended sediment during such disturbance.

The applicable aquatic-life water-quality criterion from the California Toxics Rule for $\mathrm{THg}$ in unfiltered water is 50 ng/L (U.S. Environmental Protection Agency, 1999). The federal drinking-water standard for THg is 2,000 ng/L (U.S. Environmental Protection Agency, 2003). Although there is currently no water-quality criterion for $\mathrm{MeHg}$ concentration in water, a reference concentration of $0.1 \mathrm{ng} / \mathrm{L}$ is noted in the literature as an indicator of non-pristine conditions likely to result in $\mathrm{MeHg}$ bioaccumulation above baseline levels (Rudd, 1995).

Comparisons of the distribution of mercury and $\mathrm{MeHg}$ concentrations in four types of water bodies (tunnels; ground sluices; pit lakes, ponds, or wetlands; and streams) are shown in figure $7 A-C$. Total mercury in unfiltered water in tunnels (fig. 7A) had the highest median concentration; more than half of the samples from tunnels had concentrations above the 50 ng/L water-quality criterion. Total mercury in filtered water (fig. 7B) had median concentrations in a narrow range (2 to $4 \mathrm{ng} / \mathrm{L})$ in all four water-body types. Elevated concentrations $(>1,000 \mathrm{ng} / \mathrm{L})$ of $\mathrm{THg}$ in filtered samples were observed in some of the samples from tunnels and ground sluices. The medians for $\mathrm{MeHg}$ concentrations in unfiltered water in samples from tunnels and from ground sluices (fig. 7C) were near the reference concentration of $0.1 \mathrm{ng} / \mathrm{L}$; the median $\mathrm{MeHg}$ concentrations in unfiltered samples from pit lakes, ponds, or wetlands and from streams were less than those for the other water-body types, but greater than the MDL of $0.04 \mathrm{ng} / \mathrm{L}$. The medians for the ratio of $\mathrm{MeHg}$ to $\mathrm{THg}(\mathrm{MeHg} / \mathrm{THg})$ in unfiltered water samples (fig. 8) ranged from 0.3 to 1.2 percent for the four water-body types. The highest value of $\mathrm{MeHg} / \mathrm{THg}$ (30 percent) was found in a sample from the wetlands at the Boston Mine (station BY21 on fig. 4).

Analyses of $\mathrm{THg}$ and $\mathrm{MeHg}$ in filtered and unfiltered subsamples provide information on the suspended particulate concentration of these constituents. Plots showing the relation between concentrations of the same analyte in unfiltered and filtered subsamples can be used to display the proportion of the analyte that passed through the filter. A secondary use of such plots is for quality control, to identify filtered values that are apparently higher than the unfiltered values. A plot of THg in unfiltered and filtered subsamples (fig. 9) shows that 10 to 100 percent of the $\mathrm{THg}$ in most samples passed through the filter. A single outlier from station BY 115 has been screened out because one of the filtered subsamples was likely contaminated. A plot of $\mathrm{MeHg}$ concentrations in unfiltered and filtered subsamples (fig. 10) indicates similarly that 10 to 100 percent of the $\mathrm{MeHg}$ in most samples passed through the filter. An outlier for MeHg (station BY59) also was screened out because the filtered subsample was likely contaminated. The screened out data points also were excluded from the station averages discussed below.

Plots showing the relation between $\mathrm{THg}$ and $\mathrm{MeHg}$ for various media, including water, sediment, and biota, show the proportion of mercury that is in the methyl form as a function of total mercury concentration. This relation is shown for unfiltered water samples in figure 11. Diagonal lines represent constant values of $\mathrm{MeHg} / \mathrm{THg}$. Values of $\mathrm{MeHg} / \mathrm{THg}$ in unfiltered water samples ranged from 0.001 to 30 percent, with most values ranging from 0.01 to 5 percent. A similar plot showing the relation between $\mathrm{THg}$ and $\mathrm{MeHg}$ in filtered water samples (fig. 12) indicates that most values of $\mathrm{MeHg} / \mathrm{THg}$ in filtered samples range from about 0.1 to less than 10 percent.

The surface waters of the Greenhorn Creek drainage had a relatively wide range of $\mathrm{pH}$ values (3.4 to 8.0, table 1). Sulfate is commonly the dominant anion in acidic waters and may also play a role in methylation of mercury through the action of sulfate-reducing bacteria (Gilmour and others, 1992). Therefore, the relations among $\mathrm{pH}$ and the concentrations of sulfate, mercury, and $\mathrm{MeHg}$ may be important in determining processes that control water quality in this environmental setting. The relation between $\mathrm{pH}$ and sulfate (fig. 13) indicates a wide scatter of values; the data trend toward higher sulfate concentrations at lower $\mathrm{pH}$, typical of acid mine drainage. The most acidic samples, which also have the highest sulfate concentration (>100 mg/L), are from the Buckeye area. The Headwaters station (BY51) has the least sulfate $(<1 \mathrm{mg} / \mathrm{L}$, table 5) and has near-neutral $\mathrm{pH}(6.7$, table 1$)$. Other samples that are near-neutral in $\mathrm{pH}$ (6 to 8 ) have sulfate concentrations in the range of 3 to $80 \mathrm{mg} / \mathrm{L} \mathrm{(fig.} \mathrm{13,} \mathrm{tables} 1$ and 5). 

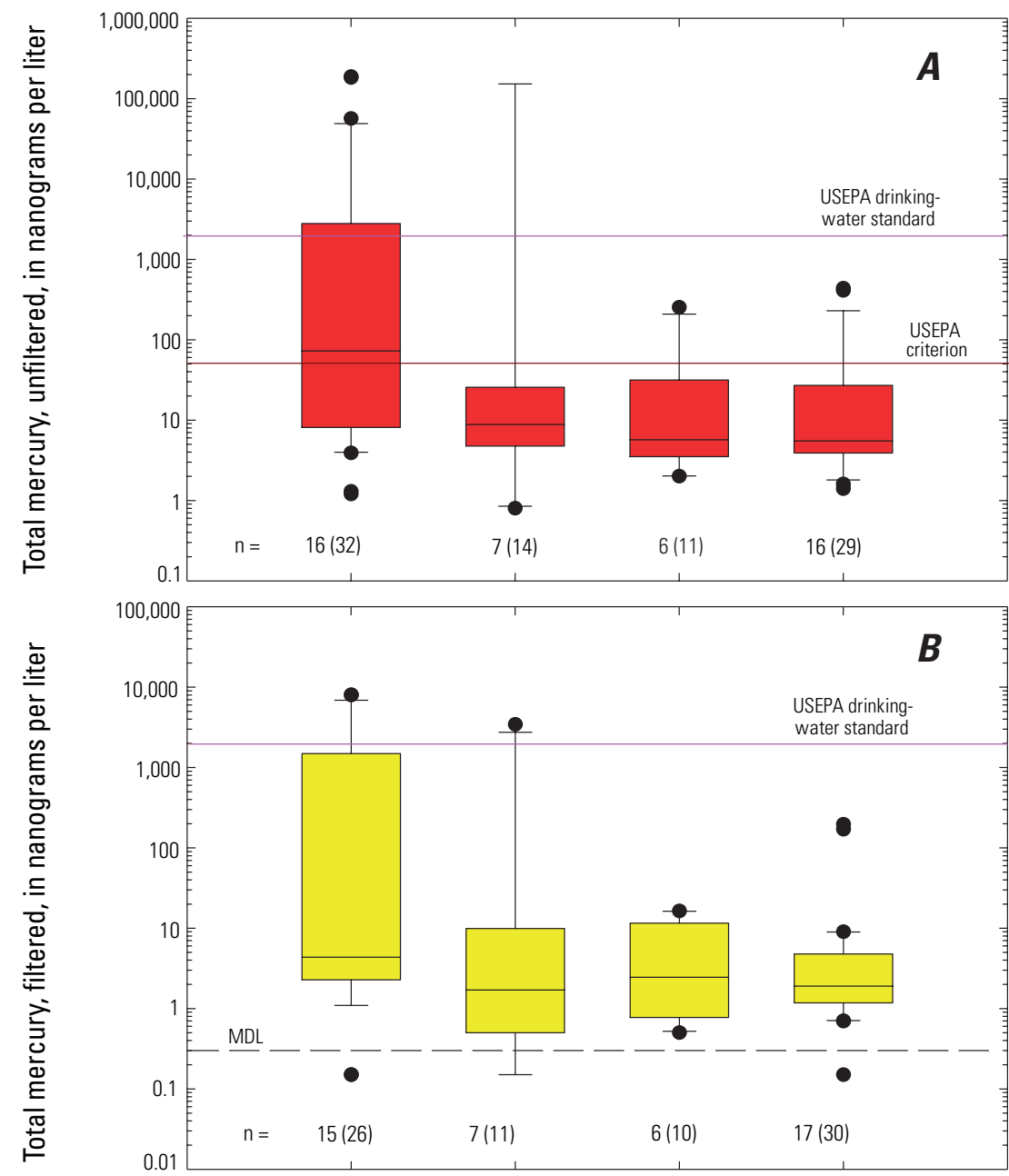

\section{EXPLANATION}

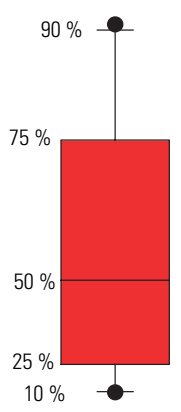

$n=$ number of samples (value in parentheses includes replicate analyses)

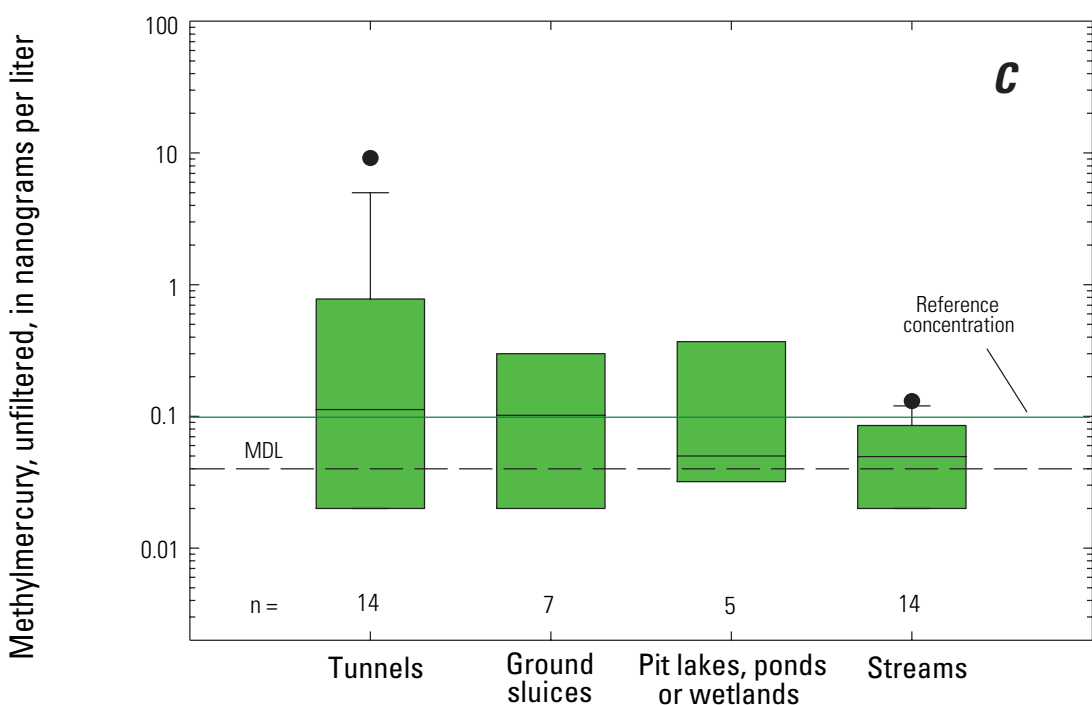

Figure 7. Box plots showing distribution of $(A)$ total mercury concentration in unfiltered water, $(B)$ total mercury concentration in filtered water, and $(C)$ methylmercury concentration in unfiltered water, grouped by type of water body, Greenhorn Creek drainage, Nevada County, California. Non-detects plotted at 50 percent of method detection limit (MDL). USEPA, U.S. Environmental Protection Agency; THg, total mercury; MeHg, methylmercury. USEPA criterion for THg-unfiltered from U.S. Environmental Protection Agency (1999). USEPA drinking water standard from U.S. Environmental Protection Agency (2003). Reference concentration for MeHg-unfiltered from Rudd (1995). 
The relations between $\mathrm{pH}$ in unfiltered water and the concentrations of various forms of mercury in unfiltered and filtered water are shown in figure 14. No clear pattern is evident in the relation between $\mathrm{pH}$ and $\mathrm{THg}$ in unfiltered water (fig. 14A); the samples with highest mercury concentrations were in the $\mathrm{pH}$ range of 4 to 5.5; the most acidic samples $(\mathrm{pH}<4)$ had relatively low $\mathrm{THg}$ concentrations $(<10 \mathrm{ng} / \mathrm{L})$. For $\mathrm{THg}$ in filtered water, the $\mathrm{pH}$ of samples having elevated concentrations of $\mathrm{THg}(>1,000 \mathrm{ng} / \mathrm{L})$ ranges from 4.0 to 6.3 (fig. 14B); it is not known whether all of the mercury passing through the $0.45-\mu \mathrm{m}$ pore-size filters is truly dissolved; some of it may in the form of colloidal particles. Relatively high concentrations of $\mathrm{MeHg}$ (unfiltered), above the reference concentration of $0.1 \mathrm{ng} / \mathrm{L}$, span the $\mathrm{pH}$ range of 4 to 7.7 , nearly the full range encountered in the Greenhorn Creek drainage, and values of $\mathrm{MeHg}$ below the detection limit $(0.04 \mathrm{ng} / \mathrm{L})$ span the entire range of $\mathrm{pH}$ (3.5 to 8.0) (fig. 14C).

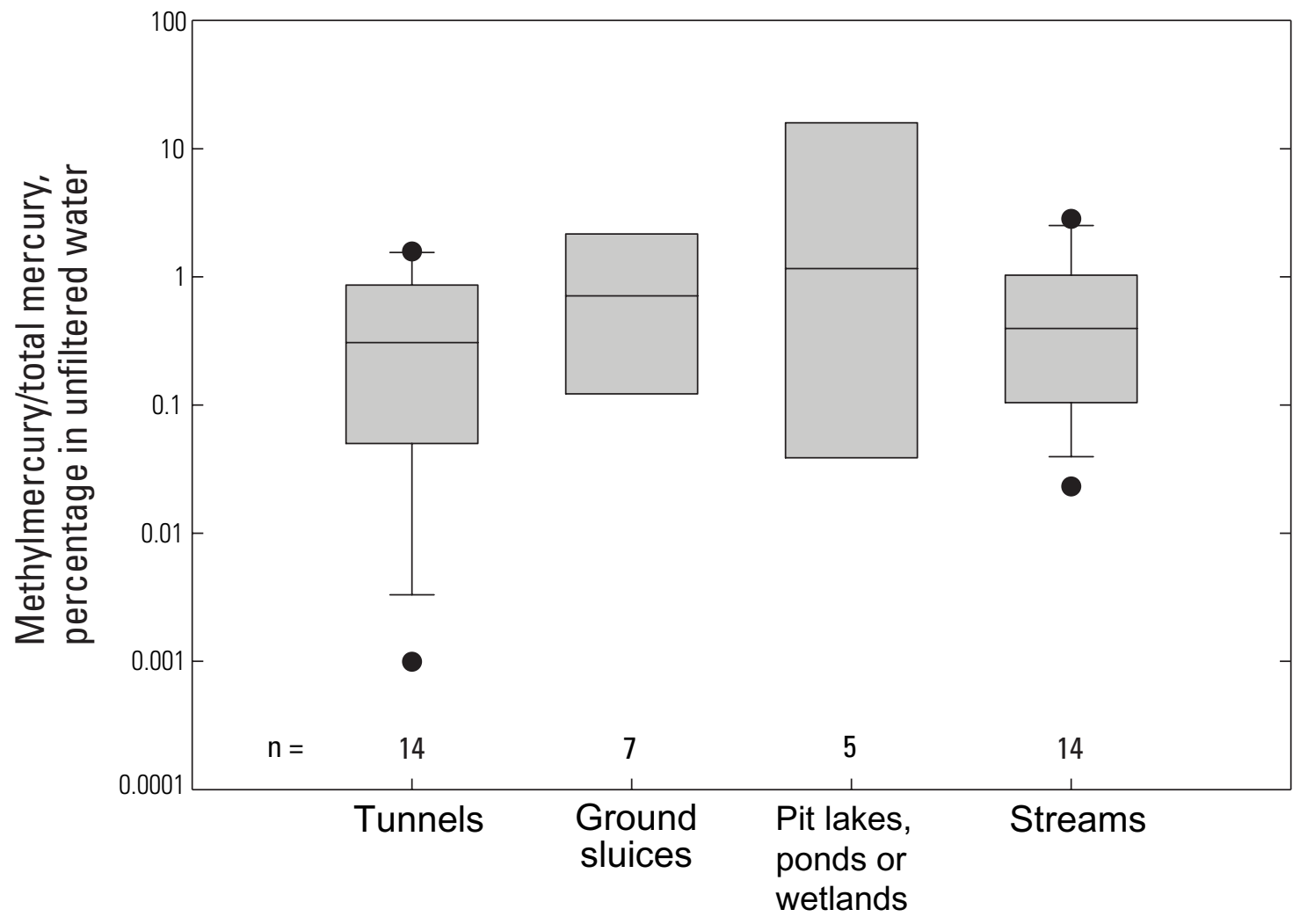

EXPLANATION

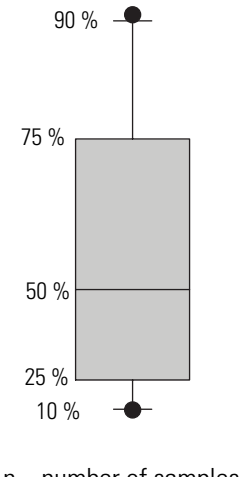

Figure 8. Box plots showing distribution of the ratio of methylmercury to total mercury in unfiltered water samples grouped by type of water body, Greenhorn Creek drainage, Nevada County, California. 
Elevated concentrations of several trace elements were found in filtered water samples from some of the sampling stations. These trace elements tend to be most highly concentrated in samples with the lowest $\mathrm{pH}$ values. Three water samples from a station in the Buckeye area (BY23) were among the most acidic ( $\mathrm{pH}$ values ranging from 3.7 to 4.2 ) and were consistently among the most metal rich, with concentrations in filtered water of aluminum from 3,900 to $4,100 \mu \mathrm{g} / \mathrm{L}$, cadmium from 1.2 to $2.6 \mu \mathrm{g} / \mathrm{L}$, copper from 28 to $48 \mu \mathrm{g} / \mathrm{L}$, iron from 130 to $4,500 \mu \mathrm{g} / \mathrm{L}$, manganese from 1,700 to 3,400 $\mu \mathrm{g} / \mathrm{L}$, nickel from 140 to $240 \mu \mathrm{g} / \mathrm{L}$, and zinc from 290 to $560 \mu \mathrm{g} / \mathrm{L}$ (table $4 B$ [see back of report]) Three other sampling stations in the Buckeye area (BY24, 25, and 58) also had relatively acidic water ( $\mathrm{pH}$ values 3.4 to 5.6 ) with some anomalously high trace metal concentrations (table $4 B$ ). Water

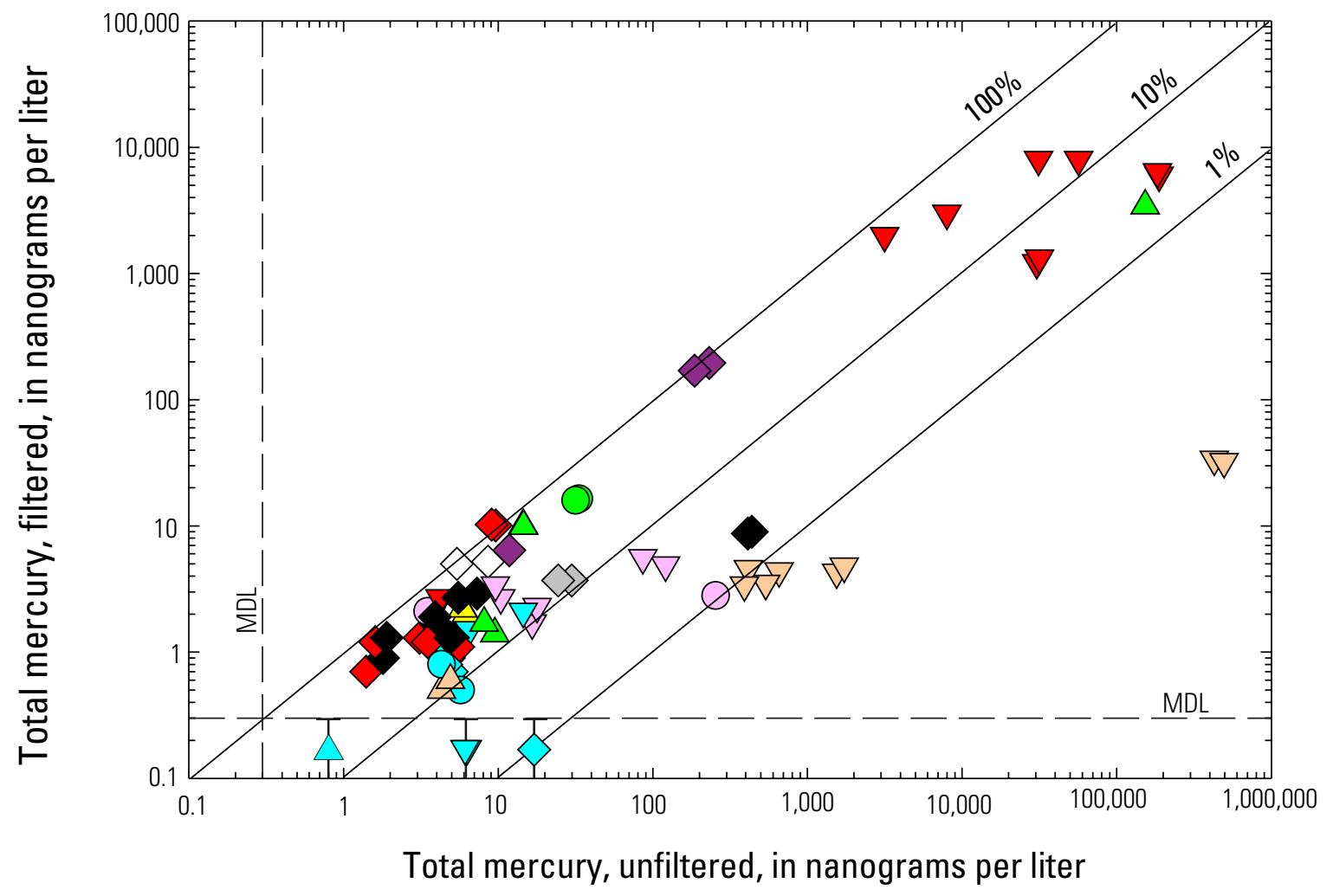

EXPLANATION

\begin{tabular}{lll}
\multicolumn{1}{c}{ Station group } & \multicolumn{2}{c}{ Type of sampling station } \\
$\square$ Boston & $\square$ Missouri Canyon & $\triangle$ Ground sluice \\
$\square$ Buckeye & $\bigcirc$ Poore & $\bigcirc$ Pit lake/pond/wetland \\
$\square$ Greenhorn, main stem & SF Greenhorn & $\searrow$ Stream \\
$\square$ Headwaters & $\square$ Sailor Flat & $\nabla$ Tunnel \\
$\square$ Integrator & $\square$ Starr &
\end{tabular}

Figure 9. Relation between total mercury concentration in unfiltered and filtered water samples, Greenhorn Creek drainage, Nevada County, California. Diagonal lines represent percentage of mercury passing through filter. Non-detects plotted at 50 percent of method detection limit (MDL). 
samples from two stations associated with a ground sluice at Poore Mine (BY88 and 90) had moderately high concentrations of iron (570 and $300 \mu \mathrm{g} / \mathrm{L}$, respectively) and manganese (390 to $400 \mu \mathrm{g} / \mathrm{L}$ ) at mildly acidic $\mathrm{pH}$ (5.8 and 5.5), but had relatively low concentrations of other trace metals. A water sample from the pit lake at Poore Mine (BY89) taken in May 2000 had a similar $\mathrm{pH}$ value (5.8) and lower iron concentration (32 to $33 \mu \mathrm{g} / \mathrm{L})$, but higher aluminum (130 $\mu \mathrm{g} / \mathrm{L})$, cadmium (1.1 $\mu \mathrm{g} / \mathrm{L})$, copper (11 to $12 \mu \mathrm{g} / \mathrm{L})$, manganese $(1,300 \mu \mathrm{g} / \mathrm{L})$, and zinc $(85 \mu \mathrm{g} / \mathrm{L})$ concentrations (table $4 B)$. Arsenic concentrations in filtered water were less than $1.0 \mu \mathrm{g} / \mathrm{L}$ at all sites except the Integrator site (BY59, Greenhorn Creek at You Bet Road), located downstream of the confluence with Little Greenhorn Creek, which drains a hardrock gold mining area including the Lava Cap Mine, where a tailings impoundment failed in January 1997, resulting in downstream arsenic contamination (CH2M Hill, 2001).

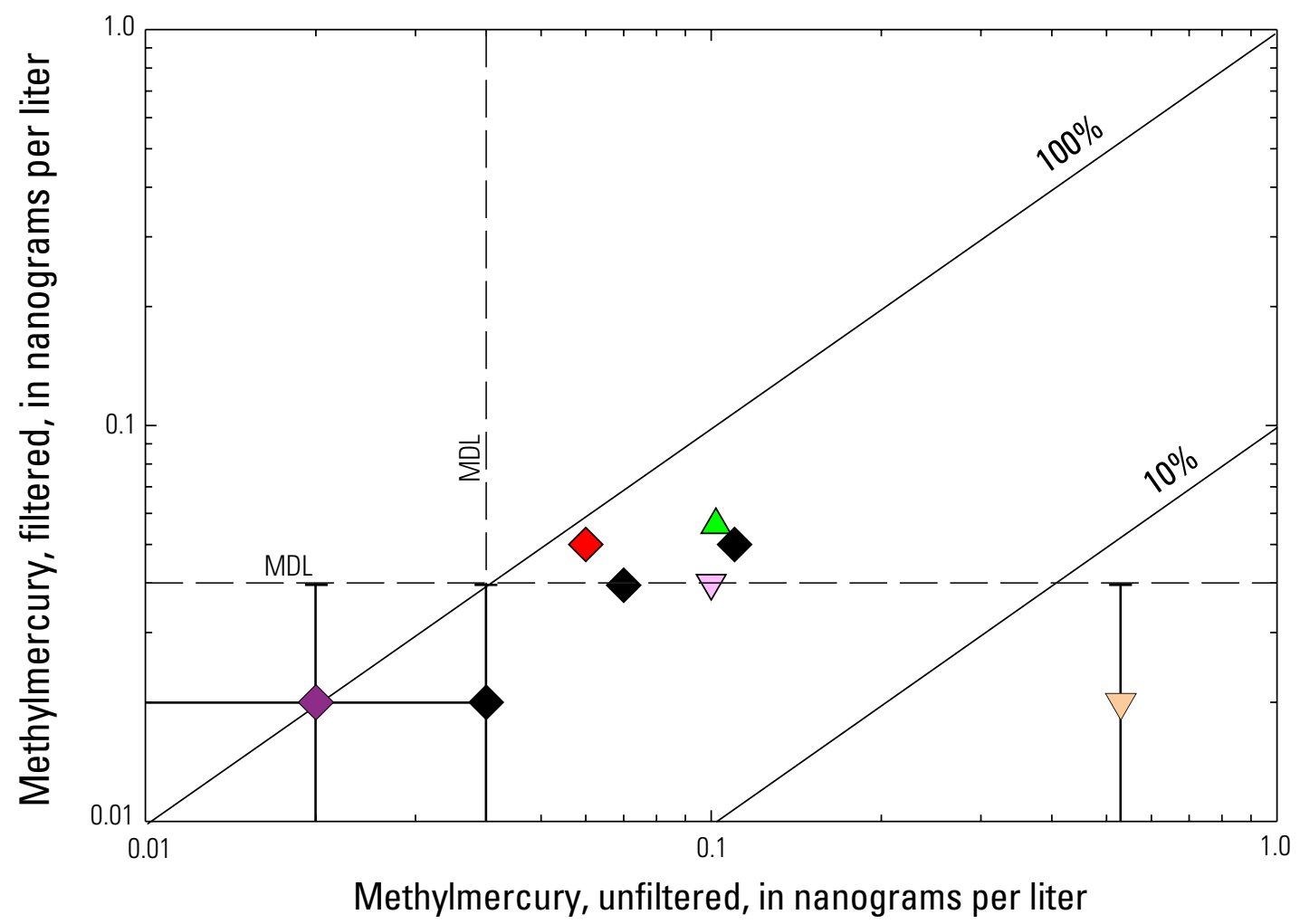

EXPLANATION

\begin{tabular}{lll}
\multicolumn{2}{c}{ Station group } & \multicolumn{2}{c}{ Type of sampling station } \\
$\square$ Boston & SF Greenhorn & $\triangle$ Ground sluice \\
Buckeye & $\square$ Sailor Flat & $\searrow$ Stream \\
Integrator & $\square$ Starr & $\nabla$ Tunnel
\end{tabular}

Figure 10. Relation between methylmercury concentration in unfiltered and filtered water samples, Greenhorn Creek drainage, Nevada County, California. Diagonal lines represent percentage of methylmercury passing through filter. Non-detects plotted at 50 percent of method detection limit (MDL). 
The relations between sulfate concentration and different forms of mercury are shown in figure 15. Concentrations of $\mathrm{THg}$ (unfiltered) above the water-quality criterion of $50 \mathrm{ng} / \mathrm{L}$ are in samples having sulfate concentrations between 3.0 and $170 \mathrm{mg} / \mathrm{L}$. Only the three most dilute water samples (two of which had replicates for $\mathrm{THg}$ ) having the lowest concentrations of sulfate $(<3 \mathrm{mg} / \mathrm{L})$, including the Headwaters sample, are in a range where elevated THg was not observed. The samples having elevated $\mathrm{THg}$ in filtered water $(>1,000 \mathrm{ng} / \mathrm{L}$; fig. $15 \mathrm{~B}$ ) had sulfate concentrations ranging from 6 to $170 \mathrm{mg} / \mathrm{L}$. Water samples with elevated $\mathrm{MeHg}$ concentrations (above the reference value of $0.1 \mathrm{ng} / \mathrm{L}$; fig. 15C) span nearly the full range of sulfate concentrations (2 to $120 \mathrm{mg} / \mathrm{L}$ ).

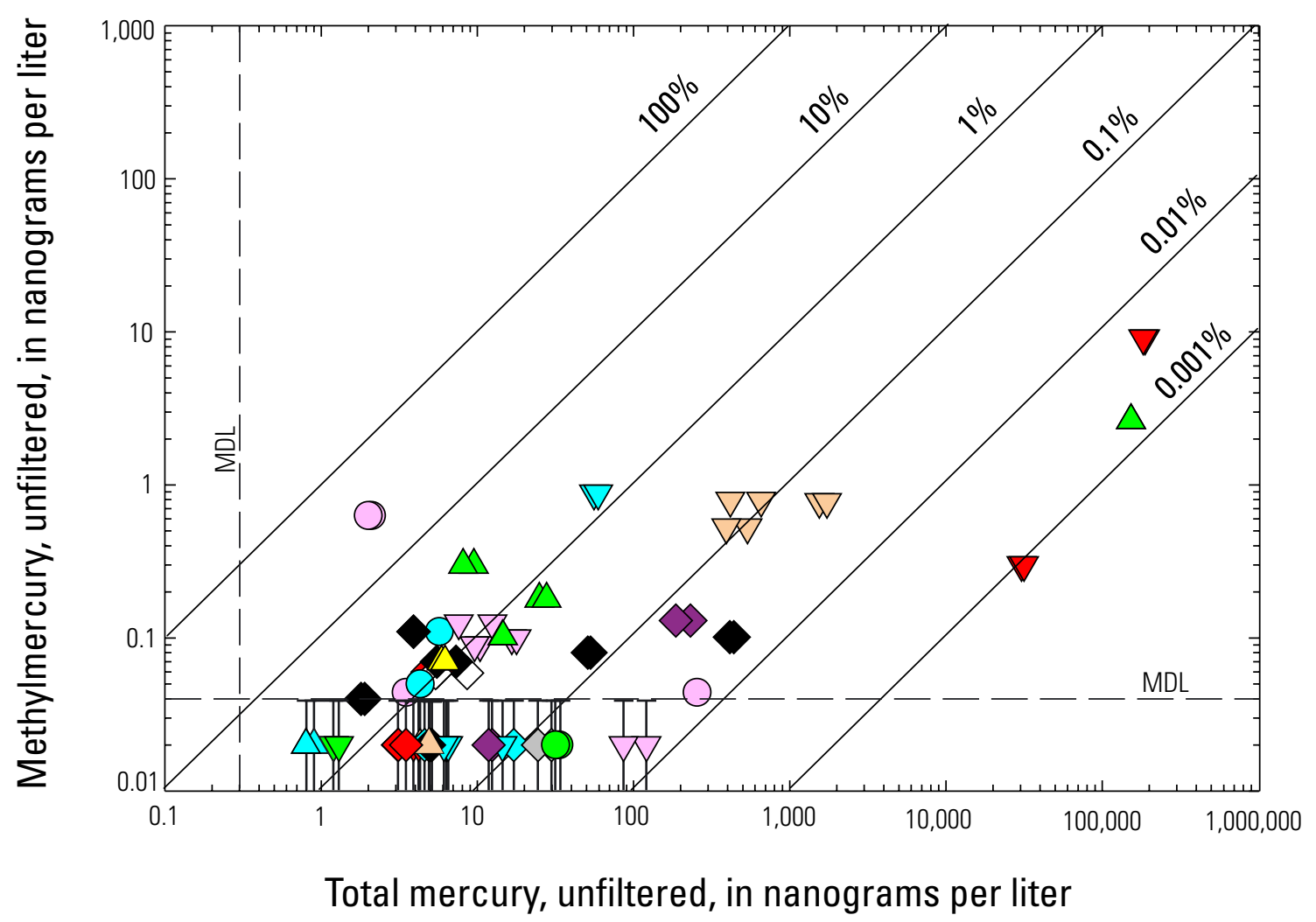

EXPLANATION

\begin{tabular}{llll}
\multicolumn{1}{c}{ Station group } & \multicolumn{2}{c}{ Type of sampling station } \\
$\square$ Boston & $\square$ Missouri Canyon & $\triangle$ Ground sluice \\
Buckeye & $\square$ Poore & $\bigcirc$ Pit lake/pond/wetland \\
$\square$ Greenhorn, main stem & $\square$ SF Greenhorn & $\searrow$ Stream \\
$\square$ Headwaters & $\begin{array}{l}\text { Sailor Flat } \\
\text { Integrator }\end{array}$ & $\nabla$ Tunnel
\end{tabular}

Figure 11. Relation between total mercury and methylmercury concentration in unfiltered water samples, Greenhorn Creek drainage, Nevada County, California. Diagonal lines represent constant values of the ratio of methylmercury to total mercury. Non-detects plotted at 50 percent of method detection limit (MDL). 
Scatter plots comparing the concentration of mercury in water with the concentration of suspended solids can be used to evaluate the range in concentration of mercury in the suspended solids. As a first approximation, one can use the concentration of mercury in unfiltered water for such plots, with the implicit assumption that all of the mercury is in particulate form (that is, that mercury passing through the filter represents fine particulates or colloids). On a plot of THg in unfiltered water versus total suspended sediment (fig. 16), the diagonal lines represent apparent concentrations of mercury in suspended material, assuming all of the mercury is in that form. Most of the data points for suspended sediment in figure 16 are clustered around the line representing 1 microgram per gram $(\mu \mathrm{g} / \mathrm{g})$, equivalent to 1 part per million (ppm) THg.
Data for nine samples (excluding replicates) plotted on figure 16 represent apparent mercury concentrations in suspended sediment in the range of $10 \mathrm{ppm}$ to $10,000 \mathrm{ppm}$ (equivalent to 1 percent by weight). Of these nine samples, six are from tunnel sites at the Boston Mine, the Starr Mine, and the Buckeye Flat Mine; the three non-tunnel sites are the wetland at Boston Mine, a ground sluice at Sailor Flat, and a stream station on South Fork Greenhorn Creek, which drains the area of the Boston Mine and the southern part of the Buckeye Flat Mine. The lowest apparent concentration of THg in suspended sediment based on the data in figure 16, represented by a pair of data points (two split replicates), is about $0.10 \mathrm{ppm}$, for a ground sluice at Sailor Flat.

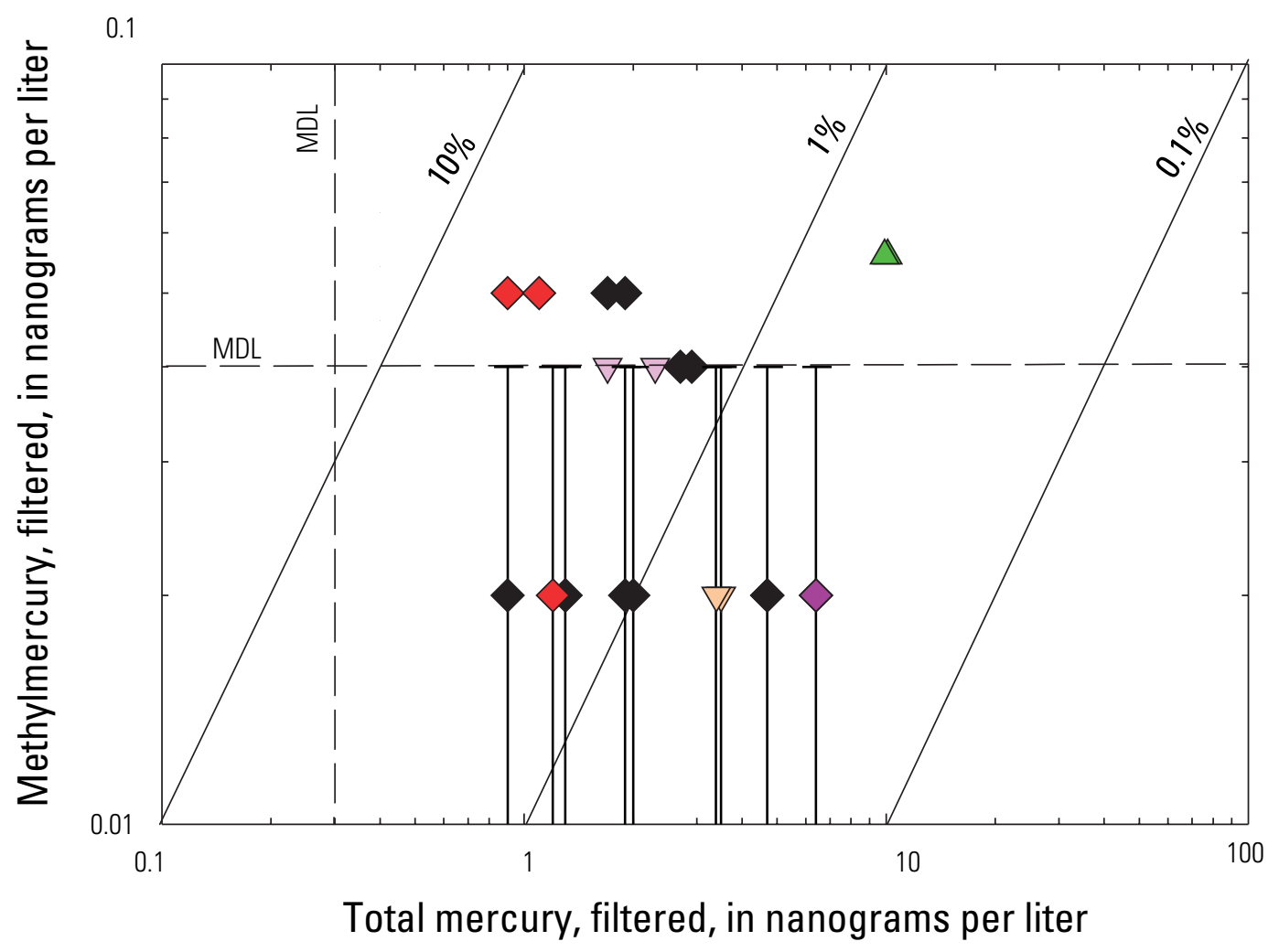

EXPLANATION

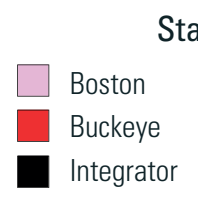

Station group

Type of sampling station

SF Greenhorn
Sailor Flat
$\square$ Starr

$\triangle$ Ground sluice
$\diamond$ Stream
$\nabla$ Tunnel

Figure 12. Relation between total mercury and methylmercury concentration in filtered water samples, Greenhorn Creek drainage, Nevada County, California. Diagonal lines represent constant values of the ratio of methylmercury to total mercury. Non-detects plotted at 50 percent of method detection limit (MDL). 
Determining the difference between the THg concentration in filtered and unfiltered samples (referred to as particulate mercury concentration) is another way to describe mercury in suspended particulate material in the Greenhorn Creek drainage. Particulate mercury concentrations are plotted as a function of total suspended sediment concentration (fig. 17) to evaluate the mercury content of particulate material that did not pass through the $0.45-\mu \mathrm{m}$ pore-diameter filters. For construction of fig. 17, geometric means of replicate analyses of $\mathrm{THg}$ in filtered and unfiltered water were calculated prior to calculating the particulate mercury concentrations. Comparison of data in figures 16 and 17 indicates a possible range of concentration for $\mathrm{THg}$ (unfiltered or particulate) in suspended sediment. No matter which assumptions are made, the apparent mercury content of suspended material at several sites, primarily tunnels, is in the range of ten to several thousand ppm.

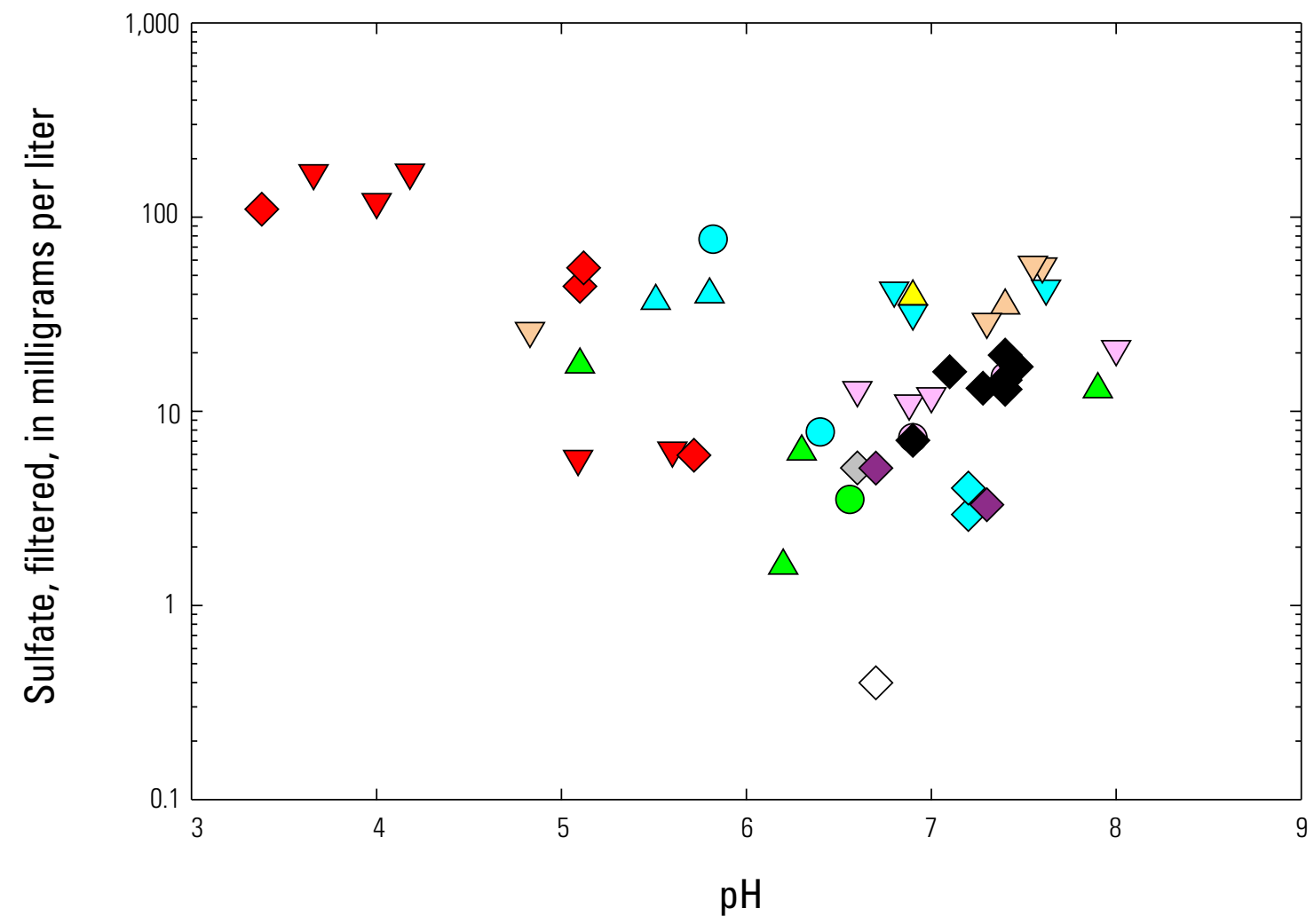

\section{EXPLANATION}

\begin{tabular}{ll}
\multicolumn{3}{c}{ Station group } & \\
$\square$ Boston & $\square$ Missouri Canyon \\
$\square$ Buckeye & $\square$ Poore \\
$\square$ Greenhorn, main stem & $\square$ SF Greenhorn \\
$\square$ Headwaters & Sailor Flat \\
$\square$ Integrator & Starr
\end{tabular}

Type of sampling station

$\triangle$ Ground sluice

Pit lake/pond/wetland

$\diamond$ Stream

$\nabla$ Tunnel

Figure 13. Relation between $\mathrm{pH}$ in unfiltered water and sulfate concentration in filtered water, Greenhorn Creek drainage, Nevada County, California. 

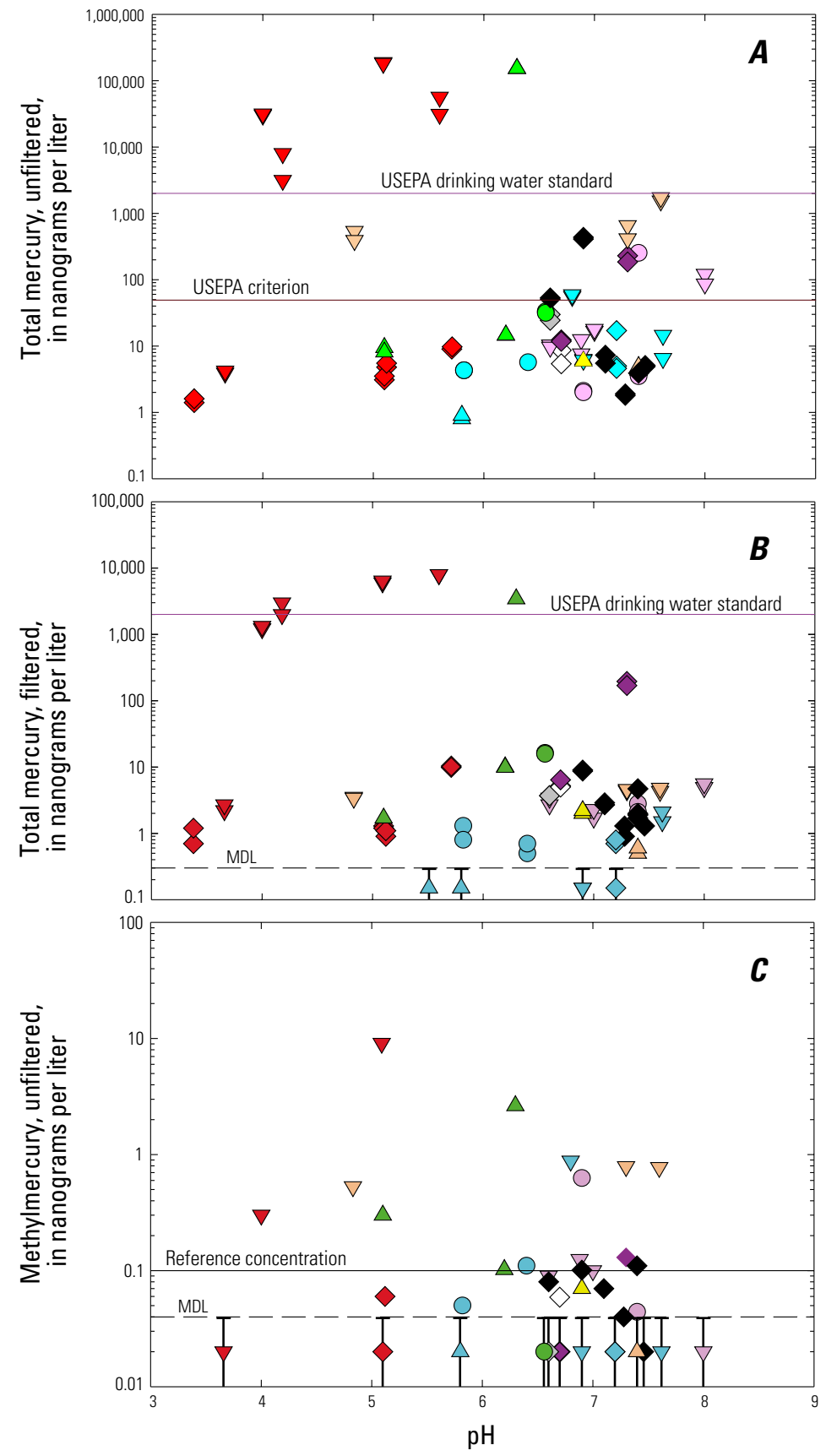

EXPLANATION

\begin{tabular}{lll}
\multicolumn{2}{c}{ Station group } & \multicolumn{2}{c}{ Type of sampling station } \\
$\square$ Boston & $\square$ Missouri Canyon & $\triangle$ Ground sluice \\
Buckeye & $\bigcirc$ Poore & $\bigcirc$ Pit lake/pond/wetland \\
$\square$ Greenhorn, main stem & SF Greenhorn & $\searrow$ Stream \\
$\square$ Headwaters & $\square$ Sailor Flat & $\nabla$ Tunnel \\
Integrator & $\square$ Starr &
\end{tabular}

Figure 14. Relation between $\mathrm{pH}$ and $(A)$ total mercury in unfiltered water, $(B)$ total mercury in filtered water, and $(C)$ methylmercury in unfiltered water, Greenhorn Creek drainage, Nevada County, California. USEPA criterion for total mercury (unfiltered) from U.S. Environmental Protection Agency (1999). USEPA drinking water standard from U.S. Environmental Protection Agency (2003). Reference concentration for MeHg (unfiltered) from Rudd (1995). 

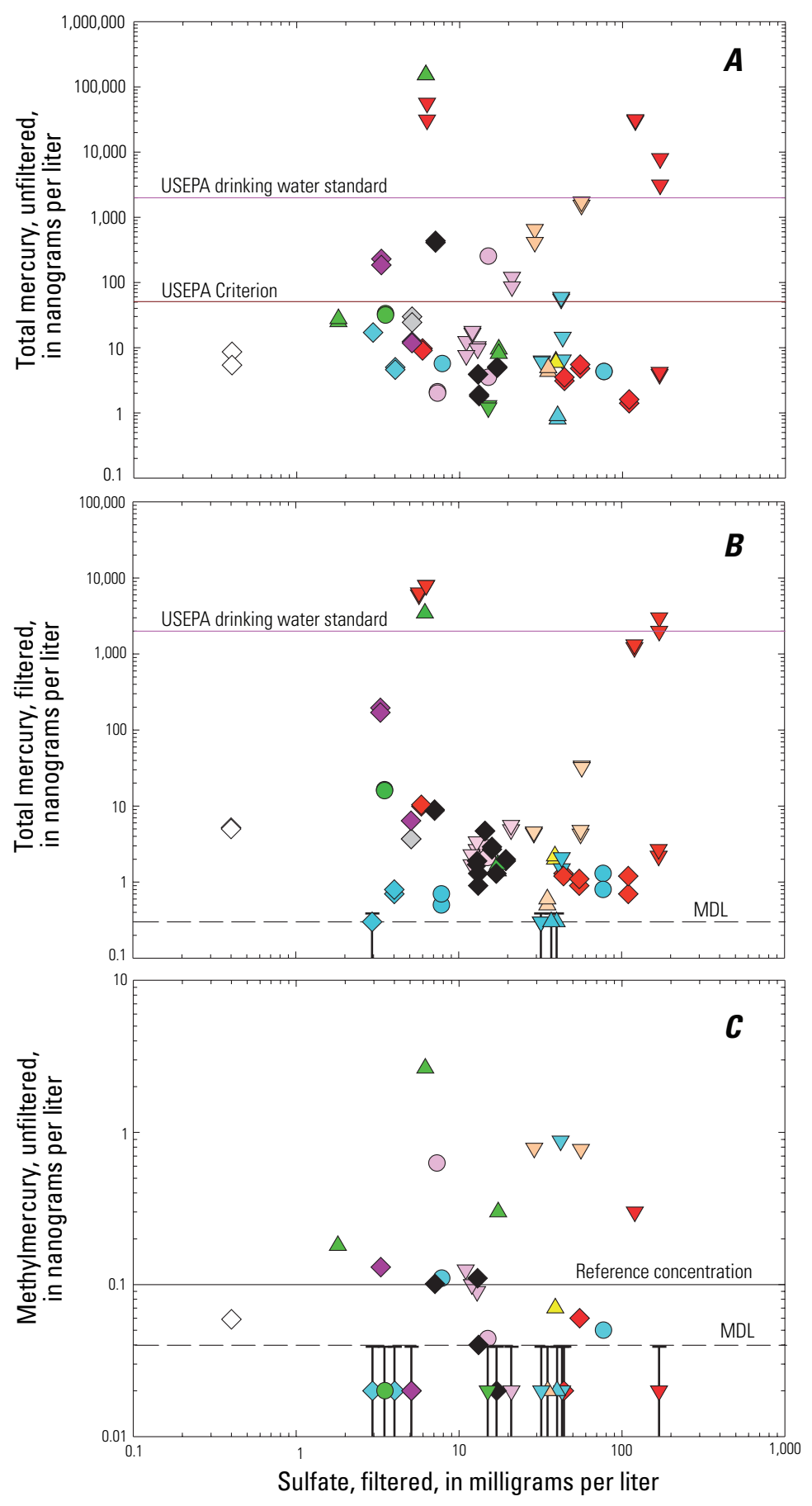

\begin{tabular}{lll}
\multicolumn{1}{c}{ Station group } & \multicolumn{1}{c}{ EXPLANATION } & \\
$\square$ Boston & & Type of sampling station \\
Buckeye & $\square$ Missouri Canyon & $\triangle$ Ground sluice \\
$\square$ Greenhorn, main stem & $\square$ S S Greenhorn & $\bigcirc$ Pit lake/pond/wetland \\
$\square$ Headwaters & $\square$ Sailor Flat & $\nabla$ Tunnel \\
$\square$ Integrator & $\square$ Starr &
\end{tabular}

Figure 15. Relation between sulfate concentration in filtered water and $(A)$ total mercury in unfiltered water, $(B)$ total mercury in filtered water, and (C) methylmercury in unfiltered water, Greenhorn Creek drainage, Nevada County, California. USEPA criterion for total mercury (unfiltered) from U.S. Environmental Protection Agency (1999). USEPA drinking water standard from U.S. Environmental Protection Agency (2003). Reference concentration for MeHg (unfiltered) from Rudd (1995). 


\section{Sediment}

Concentration data for $\mathrm{MeHg}$ and $\mathrm{THg}$ in sediment samples are given in tables 9 and 10. Laboratory analyses of $\mathrm{THg}$ and $\mathrm{MeHg}$ (table 9) are available for ten samples from eight stations. Total mercury concentrations ranged from about 0.0044 to $12 \mu \mathrm{g} / \mathrm{g}$ (microgram per gram, equivalent to $\mathrm{ppm}$ ). Methylmercury concentrations ranged from less than detection to $0.0095 \mu \mathrm{g} / \mathrm{g}$. Field panning methods were used to deter- mine the concentration of free, elemental mercury in sediment samples where the mercury was visible. The detection limit of the field balance was $100 \mathrm{mg}$, and the amount of mercury present when trace amounts of free mercury were observed is estimated at $100 \mathrm{mg} / \mathrm{kg}$ (milligram per kilogram, equivalent to part per million or ppm). Measured quantities of elemental mercury recovered by panning were as high as $45,000 \mathrm{mg} / \mathrm{kg}$ (4.5 per cent, by weight) at the Boston Mine tunnel outlet (station BY20).

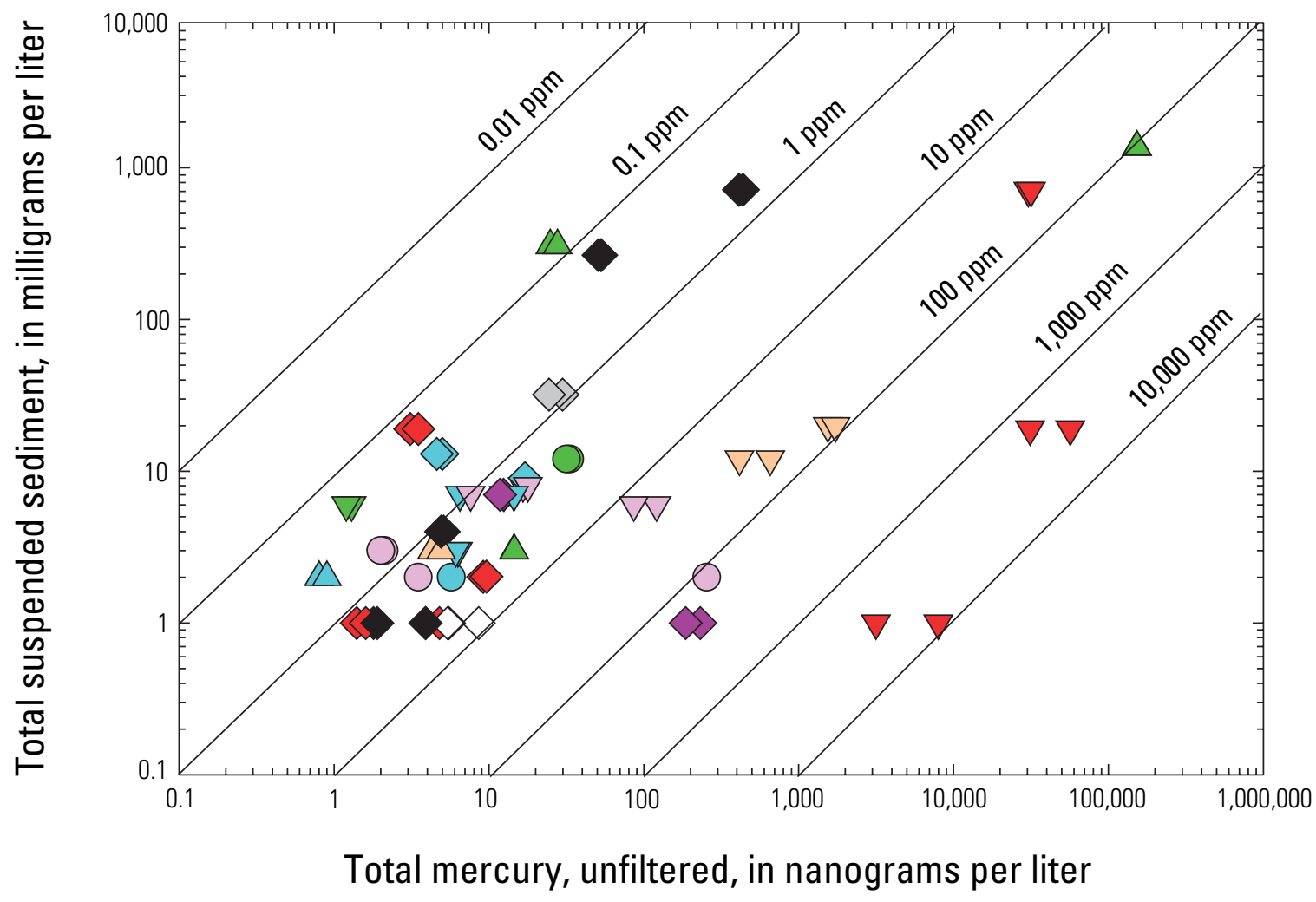

EXPLANATION

\begin{tabular}{lll}
\multicolumn{1}{c}{ Station group } & \multicolumn{2}{c}{ Type of sampling station } \\
$\square$ Boston & $\square$ Poore & $\triangle$ Ground sluice \\
$\square$ Buckeye & SF Greenhorn & $\bigcirc$ Pit lake/pond/wetland \\
$\square$ Greenhorn, main stem & $\square$ Sailor Flat & $\diamond$ Stream \\
$\square$ Headwaters & $\square$ Starr & $\nabla$ Tunnel \\
$\square$ Integrator & &
\end{tabular}

Figure 16. Relation between total mercury concentration in unfiltered water and total suspended sediment concentration, Greenhorn Creek drainage, Nevada County, California. The diagonal lines represent the apparent concentrations of mercury in suspended material. ppm, part per million. 
Box plots using a logarithmic vertical scale (fig. 18) show the wide range of concentrations: more than three orders of magnitude for $\mathrm{MeHg}$ and more than six orders of magnitude for THg (laboratory and field values). A scatter plot showing the relation between $\mathrm{THg}$ and $\mathrm{MeHg}$ in sediment samples (fig. 19) indicates that the ratio of $\mathrm{MeHg}$ to $\mathrm{THg}$ in the sediment samples ranged from less than 0.001 percent to about 3 percent. The station with the highest $\mathrm{MeHg}$ to $\mathrm{THg}$ ratio was associated with a wetland environment in the Boston Mine area (BY21).

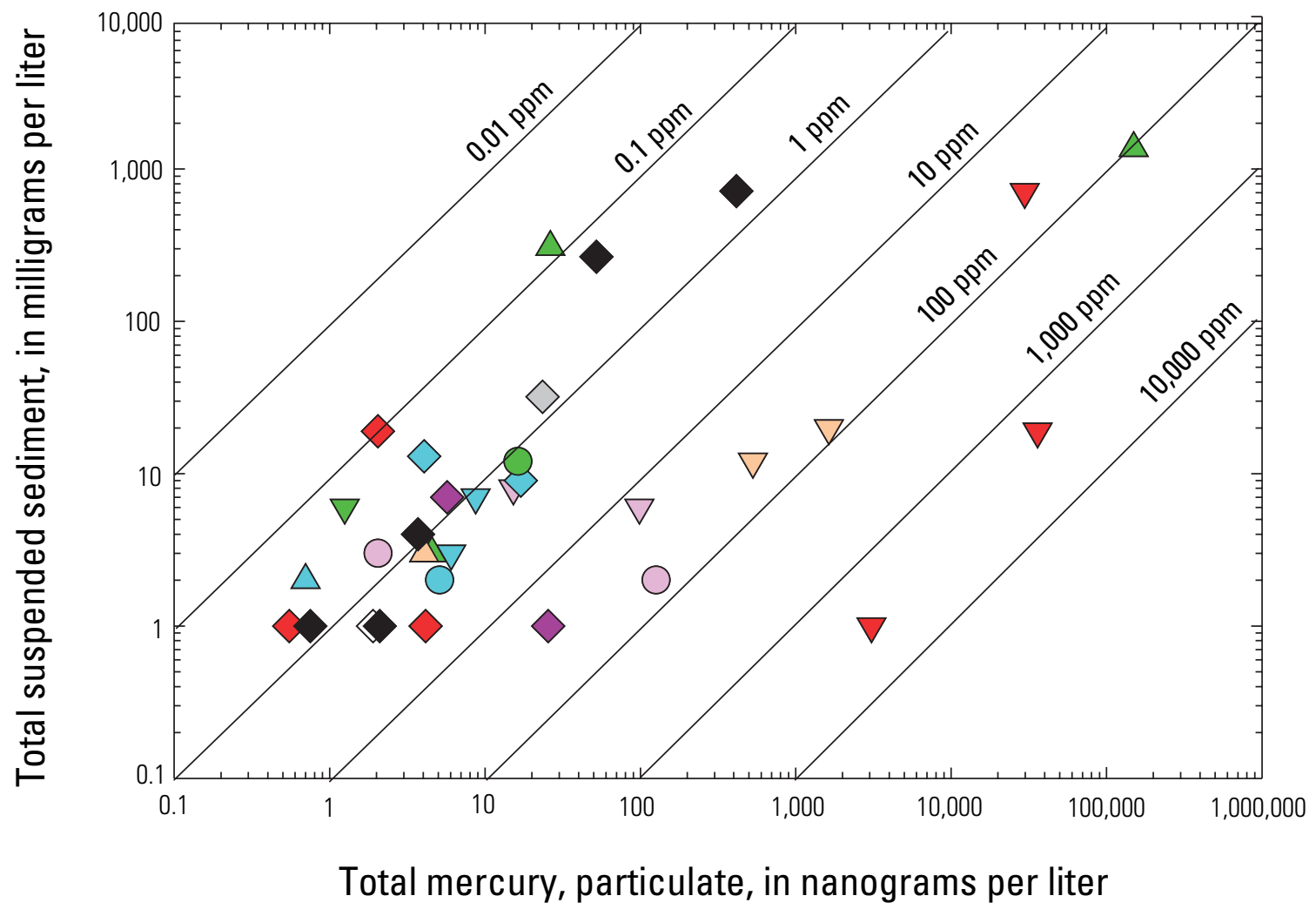

EXPLANATION

\begin{tabular}{lll}
\multicolumn{1}{c}{ Station group } & Type of sampling station \\
$\square$ Boston & $\square$ Poore & $\triangle$ Ground sluice \\
Buckeye & SF Greenhorn & $\bigcirc$ Pit lake/pond/wetland \\
$\square$ Greenhorn, main stem & $\square$ Sailor Flat & $\searrow$ Stream \\
$\square$ Headwaters & $\square$ Starr & $\nabla$ Tunnel \\
$\square$ Integrator & &
\end{tabular}

Figure 17. Relation between particulate total mercury concentration and total suspended sediment concentration, Greenhorn Creek drainage, Nevada County, California. The diagonal lines represent apparent concentrations of mercury in suspended material. ppm, parts per million. 
Table 9. Concentrations of mercury and methylmercury in sediment samples, determined by laboratory methods, Greenhorn Creek drainage, Nevada County, California.

[WI, Wisconsin; CO, Colorado. Station name abbreviations: ab, above; Cr, Creek; Cyn, Canyon; mi, mile; N, North; nr, near; S, South; SF, South Fork. $\mu$ g/g, microgram per gram (equivalent to part per million); s.d., standard deviation (three or more analyses); —, not determined; >>, much greater than; <, less than]

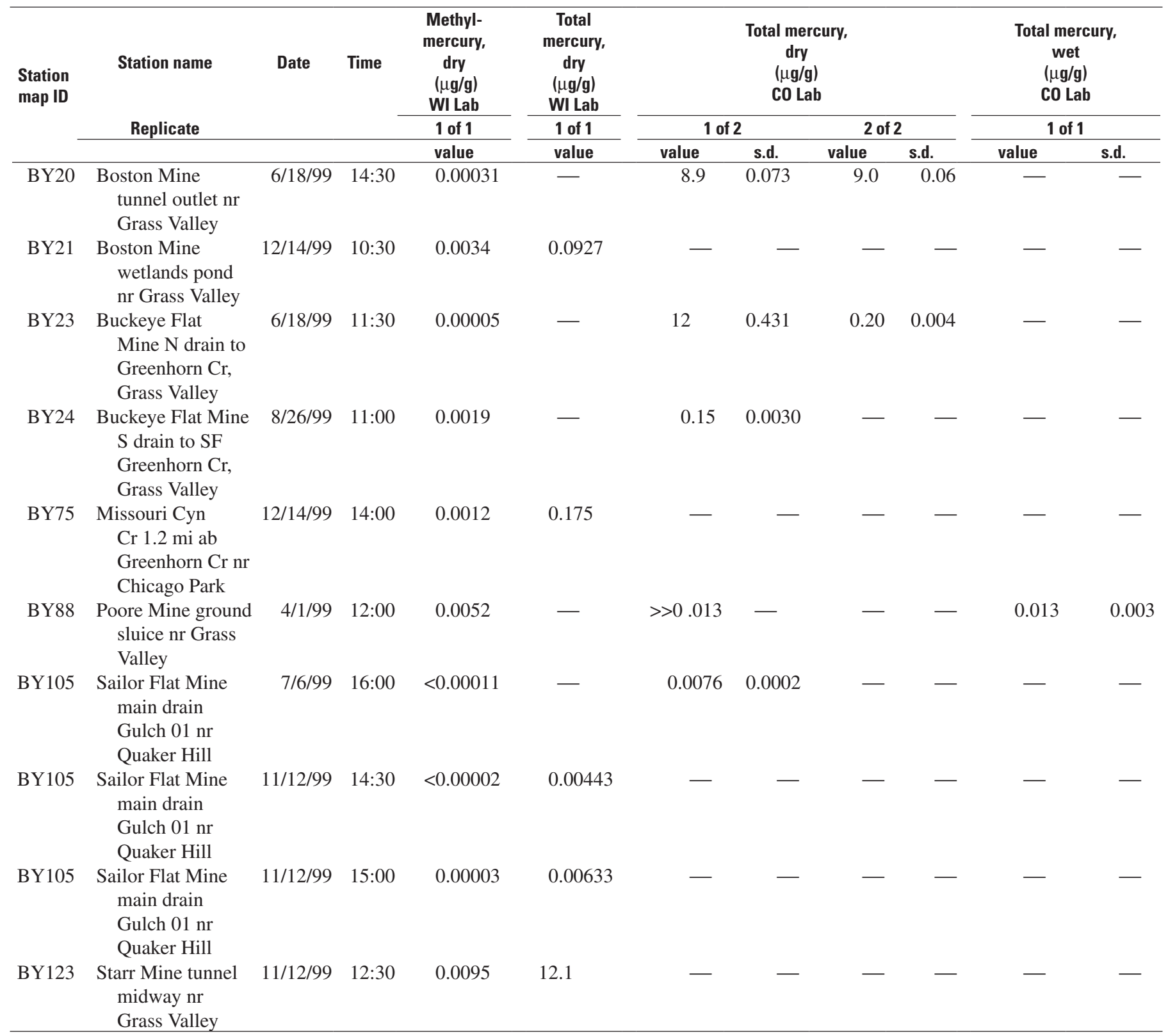


Table 10. Concentrations of visible total mercury in sediment samples, determined by field panning methods, Greenhorn Creek drainage, Nevada County, California.

[NOTE: Table 11 begins on page 204. All samples were measured "wet" after water had been allowed to drain. Water was then added during panning to facilitate recovery. Mercury was separated from concentrate and weighed after residual water was removed. Station name abbreviations: ab, above; bl, below; Cr, Creek; Cyn, Canyon; mi, mile; nr, near; Rd, Road; SF, South Fork. gal/min, gallon per minute; mg/kg, milligram per kilogram (equivalent to parts per million); ft, foot; trace, trace mercury, less than or equal to $100 \mathrm{mg} / \mathrm{kg} ;-$, not determined]

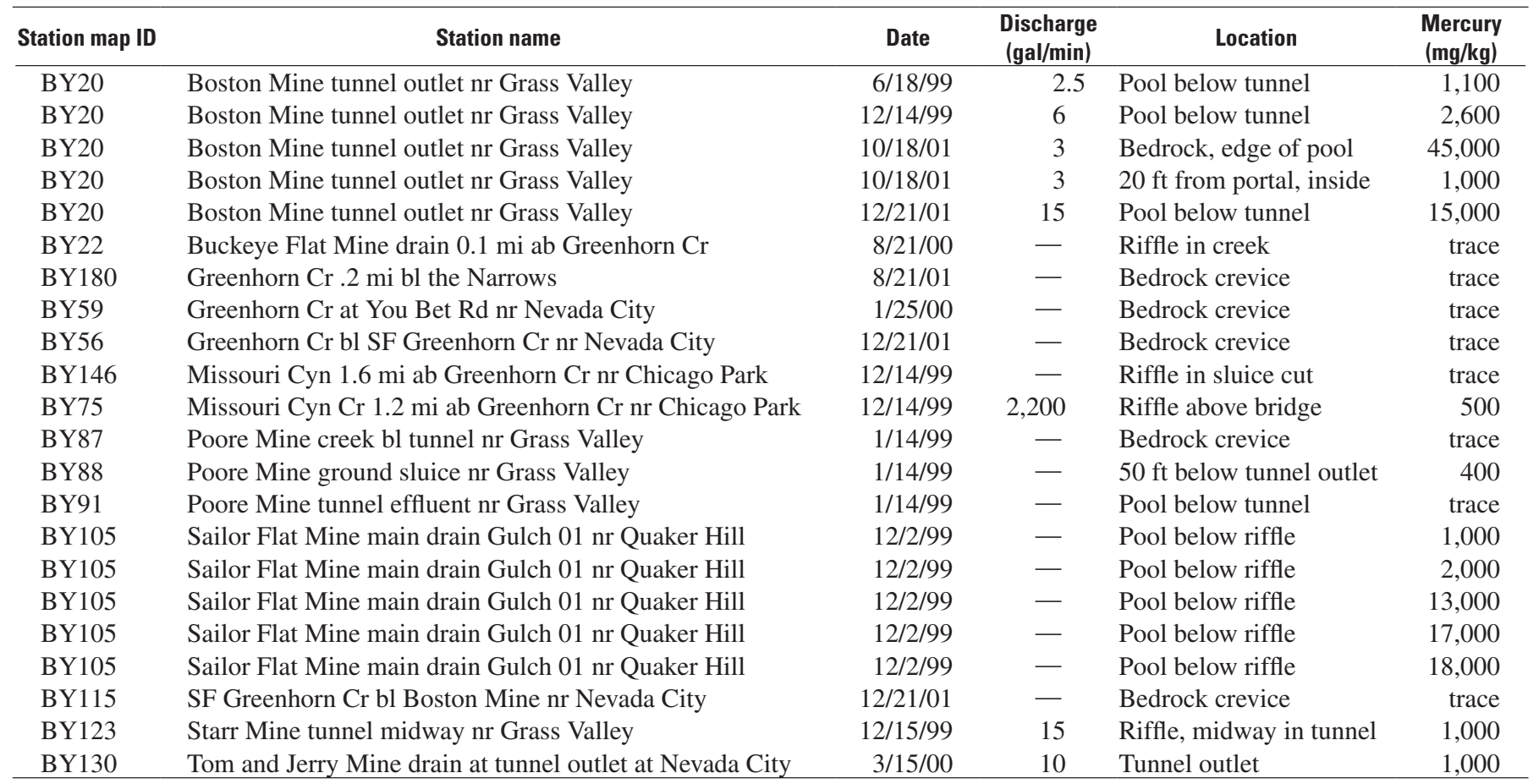




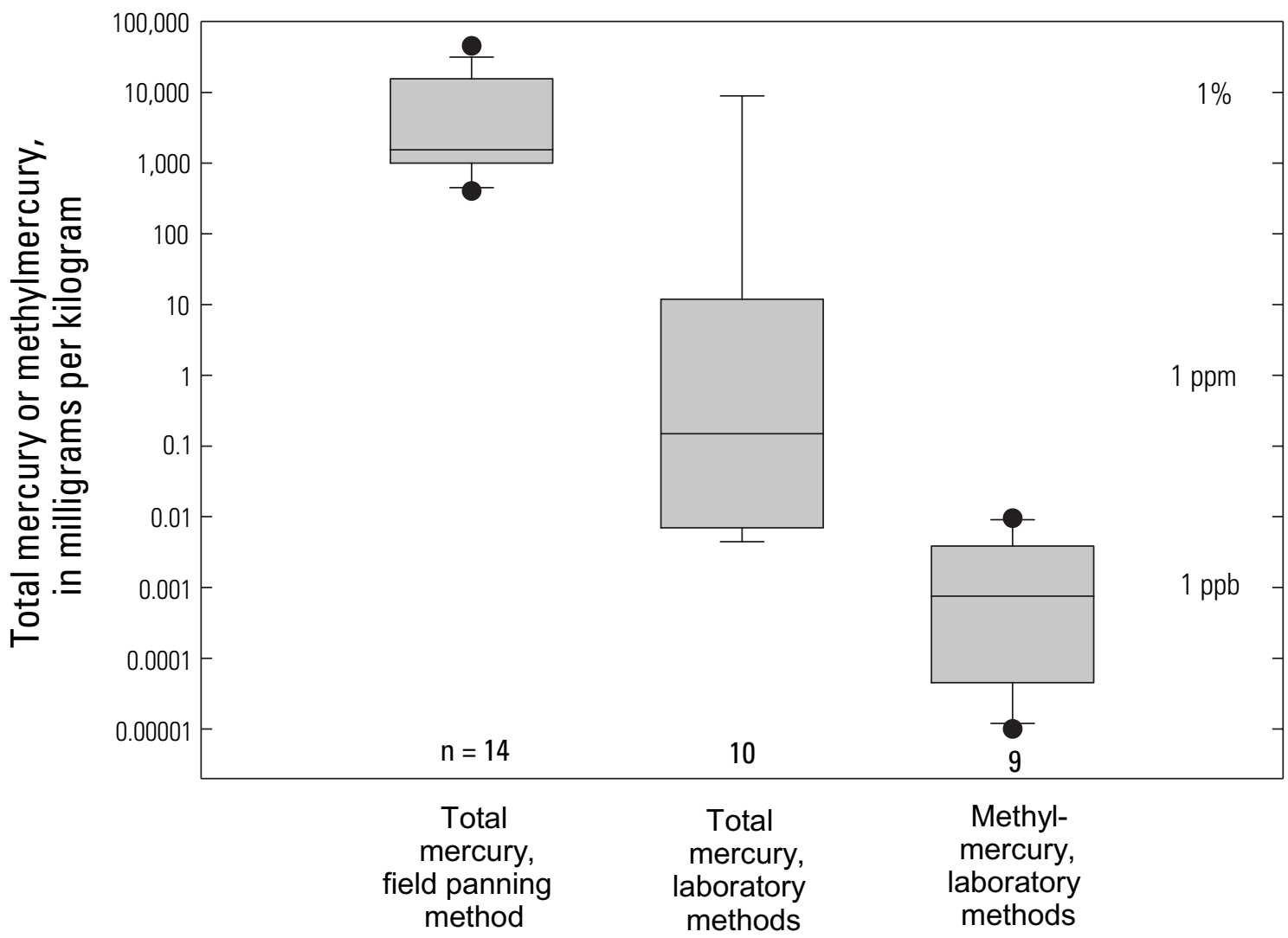

\section{EXPLANATION}

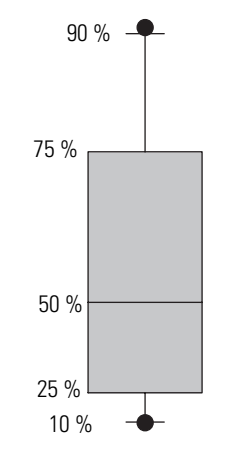

$\mathrm{n}=$ number of samples

Figure 18. Box plots showing distribution of total mercury and methylmercury concentration in sediment samples from the Greenhorn Creek drainage, Nevada County, California. ppm, part per million; ppb, part per billion. 


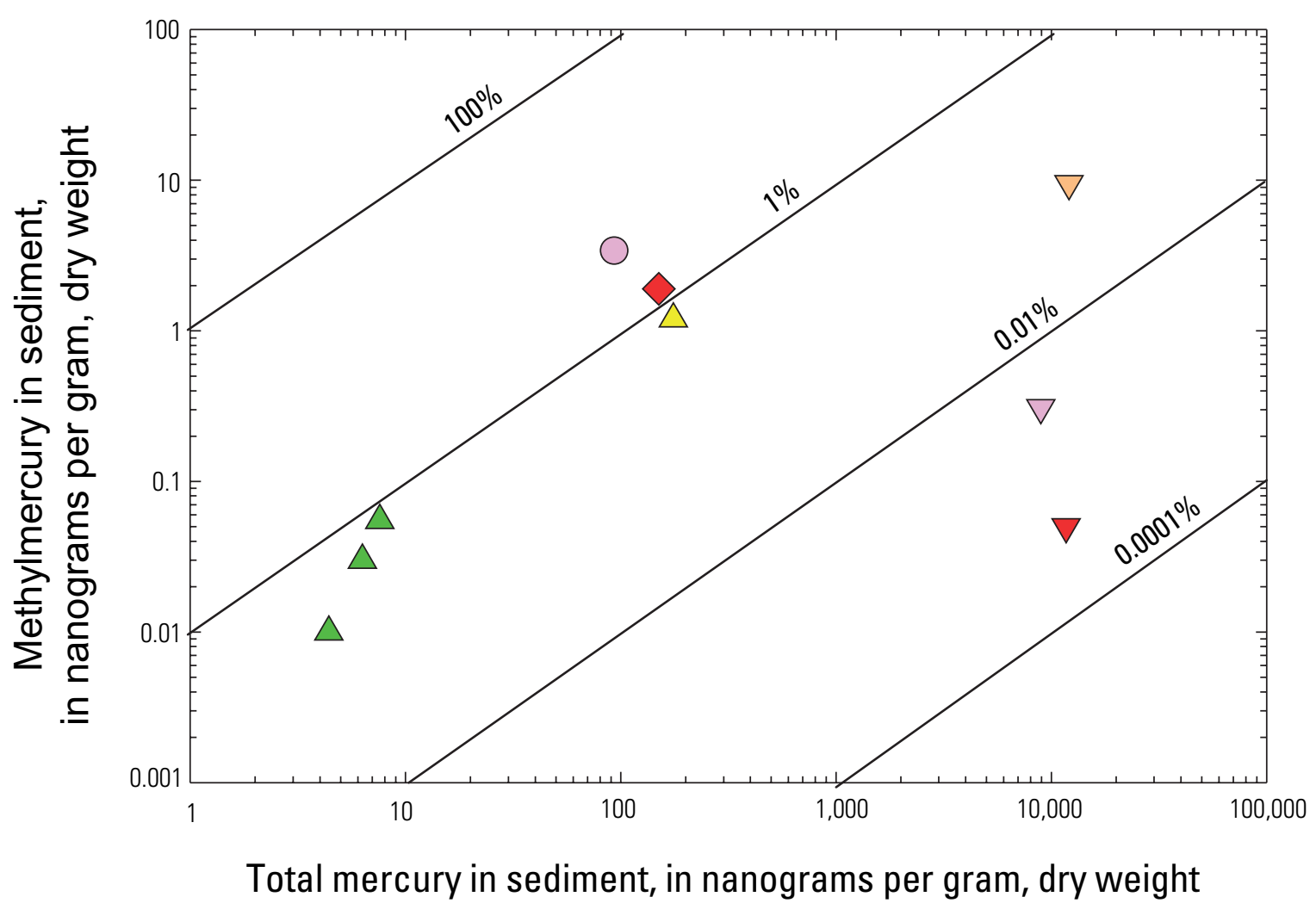

EXPLANATION

\begin{tabular}{|c|c|c|}
\hline \multicolumn{2}{|c|}{ Station group } & Type of sampling station \\
\hline Boston & Sailor Flat & $\triangle$ Ground sluice \\
\hline Buckeye & $\square$ Starr & Pit Lake/pond/wetland \\
\hline Missouri Canyon & & $\diamond$ Stream \\
\hline & & $\nabla$ Tunnel \\
\hline
\end{tabular}

Figure 19. Relation between total mercury and methylmercury concentration in sediment samples from the Greenhorn Creek drainage, Nevada County, California. The diagonal lines represent constant values of the ratio of methylmercury to total mercury.

\section{Invertebrates}

A total of 194 invertebrate samples were collected at 31 stations in the study area. Eighty of the samples were analyzed for concentrations of $\mathrm{THg}$ and $\mathrm{MeHg}$ and used to calculate $\mathrm{MeHg}$ to $\mathrm{THg}$ ratios (table $11 \mathrm{~A}$ in back of report). Sixty-nine frog samples were collected at 19 stations, and all were analyzed only for $\mathrm{THg}$ (table 12). Taxa collected (order: family) included banana slugs (Gastropoda: Arionidae, $\mathrm{n}=27 \mathrm{n}$ is number of samples] ), dobsonflies (Megaloptera: Corydalidae, $\mathrm{n}=14$ ), dragonflies (Odonata: Aeshnidae, $\mathrm{n}=19$; Cordulegastridae, $n=21$; Gomphidae, $n=4$; Libellulidae, $n=2$ ), giant water bugs (Hemiptera: Belostomatidae, $\mathrm{n}=2$ ), predaceous diving beetles (Coleoptera: Dytiscidae, $\mathrm{n}=31$ ), predaceous stoneflies (Plecoptera: Perlidae, $\mathrm{n}=18$ ), and water striders (Hemiptera: Gerridae, $\mathrm{n}=56$ ) (table 11A). An additional 19 samples of six invertebrate taxa were collected at the baseline reference site (table $11 B$ in back of report). 


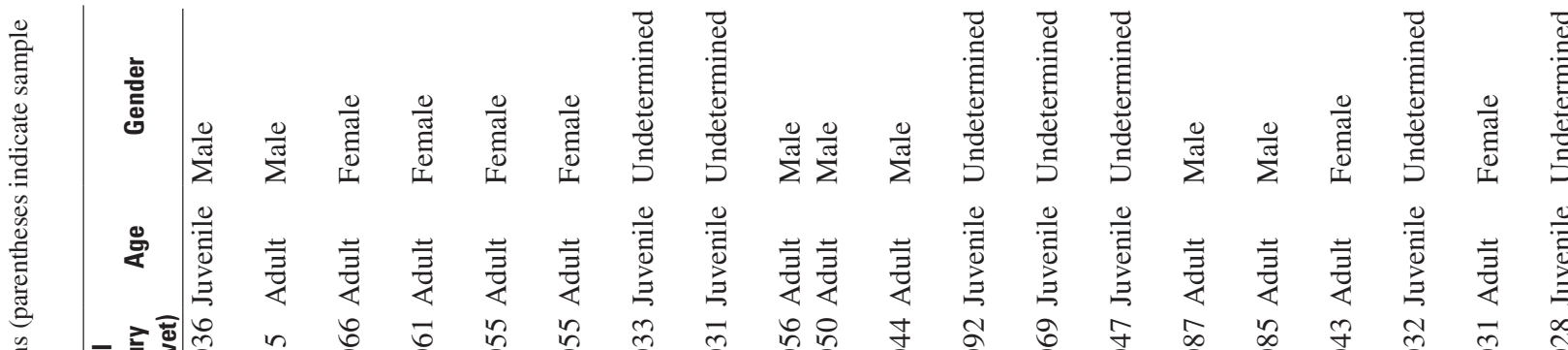

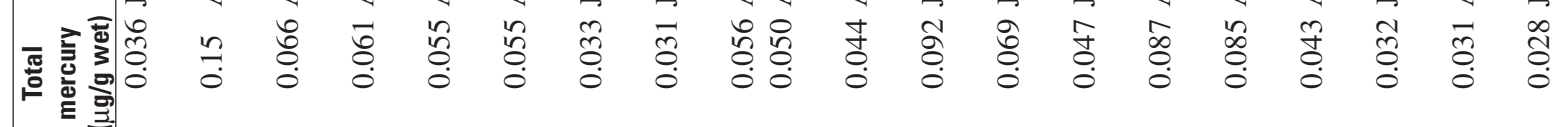

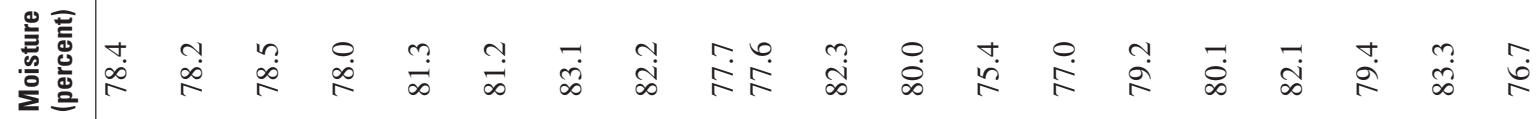

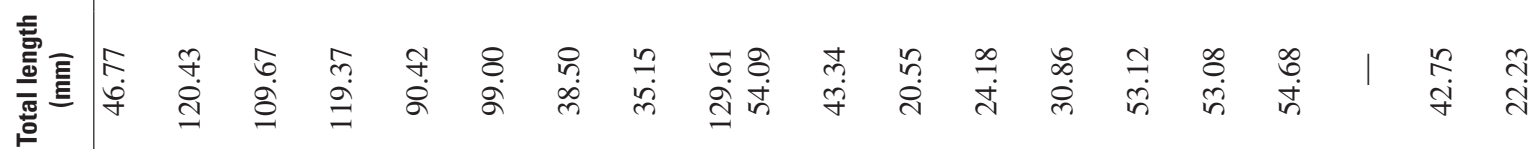

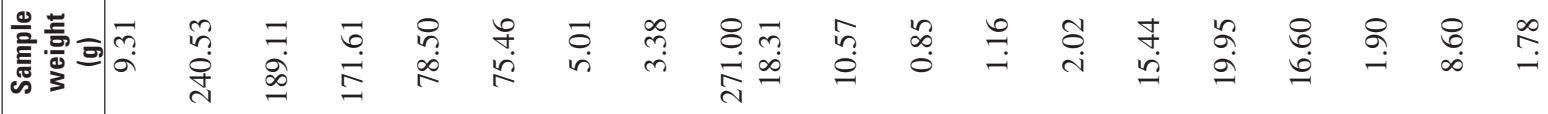

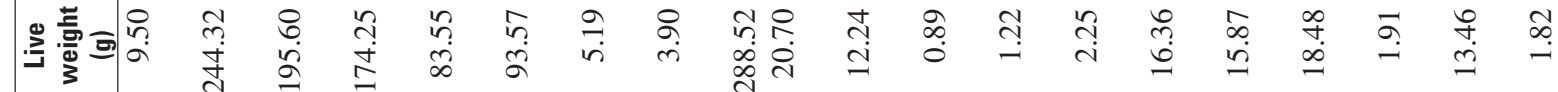

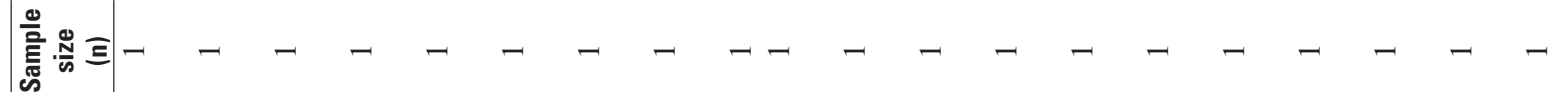

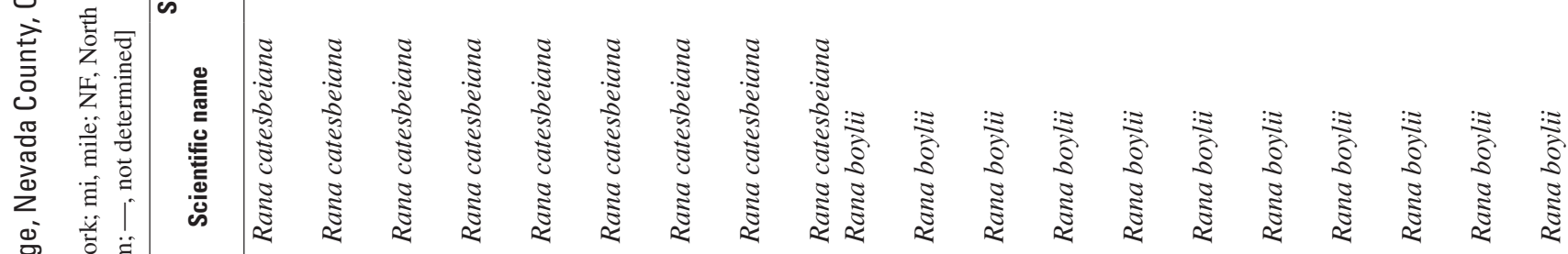

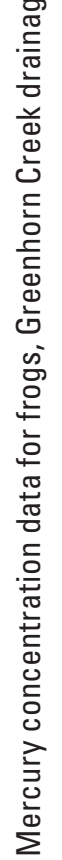

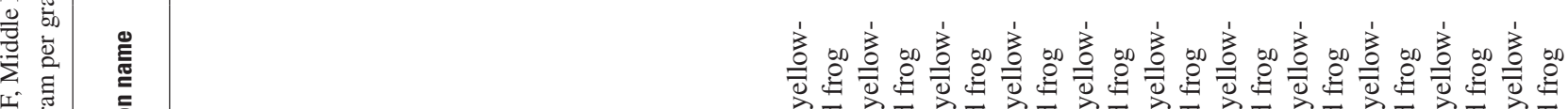

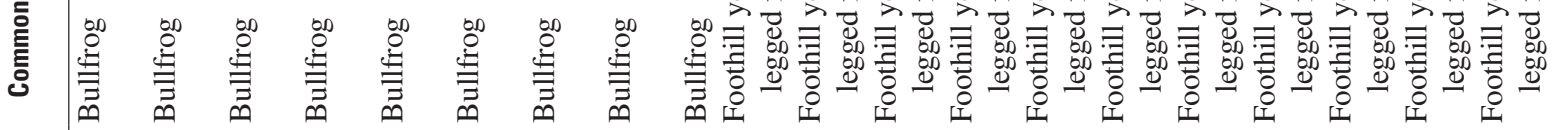

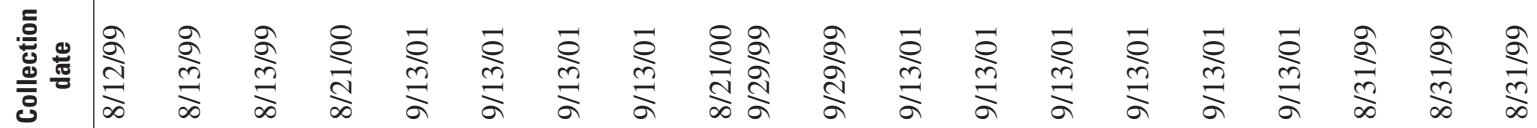

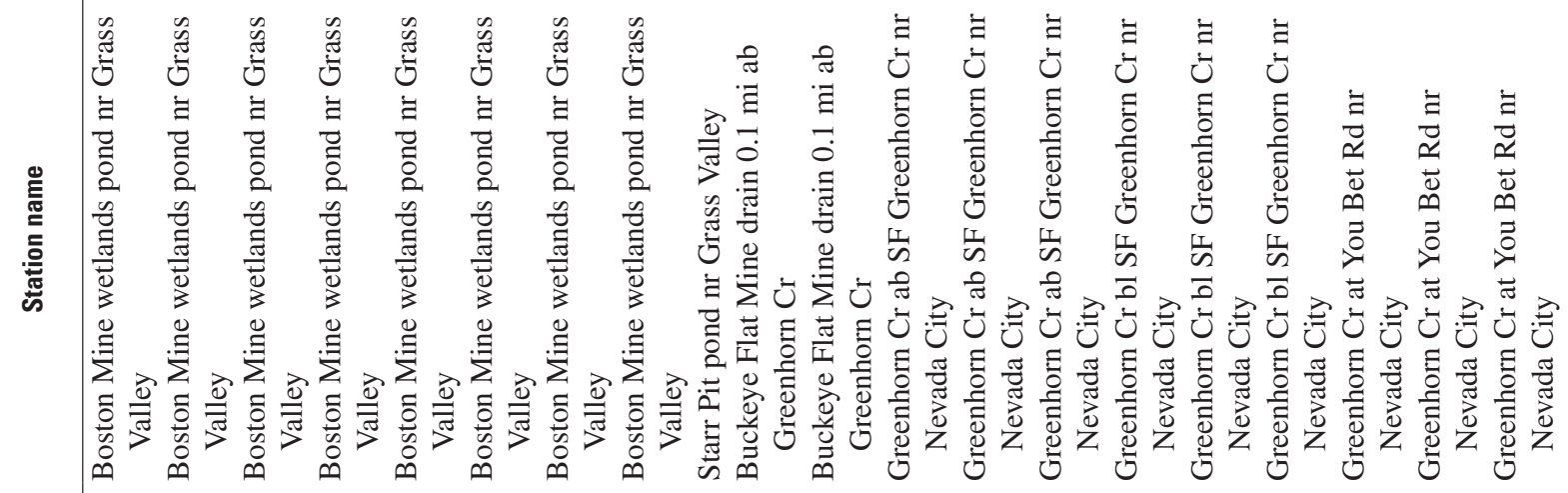

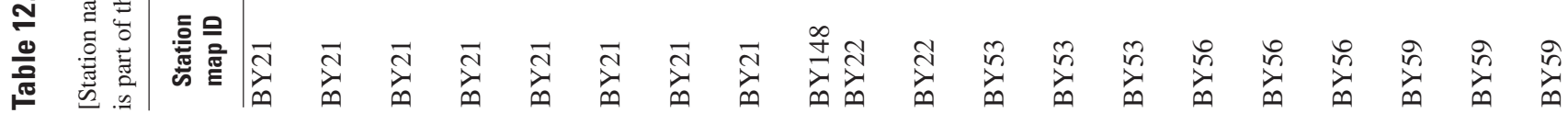




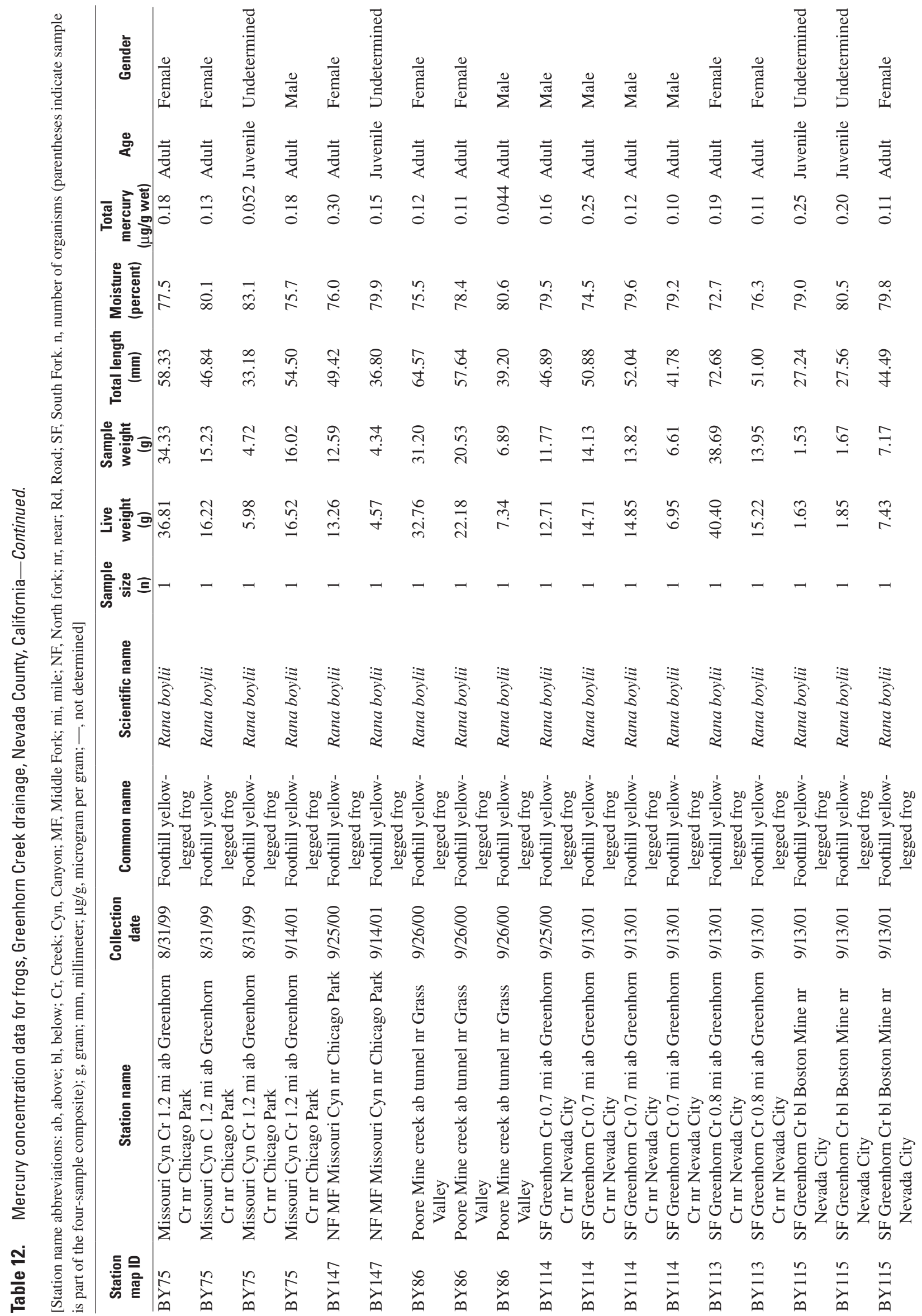




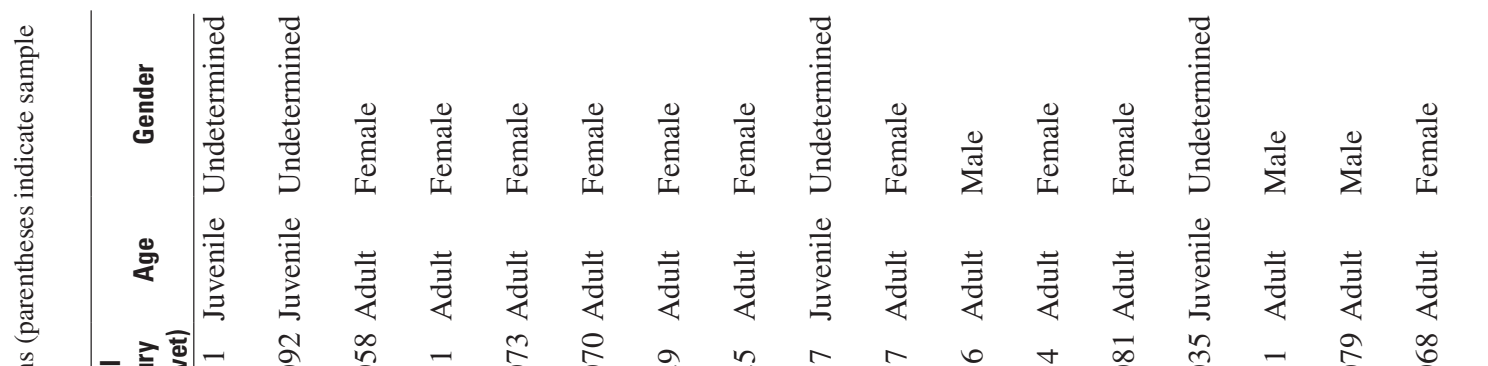

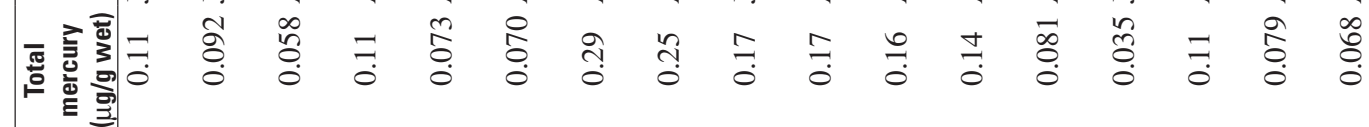

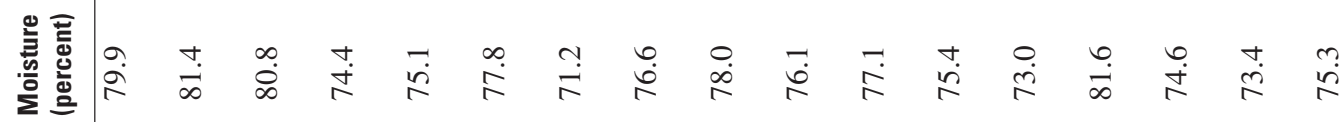

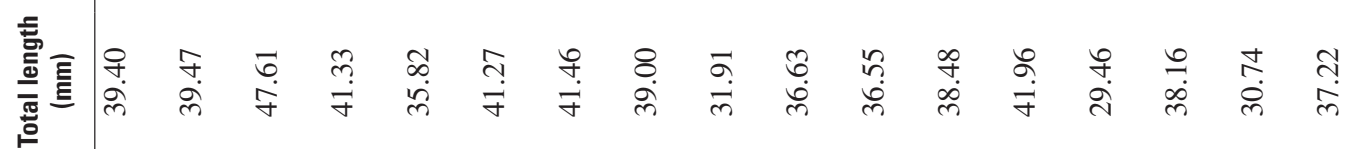

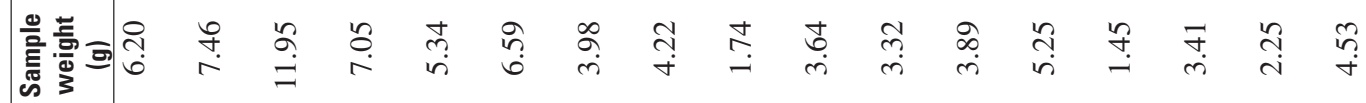

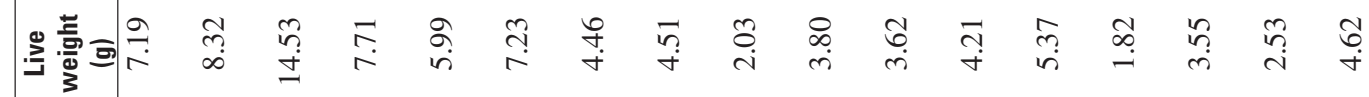

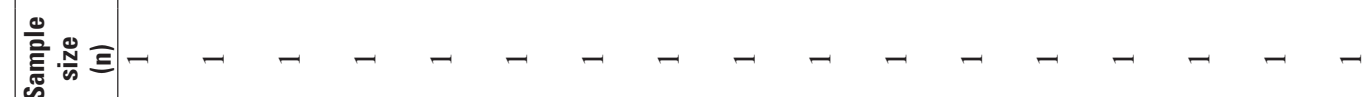

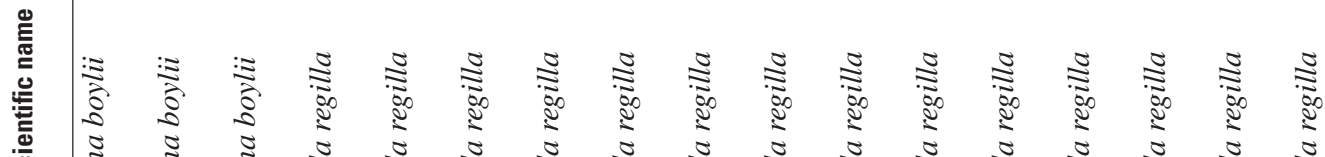

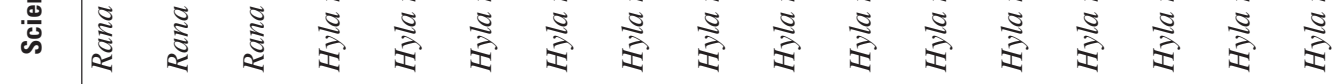

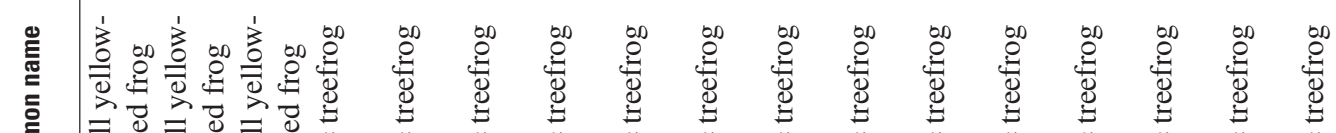

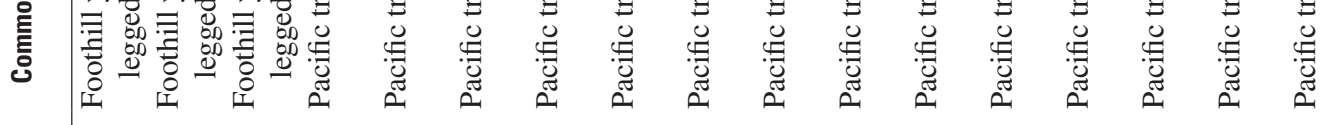

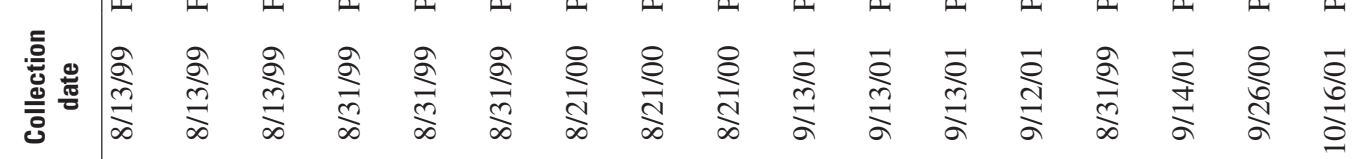

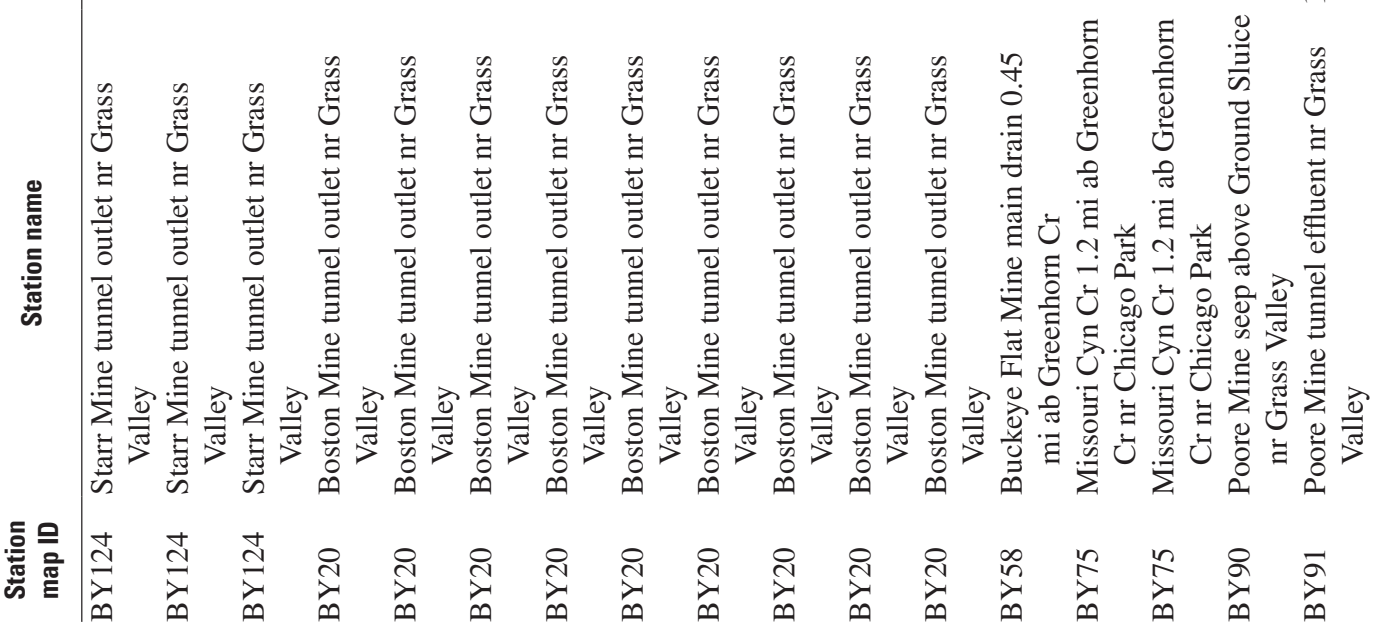




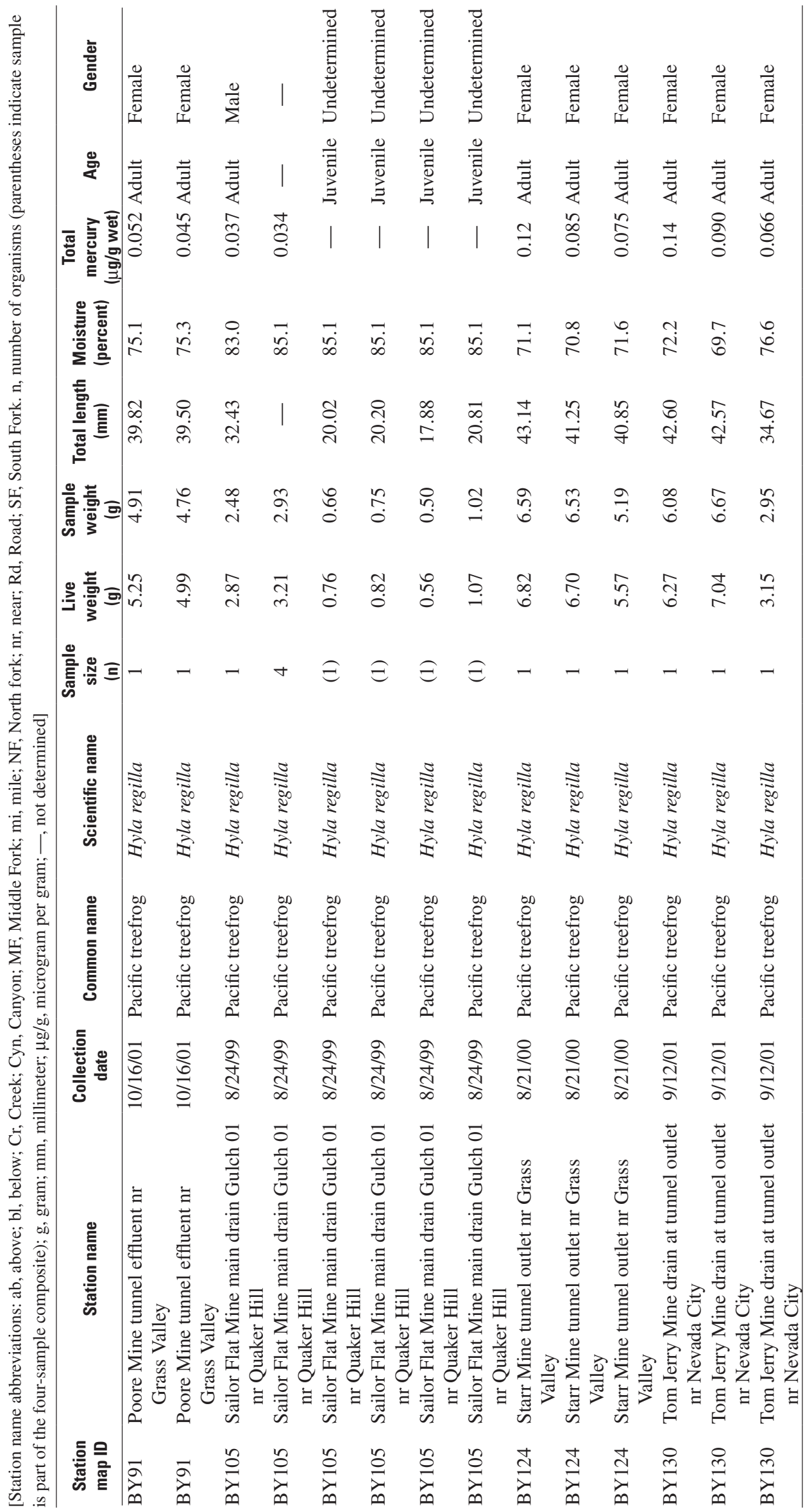


Ranges of $\mathrm{MeHg}$ concentrations ( $\mu \mathrm{g} / \mathrm{g}$, wet weight) in invertebrate samples were $0.0012-0.048$ for banana slugs, 0.027-0.39 for dobsonflies, 0.011-1.6 for dragonflies, $0.029-0.50$ for predaceous diving beetles, $0.026-0.52$ for predaceous stoneflies, and 0.061-0.55 for water striders (fig. 20, table 11A). As expected, the $\mathrm{MeHg}$ concentrations in banana slugs, which are detritivores, were dramatically less than those in predatory insects (fig. 20). Average values for invertebrate samples from the baseline reference station (BY199, fig. 1, table $11 B$ ) are lower than most of the values for samples from the study area (fig. 20, table 11A). For three taxa (dragonflies, giant water bugs, and water striders), average values from the baseline station were less than the 25 th percentile of the sample distribution from the study area. For two of the taxa (dobsonflies and predaceous stoneflies), the average values from the baseline station were approximately equal to the 25 th percentile values, and for one taxon (predaceous diving beetles) the average baseline value was approximately equal to the median value for the study area (fig. 20).

The mean value of $\mathrm{MeHg} / \mathrm{THg}$ for predatory insects collected during 1999-2001 in the Greenhorn Creek drainage was 76 percent, with a standard deviation of 22 percent. A plot of the relation between amounts of $\mathrm{THg}$ and $\mathrm{MeHg}$ in the predatory insects (fig. 21A) indicates that the value of $\mathrm{MeHg} / \mathrm{THg}$ was greater than 50 percent for 74 of 78 samples. The ranges of $\mathrm{MeHg} / \mathrm{THg}$ values per taxon are shown in parentheses: dobsonflies (27-65 percent), dragonflies (65-120 percent), predaceous diving beetles (36-94 percent), predaceous stoneflies (55-109 percent), and water striders (58-127 percent). Box plots of $\mathrm{MeHg} / \mathrm{THg}$ for five invertebrate taxa (fig. 21B) indicate that dragonflies had consistently higher values of $\mathrm{MeHg} / \mathrm{THg}$ relative to the other taxa.

Plots of $\mathrm{MeHg}$ concentration relative to the mass of individual organisms can be used to evaluate whether the mass (a proxy for age) of the organisms is more significant than other factors in determining the $\mathrm{MeHg}$ content. This approach is best applied when comparing multiple samples of different size (age) organisms of a single taxon from the same station, preferably collected at the same time. It has been shown in other studies (for example, Wong and others, 1997) that larger (older) individuals of a given invertebrate taxon will tend to have higher $\mathrm{MeHg}$ concentrations than smaller (younger) individuals from the same location. However, in this study, we did not collect a sufficient number of samples to evaluate the relationship between organism size and $\mathrm{MeHg}$ bioaccumulation; our main objective was to evaluate variations between sites. In this report, plots of organism mass versus $\mathrm{MeHg}$ content in biota show data for individual taxa from multiple sites so that variability in organism size can be assessed in the context of evaluating site-to-site variations in $\mathrm{MeHg}$ concentrations in biota. The relation between organism mass and $\mathrm{MeHg}$ content for each of the invertebrate taxa are plotted in figures 22-27; the relations between mass and $\mathrm{THg}$ for frog taxa are plotted in figures $28-31$.

Although MeHg concentrations in banana slugs (fig. 22) were lower than in other, more predatory invertebrate taxa (fig. 20), the sites with relatively high concentrations of $\mathrm{MeHg}$ and THg in the slugs correspond with "hot spot" stations having other taxa that contain higher than usual concentrations of $\mathrm{MeHg}$ or $\mathrm{THg}$. A composite sample of banana slugs collected in 2001 at a station in the Buckeye area (BY116) had the highest concentration of $\mathrm{MeHg}$, although it was the lowest in terms of average mass. The second-highest $\mathrm{MeHg}$ concentration in a banana slug sample was from a composite sample taken at a ground sluice station in Missouri Canyon (BY 147).

The MeHg concentrations in dragonflies at the Buckeye South station (BY24) and the Boston Mine tunnel outlet (BY 20) were much higher than those at all other stations (fig. 23). The dragonfly sample taken at station BY24 in 1999 had a $\mathrm{MeHg}$ concentration of $1.6 \mu \mathrm{g} / \mathrm{g}$ (wet basis), the highest concentration observed for any invertebrate sample in this study. Dragonfly samples taken at station BY24 had lower concentrations of $\mathrm{MeHg}$ in $2000(0.32 \mu \mathrm{g} / \mathrm{g})$ and in 2001 (0.079 and $0.10 \mu \mathrm{g} / \mathrm{g}$ ); however the organisms in these samples were smaller in mass than the 1999 sample. At other stations in the study area, $\mathrm{MeHg}$ concentrations in dragonflies ranged between 0.011 and $0.89 \mu \mathrm{g} / \mathrm{g}$, a range similar to that observed for other taxa. The average $\mathrm{MeHg}$ concentration for two dragonfly samples from the baseline station was $0.02 \mu \mathrm{g} / \mathrm{g}$, lower than more than 75 percent of the dragonfly samples from the study area (fig. 20).

The highest $\mathrm{MeHg}$ concentration $(0.39 \mu \mathrm{g} / \mathrm{g})$ in dobsonflies (fig. 24) was from a station in the Missouri Canyon area (BY147). Dobsonflies collected from another Missouri Canyon site (BY 75) ranged from 0.08 to $0.20 \mu \mathrm{g} / \mathrm{g}$. Data for the stations in the South Fork Greenhorn Creek area (BY 113, 114, and 115; fig. 4) show that $\mathrm{MeHg}$ content in dobsonflies increases in the downstream direction (table 11A). The average $\mathrm{MeHg}$ concentration for four dobsonfly samples from the baseline site was $0.039 \mu \mathrm{g} / \mathrm{g}$. 


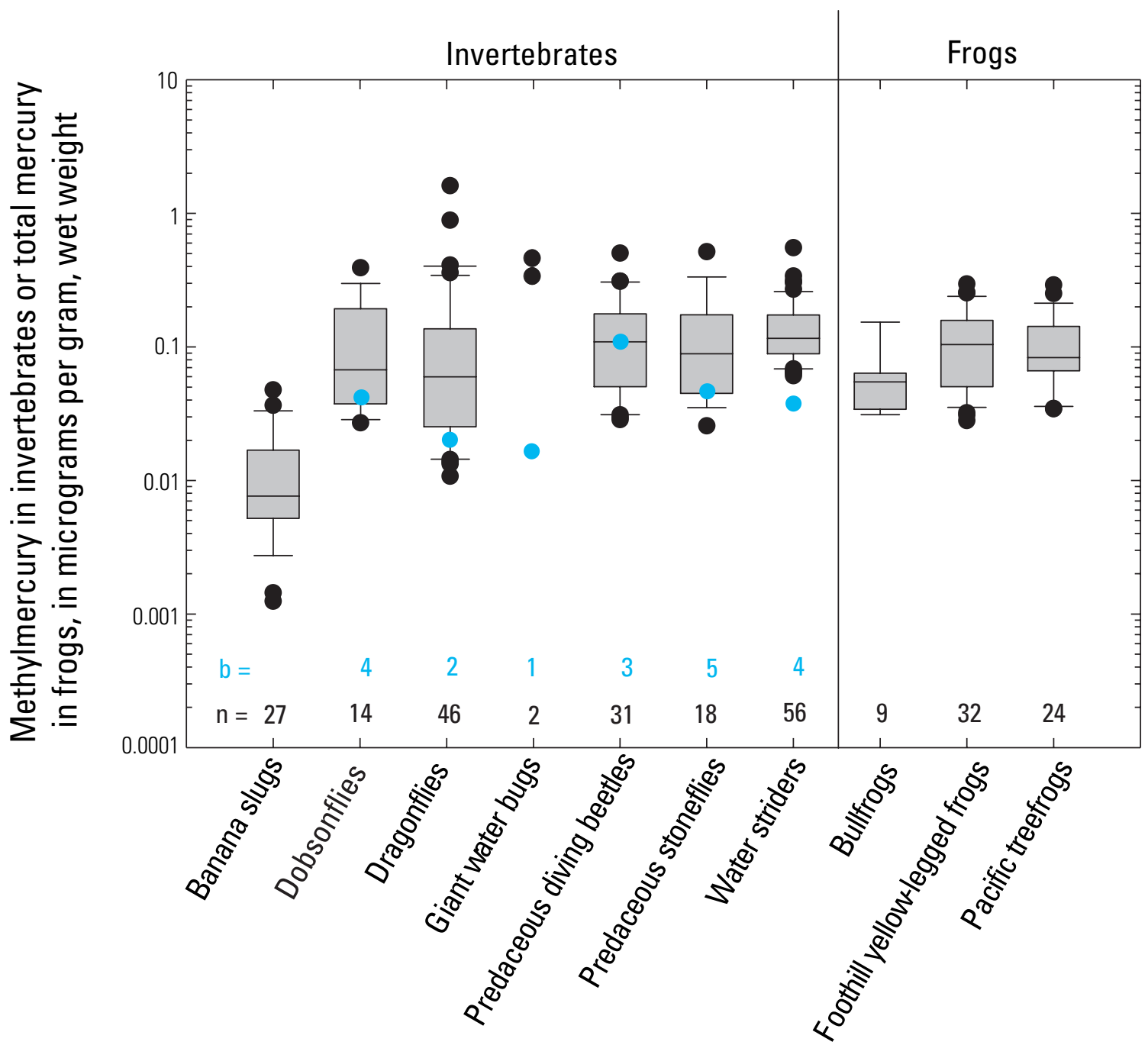

EXPLANATION

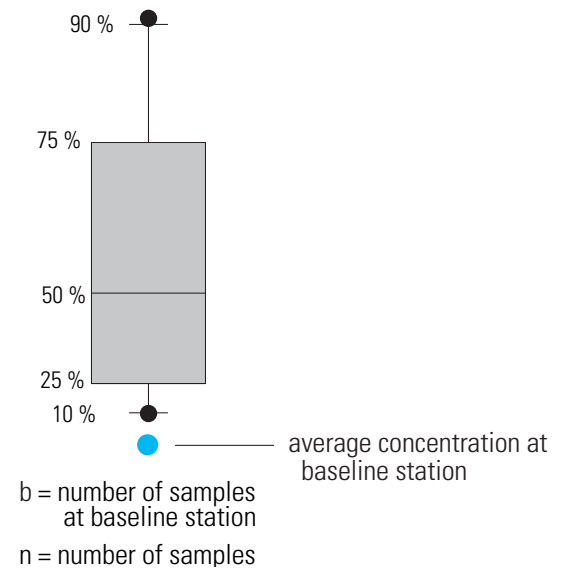

Figure 20. Box plots showing distribution of methylmercury concentrations in invertebrates and total mercury concentrations in frogs from the Greenhorn Creek drainage, Nevada County, California. $n$ is coordinate; $b$ is number of analyses from a baseline station, Bear River at Highway 20 near Emigrant Gap. 


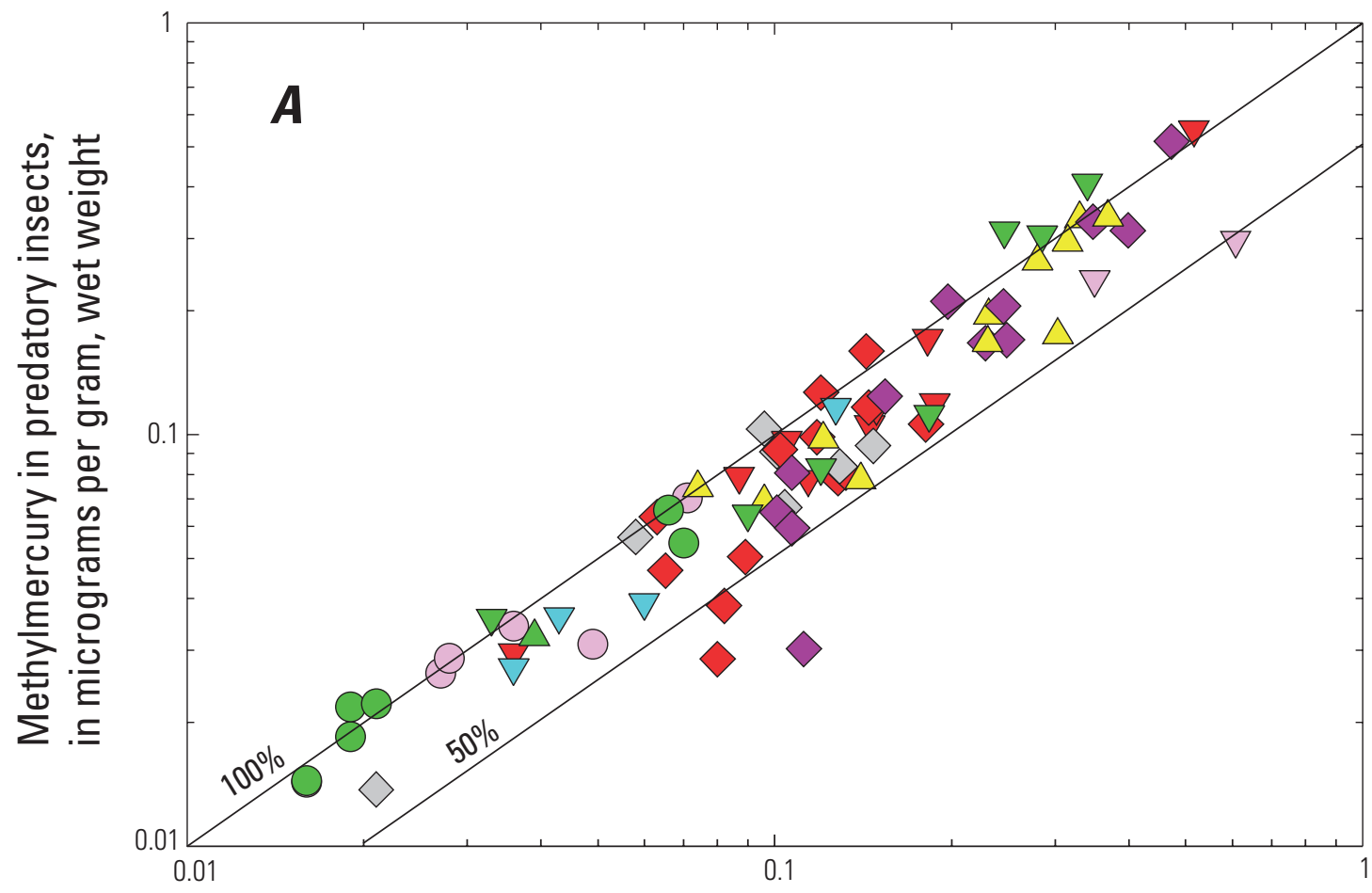

Total mercury in predatory insects, in micrograms per gram, wet weight

EXPLANATION

\begin{tabular}{l}
\multicolumn{2}{c}{ Station group } \\
$\square$ Boston \\
Buckeye \\
$\square$ Greenhorn, main stem $\quad \square$ Soore \\
$\square$ Missouri Canyon \\
$\square$
\end{tabular}

Type of sampling station

$\triangle$ Ground sluice

$\bigcirc$ Pit lake/pond/wetland

$\diamond$ Stream

$\nabla$ Tunnel

Figure 21. Data for total mercury and methylmercury in predatory insects: $(A)$ scatter plot showing relation between total mercury and methylmercury concentration; diagonal lines indicate constant values of the ratio methylmercury to total mercury; $(B)$ box plots showing ratio of methylmercury to total mercury for individual insect taxa from the Greenhorn Creek drainage, Nevada County, California. 
52 Mercury Contamination from Historical Gold Mining, Greenhorn Creek, Nevada County, Calif., 1999-2001

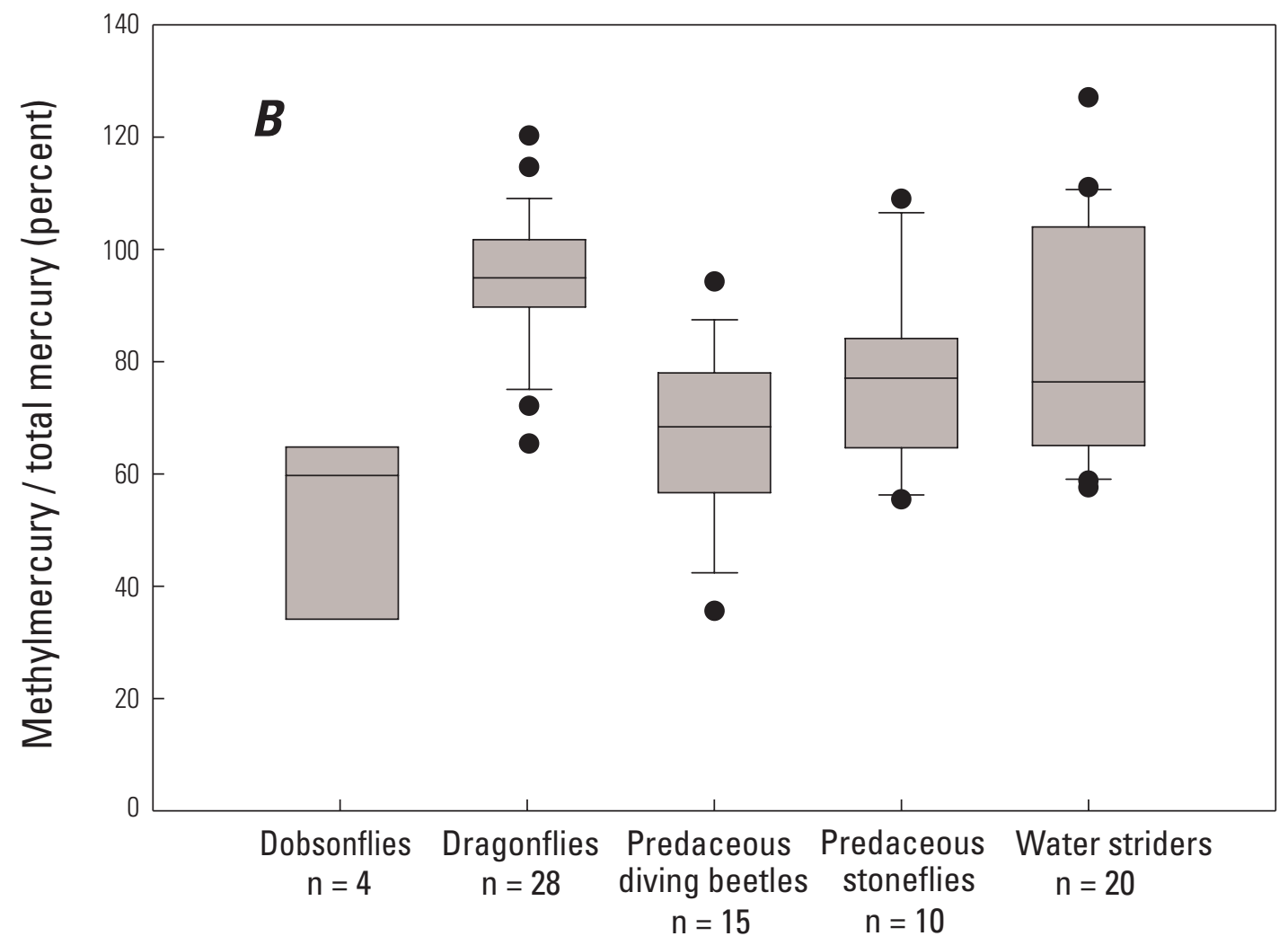

EXPLANATION

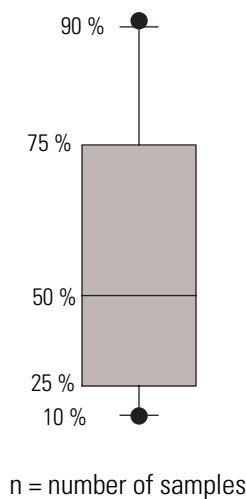

Figure 21.-Continued. 
The highest $\mathrm{MeHg}$ concentrations in predaceous diving beetles (fig. 25, table 11A) were at the Boston Mine tunnel outlet (BY20). Of the stations in the Missouri Canyon area, the station at the North Fork of the Middle Fork (BY147) had diving beetles with the highest $\mathrm{MeHg}$ concentration. The Buckeye samples ranged from 0.03 to $0.17 \mu \mathrm{g} / \mathrm{g}$; the beetles at station BY24 (Buckeye South drain) had the highest MeHg concentration. The average $\mathrm{MeHg}$ concentration of three beetle samples from the baseline station (BY199) was 0.113 $\mu \mathrm{g} / \mathrm{g}$, approximately equal to the median value of samples from the study area.
The predaceous stoneflies at the South Fork Greenhorn Creek stations (BY113, 114, and 115) showed generally increasing $\mathrm{MeHg}$ concentrations in the downstream direction (fig. 26, table 11A). Average MeHg concentrations $(\mu \mathrm{g} / \mathrm{g})$ for three composite samples per station varied from 0.06 at BY113, to 0.14 at BY114, and to 0.34 at BY115. This spatial trend is similar to that observed for dobsonflies, as discussed above. The average value of five samples of predaceous stoneflies from the baseline station (BY199) was $0.054 \mu \mathrm{g} / \mathrm{g}$, approximately equal to the 25 th percentile of samples from the study area.

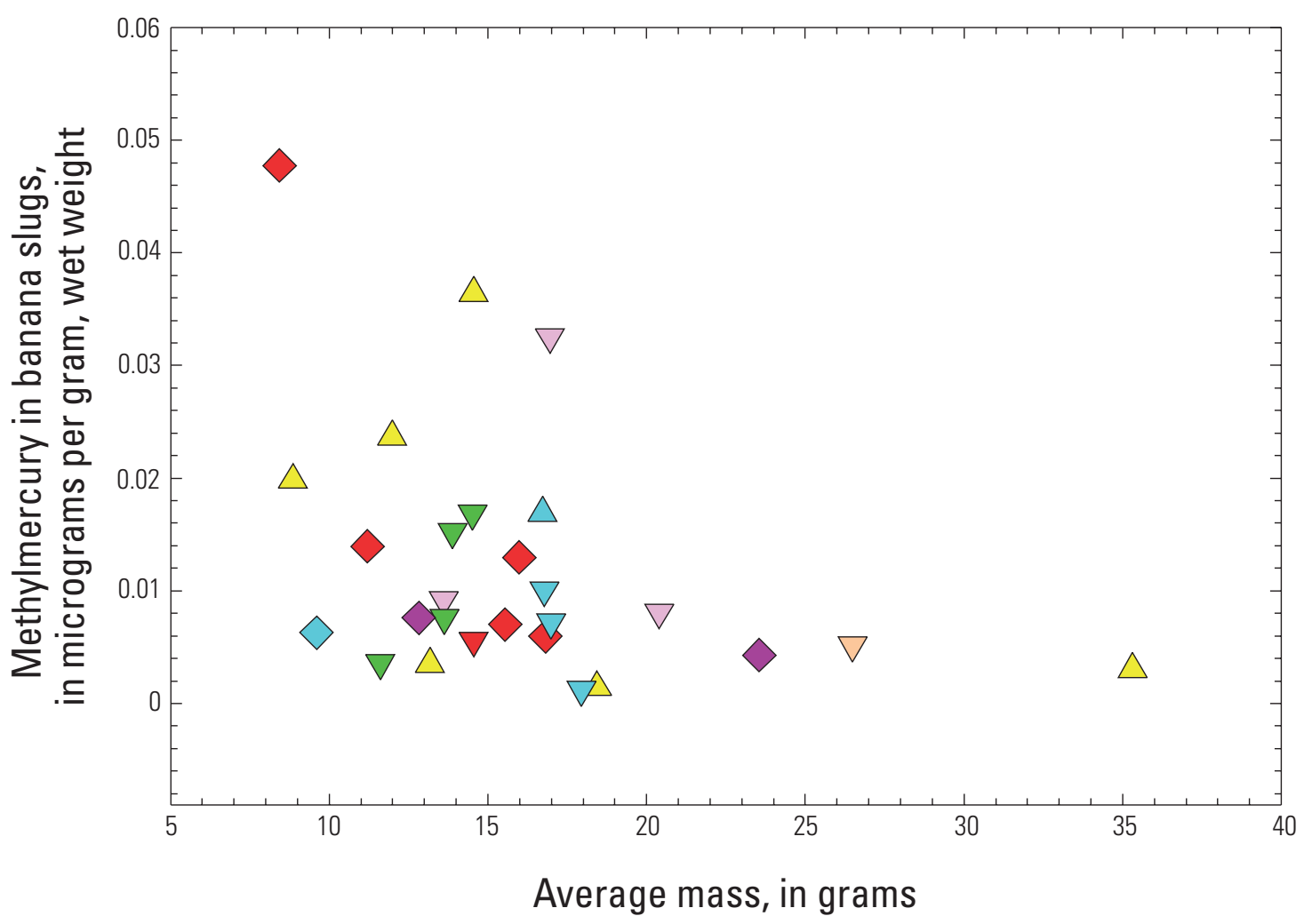

EXPLANATION

\begin{tabular}{lll}
\multicolumn{1}{c}{ Station group } & Type of sampling station \\
$\square$ Boston & SF Greenhorn & $\triangle$ Ground sluice \\
Buckeye & $\square$ Sailor Flat & $\bigcirc$ Pit lake/pond/wetland \\
$\square$ Missouri Canyon & $\square$ Starr & $\searrow$ Stream \\
$\square$ Poore & & $\nabla$ Tunnel
\end{tabular}

Figure 22. Relation between methylmercury concentration and average mass of banana slugs from the Greenhorn Creek drainage, Nevada County, California. 
The highest $\mathrm{MeHg}$ concentration in water striders (fig. 27) was $0.55 \mu \mathrm{g} / \mathrm{g}$ in a sample taken during 1999 from station BY 24 (Buckeye South drain). Similar samples of water striders from this site in 2000 and 2001 had much lower concentrations, ranging from 0.11 to $0.16 \mu \mathrm{g} / \mathrm{g}$ (table $11 A$ ). This decline was similar to that observed for the dragonflies from this site. Similarly, the MeHg concentration in water striders at two Missouri Canyon stations (BY75 and 147) varied greatly year-to-year, with a trend toward lower concentrations of $\mathrm{MeHg}$ in 2001. Water striders at station BY20 (Boston Mine tunnel outlet) had relatively consistent $\mathrm{MeHg}$ concentrations through time, which is probably related to the consistency of the microhabitat and the hydrogeology at that site. $\mathrm{MeHg}$ concentrations in water striders at stations in South Fork Greenhorn Creek (BY113, 114, 115) increased in the downstream direction toward the confluence of South Fork Greenhorn Creek with the main stem of Greenhorn Creek, a trend also observed for dobsonflies and predaceous stoneflies, as noted above. Station BY115, the most downstream station on South Fork Greenhorn Creek, serves as an integrator of multiple contaminated sites, including the Boston Mine tunnel outlet (BY20) and the southern Buckeye sites (BY24 and 116). The average $\mathrm{MeHg}$ concentration of four samples of water striders from the baseline station (BY199) is $0.036 \mu \mathrm{g} / \mathrm{g}$, which is lower than all 56 of the water strider samples from the study area.

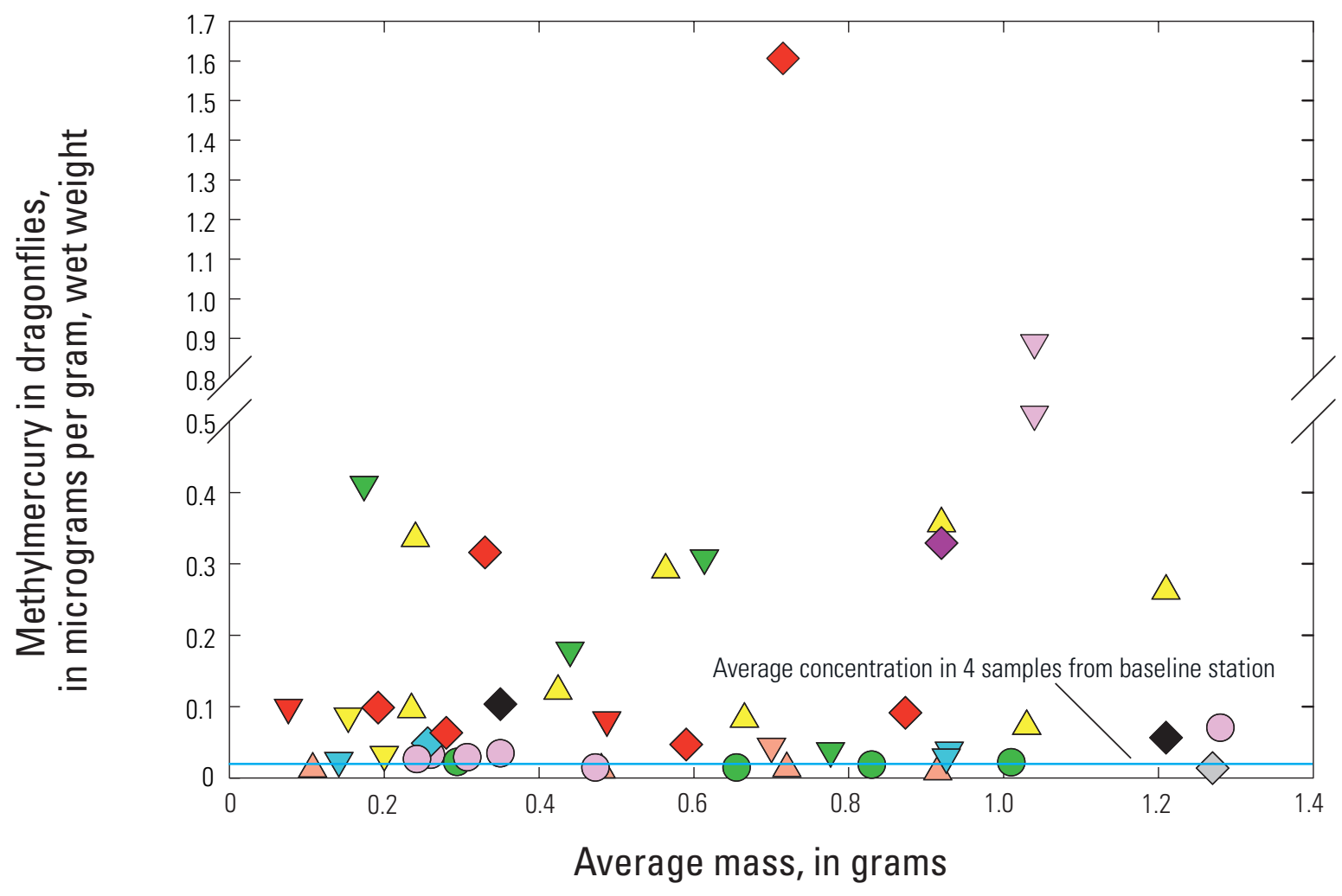

\section{EXPLANATION}

\begin{tabular}{|c|c|}
\hline \multicolumn{2}{|c|}{ Station group } \\
\hline Boston & Poore \\
\hline Buckeye & SF Greenhorn \\
\hline Integrator & Sailor Flat \\
\hline Missouri Canyon & Starr \\
\hline
\end{tabular}

Type of sampling station

$\triangle$ Ground sluice

Pit lake/pond/wetland

$\diamond$ Stream

$\nabla$ Tunnel

Figure 23. Relation between methylmercury concentration and average mass of dragonflies from the Greenhorn Creek drainage, Nevada County, California. 


\section{Frogs}

During 1999-2001, a total of 9 bullfrogs, 32 foothill yellow-legged frogs, and 24 Pacific treefrogs were collected from the study area. Ranges of THg concentrations (wet weight) are in parentheses: bullfrogs $(0.031-0.15 \mu \mathrm{g} / \mathrm{g})$, foothill yellow-legged frogs $(0.028-0.30 \mu \mathrm{g} / \mathrm{g})$, and Pacific treefrogs $(0.034-0.29 \mu \mathrm{g} / \mathrm{g})$ (figs. $28-31$, table 12).

Total mercury was plotted against mass for all three species of frogs on the same graph (fig. 28). The mass of the frogs is displayed on a logarithmic scale because of the wide range of sample size among the frogs sampled (from less than $1 \mathrm{~g}$ for Pacific treefrogs to nearly $300 \mathrm{~g}$ for the largest bullfrog). Given the diversity of sampling stations, no clear relation between frog size and $\mathrm{THg}$ would be expected. In fact, the frog with the second-highest concentration of THg $(0.29 \mu \mathrm{g} / \mathrm{g}$, table 12) was a relatively small Pacific treefrog (4.46 g) collected from one of the most contaminated sites, the Boston Mine tunnel outlet (BY20). The frog with the highest concentration of THg $(0.30 \mu \mathrm{g} / \mathrm{g})$ was a foothill yellow-legged frog of $13.36 \mathrm{~g}$, much smaller than most of the bullfrogs collected; the bullfrogs had lower concentrations of $\mathrm{THg}(0.03$ to $0.15 \mu \mathrm{g} / \mathrm{g})$. In other watersheds, bullfrogs bioaccumulated extremely high concentrations of $\mathrm{THg}$ (for example, $2.8 \mu \mathrm{g} / \mathrm{g}$ in an adult bullfrog from Cache Creek, California; Roger L. Hothem, written commun., 2002). In this study, bullfrogs were sampled from only two stations, and 8 of the 9 samples came from a single station, the Boston Mine wetlands (BY21). Apparently, the stations in the Greenhorn Creek drainage where bullfrogs were sampled in this study were less contaminated by $\mathrm{MeHg}$ than some of the other stations. Therefore, it appears that spatial variation in $\mathrm{MeHg}$ exposure, rather than mass of the organism, is the primary factor influencing $\mathrm{MeHg}$ bioaccumulation in the study area.

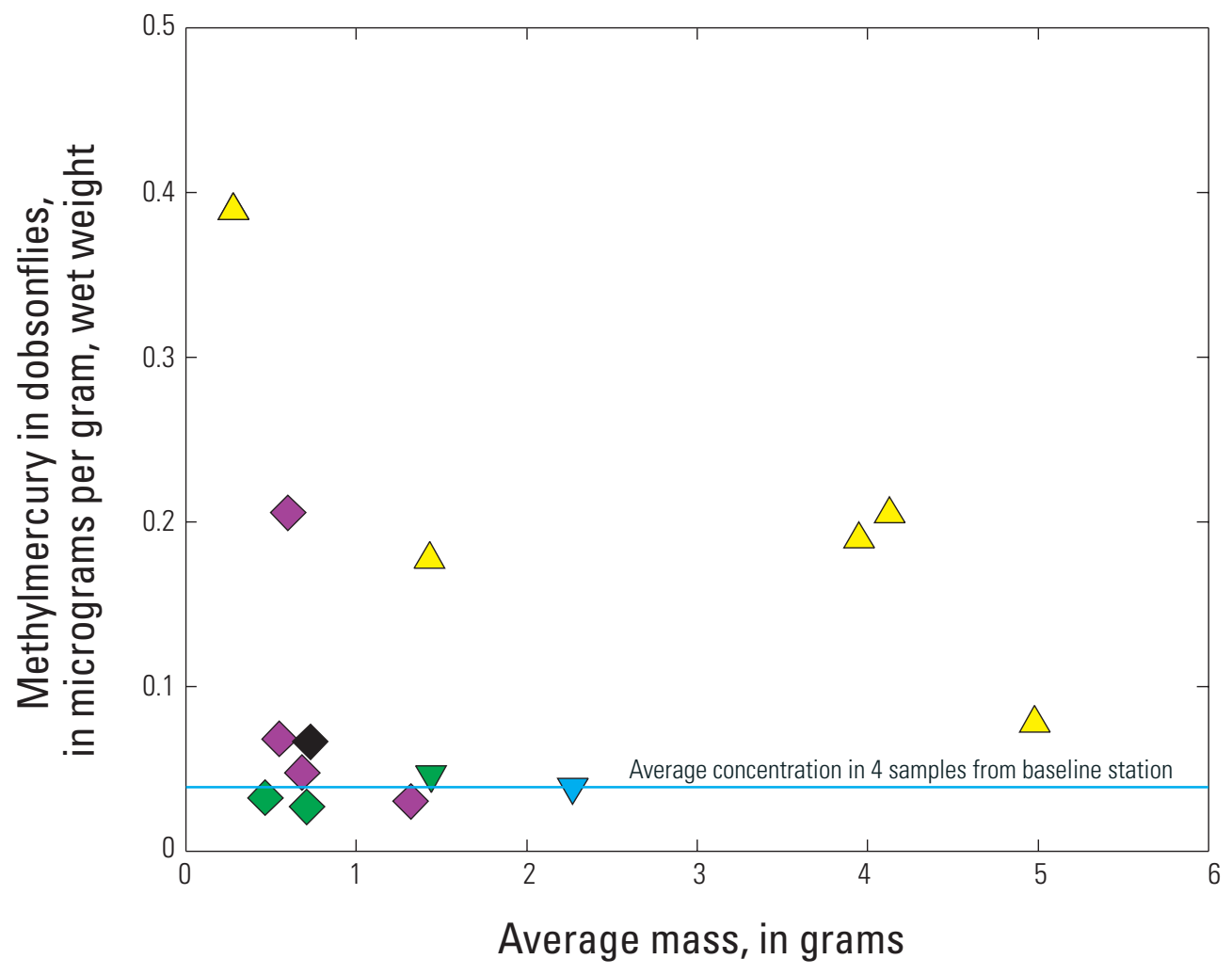

\section{EXPLANATION}

\begin{tabular}{|c|c|c|}
\hline \multicolumn{2}{|c|}{ Station group } & Type of sampling station \\
\hline Integrator & SF Greenhorn & $\triangle$ Ground sluice \\
\hline Missouri Canyon & Sailor Flat & $\diamond$ Stream \\
\hline Poore & & $\nabla$ Tunnel \\
\hline
\end{tabular}

Figure 24. Relation between methylmercury concentration and average mass of dobsonflies from the Greenhorn Creek drainage, Nevada County, California. 
Although there is no clear relation between sizes of all frogs and $\mathrm{THg}$, the $\mathrm{THg}$ concentrations in bullfrogs from the Boston wetlands (station BY21) do appear to increase in relation to sample mass (fig. 29). No clear relation between size (age) and $\mathrm{Hg}$ content is apparent for the other two frog species (figs. 30 and 31). Unlike the bullfrogs, which were nearly all from one site, the treefrogs and the yellow-legged frogs were from multiple site and year combinations, representing a wide variation in mercury exposure. Total mercury concentrations were compared with the total mass of individual bullfrogs from the Boston Mine wetlands (BY21) (fig. 29). The plot shows an increasing trend in $\mathrm{THg}$ relative to mass for the eight samples. The foothill yellow-legged frogs with the highest concentrations of $\mathrm{THg}$ were from the Missouri Canyon (BY147) and the South Fork Greenhorn Creek areas (BY113-115) (fig. 30, table 12). A yellow-legged frog sample from the North Fork of the Middle Fork of Missouri Canyon (station BY147) had the most elevated concentration of THg in this species. At two stations in Missouri Canyon (BY75 and 147), adult foothill yellow-legged frogs had higher concentrations of THg than did the juveniles from the same sites. Foothill yellow-legged frogs from the South Fork Greenhorn Creek area showed a trend of increasing $\mathrm{THg}$ concentration closer to Greenhorn Creek, but, likely because these animals are more

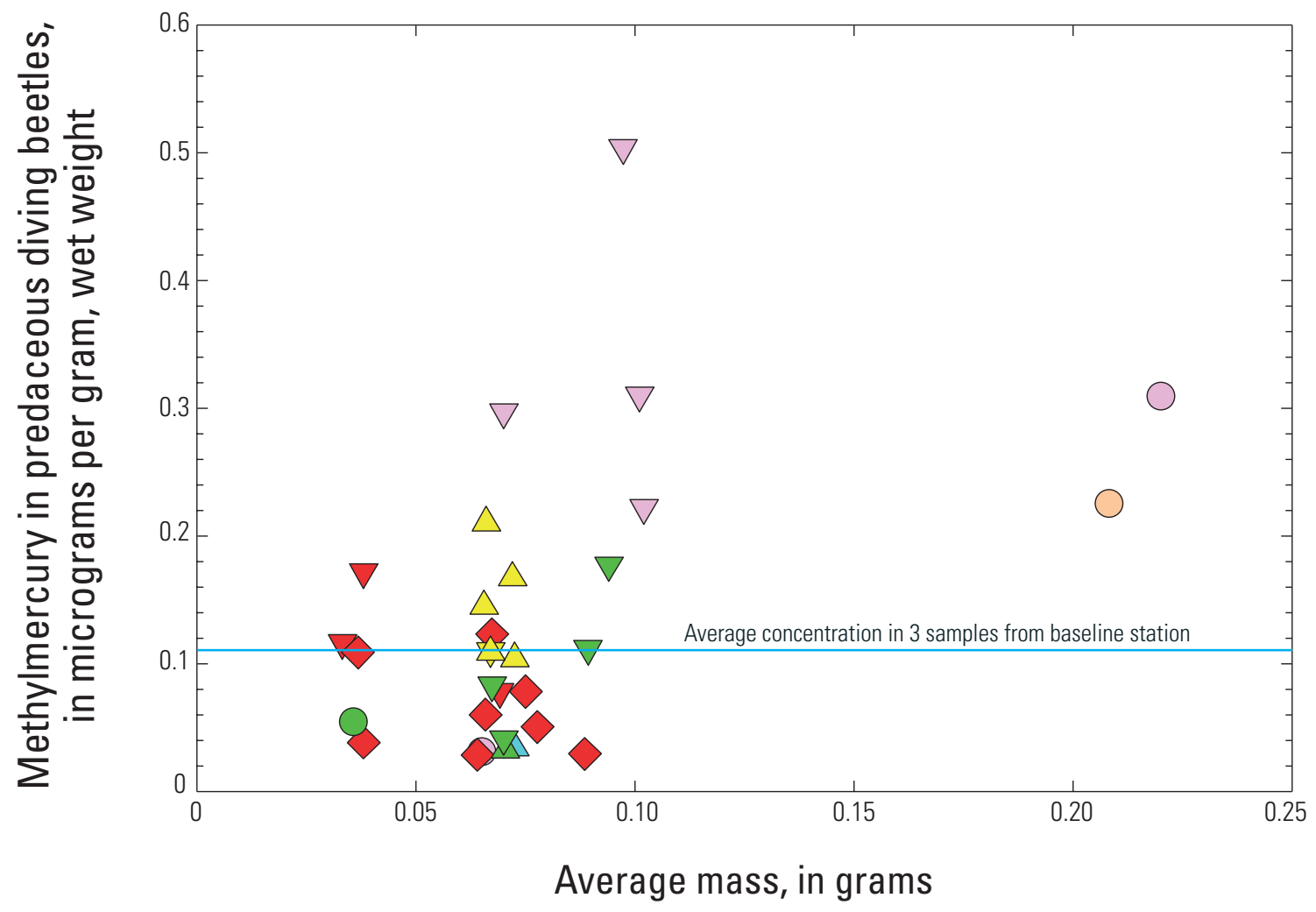

EXPLANATION

\begin{tabular}{lll}
\multicolumn{2}{c}{ Station group } & \multicolumn{2}{c}{ Type of sampling station } \\
$\square$ Boston & $\triangle$ Poore & $\triangle$ Ground sluice \\
$\square$ Buckeye & $\bigcirc$ Sailor Flat & $\bigcirc$ Pit lake/pond/wetland \\
$\square$ Missouri Canyon & $\square$ Starr & $\searrow$ Stream \\
& & $\nabla$ Tunnel
\end{tabular}

Figure 25. Relation between methylmercury concentration and average mass of predaceous diving beetles from the Greenhorn Creek drainage, Nevada County, California. 
mobile and the sites were very close together, this trend was less distinct than that shown by the invertebrates. Foothill yellow-legged frog samples from the main stem of Greenhorn Creek (BY53 and BY56), the Buckeye area (BY22), the Poore Mine area (BY86), and the Integrator station (BY59) generally had lower concentrations of THg compared with samples from other station groups. All six of the Pacific treefrogs collected from the Boston Mine tunnel outlet (BY20) in 2000 and
2001 had higher THg concentrations than the other treefrogs collected from the Greenhorn Creek study area (fig. 31). The relative stability of the habitat at station BY20 likely contributed to both the high $\mathrm{THg}$ values and the consistency of these values. The relatively high concentration of $\mathrm{MeHg}$ observed in the invertebrates collected from this site (table 11A) caused us to expect a high concentration of $\mathrm{THg}$ in the frogs that consume these invertebrates.

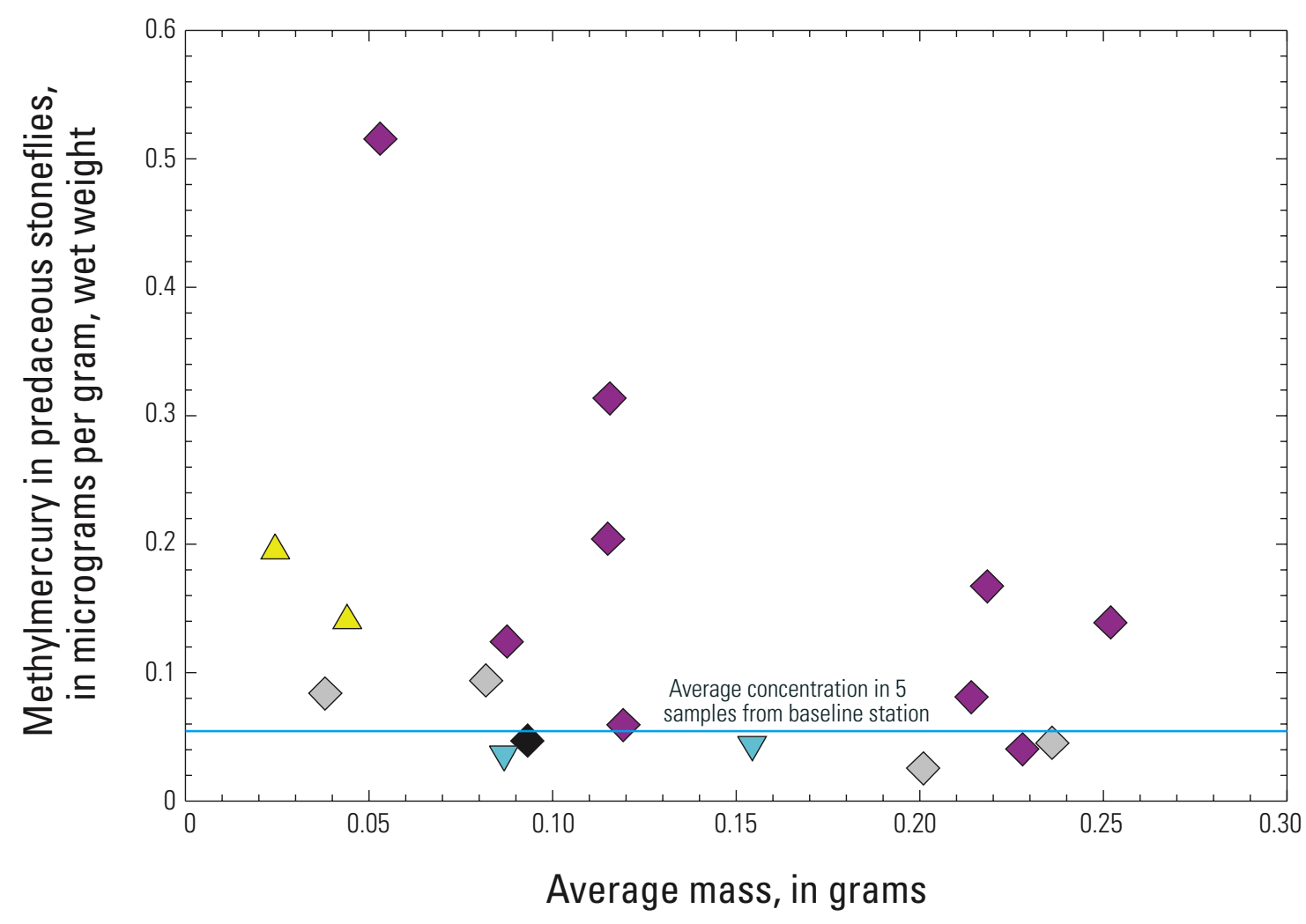

EXPLANATION

\begin{tabular}{|c|c|c|}
\hline \multicolumn{2}{|c|}{ Station group } & Type of sampling station \\
\hline Greenhorn, main stem & Poore & $\triangle$ Ground sluice \\
\hline Integrator & SF Greenhorn & $\diamond$ Stream \\
\hline Missouri Canyon & & $\nabla$ Tunnel \\
\hline
\end{tabular}

Figure 26. Relation between methylmercury concentration and average mass of predaceous stoneflies from the Greenhorn Creek drainage, Nevada County, California. 


\section{Summary of Results by Geographic Area}

Normalizing concentration data using medians for each constituent allows comparison of the relative degree of mercury contamination at the various sampling stations. For each analyte, a single value was derived for each sampling station by taking the geometric mean of available data. The median for each analyte among the various stations included in this study (referred to as the "study median") was then computed using the geometric means corresponding to each station for which data were available. When values were reported as less than the detection limit (in water and sediment data), a value equal to one-half the detection limit was used to compute the geometric mean.
To compare different analytes among stations, the normalized data were split into five categories, shaded in different colors on a summary chart (fig. 32). For the study area, values less than half of the median for each analyte were considered very low (blue). Values greater than or equal to half of the median and less than the median were considered low (green). Values greater than or equal to the median and less than twice the median were considered moderately high (grey); values greater than or equal to twice the median and less than 10 times the median were high (yellow); and values greater than 10 times the median were considered extremely high (red). The summary chart illustrates the values of the normalized concentrations for the following analytes: (1) four types of mercury in water (unfiltered and filtered $\mathrm{THg}$ [table 2], and

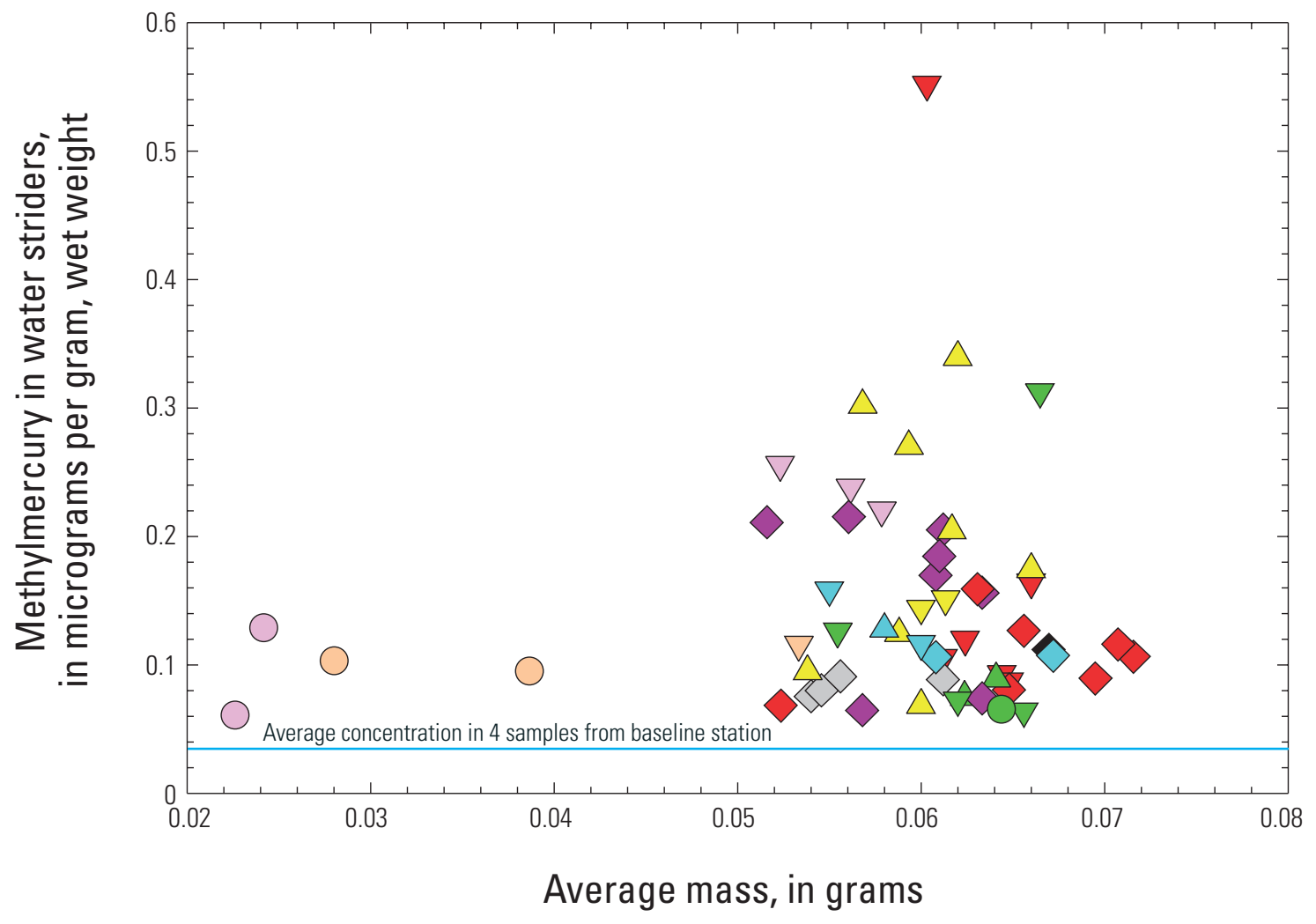

EXPLANATION

\begin{tabular}{lll}
\multicolumn{2}{c}{ Station group } & Type of sampling station \\
$\square$ Boston & $\square$ Poore & $\triangle$ Ground sluice \\
Buckeye & SF Greenhorn & $\bigcirc$ Pit lake/pond/wetland \\
$\square$ Greenhorn, main stem & $\square$ Sailor Flat & $\searrow$ Stream \\
Integrator & $\square$ Starr & $\nabla$ Tunnel \\
$\square$ Missouri Canyon & &
\end{tabular}

Figure 27. Relation between methylmercury concentration and average mass of water striders from the Greenhorn Creek drainage, Nevada County, California. 
unfiltered and filtered $\mathrm{MeHg}$ [table 3]), (2) three types of mercury in sediment (visible $\mathrm{THg}$ [table 10]) and $\mathrm{THg}$ and $\mathrm{MeHg}$ from laboratory analysis [table 9]), (3) six invertebrate taxa (table 11A), and (4) two frog taxa (table 12). Giant water bugs and bullfrogs were not included in figure 32 because the number of stations sampled for these taxa was too small $(n=2)$.

Study median values for all mercury-bearing constituents (water, sediment, and biota) are described below. For the entire study area, the medians for the four types of mercury in water were as follows: $14.6 \mathrm{ng} / \mathrm{L}$ in unfiltered THg, $2.8 \mathrm{ng} / \mathrm{L}$ in filtered THg, $0.07 \mathrm{ng} / \mathrm{L}$ in unfiltered $\mathrm{MeHg}$, and $0.03 \mathrm{ng} / \mathrm{L}$ in filtered $\mathrm{MeHg}$. (Note that the median value for filtered $\mathrm{MeHg}$ was less than the MDL of $0.04 \mathrm{ng} / \mathrm{L}$ because one-half the MDL was used for samples below the MDL.) In the case of visible mercury (table 10), it was assumed that a trace amount corresponded to $100 \mathrm{mg} / \mathrm{kg}$ (equivalent to $100 \mathrm{ppm}$ ); $100 \mathrm{mg}$ was the detection limit of the field balance used to evaluate the results of panning $1-\mathrm{kg}$ sediment samples. The median for this analyte turned out to be the trace amount; therefore, the trace amount has a normalized concentration of 1.0 (cells shaded yellow on figure 32).

The study median for THg in sediment analyzed in the laboratory is $0.175 \mathrm{mg} / \mathrm{kg}$ (equivalent to $\mathrm{ppm}$ ) and for $\mathrm{MeHg}$ in sediment, $0.00155 \mathrm{mg} / \mathrm{kg}$. For invertebrates, the study medians for $\mathrm{MeHg}$ concentration were as follows: banana slugs, $0.0076 \mu \mathrm{g} / \mathrm{g}$; dragonflies, $0.065 \mu \mathrm{g} / \mathrm{g}$; dobsonflies, $0.067 \mu \mathrm{g} / \mathrm{g}$; stoneflies, $0.084 \mu \mathrm{g} / \mathrm{g}$; diving beetles, $0.10 \mu \mathrm{g} / \mathrm{g}$; and water striders, $0.11 \mu \mathrm{g} / \mathrm{g}$. For frogs, the median concentrations for THg were $0.080 \mu \mathrm{g} / \mathrm{g}$ for Pacific treefrogs and $0.083 \mu \mathrm{g} / \mathrm{g}$ for Foothill yellow-legged frogs.

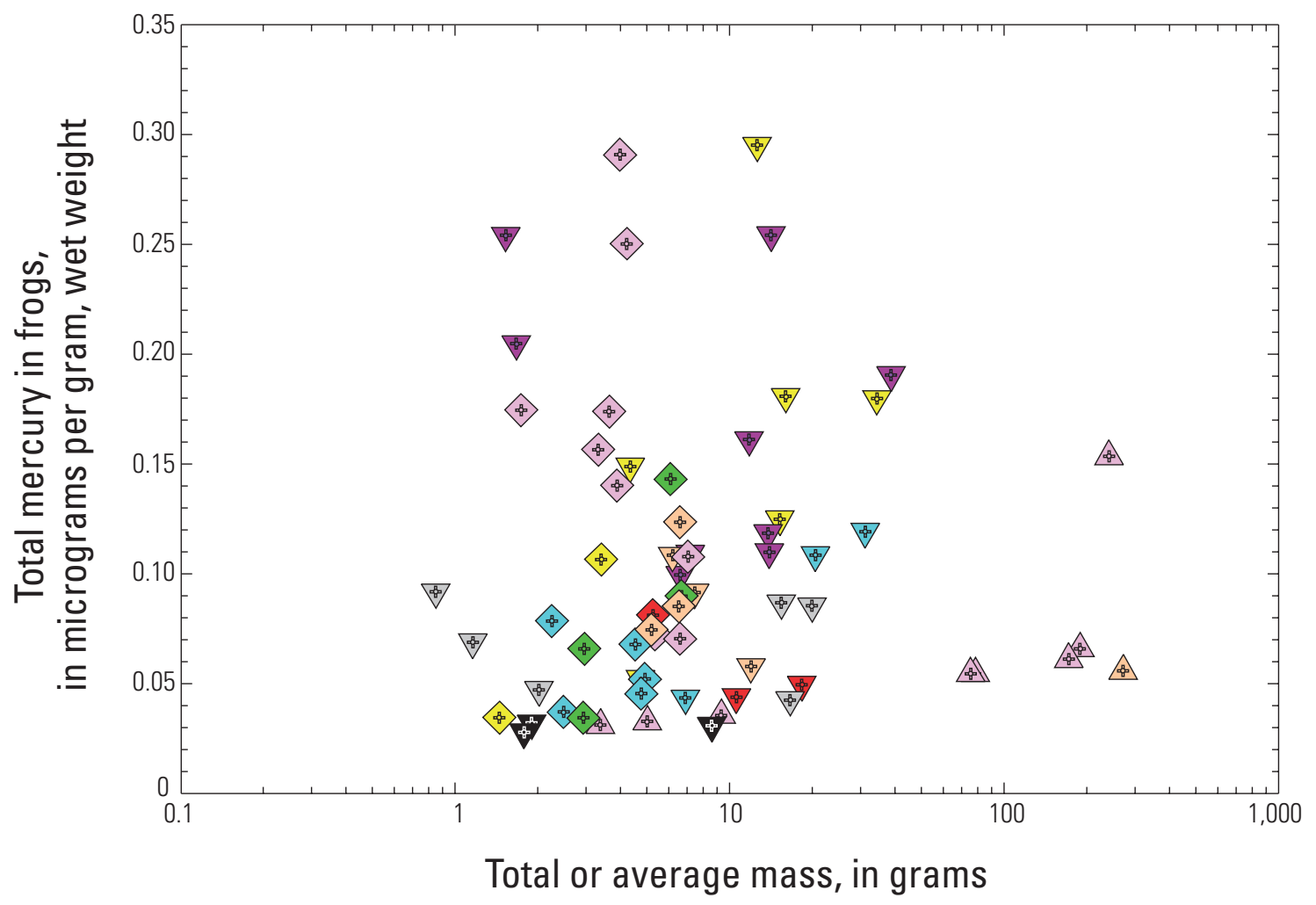

EXPLANATION

\begin{tabular}{llll}
\multicolumn{2}{c}{ Station group } & & \\
$\square$ Boston & $\square$ Poore & A Bullfrogs \\
Buckeye & SF Greenhorn & $\$$ Pacific treefrogs \\
$\square$ Greenhorn, main stem & $\square$ Sailor Flat & $\$$ Foothill yellow-legged frogs \\
Integrator & & Starr \\
$\square$ Missouri Canyon & &
\end{tabular}

Figure 28. Relation between total mercury concentration and total or average mass of all frog species from the Greenhorn Creek drainage, Nevada County, California. 
In the remainder of this section, results for each of the ten geographic areas identified in figures 2-5 are discussed. The discussion begins with the Headwaters station (BY51), then covers each of the subwatersheds with mining inputs in approximate downstream order, and then progresses to the Integrator station (Greenhorn Creek at You Bet Road, BY59).

\section{Greenhorn Creek—Headwaters}

Relatively few water and sediment samples were collected from the Headwaters reach of Greenhorn Creek (station BY51). The water sample that was collected from this station in January 2000 was rated very low for unfiltered $\mathrm{THg}$, and low for unfiltered $\mathrm{MeHg}$ (fig. 32). However, the filtered $\mathrm{THg}$ value (about $5 \mathrm{ng} / \mathrm{L}$ ) was unexpectedly rated in the moderately high category. Although there are no known mine wastes upstream of this station, soils in this area may have been contaminated by locally or regionally derived atmospheric deposition of mercury. Locally derived atmospheric mercury sources may have included retort activity during the period of active hydraulic and underground gold mining (1850-1942); regional sources included retort activity associated with mercury mining in the Coast Range (Alpers and Hunerlach, 2000); globally derived atmospheric deposition of mercury may also be a factor. The baseline station (BY199, fig. 1) is used in this report as a reference site for invertebrate samples. Although it is also upstream of known mining sources, it may also have been affected by atmospheric deposition of mercury, as described above.

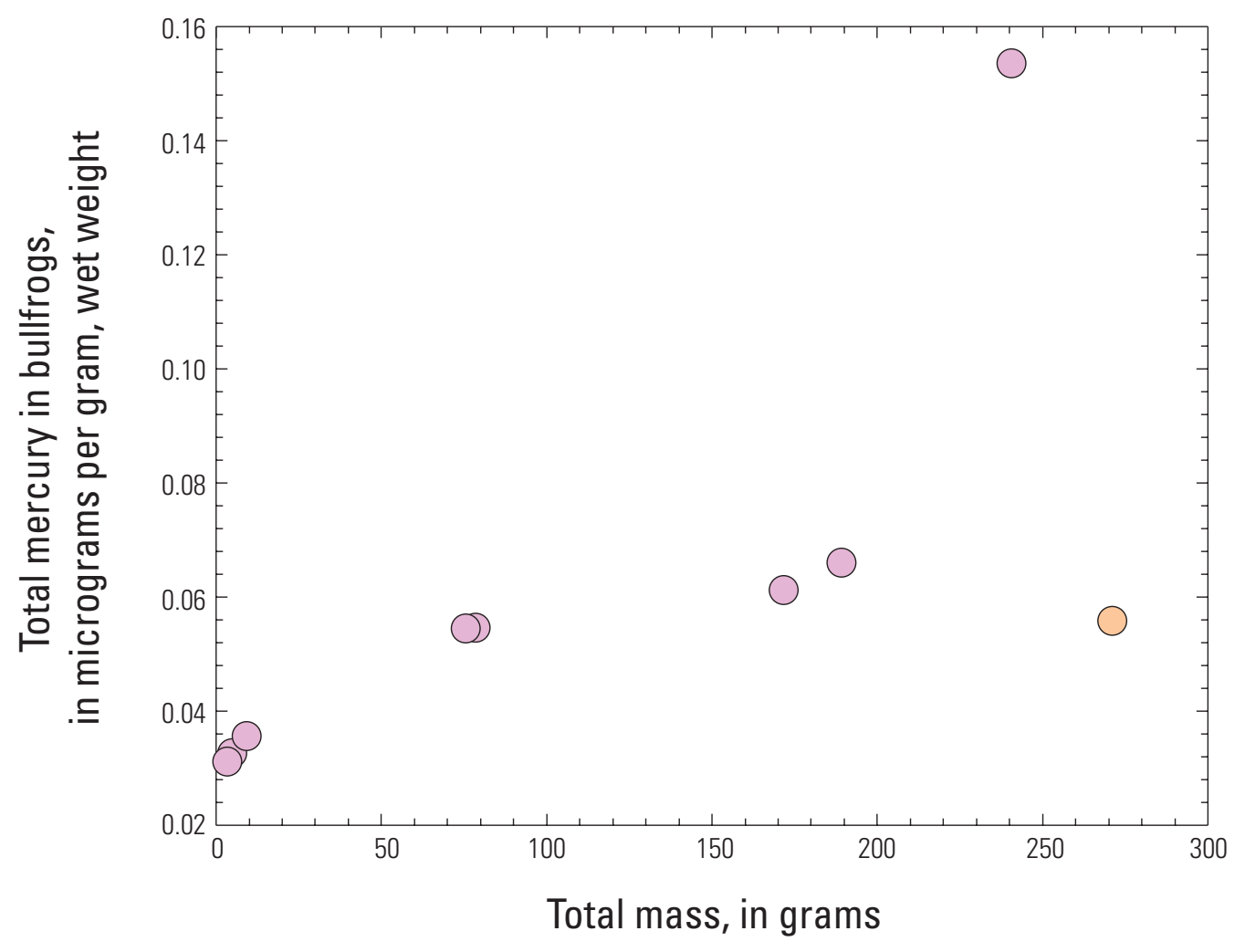

EXPLANATION

\begin{tabular}{|c|c|}
\hline & \\
\hline Boston & Starr \\
\hline
\end{tabular}

Figure 29. Relation between total mercury concentration and mass of individual bullfrogs from the Greenhorn Creek drainage, Nevada County, California. 


\section{Sailor Flat Area}

Biological samples of six taxa were collected at the outlet of the Tom and Jerry tunnel (BY130) in 2000 and 2001 (fig. 32); four of the six taxa rated in the moderately high and high categories and the other two were in the low category, falling somewhat below study median values. Mercury was visible at this station, rating in the extremely high category. In contrast, results for a water sample collected in June 2000 rated in the low category for both unfiltered THg and unfiltered $\mathrm{MeHg}$. Concentrations of $\mathrm{THg}$ and $\mathrm{MeHg}$ in unfiltered water from a water sample collected at the inlet to the Tom and Jerry tunnel (BY129) in June 2000 were higher than the study medians. A disturbed water sample collected at this station (not used in the summary table) had approximately 10 times more THg in both filtered and unfiltered water than an undisturbed sample collected on the same date (table 2). A water sample collected from a pond at the Tom and Jerry Mine station (BY131) in August 2000 rated high with regard to $\mathrm{THg}$ in both filtered and unfiltered water, and dragonfly samples from this station rated moderately high. The Tom and Jerry area (also known as Sailor Flat East) was chosen by the USDA-FS as a pilot remediation project (U.S. Department of Agriculture-Forest Service, 2002) and underwent restoration activity during November 2003.

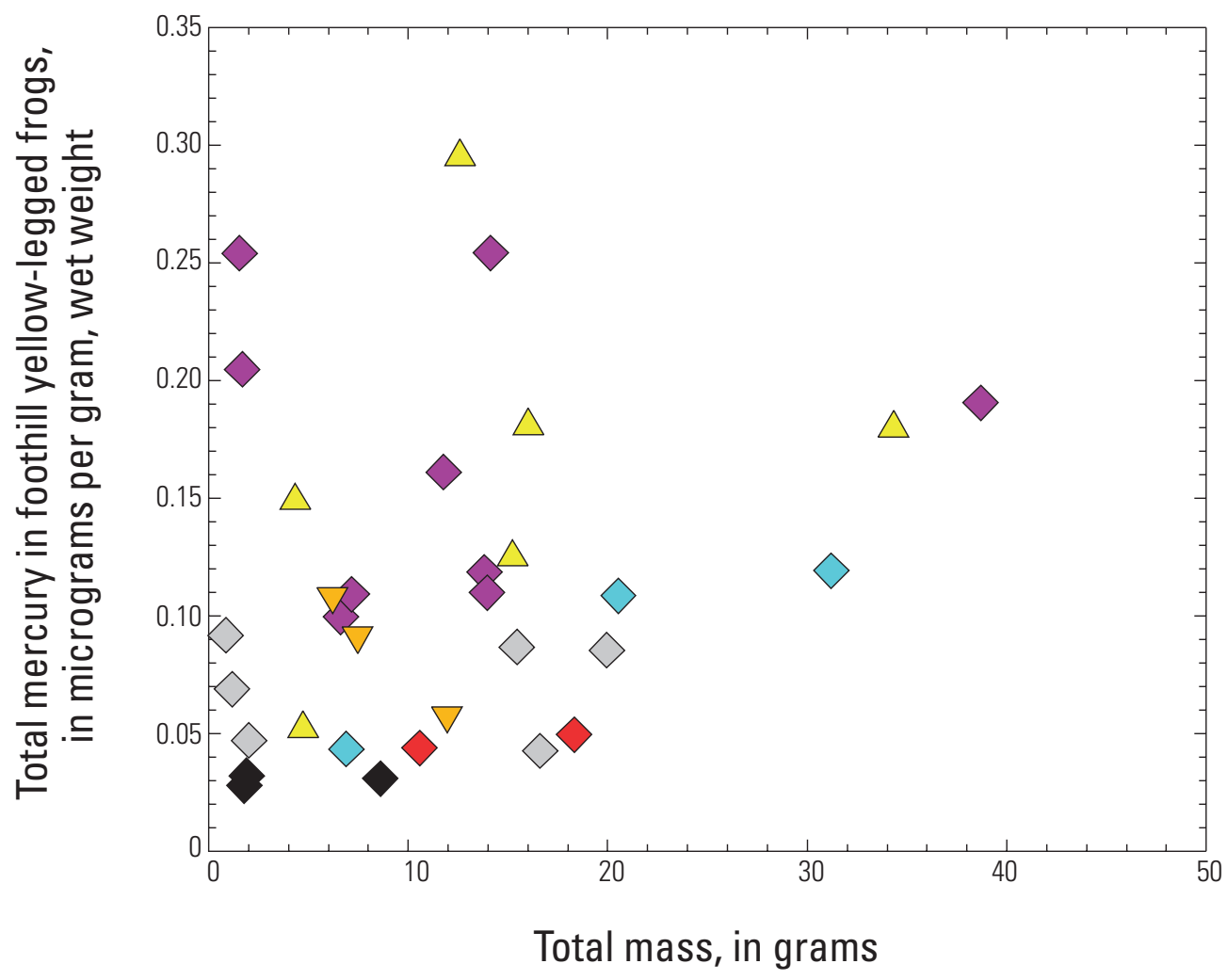

EXPLANATION

\begin{tabular}{lll}
\multicolumn{2}{c}{ Station group } & \multicolumn{2}{c}{ Type of sampling station } \\
Buckeye & $\square$ Missouri Canyon & $\triangle$ Ground sluice \\
$\square$ Greenhorn, main stem $\quad \square$ Poore & $\diamond$ Stream \\
Integrator & SF Greenhorn & $\nabla$ Tunnel
\end{tabular}

Figure 30. Relation between total mercury concentration and mass of individual foothill yellowlegged frogs from the Greenhorn Creek drainage, Nevada County, California. 
The main drainage gulch on the western side of the Sailor Flat area (BY105) had extremely high concentrations of mercury and $\mathrm{MeHg}$ in water, and consistently high concentrations of visible mercury were panned from bottom sediments in the plunge pools of the deeply incised bedrock drainage below the mine. Although no quantitative discharge measurements were made, high flow during a storm event was estimated to be about two to five cubic feet per second. Large quantities of sediment were observed being transported during this storm event. Subsequent sediment sampling yielded visible mercury in the bottom of a plunge pool after waters had receded. Sediment samples sent to the laboratory from this station were generally low in both $\mathrm{THg}$ and $\mathrm{MeHg}$, and biological samples from three taxa were relatively low compared with those from other stations.

Water samples from the drainage of the western side of the Sailor Flat area toward Greenhorn Creek (BY106) were moderately high in both filtered and unfiltered $\mathrm{MeHg}$ and high in filtered $\mathrm{THg}$, but relatively low in unfiltered $\mathrm{THg}$. Although water in the western side of the Sailor Flat area appears to be contaminated with relatively high concentrations of $\mathrm{THg}$ and $\mathrm{MeHg}$, the lack of well defined remediation targets such as tunnels or ground sluices makes this station challenging in terms of formulating a remediation strategy.

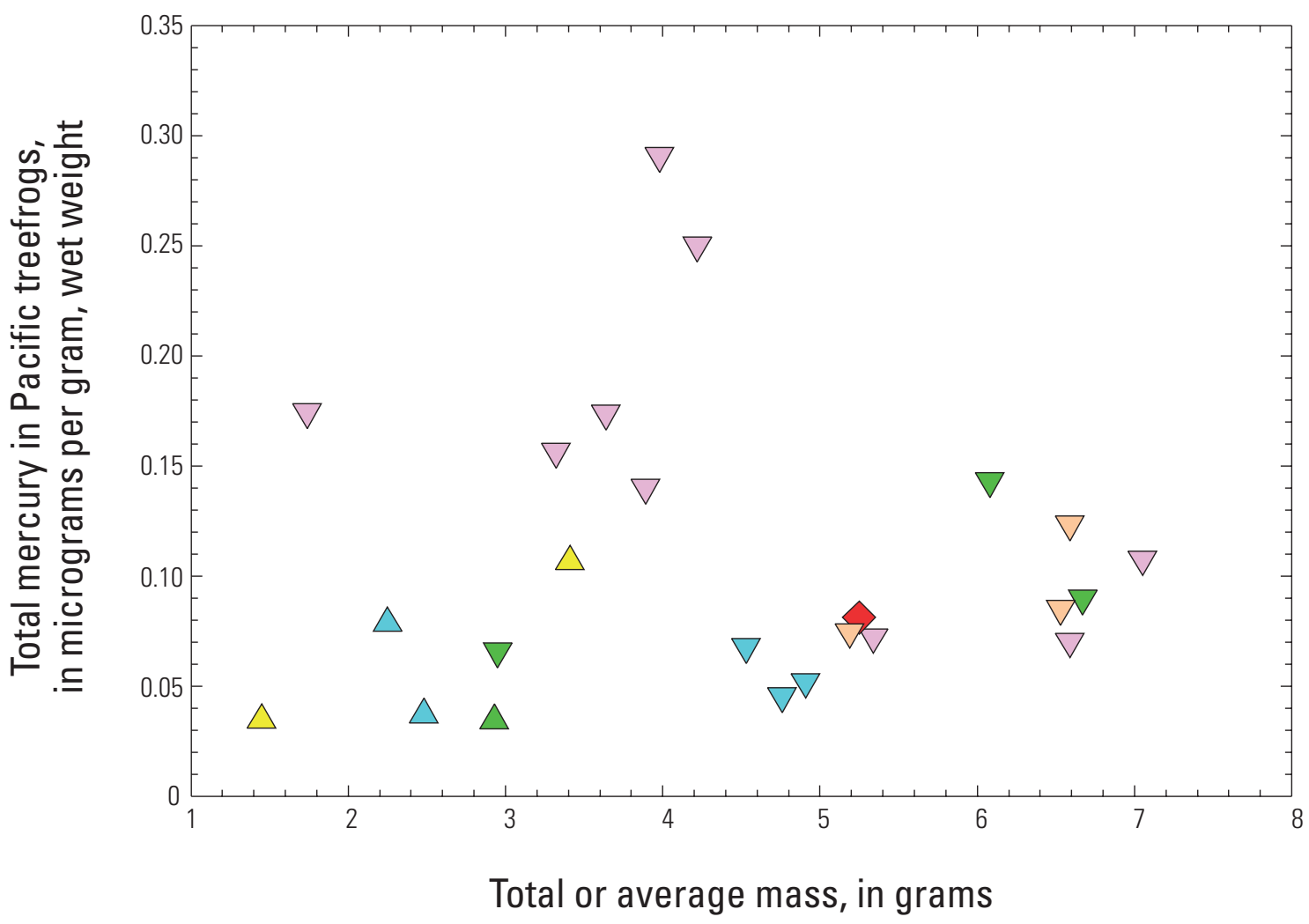

EXPLANATION

\begin{tabular}{lll}
\multicolumn{2}{c}{ Sub-watershed } & \multicolumn{2}{c}{ Type of sampling station } \\
Boston & $\triangle$ Poore & $\triangle$ Ground sluice \\
Buckeye & $\square$ Sailor Flat & $\searrow$ Stream \\
Missouri Canyon & $\square$ Starr & $\nabla$ Tunnel
\end{tabular}

Figure 31. Relation between total mercury concentration and total or average mass of Pacific treefrogs from the Greenhorn Creek drainage, Nevada County, California. 


\begin{tabular}{|c|c|c|c|c|c|c|c|c|c|c|c|c|c|c|c|c|}
\hline \multirow[b]{2}{*}{$\begin{array}{l}\text { Station } \\
\text { map ID }\end{array}$} & \multirow[b]{2}{*}{ Station Name } & \multicolumn{4}{|c|}{ Water } & \multicolumn{3}{|c|}{ Sediment } & \multicolumn{6}{|c|}{ Invertebrates } & \multicolumn{2}{|c|}{ Frogs } \\
\hline & & $\begin{array}{l}\text { 志 } \\
1 \\
\text { ㅁㅗㅗ } \\
\end{array}$ & $\begin{array}{l}\equiv \\
1 \\
1 \\
\text { 뫁 } \\
\end{array}$ & 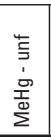 & 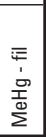 & 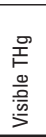 & 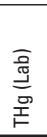 & 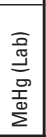 & 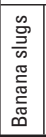 & 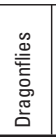 & 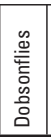 & 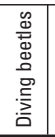 & 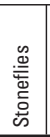 & 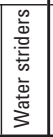 & 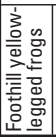 & 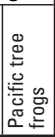 \\
\hline \multicolumn{17}{|c|}{ Headwaters } \\
\hline BY51 & Greenhorn Cr headwaters nr Scotts Flat Reservoir & 0.48 & 1.8 & 0.84 & & & & & & & & & & & & \\
\hline \multicolumn{17}{|c|}{ Sailor Flat / Tom and Jerry } \\
\hline BY105 & Sailor Flat Mine main drain Gulch 01 nr Quaker Hill & 80 & 26 & 13 & & 60 & 0.03 & 0.02 & & & & 0.33 & & 0.73 & & 0.45 \\
\hline BY106 & Sailor Flat Mine main drain to Greenhorn Cr, Gulch 03 & 1.0 & 3.5 & 1.5 & 1.8 & & & & & & & & & & & \\
\hline BY129 & Tom and Jerry Mine drain at tunnel inlet nr Nevada City & 1.8 & & 2.6 & & & & & & & & & & & & \\
\hline BY130 & Tom and Jerry Mine drain at tunnel outlet nr Nevada City & 0.09 & & 0.28 & & 10 & & & 1.2 & 2.6 & 0.70 & 0.93 & & 1.0 & & 1.2 \\
\hline BY131 & Tom and Jerry Mine drainage pond nr Nevada City & 2.2 & 5.7 & 0.28 & & & & & & 0.29 & & 0.56 & & 0.59 & & \\
\hline \multicolumn{17}{|l|}{ Buckeye } \\
\hline BY22 & Buckeye Flat Mine drain $0.1 \mathrm{mi}$ ab Greenhorn $\mathrm{Cr}$ & & & & & 1.0 & & & & & & 0.30 & & 0.80 & 0.56 & \\
\hline BY58 & Buckeye Flat Mine main drain $0.45 \mathrm{mi}$ ab Greenhorn $\mathrm{Cr}$ & 0.1 & 0.34 & & & & & & 0.85 & & & 0.53 & & 0.87 & & 1.0 \\
\hline BY23 & Buckeye Flat Mine N drain to Greenhorn Cr Grass Valley & 61 & 70 & 1.1 & & & 5.7 & 0.03 & 0.74 & & & & & 0.81 & & \\
\hline BY116 & Buckeye Flat Mine pond drain $0.15 \mathrm{mi}$ ab SF Greenhorn $\mathrm{Cr}$ & 0.65 & 3.55 & & & & & & 4.1 & 1.1 & & 0.89 & & 1.1 & & \\
\hline BY24 & Buckeye Flat Mine S drain to SF Greenhorn Cr Grass Valley & 6,200 & 2,500 & 130 & & & 0.86 & 1.2 & & 3.9 & & 1.2 & & 1.6 & & \\
\hline BY25 & Buckeye Flat Mine upper drain & 0.28 & 0.4 & 0.49 & 1.00 & & & & 1.7 & & & 0.80 & & 0.72 & & \\
\hline \multicolumn{17}{|c|}{ Boston Mine } \\
\hline BY20 & Boston Mine tunnel outlet nr Grass Valley & 1.4 & 1.1 & 0.98 & 1.3 & 45 & 51 & 0.20 & 1.8 & 14 & & 3.3 & & 2.1 & & 1.8 \\
\hline BY21 & Boston Mine wetlands pond $\mathrm{nr}$ Grass Valley & 1.1 & 0.87 & 2.4 & & & 0.53 & 2.2 & & 0.47 & & 1.7 & & 0.85 & & \\
\hline \multicolumn{17}{|c|}{ South Fork Greenhorn Creek } \\
\hline BY113 & SF Greenhorn Cr 0.8 mi ab Greenhorn Cr nr Nevada City & & & & & & & & 1.0 & & 0.58 & & 0.69 & 0.62 & 1.8 & \\
\hline BY114 & SF Greenhorn Cr 0.7 mi ab Greenhorn Cr nr Nevada City & 14 & 65 & 1.9 & 8.5 & & & & 0.57 & & 1.0 & & 1.7 & 1.6 & 1.8 & \\
\hline BY115 & SF Greenhorn bl Boston Mine nr Nevada City & 0.83 & 2.3 & 0.28 & 0.63 & 1.0 & & & & 5.1 & 3.1 & & 3.8 & 1.8 & 2.1 & \\
\hline \multicolumn{17}{|c|}{ Poore Mine } \\
\hline BY86 & Poore Mine creek ab tunnel nr Grass Valley & 1.2 & 0.05 & 0.28 & & & & & 0.83 & 0.75 & & & & 0.95 & 0.99 & \\
\hline BY87 & Poore Mine creek bl tunnel nr Grass Valley & 0.33 & 0.27 & 0.28 & & 1.0 & & & & & & & & & & \\
\hline BY88 & Poore Mine ground sluice nr Grass Valley & 0.06 & 0.05 & 0.28 & & 4.0 & & 3.4 & & & & & & & & \\
\hline BY89 & Poore Mine pit lake nr Grass Valley & 0.34 & 0.28 & 1.1 & & 1.0 & & & & & & & & & & \\
\hline BY90 & Poore Mine seep above ground sluice nr Grass Valley & & 0.05 & & & & & & 2.2 & & & 0.35 & & 1.1 & & 0.98 \\
\hline BY91 & Poore Mine tunnel effluent nr Grass Valley & 1.1 & 0.18 & 1.0 & & & & & 0.59 & 0.43 & 0.59 & & 0.48 & 1.2 & & 0.68 \\
\hline \multicolumn{17}{|c|}{ Starr Mine } \\
\hline BY122 & Starr Mine tunnel inflow nr Grass Valley & 0.32 & 0.19 & 0.28 & & & & & & & & & & & & \\
\hline BY123 & Starr Mine tunnel midway nr Grass Valley & 64 & 1.6 & 11 & & 10 & 69 & 6.1 & & & & & & & & \\
\hline BY124 & Starr Mine tunnel outlet nr Grass Valley & 32 & 1.2 & 7.6 & 0.63 & & & & 0.68 & 0.65 & & & & 1.0 & 1.0 & 1.2 \\
\hline BY148 & Starr Pit Pond nr Grass Valley & & & & & & & & & 0.21 & & 2.3 & & 0.89 & & \\
\hline \multicolumn{17}{|c|}{ Missouri Canyon } \\
\hline \multirow{5}{*}{$\begin{array}{l}\text { BY144 } \\
\text { BY146 } \\
\text { BY75 } \\
\text { BY145 } \\
\text { BY147 } \\
\end{array}$} & Coon Hollow Cr drain tunnel outlet nr Dutch Flat & & & & & & & & & 0.89 & & 1.1 & & 1.3 & & \\
\hline & Missouri Cyn 1.6 mi ab Greenhorn Cr nr Chicago Park & & & & & 1.0 & & & 3.1 & & & 1.1 & & 1.8 & & \\
\hline & Missouri Cyn Cr 1.2 mi ab Greenhorn Cr nr Chicago Park & 0.42 & 0.74 & 1.0 & & 5.0 & 1.0 & 0.77 & 0.33 & 1.4 & 2.3 & & 2.0 & 1.1 & 1.5 & 0.88 \\
\hline & Missouri Cyn Cr tributary nr Chicago Park & & & & & & & & & & & 1.1 & & 3.0 & & \\
\hline & NF MF Missouri Cyn nr Chicago Park & & & & & & & & 3.7 & 4.8 & 5.9 & 1.8 & & 2.0 & 2.7 & \\
\hline Greenhor & Creek - Main stem & & & & & & & & & & & & & & & \\
\hline BY55 & Greenhorn Cr 0.1 mi bl Sailor Flat west drain nr Nevada City & & & & & & & & & & & & & 0.68 & & \\
\hline BY57 & Greenhorn Cr 0.3 mi bl Sailor Flat west drain nr Nevada City & & & & & & & & & & 0.45 & & 0.42 & 0.72 & & \\
\hline BY52 & Greenhorn Cr 0.3 mi ab Buckeye Ford nr Nevada City & 1.9 & 1.3 & 0.28 & & & & & & & & & & & & \\
\hline BY60 & Greenhorn Cr bl Buckeye drain nr Nevada City & & & & & & & & & & & & & 0.79 & & \\
\hline BY53 & Greenhorn Cr ab SF Greenhorn Cr nr Nevada City & & & & & & & & & & & & 1.0 & & 0.80 & \\
\hline BY56 & Greenhorn Cr bl SF Greenhorn Cr nr Nevada City & & & & & 1.0 & & & & 0.21 & & & 1.1 & & 0.82 & \\
\hline BY180 & Greenhorn $\mathrm{Cr} 0.2 \mathrm{mi} b \mathrm{l}$ The Narrows & & & & & 1.0 & & & & 1.2 & 1.0 & & & 0.81 & & \\
\hline Integrator & & & & & & & & & & & & & & & & \\
\hline BY59 & Greenhorn Cr at You Bet Rd nr Nevada City & 0.90 & 0.88 & 1.0 & 0.87 & 1.0 & & & & & & & 0.56 & 1.0 & 0.36 & \\
\hline
\end{tabular}

EXPLANATION

Mercury ratings

$\begin{aligned} & \text { Extremely high } \square \quad \begin{array}{c}\square \\ c^{\prime}\end{array} \geq 10 \\ & \text { High } \square 10>c^{\prime} \geq 2 \\ & \text { Moderately high } \square \quad 2>c^{\prime} \geq 1 \\ & \text { Low } \square \quad 1>c^{\prime} \geq 0.5 \\ & \text { Very low } \square \quad 0.5>c^{\prime} \\ & c^{\prime} \text {, concentration values normalized }\end{aligned}$

c', concentration values normalized
to median for individual sites
Abbreviations

$\begin{array}{llll}\text { ab, above } & \text { Lab, laboratory } & \text { NF, North Fork } & \text { S, south } \\ \text { bl, below } & \text { MeHg, methylmercury } & \text { nr, near } & \text { SF, South Fork } \\ \text { Cr, Creek } & \text { MF, Middle Fork } & \text { Pk, Park } & \text { THg, total mercury } \\ \text { Cyn, Canyon } & \text { mi, mile } & \text { Rd, Road } & \text { unf, unfiltered } \\ \text { fil, filtered } & \text { N, north } & \text { Res, Reservoir } & \end{array}$

Figure 32. Summary of data for mercury and methylmercury in water, sediment, and biota, Greenhorn Creek drainage, Nevada County, California. 


\section{Buckeye Area}

Samples from the Buckeye Flat complex (6 stations) yielded some of the highest concentrations of $\mathrm{MeHg}$ and $\mathrm{THg}$ in water, sediment, and biota (fig. 32). The Buckeye South station (BY24) ranked among the five most contaminated locations with regard to both water and invertebrates, although sediment samples from the station were near median values in terms of $\mathrm{THg}$ and $\mathrm{MeHg}$ concentrations. Water samples collected from station BY24 during August 1999 and August 2000 had extremely elevated levels of THg (filtered and unfiltered) and $\mathrm{MeHg}$ (unfiltered), with concentrations several orders of magnitude greater than those in samples from most other stations. Concentrations of $\mathrm{THg}$ and $\mathrm{MeHg}$ in water and biota were highly variable from year to year at this station, resulting in a moderate overall rank for biota. Buckeye North drain (BY23) ranked extremely high for THg in water, high for $\mathrm{THg}$ in sediment, and moderately high for $\mathrm{MeHg}$ in unfiltered water, based on water samples collected during 1999 and 2000 and a sediment sample collected during 1999, but ranked very low for $\mathrm{MeHg}$ in sediment and low for $\mathrm{MeHg}$ in two invertebrate taxa sampled during 2000. The acidic conditions of the water at BY23 (pH 3.7 to 4.2 ; table 1) may have affected rates of $\mathrm{Hg}$ methylation and bioaccumulation and certainly affected ecological diversity.

Measured flows at station BY24 during sampling in August 1999 and August 2000 were approximately $1 \mathrm{gal} / \mathrm{min}$; however, evidence of higher flow was observed. Deep plunge pools in the incised drainage below BY24 contained small amounts of both fine- and coarse-grained sediments with abundant organic material. These plunge pools are isolated during low flow and contain water with temperatures as high as $24^{\circ} \mathrm{C}$. Waters draining the hydraulic pit toward the south travel through a network of ground sluices. Because of a moderate hydraulic gradient, the sediment transported through the BY24 station is mostly sand- and silt-sized particles.

The Buckeye Flat North drain (BY23) drains a smaller area and has a steeper gradient than station BY24. The drainage is controlled by a network of deeply incised bedrock sluices that drain through a tunnel. A large concentration of debris and sediment has accumulated in the steeply incised drainage below the tunnel. Flows were relatively consistent, between 5 and $8 \mathrm{gal} / \mathrm{min}$. Some evidence of disturbance from recreational mining was observed just below the tunnel discharge. There is a large concentration of organic material from the forest canopy. The low $\mathrm{pH}$ values (3.7-4.2) measured at station BY23 may have been caused by the oxidation of abundant pyrite exposed in the slate bedrock throughout this portion of the hydraulic pit and pyrite in residual gravel deposits.

\section{Boston Mine Area}

The Boston Mine tunnel (BY20) contains a remnant section of an old sluice box that is partially filled with mining debris. Elemental mercury was found in the sediment, with the largest concentrations at and near the bedrock contact. Precipitation and storage of water in the hydraulic pit above the tunnel leads to flow through the tunnel, where water can interact with mercury-contaminated sediments. The upstream entrance to the tunnel is caved or blocked. Measured discharge at the tunnel outlet ranged from 2.5 to $15 \mathrm{gal} / \mathrm{min}$. On the basis of several site visits, it was evident that recreational miners had frequently disturbed sediment within the tunnel and below the tunnel. The tunnel outlet is located in bedrock about 20 feet above ground surface, leading to a cascade of the outfall down a fractured bedrock face. At the higher flows, fine-grained sediment and particulate mercury are probably transported out of the tunnel. Bedrock fractures alongside the deep plunge pool below the tunnel yielded sediment containing the highest measured visible mercury concentration within the study area $(45,000 \mathrm{mg} / \mathrm{kg}$, equivalent to 4.5 percent by weight).

Samples of biota and water were collected from the Boston Mine tunnel outlet (BY20) during 1999, 2000, and 2001. Samples of biota (four taxa of invertebrates, and Pacific treefrogs) consistently had elevated concentrations of $\mathrm{MeHg}$ and THg (fig. 32). Dragonflies from station BY20 are the only example of an extremely high normalized concentration (greater than 10 times the study median) for all of the biological samples in the study area. Water samples from the Boston Mine tunnel outlet were moderately high in filtered and unfiltered $\mathrm{THg}$ and in filtered $\mathrm{MeHg}$, and just below the study median value in unfiltered $\mathrm{MeHg}$.

The ponded wetlands at the Boston Mine (BY21, fig. 4) are located upstream of the mine tunnel. Water and sediment samples from this station had moderately high to high levels of $\mathrm{MeHg}$ and somewhat lower levels of THg compared with those from the tunnel outlet (BY20). Samples of three taxa of invertebrates from these wetlands had lower levels of $\mathrm{MeHg}$ than equivalent samples from the mine tunnel outlet, but the levels for the predaceous diving beetles were moderately high. Caution must be used in the interpretation of data for predaceous diving beetles, as values even from the baseline reference station (BY199) were relatively high (near the median for all samples from the study area). Eight bullfrog samples from the Boston Mine wetlands varied in size and THg content; the concentration of $\mathrm{MeHg}$ in the frog generally corresponded to the size of the frog (fig. 29). The largest bullfrog had a THg content of about $0.15 \mu \mathrm{g} / \mathrm{g}$ (wet basis).

The Bureau of Land Management selected the Boston Mine tunnel for a pilot remediation project. Environmental compliance documents were prepared, and remediation is scheduled for 2005. 


\section{South Fork Greenhorn Creek}

The South Fork Greenhorn Creek tributary to Greenhorn Creek is the initial water body to receive discharge from the Buckeye South drainage (BY 24), the Buckeye East drain (BY 116), and the Boston Mine tunnel outlet (BY20) (fig. 4). Three sampling stations (BY113, BY114, and BY115) were located longitudinally along a reach of the South Fork of Greenhorn Creek. Of these three, station BY115 is farthest downstream, near the confluence with the main stem of Greenhorn Creek; station BY114 is located upstream of the Boston Mine tunnel outlet (BY20) but downstream of the Buckeye Drain inputs (BY24 and BY116). Station BY113 is the farthest upstream station, but is still downstream of the Buckeye East Drain (BY116). MeHg in dobsonflies, stoneflies, and water striders, and $\mathrm{THg}$ in foothill yellow-legged frogs, collected from this reach of South Fork Greenhorn Creek (tables 11, 12; fig. 32) show a systematic increase apparently associated with distance downstream and the cumulative effects of the inputs from the Buckeye and Boston Mine areas.

\section{Poore Mine Area}

Samples of water from six stations in the Poore Mine area were among the least contaminated samples from the mine sites sampled for this study. All of the normalized values for filtered $\mathrm{THg}$ are in the very low range (fig. 32). Two of five values of both unfiltered $\mathrm{THg}$ and unfiltered $\mathrm{MeHg}$ are in the moderately high range, just above the study median values for these analytes, whereas the remaining three values for both analytes are in the very low range. Sediment contained a high concentration of visible mercury in a ground sluice and a trace amount (considered moderately high, equal to the median) at two other stations in the area. The $\mathrm{MeHg}$ concentration in a sediment sample from the ground sluice area was also moderately high.

Mercury and $\mathrm{MeHg}$ in most of the biological samples from the Poore Mine area were in the very low to low range. Exceptions to this generalization were a banana slug sample from a seepage area above the ground sluice (BY90) that had high $\mathrm{MeHg}$ and water striders from BY90 and the tunnel effluent station (BY91) that had moderately high MeHg amounts just above the study median.

\section{Starr Mine Area}

Samples of water and sediment from a point near the middle of the Starr Mine tunnel (BY123) and samples of water from the tunnel outlet (BY124) indicate that this area is heavily contaminated with $\mathrm{THg}$ and $\mathrm{MeHg}$ (fig. 32). In contrast, the inflow water to the Starr Mine tunnel (BY122) was very low in both $\mathrm{THg}$ and $\mathrm{MeHg}$, which suggests that the water flowing through and out of the tunnel acquired its $\mathrm{THg}$ and $\mathrm{MeHg}$ by interacting with contaminated sediments in the tunnel.

Biological sampling in the middle of the Starr Mine tunnel was not possible because target species were not present. Samples of five taxa of biota from the tunnel outlet area were low to moderately high in MeHg. Samples of three taxa of biota from a pond in the Starr Mine pit (BY148) were rated very low (dragonflies) to high (diving beetles). Although not shown in fig. 32, a bullfrog sample from the Starr Mine pit was relatively low in $\mathrm{THg}$ compared with samples of comparable mass from the Boston Mine wetlands (BY21) (fig. 29, table 12).

\section{Missouri Canyon Area}

$\mathrm{MeHg}$ in biological samples from most stations in the Missouri Canyon area were rated moderately high to high (fig. 32). All of the sampling stations in this area are interpreted to be ground sluices except the Coon Hollow tunnel outlet (BY144). A ground sluice station at the North Fork of the Middle Fork of Missouri Canyon (BY147) had four taxa in the high category and two in the moderately high category; combining these data gives the highest overall ranking of $\mathrm{MeHg}$ in biota of all stations in the study area. A foothill yellow-legged frog sample from station BY147 had a THg concentration of $0.30 \mu \mathrm{g} / \mathrm{g}$, the highest of all frog samples from the study area (fig. 28, table 12).

Few samples of water and sediment were collected from Missouri Canyon during the study period. A water sample from station BY75 collected in December 1999 was rated very low for unfiltered $\mathrm{THg}$, low for filtered $\mathrm{THg}$, and was near the study median (moderately high) for unfiltered MeHg. Sediment from station BY75 contained a high concentration of visible mercury; $\mathrm{MeHg}$ and $\mathrm{THg}$ concentrations in a sediment sample analyzed in the laboratory were rated low and moderately high, respectively. A trace (moderately high) of visible mercury was found at another ground sluice station (BY146). Several of the sampling stations in the Missouri Canyon area contained elevated levels of $\mathrm{MeHg}$ in biota, which qualifies this area as a "hot spot." However, more sampling and hydrogeological analysis is needed to determine whether a suitable strategy for remediation can be identified. 


\section{Greenhorn Creek-Main Stem}

Samples were collected from the part of the main-stem reach of Greenhorn Creek located between the Sailor Flat area (BY55) and a point below the Narrows (BY180), a distance of about 4 river miles. A single water sample from the station above Buckeye Ford (BY52) had moderately high THg in filtered and unfiltered water, but very low MeHg in unfiltered water (fig. 32). Trace amounts of visible mercury (moderately high) were documented at two of the main-stem stations. Biological data for this area ranged from very low to moderately high. Water striders and Foothill yellow-legged frogs had low values at all six stations at which samples were collected. Three of the main-stem Greenhorn Creek stations had taxa with moderately high values, ranging from 1.0 to 1.2 times the study median for dragonflies, dobsonflies, and stoneflies. Concentrations of $\mathrm{MeHg}$ tended to increase with distance downstream for each of the five biological taxa for which data are available (fig. 32), suggesting a systematic, cumulative increase of bioavilable $\mathrm{MeHg}$ downstream.

\section{Greenhorn Creek at You Bet Road—Integrator Site}

The Integrator station at the bottom of the Greenhorn Creek drainage was generally rated low for $\mathrm{THg}$ and $\mathrm{MeHg}$ in water and biota (fig. 32). Three taxa of biota (stoneflies, water striders, and foothill yellow-legged frogs) were sampled in August 1999. Water was sampled monthly from July to December 2001. A trace of visible mercury was found by panning methods, but no sediment samples were analyzed quantitatively for $\mathrm{THg}$ and $\mathrm{MeHg}$. The relatively low $\mathrm{THg}$ and $\mathrm{MeHg}$ concentrations in biota from this station could have been caused in part by dilution, as well as by intense in-stream gravel mining activity upstream which continually disturbs the area during the summer and fall months each year. The high degree of disturbance degraded the aquatic habitat, making it difficult to compare with other sites that were not as disturbed. Mercury that was mobilized in this area because of the gravel mining activity may have accumulated in Rollins Reservoir, or may have been transported farther downstream in the Bear River watershed.

\section{Summary and Conclusions}

Reconnaissance sampling of water, sediment, and biota was done at 40 stations in the Greenhorn Creek drainage during 1999-2001 to characterize the degree of mercury and methylmercury $(\mathrm{MeHg})$ contamination associated with historical gold mining and to identify potential remediation sites. The distribution of mercury and methylmercury is highly variable in time and space. Results identified two remediation sites for federal land management agencies: abandoned sluice tunnels in the Sailor Flat Mine area (U.S. Department of AgricultureForest Service) and the Boston Mine area (Bureau of Land Management).

The range in concentrations of $\mathrm{THg}$ in unfiltered water spanned nearly seven orders of magnitude; more than 25 percent of the analyzed concentrations exceeded $50 \mathrm{ng} / \mathrm{L}$ (nanogram per liter), the applicable water-quality criterion for protection of aquatic life. Total mercury $(\mathrm{THg}$ ) concentrations in unfiltered water exceeded 2,000 ng/L, the national drinking water standard, in a small number of the water samples. The highest concentrations of $\mathrm{THg}$ in water were found in ground sluices and tunnel sluices as opposed to ponds, wetlands, pit lakes, or streams. The concentration of $\mathrm{MeHg}$ in several of the unfiltered water samples was greater than $0.1 \mathrm{ng} / \mathrm{L}$, a reference concentration above which significant bioaccumulation of $\mathrm{MeHg}$ has been observed in other systems. Values of the ratio $\mathrm{MeHg}$ to $\mathrm{THg}$ in unfiltered water samples spanned a wide range, from 0.001 to 30 percent, with most values in the range of 0.1 to 5 percent. The highest value of $\mathrm{MeHg} / \mathrm{THg}$ in water was associated with a sample from a wetland environment.

Acidic drainage in some of the mined areas had $\mathrm{pH}$ values as low as 3.4. Data for major and trace elements are reported for filtered and unfiltered water samples collected at 29 of the stations. Elevated concentrations of aluminum, cadmium, copper, iron, manganese, nickel, and zinc were found at several stations, especially in the more acidic water samples.

Visible mercury in sediment was observed in several areas, and concentrations were quantified using a panning method. Concentrations of elemental mercury documented by the panning method ranged from trace amounts (about $100 \mathrm{mg} / \mathrm{kg}$ [milligram per kilogram]) to large beads $(45,000 \mathrm{mg} / \mathrm{kg}$ or 4.5 percent by weight). The median for $\mathrm{THg}$ in sediment samples analyzed by laboratory methods was around $1 \mathrm{mg} / \mathrm{kg}$, and the median for $\mathrm{MeHg}$ in the sediment was around $0.001 \mathrm{mg} / \mathrm{kg}$ (dry basis). The range of values of the ratio of MeHg to THg in the sediment samples was about 0.01 to 3 percent. The sediment sample with the highest value of $\mathrm{MeHg} / \mathrm{THg}$ was from a wetland environment.

For biological samples, annual variations in $\mathrm{MeHg}$ concentrations by taxon appear to be related primarily to the degree of habitat stability, with intermittent habitats being more variable, and permanent features having relatively consistent $\mathrm{MeHg}$ concentrations. Values of $\mathrm{MeHg} / \mathrm{THg}$ for invertebrate samples ranged from about 35 to 125 percent. Median values for five taxa were in the range of 60 (dobsonflies) to 95 percent (dragonflies). Other studies have found similar values of $\mathrm{MeHg} / \mathrm{THg}$ in predatory insects; variations typically are related to trophic position-upper trophic level taxa (predators) tend to have higher percentages of $\mathrm{MeHg}$ than lower level taxa (herbivores, detritivores, and omnivores). 
Insects have often been used in studies to document elevated levels of metals from historic and ongoing mining operations. In this study, concentrations of $\mathrm{MeHg}$ in several taxa of predatory insects, with the exception of predaceous diving beetles, served as a consistent and reproducible criterion for prioritizing remediation projects at abandoned hydraulic gold mine sites. Methylmercury concentrations were highly variable within families of insects across the study area, but were consistent among families from a given site, indicating localized contamination.

\section{References}

Alpers, C.N., and Hunerlach, M.P., 2000, Mercury contamination from historic gold mining in California: U.S. Geological Survey Fact Sheet 061-00, 6 p. http://ca.water.usgs. gov/mercury/fs06100.html

Alpers, C.N., Hunerlach, M.P., May., J.T., and Hothem, R.L., 2005, Mercury contamination from historical gold mining in California: U.S. Geological Survey Fact Sheet 2005-3014, 6 p. http://pubs.water.usgs.gov/fs2005-3014

Alpers, C.N., Taylor, H.E., and Domagalski, J.L., eds., 2000, Metals transport in the Sacramento River, California, 1996-1997: Volume 1. Methods and Data: U.S. Geological Survey Water-Resources Investigations Report 99-4286, 430 p. http://pubs.water.usgs.gov/wrir99-4286

Bloom, N.S., Colman, J.A., and Barber, L., 1997, Artifact formation of methylmercury during aqueous distillation and alternative techniques for the extraction of methylmercury for environmental samples: Fresenius' Journal of Analytical Chemistry, v. 358, p. 371-377.

Brenton, R.W., and Arnett, T.L., 1993, Methods of analysis by the U.S. Geological Survey National Water Quality Laboratory: Determination of dissolved organic carbon by UV-promoted persulfate oxidation and infrared spectrometry: U.S. Geological Survey Open-File Report 92-480, 12 p.

Cain, D.J., Luoma, S.N., Carter, J.L., and Fend, S.V., 1992 Aquatic insects as bioindicators of trace element contamination in cobble-bottom rivers and streams: Canadian Journal of Fisheries and Aquatic Sciences, v. 49, p. 2141-2154.

California Regional Water Quality Control Board, Central Valley Region, 2003, 2002 CWA (Clean Water Act) Section 303(d) List of Water Quality Limited Segment (approved July 2003 by the U.S. Environmental Protection Agency), 23 p. Accessed July 28, 2004 at URL: http://www.swrcb. ca.gov/tmdl/docs/2002reg5303dlist.pdf
CH2M Hill, 2001, Public release draft remedial investigation report, Lava Cap Mine Superfund Site, Nevada County, California, Volume 1: Report and Appendices A-D, Prepared for U.S. Environmental Protection Agency, Region IX, San Francisco, Calif. Accessed January 12, 2005 at URL: http:// www.epa.gov/region09/cleanup/california.html

Clark, W.B., 1963, Gold districts of California: California Department of Conservation, Division of Mines and Geology: Bulletin 193, 199 p. plus one plate (some revisions through 1969; seventh printing, 1998).

De Wild, J.F., Olson, M.L., and Olund, S.D., 2002, Determination of methylmercury by aqueous phase ethylation, followed by gas chromatographic separation with cold vapor atomic fluorescence detection: U.S. Geological Survey Open-File Report 01-445. http://wi.water.usgs.gov/pubs/ofr01-445/ofr-01-445.pdf

Fishman, M.J., 1993, Methods of analysis by the U.S. Geological Survey National Water Quality Laboratory: Determination of inorganic and organic constituents in water and fluvial sediments: U.S. Geological Survey Open-File Report 93-125, 217 p.

Fishman, M.J., and Friedman, L.C., eds., 1989, Methods of determination of inorganic substances in water and fluvial sediments: U.S. Geological Survey Techniques of WaterResources Investigations, book 5, chap. A-1, 545 p.

Garbarino, J.R., and Taylor, H.E., 1996, Inductively coupled plasma-mass spectrometric method for the determination of dissolved trace elements in natural water: U.S. Geological Survey Open-File Report No. 94-358, 88 p.

Gilbert, G.K., 1917, Hydraulic-mining debris in the Sierra Nevada: U.S. Geological Survey Professional Paper 105, $154 \mathrm{p}$.

Gilmour, C.C., Henry, E.A., and Mitchell, R., 1992, Sulfate stimulation of mercury methylation in freshwater sediments: Environmental Science and Technology, v. 26, p. 2281-2287.

Guerin-Place, R., Roefs, L., and Twomey, K. (compilers), 1998, California gold mines: A Sesquicentennial photograph collection: California Department of Conservation, Division of Mines and Geology, DMG CD 98-001.

Hall, B.D., Rosenberg, D.M., and Wiens, A.P., 1998, Methylmercury in aquatic insects from an experimental reservoir: Canadian Journal of Fisheries and Aquatic Sciences, v. 55, p. 2036-2047.

Hammerschmidt, C.R., and Fitzgerald, W.F., 2001, Formation of artifact methylmercury during extraction from a sediment reference material: Analytical Chemistry, v. 73, no. 24, p. 5930-5936. 
Hare, L., 1992, Aquatic insects and trace metals: bioavailability, bioaccumulation, and toxicity: Critical Reviews in Toxicology, v. 22, no. 5/6, p. 327-369.

Hayes, H.C., 1993, Metal associations in suspended sediments and bed sediments from the Mississippi River: Golden, Colo., Colorado School of Mines, M.S. thesis, 131 p.

Hintelmann, H., Falter, R., Ilgen, G., and Evans, R.D., 1997, Determination of artifactual formation of monomethylmercury in environmental samples using stable $\mathrm{Hg}^{2+}$ isotopes with ICP-MS detection: calculation of contents applying species specific isotope addition: Fresenius' Journal of Analytic Chemistry, v. 358, p. 363-370.

Hintelmann, H., 1999, Comparison of different extraction techniques used for methylmercury analysis with respect to accidental formation of methylmercury during sample preparation: Chemosphere, v. 39, p. 1093-1105.

Hobson, E.M., and Wiltsee, E.M., 1893, Eleventh Report of the state mineralogist: California State Mining Bureau, $612 \mathrm{p}$.

Hunerlach, M.P., and Alpers, C.N., 2003, Mercury contamination from hydraulic gold mining in the Sierra Nevada, California, in Gray, J.E., ed., Geologic Studies of Mercury by the U.S. Geological Survey: U.S. Geological Survey Circular 1248, 41 p. http://pubs.usgs.gov/circ/2003/c1248/

Hunerlach, M.P., Rytuba, J.J., and Alpers, C.N., 1999, Mercury contamination from hydraulic placer-gold mining in the Dutch Flat mining district, California, in Morganwalp, D.W., and Buxton, H.T., eds., U.S. Geological Survey Toxic Substances Hydrology Program - Proceedings of the Technical Meeting, Charleston, South Carolina, March 8-12, 1999: U.S. Geological Survey Water-Resources Investigations Report 99-4018B, p. 179-189. http://ca.water.usgs. gov/mercury/dutch/wrir994018b.pdf

Jarmin, A., 1927, An investigation of "The feasibility of any plan or plans whereby hydraulic mining operations can be resumed in the state" in Report 23 of the State Mineralogist: California State Mining Bureau, v. 23, no. 1, 130 p.

Kelley, R.L., 1959, Gold vs. grain: The hydraulic mining controversy in California's Sacramento Valley: Glendale, Calif., The Arthur Clark Company, 309 p.

Klasing, S., and Brodberg, R., 2003, Evaluation of potential health effects of eating fish from selected water bodies in the northern Sierra Nevada Foothills (Nevada, Placer, and Yuba Counties): Guidelines for Sport Fish Consumption, December 2003, 46 p. http://www.oehha.ca.gov/fish/so_cal/ nosierra.html

Lindgren, W., 1911, The Tertiary gravels of the Sierra Nevada of California: U.S. Geological Survey Professional Paper $73,226 \mathrm{p}$.
Marvin-DiPasquale, M., Agee, J., McGowan, C., Oremland, R.S., Thomas, M., Krabbenhoft, D., and Gilmour, C., 2000, Methyl-mercury degradation pathways: A comparison among three mercury-impacted ecosystems: Environmental Science and Technology, v. 34, p. 4908-4916.

Marshack, J.B., 2003, A compilation of water-quality goals: California Environmental Protection Agency, Regional Water Quality Control Board - Central Valley Region. August 2003, 186 p.

Mason, R.P., Laporte, J.M., and Andres, S., 2000, Factors controlling the bioaccumulation of mercury, methylmercury, arsenic, selenium, and cadmium by freshwater invertebrates and fish: Archives of Environmental Contamination and Toxicology, v. 38, p. 283-297.

May, J.T., Hothem, R.L., Alpers, C.N., and Law, M.A., 2000, Mercury bioaccumulation in fish in a region affected by historic gold mining: The South Yuba River, Deer Creek, and Bear River watersheds, California, 1999: U.S. Geological Survey Open-File Report 00-367, 30 p. http://ca.water.usgs. gov/archive/reports/ofr00367/index.html

May, P.R., 1970, Origins of hydraulic mining: Oakland, Calif., The Holmes Book Company, 88 p.

McCafferty, W.P., 1981, Aquatic entomology: The fishermen's and ecologists' illustrated guide to insects and their relatives: Boston, Mass., Science Books International, 448 p.

Merritt, R.W., and Cummins, K.W., 1996, An introduction to the aquatic insects of North America (3rd ed.): Dubuque, Iowa, Kandall/Hunt Publishing Co.

Mitko, K., and Bebek, M., 1999, ICP-OES determination of trace elements in salinated water: Atomic Spectroscopy, v. 20, p. 217-223.

Mitko, K., and Bebek, M., 2000, Determination of major elements in saline water samples using a dual-view ICP-OES: Atomic Spectroscopy, v. 21, p. 77-85.

Olson, M.L., and De Wild, J.F., 1999, Techniques for the collection and species-specific analysis of low levels of mercury in water, sediment, and biota, in Morganwalp, D.W., and Buxton, H.T., eds., U.S. Geological Survey Toxic Substances Hydrology Program-Proceedings of the Technical Meeting, Charleston, South Carolina, March 8-12, 1999: U.S. Geological Survey Water-Resources Investigations Report 99-4018B, p. 191-199. http://wi.water.usgs. gov/pubs/WRIR-99-4018-B/index.html

Patton, C.J., and Truitt, E.P., 1992, Methods of analysis by the U.S. Geological Survey National Water Quality Laboratory-Determination of total phosphorous by a Kjeldahl digestion method and an automated colorimetric finish that includes dialysis: U.S. Geological Survey Open-File Report 92-146, 39 p. 
Roth, D.A., 1994, Ultratrace analysis of mercury and its distribution in some natural waters of the United States: Fort Collins, Colo., Colorado State University, Ph.D. dissertation, 309 p.

Rudd, J.W.M., 1995, Sources of methylmercury to freshwater ecosystems, a review: Water, Air, and Soil Pollution, v. 80, p. 697-713.

Schwarzbach, S., Thompson, L., and Adelsbach, T., 2001, Cache Creek mercury investigations USFWS Final Report. Off Refuge Investigations Report: FFS \#11301F22, 55 p.

Shelton, L.R., 1994, Field guide for collecting and processing stream-water samples for the National Water-Quality Assessment Program: U.S. Geological Survey Open-File Report 94-455, 42 p. http://ca.water.usgs.gov/pnsp/pest.rep/sw-t.html

Shelton, L.R., and Capel, P.D., 1994, Guidelines for collecting and processing samples of stream bed sediment for analysis of trace elements and organic contaminants for the National Water-Quality Assessment Program: U.S. Geological Survey Open-File Report 94-458, 20 p. http://ca.water.usgs.gov/pnsp/pest.rep/bs-t.html

Slotton, D.G., Ayers, S.M., Reuter, J.E., and Goldman, C.R., 1997, Gold mining impacts on food chain mercury in northwestern Sierra Nevada streams. Appendix B, in Larry Walker Associate, Sacramento River Watershed mercury control planning project-report for the Sacramento Regional County Sanitation District, 74 p.

Stebbins, R.C., 1985, A field guide to western reptiles and amphibians: field marks of all species in western North America, including Baja California (2nd ed.) rev.: Boston, Houghton Mifflin, 533 p.

Taylor, H.E., 2001, Inductively coupled plasma-mass spectrometry—practices and techniques: San Diego, Calif., Academic Press, 294 p.

Tremblay, A., Lucotte, M., and Rheault, I., 1996, Methylmercury in a benthic food web of two hydroelectric reservoirs and a natural lake of Northern Quebec (Canada): Water, Air, and Soil Pollution, v. 91, p. 255-269.

Tremblay, A., and Lucotte, M., 1997, Accumulation of total mercury and methylmercury in insect larvae of hydroelectric reservoirs: Canadian Journal of Fisheries and Aquatic Sciences, v. 54, p. 832-841.
U.S. Department of Agriculture-Forest Service, 2002, Preliminary assessment of the Buckeye Diggings and Sailor Flat Hydraulic Mines, prepared by CDM Federal, Walnut Creek, Calif. for the Tahoe National Forest, Nevada City, Calif., April 2002.

U.S. Environmental Protection Agency, 1990, Guidelines establishing test procedures for the analysis of pollutants (Appendix B, Part 136, Definition of procedures for the determination of the MDL-Revision 1.11), revised July 1, 1999, p. 537-539.

U.S. Environmental Protection Agency, 1999, Title 40 - Protection of Environment. Chapter 1. Environmental Protection Agency. Part 131-Water Quality Criteria: U.S. Code of Federal Regulations, Title 40, Part 131: 40 CFR 131, revised July 1, 1999.

U.S. Environmental Protection Agency, 2003, Title 40 - Protection of Environment. Chapter 1. Environmental Protection Agency. Part 141 - National Primary Drinking Water Regulations: U.S. Code of Federal Regulations, Title 40, Part 141: 40 CFR 141, revised July 1, 2003.

U.S. Fish and Wildlife Service, 2003, Evaluation of the Clean Water Act, section 304(a) human health criterion: protectiveness for threatened and endangered wildlife in California: Sacramento, Calif., U. S. Fish and Wildlife Service, Sacramento Fish and Wildlife Office, Environmental Contaminants Division, 96 p.

Wershaw, R.L., Fishman, M.J., Grabbe, R.R., and Lowe, L.E., 1987, Methods for the determination of organic substances in water and fluvial sediments: U.S. Geological Survey Techniques of Water-Resources Investigations, book 5, chap. A3, 80 p.

Western Regional Climate Center, 2004, Period of record monthly climate summary, Grass Valley No. 2 California. Accessed October 4, 2004 at URL http://www.wrcc.dri. edu/cgi-bin/cliRECtM.pl?cagras

Wong, H.K., McQueen, D.J., Williams, D.D., and Demers, E., 1997, Transfer of mercury from benthic invertebrates to fishes in lakes with contrasting fish community structures: Canadian Journal of Fisheries and Aquatic Sciences, v. 54, p. $1320-1330$.

Yeend, W.E., 1974, Gold-bearing gravels of the ancestral Yuba River, Sierra Nevada, California: U.S. Geological Survey Professional Paper 772, 44 p. 
THIS PAGE INTENTIONALLY LEFT BLANK 
Table 4A. Concentrations of trace metals and selected major elements in unfiltered water samples, Greenhorn Creek drainage, Nevada County, California.

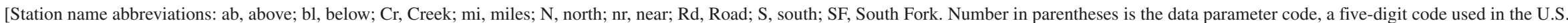
Geological Survey computerized data system. $\mu \mathrm{g} / \mathrm{L}$, microgram per liter (equivalent to part per billion); $\mathrm{mg} / \mathrm{L}$, milligram per liter (at low concentration, equiivalent to part per million); s.d., standard deviation;

$\leq$ less than; - , not determined]

\begin{tabular}{|c|c|c|c|c|c|c|c|c|c|c|c|c|c|c|c|c|c|c|}
\hline \multirow[t]{2}{*}{$\begin{array}{l}\text { Station } \\
\text { map ID }\end{array}$} & \multirow[t]{2}{*}{ Station name } & \multirow[t]{2}{*}{ Date } & \multirow[t]{2}{*}{ Time } & \multirow[t]{2}{*}{$\begin{array}{l}\text { Rep- } \\
\text { licate }\end{array}$} & \multicolumn{2}{|c|}{$\begin{array}{l}\text { Aluminum }(\mathrm{AI}), \\
\text { unfiltered } \\
(\mu \mathrm{g} / \mathrm{L}) \\
(\mathbf{0 1 1 0 4 )}\end{array}$} & \multicolumn{2}{|c|}{$\begin{array}{l}\text { Arsenic (As), } \\
\text { unfiltered } \\
(\mu \mathrm{g} / \mathrm{L}) \\
(01002)\end{array}$} & \multicolumn{2}{|c|}{$\begin{array}{c}\text { Boron (B), } \\
\text { unfiltered } \\
(\mu \mathrm{g} / \mathrm{L}) \\
(00999)\end{array}$} & \multicolumn{2}{|c|}{$\begin{array}{c}\text { Barium (Ba), } \\
\text { unfiltered } \\
(\mu \mathrm{g} / \mathrm{L}) \\
(01007)\end{array}$} & \multicolumn{2}{|c|}{$\begin{array}{c}\text { Beryllium }(\mathrm{Be}), \\
\text { unfiltered } \\
(\mu \mathrm{g} / \mathrm{L}) \\
(00998) \\
\end{array}$} & \multicolumn{2}{|c|}{$\begin{array}{c}\text { Bismuth }(\mathrm{Bi}), \\
\text { unfiltered } \\
(\mu \mathrm{g} / \mathrm{L}) \\
(01017) \\
\end{array}$} & \multicolumn{2}{|c|}{$\begin{array}{c}\text { Calcium (Ca), } \\
\text { unfiltered } \\
(\mathrm{mg} / \mathrm{L}) \\
(00916)\end{array}$} \\
\hline & & & & & value & s.d. & value & s.d. & value & s.d. & value & s.d. & value & s.d. & value & s.d. & value & s.d. \\
\hline \multicolumn{19}{|c|}{ Environmental samples } \\
\hline BY20 & $\begin{array}{l}\text { Boston Mine tunnel } \\
\text { outlet nr Grass } \\
\text { Valley }\end{array}$ & $6 / 18 / 1999$ & $14: 30$ & 1 of 2 & 14 & 3 & $<50$ & 10 & $<6$ & 3 & 54 & 3 & $<0.05$ & 0.01 & 0.007 & 0.001 & 13 & 0 \\
\hline BY20 & $\begin{array}{l}\text { Boston Mine tunnel } \\
\text { outlet nr Grass } \\
\text { Valley }\end{array}$ & $6 / 18 / 1999$ & $14: 30$ & $2 \mathrm{of} 2$ & 16 & 6 & $<50$ & 0 & $<6$ & 3 & 55 & 1 & $<0.05$ & 0.03 & 0.009 & 0.006 & 13 & 0 \\
\hline BY20 & $\begin{array}{l}\text { Boston Mine tunnel } \\
\text { outlet nr Grass } \\
\text { Valley }\end{array}$ & $12 / 14 / 1999$ & $12: 00$ & 1 of 2 & 25 & 3 & $<20$ & 8 & $<9$ & 3 & 96 & 8 & $<0.03$ & 0.04 & 0.006 & 0.003 & 18 & 0 \\
\hline BY20 & $\begin{array}{l}\text { Boston Mine tunnel } \\
\text { outlet nr Grass } \\
\text { Valley }\end{array}$ & $12 / 14 / 1999$ & $12: 00$ & 2 of 2 & 17 & 2 & $<20$ & 11 & $<9$ & 4 & 101 & 6 & $<0.03$ & 0.03 & 0.005 & 0.004 & 18 & 2 \\
\hline BY20 & $\begin{array}{l}\text { Boston Mine tunnel } \\
\text { outlet nr Grass } \\
\text { Valley }\end{array}$ & $5 / 24 / 2000$ & $16: 00$ & 1 of 2 & 17 & 4 & $<35$ & 29 & 19 & 16 & 56 & 0 & $<0.07$ & 0.02 & 0.003 & 0.001 & 12 & 0 \\
\hline BY20 & $\begin{array}{l}\text { Boston Mine tunnel } \\
\text { outlet nr Grass } \\
\text { Valley }\end{array}$ & $5 / 24 / 2000$ & $16: 00$ & 2 of 2 & 21 & 2 & $<35$ & 19 & $<16$ & 13 & 75 & 2 & $<0.07$ & 0.03 & 0.002 & 0.001 & 12 & 0 \\
\hline BY21 & $\begin{array}{l}\text { Boston Mine wetlands } \\
\text { pond nr Grass } \\
\text { Valley }\end{array}$ & 12/14/1999 & $10: 30$ & 1 of 2 & 5.7 & 0.5 & $<20$ & 15 & 15 & 14 & 50 & 0 & $<0.07$ & 0.02 & 0.005 & 0.004 & 19 & 0 \\
\hline BY21 & $\begin{array}{l}\text { Boston Mine wetlands } \\
\text { pond nr Grass } \\
\text { Valley }\end{array}$ & $12 / 14 / 1999$ & $10: 30$ & 2 of 2 & 8.4 & 2.2 & $<20$ & 12 & 11 & 8 & 52 & 3 & $<0.07$ & 0.01 & 0.006 & 0.004 & 19 & 0 \\
\hline BY21 & $\begin{array}{l}\text { Boston Mine wetlands } \\
\text { pond nr Grass } \\
\text { Valley }\end{array}$ & $5 / 24 / 2000$ & $16: 30$ & 1 of 2 & 6.8 & 2.6 & $<35$ & 16 & $<16$ & 11 & 43 & 1 & $<0.07$ & 0.05 & 0.006 & 0.001 & 11 & 0 \\
\hline BY21 & $\begin{array}{l}\text { Boston Mine wetlands } \\
\text { pond nr Grass } \\
\text { Valley }\end{array}$ & $5 / 24 / 2000$ & $16: 30$ & 2 of 2 & 15 & 4 & $<35$ & 9 & $<16$ & 7 & 45 & 2 & $<0.07$ & 0.04 & 0.007 & 0.006 & 11 & 0 \\
\hline BY58 & $\begin{array}{l}\text { Buckeye Flat Mine } \\
\text { main drain } 0.45 \mathrm{mi} \\
\text { ab Greenhorn } \mathrm{Cr}\end{array}$ & $8 / 21 / 2000$ & $11: 00$ & 1 of 2 & 1,900 & 0 & $<20$ & 0 & $<1,000$ & 0 & 36 & 7 & $<1$ & 1 & $<0.1$ & 0.0 & 12 & 1 \\
\hline BY58 & $\begin{array}{l}\text { Buckeye Flat Mine } \\
\text { main drain } 0.45 \mathrm{mi} \\
\text { ab Greenhorn } \mathrm{Cr}\end{array}$ & $8 / 21 / 2000$ & $11: 00$ & 2 of 2 & 1,600 & 0 & $<20$ & 10 & $<1,000$ & 1,000 & 34 & 7 & $<1$ & 1 & $<0.1$ & 0.1 & 12 & 3 \\
\hline BY23 & $\begin{array}{c}\text { Buckeye Flat Mine N } \\
\text { drain to Greenhorn } \\
\text { Cr, Grass Valley }\end{array}$ & $6 / 18 / 1999$ & $11: 30$ & 1 of 2 & 4,000 & 100 & $<50$ & 10 & $<6$ & 5 & 23 & 1 & 0.80 & 0.06 & 0.006 & 0.004 & 19 & 0 \\
\hline
\end{tabular}


Table 4A. Concentrations of trace metals and selected major elements in unfiltered water samples, Greenhorn Creek drainage, Nevada County, California—Continued.

[Station name abbreviations: ab, above; bl, below; Cr, Creek; mi, miles; N, north; nr, near; Rd, Road; S, south; SF, South Fork. Number in parentheses is the data parameter code, a five-digit code used in the

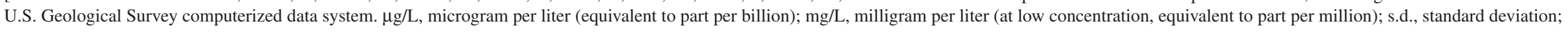
$<$, less than; - - not determined]

\begin{tabular}{|c|c|c|c|c|c|c|c|c|c|c|c|c|c|c|c|c|c|c|}
\hline \multirow[t]{2}{*}{$\begin{array}{l}\text { Station } \\
\text { map ID }\end{array}$} & \multirow[t]{2}{*}{ Station name } & \multirow[t]{2}{*}{ Date } & \multirow[t]{2}{*}{ Time } & \multirow[t]{2}{*}{$\begin{array}{l}\text { Rep- } \\
\text { licate }\end{array}$} & \multicolumn{2}{|c|}{$\begin{array}{c}\text { Aluminum }(\mathrm{AI}), \\
\text { unfiltered } \\
(\mu \mathrm{g} / \mathrm{L}) \\
(01104)\end{array}$} & \multicolumn{2}{|c|}{$\begin{array}{c}\text { Arsenic (As), } \\
\text { unfiltered } \\
(\mu \mathrm{g} / \mathrm{L}) \\
(\mathbf{0 1 0 0 2 )} \\
\end{array}$} & \multicolumn{2}{|c|}{$\begin{array}{c}\text { Boron }(\mathrm{B}) \text {, } \\
\text { unfiltered } \\
(\mu \mathrm{g} / \mathrm{L}) \\
(00999)\end{array}$} & \multicolumn{2}{|c|}{$\begin{array}{c}\text { Barium (Ba), } \\
\text { unfiltered } \\
(\mu \mathrm{g} / \mathrm{L}) \\
(01007)\end{array}$} & \multicolumn{2}{|c|}{$\begin{array}{c}\text { Beryllium }(\mathrm{Be}), \\
\text { unfiltered } \\
(\mu \mathrm{g} / \mathrm{L}) \\
(00998) \\
\end{array}$} & \multicolumn{2}{|c|}{$\begin{array}{c}\text { Bismuth }(\mathrm{Bi}), \\
\text { unfiltered } \\
(\mu \mathrm{g} / \mathrm{L}) \\
(01017)\end{array}$} & \multicolumn{2}{|c|}{$\begin{array}{c}\text { Calcium (Ca), } \\
\text { unfiltered } \\
(\mathrm{mg} / \mathrm{L}) \\
(00916)\end{array}$} \\
\hline & & & & & value & s.d. & value & s.d. & value & s.d. & value & s.d. & value & s.d. & value & s.d. & value & s.d. \\
\hline BY23 & $\begin{array}{c}\text { Buckeye Flat Mine N } \\
\text { drain to Greenhorn } \\
\text { Cr, Grass Valley }\end{array}$ & 6/18/1999 & $11: 30$ & $2 o f 2$ & 4,000 & 0 & $<50$ & 30 & $<6$ & 4 & 22 & 1 & 0.87 & 0.02 & 0.006 & 0.004 & 20 & 0 \\
\hline BY23 & $\begin{array}{c}\text { Buckeye Flat Mine N } \\
\text { drain to Greenhorn } \\
\text { Cr, Grass Valley }\end{array}$ & $12 / 2 / 1999$ & 11:00 & 1 of 2 & 3,800 & 400 & $<20$ & 13 & $<9$ & 5 & 30 & 6 & 0.58 & 0.11 & 0.010 & 0.001 & 12 & 1 \\
\hline BY23 & $\begin{array}{c}\text { Buckeye Flat Mine N } \\
\text { drain to Greenhorn } \\
\text { Cr, Grass Valley }\end{array}$ & $12 / 2 / 1999$ & $11: 00$ & $2 o f 2$ & 3,600 & 0 & $<20$ & 6 & $<9$ & 4 & 39 & 3 & 0.54 & 0.08 & 0.014 & 0.008 & 11 & 0 \\
\hline BY23 & $\begin{array}{c}\text { Buckeye Flat Mine N } \\
\text { drain to Greenhorn } \\
\text { Cr, Grass Valley }\end{array}$ & $8 / 22 / 2000$ & $10: 45$ & 1 of 2 & 4,100 & 100 & $<20$ & 10 & $<1,000$ & 1,000 & 38 & 9 & $<1$ & 0 & $<0.1$ & 0.1 & 23 & 1 \\
\hline BY23 & $\begin{array}{l}\text { Buckeye Flat Mine N } \\
\text { drain to Greenhorn } \\
\text { Cr, Grass Valley }\end{array}$ & $8 / 22 / 2000$ & $10: 45$ & $2 o f 2$ & 3,800 & 0 & $<20$ & 10 & $<1,000$ & 1,000 & 38 & 7 & $<1$ & 1 & $<0.1$ & 0.1 & 21 & 2 \\
\hline BY116 & $\begin{array}{l}\text { Buckeye Flat Mine } \\
\text { pond drain } 0.15 \mathrm{mi} \\
\text { ab SF Greenhorn } \mathrm{Cr}\end{array}$ & $8 / 21 / 2000$ & $14: 10$ & 1 of 2 & 43 & 1 & $<20$ & 10 & $<9$ & 6 & 61 & 4 & $<0.1$ & 0.1 & 0.015 & 0.004 & 1.0 & 0.2 \\
\hline BY116 & $\begin{array}{l}\text { Buckeye Flat Mine } \\
\text { pond drain } 0.15 \mathrm{mi} \\
\text { ab SF Greenhorn Cr }\end{array}$ & $8 / 21 / 2000$ & $14: 10$ & $2 o f 2$ & 50 & 2 & $<20$ & 0 & $<9$ & 5 & 57 & 2 & $<0.1$ & 0.1 & 0.026 & 0.011 & 0.87 & 0.05 \\
\hline BY24 & $\begin{array}{l}\text { Buckeye Flat Mine } \\
\text { S drain to } \mathrm{SF} \\
\text { Greenhorn } \mathrm{Cr} \\
\text { Grass Valley }\end{array}$ & $8 / 26 / 1999$ & 11:00 & 1 of 2 & 72 & 7 & $<50$ & 40 & 7 & 13 & 19 & 0 & $<0.05$ & 0.02 & 0.50 & 0.09 & 0.48 & 0.03 \\
\hline BY24 & $\begin{array}{l}\text { Buckeye Flat Mine } \\
\text { S drain to SF } \\
\text { Greenhorn } \mathrm{Cr} \text {, } \\
\text { Grass Valley }\end{array}$ & $8 / 26 / 1999$ & $11: 00$ & $2 o f 2$ & 67 & 4 & $<50$ & 20 & $<6$ & 1 & 19 & 0 & $<0.05$ & 0.01 & 0.50 & 0.13 & 0.45 & 0.01 \\
\hline BY24 & $\begin{array}{l}\text { Buckeye Flat Mine } \\
\text { S drain to SF } \\
\text { Greenhorn Cr, } \\
\text { Grass Valley }\end{array}$ & $8 / 22 / 2000$ & $12: 10$ & 1 of 2 & 250 & 40 & $<20$ & 10 & $<9$ & 5 & 23 & 1 & $<0.1$ & 0.1 & 0.025 & 0.008 & 0.43 & 0.15 \\
\hline BY24 & $\begin{array}{l}\text { Buckeye Flat Mine } \\
\text { S drain to SF } \\
\text { Greenhorn Cr, } \\
\text { Grass Valley }\end{array}$ & $8 / 22 / 2000$ & $12: 10$ & $2 o f 2$ & 260 & 40 & $<20$ & 10 & $<9$ & 9 & 26 & 3 & $<0.1$ & 0.1 & 0.035 & 0.002 & 0.53 & 0.12 \\
\hline BY25 & $\begin{array}{c}\text { Buckeye Flat Mine } \\
\text { upper drain }\end{array}$ & 4/9/2001 & $10: 00$ & 1 of 2 & 630 & 40 & $<20$ & 10 & $<6$ & 1 & 41 & 3 & 0.10 & 0.06 & 0.010 & 0.005 & 4.7 & 0.9 \\
\hline
\end{tabular}


Table 4A. Concentrations of trace metals and selected major elements in unfiltered water samples, Greenhorn Creek drainage, Nevada County, California—Continued.

[Station name abbreviations: ab, above; bl, below; Cr, Creek; mi, miles; N, north; nr, near; Rd, Road; S, south; SF, South Fork. Number in parentheses is the data parameter code, a five-digit code used in the

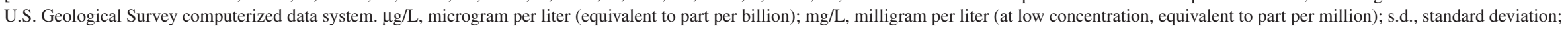
$<$, less than; - - not determined]

\begin{tabular}{|c|c|c|c|c|c|c|c|c|c|c|c|c|c|c|c|c|c|c|}
\hline \multirow[t]{2}{*}{$\begin{array}{l}\text { Station } \\
\text { map ID }\end{array}$} & \multirow[t]{2}{*}{ Station name } & \multirow[t]{2}{*}{ Date } & \multirow[t]{2}{*}{ Time } & \multirow[t]{2}{*}{$\begin{array}{l}\text { Rep- } \\
\text { licate }\end{array}$} & \multicolumn{2}{|c|}{$\begin{array}{c}\text { Aluminum (AI), } \\
\text { unfiltered } \\
(\mu \mathrm{g} / \mathrm{L}) \\
(01104)\end{array}$} & \multicolumn{2}{|c|}{$\begin{array}{c}\text { Arsenic (As), } \\
\text { unfiltered } \\
(\mu \mathrm{g} / \mathrm{L}) \\
(01002)\end{array}$} & \multicolumn{2}{|c|}{$\begin{array}{c}\text { Boron }(\mathrm{B}) \text {, } \\
\text { unfiltered } \\
(\mu \mathrm{g} / \mathrm{L}) \\
(00999)\end{array}$} & \multicolumn{2}{|c|}{$\begin{array}{c}\text { Barium }(\mathrm{Ba}), \\
\text { unfiltered } \\
(\mu \mathrm{g} / \mathrm{L}) \\
(01007)\end{array}$} & \multicolumn{2}{|c|}{$\begin{array}{c}\text { Beryllium }(\mathrm{Be}), \\
\text { unfiltered } \\
(\mu \mathrm{g} / \mathrm{L}) \\
(00998) \\
\end{array}$} & \multicolumn{2}{|c|}{$\begin{array}{c}\text { Bismuth }(\mathrm{Bi}), \\
\text { unfiltered } \\
(\mu \mathrm{g} / \mathrm{L}) \\
(\mathbf{0 1 0 1 7 )}\end{array}$} & \multicolumn{2}{|c|}{$\begin{array}{c}\text { Calcium (Ca), } \\
\text { unfiltered } \\
(\mathrm{mg} / \mathrm{L}) \\
(00916)\end{array}$} \\
\hline & & & & & value & s.d. & value & s.d. & value & s.d. & value & s.d. & value & s.d. & value & s.d. & value & s.d. \\
\hline BY25 & $\begin{array}{l}\text { Buckeye Flat Mine } \\
\text { upper drain }\end{array}$ & $4 / 9 / 2001$ & $10: 00$ & 2 of 2 & 620 & 10 & $<20$ & 10 & $<6$ & 7 & 40 & 1 & 0.11 & 0.02 & 0.009 & 0.006 & 4.6 & 0.7 \\
\hline BY52 & $\begin{array}{l}\text { Greenhorn Cr } 0.3 \mathrm{mi} \\
\text { ab Buckeye Ford nr } \\
\text { Nevada City }\end{array}$ & $1 / 25 / 2000$ & $10: 45$ & 1 of 2 & 460 & 20 & $<20$ & 25 & 14 & 1 & 23 & 1 & 0.07 & 0.01 & 0.011 & 0.003 & 2.8 & 0.1 \\
\hline BY52 & $\begin{array}{l}\text { Greenhorn Cr } 0.3 \mathrm{mi} \\
\text { ab Buckeye Ford nr } \\
\text { Nevada City }\end{array}$ & $1 / 25 / 2000$ & $10: 45$ & 2of 2 & 420 & 10 & $<20$ & 9 & 9 & 3 & 23 & 0 & $<0.07$ & 0.00 & 0.010 & 0.004 & 2.8 & 0.1 \\
\hline BY59 & $\begin{array}{l}\text { Greenhorn Cr at You } \\
\text { Bet Rd nr Nevada } \\
\text { City }\end{array}$ & $7 / 24 / 2001$ & 11:00 & 1 of 2 & 38 & 3 & $<60$ & 42 & $<10$ & 5 & 34 & 3 & $<0.06$ & 0.01 & 0.01 & 0.01 & 5.1 & 0.4 \\
\hline BY59 & $\begin{array}{l}\text { Greenhorn Cr at You } \\
\text { Bet Rd nr Nevada } \\
\text { City }\end{array}$ & $7 / 24 / 2001$ & 11:00 & 2 of 2 & 4 & 2 & $<60$ & 12 & $<10$ & 6 & 35 & 2 & $<0.06$ & 0.03 & 0.02 & 0.02 & 5.5 & 0.5 \\
\hline BY59 & $\begin{array}{l}\text { Greenhorn Cr at You } \\
\text { Bet Rd nr Nevada } \\
\text { City }\end{array}$ & $8 / 29 / 2001$ & $15: 00$ & 1 of 2 & 23 & 1 & $<60$ & 21 & $<10$ & 4 & 25 & 1 & $<0.06$ & 0.02 & 0.01 & 0.01 & 5.4 & 0.3 \\
\hline BY59 & $\begin{array}{l}\text { Greenhorn } \mathrm{Cr} \text { at You } \\
\text { Bet Rd nr Nevada } \\
\text { City }\end{array}$ & $8 / 29 / 2001$ & $15: 00$ & $2 \mathrm{of} 2$ & 25 & 2 & $<60$ & 32 & $<10$ & 2 & 27 & 3 & $<0.06$ & 0.02 & 0.02 & 0.00 & 5.6 & 0.4 \\
\hline BY59 & $\begin{array}{l}\text { Greenhorn Cr at You } \\
\text { Bet Rd nr Nevada } \\
\text { City }\end{array}$ & $9 / 14 / 2001$ & 13:00 & 1 of 2 & 29 & 1 & $<60$ & 23 & $<8$ & 5 & 30 & 1 & $<0.04$ & 0.01 & $<0.02$ & 0.01 & 5.1 & 0.2 \\
\hline BY59 & $\begin{array}{l}\text { Greenhorn Cr at You } \\
\text { Bet Rd nr Nevada } \\
\text { City }\end{array}$ & $9 / 14 / 2001$ & 13:00 & 2 of 2 & 28 & 1 & $<60$ & 5 & $<8$ & 1 & 32 & 2 & $<0.04$ & 0.01 & $<0.02$ & 0.00 & 5.2 & 0.5 \\
\hline BY59 & $\begin{array}{l}\text { Greenhorn Cr at You } \\
\text { Bet Rd nr Nevada } \\
\text { City }\end{array}$ & $10 / 15 / 2001$ & $12: 00$ & 1 of 2 & 12 & 2 & $<60$ & 16 & $<10$ & 5 & 34 & 1 & 0.05 & 0.11 & $<0.02$ & 0.01 & 5.6 & 0.4 \\
\hline BY59 & $\begin{array}{l}\text { Greenhorn Cr at You } \\
\text { Bet Rd nr Nevada } \\
\text { City }\end{array}$ & $10 / 15 / 2001$ & $12: 00$ & 2 of 2 & 13 & 2 & $<60$ & 22 & $<10$ & 1 & 36 & 3 & $<0.03$ & 0.03 & $<0.02$ & 0.00 & 5.6 & 0.2 \\
\hline BY59 & $\begin{array}{l}\text { Greenhorn } \mathrm{Cr} \text { at You } \\
\text { Bet Rd nr Nevada } \\
\text { City }\end{array}$ & $11 / 20 / 2001$ & $13: 20$ & 1 of 2 & 57 & 3 & $<30$ & 7 & $<8$ & 3 & 37 & 1 & $<0.04$ & 0.02 & 0.03 & 0.00 & 6.5 & 0.7 \\
\hline BY59 & $\begin{array}{l}\text { Greenhorn Cr at You } \\
\text { Bet Rd nr Nevada } \\
\text { City }\end{array}$ & $11 / 20 / 2001$ & $13: 20$ & $2 \mathrm{of} 2$ & 56 & 2 & $<30$ & 4 & $<8$ & 1 & 40 & 1 & $<0.04$ & 0.01 & $<0.02$ & 0.00 & 6.5 & 0.5 \\
\hline
\end{tabular}


Table 4A. Concentrations of trace metals and selected major elements in unfiltered water samples, Greenhorn Creek drainage, Nevada County, California—Continued.

[Station name abbreviations: ab, above; bl, below; Cr, Creek; mi, miles; N, north; nr, near; Rd, Road; S, south; SF, South Fork. Number in parentheses is the data parameter code, a five-digit code used in the

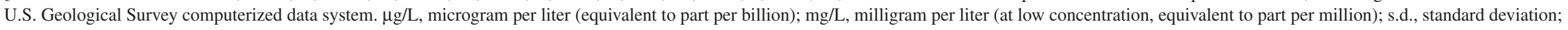
$<$, less than; - - not determined]

\begin{tabular}{|c|c|c|c|c|c|c|c|c|c|c|c|c|c|c|c|c|c|c|}
\hline \multirow[t]{2}{*}{$\begin{array}{l}\text { Station } \\
\text { map ID }\end{array}$} & \multirow[t]{2}{*}{ Station name } & \multirow[t]{2}{*}{ Date } & \multirow[t]{2}{*}{ Time } & \multirow[t]{2}{*}{$\begin{array}{l}\text { Rep- } \\
\text { licate }\end{array}$} & \multicolumn{2}{|c|}{$\begin{array}{c}\text { Aluminum (AI), } \\
\text { unfiltered } \\
(\mu \mathrm{g} / \mathrm{L}) \\
(01104)\end{array}$} & \multicolumn{2}{|c|}{$\begin{array}{l}\text { Arsenic (As), } \\
\text { unfiltered } \\
(\mu \mathrm{g} / \mathrm{L}) \\
(01002)\end{array}$} & \multicolumn{2}{|c|}{$\begin{array}{c}\text { Boron (B), } \\
\text { unfiltered } \\
(\mu \mathrm{g} / \mathrm{L}) \\
(00999)\end{array}$} & \multicolumn{2}{|c|}{$\begin{array}{c}\text { Barium (Ba), } \\
\text { unfiltered } \\
(\mu \mathrm{g} / \mathrm{L}) \\
(01007)\end{array}$} & \multicolumn{2}{|c|}{$\begin{array}{c}\text { Beryllium }(\mathrm{Be}), \\
\text { unfiltered } \\
(\mu \mathrm{g} / \mathrm{L}) \\
(00998) \\
\end{array}$} & \multicolumn{2}{|c|}{$\begin{array}{c}\text { Bismuth }(\mathrm{Bi}) \\
\text { unfiltered } \\
(\mu \mathrm{g} / \mathrm{L}) \\
(\mathbf{0 1 0 1 7})\end{array}$} & \multicolumn{2}{|c|}{$\begin{array}{l}\text { Calcium (Ca), } \\
\text { unfiltered } \\
(\mathrm{mg} / \mathrm{L}) \\
(00916)\end{array}$} \\
\hline & & & & & value & s.d. & value & s.d. & value & s.d. & value & s.d. & value & s.d. & value & s.d. & value & s.d. \\
\hline BY59 & $\begin{array}{l}\text { Greenhorn Cr at You } \\
\text { Bet Rd nr Nevada } \\
\text { City }\end{array}$ & $12 / 10 / 2001$ & $12: 20$ & 1 of 2 & 910 & 48 & $<100$ & 24 & $<10$ & 2 & 35 & 0 & 0.06 & 0.01 & $<0.02$ & 0.00 & 6.3 & 0.7 \\
\hline BY59 & $\begin{array}{l}\text { Greenhorn Cr at You } \\
\text { Bet Rd nr Nevada } \\
\text { City }\end{array}$ & $12 / 10 / 2001$ & $12: 20$ & $2 o f 2$ & 880 & 16 & $<100$ & 13 & $<10$ & 2 & 38 & 3 & $<0.03$ & 0.03 & $<0.02$ & 0.01 & 6.5 & 0.7 \\
\hline BY59 & $\begin{array}{l}\text { Greenhorn Cr at You } \\
\text { Bet Rd nr Nevada } \\
\text { City }\end{array}$ & $12 / 21 / 2001$ & 13:00 & 1 of 2 & 4,500 & 420 & $<100$ & 46 & $<9$ & 20 & 79 & 8 & 0.16 & 0.03 & $<0.04$ & 0.01 & 5.0 & 0.6 \\
\hline BY59 & $\begin{array}{l}\text { Greenhorn Cr at You } \\
\text { Bet Rd nr Nevada } \\
\text { City }\end{array}$ & $12 / 21 / 2001$ & 13:00 & $2 o f 2$ & 4,700 & 120 & $<100$ & 18 & 15 & 19 & 81 & 3 & 0.18 & 0.04 & $<0.04$ & 0.01 & 5.1 & 0.5 \\
\hline BY51 & $\begin{array}{l}\text { Greenhorn } \mathrm{Cr} n r \\
\text { headwaters nr } \\
\text { Scotts Flat Res }\end{array}$ & $1 / 25 / 2000$ & $9: 30$ & 1 of 2 & 77 & 3 & $<20$ & 10 & 3 & 3 & 16 & 1 & $<0.07$ & 0.03 & 0.006 & 0.005 & 1.4 & 0.2 \\
\hline BY51 & $\begin{array}{l}\text { Greenhorn Cr nr } \\
\text { headwaters nr } \\
\text { Scotts Flat Res }\end{array}$ & $1 / 25 / 2000$ & $9: 30$ & $2 o f 2$ & 78 & 3 & $<20$ & 16 & 4 & 2 & 16 & 0 & $<0.07$ & 0.02 & $<0.002$ & 0.001 & 1.2 & 0.1 \\
\hline BY75 & $\begin{array}{l}\text { Missouri Cyn } \mathrm{Cr} \\
\text { 1.2mi ab Greenhorn } \\
\text { Cr nr Chicago Park }\end{array}$ & $12 / 14 / 1999$ & $14: 00$ & 1 of 2 & 52 & 7 & $<20$ & 9 & $<9$ & 7 & 39 & 10 & 0.10 & 0.02 & $<0.004$ & 0.003 & 13 & 1 \\
\hline BY75 & $\begin{array}{l}\text { Missouri Cyn } \mathrm{Cr} \\
\text { 1.2mi ab Greenhorn } \\
\text { Cr nr Chicago Park }\end{array}$ & $12 / 14 / 1999$ & $14: 00$ & $2 o f 2$ & 55 & 2 & $<20$ & 3 & $<9$ & 3 & 37 & 9 & 0.14 & 0.03 & 0.008 & 0.003 & 12 & 0 \\
\hline BY86 & $\begin{array}{l}\text { Poore Mine Creek } \\
\text { ab tunnel nr Grass } \\
\text { Valley }\end{array}$ & 3/31/1999 & $17: 40$ & 1 of 2 & 660 & 10 & 12 & 10 & $<13$ & 19 & 20 & 0 & $<0.04$ & 0.01 & 0.010 & 0.003 & 3.3 & 0.1 \\
\hline BY86 & $\begin{array}{l}\text { Poore Mine Creek } \\
\text { ab tunnel nr Grass } \\
\text { Valley }\end{array}$ & $3 / 31 / 1999$ & $17: 40$ & $2 o f 2$ & 640 & 10 & $<10$ & 10 & $<13$ & 8 & 20 & 1 & 0.07 & 0.02 & 0.007 & 0.001 & 3.2 & 0.1 \\
\hline BY87 & $\begin{array}{l}\text { Poore Mine Creek } \\
\text { bl tunnel nr Grass } \\
\text { Valley }\end{array}$ & $3 / 31 / 1999$ & $13: 30$ & 1 of 2 & 540 & 30 & $<10$ & 10 & $<13$ & 19 & 20 & 1 & $<0.04$ & 0.03 & 0.008 & 0.002 & 3.6 & 0.2 \\
\hline BY87 & $\begin{array}{l}\text { Poore Mine Creek } \\
\text { bl tunnel nr Grass } \\
\text { Valley }\end{array}$ & 3/31/1999 & $13: 30$ & $2 o f 2$ & 580 & 30 & $<10$ & 10 & $<13$ & 18 & 20 & 1 & $<0.04$ & 0.04 & 0.008 & 0.002 & 3.6 & 0.2 \\
\hline BY88 & $\begin{array}{l}\text { Poore Mine ground } \\
\text { sluice nr Grass } \\
\text { Valley }\end{array}$ & 4/1/1999 & $15: 40$ & 1 of 2 & 95 & 7 & $<10$ & 10 & $<13$ & 8 & 27 & 1 & 0.07 & 0.02 & 0.005 & 0.003 & 6.7 & 0.0 \\
\hline
\end{tabular}


Table 4A. Concentrations of trace metals and selected major elements in unfiltered water samples, Greenhorn Creek drainage, Nevada County, California—Continued.

[Station name abbreviations: ab, above; bl, below; Cr, Creek; mi, miles; N, north; nr, near; Rd, Road; S, south; SF, South Fork. Number in parentheses is the data parameter code, a five-digit code used in the

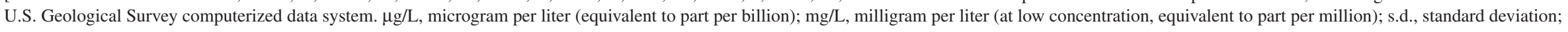
$<$, less than; - - not determined]

\begin{tabular}{|c|c|c|c|c|c|c|c|c|c|c|c|c|c|c|c|c|c|c|}
\hline \multirow[t]{2}{*}{$\begin{array}{l}\text { Station } \\
\text { map ID }\end{array}$} & \multirow[t]{2}{*}{ Station name } & \multirow[t]{2}{*}{ Date } & \multirow[t]{2}{*}{ Time } & \multirow[t]{2}{*}{$\begin{array}{l}\text { Rep- } \\
\text { licate }\end{array}$} & \multicolumn{2}{|c|}{$\begin{array}{l}\text { Aluminum (AI), } \\
\text { unfiltered } \\
(\mu \mathrm{g} / \mathrm{L}) \\
(\mathbf{0 1 1 0 4 )}\end{array}$} & \multicolumn{2}{|c|}{$\begin{array}{l}\text { Arsenic (As), } \\
\text { unfiltered } \\
(\mu \mathrm{g} / \mathrm{L}) \\
(01002)\end{array}$} & \multicolumn{2}{|c|}{$\begin{array}{c}\text { Boron }(B) \\
\text { unfiltered } \\
(\mu \mathrm{g} / \mathrm{L}) \\
(00999)\end{array}$} & \multicolumn{2}{|c|}{$\begin{array}{c}\text { Barium (Ba), } \\
\text { unfiltered } \\
(\mu \mathrm{g} / \mathrm{L}) \\
(01007)\end{array}$} & \multicolumn{2}{|c|}{$\begin{array}{c}\text { Beryllium (Be), } \\
\text { unfiltered } \\
(\mu \mathrm{g} / \mathrm{L}) \\
(00998) \\
\end{array}$} & \multicolumn{2}{|c|}{$\begin{array}{c}\text { Bismuth }(\mathrm{Bi}), \\
\text { unfiltered } \\
(\mu \mathrm{g} / \mathrm{L}) \\
(01017)\end{array}$} & \multicolumn{2}{|c|}{$\begin{array}{c}\text { Calcium (Ca), } \\
\text { unfiltered } \\
(\mathrm{mg} / \mathrm{L}) \\
(00916)\end{array}$} \\
\hline & & & & & value & s.d. & value & s.d. & value & s.d. & value & s.d. & value & s.d. & value & s.d. & value & s.d. \\
\hline BY88 & $\begin{array}{l}\text { Poore Mine ground } \\
\text { sluice nr Grass } \\
\text { Valley }\end{array}$ & 4/1/1999 & $15: 40$ & 2 of 2 & 87 & 7 & $<10$ & 10 & $<13$ & 2 & 26 & 1 & 0.06 & 0.03 & $<0.003$ & 0.001 & 6.7 & 0.3 \\
\hline BY89 & $\begin{array}{l}\text { Poore Mine pit lake nr } \\
\text { Grass Valley }\end{array}$ & 4/1/1999 & $16: 50$ & 1 of 2 & 52 & 4 & $<10$ & 0 & $<13$ & 4 & 39 & 4 & $<0.04$ & 0.00 & 0.007 & 0.002 & 2.6 & 0.3 \\
\hline BY89 & $\begin{array}{l}\text { Poore Mine pit lake nr } \\
\text { Grass Valley }\end{array}$ & 4/1/1999 & $16: 50$ & $2 \mathrm{of} 2$ & 58 & 6 & $<10$ & 0 & $<13$ & 7 & 39 & 1 & $<0.04$ & 0.01 & 0.011 & 0.005 & 2.6 & 0.1 \\
\hline BY89 & $\begin{array}{l}\text { Poore Mine pit lake nr } \\
\text { Grass Valley }\end{array}$ & $5 / 23 / 2000$ & $10: 45$ & 1 of 2 & 139 & 1 & $<35$ & 21 & $<16$ & 6 & 110 & 1 & 0.53 & 0.00 & 0.006 & 0.001 & 14 & 1 \\
\hline BY89 & $\begin{array}{l}\text { Poore Mine pit lake nr } \\
\text { Grass Valley }\end{array}$ & $5 / 23 / 2000$ & $10: 45$ & 2 of 2 & 144 & 5 & $<35$ & 6 & $<16$ & 7 & 106 & 1 & 0.60 & 0.03 & 0.006 & 0.002 & 14 & 0 \\
\hline BY90 & $\begin{array}{l}\text { Poore Mine seep } \\
\text { above ground sluice } \\
\text { nr Grass Valley }\end{array}$ & 4/1/1999 & $16: 00$ & 1of 2 & - & & - & & - & & - & & - & & - & & - & \\
\hline BY90 & $\begin{array}{l}\text { Poore Mine seep } \\
\text { above ground sluice } \\
\text { nr Grass Valley }\end{array}$ & 4/1/1999 & $16: 00$ & 2 of 2 & - & & $\longrightarrow$ & & - & & - & & - & & - & & - & \\
\hline BY91 & $\begin{array}{l}\text { Poore Mine tunnel } \\
\text { effluent nr Grass } \\
\text { Valley }\end{array}$ & $3 / 31 / 1999$ & $15: 20$ & 1of 2 & 55 & 7 & $<10$ & 10 & 14 & 10 & 28 & 1 & 0.06 & 0.04 & 0.010 & 0.003 & 12 & 1 \\
\hline BY91 & $\begin{array}{l}\text { Poore Mine tunnel } \\
\text { effluent nr Grass } \\
\text { Valley }\end{array}$ & $3 / 31 / 1999$ & $15: 20$ & 2 of 2 & 54 & 11 & $<10$ & 10 & 20 & 13 & 29 & 0 & $<0.04$ & 0.03 & 0.011 & 0.003 & 12 & 0 \\
\hline BY91 & $\begin{array}{l}\text { Poore Mine tunnel } \\
\text { effluent nr Grass } \\
\text { Valley }\end{array}$ & $12 / 15 / 1999$ & 13:00 & 1 of 2 & 61 & 1 & $<20$ & 2 & $<9$ & 7 & 31 & 4 & $<0.03$ & 0.02 & 0.009 & 0.001 & 18 & 0 \\
\hline BY91 & $\begin{array}{l}\text { Poore Mine tunnel } \\
\text { effluent nr Grass } \\
\text { Valley }\end{array}$ & $12 / 15 / 1999$ & $13: 00$ & 2of 2 & 78 & 3 & $<20$ & 10 & $<9$ & 1 & 28 & 6 & $<0.03$ & 0.03 & $<0.004$ & 0.001 & 19 & 1 \\
\hline BY91 & $\begin{array}{l}\text { Poore Mine tunnel } \\
\text { effluent nr Grass } \\
\text { Valley }\end{array}$ & $5 / 23 / 2000$ & $12: 00$ & 1 of 2 & 439 & 11 & $<35$ & 32 & $<16$ & 6 & 44 & 0 & 0.22 & 0.04 & 0.013 & 0.002 & 17 & 1 \\
\hline BY91 & $\begin{array}{l}\text { Poore Mine tunnel } \\
\text { effluent nr Grass } \\
\text { Valley }\end{array}$ & $5 / 23 / 2000$ & $12: 00$ & 2of 2 & 438 & 9 & $<35$ & 26 & $<16$ & 3 & 40 & 1 & 0.24 & 0.04 & 0.013 & 0.000 & 18 & 0 \\
\hline BY105 & $\begin{array}{l}\text { Sailor Flat Mine main } \\
\text { drain gulch 01nr } \\
\text { Quaker Hill }\end{array}$ & 7/6/1999 & $16: 00$ & 1 of 2 & 600 & 10 & $<50$ & 10 & $<6$ & 5 & 97 & 4 & 0.36 & 0.02 & 0.010 & 0.003 & 3.1 & 0.0 \\
\hline
\end{tabular}


Table 4A. Concentrations of trace metals and selected major elements in unfiltered water samples, Greenhorn Creek drainage, Nevada County, California—Continued.

[Station name abbreviations: ab, above; bl, below; Cr, Creek; mi, miles; N, north; nr, near; Rd, Road; S, south; SF, South Fork. Number in parentheses is the data parameter code, a five-digit code used in the

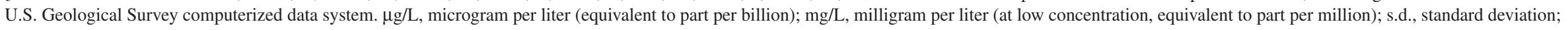

$<$, less than; - , not determined]

\begin{tabular}{|c|c|c|c|c|c|c|c|c|c|c|c|c|c|c|c|c|c|c|}
\hline \multirow[t]{2}{*}{$\begin{array}{l}\text { Station } \\
\text { map ID }\end{array}$} & \multirow[t]{2}{*}{ Station name } & \multirow[t]{2}{*}{ Date } & \multirow[t]{2}{*}{ Time } & \multirow[t]{2}{*}{$\begin{array}{l}\text { Rep- } \\
\text { licate }\end{array}$} & \multicolumn{2}{|c|}{$\begin{array}{c}\text { Aluminum (AI), } \\
\text { unfiltered } \\
(\mu \mathrm{g} / \mathrm{L}) \\
(\mathbf{0 1 1 0 4 )} \\
\end{array}$} & \multicolumn{2}{|c|}{$\begin{array}{c}\text { Arsenic (As), } \\
\text { unfiltered } \\
(\mu \mathrm{g} / \mathrm{L}) \\
(01002) \\
\end{array}$} & \multicolumn{2}{|c|}{$\begin{array}{c}\text { Boron (B), } \\
\text { unfiltered } \\
(\mu \mathrm{g} / \mathrm{L}) \\
(00999) \\
\end{array}$} & \multicolumn{2}{|c|}{$\begin{array}{c}\text { Barium (Ba), } \\
\text { unfiltered } \\
(\mu \mathrm{g} / \mathrm{L}) \\
(01007) \\
\end{array}$} & \multicolumn{2}{|c|}{$\begin{array}{c}\text { Beryllium (Be), } \\
\text { unfiltered } \\
(\mu \mathrm{g} / \mathrm{L}) \\
(00998) \\
\end{array}$} & \multicolumn{2}{|c|}{$\begin{array}{c}\text { Bismuth }(\mathrm{Bi}), \\
\text { unfiltered } \\
(\mu \mathrm{g} / \mathrm{L}) \\
(01017) \\
\end{array}$} & \multicolumn{2}{|c|}{$\begin{array}{c}\text { Calcium (Ca), } \\
\text { unfiltered } \\
(\mathrm{mg} / \mathrm{L}) \\
(00916)\end{array}$} \\
\hline & & & & & value & s.d. & value & s.d. & value & s.d. & value & s.d. & value & s.d. & value & s.d. & value & s.d. \\
\hline BY105 & $\begin{array}{l}\text { Sailor Flat Mine main } \\
\text { drain gulch 01nr } \\
\text { Quaker Hill }\end{array}$ & 7/6/1999 & $16: 00$ & 2 of 2 & 610 & 10 & $<50$ & 10 & $<6$ & 2 & 97 & 3 & 0.41 & 0.03 & 0.015 & 0.011 & 3.1 & 0.1 \\
\hline BY105 & $\begin{array}{l}\text { Sailor Flat Mine main } \\
\text { drain Gulch 01nr } \\
\text { Quaker Hill }\end{array}$ & $12 / 2 / 1999$ & $14: 30$ & 1 of 2 & 5,400 & 100 & $<20$ & 7 & $<9$ & 5 & 150 & 10 & 0.42 & 0.06 & 0.37 & 0.00 & 2.9 & 0.3 \\
\hline BY105 & $\begin{array}{l}\text { Sailor Flat Mine main } \\
\text { drain Gulch 01nr } \\
\text { Quaker Hill }\end{array}$ & $12 / 2 / 1999$ & $14: 30$ & $2 \mathrm{of} 2$ & 5,200 & 0 & $<20$ & 5 & $<9$ & 1 & 180 & 10 & 0.45 & 0.04 & 0.28 & 0.00 & 2.7 & 0.1 \\
\hline BY106 & $\begin{array}{l}\text { Sailor Flat Mine } \\
\text { main drainage to } \\
\text { Greenhorn Cr, } \\
\text { Gulch } 03\end{array}$ & 4/9/2001 & $12: 30$ & 1 of 2 & 290 & 30 & $<20$ & 10 & $<6$ & 6 & 33 & 3 & $<0.08$ & 0.06 & 0.014 & 0.007 & 0.55 & 0.04 \\
\hline BY106 & $\begin{array}{l}\text { Sailor Flat Mine } \\
\text { main drainage to } \\
\text { Greenhorn Cr, } \\
\text { Gulch } 03\end{array}$ & $4 / 9 / 2001$ & $12: 30$ & 2 of 2 & 290 & 0 & $<20$ & 10 & $<6$ & 5 & 34 & 1 & 0.09 & 0.05 & 0.019 & 0.013 & 0.56 & 0.10 \\
\hline BY114 & $\begin{array}{l}\text { SF Greenhorn Cr } \\
\text { 0.7mi ab Greenhorn } \\
\text { Cr nr Nevada City }\end{array}$ & $8 / 22 / 2000$ & $15: 10$ & 1 of 2 & 31 & 2 & $<20$ & 0 & $<9$ & 13 & 18 & 4 & $<0.1$ & 0.1 & 0.019 & 0.006 & 8.7 & 0.7 \\
\hline BY114 & $\begin{array}{l}\text { SF Greenhorn Cr } \\
\text { 0.7mi ab Greenhorn } \\
\text { Cr nr Nevada City }\end{array}$ & $8 / 22 / 2000$ & $15: 10$ & $2 \mathrm{of} 2$ & 32 & 2 & $<20$ & 10 & 12 & 8 & 17 & 4 & $<0.1$ & 0.0 & 0.023 & 0.004 & 8.1 & 2.0 \\
\hline BY122 & $\begin{array}{l}\text { Starr Mine tunnel } \\
\text { inflow nr Grass } \\
\text { Valley }\end{array}$ & 4/23/1999 & $15: 00$ & 1 of 2 & 92 & 9 & $<10$ & 10 & $<13$ & 3 & 21 & 1 & $<0.04$ & 0.01 & $<0.003$ & 0.001 & 9.8 & 0.9 \\
\hline BY122 & $\begin{array}{l}\text { Starr Mine tunnel } \\
\text { inflow nr Grass } \\
\text { Valley }\end{array}$ & 4/23/1999 & $15: 00$ & $2 \mathrm{of} 2$ & 100 & 10 & $<10$ & 10 & $<13$ & 7 & 23 & 0 & $<0.04$ & 0.01 & 0.008 & 0.002 & 11 & 0 \\
\hline BY123 & $\begin{array}{l}\text { Starr Mine tunnel } \\
\text { midway nr Grass } \\
\text { Valley }\end{array}$ & 4/23/1999 & $13: 40$ & 1 of 2 & 85 & 7 & $<10$ & 10 & $<13$ & 15 & 60 & 2 & $<0.04$ & 0.01 & 0.008 & 0.004 & 27 & 0 \\
\hline BY123 & $\begin{array}{l}\text { Starr Mine tunnel } \\
\text { midway nr Grass } \\
\text { Valley }\end{array}$ & 4/23/1999 & $13: 40$ & $2 \mathrm{of} 2$ & 95 & 4 & $<10$ & 10 & $<13$ & 9 & 59 & 3 & $<0.04$ & 0.02 & 0.010 & 0.002 & 26 & 1 \\
\hline BY123 & $\begin{array}{l}\text { Starr Mine tunnel } \\
\text { midway nr Grass } \\
\text { Valley }\end{array}$ & $12 / 15 / 1999$ & $10: 45$ & 1 of 2 & 200 & 20 & $<20$ & 3 & $<9$ & 1 & 70 & 4 & $<0.03$ & 0.02 & 0.005 & 0.002 & 34 & 0 \\
\hline BY123 & $\begin{array}{l}\text { Starr Mine tunnel } \\
\text { midway nr Grass } \\
\text { Valley }\end{array}$ & $12 / 15 / 1999$ & $10: 45$ & $2 \mathrm{of} 2$ & 200 & 0 & $<20$ & 1 & $<9$ & 1 & 74 & 14 & $<0.03$ & 0.02 & 0.008 & 0.002 & 33 & 1 \\
\hline
\end{tabular}


Table 4A. Concentrations of trace metals and selected major elements in unfiltered water samples, Greenhorn Creek drainage, Nevada County, California—Continued

[Station name abbreviations: ab, above; bl, below; Cr, Creek; mi, miles; N, north; nr, near; Rd, Road; S, south; SF, South Fork. Number in parentheses is the data parameter code, a five-digit code used in the

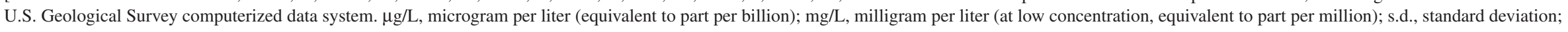
$<$, less than; - - not determined]

\begin{tabular}{|c|c|c|c|c|c|c|c|c|c|c|c|c|c|c|c|c|c|c|}
\hline \multirow[t]{2}{*}{$\begin{array}{l}\text { Station } \\
\text { map ID }\end{array}$} & \multirow[t]{2}{*}{ Station name } & \multirow[t]{2}{*}{ Date } & \multirow[t]{2}{*}{ Time } & \multirow[t]{2}{*}{$\begin{array}{l}\text { Rep- } \\
\text { licate }\end{array}$} & \multicolumn{2}{|c|}{$\begin{array}{c}\text { Aluminum (AI), } \\
\text { unfiltered } \\
(\mu \mathrm{g} / \mathrm{L}) \\
(01104)\end{array}$} & \multicolumn{2}{|c|}{$\begin{array}{c}\text { Arsenic (As), } \\
\text { unfiltered } \\
(\mu \mathrm{g} / \mathrm{L}) \\
(01002)\end{array}$} & \multicolumn{2}{|c|}{$\begin{array}{c}\text { Boron }(\mathrm{B}) \\
\text { unfiltered } \\
(\mu \mathrm{g} / \mathrm{L}) \\
(00999)\end{array}$} & \multicolumn{2}{|c|}{$\begin{array}{c}\text { Barium (Ba), } \\
\text { unfiltered } \\
(\mu \mathrm{g} / \mathrm{L}) \\
(01007)\end{array}$} & \multicolumn{2}{|c|}{$\begin{array}{c}\text { Beryllium }(\mathrm{Be}), \\
\text { unfiltered } \\
(\mu \mathrm{g} / \mathrm{L}) \\
(00998) \\
\end{array}$} & \multicolumn{2}{|c|}{$\begin{array}{c}\text { Bismuth }(\mathrm{Bi}), \\
\text { unfiltered } \\
(\mu \mathrm{g} / \mathrm{L}) \\
(\mathbf{0 1 0 1 7}) \\
\end{array}$} & \multicolumn{2}{|c|}{$\begin{array}{c}\text { Calcium (Ca) } \\
\text { unfiltered } \\
(\mathrm{mg} / \mathrm{L}) \\
(00916)\end{array}$} \\
\hline & & & & & value & s.d. & value & s.d. & value & s.d. & value & s.d. & value & s.d. & value & s.d. & value & s.d. \\
\hline BY124 & $\begin{array}{l}\text { Starr Mine tunnel } \\
\text { outlet nr Grass } \\
\text { Valley }\end{array}$ & $8 / 23 / 2000$ & $17: 30$ & 1 of 2 & 530 & 0 & $<20$ & 0 & 17 & 5 & 68 & 5 & $<0.1$ & 0.0 & 0.048 & 0.023 & 33 & 0 \\
\hline BY124 & $\begin{array}{l}\text { Starr Mine tunnel } \\
\text { outlet nr Grass } \\
\text { Valley }\end{array}$ & $8 / 23 / 2000$ & $17: 30$ & 2 of 2 & 620 & 10 & $<20$ & 0 & 12 & 10 & 61 & 3 & $<0.1$ & 0.1 & 0.045 & 0.012 & 32 & 0 \\
\hline BY129 & $\begin{array}{l}\text { Tom and Jerry Mine } \\
\text { drain at tunnel inlet } \\
\text { nr Nevada City }\end{array}$ & $6 / 28 / 2000$ & $12: 00$ & 1 of 2 & 1,900 & 300 & $<20$ & 0 & $<9$ & 13 & 94 & 5 & 0.2 & 0.1 & 0.053 & 0.019 & 4.0 & 0.9 \\
\hline BY129 & $\begin{array}{l}\text { Tom and Jerry Mine } \\
\text { drain at tunnel inlet } \\
\text { nr Nevada City }\end{array}$ & $6 / 28 / 2000$ & $12: 00$ & 2 of 2 & 1,800 & 300 & $<20$ & 0 & $<9$ & 14 & 110 & 10 & 0.2 & 0.1 & 0.082 & 0.052 & 4.2 & 0.2 \\
\hline BY130 & $\begin{array}{l}\text { Tom and Jerry Mine } \\
\text { drain at tunnel } \\
\text { outlet nr Nevada } \\
\text { City }\end{array}$ & $6 / 28 / 2000$ & $11: 30$ & 1 of 2 & 41 & 5 & $<20$ & 10 & 10 & 9 & 67 & 8 & $<0.1$ & 0.1 & 0.021 & 0.009 & 11 & 1 \\
\hline BY130 & $\begin{array}{l}\text { Tom and Jerry Mine } \\
\text { drain at tunnel } \\
\text { outlet nr Nevada } \\
\text { City }\end{array}$ & $6 / 28 / 2000$ & $11: 30$ & 2 of 2 & 42 & 6 & $<20$ & 0 & 14 & 4 & 69 & 10 & $<0.1$ & 0.0 & 0.021 & 0.010 & 12 & 2 \\
\hline BY131 & $\begin{array}{l}\text { Tom and Jerry Mine } \\
\text { drainage pond nr } \\
\text { Nevada City }\end{array}$ & $8 / 23 / 2000$ & $10: 45$ & 1 of 2 & 98 & 1 & $<20$ & 0 & 9 & 8 & 57 & 1 & $<0.1$ & 0.0 & 0.018 & 0.006 & 4.5 & 0.2 \\
\hline BY131 & $\begin{array}{l}\text { Tom and Jerry Mine } \\
\text { drainage pond nr } \\
\text { Nevada City }\end{array}$ & $8 / 23 / 2000$ & $10: 45$ & 2 of 2 & 100 & 11 & $<20$ & 0 & 16 & 8 & 47 & 4 & $<0.1$ & 0.0 & 0.013 & 0.002 & 4.2 & 1.0 \\
\hline \multicolumn{19}{|c|}{ Disturbed samples } \\
\hline BY123 & $\begin{array}{l}\text { Starr Mine tunnel } \\
\text { midway nr Grass } \\
\text { Valley }\end{array}$ & $12 / 15 / 1999$ & $11: 30$ & 1 of 2 & 40,000 & 1,000 & 170 & 10 & 17 & 16 & 11,000 & 0 & 5.3 & 0.5 & 1.0 & 0.0 & 130 & 0 \\
\hline BY123 & $\begin{array}{l}\text { Starr Mine tunnel } \\
\text { midway nr Grass } \\
\text { Valley }\end{array}$ & $12 / 15 / 1999$ & $11: 30$ & 2 of 2 & 38,000 & 2,000 & 160 & 10 & $<10$ & 5 & 11,000 & 0 & 5.5 & 0.1 & 0.96 & 0.03 & 120 & 0 \\
\hline BY129 & $\begin{array}{l}\text { Tom and Jerry Mine } \\
\text { drain at tunnel inlet } \\
\text { nr Nevada City }\end{array}$ & $6 / 28 / 2000$ & $12: 30$ & 1 of 2 & 23,000 & 1,000 & $<20$ & 10 & $<9$ & 6 & 550 & 10 & 1.9 & 0.2 & 0.26 & 0.02 & 20 & 0 \\
\hline BY129 & $\begin{array}{l}\text { Tom and Jerry Mine } \\
\text { drain at tunnel inlet } \\
\text { nr Nevada City }\end{array}$ & $6 / 28 / 2000$ & $12: 30$ & 2 of 2 & 24,000 & 0 & $<20$ & 0 & $<9$ & 2 & 690 & 110 & 1.8 & 0.1 & 0.27 & 0.01 & 26 & 5 \\
\hline
\end{tabular}


Table 4A. Concentrations of trace metals and selected major elements in unfiltered water samples, Greenhorn Creek drainage, Nevada County, California—Continued.

[Station name abbreviations: ab, above; bl, below; Cr, Creek; mi, miles; N, north; nr, near; Rd, Road; S, south; SF, South Fork. Number in parentheses is the data parameter code, a five-digit code used in the

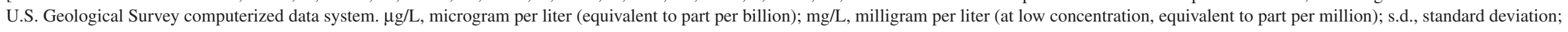

$<$, less than; - , not determined]

\begin{tabular}{|c|c|c|c|c|c|c|c|c|c|c|c|c|c|c|c|c|c|c|}
\hline \multirow[t]{2}{*}{$\begin{array}{l}\text { Station } \\
\text { map ID }\end{array}$} & \multirow[t]{2}{*}{ Station name } & \multirow[t]{2}{*}{ Date } & \multirow[t]{2}{*}{ Time } & \multirow[t]{2}{*}{$\begin{array}{l}\text { Rep- } \\
\text { licate }\end{array}$} & \multicolumn{2}{|c|}{$\begin{array}{c}\text { Cadmium (Cd), } \\
\text { unfiltered } \\
(\mu \mathrm{g} / \mathrm{L}) \\
(01027)\end{array}$} & \multicolumn{2}{|c|}{$\begin{array}{c}\text { Cerium (Ce), } \\
\text { unfiltered } \\
(\mu \mathrm{g} / \mathrm{L}) \\
(01112) \\
\end{array}$} & \multicolumn{2}{|c|}{$\begin{array}{c}\text { Cobalt (Co), } \\
\text { unfiltered } \\
(\mu \mathrm{g} / \mathrm{L}) \\
(01037)\end{array}$} & \multicolumn{2}{|c|}{$\begin{array}{c}\text { Chromium }(\mathrm{Cr}), \\
\text { unfiltered } \\
(\mu \mathrm{g} / \mathrm{L}) \\
(\mathbf{0 1 0 3 4})\end{array}$} & \multicolumn{2}{|c|}{$\begin{array}{c}\text { Cesium (Cs), } \\
\text { unfiltered } \\
(\mu \mathrm{g} / \mathrm{L}) \\
(01117)\end{array}$} & \multicolumn{2}{|c|}{$\begin{array}{c}\text { Copper }(\mathrm{Cu}) \text {, } \\
\text { unfiltered } \\
(\mu \mathrm{g} / \mathrm{L}) \\
(01042)\end{array}$} & \multicolumn{2}{|c|}{$\begin{array}{l}\text { Dysprosium (Dy), } \\
\text { unfiltered } \\
(\mu \mathrm{g} / \mathrm{L}) \\
(\mathbf{8 2 3 3 0}) \\
\end{array}$} \\
\hline & & & & & value & s.d. & value & s.d. & value & s.d. & value & s.d. & value & s.d. & value & s.d. & value & s.d. \\
\hline \multicolumn{19}{|c|}{ Environmental samples } \\
\hline BY20 & $\begin{array}{l}\text { Boston Mine tunnel } \\
\text { outlet nr Grass } \\
\text { Valley }\end{array}$ & $6 / 18 / 1999$ & $14: 30$ & 1 of 2 & 0.083 & 0.000 & 0.12 & 0.03 & 1.9 & 0.0 & 5 & 3 & $<0.06$ & 0.03 & 2.3 & 0.6 & 0.017 & 0.002 \\
\hline BY20 & $\begin{array}{l}\text { Boston Mine tunnel } \\
\text { outlet nr Grass } \\
\text { Valley }\end{array}$ & $6 / 18 / 1999$ & $14: 30$ & 2 of 2 & 0.081 & 0.007 & 0.086 & 0.007 & 2.1 & 0.1 & $<3$ & 1 & $<0.06$ & 0.02 & 1.8 & 0.4 & 0.012 & 0.001 \\
\hline BY20 & $\begin{array}{l}\text { Boston Mine tunnel } \\
\text { outlet nr Grass } \\
\text { Valley }\end{array}$ & $12 / 14 / 1999$ & $12: 00$ & 1 of 2 & 0.72 & 0.00 & 0.63 & 0.09 & 4.1 & 0.1 & $<2$ & 0 & $<2$ & 0 & 5.1 & 0.6 & 0.15 & 0.03 \\
\hline BY20 & $\begin{array}{l}\text { Boston Mine tunnel } \\
\text { outlet nr Grass } \\
\text { Valley }\end{array}$ & $12 / 14 / 1999$ & $12: 00$ & 2 of 2 & 0.36 & 0.05 & 0.42 & 0.06 & 3.9 & 0.0 & $<2$ & 1 & $<2$ & 1 & 2.2 & 0.2 & 0.086 & 0.011 \\
\hline BY20 & $\begin{array}{l}\text { Boston Mine tunnel } \\
\text { outlet nr Grass } \\
\text { Valley }\end{array}$ & $5 / 24 / 2000$ & $16: 00$ & 1 of 2 & $<0.03$ & 0.06 & 0.17 & 0.01 & 2.1 & 0.0 & $<1$ & 1 & $<0.06$ & 0.03 & 3.5 & 0.2 & 0.028 & 0.002 \\
\hline BY20 & $\begin{array}{l}\text { Boston Mine tunnel } \\
\text { outlet nr Grass } \\
\text { Valley }\end{array}$ & $5 / 24 / 2000$ & $16: 00$ & 2 of 2 & 0.08 & 0.02 & 0.33 & 0.01 & 3.6 & 0.2 & $<1$ & 1 & $<0.06$ & 0.02 & 4.6 & 0.3 & 0.064 & 0.007 \\
\hline BY21 & $\begin{array}{l}\text { Boston Mine wetlands } \\
\text { pond nr Grass } \\
\text { Valley }\end{array}$ & $12 / 14 / 1999$ & $10: 30$ & 1 of 2 & 0.034 & 0.010 & 0.037 & 0.002 & 0.14 & 0.01 & $<0.4$ & 0.2 & $<0.2$ & 0.1 & 0.26 & 0.02 & $<0.003$ & 0.001 \\
\hline BY21 & $\begin{array}{l}\text { Boston Mine wetlands } \\
\text { pond nr Grass } \\
\text { Valley }\end{array}$ & $12 / 14 / 1999$ & $10: 30$ & 2 of 2 & 0.036 & 0.005 & 0.043 & 0.002 & 0.17 & 0.02 & $<0.4$ & 0.5 & $<0.2$ & 0.1 & 0.28 & 0.05 & 0.011 & 0.003 \\
\hline BY21 & $\begin{array}{l}\text { Boston Mine wetlands } \\
\text { pond nr Grass } \\
\text { Valley }\end{array}$ & $5 / 24 / 2000$ & $16: 30$ & 1 of 2 & $<0.03$ & 0.03 & 0.043 & 0.000 & 0.17 & 0.03 & $<1$ & 0 & 0.08 & 0.04 & 1.1 & 0.1 & 0.009 & 0.001 \\
\hline BY21 & $\begin{array}{l}\text { Boston Mine wetlands } \\
\text { pond nr Grass } \\
\text { Valley }\end{array}$ & $5 / 24 / 2000$ & $16: 30$ & 2 of 2 & $<0.03$ & 0.00 & 0.046 & 0.004 & 0.19 & 0.01 & $<1$ & 0 & $<0.06$ & 0.01 & 1.4 & 0.3 & 0.009 & 0.003 \\
\hline BY58 & $\begin{array}{l}\text { Buckeye Flat Mine } \\
\text { main drain } 0.45 \mathrm{mi} \\
\text { ab Greenhorn } \mathrm{Cr}\end{array}$ & $8 / 21 / 2000$ & $11: 00$ & 1 of 2 & $<1$ & 0 & 29 & 0 & 76 & 0 & 2 & 0 & 0.6 & 0.6 & 7 & 0 & 3.6 & 0.1 \\
\hline BY58 & $\begin{array}{l}\text { Buckeye Flat Mine } \\
\text { main drain } 0.45 \mathrm{mi} \\
\text { ab Greenhorn } \mathrm{Cr}\end{array}$ & $8 / 21 / 2000$ & $11: 00$ & 2 of 2 & $<1$ & 1 & 23 & 1 & 60 & 4 & $<1$ & 1 & 0.4 & 0.5 & 5 & 0 & 2.8 & 0.1 \\
\hline
\end{tabular}


Table 4A. Concentrations of trace metals and selected major elements in unfiltered water samples, Greenhorn Creek drainage, Nevada County, California-Continued.

[Station name abbreviations: ab, above; bl, below; Cr, Creek; mi, miles; N, north; nr, near; Rd, Road; S, south; SF, South Fork. Number in parentheses is the data parameter code, a five-digit code used in the

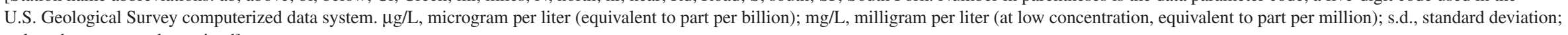

$<$, less than; - , not determined]

\begin{tabular}{|c|c|c|c|c|c|c|c|c|c|c|c|c|c|c|c|c|c|c|}
\hline \multirow[t]{2}{*}{$\begin{array}{l}\text { Station } \\
\text { map ID }\end{array}$} & \multirow[t]{2}{*}{ Station name } & \multirow[t]{2}{*}{ Date } & \multirow[t]{2}{*}{ Time } & \multirow[t]{2}{*}{$\begin{array}{l}\text { Rep- } \\
\text { licate }\end{array}$} & \multicolumn{2}{|c|}{$\begin{array}{c}\text { Cadmium }(\mathrm{Cd}), \\
\text { unfiltered } \\
(\mu \mathrm{g} / \mathrm{L}) \\
(01027) \\
\end{array}$} & \multicolumn{2}{|c|}{$\begin{array}{c}\text { Cerium (Ce), } \\
\text { unfiltered } \\
(\mu \mathrm{g} / \mathrm{L}) \\
(\mathbf{0 1 1 1 2}) \\
\end{array}$} & \multicolumn{2}{|c|}{$\begin{array}{c}\text { Cobalt (Co), } \\
\text { unfiltered } \\
(\mu \mathrm{g} / \mathrm{L}) \\
(01037)\end{array}$} & \multicolumn{2}{|c|}{$\begin{array}{c}\text { Chromium }(\mathrm{Cr}), \\
\text { unfiltered } \\
(\mu \mathrm{g} / \mathrm{L}) \\
(\mathbf{0 1 0 3 4}) \\
\end{array}$} & \multicolumn{2}{|c|}{$\begin{array}{c}\text { Cesium (Cs), } \\
\text { unfiltered } \\
(\mu \mathrm{g} / \mathrm{L}) \\
(\mathbf{0 1 1 1 7}) \\
\end{array}$} & \multicolumn{2}{|c|}{$\begin{array}{c}\text { Copper (Cu), } \\
\text { unfiltered } \\
(\mu \mathrm{g} / \mathrm{L}) \\
(01042) \\
\end{array}$} & \multicolumn{2}{|c|}{$\begin{array}{c}\text { Dysprosium (Dy), } \\
\text { unfiltered } \\
(\mu \mathrm{g} / \mathrm{L}) \\
(\mathbf{8 2 3 3 0}) \\
\end{array}$} \\
\hline & & & & & value & s.d. & value & s.d. & value & s.d. & value & s.d. & value & s.d. & value & s.d. & value & s.d. \\
\hline BY23 & $\begin{array}{c}\text { Buckeye Flat Mine N } \\
\text { drain to Greenhorn } \\
\text { Cr, Grass Valley }\end{array}$ & $6 / 18 / 1999$ & $11: 30$ & 1 of 2 & 2.3 & 0.0 & 38 & 1 & 85 & 3 & 4 & 2 & 0.09 & 0.04 & 38 & 0 & 4.8 & 0.0 \\
\hline BY23 & $\begin{array}{c}\text { Buckeye Flat Mine N } \\
\text { drain to Greenhorn } \\
\text { Cr, Grass Valley }\end{array}$ & $6 / 18 / 1999$ & $11: 30$ & 2 of 2 & 2.1 & 0.4 & 37 & 6 & 89 & 3 & 3 & 2 & 0.08 & 0.04 & 33 & 2 & 4.6 & 0.5 \\
\hline BY23 & $\begin{array}{c}\text { Buckeye Flat Mine N } \\
\text { drain to Greenhorn } \\
\text { Cr, Grass Valley }\end{array}$ & $12 / 2 / 1999$ & 11:00 & 1 of 2 & 2.6 & 0.1 & 26 & 3 & 78 & 3 & $<2$ & 1 & $<2$ & 1 & 37 & 3 & 3.2 & 0.0 \\
\hline BY23 & $\begin{array}{c}\text { Buckeye Flat Mine N } \\
\text { drain to Greenhorn } \\
\text { Cr, Grass Valley }\end{array}$ & $12 / 2 / 1999$ & $11: 00$ & 2 of 2 & 2.2 & 0.1 & 32 & 2 & 76 & 0 & $<2$ & 1 & $<2$ & 1 & 35 & 1 & 4.2 & 0.3 \\
\hline BY23 & $\begin{array}{c}\text { Buckeye Flat Mine N } \\
\text { drain to Greenhorn } \\
\text { Cr, Grass Valley }\end{array}$ & $8 / 22 / 2000$ & $10: 45$ & 1 of 2 & $<1$ & 1 & 46 & 0 & 70 & 0 & 2 & 1 & 0.4 & 0.3 & 33 & 1 & 4.9 & 0.2 \\
\hline BY23 & $\begin{array}{c}\text { Buckeye Flat Mine N } \\
\text { drain to Greenhorn } \\
\text { Cr, Grass Valley }\end{array}$ & $8 / 22 / 2000$ & $10: 45$ & 2of 2 & $<1$ & 1 & 41 & 0 & 67 & 8 & 3 & 1 & $<0.4$ & 0.3 & 31 & 2 & 4.7 & 0.1 \\
\hline BY116 & $\begin{array}{l}\text { Buckeye Flat Mine } \\
\text { pond drain } 0.15 \mathrm{mi} \\
\text { ab SF Greenhorn } \mathrm{Cr}\end{array}$ & $8 / 21 / 2000$ & $14: 10$ & 1 of 2 & $<0.08$ & 0.02 & 0.27 & 0.02 & 0.93 & 0.00 & $<2$ & 1 & 0.09 & 0.09 & 1.2 & 0.1 & 0.042 & 0.000 \\
\hline BY116 & $\begin{array}{l}\text { Buckeye Flat Mine } \\
\text { pond drain } 0.15 \mathrm{mi} \\
\text { ab SF Greenhorn } \mathrm{Cr}\end{array}$ & $8 / 21 / 2000$ & $14: 10$ & 2 of 2 & $<0.08$ & 0.04 & 0.32 & 0.01 & 1.0 & 0.0 & $<2$ & 0 & 0.10 & 0.06 & 1.4 & 0.2 & 0.030 & 0.005 \\
\hline BY24 & $\begin{array}{l}\text { Buckeye Flat Mine } \\
\text { S drain to SF } \\
\text { Greenhorn } \mathrm{Cr} \text {, } \\
\text { Grass Valley }\end{array}$ & $8 / 26 / 1999$ & 11:00 & 1 of 2 & 0.13 & 0.01 & 0.76 & 0.09 & 6.5 & 0.2 & 3 & 3 & $<0.06$ & 0.01 & 38 & 10 & 0.095 & 0.012 \\
\hline BY24 & $\begin{array}{l}\text { Buckeye Flat Mine } \\
\text { S drain to SF } \\
\text { Greenhorn Cr, } \\
\text { Grass Valley }\end{array}$ & $8 / 26 / 1999$ & 11:00 & 2 of 2 & 0.15 & 0.07 & 0.75 & 0.07 & 7.6 & 0.8 & 3 & 2 & $<0.06$ & 0.04 & 33 & 7 & 0.087 & 0.005 \\
\hline BY24 & $\begin{array}{l}\text { Buckeye Flat Mine } \\
\text { S drain to SF } \\
\text { Greenhorn Cr, } \\
\text { Grass Valley }\end{array}$ & $8 / 22 / 2000$ & $12: 10$ & 1 of 2 & 0.11 & 0.02 & 2.2 & 0.4 & 8.2 & 1.4 & $<3$ & 1 & 0.21 & 0.03 & 8.2 & 1.3 & 0.25 & 0.05 \\
\hline BY24 & $\begin{array}{l}\text { Buckeye Flat Mine } \\
\text { S drain to SF } \\
\text { Greenhorn Cr, } \\
\text { Grass Valley }\end{array}$ & $8 / 22 / 2000$ & $12: 10$ & 2 of 2 & 0.11 & 0.03 & 2.3 & 0.3 & 8.6 & 1.0 & $<3$ & 1 & 0.23 & 0.07 & 7.9 & 0.2 & 0.23 & 0.01 \\
\hline
\end{tabular}


Table 4A. Concentrations of trace metals and selected major elements in unfiltered water samples, Greenhorn Creek drainage, Nevada County, California-Continued.

[Station name abbreviations: ab, above; bl, below; Cr, Creek; mi, miles; N, north; nr, near; Rd, Road; S, south; SF, South Fork. Number in parentheses is the data parameter code, a five-digit code used in the

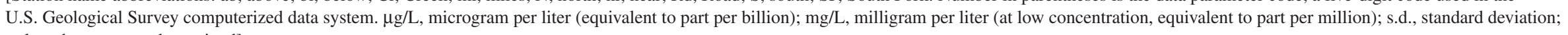
$<$, less than; - - not determined]

\begin{tabular}{|c|c|c|c|c|c|c|c|c|c|c|c|c|c|c|c|c|c|c|}
\hline \multirow[t]{2}{*}{$\begin{array}{l}\text { Station } \\
\text { map ID }\end{array}$} & \multirow[t]{2}{*}{ Station name } & \multirow[t]{2}{*}{ Date } & \multirow[t]{2}{*}{ Time } & \multirow[t]{2}{*}{$\begin{array}{l}\text { Rep- } \\
\text { licate }\end{array}$} & \multicolumn{2}{|c|}{$\begin{array}{c}\text { Cadmium }(C d) \\
\text { unfiltered } \\
(\mu \mathrm{g} / \mathrm{L}) \\
(01027) \\
\end{array}$} & \multicolumn{2}{|c|}{$\begin{array}{c}\text { Cerium (Ce), } \\
\text { unfiltered } \\
(\mu \mathrm{g} / \mathrm{L}) \\
(\mathbf{0 1 1 1 2})\end{array}$} & \multicolumn{2}{|c|}{$\begin{array}{c}\text { Cobalt (Co), } \\
\text { unfiltered } \\
(\mu \mathrm{g} / \mathrm{L}) \\
(01037) \\
\end{array}$} & \multicolumn{2}{|c|}{$\begin{array}{c}\text { Chromium }(\mathrm{Cr}), \\
\text { unfiltered } \\
(\mu \mathrm{g} / \mathrm{L}) \\
(\mathbf{0 1 0 3 4}) \\
\end{array}$} & \multicolumn{2}{|c|}{$\begin{array}{c}\text { Cesium (Cs), } \\
\text { unfiltered } \\
(\mu \mathrm{g} / \mathrm{L}) \\
(01117)\end{array}$} & \multicolumn{2}{|c|}{$\begin{array}{c}\text { Copper (Cu), } \\
\text { unfiltered } \\
(\mu \mathrm{g} / \mathrm{L}) \\
(01042)\end{array}$} & \multicolumn{2}{|c|}{$\begin{array}{c}\text { Dysprosium (Dy), } \\
\text { unfiltered } \\
(\mu \mathrm{g} / \mathrm{L}) \\
(\mathbf{8 2 3 3 0}) \\
\end{array}$} \\
\hline & & & & & value & s.d. & value & s.d. & value & s.d. & value & s.d. & value & s.d. & value & s.d. & value & s.d. \\
\hline BY25 & $\begin{array}{l}\text { Buckeye Flat Mine } \\
\text { upper drain }\end{array}$ & $4 / 9 / 2001$ & $10: 00$ & 1of 2 & 0.91 & 0.09 & 8.6 & 0.6 & 19 & 1 & 1 & 0 & 0.64 & 0.30 & 34 & 2 & 0.83 & 0.07 \\
\hline BY25 & $\begin{array}{l}\text { Buckeye Flat Mine } \\
\text { upper drain }\end{array}$ & $4 / 9 / 2001$ & $10: 00$ & 2of2 & 0.89 & 0.05 & 8.6 & 0.3 & 19 & 0 & $<1$ & 0 & 0.25 & 0.07 & 33 & 0 & 0.88 & 0.01 \\
\hline BY52 & $\begin{array}{l}\text { Greenhorn Cr } 0.3 \mathrm{mi} \\
\text { ab Buckeye Ford nr } \\
\text { Nevada City }\end{array}$ & $1 / 25 / 2000$ & $10: 45$ & 1of 2 & 0.089 & 0.009 & 2.0 & 0.0 & 2.5 & 0.0 & $<0.4$ & 0.4 & $<0.2$ & 0.0 & 2.4 & 0.1 & 0.21 & 0.00 \\
\hline BY52 & $\begin{array}{l}\text { Greenhorn Cr } 0.3 \mathrm{mi} \\
\text { ab Buckeye Ford nr } \\
\text { Nevada City }\end{array}$ & $1 / 25 / 2000$ & $10: 45$ & 2of 2 & 0.093 & 0.008 & 2.0 & 0.1 & 2.5 & 0.0 & $<0.4$ & 0.4 & $<0.2$ & 0.2 & 2.3 & 0.1 & 0.20 & 0.01 \\
\hline BY59 & $\begin{array}{l}\text { Greenhorn Cr at You } \\
\text { Bet Rd nr Nevada } \\
\text { City }\end{array}$ & $7 / 24 / 2001$ & 11:00 & 1of 2 & 0.05 & 0.02 & 0.098 & 0.014 & 0.46 & 0.01 & $<4$ & 2 & 0.14 & 0.03 & 0.6 & 0.0 & $<0.009$ & 0.004 \\
\hline BY59 & $\begin{array}{l}\text { Greenhorn } \mathrm{Cr} \text { at You } \\
\text { Bet Rd nr Nevada } \\
\text { City }\end{array}$ & $7 / 24 / 2001$ & 11:00 & 2of2 & 0.06 & 0.01 & 0.12 & 0.00 & 0.48 & 0.02 & $<4$ & 0 & 1.6 & 0.0 & 0.5 & 0.1 & 0.013 & 0.002 \\
\hline BY59 & $\begin{array}{l}\text { Greenhorn } \mathrm{Cr} \text { at You } \\
\text { Bet Rd nr Nevada } \\
\text { City }\end{array}$ & $8 / 29 / 2001$ & $15: 00$ & 1of 2 & 0.03 & 0.03 & 0.060 & 0.003 & 0.10 & 0.02 & $<4$ & 2 & 0.084 & 0.019 & 0.4 & 0.1 & $<0.009$ & 0.002 \\
\hline BY59 & $\begin{array}{l}\text { Greenhorn } \mathrm{Cr} \text { at You } \\
\text { Bet Rd nr Nevada } \\
\text { City }\end{array}$ & $8 / 29 / 2001$ & $15: 00$ & 2of 2 & 0.02 & 0.01 & 0.064 & 0.004 & 0.10 & 0.01 & 7 & 0 & 0.11 & 0.01 & 0.5 & 0.1 & $<0.009$ & 0.002 \\
\hline BY59 & $\begin{array}{l}\text { Greenhorn Cr at You } \\
\text { Bet Rd nr Nevada } \\
\text { City }\end{array}$ & $9 / 14 / 2001$ & 13:00 & 1of 2 & 0.04 & 0.01 & 0.066 & 0.005 & 0.19 & 0.03 & $<4$ & 1 & $<0.04$ & 0.02 & 1.2 & 0.1 & 0.006 & 0.001 \\
\hline BY59 & $\begin{array}{l}\text { Greenhorn Cr at You } \\
\text { Bet Rd nr Nevada } \\
\text { City }\end{array}$ & $9 / 14 / 2001$ & $13: 00$ & 2of 2 & 0.07 & 0.04 & 0.064 & 0.007 & 0.18 & 0.01 & $<4$ & 1 & $<0.04$ & 0.02 & 0.73 & 0.03 & 0.008 & 0.002 \\
\hline BY59 & $\begin{array}{l}\text { Greenhorn Cr at You } \\
\text { Bet Rd nr Nevada } \\
\text { City }\end{array}$ & $10 / 15 / 2001$ & $12: 00$ & 1of 2 & 0.12 & 0.13 & 0.027 & 0.002 & 0.15 & 0.06 & $<3$ & 2 & $<0.02$ & 0.01 & 0.4 & 0.1 & 0.008 & 0.002 \\
\hline BY59 & $\begin{array}{l}\text { Greenhorn } \mathrm{Cr} \text { at You } \\
\text { Bet Rd nr Nevada } \\
\text { City }\end{array}$ & $10 / 15 / 2001$ & $12: 00$ & 2of2 & 0.06 & 0.06 & 0.032 & 0.004 & 0.11 & 0.02 & $<3$ & 0 & 0.02 & 0.01 & 0.3 & 0.1 & $<0.006$ & 0.002 \\
\hline BY59 & $\begin{array}{l}\text { Greenhorn Cr at You } \\
\text { Bet Rd nr Nevada } \\
\text { City }\end{array}$ & $11 / 20 / 2001$ & $13: 20$ & 1of 2 & 0.09 & 0.01 & 0.21 & 0.00 & 1.3 & 0.1 & $<1$ & 0 & $<0.04$ & 0.01 & 0.97 & 0.15 & 0.029 & 0.003 \\
\hline BY59 & $\begin{array}{l}\text { Greenhorn Cr at You } \\
\text { Bet Rd nr Nevada } \\
\text { City }\end{array}$ & $11 / 20 / 2001$ & $13: 20$ & 2of 2 & 0.14 & 0.03 & 0.23 & 0.01 & 1.3 & 0.0 & $<1$ & 0 & $<0.04$ & 0.01 & 1.0 & 0.2 & 0.032 & 0.001 \\
\hline
\end{tabular}


Table 4A. Concentrations of trace metals and selected major elements in unfiltered water samples, Greenhorn Creek drainage, Nevada County, California-Continued.

[Station name abbreviations: ab, above; bl, below; Cr, Creek; mi, miles; N, north; nr, near; Rd, Road; S, south; SF, South Fork. Number in parentheses is the data parameter code, a five-digit code used in the

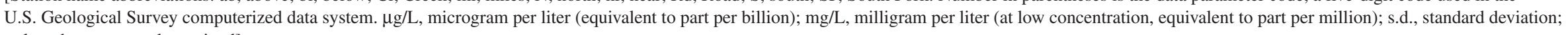
$<$, less than; - - not determined]

\begin{tabular}{|c|c|c|c|c|c|c|c|c|c|c|c|c|c|c|c|c|c|c|}
\hline \multirow[t]{2}{*}{$\begin{array}{l}\text { Station } \\
\text { map ID }\end{array}$} & \multirow[t]{2}{*}{ Station name } & \multirow[t]{2}{*}{ Date } & \multirow[t]{2}{*}{ Time } & \multirow[t]{2}{*}{$\begin{array}{l}\text { Rep- } \\
\text { licate }\end{array}$} & \multicolumn{2}{|c|}{$\begin{array}{c}\text { Cadmium }(C d), \\
\text { unfiltered } \\
(\mu \mathrm{g} / \mathrm{L}) \\
(01027) \\
\end{array}$} & \multicolumn{2}{|c|}{$\begin{array}{c}\text { Cerium (Ce), } \\
\text { unfiltered } \\
(\mu \mathrm{g} / \mathrm{L}) \\
(\mathbf{0 1 1 1 2})\end{array}$} & \multicolumn{2}{|c|}{$\begin{array}{c}\text { Cobalt (Co), } \\
\text { unfiltered } \\
(\mu \mathrm{g} / \mathrm{L}) \\
(01037)\end{array}$} & \multicolumn{2}{|c|}{$\begin{array}{c}\text { Chromium }(\mathrm{Cr}), \\
\text { unfiltered } \\
(\mu \mathrm{g} / \mathrm{L}) \\
(\mathbf{0 1 0 3 4 )} \\
\end{array}$} & \multicolumn{2}{|c|}{$\begin{array}{c}\text { Cesium (Cs), } \\
\text { unfiltered } \\
(\mu \mathrm{g} / \mathrm{L}) \\
(01117) \\
\end{array}$} & \multicolumn{2}{|c|}{$\begin{array}{c}\text { Copper }(\mathrm{Cu}), \\
\text { unfiltered } \\
(\mu \mathrm{g} / \mathrm{L}) \\
(\mathbf{0 1 0 4 2 )} \\
\end{array}$} & \multicolumn{2}{|c|}{$\begin{array}{c}\text { Dysprosium (Dy), } \\
\text { unfiltered } \\
(\mu \mathrm{g} / \mathrm{L}) \\
(\mathbf{8 2 3 3 0}) \\
\end{array}$} \\
\hline & & & & & value & s.d. & value & s.d. & value & s.d. & value & s.d. & value & s.d. & value & s.d. & value & s.d. \\
\hline BY59 & $\begin{array}{l}\text { Greenhorn Cr at You } \\
\text { Bet Rd nr Nevada } \\
\text { City }\end{array}$ & $12 / 10 / 2001$ & $12: 20$ & 1 of 2 & 0.14 & 0.02 & 2.0 & 0.0 & 2.5 & 0.2 & 1.9 & 0.1 & 0.11 & 0.00 & 2.8 & 0.1 & 0.23 & 0.01 \\
\hline BY59 & $\begin{array}{l}\text { Greenhorn } \mathrm{Cr} \text { at You } \\
\text { Bet Rd nr Nevada } \\
\text { City }\end{array}$ & $12 / 10 / 2001$ & $12: 20$ & 2 of 2 & 0.14 & 0.02 & 2.0 & 0.1 & 2.5 & 0.2 & 2.0 & 0.6 & 0.10 & 0.01 & 2.5 & 0.2 & 0.22 & 0.00 \\
\hline BY59 & $\begin{array}{l}\text { Greenhorn } \mathrm{Cr} \text { at You } \\
\text { Bet Rd nr Nevada } \\
\text { City }\end{array}$ & $12 / 21 / 2001$ & 13:00 & 1 of 2 & 0.13 & 0.06 & 8.2 & 0.8 & 5.2 & 0.5 & 5 & 1 & 0.39 & 0.06 & 14 & 3.9 & 0.87 & 0.10 \\
\hline BY59 & $\begin{array}{l}\text { Greenhorn } \mathrm{Cr} \text { at You } \\
\text { Bet Rd nr Nevada } \\
\text { City }\end{array}$ & $12 / 21 / 2001$ & $13: 00$ & 2 of 2 & $<0.05$ & 0.02 & 8.1 & 0.7 & 5.6 & 0.0 & 6 & 2 & 0.39 & 0.04 & 12 & 0.1 & 0.95 & 0.03 \\
\hline BY51 & $\begin{array}{l}\text { Greenhorn } \mathrm{Cr} n r \\
\text { headwaters nr } \\
\text { Scotts Flat Res }\end{array}$ & $1 / 25 / 2000$ & $9: 30$ & 1 of 2 & 0.057 & 0.002 & 0.30 & 0.01 & 0.22 & 0.01 & $<0.4$ & 0.5 & $<0.2$ & 0.0 & 5.0 & 0.1 & 0.060 & 0.007 \\
\hline BY51 & $\begin{array}{l}\text { Greenhorn } \mathrm{Cr} n \mathrm{r} \\
\text { headwaters } \mathrm{nr} \\
\text { Scotts Flat Res }\end{array}$ & $1 / 25 / 2000$ & $9: 30$ & $2 \mathrm{of} 2$ & 0.031 & 0.008 & 0.30 & 0.01 & 0.21 & 0.00 & $<0.4$ & 0.4 & $<0.2$ & 0.1 & 5.0 & 0.1 & 0.057 & 0.005 \\
\hline BY75 & $\begin{array}{l}\text { Missouri Cyn } \mathrm{Cr} \\
\text { 1.2mi ab Greenhorn } \\
\text { Cr nr Chicago Park }\end{array}$ & $12 / 14 / 1999$ & $14: 00$ & 1 of 2 & 0.16 & 0.04 & 0.64 & 0.20 & 4.0 & 0.1 & $<2$ & 1 & $<2$ & 1 & 1.3 & 0.1 & 0.077 & 0.003 \\
\hline BY75 & $\begin{array}{l}\text { Missouri Cyn } \mathrm{Cr} \\
\text { 1.2mi ab Greenhorn } \\
\text { Cr nr Chicago Park }\end{array}$ & $12 / 14 / 1999$ & $14: 00$ & $2 \mathrm{of} 2$ & 0.15 & 0.03 & 0.68 & 0.15 & 3.6 & 0.1 & $<2$ & 1 & $<2$ & 1 & 1.4 & 0.1 & 0.11 & 0.02 \\
\hline BY86 & $\begin{array}{l}\text { Poore Mine Creek } \\
\text { ab tunnel nr Grass } \\
\text { Valley }\end{array}$ & $3 / 31 / 1999$ & $17: 40$ & 1 of 2 & 0.024 & 0.009 & 0.83 & 0.14 & 0.24 & 0.01 & $<2$ & 2 & $<0.04$ & 0.03 & 1.3 & 0.2 & 0.051 & 0.003 \\
\hline BY86 & $\begin{array}{l}\text { Poore Mine Creek } \\
\text { ab tunnel nr Grass } \\
\text { Valley }\end{array}$ & $3 / 31 / 1999$ & $17: 40$ & $2 o f 2$ & 0.018 & 0.005 & 0.73 & 0.06 & 0.22 & 0.01 & $<2$ & 2 & $<0.04$ & 0.01 & 1.6 & 0.5 & 0.047 & 0.002 \\
\hline BY87 & $\begin{array}{l}\text { Poore Mine Creek } \\
\text { bl tunnel nr Grass } \\
\text { Valley }\end{array}$ & $3 / 31 / 1999$ & $13: 30$ & 1 of 2 & 0.015 & 0.002 & 0.58 & 0.03 & 0.21 & 0.01 & $<2$ & 1 & $<0.04$ & 0.03 & 1.1 & 0.1 & 0.055 & 0.002 \\
\hline BY87 & $\begin{array}{l}\text { Poore Mine Creek } \\
\text { bl tunnel nr Grass } \\
\text { Valley }\end{array}$ & $3 / 31 / 1999$ & $13: 30$ & $2 o f 2$ & 0.012 & 0.006 & 0.79 & 0.03 & 0.22 & 0.03 & $<2$ & 1 & $<0.04$ & 0.02 & 1.1 & 0.2 & 0.064 & 0.002 \\
\hline
\end{tabular}


Table 4A. Concentrations of trace metals and selected major elements in unfiltered water samples, Greenhorn Creek drainage, Nevada County, California—Continued.

[Station name abbreviations: ab, above; bl, below; Cr, Creek; mi, miles; N, north; nr, near; Rd, Road; S, south; SF, South Fork. Number in parentheses is the data parameter code, a five-digit code used in the

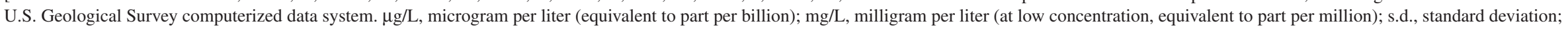
$<$, less than; - , not determined]

\begin{tabular}{|c|c|c|c|c|c|c|c|c|c|c|c|c|c|c|c|c|c|c|}
\hline \multirow[t]{2}{*}{$\begin{array}{l}\text { Station } \\
\text { map ID }\end{array}$} & \multirow[t]{2}{*}{ Station name } & \multirow[t]{2}{*}{ Date } & \multirow[t]{2}{*}{ Time } & \multirow[t]{2}{*}{$\begin{array}{l}\text { Rep- } \\
\text { licate }\end{array}$} & \multicolumn{2}{|c|}{$\begin{array}{c}\text { Cadmium }(\mathrm{Cd}), \\
\text { unfiltered } \\
(\mu \mathrm{g} / \mathrm{L}) \\
(01027) \\
\end{array}$} & \multicolumn{2}{|c|}{$\begin{array}{c}\text { Cerium (Ce), } \\
\text { unfiltered } \\
(\mu \mathrm{g} / \mathrm{L}) \\
(\mathbf{0 1 1 1 2}) \\
\end{array}$} & \multicolumn{2}{|c|}{$\begin{array}{c}\text { Cobalt (Co), } \\
\text { unfiltered } \\
(\mu \mathrm{g} / \mathrm{L}) \\
(01037)\end{array}$} & \multicolumn{2}{|c|}{$\begin{array}{c}\text { Chromium }(\mathrm{Cr}) \text {, } \\
\text { unfiltered } \\
(\mu \mathrm{g} / \mathrm{L}) \\
(01034) \\
\end{array}$} & \multicolumn{2}{|c|}{$\begin{array}{c}\text { Cesium (Cs), } \\
\text { unfiltered } \\
(\mu \mathrm{g} / \mathrm{L}) \\
(01117) \\
\end{array}$} & \multicolumn{2}{|c|}{$\begin{array}{c}\text { Copper }(\mathrm{Cu}), \\
\text { unfiltered } \\
(\mu \mathrm{g} / \mathrm{L}) \\
(01042) \\
\end{array}$} & \multicolumn{2}{|c|}{$\begin{array}{c}\text { Dysprosium (Dy), } \\
\text { unfiltered } \\
(\mu \mathrm{g} / \mathrm{L}) \\
(\mathbf{8 2 3 3 0}) \\
\end{array}$} \\
\hline & & & & & value & s.d. & value & s.d. & value & s.d. & value & s.d. & value & s.d. & value & s.d. & value & s.d. \\
\hline BY88 & $\begin{array}{l}\text { Poore Mine ground } \\
\text { sluice nr Grass } \\
\text { Valley }\end{array}$ & 4/1/1999 & $15: 40$ & 1 of 2 & 0.056 & 0.021 & 0.14 & 0.02 & 1.1 & 0.2 & $<2$ & 2 & 0.05 & 0.02 & 1.0 & 0.4 & 0.026 & 0.000 \\
\hline BY88 & $\begin{array}{l}\text { Poore Mine ground } \\
\text { sluice nr Grass } \\
\text { Valley }\end{array}$ & 4/1/1999 & $15: 40$ & 2of 2 & 0.042 & 0.011 & 0.16 & 0.02 & 1.1 & 0.0 & $<2$ & 1 & $<0.04$ & 0.01 & 0.78 & 0.03 & 0.040 & 0.005 \\
\hline BY89 & $\begin{array}{l}\text { Poore Mine pit lake nr } \\
\text { Grass Valley }\end{array}$ & 4/1/1999 & $16: 50$ & 1 of 2 & 0.063 & 0.009 & 0.11 & 0.01 & 0.77 & 0.03 & $<2$ & 1 & $<0.04$ & 0.04 & 1.7 & 0.2 & 0.017 & 0.001 \\
\hline BY89 & $\begin{array}{l}\text { Poore Mine pit lake nr } \\
\text { Grass Valley }\end{array}$ & 4/1/1999 & $16: 50$ & 2 of 2 & 0.072 & 0.005 & 0.12 & 0.02 & 0.83 & 0.04 & $<2$ & 2 & $<0.04$ & 0.03 & 1.5 & 0.3 & 0.018 & 0.004 \\
\hline BY89 & $\begin{array}{l}\text { Poore Mine pit lake } \mathrm{nr} \\
\text { Grass Valley }\end{array}$ & $5 / 23 / 2000$ & $10: 45$ & 1 of 2 & 1.1 & 0.0 & 0.26 & 0.01 & 9.4 & 0.1 & $<1$ & 0 & $<0.06$ & 0.03 & 15 & 0 & 0.045 & 0.004 \\
\hline BY89 & $\begin{array}{l}\text { Poore Mine pit lake nr } \\
\text { Grass Valley }\end{array}$ & $5 / 23 / 2000$ & $10: 45$ & 2 of 2 & $<0.03$ & 0.14 & 0.26 & 0.01 & 9.7 & 0.2 & $<1$ & 0 & $<0.06$ & 0.02 & 16 & 0 & 0.048 & 0.003 \\
\hline BY90 & $\begin{array}{l}\text { Poore Mine seep } \\
\text { above ground sluice } \\
\text { nr Grass Valley }\end{array}$ & 4/1/1999 & $16: 00$ & 1 of 2 & - & & - & & - & & - & & - & & - & & - & \\
\hline BY90 & $\begin{array}{l}\text { Poore Mine seep } \\
\text { above ground sluice } \\
\text { nr Grass Valley }\end{array}$ & 4/1/1999 & $16: 00$ & 2of 2 & - & & - & & - & & - & & - & & - & & - & \\
\hline BY91 & $\begin{array}{l}\text { Poore Mine tunnel } \\
\text { effluent nr Grass } \\
\text { Valley }\end{array}$ & 3/31/1999 & $15: 20$ & 1of 2 & 0.064 & 0.006 & 0.16 & 0.00 & 0.057 & 0.016 & $<2$ & 1 & 0.22 & 0.03 & 1.3 & 0.2 & 0.016 & 0.002 \\
\hline BY91 & $\begin{array}{l}\text { Poore Mine tunnel } \\
\text { effluent nr Grass } \\
\text { Valley }\end{array}$ & 3/31/1999 & $15: 20$ & 2 of 2 & 0.044 & 0.005 & 0.19 & 0.02 & 0.075 & 0.022 & $<2$ & 1 & 0.17 & 0.01 & 1.1 & 0.2 & 0.019 & 0.005 \\
\hline BY91 & $\begin{array}{l}\text { Poore Mine tunnel } \\
\text { effluent nr Grass } \\
\text { Valley }\end{array}$ & $12 / 15 / 1999$ & $13: 00$ & 1of 2 & 0.021 & 0.004 & 0.24 & 0.01 & 0.24 & 0.02 & $<2$ & 2 & $<2$ & 0 & 0.88 & 0.08 & 0.029 & 0.004 \\
\hline BY91 & $\begin{array}{l}\text { Poore Mine tunnel } \\
\text { effluent nr Grass } \\
\text { Valley }\end{array}$ & $12 / 15 / 1999$ & $13: 00$ & 2 of 2 & 0.037 & 0.007 & 0.21 & 0.05 & 0.29 & 0.12 & $<2$ & 1 & $<2$ & 0 & 0.84 & 0.07 & 0.026 & 0.003 \\
\hline BY91 & $\begin{array}{l}\text { Poore Mine tunnel } \\
\text { effluent nr Grass } \\
\text { Valley }\end{array}$ & $5 / 23 / 2000$ & $12: 00$ & 1of 2 & $<0.03$ & 0.02 & 2.7 & 0.0 & 2.1 & 0.0 & $<1$ & 1 & 0.37 & 0.05 & 13 & 0 & 0.31 & 0.01 \\
\hline BY91 & $\begin{array}{l}\text { Poore Mine tunnel } \\
\text { effluent nr Grass } \\
\text { Valley }\end{array}$ & $5 / 23 / 2000$ & $12: 00$ & 2 of 2 & $<0.03$ & 0.04 & 2.5 & 0.1 & 2.0 & 0.0 & $<1$ & 0 & 0.34 & 0.04 & 12 & 0 & 0.30 & 0.02 \\
\hline
\end{tabular}


Table 4A. Concentrations of trace metals and selected major elements in unfiltered water samples, Greenhorn Creek drainage, Nevada County, California-Continued.

[Station name abbreviations: ab, above; bl, below; Cr, Creek; mi, miles; N, north; nr, near; Rd, Road; S, south; SF, South Fork. Number in parentheses is the data parameter code, a five-digit code used in the

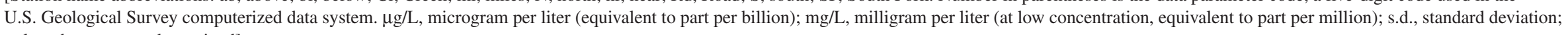

$<$, less than; - , not determined]

\begin{tabular}{|c|c|c|c|c|c|c|c|c|c|c|c|c|c|c|c|c|c|c|}
\hline \multirow[t]{2}{*}{$\begin{array}{l}\text { Station } \\
\text { map ID }\end{array}$} & \multirow[t]{2}{*}{ Station name } & \multirow[t]{2}{*}{ Date } & \multirow[t]{2}{*}{ Time } & \multirow[t]{2}{*}{$\begin{array}{l}\text { Rep- } \\
\text { licate }\end{array}$} & \multicolumn{2}{|c|}{$\begin{array}{l}\text { Cadmium }(C d), \\
\text { unfiltered } \\
(\mu g / L) \\
(01027)\end{array}$} & \multicolumn{2}{|c|}{$\begin{array}{c}\text { Cerium (Ce), } \\
\text { unfiltered } \\
(\mu \mathrm{g} / \mathrm{L}) \\
(\mathbf{0 1 1 1 2}) \\
\end{array}$} & \multicolumn{2}{|c|}{$\begin{array}{c}\text { Cobalt (Co), } \\
\text { unfiltered } \\
(\mu \mathrm{g} / \mathrm{L}) \\
(01037)\end{array}$} & \multicolumn{2}{|c|}{$\begin{array}{c}\text { Chromium }(\mathrm{Cr}) \text {, } \\
\text { unfiltered } \\
(\mu \mathrm{g} / \mathrm{L}) \\
(01034)\end{array}$} & \multicolumn{2}{|c|}{$\begin{array}{c}\text { Cesium (Cs), } \\
\text { unfiltered } \\
(\mu \mathrm{g} / \mathrm{L}) \\
(\mathbf{0 1 1 1 7}) \\
\end{array}$} & \multicolumn{2}{|c|}{$\begin{array}{c}\text { Copper }(\mathrm{Cu}), \\
\text { unfiltered } \\
(\mu \mathrm{g} / \mathrm{L}) \\
(\mathbf{0 1 0 4 2}) \\
\end{array}$} & \multicolumn{2}{|c|}{$\begin{array}{c}\text { Dysprosium (Dy), } \\
\text { unfiltered } \\
(\mu \mathrm{g} / \mathrm{L}) \\
(\mathbf{8 2 3 3 0}) \\
\end{array}$} \\
\hline & & & & & value & s.d. & value & s.d. & value & s.d. & value & s.d. & value & s.d. & value & s.d. & value & s.d. \\
\hline BY105 & $\begin{array}{l}\text { Sailor Flat Mine main } \\
\text { drain gulch 01nr } \\
\text { Quaker Hill }\end{array}$ & $7 / 6 / 1999$ & $16: 00$ & 1of 2 & 0.11 & 0.00 & 12 & 2 & 21 & 2 & 4 & 3 & 0.06 & 0.03 & 10 & 2 & 1.7 & 0.1 \\
\hline BY105 & $\begin{array}{l}\text { Sailor Flat Mine main } \\
\text { drain gulch 01nr } \\
\text { Quaker Hill }\end{array}$ & 7/6/1999 & $16: 00$ & $2 o f 2$ & 0.18 & 0.01 & 8.0 & 0.8 & 20 & 0 & 5 & 3 & 0.15 & 0.01 & 13 & 2 & 1.1 & 0.1 \\
\hline BY105 & $\begin{array}{l}\text { Sailor Flat Mine main } \\
\text { drain Gulch 01nr } \\
\text { Quaker Hill }\end{array}$ & $12 / 2 / 1999$ & $14: 30$ & 1of 2 & 0.21 & 0.03 & 44 & 6 & 8.0 & 0.2 & 3 & 1 & 2 & 1 & 43 & 0 & 3.9 & 0.0 \\
\hline BY105 & $\begin{array}{l}\text { Sailor Flat Mine main } \\
\text { drain Gulch 01nr } \\
\text { Quaker Hill }\end{array}$ & $12 / 2 / 1999$ & $14: 30$ & $2 o f 2$ & 0.21 & 0.01 & 40 & 1 & 8.0 & 0.2 & $<2$ & 2 & 2 & 1 & 32 & 1 & 2.8 & 0.1 \\
\hline BY106 & $\begin{array}{l}\text { Sailor Flat Mine } \\
\text { main drainage to } \\
\text { Greenhorn Cr, } \\
\text { Gulch } 03\end{array}$ & 4/9/2001 & $12: 30$ & 1 of 2 & 0.07 & 0.04 & 2.4 & 0.2 & 1.4 & 0.1 & 3 & 0 & 0.41 & 0.38 & 3.1 & 0.2 & 0.25 & 0.02 \\
\hline BY106 & $\begin{array}{l}\text { Sailor Flat Mine } \\
\text { main drainage to } \\
\text { Greenhorn Cr, } \\
\text { Gulch } 03\end{array}$ & $4 / 9 / 2001$ & $12: 30$ & 2 of 2 & 0.05 & 0.02 & 2.4 & 0.0 & 1.4 & 0.0 & 3 & 0 & 0.36 & 0.19 & 3.2 & 0.3 & 0.26 & 0.01 \\
\hline BY114 & $\begin{array}{l}\text { SF Greenhorn Cr } \\
\text { 0.7mi ab Greenhorn } \\
\text { Cr nr Nevada City }\end{array}$ & $8 / 22 / 2000$ & $15: 10$ & 1of 2 & $<0.08$ & 0.04 & 0.059 & 0.010 & $<0.03$ & 0.01 & $<3$ & 1 & $<0.08$ & 0.05 & 0.7 & 0.0 & 0.006 & 0.002 \\
\hline BY114 & $\begin{array}{l}\text { SF Greenhorn Cr } \\
\text { 0.7mi ab Greenhorn } \\
\text { Cr nr Nevada City }\end{array}$ & $8 / 22 / 2000$ & $15: 10$ & $2 \mathrm{of} 2$ & $<0.08$ & 0.02 & 0.058 & 0.004 & $<0.03$ & 0.00 & $<3$ & 1 & 0.36 & 0.11 & 3.4 & 0.2 & $<0.006$ & 0.002 \\
\hline BY122 & $\begin{array}{l}\text { Starr Mine tunnel } \\
\text { inflow nr Grass } \\
\text { Valley }\end{array}$ & $4 / 23 / 1999$ & $15: 00$ & 1 of 2 & 0.078 & 0.003 & 0.13 & 0.01 & 0.35 & 0.04 & $<2$ & 0 & $<0.04$ & 0.01 & 4.9 & 0.1 & 0.036 & 0.002 \\
\hline BY122 & $\begin{array}{l}\text { Starr Mine tunnel } \\
\text { inflow nr Grass } \\
\text { Valley }\end{array}$ & $4 / 23 / 1999$ & $15: 00$ & $2 o f 2$ & 0.073 & 0.011 & 0.18 & 0.03 & 0.38 & 0.02 & $<2$ & 0 & $<0.04$ & 0.01 & 4.9 & 0.2 & 0.048 & 0.005 \\
\hline BY123 & $\begin{array}{l}\text { Starr Mine tunnel } \\
\text { midway nr Grass } \\
\text { Valley }\end{array}$ & $4 / 23 / 1999$ & $13: 40$ & 1 of 2 & 0.022 & 0.011 & 0.24 & 0.05 & 0.79 & 0.01 & $<2$ & 1 & 0.11 & 0.01 & 1.2 & 0.1 & 0.037 & 0.003 \\
\hline BY123 & $\begin{array}{l}\text { Starr Mine tunnel } \\
\text { midway nr Grass } \\
\text { Valley }\end{array}$ & $4 / 23 / 1999$ & $13: 40$ & $2 \mathrm{of} 2$ & 0.020 & 0.006 & 0.16 & 0.02 & 0.71 & 0.02 & 2 & 0 & 0.15 & 0.03 & 1.2 & 0.2 & 0.019 & 0.004 \\
\hline
\end{tabular}


Table 4A. Concentrations of trace metals and selected major elements in unfiltered water samples, Greenhorn Creek drainage, Nevada County, California—Continued.

[Station name abbreviations: ab, above; bl, below; Cr, Creek; mi, miles; N, north; nr, near; Rd, Road; S, south; SF, South Fork. Number in parentheses is the data parameter code, a five-digit code used in the

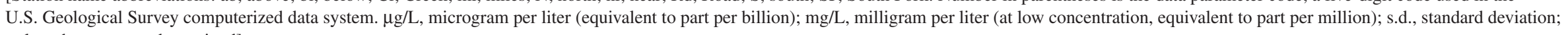
$<$, less than; - , not determined]

\begin{tabular}{|c|c|c|c|c|c|c|c|c|c|c|c|c|c|c|c|c|c|c|}
\hline \multirow[t]{2}{*}{$\begin{array}{l}\text { Station } \\
\text { map ID }\end{array}$} & \multirow[t]{2}{*}{ Station name } & \multirow[t]{2}{*}{ Date } & \multirow[t]{2}{*}{ Time } & \multirow[t]{2}{*}{$\begin{array}{l}\text { Rep- } \\
\text { licate }\end{array}$} & \multicolumn{2}{|c|}{$\begin{array}{l}\text { Cadmium (Cd), } \\
\text { unfiltered } \\
(\mu \mathrm{g} / \mathrm{L}) \\
(01027) \\
\end{array}$} & \multicolumn{2}{|c|}{$\begin{array}{c}\text { Cerium (Ce), } \\
\text { unfiltered } \\
(\mu \mathrm{g} / \mathrm{L}) \\
(\mathbf{0 1 1 1 2}) \\
\end{array}$} & \multicolumn{2}{|c|}{$\begin{array}{c}\text { Cobalt (Co), } \\
\text { unfiltered } \\
(\mu \mathrm{g} / \mathrm{L}) \\
(01037)\end{array}$} & \multicolumn{2}{|c|}{$\begin{array}{c}\text { Chromium }(\mathrm{Cr}), \\
\text { unfiltered } \\
(\mu \mathrm{g} / \mathrm{L}) \\
(\mathbf{0 1 0 3 4 )} \\
\end{array}$} & \multicolumn{2}{|c|}{$\begin{array}{c}\text { Cesium (Cs), } \\
\text { unfiltered } \\
(\mu \mathrm{g} / \mathrm{L}) \\
(\mathbf{0 1 1 1 7})\end{array}$} & \multicolumn{2}{|c|}{$\begin{array}{c}\text { Copper (Cu), } \\
\text { unfiltered } \\
(\mu \mathrm{g} / \mathrm{L}) \\
(\mathbf{0 1 0 4 2 )} \\
\end{array}$} & \multicolumn{2}{|c|}{$\begin{array}{c}\text { Dysprosium (Dy), } \\
\text { unfiltered } \\
(\mu \mathrm{g} / \mathrm{L}) \\
(\mathbf{8 2 3 3 0}) \\
\end{array}$} \\
\hline & & & & & value & s.d. & value & s.d. & value & s.d. & value & s.d. & value & s.d. & value & s.d. & value & s.d. \\
\hline BY123 & $\begin{array}{l}\text { Starr Mine tunnel } \\
\text { midway nr Grass } \\
\text { Valley }\end{array}$ & $12 / 15 / 1999$ & $10: 45$ & 1 of 2 & 0.059 & 0.015 & 0.36 & 0.06 & 2.5 & 0.0 & $<2$ & 0 & $<2$ & 1 & 2.5 & 0.2 & 0.064 & 0.019 \\
\hline BY123 & $\begin{array}{l}\text { Starr Mine tunnel } \\
\text { midway nr Grass } \\
\text { Valley }\end{array}$ & $12 / 15 / 1999$ & $10: 45$ & 2 of 2 & 0.071 & 0.007 & 0.40 & 0.03 & 2.5 & 0.1 & $<2$ & 0 & $<2$ & 0 & 2.7 & 0.4 & 0.063 & 0.005 \\
\hline BY124 & $\begin{array}{l}\text { Starr Mine tunnel } \\
\text { outlet nr Grass } \\
\text { Valley }\end{array}$ & $8 / 23 / 2000$ & $17: 30$ & 1 of 2 & $<0.08$ & 0.03 & 1.6 & 0.0 & 2.2 & 0.3 & $<3$ & 1 & 1.0 & 0.1 & 6.1 & 0.2 & 0.13 & 0.00 \\
\hline BY124 & $\begin{array}{l}\text { Starr Mine tunnel } \\
\text { outlet nr Grass } \\
\text { Valley }\end{array}$ & $8 / 23 / 2000$ & $17: 30$ & 2 of 2 & 0.08 & 0.02 & 1.8 & 0.0 & 2.4 & 0.0 & $<3$ & 1 & 0.36 & 0.08 & 6.4 & 0.2 & 0.14 & 0.00 \\
\hline BY129 & $\begin{array}{l}\text { Tom and Jerry Mine } \\
\text { drain at tunnel inlet } \\
\text { nr Nevada City }\end{array}$ & $6 / 28 / 2000$ & $12: 00$ & 1 of 2 & 0.15 & 0.04 & 20 & 4 & 12 & 2 & 19 & 4 & 0.53 & 0.10 & 21 & 1 & 2.0 & 0.4 \\
\hline BY129 & $\begin{array}{l}\text { Tom and Jerry Mine } \\
\text { drain at tunnel inlet } \\
\text { nr Nevada City }\end{array}$ & $6 / 28 / 2000$ & $12: 00$ & $2 o f 2$ & 0.17 & 0.07 & 19 & 3 & 10 & 1 & 18 & 2 & 0.61 & 0.07 & 20 & 2 & 2.0 & 0.3 \\
\hline BY130 & $\begin{array}{l}\text { Tom and Jerry Mine } \\
\text { drain at tunnel } \\
\text { outlet nr Nevada } \\
\text { City }\end{array}$ & $6 / 28 / 2000$ & $11: 30$ & 1 of 2 & $<0.08$ & 0.00 & 0.27 & 0.01 & 0.40 & 0.02 & $<3$ & 0 & 0.20 & 0.12 & 1.1 & 0.3 & 0.030 & 0.008 \\
\hline BY130 & $\begin{array}{l}\text { Tom and Jerry Mine } \\
\text { drain at tunnel } \\
\text { outlet nr Nevada } \\
\text { City }\end{array}$ & $6 / 28 / 2000$ & $11: 30$ & 2 of 2 & $<0.08$ & 0.04 & 0.31 & 0.01 & 0.50 & 0.01 & $<3$ & 1 & 0.15 & 0.06 & 1.0 & 0.0 & 0.036 & 0.008 \\
\hline BY131 & $\begin{array}{l}\text { Tom and Jerry Mine } \\
\text { drainage pond nr } \\
\text { Nevada City }\end{array}$ & $8 / 23 / 2000$ & $10: 45$ & 1of 2 & $<0.08$ & 0.03 & 0.28 & 0.01 & 0.06 & 0.02 & $<3$ & 2 & 0.11 & 0.10 & 1.8 & 0.0 & 0.042 & 0.009 \\
\hline BY131 & $\begin{array}{l}\text { Tom and Jerry Mine } \\
\text { drainage pond nr } \\
\text { Nevada City }\end{array}$ & $8 / 23 / 2000$ & $10: 45$ & 2of 2 & $<0.08$ & 0.03 & 0.28 & 0.04 & 0.04 & 0.02 & $<3$ & 1 & $<0.08$ & 0.02 & 1.8 & 0.2 & 0.051 & 0.012 \\
\hline
\end{tabular}


Table 4A. Concentrations of trace metals and selected major elements in unfiltered water samples, Greenhorn Creek drainage, Nevada County, California—Continued

[Station name abbreviations: ab, above; bl, below; Cr, Creek; mi, miles; N, north; nr, near; Rd, Road; S, south; SF, South Fork. Number in parentheses is the data parameter code, a five-digit code used in the

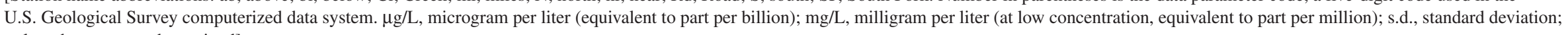

$<$, less than; - , not determined

\begin{tabular}{|c|c|c|c|c|c|c|c|c|c|c|c|c|c|c|c|c|c|c|}
\hline \multirow[t]{2}{*}{$\begin{array}{l}\text { Station } \\
\text { map ID }\end{array}$} & \multirow[t]{2}{*}{ Station name } & \multirow[t]{2}{*}{ Date } & \multirow[t]{2}{*}{ Time } & \multirow[t]{2}{*}{$\begin{array}{l}\text { Rep- } \\
\text { licate }\end{array}$} & \multicolumn{2}{|c|}{$\begin{array}{c}\text { Cadmium }(\mathbf{C d}), \\
\text { unfiltered } \\
(\mu \mathrm{g} / \mathrm{L}) \\
(01027)\end{array}$} & \multicolumn{2}{|c|}{$\begin{array}{c}\text { Cerium }(\mathrm{Ce}), \\
\text { unfiltered } \\
(\mu \mathrm{g} / \mathrm{L}) \\
(01112)\end{array}$} & \multicolumn{2}{|c|}{$\begin{array}{c}\text { Cobalt (Co), } \\
\text { unfiltered } \\
(\mu \mathrm{g} / \mathrm{L}) \\
(01037)\end{array}$} & \multicolumn{2}{|c|}{$\begin{array}{c}\text { Chromium }(\mathrm{Cr}), \\
\text { unfiltered } \\
(\mu \mathrm{g} / \mathrm{L}) \\
(01034)\end{array}$} & \multicolumn{2}{|c|}{$\begin{array}{c}\text { Cesium (Cs), } \\
\text { unfiltered } \\
(\mu \mathrm{g} / \mathrm{L}) \\
(0111)\end{array}$} & \multicolumn{2}{|c|}{$\begin{array}{c}\text { Copper (Cu), } \\
\text { unfiltered } \\
(\mu \mathrm{g} / \mathrm{L}) \\
(\mathbf{0 1 0 4 2 )}\end{array}$} & \multicolumn{2}{|c|}{$\begin{array}{c}\text { Dysprosium (Dy), } \\
\text { unfiltered } \\
(\mu \mathrm{g} / \mathrm{L}) \\
(\mathbf{8 2 3 3 0})\end{array}$} \\
\hline & & & & & value & s.d. & value & s.d. & value & s.d. & value & s.d. & value & s.d. & value & s.d. & value & s.d. \\
\hline \multicolumn{19}{|c|}{ Disturbed samples } \\
\hline BY123 & $\begin{array}{l}\text { Starr Mine tunnel } \\
\text { midway nr Grass } \\
\text { Valley }\end{array}$ & $12 / 15 / 1999$ & $11: 30$ & 1 of 2 & 21 & 0 & 131 & 2 & 1,200 & 0 & 80 & 11 & 16 & 0 & 1,100 & 200 & 23 & 3 \\
\hline BY123 & $\begin{array}{l}\text { Starr Mine tunnel } \\
\text { midway nr Grass } \\
\text { Valley }\end{array}$ & $12 / 15 / 1999$ & $11: 30$ & $20 f 2$ & 21 & 1 & 113 & 7 & 1,100 & 100 & 71 & 1 & 16 & 1 & 910 & 0 & 19 & 0 \\
\hline BY129 & $\begin{array}{l}\text { Tom and Jerry Mine } \\
\text { drain at tunnel inlet } \\
\text { nr Nevada City }\end{array}$ & $6 / 28 / 2000$ & $12: 30$ & 1 of 2 & 2.1 & 0.0 & 220 & 30 & 87 & 1 & 76 & 9 & 7.1 & 0.1 & 200 & 0 & 19 & 3 \\
\hline BY129 & $\begin{array}{l}\text { Tom and Jerry Mine } \\
\text { drain at tunnel inlet } \\
\text { nr Nevada City }\end{array}$ & $6 / 28 / 2000$ & $12: 30$ & 2of 2 & 1.9 & 0.3 & 240 & 10 & 94 & 14 & 77 & 9 & 6.6 & 1.1 & 210 & 30 & 21 & 1 \\
\hline
\end{tabular}


Table 4A. Concentrations of trace metals and selected major elements in unfiltered water samples, Greenhorn Creek drainage, Nevada County, California—Continued.

[Station name abbreviations: ab, above; bl, below; Cr, Creek; mi, miles; N, north; nr, near; Rd, Road; S, south; SF, South Fork. Number in parentheses is the data parameter code, a five-digit code used in the

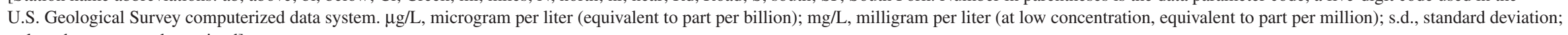
$<$, less than; - - not determined]

\begin{tabular}{|c|c|c|c|c|c|c|c|c|c|c|c|c|c|c|c|c|}
\hline \multirow[t]{2}{*}{$\begin{array}{c}\text { Station map } \\
\text { ID }\end{array}$} & \multirow[t]{2}{*}{ Station name } & \multirow[t]{2}{*}{ Date } & \multirow[t]{2}{*}{ Time } & \multirow[t]{2}{*}{$\begin{array}{l}\text { Rep- } \\
\text { licate }\end{array}$} & \multicolumn{2}{|c|}{$\begin{array}{c}\text { Erbium }(\mathrm{Er}), \\
\text { unfiltered } \\
(\mu \mathrm{g} / \mathrm{L}) \\
(01246)\end{array}$} & \multicolumn{2}{|c|}{$\begin{array}{c}\text { Europium (Eu), } \\
\text { unfiltered } \\
(\mu \mathrm{g} / \mathrm{L}) \\
(01236) \\
\end{array}$} & \multicolumn{2}{|c|}{$\begin{array}{c}\text { Iron (Fe), } \\
\text { unfiltered } \\
(\mu \mathrm{g} / \mathrm{L}) \\
(01045)\end{array}$} & \multicolumn{2}{|c|}{$\begin{array}{c}\text { Gadolinium }(\mathbf{G d}), \\
\text { unfiltered } \\
(\mu \mathrm{g} / \mathrm{L}) \\
(\mathbf{0 1 2 1 9}) \\
\end{array}$} & \multicolumn{2}{|c|}{$\begin{array}{c}\text { Holmium (Ho), } \\
\text { unfiltered } \\
(\mu \mathrm{g} / \mathrm{L}) \\
(01247) \\
\end{array}$} & \multicolumn{2}{|c|}{$\begin{array}{c}\text { Potassium }(\mathrm{K}), \\
\text { unfiltered } \\
(\mu \mathrm{g} / \mathrm{L}) \\
(00937) \\
\end{array}$} \\
\hline & & & & & value & s.d. & value & s.d. & value & s.d. & value & s.d. & value & s.d. & value & s.d. \\
\hline \multicolumn{17}{|c|}{ Environmental samples } \\
\hline BY20 & $\begin{array}{l}\text { Boston Mine tunnel } \\
\text { outlet nr Grass } \\
\text { Valley }\end{array}$ & 6/18/1999 & $14: 30$ & 1of 2 & 0.011 & 0.002 & 0.0056 & 0.0039 & 290 & 0 & 0.016 & 0.003 & 0.0038 & 0.0010 & 1.7 & 0.0 \\
\hline BY20 & $\begin{array}{l}\text { Boston Mine tunnel } \\
\text { outlet nr Grass } \\
\text { Valley }\end{array}$ & 6/18/1999 & $14: 30$ & 2of2 & 0.012 & 0.002 & 0.0050 & 0.0032 & 280 & 0 & 0.014 & 0.004 & 0.0029 & 0.0008 & 1.7 & 0.0 \\
\hline BY20 & $\begin{array}{l}\text { Boston Mine tunnel } \\
\text { outlet nr Grass } \\
\text { Valley }\end{array}$ & $12 / 14 / 1999$ & $12: 00$ & 1of 2 & 0.085 & 0.005 & 0.032 & 0.001 & 290 & 40 & 0.15 & 0.01 & 0.030 & 0.004 & 1.9 & 0.0 \\
\hline BY20 & $\begin{array}{l}\text { Boston Mine tunnel } \\
\text { outlet nr Grass } \\
\text { Valley }\end{array}$ & $12 / 14 / 1999$ & $12: 00$ & 2of2 & 0.046 & 0.004 & 0.026 & 0.005 & 270 & 10 & 0.11 & 0.01 & 0.016 & 0.001 & 1.9 & 0.1 \\
\hline BY20 & $\begin{array}{l}\text { Boston Mine tunnel } \\
\text { outlet nr Grass } \\
\text { Valley }\end{array}$ & $5 / 24 / 2000$ & $16: 00$ & 1of 2 & 0.014 & 0.003 & 0.005 & 0.001 & 373 & 24 & 0.036 & 0.001 & 0.0061 & 0.0005 & 1.6 & 0.0 \\
\hline BY20 & $\begin{array}{l}\text { Boston Mine tunnel } \\
\text { outlet nr Grass } \\
\text { Valley }\end{array}$ & $5 / 24 / 2000$ & $16: 00$ & 2of2 & 0.044 & 0.004 & 0.012 & 0.001 & 584 & 50 & 0.071 & 0.005 & 0.013 & 0.001 & 1.7 & 0.0 \\
\hline BY21 & $\begin{array}{l}\text { Boston Mine wetlands } \\
\text { pond nr Grass } \\
\text { Valley }\end{array}$ & $12 / 14 / 1999$ & $10: 30$ & 1of 2 & $<0.006$ & 0.001 & $<0.001$ & 0.001 & 120 & 10 & $<0.004$ & 0.003 & 0.0008 & 0.0008 & 2.7 & 0.2 \\
\hline BY21 & $\begin{array}{l}\text { Boston Mine wetlands } \\
\text { pond nr Grass } \\
\text { Valley }\end{array}$ & $12 / 14 / 1999$ & $10: 30$ & 2of2 & $<0.006$ & 0.003 & $<0.001$ & 0.001 & 140 & 0 & 0.004 & 0.003 & 0.0011 & 0.0001 & 2.5 & 0.0 \\
\hline BY21 & $\begin{array}{l}\text { Boston Mine wetlands } \\
\text { pond nr Grass } \\
\text { Valley }\end{array}$ & $5 / 24 / 2000$ & $16: 30$ & 1of 2 & 0.006 & 0.002 & $<0.001$ & 0.001 & 257 & 13 & 0.008 & 0.002 & 0.0016 & 0.0006 & 1.7 & 0.1 \\
\hline BY21 & $\begin{array}{l}\text { Boston Mine wetlands } \\
\text { pond nr Grass } \\
\text { Valley }\end{array}$ & $5 / 24 / 2000$ & $16: 30$ & 2of2 & $<0.005$ & 0.003 & 0.002 & 0.004 & 258 & 25 & 0.006 & 0.001 & 0.0016 & 0.0003 & 1.7 & 0.0 \\
\hline BY58 & $\begin{array}{l}\text { Buckeye Flat Mine } \\
\text { main drain } 0.45 \mathrm{mi} \\
\text { ab Greenhorn } \mathrm{Cr}\end{array}$ & $8 / 21 / 2000$ & 11:00 & 1 of 2 & 1.9 & 0.0 & 0.86 & 0.05 & 1,900 & 0 & 3.8 & 0.1 & 0.70 & 0.00 & 2.2 & 0.7 \\
\hline BY58 & $\begin{array}{l}\text { Buckeye Flat Mine } \\
\text { main drain } 0.45 \mathrm{mi} \\
\text { ab Greenhorn } \mathrm{Cr}\end{array}$ & $8 / 21 / 2000$ & 11:00 & 2of2 & 1.5 & 0.1 & 0.66 & 0.02 & 2,300 & 0 & 3.1 & 0.2 & 0.54 & 0.01 & 2.4 & 0.9 \\
\hline
\end{tabular}


Table 4A. Concentrations of trace metals and selected major elements in unfiltered water samples, Greenhorn Creek drainage, Nevada County, California-Continued.

[Station name abbreviations: ab, above; bl, below; Cr, Creek; mi, miles; N, north; nr, near; Rd, Road; S, south; SF, South Fork. Number in parentheses is the data parameter code, a five-digit code used in the

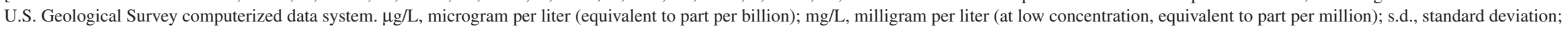
$<$, less than; - - not determined]

\begin{tabular}{|c|c|c|c|c|c|c|c|c|c|c|c|c|c|c|c|c|}
\hline \multirow[t]{2}{*}{$\begin{array}{c}\text { Station map } \\
\text { ID }\end{array}$} & \multirow[t]{2}{*}{ Station name } & \multirow[t]{2}{*}{ Date } & \multirow[t]{2}{*}{ Time } & \multirow[t]{2}{*}{$\begin{array}{l}\text { Rep- } \\
\text { licate }\end{array}$} & \multicolumn{2}{|c|}{$\begin{array}{c}\text { Erbium (Er), } \\
\text { unfiltered } \\
(\mu \mathrm{g} / \mathrm{L}) \\
(01246) \\
\end{array}$} & \multicolumn{2}{|c|}{$\begin{array}{c}\text { Europium (Eu), } \\
\text { unfiltered } \\
(\mu \mathrm{g} / \mathrm{L}) \\
(\mathbf{0 1 2 3 6}) \\
\end{array}$} & \multicolumn{2}{|c|}{$\begin{array}{c}\text { Iron (Fe), } \\
\text { unfiltered } \\
(\mu \mathrm{g} / \mathrm{L}) \\
(01045)\end{array}$} & \multicolumn{2}{|c|}{$\begin{array}{c}\text { Gadolinium }(\mathbf{G d}) \text {, } \\
\text { unfiltered } \\
(\mu \mathrm{g} / \mathrm{L}) \\
(01219) \\
\end{array}$} & \multicolumn{2}{|c|}{$\begin{array}{c}\text { Holmium (Ho), } \\
\text { unfiltered } \\
(\mu \mathrm{g} / \mathrm{L}) \\
(01247) \\
\end{array}$} & \multicolumn{2}{|c|}{$\begin{array}{l}\text { Potassium (K), } \\
\text { unfiltered } \\
(\mu \mathrm{g} / \mathrm{L}) \\
(00937) \\
\end{array}$} \\
\hline & & & & & value & s.d. & value & s.d. & value & s.d. & value & s.d. & value & s.d. & value & s.d. \\
\hline BY23 & $\begin{array}{c}\text { Buckeye Flat Mine N } \\
\text { drain to Greenhorn } \\
\text { Cr, Grass Valley }\end{array}$ & $6 / 18 / 1999$ & $11: 30$ & 1 of 2 & 2.7 & 0.2 & 1.2 & 0.1 & 4,500 & 100 & 4.2 & 0.8 & 0.95 & 0.09 & 1.3 & 0.0 \\
\hline BY23 & $\begin{array}{c}\text { Buckeye Flat Mine N } \\
\text { drain to Greenhorn } \\
\text { Cr, Grass Valley }\end{array}$ & 6/18/1999 & $11: 30$ & $2 \mathrm{of} 2$ & 2.8 & 0.1 & 1.3 & 0.3 & 4,400 & 0 & 5.7 & 0.3 & 0.93 & 0.02 & 1.3 & 0.0 \\
\hline BY23 & $\begin{array}{c}\text { Buckeye Flat Mine N } \\
\text { drain to Greenhorn } \\
\text { Cr, Grass Valley }\end{array}$ & $12 / 2 / 1999$ & 11:00 & 1 of 2 & 1.9 & 0.1 & 0.90 & 0.01 & 220 & 30 & 3.7 & 0.6 & 0.68 & 0.00 & 1.2 & 0.1 \\
\hline BY23 & $\begin{array}{c}\text { Buckeye Flat Mine N } \\
\text { drain to Greenhorn } \\
\text { Cr, Grass Valley }\end{array}$ & $12 / 2 / 1999$ & 11:00 & $2 \mathrm{of} 2$ & 2.0 & 0.0 & 1.1 & 0.2 & 250 & 70 & 5.8 & 0.3 & 0.74 & 0.04 & 1.3 & 0.0 \\
\hline BY23 & $\begin{array}{c}\text { Buckeye Flat Mine N } \\
\text { drain to Greenhorn } \\
\text { Cr, Grass Valley }\end{array}$ & $8 / 22 / 2000$ & $10: 45$ & $1 \mathrm{of} 2$ & 2.7 & 0.0 & 1.3 & 0.1 & 270 & 10 & 5.6 & 0.2 & 1.0 & 0.1 & 1.2 & 1.0 \\
\hline BY23 & $\begin{array}{c}\text { Buckeye Flat Mine N } \\
\text { drain to Greenhorn } \\
\text { Cr, Grass Valley }\end{array}$ & $8 / 22 / 2000$ & $10: 45$ & $2 \mathrm{of} 2$ & 2.4 & 0.1 & 1.3 & 0.1 & 240 & 30 & 5.1 & 0.0 & 1.0 & 0.0 & 1.5 & 1.4 \\
\hline BY116 & $\begin{array}{l}\text { Buckeye Flat Mine } \\
\text { pond drain } 0.15 \mathrm{mi} \\
\text { ab SF Greenhorn Cr }\end{array}$ & $8 / 21 / 2000$ & $14: 10$ & 1 of 2 & 0.017 & 0.005 & 0.015 & 0.005 & 330 & 10 & 0.044 & 0.007 & 0.007 & 0.002 & 0.6 & 0.1 \\
\hline BY116 & $\begin{array}{l}\text { Buckeye Flat Mine } \\
\text { pond drain } 0.15 \mathrm{mi} \\
\text { ab SF Greenhorn } \mathrm{Cr}\end{array}$ & $8 / 21 / 2000$ & $14: 10$ & $2 \mathrm{of} 2$ & 0.020 & 0.007 & 0.009 & 0.000 & 330 & 20 & 0.043 & 0.007 & 0.007 & 0.000 & 0.5 & 0.1 \\
\hline BY24 & $\begin{array}{l}\text { Buckeye Flat Mine } \\
\text { S drain to SF } \\
\text { Greenhorn } \mathrm{Cr}, \\
\text { Grass Valley }\end{array}$ & $8 / 26 / 1999$ & 11:00 & 1 of 2 & 0.065 & 0.000 & 0.024 & 0.004 & 150 & 10 & 0.067 & 0.005 & 0.019 & 0.000 & 0.40 & 0.02 \\
\hline BY24 & $\begin{array}{l}\text { Buckeye Flat Mine } \\
\text { S drain to SF } \\
\text { Greenhorn Cr, } \\
\text { Grass Valley }\end{array}$ & $8 / 26 / 1999$ & 11:00 & $2 \mathrm{of} 2$ & 0.076 & 0.003 & 0.029 & 0.007 & 140 & 0 & 0.11 & 0.03 & 0.024 & 0.004 & 0.39 & 0.00 \\
\hline BY24 & $\begin{array}{l}\text { Buckeye Flat Mine } \\
\text { S drain to SF } \\
\text { Greenhorn Cr, } \\
\text { Grass Valley }\end{array}$ & $8 / 22 / 2000$ & $12: 10$ & 1 of 2 & 0.12 & 0.01 & 0.076 & 0.003 & 790 & 10 & 0.29 & 0.00 & 0.051 & 0.007 & 0.2 & 0.0 \\
\hline BY24 & $\begin{array}{l}\text { Buckeye Flat Mine } \\
\text { S drain to SF } \\
\text { Greenhorn Cr, } \\
\text { Grass Valley }\end{array}$ & $8 / 22 / 2000$ & $12: 10$ & $2 o f 2$ & 0.14 & 0.03 & 0.066 & 0.006 & 970 & 170 & 0.28 & 0.04 & 0.050 & 0.001 & 0.2 & 0.1 \\
\hline
\end{tabular}


Table 4A. Concentrations of trace metals and selected major elements in unfiltered water samples, Greenhorn Creek drainage, Nevada County, California—Continued.

[Station name abbreviations: ab, above; bl, below; Cr, Creek; mi, miles; N, north; nr, near; Rd, Road; S, south; SF, South Fork. Number in parentheses is the data parameter code, a five-digit code used in the

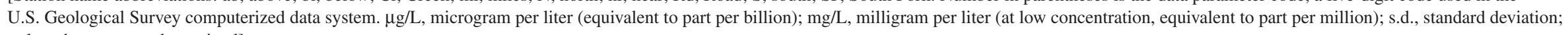
$<$ less than; - - not determined]

\begin{tabular}{|c|c|c|c|c|c|c|c|c|c|c|c|c|c|c|c|c|}
\hline \multirow[t]{2}{*}{$\begin{array}{l}\text { Station map } \\
\text { ID }\end{array}$} & \multirow[t]{2}{*}{ Station name } & \multirow[t]{2}{*}{ Date } & \multirow[t]{2}{*}{ Time } & \multirow[t]{2}{*}{$\begin{array}{l}\text { Rep- } \\
\text { licate }\end{array}$} & \multicolumn{2}{|c|}{$\begin{array}{c}\text { Erbium }(\mathrm{Er}), \\
\text { unfiltered } \\
(\mu \mathrm{g} / \mathrm{L}) \\
(01246)\end{array}$} & \multicolumn{2}{|c|}{$\begin{array}{c}\text { Europium (Eu), } \\
\text { unfiltered } \\
(\mu \mathrm{g} / \mathrm{L}) \\
(01236) \\
\end{array}$} & \multicolumn{2}{|c|}{$\begin{array}{c}\text { Iron }(\mathrm{Fe}), \\
\text { unfiltered } \\
(\mu \mathrm{g} / \mathrm{L}) \\
(01045)\end{array}$} & \multicolumn{2}{|c|}{$\begin{array}{c}\text { Gadolinium (Gd), } \\
\text { unfiltered } \\
(\mu \mathrm{g} / \mathrm{L}) \\
(01219) \\
\end{array}$} & \multicolumn{2}{|c|}{$\begin{array}{c}\text { Holmium (Ho), } \\
\text { unfiltered } \\
(\mu \mathrm{g} / \mathrm{L}) \\
(01247) \\
\end{array}$} & \multicolumn{2}{|c|}{$\begin{array}{c}\text { Potassium }(\mathrm{K}), \\
\text { unfiltered } \\
(\mu \mathrm{g} / \mathrm{L}) \\
(00937) \\
\end{array}$} \\
\hline & & & & & value & s.d. & value & s.d. & value & s.d. & value & s.d. & value & s.d. & value & s.d. \\
\hline BY25 & $\begin{array}{l}\text { Buckeye Flat Mine } \\
\text { upper drain }\end{array}$ & $4 / 9 / 2001$ & $10: 00$ & 1of 2 & 0.43 & 0.04 & 0.19 & 0.01 & 100 & 20 & 0.82 & 0.02 & 0.16 & 0.00 & 0.41 & 0.07 \\
\hline BY25 & $\begin{array}{l}\text { Buckeye Flat Mine } \\
\text { upper drain }\end{array}$ & $4 / 9 / 2001$ & 10:00 & 2of2 & 0.42 & 0.01 & 0.20 & 0.01 & 110 & 30 & 0.86 & 0.00 & 0.17 & 0.00 & 0.39 & 0.06 \\
\hline BY52 & $\begin{array}{l}\text { Greenhorn Cr } 0.3 \mathrm{mi} \\
\text { ab Buckeye Ford nr } \\
\text { Nevada City }\end{array}$ & $1 / 25 / 2000$ & $10: 45$ & 1of 2 & 0.11 & 0.007 & 0.056 & 0.000 & 460 & 20 & 0.22 & 0.02 & 0.040 & 0.003 & 0.61 & 0.01 \\
\hline BY52 & $\begin{array}{l}\text { Greenhorn Cr } 0.3 \mathrm{mi} \\
\text { ab Buckeye Ford nr } \\
\text { Nevada City }\end{array}$ & $1 / 25 / 2000$ & $10: 45$ & 2of2 & 0.11 & 0.021 & 0.058 & 0.006 & 430 & 20 & 0.23 & 0.02 & 0.043 & 0.003 & 0.62 & 0.03 \\
\hline BY59 & $\begin{array}{l}\text { Greenhorn Cr at You } \\
\text { Bet Rd nr Nevada } \\
\text { City }\end{array}$ & $7 / 24 / 2001$ & 11:00 & 1of 2 & $<0.008$ & 0.002 & 0.002 & 0.002 & 86 & 14 & 0.013 & 0.008 & 0.003 & 0.002 & 0.73 & 0.04 \\
\hline BY59 & $\begin{array}{l}\text { Greenhorn Cr at You } \\
\text { Bet Rd nr Nevada } \\
\text { City }\end{array}$ & $7 / 24 / 2001$ & $11: 00$ & 2of2 & $<0.008$ & 0.001 & 0.003 & 0.001 & 104 & 5 & 0.018 & 0.003 & 0.003 & 0.001 & 0.69 & 0.05 \\
\hline BY59 & $\begin{array}{l}\text { Greenhorn Cr at You } \\
\text { Bet Rd nr Nevada } \\
\text { City }\end{array}$ & $8 / 29 / 2001$ & $15: 00$ & 1of 2 & $<0.008$ & 0.003 & $<0.001$ & 0.001 & 75 & 15 & 0.011 & 0.002 & $<0.002$ & 0.001 & 0.71 & 0.02 \\
\hline BY59 & $\begin{array}{l}\text { Greenhorn Cr at You } \\
\text { Bet Rd nr Nevada } \\
\text { City }\end{array}$ & $8 / 29 / 2001$ & $15: 00$ & 2of2 & $<0.008$ & 0.002 & 0.003 & 0.002 & 84 & 6 & $<0.007$ & 0.003 & $<0.002$ & 0.001 & 0.73 & 0.08 \\
\hline BY59 & $\begin{array}{l}\text { Greenhorn Cr at You } \\
\text { Bet Rd nr Nevada } \\
\text { City }\end{array}$ & $9 / 14 / 2001$ & $13: 00$ & 1of 2 & 0.006 & 0.003 & $<0.002$ & 0.001 & 76 & 0 & 0.009 & 0.003 & 0.002 & 0.001 & 0.8 & 0.1 \\
\hline BY59 & $\begin{array}{l}\text { Greenhorn } \mathrm{Cr} \text { at You } \\
\text { Bet Rd nr Nevada } \\
\text { City }\end{array}$ & $9 / 14 / 2001$ & $13: 00$ & 2of2 & 0.005 & 0.001 & $<0.002$ & 0.000 & 77 & 6 & 0.010 & 0.004 & 0.001 & 0.001 & 0.9 & 0.1 \\
\hline BY59 & $\begin{array}{l}\text { Greenhorn Cr at You } \\
\text { Bet Rd nr Nevada } \\
\text { City }\end{array}$ & $10 / 15 / 2001$ & $12: 00$ & 1of 2 & 0.003 & 0.002 & 0.003 & 0.002 & 44 & 6 & 0.006 & 0.004 & 0.0014 & 0.0006 & 0.7 & 0.1 \\
\hline BY59 & $\begin{array}{l}\text { Greenhorn Cr at You } \\
\text { Bet Rd nr Nevada } \\
\text { City }\end{array}$ & $10 / 15 / 2001$ & $12: 00$ & 2of2 & $<0.002$ & 0.002 & 0.003 & 0.003 & 48 & 3 & 0.005 & 0.002 & $<0.0008$ & 0.0001 & 0.8 & 0.1 \\
\hline BY59 & $\begin{array}{l}\text { Greenhorn Cr at You } \\
\text { Bet Rd nr Nevada } \\
\text { City }\end{array}$ & $11 / 20 / 2001$ & $13: 20$ & 1of 2 & 0.016 & 0.000 & 0.007 & 0.002 & 110 & 18 & 0.032 & 0.010 & 0.006 & 0.001 & 1.0 & 0.1 \\
\hline BY59 & $\begin{array}{l}\text { Greenhorn } \mathrm{Cr} \text { at You } \\
\text { Bet Rd nr Nevada } \\
\text { City }\end{array}$ & $11 / 20 / 2001$ & $13: 20$ & 2of2 & 0.022 & 0.005 & 0.008 & 0.000 & 109 & 13 & 0.035 & 0.003 & 0.006 & 0.000 & 1.0 & 0.0 \\
\hline
\end{tabular}


Table 4A. Concentrations of trace metals and selected major elements in unfiltered water samples, Greenhorn Creek drainage, Nevada County, California-Continued.

[Station name abbreviations: ab, above; bl, below; Cr, Creek; mi, miles; N, north; nr, near; Rd, Road; S, south; SF, South Fork. Number in parentheses is the data parameter code, a five-digit code used in the

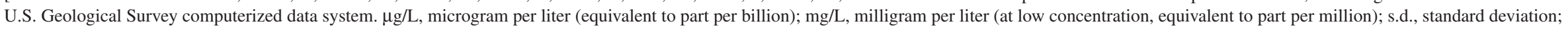
$<$ less than; - - not determined]

\begin{tabular}{|c|c|c|c|c|c|c|c|c|c|c|c|c|c|c|c|c|}
\hline \multirow[t]{2}{*}{$\begin{array}{c}\text { Station map } \\
\text { ID }\end{array}$} & \multirow[t]{2}{*}{ Station name } & \multirow[t]{2}{*}{ Date } & \multirow[t]{2}{*}{ Time } & \multirow[t]{2}{*}{$\begin{array}{l}\text { Rep- } \\
\text { licate }\end{array}$} & \multicolumn{2}{|c|}{$\begin{array}{c}\text { Erbium (Er), } \\
\text { unfiltered } \\
(\mu \mathrm{g} / \mathrm{L}) \\
(\mathbf{0 1 2 4 6 )}\end{array}$} & \multicolumn{2}{|c|}{$\begin{array}{c}\text { Europium (Eu), } \\
\text { unfiltered } \\
(\mu \mathrm{g} / \mathrm{L}) \\
(\mathbf{0 1 2 3 6}) \\
\end{array}$} & \multicolumn{2}{|c|}{$\begin{array}{c}\text { Iron (Fe), } \\
\text { unfiltered } \\
(\mu \mathrm{g} / \mathrm{L}) \\
(01045)\end{array}$} & \multicolumn{2}{|c|}{$\begin{array}{c}\text { Gadolinium (Gd), } \\
\text { unfiltered } \\
(\mu \mathrm{g} / \mathrm{L}) \\
(\mathbf{0 1 2 1 9}) \\
\end{array}$} & \multicolumn{2}{|c|}{$\begin{array}{c}\text { Holmium }(\mathrm{Ho}), \\
\text { unfiltered } \\
(\mu \mathrm{g} / \mathrm{L}) \\
(01247) \\
\end{array}$} & \multicolumn{2}{|c|}{$\begin{array}{c}\text { Potassium }(\mathrm{K}), \\
\text { unfiltered } \\
(\mu \mathrm{g} / \mathrm{L}) \\
(00937) \\
\end{array}$} \\
\hline & & & & & value & s.d. & value & s.d. & value & s.d. & value & s.d. & value & s.d. & value & s.d. \\
\hline BY59 & $\begin{array}{l}\text { Greenhorn Cr at You } \\
\text { Bet Rd nr Nevada } \\
\text { City }\end{array}$ & $12 / 10 / 2001$ & $12: 20$ & 1 of 2 & 0.13 & 0.01 & 0.061 & 0.000 & 963 & 109 & 0.22 & 0.01 & 0.037 & 0.001 & 0.8 & 0.1 \\
\hline BY59 & $\begin{array}{l}\text { Greenhorn } \mathrm{Cr} \text { at You } \\
\text { Bet Rd nr Nevada } \\
\text { City }\end{array}$ & $12 / 10 / 2001$ & $12: 20$ & 2of2 & 0.11 & 0.00 & 0.057 & 0.002 & 922 & 111 & 0.25 & 0.00 & 0.047 & 0.001 & 0.9 & 0.1 \\
\hline BY59 & $\begin{array}{l}\text { Greenhorn } \mathrm{Cr} \text { at You } \\
\text { Bet Rd nr Nevada } \\
\text { City }\end{array}$ & $12 / 21 / 2001$ & $13: 00$ & 1of 2 & 0.44 & 0.01 & 0.24 & 0.03 & 4,630 & 537 & 1.03 & 0.00 & 0.171 & 0.023 & 1.25 & 0.13 \\
\hline BY59 & $\begin{array}{l}\text { Greenhorn } \mathrm{Cr} \text { at You } \\
\text { Bet Rd nr Nevada } \\
\text { City }\end{array}$ & $12 / 21 / 2001$ & $13: 00$ & 2of 2 & 0.50 & 0.01 & 0.24 & 0.02 & 4,630 & 465 & 0.85 & 0.02 & 0.173 & 0.012 & 1.25 & 0.14 \\
\hline BY51 & $\begin{array}{l}\text { Greenhorn } \mathrm{Cr} n r \\
\text { headwaters nr } \\
\text { Scotts Flat Res }\end{array}$ & $1 / 25 / 2000$ & $9: 30$ & 1 of 2 & 0.330 & 0.002 & 0.017 & 0.004 & 56 & 4 & 0.064 & 0.006 & 0.013 & 0.002 & 0.48 & 0.02 \\
\hline BY51 & $\begin{array}{c}\text { Greenhorn } \mathrm{Cr} n r \\
\text { headwaters nr } \\
\text { Scotts Flat Res }\end{array}$ & $1 / 25 / 2000$ & $9: 30$ & 2of 2 & 0.033 & 0.003 & 0.018 & 0.002 & 52 & 8 & 0.067 & 0.009 & 0.013 & 0.000 & 0.43 & 0.00 \\
\hline BY75 & $\begin{array}{l}\text { Missouri Cyn Cr } \\
\text { 1.2mi ab Greenhorn } \\
\text { Cr nr Chicago Park }\end{array}$ & $12 / 14 / 1999$ & $14: 00$ & 1 of 2 & 0.057 & 0.003 & 0.027 & 0.007 & 160 & 20 & 0.12 & 0.04 & 0.020 & 0.005 & 1.5 & 0.0 \\
\hline BY75 & $\begin{array}{l}\text { Missouri Cyn Cr } \\
\text { 1.2mi ab Greenhorn } \\
\text { Cr nr Chicago Park }\end{array}$ & $12 / 14 / 1999$ & $14: 00$ & 2of 2 & 0.057 & 0.006 & 0.018 & 0.001 & 200 & 50 & 0.098 & 0.035 & 0.021 & 0.001 & 1.5 & 0.0 \\
\hline BY86 & $\begin{array}{l}\text { Poore Mine Creek } \\
\text { ab tunnel nr Grass } \\
\text { Valley }\end{array}$ & 3/31/1999 & $17: 40$ & 1 of 2 & 0.035 & 0.003 & 0.020 & 0.001 & 460 & 20 & 0.087 & 0.005 & 0.012 & 0.000 & 0.53 & 0.00 \\
\hline BY86 & $\begin{array}{l}\text { Poore Mine Creek } \\
\text { ab tunnel nr Grass } \\
\text { Valley }\end{array}$ & $3 / 31 / 1999$ & $17: 40$ & 2of 2 & 0.033 & 0.002 & 0.018 & 0.004 & 450 & 30 & 0.059 & 0.011 & 0.013 & 0.001 & 0.53 & 0.02 \\
\hline BY87 & $\begin{array}{l}\text { Poore Mine Creek } \\
\text { bl tunnel nr Grass } \\
\text { Valley }\end{array}$ & $3 / 31 / 1999$ & $13: 30$ & 1of 2 & 0.034 & 0.002 & 0.022 & 0.003 & 380 & 30 & 0.072 & 0.010 & 0.010 & 0.000 & 0.55 & 0.04 \\
\hline BY87 & $\begin{array}{l}\text { Poore Mine Creek } \\
\text { bl tunnel nr Grass } \\
\text { Valley }\end{array}$ & 3/31/1999 & $13: 30$ & 2of 2 & 0.038 & 0.010 & 0.020 & 0.003 & 410 & 40 & 0.077 & 0.005 & 0.011 & 0.001 & 0.56 & 0.04 \\
\hline
\end{tabular}


Table 4A. Concentrations of trace metals and selected major elements in unfiltered water samples, Greenhorn Creek drainage, Nevada County, California—Continued.

[Station name abbreviations: ab, above; bl, below; Cr, Creek; mi, miles; N, north; nr, near; Rd, Road; S, south; SF, South Fork. Number in parentheses is the data parameter code, a five-digit code used in the

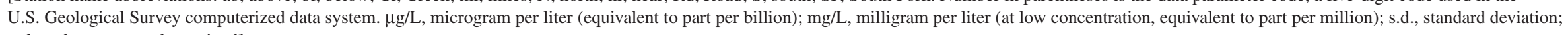
$<$, less than; - - not determined]

\begin{tabular}{|c|c|c|c|c|c|c|c|c|c|c|c|c|c|c|c|c|}
\hline \multirow[t]{2}{*}{$\begin{array}{l}\text { Station map } \\
\text { ID }\end{array}$} & \multirow[t]{2}{*}{ Station name } & \multirow[t]{2}{*}{ Date } & \multirow[t]{2}{*}{ Time } & \multirow[t]{2}{*}{$\begin{array}{l}\text { Rep- } \\
\text { licate }\end{array}$} & \multicolumn{2}{|c|}{$\begin{array}{c}\text { Erbium (Er), } \\
\text { unfiltered } \\
(\mu \mathrm{g} / \mathrm{L}) \\
(\mathbf{0 1 2 4 6 )}\end{array}$} & \multicolumn{2}{|c|}{$\begin{array}{c}\text { Europium (Eu), } \\
\text { unfiltered } \\
(\mu \mathrm{g} / \mathrm{L}) \\
(01236) \\
\end{array}$} & \multicolumn{2}{|c|}{$\begin{array}{c}\text { Iron (Fe), } \\
\text { unfiltered } \\
(\mu \mathrm{g} / \mathrm{L}) \\
(01045)\end{array}$} & \multicolumn{2}{|c|}{$\begin{array}{c}\text { Gadolinium (Gd), } \\
\text { unfiltered } \\
(\mu \mathrm{g} / \mathrm{L}) \\
(\mathbf{0 1 2 1 9}) \\
\end{array}$} & \multicolumn{2}{|c|}{$\begin{array}{c}\text { Holmium (Ho), } \\
\text { unfiltered } \\
(\mu \mathrm{g} / \mathrm{L}) \\
(01247) \\
\end{array}$} & \multicolumn{2}{|c|}{$\begin{array}{c}\text { Potassium }(\mathrm{K}), \\
\text { unfiltered } \\
(\mu \mathrm{g} / \mathrm{L}) \\
(00937) \\
\end{array}$} \\
\hline & & & & & value & s.d. & value & s.d. & value & s.d. & value & s.d. & value & s.d. & value & s.d. \\
\hline BY88 & $\begin{array}{l}\text { Poore Mine ground } \\
\text { sluice nr Grass } \\
\text { Valley }\end{array}$ & 4/1/1999 & $15: 40$ & 1 of 2 & 0.018 & 0.000 & 0.011 & 0.003 & 770 & 10 & 0.031 & 0.007 & 0.0068 & 0.0019 & 1.6 & 0.1 \\
\hline BY88 & $\begin{array}{l}\text { Poore Mine ground } \\
\text { sluice nr Grass } \\
\text { Valley }\end{array}$ & 4/1/1999 & $15: 40$ & 2of2 & 0.021 & 0.003 & 0.008 & 0.002 & 780 & 50 & 0.043 & 0.005 & 0.0069 & 0.0003 & 1.5 & 0.1 \\
\hline BY89 & $\begin{array}{l}\text { Poore Mine pit lake nr } \\
\text { Grass Valley }\end{array}$ & 4/1/1999 & $16: 50$ & 1 of 2 & 0.012 & 0.001 & 0.006 & 0.002 & 340 & 50 & 0.022 & 0.001 & 0.0044 & 0.0007 & 1.3 & 0.1 \\
\hline BY89 & $\begin{array}{l}\text { Poore Mine pit lake nr } \\
\text { Grass Valley }\end{array}$ & 4/1/1999 & $16: 50$ & 2of2 & 0.011 & 0.002 & 0.007 & 0.003 & 350 & 20 & 0.024 & 0.007 & 0.0038 & 0.0005 & 1.3 & 0.0 \\
\hline BY89 & $\begin{array}{l}\text { Poore Mine pit lake } \mathrm{nr} \\
\text { Grass Valley }\end{array}$ & $5 / 23 / 2000$ & $10: 45$ & 1of 2 & 0.019 & 0.002 & 0.006 & 0.003 & 64 & 6 & 0.045 & 0.006 & 0.0095 & 0.0014 & 2.4 & 0.1 \\
\hline BY89 & $\begin{array}{l}\text { Poore Mine pit lake nr } \\
\text { Grass Valley }\end{array}$ & $5 / 23 / 2000$ & $10: 45$ & 2of2 & 0.025 & 0.003 & 0.008 & 0.005 & 62 & 1 & 0.052 & 0.002 & 0.0089 & 0.0010 & 2.4 & 0.0 \\
\hline BY90 & $\begin{array}{l}\text { Poore Mine seep } \\
\text { above ground sluice } \\
\text { nr Grass Valley }\end{array}$ & 4/1/1999 & $16: 00$ & 1of 2 & - & & - & & - & & - & & - & & - & \\
\hline BY90 & $\begin{array}{l}\text { Poore Mine seep } \\
\text { above ground sluice } \\
\text { nr Grass Valley }\end{array}$ & 4/1/1999 & $16: 00$ & 2of2 & - & & - & & - & & - & & - & & - & \\
\hline BY91 & $\begin{array}{l}\text { Poore Mine tunnel } \\
\text { effluent nr Grass } \\
\text { Valley }\end{array}$ & 3/31/1999 & $15: 20$ & 1of 2 & 0.007 & 0.002 & 0.006 & 0.000 & 94 & 6 & 0.018 & 0.004 & 0.0032 & 0.0004 & 1.4 & 0.1 \\
\hline BY91 & $\begin{array}{l}\text { Poore Mine tunnel } \\
\text { effluent nr Grass } \\
\text { Valley }\end{array}$ & 3/31/1999 & $15: 20$ & 2of2 & 0.011 & 0.002 & 0.007 & 0.004 & 97 & 9 & 0.021 & 0.007 & 0.0043 & 0.0007 & 1.5 & 0.0 \\
\hline BY91 & $\begin{array}{l}\text { Poore Mine tunnel } \\
\text { effluent nr Grass } \\
\text { Valley }\end{array}$ & $12 / 15 / 1999$ & 13:00 & 1of 2 & 0.012 & 0.004 & 0.006 & 0.002 & 200 & 60 & 0.028 & 0.004 & 0.0044 & 0.0009 & 1.5 & 0.0 \\
\hline BY91 & $\begin{array}{l}\text { Poore Mine tunnel } \\
\text { effluent nr Grass } \\
\text { Valley }\end{array}$ & $12 / 15 / 1999$ & 13:00 & 2of2 & 0.014 & 0.002 & 0.008 & 0.002 & 350 & 10 & 0.034 & 0.014 & 0.0044 & 0.0009 & 1.7 & 0.1 \\
\hline BY91 & $\begin{array}{l}\text { Poore Mine tunnel } \\
\text { effluent nr Grass } \\
\text { Valley }\end{array}$ & $5 / 23 / 2000$ & $12: 00$ & 1of 2 & 0.16 & 0.01 & 0.078 & 0.001 & 1,403 & 59 & 0.36 & 0.00 & 0.057 & 0.001 & 1.8 & 0.0 \\
\hline BY91 & $\begin{array}{l}\text { Poore Mine tunnel } \\
\text { effluent nr Grass } \\
\text { Valley }\end{array}$ & $5 / 23 / 2000$ & $12: 00$ & 2of2 & 0.14 & 0.00 & 0.071 & 0.001 & 1,420 & 62 & 0.32 & 0.01 & 0.055 & 0.002 & 1.8 & 0.0 \\
\hline
\end{tabular}


Table 4A. Concentrations of trace metals and selected major elements in unfiltered water samples, Greenhorn Creek drainage, Nevada County, California-Continued.

[Station name abbreviations: ab, above; bl, below; Cr, Creek; mi, miles; N, north; nr, near; Rd, Road; S, south; SF, South Fork. Number in parentheses is the data parameter code, a five-digit code used in the

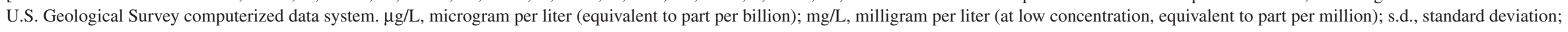
$<$ less than; - - not determined]

\begin{tabular}{|c|c|c|c|c|c|c|c|c|c|c|c|c|c|c|c|c|}
\hline \multirow[t]{2}{*}{$\begin{array}{c}\text { Station map } \\
\text { ID }\end{array}$} & \multirow[t]{2}{*}{ Station name } & \multirow[t]{2}{*}{ Date } & \multirow[t]{2}{*}{ Time } & \multirow[t]{2}{*}{$\begin{array}{l}\text { Rep- } \\
\text { licate }\end{array}$} & \multicolumn{2}{|c|}{$\begin{array}{c}\text { Erbium (Er), } \\
\text { unfiltered } \\
(\mu \mathrm{g} / \mathrm{L}) \\
(\mathbf{0 1 2 4 6 )}\end{array}$} & \multicolumn{2}{|c|}{$\begin{array}{c}\text { Europium (Eu), } \\
\text { unfiltered } \\
(\mu \mathrm{g} / \mathrm{L}) \\
(01236) \\
\end{array}$} & \multicolumn{2}{|c|}{$\begin{array}{c}\text { Iron (Fe), } \\
\text { unfiltered } \\
(\mu \mathrm{g} / \mathrm{L}) \\
(01045)\end{array}$} & \multicolumn{2}{|c|}{$\begin{array}{c}\text { Gadolinium (Gd), } \\
\text { unfiltered } \\
(\mu \mathbf{g} / \mathrm{L}) \\
(\mathbf{0 1 2 1 9}) \\
\end{array}$} & \multicolumn{2}{|c|}{$\begin{array}{c}\text { Holmium (Ho), } \\
\text { unfiltered } \\
(\mu \mathrm{g} / \mathrm{L}) \\
(01247) \\
\end{array}$} & \multicolumn{2}{|c|}{$\begin{array}{c}\text { Potassium }(\mathrm{K}), \\
\text { unfiltered } \\
(\mu \mathrm{g} / \mathrm{L}) \\
(00937) \\
\end{array}$} \\
\hline & & & & & value & s.d. & value & s.d. & value & s.d. & value & s.d. & value & s.d. & value & s.d. \\
\hline BY105 & $\begin{array}{l}\text { Sailor Flat Mine main } \\
\text { drain gulch } 01 \mathrm{nr} \\
\text { Quaker Hill }\end{array}$ & 7/6/1999 & $16: 00$ & 1 of 2 & 0.87 & 0.01 & 0.46 & 0.05 & 3,100 & 0 & 1.5 & 0.1 & 0.30 & 0.01 & 1.4 & 0.1 \\
\hline BY105 & $\begin{array}{l}\text { Sailor Flat Mine main } \\
\text { drain gulch } 01 \mathrm{nr} \\
\text { Quaker Hill }\end{array}$ & 7/6/1999 & 16:00 & 2of 2 & 0.84 & 0.03 & 0.37 & 0.00 & 3,100 & 0 & 0.92 & 0.02 & 0.27 & 0.04 & 1.4 & 0.1 \\
\hline BY105 & $\begin{array}{l}\text { Sailor Flat Mine main } \\
\text { drain Gulch 01nr } \\
\text { Quaker Hill }\end{array}$ & $12 / 2 / 1999$ & $14: 30$ & 1 of 2 & 1.7 & 0.0 & 0.97 & 0.09 & 5,500 & 200 & 3.5 & 0.1 & 0.67 & 0.00 & 2.2 & 0.1 \\
\hline BY105 & $\begin{array}{l}\text { Sailor Flat Mine main } \\
\text { drain Gulch 01nr } \\
\text { Quaker Hill }\end{array}$ & $12 / 2 / 1999$ & $14: 30$ & $2 o f 2$ & 1.6 & 0.0 & 1.1 & 0.1 & 5,300 & 0 & 4.3 & 0.1 & 0.53 & 0.02 & 2.0 & 0.1 \\
\hline BY106 & $\begin{array}{l}\text { Sailor Flat Mine } \\
\text { main drainage to } \\
\text { Greenhorn } \mathrm{Cr} \text {, } \\
\text { Gulch } 03\end{array}$ & $4 / 9 / 2001$ & $12: 30$ & 1 of 2 & 0.15 & 0.01 & 0.081 & 0.002 & 180 & 10 & 0.29 & 0.00 & 0.054 & 0.008 & 0.42 & 0.03 \\
\hline BY106 & $\begin{array}{l}\text { Sailor Flat Mine } \\
\text { main drainage to } \\
\text { Greenhorn Cr, } \\
\text { Gulch } 03\end{array}$ & $4 / 9 / 2001$ & $12: 30$ & 2 of 2 & 0.14 & 0.01 & 0.069 & 0.003 & 190 & 30 & 0.28 & 0.02 & 0.052 & 0.001 & 0.44 & 0.08 \\
\hline BY114 & $\begin{array}{l}\text { SF Greenhorn Cr } \\
\text { 0.7mi ab Greenhorn } \\
\text { Cr nr Nevada City }\end{array}$ & $8 / 22 / 2000$ & $15: 10$ & 1 of 2 & $<0.008$ & 0.005 & 0.003 & 0.002 & 38 & 9 & 0.007 & 0.003 & $<0.001$ & 0.001 & 0.2 & 0.2 \\
\hline BY114 & $\begin{array}{l}\text { SF Greenhorn Cr } \\
\text { 0.7mi ab Greenhorn } \\
\text { Cr nr Nevada City }\end{array}$ & $8 / 22 / 2000$ & $15: 10$ & $2 o f 2$ & $<0.008$ & 0.003 & 0.003 & 0.002 & 31 & 9 & $<0.005$ & 0.002 & $<0.001$ & 0.001 & 0.3 & 0.1 \\
\hline BY122 & $\begin{array}{l}\text { Starr Mine tunnel } \\
\text { inflow nr Grass } \\
\text { Valley }\end{array}$ & 4/23/1999 & 15:00 & 1 of 2 & 0.020 & 0.001 & 0.012 & 0.001 & 94 & 19 & 0.051 & 0.002 & 0.0069 & 0.0004 & 1.2 & 0.1 \\
\hline BY122 & $\begin{array}{l}\text { Starr Mine tunnel } \\
\text { inflow nr Grass } \\
\text { Valley }\end{array}$ & 4/23/1999 & 15:00 & $2 \mathrm{of} 2$ & 0.022 & 0.004 & 0.012 & 0.002 & 98 & 2 & 0.062 & 0.005 & 0.0082 & 0.0008 & 1.3 & 0.0 \\
\hline BY123 & $\begin{array}{l}\text { Starr Mine tunnel } \\
\text { midway nr Grass } \\
\text { Valley }\end{array}$ & $4 / 23 / 1999$ & $13: 40$ & 1 of 2 & 0.015 & 0.003 & 0.009 & 0.002 & 700 & 40 & 0.037 & 0.007 & 0.0062 & 0.0001 & 2.2 & 0.1 \\
\hline BY123 & $\begin{array}{l}\text { Starr Mine tunnel } \\
\text { midway nr Grass } \\
\text { Valley }\end{array}$ & 4/23/1999 & $13: 40$ & $2 \mathrm{of} 2$ & 0.009 & 0.001 & 0.007 & 0.002 & 660 & 60 & 0.024 & 0.006 & 0.0044 & 0.0005 & 2.1 & 0.1 \\
\hline
\end{tabular}


Table 4A. Concentrations of trace metals and selected major elements in unfiltered water samples, Greenhorn Creek drainage, Nevada County, California—Continued.

[Station name abbreviations: ab, above; bl, below; Cr, Creek; mi, miles; N, north; nr, near; Rd, Road; S, south; SF, South Fork. Number in parentheses is the data parameter code, a five-digit code used in the

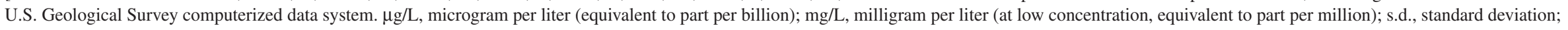
$<$, less than; - , not determined]

\begin{tabular}{|c|c|c|c|c|c|c|c|c|c|c|c|c|c|c|c|c|}
\hline \multirow[t]{2}{*}{$\begin{array}{c}\text { Station map } \\
\text { ID }\end{array}$} & \multirow[t]{2}{*}{ Station name } & \multirow[t]{2}{*}{ Date } & \multirow[t]{2}{*}{ Time } & \multirow[t]{2}{*}{$\begin{array}{l}\text { Rep- } \\
\text { licate }\end{array}$} & \multicolumn{2}{|c|}{$\begin{array}{c}\text { Erbium (Er), } \\
\text { unfiltered } \\
(\mu \mathrm{g} / \mathrm{L}) \\
(01246)\end{array}$} & \multicolumn{2}{|c|}{$\begin{array}{c}\text { Europium (Eu), } \\
\text { unfiltered } \\
(\mu \mathrm{g} / \mathrm{L}) \\
(\mathbf{0 1 2 3 6}) \\
\end{array}$} & \multicolumn{2}{|c|}{$\begin{array}{c}\text { Iron (Fe), } \\
\text { unfiltered } \\
(\mu \mathrm{g} / \mathrm{L}) \\
(01045)\end{array}$} & \multicolumn{2}{|c|}{$\begin{array}{c}\text { Gadolinium (Gd), } \\
\text { unfiltered } \\
(\mu \mathrm{g} / \mathrm{L}) \\
(\mathbf{0 1 2 1 9}) \\
\end{array}$} & \multicolumn{2}{|c|}{$\begin{array}{c}\text { Holmium }(\mathrm{Ho}), \\
\text { unfiltered } \\
(\mu \mathrm{g} / \mathrm{L}) \\
(01247) \\
\end{array}$} & \multicolumn{2}{|c|}{$\begin{array}{c}\text { Potassium (K), } \\
\text { unfiltered } \\
(\mu \mathrm{g} / \mathrm{L}) \\
(\mathbf{0 0 9 3 7}) \\
\end{array}$} \\
\hline & & & & & value & s.d. & value & s.d. & value & s.d. & value & s.d. & value & s.d. & value & s.d. \\
\hline BY123 & $\begin{array}{l}\text { Starr Mine tunnel } \\
\text { midway nr Grass } \\
\text { Valley }\end{array}$ & $12 / 15 / 1999$ & $10: 45$ & 1 of 2 & 0.038 & 0.005 & 0.017 & 0.008 & 1,700 & 100 & 0.077 & 0.027 & 0.012 & 0.001 & 2.3 & 0.1 \\
\hline BY123 & $\begin{array}{l}\text { Starr Mine tunnel } \\
\text { midway nr Grass } \\
\text { Valley }\end{array}$ & $12 / 15 / 1999$ & $10: 45$ & 2of 2 & 0.033 & 0.005 & 0.008 & 0.005 & 1,600 & 0 & 0.068 & 0.021 & 0.013 & 0.001 & 2.2 & 0.0 \\
\hline BY124 & $\begin{array}{l}\text { Starr Mine tunnel } \\
\text { outlet nr Grass } \\
\text { Valley }\end{array}$ & $8 / 23 / 2000$ & $17: 30$ & 1of 2 & 0.087 & 0.017 & 0.043 & 0.009 & 2,300 & 200 & 0.16 & 0.02 & 0.027 & 0.001 & 1.8 & 0.3 \\
\hline BY124 & $\begin{array}{l}\text { Starr Mine tunnel } \\
\text { outlet nr Grass } \\
\text { Valley }\end{array}$ & $8 / 23 / 2000$ & $17: 30$ & 2of 2 & 0.091 & 0.005 & 0.035 & 0.001 & 2,100 & 0 & 0.15 & 0.00 & 0.031 & 0.002 & 1.8 & 0.3 \\
\hline BY129 & $\begin{array}{l}\text { Tom and Jerry Mine } \\
\text { drain at tunnel inlet } \\
\text { nr Nevada City }\end{array}$ & $6 / 28 / 2000$ & $12: 00$ & 1of 2 & 1.1 & 0.2 & 0.66 & 0.11 & 8,900 & 400 & 2.4 & 0.5 & 0.38 & 0.07 & 0.5 & 0.1 \\
\hline BY129 & $\begin{array}{l}\text { Tom and Jerry Mine } \\
\text { drain at tunnel inlet } \\
\text { nr Nevada City }\end{array}$ & $6 / 28 / 2000$ & 12:00 & 2of 2 & 0.98 & 0.19 & 0.64 & 0.10 & 10,000 & 1,000 & 2.3 & 0.4 & 0.37 & 0.05 & 0.4 & 0.1 \\
\hline BY130 & $\begin{array}{l}\text { Tom and Jerry Mine } \\
\text { drain at tunnel } \\
\text { outlet nr Nevada } \\
\text { City }\end{array}$ & $6 / 28 / 2000$ & $11: 30$ & 1 of 2 & 0.015 & 0.006 & 0.011 & 0.003 & 630 & 70 & 0.046 & 0.010 & 0.006 & 0.002 & 0.6 & 0.1 \\
\hline BY130 & $\begin{array}{l}\text { Tom and Jerry Mine } \\
\text { drain at tunnel } \\
\text { outlet nr Nevada } \\
\text { City }\end{array}$ & $6 / 28 / 2000$ & $11: 30$ & 2of2 & 0.018 & 0.004 & 0.011 & 0.003 & 630 & 120 & 0.038 & 0.007 & 0.009 & 0.000 & 0.6 & 0.2 \\
\hline BY131 & $\begin{array}{l}\text { Tom and Jerry Mine } \\
\text { drainage pond nr } \\
\text { Nevada City }\end{array}$ & $8 / 23 / 2000$ & $10: 45$ & 1 of 2 & 0.025 & 0.004 & 0.013 & 0.003 & 95 & 6 & 0.050 & 0.013 & 0.010 & 0.003 & 0.7 & 0.1 \\
\hline BY131 & $\begin{array}{l}\text { Tom and Jerry Mine } \\
\text { drainage pond nr } \\
\text { Nevada City }\end{array}$ & $8 / 23 / 2000$ & $10: 45$ & 2of 2 & 0.026 & 0.005 & 0.013 & 0.003 & 98 & 32 & 0.057 & 0.009 & 0.009 & 0.001 & 0.6 & 0.1 \\
\hline
\end{tabular}


Table 4A. Concentrations of trace metals and selected major elements in unfiltered water samples, Greenhorn Creek drainage, Nevada County, California-Continued.

[Station name abbreviations: ab, above; bl, below; Cr, Creek; mi, miles; N, north; nr, near; Rd, Road; S, south; SF, South Fork. Number in parentheses is the data parameter code, a five-digit code used in the

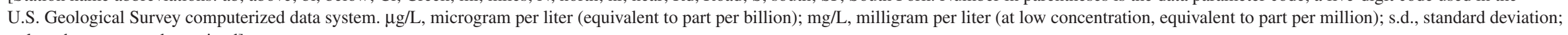
$<$ less than; - , not determined]

\begin{tabular}{|c|c|c|c|c|c|c|c|c|c|c|c|c|c|c|c|c|}
\hline \multirow[t]{2}{*}{$\begin{array}{l}\text { Station map } \\
\text { ID }\end{array}$} & \multirow[t]{2}{*}{ Station name } & \multirow[t]{2}{*}{ Date } & \multirow[t]{2}{*}{ Time } & \multirow[t]{2}{*}{$\begin{array}{l}\text { Rep- } \\
\text { licate }\end{array}$} & \multicolumn{2}{|c|}{$\begin{array}{c}\text { Erbium (Er), } \\
\text { unfiltered } \\
(\mu \mathrm{g} / \mathrm{L}) \\
(01246)\end{array}$} & \multicolumn{2}{|c|}{$\begin{array}{c}\text { Europium (Eu), } \\
\text { unfiltered } \\
(\mu \mathrm{g} / \mathrm{L}) \\
(01236)\end{array}$} & \multicolumn{2}{|c|}{$\begin{array}{c}\text { Iron (Fe), } \\
\text { unfiltered } \\
(\mu \mathrm{g} / \mathrm{L}) \\
(01045)\end{array}$} & \multicolumn{2}{|c|}{$\begin{array}{c}\text { Gadolinium }(\mathbf{G d}), \\
\text { unfiltered } \\
(\mu \mathrm{g} / \mathrm{L}) \\
(\mathbf{0 1 2 1 9}) \\
\end{array}$} & \multicolumn{2}{|c|}{$\begin{array}{c}\text { Holmium (Ho), } \\
\text { unfiltered } \\
(\mu \mathrm{g} / \mathrm{L}) \\
(01247) \\
\end{array}$} & \multicolumn{2}{|c|}{$\begin{array}{c}\text { Potassium }(\mathrm{K}) \\
\text { unfiltered } \\
(\mu \mathrm{g} / \mathrm{L}) \\
(00937) \\
\end{array}$} \\
\hline & & & & & value & s.d. & value & s.d. & value & s.d. & value & s.d. & value & s.d. & value & s.d. \\
\hline \multicolumn{17}{|c|}{ Disturbed samples } \\
\hline BY123 & $\begin{array}{l}\text { Starr Mine tunnel } \\
\text { midway nr Grass } \\
\text { Valley }\end{array}$ & $12 / 15 / 1999$ & $11: 30$ & 1 of 2 & 15 & 1 & 5.1 & 0.2 & 920,000 & 10,000 & 24 & 5 & 4.7 & 0.0 & 10 & 0 \\
\hline BY123 & $\begin{array}{l}\text { Starr Mine tunnel } \\
\text { midway nr Grass } \\
\text { Valley }\end{array}$ & $12 / 15 / 1999$ & $11: 30$ & $2 o f 2$ & 14 & 0 & 5.8 & 0.3 & 780,000 & 10,000 & 23 & 2 & 4.4 & 0.1 & 9.6 & 0.1 \\
\hline BY129 & $\begin{array}{l}\text { Tom and Jerry Mine } \\
\text { drain at tunnel inlet } \\
\text { nr Nevada City }\end{array}$ & $6 / 28 / 2000$ & $12: 30$ & 1 of 2 & 8.9 & 1.4 & 6.7 & 1.0 & 72,000 & 1,000 & 24 & 3 & 3.4 & 0.6 & 1.6 & 0.1 \\
\hline BY129 & $\begin{array}{l}\text { Tom and Jerry Mine } \\
\text { drain at tunnel inlet } \\
\text { nr Nevada City }\end{array}$ & $6 / 28 / 2000$ & $12: 30$ & $2 o f 2$ & 9.8 & 0.5 & 7.4 & 0.3 & 93,000 & 20,000 & 24 & 4 & 3.8 & 0.2 & 2.1 & 0.6 \\
\hline
\end{tabular}


Table 4A. Concentrations of trace metals and selected major elements in unfiltered water samples, Greenhorn Creek drainage, Nevada County, California—Continued.

[Station name abbreviations: ab, above; bl, below; Cr, Creek; mi, miles; N, north; nr, near; Rd, Road; S, south; SF, South Fork. Number in parentheses is the data parameter code, a five-digit code used in the

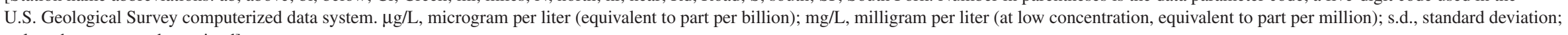
$<$, less than; - , not determined]

\begin{tabular}{|c|c|c|c|c|c|c|c|c|c|c|c|c|c|c|c|c|}
\hline \multirow[t]{2}{*}{$\begin{array}{l}\text { Station } \\
\text { map ID }\end{array}$} & \multirow[t]{2}{*}{ Station name } & \multirow[t]{2}{*}{ Date } & \multirow[t]{2}{*}{ Time } & \multirow[t]{2}{*}{$\begin{array}{l}\text { Rep- } \\
\text { licate }\end{array}$} & \multicolumn{2}{|c|}{$\begin{array}{c}\text { Lanthanum (La), } \\
\text { unfiltered } \\
(\mu \mathrm{g} / \mathrm{L}) \\
(\mathbf{0 1 1 8 2}) \\
\end{array}$} & \multicolumn{2}{|c|}{$\begin{array}{c}\text { Lithium (Li), } \\
\text { unfiltered } \\
(\mu \mathrm{g} / \mathrm{L}) \\
(\mathbf{0 1 1 3 2}) \\
\end{array}$} & \multicolumn{2}{|c|}{$\begin{array}{c}\text { Lutetium (Lu), } \\
\text { unfiltered } \\
(\mu \mathrm{g} / \mathrm{L}) \\
(01244) \\
\end{array}$} & \multicolumn{2}{|c|}{$\begin{array}{l}\text { Magnesium }(\mathrm{Mg}) \text {, } \\
\text { unfiltered } \\
(\mathrm{mg} / \mathrm{L}) \\
(00921) \\
\end{array}$} & \multicolumn{2}{|c|}{$\begin{array}{c}\text { Manganese }(\mathrm{Mn}), \\
\text { unfiltered } \\
(\mu \mathrm{g} / \mathrm{L}) \\
(01055) \\
\end{array}$} & \multicolumn{2}{|c|}{$\begin{array}{c}\text { Molybdenum (Mo), } \\
\text { unfiltered } \\
(\mu \mathrm{g} / \mathrm{L}) \\
(01062)\end{array}$} \\
\hline & & & & & value & s.d. & value & s.d. & value & s.d. & value & s.d. & value & s.d. & value & s.d. \\
\hline \multicolumn{17}{|c|}{ Environmental samples } \\
\hline BY20 & $\begin{array}{l}\text { Boston Mine tunnel } \\
\text { outlet nr Grass } \\
\text { Valley }\end{array}$ & $6 / 18 / 1999$ & $14: 30$ & 1 of 2 & 0.057 & 0.008 & 2.6 & 0.1 & 0.0016 & 0.0003 & 4.6 & 0.0 & 220 & 0 & 0.11 & 0.03 \\
\hline BY20 & $\begin{array}{l}\text { Boston Mine tunnel } \\
\text { outlet nr Grass } \\
\text { Valley }\end{array}$ & $6 / 18 / 1999$ & $14: 30$ & 2 of 2 & 0.044 & 0.010 & 2.4 & 0.1 & 0.0017 & 0.0003 & 4.6 & 0.1 & 220 & 0 & 0.21 & 0.10 \\
\hline BY20 & $\begin{array}{l}\text { Boston Mine tunnel } \\
\text { outlet nr Grass } \\
\text { Valley }\end{array}$ & $12 / 14 / 1999$ & 12:00 & 1of 2 & 0.41 & 0.05 & 3.2 & 0.1 & 0.010 & 0.002 & 6.1 & 0.1 & 540 & 90 & 0.10 & 0.04 \\
\hline BY20 & $\begin{array}{l}\text { Boston Mine tunnel } \\
\text { outlet nr Grass } \\
\text { Valley }\end{array}$ & $12 / 14 / 1999$ & $12: 00$ & 2of 2 & 0.29 & 0.01 & 2.7 & 0.3 & 0.007 & 0.001 & 6.2 & 0.6 & 500 & 60 & 0.09 & 0.07 \\
\hline BY20 & $\begin{array}{l}\text { Boston Mine tunnel } \\
\text { outlet nr Grass } \\
\text { Valley }\end{array}$ & $5 / 24 / 2000$ & $16: 00$ & 1of 2 & 0.095 & 0.005 & 2.3 & 0.3 & 0.0019 & 0.0011 & 3.9 & 0.2 & 302 & 13 & 0.09 & 0.04 \\
\hline BY20 & $\begin{array}{l}\text { Boston Mine tunnel } \\
\text { outlet nr Grass } \\
\text { Valley }\end{array}$ & $5 / 24 / 2000$ & $16: 00$ & 2of 2 & 0.21 & 0.01 & 2.3 & 0.2 & 0.0050 & 0.0003 & 4.1 & 0.0 & 510 & 4 & 0.07 & 0.01 \\
\hline BY21 & $\begin{array}{l}\text { Boston Mine wetlands } \\
\text { pond nr Grass } \\
\text { Valley }\end{array}$ & $12 / 14 / 1999$ & $10: 30$ & 1of 2 & 0.015 & 0.002 & 0.92 & 0.06 & $<0.001$ & 0.001 & 5.7 & 0.0 & 15 & 1 & 0.33 & 0.02 \\
\hline BY21 & $\begin{array}{l}\text { Boston Mine wetlands } \\
\text { pond nr Grass } \\
\text { Valley }\end{array}$ & $12 / 14 / 1999$ & $10: 30$ & 2 of 2 & 0.017 & 0.003 & 0.91 & 0.02 & 0.001 & 0.001 & 5.7 & 0.3 & 27 & 1 & 0.26 & 0.13 \\
\hline BY21 & $\begin{array}{l}\text { Boston Mine wetlands } \\
\text { pond nr Grass } \\
\text { Valley }\end{array}$ & $5 / 24 / 2000$ & $16: 30$ & 1of 2 & 0.024 & 0.000 & 3.0 & 0.2 & $<0.0009$ & 0.0004 & 2.8 & 0.1 & 56 & 2 & 0.10 & 0.03 \\
\hline BY21 & $\begin{array}{l}\text { Boston Mine wetlands } \\
\text { pond nr Grass } \\
\text { Valley }\end{array}$ & $5 / 24 / 2000$ & $16: 30$ & 2of 2 & 0.026 & 0.003 & 2.8 & 0.2 & $<0.0009$ & 0.0003 & 2.8 & 0.1 & 56 & 3 & 0.09 & 0.01 \\
\hline BY58 & $\begin{array}{l}\text { Buckeye Flat Mine } \\
\text { main drain } 0.45 \mathrm{mi} \\
\text { ab Greenhorn } \mathrm{Cr}\end{array}$ & $8 / 21 / 2000$ & 11:00 & 1of 2 & 16 & 1 & 21 & 1 & 0.18 & 0.01 & 12 & 2 & 1,800 & 100 & $<4$ & 0 \\
\hline BY58 & $\begin{array}{l}\text { Buckeye Flat Mine } \\
\text { main drain } 0.45 \mathrm{mi} \\
\text { ab Greenhorn } \mathrm{Cr}\end{array}$ & $8 / 21 / 2000$ & 11:00 & 2 of 2 & 12 & 0 & 17 & 1 & 0.13 & 0.00 & 11 & 3 & 1,400 & 100 & $<4$ & 2 \\
\hline
\end{tabular}


Table 4A. Concentrations of trace metals and selected major elements in unfiltered water samples, Greenhorn Creek drainage, Nevada County, California-Continued.

[Station name abbreviations: ab, above; bl, below; Cr, Creek; mi, miles; N, north; nr, near; Rd, Road; S, south; SF, South Fork. Number in parentheses is the data parameter code, a five-digit code used in the

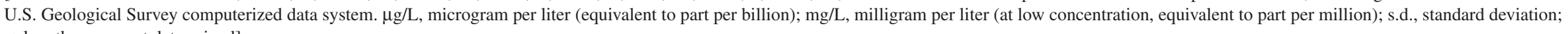
$<$, less than; - - not determined]

\begin{tabular}{|c|c|c|c|c|c|c|c|c|c|c|c|c|c|c|c|c|}
\hline \multirow[t]{2}{*}{$\begin{array}{l}\text { Station } \\
\text { map ID }\end{array}$} & \multirow[t]{2}{*}{ Station name } & \multirow[t]{2}{*}{ Date } & \multirow[t]{2}{*}{ Time } & \multirow[t]{2}{*}{$\begin{array}{l}\text { Rep- } \\
\text { licate }\end{array}$} & \multicolumn{2}{|c|}{$\begin{array}{c}\text { Lanthanum (La), } \\
\text { unfiltered } \\
(\mu \mathrm{g} / \mathrm{L}) \\
(\mathbf{0 1 1 8 2})\end{array}$} & \multicolumn{2}{|c|}{$\begin{array}{c}\text { Lithium }(\mathrm{Li}), \\
\text { unfiltered } \\
(\mu \mathrm{g} / \mathrm{L}) \\
(\mathbf{0 1 1 3 2}) \\
\end{array}$} & \multicolumn{2}{|c|}{$\begin{array}{c}\text { Lutetium (Lu), } \\
\text { unfiltered } \\
(\mu \mathrm{g} / \mathrm{L}) \\
(01244) \\
\end{array}$} & \multicolumn{2}{|c|}{$\begin{array}{l}\text { Magnesium (Mg), } \\
\text { unfiltered } \\
(\mathrm{mg} / \mathrm{L}) \\
(00921) \\
\end{array}$} & \multicolumn{2}{|c|}{$\begin{array}{c}\text { Manganese (Mn), } \\
\text { unfiltered } \\
(\mu \mathrm{g} / \mathrm{L}) \\
(01055)\end{array}$} & \multicolumn{2}{|c|}{$\begin{array}{c}\text { Molybdenum (Mo), } \\
\text { unfiltered } \\
(\mu \mathrm{g} / \mathrm{L}) \\
(\mathbf{0 1 0 6 2 )}\end{array}$} \\
\hline & & & & & value & s.d. & value & s.d. & value & s.d. & value & s.d. & value & s.d. & value & s.d. \\
\hline BY23 & $\begin{array}{c}\text { Buckeye Flat Mine N } \\
\text { drain to Greenhorn } \\
\text { Cr, Grass Valley }\end{array}$ & 6/18/1999 & $11: 30$ & 1 of 2 & 16 & 0 & 22 & 0 & 0.20 & 0.02 & 17 & 0 & 2,700 & 100 & 0.10 & 0.02 \\
\hline BY23 & $\begin{array}{c}\text { Buckeye Flat Mine N } \\
\text { drain to Greenhorn } \\
\text { Cr, Grass Valley }\end{array}$ & 6/18/1999 & $11: 30$ & $2 \mathrm{of} 2$ & 21 & 1 & 22 & 1 & 0.25 & 0.02 & 17 & 0 & 2,600 & 200 & 0.10 & 0.02 \\
\hline BY23 & $\begin{array}{c}\text { Buckeye Flat Mine N } \\
\text { drain to Greenhorn } \\
\text { Cr, Grass Valley }\end{array}$ & $12 / 2 / 1999$ & $11: 00$ & $1 \mathrm{of} 2$ & 13 & 2 & 16 & 1 & 0.18 & 0.03 & 10 & 1 & 1,600 & 100 & 0.21 & 0.12 \\
\hline BY23 & $\begin{array}{c}\text { Buckeye Flat Mine N } \\
\text { drain to Greenhorn } \\
\text { Cr, Grass Valley }\end{array}$ & $12 / 2 / 1999$ & $11: 00$ & $2 \mathrm{of} 2$ & 17 & 1 & 14 & 0 & 0.25 & 0.05 & 10 & 0 & 1,600 & 0 & 0.08 & 0.01 \\
\hline BY23 & $\begin{array}{c}\text { Buckeye Flat Mine N } \\
\text { drain to Greenhorn } \\
\text { Cr, Grass Valley }\end{array}$ & $8 / 22 / 2000$ & $10: 45$ & 1 of 2 & 23 & 0 & 20 & 4 & 0.26 & 0.01 & 17 & 4 & 2,100 & 0 & $<4$ & 1 \\
\hline BY23 & $\begin{array}{c}\text { Buckeye Flat Mine N } \\
\text { drain to Greenhorn } \\
\text { Cr, Grass Valley }\end{array}$ & $8 / 22 / 2000$ & $10: 45$ & 2 of 2 & 21 & 0 & 17 & 1 & 0.24 & 0.02 & 17 & 2 & 1,900 & 0 & $<4$ & 1 \\
\hline BY116 & $\begin{array}{l}\text { Buckeye Flat Mine } \\
\text { pond drain } 0.15 \mathrm{mi} \\
\text { ab SF Greenhorn } \mathrm{Cr}\end{array}$ & $8 / 21 / 2000$ & $14: 10$ & 1 of 2 & 0.13 & 0.01 & 2.5 & 0.2 & 0.002 & 0.001 & 0.8 & 0.0 & 68 & 1 & $<0.1$ & 0.0 \\
\hline BY116 & $\begin{array}{l}\text { Buckeye Flat Mine } \\
\text { pond drain } 0.15 \mathrm{mi} \\
\text { ab SF Greenhorn } \mathrm{Cr}\end{array}$ & $8 / 21 / 2000$ & $14: 10$ & $2 \mathrm{of} 2$ & 0.15 & 0.00 & 2.5 & 0.0 & 0.002 & 0.001 & 0.6 & 0.1 & 67 & 5 & $<0.1$ & 0.2 \\
\hline BY24 & $\begin{array}{l}\text { Buckeye Flat Mine } \\
\text { S drain to SF } \\
\text { Greenhorn Cr, } \\
\text { Grass Valley }\end{array}$ & 8/26/1999 & $11: 00$ & 1 of 2 & 0.33 & 0.02 & 1.4 & 0.1 & 0.0060 & 0.0003 & 0.58 & 0.02 & 150 & 10 & 0.04 & 0.01 \\
\hline BY24 & $\begin{array}{l}\text { Buckeye Flat Mine } \\
\text { S drain to SF } \\
\text { Greenhorn Cr, } \\
\text { Grass Valley }\end{array}$ & $8 / 26 / 1999$ & $11: 00$ & $2 \mathrm{of} 2$ & 0.33 & 0.00 & 1.3 & 0.1 & 0.0058 & 0.0003 & 0.57 & 0.01 & 150 & 0 & 0.04 & 0.01 \\
\hline BY24 & $\begin{array}{l}\text { Buckeye Flat Mine } \\
\text { S drain to SF } \\
\text { Greenhorn Cr, } \\
\text { Grass Valley }\end{array}$ & $8 / 22 / 2000$ & $12: 10$ & 1 of 2 & 0.99 & 0.19 & 2.4 & 0.2 & 0.015 & 0.003 & 0.7 & 0.3 & 110 & 0 & $<0.1$ & 0.1 \\
\hline BY24 & $\begin{array}{l}\text { Buckeye Flat Mine } \\
\text { S drain to SF } \\
\text { Greenhorn Cr, } \\
\text { Grass Valley }\end{array}$ & $8 / 22 / 2000$ & $12: 10$ & $2 \mathrm{of} 2$ & 0.99 & 0.14 & 2.5 & 0.1 & 0.016 & 0.000 & 0.7 & 0.2 & 100 & 10 & $<0.1$ & 0.1 \\
\hline
\end{tabular}


Table 4A. Concentrations of trace metals and selected major elements in unfiltered water samples, Greenhorn Creek drainage, Nevada County, California—Continued.

[Station name abbreviations: ab, above; bl, below; Cr, Creek; mi, miles; N, north; nr, near; Rd, Road; S, south; SF, South Fork. Number in parentheses is the data parameter code, a five-digit code used in the

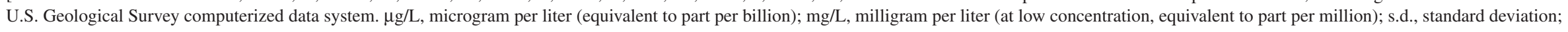
$<$, less than; - - not determined]

\begin{tabular}{|c|c|c|c|c|c|c|c|c|c|c|c|c|c|c|c|c|}
\hline \multirow[t]{2}{*}{$\begin{array}{l}\text { Station } \\
\text { map ID }\end{array}$} & \multirow[t]{2}{*}{ Station name } & \multirow[t]{2}{*}{ Date } & \multirow[t]{2}{*}{ Time } & \multirow[t]{2}{*}{$\begin{array}{l}\text { Rep- } \\
\text { licate }\end{array}$} & \multicolumn{2}{|c|}{$\begin{array}{c}\text { Lanthanum (La), } \\
\text { unfiltered } \\
(\mu \mathrm{g} / \mathrm{L}) \\
(\mathbf{0 1 1 8 2})\end{array}$} & \multicolumn{2}{|c|}{$\begin{array}{c}\text { Lithium (Li), } \\
\text { unfiltered } \\
(\mu \mathrm{g} / \mathrm{L}) \\
(\mathbf{0 1 1 3 2})\end{array}$} & \multicolumn{2}{|c|}{$\begin{array}{c}\text { Lutetium (Lu), } \\
\text { unfiltered } \\
(\mu \mathrm{g} / \mathrm{L}) \\
(01244)\end{array}$} & \multicolumn{2}{|c|}{$\begin{array}{l}\text { Magnesium }(\mathrm{Mg}) \text {, } \\
\text { unfiltered } \\
(\mathrm{mg} / \mathrm{L}) \\
(00921) \\
\end{array}$} & \multicolumn{2}{|c|}{$\begin{array}{c}\text { Manganese }(\mathbf{M n}), \\
\text { unfiltered } \\
(\mu \mathrm{g} / \mathrm{L}) \\
(01055)\end{array}$} & \multicolumn{2}{|c|}{$\begin{array}{c}\text { Molybdenum (Mo), } \\
\text { unfiltered } \\
(\mu \mathrm{g} / \mathrm{L}) \\
(01062)\end{array}$} \\
\hline & & & & & value & s.d. & value & s.d. & value & s.d. & value & s.d. & value & s.d. & value & s.d. \\
\hline BY25 & $\begin{array}{l}\text { Buckeye Flat Mine } \\
\text { upper drain }\end{array}$ & 4/9/2001 & $10: 00$ & 1of 2 & 4.4 & 0.3 & 4.6 & 0.4 & 0.039 & 0.001 & 4.6 & 0.4 & 360 & 70 & $<0.3$ & 0.1 \\
\hline BY25 & $\begin{array}{l}\text { Buckeye Flat Mine } \\
\text { upper drain }\end{array}$ & 4/9/2001 & $10: 00$ & 2of 2 & 4.4 & 0.0 & 4.6 & 0.1 & 0.038 & 0.001 & 5.5 & 0.1 & 370 & 0 & $<0.3$ & 0.1 \\
\hline BY52 & $\begin{array}{l}\text { Greenhorn Cr } 0.3 \mathrm{mi} \\
\text { ab Buckeye Ford nr } \\
\text { Nevada City }\end{array}$ & $1 / 25 / 2000$ & $10: 45$ & 1of 2 & 0.87 & 0.01 & 1.2 & 0.0 & 0.015 & 0.000 & 1.3 & 0.1 & 36 & 2 & 0.17 & 0.05 \\
\hline BY52 & $\begin{array}{l}\text { Greenhorn Cr } 0.3 \mathrm{mi} \\
\text { ab Buckeye Ford nr } \\
\text { Nevada City }\end{array}$ & $1 / 25 / 2000$ & $10: 45$ & 2 of 2 & 0.87 & 0.01 & 1.2 & 0.1 & 0.014 & 0.001 & 1.3 & 0.1 & 36 & 1 & 0.40 & 0.05 \\
\hline BY59 & $\begin{array}{l}\text { Greenhorn Cr at You } \\
\text { Bet Rd nr Nevada } \\
\text { City }\end{array}$ & $7 / 24 / 2001$ & 11:00 & 1of 2 & 0.051 & 0.002 & 0.9 & 0.1 & $<0.002$ & 0.000 & 2.2 & 0.2 & 113 & 3 & $<0.5$ & 0.3 \\
\hline BY59 & $\begin{array}{l}\text { Greenhorn Cr at You } \\
\text { Bet Rd nr Nevada } \\
\text { City }\end{array}$ & $7 / 24 / 2001$ & 11:00 & 2 of 2 & 0.051 & 0.002 & 0.7 & 0.3 & $<0.002$ & 0.000 & 2.3 & 0.2 & 124 & 0 & $<0.5$ & 0.3 \\
\hline BY59 & $\begin{array}{l}\text { Greenhorn Cr at You } \\
\text { Bet Rd nr Nevada } \\
\text { City }\end{array}$ & $8 / 29 / 2001$ & $15: 00$ & 1 of 2 & 0.029 & 0.001 & 0.9 & 0.0 & $<0.002$ & 0.001 & 2.2 & 0.2 & 36 & 2 & $<0.5$ & 0.4 \\
\hline BY59 & $\begin{array}{l}\text { Greenhorn Cr at You } \\
\text { Bet Rd nr Nevada } \\
\text { City }\end{array}$ & $8 / 29 / 2001$ & $15: 00$ & 2of 2 & 0.038 & 0.004 & 0.9 & 0.3 & $<0.002$ & 0.001 & 2.3 & 0.2 & 37 & 0 & $<0.5$ & 0.0 \\
\hline BY59 & $\begin{array}{l}\text { Greenhorn Cr at You } \\
\text { Bet Rd nr Nevada } \\
\text { City }\end{array}$ & $9 / 14 / 2001$ & $13: 00$ & 1of 2 & 0.040 & 0.001 & 0.77 & 0.02 & $<0.001$ & 0.000 & 2.2 & 0.1 & 61 & 1 & $<0.5$ & 0.0 \\
\hline BY59 & $\begin{array}{l}\text { Greenhorn } \mathrm{Cr} \text { at You } \\
\text { Bet Rd nr Nevada } \\
\text { City }\end{array}$ & $9 / 14 / 2001$ & $13: 00$ & 2of 2 & 0.037 & 0.002 & 0.91 & 0.13 & $<0.001$ & 0.000 & 2.2 & 0.2 & 59 & 1 & $<0.5$ & 0.4 \\
\hline BY59 & $\begin{array}{l}\text { Greenhorn Cr at You } \\
\text { Bet Rd nr Nevada } \\
\text { City }\end{array}$ & $10 / 15 / 2001$ & $12: 00$ & 1of 2 & 0.017 & 0.001 & 0.8 & 0.3 & $<0.0005$ & 0.0003 & 2.4 & 0.2 & 54 & 1 & 0.5 & 0.6 \\
\hline BY59 & $\begin{array}{l}\text { Greenhorn Cr at You } \\
\text { Bet Rd nr Nevada } \\
\text { City }\end{array}$ & $10 / 15 / 2001$ & $12: 00$ & $2 \mathrm{of} 2$ & 0.017 & 0.001 & 0.7 & 0.1 & 0.0005 & 0.0006 & 2.4 & 0.1 & 57 & 2 & $<0.5$ & 0.0 \\
\hline BY59 & $\begin{array}{l}\text { Greenhorn } \mathrm{Cr} \text { at You } \\
\text { Bet Rd nr Nevada } \\
\text { City }\end{array}$ & $11 / 20 / 2001$ & $13: 20$ & 1of 2 & 0.100 & 0.010 & 0.87 & 0.06 & 0.002 & 0.001 & 2.7 & 0.4 & 200 & 22 & $<0.5$ & 0.0 \\
\hline BY59 & $\begin{array}{l}\text { Greenhorn Cr at You } \\
\text { Bet Rd nr Nevada } \\
\text { City }\end{array}$ & $11 / 20 / 2001$ & $13: 20$ & 2 of 2 & 0.10 & 0.01 & 0.84 & 0.08 & 0.003 & 0.000 & 2.7 & 0.3 & 200 & 14 & $<0.5$ & 0.0 \\
\hline
\end{tabular}


Table 4A. Concentrations of trace metals and selected major elements in unfiltered water samples, Greenhorn Creek drainage, Nevada County, California—Continued.

[Station name abbreviations: ab, above; bl, below; Cr, Creek; mi, miles; N, north; nr, near; Rd, Road; S, south; SF, South Fork. Number in parentheses is the data parameter code, a five-digit code used in the

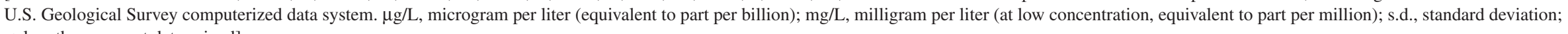
$<$, less than; - - not determined]

\begin{tabular}{|c|c|c|c|c|c|c|c|c|c|c|c|c|c|c|c|c|}
\hline \multirow[t]{2}{*}{$\begin{array}{l}\text { Station } \\
\text { map ID }\end{array}$} & \multirow[t]{2}{*}{ Station name } & \multirow[t]{2}{*}{ Date } & \multirow[t]{2}{*}{ Time } & \multirow[t]{2}{*}{$\begin{array}{l}\text { Rep- } \\
\text { licate }\end{array}$} & \multicolumn{2}{|c|}{$\begin{array}{c}\text { Lanthanum (La), } \\
\text { unfiltered } \\
(\mu \mathrm{g} / \mathrm{L}) \\
(01182)\end{array}$} & \multicolumn{2}{|c|}{$\begin{array}{c}\text { Lithium (Li), } \\
\text { unfiltered } \\
(\mu \mathrm{g} / \mathrm{L}) \\
(\mathbf{0 1 1 3 2})\end{array}$} & \multicolumn{2}{|c|}{$\begin{array}{c}\text { Lutetium (Lu), } \\
\text { unfiltered } \\
(\mu \mathrm{g} / \mathrm{L}) \\
(01244) \\
\end{array}$} & \multicolumn{2}{|c|}{$\begin{array}{c}\text { Magnesium }(\mathrm{Mg}) \text {, } \\
\text { unfiltered } \\
(\mathrm{mg} / \mathrm{L}) \\
(00921) \\
\end{array}$} & \multicolumn{2}{|c|}{$\begin{array}{c}\text { Manganese }(\mathrm{Mn}), \\
\text { unfiltered } \\
(\mu \mathrm{g} / \mathrm{L}) \\
(01055) \\
\end{array}$} & \multicolumn{2}{|c|}{$\begin{array}{c}\text { Molybdenum (Mo), } \\
\text { unfiltered } \\
(\mu \mathrm{g} / \mathrm{L}) \\
(01062)\end{array}$} \\
\hline & & & & & value & s.d. & value & s.d. & value & s.d. & value & s.d. & value & s.d. & value & s.d. \\
\hline BY59 & $\begin{array}{l}\text { Greenhorn Cr at You } \\
\text { Bet Rd nr Nevada } \\
\text { City }\end{array}$ & $12 / 10 / 2001$ & $12: 20$ & 1 of 2 & 0.85 & 0.02 & 1.3 & 0.2 & 0.016 & 0.002 & 2.6 & 0.3 & 110 & 13 & $<0.5$ & 0.1 \\
\hline BY59 & $\begin{array}{l}\text { Greenhorn Cr at You } \\
\text { Bet Rd nr Nevada } \\
\text { City }\end{array}$ & $12 / 10 / 2001$ & $12: 20$ & 2 of 2 & 0.80 & 0.08 & 1.2 & 0.1 & 0.016 & 0.003 & 2.6 & 0.3 & 111 & 14 & $<0.5$ & 0.1 \\
\hline BY59 & $\begin{array}{l}\text { Greenhorn Cr at You } \\
\text { Bet Rd nr Nevada } \\
\text { City }\end{array}$ & $12 / 21 / 2001$ & $13: 00$ & 1 of 2 & 3.4 & 0.4 & 2.5 & 0.2 & 0.059 & 0.009 & 2.7 & 0.3 & 190 & 24 & $<0.7$ & 1.6 \\
\hline BY59 & $\begin{array}{l}\text { Greenhorn Cr at You } \\
\text { Bet Rd nr Nevada } \\
\text { City }\end{array}$ & $12 / 21 / 2001$ & $13: 00$ & 2 of 2 & 3.3 & 0.4 & 2.8 & 0.0 & 0.060 & 0.014 & 2.8 & 0.4 & 191 & 18 & 1.0 & 1.7 \\
\hline BY51 & $\begin{array}{l}\text { Greenhorn } \mathrm{Cr} \mathrm{nr} \\
\text { headwaters } \mathrm{nr} \\
\text { Scotts Flat Res }\end{array}$ & $1 / 25 / 2000$ & $9: 30$ & 1 of 2 & 0.23 & 0.01 & 0.31 & 0.05 & 0.005 & 0.001 & 0.76 & 0.07 & 3 & 0 & 0.11 & 0.12 \\
\hline BY51 & $\begin{array}{l}\text { Greenhorn Cr nr } \\
\text { headwaters nr } \\
\text { Scotts Flat Res }\end{array}$ & $1 / 25 / 2000$ & $9: 30$ & $2 o f 2$ & 0.22 & 0.01 & 0.35 & 0.03 & 0.005 & 0.000 & 0.68 & 0.05 & 3 & 0 & 0.07 & 0.06 \\
\hline BY75 & $\begin{array}{l}\text { Missouri Cyn Cr } 1.2 \\
\text { mi ab Greenhorn Cr } \\
\text { nr Chicago Park }\end{array}$ & $12 / 14 / 1999$ & $14: 00$ & 1 of 2 & 0.40 & 0.13 & 8.1 & 2.1 & 0.008 & 0.004 & 4.4 & 0.2 & 89 & 21 & 0.18 & 0.06 \\
\hline BY75 & $\begin{array}{l}\text { Missouri Cyn Cr } 1.2 \\
\text { mi ab Greenhorn Cr } \\
\text { nr Chicago Park }\end{array}$ & $12 / 14 / 1999$ & $14: 00$ & $2 \mathrm{of} 2$ & 0.40 & 0.12 & 6.8 & 0.1 & 0.008 & 0.003 & 4.0 & 0.1 & 78 & 11 & 0.17 & 0.02 \\
\hline BY86 & $\begin{array}{l}\text { Poore Mine Creek } \\
\text { ab tunnel nr Grass } \\
\text { Valley }\end{array}$ & 3/31/1999 & $17: 40$ & 1 of 2 & 0.38 & 0.01 & 0.6 & 0.1 & 0.0048 & 0.0010 & 1.5 & 0.0 & 10 & 0 & 0.05 & 0.01 \\
\hline BY86 & $\begin{array}{l}\text { Poore Mine Creek } \\
\text { ab tunnel nr Grass } \\
\text { Valley }\end{array}$ & 3/31/1999 & $17: 40$ & 2 of 2 & 0.30 & 0.03 & 0.7 & 0.1 & 0.0039 & 0.0006 & 1.5 & 0.0 & 10 & 0 & 0.06 & 0.00 \\
\hline BY87 & $\begin{array}{l}\text { Poore Mine Creek } \\
\text { bl tunnel nr Grass } \\
\text { Valley }\end{array}$ & 3/31/1999 & $13: 30$ & 1 of 2 & 0.30 & 0.05 & 0.8 & 0.2 & 0.0044 & 0.0008 & 1.6 & 0.1 & 8 & 1 & 0.11 & 0.03 \\
\hline BY87 & $\begin{array}{l}\text { Poore Mine Creek } \\
\text { bl tunnel nr Grass } \\
\text { Valley }\end{array}$ & 3/31/1999 & $13: 30$ & 2 of 2 & 0.37 & 0.03 & 0.9 & 0.1 & 0.0063 & 0.0006 & 1.6 & 0.1 & 9 & 1 & 0.07 & 0.01 \\
\hline
\end{tabular}


Table 4A. Concentrations of trace metals and selected major elements in unfiltered water samples, Greenhorn Creek drainage, Nevada County, California-Continued.

[Station name abbreviations: ab, above; bl, below; Cr, Creek; mi, miles; N, north; nr, near; Rd, Road; S, south; SF, South Fork. Number in parentheses is the data parameter code, a five-digit code used in the

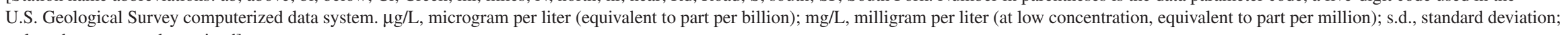
$<$, less than; - - not determined]

\begin{tabular}{|c|c|c|c|c|c|c|c|c|c|c|c|c|c|c|c|c|}
\hline \multirow[t]{2}{*}{$\begin{array}{l}\text { Station } \\
\text { map ID }\end{array}$} & \multirow[t]{2}{*}{ Station name } & \multirow[t]{2}{*}{ Date } & \multirow[t]{2}{*}{ Time } & \multirow[t]{2}{*}{$\begin{array}{l}\text { Rep- } \\
\text { licate }\end{array}$} & \multicolumn{2}{|c|}{$\begin{array}{c}\text { Lanthanum (La), } \\
\text { unfiltered } \\
(\mu \mathrm{g} / \mathrm{L}) \\
(\mathbf{0 1 1 8 2})\end{array}$} & \multicolumn{2}{|c|}{$\begin{array}{c}\text { Lithium (Li), } \\
\text { unfiltered } \\
(\mu \mathrm{g} / \mathrm{L}) \\
(\mathbf{0 1 1 3 2})\end{array}$} & \multicolumn{2}{|c|}{$\begin{array}{c}\text { Lutetium (Lu), } \\
\text { unfiltered } \\
(\mu \mathrm{g} / \mathrm{L}) \\
(01244)\end{array}$} & \multicolumn{2}{|c|}{$\begin{array}{l}\text { Magnesium }(\mathrm{Mg}) \text {, } \\
\text { unfiltered } \\
(\mathrm{mg} / \mathrm{L}) \\
(00921) \\
\end{array}$} & \multicolumn{2}{|c|}{$\begin{array}{c}\text { Manganese }(\mathrm{Mn}), \\
\text { unfiltered } \\
(\mu \mathrm{g} / \mathrm{L}) \\
(01055)\end{array}$} & \multicolumn{2}{|c|}{$\begin{array}{c}\text { Molybdenum (Mo), } \\
\text { unfiltered } \\
(\mu \mathrm{g} / \mathrm{L}) \\
(01062)\end{array}$} \\
\hline & & & & & value & s.d. & value & s.d. & value & s.d. & value & s.d. & value & s.d. & value & s.d. \\
\hline BY88 & $\begin{array}{l}\text { Poore Mine ground } \\
\text { sluice nr Grass } \\
\text { Valley }\end{array}$ & $4 / 1 / 1999$ & $15: 40$ & 1of 2 & 0.089 & 0.007 & 10 & 0 & 0.0041 & 0.0003 & 3.4 & 0.2 & 370 & 0 & 0.05 & 0.01 \\
\hline BY88 & $\begin{array}{l}\text { Poore Mine ground } \\
\text { sluice nr Grass } \\
\text { Valley }\end{array}$ & 4/1/1999 & $15: 40$ & 2of 2 & 0.11 & 0.02 & 10 & 1 & 0.0037 & 0.0002 & 3.2 & 0.1 & 380 & 0 & 0.05 & 0.02 \\
\hline BY89 & $\begin{array}{l}\text { Poore Mine pit lake nr } \\
\text { Grass Valley }\end{array}$ & 4/1/1999 & $16: 50$ & 1of 2 & 0.061 & 0.004 & 2.4 & 0.2 & 0.0016 & 0.0010 & 1.1 & 0.1 & 120 & 0 & 0.04 & 0.02 \\
\hline BY89 & $\begin{array}{l}\text { Poore Mine pit lake nr } \\
\text { Grass Valley }\end{array}$ & 4/1/1999 & $16: 50$ & 2of 2 & 0.067 & 0.012 & 2.4 & 0.2 & 0.0017 & 0.0003 & 1.1 & 0.0 & 120 & 10 & 0.04 & 0.00 \\
\hline BY89 & $\begin{array}{l}\text { Poore Mine pit lake nr } \\
\text { Grass Valley }\end{array}$ & $5 / 23 / 2000$ & $10: 45$ & 1 of 2 & 0.20 & 0.01 & 11 & 1 & 0.0027 & 0.0013 & 5.9 & 0.1 & 1,300 & 8 & 0.05 & 0.02 \\
\hline BY89 & $\begin{array}{l}\text { Poore Mine pit lake nr } \\
\text { Grass Valley }\end{array}$ & $5 / 23 / 2000$ & $10: 45$ & 2of 2 & 0.20 & 0.01 & 11 & 0 & 0.0030 & 0.0003 & 6.1 & 0.1 & 1,300 & 6 & 0.08 & 0.00 \\
\hline BY90 & $\begin{array}{l}\text { Poore Mine seep } \\
\text { above ground sluice } \\
\text { nr Grass Valley }\end{array}$ & 4/1/1999 & $16: 00$ & 1 of 2 & - & & - & & - & & - & & - & & - & \\
\hline BY90 & $\begin{array}{l}\text { Poore Mine seep } \\
\text { above ground sluice } \\
\text { nr Grass Valley }\end{array}$ & 4/1/1999 & $16: 00$ & 2of 2 & - & & - & & - & & - & & - & & - & \\
\hline BY91 & $\begin{array}{l}\text { Poore Mine tunnel } \\
\text { effluent nr Grass } \\
\text { Valley }\end{array}$ & 3/31/1999 & $15: 20$ & 1of 2 & 0.095 & 0.003 & 8.0 & 0.4 & $<0.0009$ & 0.0005 & 3.5 & 0.1 & 10 & 0 & 0.12 & 0.05 \\
\hline BY91 & $\begin{array}{l}\text { Poore Mine tunnel } \\
\text { effluent nr Grass } \\
\text { Valley }\end{array}$ & $3 / 31 / 1999$ & $15: 20$ & 2 of 2 & 0.11 & 0.01 & 8.2 & 0.2 & 0.0010 & 0.0002 & 3.6 & 0.1 & 10 & 0 & 0.10 & 0.02 \\
\hline BY91 & $\begin{array}{l}\text { Poore Mine tunnel } \\
\text { effluent nr Grass } \\
\text { Valley }\end{array}$ & $12 / 15 / 1999$ & 13:00 & 1of 2 & 0.13 & 0.02 & 8.3 & 0.4 & 0.002 & 0.001 & 5.1 & 0.0 & 9 & 1 & 0.42 & 0.11 \\
\hline BY91 & $\begin{array}{l}\text { Poore Mine tunnel } \\
\text { effluent nr Grass } \\
\text { Valley }\end{array}$ & $12 / 15 / 1999$ & $13: 00$ & 2of2 & 0.12 & 0.04 & 11 & 0 & 0.001 & 0.001 & 5.7 & 0.1 & 31 & 38 & 0.47 & 0.05 \\
\hline BY91 & $\begin{array}{l}\text { Poore Mine tunnel } \\
\text { effluent nr Grass } \\
\text { Valley }\end{array}$ & $5 / 23 / 2000$ & $12: 00$ & 1of 2 & 1.5 & 0.0 & 10 & 0 & 0.016 & 0.001 & 5.1 & 0.1 & 130 & 7 & 0.17 & 0.01 \\
\hline BY91 & $\begin{array}{l}\text { Poore Mine tunnel } \\
\text { effluent nr Grass } \\
\text { Valley }\end{array}$ & $5 / 23 / 2000$ & $12: 00$ & 2of 2 & 1.4 & 0.1 & 10 & 1 & 0.016 & 0.001 & 5.2 & 0.1 & 120 & 4 & 0.17 & 0.04 \\
\hline
\end{tabular}


Table 4A. Concentrations of trace metals and selected major elements in unfiltered water samples, Greenhorn Creek drainage, Nevada County, California-Continued.

[Station name abbreviations: ab, above; bl, below; Cr, Creek; mi, miles; N, north; nr, near; Rd, Road; S, south; SF, South Fork. Number in parentheses is the data parameter code, a five-digit code used in the

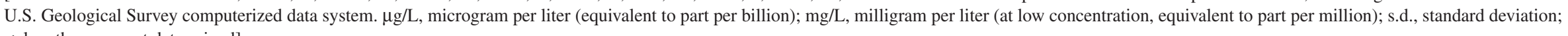
$<$, less than; - - not determined]

\begin{tabular}{|c|c|c|c|c|c|c|c|c|c|c|c|c|c|c|c|c|}
\hline \multirow[t]{2}{*}{$\begin{array}{l}\text { Station } \\
\text { map ID }\end{array}$} & \multirow[t]{2}{*}{ Station name } & \multirow[t]{2}{*}{ Date } & \multirow[t]{2}{*}{ Time } & \multirow[t]{2}{*}{$\begin{array}{l}\text { Rep- } \\
\text { licate }\end{array}$} & \multicolumn{2}{|c|}{$\begin{array}{c}\text { Lanthanum (La), } \\
\text { unfiltered } \\
(\mu \mathrm{g} / \mathrm{L}) \\
(\mathbf{0 1 1 8 2}) \\
\end{array}$} & \multicolumn{2}{|c|}{$\begin{array}{c}\text { Lithium (Li), } \\
\text { unfiltered } \\
(\mu \mathrm{g} / \mathrm{L}) \\
(\mathbf{0 1 1 3 2}) \\
\end{array}$} & \multicolumn{2}{|c|}{$\begin{array}{c}\text { Lutetium (Lu), } \\
\text { unfiltered } \\
(\mu \mathrm{g} / \mathrm{L}) \\
(\mathbf{0 1 2 4 4 )} \\
\end{array}$} & \multicolumn{2}{|c|}{$\begin{array}{l}\text { Magnesium }(\mathrm{Mg}) \text {, } \\
\text { unfiltered } \\
(\mathrm{mg} / \mathrm{L}) \\
(00921) \\
\end{array}$} & \multicolumn{2}{|c|}{$\begin{array}{c}\text { Manganese }(\mathrm{Mn}), \\
\text { unfiltered } \\
(\mu \mathrm{g} / \mathrm{L}) \\
(01055) \\
\end{array}$} & \multicolumn{2}{|c|}{$\begin{array}{c}\text { Molybdenum (Mo), } \\
\text { unfiltered } \\
(\mu \mathrm{g} / \mathrm{L}) \\
(01062)\end{array}$} \\
\hline & & & & & value & s.d. & value & s.d. & value & s.d. & value & s.d. & value & s.d. & value & s.d. \\
\hline BY105 & $\begin{array}{l}\text { Sailor Flat Mine main } \\
\text { drain gulch } 01 \mathrm{nr} \\
\text { Quaker Hill }\end{array}$ & 7/6/1999 & $16: 00$ & 1of 2 & 3.8 & 0.1 & 7.8 & 0.2 & 0.12 & 0.01 & 2.0 & 0.0 & 150 & 0 & 0.18 & 0.05 \\
\hline BY105 & $\begin{array}{l}\text { Sailor Flat Mine main } \\
\text { drain gulch } 01 \mathrm{nr} \\
\text { Quaker Hill }\end{array}$ & 7/6/1999 & $16: 00$ & 2of 2 & 2.7 & 0.1 & 7.8 & 0.1 & 0.083 & 0.005 & 2.0 & 0.0 & 150 & 0 & 0.18 & 0.02 \\
\hline BY105 & $\begin{array}{l}\text { Sailor Flat Mine main } \\
\text { drain Gulch } 01 \mathrm{nr} \\
\text { Quaker Hill }\end{array}$ & $12 / 2 / 1999$ & $14: 30$ & 1of 2 & 19 & 1 & 3.8 & 0.1 & 0.20 & 0.00 & 2.2 & 0.2 & 91 & 29 & 0.27 & 0.05 \\
\hline BY105 & $\begin{array}{l}\text { Sailor Flat Mine main } \\
\text { drain Gulch } 01 \mathrm{nr} \\
\text { Quaker Hill }\end{array}$ & $12 / 2 / 1999$ & $14: 30$ & 2of2 & 21 & 1 & 4.2 & 0.5 & 0.21 & 0.00 & 2.0 & 0.1 & 80 & 16 & 0.21 & 0.01 \\
\hline BY106 & $\begin{array}{l}\text { Sailor Flat Mine } \\
\text { main drainage to } \\
\text { Greenhorn Cr, } \\
\text { Gulch } 03\end{array}$ & $4 / 9 / 2001$ & $12: 30$ & 1 of 2 & 0.99 & 0.03 & 1.2 & 0.1 & 0.025 & 0.000 & 0.39 & 0.01 & 21 & 2 & $<0.3$ & 0.0 \\
\hline BY106 & $\begin{array}{l}\text { Sailor Flat Mine } \\
\text { main drainage to } \\
\text { Greenhorn } \mathrm{Cr} \text {, } \\
\text { Gulch } 03\end{array}$ & $4 / 9 / 2001$ & $12: 30$ & 2 of 2 & 0.96 & 0.02 & 1.1 & 0.1 & 0.023 & 0.000 & 0.39 & 0.06 & 20 & 1 & $<0.3$ & 0.2 \\
\hline BY114 & $\begin{array}{l}\text { SF Greenhorn Cr } 0.7 \\
\text { mi ab Greenhorn Cr } \\
\text { nr Nevada City }\end{array}$ & $8 / 22 / 2000$ & $15: 10$ & 1of 2 & 0.032 & 0.004 & 0.6 & 0.1 & 0.001 & 0.001 & 2.3 & 0.2 & $<0.6$ & 0 & $<0.1$ & 0.1 \\
\hline BY114 & $\begin{array}{l}\text { SF Greenhorn Cr } 0.7 \\
\text { mi ab Greenhorn Cr } \\
\text { nr Nevada City }\end{array}$ & $8 / 22 / 2000$ & $15: 10$ & 2of2 & 0.037 & 0.005 & 0.7 & 0.2 & $<0.001$ & 0.001 & 2.1 & 0.6 & $<0.6$ & 0 & 0.3 & 0.2 \\
\hline BY122 & $\begin{array}{l}\text { Starr Mine tunnel } \\
\text { inflow nr Grass } \\
\text { Valley }\end{array}$ & $4 / 23 / 1999$ & $15: 00$ & 1of 2 & 0.14 & 0.01 & 4.7 & 0.2 & 0.0014 & 0.0005 & 4.5 & 0.4 & 51 & 4 & 0.06 & 0.02 \\
\hline BY122 & $\begin{array}{l}\text { Starr Mine tunnel } \\
\text { inflow nr Grass } \\
\text { Valley }\end{array}$ & $4 / 23 / 1999$ & $15: 00$ & 2of2 & 0.17 & 0.01 & 5.7 & 0.3 & 0.0025 & 0.0004 & 4.9 & 0.1 & 58 & 1 & 0.06 & 0.02 \\
\hline BY123 & $\begin{array}{l}\text { Starr Mine tunnel } \\
\text { midway nr Grass } \\
\text { Valley }\end{array}$ & 4/23/1999 & $13: 40$ & 1of 2 & 0.12 & 0.03 & 10 & 1 & 0.0018 & 0.0007 & 8.1 & 0.1 & 300 & 10 & 0.23 & 0.01 \\
\hline BY123 & $\begin{array}{l}\text { Starr Mine tunnel } \\
\text { midway nr Grass } \\
\text { Valley }\end{array}$ & 4/23/1999 & $13: 40$ & 2of2 & 0.080 & 0.014 & 9.8 & 0.3 & 0.0017 & 0.0006 & 8.3 & 0.2 & 280 & 10 & 0.25 & 0.01 \\
\hline
\end{tabular}


Table 4A. Concentrations of trace metals and selected major elements in unfiltered water samples, Greenhorn Creek drainage, Nevada County, California—Continued.

[Station name abbreviations: ab, above; bl, below; Cr, Creek; mi, miles; N, north; nr, near; Rd, Road; S, south; SF, South Fork. Number in parentheses is the data parameter code, a five-digit code used in the

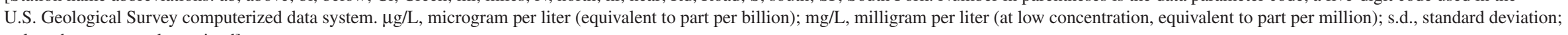
$<$, less than; - , not determined

\begin{tabular}{|c|c|c|c|c|c|c|c|c|c|c|c|c|c|c|c|c|}
\hline \multirow[t]{2}{*}{$\begin{array}{l}\text { Station } \\
\text { map ID }\end{array}$} & \multirow[t]{2}{*}{ Station name } & \multirow[t]{2}{*}{ Date } & \multirow[t]{2}{*}{ Time } & \multirow[t]{2}{*}{$\begin{array}{l}\text { Rep- } \\
\text { licate }\end{array}$} & \multicolumn{2}{|c|}{$\begin{array}{c}\text { Lanthanum (La), } \\
\text { unfiltered } \\
(\mu \mathrm{g} / \mathrm{L}) \\
(\mathbf{0 1 1 8 2}) \\
\end{array}$} & \multicolumn{2}{|c|}{$\begin{array}{c}\text { Lithium }(\mathrm{Li}), \\
\text { unfiltered } \\
(\mu \mathrm{g} / \mathrm{L}) \\
(\mathbf{0 1 1 3 2}) \\
\end{array}$} & \multicolumn{2}{|c|}{$\begin{array}{c}\text { Lutetium (Lu), } \\
\text { unfiltered } \\
(\mu \mathrm{g} / \mathrm{L}) \\
(01244) \\
\end{array}$} & \multicolumn{2}{|c|}{$\begin{array}{c}\text { Magnesium (Mg), } \\
\text { unfiltered } \\
(\mathrm{mg} / \mathrm{L}) \\
(00921) \\
\end{array}$} & \multicolumn{2}{|c|}{$\begin{array}{c}\text { Manganese (Mn), } \\
\text { unfiltered } \\
(\mu \mathrm{g} / \mathrm{L}) \\
(01055) \\
\end{array}$} & \multicolumn{2}{|c|}{$\begin{array}{c}\text { Molybdenum (Mo), } \\
\text { unfiltered } \\
(\mu \mathrm{g} / \mathrm{L}) \\
(\mathbf{0 1 0 6 2}) \\
\end{array}$} \\
\hline & & & & & value & s.d. & value & s.d. & value & s.d. & value & s.d. & value & s.d. & value & s.d. \\
\hline BY123 & $\begin{array}{l}\text { Starr Mine tunnel } \\
\text { midway nr Grass } \\
\text { Valley }\end{array}$ & $12 / 15 / 1999$ & $10: 45$ & 1of 2 & 0.16 & 0.04 & 11 & 1 & 0.005 & 0.001 & 10 & 0 & 730 & 20 & 0.38 & 0.05 \\
\hline BY123 & $\begin{array}{l}\text { Starr Mine tunnel } \\
\text { midway nr Grass } \\
\text { Valley }\end{array}$ & $12 / 15 / 1999$ & $10: 45$ & 2 of 2 & 0.17 & 0.01 & 8.8 & 0.6 & 0.005 & 0.001 & 9.9 & 0.4 & 710 & 20 & 0.29 & 0.01 \\
\hline BY124 & $\begin{array}{l}\text { Starr Mine tunnel } \\
\text { outlet nr Grass } \\
\text { Valley }\end{array}$ & $8 / 23 / 2000$ & $17: 30$ & 1of 2 & 0.56 & 0.01 & 8.4 & 0.7 & 0.007 & 0.001 & 8.7 & 0.2 & 560 & 10 & 0.6 & 0.2 \\
\hline BY124 & $\begin{array}{l}\text { Starr Mine tunnel } \\
\text { outlet nr Grass } \\
\text { Valley }\end{array}$ & $8 / 23 / 2000$ & $17: 30$ & 2 of 2 & 0.66 & 0.00 & 8.6 & 0.5 & 0.011 & 0.000 & 8.4 & 0.2 & 630 & 30 & 0.6 & 0.4 \\
\hline BY129 & $\begin{array}{l}\text { Tom and Jerry Mine } \\
\text { drain at tunnel inlet } \\
\text { nr Nevada City }\end{array}$ & $6 / 28 / 2000$ & $12: 00$ & 1 of 2 & 9.5 & 1.7 & 6.3 & 1.2 & 0.087 & 0.015 & 2.4 & 0.6 & 650 & 20 & $<0.1$ & 0.2 \\
\hline BY129 & $\begin{array}{l}\text { Tom and Jerry Mine } \\
\text { drain at tunnel inlet } \\
\text { nr Nevada City }\end{array}$ & $6 / 28 / 2000$ & $12: 00$ & 2of 2 & 9.1 & 1.3 & 6.6 & 0.3 & 0.091 & 0.015 & 2.6 & 0.3 & 740 & 40 & $<0.1$ & 0.0 \\
\hline BY130 & $\begin{array}{l}\text { Tom and Jerry Mine } \\
\text { drain at tunnel } \\
\text { outlet nr Nevada } \\
\text { City }\end{array}$ & $6 / 28 / 2000$ & $11: 30$ & 1of 2 & 0.14 & 0.01 & 11 & 2 & $<0.001$ & 0.001 & 5.2 & 0.5 & 89 & 1 & $<0.1$ & 0.0 \\
\hline BY130 & $\begin{array}{l}\text { Tom and Jerry Mine } \\
\text { drain at tunnel } \\
\text { outlet nr Nevada } \\
\text { City }\end{array}$ & $6 / 28 / 2000$ & $11: 30$ & 2 of 2 & 0.16 & 0.02 & 13 & 0 & 0.002 & 0.001 & 5.4 & 1.0 & 96 & 9 & 0.1 & 0.2 \\
\hline BY131 & $\begin{array}{l}\text { Tom and Jerry Mine } \\
\text { drainage pond nr } \\
\text { Nevada City }\end{array}$ & $8 / 23 / 2000$ & $10: 45$ & 1of 2 & 0.18 & 0.02 & 1.2 & 0.1 & 0.002 & 0.001 & 2.6 & 0.2 & 7 & 0 & $<0.1$ & 0.2 \\
\hline BY131 & $\begin{array}{l}\text { Tom and Jerry Mine } \\
\text { drainage pond nr } \\
\text { Nevada City }\end{array}$ & $8 / 23 / 2000$ & $10: 45$ & 2of 2 & 0.18 & 0.02 & 1.5 & 0.0 & 0.004 & 0.001 & 2.4 & 0.6 & 7 & 1 & $<0.1$ & 0.1 \\
\hline
\end{tabular}


Table 4A. Concentrations of trace metals and selected major elements in unfiltered water samples, Greenhorn Creek drainage, Nevada County, California—Continued

[Station name abbreviations: ab, above; bl, below; Cr, Creek; mi, miles; N, north; nr, near; Rd, Road; S, south; SF, South Fork. Number in parentheses is the data parameter code, a five-digit code used in the

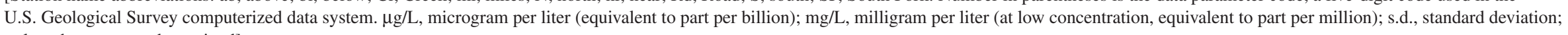
$<$, less than; - , not determined

\begin{tabular}{|c|c|c|c|c|c|c|c|c|c|c|c|c|c|c|c|c|}
\hline \multirow[t]{2}{*}{$\begin{array}{l}\text { Station } \\
\text { map ID }\end{array}$} & \multirow[t]{2}{*}{ Station name } & \multirow[t]{2}{*}{ Date } & \multirow[t]{2}{*}{ Time } & \multirow[t]{2}{*}{$\begin{array}{l}\text { Rep- } \\
\text { licate }\end{array}$} & \multicolumn{2}{|c|}{$\begin{array}{c}\text { Lanthanum (La), } \\
\text { unfiltered } \\
(\mu \mathrm{g} / \mathrm{L}) \\
(\mathbf{0 1 1 8 2})\end{array}$} & \multicolumn{2}{|c|}{$\begin{array}{c}\text { Lithium }(\mathrm{Li}), \\
\text { unfiltered } \\
(\mu \mathrm{g} / \mathrm{L}) \\
(\mathbf{0 1 1 3 2})\end{array}$} & \multicolumn{2}{|c|}{$\begin{array}{c}\text { Lutetium (Lu), } \\
\text { unfiltered } \\
(\mu \mathrm{g} / \mathrm{L}) \\
(\mathbf{0 1 2 4 4 )} \\
\end{array}$} & \multicolumn{2}{|c|}{$\begin{array}{l}\text { Magnesium }(\mathrm{Mg}) \text {, } \\
\text { unfiltered } \\
(\mathrm{mg} / \mathrm{L}) \\
(00921) \\
\end{array}$} & \multicolumn{2}{|c|}{$\begin{array}{c}\text { Manganese }(\mathrm{Mn}), \\
\text { unfiltered } \\
(\mu \mathrm{g} / \mathrm{L}) \\
(01055) \\
\end{array}$} & \multicolumn{2}{|c|}{$\begin{array}{c}\text { Molybdenum (Mo), } \\
\text { unfiltered } \\
(\mu \mathrm{g} / \mathrm{L}) \\
(01062)\end{array}$} \\
\hline & & & & & value & s.d. & value & s.d. & value & s.d. & value & s.d. & value & s.d. & value & s.d. \\
\hline \multicolumn{17}{|c|}{ Disturbed samples } \\
\hline BY123 & $\begin{array}{l}\text { Starr Mine tunnel } \\
\text { midway nr Grass } \\
\text { Valley }\end{array}$ & $12 / 15 / 1999$ & $11: 30$ & 1 of 2 & 48 & 9 & 74 & 1 & 1.7 & 0.4 & 36 & 0 & 430,000 & 0 & 18 & 0 \\
\hline BY123 & $\begin{array}{l}\text { Starr Mine tunnel } \\
\text { midway nr Grass } \\
\text { Valley }\end{array}$ & $12 / 15 / 1999$ & $11: 30$ & 2 of 2 & 48 & 1 & 67 & 3 & 1.9 & 0.0 & 33 & 1 & 390,000 & 10,000 & 19 & 1 \\
\hline BY129 & $\begin{array}{l}\text { Tom and Jerry Mine } \\
\text { drain at tunnel inlet } \\
\text { nr Nevada City }\end{array}$ & $6 / 28 / 2000$ & $12: 30$ & 1 of 2 & 84 & 12 & 32 & 5 & 0.81 & 0.13 & 12 & 0 & 7,600 & 400 & 0.2 & 0.0 \\
\hline BY129 & $\begin{array}{l}\text { Tom and Jerry Mine } \\
\text { drain at tunnel inlet } \\
\text { nr Nevada City }\end{array}$ & $6 / 28 / 2000$ & $12: 30$ & $2 \mathrm{of} 2$ & 93 & 4 & 35 & 1 & 0.80 & 0.13 & 16 & 3 & 8,100 & 0 & 0.1 & 0.1 \\
\hline
\end{tabular}


Table 4A. Concentrations of trace metals and selected major elements in unfiltered water samples, Greenhorn Creek drainage, Nevada County, California—Continued.

[Station name abbreviations: ab, above; bl, below; Cr, Creek; mi, miles; N, north; nr, near; Rd, Road; S, south; SF, South Fork. Number in parentheses is the data parameter code, a five-digit code used in the

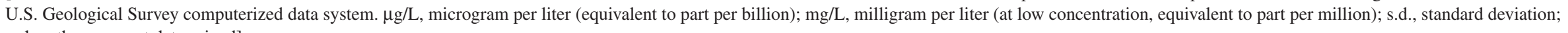

$<$, less than; - , not determined]

\begin{tabular}{|c|c|c|c|c|c|c|c|c|c|c|c|c|c|c|c|c|c|c|}
\hline \multirow[t]{2}{*}{$\begin{array}{l}\text { Station } \\
\text { map ID }\end{array}$} & \multirow[t]{2}{*}{ Station name } & \multirow[t]{2}{*}{ Date } & \multirow[t]{2}{*}{ Time } & \multirow[t]{2}{*}{$\begin{array}{l}\text { Rep- } \\
\text { licate }\end{array}$} & \multicolumn{2}{|c|}{$\begin{array}{c}\text { Sodium (Na), } \\
\text { unfiltered } \\
(\mathrm{mg} / \mathrm{L}) \\
(00923)\end{array}$} & \multicolumn{2}{|c|}{$\begin{array}{c}\text { Neodymium } \\
\text { (Nd), } \\
\text { unfiltered } \\
(\mu \mathrm{g} / \mathrm{L}) \\
(01237)\end{array}$} & \multicolumn{2}{|c|}{$\begin{array}{c}\text { Nickel (Ni), } \\
\text { unfiltered } \\
(\mu \mathrm{g} / \mathrm{L}) \\
(01067)\end{array}$} & \multicolumn{2}{|c|}{$\begin{array}{c}\text { Lead }(\mathrm{Pb}) \text {, } \\
\text { unfiltered } \\
(\mu \mathrm{g} / \mathrm{L}) \\
(\mathbf{0 1 0 5 1 )}\end{array}$} & \multicolumn{2}{|c|}{$\begin{array}{c}\text { Praesodymium } \\
\text { (Pr), } \\
\text { unfiltered } \\
(\mu \mathrm{g} / \mathrm{L}) \\
(010238)\end{array}$} & \multicolumn{2}{|c|}{$\begin{array}{c}\text { Rubidium (Rb), } \\
\text { unfiltered } \\
(\mu \mathrm{g} / \mathrm{L}) \\
(\mathbf{0 1 1 3 7})\end{array}$} & \multicolumn{2}{|c|}{$\begin{array}{c}\text { Rhenium }(\mathrm{Re}), \\
\text { unfiltered } \\
(\mu \mathrm{g} / \mathrm{L}) \\
(\mathbf{0 1 2 4 2})\end{array}$} \\
\hline & & & & & value & s.d. & value & s.d. & value & s.d. & value & s.d. & value & s.d. & value & s.d. & value & s.d. \\
\hline \multicolumn{19}{|c|}{ Environmental samples } \\
\hline BY20 & $\begin{array}{l}\text { Boston Mine tunnel } \\
\text { outlet nr Grass } \\
\text { Valley }\end{array}$ & $6 / 18 / 1999$ & $14: 30$ & 1 of 2 & 1.2 & 0.0 & 0.090 & 0.010 & 10 & 0 & 0.05 & 0.01 & 0.021 & 0.004 & 2.0 & 0.3 & 0.0038 & 0.0006 \\
\hline BY20 & $\begin{array}{l}\text { Boston Mine tunnel } \\
\text { outlet nr Grass } \\
\text { Valley }\end{array}$ & 6/18/1999 & $14: 30$ & $2 o f 2$ & 1.4 & 0.2 & 0.074 & 0.002 & 7.1 & 0.4 & 0.06 & 0.02 & 0.014 & 0.001 & 1.6 & 0.2 & 0.0024 & 0.0011 \\
\hline BY20 & $\begin{array}{l}\text { Boston Mine tunnel } \\
\text { outlet nr Grass } \\
\text { Valley }\end{array}$ & $12 / 14 / 1999$ & $12: 00$ & 1 of 2 & 1.4 & 0.0 & 0.70 & 0.06 & 37 & 3 & 0.16 & 0.12 & 0.15 & 0.02 & 2.2 & 0.2 & 0.004 & 0.000 \\
\hline BY20 & $\begin{array}{l}\text { Boston Mine tunnel } \\
\text { outlet nr Grass } \\
\text { Valley }\end{array}$ & $12 / 14 / 1999$ & $12: 00$ & 2 of 2 & 1.5 & 0.1 & 0.35 & 0.01 & 22 & 1 & 0.14 & 0.02 & 0.080 & 0.008 & 1.7 & 0.1 & 0.003 & 0.001 \\
\hline BY20 & $\begin{array}{l}\text { Boston Mine tunnel } \\
\text { outlet nr Grass } \\
\text { Valley }\end{array}$ & $5 / 24 / 2000$ & $16: 00$ & 1 of 2 & 1.4 & 0.1 & 0.14 & 0.00 & 8.8 & 0.2 & 0.08 & 0.02 & 0.030 & 0.001 & 1.7 & 0.0 & 0.005 & 0.000 \\
\hline BY20 & $\begin{array}{l}\text { Boston Mine tunnel } \\
\text { outlet nr Grass } \\
\text { Valley }\end{array}$ & $5 / 24 / 2000$ & $16: 00$ & $2 \mathrm{of} 2$ & 1.4 & 0.1 & 0.31 & 0.03 & 17 & 1 & 0.07 & 0.01 & 0.065 & 0.001 & 1.8 & 0.1 & $<0.002$ & 0.000 \\
\hline BY21 & $\begin{array}{l}\text { Boston Mine wetlands } \\
\text { pond nr Grass } \\
\text { Valley }\end{array}$ & $12 / 14 / 1999$ & $10: 30$ & 1 of 2 & 3.0 & 0.3 & 0.024 & 0.005 & 0.92 & 0.07 & 0.11 & 0.02 & 0.006 & 0.001 & 2.6 & 0.0 & 0.011 & 0.000 \\
\hline BY21 & $\begin{array}{l}\text { Boston Mine wetlands } \\
\text { pond nr Grass } \\
\text { Valley }\end{array}$ & $12 / 14 / 1999$ & $10: 30$ & 2of 2 & 1.4 & 0.1 & 0.027 & 0.006 & 0.95 & 0.07 & 0.14 & 0.01 & 0.007 & 0.001 & 2.6 & 0.2 & 0.007 & 0.002 \\
\hline BY21 & $\begin{array}{l}\text { Boston Mine wetlands } \\
\text { pond nr Grass } \\
\text { Valley }\end{array}$ & $5 / 24 / 2000$ & $16: 30$ & 1 of 2 & 1.3 & 0.1 & 0.032 & 0.006 & 2.2 & 0.0 & 0.12 & 0.03 & 0.007 & 0.001 & 2.2 & 0.1 & 0.005 & 0.002 \\
\hline BY21 & $\begin{array}{l}\text { Boston Mine wetlands } \\
\text { pond nr Grass } \\
\text { Valley }\end{array}$ & $5 / 24 / 2000$ & $16: 30$ & $2 \mathrm{of} 2$ & 1.3 & 0.1 & 0.033 & 0.007 & 2.2 & 0.1 & 0.10 & 0.01 & 0.007 & 0.000 & 2.2 & 0.1 & 0.003 & 0.001 \\
\hline BY58 & $\begin{array}{l}\text { Buckeye Flat Mine } \\
\text { main drain } 0.45 \mathrm{mi} \\
\text { ab Greenhorn } \mathrm{Cr}\end{array}$ & $8 / 21 / 2000$ & 11:00 & 1 of 2 & 3 & 1 & 16 & 0 & 130 & 10 & $<1$ & 0 & 4.0 & 0.3 & 9.3 & 0.2 & $<0.02$ & 0.01 \\
\hline BY58 & $\begin{array}{l}\text { Buckeye Flat Mine } \\
\text { main drain } 0.45 \mathrm{mi} \\
\text { ab Greenhorn } \mathrm{Cr}\end{array}$ & $8 / 21 / 2000$ & 11:00 & 2 of 2 & 2 & 0 & 13 & 0 & 93 & 3 & $<1$ & 1 & 3.2 & 0.0 & 7.2 & 0.3 & $<0.02$ & 0.02 \\
\hline
\end{tabular}


Table 4A. Concentrations of trace metals and selected major elements in unfiltered water samples, Greenhorn Creek drainage, Nevada County, California-Continued.

[Station name abbreviations: ab, above; bl, below; Cr, Creek; mi, miles; N, north; nr, near; Rd, Road; S, south; SF, South Fork. Number in parentheses is the data parameter code, a five-digit code used in the

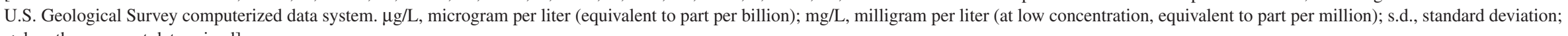
$<$, less than; - - not determined]

\begin{tabular}{|c|c|c|c|c|c|c|c|c|c|c|c|c|c|c|c|c|c|c|}
\hline \multirow[t]{2}{*}{$\begin{array}{l}\text { Station } \\
\text { map ID }\end{array}$} & \multirow[t]{2}{*}{ Station name } & \multirow[t]{2}{*}{ Date } & \multirow[t]{2}{*}{ Time } & \multirow[t]{2}{*}{$\begin{array}{l}\text { Rep- } \\
\text { licate }\end{array}$} & \multicolumn{2}{|c|}{$\begin{array}{c}\text { Sodium (Na), } \\
\text { unfiltered } \\
(\mathrm{mg} / \mathrm{L}) \\
(00923)\end{array}$} & \multicolumn{2}{|c|}{$\begin{array}{l}\text { Neodymium } \\
(\mathrm{Nd}), \\
\text { unfiltered } \\
(\mu \mathrm{g} / \mathrm{L}) \\
(01237)\end{array}$} & \multicolumn{2}{|c|}{$\begin{array}{c}\text { Nickel (Ni), } \\
\text { unfiltered } \\
(\mu \mathrm{g} / \mathrm{L}) \\
(\mathbf{0 1 0 6 7 )}\end{array}$} & \multicolumn{2}{|c|}{$\begin{array}{c}\text { Lead }(\mathrm{Pb}) \text {, } \\
\text { unfiltered } \\
(\mu \mathrm{g} / \mathrm{L}) \\
(01051)\end{array}$} & \multicolumn{2}{|c|}{$\begin{array}{c}\text { Praesodymium } \\
(\mathrm{Pr}), \\
\text { unfiltered } \\
(\mu \mathrm{g} / \mathrm{L}) \\
(\mathbf{0 1 0 2 3 8 )}\end{array}$} & \multicolumn{2}{|c|}{$\begin{array}{c}\text { Rubidium (Rb), } \\
\text { unfiltered } \\
(\mu \mathrm{g} / \mathrm{L}) \\
(\mathbf{0 1 1 3 7})\end{array}$} & \multicolumn{2}{|c|}{$\begin{array}{c}\text { Rhenium (Re), } \\
\text { unfiltered } \\
(\mu \mathrm{g} / \mathrm{L}) \\
(\mathbf{0 1 2 4 2})\end{array}$} \\
\hline & & & & & value & s.d. & value & s.d. & value & s.d. & value & s.d. & value & s.d. & value & s.d. & value & s.d. \\
\hline BY23 & $\begin{array}{c}\text { Buckeye Flat Mine N } \\
\text { drain to Greenhorn } \\
\text { Cr, Grass Valley }\end{array}$ & 6/18/1999 & $11: 30$ & 1 of 2 & 2.4 & 0.0 & 22 & 1 & 220 & 0 & 0.46 & 0.01 & 5.1 & 0.5 & 4.3 & 0.4 & 0.0027 & 0.0012 \\
\hline BY23 & $\begin{array}{c}\text { Buckeye Flat Mine N } \\
\text { drain to Greenhorn } \\
\text { Cr, Grass Valley }\end{array}$ & 6/18/1999 & $11: 30$ & $2 o f 2$ & 2.3 & 0.0 & 22 & 0 & 220 & 0 & 0.64 & 0.04 & 4.8 & 0.0 & 3.7 & 0.1 & 0.0034 & 0.0007 \\
\hline BY23 & $\begin{array}{c}\text { Buckeye Flat Mine N } \\
\text { drain to Greenhorn } \\
\text { Cr, Grass Valley }\end{array}$ & $12 / 2 / 1999$ & 11:00 & 1of 2 & 1.9 & 0.2 & 16 & 0 & 130 & 10 & 0.61 & 0.08 & 3.6 & 0.1 & 3.5 & 0.3 & 0.007 & 0.001 \\
\hline BY23 & $\begin{array}{c}\text { Buckeye Flat Mine N } \\
\text { drain to Greenhorn } \\
\text { Cr, Grass Valley }\end{array}$ & $12 / 2 / 1999$ & $11: 00$ & $2 o f 2$ & 2.1 & 0.2 & 16 & 0 & 130 & 0 & 0.84 & 0.12 & 4.4 & 0.1 & 2.9 & 0.0 & 0.006 & 0.000 \\
\hline BY23 & $\begin{array}{c}\text { Buckeye Flat Mine N } \\
\text { drain to Greenhorn } \\
\text { Cr, Grass Valley }\end{array}$ & $8 / 22 / 2000$ & $10: 45$ & 1 of 2 & 4 & 1 & 25 & 0 & 170 & 10 & $<1$ & 0 & 6.0 & 0.1 & 2.1 & 0.1 & $<0.02$ & 0.01 \\
\hline BY23 & $\begin{array}{c}\text { Buckeye Flat Mine N } \\
\text { drain to Greenhorn } \\
\text { Cr, Grass Valley }\end{array}$ & $8 / 22 / 2000$ & $10: 45$ & $2 o f 2$ & 4 & 1 & 22 & 0 & 160 & 10 & $<1$ & 0 & 5.6 & 0.0 & 2.0 & 0.3 & $<0.02$ & 0.02 \\
\hline BY116 & $\begin{array}{l}\text { Buckeye Flat Mine } \\
\text { pond drain } 0.15 \mathrm{mi} \\
\text { ab SF Greenhorn } \mathrm{Cr}\end{array}$ & $8 / 21 / 2000$ & $14: 10$ & 1 of 2 & 0.55 & 0.03 & 0.19 & 0.01 & 4.5 & 0.3 & $<0.1$ & 0.1 & 0.047 & 0.002 & 2.0 & 0.0 & 0.003 & 0.001 \\
\hline BY116 & $\begin{array}{l}\text { Buckeye Flat Mine } \\
\text { pond drain } 0.15 \mathrm{mi} \\
\text { ab SF Greenhorn } \mathrm{Cr}\end{array}$ & $8 / 21 / 2000$ & $14: 10$ & $2 o f 2$ & 0.54 & 0.03 & 0.20 & 0.01 & 5.2 & 0.3 & $<0.1$ & 0.1 & 0.048 & 0.002 & 2.0 & 0.0 & $<0.003$ & 0.002 \\
\hline BY24 & $\begin{array}{l}\text { Buckeye Flat Mine } \\
\text { S drain to SF } \\
\text { Greenhorn } \mathrm{Cr} \text {, } \\
\text { Grass Valley }\end{array}$ & $8 / 26 / 1999$ & 11:00 & 1 of 2 & 0.66 & 0.11 & 0.50 & 0.01 & 8.5 & 0.7 & 3.9 & 0.2 & 0.11 & 0.01 & 1.2 & 0.3 & 0.0015 & 0.0010 \\
\hline BY24 & $\begin{array}{l}\text { Buckeye Flat Mine } \\
\text { S drain to SF } \\
\text { Greenhorn } \mathrm{Cr} \text {, } \\
\text { Grass Valley }\end{array}$ & $8 / 26 / 1999$ & 11:00 & $2 o f 2$ & 0.62 & 0.01 & 0.50 & 0.01 & 9.2 & 1.0 & 4.0 & 0.3 & 0.11 & 0.01 & 1.0 & 0.2 & 0.0016 & 0.0008 \\
\hline BY24 & $\begin{array}{l}\text { Buckeye Flat Mine } \\
\text { S drain to SF } \\
\text { Greenhorn } \mathrm{Cr} \text {, } \\
\text { Grass Valley }\end{array}$ & $8 / 22 / 2000$ & $12: 10$ & 1 of 2 & 0.64 & 0.12 & 1.4 & 0.1 & 10.0 & 1.6 & 0.9 & 0.0 & 0.31 & 0.05 & 1.1 & 0.1 & $<0.003$ & 0.001 \\
\hline BY24 & $\begin{array}{l}\text { Buckeye Flat Mine } \\
\text { S drain to SF } \\
\text { Greenhorn } \mathrm{Cr} \text {, } \\
\text { Grass Valley }\end{array}$ & $8 / 22 / 2000$ & $12: 10$ & $2 o f 2$ & 0.70 & 0.02 & 1.3 & 0.2 & 9.6 & 0.5 & 0.9 & 0.1 & 0.30 & 0.05 & 0.99 & 0.05 & 0.004 & 0.001 \\
\hline
\end{tabular}


Table 4A. Concentrations of trace metals and selected major elements in unfiltered water samples, Greenhorn Creek drainage, Nevada County, California—Continued.

[Station name abbreviations: ab, above; bl, below; Cr, Creek; mi, miles; N, north; nr, near; Rd, Road; S, south; SF, South Fork. Number in parentheses is the data parameter code, a five-digit code used in the

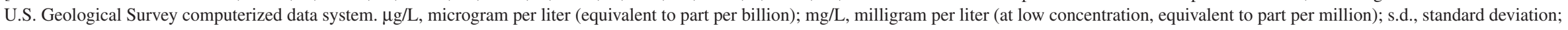
$<$, less than; - - not determined]

\begin{tabular}{|c|c|c|c|c|c|c|c|c|c|c|c|c|c|c|c|c|c|c|}
\hline \multirow[t]{2}{*}{$\begin{array}{l}\text { Station } \\
\text { map ID }\end{array}$} & \multirow[t]{2}{*}{ Station name } & \multirow[t]{2}{*}{ Date } & \multirow[t]{2}{*}{ Time } & \multirow[t]{2}{*}{$\begin{array}{l}\text { Rep- } \\
\text { licate }\end{array}$} & \multicolumn{2}{|c|}{$\begin{array}{c}\text { Sodium }(\mathrm{Na}) \\
\text { unfiltered } \\
(\mathrm{mg} / \mathrm{L}) \\
(00923)\end{array}$} & \multicolumn{2}{|c|}{$\begin{array}{l}\text { Neodymium } \\
(\mathbf{N d}), \\
\text { unfiltered } \\
(\mu \mathrm{g} / \mathrm{L}) \\
(\mathbf{0 1 2 3 7})\end{array}$} & \multicolumn{2}{|c|}{$\begin{array}{c}\text { Nickel (Ni), } \\
\text { unfiltered } \\
(\mu \mathrm{g} / \mathrm{L}) \\
(\mathbf{0 1 0 6 7 )}\end{array}$} & \multicolumn{2}{|c|}{$\begin{array}{c}\text { Lead }(\mathrm{Pb}) \\
\text { unfiltered } \\
(\mu \mathrm{g} / \mathrm{L}) \\
(01051)\end{array}$} & \multicolumn{2}{|c|}{$\begin{array}{c}\text { Praesodymium } \\
(\mathrm{Pr}), \\
\text { unfiltered } \\
(\mu \mathrm{g} / \mathrm{L}) \\
(\mathbf{0 1 0 2 3 8 )}\end{array}$} & \multicolumn{2}{|c|}{$\begin{array}{c}\text { Rubidium (Rb), } \\
\text { unfiltered } \\
(\mu \mathrm{g} / \mathrm{L}) \\
(01137)\end{array}$} & \multicolumn{2}{|c|}{$\begin{array}{c}\text { Rhenium (Re), } \\
\text { unfiltered } \\
(\mu \mathrm{g} / \mathrm{L}) \\
(01242)\end{array}$} \\
\hline & & & & & value & s.d. & value & s.d. & value & s.d. & value & s.d. & value & s.d. & value & s.d. & value & s.d. \\
\hline BY25 & $\begin{array}{l}\text { Buckeye Flat Mine } \\
\text { upper drain }\end{array}$ & $4 / 9 / 2001$ & $10: 00$ & 1of 2 & 1.3 & 0.3 & 4.1 & 0.3 & 65 & 4 & $<0.2$ & 0.1 & 1.0 & 0.1 & 1.4 & 0.1 & $<0.003$ & 0.001 \\
\hline BY25 & $\begin{array}{l}\text { Buckeye Flat Mine } \\
\text { upper drain }\end{array}$ & $4 / 9 / 2001$ & $10: 00$ & 2 of 2 & 1.4 & 0.4 & 4.0 & 0.1 & 66 & 0 & $<0.2$ & 0.2 & 1.0 & 0.0 & 1.4 & 0.0 & $<0.003$ & 0.001 \\
\hline BY52 & $\begin{array}{l}\text { Greenhorn Cr } 0.3 \mathrm{mi} \\
\text { ab Buckeye Ford nr } \\
\text { Nevada City }\end{array}$ & $1 / 25 / 2000$ & $10: 45$ & 1 of 2 & 1.4 & 0.1 & 1.2 & 0.0 & 5.8 & 0.2 & 0.55 & 0.01 & 0.27 & 0.00 & 1.7 & 0.0 & $<0.002$ & 0.002 \\
\hline BY52 & $\begin{array}{l}\text { Greenhorn Cr } 0.3 \mathrm{mi} \\
\text { ab Buckeye Ford nr } \\
\text { Nevada City }\end{array}$ & $1 / 25 / 2000$ & $10: 45$ & $2 \mathrm{of} 2$ & 1.4 & 0.0 & 1.1 & 0.0 & 6.0 & 0.1 & 0.51 & 0.02 & 0.27 & 0.01 & 1.7 & 0.0 & $<0.002$ & 0.001 \\
\hline BY59 & $\begin{array}{l}\text { Greenhorn Cr at You } \\
\text { Bet Rd nr Nevada } \\
\text { City }\end{array}$ & $7 / 24 / 2001$ & $11: 00$ & 1 of 2 & 2.4 & 0.1 & 0.05 & 0.00 & 1.9 & 0.1 & 0.10 & 0.04 & 0.013 & 0.002 & 1.5 & 0.1 & 0.005 & 0.001 \\
\hline BY59 & $\begin{array}{l}\text { Greenhorn } \mathrm{Cr} \text { at You } \\
\text { Bet Rd nr Nevada } \\
\text { City }\end{array}$ & $7 / 24 / 2001$ & 11:00 & 2 of 2 & 2.4 & 0.2 & 0.08 & 0.00 & 2.1 & 0.1 & 0.11 & 0.00 & 0.014 & 0.002 & 1.5 & 0.0 & 0.006 & 0.001 \\
\hline BY59 & $\begin{array}{l}\text { Greenhorn } \mathrm{Cr} \text { at You } \\
\text { Bet Rd nr Nevada } \\
\text { City }\end{array}$ & $8 / 29 / 2001$ & $15: 00$ & 1 of 2 & 2.5 & 0.1 & 0.03 & 0.01 & 0.96 & 0.06 & $<0.06$ & 0.02 & 0.011 & 0.001 & 1.4 & 0.1 & 0.007 & 0.001 \\
\hline BY59 & $\begin{array}{l}\text { Greenhorn Cr at You } \\
\text { Bet Rd nr Nevada } \\
\text { City }\end{array}$ & $8 / 29 / 2001$ & $15: 00$ & 2 of 2 & 2.5 & 0.3 & 0.03 & 0.01 & 1.0 & 0.0 & 0.06 & 0.05 & 0.009 & 0.002 & 1.5 & 0.1 & 0.007 & 0.002 \\
\hline BY59 & $\begin{array}{l}\text { Greenhorn Cr at You } \\
\text { Bet Rd nr Nevada } \\
\text { City }\end{array}$ & $9 / 14 / 2001$ & 13:00 & 1of 2 & 2.0 & 0.1 & 0.048 & 0.008 & 1.6 & 0.1 & 0.07 & 0.00 & 0.011 & 0.003 & 1.5 & 0.1 & 0.005 & 0.001 \\
\hline BY59 & $\begin{array}{l}\text { Greenhorn } \mathrm{Cr} \text { at You } \\
\text { Bet Rd nr Nevada } \\
\text { City }\end{array}$ & $9 / 14 / 2001$ & $13: 00$ & 2 of 2 & 2.1 & 0.2 & 0.042 & 0.008 & 1.5 & 0.0 & 0.06 & 0.01 & 0.011 & 0.002 & 1.5 & 0.1 & 0.007 & 0.004 \\
\hline BY59 & $\begin{array}{l}\text { Greenhorn Cr at You } \\
\text { Bet Rd nr Nevada } \\
\text { City }\end{array}$ & $10 / 15 / 2001$ & $12: 00$ & 1 of 2 & 3.0 & 0.4 & 0.020 & 0.010 & 1.5 & 0.2 & 0.19 & 0.18 & 0.004 & 0.003 & 1.2 & 0.1 & 0.005 & 0.002 \\
\hline BY59 & $\begin{array}{l}\text { Greenhorn } \mathrm{Cr} \text { at You } \\
\text { Bet Rd nr Nevada } \\
\text { City }\end{array}$ & $10 / 15 / 2001$ & $12: 00$ & 2 of 2 & 3.0 & 0.3 & 0.021 & 0.008 & 1.4 & 0.1 & $<0.07$ & 0.04 & 0.005 & 0.001 & 1.2 & 0.1 & 0.005 & 0.002 \\
\hline BY59 & $\begin{array}{l}\text { Greenhorn } \mathrm{Cr} \text { at You } \\
\text { Bet Rd nr Nevada } \\
\text { City }\end{array}$ & $11 / 20 / 2001$ & $13: 20$ & 1 of 2 & 2.8 & 0.4 & 0.16 & 0.02 & 3.5 & 0.0 & 0.19 & 0.03 & 0.050 & 0.005 & 1.6 & 0.0 & 0.006 & 0.000 \\
\hline BY59 & $\begin{array}{l}\text { Greenhorn } \mathrm{Cr} \text { at You } \\
\text { Bet Rd nr Nevada } \\
\text { City }\end{array}$ & $11 / 20 / 2001$ & $13: 20$ & 2 of 2 & 2.8 & 0.3 & 0.14 & 0.01 & 3.5 & 0.1 & 0.23 & 0.08 & 0.035 & 0.000 & 1.7 & 0.1 & 0.006 & 0.001 \\
\hline
\end{tabular}


Table 4A. Concentrations of trace metals and selected major elements in unfiltered water samples, Greenhorn Creek drainage, Nevada County, California-Continued.

[Station name abbreviations: ab, above; bl, below; Cr, Creek; mi, miles; N, north; nr, near; Rd, Road; S, south; SF, South Fork. Number in parentheses is the data parameter code, a five-digit code used in the

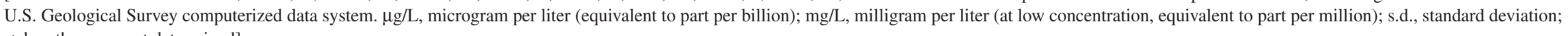
$<$, less than; - - not determined]

\begin{tabular}{|c|c|c|c|c|c|c|c|c|c|c|c|c|c|c|c|c|c|c|}
\hline \multirow[t]{2}{*}{$\begin{array}{l}\text { Station } \\
\text { map ID }\end{array}$} & \multirow[t]{2}{*}{ Station name } & \multirow[t]{2}{*}{ Date } & \multirow[t]{2}{*}{ Time } & \multirow[t]{2}{*}{$\begin{array}{l}\text { Rep- } \\
\text { licate }\end{array}$} & \multicolumn{2}{|c|}{$\begin{array}{c}\text { Sodium (Na), } \\
\text { unfiltered } \\
(\mathrm{mg} / \mathrm{L}) \\
(00923)\end{array}$} & \multicolumn{2}{|c|}{$\begin{array}{l}\text { Neodymium } \\
(\mathrm{Nd}), \\
\text { unfiltered } \\
(\mu \mathrm{g} / \mathrm{L}) \\
(\mathbf{0 1 2 3 7})\end{array}$} & \multicolumn{2}{|c|}{$\begin{array}{c}\text { Nickel (Ni), } \\
\text { unfiltered } \\
(\mu \mathrm{g} / \mathrm{L}) \\
(01067)\end{array}$} & \multicolumn{2}{|c|}{$\begin{array}{c}\text { Lead }(\mathrm{Pb}) \text {, } \\
\text { unfiltered } \\
(\mu \mathrm{g} / \mathrm{L}) \\
(01051)\end{array}$} & \multicolumn{2}{|c|}{$\begin{array}{c}\text { Praesodymium } \\
(\mathrm{Pr}), \\
\text { unfiltered } \\
(\mu \mathrm{g} / \mathrm{L}) \\
(\mathbf{0 1 0 2 3 8})\end{array}$} & \multicolumn{2}{|c|}{$\begin{array}{c}\text { Rubidium (Rb), } \\
\text { unfiltered } \\
(\mu \mathbf{g} / \mathbf{L}) \\
(\mathbf{0 1 1 3 7})\end{array}$} & \multicolumn{2}{|c|}{$\begin{array}{c}\text { Rhenium (Re), } \\
\text { unfiltered } \\
(\mu \mathrm{g} / \mathrm{L}) \\
(\mathbf{0 1 2 4 2})\end{array}$} \\
\hline & & & & & value & s.d. & value & s.d. & value & s.d. & value & s.d. & value & s.d. & value & s.d. & value & s.d. \\
\hline BY59 & $\begin{array}{l}\text { Greenhorn Cr at You } \\
\text { Bet Rd nr Nevada } \\
\text { City }\end{array}$ & $12 / 10 / 2001$ & $12: 20$ & 1of 2 & 2.5 & 0.2 & 1.2 & 0.0 & 6.6 & 0.5 & 0.57 & 0.01 & 0.28 & 0.00 & 2.1 & 0.0 & 0.005 & 0.002 \\
\hline BY59 & $\begin{array}{l}\text { Greenhorn Cr at You } \\
\text { Bet Rd nr Nevada } \\
\text { City }\end{array}$ & $12 / 10 / 2001$ & $12: 20$ & 2 of 2 & 2.6 & 0.2 & 1.2 & 0.1 & 6.8 & 0.5 & 0.46 & 0.01 & 0.27 & 0.01 & 2.2 & 0.1 & 0.005 & 0.000 \\
\hline BY59 & $\begin{array}{l}\text { Greenhorn Cr at You } \\
\text { Bet Rd nr Nevada } \\
\text { City }\end{array}$ & $12 / 21 / 2001$ & $13: 00$ & 1of 2 & 1.9 & 0.2 & 4.7 & 0.3 & 11.4 & 0.7 & 2.8 & 0.0 & 1.06 & 0.09 & 6.6 & 0.5 & $<0.004$ & 0.002 \\
\hline BY59 & $\begin{array}{l}\text { Greenhorn Cr at You } \\
\text { Bet Rd nr Nevada } \\
\text { City }\end{array}$ & $12 / 21 / 2001$ & $13: 00$ & $2 \mathrm{of} 2$ & 1.9 & 0.3 & 4.9 & 0.0 & 11.8 & 0.1 & 2.7 & 0.0 & 1.04 & 0.09 & 6.5 & 0.7 & $<0.004$ & 0.001 \\
\hline BY51 & $\begin{array}{l}\text { Greenhorn } \mathrm{Cr} n r \\
\text { headwaters nr } \\
\text { Scotts Flat Res }\end{array}$ & $1 / 25 / 2000$ & $9: 30$ & 1 of 2 & 1.4 & 0.0 & 0.35 & 0.01 & 2.0 & 0.0 & 0.17 & 0.02 & 0.079 & 0.001 & 0.53 & 0.02 & $<0.002$ & 0.001 \\
\hline BY51 & $\begin{array}{l}\text { Greenhorn } \mathrm{Cr} n r \\
\text { headwaters nr } \\
\text { Scotts Flat Res }\end{array}$ & $1 / 25 / 2000$ & $9: 30$ & $2 \mathrm{of} 2$ & 0.85 & 0.07 & 0.33 & 0.01 & 2.0 & 0.1 & 0.13 & 0.01 & 0.078 & 0.003 & 0.54 & 0.01 & $<0.002$ & 0.001 \\
\hline BY75 & $\begin{array}{l}\text { Missouri Cyn Cr } 1.2 \\
\text { mi ab Greenhorn Cr } \\
\text { nr Chicago Park }\end{array}$ & $12 / 14 / 1999$ & $14: 00$ & 1of 2 & 3.0 & 0.1 & 0.43 & 0.05 & 29 & 1 & 0.11 & 0.04 & 0.10 & 0.02 & 3.3 & 0.0 & 0.006 & 0.001 \\
\hline BY75 & $\begin{array}{l}\text { Missouri Cyn Cr } 1.2 \\
\text { mi ab Greenhorn Cr } \\
\text { nr Chicago Park }\end{array}$ & $12 / 14 / 1999$ & $14: 00$ & 2of 2 & 2.7 & 0.0 & 0.44 & 0.02 & 29 & 0 & 0.12 & 0.03 & 0.11 & 0.01 & 4.0 & 0.7 & 0.007 & 0.001 \\
\hline BY86 & $\begin{array}{l}\text { Poore Mine Creek } \\
\text { ab tunnel nr Grass } \\
\text { Valley }\end{array}$ & 3/31/1999 & $17: 40$ & 1of 2 & 1.9 & 0.1 & 0.35 & 0.01 & 3.0 & 0.4 & 0.25 & 0.05 & 0.085 & 0.009 & 1.2 & 0.2 & $<0.001$ & 0.0010 \\
\hline BY86 & $\begin{array}{l}\text { Poore Mine Creek } \\
\text { ab tunnel nr Grass } \\
\text { Valley }\end{array}$ & 3/31/1999 & $17: 40$ & $2 \mathrm{of} 2$ & 2.0 & 0.2 & 0.33 & 0.01 & 2.5 & 0.2 & 0.20 & 0.02 & 0.077 & 0.006 & 1.3 & 0.2 & $<0.001$ & 0.0012 \\
\hline BY87 & $\begin{array}{l}\text { Poore Mine Creek } \\
\text { bl tunnel nr Grass } \\
\text { Valley }\end{array}$ & 3/31/1999 & $13: 30$ & 1 of 2 & 1.8 & 0.0 & 0.34 & 0.01 & 2.5 & 0.3 & 0.21 & 0.04 & 0.078 & 0.004 & 1.2 & 0.0 & $<0.001$ & 0.0008 \\
\hline BY87 & $\begin{array}{l}\text { Poore Mine Creek } \\
\text { bl tunnel nr Grass } \\
\text { Valley }\end{array}$ & 3/31/1999 & $13: 30$ & 2 of 2 & 1.9 & 0.1 & 0.36 & 0.00 & 2.7 & 0.2 & 0.29 & 0.01 & 0.086 & 0.001 & 1.1 & 0.2 & 0.0015 & 0.0013 \\
\hline BY88 & $\begin{array}{l}\text { Poore Mine ground } \\
\text { sluice nr Grass } \\
\text { Valley }\end{array}$ & 4/1/1999 & $15: 40$ & 1 of 2 & 1.7 & 0.2 & 0.11 & 0.00 & 10 & 1 & $<0.01$ & 0.03 & 0.024 & 0.004 & 7.2 & 1.8 & 0.011 & 0.000 \\
\hline
\end{tabular}


Table 4A. Concentrations of trace metals and selected major elements in unfiltered water samples, Greenhorn Creek drainage, Nevada County, California—Continued.

[Station name abbreviations: ab, above; bl, below; Cr, Creek; mi, miles; N, north; nr, near; Rd, Road; S, south; SF, South Fork. Number in parentheses is the data parameter code, a five-digit code used in the

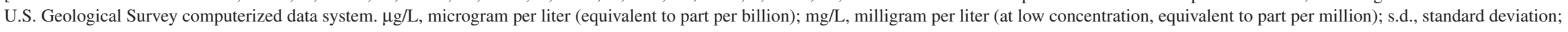

$<$, less than; - , not determined

\begin{tabular}{|c|c|c|c|c|c|c|c|c|c|c|c|c|c|c|c|c|c|c|}
\hline \multirow[t]{2}{*}{$\begin{array}{l}\text { Station } \\
\text { map ID }\end{array}$} & \multirow[t]{2}{*}{ Station name } & \multirow[t]{2}{*}{ Date } & \multirow[t]{2}{*}{ Time } & \multirow[t]{2}{*}{$\begin{array}{l}\text { Rep- } \\
\text { licate }\end{array}$} & \multicolumn{2}{|c|}{$\begin{array}{l}\text { Sodium }(\mathrm{Na}), \\
\text { unfiltered } \\
(\mathrm{mg} / \mathrm{L}) \\
(00923)\end{array}$} & \multicolumn{2}{|c|}{$\begin{array}{c}\text { Neodymium } \\
\text { (Nd), } \\
\text { unfiltered } \\
(\mu \mathrm{g} / \mathrm{L}) \\
(01237) \\
\end{array}$} & \multicolumn{2}{|c|}{$\begin{array}{c}\text { Nickel (Ni), } \\
\text { unfiltered } \\
(\mu \mathrm{g} / \mathrm{L}) \\
(01067)\end{array}$} & \multicolumn{2}{|c|}{$\begin{array}{l}\text { Lead }(\mathrm{Pb}) \\
\text { unfiltered } \\
(\mu \mathrm{g} / \mathrm{L}) \\
(01051)\end{array}$} & \multicolumn{2}{|c|}{$\begin{array}{c}\text { Praesodymium } \\
\text { (Pr), } \\
\text { unfiltered } \\
(\mu \mathrm{g} / \mathrm{L}) \\
(\mathbf{0 1 0 2 3 8 )}\end{array}$} & \multicolumn{2}{|c|}{$\begin{array}{c}\text { Rubidium }(\mathbf{R b}), \\
\text { unfiltered } \\
(\mu \mathrm{g} / \mathrm{L}) \\
(\mathbf{0 1 1 3 7})\end{array}$} & \multicolumn{2}{|c|}{$\begin{array}{c}\text { Rhenium (Re), } \\
\text { unfiltered } \\
(\mu \mathrm{g} / \mathrm{L}) \\
(01242)\end{array}$} \\
\hline & & & & & value & s.d. & value & s.d. & value & s.d. & value & s.d. & value & s.d. & value & s.d. & value & s.d. \\
\hline BY88 & $\begin{array}{l}\text { Poore Mine ground } \\
\text { sluice nr Grass } \\
\text { Valley }\end{array}$ & $4 / 1 / 1999$ & $15: 40$ & 2 of 2 & 1.6 & 0.1 & 0.12 & 0.00 & 11 & 1 & $<0.01$ & 0.02 & 0.026 & 0.002 & 6.1 & 0.1 & 0.011 & 0.003 \\
\hline BY89 & $\begin{array}{l}\text { Poore Mine pit lake nr } \\
\text { Grass Valley }\end{array}$ & 4/1/1999 & $16: 50$ & 1 of 2 & 0.9 & 0.1 & 0.10 & 0.00 & 3.4 & 0.3 & 0.07 & 0.02 & 0.021 & 0.002 & 2.4 & 0.3 & $<0.001$ & 0.0013 \\
\hline BY89 & $\begin{array}{l}\text { Poore Mine pit lake nr } \\
\text { Grass Valley }\end{array}$ & 4/1/1999 & $16: 50$ & 2 of 2 & 0.9 & 0.2 & 0.10 & 0.00 & 3.5 & 0.3 & 0.09 & 0.05 & 0.022 & 0.004 & 2.2 & 0.3 & 0.0015 & 0.0003 \\
\hline BY89 & $\begin{array}{l}\text { Poore Mine pit lake nr } \\
\text { Grass Valley }\end{array}$ & $5 / 23 / 2000$ & $10: 45$ & 1 of 2 & 1.4 & 0.1 & 0.15 & 0.01 & 61 & 0 & 0.07 & 0.02 & 0.037 & 0.003 & 7.7 & 0.1 & 0.005 & 0.000 \\
\hline BY89 & $\begin{array}{l}\text { Poore Mine pit lake nr } \\
\text { Grass Valley }\end{array}$ & $5 / 23 / 2000$ & $10: 45$ & 2 of 2 & 1.5 & 0.2 & 0.16 & 0.01 & 64 & 1 & 0.06 & 0.01 & 0.037 & 0.001 & 7.9 & 0.5 & 0.008 & 0.001 \\
\hline BY90 & $\begin{array}{l}\text { Poore Mine seep } \\
\text { above ground sluice } \\
\text { nr Grass Valley }\end{array}$ & 4/1/1999 & $16: 00$ & 1 of 2 & - & & - & & - & & - & & - & & - & & - & \\
\hline BY90 & $\begin{array}{l}\text { Poore Mine seep } \\
\text { above ground sluice } \\
\text { nr Grass Valley }\end{array}$ & 4/1/1999 & $16: 00$ & 2 of 2 & - & & - & & - & & - & & - & & - & & - & \\
\hline BY91 & $\begin{array}{l}\text { Poore Mine tunnel } \\
\text { effluent nr Grass } \\
\text { Valley }\end{array}$ & $3 / 31 / 1999$ & $15: 20$ & 1 of 2 & 1.7 & 0.1 & 0.094 & 0.005 & 5.2 & 0.2 & 0.28 & 0.03 & 0.024 & 0.001 & 6.3 & 0.3 & 0.0063 & 0.0019 \\
\hline BY91 & $\begin{array}{l}\text { Poore Mine tunnel } \\
\text { effluent nr Grass } \\
\text { Valley }\end{array}$ & $3 / 31 / 1999$ & $15: 20$ & 2 of 2 & 1.7 & 0.1 & 0.099 & 0.007 & 6.3 & 0.5 & 0.35 & 0.06 & 0.024 & 0.001 & 5.7 & 0.7 & 0.0099 & 0.0003 \\
\hline BY91 & $\begin{array}{l}\text { Poore Mine tunnel } \\
\text { effluent nr Grass } \\
\text { Valley }\end{array}$ & $12 / 15 / 1999$ & $13: 00$ & 1 of 2 & 2.4 & 0.2 & 0.11 & 0.00 & 3.1 & 0.1 & 0.46 & 0.03 & 0.031 & 0.002 & 5.5 & 0.0 & 0.013 & 0.003 \\
\hline BY91 & $\begin{array}{l}\text { Poore Mine tunnel } \\
\text { effluent nr Grass } \\
\text { Valley }\end{array}$ & $12 / 15 / 1999$ & $13: 00$ & 2 of 2 & 2.9 & 0.3 & 0.13 & 0.01 & 2.7 & 0.4 & 0.44 & 0.18 & 0.028 & 0.003 & 4.7 & 0.6 & 0.009 & 0.001 \\
\hline BY91 & $\begin{array}{l}\text { Poore Mine tunnel } \\
\text { effluent nr Grass } \\
\text { Valley }\end{array}$ & $5 / 23 / 2000$ & $12: 00$ & 1 of 2 & 3.1 & 0.3 & 1.5 & 0.0 & 15 & 0 & 6.5 & 0.0 & 0.37 & 0.00 & 6.1 & 0.2 & 0.010 & 0.001 \\
\hline BY91 & $\begin{array}{l}\text { Poore Mine tunnel } \\
\text { effluent nr Grass } \\
\text { Valley }\end{array}$ & $5 / 23 / 2000$ & $12: 00$ & 2 of 2 & 3.0 & 0.2 & 1.4 & 0.0 & 14 & 0 & 6.2 & 0.1 & 0.35 & 0.00 & 6.1 & 0.0 & 0.010 & 0.001 \\
\hline BY105 & $\begin{array}{l}\text { Sailor Flat Mine main } \\
\text { drain gulch } 01 \mathrm{nr} \\
\text { Quaker Hill }\end{array}$ & 7/6/1999 & $16: 00$ & 1 of 2 & 1.7 & 0.1 & 6.5 & 0.0 & 42 & 5 & 1.0 & 0.1 & 1.6 & 0.3 & 4.1 & 0.8 & 0.0038 & 0.0005 \\
\hline
\end{tabular}


Table 4A. Concentrations of trace metals and selected major elements in unfiltered water samples, Greenhorn Creek drainage, Nevada County, California—Continued.

[Station name abbreviations: ab, above; bl, below; Cr, Creek; mi, miles; N, north; nr, near; Rd, Road; S, south; SF, South Fork. Number in parentheses is the data parameter code, a five-digit code used in the

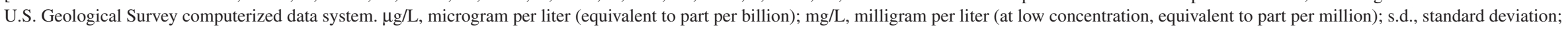
$<$, less than; - - not determined]

\begin{tabular}{|c|c|c|c|c|c|c|c|c|c|c|c|c|c|c|c|c|c|c|}
\hline \multirow[t]{2}{*}{$\begin{array}{l}\text { Station } \\
\text { map ID }\end{array}$} & \multirow[t]{2}{*}{ Station name } & \multirow[t]{2}{*}{ Date } & \multirow[t]{2}{*}{ Time } & \multirow[t]{2}{*}{$\begin{array}{l}\text { Rep- } \\
\text { licate }\end{array}$} & \multicolumn{2}{|c|}{$\begin{array}{c}\text { Sodium (Na), } \\
\text { unfiltered } \\
(\mathrm{mg} / \mathrm{L}) \\
(00923)\end{array}$} & \multicolumn{2}{|c|}{$\begin{array}{l}\text { Neodymium } \\
(\mathrm{Nd}), \\
\text { unfiltered } \\
(\mu \mathrm{g} / \mathrm{L}) \\
(\mathbf{0 1 2 3 7})\end{array}$} & \multicolumn{2}{|c|}{$\begin{array}{c}\text { Nickel (Ni), } \\
\text { unfiltered } \\
(\mu \mathrm{g} / \mathrm{L}) \\
(01067)\end{array}$} & \multicolumn{2}{|c|}{$\begin{array}{c}\text { Lead }(\mathrm{Pb}) \\
\text { unfiltered } \\
(\mu \mathrm{g} / \mathrm{L}) \\
(\mathbf{0 1 0 5 1 )}\end{array}$} & \multicolumn{2}{|c|}{$\begin{array}{c}\text { Praesodymium } \\
(\mathrm{Pr}), \\
\text { unfiltered } \\
(\mu \mathrm{g} / \mathrm{L}) \\
(\mathbf{0 1 0 2 3 8})\end{array}$} & \multicolumn{2}{|c|}{$\begin{array}{c}\text { Rubidium (Rb), } \\
\text { unfiltered } \\
(\mu \mathrm{g} / \mathrm{L}) \\
(\mathbf{0 1 1 3 7})\end{array}$} & \multicolumn{2}{|c|}{$\begin{array}{c}\text { Rhenium (Re), } \\
\text { unfiltered } \\
(\mu \mathrm{g} / \mathrm{L}) \\
(\mathbf{0 1 2 4 2 )}\end{array}$} \\
\hline & & & & & value & s.d. & value & s.d. & value & s.d. & value & s.d. & value & s.d. & value & s.d. & value & s.d. \\
\hline BY105 & $\begin{array}{l}\text { Sailor Flat Mine main } \\
\text { drain gulch } 01 \mathrm{nr} \\
\text { Quaker Hill }\end{array}$ & $7 / 6 / 1999$ & $16: 00$ & 2of 2 & 1.8 & 0.1 & 6.8 & 0.0 & 39 & 6 & 0.66 & 0.05 & 1.4 & 0.3 & 5.4 & 0.9 & 0.0025 & 0.0007 \\
\hline BY105 & $\begin{array}{l}\text { Sailor Flat Mine main } \\
\text { drain Gulch } 01 \mathrm{nr} \\
\text { Quaker Hill }\end{array}$ & $12 / 2 / 1999$ & $14: 30$ & 1 of 2 & 1.3 & 0.3 & 22 & 0 & 12 & 1 & 10 & 0 & 6.1 & 0.1 & 17 & 0 & $<0.002$ & 0.000 \\
\hline BY105 & $\begin{array}{l}\text { Sailor Flat Mine main } \\
\text { drain Gulch } 01 \mathrm{nr} \\
\text { Quaker Hill }\end{array}$ & $12 / 2 / 1999$ & $14: 30$ & 2 of 2 & 1.2 & 0.0 & 20 & 0 & 12 & 0 & 10 & 0 & 4.9 & 0.1 & 13 & 0 & $<0.002$ & 0.001 \\
\hline BY106 & $\begin{array}{l}\text { Sailor Flat Mine } \\
\text { main drainage to } \\
\text { Greenhorn Cr, } \\
\text { Gulch } 03\end{array}$ & $4 / 9 / 2001$ & $12: 30$ & 1 of 2 & 0.70 & 0.07 & 1.4 & 0.1 & 4.5 & 0.3 & $<0.2$ & 0.1 & 0.34 & 0.03 & 1.8 & 0.2 & $<0.003$ & 0.001 \\
\hline BY106 & $\begin{array}{l}\text { Sailor Flat Mine } \\
\text { main drainage to } \\
\text { Greenhorn Cr, } \\
\text { Gulch } 03\end{array}$ & 4/9/2001 & $12: 30$ & 2 of 2 & 0.73 & 0.16 & 1.4 & 0.0 & 4.7 & 0.9 & $<0.2$ & 0.1 & 0.34 & 0.01 & 1.8 & 0.1 & $<0.003$ & 0.002 \\
\hline BY114 & $\begin{array}{l}\text { SF Greenhorn Cr } 0.7 \\
\text { mi ab Greenhorn Cr } \\
\text { nr Nevada City }\end{array}$ & $8 / 22 / 2000$ & $15: 10$ & 1 of 2 & 2.0 & 0.3 & 0.03 & 0.01 & 0.7 & 0.1 & $<0.1$ & 0.1 & 0.007 & 0.002 & 0.44 & 0.06 & $<0.003$ & 0.000 \\
\hline BY114 & $\begin{array}{l}\text { SF Greenhorn Cr } 0.7 \\
\text { mi ab Greenhorn Cr } \\
\text { nr Nevada City }\end{array}$ & $8 / 22 / 2000$ & $15: 10$ & 2 of 2 & 2.0 & 0.2 & 0.03 & 0.00 & 1.2 & 0.1 & 0.4 & 0.0 & 0.008 & 0.001 & 0.45 & 0.06 & 0.004 & 0.002 \\
\hline BY122 & $\begin{array}{l}\text { Starr Mine tunnel } \\
\text { inflow nr Grass } \\
\text { Valley }\end{array}$ & $4 / 23 / 1999$ & $15: 00$ & 1of 2 & 1.5 & 0.1 & 0.17 & 0.00 & 11 & 1 & 1.5 & 0.0 & 0.037 & 0.003 & 3.0 & 0.1 & 0.0057 & 0.0005 \\
\hline BY122 & $\begin{array}{l}\text { Starr Mine tunnel } \\
\text { inflow nr Grass } \\
\text { Valley }\end{array}$ & 4/23/1999 & $15: 00$ & 2of 2 & 1.6 & 0.1 & 0.19 & 0.00 & 13 & 0 & 1.8 & 0.0 & 0.043 & 0.005 & 3.0 & 0.3 & 0.0067 & 0.0013 \\
\hline BY123 & $\begin{array}{l}\text { Starr Mine tunnel } \\
\text { midway nr Grass } \\
\text { Valley }\end{array}$ & 4/23/1999 & $13: 40$ & 1 of 2 & 2.2 & 0.1 & 0.13 & 0.01 & 3.5 & 0.2 & 0.79 & 0.01 & 0.032 & 0.004 & 4.6 & 0.1 & 0.0092 & 0.0012 \\
\hline BY123 & $\begin{array}{l}\text { Starr Mine tunnel } \\
\text { midway nr Grass } \\
\text { Valley }\end{array}$ & $4 / 23 / 1999$ & $13: 40$ & 2of 2 & 2.2 & 0.1 & 0.086 & 0.003 & 3.0 & 0.6 & 0.60 & 0.08 & 0.020 & 0.002 & 4.7 & 0.1 & 0.010 & 0.003 \\
\hline BY123 & $\begin{array}{l}\text { Starr Mine tunnel } \\
\text { midway nr Grass } \\
\text { Valley }\end{array}$ & $12 / 15 / 1999$ & $10: 45$ & 1of 2 & 2.2 & 0.1 & 0.23 & 0.00 & 7.5 & 0.5 & 2.1 & 0.1 & 0.050 & 0.001 & 6.2 & 0.2 & 0.021 & 0.002 \\
\hline
\end{tabular}


Table 4A. Concentrations of trace metals and selected major elements in unfiltered water samples, Greenhorn Creek drainage, Nevada County, California—Continued.

[Station name abbreviations: ab, above; bl, below; Cr, Creek; mi, miles; N, north; nr, near; Rd, Road; S, south; SF, South Fork. Number in parentheses is the data parameter code, a five-digit code used in the

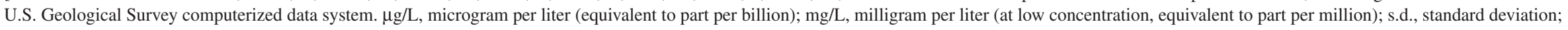

$<$, less than; - , not determined]

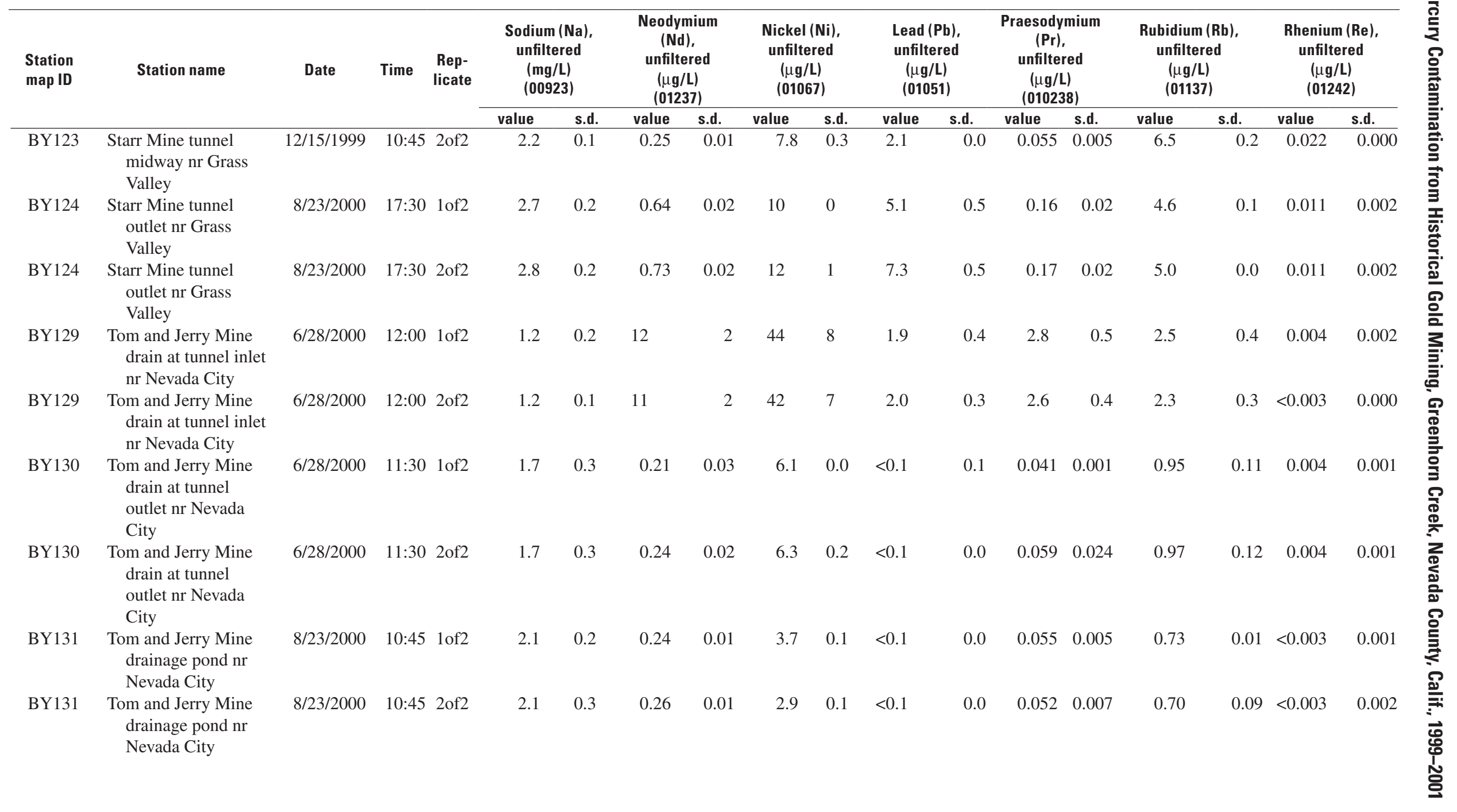


Table 4A. Concentrations of trace metals and selected major elements in unfiltered water samples, Greenhorn Creek drainage, Nevada County, California—Continued

[Station name abbreviations: ab, above; bl, below; Cr, Creek; mi, miles; N, north; nr, near; Rd, Road; S, south; SF, South Fork. Number in parentheses is the data parameter code, a five-digit code used in the

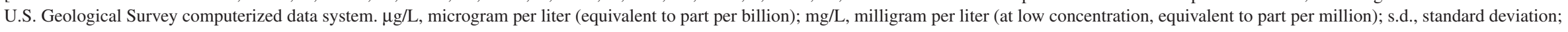
$<$, less than; - - not determined]

\begin{tabular}{|c|c|c|c|c|c|c|c|c|c|c|c|c|c|c|c|c|c|c|}
\hline \multirow[t]{2}{*}{$\begin{array}{l}\text { Station } \\
\text { map ID }\end{array}$} & \multirow[t]{2}{*}{ Station name } & \multirow[t]{2}{*}{ Date } & \multirow[t]{2}{*}{ Time } & \multirow[t]{2}{*}{$\begin{array}{l}\text { Rep- } \\
\text { licate }\end{array}$} & \multicolumn{2}{|c|}{$\begin{array}{c}\text { Sodium (Na), } \\
\text { unfiltered } \\
(\mathrm{mg} / \mathrm{L}) \\
(00923)\end{array}$} & \multicolumn{2}{|c|}{$\begin{array}{l}\text { Neodymium } \\
(\mathrm{Nd}), \\
\text { unfiltered } \\
(\mu \mathrm{g} / \mathrm{L}) \\
(\mathbf{0 1 2 3 7})\end{array}$} & \multicolumn{2}{|c|}{$\begin{array}{c}\text { Nickel (Ni), } \\
\text { unfiltered } \\
(\mu \mathrm{g} / \mathrm{L}) \\
(01067)\end{array}$} & \multicolumn{2}{|c|}{$\begin{array}{c}\text { Lead }(\mathrm{Pb}) \text {, } \\
\text { unfiltered } \\
(\mu \mathrm{g} / \mathrm{L}) \\
(\mathbf{0 1 0 5 1 )}\end{array}$} & \multicolumn{2}{|c|}{$\begin{array}{c}\text { Praesodymium } \\
(\mathrm{Pr}), \\
\text { unfiltered } \\
(\mu \mathrm{g} / \mathrm{L}) \\
(\mathbf{0 1 0 2 3 8 )}\end{array}$} & \multicolumn{2}{|c|}{$\begin{array}{c}\text { Rubidium (Rb), } \\
\text { unfiltered } \\
(\mu \mathrm{g} / \mathrm{L}) \\
(\mathbf{0 1 1 3 7})\end{array}$} & \multicolumn{2}{|c|}{$\begin{array}{c}\text { Rhenium (Re), } \\
\text { unfiltered } \\
(\mu \mathrm{g} / \mathrm{L}) \\
(\mathbf{0 1 2 4 2})\end{array}$} \\
\hline & & & & & value & s.d. & value & s.d. & value & s.d. & value & s.d. & value & s.d. & value & s.d. & value & s.d. \\
\hline \multicolumn{19}{|c|}{ Disturbed samples } \\
\hline BY123 & $\begin{array}{l}\text { Starr Mine tunnel } \\
\text { midway nr Grass } \\
\text { Valley }\end{array}$ & $12 / 15 / 1999$ & $11: 30$ & 1 of 2 & 2.9 & 0.0 & 85 & 0 & 3,700 & 0 & 980 & 140 & 17 & 0 & 92 & 18 & 0.036 & 0.003 \\
\hline BY123 & $\begin{array}{l}\text { Starr Mine tunnel } \\
\text { midway nr Grass } \\
\text { Valley }\end{array}$ & $12 / 15 / 1999$ & $11: 30$ & 2of 2 & 2.6 & 0.1 & 85 & 3 & 3,300 & 200 & 1,000 & 0 & 16 & 0 & 86 & 0 & 0.030 & 0.004 \\
\hline BY129 & $\begin{array}{l}\text { Tom and Jerry Mine } \\
\text { drain at tunnel inlet } \\
\text { nr Nevada City }\end{array}$ & $6 / 28 / 2000$ & $12: 30$ & 1 of 2 & 1.6 & 0.2 & 120 & 20 & 350 & 10 & 25 & 4 & 28 & 4 & 23 & 3 & 0.003 & 0.000 \\
\hline BY129 & $\begin{array}{l}\text { Tom and Jerry Mine } \\
\text { drain at tunnel inlet } \\
\text { nr Nevada City }\end{array}$ & $6 / 28 / 2000$ & $12: 30$ & 2of 2 & 1.5 & 0.3 & 140 & 10 & 370 & 50 & 28 & 1 & 32 & 2 & 23 & 3 & 0.003 & 0.002 \\
\hline
\end{tabular}


Table 4A. Concentrations of trace metals and selected major elements in unfiltered water samples, Greenhorn Creek drainage, Nevada County, California—Continued.

[Station name abbreviations: ab, above; bl, below; Cr, Creek; mi, miles; N, north; nr, near; Rd, Road; S, south; SF, South Fork. Number in parentheses is the data parameter code, a five-digit code used in the

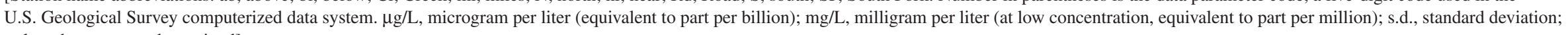

$<$, less than; - , not determined]

\begin{tabular}{|c|c|c|c|c|c|c|c|c|c|c|c|c|c|c|c|c|c|c|}
\hline \multirow[t]{2}{*}{$\begin{array}{l}\text { Station } \\
\text { map ID }\end{array}$} & \multirow[t]{2}{*}{ Station name } & \multirow[t]{2}{*}{ Date } & \multirow[t]{2}{*}{ Time } & \multirow[t]{2}{*}{$\begin{array}{l}\text { Rep- } \\
\text { licate }\end{array}$} & \multicolumn{2}{|c|}{$\begin{array}{c}\text { Sulfur }(\mathrm{S}) \text {, } \\
\text { unfiltered } \\
(\mathbf{m g} / \mathrm{L}) \\
(\mathbf{8 0 1 0 7 )}\end{array}$} & \multicolumn{2}{|c|}{$\begin{array}{c}\text { Antimony }(\mathrm{Sb}), \\
\text { unfiltered } \\
(\mu \mathrm{g} / \mathrm{L}) \\
(\mathbf{0 1 0 9 7 )} \\
\end{array}$} & \multicolumn{2}{|c|}{$\begin{array}{c}\text { Selenium (Se), } \\
\text { unfiltered } \\
(\mu \mathrm{g} / \mathrm{L}) \\
(01147) \\
\end{array}$} & \multicolumn{2}{|c|}{$\begin{array}{c}\text { Silica }(\mathrm{Si}) \\
\text { unfiltered } \\
(\mathrm{mg} / \mathrm{L} \text { as } \mathrm{SiO}) \\
(00956)\end{array}$} & \multicolumn{2}{|c|}{$\begin{array}{c}\text { Samarium }(\mathrm{Sm}), \\
\text { unfiltered } \\
(\mu \mathrm{g} / \mathrm{L}) \\
(\mathbf{8 2 3 2 2}) \\
\end{array}$} & \multicolumn{2}{|c|}{$\begin{array}{c}\text { Strontium }(\mathrm{Sr}), \\
\text { unfiltered } \\
(\mu \mathrm{g} / \mathrm{L}) \\
(\mathbf{0 1 0 8 4}) \\
\end{array}$} & \multicolumn{2}{|c|}{$\begin{array}{c}\text { Terbium (Tb), } \\
\text { unfiltered } \\
(\mu \mathrm{g} / \mathrm{L}) \\
(\mathbf{0 1 2 1 8}) \\
\end{array}$} \\
\hline & & & & & value & s.d. & value & s.d. & value & s.d. & value & s.d. & value & s.d. & value & s.d. & value & s.d. \\
\hline \multicolumn{19}{|c|}{ Environmental samples } \\
\hline BY20 & $\begin{array}{l}\text { Boston Mine tunnel } \\
\text { outlet nr Grass } \\
\text { Valley }\end{array}$ & $6 / 18 / 1999$ & $14: 30$ & 1 of 2 & 4.9 & 0.1 & 0.16 & 0.04 & $<2$ & 2 & 14 & 0 & 0.018 & 0.003 & 86 & 2 & 0.0023 & 0.0003 \\
\hline BY20 & $\begin{array}{l}\text { Boston Mine tunnel } \\
\text { outlet nr Grass } \\
\text { Valley }\end{array}$ & $6 / 18 / 1999$ & $14: 30$ & 2 of 2 & 4.9 & 0.0 & 0.19 & 0.01 & $<2$ & 1 & 14 & 0 & 0.017 & 0.001 & 86 & 3 & 0.0021 & 0.0007 \\
\hline BY20 & $\begin{array}{l}\text { Boston Mine tunnel } \\
\text { outlet nr Grass } \\
\text { Valley }\end{array}$ & $12 / 14 / 1999$ & $12: 00$ & 1 of 2 & 6.6 & 0.0 & 0.07 & 0.02 & 0.7 & 0.4 & 14 & 3 & 0.13 & 0.01 & 132 & 5 & 0.021 & 0.004 \\
\hline BY20 & $\begin{array}{l}\text { Boston Mine tunnel } \\
\text { outlet nr Grass } \\
\text { Valley }\end{array}$ & 12/14/1999 & $12: 00$ & 2of2 & 6.8 & 0.4 & 0.05 & 0.01 & $<0.6$ & 0.4 & 14 & 1 & 0.084 & 0.010 & 102 & 4 & 0.014 & 0.003 \\
\hline BY20 & $\begin{array}{l}\text { Boston Mine tunnel } \\
\text { outlet nr Grass } \\
\text { Valley }\end{array}$ & $5 / 24 / 2000$ & $16: 00$ & 1 of 2 & 4.2 & 0.2 & 0.088 & 0.017 & $<86$ & 8 & 14 & 0 & 0.030 & 0.005 & 81 & 2 & 0.0052 & 0.0013 \\
\hline BY20 & $\begin{array}{l}\text { Boston Mine tunnel } \\
\text { outlet nr Grass } \\
\text { Valley }\end{array}$ & $5 / 24 / 2000$ & $16: 00$ & 2 of 2 & 4.2 & 0.2 & 0.085 & 0.005 & $<86$ & 14 & 14 & 1 & 0.062 & 0.003 & 83 & 3 & 0.011 & 0.001 \\
\hline BY21 & $\begin{array}{l}\text { Boston Mine wetlands } \\
\text { pond nr Grass } \\
\text { Valley }\end{array}$ & $12 / 14 / 1999$ & $10: 30$ & 1 of 2 & 5.7 & 0.3 & 0.049 & 0.002 & $<0.2$ & 0.3 & 4.3 & 0.3 & $<0.006$ & 0.002 & 122 & 1 & 0.0011 & 0.0007 \\
\hline BY21 & $\begin{array}{l}\text { Boston Mine wetlands } \\
\text { pond nr Grass } \\
\text { Valley }\end{array}$ & $12 / 14 / 1999$ & $10: 30$ & 2of2 & 5.4 & 0.1 & 0.055 & 0.001 & $<0.2$ & 0.4 & 4.3 & 0.2 & 0.006 & 0.004 & 121 & 7 & 0.0010 & 0.0000 \\
\hline BY21 & $\begin{array}{l}\text { Boston Mine wetlands } \\
\text { pond nr Grass } \\
\text { Valley }\end{array}$ & $5 / 24 / 2000$ & $16: 30$ & 1 of 2 & 2.7 & 0.1 & 0.079 & 0.008 & $<86$ & 16 & 11 & 0 & 0.011 & 0.007 & 73 & 1 & 0.0019 & 0.0004 \\
\hline BY21 & $\begin{array}{l}\text { Boston Mine wetlands } \\
\text { pond nr Grass } \\
\text { Valley }\end{array}$ & $5 / 24 / 2000$ & $16: 30$ & 2 of 2 & 2.8 & 0.1 & 0.081 & 0.000 & $<86$ & 12 & 11 & 0 & $<0.007$ & 0.003 & 71 & 4 & 0.0012 & 0.0003 \\
\hline BY58 & $\begin{array}{l}\text { Buckeye Flat Mine } \\
\text { main drain } 0.45 \mathrm{mi} \\
\text { ab Greenhorn } \mathrm{Cr}\end{array}$ & $8 / 21 / 2000$ & 11:00 & 1 of 2 & 42 & 5 & 0.4 & 0.1 & $<8$ & 3 & 30 & 5 & 3.4 & 0.4 & 87 & 22 & 0.60 & 0.05 \\
\hline
\end{tabular}


Table 4A. Concentrations of trace metals and selected major elements in unfiltered water samples, Greenhorn Creek drainage, Nevada County, California—Continued.

[Station name abbreviations: ab, above; bl, below; Cr, Creek; mi, miles; N, north; nr, near; Rd, Road; S, south; SF, South Fork. Number in parentheses is the data parameter code, a five-digit code used in the

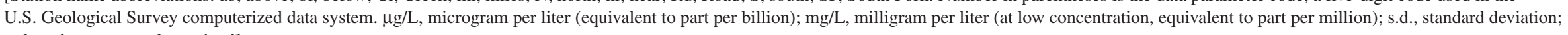

$<$, less than; - , not determined]

\begin{tabular}{|c|c|c|c|c|c|c|c|c|c|c|c|c|c|c|c|c|c|c|}
\hline \multirow[t]{2}{*}{$\begin{array}{l}\text { Station } \\
\text { map ID }\end{array}$} & \multirow[t]{2}{*}{ Station name } & \multirow[t]{2}{*}{ Date } & \multirow[t]{2}{*}{ Time } & \multirow[t]{2}{*}{$\begin{array}{l}\text { Rep- } \\
\text { licate }\end{array}$} & \multicolumn{2}{|c|}{$\begin{array}{c}\text { Sulfur (S), } \\
\text { unfiltered } \\
(\mathrm{mg} / \mathrm{L}) \\
(\mathbf{8 0 1 0 7 )}\end{array}$} & \multicolumn{2}{|c|}{$\begin{array}{c}\text { Antimony }(S b) \\
\text { unfiltered } \\
(\mu \mathrm{g} / \mathrm{L}) \\
(01097) \\
\end{array}$} & \multicolumn{2}{|c|}{$\begin{array}{c}\text { Selenium }(\mathrm{Se}), \\
\text { unfiltered } \\
(\mu \mathrm{g} / \mathrm{L}) \\
(01147) \\
\end{array}$} & \multicolumn{2}{|c|}{$\begin{array}{c}\text { Silica }(\mathrm{Si}), \\
\text { unfiltered } \\
\left(\mathrm{mg} / \mathrm{L} \text { as } \mathrm{SiO}_{2}\right) \\
(00956)\end{array}$} & \multicolumn{2}{|c|}{$\begin{array}{c}\text { Samarium }(\mathrm{Sm}), \\
\text { unfiltered } \\
(\mu \mathrm{g} / \mathrm{L}) \\
(\mathbf{8 2 3 2 2}) \\
\end{array}$} & \multicolumn{2}{|c|}{$\begin{array}{c}\text { Strontium }(\mathrm{Sr}) \\
\text { unfiltered } \\
(\mu \mathrm{g} / \mathrm{L}) \\
(\mathbf{0 1 0 8 4}) \\
\end{array}$} & \multicolumn{2}{|c|}{$\begin{array}{c}\text { Terbium }(\mathrm{Tb}), \\
\text { unfiltered } \\
(\mu \mathrm{g} / \mathrm{L}) \\
(\mathbf{0 1 2 1 8}) \\
\end{array}$} \\
\hline & & & & & value & s.d. & value & s.d. & value & s.d. & value & s.d. & value & s.d. & value & s.d. & value & s.d. \\
\hline BY23 & $\begin{array}{c}\text { Buckeye Flat Mine N } \\
\text { drain to Greenhorn } \\
\text { Cr, Grass Valley }\end{array}$ & 6/18/1999 & $11: 30$ & 1 of 2 & 61 & 0 & 0.10 & 0.02 & $<2$ & 2 & 36 & 2 & 4.3 & 0.1 & 140 & 0 & 0.67 & 0.10 \\
\hline BY23 & $\begin{array}{c}\text { Buckeye Flat Mine N } \\
\text { drain to Greenhorn } \\
\text { Cr, Grass Valley }\end{array}$ & 6/18/1999 & $11: 30$ & $2 \mathrm{of} 2$ & 62 & 0 & 0.095 & 0.035 & $<2$ & 1 & 35 & 1 & 4.8 & 0.5 & 140 & 0 & 0.82 & 0.03 \\
\hline BY23 & $\begin{array}{c}\text { Buckeye Flat Mine N } \\
\text { drain to Greenhorn } \\
\text { Cr, Grass Valley }\end{array}$ & 12/2/1999 & $11: 00$ & 1 of 2 & 39 & 0 & 0.04 & 0.01 & $<0.6$ & 0.4 & 28 & 2 & 3.5 & 0.3 & 88 & 10 & 0.53 & 0.09 \\
\hline BY23 & $\begin{array}{l}\text { Buckeye Flat Mine N } \\
\text { drain to Greenhorn } \\
\text { Cr, Grass Valley }\end{array}$ & $12 / 2 / 1999$ & $11: 00$ & $2 \mathrm{of} 2$ & 37 & 0 & 0.04 & 0.01 & $<0.6$ & 0.5 & 28 & 1 & 3.6 & 0.2 & 65 & 3 & 0.76 & 0.09 \\
\hline BY23 & $\begin{array}{c}\text { Buckeye Flat Mine N } \\
\text { drain to Greenhorn } \\
\text { Cr, Grass Valley }\end{array}$ & $8 / 22 / 2000$ & $10: 45$ & 1 of 2 & 65 & 3 & $<0.4$ & 0.2 & $<8$ & 6 & 46 & 0 & 5.5 & 0.2 & 130 & 40 & 0.92 & 0.00 \\
\hline BY23 & $\begin{array}{l}\text { Buckeye Flat Mine N } \\
\text { drain to Greenhorn } \\
\text { Cr, Grass Valley }\end{array}$ & $8 / 22 / 2000$ & $10: 45$ & $2 \mathrm{of} 2$ & 59 & 6 & $<0.4$ & 0.2 & $<8$ & 2 & 41 & 5 & 5.2 & 0.1 & 130 & 30 & 0.82 & 0.02 \\
\hline BY116 & $\begin{array}{l}\text { Buckeye Flat Mine } \\
\text { pond drain } 0.15 \mathrm{mi} \\
\text { ab SF Greenhorn } \mathrm{Cr}\end{array}$ & $8 / 21 / 2000$ & $14: 10$ & 1 of 2 & 2.3 & 0.1 & 0.06 & 0.02 & $<1$ & 1 & 5.2 & 0.6 & 0.028 & 0.004 & 14 & 2 & 0.006 & 0.001 \\
\hline BY116 & $\begin{array}{l}\text { Buckeye Flat Mine } \\
\text { pond drain } 0.15 \mathrm{mi} \\
\text { ab SF Greenhorn } \mathrm{Cr}\end{array}$ & $8 / 21 / 2000$ & $14: 10$ & $2 \mathrm{of} 2$ & 2.1 & 0.1 & 0.07 & 0.03 & $<1$ & 1 & 4.7 & 0.3 & 0.050 & 0.004 & 14 & 0 & 0.007 & 0.001 \\
\hline BY24 & $\begin{array}{l}\text { Buckeye Flat Mine } \\
\text { S drain to SF } \\
\text { Greenhorn } \mathrm{Cr}, \\
\text { Grass Valley }\end{array}$ & $8 / 26 / 1999$ & 11:00 & 1 of 2 & 2.0 & 0.1 & 2.0 & 0.0 & $<2$ & 2 & 6.9 & 0.2 & 0.11 & 0.00 & 5.6 & 0.2 & 0.014 & 0.002 \\
\hline BY24 & $\begin{array}{l}\text { Buckeye Flat Mine } \\
\text { S drain to SF } \\
\text { Greenhorn Cr, } \\
\text { Grass Valley }\end{array}$ & $8 / 26 / 1999$ & $11: 00$ & $2 \mathrm{of} 2$ & 1.9 & 0.0 & 1.4 & 0.0 & $<2$ & 2 & 6.6 & 0.1 & 0.11 & 0.01 & 5.3 & 0.0 & 0.013 & 0.000 \\
\hline BY24 & $\begin{array}{l}\text { Buckeye Flat Mine } \\
\text { S drain to SF } \\
\text { Greenhorn Cr, } \\
\text { Grass Valley }\end{array}$ & $8 / 22 / 2000$ & $12: 10$ & 1 of 2 & 1.6 & 0.1 & 0.07 & 0.02 & $<1$ & 1 & 8.3 & 2.9 & 0.26 & 0.01 & 6.1 & 1.0 & 0.044 & 0.007 \\
\hline BY24 & $\begin{array}{l}\text { Buckeye Flat Mine } \\
\text { S drain to SF } \\
\text { Greenhorn Cr, } \\
\text { Grass Valley }\end{array}$ & $8 / 22 / 2000$ & $12: 10$ & $2 \mathrm{of} 2$ & 2.0 & 0.4 & 0.07 & 0.01 & $<1$ & 1 & 7.9 & 1.6 & 0.29 & 0.00 & 6.6 & 1.7 & 0.045 & 0.002 \\
\hline
\end{tabular}


Table 4A. Concentrations of trace metals and selected major elements in unfiltered water samples, Nevada County, California-Continued.

[Station name abbreviations: ab, above; bl, below; Cr, Creek; mi, miles; N, north; nr, near; Rd, Road; S, south; SF, South Fork. Number in parentheses is the data parameter code, a five-digit code used in the

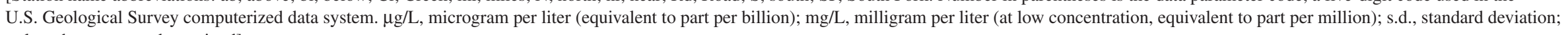
$<$, less than; - , not determined]

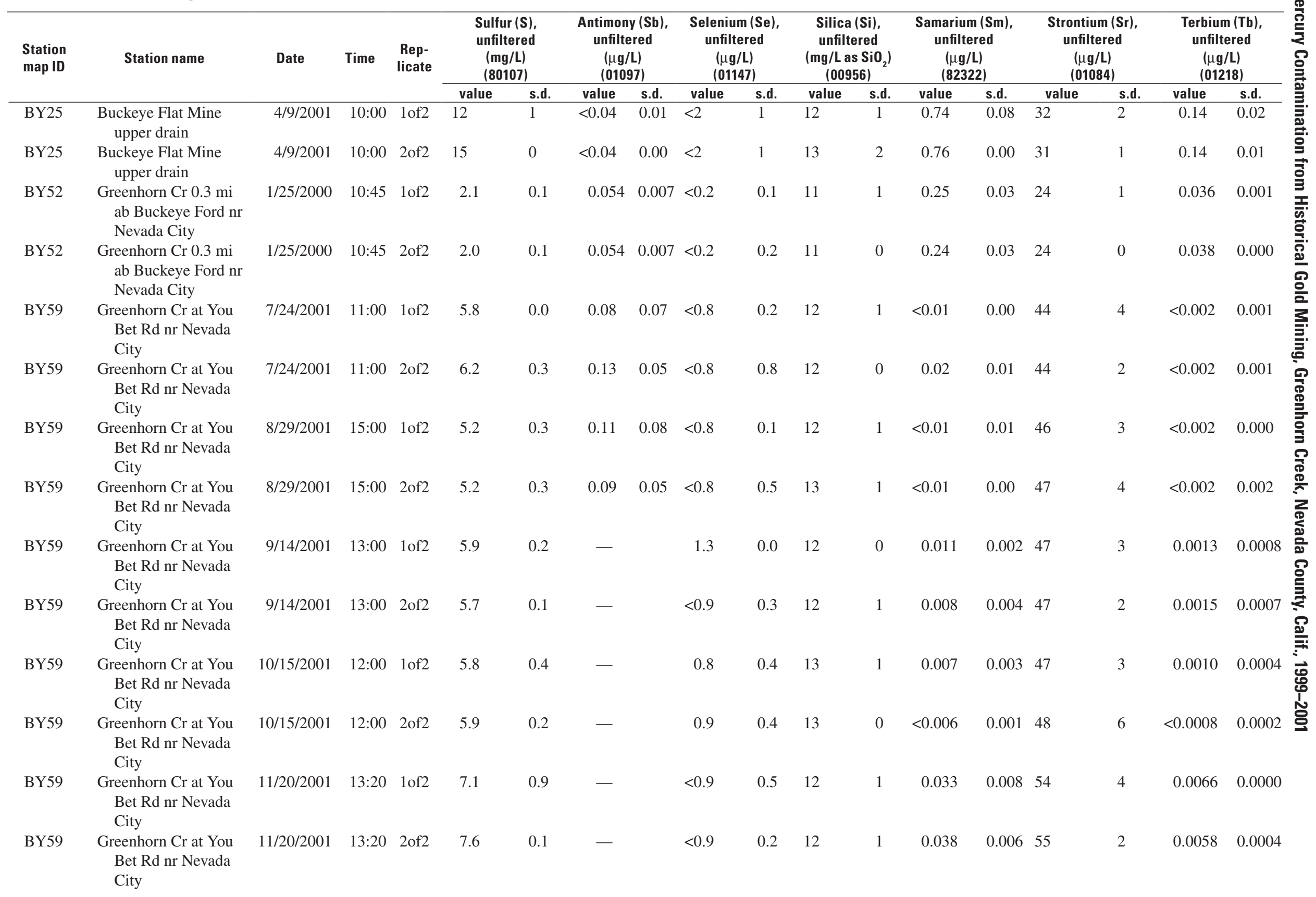


Table 4A. Concentrations of trace metals and selected major elements in unfiltered water samples, Greenhorn Creek drainage, Nevada County, California-Continued.

[Station name abbreviations: ab, above; bl, below; Cr, Creek; mi, miles; N, north; nr, near; Rd, Road; S, south; SF, South Fork. Number in parentheses is the data parameter code, a five-digit code used in the

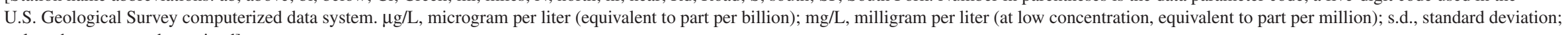

$<$, less than; - - not determined]

\begin{tabular}{|c|c|c|c|c|c|c|c|c|c|c|c|c|c|c|c|c|c|c|}
\hline \multirow[t]{2}{*}{$\begin{array}{l}\text { Station } \\
\text { map ID }\end{array}$} & \multirow[t]{2}{*}{ Station name } & \multirow[t]{2}{*}{ Date } & \multirow[t]{2}{*}{ Time } & \multirow[t]{2}{*}{$\begin{array}{l}\text { Rep- } \\
\text { licate }\end{array}$} & \multicolumn{2}{|c|}{$\begin{array}{c}\text { Sulfur }(\mathrm{S}), \\
\text { unfiltered } \\
(\mathrm{mg} / \mathrm{L}) \\
(\mathbf{8 0 1 0 7 )}\end{array}$} & \multicolumn{2}{|c|}{$\begin{array}{c}\text { Antimony (Sb), } \\
\text { unfiltered } \\
(\mu \mathrm{g} / \mathrm{L}) \\
(\mathbf{0 1 0 9 7 )}\end{array}$} & \multicolumn{2}{|c|}{$\begin{array}{c}\text { Selenium }(\mathrm{Se}), \\
\text { unfiltered } \\
(\mu \mathrm{g} / \mathrm{L}) \\
(\mathbf{0 1 1 4 7}) \\
\end{array}$} & \multicolumn{2}{|c|}{$\begin{array}{c}\text { Silica }(\mathrm{Si}), \\
\text { unfiltered } \\
\left(\mathrm{mg} / \mathrm{L} \text { as } \mathrm{SiO}_{2}\right) \\
(00956)\end{array}$} & \multicolumn{2}{|c|}{$\begin{array}{c}\text { Samarium }(\mathrm{Sm}), \\
\text { unfiltered } \\
(\mu \mathrm{g} / \mathrm{L}) \\
(\mathbf{8 2 3 2 2}) \\
\end{array}$} & \multicolumn{2}{|c|}{$\begin{array}{c}\text { Strontium }(\mathrm{Sr}), \\
\text { unfiltered } \\
(\mu \mathrm{g} / \mathrm{L}) \\
(\mathbf{0 1 0 8 4}) \\
\end{array}$} & \multicolumn{2}{|c|}{$\begin{array}{c}\text { Terbium }(\mathrm{Tb}), \\
\text { unfiltered } \\
(\mu \mathrm{g} / \mathrm{L}) \\
(\mathbf{0 1 2 1 8})\end{array}$} \\
\hline & & & & & value & s.d. & value & s.d. & value & s.d. & value & s.d. & value & s.d. & value & s.d. & value & s.d. \\
\hline BY59 & $\begin{array}{l}\text { Greenhorn Cr at You } \\
\text { Bet Rd nr Nevada } \\
\text { City }\end{array}$ & $12 / 10 / 2001$ & $12: 20$ & 1 of 2 & 6.1 & 0.7 & - & & 0.6 & 0.2 & 16 & 2 & 0.26 & 0.00 & 51 & 4 & 0.039 & 0.004 \\
\hline BY59 & $\begin{array}{l}\text { Greenhorn Cr at You } \\
\text { Bet Rd nr Nevada } \\
\text { City }\end{array}$ & $12 / 10 / 2001$ & $12: 20$ & $2 \mathrm{of} 2$ & 6.2 & 0.8 & 0.20 & 0.13 & 0.6 & 0.2 & 16 & 2 & 0.26 & 0.01 & 53 & 4 & 0.037 & 0.002 \\
\hline BY59 & $\begin{array}{l}\text { Greenhorn Cr at You } \\
\text { Bet Rd nr Nevada } \\
\text { City }\end{array}$ & $12 / 21 / 2001$ & 13:00 & 1 of 2 & 4.2 & 0.4 & 0.28 & 0.25 & $<0.9$ & 0.6 & 26 & 3 & 1.02 & 0.11 & 45 & 4 & 0.160 & 0.005 \\
\hline BY59 & $\begin{array}{l}\text { Greenhorn Cr at You } \\
\text { Bet Rd nr Nevada } \\
\text { City }\end{array}$ & $12 / 21 / 2001$ & $13: 00$ & $2 \mathrm{of} 2$ & 4.3 & 0.5 & 0.26 & 0.15 & $<0.9$ & 0.3 & 26 & 3 & 1.02 & 0.01 & 45 & 4 & 0.139 & 0.011 \\
\hline BY51 & $\begin{array}{l}\text { Greenhorn } \mathrm{Cr} n r \\
\text { headwaters nr } \\
\text { Scotts Flat Res }\end{array}$ & $1 / 25 / 2000$ & $9: 30$ & 1 of 2 & 0.2 & 0.0 & 0.022 & 0.003 & $<0.2$ & 0.4 & 7.9 & 0.3 & 0.081 & 0.000 & 17 & 0 & 0.0099 & 0.0004 \\
\hline BY51 & $\begin{array}{l}\text { Greenhorn } \mathrm{Cr} n r \\
\text { headwaters } \mathrm{nr} \\
\text { Scotts Flat Res }\end{array}$ & $1 / 25 / 2000$ & $9: 30$ & $2 \mathrm{of} 2$ & 0.1 & 0.1 & 0.024 & 0.009 & 0.4 & 0.1 & 7.6 & 0.2 & 0.080 & 0.010 & 17 & 0 & 0.011 & 0.002 \\
\hline BY75 & $\begin{array}{l}\text { Missouri Cyn Cr } 1.2 \\
\text { mi ab Greenhorn Cr } \\
\text { nr Chicago Park }\end{array}$ & $12 / 14 / 1999$ & $14: 00$ & 1 of 2 & 16 & 1 & 0.08 & 0.01 & $<0.6$ & 0.6 & 26 & 1 & 0.080 & 0.007 & 96 & 22 & 0.018 & 0.007 \\
\hline BY75 & $\begin{array}{l}\text { Missouri Cyn Cr } 1.2 \\
\text { mi ab Greenhorn Cr } \\
\text { nr Chicago Park }\end{array}$ & $12 / 14 / 1999$ & $14: 00$ & $2 \mathrm{of} 2$ & 15 & 0 & 0.09 & 0.01 & $<0.6$ & 0.4 & 24 & 0 & 0.084 & 0.017 & 102 & 26 & 0.017 & 0.005 \\
\hline BY86 & $\begin{array}{l}\text { Poore Mine Creek } \\
\text { ab tunnel nr Grass } \\
\text { Valley }\end{array}$ & 3/31/1999 & $17: 40$ & 1 of 2 & 1.2 & 0.0 & 0.096 & 0.007 & $<2$ & 0 & 17 & 0 & 0.073 & 0.005 & 39 & 0 & 0.012 & 0.000 \\
\hline BY86 & $\begin{array}{l}\text { Poore Mine Creek } \\
\text { ab tunnel nr Grass } \\
\text { Valley }\end{array}$ & 3/31/1999 & $17: 40$ & $2 \mathrm{of} 2$ & 1.2 & 0.1 & 0.10 & 0.02 & $<2$ & 1 & 17 & 1 & 0.069 & 0.008 & 39 & 2 & 0.0092 & 0.0017 \\
\hline BY87 & $\begin{array}{l}\text { Poore Mine Creek } \\
\text { bl tunnel nr Grass } \\
\text { Valley }\end{array}$ & $3 / 31 / 1999$ & $13: 30$ & 1 of 2 & 1.7 & 0.2 & 0.094 & 0.013 & $<2$ & 1 & 17 & 1 & 0.070 & 0.003 & 39 & 2 & 0.010 & 0.002 \\
\hline BY87 & $\begin{array}{l}\text { Poore Mine Creek } \\
\text { bl tunnel nr Grass } \\
\text { Valley }\end{array}$ & $3 / 31 / 1999$ & $13: 30$ & $2 \mathrm{of} 2$ & 1.7 & 0.2 & 0.093 & 0.009 & $<2$ & 1 & 16 & 0 & 0.081 & 0.005 & 40 & 2 & 0.011 & 0.001 \\
\hline
\end{tabular}


Table 4A. Concentrations of trace metals and selected major elements in unfiltered water samples, Greenhorn Creek drainage, Nevada County, California—Continued.

[Station name abbreviations: ab, above; bl, below; Cr, Creek; mi, miles; N, north; nr, near; Rd, Road; S, south; SF, South Fork. Number in parentheses is the data parameter code, a five-digit code used in the

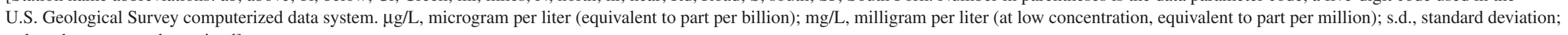

$<$, less than; - - not determined]

\begin{tabular}{|c|c|c|c|c|c|c|c|c|c|c|c|c|c|c|c|c|c|c|}
\hline \multirow[t]{2}{*}{$\begin{array}{l}\text { Station } \\
\text { map ID }\end{array}$} & \multirow[t]{2}{*}{ Station name } & \multirow[t]{2}{*}{ Date } & \multirow[t]{2}{*}{ Time } & \multirow[t]{2}{*}{$\begin{array}{l}\text { Rep- } \\
\text { licate }\end{array}$} & \multicolumn{2}{|c|}{$\begin{array}{c}\text { Sulfur (S), } \\
\text { unfiltered } \\
(\mathrm{mg} / \mathrm{L}) \\
(\mathbf{8 0 1 0 7 )}\end{array}$} & \multicolumn{2}{|c|}{$\begin{array}{c}\text { Antimony }(\mathrm{Sb}), \\
\text { unfiltered } \\
(\mu \mathrm{g} / \mathrm{L}) \\
(01097) \\
\end{array}$} & \multicolumn{2}{|c|}{$\begin{array}{c}\text { Selenium }(\text { Se }), \\
\text { unfiltered } \\
(\mu \mathrm{g} / \mathrm{L}) \\
(\mathbf{0 1 1 4 7 )} \\
\end{array}$} & \multicolumn{2}{|c|}{$\begin{array}{c}\text { Silica }(\mathrm{Si}), \\
\text { unfiltered } \\
\left(\mathrm{mg} / \mathrm{L} \text { as } \mathrm{SiO}_{2}\right) \\
(00956)\end{array}$} & \multicolumn{2}{|c|}{$\begin{array}{c}\text { Samarium }(\mathrm{Sm}), \\
\text { unfiltered } \\
(\mu \mathrm{g} / \mathrm{L}) \\
(\mathbf{8 2 3 2 2}) \\
\end{array}$} & \multicolumn{2}{|c|}{$\begin{array}{c}\text { Strontium }(\mathrm{Sr}), \\
\text { unfiltered } \\
(\mu \mathrm{g} / \mathrm{L}) \\
(\mathbf{0 1 0 8 4}) \\
\end{array}$} & \multicolumn{2}{|c|}{$\begin{array}{c}\text { Terbium }(\mathrm{Tb}), \\
\text { unfiltered } \\
(\mu \mathrm{g} / \mathrm{L}) \\
(\mathbf{0 1 2 1 8}) \\
\end{array}$} \\
\hline & & & & & value & s.d. & value & s.d. & value & s.d. & value & s.d. & value & s.d. & value & s.d. & value & s.d. \\
\hline BY88 & $\begin{array}{l}\text { Poore Mine ground } \\
\text { sluice nr Grass } \\
\text { Valley }\end{array}$ & 4/1/1999 & $15: 40$ & 1 of 2 & 13 & 0 & 0.080 & 0.023 & $<2$ & 1 & 25 & 0 & 0.025 & 0.001 & 43 & 2 & 0.0049 & 0.0001 \\
\hline BY88 & $\begin{array}{l}\text { Poore Mine ground } \\
\text { sluice nr Grass } \\
\text { Valley }\end{array}$ & 4/1/1999 & $15: 40$ & $2 \mathrm{of} 2$ & 13 & 1 & 0.071 & 0.003 & $<2$ & 0 & 26 & 0 & 0.031 & 0.004 & 41 & 2 & 0.0064 & 0.0012 \\
\hline BY89 & $\begin{array}{l}\text { Poore Mine pit lake nr } \\
\text { Grass Valley }\end{array}$ & 4/1/1999 & $16: 50$ & 1 of 2 & 3.3 & 0.2 & 0.20 & 0.00 & $<2$ & 1 & 15 & 0 & 0.025 & 0.004 & 24 & 2 & 0.0033 & 0.0003 \\
\hline BY89 & $\begin{array}{l}\text { Poore Mine pit lake nr } \\
\text { Grass Valley }\end{array}$ & 4/1/1999 & $16: 50$ & 2of2 & 3.2 & 0.1 & 0.23 & 0.05 & $<2$ & 0 & 15 & 1 & 0.026 & 0.006 & 25 & 1 & 0.0039 & 0.0012 \\
\hline BY89 & $\begin{array}{l}\text { Poore Mine pit lake nr } \\
\text { Grass Valley }\end{array}$ & $5 / 23 / 2000$ & $10: 45$ & 1 of 2 & 25 & 1 & 0.058 & 0.008 & $<86$ & 22 & 21 & 0 & 0.029 & 0.004 & 122 & 2 & 0.0068 & 0.0012 \\
\hline BY89 & $\begin{array}{l}\text { Poore Mine pit lake nr } \\
\text { Grass Valley }\end{array}$ & $5 / 23 / 2000$ & $10: 45$ & 2of2 & 25 & 0 & 0.054 & 0.004 & $<86$ & 11 & 21 & 0 & 0.029 & 0.002 & 126 & 6 & 0.0078 & 0.0006 \\
\hline BY90 & $\begin{array}{l}\text { Poore Mine seep } \\
\text { above ground sluice } \\
\text { nr Grass Valley }\end{array}$ & 4/1/1999 & $16: 00$ & 2of2 & - & & - & & - & & - & & - & & - & & - & \\
\hline BY91 & $\begin{array}{l}\text { Poore Mine tunnel } \\
\text { effluent nr Grass } \\
\text { Valley }\end{array}$ & $3 / 31 / 1999$ & $15: 20$ & 1of 2 & 11 & 0 & 0.16 & 0.01 & $<2$ & 1 & 28 & 1 & 0.017 & 0.001 & 80 & 3 & 0.0031 & 0.0002 \\
\hline BY91 & $\begin{array}{l}\text { Poore Mine tunnel } \\
\text { effluent nr Grass } \\
\text { Valley }\end{array}$ & $3 / 31 / 1999$ & $15: 20$ & 2of 2 & 11 & 0 & 0.20 & 0.01 & $<2$ & 1 & 29 & 0 & 0.019 & 0.003 & 82 & 1 & 0.0036 & 0.0008 \\
\hline BY91 & $\begin{array}{l}\text { Poore Mine tunnel } \\
\text { effluent nr Grass } \\
\text { Valley }\end{array}$ & $12 / 15 / 1999$ & $13: 00$ & 1of 2 & 15 & 0 & 0.09 & 0.01 & $<0.6$ & 0.4 & 34 & 1 & 0.022 & 0.004 & 110 & 0 & 0.0053 & 0.0005 \\
\hline BY91 & $\begin{array}{l}\text { Poore Mine tunnel } \\
\text { effluent nr Grass } \\
\text { Valley }\end{array}$ & $12 / 15 / 1999$ & $13: 00$ & 2of2 & 17 & 0 & 0.07 & 0.01 & $<0.6$ & 0.3 & 38 & 1 & 0.033 & 0.010 & 110 & 20 & 0.0038 & 0.0015 \\
\hline BY91 & $\begin{array}{l}\text { Poore Mine tunnel } \\
\text { effluent nr Grass } \\
\text { Valley }\end{array}$ & $5 / 23 / 2000$ & $12: 00$ & 1 of 2 & 15 & 0 & 0.14 & 0.02 & $<86$ & 30 & 36 & 0 & 0.32 & 0.01 & 123 & 4 & 0.050 & 0.004 \\
\hline BY91 & $\begin{array}{l}\text { Poore Mine tunnel } \\
\text { effluent nr Grass } \\
\text { Valley }\end{array}$ & $5 / 23 / 2000$ & $12: 00$ & 2of 2 & 16 & 0 & 0.14 & 0.01 & $<86$ & 21 & 37 & 1 & 0.29 & 0.02 & 121 & 6 & 0.044 & 0.003 \\
\hline
\end{tabular}


Table 4A. Concentrations of trace metals and selected major elements in unfiltered water samples, Greenhorn Creek drainage, Nevada County, California-Continued.

[Station name abbreviations: ab, above; bl, below; Cr, Creek; mi, miles; N, north; nr, near; Rd, Road; S, south; SF, South Fork. Number in parentheses is the data parameter code, a five-digit code used in the

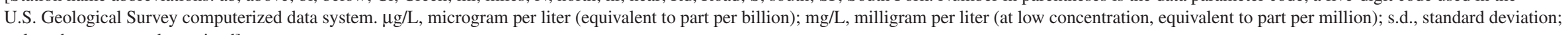

$<$, less than; - - not determined]

\begin{tabular}{|c|c|c|c|c|c|c|c|c|c|c|c|c|c|c|c|c|c|c|}
\hline \multirow[t]{2}{*}{$\begin{array}{l}\text { Station } \\
\text { map ID }\end{array}$} & \multirow[t]{2}{*}{ Station name } & \multirow[t]{2}{*}{ Date } & \multirow[t]{2}{*}{ Time } & \multirow[t]{2}{*}{$\begin{array}{l}\text { Rep- } \\
\text { licate }\end{array}$} & \multicolumn{2}{|c|}{$\begin{array}{c}\text { Sulfur }(\mathrm{S}) \\
\text { unfiltered } \\
(\mathrm{mg} / \mathrm{L}) \\
(\mathbf{8 0 1 0 7 )}\end{array}$} & \multicolumn{2}{|c|}{$\begin{array}{c}\text { Antimony }(\mathbf{S b}), \\
\text { unfiltered } \\
(\mu \mathrm{g} / \mathrm{L}) \\
(01097)\end{array}$} & \multicolumn{2}{|c|}{$\begin{array}{c}\text { Selenium (Se), } \\
\text { unfiltered } \\
(\mu \mathrm{g} / \mathrm{L}) \\
(01147)\end{array}$} & \multicolumn{2}{|c|}{$\begin{array}{c}\text { Silica }(\mathrm{Si}), \\
\text { unfiltered } \\
\left(\mathrm{mg} / \mathrm{L} \text { as } \mathrm{SiO}_{2}\right) \\
(00956)\end{array}$} & \multicolumn{2}{|c|}{$\begin{array}{c}\text { Samarium }(\mathrm{Sm}), \\
\text { unfiltered } \\
(\mu \mathrm{g} / \mathrm{L}) \\
(\mathbf{8 2 3 2 2})\end{array}$} & \multicolumn{2}{|c|}{$\begin{array}{c}\text { Strontium }(\mathrm{Sr}), \\
\text { unfiltered } \\
(\mu \mathrm{g} / \mathrm{L}) \\
(\mathbf{0 1 0 8 4 )} \\
\end{array}$} & \multicolumn{2}{|c|}{$\begin{array}{c}\text { Terbium }(\mathrm{Tb}), \\
\text { unfiltered } \\
(\mu \mathrm{g} / \mathrm{L}) \\
(\mathbf{0 1 2 1 8})\end{array}$} \\
\hline & & & & & value & s.d. & value & s.d. & value & s.d. & value & s.d. & value & s.d. & value & s.d. & value & s.d. \\
\hline BY105 & $\begin{array}{l}\text { Sailor Flat Mine main } \\
\text { drain gulch } 01 \mathrm{nr} \\
\text { Quaker Hill }\end{array}$ & $7 / 6 / 1999$ & $16: 00$ & 1 of 2 & 6.5 & 0.1 & 0.15 & 0.02 & $<2$ & 2 & 27 & 1 & 1.7 & 0.2 & 56 & 1 & 0.25 & 0.03 \\
\hline BY105 & $\begin{array}{l}\text { Sailor Flat Mine main } \\
\text { drain gulch } 01 \mathrm{nr} \\
\text { Quaker Hill }\end{array}$ & 7/6/1999 & $16: 00$ & $2 \mathrm{of} 2$ & 6.5 & 0.1 & 0.19 & 0.01 & $<2$ & 3 & 26 & 1 & 1.6 & 0.1 & 56 & 1 & 0.16 & 0.02 \\
\hline BY105 & $\begin{array}{l}\text { Sailor Flat Mine main } \\
\text { drain Gulch } 01 \mathrm{nr} \\
\text { Quaker Hill }\end{array}$ & $12 / 2 / 1999$ & $14: 30$ & 1 of 2 & 2.7 & 0.2 & 0.55 & 0.00 & $<0.6$ & 0.0 & 32 & 1 & 4.0 & 0.0 & 61 & 0 & 0.57 & 0.09 \\
\hline BY105 & $\begin{array}{l}\text { Sailor Flat Mine main } \\
\text { drain Gulch } 01 \mathrm{nr} \\
\text { Quaker Hill }\end{array}$ & $12 / 2 / 1999$ & $14: 30$ & $2 \mathrm{of} 2$ & 2.6 & 0.2 & 0.45 & 0.01 & $<0.6$ & 0.1 & 31 & 1 & 4.5 & 0.0 & 55 & 8 & 0.55 & 0.04 \\
\hline BY106 & $\begin{array}{l}\text { Sailor Flat Mine } \\
\text { main drainage to } \\
\text { Greenhorn Cr, } \\
\text { Gulch } 03\end{array}$ & $4 / 9 / 2001$ & $12: 30$ & 1 of 2 & 0.59 & 0.07 & 0.05 & 0.03 & $<2$ & 0 & 12 & 1 & 0.32 & 0.00 & 11 & 1 & 0.048 & 0.002 \\
\hline BY106 & $\begin{array}{l}\text { Sailor Flat Mine } \\
\text { main drainage to } \\
\text { Greenhorn Cr, } \\
\text { Gulch } 03\end{array}$ & $4 / 9 / 2001$ & $12: 30$ & 2 of 2 & 0.54 & 0.08 & $<0.04$ & 0.01 & $<2$ & 0 & 12 & 3 & 0.31 & 0.01 & 11 & 0 & 0.044 & 0.001 \\
\hline BY114 & $\begin{array}{l}\text { SF Greenhorn Cr } 0.7 \\
\text { mi ab Greenhorn Cr } \\
\text { nr Nevada City }\end{array}$ & $8 / 22 / 2000$ & $15: 10$ & 1 of 2 & 1.6 & 0.1 & 0.05 & 0.01 & $<1$ & 1 & 13 & 3 & $<0.008$ & 0.002 & 43 & 6 & $<0.002$ & 0.001 \\
\hline BY114 & $\begin{array}{l}\text { SF Greenhorn Cr } 0.7 \\
\text { mi ab Greenhorn Cr } \\
\text { nr Nevada City }\end{array}$ & $8 / 22 / 2000$ & $15: 10$ & $2 \mathrm{of} 2$ & 1.5 & 0.4 & 0.10 & 0.02 & $<1$ & 1 & 11 & 1 & $<0.008$ & 0.005 & 40 & 16 & $<0.002$ & 0.000 \\
\hline BY122 & $\begin{array}{l}\text { Starr Mine tunnel } \\
\text { inflow nr Grass } \\
\text { Valley }\end{array}$ & 4/23/1999 & $15: 00$ & 1 of 2 & 13 & 1 & 0.22 & 0.01 & $<2$ & 0 & 15 & 1 & 0.044 & 0.003 & 54 & 4 & 0.0073 & 0.0009 \\
\hline BY122 & $\begin{array}{l}\text { Starr Mine tunnel } \\
\text { inflow nr Grass } \\
\text { Valley }\end{array}$ & 4/23/1999 & $15: 00$ & $2 \mathrm{of} 2$ & 14 & 0 & 0.23 & 0.01 & $<2$ & 1 & 17 & 0 & 0.053 & 0.008 & 59 & 1 & 0.0095 & 0.0020 \\
\hline BY123 & $\begin{array}{l}\text { Starr Mine tunnel } \\
\text { midway nr Grass } \\
\text { Valley }\end{array}$ & 4/23/1999 & $13: 40$ & $1 \mathrm{of} 2$ & 12 & 1 & 0.15 & 0.01 & $<2$ & 1 & 20 & 1 & 0.032 & 0.005 & 200 & 10 & 0.0055 & 0.0017 \\
\hline BY123 & $\begin{array}{l}\text { Starr Mine tunnel } \\
\text { midway nr Grass } \\
\text { Valley }\end{array}$ & 4/23/1999 & $13: 40$ & 2of 2 & 12 & 1 & 0.14 & 0.01 & $<2$ & 1 & 20 & 1 & 0.016 & 0.006 & 200 & 10 & 0.0045 & 0.0011 \\
\hline
\end{tabular}


Table 4A. Concentrations of trace metals and selected major elements in unfiltered water samples, Greenhorn Creek drainage, Nevada County, California—Continued.

[Station name abbreviations: ab, above; bl, below; Cr, Creek; mi, miles; N, north; nr, near; Rd, Road; S, south; SF, South Fork. Number in parentheses is the data parameter code, a five-digit code used in the

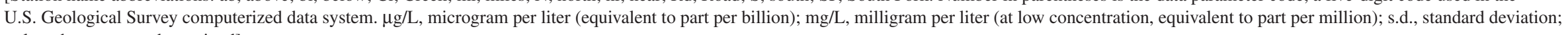
$<$, less than; - , not determined]

\begin{tabular}{|c|c|c|c|c|c|c|c|c|c|c|c|c|c|c|c|c|c|c|}
\hline \multirow[t]{2}{*}{$\begin{array}{l}\text { Station } \\
\text { map ID }\end{array}$} & \multirow[t]{2}{*}{ Station name } & \multirow[t]{2}{*}{ Date } & \multirow[t]{2}{*}{ Time } & \multirow[t]{2}{*}{$\begin{array}{l}\text { Rep- } \\
\text { licate }\end{array}$} & \multicolumn{2}{|c|}{$\begin{array}{c}\text { Sulfur (S), } \\
\text { unfiltered } \\
(\mathrm{mg} / \mathrm{L}) \\
(\mathbf{8 0 1 0 7 )}\end{array}$} & \multicolumn{2}{|c|}{$\begin{array}{c}\text { Antimony }(S b) \\
\text { unfiltered } \\
(\mu \mathrm{g} / \mathrm{L}) \\
(01097) \\
\end{array}$} & \multicolumn{2}{|c|}{$\begin{array}{c}\text { Selenium (Se), } \\
\text { unfiltered } \\
(\mu \mathrm{g} / \mathrm{L}) \\
(01147) \\
\end{array}$} & \multicolumn{2}{|c|}{$\begin{array}{c}\text { Silica }(\mathrm{Si}), \\
\text { unfiltered } \\
\left(\mathrm{mg} / \mathrm{L} \text { as } \mathrm{SiO}_{2}\right) \\
(00956)\end{array}$} & \multicolumn{2}{|c|}{$\begin{array}{c}\text { Samarium }(\mathrm{Sm}), \\
\text { unfiltered } \\
(\mu \mathrm{g} / \mathrm{L}) \\
(\mathbf{8 2 3 2 2}) \\
\end{array}$} & \multicolumn{2}{|c|}{$\begin{array}{c}\text { Strontium }(\mathrm{Sr}) \\
\text { unfiltered } \\
(\mu \mathrm{g} / \mathrm{L}) \\
(\mathbf{0 1 0 8 4}) \\
\end{array}$} & \multicolumn{2}{|c|}{$\begin{array}{c}\text { Terbium }(\mathrm{Tb}) \\
\text { unfiltered } \\
(\mu \mathrm{g} / \mathrm{L}) \\
(\mathbf{0 1 2 1 8}) \\
\end{array}$} \\
\hline & & & & & value & s.d. & value & s.d. & value & s.d. & value & s.d. & value & s.d. & value & s.d. & value & s.d. \\
\hline BY123 & $\begin{array}{l}\text { Starr Mine tunnel } \\
\text { midway nr Grass } \\
\text { Valley }\end{array}$ & $12 / 15 / 1999$ & $10: 45$ & 1 of 2 & 21 & 1 & 0.13 & 0.00 & 1.0 & 0.1 & 19 & 1 & 0.061 & 0.007 & 280 & 20 & 0.010 & 0.004 \\
\hline BY123 & $\begin{array}{l}\text { Starr Mine tunnel } \\
\text { midway nr Grass } \\
\text { Valley }\end{array}$ & $12 / 15 / 1999$ & $10: 45$ & 2of 2 & 21 & 0 & 0.12 & 0.02 & 1.2 & 0.2 & 18 & 0 & 0.057 & 0.001 & 270 & 0 & 0.011 & 0.002 \\
\hline BY124 & $\begin{array}{l}\text { Starr Mine tunnel } \\
\text { outlet nr Grass } \\
\text { Valley }\end{array}$ & $8 / 23 / 2000$ & $17: 30$ & 1 of 2 & 9.2 & 0.0 & 0.28 & 0.02 & $<1$ & 0 & 22 & 3 & 0.15 & 0.00 & 200 & 30 & 0.026 & 0.002 \\
\hline BY124 & $\begin{array}{l}\text { Starr Mine tunnel } \\
\text { outlet nr Grass } \\
\text { Valley }\end{array}$ & $8 / 23 / 2000$ & $17: 30$ & 2of2 & 8.9 & 0.3 & 0.30 & 0.04 & $<1$ & 1 & 20 & 1 & 0.15 & 0.01 & 190 & 40 & 0.027 & 0.000 \\
\hline BY129 & $\begin{array}{l}\text { Tom and Jerry Mine } \\
\text { drain at tunnel inlet } \\
\text { nr Nevada City }\end{array}$ & $6 / 28 / 2000$ & $12: 00$ & 1of 2 & 0.74 & 0.19 & 0.25 & 0.05 & $<1$ & 1 & 19 & 1 & 2.5 & 0.5 & 60 & 16 & 0.37 & 0.07 \\
\hline BY129 & $\begin{array}{l}\text { Tom and Jerry Mine } \\
\text { drain at tunnel inlet } \\
\text { nr Nevada City }\end{array}$ & $6 / 28 / 2000$ & $12: 00$ & 2of 2 & 0.86 & 0.08 & 0.23 & 0.01 & $<1$ & 1 & 22 & 2 & 2.4 & 0.4 & 61 & 11 & 0.35 & 0.05 \\
\hline BY130 & $\begin{array}{l}\text { Tom and Jerry Mine } \\
\text { drain at tunnel } \\
\text { outlet nr Nevada } \\
\text { City }\end{array}$ & $6 / 28 / 2000$ & $11: 30$ & 1of 2 & 6.1 & 0.2 & 0.12 & 0.04 & $<1$ & 1 & 18 & 2 & 0.037 & 0.006 & 110 & 0 & 0.004 & 0.000 \\
\hline BY130 & $\begin{array}{l}\text { Tom and Jerry Mine } \\
\text { drain at tunnel } \\
\text { outlet nr Nevada } \\
\text { City }\end{array}$ & $6 / 28 / 2000$ & $11: 30$ & 2of2 & 6.7 & 0.4 & 0.11 & 0.03 & $<1$ & 1 & 19 & 4 & 0.046 & 0.017 & 120 & 10 & 0.007 & 0.002 \\
\hline BY131 & $\begin{array}{l}\text { Tom and Jerry Mine } \\
\text { drainage pond nr } \\
\text { Nevada City }\end{array}$ & $8 / 23 / 2000$ & $10: 45$ & 1 of 2 & 1.5 & 0.1 & 0.07 & 0.02 & $<1$ & 1 & 16 & 1 & 0.055 & 0.007 & 43 & 4 & 0.008 & 0.001 \\
\hline BY131 & $\begin{array}{l}\text { Tom and Jerry Mine } \\
\text { drainage pond nr } \\
\text { Nevada City }\end{array}$ & $8 / 23 / 2000$ & $10: 45$ & 2of 2 & 1.4 & 0.4 & 0.06 & 0.02 & $<1$ & 2 & 15 & 4 & 0.042 & 0.002 & 39 & 8 & 0.009 & 0.001 \\
\hline
\end{tabular}


Table 4A. Concentrations of trace metals and selected major elements in unfiltered water samples, Greenhorn Creek drainage, Nevada County, California—Continued

[Station name abbreviations: ab, above; bl, below; Cr, Creek; mi, miles; N, north; nr, near; Rd, Road; S, south; SF, South Fork. Number in parentheses is the data parameter code, a five-digit code used in the

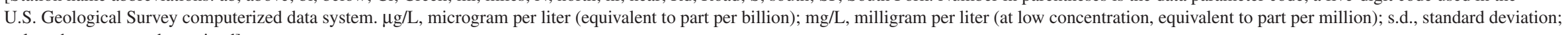

$<$ less than; - - not determined]

\begin{tabular}{|c|c|c|c|c|c|c|c|c|c|c|c|c|c|c|c|c|c|c|}
\hline \multirow[t]{2}{*}{$\begin{array}{l}\text { Station } \\
\text { map ID }\end{array}$} & \multirow[t]{2}{*}{ Station name } & \multirow[t]{2}{*}{ Date } & \multirow[t]{2}{*}{ Time } & \multirow[t]{2}{*}{$\begin{array}{l}\text { Rep- } \\
\text { licate }\end{array}$} & \multicolumn{2}{|c|}{$\begin{array}{c}\text { Sulfur }(\mathrm{S}) \text {, } \\
\text { unfiltered } \\
(\mathrm{mg} / \mathrm{L}) \\
(\mathbf{8 0 1 0 7 )}\end{array}$} & \multicolumn{2}{|c|}{$\begin{array}{c}\text { Antimony }(\mathbf{S b}), \\
\text { unfiltered } \\
(\mu \mathrm{g} / \mathrm{L}) \\
(01097)\end{array}$} & \multicolumn{2}{|c|}{$\begin{array}{c}\text { Selenium (Se), } \\
\text { unfiltered } \\
(\mu \mathrm{g} / \mathrm{L}) \\
(01147)\end{array}$} & \multicolumn{2}{|c|}{$\begin{array}{c}\text { Silica }(\mathrm{Si}), \\
\text { unfiltered } \\
\left(\mathrm{mg} / \mathrm{L} \text { as } \mathrm{SiO}_{2}\right) \\
(00956)\end{array}$} & \multicolumn{2}{|c|}{$\begin{array}{c}\text { Samarium }(\mathrm{Sm}), \\
\text { unfiltered } \\
(\mu \mathrm{g} / \mathrm{L}) \\
(\mathbf{8 2 3 2 2})\end{array}$} & \multicolumn{2}{|c|}{$\begin{array}{c}\text { Strontium }(\mathrm{Sr}), \\
\text { unfiltered } \\
(\mu \mathrm{g} / \mathrm{L}) \\
(01084)\end{array}$} & \multicolumn{2}{|c|}{$\begin{array}{c}\text { Terbium }(\mathrm{Tb}), \\
\text { unfiltered } \\
(\mu \mathrm{g} / \mathrm{L}) \\
(\mathbf{0 1 2 1 8})\end{array}$} \\
\hline & & & & & value & s.d. & value & s.d. & value & s.d. & value & s.d. & value & s.d. & value & s.d. & value & s.d. \\
\hline \multicolumn{19}{|c|}{ Disturbed samples } \\
\hline BY123 & $\begin{array}{l}\text { Starr Mine tunnel } \\
\text { midway nr Grass } \\
\text { Valley }\end{array}$ & $12 / 15 / 1999$ & $11: 30$ & 1 of 2 & 36 & 0 & 2.9 & 0.4 & 15 & 0 & 280 & 20 & 20 & 0 & 1,300 & 0 & 3.7 & 0.0 \\
\hline BY123 & $\begin{array}{l}\text { Starr Mine tunnel } \\
\text { midway nr Grass } \\
\text { Valley }\end{array}$ & $12 / 15 / 1999$ & $11: 30$ & $2 \mathrm{of} 2$ & 33 & 1 & 2.8 & 0.0 & 15 & 0 & 270 & 20 & 21 & 0 & 1,100 & 0 & 3.3 & 0.1 \\
\hline BY129 & $\begin{array}{l}\text { Tom and Jerry Mine } \\
\text { drain at tunnel inlet } \\
\text { nr Nevada City }\end{array}$ & $6 / 28 / 2000$ & $12: 30$ & 1 of 2 & 4.8 & 0.2 & 0.19 & 0.01 & $<1$ & 1 & 84 & 2 & 26 & 4 & 280 & 40 & 3.6 & 0.5 \\
\hline BY129 & $\begin{array}{l}\text { Tom and Jerry Mine } \\
\text { drain at tunnel inlet } \\
\text { nr Nevada City }\end{array}$ & $6 / 28 / 2000$ & $12: 30$ & $2 \mathrm{of} 2$ & 6.3 & 1.4 & 0.19 & 0.03 & $<1$ & 1 & 110 & 30 & 29 & 1 & 300 & 20 & 4.0 & 0.2 \\
\hline
\end{tabular}


Table 4A. Concentrations of trace metals and selected major elements in unfiltered water samples, Greenhorn Creek drainage, Nevada County, California—Continued.

[Station name abbreviations: ab, above; bl, below; Cr, Creek; mi, miles; N, north; nr, near; Rd, Road; S, south; SF, South Fork. Number in parentheses is the data parameter code, a five-digit code used in the U.S. Geological Survey computerized data system. $\mu \mathrm{g} / \mathrm{L}$, microgram per liter (equivalent to part per billion); $\mathrm{mg} / \mathrm{L}$, milligram per liter (at low concentration, equivalent to part per million); s.d., standard deviation; $<$, less than; - , not determined]

\begin{tabular}{|c|c|c|c|c|c|c|c|c|c|c|c|c|c|c|}
\hline \multirow[t]{2}{*}{$\begin{array}{l}\text { Station } \\
\text { map ID }\end{array}$} & \multirow[t]{2}{*}{ Station name } & \multirow[t]{2}{*}{ Date } & \multirow[t]{2}{*}{ Time } & \multirow[t]{2}{*}{$\begin{array}{l}\text { Rep- } \\
\text { licate }\end{array}$} & \multicolumn{2}{|c|}{$\begin{array}{c}\text { Tellurium }(\mathrm{Te}) \\
\text { unfiltered } \\
(\mu \mathrm{g} / \mathrm{L}) \\
(01064)\end{array}$} & \multicolumn{2}{|c|}{$\begin{array}{c}\text { Thorium }(T h), \\
\text { unfiltered } \\
(\mu \mathrm{g} / \mathrm{L}) \\
(\mathbf{8 2 3 6 4 )}\end{array}$} & \multicolumn{2}{|c|}{$\begin{array}{c}\text { Thallium (TI), } \\
\text { unfiltered } \\
(\mu \mathrm{g} / \mathrm{L}) \\
(01059)\end{array}$} & \multicolumn{2}{|c|}{$\begin{array}{c}\text { Thulium (Tm), } \\
\text { unfiltered } \\
(\mu \mathrm{g} / \mathrm{L})\end{array}$} & \multicolumn{2}{|c|}{$\begin{array}{c}\text { Uranium }(\mathrm{U}) \text {, } \\
\text { unfiltered } \\
(\mu \mathrm{g} / \mathrm{L}) \\
(28011)\end{array}$} \\
\hline & & & & & value & s.d. & value & s.d. & value & s.d. & value & s.d. & value & s.d. \\
\hline \multicolumn{15}{|c|}{ Environmental samples } \\
\hline BY20 & $\begin{array}{l}\text { Boston Mine tunnel } \\
\text { outlet nr Grass } \\
\text { Valley }\end{array}$ & $6 / 18 / 1999$ & $14: 30$ & 1of 2 & $<0.02$ & 0.01 & 0.0054 & 0.0014 & 0.017 & 0.006 & 0.0016 & 0.0003 & 0.0078 & 0.0012 \\
\hline BY20 & $\begin{array}{l}\text { Boston Mine tunnel } \\
\text { outlet nr Grass } \\
\text { Valley }\end{array}$ & $6 / 18 / 1999$ & $14: 30$ & 2 of 2 & $<0.02$ & 0.01 & 0.0027 & 0.0006 & 0.016 & 0.003 & 0.0015 & 0.0002 & 0.012 & 0.005 \\
\hline BY20 & $\begin{array}{l}\text { Boston Mine tunnel } \\
\text { outlet nr Grass } \\
\text { Valley }\end{array}$ & $12 / 14 / 1999$ & $12: 00$ & 1 of 2 & $<0.02$ & 0.00 & 0.015 & 0.008 & 0.024 & 0.003 & 0.011 & 0.000 & 0.019 & 0.003 \\
\hline BY20 & $\begin{array}{l}\text { Boston Mine tunnel } \\
\text { outlet nr Grass } \\
\text { Valley }\end{array}$ & $12 / 14 / 1999$ & $12: 00$ & $2 \mathrm{of} 2$ & $<0.02$ & 0.01 & 0.019 & 0.006 & 0.021 & 0.007 & 0.0068 & 0.0012 & 0.018 & 0.001 \\
\hline BY20 & $\begin{array}{l}\text { Boston Mine tunnel } \\
\text { outlet nr Grass } \\
\text { Valley }\end{array}$ & $5 / 24 / 2000$ & $16: 00$ & 1 of 2 & $<0.04$ & 0.04 & 0.003 & 0.001 & 0.016 & 0.004 & 0.0033 & 0.0015 & 0.011 & 0.001 \\
\hline BY20 & $\begin{array}{l}\text { Boston Mine tunnel } \\
\text { outlet nr Grass } \\
\text { Valley }\end{array}$ & $5 / 24 / 2000$ & $16: 00$ & $2 \mathrm{of} 2$ & $<0.04$ & 0.01 & 0.004 & 0.002 & 0.020 & 0.010 & 0.0061 & 0.0004 & 0.013 & 0.003 \\
\hline BY21 & $\begin{array}{l}\text { Boston Mine wetlands } \\
\text { pond nr Grass } \\
\text { Valley }\end{array}$ & $12 / 14 / 1999$ & $10: 30$ & 1 of 2 & $<0.05$ & 0.03 & $<0.005$ & 0.004 & 0.013 & 0.009 & $<0.001$ & 0.001 & 0.006 & 0.001 \\
\hline BY21 & $\begin{array}{l}\text { Boston Mine wetlands } \\
\text { pond nr Grass } \\
\text { Valley }\end{array}$ & $12 / 14 / 1999$ & $10: 30$ & 2 of 2 & $<0.05$ & 0.02 & 0.006 & 0.003 & 0.007 & 0.002 & $<0.001$ & 0.000 & 0.008 & 0.001 \\
\hline BY21 & $\begin{array}{l}\text { Boston Mine wetlands } \\
\text { pond nr Grass } \\
\text { Valley }\end{array}$ & $5 / 24 / 2000$ & $16: 30$ & 1of 2 & $<0.04$ & 0.04 & 0.005 & 0.002 & 0.017 & 0.010 & 0.0012 & 0.0003 & 0.008 & 0.002 \\
\hline BY21 & $\begin{array}{l}\text { Boston Mine wetlands } \\
\text { pond nr Grass } \\
\text { Valley }\end{array}$ & $5 / 24 / 2000$ & $16: 30$ & $2 \mathrm{of} 2$ & $<0.04$ & 0.02 & 0.004 & 0.001 & 0.008 & 0.005 & 0.0009 & 0.0002 & 0.008 & 0.002 \\
\hline BY58 & $\begin{array}{l}\text { Buckeye Flat Mine } \\
\text { main drain } 0.45 \mathrm{mi} \\
\text { ab Greenhorn } \mathrm{Cr}\end{array}$ & $8 / 21 / 2000$ & $11: 00$ & 1 of 2 & $<0.6$ & 0.3 & $<0.02$ & 0.01 & $<0.3$ & 0.1 & 0.25 & 0.01 & 0.31 & 0.04 \\
\hline BY58 & $\begin{array}{l}\text { Buckeye Flat Mine } \\
\text { main drain } 0.45 \mathrm{mi} \\
\text { ab Greenhorn } \mathrm{Cr}\end{array}$ & $8 / 21 / 2000$ & $11: 00$ & 2 of 2 & $<0.6$ & 0.3 & $<0.02$ & 0.00 & $<0.3$ & 0.1 & 0.17 & 0.02 & 0.25 & 0.02 \\
\hline
\end{tabular}


Table 4A. Concentrations of trace metals and selected major elements in unfiltered water samples, Greenhorn Creek drainage, Nevada County, California-Continued.

[Station name abbreviations: ab, above; bl, below; Cr, Creek; mi, miles; N, north; nr, near; Rd, Road; S, south; SF, South Fork. Number in parentheses is the data parameter code, a five-digit code used in the U.S. Geological Survey computerized data system. $\mu \mathrm{g} / \mathrm{L}$, microgram per liter (equivalent to part per billion); mg/L, milligram per liter (at low concentration, equivalent to part per million); s.d., standard deviation; $<$, less than; - , not determined]

\begin{tabular}{|c|c|c|c|c|c|c|c|c|c|c|c|c|c|c|}
\hline \multirow[t]{2}{*}{$\begin{array}{l}\text { Station } \\
\text { map ID }\end{array}$} & \multirow[t]{2}{*}{ Station name } & \multirow[t]{2}{*}{ Date } & \multirow[t]{2}{*}{ Time } & \multirow[t]{2}{*}{$\begin{array}{l}\text { Rep- } \\
\text { licate }\end{array}$} & \multicolumn{2}{|c|}{$\begin{array}{c}\text { Tellurium (Te), } \\
\text { unfiltered } \\
(\mu \mathrm{g} / \mathrm{L}) \\
(01064) \\
\end{array}$} & \multicolumn{2}{|c|}{$\begin{array}{c}\text { Thorium (Th), } \\
\text { unfiltered } \\
(\mu \mathrm{g} / \mathrm{L}) \\
(\mathbf{8 2 3 6 4 )} \\
\end{array}$} & \multicolumn{2}{|c|}{$\begin{array}{c}\text { Thallium }(\mathrm{TI}), \\
\text { unfiltered } \\
(\mu \mathrm{g} / \mathrm{L}) \\
(01059)\end{array}$} & \multicolumn{2}{|c|}{$\begin{array}{c}\text { Thulium (Tm), } \\
\text { unfiltered } \\
(\mu \mathrm{g} / \mathrm{L}) \\
-\end{array}$} & \multicolumn{2}{|c|}{$\begin{array}{c}\text { Uranium (U), } \\
\text { unfiltered } \\
(\mu \mathrm{g} / \mathrm{L}) \\
(28011) \\
\end{array}$} \\
\hline & & & & & value & s.d. & value & s.d. & value & s.d. & value & s.d. & value & s.d. \\
\hline BY23 & $\begin{array}{c}\text { Buckeye Flat Mine N } \\
\text { drain to Greenhorn } \\
\text { Cr, Grass Valley }\end{array}$ & $6 / 18 / 1999$ & $11: 30$ & 1of 2 & $<0.02$ & 0.01 & 0.014 & 0.000 & 0.13 & 0.00 & 0.31 & 0.03 & 0.39 & 0.00 \\
\hline BY23 & $\begin{array}{c}\text { Buckeye Flat Mine N } \\
\text { drain to Greenhorn } \\
\text { Cr, Grass Valley }\end{array}$ & $6 / 18 / 1999$ & $11: 30$ & 2of 2 & $<0.02$ & 0.02 & 0.015 & 0.002 & 0.15 & 0.00 & 0.32 & 0.04 & 0.42 & 0.01 \\
\hline BY23 & $\begin{array}{c}\text { Buckeye Flat Mine N } \\
\text { drain to Greenhorn } \\
\text { Cr, Grass Valley }\end{array}$ & $12 / 2 / 1999$ & 11:00 & 1 of 2 & $<0.02$ & 0.02 & 0.14 & 0.00 & 0.13 & 0.00 & 0.27 & 0.03 & 0.41 & 0.01 \\
\hline BY23 & $\begin{array}{c}\text { Buckeye Flat Mine N } \\
\text { drain to Greenhorn } \\
\text { Cr, Grass Valley }\end{array}$ & $12 / 2 / 1999$ & $11: 00$ & 2 of 2 & $<0.02$ & 0.01 & 0.12 & 0.01 & 0.15 & 0.01 & 0.29 & 0.02 & 0.41 & 0.00 \\
\hline BY23 & $\begin{array}{c}\text { Buckeye Flat Mine N } \\
\text { drain to Greenhorn } \\
\text { Cr, Grass Valley }\end{array}$ & $8 / 22 / 2000$ & $10: 45$ & 1 of 2 & $<0.6$ & 0.2 & $<0.02$ & 0.01 & $<0.3$ & 0.3 & 0.34 & 0.00 & 0.38 & 0.01 \\
\hline BY23 & $\begin{array}{c}\text { Buckeye Flat Mine N } \\
\text { drain to Greenhorn } \\
\text { Cr, Grass Valley }\end{array}$ & $8 / 22 / 2000$ & $10: 45$ & 2of 2 & $<0.6$ & 0.5 & $<0.02$ & 0.01 & $<0.3$ & 0.6 & 0.32 & 0.01 & 0.41 & 0.09 \\
\hline BY116 & $\begin{array}{l}\text { Buckeye Flat Mine } \\
\text { pond drain } 0.15 \mathrm{mi} \\
\text { ab SF Greenhorn } \mathrm{Cr}\end{array}$ & $8 / 21 / 2000$ & $14: 10$ & 1of 2 & $<0.07$ & 0.05 & 0.020 & 0.013 & $<0.07$ & 0.01 & 0.002 & 0.001 & 0.008 & 0.001 \\
\hline BY116 & $\begin{array}{l}\text { Buckeye Flat Mine } \\
\text { pond drain } 0.15 \mathrm{mi} \\
\text { ab SF Greenhorn } \mathrm{Cr}\end{array}$ & $8 / 21 / 2000$ & $14: 10$ & 2of 2 & $<0.07$ & 0.01 & 0.042 & 0.016 & $<0.07$ & 0.01 & 0.003 & 0.001 & 0.014 & 0.002 \\
\hline BY24 & $\begin{array}{l}\text { Buckeye Flat Mine } \\
\text { S drain to SF } \\
\text { Greenhorn } \mathrm{Cr}, \\
\text { Grass Valley }\end{array}$ & $8 / 26 / 1999$ & 11:00 & 1 of 2 & 0.22 & 0.05 & 0.0077 & 0.0016 & 0.012 & 0.005 & 0.0078 & 0.0008 & 0.031 & 0.001 \\
\hline BY24 & $\begin{array}{l}\text { Buckeye Flat Mine } \\
\text { S drain to SF } \\
\text { Greenhorn Cr, } \\
\text { Grass Valley }\end{array}$ & $8 / 26 / 1999$ & 11:00 & 2 of 2 & 0.18 & 0.06 & 0.0094 & 0.0006 & 0.015 & 0.003 & 0.0099 & 0.0014 & 0.034 & 0.002 \\
\hline BY24 & $\begin{array}{l}\text { Buckeye Flat Mine } \\
\text { S drain to SF } \\
\text { Greenhorn Cr, } \\
\text { Grass Valley }\end{array}$ & $8 / 22 / 2000$ & $12: 10$ & 1of 2 & $<0.07$ & 0.03 & 0.029 & 0.002 & $<0.07$ & 0.02 & 0.017 & 0.003 & 0.10 & 0.00 \\
\hline BY24 & $\begin{array}{l}\text { Buckeye Flat Mine } \\
\text { S drain to SF } \\
\text { Greenhorn Cr, } \\
\text { Grass Valley }\end{array}$ & $8 / 22 / 2000$ & $12: 10$ & 2of2 & $<0.07$ & 0.02 & 0.021 & 0.004 & $<0.07$ & 0.03 & 0.016 & 0.003 & 0.098 & 0.005 \\
\hline
\end{tabular}


Table 4A. Concentrations of trace metals and selected major elements in unfiltered water samples, Greenhorn Creek drainage, Nevada County, California—Continued.

[Station name abbreviations: ab, above; bl, below; Cr, Creek; mi, miles; N, north; nr, near; Rd, Road; S, south; SF, South Fork. Number in parentheses is the data parameter code, a five-digit code used in the U.S. Geological Survey computerized data system. $\mu \mathrm{g} / \mathrm{L}$, microgram per liter (equivalent to part per billion); $\mathrm{mg} / \mathrm{L}$, milligram per liter (at low concentration, equivalent to part per million); s.d., standard deviation; <, less than; - , not determined]

\begin{tabular}{|c|c|c|c|c|c|c|c|c|c|c|c|c|c|c|}
\hline \multirow[t]{2}{*}{$\begin{array}{l}\text { Station } \\
\text { map ID }\end{array}$} & \multirow[t]{2}{*}{ Station name } & \multirow[t]{2}{*}{ Date } & \multirow[t]{2}{*}{ Time } & \multirow[t]{2}{*}{$\begin{array}{l}\text { Rep- } \\
\text { licate }\end{array}$} & \multicolumn{2}{|c|}{$\begin{array}{c}\text { Tellurium (Te), } \\
\text { unfiltered } \\
(\mu \mathrm{g} / \mathrm{L}) \\
(01064) \\
\end{array}$} & \multicolumn{2}{|c|}{$\begin{array}{c}\text { Thorium }(\mathrm{Th}), \\
\text { unfiltered } \\
(\mu \mathrm{g} / \mathrm{L}) \\
(82364) \\
\end{array}$} & \multicolumn{2}{|c|}{$\begin{array}{c}\text { Thallium (TI), } \\
\text { unfiltered } \\
(\mu \mathrm{g} / \mathrm{L}) \\
(01059)\end{array}$} & \multicolumn{2}{|c|}{$\begin{array}{c}\text { Thulium (Tm), } \\
\text { unfiltered } \\
(\mu \mathrm{g} / \mathrm{L}) \\
\end{array}$} & \multicolumn{2}{|c|}{$\begin{array}{c}\text { Uranium }(\mathrm{U}), \\
\text { unfiltered } \\
(\mu \mathrm{g} / \mathrm{L}) \\
(28011)\end{array}$} \\
\hline & & & & & value & s.d. & value & s.d. & value & s.d. & value & s.d. & value & s.d. \\
\hline BY25 & $\begin{array}{l}\text { Buckeye Flat Mine } \\
\text { upper drain }\end{array}$ & $4 / 9 / 2001$ & $10: 00$ & 1of 2 & $<0.1$ & 0.0 & $<0.02$ & 0.00 & 0.01 & 0.00 & 0.054 & 0.002 & 0.044 & 0.001 \\
\hline BY25 & $\begin{array}{l}\text { Buckeye Flat Mine } \\
\text { upper drain }\end{array}$ & $4 / 9 / 2001$ & $10: 00$ & 2 of 2 & $<0.1$ & 0.0 & $<0.02$ & 0.00 & $<0.01$ & 0.02 & 0.055 & 0.000 & 0.042 & 0.001 \\
\hline BY52 & $\begin{array}{l}\text { Greenhorn Cr } 0.3 \mathrm{mi} \\
\text { ab Buckeye Ford nr } \\
\text { Nevada City }\end{array}$ & $1 / 25 / 2000$ & $10: 45$ & 1 of 2 & $<0.05$ & 0.03 & 0.18 & 0.01 & 0.016 & 0.003 & 0.013 & 0.002 & 0.11 & 0.00 \\
\hline BY52 & $\begin{array}{l}\text { Greenhorn Cr } 0.3 \mathrm{mi} \\
\text { ab Buckeye Ford nr } \\
\text { Nevada City }\end{array}$ & $1 / 25 / 2000$ & $10: 45$ & $2 o f 2$ & $<0.05$ & 0.01 & 0.19 & 0.00 & 0.018 & 0.006 & 0.015 & 0.003 & 0.11 & 0.00 \\
\hline BY59 & $\begin{array}{l}\text { Greenhorn Cr at You } \\
\text { Bet Rd nr Nevada } \\
\text { City }\end{array}$ & $7 / 24 / 2001$ & $11: 00$ & 1of 2 & $<0.08$ & 0.03 & $<0.006$ & 0.000 & $<0.04$ & 0.01 & 0.002 & 0.001 & 0.054 & 0.009 \\
\hline BY59 & $\begin{array}{l}\text { Greenhorn Cr at You } \\
\text { Bet Rd nr Nevada } \\
\text { City }\end{array}$ & $7 / 24 / 2001$ & 11:00 & 2 of 2 & $<0.08$ & 0.02 & $<0.006$ & 0.001 & $<0.04$ & 0.02 & $<0.002$ & 0.000 & 0.013 & 0.001 \\
\hline BY59 & $\begin{array}{l}\text { Greenhorn Cr at You } \\
\text { Bet Rd nr Nevada } \\
\text { City }\end{array}$ & $8 / 29 / 2001$ & $15: 00$ & 1 of 2 & $<0.08$ & 0.01 & $<0.006$ & 0.001 & $<0.04$ & 0.00 & $<0.002$ & 0.001 & $<0.007$ & 0.000 \\
\hline BY59 & $\begin{array}{l}\text { Greenhorn Cr at You } \\
\text { Bet Rd nr Nevada } \\
\text { City }\end{array}$ & $8 / 29 / 2001$ & $15: 00$ & 2 of 2 & $<0.08$ & 0.02 & $<0.006$ & 0.001 & $<0.04$ & 0.01 & $<0.002$ & 0.001 & $<0.007$ & 0.003 \\
\hline BY59 & $\begin{array}{l}\text { Greenhorn Cr at You } \\
\text { Bet Rd nr Nevada } \\
\text { City }\end{array}$ & $9 / 14 / 2001$ & $13: 00$ & 1of 2 & $<0.03$ & 0.02 & $<0.004$ & 0.001 & $<0.06$ & 0.02 & 0.0012 & 0.0007 & $<0.008$ & 0.004 \\
\hline BY59 & $\begin{array}{l}\text { Greenhorn Cr at You } \\
\text { Bet Rd nr Nevada } \\
\text { City }\end{array}$ & $9 / 14 / 2001$ & $13: 00$ & 2of 2 & $<0.03$ & 0.02 & $<0.004$ & 0.001 & $<0.06$ & 0.04 & $<0.001$ & 0.000 & $<0.008$ & 0.002 \\
\hline BY59 & $\begin{array}{l}\text { Greenhorn Cr at You } \\
\text { Bet Rd nr Nevada } \\
\text { City }\end{array}$ & $10 / 15 / 2001$ & $12: 00$ & 1 of 2 & $<0.05$ & 0.02 & $<0.006$ & 0.002 & $<0.05$ & 0.02 & $<0.001$ & 0.001 & $<0.006$ & 0.001 \\
\hline BY59 & $\begin{array}{l}\text { Greenhorn Cr at You } \\
\text { Bet Rd nr Nevada } \\
\text { City }\end{array}$ & $10 / 15 / 2001$ & $12: 00$ & 2 of 2 & $<0.05$ & 0.04 & $<0.006$ & 0.001 & $<0.05$ & 0.00 & $<0.001$ & 0.001 & $<0.006$ & 0.004 \\
\hline BY59 & $\begin{array}{l}\text { Greenhorn } \mathrm{Cr} \text { at You } \\
\text { Bet Rd nr Nevada } \\
\text { City }\end{array}$ & $11 / 20 / 2001$ & $13: 20$ & 1of 2 & $<0.03$ & 0.02 & $<0.004$ & 0.000 & $<0.06$ & 0.03 & 0.0020 & 0.0003 & 0.011 & 0.005 \\
\hline BY59 & $\begin{array}{l}\text { Greenhorn Cr at You } \\
\text { Bet Rd nr Nevada } \\
\text { City }\end{array}$ & $11 / 20 / 2001$ & $13: 20$ & 2 of 2 & $<0.03$ & 0.03 & $<0.004$ & 0.000 & $<0.06$ & 0.01 & 0.0034 & 0.0006 & $<0.008$ & 0.002 \\
\hline
\end{tabular}


Table 4A. Concentrations of trace metals and selected major elements in unfiltered water samples, Greenhorn Creek drainage, Nevada County, California—Continued.

[Station name abbreviations: ab, above; bl, below; Cr, Creek; mi, miles; N, north; nr, near; Rd, Road; S, south; SF, South Fork. Number in parentheses is the data parameter code, a five-digit code used in the U.S. Geological Survey computerized data system. $\mu \mathrm{g} / \mathrm{L}$, microgram per liter (equivalent to part per billion); mg/L, milligram per liter (at low concentration, equivalent to part per million); s.d., standard deviation; <, less than; - , not determined]

\begin{tabular}{|c|c|c|c|c|c|c|c|c|c|c|c|c|c|c|}
\hline \multirow[t]{2}{*}{$\begin{array}{l}\text { Station } \\
\text { map ID }\end{array}$} & \multirow[t]{2}{*}{ Station name } & \multirow[t]{2}{*}{ Date } & \multirow[t]{2}{*}{ Time } & \multirow[t]{2}{*}{$\begin{array}{l}\text { Rep- } \\
\text { licate }\end{array}$} & \multicolumn{2}{|c|}{$\begin{array}{c}\text { Tellurium (Te), } \\
\text { unfiltered } \\
(\mu \mathrm{g} / \mathrm{L}) \\
(\mathbf{0 1 0 6 4 )}\end{array}$} & \multicolumn{2}{|c|}{$\begin{array}{c}\text { Thorium (Th), } \\
\text { unfiltered } \\
(\mu \mathrm{g} / \mathrm{L}) \\
(\mathbf{8 2 3 6 4 )}\end{array}$} & \multicolumn{2}{|c|}{$\begin{array}{c}\text { Thallium (TI), } \\
\text { unfiltered } \\
(\mu \mathrm{g} / \mathrm{L}) \\
(01059)\end{array}$} & \multicolumn{2}{|c|}{$\begin{array}{c}\text { Thulium (Tm), } \\
\text { unfiltered } \\
(\mu \mathrm{g} / \mathrm{L}) \\
\end{array}$} & \multicolumn{2}{|c|}{$\begin{array}{c}\text { Uranium }(\mathrm{U}), \\
\text { unfiltered } \\
(\mu \mathrm{g} / \mathrm{L}) \\
(28011)\end{array}$} \\
\hline & & & & & value & s.d. & value & s.d. & value & s.d. & value & s.d. & value & s.d. \\
\hline BY59 & $\begin{array}{l}\text { Greenhorn Cr at You } \\
\text { Bet Rd nr Nevada } \\
\text { City }\end{array}$ & $12 / 10 / 2001$ & $12: 20$ & 1 of 2 & $<0.05$ & 0.01 & 0.076 & 0.001 & $<0.05$ & 0.02 & 0.016 & 0.000 & 0.11 & 0.01 \\
\hline BY59 & $\begin{array}{l}\text { Greenhorn Cr at You } \\
\text { Bet Rd nr Nevada } \\
\text { City }\end{array}$ & $12 / 10 / 2001$ & $12: 20$ & $2 \mathrm{of} 2$ & $<0.05$ & 0.04 & 0.075 & 0.001 & $<0.05$ & 0.02 & 0.017 & 0.002 & 0.10 & 0.01 \\
\hline BY59 & $\begin{array}{l}\text { Greenhorn Cr at You } \\
\text { Bet Rd nr Nevada } \\
\text { City }\end{array}$ & $12 / 21 / 2001$ & $13: 00$ & 1 of 2 & $<0.11$ & 0.08 & 0.40 & 0.08 & $<0.11$ & 0.08 & 0.059 & 0.001 & 0.49 & 0.00 \\
\hline BY59 & $\begin{array}{l}\text { Greenhorn Cr at You } \\
\text { Bet Rd nr Nevada } \\
\text { City }\end{array}$ & $12 / 21 / 2001$ & 13:00 & $2 \mathrm{of} 2$ & $<0.11$ & 0.06 & 0.41 & 0.04 & 0.23 & 0.35 & 0.065 & 0.002 & 0.47 & 0.01 \\
\hline BY51 & $\begin{array}{l}\text { Greenhorn } \mathrm{Cr} n r \\
\text { headwaters nr } \\
\text { Scotts Flat Res }\end{array}$ & $1 / 25 / 2000$ & $9: 30$ & 1 of 2 & $<0.05$ & 0.02 & 0.017 & 0.004 & 0.003 & 0.005 & 0.003 & 0.001 & 0.012 & 0.001 \\
\hline BY51 & $\begin{array}{l}\text { Greenhorn } \mathrm{Cr} \mathrm{nr} \\
\text { headwaters nr } \\
\text { Scotts Flat Res }\end{array}$ & $1 / 25 / 2000$ & $9: 30$ & $2 \mathrm{of} 2$ & $<0.05$ & 0.00 & 0.019 & 0.010 & $<0.003$ & 0.003 & 0.005 & 0.001 & 0.009 & 0.000 \\
\hline BY75 & $\begin{array}{l}\text { Missouri Cyn Cr } 1.2 \\
\text { mi ab Greenhorn Cr } \\
\text { nr Chicago Park }\end{array}$ & $12 / 14 / 1999$ & $14: 00$ & 1 of 2 & $<0.02$ & 0.00 & 0.016 & 0.014 & 0.019 & 0.010 & 0.0088 & 0.0013 & 0.017 & 0.003 \\
\hline BY75 & $\begin{array}{l}\text { Missouri Cyn Cr } 1.2 \\
\text { mi ab Greenhorn Cr } \\
\text { nr Chicago Park }\end{array}$ & $12 / 14 / 1999$ & $14: 00$ & $2 \mathrm{of} 2$ & $<0.02$ & 0.02 & 0.010 & 0.002 & 0.009 & 0.006 & 0.0074 & 0.0006 & 0.018 & 0.001 \\
\hline BY86 & $\begin{array}{l}\text { Poore Mine Creek } \\
\text { ab tunnel nr Grass } \\
\text { Valley }\end{array}$ & $3 / 31 / 1999$ & $17: 40$ & 1 of 2 & $<0.02$ & 0.01 & 0.057 & 0.006 & $<0.004$ & 0.002 & 0.0043 & 0.0001 & 0.029 & 0.003 \\
\hline BY86 & $\begin{array}{l}\text { Poore Mine Creek } \\
\text { ab tunnel nr Grass } \\
\text { Valley }\end{array}$ & 3/31/1999 & $17: 40$ & $2 \mathrm{of} 2$ & $<0.02$ & 0.03 & 0.061 & 0.000 & $<0.004$ & 0.005 & 0.0043 & 0.0003 & 0.028 & 0.001 \\
\hline BY87 & $\begin{array}{l}\text { Poore Mine Creek } \\
\text { bl tunnel nr Grass } \\
\text { Valley }\end{array}$ & $3 / 31 / 1999$ & $13: 30$ & 1 of 2 & $<0.02$ & 0.03 & 0.049 & 0.002 & $<0.004$ & 0.008 & 0.0042 & 0.0007 & 0.025 & 0.001 \\
\hline BY87 & $\begin{array}{l}\text { Poore Mine Creek } \\
\text { bl tunnel nr Grass } \\
\text { Valley }\end{array}$ & $3 / 31 / 1999$ & $13: 30$ & $2 \mathrm{of} 2$ & $<0.02$ & 0.01 & 0.054 & 0.010 & $<0.004$ & 0.003 & 0.0055 & 0.0018 & 0.024 & 0.000 \\
\hline
\end{tabular}


Table 4A. Concentrations of trace metals and selected major elements in unfiltered water samples, Greenhorn Creek drainage, Nevada County, California—Continued.

[Station name abbreviations: ab, above; bl, below; Cr, Creek; mi, miles; N, north; nr, near; Rd, Road; S, south; SF, South Fork. Number in parentheses is the data parameter code, a five-digit code used in the U.S. Geological Survey computerized data system. $\mu \mathrm{g} / \mathrm{L}$, microgram per liter (equivalent to part per billion); $\mathrm{mg} / \mathrm{L}$, milligram per liter (at low concentration, equivalent to part per million); s.d., standard deviation; <, less than; - - not determined]

\begin{tabular}{|c|c|c|c|c|c|c|c|c|c|c|c|c|c|c|}
\hline \multirow[t]{2}{*}{$\begin{array}{l}\text { Station } \\
\text { map ID }\end{array}$} & \multirow[t]{2}{*}{ Station name } & \multirow[t]{2}{*}{ Date } & \multirow[t]{2}{*}{ Time } & \multirow[t]{2}{*}{$\begin{array}{l}\text { Rep- } \\
\text { licate }\end{array}$} & \multicolumn{2}{|c|}{$\begin{array}{c}\text { Tellurium }(\mathrm{Te}), \\
\text { unfiltered } \\
(\mu \mathrm{g} / \mathrm{L}) \\
(01064)\end{array}$} & \multicolumn{2}{|c|}{$\begin{array}{c}\text { Thorium }(\mathrm{Th}), \\
\text { unfiltered } \\
(\mu \mathrm{g} / \mathrm{L}) \\
(82364)\end{array}$} & \multicolumn{2}{|c|}{$\begin{array}{c}\text { Thallium }(\mathrm{TI}), \\
\text { unfiltered } \\
(\mu \mathrm{g} / \mathrm{L}) \\
(01059)\end{array}$} & \multicolumn{2}{|c|}{$\begin{array}{c}\text { Thulium (Tm), } \\
\text { unfiltered } \\
(\mu \mathrm{g} / \mathrm{L})\end{array}$} & \multicolumn{2}{|c|}{$\begin{array}{c}\text { Uranium }(\mathrm{U}), \\
\text { unfiltered } \\
(\mu \mathrm{g} / \mathrm{L}) \\
(\mathbf{2 8 0 1 1 )}\end{array}$} \\
\hline & & & & & value & s.d. & value & s.d. & value & s.d. & value & s.d. & value & s.d. \\
\hline BY88 & $\begin{array}{l}\text { Poore Mine ground } \\
\text { sluice nr Grass } \\
\text { Valley }\end{array}$ & $4 / 1 / 1999$ & $15: 40$ & 1of 2 & $<0.02$ & 0.03 & 0.004 & 0.001 & $<0.004$ & 0.001 & 0.0026 & 0.0005 & 0.012 & 0.004 \\
\hline BY88 & $\begin{array}{l}\text { Poore Mine ground } \\
\text { sluice nr Grass } \\
\text { Valley }\end{array}$ & 4/1/1999 & $15: 40$ & 2 of 2 & $<0.02$ & 0.02 & 0.005 & 0.003 & $<0.004$ & 0.003 & 0.0035 & 0.0001 & 0.0097 & 0.0012 \\
\hline BY89 & $\begin{array}{l}\text { Poore Mine pit lake nr } \\
\text { Grass Valley }\end{array}$ & 4/1/1999 & $16: 50$ & 1of 2 & $<0.02$ & 0.02 & 0.007 & 0.000 & $<0.004$ & 0.003 & 0.0018 & 0.0006 & 0.0082 & 0.0013 \\
\hline BY89 & $\begin{array}{l}\text { Poore Mine pit lake nr } \\
\text { Grass Valley }\end{array}$ & 4/1/1999 & $16: 50$ & $2 o f 2$ & $<0.02$ & 0.01 & 0.007 & 0.002 & $<0.004$ & 0.007 & 0.0023 & 0.0005 & 0.0088 & 0.0015 \\
\hline BY89 & $\begin{array}{l}\text { Poore Mine pit lake nr } \\
\text { Grass Valley }\end{array}$ & $5 / 23 / 2000$ & $10: 45$ & 1of 2 & $<0.04$ & 0.02 & 0.004 & 0.001 & 0.029 & 0.004 & 0.0035 & 0.0007 & 0.031 & 0.002 \\
\hline BY89 & $\begin{array}{l}\text { Poore Mine pit lake nr } \\
\text { Grass Valley }\end{array}$ & $5 / 23 / 2000$ & $10: 45$ & $2 o f 2$ & $<0.04$ & 0.02 & 0.005 & 0.001 & 0.028 & 0.000 & 0.0027 & 0.0006 & 0.030 & 0.002 \\
\hline BY90 & $\begin{array}{l}\text { Poore Mine seep } \\
\text { above ground sluice } \\
\text { nr Grass Valley }\end{array}$ & 4/1/1999 & $16: 00$ & 1of 2 & - & & - & & - & & - & & - & \\
\hline BY90 & $\begin{array}{l}\text { Poore Mine seep } \\
\text { above ground sluice } \\
\text { nr Grass Valley }\end{array}$ & 4/1/1999 & $16: 00$ & 2 of 2 & - & & - & & - & & - & & - & \\
\hline BY91 & $\begin{array}{l}\text { Poore Mine tunnel } \\
\text { effluent nr Grass } \\
\text { Valley }\end{array}$ & 3/31/1999 & $15: 20$ & 1of 2 & $<0.02$ & 0.03 & 0.006 & 0.002 & $<0.004$ & 0.006 & 0.0011 & 0.0005 & 0.048 & 0.001 \\
\hline BY91 & $\begin{array}{l}\text { Poore Mine tunnel } \\
\text { effluent nr Grass } \\
\text { Valley }\end{array}$ & 3/31/1999 & $15: 20$ & $2 o f 2$ & $<0.02$ & 0.00 & 0.010 & 0.002 & $<0.004$ & 0.001 & 0.0012 & 0.0006 & 0.045 & 0.001 \\
\hline BY91 & $\begin{array}{l}\text { Poore Mine tunnel } \\
\text { effluent nr Grass } \\
\text { Valley }\end{array}$ & $12 / 15 / 1999$ & $13: 00$ & 1 of 2 & $<0.02$ & 0.02 & 0.013 & 0.003 & $<0.006$ & 0.001 & 0.0014 & 0.0012 & 0.079 & 0.004 \\
\hline BY91 & $\begin{array}{l}\text { Poore Mine tunnel } \\
\text { effluent nr Grass } \\
\text { Valley }\end{array}$ & $12 / 15 / 1999$ & 13:00 & $2 o f 2$ & $<0.02$ & 0.01 & 0.046 & 0.004 & $<0.006$ & 0.004 & 0.0019 & 0.0013 & 0.079 & 0.001 \\
\hline BY91 & $\begin{array}{l}\text { Poore Mine tunnel } \\
\text { effluent nr Grass } \\
\text { Valley }\end{array}$ & $5 / 23 / 2000$ & $12: 00$ & 1of 2 & $<0.04$ & 0.01 & 0.032 & 0.001 & 0.011 & 0.008 & 0.019 & 0.002 & 0.35 & 0.01 \\
\hline BY91 & $\begin{array}{l}\text { Poore Mine tunnel } \\
\text { effluent nr Grass } \\
\text { Valley }\end{array}$ & $5 / 23 / 2000$ & $12: 00$ & $2 o f 2$ & $<0.04$ & 0.02 & 0.027 & 0.001 & 0.008 & 0.002 & 0.019 & 0.001 & 0.32 & 0.00 \\
\hline
\end{tabular}


Table 4A. Concentrations of trace metals and selected major elements in unfiltered water samples, Greenhorn Creek drainage, Nevada County, California—Continued.

[Station name abbreviations: ab, above; bl, below; Cr, Creek; mi, miles; N, north; nr, near; Rd, Road; S, south; SF, South Fork. Number in parentheses is the data parameter code, a five-digit code used in the U.S. Geological Survey computerized data system. $\mu \mathrm{g} / \mathrm{L}$, microgram per liter (equivalent to part per billion); $\mathrm{mg} / \mathrm{L}$, milligram per liter (at low concentration, equivalent to part per million); s.d., standard deviation; <, less than; - , not determined]

\begin{tabular}{|c|c|c|c|c|c|c|c|c|c|c|c|c|c|c|}
\hline \multirow[t]{2}{*}{$\begin{array}{l}\text { Station } \\
\text { map ID }\end{array}$} & \multirow[t]{2}{*}{ Station name } & \multirow[t]{2}{*}{ Date } & \multirow[t]{2}{*}{ Time } & \multirow[t]{2}{*}{$\begin{array}{l}\text { Rep- } \\
\text { licate }\end{array}$} & \multicolumn{2}{|c|}{$\begin{array}{c}\text { Tellurium }(\mathrm{Te}) \\
\text { unfiltered } \\
(\mu \mathrm{g} / \mathrm{L}) \\
(01064)\end{array}$} & \multicolumn{2}{|c|}{$\begin{array}{c}\text { Thorium }(T h), \\
\text { unfiltered } \\
(\mu \mathrm{g} / \mathrm{L}) \\
(82364)\end{array}$} & \multicolumn{2}{|c|}{$\begin{array}{c}\text { Thallium }(\mathrm{TI}), \\
\text { unfiltered } \\
(\mu \mathrm{g} / \mathrm{L}) \\
(01059)\end{array}$} & \multicolumn{2}{|c|}{$\begin{array}{c}\text { Thulium (Tm), } \\
\text { unfiltered } \\
(\mu \mathrm{g} / \mathrm{L})\end{array}$} & \multicolumn{2}{|c|}{$\begin{array}{c}\text { Uranium }(\mathrm{U}), \\
\text { unfiltered } \\
(\mu \mathrm{g} / \mathrm{L}) \\
(28011)\end{array}$} \\
\hline & & & & & value & s.d. & value & s.d. & value & s.d. & value & s.d. & value & s.d. \\
\hline BY105 & $\begin{array}{l}\text { Sailor Flat Mine main } \\
\text { drain gulch } 01 \mathrm{nr} \\
\text { Quaker Hill }\end{array}$ & $7 / 6 / 1999$ & $16: 00$ & 1 of 2 & $<0.02$ & 0.01 & 0.22 & 0.03 & 0.047 & 0.013 & 0.11 & 0.00 & 0.66 & 0.05 \\
\hline BY105 & $\begin{array}{l}\text { Sailor Flat Mine main } \\
\text { drain gulch } 01 \mathrm{nr} \\
\text { Quaker Hill }\end{array}$ & 7/6/1999 & $16: 00$ & $2 \mathrm{of} 2$ & 0.03 & 0.01 & 0.23 & 0.03 & 0.045 & 0.003 & 0.11 & 0.00 & 0.72 & 0.00 \\
\hline BY105 & $\begin{array}{l}\text { Sailor Flat Mine main } \\
\text { drain Gulch } 01 \mathrm{nr} \\
\text { Quaker Hill }\end{array}$ & $12 / 2 / 1999$ & $14: 30$ & 1 of 2 & 0.06 & 0.03 & 13 & 0 & 0.17 & 0.01 & 0.23 & 0.02 & 5.7 & 0.2 \\
\hline BY105 & $\begin{array}{l}\text { Sailor Flat Mine main } \\
\text { drain Gulch } 01 \mathrm{nr} \\
\text { Quaker Hill }\end{array}$ & $12 / 2 / 1999$ & $14: 30$ & 2 of 2 & 0.03 & 0.02 & 11 & 0 & 0.19 & 0.01 & 0.24 & 0.02 & 6.0 & 0.1 \\
\hline BY106 & $\begin{array}{l}\text { Sailor Flat Mine } \\
\text { main drainage to } \\
\text { Greenhorn Cr, } \\
\text { Gulch } 03\end{array}$ & 4/9/2001 & $12: 30$ & 1 of 2 & $<0.1$ & 0.0 & 0.07 & 0.00 & $<0.01$ & 0.00 & 0.023 & 0.004 & 0.43 & 0.05 \\
\hline BY106 & $\begin{array}{l}\text { Sailor Flat Mine } \\
\text { main drainage to } \\
\text { Greenhorn Cr, } \\
\text { Gulch } 03\end{array}$ & $4 / 9 / 2001$ & $12: 30$ & 2 of 2 & $<0.1$ & 0.0 & 0.09 & 0.02 & $<0.01$ & 0.01 & 0.023 & 0.002 & 0.43 & 0.00 \\
\hline BY114 & $\begin{array}{l}\text { SF Greenhorn Cr } 0.7 \\
\text { mi ab Greenhorn Cr } \\
\text { nr Nevada City }\end{array}$ & $8 / 22 / 2000$ & $15: 10$ & 1of 2 & $<0.07$ & 0.04 & 0.007 & 0.003 & $<0.07$ & 0.01 & $<0.001$ & 0.000 & 0.011 & 0.003 \\
\hline BY114 & $\begin{array}{l}\text { SF Greenhorn Cr } 0.7 \\
\text { mi ab Greenhorn Cr } \\
\text { nr Nevada City }\end{array}$ & $8 / 22 / 2000$ & $15: 10$ & $2 \mathrm{of} 2$ & $<0.07$ & 0.02 & 0.008 & 0.005 & $<0.07$ & 0.01 & $<0.001$ & 0.000 & 0.010 & 0.004 \\
\hline BY122 & $\begin{array}{l}\text { Starr Mine tunnel } \\
\text { inflow nr Grass } \\
\text { Valley }\end{array}$ & 4/23/1999 & $15: 00$ & 1 of 2 & $<0.02$ & 0.01 & 0.006 & 0.001 & $<0.004$ & 0.003 & 0.0032 & 0.0008 & 0.0085 & 0.0012 \\
\hline BY122 & $\begin{array}{l}\text { Starr Mine tunnel } \\
\text { inflow nr Grass } \\
\text { Valley }\end{array}$ & 4/23/1999 & $15: 00$ & 2of2 & $<0.02$ & 0.02 & 0.008 & 0.002 & $<0.004$ & 0.001 & 0.0035 & 0.0008 & 0.0086 & 0.0011 \\
\hline BY123 & $\begin{array}{l}\text { Starr Mine tunnel } \\
\text { midway nr Grass } \\
\text { Valley }\end{array}$ & 4/23/1999 & $13: 40$ & 1 of 2 & $<0.02$ & 0.02 & 0.12 & 0.00 & $<0.004$ & 0.004 & 0.0024 & 0.0004 & 0.046 & 0.002 \\
\hline BY123 & $\begin{array}{l}\text { Starr Mine tunnel } \\
\text { midway nr Grass } \\
\text { Valley }\end{array}$ & 4/23/1999 & $13: 40$ & 2of2 & $<0.02$ & 0.03 & 0.013 & 0.003 & $<0.004$ & 0.005 & 0.0017 & 0.0002 & 0.040 & 0.001 \\
\hline
\end{tabular}


Table 4A. Concentrations of trace metals and selected major elements in unfiltered water samples, Greenhorn Creek drainage, Nevada County, California—Continued.

[Station name abbreviations: ab, above; bl, below; Cr, Creek; mi, miles; N, north; nr, near; Rd, Road; S, south; SF, South Fork. Number in parentheses is the data parameter code, a five-digit code used in the U.S. Geological Survey computerized data system. $\mu \mathrm{g} / \mathrm{L}$, microgram per liter (equivalent to part per billion); mg/L, milligram per liter (at low concentration, equivalent to part per million); s.d., standard deviation; $<$, less than; —, not determined]

\begin{tabular}{|c|c|c|c|c|c|c|c|c|c|c|c|c|c|c|}
\hline \multirow[t]{2}{*}{$\begin{array}{l}\text { Station } \\
\text { map ID }\end{array}$} & \multirow[t]{2}{*}{ Station name } & \multirow[t]{2}{*}{ Date } & \multirow[t]{2}{*}{ Time } & \multirow[t]{2}{*}{$\begin{array}{l}\text { Rep- } \\
\text { licate }\end{array}$} & \multicolumn{2}{|c|}{$\begin{array}{c}\text { Tellurium }(\mathrm{Te}), \\
\text { unfiltered } \\
(\mu \mathrm{g} / \mathrm{L}) \\
(01064)\end{array}$} & \multicolumn{2}{|c|}{$\begin{array}{c}\text { Thorium (Th), } \\
\text { unfiltered } \\
(\mu \mathrm{g} / \mathrm{L}) \\
(82364) \\
\end{array}$} & \multicolumn{2}{|c|}{$\begin{array}{c}\text { Thallium (TI), } \\
\text { unfiltered } \\
(\mu \mathrm{g} / \mathrm{L}) \\
(01059) \\
\end{array}$} & \multicolumn{2}{|c|}{$\begin{array}{c}\text { Thulium (Tm), } \\
\text { unfiltered } \\
(\mu \mathrm{g} / \mathrm{L}) \\
\end{array}$} & \multicolumn{2}{|c|}{$\begin{array}{c}\text { Uranium }(\mathrm{U}), \\
\text { unfiltered } \\
(\mu \mathrm{g} / \mathrm{L}) \\
(\mathbf{2 8 0 1 1 )} \\
\end{array}$} \\
\hline & & & & & value & s.d. & value & s.d. & value & s.d. & value & s.d. & value & s.d. \\
\hline BY123 & $\begin{array}{l}\text { Starr Mine tunnel } \\
\text { midway nr Grass } \\
\text { Valley }\end{array}$ & $12 / 15 / 1999$ & $10: 45$ & 1 of 2 & $<0.02$ & 0.03 & 0.031 & 0.003 & $<0.006$ & 0.003 & 0.0057 & 0.0012 & 0.062 & 0.002 \\
\hline BY123 & $\begin{array}{l}\text { Starr Mine tunnel } \\
\text { midway nr Grass } \\
\text { Valley }\end{array}$ & $12 / 15 / 1999$ & $10: 45$ & $2 \mathrm{of} 2$ & $<0.02$ & 0.01 & 0.031 & 0.000 & $<0.006$ & 0.007 & 0.0051 & 0.0009 & 0.061 & 0.002 \\
\hline BY124 & $\begin{array}{l}\text { Starr Mine tunnel } \\
\text { outlet nr Grass } \\
\text { Valley }\end{array}$ & $8 / 23 / 2000$ & $17: 30$ & 1 of 2 & $<0.07$ & 0.08 & 0.022 & 0.003 & $<0.07$ & 0.05 & 0.010 & 0.002 & 0.19 & 0.00 \\
\hline BY124 & $\begin{array}{l}\text { Starr Mine tunnel } \\
\text { outlet nr Grass } \\
\text { Valley }\end{array}$ & $8 / 23 / 2000$ & $17: 30$ & 2 of 2 & $<0.07$ & 0.03 & 0.021 & 0.003 & $<0.07$ & 0.02 & 0.013 & 0.002 & 0.19 & 0.00 \\
\hline BY129 & $\begin{array}{l}\text { Tom and Jerry Mine } \\
\text { drain at tunnel inlet } \\
\text { nr Nevada City }\end{array}$ & $6 / 28 / 2000$ & $12: 00$ & 1 of 2 & $<0.07$ & 0.00 & 0.25 & 0.00 & $<0.07$ & 0.03 & 0.13 & 0.03 & 0.13 & 0.03 \\
\hline BY129 & $\begin{array}{l}\text { Tom and Jerry Mine } \\
\text { drain at tunnel inlet } \\
\text { nr Nevada City }\end{array}$ & $6 / 28 / 2000$ & $12: 00$ & $2 \mathrm{of} 2$ & $<0.07$ & 0.01 & 0.24 & 0.00 & $<0.07$ & 0.00 & 0.13 & 0.02 & 0.14 & 0.01 \\
\hline BY130 & $\begin{array}{l}\text { Tom and Jerry Mine } \\
\text { drain at tunnel } \\
\text { outlet nr Nevada } \\
\text { City }\end{array}$ & $6 / 28 / 2000$ & $11: 30$ & 1 of 2 & $<0.07$ & 0.04 & 0.020 & 0.012 & $<0.07$ & 0.00 & 0.002 & 0.001 & 0.006 & 0.000 \\
\hline BY130 & $\begin{array}{l}\text { Tom and Jerry Mine } \\
\text { drain at tunnel } \\
\text { outlet nr Nevada } \\
\text { City }\end{array}$ & $6 / 28 / 2000$ & $11: 30$ & $2 \mathrm{of} 2$ & $<0.07$ & 0.05 & 0.014 & 0.005 & $<0.07$ & 0.01 & 0.002 & 0.000 & 0.007 & 0.002 \\
\hline BY131 & $\begin{array}{l}\text { Tom and Jerry Mine } \\
\text { drainage pond nr } \\
\text { Nevada City }\end{array}$ & $8 / 23 / 2000$ & $10: 45$ & 1 of 2 & $<0.07$ & 0.05 & 0.013 & 0.002 & $<0.07$ & 0.01 & 0.003 & 0.000 & 0.008 & 0.001 \\
\hline BY131 & $\begin{array}{l}\text { Tom and Jerry Mine } \\
\text { drainage pond nr } \\
\text { Nevada City }\end{array}$ & $8 / 23 / 2000$ & $10: 45$ & $2 \mathrm{of} 2$ & $<0.07$ & 0.06 & 0.010 & 0.003 & $<0.07$ & 0.01 & 0.005 & 0.000 & 0.009 & 0.002 \\
\hline
\end{tabular}


Table 4A. Concentrations of trace metals and selected major elements in unfiltered water samples, Greenhorn Creek drainage, Nevada County, California-Continued.

[Station name abbreviations: ab, above; bl, below; Cr, Creek; mi, miles; N, north; nr, near; Rd, Road; S, south; SF, South Fork. Number in parentheses is the data parameter code, a five-digit code used in the U.S. Geological Survey computerized data system. $\mu \mathrm{g} / \mathrm{L}$, microgram per liter (equivalent to part per billion); mg/L, milligram per liter (at low concentration, equivalent to part per million); s.d., standard deviation; <, less than; - , not determined]

\begin{tabular}{|c|c|c|c|c|c|c|c|c|c|c|c|c|c|c|}
\hline \multirow[t]{2}{*}{$\begin{array}{l}\text { Station } \\
\text { map ID }\end{array}$} & \multirow[t]{2}{*}{ Station name } & \multirow[t]{2}{*}{ Date } & \multirow[t]{2}{*}{ Time } & \multirow[t]{2}{*}{$\begin{array}{l}\text { Rep- } \\
\text { licate }\end{array}$} & \multicolumn{2}{|c|}{$\begin{array}{c}\text { Tellurium }(\mathrm{Te}), \\
\text { unfiltered } \\
(\mu \mathrm{g} / \mathrm{L}) \\
(\mathbf{0 1 0 6 4 )}\end{array}$} & \multicolumn{2}{|c|}{$\begin{array}{c}\text { Thorium (Th), } \\
\text { unfiltered } \\
(\mu \mathrm{g} / \mathrm{L}) \\
(\mathbf{8 2 3 6 4 )}\end{array}$} & \multicolumn{2}{|c|}{$\begin{array}{c}\text { Thallium }(\mathrm{TI}), \\
\text { unfiltered } \\
(\mu \mathrm{g} / \mathrm{L}) \\
(01059)\end{array}$} & \multicolumn{2}{|c|}{$\begin{array}{c}\text { Thulium (Tm), } \\
\text { unfiltered } \\
(\mu \mathrm{g} / \mathrm{L}) \\
\end{array}$} & \multicolumn{2}{|c|}{$\begin{array}{c}\text { Uranium }(U), \\
\text { unfiltered } \\
(\mu \mathrm{g} / \mathrm{L}) \\
(28011)\end{array}$} \\
\hline & & & & & value & s.d. & value & s.d. & value & s.d. & value & s.d. & value & s.d \\
\hline \multicolumn{15}{|c|}{ Disturbed samples } \\
\hline BY123 & $\begin{array}{l}\text { Starr Mine tunnel } \\
\text { midway nr Grass } \\
\text { Valley }\end{array}$ & $12 / 15 / 1999$ & $11: 30$ & 1 of 2 & 0.75 & 0.06 & 14 & 0 & 3.1 & 0.1 & 2.0 & 0.1 & 7.6 & 0.3 \\
\hline BY123 & $\begin{array}{l}\text { Starr Mine tunnel } \\
\text { midway nr Grass } \\
\text { Valley }\end{array}$ & $12 / 15 / 1999$ & $11: 30$ & 2 of 2 & 0.61 & 0.08 & 12 & 0 & 3.4 & 0.1 & 2.2 & 0.0 & 7.1 & 0.2 \\
\hline BY129 & $\begin{array}{l}\text { Tom and Jerry Mine } \\
\text { drain at tunnel inlet } \\
\text { nr Nevada City }\end{array}$ & $6 / 28 / 2000$ & $12: 30$ & 1 of 2 & 0.10 & 0.08 & 5.6 & 0.2 & 0.16 & 0.06 & 1.1 & 0.2 & 4.7 & 0.7 \\
\hline BY129 & $\begin{array}{l}\text { Tom and Jerry Mine } \\
\text { drain at tunnel inlet } \\
\text { nr Nevada City }\end{array}$ & $6 / 28 / 2000$ & $12: 30$ & 2 of 2 & 0.07 & 0.05 & 5.9 & 0.0 & 0.21 & 0.03 & 1.2 & 0.0 & 5.2 & 0.2 \\
\hline
\end{tabular}


Table 4A. Concentrations of trace metals and selected major elements in unfiltered water samples, Greenhorn Creek drainage, Nevada County, California—Continued.

[Station name abbreviations: ab, above; bl, below; Cr, Creek; mi, miles; N, north; nr, near; Rd, Road; S, south; SF, South Fork. Number in parentheses is the data parameter code, a five-digit code used in the U.S. Geological Survey computerized data system. $\mu \mathrm{g} / \mathrm{L}$, microgram per liter (equivalent to part per billion); $\mathrm{mg} / \mathrm{L}$, milligram per liter (at low concentration, equivalent to part per million); s.d., standard deviation; $<$, less than; —, not determined]

\begin{tabular}{|c|c|c|c|c|c|c|c|c|c|c|c|c|c|c|}
\hline \multirow[t]{2}{*}{$\begin{array}{l}\text { Station } \\
\text { map ID }\end{array}$} & \multirow[t]{2}{*}{ Station name } & \multirow[t]{2}{*}{ Date } & \multirow[t]{2}{*}{ Time } & \multirow[t]{2}{*}{$\begin{array}{l}\text { Rep- } \\
\text { licate }\end{array}$} & \multicolumn{2}{|c|}{$\begin{array}{c}\text { Vanadium }(\mathrm{V}), \\
\text { unfiltered } \\
(\mu \mathrm{g} / \mathrm{L}) \\
(\mathbf{0 1 0 8 7}) \\
\end{array}$} & \multicolumn{2}{|c|}{$\begin{array}{c}\text { Yttrium }(\mathrm{Y}) \text {, } \\
\text { unfiltered } \\
(\mu \mathrm{g} / \mathrm{L}) \\
(01203)\end{array}$} & \multicolumn{2}{|c|}{$\begin{array}{c}\text { Ytterbium }(Y \mathbf{b}) \text {, } \\
\text { unfiltered } \\
(\mu \mathrm{g} / \mathrm{L}) \\
(01196)\end{array}$} & \multicolumn{2}{|c|}{$\begin{array}{c}\text { Zinc }(Z n), \\
\text { unfiltered } \\
(\mu \mathrm{g} / \mathrm{L}) \\
(01092)\end{array}$} & \multicolumn{2}{|c|}{$\begin{array}{c}\text { Zirconium }(\mathrm{Zr}) \\
\text { unfiltered } \\
(\mu \mathrm{g} / \mathrm{L}) \\
(01162)\end{array}$} \\
\hline & & & & & value & s.d. & value & s.d. & value & s.d. & value & s.d. & value & s.d. \\
\hline \multicolumn{15}{|c|}{ Environmental samples } \\
\hline BY20 & $\begin{array}{l}\text { Boston Mine tunnel } \\
\text { outlet nr Grass } \\
\text { Valley }\end{array}$ & 6/18/1999 & $14: 30$ & 1 of 2 & $<3$ & 2 & 0.16 & 0.03 & 0.009 & 0.001 & 5.1 & 0.9 & 0.006 & 0.003 \\
\hline BY20 & $\begin{array}{l}\text { Boston Mine tunnel } \\
\text { outlet nr Grass } \\
\text { Valley }\end{array}$ & $6 / 18 / 1999$ & $14: 30$ & $2 o f 2$ & $<3$ & 2 & 0.16 & 0.02 & 0.010 & 0.002 & 4.7 & 1.9 & 0.011 & 0.003 \\
\hline BY20 & $\begin{array}{l}\text { Boston Mine tunnel } \\
\text { outlet nr Grass } \\
\text { Valley }\end{array}$ & $12 / 14 / 1999$ & $12: 00$ & 1 of 2 & $<4$ & 1 & 1.1 & 0.0 & 0.060 & 0.003 & 43 & 11 & 0.018 & 0.006 \\
\hline BY20 & $\begin{array}{l}\text { Boston Mine tunnel } \\
\text { outlet nr Grass } \\
\text { Valley }\end{array}$ & $12 / 14 / 1999$ & $12: 00$ & $2 o f 2$ & $<4$ & 3 & 0.47 & 0.05 & 0.044 & 0.002 & 29 & 3 & 0.012 & 0.004 \\
\hline BY20 & $\begin{array}{l}\text { Boston Mine tunnel } \\
\text { outlet nr Grass } \\
\text { Valley }\end{array}$ & $5 / 24 / 2000$ & $16: 00$ & 1 of 2 & $<1$ & 0 & 0.22 & 0.01 & 0.015 & 0.003 & 5.0 & 0.4 & 0.006 & 0.001 \\
\hline BY20 & $\begin{array}{l}\text { Boston Mine tunnel } \\
\text { outlet nr Grass } \\
\text { Valley }\end{array}$ & $5 / 24 / 2000$ & $16: 00$ & $2 o f 2$ & $<1$ & 1 & 0.46 & 0.01 & 0.030 & 0.007 & 13 & 1 & 0.009 & 0.005 \\
\hline BY21 & $\begin{array}{l}\text { Boston Mine wetlands } \\
\text { pond nr Grass } \\
\text { Valley }\end{array}$ & $12 / 14 / 1999$ & $10: 30$ & 1of 2 & $<2$ & 1 & 0.028 & 0.001 & $<0.002$ & 0.001 & 15 & 2 & 0.011 & 0.006 \\
\hline BY21 & $\begin{array}{l}\text { Boston Mine wetlands } \\
\text { pond nr Grass } \\
\text { Valley }\end{array}$ & $12 / 14 / 1999$ & $10: 30$ & $2 o f 2$ & $<2$ & 1 & 0.037 & 0.001 & 0.004 & 0.001 & 14 & 3 & 0.011 & 0.002 \\
\hline BY21 & $\begin{array}{l}\text { Boston Mine wetlands } \\
\text { pond nr Grass } \\
\text { Valley }\end{array}$ & $5 / 24 / 2000$ & $16: 30$ & 1 of 2 & $<1$ & 1 & 0.058 & 0.005 & 0.003 & 0.000 & 4.4 & 0.1 & 0.013 & 0.002 \\
\hline BY21 & $\begin{array}{l}\text { Boston Mine wetlands } \\
\text { pond nr Grass } \\
\text { Valley }\end{array}$ & $5 / 24 / 2000$ & $16: 30$ & $2 o f 2$ & $<1$ & 0 & 0.054 & 0.004 & 0.004 & 0.002 & 7.4 & 0.6 & 1.0 & 0.1 \\
\hline BY58 & $\begin{array}{l}\text { Buckeye Flat Mine } \\
\text { main drain } 0.45 \mathrm{mi} \\
\text { ab Greenhorn } \mathrm{Cr}\end{array}$ & $8 / 21 / 2000$ & 11:00 & 1 of 2 & $<1$ & 1 & 22 & 1 & 1.4 & 0.1 & 290 & 10 & $<0.3$ & 0.1 \\
\hline BY58 & $\begin{array}{l}\text { Buckeye Flat Mine } \\
\text { main drain } 0.45 \mathrm{mi} \\
\text { ab Greenhorn } \mathrm{Cr}\end{array}$ & $8 / 21 / 2000$ & 11:00 & $2 o f 2$ & $<1$ & 1 & 17 & 1 & 0.99 & 0.01 & 220 & 10 & $<0.3$ & 0.2 \\
\hline
\end{tabular}


Table 4A. Concentrations of trace metals and selected major elements in unfiltered water samples, Greenhorn Creek drainage, Nevada County, California—Continued

[Station name abbreviations: ab, above; bl, below; Cr, Creek; mi, miles; N, north; nr, near; Rd, Road; S, south; SF, South Fork. Number in parentheses is the data parameter code, a five-digit code used in the U.S. Geological Survey computerized data system. $\mu \mathrm{g} / \mathrm{L}$, microgram per liter (equivalent to part per billion); mg/L, milligram per liter (at low concentration, equivalent to part per million); s.d., standard deviation; $<$, less than; —, not determined]

\begin{tabular}{|c|c|c|c|c|c|c|c|c|c|c|c|c|c|c|}
\hline \multirow[t]{2}{*}{$\begin{array}{l}\text { Station } \\
\text { map ID }\end{array}$} & \multirow[t]{2}{*}{ Station name } & \multirow[t]{2}{*}{ Date } & \multirow[t]{2}{*}{ Time } & \multirow[t]{2}{*}{$\begin{array}{c}\text { Rep- } \\
\text { licate }\end{array}$} & \multicolumn{2}{|c|}{$\begin{array}{c}\text { Vanadium }(\mathrm{V}), \\
\text { unfiltered } \\
(\mu \mathrm{g} / \mathrm{L}) \\
(01087)\end{array}$} & \multicolumn{2}{|c|}{$\begin{array}{c}\text { Yttrium }(\mathrm{Y}) \text {, } \\
\text { unfiltered } \\
(\mu \mathrm{g} / \mathrm{L}) \\
(01203)\end{array}$} & \multicolumn{2}{|c|}{$\begin{array}{c}\text { Ytterbium }(Y \mathbf{b}), \\
\text { unfiltered } \\
(\mu \mathrm{g} / \mathrm{L}) \\
(01196)\end{array}$} & \multicolumn{2}{|c|}{$\begin{array}{c}\text { Zinc }(Z n) \\
\text { unfiltered } \\
(\mu \mathrm{g} / \mathrm{L}) \\
(\mathbf{0 1 0 9 2 )}\end{array}$} & \multicolumn{2}{|c|}{$\begin{array}{c}\text { Zirconium }(\mathrm{Zr}) \\
\text { unfiltered } \\
(\mu \mathrm{g} / \mathrm{L}) \\
(01162)\end{array}$} \\
\hline & & & & & value & s.d. & value & s.d. & value & s.d. & value & s.d. & value & s.d. \\
\hline BY23 & $\begin{array}{c}\text { Buckeye Flat Mine N } \\
\text { drain to Greenhorn } \\
\text { Cr, Grass Valley }\end{array}$ & $6 / 18 / 1999$ & $11: 30$ & 1of 2 & $<3$ & 2 & 44 & 1 & 1.8 & 0.1 & 550 & 30 & 0.021 & 0.000 \\
\hline BY23 & $\begin{array}{c}\text { Buckeye Flat Mine N } \\
\text { drain to Greenhorn } \\
\text { Cr, Grass Valley }\end{array}$ & $6 / 18 / 1999$ & $11: 30$ & $2 o f 2$ & $<3$ & 2 & 31 & 5 & 2.0 & 0.1 & 550 & 10 & 0.014 & 0.004 \\
\hline BY23 & $\begin{array}{c}\text { Buckeye Flat Mine N } \\
\text { drain to Greenhorn } \\
\text { Cr, Grass Valley }\end{array}$ & $12 / 2 / 1999$ & 11:00 & 1of 2 & $<4$ & 2 & 25 & 1 & 1.5 & 0.0 & 270 & 20 & 0.093 & 0.005 \\
\hline BY23 & $\begin{array}{c}\text { Buckeye Flat Mine N } \\
\text { drain to Greenhorn } \\
\text { Cr, Grass Valley }\end{array}$ & $12 / 2 / 1999$ & 11:00 & $2 o f 2$ & $<4$ & 0 & 18 & 1 & 1.9 & 0.0 & 270 & 0 & 0.091 & 0.007 \\
\hline BY23 & $\begin{array}{c}\text { Buckeye Flat Mine N } \\
\text { drain to Greenhorn } \\
\text { Cr, Grass Valley }\end{array}$ & $8 / 22 / 2000$ & $10: 45$ & 1 of 2 & $<1$ & 1 & 32 & 0 & 1.9 & 0.2 & 400 & 0 & 19 & 1 \\
\hline BY23 & $\begin{array}{c}\text { Buckeye Flat Mine N } \\
\text { drain to Greenhorn } \\
\text { Cr, Grass Valley }\end{array}$ & $8 / 22 / 2000$ & $10: 45$ & $2 o f 2$ & $<1$ & 1 & 29 & 3 & 1.8 & 0.0 & 360 & 10 & 8.0 & 7.9 \\
\hline BY116 & $\begin{array}{l}\text { Buckeye Flat Mine } \\
\text { pond drain } 0.15 \mathrm{mi} \\
\text { ab SF Greenhorn } \mathrm{Cr}\end{array}$ & $8 / 21 / 2000$ & $14: 10$ & 1 of 2 & $<2$ & 1 & 0.17 & 0.02 & 0.016 & 0.001 & 9 & 0 & 0.011 & 0.007 \\
\hline BY116 & $\begin{array}{l}\text { Buckeye Flat Mine } \\
\text { pond drain } 0.15 \mathrm{mi} \\
\text { ab SF Greenhorn } \mathrm{Cr}\end{array}$ & $8 / 21 / 2000$ & $14: 10$ & $2 \mathrm{of} 2$ & $<2$ & 1 & 0.17 & 0.00 & 0.015 & 0.004 & 9 & 1 & 0.016 & 0.009 \\
\hline BY24 & $\begin{array}{l}\text { Buckeye Flat Mine } \\
\text { S drain to SF } \\
\text { Greenhorn Cr, } \\
\text { Grass Valley }\end{array}$ & $8 / 26 / 1999$ & 11:00 & 1 of 2 & $<3$ & 2 & 0.98 & 0.06 & 0.048 & 0.004 & 23 & 1 & 0.015 & 0.002 \\
\hline BY24 & $\begin{array}{l}\text { Buckeye Flat Mine } \\
\text { S drain to SF } \\
\text { Greenhorn Cr, } \\
\text { Grass Valley }\end{array}$ & $8 / 26 / 1999$ & 11:00 & $2 o f 2$ & $<3$ & 1 & 0.94 & 0.08 & 0.056 & 0.010 & 21 & 8 & 0.014 & 0.006 \\
\hline BY24 & $\begin{array}{l}\text { Buckeye Flat Mine } \\
\text { S drain to SF } \\
\text { Greenhorn } \mathrm{Cr}, \\
\text { Grass Valley }\end{array}$ & $8 / 22 / 2000$ & $12: 10$ & 1of 2 & $<2$ & 1 & 1.4 & 0.1 & 0.11 & 0.03 & 17 & 2 & 0.035 & 0.011 \\
\hline BY24 & $\begin{array}{l}\text { Buckeye Flat Mine } \\
\text { S drain to SF } \\
\text { Greenhorn Cr, } \\
\text { Grass Valley }\end{array}$ & $8 / 22 / 2000$ & $12: 10$ & $2 o f 2$ & $<2$ & 1 & 1.3 & 0.2 & 0.11 & 0.02 & 19 & 0 & 0.079 & 0.028 \\
\hline
\end{tabular}


Table 4A. Concentrations of trace metals and selected major elements in unfiltered water samples, Greenhorn Creek drainage, Nevada County, California—Continued.

[Station name abbreviations: ab, above; bl, below; Cr, Creek; mi, miles; N, north; nr, near; Rd, Road; S, south; SF, South Fork. Number in parentheses is the data parameter code, a five-digit code used in the

U.S. Geological Survey computerized data system. $\mu \mathrm{g} / \mathrm{L}$, microgram per liter (equivalent to part per billion); mg/L, milligram per liter (at low concentration, equivalent to part per million); s.d., standard deviation; <, less than; —, not determined]

\begin{tabular}{|c|c|c|c|c|c|c|c|c|c|c|c|c|c|c|}
\hline \multirow[t]{2}{*}{$\begin{array}{l}\text { Station } \\
\text { map ID }\end{array}$} & \multirow[t]{2}{*}{ Station name } & \multirow[t]{2}{*}{ Date } & \multirow[t]{2}{*}{ Time } & \multirow[t]{2}{*}{$\begin{array}{l}\text { Rep- } \\
\text { licate }\end{array}$} & \multicolumn{2}{|c|}{$\begin{array}{c}\text { Vanadium }(\mathrm{V}), \\
\text { unfiltered } \\
(\mu \mathrm{g} / \mathrm{L}) \\
(\mathbf{0 1 0 8 7}) \\
\end{array}$} & \multicolumn{2}{|c|}{$\begin{array}{c}\text { Yttrium }(\mathrm{Y}) \text {, } \\
\text { unfiltered } \\
(\mu \mathrm{g} / \mathrm{L}) \\
(01203)\end{array}$} & \multicolumn{2}{|c|}{$\begin{array}{c}\text { Ytterbium }(Y \mathbf{Y b}), \\
\text { unfiltered } \\
(\mu \mathrm{g} / \mathrm{L}) \\
(\mathbf{0 1 1 9 6 )} \\
\end{array}$} & \multicolumn{2}{|c|}{$\begin{array}{c}\text { Zinc }(\mathrm{Zn}) \text {, } \\
\text { unfiltered } \\
(\mu \mathrm{g} / \mathrm{L}) \\
(01092)\end{array}$} & \multicolumn{2}{|c|}{$\begin{array}{c}\text { Zirconium }(\mathrm{Zr}) \\
\text { unfiltered } \\
(\mu \mathrm{g} / \mathrm{L}) \\
(\mathbf{0 1 1 6 2 )} \\
\end{array}$} \\
\hline & & & & & value & s.d. & value & s.d. & value & s.d. & value & s.d. & value & s.d. \\
\hline BY25 & $\begin{array}{l}\text { Buckeye Flat Mine } \\
\text { upper drain }\end{array}$ & $4 / 9 / 2001$ & $10: 00$ & 1of 2 & $<1$ & 1 & 4.9 & 0.2 & 0.29 & 0.00 & 80 & 6 & $<0.02$ & 0.01 \\
\hline BY25 & $\begin{array}{l}\text { Buckeye Flat Mine } \\
\text { upper drain }\end{array}$ & $4 / 9 / 2001$ & $10: 00$ & 2of2 & $<1$ & 1 & 5.2 & 0.0 & 0.30 & 0.01 & 81 & 1 & $<0.02$ & 0.00 \\
\hline BY52 & $\begin{array}{l}\text { Greenhorn Cr } 0.3 \mathrm{mi} \\
\text { ab Buckeye Ford nr } \\
\text { Nevada City }\end{array}$ & $1 / 25 / 2000$ & $10: 45$ & 1of 2 & $<2$ & 1 & 1.0 & 0.0 & 0.092 & 0.001 & 17 & 2 & 0.091 & 0.011 \\
\hline BY52 & $\begin{array}{l}\text { Greenhorn Cr } 0.3 \mathrm{mi} \\
\text { ab Buckeye Ford nr } \\
\text { Nevada City }\end{array}$ & $1 / 25 / 2000$ & $10: 45$ & 2of 2 & $<2$ & 1 & 1.0 & 0.0 & 0.092 & 0.001 & 19 & 1 & 0.090 & 0.012 \\
\hline BY59 & $\begin{array}{l}\text { Greenhorn } \mathrm{Cr} \text { at You } \\
\text { Bet Rd nr Nevada } \\
\text { City }\end{array}$ & $7 / 24 / 2001$ & $11: 00$ & 1 of 2 & $<2$ & 1 & 0.052 & 0.003 & 0.005 & 0.002 & 3.1 & 0.3 & $<0.01$ & 0.00 \\
\hline BY59 & $\begin{array}{l}\text { Greenhorn } \mathrm{Cr} \text { at You } \\
\text { Bet Rd nr Nevada } \\
\text { City }\end{array}$ & $7 / 24 / 2001$ & $11: 00$ & 2 of 2 & $<2$ & 1 & 0.066 & 0.007 & 0.006 & 0.002 & 2.4 & 0.6 & $<0.01$ & 0.00 \\
\hline BY59 & $\begin{array}{l}\text { Greenhorn } \mathrm{Cr} \text { at You } \\
\text { Bet Rd nr Nevada } \\
\text { City }\end{array}$ & $8 / 29 / 2001$ & $15: 00$ & 1of 2 & $<2$ & 1 & 0.038 & 0.007 & $<0.004$ & 0.001 & 1.3 & 0.4 & $<0.01$ & 0.01 \\
\hline BY59 & $\begin{array}{l}\text { Greenhorn } \mathrm{Cr} \text { at You } \\
\text { Bet Rd nr Nevada } \\
\text { City }\end{array}$ & $8 / 29 / 2001$ & $15: 00$ & 2of 2 & $<2$ & 1 & 0.043 & 0.002 & 0.006 & 0.003 & 1.9 & 0.4 & $<0.01$ & 0.01 \\
\hline BY59 & $\begin{array}{l}\text { Greenhorn Cr at You } \\
\text { Bet Rd nr Nevada } \\
\text { City }\end{array}$ & $9 / 14 / 2001$ & $13: 00$ & 1of 2 & $<2$ & 2 & 0.047 & 0.003 & 0.005 & 0.002 & 4.3 & 0.8 & $<0.02$ & 0.00 \\
\hline BY59 & $\begin{array}{l}\text { Greenhorn Cr at You } \\
\text { Bet Rd nr Nevada } \\
\text { City }\end{array}$ & $9 / 14 / 2001$ & $13: 00$ & 2of 2 & $<2$ & 2 & 0.049 & 0.001 & $<0.003$ & 0.002 & 4.5 & 0.6 & $<0.02$ & 0.01 \\
\hline BY59 & $\begin{array}{l}\text { Greenhorn } \mathrm{Cr} \text { at You } \\
\text { Bet Rd nr Nevada } \\
\text { City }\end{array}$ & $10 / 15 / 2001$ & $12: 00$ & 1of 2 & $<1$ & 1 & 0.032 & 0.012 & 0.004 & 0.003 & $<2$ & 0 & $<0.02$ & 0.01 \\
\hline BY59 & $\begin{array}{l}\text { Greenhorn } \mathrm{Cr} \text { at You } \\
\text { Bet Rd nr Nevada } \\
\text { City }\end{array}$ & $10 / 15 / 2001$ & $12: 00$ & 2of2 & $<1$ & 2 & 0.029 & 0.002 & 0.004 & 0.002 & $<2$ & 1 & $<0.02$ & 0.00 \\
\hline BY59 & $\begin{array}{l}\text { Greenhorn } \mathrm{Cr} \text { at You } \\
\text { Bet Rd nr Nevada } \\
\text { City }\end{array}$ & $11 / 20 / 2001$ & $13: 20$ & 1of 2 & $<2$ & 1 & 0.17 & 0.01 & 0.015 & 0.002 & 13 & 2 & $<0.02$ & 0.01 \\
\hline BY59 & $\begin{array}{l}\text { Greenhorn } \mathrm{Cr} \text { at You } \\
\text { Bet Rd nr Nevada } \\
\text { City }\end{array}$ & $11 / 20 / 2001$ & $13: 20$ & 2of2 & $<2$ & 1 & 0.19 & 0.00 & 0.016 & 0.001 & 16 & 0 & $<0.02$ & 0.00 \\
\hline
\end{tabular}


Table 4A. Concentrations of trace metals and selected major elements in unfiltered water samples, Greenhorn Creek drainage, Nevada County, California—Continued

[Station name abbreviations: ab, above; bl, below; Cr, Creek; mi, miles; N, north; nr, near; Rd, Road; S, south; SF, South Fork. Number in parentheses is the data parameter code, a five-digit code used in the U.S. Geological Survey computerized data system. $\mu \mathrm{g} / \mathrm{L}$, microgram per liter (equivalent to part per billion); mg/L, milligram per liter (at low concentration, equivalent to part per million); s.d., standard deviation; <, less than; - , not determined]

\begin{tabular}{|c|c|c|c|c|c|c|c|c|c|c|c|c|c|c|}
\hline \multirow[t]{2}{*}{$\begin{array}{l}\text { Station } \\
\text { map ID }\end{array}$} & \multirow[t]{2}{*}{ Station name } & \multirow[t]{2}{*}{ Date } & \multirow[t]{2}{*}{ Time } & \multirow[t]{2}{*}{$\begin{array}{l}\text { Rep- } \\
\text { licate }\end{array}$} & \multicolumn{2}{|c|}{$\begin{array}{c}\text { Vanadium }(\mathrm{V}), \\
\text { unfiltered } \\
(\mu \mathrm{g} / \mathrm{L}) \\
(\mathbf{0 1 0 8 7}) \\
\end{array}$} & \multicolumn{2}{|c|}{$\begin{array}{c}\text { Yttrium }(Y), \\
\text { unfiltered } \\
(\mu \mathrm{g} / \mathrm{L}) \\
(01203)\end{array}$} & \multicolumn{2}{|c|}{$\begin{array}{c}\text { Ytterbium }(Y \mathbf{Y b}), \\
\text { unfiltered } \\
(\mu \mathrm{g} / \mathrm{L}) \\
(\mathbf{0 1 1 9 6 )} \\
\end{array}$} & \multicolumn{2}{|c|}{$\begin{array}{c}\text { Zinc }(\mathrm{Zn}), \\
\text { unfiltered } \\
(\mu \mathrm{g} / \mathrm{L}) \\
(01092) \\
\end{array}$} & \multicolumn{2}{|c|}{$\begin{array}{c}\text { Zirconium }(\mathrm{Zr}) \\
\text { unfiltered } \\
(\mu \mathrm{g} / \mathrm{L}) \\
(\mathbf{0 1 1 6 2}) \\
\end{array}$} \\
\hline & & & & & value & s.d. & value & s.d. & value & s.d. & value & s.d. & value & s.d. \\
\hline BY59 & $\begin{array}{l}\text { Greenhorn Cr at You } \\
\text { Bet Rd nr Nevada } \\
\text { City }\end{array}$ & $12 / 10 / 2001$ & $12: 20$ & 1 of 2 & 3 & 1 & 1.2 & 0.0 & 0.087 & 0.006 & 3 & 1 & 0.05 & 0.01 \\
\hline BY59 & $\begin{array}{l}\text { Greenhorn } \mathrm{Cr} \text { at You } \\
\text { Bet Rd nr Nevada } \\
\text { City }\end{array}$ & $12 / 10 / 2001$ & $12: 20$ & $2 \mathrm{of} 2$ & 4 & 2 & 1.2 & 0.1 & 0.10 & 0.00 & 5 & 0 & 0.06 & 0.01 \\
\hline BY59 & $\begin{array}{l}\text { Greenhorn Cr at You } \\
\text { Bet Rd nr Nevada } \\
\text { City }\end{array}$ & $12 / 21 / 2001$ & $13: 00$ & 1 of 2 & 17 & 1 & 4.6 & 0.3 & 0.37 & 0.01 & 25 & 4 & 0.07 & 0.01 \\
\hline BY59 & $\begin{array}{l}\text { Greenhorn } \mathrm{Cr} \text { at You } \\
\text { Bet Rd nr Nevada } \\
\text { City }\end{array}$ & $12 / 21 / 2001$ & $13: 00$ & $2 \mathrm{of} 2$ & 17 & 5 & 4.9 & 0.1 & 0.46 & 0.01 & 25 & 1 & 0.06 & 0.00 \\
\hline BY51 & $\begin{array}{l}\text { Greenhorn Cr nr } \\
\text { headwaters } \\
\text { nr Scotts Flat } \\
\text { Reservoir }\end{array}$ & $1 / 25 / 2000$ & $9: 30$ & 1 of 2 & $<2$ & 1 & 0.30 & 0.02 & 0.032 & 0.006 & 13 & 1 & 0.040 & 0.002 \\
\hline BY51 & $\begin{array}{l}\text { Greenhorn } \mathrm{Cr} n \mathrm{nr} \\
\text { headwaters } \\
\text { nr Scotts Flat } \\
\text { Reservoir }\end{array}$ & $1 / 25 / 2000$ & $9: 30$ & $2 \mathrm{of} 2$ & $<2$ & 1 & 0.29 & 0.01 & 0.027 & 0.005 & 14 & 0 & 0.043 & 0.008 \\
\hline BY75 & $\begin{array}{l}\text { Missouri Cyn Cr } 1.2 \\
\quad \text { mi ab Greenhorn Cr } \\
\text { nr Chicago Park }\end{array}$ & 12/14/1999 & $14: 00$ & 1 of 2 & $<4$ & 1 & 0.70 & 0.16 & 0.045 & 0.013 & 45 & 1 & 0.019 & 0.004 \\
\hline BY75 & $\begin{array}{l}\text { Missouri Cyn Cr } 1.2 \\
\quad \text { mi ab Greenhorn Cr } \\
\text { nr Chicago Park }\end{array}$ & $12 / 14 / 1999$ & $14: 00$ & $2 \mathrm{of} 2$ & $<4$ & 1 & 0.60 & 0.06 & 0.045 & 0.007 & 44 & 1 & 0.017 & 0.008 \\
\hline BY86 & $\begin{array}{l}\text { Poore Mine Creek } \\
\text { ab tunnel nr Grass } \\
\text { Valley }\end{array}$ & 3/31/1999 & $17: 40$ & $1 \mathrm{of} 2$ & $<4$ & 0 & 0.26 & 0.01 & 0.032 & 0.003 & 4.1 & 1.3 & 0.071 & 0.002 \\
\hline BY86 & $\begin{array}{l}\text { Poore Mine Creek } \\
\text { ab tunnel nr Grass } \\
\text { Valley }\end{array}$ & 3/31/1999 & $17: 40$ & $2 \mathrm{of} 2$ & $<4$ & 1 & 0.37 & 0.05 & 0.028 & 0.005 & 5.3 & 1.9 & 0.087 & 0.007 \\
\hline BY87 & $\begin{array}{l}\text { Poore Mine Creek } \\
\text { bl tunnel nr Grass } \\
\text { Valley }\end{array}$ & $3 / 31 / 1999$ & $13: 30$ & 1 of 2 & $<4$ & 1 & 0.40 & 0.02 & 0.033 & 0.003 & 3.9 & 0.8 & 0.089 & 0.016 \\
\hline
\end{tabular}


Table 4A. Concentrations of trace metals and selected major elements in unfiltered water samples, Greenhorn Creek drainage, Nevada County, California—Continued.

[Station name abbreviations: ab, above; bl, below; Cr, Creek; mi, miles; N, north; nr, near; Rd, Road; S, south; SF, South Fork. Number in parentheses is the data parameter code, a five-digit code used in the U.S. Geological Survey computerized data system. $\mu \mathrm{g} / \mathrm{L}$, microgram per liter (equivalent to part per billion); $\mathrm{mg} / \mathrm{L}$, milligram per liter (at low concentration, equivalent to part per million); s.d., standard deviation; $<$, less than; - , not determined]

\begin{tabular}{|c|c|c|c|c|c|c|c|c|c|c|c|c|c|c|}
\hline \multirow[t]{2}{*}{$\begin{array}{l}\text { Station } \\
\text { map ID }\end{array}$} & \multirow[t]{2}{*}{ Station name } & \multirow[t]{2}{*}{ Date } & \multirow[t]{2}{*}{ Time } & \multirow[t]{2}{*}{$\begin{array}{l}\text { Rep- } \\
\text { licate }\end{array}$} & \multicolumn{2}{|c|}{$\begin{array}{c}\text { Vanadium }(\mathrm{V}), \\
\text { unfiltered } \\
(\mu \mathrm{g} / \mathrm{L}) \\
(\mathbf{0 1 0 8 7}) \\
\end{array}$} & \multicolumn{2}{|c|}{$\begin{array}{c}\text { Yttrium }(\mathrm{Y}) \text {, } \\
\text { unfiltered } \\
(\mu \mathrm{g} / \mathrm{L}) \\
(01203)\end{array}$} & \multicolumn{2}{|c|}{$\begin{array}{c}\text { Ytterbium }(Y b), \\
\text { unfiltered } \\
(\mu \mathrm{g} / \mathrm{L}) \\
(01196) \\
\end{array}$} & \multicolumn{2}{|c|}{$\begin{array}{c}\text { Zinc }(\mathrm{Zn}), \\
\text { unfiltered } \\
(\mu \mathrm{g} / \mathrm{L}) \\
(01092)\end{array}$} & \multicolumn{2}{|c|}{$\begin{array}{c}\text { Zirconium }(\mathrm{Zr}) \\
\text { unfiltered } \\
(\mu \mathrm{g} / \mathrm{L}) \\
(01162)\end{array}$} \\
\hline & & & & & value & s.d. & value & s.d. & value & s.d. & value & s.d. & value & s.d. \\
\hline BY87 & $\begin{array}{l}\text { Poore Mine Creek } \\
\text { bl tunnel nr Grass } \\
\text { Valley }\end{array}$ & $3 / 31 / 1999$ & $13: 30$ & 2 of 2 & $<4$ & 1 & 0.31 & 0.03 & 0.039 & 0.002 & 2.8 & 0.4 & 0.080 & 0.000 \\
\hline BY88 & $\begin{array}{l}\text { Poore Mine ground } \\
\text { sluice nr Grass } \\
\text { Valley }\end{array}$ & 4/1/1999 & $15: 40$ & $1 \mathrm{of} 2$ & $<4$ & 2 & 0.25 & 0.02 & 0.019 & 0.002 & 11 & 1 & 0.010 & 0.002 \\
\hline BY88 & $\begin{array}{l}\text { Poore Mine ground } \\
\text { sluice nr Grass } \\
\text { Valley }\end{array}$ & 4/1/1999 & $15: 40$ & $2 \mathrm{of} 2$ & $<4$ & 2 & 0.20 & 0.01 & 0.024 & 0.002 & 8.9 & 0.1 & 0.005 & 0.001 \\
\hline BY89 & $\begin{array}{l}\text { Poore Mine pit lake nr } \\
\text { Grass Valley }\end{array}$ & 4/1/1999 & $16: 50$ & 1 of 2 & $<4$ & 0 & 0.13 & 0.03 & 0.010 & 0.003 & 16 & 3 & 0.028 & 0.002 \\
\hline BY89 & $\begin{array}{l}\text { Poore Mine pit lake nr } \\
\text { Grass Valley }\end{array}$ & 4/1/1999 & $16: 50$ & $2 \mathrm{of} 2$ & $<4$ & 0 & 0.12 & 0.01 & 0.013 & 0.001 & 14 & 3 & 0.009 & 0.002 \\
\hline BY89 & $\begin{array}{l}\text { Poore Mine pit lake nr } \\
\text { Grass Valley }\end{array}$ & $5 / 23 / 2000$ & $10: 45$ & 1 of 2 & 2 & 0 & 0.36 & 0.01 & 0.015 & 0.002 & 85 & 0 & 0.023 & 0.005 \\
\hline BY89 & $\begin{array}{l}\text { Poore Mine pit lake nr } \\
\text { Grass Valley }\end{array}$ & $5 / 23 / 2000$ & $10: 45$ & $2 \mathrm{of} 2$ & $<1$ & 1 & 0.37 & 0.01 & 0.016 & 0.001 & 84 & 1 & 0.016 & 0.003 \\
\hline BY90 & $\begin{array}{l}\text { Poore Mine seep } \\
\text { above ground sluice } \\
\text { nr Grass Valley }\end{array}$ & 4/1/1999 & $16: 00$ & 1 of 2 & - & & - & & - & & - & & - & \\
\hline BY90 & $\begin{array}{l}\text { Poore Mine seep } \\
\text { above ground sluice } \\
\text { nr Grass Valley }\end{array}$ & 4/1/1999 & $16: 00$ & $2 \mathrm{of} 2$ & - & & - & & - & & - & & - & \\
\hline BY91 & $\begin{array}{l}\text { Poore Mine tunnel } \\
\text { effluent nr Grass } \\
\text { Valley }\end{array}$ & 3/31/1999 & $15: 20$ & 1 of 2 & $<4$ & 1 & 0.14 & 0.02 & 0.007 & 0.002 & 13 & 2 & 0.010 & 0.001 \\
\hline BY91 & $\begin{array}{l}\text { Poore Mine tunnel } \\
\text { effluent nr Grass } \\
\text { Valley }\end{array}$ & $3 / 31 / 1999$ & $15: 20$ & $2 \mathrm{of} 2$ & $<4$ & 3 & 0.11 & 0.01 & 0.009 & 0.001 & 10 & 2 & 0.011 & 0.007 \\
\hline BY91 & $\begin{array}{l}\text { Poore Mine tunnel } \\
\text { effluent nr Grass } \\
\text { Valley }\end{array}$ & $12 / 15 / 1999$ & $13: 00$ & $1 \mathrm{of} 2$ & $<4$ & 2 & 0.10 & 0.01 & 0.010 & 0.003 & 14 & 1 & 0.011 & 0.004 \\
\hline BY91 & $\begin{array}{l}\text { Poore Mine tunnel } \\
\text { effluent nr Grass } \\
\text { Valley }\end{array}$ & $12 / 15 / 1999$ & $13: 00$ & $2 \mathrm{of} 2$ & $<4$ & 1 & 0.16 & 0.00 & 0.012 & 0.004 & 16 & 2 & 0.015 & 0.004 \\
\hline BY91 & $\begin{array}{l}\text { Poore Mine tunnel } \\
\text { effluent nr Grass } \\
\text { Valley }\end{array}$ & $5 / 23 / 2000$ & $12: 00$ & $1 \mathrm{of} 2$ & 2 & 1 & 2.0 & 0.0 & 0.11 & 0.00 & 28 & 0 & 0.008 & 0.003 \\
\hline
\end{tabular}


Table 4A. Concentrations of trace metals and selected major elements in unfiltered water samples, Greenhorn Creek drainage, Nevada County, California—Continued

[Station name abbreviations: ab, above; bl, below; Cr, Creek; mi, miles; N, north; nr, near; Rd, Road; S, south; SF, South Fork. Number in parentheses is the data parameter code, a five-digit code used in the U.S. Geological Survey computerized data system. $\mu \mathrm{g} / \mathrm{L}$, microgram per liter (equivalent to part per billion); mg/L, milligram per liter (at low concentration, equivalent to part per million); s.d., standard deviation; $<$, less than; —, not determined]

\begin{tabular}{|c|c|c|c|c|c|c|c|c|c|c|c|c|c|c|}
\hline \multirow[t]{2}{*}{$\begin{array}{l}\text { Station } \\
\text { map ID }\end{array}$} & \multirow[t]{2}{*}{ Station name } & \multirow[t]{2}{*}{ Date } & \multirow[t]{2}{*}{ Time } & \multirow[t]{2}{*}{$\begin{array}{c}\text { Rep- } \\
\text { licate }\end{array}$} & \multicolumn{2}{|c|}{$\begin{array}{c}\text { Vanadium }(\mathrm{V}), \\
\text { unfiltered } \\
(\mu \mathrm{g} / \mathrm{L}) \\
(\mathbf{0 1 0 8 7}) \\
\end{array}$} & \multicolumn{2}{|c|}{$\begin{array}{c}\text { Yttrium }(\mathrm{Y}) \text {, } \\
\text { unfiltered } \\
(\mu \mathrm{g} / \mathrm{L}) \\
(\mathbf{0 1 2 0 3})\end{array}$} & \multicolumn{2}{|c|}{$\begin{array}{c}\text { Ytterbium }(Y \mathbf{Y b}), \\
\text { unfiltered } \\
(\mu \mathrm{g} / \mathrm{L}) \\
(\mathbf{0 1 1 9 6 )} \\
\end{array}$} & \multicolumn{2}{|c|}{$\begin{array}{c}\text { Zinc }(Z n) \\
\text { unfiltered } \\
(\mu \mathrm{g} / \mathrm{L}) \\
(01092) \\
\end{array}$} & \multicolumn{2}{|c|}{$\begin{array}{c}\text { Zirconium }(\mathrm{Zr}) \\
\text { unfiltered } \\
(\mu \mathrm{g} / \mathrm{L}) \\
(\mathbf{0 1 1 6 2 )} \\
\end{array}$} \\
\hline & & & & & value & s.d. & value & s.d. & value & s.d. & value & s.d. & value & s.d. \\
\hline BY91 & $\begin{array}{l}\text { Poore Mine tunnel } \\
\text { effluent nr Grass } \\
\text { Valley }\end{array}$ & $5 / 23 / 2000$ & $12: 00$ & 2 of 2 & $<1$ & 0 & 1.9 & 0.0 & 0.11 & 0.00 & 30 & 2 & 0.069 & 0.007 \\
\hline BY105 & $\begin{array}{l}\text { Sailor Flat Mine main } \\
\text { drain gulch } 01 \mathrm{nr} \\
\text { Quaker Hill }\end{array}$ & 7/6/1999 & $16: 00$ & 1 of 2 & $<3$ & 1 & 5.7 & 0.0 & 0.84 & 0.12 & 41 & 1 & 0.19 & 0.03 \\
\hline BY105 & $\begin{array}{l}\text { Sailor Flat Mine main } \\
\text { drain gulch } 01 \mathrm{nr} \\
\text { Quaker Hill }\end{array}$ & 7/6/1999 & $16: 00$ & $2 \mathrm{of} 2$ & 3 & 1 & 8.5 & 1.9 & 0.65 & 0.00 & 42 & 0 & 0.24 & 0.06 \\
\hline BY105 & $\begin{array}{l}\text { Sailor Flat Mine main } \\
\text { drain Gulch } 01 \mathrm{nr} \\
\text { Quaker Hill }\end{array}$ & $12 / 2 / 1999$ & $14: 30$ & 1 of 2 & 19 & 1 & 14 & 0 & 1.3 & 0.0 & 38 & 2 & 0.32 & 0.03 \\
\hline BY105 & $\begin{array}{l}\text { Sailor Flat Mine main } \\
\text { drain Gulch } 01 \mathrm{nr} \\
\text { Quaker Hill }\end{array}$ & $12 / 2 / 1999$ & $14: 30$ & 2 of 2 & 18 & 4 & 14 & 1 & 1.5 & 0.2 & 37 & 2 & 0.32 & 0.00 \\
\hline BY106 & $\begin{array}{l}\text { Sailor Flat Mine } \\
\text { main drainage to } \\
\text { Greenhorn Cr, } \\
\text { Gulch } 03\end{array}$ & 4/9/2001 & $12: 30$ & 1 of 2 & 2 & 1 & 1.3 & 0.2 & 0.18 & 0.00 & 6.3 & 3.1 & 0.23 & 0.00 \\
\hline BY106 & $\begin{array}{l}\text { Sailor Flat Mine } \\
\text { main drainage to } \\
\text { Greenhorn Cr, } \\
\text { Gulch } 03\end{array}$ & 4/9/2001 & $12: 30$ & 2 of 2 & $<1$ & 0 & 1.3 & 0.0 & 0.18 & 0.01 & 5.3 & 1.0 & 0.22 & 0.05 \\
\hline BY114 & $\begin{array}{l}\text { SF Greenhorn Cr } 0.7 \\
\text { mi ab Greenhorn Cr } \\
\text { nr Nevada City }\end{array}$ & $8 / 22 / 2000$ & $15: 10$ & 1 of 2 & $<2$ & 1 & 0.025 & 0.005 & $<0.002$ & 0.001 & 2 & 0 & 0.015 & 0.003 \\
\hline BY114 & $\begin{array}{l}\text { SF Greenhorn Cr } 0.7 \\
\quad \text { mi ab Greenhorn Cr } \\
\text { nr Nevada City }\end{array}$ & $8 / 22 / 2000$ & $15: 10$ & $2 \mathrm{of} 2$ & $<2$ & 2 & 0.025 & 0.003 & 0.004 & 0.001 & 15 & 1 & 0.035 & 0.005 \\
\hline BY122 & $\begin{array}{l}\text { Starr Mine tunnel } \\
\text { inflow nr Grass } \\
\text { Valley }\end{array}$ & $4 / 23 / 1999$ & $15: 00$ & 1 of 2 & $<4$ & 1 & 0.27 & 0.00 & 0.019 & 0.005 & 21 & 1 & 0.008 & 0.003 \\
\hline BY122 & $\begin{array}{l}\text { Starr Mine tunnel } \\
\text { inflow nr Grass } \\
\text { Valley }\end{array}$ & $4 / 23 / 1999$ & $15: 00$ & $2 \mathrm{of} 2$ & $<4$ & 1 & 0.23 & 0.03 & 0.021 & 0.001 & 20 & 3 & 0.010 & 0.006 \\
\hline BY123 & $\begin{array}{l}\text { Starr Mine tunnel } \\
\text { midway nr Grass } \\
\text { Valley }\end{array}$ & 4/23/1999 & $13: 40$ & 1 of 2 & $<4$ & 0 & 0.16 & 0.05 & 0.014 & 0.003 & 2.8 & 1.1 & 0.006 & 0.004 \\
\hline
\end{tabular}


Table 4A. Concentrations of trace metals and selected major elements in unfiltered water samples, Greenhorn Creek drainage, Nevada County, California—Continued.

[Station name abbreviations: ab, above; bl, below; Cr, Creek; mi, miles; N, north; nr, near; Rd, Road; S, south; SF, South Fork. Number in parentheses is the data parameter code, a five-digit code used in the U.S. Geological Survey computerized data system. $\mu \mathrm{g} / \mathrm{L}$, microgram per liter (equivalent to part per billion); mg/L, milligram per liter (at low concentration, equivalent to part per million); s.d., standard deviation; $<$, less than; - - not determined]

\begin{tabular}{|c|c|c|c|c|c|c|c|c|c|c|c|c|c|c|}
\hline \multirow[t]{2}{*}{$\begin{array}{l}\text { Station } \\
\text { map ID }\end{array}$} & \multirow[t]{2}{*}{ Station name } & \multirow[t]{2}{*}{ Date } & \multirow[t]{2}{*}{ Time } & \multirow[t]{2}{*}{$\begin{array}{l}\text { Rep- } \\
\text { licate }\end{array}$} & \multicolumn{2}{|c|}{$\begin{array}{c}\text { Vanadium }(\mathrm{V}), \\
\text { unfiltered } \\
(\mu \mathrm{g} / \mathrm{L}) \\
(01087) \\
\end{array}$} & \multicolumn{2}{|c|}{$\begin{array}{c}\text { Yttrium }(\mathbf{Y}), \\
\text { unfiltered } \\
(\mu \mathrm{g} / \mathrm{L}) \\
(01203)\end{array}$} & \multicolumn{2}{|c|}{$\begin{array}{c}\text { Ytterbium }(Y b), \\
\text { unfiltered } \\
(\mu \mathrm{g} / \mathrm{L}) \\
(\mathbf{0 1 1 9 6 )} \\
\end{array}$} & \multicolumn{2}{|c|}{$\begin{array}{c}\text { Zinc }(Z n), \\
\text { unfiltered } \\
(\mu \mathrm{g} / \mathrm{L}) \\
(01092)\end{array}$} & \multicolumn{2}{|c|}{$\begin{array}{c}\text { Zirconium }(\mathrm{Zr}) \\
\text { unfiltered } \\
(\mu \mathrm{g} / \mathrm{L}) \\
(\mathbf{0 1 1 6 2 )} \\
\end{array}$} \\
\hline & & & & & value & s.d. & value & s.d. & value & s.d. & value & s.d. & value & s.d. \\
\hline BY123 & $\begin{array}{l}\text { Starr Mine tunnel } \\
\text { midway nr Grass } \\
\text { Valley }\end{array}$ & 4/23/1999 & $13: 40$ & 2of2 & $<4$ & 0 & 0.14 & 0.01 & 0.011 & 0.004 & 3.3 & 1.0 & 0.006 & 0.001 \\
\hline BY123 & $\begin{array}{l}\text { Starr Mine tunnel } \\
\text { midway nr Grass } \\
\text { Valley }\end{array}$ & $12 / 15 / 1999$ & $10: 45$ & 1 of 2 & $<4$ & 1 & 0.44 & 0.10 & 0.032 & 0.010 & 17 & 1 & 0.020 & 0.009 \\
\hline BY123 & $\begin{array}{l}\text { Starr Mine tunnel } \\
\text { midway nr Grass } \\
\text { Valley }\end{array}$ & $12 / 15 / 1999$ & $10: 45$ & $2 o f 2$ & $<4$ & 2 & 0.43 & 0.05 & 0.032 & 0.013 & 19 & 2 & 0.017 & 0.003 \\
\hline BY124 & $\begin{array}{l}\text { Starr Mine tunnel } \\
\text { outlet nr Grass } \\
\text { Valley }\end{array}$ & $8 / 23 / 2000$ & $17: 30$ & 1 of 2 & 3 & 1 & 0.83 & 0.03 & 0.074 & 0.004 & 25 & 1 & 0.030 & 0.007 \\
\hline BY124 & $\begin{array}{l}\text { Starr Mine tunnel } \\
\text { outlet nr Grass } \\
\text { Valley }\end{array}$ & $8 / 23 / 2000$ & $17: 30$ & $2 \mathrm{of} 2$ & 5 & 0 & 0.92 & 0.02 & 0.063 & 0.003 & 21 & 1 & 0.028 & 0.007 \\
\hline BY129 & $\begin{array}{l}\text { Tom and Jerry Mine } \\
\text { drain at tunnel inlet } \\
\text { nr Nevada City }\end{array}$ & $6 / 28 / 2000$ & $12: 00$ & 1 of 2 & 9 & 2 & 9.8 & 1.6 & 0.73 & 0.14 & 60 & 10 & 0.032 & 0.011 \\
\hline BY129 & $\begin{array}{l}\text { Tom and Jerry Mine } \\
\text { drain at tunnel inlet } \\
\text { nr Nevada City }\end{array}$ & $6 / 28 / 2000$ & $12: 00$ & $2 o f 2$ & 10 & 2 & 9.4 & 1.5 & 0.71 & 0.14 & 49 & 8 & 0.039 & 0.009 \\
\hline BY130 & $\begin{array}{l}\text { Tom and Jerry Mine } \\
\text { drain at tunnel } \\
\text { outlet nr Nevada } \\
\text { City }\end{array}$ & $6 / 28 / 2000$ & $11: 30$ & 1 of 2 & $<2$ & 0 & 0.17 & 0.00 & 0.016 & 0.005 & 4 & 1 & 0.028 & 0.010 \\
\hline BY130 & $\begin{array}{l}\text { Tom and Jerry Mine } \\
\text { drain at tunnel } \\
\text { outlet nr Nevada } \\
\text { City }\end{array}$ & $6 / 28 / 2000$ & $11: 30$ & $2 \mathrm{of} 2$ & $<2$ & 0 & 0.20 & 0.00 & 0.014 & 0.006 & 12 & 2 & 0.029 & 0.011 \\
\hline BY131 & $\begin{array}{l}\text { Tom and Jerry Mine } \\
\text { drainage pond nr } \\
\text { Nevada City }\end{array}$ & $8 / 23 / 2000$ & $10: 45$ & 1 of 2 & $<2$ & 0 & 0.24 & 0.02 & 0.017 & 0.004 & 3 & 0 & 0.025 & 0.002 \\
\hline
\end{tabular}


Table 4A. Concentrations of trace metals and selected major elements in unfiltered water samples, Greenhorn Creek drainage, Nevada County, California—Continued

[Station name abbreviations: ab, above; bl, below; Cr, Creek; mi, miles; N, north; nr, near; Rd, Road; S, south; SF, South Fork. Number in parentheses is the data parameter code, a five-digit code used in the U.S. Geological Survey computerized data system. $\mu \mathrm{g} / \mathrm{L}$, microgram per liter (equivalent to part per billion); mg/L, milligram per liter (at low concentration, equivalent to part per million); s.d., standard deviation; $<$, less than; —, not determined]

\begin{tabular}{|c|c|c|c|c|c|c|c|c|c|c|c|c|c|c|}
\hline \multirow[t]{2}{*}{$\begin{array}{l}\text { Station } \\
\text { map ID }\end{array}$} & \multirow[t]{2}{*}{ Station name } & \multirow[t]{2}{*}{ Date } & \multirow[t]{2}{*}{ Time } & \multirow[t]{2}{*}{$\begin{array}{l}\text { Rep- } \\
\text { licate }\end{array}$} & \multicolumn{2}{|c|}{$\begin{array}{c}\text { Vanadium }(\mathrm{V}), \\
\text { unfiltered } \\
(\mu \mathrm{g} / \mathrm{L}) \\
(\mathbf{0 1 0 8 7}) \\
\end{array}$} & \multicolumn{2}{|c|}{$\begin{array}{c}\text { Yttrium }(\mathbf{Y}), \\
\text { unfiltered } \\
(\mu \mathrm{g} / \mathrm{L}) \\
(01203) \\
\end{array}$} & \multicolumn{2}{|c|}{$\begin{array}{c}\text { Ytterbium }(Y \mathbf{Y b}), \\
\text { unfiltered } \\
(\mu \mathrm{g} / \mathrm{L}) \\
(\mathbf{0 1 1 9 6 )} \\
\end{array}$} & \multicolumn{2}{|c|}{$\begin{array}{c}\text { Zinc }(\mathrm{Zn}), \\
\text { unfiltered } \\
(\mu \mathrm{g} / \mathrm{L}) \\
(01092)\end{array}$} & \multicolumn{2}{|c|}{$\begin{array}{c}\text { Zirconium }(\mathrm{Zr}) \\
\text { unfiltered } \\
(\mu \mathrm{g} / \mathrm{L}) \\
(\mathbf{0 1 1 6 2 )} \\
\end{array}$} \\
\hline & & & & & value & s.d. & value & s.d. & value & s.d. & value & s.d. & value & s.d. \\
\hline BY131 & $\begin{array}{l}\text { Tom and Jerry Mine } \\
\text { drainage pond nr } \\
\text { Nevada City }\end{array}$ & $8 / 23 / 2000$ & $10: 45$ & 2of2 & $<2$ & 1 & 0.26 & 0.04 & 0.031 & 0.003 & 3 & 1 & 0.053 & 0.034 \\
\hline \multicolumn{15}{|c|}{ Disturbed samples } \\
\hline BY123 & $\begin{array}{l}\text { Starr Mine tunnel } \\
\text { midway nr Grass } \\
\text { Valley }\end{array}$ & $12 / 15 / 1999$ & $11: 30$ & 1 of 2 & 290 & 0 & 194 & 4 & 10 & 1 & 2,700 & 0 & 0.68 & 0.01 \\
\hline BY123 & $\begin{array}{l}\text { Starr Mine tunnel } \\
\text { midway nr Grass } \\
\text { Valley }\end{array}$ & $12 / 15 / 1999$ & $11: 30$ & 2of 2 & 280 & 10 & 176 & 11 & 13 & 1 & 2,500 & 100 & 0.68 & 0.03 \\
\hline BY129 & $\begin{array}{l}\text { Tom and Jerry Mine } \\
\text { drain at tunnel inlet } \\
\text { nr Nevada City }\end{array}$ & $6 / 28 / 2000$ & $12: 30$ & 1of 2 & 100 & 0 & 86 & 12 & 6.2 & 1.0 & 230 & 10 & 0.28 & 0.04 \\
\hline BY129 & $\begin{array}{l}\text { Tom and Jerry Mine } \\
\text { drain at tunnel inlet } \\
\text { nr Nevada City }\end{array}$ & $6 / 28 / 2000$ & $12: 30$ & 2of2 & 130 & 20 & 93 & 5 & 6.8 & 0.3 & 240 & 40 & 0.35 & 0.02 \\
\hline
\end{tabular}


Table 4B. Concentrations of trace metals and selected major elements in filtered water samples, Greenhorn Creek drainage, Nevada County, California.

[Station name abbreviations: ab, above; bl, below; Cr, creek; Cyn, Canyon; mi, miles; N, north; nr, near; Rd, Road; S, south; SF, South Fork. Number in parentheses is the data parameter code, a five-digit code

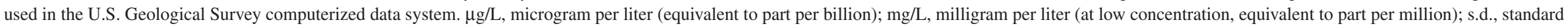
deviation; <, less than; - , not determined]

\begin{tabular}{|c|c|c|c|c|c|c|c|c|c|c|c|c|c|c|c|c|}
\hline \multirow[t]{2}{*}{$\begin{array}{l}\text { Station } \\
\text { map ID }\end{array}$} & \multirow[t]{2}{*}{ Station name } & \multirow[t]{2}{*}{ Date } & \multirow[t]{2}{*}{ Time } & \multirow[t]{2}{*}{$\begin{array}{l}\text { Rep- } \\
\text { licate }\end{array}$} & \multicolumn{2}{|c|}{$\begin{array}{l}\text { Aluminum (AI), } \\
\text { filtered } \\
(\mu \mathrm{g} / \mathrm{L}) \\
(01105)\end{array}$} & \multicolumn{2}{|c|}{$\begin{array}{l}\text { Arsenic (As), } \\
\text { filtered } \\
(\mu \mathrm{g} / \mathrm{L}) \\
(01000)\end{array}$} & \multicolumn{2}{|c|}{$\begin{array}{c}\text { Boron (B), } \\
\text { filtered } \\
(\mu \mathrm{g} / \mathrm{L}) \\
(01020)\end{array}$} & \multicolumn{2}{|c|}{$\begin{array}{c}\text { Barium (Ba), } \\
\text { filtered } \\
(\mu \mathrm{g} / \mathrm{L}) \\
(01005\end{array}$} & \multicolumn{2}{|c|}{$\begin{array}{l}\text { Beryllium }(\mathrm{Be}), \\
\text { filtered } \\
(\mu \mathrm{g} / \mathrm{L}) \\
(\mathbf{0 1 0 1 0})\end{array}$} & \multicolumn{2}{|c|}{$\begin{array}{c}\text { Bismuth (Bi), } \\
\text { filtered } \\
(\mu \mathrm{g} / \mathrm{L}) \\
(\mathbf{0 1 0 1 5 )}\end{array}$} \\
\hline & & & & & value & s.d. & value & s.d. & value & s.d. & value & s.d. & value & s.d. & value & s.d. \\
\hline \multicolumn{17}{|c|}{ Environmental samples } \\
\hline BY20 & $\begin{array}{l}\text { Boston Mine tunnel outlet } \\
\text { nr Grass Valley }\end{array}$ & $6 / 18 / 1999$ & $14: 30$ & 1 of 2 & 1.2 & 0.1 & 0.35 & 0.01 & 3 & 1 & 52 & 0 & $<0.005$ & 0.002 & $<0.0008$ & 0.0006 \\
\hline BY20 & $\begin{array}{l}\text { Boston Mine tunnel outlet } \\
\text { nr Grass Valley }\end{array}$ & $6 / 18 / 1999$ & $14: 30$ & $2 \mathrm{of} 2$ & 1.1 & 0.3 & 0.35 & 0.01 & 2 & 0 & 54 & 0 & $<0.005$ & 0.004 & $<0.0008$ & 0.0001 \\
\hline BY20 & $\begin{array}{l}\text { Boston Mine tunnel outlet } \\
\text { nr Grass Valley }\end{array}$ & $12 / 14 / 1999$ & $12: 00$ & 1 of 2 & 0.70 & 0.17 & $<0.6$ & 0.4 & 4 & 6 & 55 & 3 & $<0.003$ & 0.001 & 0.015 & 0.018 \\
\hline BY20 & $\begin{array}{l}\text { Boston Mine tunnel outlet } \\
\text { nr Grass Valley }\end{array}$ & 12/14/1999 & $12: 00$ & $2 \mathrm{of} 2$ & 0.55 & 0.27 & $<0.6$ & 0.5 & 5 & 3 & 56 & 3 & $<0.003$ & 0.001 & $<0.002$ & 0.001 \\
\hline BY20 & $\begin{array}{l}\text { Boston Mine tunnel outlet } \\
\text { nr Grass Valley }\end{array}$ & $5 / 24 / 2000$ & $16: 00$ & 1 of 2 & 9.2 & 0.1 & $<0.2$ & 0.1 & $<20$ & 10 & 53 & 1 & $<0.06$ & 0.04 & 0.011 & 0.001 \\
\hline BY20 & $\begin{array}{l}\text { Boston Mine tunnel outlet } \\
\text { nr Grass Valley }\end{array}$ & $5 / 24 / 2000$ & $16: 00$ & $2 \mathrm{of} 2$ & 9.5 & 0.2 & $<0.2$ & 0.1 & $<20$ & 10 & 52 & 0 & $<0.06$ & 0.04 & 0.003 & 0.001 \\
\hline BY21 & $\begin{array}{l}\text { Boston Mine wetlands pond } \\
\text { nr Grass Valley }\end{array}$ & 12/14/1999 & $10: 30$ & 1 of 2 & 0.77 & 0.08 & $<0.6$ & 0.3 & 6 & 4 & 50 & 1 & 0.005 & 0.004 & $<0.002$ & 0.001 \\
\hline BY21 & $\begin{array}{l}\text { Boston Mine wetlands pond } \\
\text { nr Grass Valley }\end{array}$ & $12 / 14 / 1999$ & $10: 30$ & $2 \mathrm{of} 2$ & 0.31 & 0.25 & $<0.6$ & 0.2 & 6 & 4 & 50 & 1 & 0.004 & 0.003 & $<0.002$ & 0.002 \\
\hline BY21 & $\begin{array}{l}\text { Boston Mine wetlands pond } \\
\text { nr Grass Valley }\end{array}$ & $5 / 24 / 2000$ & $16: 30$ & 1 of 2 & 2.3 & 0.4 & 0.3 & 0.0 & $<20$ & 20 & 50 & 0 & $<0.06$ & 0.01 & 0.003 & 0.001 \\
\hline BY21 & $\begin{array}{l}\text { Boston Mine wetlands pond } \\
\text { nr Grass Valley }\end{array}$ & $5 / 24 / 2000$ & $16: 30$ & $2 \mathrm{of} 2$ & 2.0 & 0.0 & 0.4 & 0.1 & $<20$ & 10 & 47 & 2 & $<0.06$ & 0.01 & $<0.003$ & 0.001 \\
\hline BY58 & $\begin{array}{l}\text { Buckeye Flat Mine } \\
\text { main drain } 0.45 \mathrm{mi} \text { ab } \\
\text { Greenhorn } \mathrm{Cr}\end{array}$ & $8 / 21 / 2000$ & $11: 00$ & 1 of 2 & 1,500 & 0 & $<0.1$ & 0.0 & $<20$ & 0 & 32 & 1 & 0.39 & 0.12 & $<0.003$ & 0.001 \\
\hline BY58 & $\begin{array}{l}\text { Buckeye Flat Mine } \\
\text { main drain } 0.45 \mathrm{mi} \text { ab } \\
\text { Greenhorn } \mathrm{Cr}\end{array}$ & $8 / 21 / 2000$ & $11: 00$ & $2 \mathrm{of} 2$ & 1,500 & 100 & $<0.1$ & 0.0 & $<20$ & 10 & 32 & 1 & 0.30 & 0.10 & 0.003 & 0.003 \\
\hline BY23 & $\begin{array}{l}\text { Buckeye Flat Mine N drain } \\
\text { to Greenhorn Cr, Grass } \\
\text { Valley }\end{array}$ & $6 / 18 / 1999$ & $11: 30$ & 1 of 2 & 4,100 & 400 & 0.44 & 0.05 & 2 & 0 & 23 & 0 & 0.76 & 0.00 & $<0.0008$ & 0.0011 \\
\hline BY23 & $\begin{array}{l}\text { Buckeye Flat Mine N drain } \\
\text { to Greenhorn Cr, Grass } \\
\text { Valley }\end{array}$ & $6 / 18 / 1999$ & $11: 30$ & $2 \mathrm{of} 2$ & 4,100 & 400 & 0.40 & 0.01 & 4 & 2 & 23 & 0 & 0.75 & 0.00 & $<0.0008$ & 0.0012 \\
\hline BY23 & $\begin{array}{l}\text { Buckeye Flat Mine N drain } \\
\text { to Greenhorn Cr, Grass } \\
\text { Valley }\end{array}$ & $12 / 2 / 1999$ & $11: 00$ & 1 of 2 & 4,000 & 100 & $<0.6$ & 0.1 & 8 & 6 & 33 & 0 & 0.52 & 0.03 & 0.005 & 0.008 \\
\hline BY23 & $\begin{array}{l}\text { Buckeye Flat Mine N drain } \\
\text { to Greenhorn Cr, Grass } \\
\text { Valley }\end{array}$ & $12 / 2 / 1999$ & 11:00 & $2 \mathrm{of} 2$ & 4,100 & 100 & $<0.6$ & 0.2 & 8 & 3 & 33 & 2 & 0.54 & 0.01 & 0.003 & 0.003 \\
\hline
\end{tabular}


Table 4B. Concentrations of trace metals and selected major elements in filtered water samples, Greenhorn Creek drainage, Greenhorn Creek drainage, Nevada County, California-Continued.

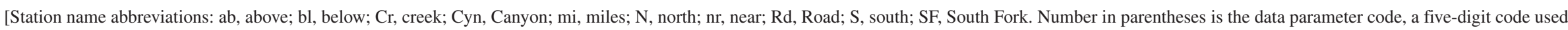
in the U.S. Geological Survey computerized data system. $\mu \mathrm{g} / \mathrm{L}$, microgram per liter (equivalent to part per billion); mg/L, milligram per liter (at low concentration, equivalent to part per million); s.d., standard deviation; <, less than; - , not determined; - , not determined]

\begin{tabular}{|c|c|c|c|c|c|c|c|c|c|c|c|c|c|c|c|c|}
\hline \multirow[t]{2}{*}{$\begin{array}{l}\text { Station } \\
\text { map ID }\end{array}$} & \multirow[t]{2}{*}{ Station name } & \multirow[t]{2}{*}{ Date } & \multirow[t]{2}{*}{ Time } & \multirow[t]{2}{*}{$\begin{array}{l}\text { Rep- } \\
\text { licate }\end{array}$} & \multicolumn{2}{|c|}{$\begin{array}{l}\text { Aluminum (AI), } \\
\text { filtered } \\
(\mu \mathrm{g} / \mathrm{L}) \\
(\mathbf{0 1 1 0 5})\end{array}$} & \multicolumn{2}{|c|}{$\begin{array}{l}\text { Arsenic (As), } \\
\text { filtered } \\
(\mu \mathrm{g} / \mathrm{L}) \\
(01000)\end{array}$} & \multicolumn{2}{|c|}{$\begin{array}{c}\text { Boron (B), } \\
\text { filtered } \\
(\mu \mathrm{g} / \mathrm{L}) \\
(\mathbf{0 1 0 2 0})\end{array}$} & \multicolumn{2}{|c|}{$\begin{array}{c}\text { Barium (Ba), } \\
\text { filtered } \\
(\mu \mathrm{g} / \mathrm{L}) \\
(01005\end{array}$} & \multicolumn{2}{|c|}{$\begin{array}{c}\text { Beryllium (Be), } \\
\text { filtered } \\
(\mu \mathrm{g} / \mathrm{L}) \\
(\mathbf{0 1 0 1 0})\end{array}$} & \multicolumn{2}{|c|}{$\begin{array}{c}\text { Bismuth }(\mathrm{Bi}), \\
\text { filtered } \\
(\mu \mathrm{g} / \mathrm{L}) \\
(01015)\end{array}$} \\
\hline & & & & & value & s.d. & value & s.d. & value & s.d. & value & s.d. & value & s.d. & value & s.d. \\
\hline$\overline{\mathrm{BY} 23}$ & $\begin{array}{l}\text { Buckeye Flat Mine N drain } \\
\text { to Greenhorn Cr, Grass } \\
\text { Valley }\end{array}$ & $8 / 22 / 2000$ & $10: 45$ & 1 of 2 & 3,900 & 100 & 0.1 & 0.0 & $<20$ & 0 & 35 & 1 & 0.43 & 0.14 & 0.004 & 0.004 \\
\hline BY23 & $\begin{array}{l}\text { Buckeye Flat Mine N drain } \\
\text { to Greenhorn Cr, Grass } \\
\text { Valley }\end{array}$ & $8 / 22 / 2000$ & $10: 45$ & $2 \mathrm{of} 2$ & 4,100 & 0 & 0.2 & 0.0 & $<20$ & 10 & 36 & 1 & 0.43 & 0.14 & $<0.003$ & 0.000 \\
\hline BY116 & $\begin{array}{l}\text { Buckeye Flat Mine pond } \\
\text { drain } 0.15 \mathrm{mi} \text { ab SF } \\
\text { Greenhorn } \mathrm{Cr}\end{array}$ & $8 / 21 / 2000$ & $14: 10$ & 1 of 2 & 9.5 & 0.9 & $<0.1$ & 0.1 & $<20$ & 20 & 59 & 2 & $<0.2$ & 0.1 & $<0.003$ & 0.001 \\
\hline BY116 & $\begin{array}{l}\text { Buckeye Flat Mine pond } \\
\text { drain } 0.15 \mathrm{mi} \text { ab SF } \\
\text { Greenhorn } \mathrm{Cr}\end{array}$ & $8 / 21 / 2000$ & $14: 10$ & $2 \mathrm{of} 2$ & 10.3 & 0.4 & $<0.1$ & 0.0 & $<20$ & 20 & 61 & 0 & $<0.2$ & 0.1 & $<0.003$ & 0.001 \\
\hline BY24 & $\begin{array}{l}\text { Buckeye Flat Mine S drain } \\
\text { to SF Greenhorn Cr, Grass } \\
\text { Valley }\end{array}$ & $8 / 26 / 1999$ & $11: 00$ & 1 of 2 & 55 & 1 & 0.32 & 0.02 & 4 & 3 & 18 & 0 & 0.04 & 0.03 & 0.0016 & 0.0002 \\
\hline BY24 & $\begin{array}{l}\text { Buckeye Flat Mine S drain } \\
\text { to SF Greenhorn Cr, Grass } \\
\text { Valley }\end{array}$ & $8 / 26 / 1999$ & $11: 00$ & $2 \mathrm{of} 2$ & 51 & 0 & 0.32 & 0.03 & 4 & 3 & 17 & 0 & $<0.03$ & 0.01 & 0.0019 & 0.0008 \\
\hline BY24 & $\begin{array}{l}\text { Buckeye Flat Mine S drain } \\
\text { to SF Greenhorn Cr, Grass } \\
\text { Valley }\end{array}$ & $8 / 22 / 2000$ & $12: 10$ & 1 of 2 & 71 & 1 & $<0.1$ & 0.0 & $<20$ & 0 & 23 & 0 & $<0.2$ & 0.1 & $<0.003$ & 0.001 \\
\hline BY24 & $\begin{array}{l}\text { Buckeye Flat Mine S drain } \\
\text { to SF Greenhorn Cr, Grass } \\
\text { Valley }\end{array}$ & $8 / 22 / 2000$ & $12: 10$ & $2 \mathrm{of} 2$ & 70 & 1 & $<0.1$ & 0.1 & $<20$ & 10 & 24 & 1 & $<0.2$ & 0.1 & $<0.003$ & 0.001 \\
\hline BY25 & $\begin{array}{l}\text { Buckeye Flat Mine upper } \\
\text { drain }\end{array}$ & 4/9/2001 & $10: 00$ & 1 of 2 & 600 & 90 & $<0.1$ & 0.0 & $<20$ & 0 & 41 & 7 & 0.10 & 0.06 & $<0.02$ & 0.00 \\
\hline BY25 & $\begin{array}{l}\text { Buckeye Flat Mine upper } \\
\text { drain }\end{array}$ & $4 / 9 / 2001$ & $10: 00$ & $2 \mathrm{of} 2$ & 580 & 0 & $<0.1$ & 0.0 & $<20$ & 10 & 41 & 2 & 0.14 & 0.03 & 0.03 & 0.00 \\
\hline BY52 & $\begin{array}{l}\text { Greenhorn } \mathrm{Cr} 0.3 \mathrm{mi} \text { ab } \\
\text { Buckeye Ford nr Nevada } \\
\text { City }\end{array}$ & $1 / 25 / 2000$ & $10: 45$ & 1 of 2 & 27 & 0 & $<0.1$ & 0.1 & $<5$ & 2 & 18 & 0 & 0.09 & 0.10 & 0.005 & 0.001 \\
\hline BY52 & $\begin{array}{l}\text { Greenhorn Cr } 0.3 \mathrm{mi} \mathrm{ab} \\
\text { Buckeye Ford nr Nevada } \\
\text { City }\end{array}$ & $1 / 25 / 2000$ & $10: 45$ & $2 \mathrm{of} 2$ & 31 & 0 & $<0.1$ & 0.1 & 5 & 3 & 18 & 1 & $<0.05$ & 0.00 & $<0.003$ & 0.001 \\
\hline BY59 & $\begin{array}{l}\text { Greenhorn Cr at You Bet Rd } \\
\text { nr Nevada City }\end{array}$ & $7 / 24 / 2001$ & $11: 00$ & 1 of 2 & 2.0 & 0.1 & 1.0 & 0.0 & 7.2 & 1.1 & 34 & 1 & 0.02 & 0.00 & 0.0035 & 0.0021 \\
\hline BY59 & $\begin{array}{l}\text { Greenhorn Cr at You Bet Rd } \\
\text { nr Nevada City }\end{array}$ & $7 / 24 / 2001$ & $11: 00$ & $2 \mathrm{of} 2$ & 2.0 & 0.0 & 1.0 & 0.0 & 6.7 & 0.4 & 33 & 0 & 0.10 & 0.14 & $<0.0009$ & 0.0003 \\
\hline
\end{tabular}


Table 4B. Concentrations of trace metals and selected major elements in filtered water samples, Greenhorn Creek drainage, Nevada County, California—Continued.

[Station name abbreviations: ab, above; bl, below; Cr, creek; Cyn, Canyon; mi, miles; N, north; nr, near; Rd, Road; S, south; SF, South Fork. Number in parentheses is the data parameter code, a five-digit code

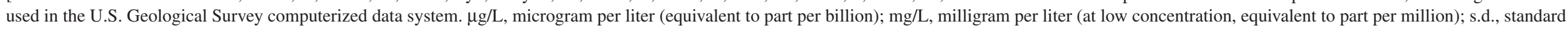
deviation; $<$, less than; - - not determined]

\begin{tabular}{|c|c|c|c|c|c|c|c|c|c|c|c|c|c|c|c|c|}
\hline \multirow[t]{2}{*}{$\begin{array}{l}\text { Station } \\
\text { map ID }\end{array}$} & \multirow[t]{2}{*}{ Station name } & \multirow[t]{2}{*}{ Date } & \multirow[t]{2}{*}{ Time } & \multirow[t]{2}{*}{$\begin{array}{l}\text { Rep- } \\
\text { licate }\end{array}$} & \multicolumn{2}{|c|}{$\begin{array}{l}\text { Aluminum (AI), } \\
\text { filtered } \\
(\mu \mathrm{g} / \mathrm{L}) \\
(01105) \\
\end{array}$} & \multicolumn{2}{|c|}{$\begin{array}{c}\text { Arsenic (As), } \\
\text { filtered } \\
(\mu \mathrm{g} / \mathrm{L}) \\
(01000) \\
\end{array}$} & \multicolumn{2}{|c|}{$\begin{array}{c}\text { Boron (B), } \\
\text { filtered } \\
(\mu \mathrm{g} / \mathrm{L}) \\
(01020) \\
\end{array}$} & \multicolumn{2}{|c|}{$\begin{array}{c}\text { Barium (Ba), } \\
\text { filtered } \\
(\mu \mathrm{g} / \mathrm{L}) \\
(01005\end{array}$} & \multicolumn{2}{|c|}{$\begin{array}{l}\text { Beryllium (Be), } \\
\text { filtered } \\
(\mu \mathrm{g} / \mathrm{L}) \\
(\mathbf{0 1 0 1 0 )} \\
\end{array}$} & \multicolumn{2}{|c|}{$\begin{array}{c}\text { Bismuth }(\mathrm{Bi}), \\
\text { filtered } \\
(\mu \mathrm{g} / \mathrm{L}) \\
(01015) \\
\end{array}$} \\
\hline & & & & & value & s.d. & value & s.d. & value & s.d. & value & s.d. & value & s.d. & value & s.d. \\
\hline BY59 & $\begin{array}{l}\text { Greenhorn Cr at You Bet Rd } \\
\text { nr Nevada City }\end{array}$ & $8 / 29 / 2001$ & $15: 00$ & 1 of 2 & 1.8 & 0.1 & 1.9 & 0.0 & 5.7 & 0.2 & 26 & 1 & $<0.01$ & 0.00 & $<0.0009$ & 0.0007 \\
\hline BY59 & $\begin{array}{l}\text { Greenhorn Cr at You Bet Rd } \\
\text { nr Nevada City }\end{array}$ & $8 / 29 / 2001$ & $15: 00$ & 2 of 2 & 1.8 & 0.0 & 2.0 & 0.0 & 6.2 & 0.3 & 26 & 0 & $<0.01$ & 0.00 & $<0.0009$ & 0.0002 \\
\hline BY59 & $\begin{array}{l}\text { Greenhorn Cr at You Bet Rd } \\
\text { nr Nevada City }\end{array}$ & $9 / 14 / 2001$ & $13: 00$ & 1 of 2 & 2.4 & 0.1 & 1.2 & 0.0 & 6 & 0 & 30 & 0 & $<0.008$ & 0.003 & $<0.001$ & 0.001 \\
\hline BY59 & $\begin{array}{l}\text { Greenhorn Cr at You Bet Rd } \\
\text { nr Nevada City }\end{array}$ & $9 / 14 / 2001$ & $13: 00$ & 2 of 2 & 2.5 & 0.0 & 1.2 & 0.0 & 6 & 0 & 30 & 0 & $<0.008$ & 0.008 & $<0.001$ & 0.001 \\
\hline BY59 & $\begin{array}{l}\text { Greenhorn Cr at You Bet Rd } \\
\text { nr Nevada City }\end{array}$ & $10 / 15 / 2001$ & $12: 00$ & 1 of 2 & 0.96 & 0.04 & 0.98 & 0.02 & 5 & 0 & 34 & 0 & $<0.008$ & 0.004 & $<0.001$ & 0.000 \\
\hline BY59 & $\begin{array}{l}\text { Greenhorn Cr at You Bet Rd } \\
\text { nr Nevada City }\end{array}$ & $10 / 15 / 2001$ & $12: 00$ & $2 \mathrm{of} 2$ & 0.96 & 0.06 & 0.95 & 0.01 & 5 & 0 & 34 & 0 & $<0.008$ & 0.003 & $<0.001$ & 0.001 \\
\hline BY59 & $\begin{array}{l}\text { Greenhorn Cr at You Bet Rd } \\
\text { nr Nevada City }\end{array}$ & $11 / 20 / 2001$ & $13: 20$ & 1 of 2 & 8.7 & 0.4 & 0.71 & 0.03 & 6 & 0 & 37 & 0 & $<0.005$ & 0.003 & $<0.0008$ & 0.0008 \\
\hline BY59 & $\begin{array}{l}\text { Greenhorn Cr at You Bet Rd } \\
\text { nr Nevada City }\end{array}$ & $11 / 20 / 2001$ & $13: 20$ & $2 \mathrm{of} 2$ & 9.2 & 0.4 & 0.73 & 0.04 & 6 & 1 & 36 & 1 & $<0.005$ & 0.003 & $<0.0008$ & 0.0015 \\
\hline BY59 & $\begin{array}{l}\text { Greenhorn Cr at You Bet Rd } \\
\text { nr Nevada City }\end{array}$ & $12 / 10 / 2001$ & $12: 20$ & 1 of 2 & 11 & 0 & 1.8 & 0.0 & 4.7 & 0.3 & 27 & 0 & $<0.008$ & 0.003 & $<0.001$ & 0.001 \\
\hline BY59 & $\begin{array}{l}\text { Greenhorn Cr at You Bet Rd } \\
\text { nr Nevada City }\end{array}$ & $12 / 10 / 2001$ & $12: 20$ & 2 of 2 & 12 & 0 & 1.8 & 0.1 & 4.3 & 0.2 & 27 & 0 & $<0.008$ & 0.005 & $<0.001$ & 0.000 \\
\hline BY59 & $\begin{array}{l}\text { Greenhorn Cr at You Bet Rd } \\
\text { nr Nevada City }\end{array}$ & $12 / 21 / 2001$ & $13: 00$ & 1 of 2 & 12 & 0 & 0.92 & 0.00 & 3.8 & 0.4 & 20 & 0 & $<0.006$ & 0.004 & $<0.001$ & 0.002 \\
\hline BY59 & $\begin{array}{l}\text { Greenhorn Cr at You Bet Rd } \\
\text { nr Nevada City }\end{array}$ & $12 / 21 / 2001$ & $13: 00$ & 2 of 2 & 11 & 0 & 0.91 & 0.01 & 3.9 & 0.6 & 21 & 0 & $<0.006$ & 0.002 & $<0.001$ & 0.001 \\
\hline BY51 & $\begin{array}{c}\text { Greenhorn Cr nr Headwaters } \\
\text { nr Scotts Flat Reservoir }\end{array}$ & $1 / 25 / 2000$ & $9: 30$ & 1 of 2 & 28 & 2 & $<0.1$ & 0.1 & $<5$ & 1 & 14 & 0 & $<0.05$ & 0.00 & 0.004 & 0.004 \\
\hline BY51 & $\begin{array}{c}\text { Greenhorn Cr nr Headwaters } \\
\text { nr Scotts Flat Reservoir }\end{array}$ & $1 / 25 / 2000$ & $9: 30$ & 2 of 2 & 24 & 1 & $<0.1$ & 0.0 & $<5$ & 1 & 16 & 1 & $<0.05$ & 0.02 & $<0.003$ & 0.002 \\
\hline BY75 & $\begin{array}{l}\text { Missouri Cyn Cr } 1.2 \text { mi ab } \\
\text { Greenhorn Cr nr Chicago } \\
\text { Park }\end{array}$ & $12 / 14 / 1999$ & $14: 00$ & 1 of 2 & 18 & 0 & $<0.6$ & 0.1 & 6 & 5 & 40 & 1 & 0.11 & 0.00 & $<0.002$ & 0.001 \\
\hline BY75 & $\begin{array}{l}\text { Missouri Cyn Cr } 1.2 \mathrm{mi} \text { ab } \\
\text { Greenhorn Cr nr Chicago } \\
\text { Park }\end{array}$ & $12 / 14 / 1999$ & $14: 00$ & $2 \mathrm{of} 2$ & 18 & 0 & $<0.6$ & 0.2 & 8 & 7 & 41 & 1 & 0.12 & 0.00 & 0.006 & 0.010 \\
\hline BY86 & $\begin{array}{l}\text { Poore Mine creek ab tunnel } \\
\text { nr Grass Valley }\end{array}$ & $3 / 31 / 1999$ & $17: 40$ & 1 of 2 & 12 & 1 & 0.12 & 0.01 & $<9$ & 0 & 16 & 0 & $<0.02$ & 0.01 & 0.0029 & 0.0035 \\
\hline BY86 & $\begin{array}{l}\text { Poore Mine creek ab tunnel } \\
\text { nr Grass Valley }\end{array}$ & 3/31/1999 & $17: 40$ & 2 of 2 & 11 & 0 & 0.14 & 0.03 & $<9$ & 0 & 16 & 0 & $<0.02$ & 0.02 & 0.0007 & 0.0008 \\
\hline
\end{tabular}


Table 4B. Concentrations of trace metals and selected major elements in filtered water samples, Greenhorn Creek drainage, Nevada County, California—Continued.

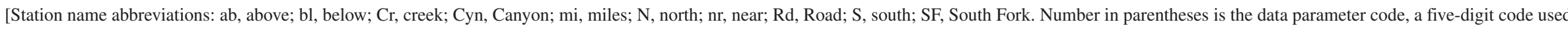
in the U.S. Geological Survey computerized data system. $\mu \mathrm{g} / \mathrm{L}$, microgram per liter (equivalent to part per billion); mg/L, milligram per liter (at low concentration, equivalent to part per million); s.d., standard deviation; $<$, less than; —, not determined]

\begin{tabular}{|c|c|c|c|c|c|c|c|c|c|c|c|c|c|c|c|c|}
\hline \multirow[t]{2}{*}{$\begin{array}{l}\text { Station } \\
\text { map ID }\end{array}$} & \multirow[t]{2}{*}{ Station name } & \multirow[t]{2}{*}{ Date } & \multirow[t]{2}{*}{ Time } & \multirow[t]{2}{*}{$\begin{array}{l}\text { Rep- } \\
\text { licate }\end{array}$} & \multicolumn{2}{|c|}{$\begin{array}{c}\text { Aluminum (AI), } \\
\text { filtered } \\
(\mu \mathrm{g} / \mathrm{L}) \\
(01105)\end{array}$} & \multicolumn{2}{|c|}{$\begin{array}{c}\text { Arsenic (As), } \\
\text { filtered } \\
(\mu \mathrm{g} / \mathrm{L}) \\
(01000)\end{array}$} & \multicolumn{2}{|c|}{$\begin{array}{c}\text { Boron (B), } \\
\text { filtered } \\
(\mu \mathrm{g} / \mathrm{L}) \\
(01020)\end{array}$} & \multicolumn{2}{|c|}{$\begin{array}{c}\text { Barium (Ba), } \\
\text { filtered } \\
(\mu / L) \\
(01005\end{array}$} & \multicolumn{2}{|c|}{$\begin{array}{c}\text { Beryllium (Be), } \\
\text { filtered } \\
(\mu \mathrm{g} / \mathrm{L}) \\
(01010) \\
\end{array}$} & \multicolumn{2}{|c|}{$\begin{array}{c}\text { Bismuth (Bi), } \\
\text { filtered } \\
(\mu \mathrm{g} / \mathrm{L}) \\
(01015) \\
\end{array}$} \\
\hline & & & & & value & s.d. & value & s.d. & value & s.d. & value & s.d. & value & s.d. & value & s.d. \\
\hline BY87 & $\begin{array}{l}\text { Poore Mine creek bl tunnel } \\
\text { nr Grass Valley }\end{array}$ & $3 / 31 / 1999$ & $13: 30$ & 1 of 2 & 6.9 & 0.3 & 0.15 & 0.01 & $<9$ & 0 & 17 & 1 & $<0.02$ & 0.00 & 0.0010 & 0.0012 \\
\hline BY87 & $\begin{array}{l}\text { Poore Mine creek bl tunnel } \\
\text { nr Grass Valley }\end{array}$ & 3/31/1999 & $13: 30$ & $2 o f 2$ & 5.9 & 0.1 & 0.15 & 0.01 & $<9$ & 0 & 16 & 2 & $<0.02$ & 0.00 & 0.0028 & 0.0015 \\
\hline BY88 & $\begin{array}{l}\text { Poore Mine ground sluice } \\
\text { nr Grass Valley }\end{array}$ & 4/1/1999 & $15: 40$ & 1 of 2 & 72 & 2 & 0.13 & 0.01 & $<9$ & 2 & 28 & 1 & 0.07 & 0.00 & $<0.0007$ & 0.0002 \\
\hline BY88 & $\begin{array}{l}\text { Poore Mine ground sluice } \\
\text { nr Grass Valley }\end{array}$ & 4/1/1999 & $15: 40$ & $2 o f 2$ & 69 & 0 & 0.15 & 0.05 & 2 & 0 & 28 & 1 & 0.071 & 0.007 & $<0.0008$ & 0.0006 \\
\hline BY89 & $\begin{array}{l}\text { Poore Mine pit lake nr Grass } \\
\text { Valley }\end{array}$ & 4/1/1999 & $16: 50$ & 1 of 2 & 4.9 & 0.2 & 0.18 & 0.02 & 3 & 0 & 37 & 0 & 0.015 & 0.001 & 0.0017 & 0.0017 \\
\hline BY89 & $\begin{array}{l}\text { Poore Mine pit lake nr Grass } \\
\text { Valley }\end{array}$ & 4/1/1999 & $16: 50$ & $2 o f 2$ & 4.0 & 0.2 & 0.16 & 0.01 & 3 & 0 & 37 & 0 & 0.013 & 0.002 & $<0.0008$ & 0.0038 \\
\hline BY89 & $\begin{array}{l}\text { Poore Mine pit lake nr Grass } \\
\text { Valley }\end{array}$ & $5 / 23 / 2000$ & $10: 45$ & 1 of 2 & 130 & 0 & $<0.2$ & 0.1 & $<20$ & 20 & 120 & 0 & 0.54 & 0.03 & $<0.003$ & 0.001 \\
\hline BY89 & $\begin{array}{l}\text { Poore Mine pit lake nr Grass } \\
\text { Valley }\end{array}$ & $5 / 23 / 2000$ & $10: 45$ & $2 o f 2$ & 130 & 0 & $<0.2$ & 0.0 & $<20$ & 10 & 120 & 0 & 0.57 & 0.05 & $<0.003$ & 0.000 \\
\hline BY90 & $\begin{array}{l}\text { Poore Mine seep ab ground } \\
\text { sluice nr Grass Valley }\end{array}$ & 4/1/1999 & $16: 00$ & 1 of 2 & 33 & 3 & 0.13 & 0.01 & 2 & 0 & 30 & 0 & 0.055 & 0.002 & 0.0009 & 0.0010 \\
\hline BY90 & $\begin{array}{l}\text { Poore Mine seep ab ground } \\
\text { sluice nr Grass Valley }\end{array}$ & 4/1/1999 & $16: 00$ & $2 o f 2$ & 33 & 0 & 0.13 & 0.02 & 2 & 0 & 28 & 0 & 0.044 & 0.001 & 0.0040 & 0.0035 \\
\hline BY91 & $\begin{array}{l}\text { Poore Mine tunnel effluent } \\
\text { nr Grass Valley }\end{array}$ & 3/31/1999 & $15: 20$ & 1 of 2 & 5.6 & 0.3 & 0.12 & 0.00 & $<9$ & 1 & 29 & 2 & 0.04 & 0.01 & 0.0024 & 0.0001 \\
\hline BY91 & $\begin{array}{l}\text { Poore Mine tunnel effluent } \\
\text { nr Grass Valley }\end{array}$ & 3/31/1999 & $15: 20$ & $2 o f 2$ & 5.2 & 0.4 & 0.10 & 0.02 & 5 & 0 & 29 & 0 & 0.041 & 0.005 & 0.0014 & 0.0014 \\
\hline BY91 & $\begin{array}{l}\text { Poore Mine tunnel effluent } \\
\text { nr Grass Valley }\end{array}$ & $12 / 15 / 1999$ & $13: 00$ & 1 of 2 & 2.9 & 0.2 & $<0.6$ & 0.1 & 13 & 1 & 27 & 1 & 0.007 & 0.001 & $<0.002$ & 0.002 \\
\hline BY91 & $\begin{array}{l}\text { Poore Mine tunnel effluent } \\
\text { nr Grass Valley }\end{array}$ & $12 / 15 / 1999$ & $13: 00$ & $2 o f 2$ & 3.9 & 1.6 & $<0.6$ & 0.2 & 10 & 3 & 27 & 1 & 0.006 & 0.002 & 0.019 & 0.025 \\
\hline BY91 & $\begin{array}{l}\text { Poore Mine tunnel effluent } \\
\text { nr Grass Valley }\end{array}$ & $5 / 23 / 2000$ & $12: 00$ & 1 of 2 & 5.2 & 0.1 & $<0.2$ & 0.1 & 64 & 42 & 37 & 1 & $<0.06$ & 0.03 & $<0.003$ & 0.001 \\
\hline BY91 & $\begin{array}{l}\text { Poore Mine tunnel effluent } \\
\text { nr Grass Valley }\end{array}$ & $5 / 23 / 2000$ & $12: 00$ & $2 o f 2$ & 7.7 & 1.9 & 0.2 & 0.1 & 6 & 1 & 37 & 0 & $<0.05$ & 0.04 & $<0.003$ & 0.003 \\
\hline BY105 & $\begin{array}{l}\text { Sailor Flat Mine main drain } \\
\text { Gulch 01nr Quaker Hill }\end{array}$ & 7/6/1999 & $16: 00$ & 1 of 2 & 11 & 0 & 0.61 & 0.01 & 4 & 1 & 85 & 1 & 0.07 & 0.00 & 0.0008 & 0.0006 \\
\hline BY105 & $\begin{array}{l}\text { Sailor Flat Mine main drain } \\
\text { Gulch } 01 \mathrm{nr} \text { Quaker Hill }\end{array}$ & 7/6/1999 & $16: 00$ & $2 o f 2$ & 10 & 0 & 0.55 & 0.02 & $<9$ & 5 & 86 & 5 & 0.06 & 0.00 & $<0.0007$ & 0.0007 \\
\hline BY105 & $\begin{array}{l}\text { Sailor Flat Mine main drain } \\
\text { Gulch 01nr Quaker Hill }\end{array}$ & 12/2/1999 & $14: 30$ & 1 of 2 & 79 & 3 & $<0.6$ & 0.2 & 5 & 1 & 32 & 0 & 0.039 & 0.006 & 0.083 & 0.111 \\
\hline
\end{tabular}


Table 4B. Concentrations of trace metals and selected major elements in filtered water samples, Greenhorn Creek drainage, Nevada County, California—Continued.

[Station name abbreviations: ab, above; bl, below; Cr, creek; Cyn, Canyon; mi, miles; N, north; nr, near; Rd, Road; S, south; SF, South Fork. Number in parentheses is the data parameter code, a five-digit code

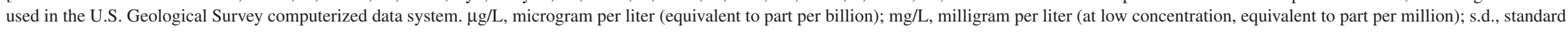
deviation; <, less than; - - not determined]

\begin{tabular}{|c|c|c|c|c|c|c|c|c|c|c|c|c|c|c|c|c|}
\hline \multirow[t]{2}{*}{$\begin{array}{l}\text { Station } \\
\text { map ID }\end{array}$} & \multirow[t]{2}{*}{ Station name } & \multirow[t]{2}{*}{ Date } & \multirow[t]{2}{*}{ Time } & \multirow[t]{2}{*}{$\begin{array}{l}\text { Rep- } \\
\text { licate }\end{array}$} & \multicolumn{2}{|c|}{$\begin{array}{c}\text { Aluminum (AI), } \\
\text { filtered } \\
(\mu \mathrm{g} / \mathrm{L}) \\
(\mathbf{0 1 1 0 5}) \\
\end{array}$} & \multicolumn{2}{|c|}{$\begin{array}{c}\text { Arsenic (As), } \\
\text { filtered } \\
(\mu \mathrm{g} / \mathrm{L}) \\
(01000)\end{array}$} & \multicolumn{2}{|c|}{$\begin{array}{c}\text { Boron (B), } \\
\text { filtered } \\
(\mu \mathrm{g} / \mathrm{L}) \\
(01020)\end{array}$} & \multicolumn{2}{|c|}{$\begin{array}{c}\text { Barium (Ba), } \\
\text { filtered } \\
(\mu / L) \\
(01005\end{array}$} & \multicolumn{2}{|c|}{$\begin{array}{c}\text { Beryllium (Be), } \\
\text { filtered } \\
(\mu \mathrm{g} / \mathrm{L}) \\
(01010) \\
\end{array}$} & \multicolumn{2}{|c|}{$\begin{array}{c}\text { Bismuth }(\mathrm{Bi}), \\
\text { filtered } \\
(\mu \mathrm{g} / \mathrm{L}) \\
(01015) \\
\end{array}$} \\
\hline & & & & & value & s.d. & value & s.d. & value & s.d. & value & s.d. & value & s.d. & value & s.d. \\
\hline$\overline{\text { BY105 }}$ & $\begin{array}{l}\text { Sailor Flat Mine main drain } \\
\text { Gulch 01nr Quaker Hill }\end{array}$ & $12 / 2 / 1999$ & $14: 30$ & 2 of 2 & 74 & 3 & $<0.6$ & 0.1 & 3 & 2 & 33 & 1 & 0.047 & 0.001 & $<0.002$ & 0.000 \\
\hline BY106 & $\begin{array}{l}\text { Sailor Flat Mine main } \\
\text { drainage to Greenhorn } \mathrm{Cr} \text {, } \\
\text { Gulch } 03\end{array}$ & $4 / 9 / 01$ & $12: 30$ & 1 of 2 & 96 & 12 & 0.54 & 0.16 & $<20$ & 0 & 30 & 7 & $<0.03$ & 0.04 & $<0.02$ & 0.00 \\
\hline BY106 & $\begin{array}{l}\text { Sailor Flat Mine main } \\
\text { drainage to Greenhorn } \mathrm{Cr} \text {, } \\
\text { Gulch } 03\end{array}$ & $4 / 9 / 2001$ & $12: 30$ & 2 of 2 & 110 & 20 & 0.50 & 0.12 & $<20$ & 10 & 30 & 6 & 0.06 & 0.07 & $<0.02$ & 0.01 \\
\hline BY114 & $\begin{array}{l}\text { SF Greenhorn Cr } 0.7 \text { mi ab } \\
\text { Greenhorn Cr nr Nevada } \\
\text { City }\end{array}$ & $8 / 22 / 2000$ & $15: 10$ & 1 of 2 & 3.8 & 0.3 & 0.1 & 0.0 & $<20$ & 0 & 17 & 1 & $<0.2$ & 0.1 & $<0.003$ & 0.001 \\
\hline BY114 & $\begin{array}{l}\text { SF Greenhorn Cr } 0.7 \text { mi ab } \\
\text { Greenhorn Cr nr Nevada } \\
\text { City }\end{array}$ & $8 / 22 / 2000$ & $15: 10$ & $2 \mathrm{of} 2$ & 4.2 & 1.1 & 0.2 & 0.1 & $<20$ & 20 & 16 & 0 & $<0.2$ & 0.1 & $<0.003$ & 0.001 \\
\hline BY122 & $\begin{array}{l}\text { Starr Mine tunnel inflow } \\
\text { nr Grass Valley }\end{array}$ & 4/23/1999 & $15: 00$ & 1 of 2 & 28 & 2 & 0.088 & 0.007 & 3 & 0 & 22 & 1 & 0.025 & 0.002 & 0.0019 & 0.0003 \\
\hline BY122 & $\begin{array}{l}\text { Starr Mine tunnel inflow } \\
\text { nr Grass Valley }\end{array}$ & 4/23/1999 & $15: 00$ & $2 \mathrm{of} 2$ & 27 & 2 & 0.083 & 0.002 & 3 & 1 & 21 & 2 & 0.018 & 0.006 & $<0.0008$ & 0.0004 \\
\hline BY123 & $\begin{array}{l}\text { Starr Mine tunnel midway } \\
\text { nr Grass Valley }\end{array}$ & 4/23/1999 & $13: 40$ & 1 of 2 & 0.90 & 0.04 & 0.17 & 0.04 & 5 & 1 & 49 & 0 & $<0.005$ & 0.000 & 0.0018 & 0.0011 \\
\hline BY123 & $\begin{array}{l}\text { Starr Mine tunnel midway } \\
\text { nr Grass Valley }\end{array}$ & 4/23/1999 & $13: 40$ & $2 \mathrm{of} 2$ & 2.3 & 0.1 & 0.18 & 0.04 & 6 & 1 & 49 & 1 & $<0.005$ & 0.004 & $<0.0008$ & 0.0002 \\
\hline BY123 & $\begin{array}{l}\text { Starr Mine tunnel midway } \\
\text { nr Grass Valley }\end{array}$ & $12 / 15 / 1999$ & $10: 45$ & 1 of 2 & 0.47 & 0.05 & $<0.6$ & 0.3 & 9 & 5 & 55 & 5 & 0.005 & 0.002 & 0.004 & 0.002 \\
\hline BY123 & $\begin{array}{l}\text { Starr Mine tunnel midway } \\
\text { nr Grass Valley }\end{array}$ & $12 / 15 / 1999$ & $10: 45$ & $2 \mathrm{of} 2$ & 0.54 & 0.21 & $<0.6$ & 0.1 & 6 & 1 & 59 & 2 & $<0.003$ & 0.002 & 0.004 & 0.003 \\
\hline BY124 & $\begin{array}{l}\text { Starr Mine tunnel outlet } \\
\text { nr Grass Valley }\end{array}$ & $8 / 23 / 2000$ & $17: 30$ & 1 of 2 & 2.9 & 0.1 & 0.4 & 0.0 & $<20$ & 10 & 42 & 1 & $<0.2$ & 0.1 & $<0.003$ & 0.001 \\
\hline BY124 & $\begin{array}{l}\text { Starr Mine tunnel outlet } \\
\text { nr Grass Valley }\end{array}$ & $8 / 23 / 2000$ & $17: 30$ & $2 o f 2$ & 2.4 & 0.4 & 0.4 & 0.1 & $<20$ & 0 & 42 & 2 & $<0.2$ & 0.1 & 0.008 & 0.009 \\
\hline BY129 & $\begin{array}{l}\text { Tom and Jerry Mine drain at } \\
\text { tunnel inlet nr Nevada City }\end{array}$ & $6 / 28 / 2000$ & $12: 00$ & 1 of 2 & 2.7 & 0.1 & $<0.4$ & 0.1 & 100 & 110 & 35 & 1 & $<0.2$ & 0.1 & $<0.003$ & 0.002 \\
\hline BY129 & $\begin{array}{l}\text { Tom and Jerry Mine drain at } \\
\text { tunnel inlet nr Nevada City }\end{array}$ & $6 / 28 / 2000$ & $12: 00$ & $2 o f 2$ & 2.8 & 0.1 & $<0.4$ & 0.1 & $<60$ & 140 & 34 & 0 & $<0.2$ & 0.0 & $<0.003$ & 0.000 \\
\hline BY130 & $\begin{array}{l}\text { Tom and Jerry Mine drain } \\
\text { at tunnel outlet nr Nevada } \\
\text { City }\end{array}$ & $6 / 28 / 2000$ & $11: 30$ & 1 of 2 & 1.4 & 0.4 & $<0.4$ & 0.3 & $<60$ & 100 & 68 & 3 & $<0.2$ & 0.1 & $<0.003$ & 0.001 \\
\hline BY130 & $\begin{array}{l}\text { Tom and Jerry Mine drain } \\
\text { at tunnel outlet nr Nevada } \\
\text { City }\end{array}$ & $6 / 28 / 2000$ & $11: 30$ & $2 o f 2$ & 1.6 & 0.7 & $<0.4$ & 0.1 & $<60$ & 110 & 68 & 0 & $<0.2$ & 0.0 & $<0.003$ & 0.002 \\
\hline
\end{tabular}


Table 4B. Concentrations of trace metals and selected major elements in filtered water samples, Greenhorn Creek drainage, Nevada County, California—Continued.

[Station name abbreviations: ab, above; bl, below; Cr, creek; Cyn, Canyon; mi, miles; N, north; nr, near; Rd, Road; S, south; SF, South Fork. Number in parentheses is the data parameter code, a five-digit code

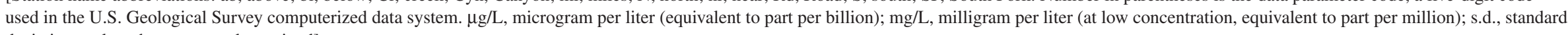
deviation; <, less than; - , not determined]

\begin{tabular}{|c|c|c|c|c|c|c|c|c|c|c|c|c|c|c|c|c|}
\hline \multirow[t]{2}{*}{$\begin{array}{l}\text { Station } \\
\text { map ID }\end{array}$} & \multirow[t]{2}{*}{ Station name } & \multirow[t]{2}{*}{ Date } & \multirow[t]{2}{*}{ Time } & \multirow[t]{2}{*}{$\begin{array}{l}\text { Rep- } \\
\text { licate }\end{array}$} & \multicolumn{2}{|c|}{$\begin{array}{c}\text { Aluminum (AI), } \\
\text { filtered } \\
(\mu \mathrm{g} / \mathrm{L}) \\
(01105)\end{array}$} & \multicolumn{2}{|c|}{$\begin{array}{c}\text { Arsenic (As), } \\
\text { filtered } \\
(\mu \mathrm{g} / \mathrm{L}) \\
(01000)\end{array}$} & \multicolumn{2}{|c|}{$\begin{array}{c}\text { Boron (B), } \\
\text { filtered } \\
(\mu \mathrm{g} / \mathrm{L}) \\
(01020)\end{array}$} & \multicolumn{2}{|c|}{$\begin{array}{c}\text { Barium (Ba), } \\
\text { filtered } \\
(\mu / L) \\
(01005\end{array}$} & \multicolumn{2}{|c|}{$\begin{array}{c}\text { Beryllium (Be), } \\
\text { filtered } \\
(\mu \mathrm{g} / \mathrm{L}) \\
(01010)\end{array}$} & \multicolumn{2}{|c|}{$\begin{array}{c}\text { Bismuth }(\mathrm{Bi}), \\
\text { filtered } \\
(\mu \mathrm{g} / \mathrm{L}) \\
(01015)\end{array}$} \\
\hline & & & & & value & s.d. & value & s.d. & value & s.d. & value & s.d. & value & s.d. & value & s.d. \\
\hline BY131 & $\begin{array}{l}\text { Tom and Jerry Mine drainage } \\
\text { pond nr Nevada City }\end{array}$ & $8 / 23 / 2000$ & $10: 45$ & 1 of 2 & 2.5 & 0.3 & $<0.1$ & 0.0 & $<20$ & 10 & 52 & 0 & $<0.2$ & 0.1 & $<0.003$ & 0.001 \\
\hline BY131 & $\begin{array}{l}\text { Tom and Jerry Mine drainage } \\
\text { pond nr Nevada City }\end{array}$ & $8 / 23 / 2000$ & $10: 45$ & $2 o f 2$ & 2.7 & 0.4 & $<0.1$ & 0.0 & $<20$ & 10 & 52 & 0 & $<0.2$ & 0.1 & 0.004 & 0.001 \\
\hline
\end{tabular}

Disturbed samples

\begin{tabular}{|c|c|c|c|c|c|c|c|c|c|c|c|c|c|c|c|c|}
\hline BY123 & $\begin{array}{l}\text { Starr Mine tunnel midway } \\
\text { nr Grass Valley }\end{array}$ & $12 / 15 / 1999$ & $11: 30$ & 1 of 2 & 0.73 & 0.23 & $<0.6$ & 0.1 & 5 & 0 & 46 & 1 & $<0.003$ & 0.003 & $<0.002$ & 0.001 \\
\hline BY123 & $\begin{array}{l}\text { Starr Mine tunnel midway } \\
\text { nr Grass Valley }\end{array}$ & $12 / 15 / 1999$ & $11: 30$ & $2 \mathrm{of} 2$ & 0.42 & 0.03 & $<0.6$ & 0.2 & 7 & 1 & 46 & 1 & $<0.003$ & 0.001 & 0.005 & 0.005 \\
\hline BY129 & $\begin{array}{l}\text { Tom and Jerry Mine drain at } \\
\text { tunnel inlet nr Nevada City }\end{array}$ & $6 / 28 / 2000$ & $12: 30$ & 1 of 2 & 1.6 & 0.3 & $<0.4$ & 0.3 & 110 & 150 & 59 & 0 & $<0.2$ & 0.0 & 0.004 & 0.007 \\
\hline BY129 & $\begin{array}{l}\text { Tom and Jerry Mine drain at } \\
\text { tunnel inlet nr Nevada City }\end{array}$ & $6 / 28 / 2000$ & $12: 30$ & $2 \mathrm{of} 2$ & 1.5 & 0.3 & $<0.4$ & 0.2 & 66 & 131 & 60 & 1 & $<0.2$ & 0.0 & $<0.003$ & 0.002 \\
\hline
\end{tabular}


Table 4B. Concentrations of trace metals and selected major elements in filtered water samples, Greenhorn Creek drainage, Nevada County, California—Continued.

[Station name abbreviations: ab, above; bl, below; Cr, creek; Cyn, Canyon; mi, miles; N, north; nr, near; Rd, Road; S, south; SF, South Fork. Number in parentheses is the data parameter code, a five-digit code

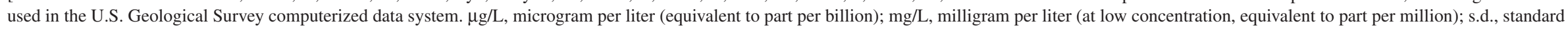
deviation; $<$, less than; - , not determined]

\begin{tabular}{|c|c|c|c|c|c|c|c|c|c|c|c|c|c|c|c|c|}
\hline \multirow[t]{2}{*}{$\begin{array}{l}\text { Station } \\
\text { map ID }\end{array}$} & \multirow[t]{2}{*}{ Station name } & \multirow[t]{2}{*}{ Date } & \multirow[t]{2}{*}{ Time } & \multirow[t]{2}{*}{$\begin{array}{l}\text { Rep- } \\
\text { licate }\end{array}$} & \multicolumn{2}{|c|}{$\begin{array}{c}\text { Calcium (Ca), } \\
\text { filtered } \\
\text { (mg/L) } \\
(00915)\end{array}$} & \multicolumn{2}{|c|}{$\begin{array}{c}\text { Cadmium (Cd), } \\
\text { filtered } \\
(\mu \mathrm{g} / \mathrm{L}) \\
(01025) \\
\end{array}$} & \multicolumn{2}{|c|}{$\begin{array}{c}\text { Cerium (Ce), } \\
\text { filtered } \\
(\mu \mathrm{g} / \mathrm{L}) \\
(\mathbf{0 1 1 1 0} \\
\end{array}$} & \multicolumn{2}{|c|}{$\begin{array}{c}\text { Cobalt (Co), } \\
\text { filtered } \\
(\mu \mathrm{g} / \mathrm{L}) \\
(01035) \\
\end{array}$} & \multicolumn{2}{|c|}{$\begin{array}{c}\text { Chromium (Cr), } \\
\text { filtered } \\
(\mu \mathrm{g} / \mathrm{L}) \\
(\mathbf{0 1 0 3 0}) \\
\end{array}$} & \multicolumn{2}{|c|}{$\begin{array}{c}\text { Cesium (Cs), } \\
\text { filtered } \\
(\mu \mathrm{g} / \mathrm{L}) \\
(\mathbf{0 1 1 1 5}) \\
\end{array}$} \\
\hline & & & & & value & s.d. & value & s.d. & value & s.d. & value & s.d. & value & s.d. & value & s.d. \\
\hline \multicolumn{17}{|c|}{ Environmental samples } \\
\hline BY20 & $\begin{array}{l}\text { Boston Mine tunnel } \\
\text { outlet nr Grass } \\
\text { Valley }\end{array}$ & 6/18/1999 & $14: 30$ & $1 \mathrm{of} 2$ & 14 & 0 & 0.024 & 0.002 & 0.018 & 0.002 & 0.95 & 0.05 & $<0.1$ & 0.0 & 0.020 & 0.001 \\
\hline BY20 & $\begin{array}{l}\text { Boston Mine tunnel } \\
\text { outlet nr Grass } \\
\text { Valley }\end{array}$ & 6/18/1999 & $14: 30$ & $2 \mathrm{of} 2$ & 14 & 0 & 0.024 & 0.002 & 0.016 & 0.003 & 0.95 & 0.06 & $<0.1$ & 0.1 & 0.021 & 0.004 \\
\hline BY20 & $\begin{array}{l}\text { Boston Mine tunnel } \\
\text { outlet nr Grass } \\
\text { Valley }\end{array}$ & $12 / 14 / 1999$ & $12: 00$ & 1 of 2 & 17 & 1 & 0.024 & 0.001 & 0.0026 & 0.0003 & 0.24 & 0.00 & $<0.1$ & 0.1 & 0.023 & 0.002 \\
\hline BY20 & $\begin{array}{l}\text { Boston Mine tunnel } \\
\text { outlet nr Grass } \\
\text { Valley }\end{array}$ & $12 / 14 / 1999$ & $12: 00$ & $2 \mathrm{of} 2$ & 21 & 0 & 0.018 & 0.003 & 0.0034 & 0.0008 & 0.24 & 0.01 & $<0.1$ & 0.1 & 0.034 & 0.021 \\
\hline BY20 & $\begin{array}{l}\text { Boston Mine tunnel } \\
\text { outlet nr Grass } \\
\text { Valley }\end{array}$ & $5 / 24 / 2000$ & $16: 00$ & $1 \mathrm{of} 2$ & 12 & 3 & 0.04 & 0.01 & 0.024 & 0.002 & 0.74 & 0.03 & $<0.6$ & 0.2 & $<0.2$ & 0.1 \\
\hline BY20 & $\begin{array}{l}\text { Boston Mine tunnel } \\
\text { outlet nr Grass } \\
\text { Valley }\end{array}$ & $5 / 24 / 2000$ & $16: 00$ & $2 \mathrm{of} 2$ & 13 & 2 & 0.03 & 0.00 & 0.025 & 0.001 & 0.75 & 0.00 & $<0.6$ & 0.1 & $<0.2$ & 0.3 \\
\hline BY21 & $\begin{array}{l}\text { Boston Mine wetlands } \\
\text { pond nr Grass Valley }\end{array}$ & $12 / 14 / 1999$ & $10: 30$ & $1 \mathrm{of} 2$ & 23 & 5 & 0.010 & 0.002 & 0.0057 & 0.0001 & $<0.006$ & 0.001 & $<0.1$ & 0.0 & 0.0071 & 0.0006 \\
\hline BY21 & $\begin{array}{l}\text { Boston Mine wetlands } \\
\text { pond nr Grass Valley }\end{array}$ & $12 / 14 / 1999$ & $10: 30$ & $2 \mathrm{of} 2$ & 21 & 1 & 0.010 & 0.001 & 0.0053 & 0.0003 & $<0.006$ & 0.003 & $<0.1$ & 0.0 & 0.0081 & 0.0010 \\
\hline BY21 & $\begin{array}{l}\text { Boston Mine wetlands } \\
\text { pond nr Grass Valley }\end{array}$ & $5 / 24 / 2000$ & $16: 30$ & 1 of 2 & 12 & 0 & 0.02 & 0.00 & 0.019 & 0.001 & 0.13 & 0.03 & $<0.6$ & 0.0 & $<0.2$ & 0.2 \\
\hline BY21 & $\begin{array}{l}\text { Boston Mine wetlands } \\
\text { pond nr Grass Valley }\end{array}$ & $5 / 24 / 2000$ & $16: 30$ & $2 \mathrm{of} 2$ & 11 & 2 & 0.03 & 0.00 & 0.016 & 0.001 & 0.10 & 0.01 & $<0.6$ & 0.3 & $<0.2$ & 0.2 \\
\hline BY58 & $\begin{array}{l}\text { Buckeye Flat Mine } \\
\text { main drain } 0.45 \mathrm{mi} \\
\text { ab Greenhorn } \mathrm{Cr}\end{array}$ & $8 / 21 / 2000$ & $11: 00$ & $1 \mathrm{of} 2$ & 12 & 0 & 0.32 & 0.03 & 21 & 0 & 55 & 0 & $<3$ & 1 & 0.06 & 0.01 \\
\hline BY58 & $\begin{array}{l}\text { Buckeye Flat Mine } \\
\text { main drain } 0.45 \mathrm{mi} \\
\text { ab Greenhorn } \mathrm{Cr}\end{array}$ & $8 / 21 / 2000$ & $11: 00$ & $2 \mathrm{of} 2$ & 12 & 0 & 0.34 & 0.03 & 22 & 1 & 58 & 1 & $<3$ & 1 & 0.06 & 0.01 \\
\hline BY23 & $\begin{array}{c}\text { Buckeye Flat Mine N } \\
\text { drain to Greenhorn } \\
\text { Cr, Grass Valley }\end{array}$ & 6/18/1999 & $11: 30$ & 1 of 2 & 20 & 0 & 2.1 & 0.0 & 42 & 0 & 110 & 0 & 0.3 & 0.1 & 0.071 & 0.001 \\
\hline BY23 & $\begin{array}{c}\text { Buckeye Flat Mine N } \\
\text { drain to Greenhorn } \\
\text { Cr, Grass Valley }\end{array}$ & $6 / 18 / 1999$ & $11: 30$ & $2 \mathrm{of} 2$ & 20 & 0 & 2.1 & 0.0 & 44 & 1 & 99 & 0 & 0.3 & 0.0 & 0.074 & 0.002 \\
\hline
\end{tabular}


Table 4B. Concentrations of trace metals and selected major elements in filtered water samples, Greenhorn Creek drainage, Nevada County, California—Continued.

[Station name abbreviations: ab, above; bl, below; Cr, creek; Cyn, Canyon; mi, miles; N, north; nr, near; Rd, Road; S, south; SF, South Fork. Number in parentheses is the data parameter code, a five-digit code

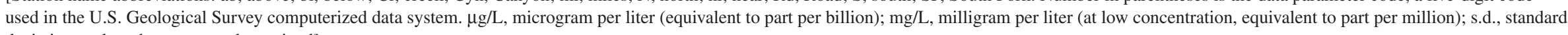
deviation; $<$, less than; - , not determined]

\begin{tabular}{|c|c|c|c|c|c|c|c|c|c|c|c|c|c|c|c|c|}
\hline \multirow[t]{2}{*}{$\begin{array}{l}\text { Station } \\
\text { map ID }\end{array}$} & \multirow[t]{2}{*}{ Station name } & \multirow[t]{2}{*}{ Date } & \multirow[t]{2}{*}{ Time } & \multirow[t]{2}{*}{$\begin{array}{l}\text { Rep- } \\
\text { licate }\end{array}$} & \multicolumn{2}{|c|}{$\begin{array}{c}\text { Calcium (Ca), } \\
\text { filtered } \\
\text { (mg/L) } \\
(00915)\end{array}$} & \multicolumn{2}{|c|}{$\begin{array}{c}\text { Cadmium (Cd), } \\
\text { filtered } \\
(\mu \mathrm{g} / \mathrm{L}) \\
(01025)\end{array}$} & \multicolumn{2}{|c|}{$\begin{array}{c}\text { Cerium (Ce), } \\
\text { filtered } \\
(\mu \mathrm{g} / \mathrm{L}) \\
(01110\end{array}$} & \multicolumn{2}{|c|}{$\begin{array}{c}\text { Cobalt (Co), } \\
\text { filtered } \\
(\mu \mathrm{g} / \mathrm{L}) \\
(01035)\end{array}$} & \multicolumn{2}{|c|}{$\begin{array}{c}\text { Chromium (Cr), } \\
\text { filtered } \\
(\mu \mathrm{g} / \mathrm{L}) \\
(\mathbf{0 1 0 3 0})\end{array}$} & \multicolumn{2}{|c|}{$\begin{array}{c}\text { Cesium (Cs), } \\
\text { filtered } \\
(\mu \mathrm{g} / \mathrm{L}) \\
(\mathbf{0 1 1 1 5})\end{array}$} \\
\hline & & & & & value & s.d. & value & s.d. & value & s.d. & value & s.d. & value & s.d. & value & s.d. \\
\hline BY23 & $\begin{array}{c}\text { Buckeye Flat Mine N } \\
\text { drain to Greenhorn } \\
\text { Cr, Grass Valley }\end{array}$ & $12 / 2 / 1999$ & $11: 00$ & 1 of 2 & 13 & 1 & 2.6 & 0.0 & 31 & 1 & 81 & 1 & 0.5 & 0.0 & 0.027 & 0.002 \\
\hline BY23 & $\begin{array}{c}\text { Buckeye Flat Mine N } \\
\text { drain to Greenhorn } \\
\text { Cr, Grass Valley }\end{array}$ & $12 / 2 / 1999$ & $11: 00$ & $2 o f 2$ & 13 & 1 & 2.5 & 0.0 & 31 & 2 & 81 & 0 & 0.4 & 0.0 & 0.027 & 0.002 \\
\hline BY23 & $\begin{array}{c}\text { Buckeye Flat Mine N } \\
\text { drain to Greenhorn } \\
\text { Cr, Grass Valley }\end{array}$ & $8 / 22 / 2000$ & $10: 45$ & 1 of 2 & 20 & 1 & 1.3 & 0.0 & 42 & 1 & 67 & 2 & $<3$ & 1 & $<0.02$ & 0.01 \\
\hline BY23 & $\begin{array}{c}\text { Buckeye Flat Mine N } \\
\text { drain to Greenhorn } \\
\text { Cr, Grass Valley }\end{array}$ & $8 / 22 / 2000$ & $10: 45$ & 2 of 2 & 20 & 1 & 1.2 & 0.0 & 44 & 0 & 69 & 0 & $<3$ & 1 & $<0.02$ & 0.01 \\
\hline BY116 & $\begin{array}{l}\text { Buckeye Flat Mine } \\
\text { pond drain } 0.15 \mathrm{mi} \\
\text { ab SF Greenhorn } \mathrm{Cr}\end{array}$ & $8 / 21 / 2000$ & $14: 10$ & 1 of 2 & 0.8 & 0.0 & 0.03 & 0.01 & 0.048 & 0.006 & 0.66 & 0.03 & $<3$ & 1 & $<0.02$ & 0.01 \\
\hline BY116 & $\begin{array}{l}\text { Buckeye Flat Mine } \\
\text { pond drain } 0.15 \mathrm{mi} \\
\text { ab SF Greenhorn } \mathrm{Cr}\end{array}$ & $8 / 21 / 2000$ & $14: 10$ & 2 of 2 & 0.8 & 0.0 & 0.05 & 0.01 & 0.053 & 0.003 & 0.72 & 0.04 & $<3$ & 1 & $<0.02$ & 0.01 \\
\hline BY24 & $\begin{array}{l}\text { Buckeye Flat Mine } \\
\text { S drain to SF } \\
\text { Greenhorn Cr, Grass } \\
\text { Valley }\end{array}$ & $8 / 26 / 1999$ & $11: 00$ & 1 of 2 & 0.47 & 0.05 & 0.11 & 0.00 & 0.82 & 0.02 & 7.4 & 0.1 & 0.1 & 0.1 & 0.006 & 0.001 \\
\hline BY24 & $\begin{array}{l}\text { Buckeye Flat Mine } \\
\text { S drain to SF } \\
\text { Greenhorn Cr, Grass } \\
\text { Valley }\end{array}$ & $8 / 26 / 1999$ & $11: 00$ & $2 \mathrm{of} 2$ & 0.51 & 0.13 & 0.12 & 0.00 & 0.76 & 0.03 & 7.0 & 0.1 & 0.1 & 0.0 & 0.008 & 0.002 \\
\hline BY24 & $\begin{array}{l}\text { Buckeye Flat Mine } \\
\text { S drain to SF } \\
\text { Greenhorn Cr, Grass } \\
\text { Valley }\end{array}$ & $8 / 22 / 2000$ & $12: 10$ & 1 of 2 & 0.4 & 0.0 & 0.15 & 0.02 & 0.60 & 0.01 & 8.7 & 0.1 & $<3$ & 1 & $<0.02$ & 0.00 \\
\hline BY24 & $\begin{array}{l}\text { Buckeye Flat Mine } \\
\text { S drain to SF } \\
\text { Greenhorn Cr, Grass } \\
\text { Valley }\end{array}$ & $8 / 22 / 2000$ & $12: 10$ & 2 of 2 & 0.4 & 0.0 & 0.15 & 0.02 & 0.63 & 0.03 & 8.7 & 0.2 & $<3$ & 1 & $<0.02$ & 0.00 \\
\hline BY25 & $\begin{array}{l}\text { Buckeye Flat Mine } \\
\text { upper drain }\end{array}$ & $4 / 9 / 2001$ & $10: 00$ & 1 of 2 & 5.1 & 0.8 & 0.85 & 0.13 & 8.9 & 1.7 & 19 & 3 & $<0.5$ & 0.2 & 0.20 & 0.04 \\
\hline BY25 & $\begin{array}{l}\text { Buckeye Flat Mine } \\
\text { upper drain }\end{array}$ & $4 / 9 / 2001$ & $10: 00$ & $2 \mathrm{of} 2$ & 5.1 & 0.3 & 0.86 & 0.07 & 8.8 & 0.4 & 19 & 1 & $<0.5$ & 0.2 & 0.25 & 0.09 \\
\hline
\end{tabular}


Table 4B. Concentrations of trace metals and selected major elements in filtered water samples, Greenhorn Creek drainage, Nevada County, California—Continued.

[Station name abbreviations: ab, above; bl, below; Cr, creek; Cyn, Canyon; mi, miles; N, north; nr, near; Rd, Road; S, south; SF, South Fork. Number in parentheses is the data parameter code, a five-digit code

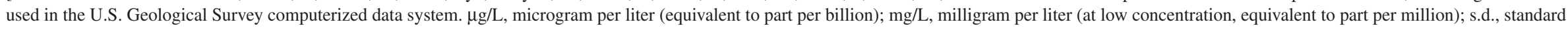
deviation; <, less than; - - not determined]

\begin{tabular}{|c|c|c|c|c|c|c|c|c|c|c|c|c|c|c|c|c|}
\hline \multirow[t]{2}{*}{$\begin{array}{l}\text { Station } \\
\text { map ID }\end{array}$} & \multirow[t]{2}{*}{ Station name } & \multirow[t]{2}{*}{ Date } & \multirow[t]{2}{*}{ Time } & \multirow[t]{2}{*}{$\begin{array}{l}\text { Rep- } \\
\text { licate }\end{array}$} & \multicolumn{2}{|c|}{$\begin{array}{l}\text { Calcium (Ca), } \\
\text { filtered } \\
(\mathrm{mg} / \mathrm{L}) \\
(00915)\end{array}$} & \multicolumn{2}{|c|}{$\begin{array}{c}\text { Cadmium (Cd), } \\
\text { filtered } \\
(\mu \mathrm{g} / \mathrm{L}) \\
(01025) \\
\end{array}$} & \multicolumn{2}{|c|}{$\begin{array}{c}\text { Cerium (Ce), } \\
\text { filtered } \\
(\mu \mathrm{g} / \mathrm{L}) \\
(01110\end{array}$} & \multicolumn{2}{|c|}{$\begin{array}{c}\text { Cobalt (Co), } \\
\text { filtered } \\
(\mu \mathrm{g} / \mathrm{L}) \\
(01035) \\
\end{array}$} & \multicolumn{2}{|c|}{$\begin{array}{l}\text { Chromium (Cr), } \\
\text { filtered } \\
(\mu \mathrm{g} / \mathrm{L}) \\
(01030) \\
\end{array}$} & \multicolumn{2}{|c|}{$\begin{array}{c}\text { Cesium (Cs), } \\
\text { filtered } \\
(\mu \mathrm{g} / \mathrm{L}) \\
(01115) \\
\end{array}$} \\
\hline & & & & & value & s.d. & value & s.d. & value & s.d. & value & s.d. & value & s.d. & value & s.d. \\
\hline BY52 & $\begin{array}{l}\text { Greenhorn Cr } 0.3 \mathrm{mi} \\
\text { ab Buckeye Ford nr } \\
\text { Nevada City }\end{array}$ & $1 / 25 / 2000$ & $10: 45$ & 1 of 2 & 2.7 & 0.2 & 0.04 & 0.01 & 0.57 & 0.02 & 1.9 & 0.1 & $<0.4$ & 0.3 & $<0.5$ & 0.2 \\
\hline BY52 & $\begin{array}{l}\text { Greenhorn Cr } 0.3 \mathrm{mi} \\
\text { ab Buckeye Ford nr } \\
\text { Nevada City }\end{array}$ & $1 / 25 / 2000$ & $10: 45$ & $2 \mathrm{of} 2$ & 2.7 & 0.1 & $<0.03$ & 0.03 & 0.54 & 0.02 & 2.1 & 0.0 & $<0.4$ & 0.1 & $<0.5$ & 0.2 \\
\hline BY59 & $\begin{array}{l}\text { Greenhorn Cr at You } \\
\text { Bet Rd nr Nevada } \\
\text { City }\end{array}$ & $7 / 24 / 2001$ & $11: 00$ & 1 of 2 & 5.6 & 0.1 & 0.040 & 0.010 & 0.013 & 0.001 & 0.47 & 0.01 & $<0.06$ & 0.06 & 0.038 & 0.003 \\
\hline BY59 & $\begin{array}{l}\text { Greenhorn } \mathrm{Cr} \text { at You } \\
\text { Bet Rd nr Nevada } \\
\text { City }\end{array}$ & $7 / 24 / 2001$ & $11: 00$ & $2 \mathrm{of} 2$ & 5.5 & 0.1 & 0.040 & 0.006 & 0.013 & 0.000 & 0.49 & 0.03 & $<0.06$ & 0.00 & 0.047 & 0.023 \\
\hline BY59 & $\begin{array}{l}\text { Greenhorn Cr at You } \\
\text { Bet Rd nr Nevada } \\
\text { City }\end{array}$ & $8 / 29 / 2001$ & $15: 00$ & 1 of 2 & 5.3 & 0.2 & 0.009 & 0.002 & 0.010 & 0.000 & 0.053 & 0.003 & $<0.06$ & 0.04 & 0.021 & 0.003 \\
\hline BY59 & $\begin{array}{l}\text { Greenhorn } \mathrm{Cr} \text { at You } \\
\text { Bet Rd nr Nevada } \\
\text { City }\end{array}$ & $8 / 29 / 2001$ & $15: 00$ & $2 \mathrm{of} 2$ & 5.4 & 0.0 & 0.009 & 0.000 & 0.0100 & 0.0001 & 0.057 & 0.003 & $<0.06$ & 0.01 & 0.013 & 0.002 \\
\hline BY59 & $\begin{array}{l}\text { Greenhorn } \mathrm{Cr} \text { at You } \\
\text { Bet Rd nr Nevada } \\
\text { City }\end{array}$ & $9 / 14 / 2001$ & 13:00 & 1 of 2 & 5.3 & 0.2 & 0.020 & 0.000 & 0.0081 & 0.0004 & 0.099 & 0.002 & 0.26 & 0.05 & 0.018 & 0.003 \\
\hline BY59 & $\begin{array}{l}\text { Greenhorn Cr at You } \\
\text { Bet Rd nr Nevada } \\
\text { City }\end{array}$ & $9 / 14 / 2001$ & $13: 00$ & $2 \mathrm{of} 2$ & 5.2 & 0.1 & 0.022 & 0.002 & 0.0086 & 0.0001 & 0.10 & 0.00 & 0.31 & 0.04 & 0.018 & 0.002 \\
\hline BY59 & $\begin{array}{l}\text { Greenhorn Cr at You } \\
\text { Bet Rd nr Nevada } \\
\text { City }\end{array}$ & $10 / 15 / 2001$ & $12: 00$ & 1 of 2 & 5.3 & 0.1 & 0.020 & 0.006 & 0.0046 & 0.0003 & 0.080 & 0.003 & $<0.07$ & 0.02 & 0.010 & 0.002 \\
\hline BY59 & $\begin{array}{l}\text { Greenhorn Cr at You } \\
\text { Bet Rd nr Nevada } \\
\text { City }\end{array}$ & $10 / 15 / 2001$ & $12: 00$ & $2 \mathrm{of} 2$ & 5.3 & 0.1 & 0.017 & 0.001 & 0.0054 & 0.0003 & 0.077 & 0.001 & $<0.07$ & 0.01 & 0.014 & 0.001 \\
\hline BY59 & $\begin{array}{l}\text { Greenhorn Cr at You } \\
\text { Bet Rd nr Nevada } \\
\text { City }\end{array}$ & $11 / 20 / 2001$ & $13: 20$ & 1 of 2 & 6.2 & 0.1 & 0.078 & 0.002 & 0.074 & 0.003 & 1.2 & 0.0 & $<0.2$ & 0.1 & 0.019 & 0.002 \\
\hline BY59 & $\begin{array}{l}\text { Greenhorn Cr at You } \\
\text { Bet Rd nr Nevada } \\
\text { City }\end{array}$ & $11 / 20 / 2001$ & $13: 20$ & $2 \mathrm{of} 2$ & 6.2 & 0.0 & 0.072 & 0.005 & 0.079 & 0.002 & 1.2 & 0.0 & $<0.2$ & 0.1 & 0.021 & 0.001 \\
\hline BY59 & $\begin{array}{l}\text { Greenhorn } \mathrm{Cr} \text { at You } \\
\text { Bet Rd nr Nevada } \\
\text { City }\end{array}$ & $12 / 10 / 2001$ & $12: 20$ & 1 of 2 & 6.0 & 0.1 & 0.094 & 0.001 & 0.24 & 0.00 & 1.9 & 0.0 & $<0.04$ & 0.03 & 0.014 & 0.002 \\
\hline
\end{tabular}


Table 4B. Concentrations of trace metals and selected major elements in filtered water samples, Greenhorn Creek drainage, Nevada County, California—Continued.

[Station name abbreviations: ab, above; bl, below; Cr, creek; Cyn, Canyon; mi, miles; N, north; nr, near; Rd, Road; S, south; SF, South Fork. Number in parentheses is the data parameter code, a five-digit code

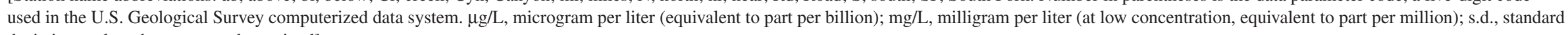
deviation; <, less than; - , not determined]

\begin{tabular}{|c|c|c|c|c|c|c|c|c|c|c|c|c|c|c|c|c|}
\hline \multirow[t]{2}{*}{$\begin{array}{l}\text { Station } \\
\text { map ID }\end{array}$} & \multirow[t]{2}{*}{ Station name } & \multirow[t]{2}{*}{ Date } & \multirow[t]{2}{*}{ Time } & \multirow[t]{2}{*}{$\begin{array}{l}\text { Rep- } \\
\text { licate }\end{array}$} & \multicolumn{2}{|c|}{$\begin{array}{c}\text { Calcium (Ca), } \\
\text { filtered } \\
\text { (mg/L) } \\
(00915)\end{array}$} & \multicolumn{2}{|c|}{$\begin{array}{c}\text { Cadmium (Cd), } \\
\text { filtered } \\
(\mu \mathrm{g} / \mathrm{L}) \\
(01025)\end{array}$} & \multicolumn{2}{|c|}{$\begin{array}{l}\text { Cerium (Ce), } \\
\text { filtered } \\
(\mu \mathrm{g} / \mathrm{L}) \\
(01110\end{array}$} & \multicolumn{2}{|c|}{$\begin{array}{c}\text { Cobalt (Co), } \\
\text { filtered } \\
(\mu \mathrm{g} / \mathrm{L}) \\
(01035)\end{array}$} & \multicolumn{2}{|c|}{$\begin{array}{c}\text { Chromium (Cr), } \\
\text { filtered } \\
(\mu \mathrm{g} / \mathrm{L}) \\
(\mathbf{0 1 0 3 0})\end{array}$} & \multicolumn{2}{|c|}{$\begin{array}{c}\text { Cesium (Cs), } \\
\text { filtered } \\
(\mu \mathrm{g} / \mathrm{L}) \\
(01115)\end{array}$} \\
\hline & & & & & value & s.d. & value & s.d. & value & s.d. & value & s.d. & value & s.d. & value & s.d. \\
\hline BY59 & $\begin{array}{l}\text { Greenhorn Cr at You } \\
\text { Bet Rd nr Nevada } \\
\text { City }\end{array}$ & $12 / 10 / 2001$ & $12: 20$ & 2of2 & 6.1 & 0.1 & 0.090 & 0.002 & 0.25 & 0.01 & 1.9 & 0.0 & $<0.04$ & 0.03 & 0.011 & 0.002 \\
\hline BY59 & $\begin{array}{l}\text { Greenhorn } \mathrm{Cr} \text { at You } \\
\text { Bet Rd nr Nevada } \\
\text { City }\end{array}$ & $12 / 21 / 2001$ & $13: 00$ & $1 \mathrm{of} 2$ & 4.4 & 0.0 & 0.054 & 0.001 & 0.49 & 0.00 & 1.2 & 0.0 & 0.07 & 0.01 & 0.013 & 0.001 \\
\hline BY59 & $\begin{array}{l}\text { Greenhorn Cr at You } \\
\text { Bet Rd nr Nevada } \\
\text { City }\end{array}$ & $12 / 21 / 2001$ & $13: 00$ & $2 \mathrm{of} 2$ & 4.3 & 0.0 & 0.054 & 0.003 & 0.47 & 0.00 & 1.2 & 0.0 & $<0.06$ & 0.01 & 0.013 & 0.005 \\
\hline BY51 & $\begin{array}{l}\text { Greenhorn Cr nr } \\
\text { Headwaters nr Scotts } \\
\text { Flat Reservoir }\end{array}$ & $1 / 25 / 2000$ & $9: 30$ & $1 \mathrm{of} 2$ & 1.3 & 0.1 & 0.03 & 0.01 & 0.15 & 0.01 & 0.07 & 0.02 & 0.6 & 0.3 & $<0.5$ & 0.2 \\
\hline BY51 & $\begin{array}{l}\text { Greenhorn Cr nr } \\
\text { Headwaters nr Scotts } \\
\text { Flat Reservoir }\end{array}$ & $1 / 25 / 2000$ & $9: 30$ & $2 \mathrm{of} 2$ & 1.3 & 0.0 & 0.01 & 0.01 & 0.15 & 0.00 & 0.06 & 0.02 & $<0.4$ & 0.1 & $<0.5$ & 0.1 \\
\hline BY75 & $\begin{array}{l}\text { Missouri Cyn Cr } 1.2 \mathrm{mi} \\
\text { ab Greenhorn Cr nr } \\
\text { Chicago Park }\end{array}$ & 12/14/1999 & $14: 00$ & $1 \mathrm{of} 2$ & 11 & 1 & 0.15 & 0.00 & 0.44 & 0.01 & 3.7 & 0.0 & $<0.1$ & 0.0 & 0.046 & 0.001 \\
\hline BY75 & $\begin{array}{l}\text { Missouri Cyn Cr } 1.2 \mathrm{mi} \\
\text { ab Greenhorn Cr nr } \\
\text { Chicago Park }\end{array}$ & 12/14/1999 & $14: 00$ & $2 \mathrm{of} 2$ & 15 & 4 & 0.15 & 0.00 & 0.44 & 0.01 & 3.6 & 0.0 & $<0.1$ & 0.0 & 0.048 & 0.001 \\
\hline BY86 & $\begin{array}{l}\text { Poore Mine creek ab } \\
\text { tunnel nr Grass } \\
\text { Valley }\end{array}$ & 3/31/1999 & $17: 40$ & $1 \mathrm{of} 2$ & 3.5 & 0.2 & 0.016 & 0.000 & 0.072 & 0.005 & 0.033 & 0.002 & $<0.3$ & 0.2 & 0.002 & 0.005 \\
\hline BY86 & $\begin{array}{l}\text { Poore Mine creek ab } \\
\text { tunnel nr Grass } \\
\text { Valley }\end{array}$ & 3/31/1999 & $17: 40$ & $2 \mathrm{of} 2$ & 3.5 & 0.0 & 0.012 & 0.002 & 0.067 & 0.002 & 0.029 & 0.002 & $<0.3$ & 0.1 & 0.004 & 0.009 \\
\hline BY87 & $\begin{array}{l}\text { Poore Mine creek bl } \\
\text { tunnel nr Grass } \\
\text { Valley }\end{array}$ & 3/31/1999 & $13: 30$ & $1 \mathrm{of} 2$ & 3.8 & 0.0 & 0.014 & 0.000 & 0.061 & 0.005 & 0.020 & 0.000 & $<0.3$ & 0.1 & 0.002 & 0.000 \\
\hline BY87 & $\begin{array}{l}\text { Poore Mine creek bl } \\
\text { tunnel nr Grass } \\
\text { Valley }\end{array}$ & 3/31/1999 & $13: 30$ & $2 \mathrm{of} 2$ & 3.7 & 0.0 & 0.013 & 0.001 & 0.051 & 0.002 & 0.026 & 0.005 & $<0.3$ & 0.2 & 0.002 & 0.000 \\
\hline BY88 & $\begin{array}{l}\text { Poore Mine ground } \\
\text { sluice nr Grass } \\
\text { Valley }\end{array}$ & 4/1/1999 & $15: 40$ & $1 \mathrm{of} 2$ & 7.5 & 0.1 & 0.048 & 0.001 & 0.13 & 0.00 & 1.1 & 0.0 & $<0.3$ & 0.1 & 0.043 & 0.000 \\
\hline BY88 & $\begin{array}{l}\text { Poore Mine ground } \\
\text { sluice nr Grass } \\
\text { Valley }\end{array}$ & 4/1/1999 & $15: 40$ & $2 \mathrm{of} 2$ & 7.6 & 0.1 & 0.047 & 0.005 & 0.13 & 0.00 & 1.1 & 0.1 & $<0.1$ & 0.1 & 0.044 & 0.003 \\
\hline
\end{tabular}


Table 4B. Concentrations of trace metals and selected major elements in filtered water samples, Greenhorn Creek drainage, Nevada County, California—Continued.

[Station name abbreviations: ab, above; bl, below; Cr, creek; Cyn, Canyon; mi, miles; N, north; nr, near; Rd, Road; S, south; SF, South Fork. Number in parentheses is the data parameter code, a five-digit code

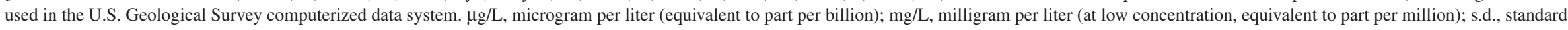
deviation; $<$, less than; —, not determined]

\begin{tabular}{|c|c|c|c|c|c|c|c|c|c|c|c|c|c|c|c|c|}
\hline \multirow[t]{2}{*}{$\begin{array}{l}\text { Station } \\
\text { map ID }\end{array}$} & \multirow[t]{2}{*}{ Station name } & \multirow[t]{2}{*}{ Date } & \multirow[t]{2}{*}{ Time } & \multirow[t]{2}{*}{$\begin{array}{l}\text { Rep- } \\
\text { licate }\end{array}$} & \multicolumn{2}{|c|}{$\begin{array}{c}\text { Calcium (Ca), } \\
\text { filtered } \\
\text { (mg/L) } \\
(00915)\end{array}$} & \multicolumn{2}{|c|}{$\begin{array}{c}\text { Cadmium (Cd), } \\
\text { filtered } \\
(\mu \mathrm{g} / \mathrm{L}) \\
(01025) \\
\end{array}$} & \multicolumn{2}{|c|}{$\begin{array}{c}\text { Cerium (Ce), } \\
\text { filtered } \\
(\mu \mathrm{g} / \mathrm{L}) \\
(01110 \\
\end{array}$} & \multicolumn{2}{|c|}{$\begin{array}{l}\text { Cobalt (Co), } \\
\text { filtered } \\
(\mu \mathrm{g} / \mathrm{L}) \\
(01035)\end{array}$} & \multicolumn{2}{|c|}{$\begin{array}{c}\text { Chromium (Cr), } \\
\text { filtered } \\
(\mu \mathrm{g} / \mathrm{L}) \\
(\mathbf{0 1 0 3 0}) \\
\end{array}$} & \multicolumn{2}{|c|}{$\begin{array}{c}\text { Cesium (Cs), } \\
\text { filtered } \\
(\mu \mathrm{g} / \mathrm{L}) \\
(01115)\end{array}$} \\
\hline & & & & & value & s.d. & value & s.d. & value & s.d. & value & s.d. & value & s.d. & value & s.d. \\
\hline BY89 & $\begin{array}{l}\text { Poore Mine pit lake } \\
\text { nr Grass Valley }\end{array}$ & 4/1/1999 & $16: 50$ & 1 of 2 & 2.6 & 0.1 & 0.055 & 0.005 & 0.037 & 0.001 & 0.85 & 0.04 & $<0.1$ & 0.0 & 0.006 & 0.001 \\
\hline BY89 & $\begin{array}{l}\text { Poore Mine pit lake } \\
\text { nr Grass Valley }\end{array}$ & 4/1/1999 & $16: 50$ & $2 \mathrm{of} 2$ & 2.6 & 0.1 & 0.056 & 0.004 & 0.032 & 0.002 & 0.80 & 0.04 & $<0.1$ & 0.2 & 0.010 & 0.005 \\
\hline BY89 & $\begin{array}{l}\text { Poore Mine pit lake } \\
\text { nr Grass Valley }\end{array}$ & $5 / 23 / 2000$ & $10: 45$ & 1 of 2 & 15 & 0 & 1.1 & 0.0 & 0.24 & 0.01 & 9.8 & 0.0 & $<0.6$ & 0.1 & $<0.2$ & 0.1 \\
\hline BY89 & $\begin{array}{l}\text { Poore Mine pit lake } \\
\text { nr Grass Valley }\end{array}$ & $5 / 23 / 2000$ & $10: 45$ & $2 \mathrm{of} 2$ & 15 & 1 & 1.1 & 0.0 & 0.25 & 0.01 & 9.5 & 0.3 & $<0.6$ & 0.2 & $<0.2$ & 0.3 \\
\hline BY90 & $\begin{array}{l}\text { Poore Mine seep } \\
\text { ab ground sluice } \\
\text { nr Grass Valley }\end{array}$ & 4/1/1999 & $16: 00$ & 1 of 2 & 7.1 & 0.1 & 0.042 & 0.003 & 0.031 & 0.001 & 1.3 & 0.1 & $<0.1$ & 0.1 & 0.041 & 0.003 \\
\hline BY90 & $\begin{array}{l}\text { Poore Mine seep } \\
\text { ab ground sluice } \\
\text { nr Grass Valley }\end{array}$ & 4/1/1999 & $16: 00$ & $2 \mathrm{of} 2$ & 7.2 & 0.1 & 0.041 & 0.002 & 0.031 & 0.000 & 1.3 & 0.0 & $<0.1$ & 0.0 & 0.038 & 0.001 \\
\hline BY91 & $\begin{array}{l}\text { Poore Mine tunnel } \\
\text { effluent nr Grass } \\
\text { Valley }\end{array}$ & 3/31/1999 & $15: 20$ & 1 of 2 & 13 & 0 & 0.041 & 0.001 & 0.013 & 0.003 & 0.021 & 0.010 & $<0.3$ & 0.1 & 0.20 & 0.00 \\
\hline BY91 & $\begin{array}{l}\text { Poore Mine tunnel } \\
\text { effluent nr Grass } \\
\text { Valley }\end{array}$ & 3/31/1999 & $15: 20$ & $2 \mathrm{of} 2$ & 12 & 0 & 0.035 & 0.000 & 0.013 & 0.001 & 0.012 & 0.022 & $<0.1$ & 0.1 & 0.21 & 0.00 \\
\hline BY91 & $\begin{array}{l}\text { Poore Mine tunnel } \\
\text { effluent nr Grass } \\
\text { Valley }\end{array}$ & $12 / 15 / 1999$ & $13: 00$ & $1 \mathrm{of} 2$ & 20 & 2 & 0.017 & 0.005 & 0.0085 & 0.0076 & $<0.006$ & 0.002 & $<0.1$ & 0.1 & 0.22 & 0.00 \\
\hline BY91 & $\begin{array}{l}\text { Poore Mine tunnel } \\
\text { effluent nr Grass } \\
\text { Valley }\end{array}$ & $12 / 15 / 1999$ & $13: 00$ & $2 o f 2$ & 23 & 2 & 0.025 & 0.013 & 0.0037 & 0.0002 & $<0.006$ & 0.003 & $<0.1$ & 0.1 & 0.22 & 0.01 \\
\hline BY91 & $\begin{array}{l}\text { Poore Mine tunnel } \\
\text { effluent nr Grass } \\
\text { Valley }\end{array}$ & $5 / 23 / 2000$ & $12: 00$ & 1 of 2 & 20 & 1 & 0.04 & 0.01 & 0.0045 & 0.0004 & $<0.02$ & 0.01 & $<0.6$ & 0.1 & 0.4 & 0.1 \\
\hline BY91 & $\begin{array}{l}\text { Poore Mine tunnel } \\
\text { effluent nr Grass } \\
\text { Valley }\end{array}$ & $5 / 23 / 2000$ & $12: 00$ & $2 o f 2$ & 18 & 0 & 0.05 & 0.00 & 0.004 & 0.001 & $<0.01$ & 0.01 & $<2$ & 0 & $<0.5$ & 0.0 \\
\hline BY105 & $\begin{array}{l}\text { Sailor Flat Mine main } \\
\text { drain Gulch 01nr } \\
\text { Quaker Hill }\end{array}$ & 7/6/1999 & $16: 00$ & 1 of 2 & 3.1 & 0.1 & 0.11 & 0.00 & 1.0 & 0.0 & 6.8 & 0.1 & 0.3 & 0.0 & 0.051 & 0.001 \\
\hline BY105 & $\begin{array}{l}\text { Sailor Flat Mine main } \\
\text { drain Gulch 01nr } \\
\text { Quaker Hill }\end{array}$ & 7/6/1999 & $16: 00$ & 2 of 2 & 2.9 & 0.0 & 0.11 & 0.00 & 0.97 & 0.09 & 6.6 & 0.6 & $<0.3$ & 0.3 & 0.051 & 0.004 \\
\hline
\end{tabular}


Table 4B. Concentrations of trace metals and selected major elements in filtered water samples, Greenhorn Creek drainage, Nevada County, California—Continued.

[Station name abbreviations: ab, above; bl, below; Cr, creek; Cyn, Canyon; mi, miles; N, north; nr, near; Rd, Road; S, south; SF, South Fork. Number in parentheses is the data parameter code, a five-digit code

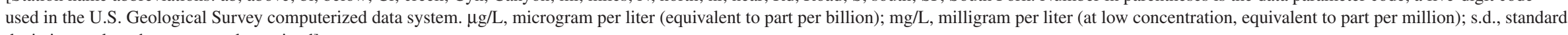
deviation; <, less than; - - not determined]

\begin{tabular}{|c|c|c|c|c|c|c|c|c|c|c|c|c|c|c|c|c|}
\hline \multirow[t]{2}{*}{$\begin{array}{l}\text { Station } \\
\text { map ID }\end{array}$} & \multirow[t]{2}{*}{ Station name } & \multirow[t]{2}{*}{ Date } & \multirow[t]{2}{*}{ Time } & \multirow[t]{2}{*}{$\begin{array}{l}\text { Rep- } \\
\text { licate }\end{array}$} & \multicolumn{2}{|c|}{$\begin{array}{c}\text { Calcium (Ca), } \\
\text { filtered } \\
\text { (mg/L) } \\
(00915) \\
\end{array}$} & \multicolumn{2}{|c|}{$\begin{array}{c}\text { Cadmium (Cd), } \\
\text { filtered } \\
(\mu \mathrm{g} / \mathrm{L}) \\
(01025) \\
\end{array}$} & \multicolumn{2}{|c|}{$\begin{array}{c}\text { Cerium (Ce), } \\
\text { filtered } \\
(\mu \mathrm{g} / \mathrm{L}) \\
(\mathbf{0 1 1 1 0} \\
\end{array}$} & \multicolumn{2}{|c|}{$\begin{array}{c}\text { Cobalt (Co), } \\
\text { filtered } \\
(\mu \mathrm{g} / \mathrm{L}) \\
(01035) \\
\end{array}$} & \multicolumn{2}{|c|}{$\begin{array}{c}\text { Chromium (Cr), } \\
\text { filtered } \\
(\mu \mathrm{g} / \mathrm{L}) \\
(01030) \\
\end{array}$} & \multicolumn{2}{|c|}{$\begin{array}{c}\text { Cesium (Cs), } \\
\text { filtered } \\
(\mu \mathrm{g} / \mathrm{L}) \\
(\mathbf{0 1 1 1 5}) \\
\end{array}$} \\
\hline & & & & & value & s.d. & value & s.d. & value & s.d. & value & s.d. & value & s.d. & value & s.d. \\
\hline BY105 & $\begin{array}{l}\text { Sailor Flat Mine main } \\
\text { drain Gulch 01nr } \\
\text { Quaker Hill }\end{array}$ & 12/2/1999 & $14: 30$ & 1 of 2 & 1.2 & 0.1 & 0.13 & 0.08 & 1.9 & 0.1 & 2.4 & 0.1 & 0.2 & 0.0 & 0.055 & 0.068 \\
\hline BY105 & $\begin{array}{l}\text { Sailor Flat Mine main } \\
\text { drain Gulch 01nr } \\
\text { Quaker Hill }\end{array}$ & 12/2/1999 & $14: 30$ & 2 of 2 & 1.1 & 0.2 & 0.067 & 0.006 & 2.0 & 0.0 & 2.3 & 0.0 & 0.2 & 0.0 & 0.0055 & 0.0008 \\
\hline BY106 & $\begin{array}{l}\text { Sailor Flat Mine } \\
\text { main drainage to } \\
\text { Greenhorn Cr, } \\
\text { Gulch } 03\end{array}$ & $4 / 9 / 2001$ & $12: 30$ & 1of 2 & 0.6 & 0.2 & $<0.03$ & 0.01 & 1.9 & 0.4 & 1.4 & 0.3 & 0.7 & 0.2 & 0.23 & 0.08 \\
\hline BY106 & $\begin{array}{l}\text { Sailor Flat Mine } \\
\text { main drainage to } \\
\text { Greenhorn Cr, } \\
\text { Gulch } 03\end{array}$ & $4 / 9 / 2001$ & $12: 30$ & 2 of 2 & 0.6 & 0.2 & $<0.03$ & 0.01 & 1.9 & 0.4 & 1.3 & 0.3 & 0.9 & 0.2 & 0.18 & 0.11 \\
\hline BY114 & $\begin{array}{l}\text { SF Greenhorn Cr } \\
0.7 \text { mi ab Greenhorn } \\
\text { Cr nr Nevada City }\end{array}$ & $8 / 22 / 2000$ & $15: 10$ & 1 of 2 & 7.6 & 0.2 & $<0.03$ & 0.01 & 0.006 & 0.000 & $<0.02$ & 0.01 & $<3$ & 1 & $<0.02$ & 0.01 \\
\hline BY114 & $\begin{array}{l}\text { SF Greenhorn Cr } \\
0.7 \text { mi ab Greenhorn } \\
\text { Cr nr Nevada City }\end{array}$ & $8 / 22 / 2000$ & $15: 10$ & 2 of 2 & 7.5 & 0.2 & $<0.03$ & 0.01 & 0.006 & 0.001 & $<0.02$ & 0.01 & $<3$ & 1 & $<0.02$ & 0.00 \\
\hline BY122 & $\begin{array}{l}\text { Starr Mine tunnel } \\
\text { inflow nr Grass } \\
\text { Valley }\end{array}$ & 4/23/1999 & $15: 00$ & 1 of 2 & 9.4 & 0.1 & 0.070 & 0.000 & 0.059 & 0.003 & 0.23 & 0.03 & $<0.1$ & 0.0 & 0.022 & 0.000 \\
\hline BY122 & $\begin{array}{l}\text { Starr Mine tunnel } \\
\text { inflow nr Grass } \\
\text { Valley }\end{array}$ & 4/23/1999 & $15: 00$ & 2 of 2 & 9.8 & 0.4 & 0.073 & 0.001 & 0.058 & 0.003 & 0.23 & 0.03 & $<0.1$ & 0.0 & 0.023 & 0.001 \\
\hline BY123 & $\begin{array}{l}\text { Starr Mine tunnel } \\
\text { midway nr Grass } \\
\text { Valley }\end{array}$ & 4/23/1999 & $13: 40$ & 1 of 2 & 29 & 1 & 0.0044 & 0.0001 & 0.0010 & 0.0002 & $<0.003$ & 0.031 & $<0.1$ & 0.1 & 0.17 & 0.01 \\
\hline BY123 & $\begin{array}{l}\text { Starr Mine tunnel } \\
\text { midway nr Grass } \\
\text { Valley }\end{array}$ & 4/23/1999 & $13: 40$ & $2 \mathrm{of} 2$ & 29 & 0 & 0.0069 & 0.0022 & 0.0011 & 0.0001 & $<0.003$ & 0.012 & $<0.1$ & 0.0 & 0.17 & 0.00 \\
\hline BY123 & $\begin{array}{l}\text { Starr Mine tunnel } \\
\text { midway nr Grass } \\
\text { Valley }\end{array}$ & $12 / 15 / 1999$ & $10: 45$ & 1 of 2 & 33 & 0 & 0.015 & 0.002 & 0.0029 & 0.0003 & 0.013 & 0.000 & $<0.1$ & 0.1 & 0.15 & 0.00 \\
\hline BY123 & $\begin{array}{l}\text { Starr Mine tunnel } \\
\text { midway nr Grass } \\
\text { Valley }\end{array}$ & $12 / 15 / 1999$ & $10: 45$ & 2 of 2 & 36 & 1 & 0.013 & 0.003 & 0.0040 & 0.0003 & 0.014 & 0.002 & $<0.1$ & 0.0 & 0.15 & 0.00 \\
\hline BY124 & $\begin{array}{l}\text { Starr Mine tunnel outlet } \\
\text { nr Grass Valley }\end{array}$ & $8 / 23 / 2000$ & $17: 30$ & 1 of 2 & 36 & 0 & $<0.03$ & 0.01 & 0.014 & 0.002 & $<0.02$ & 0.01 & $<3$ & 0 & 0.07 & 0.02 \\
\hline
\end{tabular}


Table 4B. Concentrations of trace metals and selected major elements in filtered water samples, Greenhorn Creek drainage, Nevada County, California—Continued.

[Station name abbreviations: ab, above; bl, below; Cr, creek; Cyn, Canyon; mi, miles; N, north; nr, near; Rd, Road; S, south; SF, South Fork. Number in parentheses is the data parameter code, a five-digit code

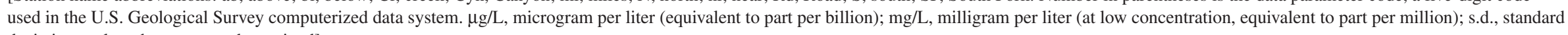
deviation; $<$, less than; - , not determined]

\begin{tabular}{|c|c|c|c|c|c|c|c|c|c|c|c|c|c|c|c|c|}
\hline \multirow[t]{2}{*}{$\begin{array}{l}\text { Station } \\
\text { map ID }\end{array}$} & \multirow[t]{2}{*}{ Station name } & \multirow[t]{2}{*}{ Date } & \multirow[t]{2}{*}{ Time } & \multirow[t]{2}{*}{$\begin{array}{l}\text { Rep- } \\
\text { licate }\end{array}$} & \multicolumn{2}{|c|}{$\begin{array}{c}\text { Calcium (Ca), } \\
\text { filtered } \\
(\mathrm{mg} / \mathrm{L}) \\
(00915)\end{array}$} & \multicolumn{2}{|c|}{$\begin{array}{c}\text { Cadmium (Cd), } \\
\text { filtered } \\
(\mu \mathrm{g} / \mathrm{L}) \\
(01025)\end{array}$} & \multicolumn{2}{|c|}{$\begin{array}{l}\text { Cerium (Ce), } \\
\text { filtered } \\
(\mu \mathrm{g} / \mathrm{L}) \\
(01110\end{array}$} & \multicolumn{2}{|c|}{$\begin{array}{l}\text { Cobalt (Co), } \\
\text { filtered } \\
(\mu \mathrm{g} / \mathrm{L}) \\
(01035)\end{array}$} & \multicolumn{2}{|c|}{$\begin{array}{c}\text { Chromium (Cr), } \\
\text { filtered } \\
(\mu \mathrm{g} / \mathrm{L}) \\
(\mathbf{0 1 0 3 0})\end{array}$} & \multicolumn{2}{|c|}{$\begin{array}{c}\text { Cesium (Cs), } \\
\text { filtered } \\
(\mu \mathrm{g} / \mathrm{L}) \\
(01115)\end{array}$} \\
\hline & & & & & value & s.d. & value & s.d. & value & s.d. & value & s.d. & value & s.d. & value & s.d. \\
\hline BY124 & $\begin{array}{l}\text { Starr Mine tunnel outlet } \\
\text { nr Grass Valley }\end{array}$ & $8 / 23 / 2000$ & $17: 30$ & 2 of 2 & 35 & 1 & $<0.03$ & 0.01 & 0.004 & 0.001 & $<0.02$ & 0.01 & $<3$ & 1 & 0.06 & 0.00 \\
\hline BY129 & $\begin{array}{l}\text { Tom and Jerry Mine } \\
\text { drain at tunnel inlet } \\
\text { nr Nevada City }\end{array}$ & $6 / 28 / 2000$ & $12: 00$ & 1 of 2 & 2.8 & 0.1 & $<0.01$ & 0.02 & 0.022 & 0.002 & 0.02 & 0.00 & $<2$ & 1 & $<0.01$ & 0.01 \\
\hline BY129 & $\begin{array}{l}\text { Tom and Jerry Mine } \\
\text { drain at tunnel inlet } \\
\text { nr Nevada City }\end{array}$ & $6 / 28 / 2000$ & $12: 00$ & 2 of 2 & 2.7 & 0.2 & $<0.01$ & 0.01 & 0.025 & 0.001 & $<0.02$ & 0.00 & $<2$ & 1 & $<0.01$ & 0.01 \\
\hline BY130 & $\begin{array}{l}\text { Tom and Jerry Mine } \\
\text { drain at tunnel outlet } \\
\text { nr Nevada City }\end{array}$ & $6 / 28 / 2000$ & $11: 30$ & 1 of 2 & 11 & 0 & $<0.01$ & 0.01 & 0.006 & 0.001 & 0.41 & 0.02 & $<2$ & 1 & $<0.01$ & 0.01 \\
\hline BY130 & $\begin{array}{l}\text { Tom and Jerry Mine } \\
\text { drain at tunnel outlet } \\
\text { nr Nevada City }\end{array}$ & $6 / 28 / 2000$ & $11: 30$ & $2 o f 2$ & 11 & 1 & $<0.01$ & 0.00 & 0.008 & 0.001 & 0.40 & 0.01 & $<2$ & 1 & $<0.01$ & 0.01 \\
\hline BY131 & $\begin{array}{l}\text { Tom and Jerry Mine } \\
\text { drainage pond nr } \\
\text { Nevada City }\end{array}$ & $8 / 23 / 2000$ & $10: 45$ & 1 of 2 & 4.0 & 0.1 & $<0.03$ & 0.01 & 0.012 & 0.000 & $<0.02$ & 0.01 & $<3$ & 1 & $<0.02$ & 0.00 \\
\hline BY131 & $\begin{array}{l}\text { Tom and Jerry Mine } \\
\text { drainage pond nr } \\
\text { Nevada City }\end{array}$ & $8 / 23 / 2000$ & $10: 45$ & $2 o f 2$ & 4.0 & 0.1 & $<0.03$ & 0.01 & 0.013 & 0.003 & $<0.02$ & 0.01 & $<3$ & 1 & $<0.02$ & 0.01 \\
\hline \multicolumn{17}{|c|}{ Disturbed samples } \\
\hline BY123 & $\begin{array}{l}\text { Starr Mine tunnel } \\
\text { midway nr Grass } \\
\text { Valley }\end{array}$ & $12 / 15 / 1999$ & $11: 30$ & 1 of 2 & 33 & 2 & 0.002 & 0.001 & 0.0009 & 0.0000 & $<0.006$ & 0.002 & $<0.1$ & 0.0 & 0.15 & 0.00 \\
\hline BY123 & $\begin{array}{l}\text { Starr Mine tunnel } \\
\text { midway nr Grass } \\
\text { Valley }\end{array}$ & $12 / 15 / 1999$ & $11: 30$ & 2 of 2 & 41 & 0 & 0.006 & 0.006 & 0.0010 & 0.0000 & $<0.006$ & 0.004 & $<0.1$ & 0.0 & 0.15 & 0.00 \\
\hline BY129 & $\begin{array}{l}\text { Tom and Jerry Mine } \\
\text { drain at tunnel inlet } \\
\text { nr Nevada City }\end{array}$ & $6 / 28 / 2000$ & $12: 30$ & 1 of 2 & 9.9 & 0.2 & $<0.01$ & 0.01 & 0.013 & 0.001 & $<0.02$ & 0.01 & $<2$ & 2 & 0.03 & 0.02 \\
\hline BY129 & $\begin{array}{l}\text { Tom and Jerry Mine } \\
\text { drain at tunnel inlet } \\
\text { nr Nevada City }\end{array}$ & $6 / 28 / 2000$ & $12: 30$ & 2 of 2 & 9.8 & 0.4 & $<0.01$ & 0.01 & 0.016 & 0.000 & $<0.02$ & 0.01 & $<2$ & 2 & 0.02 & 0.01 \\
\hline
\end{tabular}


Table 4B. Concentrations of trace metals and selected major elements in filtered water samples, Greenhorn Creek drainage, Nevada County, California—Continued.

[Station name abbreviations: ab, above; bl, below; Cr, creek; Cyn, Canyon; mi, miles; N, north; nr, near; Rd, Road; S, south; SF, South Fork. Number in parentheses is the data parameter code, a five-digit code used in the U.S. Geological Survey computerized data system. $\mu \mathrm{g} / \mathrm{L}$, microgram per liter (equivalent to part per billion); $\mathrm{mg} / \mathrm{L}$, milligram per liter (at low concentration, equivalent to part per million); s.d., standard deviation; <, less than; - , not determined]

\begin{tabular}{|c|c|c|c|c|c|c|c|c|c|c|c|c|c|c|}
\hline \multirow[t]{2}{*}{$\begin{array}{l}\text { Station } \\
\text { map ID }\end{array}$} & \multirow[t]{2}{*}{ Station name } & \multirow[t]{2}{*}{ Date } & \multirow[t]{2}{*}{ Time } & \multirow[t]{2}{*}{$\begin{array}{l}\text { Rep- } \\
\text { licate }\end{array}$} & \multicolumn{2}{|c|}{$\begin{array}{c}\text { Copper }(\mathrm{Cu}), \\
\text { filtered } \\
(\mu \mathrm{g} / \mathrm{L}) \\
(01040)\end{array}$} & \multicolumn{2}{|c|}{$\begin{array}{c}\text { Dysprosium (Dy), } \\
\text { filtered } \\
(\mu \mathrm{g} / \mathrm{L}) \\
(\mathbf{8 2 3 3 1}) \\
\end{array}$} & \multicolumn{2}{|c|}{$\begin{array}{c}\text { Erbium (Er), } \\
\text { filtered } \\
(\mu \mathrm{g} / \mathrm{L}) \\
(50573\end{array}$} & \multicolumn{2}{|c|}{$\begin{array}{c}\text { Europium (Eu), } \\
\text { filtered } \\
(\mu \mathrm{g} / \mathrm{L}) \\
(50574)\end{array}$} & \multicolumn{2}{|c|}{$\begin{array}{c}\text { Iron (Fe), } \\
\text { filtered } \\
(\mu \mathrm{g} / \mathrm{L}) \\
(01046)\end{array}$} \\
\hline & & & & & value & s.d. & value & s.d. & value & s.d. & value & s.d. & value & s.d. \\
\hline \multicolumn{15}{|c|}{ Environmental samples } \\
\hline BY20 & $\begin{array}{l}\text { Boston Mine tunnel } \\
\text { outlet nr Grass } \\
\text { Valley }\end{array}$ & $6 / 18 / 1999$ & $14: 30$ & 1 of 2 & 0.63 & 0.04 & 0.0032 & 0.0001 & 0.0023 & 0.0005 & $<0.0001$ & 0.0037 & 12 & 1 \\
\hline BY20 & $\begin{array}{l}\text { Boston Mine tunnel } \\
\text { outlet nr Grass } \\
\text { Valley }\end{array}$ & $6 / 18 / 1999$ & $14: 30$ & $2 \mathrm{of} 2$ & 0.58 & 0.03 & 0.0024 & 0.0003 & 0.0024 & 0.0003 & $<0.0001$ & 0.0031 & 9.0 & 1.3 \\
\hline BY20 & $\begin{array}{l}\text { Boston Mine tunnel } \\
\text { outlet nr Grass } \\
\text { Valley }\end{array}$ & $12 / 14 / 1999$ & $12: 00$ & 1 of 2 & 0.24 & 0.03 & 0.0009 & 0.0000 & 0.0007 & 0.0005 & $<0.0004$ & 0.0002 & 4 & 0 \\
\hline BY20 & $\begin{array}{l}\text { Boston Mine tunnel } \\
\text { outlet nr Grass } \\
\text { Valley }\end{array}$ & $12 / 14 / 1999$ & $12: 00$ & $2 \mathrm{of} 2$ & 0.23 & 0.00 & 0.0011 & 0.0002 & 0.0007 & 0.0010 & $<0.0004$ & 0.0003 & 3 & 1 \\
\hline BY20 & $\begin{array}{l}\text { Boston Mine tunnel } \\
\text { outlet nr Grass } \\
\text { Valley }\end{array}$ & $5 / 24 / 2000$ & $16: 00$ & 1 of 2 & 1.5 & 0.2 & 0.006 & 0.003 & $<0.004$ & 0.001 & $<0.001$ & 0.002 & 24 & 0 \\
\hline BY20 & $\begin{array}{l}\text { Boston Mine tunnel } \\
\text { outlet nr Grass } \\
\text { Valley }\end{array}$ & $5 / 24 / 2000$ & $16: 00$ & $2 \mathrm{of} 2$ & 1.5 & 0.1 & 0.004 & 0.001 & $<0.004$ & 0.001 & $<0.001$ & 0.001 & 23 & 0 \\
\hline BY21 & $\begin{array}{l}\text { Boston Mine wetlands } \\
\text { pond nr Grass Valley }\end{array}$ & $12 / 14 / 1999$ & $10: 30$ & $1 \mathrm{of} 2$ & 0.35 & 0.02 & 0.0014 & 0.0002 & 0.0009 & 0.0002 & 0.0007 & 0.0004 & 11 & 4 \\
\hline BY21 & $\begin{array}{l}\text { Boston Mine wetlands } \\
\text { pond nr Grass Valley }\end{array}$ & $12 / 14 / 1999$ & $10: 30$ & $2 \mathrm{of} 2$ & 0.34 & 0.01 & 0.0013 & 0.0001 & 0.0008 & 0.0001 & 0.0005 & 0.0003 & 8 & 0 \\
\hline BY21 & $\begin{array}{l}\text { Boston Mine wetlands } \\
\text { pond nr Grass Valley }\end{array}$ & $5 / 24 / 2000$ & $16: 30$ & 1 of 2 & 1.1 & 0.2 & 0.003 & 0.000 & $<0.004$ & 0.001 & $<0.001$ & 0.001 & 77 & 6 \\
\hline BY21 & $\begin{array}{l}\text { Boston Mine wetlands } \\
\text { pond nr Grass Valley }\end{array}$ & $5 / 24 / 2000$ & $16: 30$ & $2 \mathrm{of} 2$ & 0.57 & 0.03 & 0.004 & 0.001 & $<0.004$ & 0.001 & $<0.001$ & 0.001 & 59 & 3 \\
\hline BY58 & $\begin{array}{l}\text { Buckeye Flat Mine } \\
\text { main drain } 0.45 \mathrm{mi} \\
\text { ab Greenhorn } \mathrm{Cr}\end{array}$ & $8 / 21 / 2000$ & 11:00 & 1 of 2 & 2.9 & 0.2 & 2.7 & 0.1 & 1.5 & 0.0 & 0.63 & 0.00 & 1,900 & 100 \\
\hline BY58 & $\begin{array}{l}\text { Buckeye Flat Mine } \\
\text { main drain } 0.45 \mathrm{mi} \\
\text { ab Greenhorn } \mathrm{Cr}\end{array}$ & $8 / 21 / 2000$ & 11:00 & $2 \mathrm{of} 2$ & 3.0 & 0.2 & 2.7 & 0.1 & 1.5 & 0.0 & 0.65 & 0.03 & 2,100 & 100 \\
\hline BY23 & $\begin{array}{c}\text { Buckeye Flat Mine N } \\
\text { drain to Greenhorn } \\
\text { Cr, Grass Valley }\end{array}$ & $6 / 18 / 1999$ & $11: 30$ & 1 of 2 & 48 & 1 & 5.3 & 0.0 & 2.9 & 0.0 & 1.3 & 0.0 & 4,500 & 0 \\
\hline BY23 & $\begin{array}{c}\text { Buckeye Flat Mine N } \\
\text { drain to Greenhorn } \\
\text { Cr, Grass Valley }\end{array}$ & $6 / 18 / 1999$ & $11: 30$ & $2 \mathrm{of} 2$ & 48 & 0 & 5.5 & 0.1 & 3.0 & 0.1 & 1.3 & 0.0 & 4,500 & 0 \\
\hline
\end{tabular}


Table 4B. Concentrations of trace metals and selected major elements in filtered water samples, Greenhorn Creek drainage, Nevada County, California—Continued.

[Station name abbreviations: ab, above; bl, below; Cr, creek; Cyn, Canyon; mi, miles; N, north; nr, near; Rd, Road; S, south; SF, South Fork. Number in parentheses is the data parameter code, a five-digit code used in the U.S. Geological Survey computerized data system. $\mu \mathrm{g} / \mathrm{L}$, microgram per liter (equivalent to part per billion); mg/L, milligram per liter (at low concentration, equivalent to part per million); s.d., standard deviation; <, less than; - , not determined]

\begin{tabular}{|c|c|c|c|c|c|c|c|c|c|c|c|c|c|c|}
\hline \multirow[t]{2}{*}{$\begin{array}{l}\text { Station } \\
\text { map ID }\end{array}$} & \multirow[t]{2}{*}{ Station name } & \multirow[t]{2}{*}{ Date } & \multirow[t]{2}{*}{ Time } & \multirow[t]{2}{*}{$\begin{array}{l}\text { Rep- } \\
\text { licate }\end{array}$} & \multicolumn{2}{|c|}{$\begin{array}{c}\text { Copper (Cu), } \\
\text { filtered } \\
(\mu \mathrm{g} / \mathrm{L}) \\
(01040)\end{array}$} & \multicolumn{2}{|c|}{$\begin{array}{c}\text { Dysprosium (Dy), } \\
\text { filtered } \\
(\mu \mathrm{g} / \mathrm{L}) \\
(\mathbf{8 2 3 3 1}) \\
\end{array}$} & \multicolumn{2}{|c|}{$\begin{array}{c}\text { Erbium (Er), } \\
\text { filtered } \\
(\mu \mathrm{g} / \mathrm{L}) \\
(50573\end{array}$} & \multicolumn{2}{|c|}{$\begin{array}{c}\text { Europium (Eu), } \\
\text { filtered } \\
(\mu \mathrm{g} / \mathrm{L}) \\
(\mathbf{5 0 5 7 4 )} \\
\end{array}$} & \multicolumn{2}{|c|}{$\begin{array}{c}\text { Iron (Fe), } \\
\text { filtered } \\
(\mu \mathrm{g} / \mathrm{L}) \\
(01046)\end{array}$} \\
\hline & & & & & value & s.d. & value & s.d. & value & s.d. & value & s.d. & value & s.d. \\
\hline BY23 & $\begin{array}{c}\text { Buckeye Flat Mine N } \\
\text { drain to Greenhorn } \\
\text { Cr, Grass Valley }\end{array}$ & $12 / 2 / 1999$ & $11: 00$ & 1 of 2 & 43 & 1 & 3.7 & 0.0 & 2.0 & 0.0 & 0.92 & 0.01 & 150 & 10 \\
\hline BY23 & $\begin{array}{c}\text { Buckeye Flat Mine N } \\
\text { drain to Greenhorn } \\
\text { Cr, Grass Valley }\end{array}$ & $12 / 2 / 1999$ & $11: 00$ & $2 \mathrm{of} 2$ & 42 & 0 & 3.7 & 0.1 & 2.0 & 0.0 & 0.89 & 0.04 & 170 & 0 \\
\hline BY23 & $\begin{array}{c}\text { Buckeye Flat Mine N } \\
\text { drain to Greenhorn } \\
\text { Cr, Grass Valley }\end{array}$ & $8 / 22 / 2000$ & $10: 45$ & 1 of 2 & 28 & 1 & 4.9 & 0.0 & 2.7 & 0.0 & 1.2 & 0.0 & 140 & 10 \\
\hline BY23 & $\begin{array}{c}\text { Buckeye Flat Mine N } \\
\text { drain to Greenhorn } \\
\text { Cr, Grass Valley }\end{array}$ & $8 / 22 / 2000$ & $10: 45$ & $2 \mathrm{of} 2$ & 28 & 1 & 4.9 & 0.0 & 2.7 & 0.1 & 1.3 & 0.0 & 130 & 20 \\
\hline BY116 & $\begin{array}{l}\text { Buckeye Flat Mine } \\
\text { pond drain } 0.15 \mathrm{mi} \\
\text { ab SF Greenhorn } \mathrm{Cr}\end{array}$ & $8 / 21 / 2000$ & $14: 10$ & 1 of 2 & 14 & 0 & 0.009 & 0.003 & 0.005 & 0.002 & 0.005 & 0.003 & $<20$ & 10 \\
\hline BY116 & $\begin{array}{l}\text { Buckeye Flat Mine } \\
\text { pond drain } 0.15 \mathrm{mi} \\
\text { ab SF Greenhorn } \mathrm{Cr}\end{array}$ & $8 / 21 / 2000$ & $14: 10$ & $2 \mathrm{of} 2$ & 16 & 1 & 0.012 & 0.002 & 0.006 & 0.004 & 0.006 & 0.003 & $<20$ & 0 \\
\hline BY24 & $\begin{array}{l}\text { Buckeye Flat Mine } \\
\text { S drain to SF } \\
\text { Greenhorn Cr, Grass } \\
\text { Valley }\end{array}$ & $8 / 26 / 1999$ & 11:00 & 1 of 2 & 22 & 0 & 0.10 & 0.00 & 0.060 & 0.002 & 0.024 & 0.001 & 68 & 2 \\
\hline BY24 & $\begin{array}{l}\text { Buckeye Flat Mine } \\
\text { S drain to SF } \\
\text { Greenhorn Cr, Grass } \\
\text { Valley }\end{array}$ & $8 / 26 / 1999$ & 11:00 & $2 \mathrm{of} 2$ & 22 & 0 & 0.095 & 0.005 & 0.055 & 0.001 & 0.022 & 0.000 & 79 & 19 \\
\hline BY24 & $\begin{array}{l}\text { Buckeye Flat Mine } \\
\text { S drain to SF } \\
\text { Greenhorn Cr, Grass } \\
\text { Valley }\end{array}$ & $8 / 22 / 2000$ & $12: 10$ & 1 of 2 & 4.6 & 0.1 & 0.075 & 0.007 & 0.048 & 0.002 & 0.016 & 0.000 & $<20$ & 10 \\
\hline BY24 & $\begin{array}{l}\text { Buckeye Flat Mine } \\
\text { S drain to SF } \\
\text { Greenhorn Cr, Grass } \\
\text { Valley }\end{array}$ & $8 / 22 / 2000$ & $12: 10$ & $2 o f 2$ & 4.8 & 0.2 & 0.081 & 0.006 & 0.049 & 0.007 & 0.017 & 0.001 & $<20$ & 10 \\
\hline BY25 & $\begin{array}{l}\text { Buckeye Flat Mine } \\
\text { upper drain }\end{array}$ & 4/9/2001 & $10: 00$ & 1 of 2 & 34 & 5 & 0.82 & 0.16 & 0.48 & 0.02 & 0.18 & 0.03 & 41 & 3 \\
\hline BY25 & $\begin{array}{l}\text { Buckeye Flat Mine } \\
\text { upper drain }\end{array}$ & 4/9/2001 & $10: 00$ & $2 \mathrm{of} 2$ & 33 & 1 & 0.86 & 0.00 & 0.46 & 0.00 & 0.19 & 0.00 & 42 & 4 \\
\hline
\end{tabular}


Table 4B. Concentrations of trace metals and selected major elements in filtered water samples, Greenhorn Creek drainage, Nevada County, California—Continued.

[Station name abbreviations: ab, above; bl, below; Cr, creek; Cyn, Canyon; mi, miles; N, north; nr, near; Rd, Road; S, south; SF, South Fork. Number in parentheses is the data parameter code, a five-digit code used in the U.S. Geological Survey computerized data system. $\mu \mathrm{g} / \mathrm{L}$, microgram per liter (equivalent to part per billion); $\mathrm{mg} / \mathrm{L}$, milligram per liter (at low concentration, equivalent to part per million); s.d., standard deviation; <, less than; - , not determined]

\begin{tabular}{|c|c|c|c|c|c|c|c|c|c|c|c|c|c|c|}
\hline \multirow[t]{2}{*}{$\begin{array}{l}\text { Station } \\
\text { map ID }\end{array}$} & \multirow[t]{2}{*}{ Station name } & \multirow[t]{2}{*}{ Date } & \multirow[t]{2}{*}{ Time } & \multirow[t]{2}{*}{$\begin{array}{l}\text { Rep- } \\
\text { licate }\end{array}$} & \multicolumn{2}{|c|}{$\begin{array}{c}\text { Copper (Cu), } \\
\text { filtered } \\
(\mu \mathrm{g} / \mathrm{L}) \\
(01040)\end{array}$} & \multicolumn{2}{|c|}{$\begin{array}{c}\text { Dysprosium (Dy), } \\
\text { filtered } \\
(\mu \mathrm{g} / \mathrm{L}) \\
(\mathbf{8 2 3 3 1}) \\
\end{array}$} & \multicolumn{2}{|c|}{$\begin{array}{c}\text { Erbium (Er), } \\
\text { filtered } \\
(\mu \mathrm{g} / \mathrm{L}) \\
(50573\end{array}$} & \multicolumn{2}{|c|}{$\begin{array}{c}\text { Europium (Eu), } \\
\text { filtered } \\
(\mu \mathrm{g} / \mathrm{L}) \\
(\mathbf{5 0 5 7 4 )}\end{array}$} & \multicolumn{2}{|c|}{$\begin{array}{c}\text { Iron (Fe), } \\
\text { filtered } \\
(\mu \mathrm{g} / \mathrm{L}) \\
(01046) \\
\end{array}$} \\
\hline & & & & & value & s.d. & value & s.d. & value & s.d. & value & s.d. & value & s.d. \\
\hline BY52 & $\begin{array}{l}\text { Greenhorn Cr } 0.3 \mathrm{mi} \\
\text { ab Buckeye Ford nr } \\
\text { Nevada City }\end{array}$ & $1 / 25 / 2000$ & $10: 45$ & 1 of 2 & 1.1 & 0.1 & 0.076 & 0.006 & 0.046 & 0.004 & 0.020 & 0.003 & 27 & 2 \\
\hline BY52 & $\begin{array}{l}\text { Greenhorn Cr } 0.3 \mathrm{mi} \\
\text { ab Buckeye Ford nr } \\
\text { Nevada City }\end{array}$ & $1 / 25 / 2000$ & $10: 45$ & $2 \mathrm{of} 2$ & 0.92 & 0.04 & 0.075 & 0.004 & 0.046 & 0.003 & 0.019 & 0.001 & 28 & 1 \\
\hline BY59 & $\begin{array}{l}\text { Greenhorn Cr at You } \\
\text { Bet Rd nr Nevada } \\
\text { City }\end{array}$ & $7 / 24 / 2001$ & $11: 00$ & 1 of 2 & 0.30 & 0.06 & 0.0027 & 0.0001 & 0.0016 & 0.0003 & 0.0009 & 0.0002 & 18 & 0 \\
\hline BY59 & $\begin{array}{l}\text { Greenhorn Cr at You } \\
\text { Bet Rd nr Nevada } \\
\text { City }\end{array}$ & $7 / 24 / 2001$ & $11: 00$ & $2 \mathrm{of} 2$ & 5.6 & 7.4 & 0.0025 & 0.0003 & 0.0016 & 0.0003 & $<0.0002$ & 0.0003 & 18 & 0 \\
\hline BY59 & $\begin{array}{l}\text { Greenhorn Cr at You } \\
\text { Bet Rd nr Nevada } \\
\text { City }\end{array}$ & $8 / 29 / 2001$ & $15: 00$ & 1 of 2 & 0.31 & 0.06 & 0.0021 & 0.0001 & 0.0012 & 0.0006 & 0.0005 & 0.0003 & 29 & 0 \\
\hline BY59 & $\begin{array}{l}\text { Greenhorn } \mathrm{Cr} \text { at You } \\
\text { Bet Rd nr Nevada } \\
\text { City }\end{array}$ & $8 / 29 / 2001$ & $15: 00$ & $2 \mathrm{of} 2$ & 0.24 & 0.01 & 0.0018 & 0.0007 & 0.0015 & 0.0004 & 0.0005 & 0.0004 & 28 & 1 \\
\hline BY59 & $\begin{array}{l}\text { Greenhorn Cr at You } \\
\text { Bet Rd nr Nevada } \\
\text { City }\end{array}$ & $9 / 14 / 2001$ & $13: 00$ & 1 of 2 & 0.36 & 0.01 & 0.0020 & 0.0002 & 0.0012 & 0.0004 & $<0.0001$ & 0.0004 & 20 & 1 \\
\hline BY59 & $\begin{array}{l}\text { Greenhorn Cr at You } \\
\text { Bet Rd nr Nevada } \\
\text { City }\end{array}$ & $9 / 14 / 2001$ & $13: 00$ & $2 \mathrm{of} 2$ & 0.39 & 0.01 & 0.0022 & 0.0001 & 0.0010 & 0.0003 & $<0.0001$ & 0.0001 & 20 & 1 \\
\hline BY59 & $\begin{array}{l}\text { Greenhorn Cr at You } \\
\text { Bet Rd nr Nevada } \\
\text { City }\end{array}$ & $10 / 15 / 2001$ & $12: 00$ & 1 of 2 & 0.11 & 0.03 & 0.0012 & 0.0003 & 0.0004 & 0.0001 & $<0.0001$ & 0.0004 & 13 & 1 \\
\hline BY59 & $\begin{array}{l}\text { Greenhorn Cr at You } \\
\text { Bet Rd nr Nevada } \\
\text { City }\end{array}$ & $10 / 15 / 2001$ & $12: 00$ & $2 \mathrm{of} 2$ & 0.08 & 0.00 & 0.0017 & 0.0003 & 0.0008 & 0.0003 & $<0.0001$ & 0.0003 & 14 & 1 \\
\hline BY59 & $\begin{array}{l}\text { Greenhorn } \mathrm{Cr} \text { at You } \\
\text { Bet Rd nr Nevada } \\
\text { City }\end{array}$ & $11 / 20 / 2001$ & $13: 20$ & 1 of 2 & 0.37 & 0.03 & 0.011 & 0.000 & 0.0068 & 0.0004 & 0.0063 & 0.0006 & 31 & 1 \\
\hline BY59 & $\begin{array}{l}\text { Greenhorn } \mathrm{Cr} \text { at You } \\
\text { Bet Rd nr Nevada } \\
\text { City }\end{array}$ & $11 / 20 / 2001$ & $13: 20$ & $2 \mathrm{of} 2$ & 0.36 & 0.00 & 0.012 & 0.000 & 0.0071 & 0.0005 & 0.0076 & 0.0002 & 34 & 1 \\
\hline BY59 & $\begin{array}{l}\text { Greenhorn Cr at You } \\
\text { Bet Rd nr Nevada } \\
\text { City }\end{array}$ & $12 / 10 / 2001$ & $12: 20$ & 1 of 2 & 0.33 & 0.02 & 0.033 & 0.002 & 0.020 & 0.001 & 0.0090 & 0.0004 & 22 & 1 \\
\hline
\end{tabular}


Table 4B. Concentrations of trace metals and selected major elements in filtered water samples, Greenhorn Creek drainage, Nevada County, California—Continued.

[Station name abbreviations: ab, above; bl, below; Cr, creek; Cyn, Canyon; mi, miles; N, north; nr, near; Rd, Road; S, south; SF, South Fork. Number in parentheses is the data parameter code, a five-digit code used in the U.S. Geological Survey computerized data system. $\mu \mathrm{g} / \mathrm{L}$, microgram per liter (equivalent to part per billion); $\mathrm{mg} / \mathrm{L}$, milligram per liter (at low concentration, equivalent to part per million); s.d., standard deviation; <, less than; - , not determined]

\begin{tabular}{|c|c|c|c|c|c|c|c|c|c|c|c|c|c|c|}
\hline \multirow[t]{2}{*}{$\begin{array}{l}\text { Station } \\
\text { map ID }\end{array}$} & \multirow[t]{2}{*}{ Station name } & \multirow[t]{2}{*}{ Date } & \multirow[t]{2}{*}{ Time } & \multirow[t]{2}{*}{$\begin{array}{l}\text { Rep- } \\
\text { licate }\end{array}$} & \multicolumn{2}{|c|}{$\begin{array}{c}\text { Copper (Cu), } \\
\text { filtered } \\
(\mu \mathrm{g} / \mathrm{L}) \\
(01040)\end{array}$} & \multicolumn{2}{|c|}{$\begin{array}{c}\text { Dysprosium (Dy), } \\
\text { filtered } \\
(\mu \mathrm{g} / \mathrm{L}) \\
(\mathbf{8 2 3 3 1}) \\
\end{array}$} & \multicolumn{2}{|c|}{$\begin{array}{c}\text { Erbium (Er), } \\
\text { filtered } \\
(\mu \mathrm{g} / \mathrm{L}) \\
(50573\end{array}$} & \multicolumn{2}{|c|}{$\begin{array}{c}\text { Europium (Eu), } \\
\text { filtered } \\
(\mu \mathrm{g} / \mathrm{L}) \\
(\mathbf{5 0 5 7 4 )}\end{array}$} & \multicolumn{2}{|c|}{$\begin{array}{c}\text { Iron (Fe), } \\
\text { filtered } \\
(\mu \mathrm{g} / \mathrm{L}) \\
(01046)\end{array}$} \\
\hline & & & & & value & s.d. & value & s.d. & value & s.d. & value & s.d. & value & s.d. \\
\hline BY59 & $\begin{array}{l}\text { Greenhorn Cr at You } \\
\text { Bet Rd nr Nevada } \\
\text { City }\end{array}$ & $12 / 10 / 2001$ & $12: 20$ & 2 of 2 & 0.34 & 0.03 & 0.029 & 0.000 & 0.021 & 0.001 & 0.0098 & 0.0003 & 23 & 0 \\
\hline BY59 & $\begin{array}{l}\text { Greenhorn Cr at You } \\
\text { Bet Rd nr Nevada } \\
\text { City }\end{array}$ & $12 / 21 / 2001$ & $13: 00$ & 1 of 2 & 0.66 & 0.04 & 0.070 & 0.002 & 0.043 & 0.000 & 0.021 & 0.001 & 18 & 0 \\
\hline BY59 & $\begin{array}{l}\text { Greenhorn } \mathrm{Cr} \text { at You } \\
\text { Bet Rd nr Nevada } \\
\text { City }\end{array}$ & $12 / 21 / 2001$ & $13: 00$ & $2 \mathrm{of} 2$ & 0.67 & 0.01 & 0.070 & 0.001 & 0.044 & 0.003 & 0.022 & 0.000 & 15 & 0 \\
\hline BY51 & $\begin{array}{l}\text { Greenhorn Cr nr } \\
\text { Headwaters nr Scotts } \\
\text { Flat Reservoir }\end{array}$ & $1 / 25 / 2000$ & $9: 30$ & 1 of 2 & 5.1 & 0.1 & 0.043 & 0.001 & 0.031 & 0.007 & 0.012 & 0.002 & 14 & 1 \\
\hline BY51 & $\begin{array}{l}\text { Greenhorn Cr nr } \\
\text { Headwaters nr Scotts } \\
\text { Flat Reservoir }\end{array}$ & $1 / 25 / 2000$ & $9: 30$ & $2 \mathrm{of} 2$ & 4.2 & 0.3 & 0.040 & 0.006 & 0.030 & 0.000 & 0.013 & 0.001 & 15 & 2 \\
\hline BY75 & $\begin{array}{l}\text { Missouri Cyn Cr } 1.2 \mathrm{mi} \\
\text { ab Greenhorn Cr nr } \\
\text { Chicago Park }\end{array}$ & $12 / 14 / 1999$ & $14: 00$ & 1 of 2 & 0.88 & 0.04 & 0.059 & 0.001 & 0.038 & 0.000 & 0.013 & 0.000 & 49 & 7 \\
\hline BY75 & $\begin{array}{l}\text { Missouri Cyn Cr } 1.2 \mathrm{mi} \\
\text { ab Greenhorn Cr nr } \\
\text { Chicago Park }\end{array}$ & $12 / 14 / 1999$ & $14: 00$ & $2 \mathrm{of} 2$ & 0.92 & 0.04 & 0.061 & 0.004 & 0.037 & 0.002 & 0.013 & 0.000 & 59 & 3 \\
\hline BY86 & $\begin{array}{l}\text { Poore Mine creek ab } \\
\text { tunnel nr Grass } \\
\text { Valley }\end{array}$ & 3/31/1999 & $17: 40$ & 1 of 2 & 0.64 & 0.01 & 0.013 & 0.001 & 0.008 & 0.002 & 0.0048 & 0.0010 & 11 & 0 \\
\hline BY86 & $\begin{array}{l}\text { Poore Mine creek ab } \\
\text { tunnel nr Grass } \\
\text { Valley }\end{array}$ & $3 / 31 / 1999$ & $17: 40$ & $2 \mathrm{of} 2$ & 0.47 & 0.00 & 0.012 & 0.001 & 0.007 & 0.001 & 0.0049 & 0.0022 & 9.3 & 1.0 \\
\hline BY87 & $\begin{array}{l}\text { Poore Mine creek bl } \\
\text { tunnel nr Grass } \\
\text { Valley }\end{array}$ & 3/31/1999 & $13: 30$ & 1 of 2 & 0.48 & 0.02 & 0.014 & 0.001 & 0.009 & 0.000 & 0.0058 & 0.0002 & 6.7 & 0.6 \\
\hline BY87 & $\begin{array}{l}\text { Poore Mine creek bl } \\
\text { tunnel nr Grass } \\
\text { Valley }\end{array}$ & 3/31/1999 & $13: 30$ & $2 \mathrm{of} 2$ & 0.58 & 0.02 & 0.014 & 0.003 & 0.010 & 0.006 & 0.0059 & 0.0008 & 5.2 & 0.5 \\
\hline BY88 & $\begin{array}{l}\text { Poore Mine ground } \\
\text { sluice nr Grass } \\
\text { Valley }\end{array}$ & 4/1/1999 & $15: 40$ & 1 of 2 & 0.82 & 0.08 & 0.030 & 0.002 & 0.019 & 0.000 & 0.0086 & 0.0003 & 570 & 10 \\
\hline BY88 & $\begin{array}{l}\text { Poore Mine ground } \\
\text { sluice nr Grass } \\
\text { Valley }\end{array}$ & 4/1/1999 & $15: 40$ & $2 \mathrm{of} 2$ & 0.74 & 0.07 & 0.028 & 0.002 & 0.019 & 0.002 & 0.0078 & 0.0004 & 580 & 20 \\
\hline
\end{tabular}


Table 4B. Concentrations of trace metals and selected major elements in filtered water samples, Greenhorn Creek drainage, Nevada County, California—Continued.

[Station name abbreviations: ab, above; bl, below; Cr, creek; Cyn, Canyon; mi, miles; N, north; nr, near; Rd, Road; S, south; SF, South Fork. Number in parentheses is the data parameter code, a five-digit code used in the U.S. Geological Survey computerized data system. $\mu \mathrm{g} / \mathrm{L}$, microgram per liter (equivalent to part per billion); mg/L, milligram per liter (at low concentration, equivalent to part per million); s.d., standard deviation; <, less than; - , not determined]

\begin{tabular}{|c|c|c|c|c|c|c|c|c|c|c|c|c|c|c|}
\hline \multirow[t]{2}{*}{$\begin{array}{l}\text { Station } \\
\text { map ID }\end{array}$} & \multirow[t]{2}{*}{ Station name } & \multirow[t]{2}{*}{ Date } & \multirow[t]{2}{*}{ Time } & \multirow[t]{2}{*}{$\begin{array}{l}\text { Rep- } \\
\text { licate }\end{array}$} & \multicolumn{2}{|c|}{$\begin{array}{l}\text { Copper (Cu), } \\
\text { filtered } \\
(\mu \mathrm{g} / \mathrm{L}) \\
(01040)\end{array}$} & \multicolumn{2}{|c|}{$\begin{array}{l}\text { Dysprosium (Dy), } \\
\text { filtered } \\
(\mu \mathrm{g} / \mathrm{L}) \\
(\mathbf{8 2 3 3 1 )}\end{array}$} & \multicolumn{2}{|c|}{$\begin{array}{c}\text { Erbium (Er), } \\
\text { filtered } \\
(\mu \mathrm{g} / \mathrm{L}) \\
(50573 \\
\end{array}$} & \multicolumn{2}{|c|}{$\begin{array}{l}\text { Europium (Eu), } \\
\text { filtered } \\
(\mu \mathrm{g} / \mathrm{L}) \\
(50574) \\
\end{array}$} & \multicolumn{2}{|c|}{$\begin{array}{c}\text { Iron (Fe), } \\
\text { filtered } \\
(\mu \mathrm{g} / \mathrm{L}) \\
(01046)\end{array}$} \\
\hline & & & & & value & s.d. & value & s.d. & value & s.d. & value & s.d. & value & s.d. \\
\hline BY89 & $\begin{array}{c}\text { Poore Mine pit lake } \\
\text { nr Grass Valley }\end{array}$ & $4 / 1 / 1999$ & $16: 50$ & 1 of 2 & 1.0 & 0.1 & 0.0071 & 0.0005 & 0.0047 & 0.0008 & 0.0011 & 0.0025 & 90 & 5 \\
\hline BY89 & $\begin{array}{l}\text { Poore Mine pit lake } \\
\text { nr Grass Valley }\end{array}$ & 4/1/1999 & $16: 50$ & $2 \mathrm{of} 2$ & 0.87 & 0.05 & 0.0047 & 0.0021 & 0.0038 & 0.0009 & 0.0011 & 0.0041 & 78 & 2 \\
\hline BY89 & $\begin{array}{l}\text { Poore Mine pit lake } \\
\text { nr Grass Valley }\end{array}$ & $5 / 23 / 2000$ & $10: 45$ & 1 of 2 & 11 & 0 & 0.039 & 0.005 & 0.021 & 0.002 & $<0.001$ & 0.001 & 33 & 2 \\
\hline BY89 & $\begin{array}{l}\text { Poore Mine pit lake } \\
\text { nr Grass Valley }\end{array}$ & $5 / 23 / 2000$ & $10: 45$ & $2 \mathrm{of} 2$ & 12 & 0 & 0.039 & 0.001 & 0.019 & 0.001 & $<0.001$ & 0.002 & 32 & 3 \\
\hline BY90 & $\begin{array}{l}\text { Poore Mine seep } \\
\text { ab ground sluice } \\
\text { nr Grass Valley }\end{array}$ & 4/1/1999 & $16: 00$ & 1 of 2 & 0.50 & 0.04 & 0.011 & 0.003 & 0.0042 & 0.0046 & 0.0015 & 0.0015 & 330 & 10 \\
\hline BY90 & $\begin{array}{l}\text { Poore Mine seep } \\
\text { ab ground sluice } \\
\text { nr Grass Valley }\end{array}$ & 4/1/1999 & $16: 00$ & $2 \mathrm{of} 2$ & 0.44 & 0.08 & 0.0085 & 0.0006 & 0.0070 & 0.0008 & 0.0016 & 0.0022 & 330 & 0 \\
\hline BY91 & $\begin{array}{l}\text { Poore Mine tunnel } \\
\text { effluent nr Grass } \\
\text { Valley }\end{array}$ & 3/31/1999 & $15: 20$ & 1 of 2 & 0.50 & 0.01 & 0.0025 & 0.0005 & 0.001 & 0.000 & 0.0011 & 0.0018 & 2.1 & 0.2 \\
\hline BY91 & $\begin{array}{l}\text { Poore Mine tunnel } \\
\text { effluent nr Grass } \\
\text { Valley }\end{array}$ & 3/31/1999 & $15: 20$ & $2 \mathrm{of} 2$ & 0.45 & 0.01 & 0.0035 & 0.0015 & 0.0028 & 0.0015 & 0.0038 & 0.0048 & 4 & 1 \\
\hline BY91 & $\begin{array}{l}\text { Poore Mine tunnel } \\
\text { effluent nr Grass } \\
\text { Valley }\end{array}$ & $12 / 15 / 1999$ & 13:00 & 1 of 2 & 0.23 & 0.09 & 0.0011 & 0.0003 & 0.0005 & 0.0006 & $<0.0004$ & 0.0000 & 26 & 27 \\
\hline BY91 & $\begin{array}{l}\text { Poore Mine tunnel } \\
\text { effluent nr Grass } \\
\text { Valley }\end{array}$ & $12 / 15 / 1999$ & $13: 00$ & $2 \mathrm{of} 2$ & 0.25 & 0.07 & 0.0011 & 0.0001 & 0.0006 & 0.0001 & $<0.0004$ & 0.0005 & 4 & 4 \\
\hline BY91 & $\begin{array}{l}\text { Poore Mine tunnel } \\
\text { effluent nr Grass } \\
\text { Valley }\end{array}$ & $5 / 23 / 2000$ & $12: 00$ & 1 of 2 & 0.51 & 0.12 & $<0.002$ & 0.000 & $<0.004$ & 0.000 & $<0.001$ & 0.001 & 1.5 & 0.0 \\
\hline BY91 & $\begin{array}{l}\text { Poore Mine tunnel } \\
\text { effluent nr Grass } \\
\text { Valley }\end{array}$ & $5 / 23 / 2000$ & $12: 00$ & $2 \mathrm{of} 2$ & 0.71 & 0.08 & $<0.003$ & 0.002 & $<0.002$ & 0.002 & $<0.001$ & 0.001 & 1.9 & 0.4 \\
\hline BY105 & $\begin{array}{l}\text { Sailor Flat Mine main } \\
\text { drain Gulch 01nr } \\
\text { Quaker Hill }\end{array}$ & 7/6/1999 & $16: 00$ & 1 of 2 & 2.8 & 0.0 & 0.18 & 0.00 & 0.12 & 0.00 & 0.052 & 0.009 & 8.5 & 0.4 \\
\hline BY105 & $\begin{array}{l}\text { Sailor Flat Mine main } \\
\text { drain Gulch 01nr } \\
\text { Quaker Hill }\end{array}$ & 7/6/1999 & $16: 00$ & $2 \mathrm{of} 2$ & 2.7 & 0.1 & 0.17 & 0.01 & 0.11 & 0.01 & 0.061 & 0.002 & 8 & 0 \\
\hline
\end{tabular}


Table 4B. Concentrations of trace metals and selected major elements in filtered water samples, Greenhorn Creek drainage, Nevada County, California—Continued.

[Station name abbreviations: ab, above; bl, below; Cr, creek; Cyn, Canyon; mi, miles; N, north; nr, near; Rd, Road; S, south; SF, South Fork. Number in parentheses is the data parameter code, a five-digit code used in the U.S. Geological Survey computerized data system. $\mu \mathrm{g} / \mathrm{L}$, microgram per liter (equivalent to part per billion); $\mathrm{mg} / \mathrm{L}$, milligram per liter (at low concentration, equivalent to part per million); s.d., standard deviation; <, less than; - , not determined]

\begin{tabular}{|c|c|c|c|c|c|c|c|c|c|c|c|c|c|c|}
\hline \multirow[t]{2}{*}{$\begin{array}{l}\text { Station } \\
\text { map ID }\end{array}$} & \multirow[t]{2}{*}{ Station name } & \multirow[t]{2}{*}{ Date } & \multirow[t]{2}{*}{ Time } & \multirow[t]{2}{*}{$\begin{array}{l}\text { Rep- } \\
\text { licate }\end{array}$} & \multicolumn{2}{|c|}{$\begin{array}{c}\text { Copper (Cu), } \\
\text { filtered } \\
(\mu \mathrm{g} / \mathrm{L}) \\
(01040)\end{array}$} & \multicolumn{2}{|c|}{$\begin{array}{c}\text { Dysprosium (Dy), } \\
\text { filtered } \\
(\mu \mathrm{g} / \mathrm{L}) \\
(82331) \\
\end{array}$} & \multicolumn{2}{|c|}{$\begin{array}{c}\text { Erbium (Er), } \\
\text { filtered } \\
(\mu \mathrm{g} / \mathrm{L}) \\
(50573 \\
\end{array}$} & \multicolumn{2}{|c|}{$\begin{array}{c}\text { Europium (Eu), } \\
\text { filtered } \\
(\mu \mathrm{g} / \mathrm{L}) \\
(\mathbf{5 0 5 7 4 )}\end{array}$} & \multicolumn{2}{|c|}{$\begin{array}{c}\text { Iron (Fe), } \\
\text { filtered } \\
(\mu \mathrm{g} / \mathrm{L}) \\
(01046)\end{array}$} \\
\hline & & & & & value & s.d. & value & s.d. & value & s.d. & value & s.d. & value & s.d. \\
\hline BY105 & $\begin{array}{l}\text { Sailor Flat Mine main } \\
\text { drain Gulch 01nr } \\
\text { Quaker Hill }\end{array}$ & $12 / 2 / 1999$ & $14: 30$ & 1 of 2 & 4.8 & 0.2 & 0.29 & 0.01 & 0.17 & 0.01 & 0.098 & 0.000 & 58 & 1 \\
\hline BY105 & $\begin{array}{l}\text { Sailor Flat Mine main } \\
\text { drain Gulch 01nr } \\
\text { Quaker Hill }\end{array}$ & $12 / 2 / 1999$ & $14: 30$ & $2 \mathrm{of} 2$ & 4.6 & 0.1 & 0.30 & 0.00 & 0.18 & 0.00 & 0.099 & 0.001 & 56 & 6 \\
\hline BY106 & $\begin{array}{l}\text { Sailor Flat Mine } \\
\text { main drainage to } \\
\text { Greenhorn Cr, } \\
\text { Gulch } 03\end{array}$ & 4/9/2001 & $12: 30$ & 1 of 2 & 2.8 & 0.3 & 0.22 & 0.04 & 0.13 & 0.02 & 0.058 & 0.010 & 67 & 2 \\
\hline BY106 & $\begin{array}{l}\text { Sailor Flat Mine } \\
\text { main drainage to } \\
\text { Greenhorn Cr, } \\
\text { Gulch } 03\end{array}$ & 4/9/2001 & $12: 30$ & 2 of 2 & 3.0 & 0.4 & 0.22 & 0.03 & 0.13 & 0.04 & 0.062 & 0.016 & 72 & 4 \\
\hline BY114 & $\begin{array}{l}\text { SF Greenhorn Cr } \\
0.7 \text { mi ab Greenhorn } \\
\text { Cr nr Nevada City }\end{array}$ & $8 / 22 / 2000$ & $15: 10$ & 1 of 2 & $<0.4$ & 0.0 & $<0.005$ & 0.002 & $<0.005$ & 0.004 & $<0.003$ & 0.001 & $<20$ & 0 \\
\hline BY114 & $\begin{array}{l}\text { SF Greenhorn Cr } \\
0.7 \text { mi ab Greenhorn } \\
\text { Cr nr Nevada City }\end{array}$ & $8 / 22 / 2000$ & $15: 10$ & $2 \mathrm{of} 2$ & $<0.4$ & 0.1 & $<0.005$ & 0.001 & $<0.005$ & 0.003 & $<0.003$ & 0.002 & $<20$ & 0 \\
\hline BY122 & $\begin{array}{l}\text { Starr Mine tunnel } \\
\text { inflow nr Grass } \\
\text { Valley }\end{array}$ & 4/23/1999 & $15: 00$ & 1 of 2 & 3.0 & 0.5 & 0.021 & 0.002 & 0.012 & 0.001 & 0.0043 & 0.0022 & 9 & 1 \\
\hline BY122 & $\begin{array}{l}\text { Starr Mine tunnel } \\
\text { inflow nr Grass } \\
\text { Valley }\end{array}$ & 4/23/1999 & $15: 00$ & $2 \mathrm{of} 2$ & 3.3 & 0.0 & 0.020 & 0.001 & 0.012 & 0.002 & 0.0050 & 0.0036 & 9 & 1 \\
\hline BY123 & $\begin{array}{l}\text { Starr Mine tunnel } \\
\text { midway nr Grass } \\
\text { Valley }\end{array}$ & 4/23/1999 & $13: 40$ & 1 of 2 & 0.25 & 0.09 & $<0.0005$ & 0.0004 & 0.0014 & 0.0019 & 0.0042 & 0.0039 & 3.0 & 0.1 \\
\hline BY123 & $\begin{array}{l}\text { Starr Mine tunnel } \\
\text { midway nr Grass } \\
\text { Valley }\end{array}$ & 4/23/1999 & $13: 40$ & $2 \mathrm{of} 2$ & 0.30 & 0.04 & $<0.0005$ & 0.0001 & $<0.0006$ & 0.0002 & 0.0036 & 0.0017 & 4.6 & 1.8 \\
\hline BY123 & $\begin{array}{l}\text { Starr Mine tunnel } \\
\text { midway nr Grass } \\
\text { Valley }\end{array}$ & $12 / 15 / 1999$ & $10: 45$ & 1 of 2 & 0.22 & 0.03 & 0.0010 & 0.0003 & 0.0004 & 0.0005 & $<0.0004$ & 0.0003 & $<3$ & 1 \\
\hline BY123 & $\begin{array}{l}\text { Starr Mine tunnel } \\
\text { midway nr Grass } \\
\text { Valley }\end{array}$ & $12 / 15 / 1999$ & $10: 45$ & $2 \mathrm{of} 2$ & 0.26 & 0.02 & 0.0011 & 0.0003 & 0.0006 & 0.0000 & $<0.0004$ & 0.0001 & $<3$ & 1 \\
\hline
\end{tabular}


Table 4B. Concentrations of trace metals and selected major elements in filtered water samples, Greenhorn Creek drainage, Nevada County, California—Continued.

[Station name abbreviations: ab, above; bl, below; Cr, creek; Cyn, Canyon; mi, miles; N, north; nr, near; Rd, Road; S, south; SF, South Fork. Number in parentheses is the data parameter code, a five-digit code used in the U.S. Geological Survey computerized data system. $\mu \mathrm{g} / \mathrm{L}$, microgram per liter (equivalent to part per billion); $\mathrm{mg} / \mathrm{L}$, milligram per liter (at low concentration, equivalent to part per million); s.d., standard deviation; <, less than; - , not determined]

\begin{tabular}{|c|c|c|c|c|c|c|c|c|c|c|c|c|c|c|}
\hline \multirow[t]{2}{*}{$\begin{array}{l}\text { Station } \\
\text { map ID }\end{array}$} & \multirow[t]{2}{*}{ Station name } & \multirow[t]{2}{*}{ Date } & \multirow[t]{2}{*}{ Time } & \multirow[t]{2}{*}{$\begin{array}{l}\text { Rep- } \\
\text { licate }\end{array}$} & \multicolumn{2}{|c|}{$\begin{array}{c}\text { Copper (Cu), } \\
\text { filtered } \\
(\mu \mathrm{g} / \mathrm{L}) \\
(01040)\end{array}$} & \multicolumn{2}{|c|}{$\begin{array}{c}\text { Dysprosium (Dy), } \\
\text { filtered } \\
(\mu \mathrm{g} / \mathrm{L}) \\
(\mathbf{8 2 3 3 1}) \\
\end{array}$} & \multicolumn{2}{|c|}{$\begin{array}{l}\text { Erbium (Er), } \\
\text { filtered } \\
(\mu \mathrm{g} / \mathrm{L}) \\
(50573 \\
\end{array}$} & \multicolumn{2}{|c|}{$\begin{array}{c}\text { Europium (Eu), } \\
\text { filtered } \\
(\mu \mathrm{g} / \mathrm{L}) \\
(50574)\end{array}$} & \multicolumn{2}{|c|}{$\begin{array}{c}\text { Iron }(\mathrm{Fe}), \\
\text { filtered } \\
(\mu \mathrm{g} / \mathrm{L}) \\
(01046)\end{array}$} \\
\hline & & & & & value & s.d. & value & s.d. & value & s.d. & value & s.d. & value & s.d. \\
\hline BY124 & $\begin{array}{l}\text { Starr Mine tunnel outlet } \\
\text { nr Grass Valley }\end{array}$ & $8 / 23 / 2000$ & $17: 30$ & 1 of 2 & $<0.4$ & 0.1 & $<0.005$ & 0.001 & $<0.005$ & 0.001 & $<0.003$ & 0.002 & $<20$ & 10 \\
\hline BY124 & $\begin{array}{l}\text { Starr Mine tunnel outlet } \\
\text { nr Grass Valley }\end{array}$ & $8 / 23 / 2000$ & $17: 30$ & $2 \mathrm{of} 2$ & 0.4 & 0.1 & $<0.005$ & 0.004 & $<0.005$ & 0.004 & $<0.003$ & 0.001 & $<20$ & 20 \\
\hline BY129 & $\begin{array}{l}\text { Tom and Jerry Mine } \\
\text { drain at tunnel inlet } \\
\text { nr Nevada City }\end{array}$ & $6 / 28 / 2000$ & $12: 00$ & 1 of 2 & 0.8 & 0.2 & 0.010 & 0.004 & 0.004 & 0.003 & $<0.002$ & 0.001 & $<7$ & 0 \\
\hline BY129 & $\begin{array}{l}\text { Tom and Jerry Mine } \\
\text { drain at tunnel inlet } \\
\text { nr Nevada City }\end{array}$ & $6 / 28 / 2000$ & $12: 00$ & $2 \mathrm{of} 2$ & 0.8 & 0.2 & 0.008 & 0.001 & 0.006 & 0.002 & $<0.002$ & 0.001 & $<7$ & 2 \\
\hline BY130 & $\begin{array}{l}\text { Tom and Jerry Mine } \\
\text { drain at tunnel outlet } \\
\text { nr Nevada City }\end{array}$ & $6 / 28 / 2000$ & $11: 30$ & 1 of 2 & $<0.2$ & 0.1 & $<0.003$ & 0.002 & $<0.003$ & 0.000 & $<0.002$ & 0.002 & 66 & 17 \\
\hline BY130 & $\begin{array}{l}\text { Tom and Jerry Mine } \\
\text { drain at tunnel outlet } \\
\text { nr Nevada City }\end{array}$ & $6 / 28 / 2000$ & $11: 30$ & $2 \mathrm{of} 2$ & 0.3 & 0.1 & $<0.003$ & 0.002 & 0.004 & 0.004 & $<0.002$ & 0.001 & 75 & 9 \\
\hline BY131 & $\begin{array}{l}\text { Tom and Jerry Mine } \\
\text { drainage pond nr } \\
\text { Nevada City }\end{array}$ & $8 / 23 / 2000$ & $10: 45$ & 1 of 2 & 2.0 & 0.0 & 0.007 & 0.000 & 0.005 & 0.002 & $<0.003$ & 0.001 & $<20$ & 10 \\
\hline BY131 & $\begin{array}{l}\text { Tom and Jerry Mine } \\
\text { drainage pond nr } \\
\text { Nevada City }\end{array}$ & $8 / 23 / 2000$ & $10: 45$ & $2 \mathrm{of} 2$ & 1.9 & 0.0 & 0.009 & 0.001 & 0.006 & 0.004 & 0.004 & 0.001 & $<20$ & 10 \\
\hline \multicolumn{15}{|c|}{ Disturbed samples } \\
\hline BY123 & $\begin{array}{l}\text { Starr Mine tunnel } \\
\text { midway nr Grass } \\
\text { Valley }\end{array}$ & $12 / 15 / 1999$ & $11: 30$ & 1 of 2 & 0.20 & 0.03 & 0.0006 & 0.0004 & 0.0005 & 0.0001 & $<0.0004$ & 0.0003 & 7 & 2 \\
\hline BY123 & $\begin{array}{l}\text { Starr Mine tunnel } \\
\text { midway nr Grass } \\
\text { Valley }\end{array}$ & $12 / 15 / 1999$ & $11: 30$ & $2 \mathrm{of} 2$ & 0.18 & 0.02 & $<0.0005$ & 0.0006 & 0.0009 & 0.0001 & 0.0005 & 0.0001 & 7 & 1 \\
\hline BY129 & $\begin{array}{l}\text { Tom and Jerry Mine } \\
\text { drain at tunnel inlet } \\
\text { nr Nevada City }\end{array}$ & $6 / 28 / 2000$ & $12: 30$ & 1 of 2 & 0.6 & 0.2 & 0.006 & 0.001 & 0.004 & 0.004 & $<0.002$ & 0.001 & $<7$ & 2 \\
\hline BY129 & $\begin{array}{l}\text { Tom and Jerry Mine } \\
\text { drain at tunnel inlet } \\
\text { nr Nevada City }\end{array}$ & $6 / 28 / 2000$ & $12: 30$ & $2 \mathrm{of} 2$ & 0.5 & 0.1 & 0.010 & 0.000 & 0.004 & 0.003 & 0.002 & 0.000 & 12 & 4 \\
\hline
\end{tabular}


Table 4B. Concentrations of trace metals and selected major elements in filtered water samples, Greenhorn Creek drainage, Nevada County, California—Continued.

[Station name abbreviations: ab, above; bl, below; Cr, creek; Cyn, Canyon; mi, miles; N, north; nr, near; Rd, Road; S, south; SF, South Fork. Number in parentheses is the data parameter code, a five-digit code used in the U.S. Geological Survey computerized data system. $\mu \mathrm{g} / \mathrm{L}$, microgram per liter (equivalent to part per billion); $\mathrm{mg} / \mathrm{L}$, milligram per liter (at low concentration, equivalen to part per million); s.d., standard deviation; <, less than; - , not determined]

\begin{tabular}{|c|c|c|c|c|c|c|c|c|c|c|c|c|c|c|}
\hline \multirow[t]{2}{*}{$\begin{array}{l}\text { Station } \\
\text { map ID }\end{array}$} & \multirow[t]{2}{*}{ Station name } & \multirow[t]{2}{*}{ Date } & \multirow[t]{2}{*}{ Time } & \multirow[t]{2}{*}{$\begin{array}{l}\text { Rep- } \\
\text { licate }\end{array}$} & \multicolumn{2}{|c|}{$\begin{array}{c}\text { Gadolinium (Gd), } \\
\text { filtered } \\
(\mu \mathrm{g} / \mathrm{L}) \\
(\mathbf{5 0 5 7 5 )} \\
\end{array}$} & \multicolumn{2}{|c|}{$\begin{array}{c}\text { Holmium (Ho), } \\
\text { filtered } \\
(\mu \mathrm{g} / \mathrm{L}) \\
(\mathbf{5 0 5 7 7 )} \\
\end{array}$} & \multicolumn{2}{|c|}{$\begin{array}{c}\text { Potassium (K), } \\
\text { filtered } \\
(\mathrm{mg} / \mathrm{L}) \\
(00935) \\
\end{array}$} & \multicolumn{2}{|c|}{$\begin{array}{c}\text { Lanthanum (La), } \\
\text { filtered } \\
(\mu \mathrm{g} / \mathrm{L}) \\
(\mathbf{0 1 1 8 0 )} \\
\end{array}$} & \multicolumn{2}{|c|}{$\begin{array}{c}\text { Lithium (Li), } \\
\text { filtered } \\
(\mu \mathrm{g} / \mathrm{L}) \\
(01130)\end{array}$} \\
\hline & & & & & value & s.d. & value & s.d. & value & s.d. & value & s.d. & value & s.d. \\
\hline BY20 & $\begin{array}{l}\text { Boston Mine tunnel } \\
\text { outlet nr Grass } \\
\text { Valley }\end{array}$ & $6 / 18 / 1999$ & $14: 30$ & 1 of 2 & 0.0033 & 0.0006 & 0.0007 & 0.0002 & 1.7 & 0.0 & 0.0087 & 0.0007 & 2.4 & 0.1 \\
\hline BY20 & $\begin{array}{l}\text { Boston Mine tunnel } \\
\text { outlet nr Grass } \\
\text { Valley }\end{array}$ & $6 / 18 / 1999$ & $14: 30$ & $2 \mathrm{of} 2$ & 0.0026 & 0.0006 & 0.0006 & 0.0000 & 1.8 & 0.1 & 0.0078 & 0.0015 & 2.4 & 0.1 \\
\hline BY20 & $\begin{array}{l}\text { Boston Mine tunnel } \\
\text { outlet nr Grass } \\
\text { Valley }\end{array}$ & $12 / 14 / 1999$ & $12: 00$ & $1 \mathrm{of} 2$ & 0.0006 & 0.0005 & 0.0003 & 0.0001 & 1.8 & 0.0 & 0.0025 & 0.0004 & 3.1 & 0.1 \\
\hline BY20 & $\begin{array}{l}\text { Boston Mine tunnel } \\
\text { outlet nr Grass } \\
\text { Valley }\end{array}$ & $12 / 14 / 1999$ & $12: 00$ & $2 \mathrm{of} 2$ & 0.0011 & 0.0002 & 0.0002 & 0.0001 & 2.2 & 0.0 & 0.0028 & 0.0005 & 2.9 & 0.1 \\
\hline BY20 & $\begin{array}{l}\text { Boston Mine tunnel } \\
\text { outlet nr Grass } \\
\text { Valley }\end{array}$ & $5 / 24 / 2000$ & $16: 00$ & $1 \mathrm{of} 2$ & 0.006 & 0.002 & 0.0012 & 0.0001 & 1.7 & 0.2 & 0.014 & 0.001 & 2.2 & 0.1 \\
\hline BY20 & $\begin{array}{l}\text { Boston Mine tunnel } \\
\text { outlet nr Grass } \\
\text { Valley }\end{array}$ & $5 / 24 / 2000$ & $16: 00$ & $2 \mathrm{of} 2$ & 0.004 & 0.002 & 0.0010 & 0.0006 & 1.6 & 0.0 & 0.013 & 0.000 & 2.2 & 0.1 \\
\hline BY21 & $\begin{array}{l}\text { Boston Mine wetlands } \\
\text { pond nr Grass Valley }\end{array}$ & $12 / 14 / 1999$ & $10: 30$ & 1 of 2 & 0.0014 & 0.0002 & 0.0004 & 0.0000 & 3.4 & 0.2 & 0.0034 & 0.0001 & 0.90 & 0.05 \\
\hline BY21 & $\begin{array}{l}\text { Boston Mine wetlands } \\
\text { pond nr Grass Valley }\end{array}$ & $12 / 14 / 1999$ & $10: 30$ & $2 \mathrm{of} 2$ & 0.0017 & 0.0003 & 0.0003 & 0.0000 & 3.0 & 0.2 & 0.0035 & 0.0004 & 0.92 & 0.08 \\
\hline BY21 & $\begin{array}{l}\text { Boston Mine wetlands } \\
\text { pond nr Grass Valley }\end{array}$ & $5 / 24 / 2000$ & $16: 30$ & $1 \mathrm{of} 2$ & 0.004 & 0.001 & 0.0009 & 0.0005 & 1.6 & 0.4 & 0.011 & 0.001 & 2.9 & 0.2 \\
\hline BY21 & $\begin{array}{l}\text { Boston Mine wetlands } \\
\text { pond nr Grass Valley }\end{array}$ & $5 / 24 / 2000$ & $16: 30$ & $2 \mathrm{of} 2$ & 0.004 & 0.002 & 0.0010 & 0.0003 & 1.6 & 0.1 & 0.011 & 0.000 & 2.8 & 0.1 \\
\hline BY58 & $\begin{array}{l}\text { Buckeye Flat Mine } \\
\text { main drain } 0.45 \mathrm{mi} \\
\text { ab Greenhorn } \mathrm{Cr}\end{array}$ & $8 / 21 / 2000$ & $11: 00$ & 1 of 2 & 2.9 & 0.0 & 0.54 & 0.00 & 1.7 & 0.1 & 11 & 0 & 14 & 0 \\
\hline BY58 & $\begin{array}{l}\text { Buckeye Flat Mine } \\
\text { main drain } 0.45 \mathrm{mi} \\
\text { ab Greenhorn } \mathrm{Cr}\end{array}$ & $8 / 21 / 2000$ & $11: 00$ & $2 \mathrm{of} 2$ & 3.0 & 0.1 & 0.54 & 0.01 & 1.8 & 0.1 & 11 & 0 & 16 & 1 \\
\hline BY23 & $\begin{array}{c}\text { Buckeye Flat Mine N } \\
\text { drain to Greenhorn } \\
\text { Cr, Grass Valley }\end{array}$ & 6/18/1999 & $11: 30$ & 2 of 2 & 5.7 & 0.3 & 1.1 & 0.0 & 1.3 & 0.0 & 21 & 0 & 25 & 0 \\
\hline
\end{tabular}


Table 4B. Concentrations of trace metals and selected major elements in filtered water samples, Greenhorn Creek drainage, Nevada County, California—Continued.

[Station name abbreviations: ab, above; bl, below; Cr, creek; Cyn, Canyon; mi, miles; N, north; nr, near; Rd, Road; S, south; SF, South Fork. Number in parentheses is the data parameter code, a five-digit code used in the U.S. Geological Survey computerized data system. $\mu \mathrm{g} / \mathrm{L}$, microgram per liter (equivalent to part per billion); mg/L, milligram per liter (at low concentration, equivalen to part per million); s.d., standard deviation; <, less than; - , not determined]

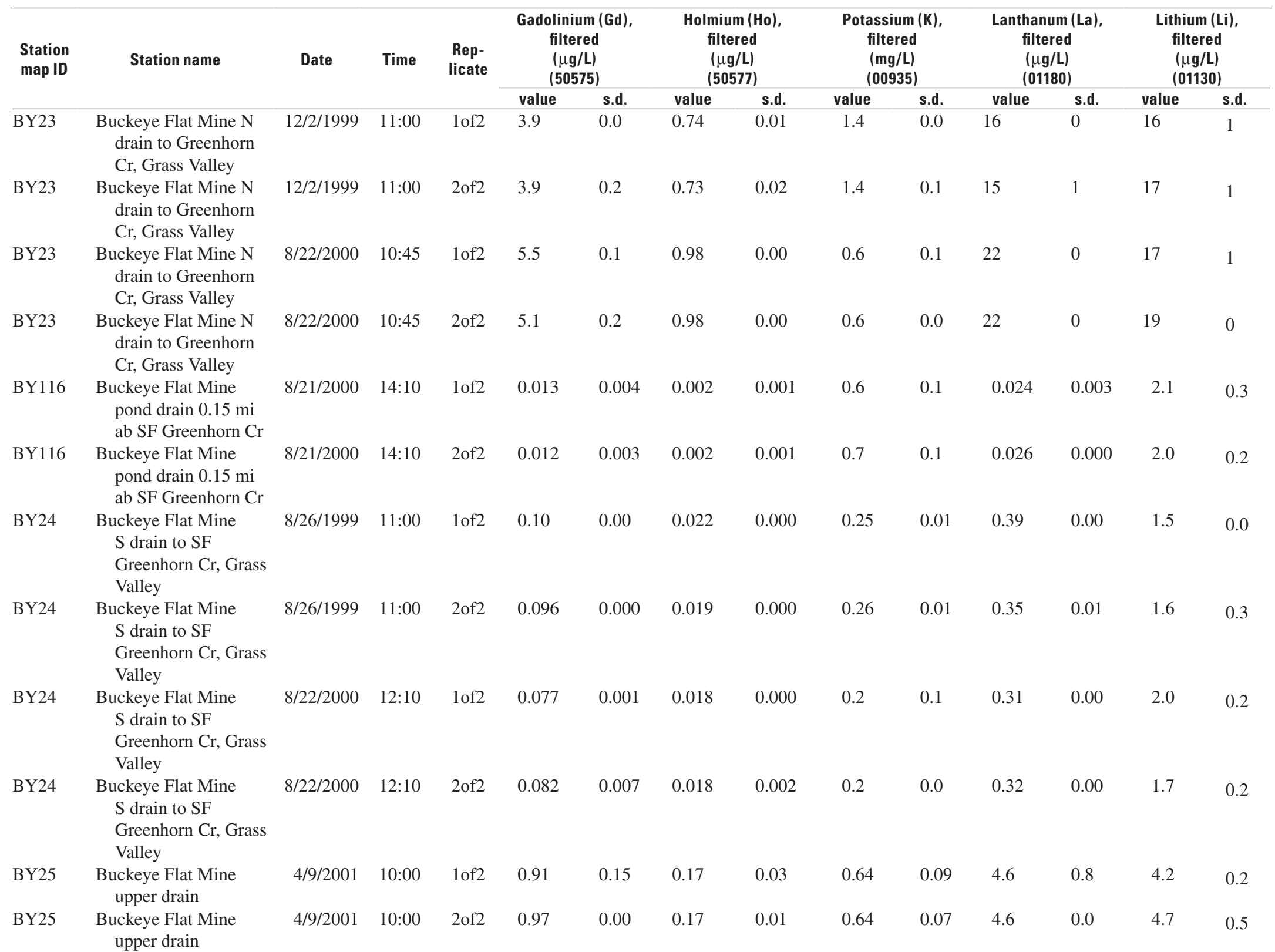


Table 4B. Concentrations of trace metals and selected major elements in filtered water samples, Greenhorn Creek drainage, Nevada County, California—Continued.

[Station name abbreviations: ab, above; bl, below; Cr, creek; Cyn, Canyon; mi, miles; N, north; nr, near; Rd, Road; S, south; SF, South Fork. Number in parentheses is the data parameter code, a five-digit code used in the U.S. Geological Survey computerized data system. $\mu \mathrm{g} / \mathrm{L}$, microgram per liter (equivalent to part per billion); $\mathrm{mg} / \mathrm{L}$, milligram per liter (at low concentration, equivalen to part per million); s.d., standard deviation; <, less than; - , not determined]

\begin{tabular}{|c|c|c|c|c|c|c|c|c|c|c|c|c|c|c|}
\hline \multirow[t]{2}{*}{$\begin{array}{l}\text { Station } \\
\text { map ID }\end{array}$} & \multirow[t]{2}{*}{ Station name } & \multirow[t]{2}{*}{ Date } & \multirow[t]{2}{*}{ Time } & \multirow[t]{2}{*}{$\begin{array}{l}\text { Rep- } \\
\text { licate }\end{array}$} & \multicolumn{2}{|c|}{$\begin{array}{c}\text { Gadolinium (Gd), } \\
\text { filtered } \\
(\mu \mathrm{g} / \mathrm{L}) \\
(\mathbf{5 0 5 7 5 )} \\
\end{array}$} & \multicolumn{2}{|c|}{$\begin{array}{c}\text { Holmium (Ho), } \\
\text { filtered } \\
(\mu \mathrm{g} / \mathrm{L}) \\
(\mathbf{5 0 5 7 7 )} \\
\end{array}$} & \multicolumn{2}{|c|}{$\begin{array}{c}\text { Potassium (K), } \\
\text { filtered } \\
(\mathrm{mg} / \mathrm{L}) \\
(00935) \\
\end{array}$} & \multicolumn{2}{|c|}{$\begin{array}{c}\text { Lanthanum (La), } \\
\text { filtered } \\
(\mu \mathrm{g} / \mathrm{L}) \\
(01180) \\
\end{array}$} & \multicolumn{2}{|c|}{$\begin{array}{c}\text { Lithium (Li), } \\
\text { filtered } \\
(\mu \mathrm{g} / \mathrm{L}) \\
(\mathbf{0 1 1 3 0}) \\
\end{array}$} \\
\hline & & & & & value & s.d. & value & s.d. & value & s.d. & value & s.d. & value & s.d. \\
\hline BY52 & $\begin{array}{l}\text { Greenhorn Cr } 0.3 \mathrm{mi} \\
\text { ab Buckeye Ford nr } \\
\text { Nevada City }\end{array}$ & $1 / 25 / 2000$ & $10: 45$ & $2 \mathrm{of} 2$ & 0.085 & 0.002 & 0.016 & 0.000 & 0.53 & 0.04 & 0.31 & 0.00 & 1.0 & 0.2 \\
\hline BY59 & $\begin{array}{l}\text { Greenhorn Cr at You } \\
\text { Bet Rd nr Nevada } \\
\text { City }\end{array}$ & $7 / 24 / 2001$ & 11:00 & 1 of 2 & 0.0026 & 0.0002 & 0.0006 & 0.0000 & 0.68 & 0.01 & 0.0094 & 0.0008 & 0.52 & 0.04 \\
\hline BY59 & $\begin{array}{l}\text { Greenhorn } \mathrm{Cr} \text { at You } \\
\text { Bet Rd nr Nevada } \\
\text { City }\end{array}$ & $7 / 24 / 2001$ & 11:00 & $2 \mathrm{of} 2$ & 0.0032 & 0.0000 & 0.0006 & 0.0003 & 0.68 & 0.01 & 0.0087 & 0.0003 & 0.56 & 0.01 \\
\hline BY59 & $\begin{array}{l}\text { Greenhorn Cr at You } \\
\text { Bet Rd nr Nevada } \\
\text { City }\end{array}$ & $8 / 29 / 2001$ & $15: 00$ & 1 of 2 & 0.0018 & 0.0000 & 0.0005 & 0.0000 & 0.72 & 0.01 & 0.0084 & 0.0001 & 0.42 & 0.03 \\
\hline BY59 & $\begin{array}{l}\text { Greenhorn Cr at You } \\
\text { Bet Rd nr Nevada } \\
\text { City }\end{array}$ & $8 / 29 / 2001$ & $15: 00$ & $2 \mathrm{of} 2$ & 0.0028 & 0.0007 & 0.0005 & 0.0001 & 0.74 & 0.01 & 0.0082 & 0.0006 & 0.45 & 0.01 \\
\hline BY59 & $\begin{array}{l}\text { Greenhorn Cr at You } \\
\text { Bet Rd nr Nevada } \\
\text { City }\end{array}$ & $9 / 14 / 2001$ & $13: 00$ & 1 of 2 & 0.0024 & 0.0006 & 0.0005 & 0.0001 & 0.69 & 0.01 & 0.0071 & 0.0004 & 0.44 & 0.01 \\
\hline BY59 & $\begin{array}{l}\text { Greenhorn Cr at You } \\
\text { Bet Rd nr Nevada } \\
\text { City }\end{array}$ & $9 / 14 / 2001$ & $13: 00$ & $2 \mathrm{of} 2$ & 0.0022 & 0.0001 & 0.0003 & 0.0000 & 0.69 & 0.01 & 0.0070 & 0.0005 & 0.45 & 0.01 \\
\hline BY59 & $\begin{array}{l}\text { Greenhorn } \mathrm{Cr} \text { at You } \\
\text { Bet Rd nr Nevada } \\
\text { City }\end{array}$ & $10 / 15 / 2001$ & $12: 00$ & $1 \mathrm{of} 2$ & 0.0015 & 0.0004 & 0.0002 & 0.0001 & 0.63 & 0.02 & 0.0049 & 0.0003 & 0.37 & 0.00 \\
\hline BY59 & $\begin{array}{l}\text { Greenhorn } \mathrm{Cr} \text { at You } \\
\text { Bet Rd nr Nevada } \\
\text { City }\end{array}$ & $10 / 15 / 2001$ & $12: 00$ & $2 \mathrm{of} 2$ & 0.0016 & 0.0002 & 0.0004 & 0.0000 & 0.63 & 0.02 & 0.0051 & 0.0005 & 0.37 & 0.01 \\
\hline BY59 & $\begin{array}{l}\text { Greenhorn } \mathrm{Cr} \text { at You } \\
\text { Bet Rd nr Nevada } \\
\text { City }\end{array}$ & $11 / 20 / 2001$ & $13: 20$ & 1 of 2 & 0.013 & 0.001 & 0.0025 & 0.0001 & 0.77 & 0.00 & 0.037 & 0.001 & 0.52 & 0.00 \\
\hline BY59 & $\begin{array}{l}\text { Greenhorn Cr at You } \\
\text { Bet Rd nr Nevada } \\
\text { City }\end{array}$ & $12 / 10 / 2001$ & $12: 20$ & 1 of 2 & 0.037 & 0.001 & 0.0067 & 0.0008 & 0.60 & 0.00 & 0.10 & 0.00 & 0.86 & 0.02 \\
\hline
\end{tabular}


Table 4B. Concentrations of trace metals and selected major elements in filtered water samples, Greenhorn Creek drainage, Nevada County, California—Continued.

[Station name abbreviations: ab, above; bl, below; Cr, creek; Cyn, Canyon; mi, miles; N, north; nr, near; Rd, Road; S, south; SF, South Fork. Number in parentheses is the data parameter code, a five-digit code used in the U.S. Geological Survey computerized data system. $\mu \mathrm{g} / \mathrm{L}$, microgram per liter (equivalent to part per billion); $\mathrm{mg} / \mathrm{L}$, milligram per liter (at low concentration, equivalen to part per million); s.d., standard deviation; <, less than; - , not determined]

\begin{tabular}{|c|c|c|c|c|c|c|c|c|c|c|c|c|c|c|}
\hline \multirow[t]{2}{*}{$\begin{array}{l}\text { Station } \\
\text { map ID }\end{array}$} & \multirow[t]{2}{*}{ Station name } & \multirow[t]{2}{*}{ Date } & \multirow[t]{2}{*}{ Time } & \multirow[t]{2}{*}{$\begin{array}{l}\text { Rep- } \\
\text { licate }\end{array}$} & \multicolumn{2}{|c|}{$\begin{array}{c}\text { Gadolinium (Gd), } \\
\text { filtered } \\
(\mu \mathbf{g} / \mathrm{L}) \\
(\mathbf{5 0 5 7 5 )} \\
\end{array}$} & \multicolumn{2}{|c|}{$\begin{array}{c}\text { Holmium (Ho), } \\
\text { filtered } \\
(\mu \mathrm{g} / \mathrm{L}) \\
(\mathbf{5 0 5 7 7 )} \\
\end{array}$} & \multicolumn{2}{|c|}{$\begin{array}{c}\text { Potassium (K), } \\
\text { filtered } \\
(\mathrm{mg} / \mathrm{L}) \\
(00935) \\
\end{array}$} & \multicolumn{2}{|c|}{$\begin{array}{c}\text { Lanthanum (La), } \\
\text { filtered } \\
(\mu \mathrm{g} / \mathrm{L}) \\
(\mathbf{0 1 1 8 0}) \\
\end{array}$} & \multicolumn{2}{|c|}{$\begin{array}{l}\text { Lithium (Li), } \\
\text { filtered } \\
(\mu \mathrm{g} / \mathrm{L}) \\
(\mathbf{0 1 1 3 0})\end{array}$} \\
\hline & & & & & value & s.d. & value & s.d. & value & s.d. & value & s.d. & value & s.d. \\
\hline BY59 & $\begin{array}{l}\text { Greenhorn Cr at You } \\
\text { Bet Rd nr Nevada } \\
\text { City }\end{array}$ & $12 / 21 / 2001$ & $13: 00$ & $1 \mathrm{of} 2$ & 0.085 & 0.005 & 0.015 & 0.000 & 0.55 & 0.00 & 0.19 & 0.00 & 0.73 & 0.03 \\
\hline BY59 & $\begin{array}{l}\text { Greenhorn Cr at You } \\
\text { Bet Rd nr Nevada } \\
\text { City }\end{array}$ & $12 / 21 / 2001$ & $13: 00$ & 2 of 2 & 0.084 & 0.001 & 0.015 & 0.001 & 0.55 & 0.01 & 0.18 & 0.00 & 0.74 & 0.04 \\
\hline BY51 & $\begin{array}{l}\text { Greenhorn Cr nr } \\
\text { Headwaters nr Scotts } \\
\text { Flat Reservoir }\end{array}$ & $1 / 25 / 2000$ & $9: 30$ & $1 \mathrm{of} 2$ & 0.058 & 0.005 & 0.011 & 0.001 & 0.44 & 0.03 & 0.15 & 0.00 & 0.3 & 0.1 \\
\hline BY51 & $\begin{array}{l}\text { Greenhorn Cr nr } \\
\text { Headwaters nr Scotts } \\
\text { Flat Reservoir }\end{array}$ & $1 / 25 / 2000$ & $9: 30$ & $2 \mathrm{of} 2$ & 0.061 & 0.004 & 0.010 & 0.000 & 0.45 & 0.02 & 0.17 & 0.00 & 0.2 & 0.1 \\
\hline BY75 & $\begin{array}{l}\text { Missouri Cyn Cr } 1.2 \mathrm{mi} \\
\text { ab Greenhorn Cr nr } \\
\text { Chicago Park }\end{array}$ & $12 / 14 / 1999$ & $14: 00$ & $1 \mathrm{of} 2$ & 0.065 & 0.002 & 0.013 & 0.000 & 1.5 & 0.2 & 0.29 & 0.00 & 7.6 & 0.1 \\
\hline BY75 & $\begin{array}{l}\text { Missouri Cyn Cr } 1.2 \mathrm{mi} \\
\text { ab Greenhorn Cr nr } \\
\text { Chicago Park }\end{array}$ & $12 / 14 / 1999$ & $14: 00$ & $2 \mathrm{of} 2$ & 0.066 & 0.004 & 0.013 & 0.001 & 1.7 & 0.2 & 0.29 & 0.01 & 7.9 & 0.3 \\
\hline BY86 & $\begin{array}{l}\text { Poore Mine creek ab } \\
\text { tunnel nr Grass } \\
\text { Valley }\end{array}$ & 3/31/1999 & $17: 40$ & $1 \mathrm{of} 2$ & 0.016 & 0.000 & 0.0028 & 0.0004 & 0.59 & 0.04 & 0.061 & 0.004 & 0.41 & 0.04 \\
\hline BY86 & $\begin{array}{l}\text { Poore Mine creek ab } \\
\text { tunnel nr Grass } \\
\text { Valley }\end{array}$ & 3/31/1999 & $17: 40$ & $2 \mathrm{of} 2$ & 0.015 & 0.001 & 0.0028 & 0.0004 & 0.57 & 0.01 & 0.059 & 0.003 & 0.41 & 0.02 \\
\hline BY87 & $\begin{array}{l}\text { Poore Mine creek bl } \\
\text { tunnel nr Grass } \\
\text { Valley }\end{array}$ & 3/31/1999 & $13: 30$ & $1 \mathrm{of} 2$ & 0.017 & 0.001 & 0.0029 & 0.0001 & 0.59 & 0.02 & 0.059 & 0.006 & 0.65 & 0.04 \\
\hline BY87 & $\begin{array}{l}\text { Poore Mine creek bl } \\
\text { tunnel nr Grass } \\
\text { Valley }\end{array}$ & 3/31/1999 & $13: 30$ & $2 \mathrm{of} 2$ & 0.016 & 0.001 & 0.0025 & 0.0003 & 0.57 & 0.01 & 0.049 & 0.003 & 0.63 & 0.02 \\
\hline BY88 & $\begin{array}{l}\text { Poore Mine ground } \\
\text { sluice nr Grass } \\
\text { Valley }\end{array}$ & 4/1/1999 & $15: 40$ & $2 \mathrm{of} 2$ & 0.029 & 0.001 & 0.0065 & 0.0006 & 1.7 & 0.0 & 0.077 & 0.001 & 11 & 0 \\
\hline
\end{tabular}


Table 4B. Concentrations of trace metals and selected major elements in filtered water samples, Greenhorn Creek drainage, Nevada County, California—Continued.

[Station name abbreviations: ab, above; bl, below; Cr, creek; Cyn, Canyon; mi, miles; N, north; nr, near; Rd, Road; S, south; SF, South Fork. Number in parentheses is the data parameter code, a five-digit code used in the U.S. Geological Survey computerized data system. $\mu \mathrm{g} / \mathrm{L}$, microgram per liter (equivalent to part per billion); $\mathrm{mg} / \mathrm{L}$, milligram per liter (at low concentration, equivalen to part per million); s.d., standard deviation; <, less than; - , not determined]

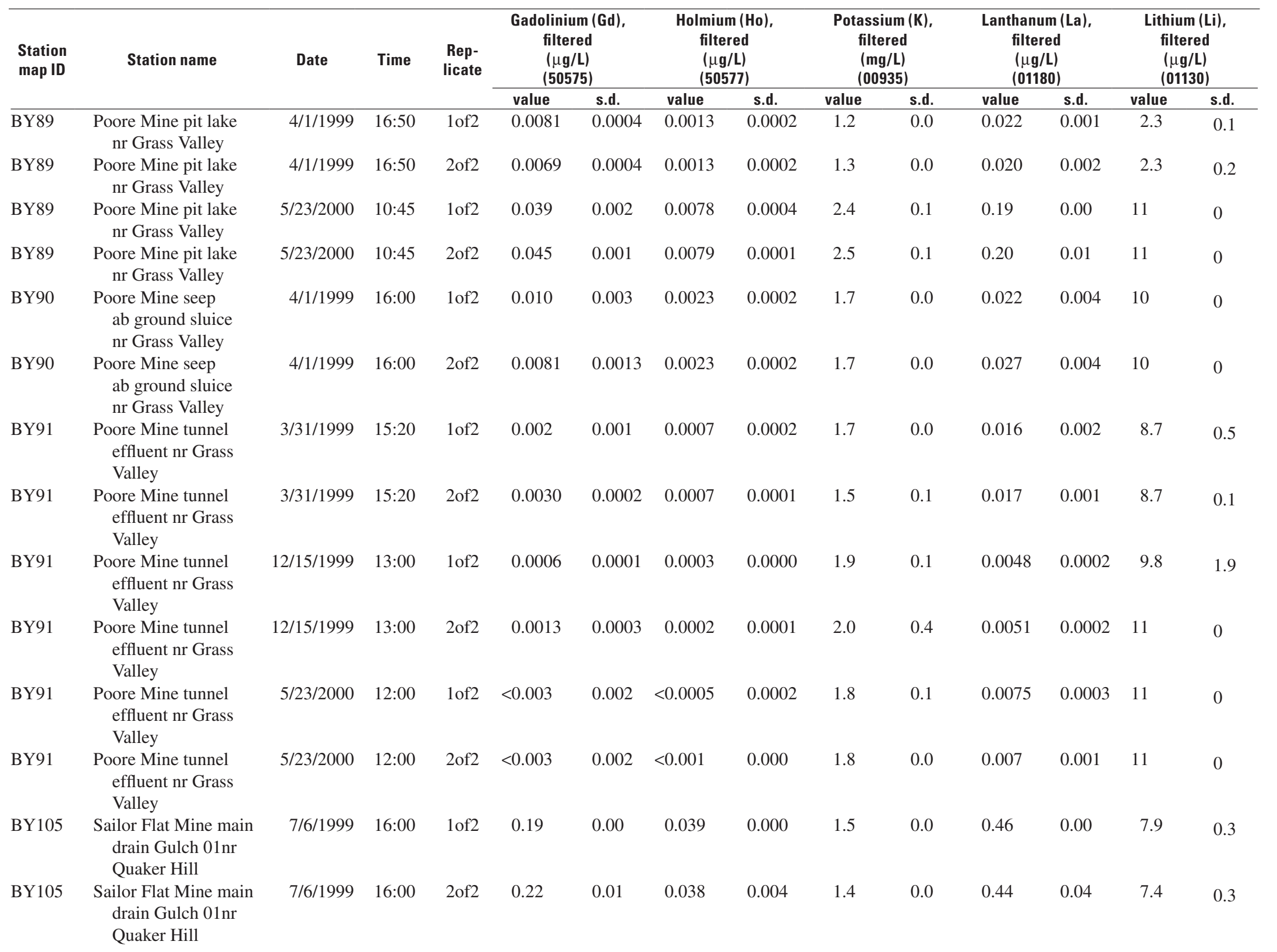


Table 4B. Concentrations of trace metals and selected major elements in filtered water samples, Greenhorn Creek drainage, Nevada County, California—Continued.

[Station name abbreviations: ab, above; bl, below; Cr, creek; Cyn, Canyon; mi, miles; N, north; nr, near; Rd, Road; S, south; SF, South Fork. Number in parentheses is the data parameter code, a five-digit code used in the U.S. Geological Survey computerized data system. $\mu \mathrm{g} / \mathrm{L}$, microgram per liter (equivalent to part per billion); $\mathrm{mg} / \mathrm{L}$, milligram per liter (at low concentration, equivalen to part per million); s.d., standard deviation; <, less than; - , not determined]

\begin{tabular}{|c|c|c|c|c|c|c|c|c|c|c|c|c|c|c|}
\hline \multirow[t]{2}{*}{$\begin{array}{l}\text { Station } \\
\text { map ID }\end{array}$} & \multirow[t]{2}{*}{ Station name } & \multirow[t]{2}{*}{ Date } & \multirow[t]{2}{*}{ Time } & \multirow[t]{2}{*}{$\begin{array}{l}\text { Rep- } \\
\text { licate }\end{array}$} & \multicolumn{2}{|c|}{$\begin{array}{c}\text { Gadolinium (Gd), } \\
\text { filtered } \\
(\mu \mathbf{g} / \mathrm{L}) \\
(\mathbf{5 0 5 7 5 )} \\
\end{array}$} & \multicolumn{2}{|c|}{$\begin{array}{c}\text { Holmium (Ho), } \\
\text { filtered } \\
(\mu \mathrm{g} / \mathrm{L}) \\
(50577) \\
\end{array}$} & \multicolumn{2}{|c|}{$\begin{array}{c}\text { Potassium (K), } \\
\text { filtered } \\
(\mathrm{mg} / \mathrm{L}) \\
(00935) \\
\end{array}$} & \multicolumn{2}{|c|}{$\begin{array}{c}\text { Lanthanum (La), } \\
\text { filtered } \\
(\mu \mathrm{g} / \mathrm{L}) \\
(\mathbf{0 1 1 8 0}) \\
\end{array}$} & \multicolumn{2}{|c|}{$\begin{array}{c}\text { Lithium (Li), } \\
\text { filtered } \\
(\mu \mathrm{g} / \mathrm{L}) \\
(01130)\end{array}$} \\
\hline & & & & & value & s.d. & value & s.d. & value & s.d. & value & s.d. & value & s.d. \\
\hline BY105 & $\begin{array}{l}\text { Sailor Flat Mine main } \\
\text { drain Gulch 01nr } \\
\text { Quaker Hill }\end{array}$ & $12 / 2 / 1999$ & $14: 30$ & $2 \mathrm{of} 2$ & 0.34 & 0.00 & 0.058 & 0.000 & 1.0 & 0.1 & 0.70 & 0.01 & 2.2 & 0.1 \\
\hline BY106 & $\begin{array}{l}\text { Sailor Flat Mine } \\
\text { main drainage to } \\
\text { Greenhorn Cr, } \\
\text { Gulch } 03\end{array}$ & 4/9/2001 & $12: 30$ & 1 of 2 & 0.21 & 0.02 & 0.045 & 0.012 & 0.64 & 0.15 & 0.73 & 0.17 & 0.9 & 0.3 \\
\hline BY106 & $\begin{array}{l}\text { Sailor Flat Mine } \\
\text { main drainage to } \\
\text { Greenhorn } \mathrm{Cr} \text {, } \\
\text { Gulch } 03\end{array}$ & 4/9/2001 & $12: 30$ & 2 of 2 & 0.21 & 0.02 & 0.048 & 0.012 & 0.67 & 0.15 & 0.74 & 0.16 & 1.0 & 0.3 \\
\hline BY114 & $\begin{array}{l}\text { SF Greenhorn Cr } \\
0.7 \text { mi ab Greenhorn } \\
\text { Cr nr Nevada City }\end{array}$ & $8 / 22 / 2000$ & $15: 10$ & $2 \mathrm{of} 2$ & $<0.004$ & 0.001 & $<0.0009$ & 0.0003 & 0.3 & 0.0 & 0.010 & 0.001 & $<0.4$ & 0.2 \\
\hline BY122 & $\begin{array}{l}\text { Starr Mine tunnel } \\
\text { inflow nr Grass } \\
\text { Valley }\end{array}$ & 4/23/1999 & $15: 00$ & 1 of 2 & 0.026 & 0.002 & 0.0041 & 0.0002 & 1.1 & 0.0 & 0.072 & 0.003 & 5.1 & 0.1 \\
\hline BY122 & $\begin{array}{l}\text { Starr Mine tunnel } \\
\text { inflow nr Grass } \\
\text { Valley }\end{array}$ & 4/23/1999 & $15: 00$ & $2 \mathrm{of} 2$ & 0.026 & 0.000 & 0.0035 & 0.0014 & 1.2 & 0.0 & 0.071 & 0.008 & 5.2 & 0.2 \\
\hline BY123 & $\begin{array}{l}\text { Starr Mine tunnel } \\
\text { midway nr Grass } \\
\text { Valley }\end{array}$ & 4/23/1999 & $13: 40$ & 1 of 2 & 0.0004 & 0.0002 & $<0.0001$ & 0.0000 & 2.2 & 0.2 & 0.0020 & 0.0002 & 10 & 0 \\
\hline BY123 & $\begin{array}{l}\text { Starr Mine tunnel } \\
\text { midway nr Grass } \\
\text { Valley }\end{array}$ & 4/23/1999 & $13: 40$ & $2 \mathrm{of} 2$ & 0.0005 & 0.0002 & $<0.0001$ & 0.0000 & 2.1 & 0.1 & 0.0015 & 0.0002 & 10 & 0 \\
\hline BY123 & $\begin{array}{l}\text { Starr Mine tunnel } \\
\text { midway nr Grass } \\
\text { Valley }\end{array}$ & $12 / 15 / 1999$ & $10: 45$ & 1 of 2 & 0.0004 & 0.0003 & 0.0002 & 0.0001 & 2.2 & 0.0 & 0.0025 & 0.0002 & 9.3 & 0.8 \\
\hline BY123 & $\begin{array}{l}\text { Starr Mine tunnel } \\
\text { midway nr Grass } \\
\text { Valley }\end{array}$ & $12 / 15 / 1999$ & $10: 45$ & $2 \mathrm{of} 2$ & 0.0010 & 0.0001 & 0.0002 & 0.0000 & 2.4 & 0.0 & 0.0030 & 0.0001 & 9.1 & 0.4 \\
\hline BY124 & $\begin{array}{l}\text { Starr Mine tunnel outlet } \\
\text { nr Grass Valley }\end{array}$ & $8 / 23 / 2000$ & $17: 30$ & $1 \mathrm{of} 2$ & $<0.004$ & 0.003 & $<0.0009$ & 0.0006 & 1.9 & 0.1 & 0.007 & 0.002 & 8.4 & 0.6 \\
\hline
\end{tabular}


Table 4B. Concentrations of trace metals and selected major elements in filtered water samples, Greenhorn Creek drainage, Nevada County, California—Continued.

[Station name abbreviations: ab, above; bl, below; Cr, creek; Cyn, Canyon; mi, miles; N, north; nr, near; Rd, Road; S, south; SF, South Fork. Number in parentheses is the data parameter code, a five-digit code used in the U.S. Geological Survey computerized data system. $\mu \mathrm{g} / \mathrm{L}$, microgram per liter (equivalent to part per billion); $\mathrm{mg} / \mathrm{L}$, milligram per liter (at low concentration, equivalen to part per million); s.d., standard deviation; <, less than; - , not determined]

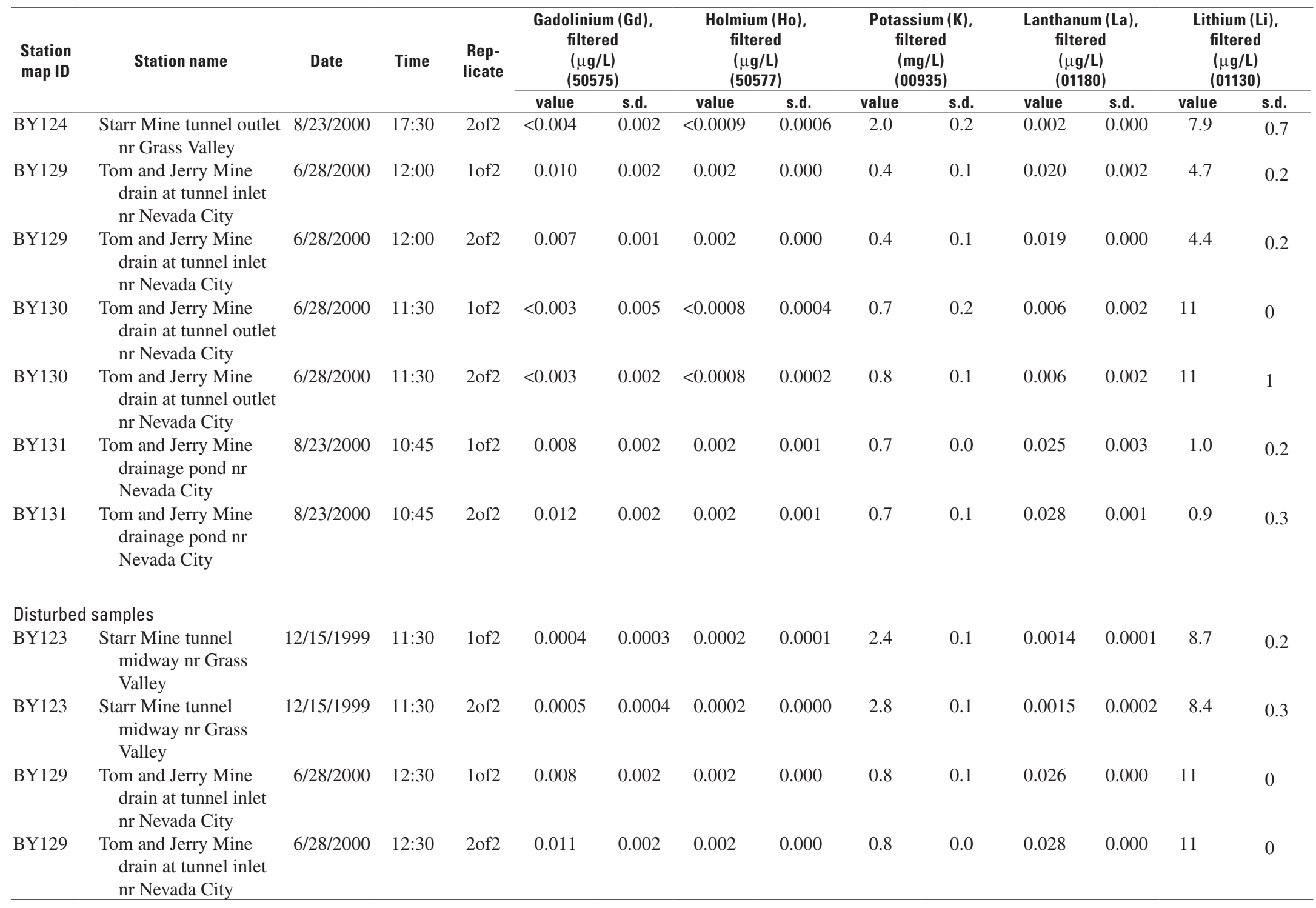


Table 4B. Concentrations of trace metals and selected major elements in filtered water samples, Greenhorn Creek drainage, Nevada County, California—Continued.

[Station name abbreviations: ab, above; bl, below; Cr, creek; Cyn, Canyon; mi, miles; N, north; nr, near; Rd, Road; S, south; SF, South Fork. Number in parentheses is the data parameter code, a five-digit code used in the U.S. Geological Survey computerized data system. $\mu \mathrm{g} / \mathrm{L}$, microgram per liter (equivalent to part per billion); mg/L, milligram per liter (at low concentration, equivalent to part per million); s.d., standard deviation; <, less than; —, not determined]

\begin{tabular}{|c|c|c|c|c|c|c|c|c|c|c|c|c|c|c|c|c|}
\hline \multirow[t]{2}{*}{$\begin{array}{l}\text { Station } \\
\text { map ID }\end{array}$} & \multirow[t]{2}{*}{ Station name } & \multirow[t]{2}{*}{ Date } & \multirow[t]{2}{*}{ Time } & \multirow[t]{2}{*}{$\begin{array}{l}\text { Rep- } \\
\text { licate }\end{array}$} & \multicolumn{2}{|c|}{$\begin{array}{l}\text { Lutetium (Lu), } \\
\text { filtered } \\
(\mu \mathrm{g} / \mathrm{L}) \\
(\mathbf{6 2 8 4 4 )}\end{array}$} & \multicolumn{2}{|c|}{$\begin{array}{c}\text { Magnesium (Mg), } \\
\text { filtered } \\
(\mathrm{mg} / \mathrm{L}) \\
(00925) \\
\end{array}$} & \multicolumn{2}{|c|}{$\begin{array}{c}\text { Manganese (Mn), } \\
\text { filtered } \\
(\mu \mathrm{g} / \mathrm{L}) \\
(01056) \\
\end{array}$} & \multicolumn{2}{|c|}{$\begin{array}{c}\text { Molybdenum (Mo), } \\
\text { filtered } \\
(\mu \mathrm{g} / \mathrm{L}) \\
(01060)\end{array}$} & \multicolumn{2}{|c|}{$\begin{array}{l}\text { Sodium (Na), } \\
\text { filtered } \\
\text { (mg/L) } \\
(00930)\end{array}$} & \multicolumn{2}{|c|}{$\begin{array}{c}\text { Neodymium (Nd), } \\
\text { filtered } \\
(\mu \mathrm{g} / \mathrm{L}) \\
(\mathbf{5 0 5 7 9 )}\end{array}$} \\
\hline & & & & & value & s.d. & value & s.d. & value & s.d. & value & s.d. & value & s.d. & value & s.d. \\
\hline \multicolumn{17}{|c|}{ Environmental samples } \\
\hline BY20 & $\begin{array}{l}\text { Boston Mine tunnel } \\
\text { outlet nr Grass } \\
\text { Valley }\end{array}$ & 6/18/1999 & $14: 30$ & 1 of 2 & 0.0004 & 0.0001 & 4.8 & 0.2 & 100 & 0 & 0.23 & 0.01 & 1.2 & 0.1 & 0.013 & 0.002 \\
\hline BY20 & $\begin{array}{l}\text { Boston Mine tunnel } \\
\text { outlet nr Grass } \\
\text { Valley }\end{array}$ & $6 / 18 / 1999$ & $14: 30$ & $2 \mathrm{of} 2$ & 0.0005 & 0.0002 & 4.8 & 0.2 & 120 & 20 & 0.19 & 0.02 & 1.2 & 0.1 & 0.013 & 0.002 \\
\hline BY20 & $\begin{array}{l}\text { Boston Mine tunnel } \\
\text { outlet nr Grass } \\
\text { Valley }\end{array}$ & $12 / 14 / 1999$ & $12: 00$ & 1 of 2 & $<0.0001$ & 0.0002 & 6.3 & 0.8 & 51 & 3 & 0.13 & 0.03 & 1.6 & 0.2 & 0.0035 & 0.0003 \\
\hline BY20 & $\begin{array}{l}\text { Boston Mine tunnel } \\
\text { outlet nr Grass } \\
\text { Valley }\end{array}$ & $12 / 14 / 1999$ & $12: 00$ & $2 \mathrm{of} 2$ & 0.0001 & 0.0000 & 7.1 & 0.0 & 52 & 2 & 0.16 & 0.00 & 1.5 & 0.1 & 0.0033 & 0.0005 \\
\hline BY20 & $\begin{array}{l}\text { Boston Mine tunnel } \\
\text { outlet nr Grass } \\
\text { Valley }\end{array}$ & $5 / 24 / 2000$ & $16: 00$ & 1 of 2 & 0.0010 & 0.0005 & 4.0 & 0.7 & 120 & 0 & 0.22 & 0.03 & 1.5 & 0.0 & 0.020 & 0.005 \\
\hline BY20 & $\begin{array}{l}\text { Boston Mine tunnel } \\
\text { outlet nr Grass } \\
\text { Valley }\end{array}$ & $5 / 24 / 2000$ & $16: 00$ & $2 \mathrm{of} 2$ & 0.0003 & 0.0004 & 4.1 & 0.4 & 120 & 0 & 0.08 & 0.00 & 1.5 & 0.1 & 0.021 & 0.003 \\
\hline BY21 & $\begin{array}{l}\text { Boston Mine wetlands } \\
\text { pond nr Grass Valley }\end{array}$ & $12 / 14 / 1999$ & $10: 30$ & 1 of 2 & $<0.0001$ & 0.0000 & 6.6 & 1.3 & 12 & 0 & $<0.05$ & 0.00 & 1.6 & 0.3 & 0.0048 & 0.0003 \\
\hline BY21 & $\begin{array}{l}\text { Boston Mine wetlands } \\
\text { pond nr Grass Valley }\end{array}$ & 12/14/1999 & $10: 30$ & $2 \mathrm{of} 2$ & 0.0001 & 0.0000 & 6.2 & 0.3 & 12 & 0 & 0.09 & 0.05 & 1.5 & 0.2 & 0.0049 & 0.0006 \\
\hline BY21 & $\begin{array}{l}\text { Boston Mine wetlands } \\
\text { pond nr Grass Valley }\end{array}$ & $5 / 24 / 2000$ & $16: 30$ & 1 of 2 & 0.0004 & 0.0002 & 2.8 & 0.8 & 45 & 2 & 0.15 & 0.08 & 1.5 & 0.1 & 0.016 & 0.002 \\
\hline BY21 & $\begin{array}{l}\text { Boston Mine wetlands } \\
\text { pond nr Grass Valley }\end{array}$ & $5 / 24 / 2000$ & $16: 30$ & $2 \mathrm{of} 2$ & 0.0002 & 0.0004 & 2.9 & 0.4 & 44 & 0 & 0.10 & 0.06 & 1.5 & 0.0 & 0.018 & 0.000 \\
\hline BY58 & $\begin{array}{l}\text { Buckeye Flat Mine } \\
\text { main drain } 0.45 \mathrm{mi} \\
\text { ab Greenhorn } \mathrm{Cr}\end{array}$ & $8 / 21 / 2000$ & 11:00 & 1 of 2 & 0.14 & 0.01 & 11 & 0 & 1,500 & 0 & 0.05 & 0.02 & 1.8 & 0.0 & 12 & 0 \\
\hline BY58 & $\begin{array}{l}\text { Buckeye Flat Mine } \\
\text { main drain } 0.45 \mathrm{mi} \\
\text { ab Greenhorn } \mathrm{Cr}\end{array}$ & $8 / 21 / 2000$ & 11:00 & $2 \mathrm{of} 2$ & 0.14 & 0.01 & 12 & 1 & 1,400 & 100 & $<0.04$ & 0.03 & 1.8 & 0.0 & 12 & 0 \\
\hline BY23 & $\begin{array}{c}\text { Buckeye Flat Mine N } \\
\text { drain to Greenhorn } \\
\text { Cr, Grass Valley }\end{array}$ & $6 / 18 / 1999$ & $11: 30$ & 1 of 2 & 0.25 & 0.00 & 17 & 0 & 3,400 & 0 & 0.079 & 0.014 & 2.3 & 0.1 & 24 & 0 \\
\hline BY23 & $\begin{array}{c}\text { Buckeye Flat Mine N } \\
\text { drain to Greenhorn } \\
\text { Cr, Grass Valley }\end{array}$ & $6 / 18 / 1999$ & $11: 30$ & $2 \mathrm{of} 2$ & 0.26 & 0.00 & 18 & 0 & 3,100 & 100 & 0.085 & 0.014 & 2.3 & 0.1 & 25 & 0 \\
\hline
\end{tabular}


Table 4B. Concentrations of trace metals and selected major elements in filtered water samples, Greenhorn Creek drainage, Nevada County, California—Continued.

[Station name abbreviations: ab, above; bl, below; Cr, creek; Cyn, Canyon; mi, miles; N, north; nr, near; Rd, Road; S, south; SF, South Fork. Number in parentheses is the data parameter code, a five-digit code used in the U.S. Geological Survey computerized data system. $\mu \mathrm{g} / \mathrm{L}$, microgram per liter (equivalent to part per billion); mg/L, milligram per liter (at low concentration, equivalent to part per million); s.d., standard deviation; <, less than; - , not determined]

\begin{tabular}{|c|c|c|c|c|c|c|c|c|c|c|c|c|c|c|c|c|}
\hline \multirow[t]{2}{*}{$\begin{array}{l}\text { Station } \\
\text { map ID }\end{array}$} & \multirow[t]{2}{*}{ Station name } & \multirow[t]{2}{*}{ Date } & \multirow[t]{2}{*}{ Time } & \multirow[t]{2}{*}{$\begin{array}{l}\text { Rep- } \\
\text { licate }\end{array}$} & \multicolumn{2}{|c|}{$\begin{array}{c}\text { Lutetium (Lu), } \\
\text { filtered } \\
(\mu \mathrm{g} / \mathrm{L}) \\
(\mathbf{6 2 8 4 4 )} \\
\end{array}$} & \multicolumn{2}{|c|}{$\begin{array}{l}\text { Magnesium (Mg), } \\
\text { filtered } \\
(\mathrm{mg} / \mathrm{L}) \\
(00925) \\
\end{array}$} & \multicolumn{2}{|c|}{$\begin{array}{c}\text { Manganese }(\mathrm{Mn}), \\
\text { filtered } \\
(\mu \mathrm{g} / \mathrm{L}) \\
(\mathbf{0 1 0 5 6 )} \\
\end{array}$} & \multicolumn{2}{|c|}{$\begin{array}{c}\text { Molybdenum (Mo), } \\
\text { filtered } \\
(\mu \mathrm{g} / \mathrm{L}) \\
(01060)\end{array}$} & \multicolumn{2}{|c|}{$\begin{array}{c}\text { Sodium (Na), } \\
\text { filtered } \\
(\mathrm{mg} / \mathrm{L}) \\
(00930)\end{array}$} & \multicolumn{2}{|c|}{$\begin{array}{c}\text { Neodymium (Nd), } \\
\text { filtered } \\
(\mu \mathrm{g} / \mathrm{L}) \\
(50579)\end{array}$} \\
\hline & & & & & value & s.d. & value & s.d. & value & s.d. & value & s.d. & value & s.d. & value & s.d. \\
\hline BY23 & $\begin{array}{c}\text { Buckeye Flat Mine N } \\
\text { drain to Greenhorn } \\
\text { Cr, Grass Valley }\end{array}$ & $12 / 2 / 1999$ & 11:00 & 1 of 2 & 0.19 & 0.00 & 12 & 1 & 1,700 & 0 & 0.10 & 0.08 & 2.1 & 0.2 & 18 & 1 \\
\hline BY23 & $\begin{array}{c}\text { Buckeye Flat Mine N } \\
\text { drain to Greenhorn } \\
\text { Cr, Grass Valley }\end{array}$ & $12 / 2 / 1999$ & 11:00 & $2 \mathrm{of} 2$ & 0.19 & 0.01 & 12 & 0 & 1,700 & 0 & $<0.05$ & 0.01 & 2.1 & 0.3 & 17 & 1 \\
\hline BY23 & $\begin{array}{c}\text { Buckeye Flat Mine N } \\
\text { drain to Greenhorn } \\
\text { Cr, Grass Valley }\end{array}$ & $8 / 22 / 2000$ & $10: 45$ & 1 of 2 & 0.26 & 0.00 & 16 & 0 & 2,200 & 100 & 0.05 & 0.01 & 3.8 & 0.0 & 24 & 0 \\
\hline BY23 & $\begin{array}{c}\text { Buckeye Flat Mine N } \\
\text { drain to Greenhorn } \\
\text { Cr, Grass Valley }\end{array}$ & $8 / 22 / 2000$ & $10: 45$ & $2 \mathrm{of} 2$ & 0.24 & 0.01 & 15 & 1 & 2,300 & 200 & 0.09 & 0.06 & 3.8 & 0.0 & 24 & 0 \\
\hline BY116 & $\begin{array}{l}\text { Buckeye Flat Mine } \\
\text { pond drain } 0.15 \mathrm{mi} \\
\text { ab SF Greenhorn } \mathrm{Cr}\end{array}$ & $8 / 21 / 2000$ & $14: 10$ & 1 of 2 & $<0.001$ & 0.000 & 0.7 & 0.2 & 54 & 2 & $<0.04$ & 0.01 & 0.60 & 0.03 & 0.047 & 0.004 \\
\hline BY116 & $\begin{array}{l}\text { Buckeye Flat Mine } \\
\text { pond drain } 0.15 \mathrm{mi} \\
\text { ab SF Greenhorn } \mathrm{Cr}\end{array}$ & $8 / 21 / 2000$ & $14: 10$ & $2 \mathrm{of} 2$ & $<0.001$ & 0.001 & 0.8 & 0.1 & 53 & 0 & 0.07 & 0.06 & 0.61 & 0.03 & 0.050 & 0.007 \\
\hline BY24 & $\begin{array}{l}\text { Buckeye Flat Mine } \\
\text { S drain to SF } \\
\text { Greenhorn Cr, Grass } \\
\text { Valley }\end{array}$ & $8 / 26 / 1999$ & $11: 00$ & 1 of 2 & 0.0073 & 0.0001 & 0.54 & 0.08 & 140 & 10 & 0.06 & 0.04 & 0.60 & 0.06 & 0.46 & 0.01 \\
\hline BY24 & $\begin{array}{l}\text { Buckeye Flat Mine } \\
\text { S drain to SF } \\
\text { Greenhorn Cr, Grass } \\
\text { Valley }\end{array}$ & $8 / 26 / 1999$ & 11:00 & $2 \mathrm{of} 2$ & 0.0070 & 0.0000 & 0.54 & 0.11 & 140 & 10 & $<0.04$ & 0.01 & 0.56 & 0.08 & 0.42 & 0.02 \\
\hline BY24 & $\begin{array}{l}\text { Buckeye Flat Mine } \\
\text { S drain to SF } \\
\text { Greenhorn Cr, Grass } \\
\text { Valley }\end{array}$ & $8 / 22 / 2000$ & $12: 10$ & 1 of 2 & 0.005 & 0.000 & 0.6 & 0.1 & 100 & 0 & $<0.04$ & 0.02 & 0.68 & 0.03 & 0.32 & 0.01 \\
\hline BY24 & $\begin{array}{l}\text { Buckeye Flat Mine } \\
\text { S drain to SF } \\
\text { Greenhorn Cr, Grass } \\
\text { Valley }\end{array}$ & $8 / 22 / 2000$ & $12: 10$ & $2 \mathrm{of} 2$ & 0.005 & 0.000 & 0.6 & 0.2 & 100 & 0 & $<0.04$ & 0.02 & 0.68 & 0.02 & 0.32 & 0.01 \\
\hline BY25 & $\begin{array}{l}\text { Buckeye Flat Mine } \\
\text { upper drain }\end{array}$ & 4/9/2001 & $10: 00$ & 1 of 2 & 0.044 & 0.007 & 5.2 & 0.3 & 370 & 20 & $<0.2$ & 0.1 & 1.6 & 0.3 & 4.1 & 0.8 \\
\hline BY25 & $\begin{array}{l}\text { Buckeye Flat Mine } \\
\text { upper drain }\end{array}$ & $4 / 9 / 2001$ & $10: 00$ & $2 \mathrm{of} 2$ & 0.043 & 0.001 & 5.4 & 0.4 & 390 & 10 & $<0.2$ & 0.1 & 1.6 & 0.1 & 4.2 & 0.1 \\
\hline
\end{tabular}
upper drain 
Table 4B. Concentrations of trace metals and selected major elements in filtered water samples, Greenhorn Creek drainage, Nevada County, California—Continued.

[Station name abbreviations: ab, above; bl, below; Cr, creek; Cyn, Canyon; mi, miles; N, north; nr, near; Rd, Road; S, south; SF, South Fork. Number in parentheses is the data parameter code, a five-digit code used in the U.S. Geological Survey computerized data system. $\mu \mathrm{g} / \mathrm{L}$, microgram per liter (equivalent to part per billion); mg/L, milligram per liter (at low concentration, equivalent to part per million); s.d., standard deviation; <, less than; —, not determined]

\begin{tabular}{|c|c|c|c|c|c|c|c|c|c|c|c|c|c|c|c|c|}
\hline \multirow[t]{2}{*}{$\begin{array}{l}\text { Station } \\
\text { map ID }\end{array}$} & \multirow[t]{2}{*}{ Station name } & \multirow[t]{2}{*}{ Date } & \multirow[t]{2}{*}{ Time } & \multirow[t]{2}{*}{$\begin{array}{l}\text { Rep- } \\
\text { licate }\end{array}$} & \multicolumn{2}{|c|}{$\begin{array}{c}\text { Lutetium (Lu), } \\
\text { filtered } \\
(\mu \mathrm{g} / \mathrm{L}) \\
(62844)\end{array}$} & \multicolumn{2}{|c|}{$\begin{array}{c}\text { Magnesium (Mg), } \\
\text { filtered } \\
(\mathrm{mg} / \mathrm{L}) \\
(00925) \\
\end{array}$} & \multicolumn{2}{|c|}{$\begin{array}{c}\text { Manganese (Mn), } \\
\text { filtered } \\
(\mu \mathrm{g} / \mathrm{L}) \\
(01056) \\
\end{array}$} & \multicolumn{2}{|c|}{$\begin{array}{c}\text { Molybdenum (Mo), } \\
\text { filtered } \\
(\mu \mathrm{g} / \mathrm{L}) \\
(01060) \\
\end{array}$} & \multicolumn{2}{|c|}{$\begin{array}{c}\text { Sodium (Na), } \\
\text { filtered } \\
(\mathrm{mg} / \mathrm{L}) \\
(00930) \\
\end{array}$} & \multicolumn{2}{|c|}{$\begin{array}{c}\text { Neodymium (Nd), } \\
\text { filtered } \\
(\mu \mathrm{g} / \mathrm{L}) \\
(\mathbf{5 0 5 7 9 )} \\
\end{array}$} \\
\hline & & & & & value & s.d. & value & s.d. & value & s.d. & value & s.d. & value & s.d. & value & s.d. \\
\hline BY52 & $\begin{array}{l}\text { Greenhorn Cr } 0.3 \mathrm{mi} \\
\text { ab Buckeye Ford nr } \\
\text { Nevada City }\end{array}$ & $1 / 25 / 2000$ & $10: 45$ & 1of 2 & 0.0063 & 0.0009 & 1.2 & 0.1 & 27 & 0 & 0.07 & 0.01 & 1.7 & 0.1 & 0.45 & 0.01 \\
\hline BY52 & $\begin{array}{l}\text { Greenhorn Cr } 0.3 \mathrm{mi} \\
\text { ab Buckeye Ford nr } \\
\text { Nevada City }\end{array}$ & $1 / 25 / 2000$ & $10: 45$ & $2 o f 2$ & 0.0062 & 0.0012 & 1.1 & 0.1 & 28 & 1 & 0.09 & 0.04 & 1.7 & 0.1 & 0.42 & 0.01 \\
\hline BY59 & $\begin{array}{l}\text { Greenhorn Cr at You } \\
\text { Bet Rd nr Nevada } \\
\text { City }\end{array}$ & $7 / 24 / 2001$ & $11: 00$ & 1 of 2 & 0.0002 & 0.0001 & 2.5 & 0.0 & 110 & 0 & 0.25 & 0.03 & 2.3 & 0.1 & 0.012 & 0.000 \\
\hline BY59 & $\begin{array}{l}\text { Greenhorn } \mathrm{Cr} \text { at You } \\
\text { Bet Rd nr Nevada } \\
\text { City }\end{array}$ & $7 / 24 / 2001$ & $11: 00$ & 2 of 2 & 0.0003 & 0.0001 & 2.5 & 0.0 & 120 & 0 & 0.18 & 0.02 & 2.3 & 0.1 & 0.0099 & 0.0016 \\
\hline BY59 & $\begin{array}{l}\text { Greenhorn } \mathrm{Cr} \text { at You } \\
\text { Bet Rd nr Nevada } \\
\text { City }\end{array}$ & $8 / 29 / 2001$ & $15: 00$ & 1 of 2 & 0.0002 & 0.0001 & 2.2 & 0.1 & 28 & 0 & 0.12 & 0.01 & 2.5 & 0.0 & 0.0095 & 0.0000 \\
\hline BY59 & $\begin{array}{l}\text { Greenhorn } \mathrm{Cr} \text { at You } \\
\text { Bet Rd nr Nevada } \\
\text { City }\end{array}$ & $8 / 29 / 2001$ & $15: 00$ & $2 \mathrm{of} 2$ & 0.0002 & 0.0001 & 2.3 & 0.0 & 29 & 0 & 0.14 & 0.02 & 2.6 & 0.1 & 0.011 & 0.001 \\
\hline BY59 & $\begin{array}{l}\text { Greenhorn Cr at You } \\
\text { Bet Rd nr Nevada } \\
\text { City }\end{array}$ & $9 / 14 / 2001$ & $13: 00$ & 1 of 2 & $<0.0001$ & 0.0001 & 2.2 & 0.0 & 53 & 0 & 0.15 & 0.04 & 2.1 & 0.1 & 0.0079 & 0.0006 \\
\hline BY59 & $\begin{array}{l}\text { Greenhorn } \mathrm{Cr} \text { at You } \\
\text { Bet Rd nr Nevada } \\
\text { City }\end{array}$ & $9 / 14 / 2001$ & $13: 00$ & $2 \mathrm{of} 2$ & 0.0002 & 0.0001 & 2.2 & 0.0 & 54 & 0 & 0.18 & 0.05 & 2.1 & 0.0 & 0.0091 & 0.0006 \\
\hline BY59 & $\begin{array}{l}\text { Greenhorn Cr at You } \\
\text { Bet Rd nr Nevada } \\
\text { City }\end{array}$ & $10 / 15 / 2001$ & $12: 00$ & 1 of 2 & $<0.0001$ & 0.0000 & 2.2 & 0.0 & 55 & 0 & 0.13 & 0.06 & 2.3 & 0.1 & 0.0062 & 0.0008 \\
\hline BY59 & $\begin{array}{l}\text { Greenhorn Cr at You } \\
\text { Bet Rd nr Nevada } \\
\text { City }\end{array}$ & $10 / 15 / 2001$ & $12: 00$ & $2 o f 2$ & 0.0001 & 0.0001 & 2.3 & 0.1 & 53 & 0 & 0.09 & 0.02 & 2.4 & 0.0 & 0.0077 & 0.0008 \\
\hline BY59 & $\begin{array}{l}\text { Greenhorn Cr at You } \\
\text { Bet Rd nr Nevada } \\
\text { City }\end{array}$ & $11 / 20 / 2001$ & $13: 20$ & 1 of 2 & 0.0009 & 0.0001 & 2.6 & 0.1 & 200 & 0 & 0.14 & 0.05 & 2.2 & 0.1 & 0.057 & 0.001 \\
\hline BY59 & $\begin{array}{l}\text { Greenhorn Cr at You } \\
\text { Bet Rd nr Nevada } \\
\text { City }\end{array}$ & $11 / 20 / 2001$ & $13: 20$ & $2 \mathrm{of} 2$ & 0.0010 & 0.0001 & 2.6 & 0.0 & 190 & 0 & 0.11 & 0.02 & 2.2 & 0.0 & 0.059 & 0.000 \\
\hline BY59 & $\begin{array}{l}\text { Greenhorn Cr at You } \\
\text { Bet Rd nr Nevada } \\
\text { City }\end{array}$ & $12 / 10 / 2001$ & $12: 20$ & 1 of 2 & 0.0026 & 0.0002 & 2.4 & 0.0 & 98 & 3 & 0.18 & 0.04 & 2.3 & 0.0 & 0.18 & 0.00 \\
\hline
\end{tabular}


Table 4B. Concentrations of trace metals and selected major elements in filtered water samples, Greenhorn Creek drainage, Nevada County, California—Continued.

[Station name abbreviations: ab, above; bl, below; Cr, creek; Cyn, Canyon; mi, miles; N, north; nr, near; Rd, Road; S, south; SF, South Fork. Number in parentheses is the data parameter code, a five-digit code used in the U.S. Geological Survey computerized data system. $\mu \mathrm{g} / \mathrm{L}$, microgram per liter (equivalent to part per billion); mg/L, milligram per liter (at low concentration, equivalent to part per million); s.d., standard deviation; <, less than; - , not determined]

\begin{tabular}{|c|c|c|c|c|c|c|c|c|c|c|c|c|c|c|c|c|}
\hline \multirow[t]{2}{*}{$\begin{array}{l}\text { Station } \\
\text { map ID }\end{array}$} & \multirow[t]{2}{*}{ Station name } & \multirow[t]{2}{*}{ Date } & \multirow[t]{2}{*}{ Time } & \multirow[t]{2}{*}{$\begin{array}{l}\text { Rep- } \\
\text { licate }\end{array}$} & \multicolumn{2}{|c|}{$\begin{array}{c}\text { Lutetium (Lu), } \\
\text { filtered } \\
(\mu \mathrm{g} / \mathrm{L}) \\
(\mathbf{6 2 8 4 4 )} \\
\end{array}$} & \multicolumn{2}{|c|}{$\begin{array}{c}\text { Magnesium (Mg), } \\
\text { filtered } \\
(\mathrm{mg} / \mathrm{L}) \\
(00925) \\
\end{array}$} & \multicolumn{2}{|c|}{$\begin{array}{c}\text { Manganese (Mn), } \\
\text { filtered } \\
(\mu \mathrm{g} / \mathrm{L}) \\
(\mathbf{0 1 0 5 6 )} \\
\end{array}$} & \multicolumn{2}{|c|}{$\begin{array}{c}\text { Molybdenum (Mo), } \\
\text { filtered } \\
(\mu \mathrm{g} / \mathrm{L}) \\
(01060)\end{array}$} & \multicolumn{2}{|c|}{$\begin{array}{c}\text { Sodium (Na), } \\
\text { filtered } \\
(\mathrm{mg} / \mathrm{L}) \\
(00930)\end{array}$} & \multicolumn{2}{|c|}{$\begin{array}{c}\text { Neodymium (Nd), } \\
\text { filtered } \\
(\mu \mathrm{g} / \mathrm{L}) \\
(\mathbf{5 0 5 7 9 )}\end{array}$} \\
\hline & & & & & value & s.d. & value & s.d. & value & s.d. & value & s.d. & value & s.d. & value & s.d. \\
\hline BY59 & $\begin{array}{l}\text { Greenhorn Cr at You } \\
\text { Bet Rd nr Nevada } \\
\text { City }\end{array}$ & $12 / 10 / 2001$ & $12: 20$ & 2 of 2 & 0.0024 & 0.0002 & 2.4 & 0.0 & 100 & 0 & 0.16 & 0.02 & 2.4 & 0.1 & 0.19 & 0.00 \\
\hline BY59 & $\begin{array}{l}\text { Greenhorn } \mathrm{Cr} \text { at You } \\
\text { Bet Rd nr Nevada } \\
\text { City }\end{array}$ & $12 / 21 / 2001$ & $13: 00$ & $1 \mathrm{of} 2$ & 0.0059 & 0.0002 & 1.9 & 0.0 & 76 & 0 & 0.16 & 0.05 & 1.7 & 0.1 & 0.39 & 0.00 \\
\hline BY59 & $\begin{array}{l}\text { Greenhorn Cr at You } \\
\text { Bet Rd nr Nevada } \\
\text { City }\end{array}$ & $12 / 21 / 2001$ & $13: 00$ & $2 \mathrm{of} 2$ & 0.0061 & 0.0009 & 1.9 & 0.0 & 73 & 0 & 0.20 & 0.11 & 1.7 & 0.1 & 0.37 & 0.00 \\
\hline BY51 & $\begin{array}{l}\text { Greenhorn Cr nr } \\
\text { Headwaters nr Scotts } \\
\text { Flat Reservoir }\end{array}$ & $1 / 25 / 2000$ & $9: 30$ & $1 \mathrm{of} 2$ & 0.0045 & 0.0001 & 0.61 & 0.03 & 1.9 & 0.1 & 0.05 & 0.02 & 1.4 & 0.1 & 0.26 & 0.00 \\
\hline BY51 & $\begin{array}{l}\text { Greenhorn Cr nr } \\
\text { Headwaters nr Scotts } \\
\text { Flat Reservoir }\end{array}$ & $1 / 25 / 2000$ & $9: 30$ & $2 \mathrm{of} 2$ & 0.0035 & 0.0009 & 0.63 & 0.02 & 1.9 & 0.5 & 0.05 & 0.03 & 1.2 & 0.1 & 0.26 & 0.00 \\
\hline BY75 & $\begin{array}{l}\text { Missouri Cyn Cr } 1.2 \mathrm{mi} \\
\text { ab Greenhorn Cr nr } \\
\text { Chicago Park }\end{array}$ & $12 / 14 / 1999$ & $14: 00$ & $1 \mathrm{of} 2$ & 0.0048 & 0.0002 & 3.9 & 0.4 & 98 & 3 & 0.14 & 0.05 & 2.7 & 0.4 & 0.30 & 0.00 \\
\hline BY75 & $\begin{array}{l}\text { Missouri Cyn Cr } 1.2 \mathrm{mi} \\
\text { ab Greenhorn Cr nr } \\
\text { Chicago Park }\end{array}$ & $12 / 14 / 1999$ & $14: 00$ & $2 \mathrm{of} 2$ & 0.0049 & 0.0001 & 5.0 & 0.9 & 99 & 3 & 0.13 & 0.04 & 2.9 & 0.3 & 0.30 & 0.03 \\
\hline BY86 & $\begin{array}{l}\text { Poore Mine creek ab } \\
\text { tunnel nr Grass } \\
\text { Valley }\end{array}$ & 3/31/1999 & $17: 40$ & $1 \mathrm{of} 2$ & 0.0013 & 0.0002 & 1.9 & 0.4 & 2.4 & 0.2 & 0.05 & 0.01 & 2.3 & 0.2 & 0.078 & 0.005 \\
\hline BY86 & $\begin{array}{l}\text { Poore Mine creek ab } \\
\text { tunnel nr Grass } \\
\text { Valley }\end{array}$ & $3 / 31 / 1999$ & $17: 40$ & $2 \mathrm{of} 2$ & 0.0013 & 0.0002 & 1.8 & 0.2 & 2.3 & 0.2 & 0.04 & 0.00 & 2.1 & 0.0 & 0.076 & 0.002 \\
\hline BY87 & $\begin{array}{l}\text { Poore Mine creek bl } \\
\text { tunnel nr Grass } \\
\text { Valley }\end{array}$ & 3/31/1999 & $13: 30$ & $1 \mathrm{of} 2$ & 0.0016 & 0.0004 & 1.8 & 0.1 & 1.4 & 0.0 & 0.09 & 0.04 & 2.0 & 0.1 & 0.082 & 0.006 \\
\hline BY87 & $\begin{array}{l}\text { Poore Mine creek bl } \\
\text { tunnel nr Grass } \\
\text { Valley }\end{array}$ & $3 / 31 / 1999$ & $13: 30$ & $2 \mathrm{of} 2$ & 0.0014 & 0.0005 & 1.6 & 0.0 & 1.4 & 0.1 & 0.07 & 0.02 & 2.0 & 0.1 & 0.078 & 0.010 \\
\hline BY88 & $\begin{array}{l}\text { Poore Mine ground } \\
\text { sluice nr Grass } \\
\text { Valley }\end{array}$ & 4/1/1999 & $15: 40$ & $1 \mathrm{of} 2$ & 0.0032 & 0.0004 & 3.7 & 0.1 & 430 & 30 & 0.03 & 0.02 & 1.8 & 0.0 & 0.10 & 0.01 \\
\hline BY88 & $\begin{array}{l}\text { Poore Mine ground } \\
\text { sluice nr Grass } \\
\text { Valley }\end{array}$ & 4/1/1999 & $15: 40$ & $2 \mathrm{of} 2$ & 0.0032 & 0.0005 & 3.7 & 0.0 & 440 & 50 & 0.069 & 0.042 & 1.9 & 0.0 & 0.11 & 0.01 \\
\hline
\end{tabular}


Table 4B. Concentrations of trace metals and selected major elements in filtered water samples, Greenhorn Creek drainage, Nevada County, California—Continued.

[Station name abbreviations: ab, above; bl, below; Cr, creek; Cyn, Canyon; mi, miles; N, north; nr, near; Rd, Road; S, south; SF, South Fork. Number in parentheses is the data parameter code, a five-digit code used in the U.S. Geological Survey computerized data system. $\mu \mathrm{g} / \mathrm{L}$, microgram per liter (equivalent to part per billion); mg/L, milligram per liter (at low concentration, equivalent to part per million); s.d., standard deviation; <, less than; —, not determined]

\begin{tabular}{|c|c|c|c|c|c|c|c|c|c|c|c|c|c|c|c|c|}
\hline \multirow[t]{2}{*}{$\begin{array}{l}\text { Station } \\
\text { map ID }\end{array}$} & \multirow[t]{2}{*}{ Station name } & \multirow[t]{2}{*}{ Date } & \multirow[t]{2}{*}{ Time } & \multirow[t]{2}{*}{$\begin{array}{l}\text { Rep- } \\
\text { licate }\end{array}$} & \multicolumn{2}{|c|}{$\begin{array}{c}\text { Lutetium (Lu), } \\
\text { filtered } \\
(\mu \mathrm{g} / \mathrm{L}) \\
(\mathbf{6 2 8 4 4 )} \\
\end{array}$} & \multicolumn{2}{|c|}{$\begin{array}{c}\text { Magnesium (Mg), } \\
\text { filtered } \\
(\mathrm{mg} / \mathrm{L}) \\
(00925) \\
\end{array}$} & \multicolumn{2}{|c|}{$\begin{array}{c}\text { Manganese }(\mathrm{Mn}), \\
\text { filtered } \\
(\mu \mathrm{g} / \mathrm{L}) \\
(01056) \\
\end{array}$} & \multicolumn{2}{|c|}{$\begin{array}{c}\text { Molybdenum (Mo), } \\
\text { filtered } \\
(\mu \mathrm{g} / \mathrm{L}) \\
(\mathbf{0 1 0 6 0 )} \\
\end{array}$} & \multicolumn{2}{|c|}{$\begin{array}{c}\text { Sodium (Na), } \\
\text { filtered } \\
(\mathrm{mg} / \mathrm{L}) \\
(00930)\end{array}$} & \multicolumn{2}{|c|}{$\begin{array}{c}\text { Neodymium (Nd), } \\
\text { filtered } \\
(\mu \mathrm{g} / \mathrm{L}) \\
(\mathbf{5 0 5 7 9 )}\end{array}$} \\
\hline & & & & & value & s.d. & value & s.d. & value & s.d. & value & s.d. & value & s.d. & value & s.d. \\
\hline BY89 & $\begin{array}{c}\text { Poore Mine pit lake } \\
\text { nr Grass Valley }\end{array}$ & $4 / 1 / 1999$ & $16: 50$ & 1of 2 & 0.0009 & 0.0002 & 1.1 & 0.0 & 130 & 0 & 0.032 & 0.025 & 0.90 & 0.08 & 0.037 & 0.003 \\
\hline BY89 & $\begin{array}{l}\text { Poore Mine pit lake } \\
\text { nr Grass Valley }\end{array}$ & 4/1/1999 & $16: 50$ & $2 \mathrm{of} 2$ & 0.0007 & 0.0000 & 1.1 & 0.0 & 99 & 9 & 0.13 & 0.02 & 0.88 & 0.11 & 0.031 & 0.004 \\
\hline BY89 & $\begin{array}{l}\text { Poore Mine pit lake } \\
\text { nr Grass Valley }\end{array}$ & $5 / 23 / 2000$ & $10: 45$ & $1 \mathrm{of} 2$ & 0.0030 & 0.0004 & 6.5 & 0.4 & 1,300 & 100 & 0.04 & 0.01 & 1.5 & 0.1 & 0.15 & 0.00 \\
\hline BY89 & $\begin{array}{l}\text { Poore Mine pit lake } \\
\text { nr Grass Valley }\end{array}$ & $5 / 23 / 2000$ & $10: 45$ & $2 \mathrm{of} 2$ & 0.0026 & 0.0007 & 6.8 & 0.3 & 1,300 & 0 & 0.02 & 0.01 & 1.4 & 0.1 & 0.15 & 0.00 \\
\hline BY90 & $\begin{array}{l}\text { Poore Mine seep } \\
\text { ab ground sluice } \\
\text { nr Grass Valley }\end{array}$ & 4/1/1999 & $16: 00$ & 1 of 2 & 0.0014 & 0.0003 & 3.5 & 0.1 & 390 & 10 & 0.052 & 0.032 & 1.8 & 0.4 & 0.031 & 0.001 \\
\hline BY90 & $\begin{array}{l}\text { Poore Mine seep } \\
\text { ab ground sluice } \\
\text { nr Grass Valley }\end{array}$ & 4/1/1999 & $16: 00$ & $2 \mathrm{of} 2$ & 0.0012 & 0.0002 & 3.5 & 0.0 & 430 & 10 & 0.033 & 0.018 & 1.8 & 0.1 & 0.031 & 0.004 \\
\hline BY91 & $\begin{array}{l}\text { Poore Mine tunnel } \\
\text { effluent nr Grass } \\
\text { Valley }\end{array}$ & 3/31/1999 & $15: 20$ & 1 of 2 & $<0.0002$ & 0.0001 & 4.1 & 0.1 & 6.9 & 0.2 & 0.14 & 0.08 & 1.9 & 0.0 & 0.013 & 0.004 \\
\hline BY91 & $\begin{array}{l}\text { Poore Mine tunnel } \\
\text { effluent nr Grass } \\
\text { Valley }\end{array}$ & 3/31/1999 & $15: 20$ & $2 \mathrm{of} 2$ & 0.0003 & 0.0002 & 3.8 & 0.1 & 7.3 & 0.6 & 0.086 & 0.020 & 1.8 & 0.0 & 0.012 & 0.000 \\
\hline BY91 & $\begin{array}{l}\text { Poore Mine tunnel } \\
\text { effluent nr Grass } \\
\text { Valley }\end{array}$ & $12 / 15 / 1999$ & $13: 00$ & 1 of 2 & $<0.0001$ & 0.0000 & 5.7 & 0.4 & 0.91 & 0.08 & 0.42 & 0.03 & 2.9 & 0.0 & 0.0031 & 0.0008 \\
\hline BY91 & $\begin{array}{l}\text { Poore Mine tunnel } \\
\text { effluent nr Grass } \\
\text { Valley }\end{array}$ & $12 / 15 / 1999$ & $13: 00$ & $2 \mathrm{of} 2$ & $<0.0001$ & 0.0001 & 6.3 & 0.6 & 0.72 & 0.01 & 0.49 & 0.08 & 3.1 & 1.0 & 0.0046 & 0.0003 \\
\hline BY91 & $\begin{array}{l}\text { Poore Mine tunnel } \\
\text { effluent nr Grass } \\
\text { Valley }\end{array}$ & $5 / 23 / 2000$ & $12: 00$ & $1 \mathrm{of} 2$ & $<0.0002$ & 0.0003 & 5.5 & 0.3 & 4.0 & 0.0 & 0.13 & 0.04 & 3.5 & 0.2 & $<0.004$ & 0.003 \\
\hline BY91 & $\begin{array}{l}\text { Poore Mine tunnel } \\
\text { effluent nr Grass } \\
\text { Valley }\end{array}$ & $5 / 23 / 2000$ & $12: 00$ & $2 \mathrm{of} 2$ & $<0.0007$ & 0.0003 & 5.2 & 0.1 & 4.4 & 0.1 & 0.12 & 0.02 & 3.6 & 0.1 & $<0.007$ & 0.002 \\
\hline BY105 & $\begin{array}{l}\text { Sailor Flat Mine main } \\
\text { drain Gulch 01nr } \\
\text { Quaker Hill }\end{array}$ & 7/6/1999 & $16: 00$ & 1 of 2 & 0.020 & 0.001 & 2.1 & 0.1 & 36 & 0 & 0.14 & 0.01 & 1.7 & 0.1 & 0.90 & 0.01 \\
\hline BY105 & $\begin{array}{l}\text { Sailor Flat Mine main } \\
\text { drain Gulch 01nr } \\
\text { Quaker Hill }\end{array}$ & 7/6/1999 & $16: 00$ & $2 \mathrm{of} 2$ & 0.020 & 0.001 & 1.9 & 0.0 & 37 & 3 & 0.14 & 0.01 & 1.6 & 0.0 & 0.90 & 0.07 \\
\hline
\end{tabular}


Table 4B. Concentrations of trace metals and selected major elements in filtered water samples, Greenhorn Creek drainage, Nevada County, California—Continued.

[Station name abbreviations: ab, above; bl, below; Cr, creek; Cyn, Canyon; mi, miles; N, north; nr, near; Rd, Road; S, south; SF, South Fork. Number in parentheses is the data parameter code, a five-digit code used in the U.S. Geological Survey computerized data system. $\mu \mathrm{g} / \mathrm{L}$, microgram per liter (equivalent to part per billion); mg/L, milligram per liter (at low concentration, equivalent to part per million); s.d., standard deviation; <, less than; - , not determined]

\begin{tabular}{|c|c|c|c|c|c|c|c|c|c|c|c|c|c|c|c|c|}
\hline \multirow[t]{2}{*}{$\begin{array}{l}\text { Station } \\
\text { map ID }\end{array}$} & \multirow[t]{2}{*}{ Station name } & \multirow[t]{2}{*}{ Date } & \multirow[t]{2}{*}{ Time } & \multirow[t]{2}{*}{$\begin{array}{l}\text { Rep- } \\
\text { licate }\end{array}$} & \multicolumn{2}{|c|}{$\begin{array}{c}\text { Lutetium (Lu), } \\
\text { filtered } \\
(\mu \mathrm{g} / \mathrm{L}) \\
(\mathbf{6 2 8 4 4 )} \\
\end{array}$} & \multicolumn{2}{|c|}{$\begin{array}{l}\text { Magnesium (Mg), } \\
\text { filtered } \\
(\mathrm{mg} / \mathrm{L}) \\
(00925) \\
\end{array}$} & \multicolumn{2}{|c|}{$\begin{array}{c}\text { Manganese (Mn), } \\
\text { filtered } \\
(\mu \mathrm{g} / \mathrm{L}) \\
(01056) \\
\end{array}$} & \multicolumn{2}{|c|}{$\begin{array}{c}\text { Molybdenum (Mo), } \\
\text { filtered } \\
(\mu \mathrm{g} / \mathrm{L}) \\
(01060)\end{array}$} & \multicolumn{2}{|c|}{$\begin{array}{c}\text { Sodium (Na), } \\
\text { filtered } \\
(\mathrm{mg} / \mathrm{L}) \\
(00930)\end{array}$} & \multicolumn{2}{|c|}{$\begin{array}{c}\text { Neodymium (Nd), } \\
\text { filtered } \\
(\mu \mathrm{g} / \mathrm{L}) \\
(50579)\end{array}$} \\
\hline & & & & & value & s.d. & value & s.d. & value & s.d. & value & s.d. & value & s.d. & value & s.d. \\
\hline BY105 & $\begin{array}{l}\text { Sailor Flat Mine main } \\
\text { drain Gulch 01nr } \\
\text { Quaker Hill }\end{array}$ & $12 / 2 / 1999$ & $14: 30$ & 1 of 2 & 0.030 & 0.002 & 0.71 & 0.03 & 25 & 0 & 0.09 & 0.00 & 1.2 & 0.1 & 1.7 & 0.1 \\
\hline BY105 & $\begin{array}{l}\text { Sailor Flat Mine main } \\
\text { drain Gulch 01nr } \\
\text { Quaker Hill }\end{array}$ & $12 / 2 / 1999$ & $14: 30$ & $2 \mathrm{of} 2$ & 0.031 & 0.002 & 0.67 & 0.10 & 24 & 0 & 0.08 & 0.03 & 1.0 & 0.0 & 1.8 & 0.0 \\
\hline BY106 & $\begin{array}{l}\text { Sailor Flat Mine } \\
\text { main drainage to } \\
\text { Greenhorn Cr, } \\
\text { Gulch } 03\end{array}$ & 4/9/2001 & $12: 30$ & 1 of 2 & 0.023 & 0.006 & 0.37 & 0.03 & 22 & 5 & $<0.2$ & 0.2 & 0.92 & 0.21 & 1.2 & 0.3 \\
\hline BY106 & $\begin{array}{l}\text { Sailor Flat Mine } \\
\text { main drainage to } \\
\text { Greenhorn Cr, } \\
\text { Gulch } 03\end{array}$ & 4/9/2001 & $12: 30$ & 2of 2 & 0.025 & 0.005 & 0.36 & 0.03 & 22 & 4 & $<0.2$ & 0.1 & 0.93 & 0.20 & 1.2 & 0.2 \\
\hline BY114 & $\begin{array}{l}\text { SF Greenhorn Cr } \\
0.7 \text { mi ab Greenhorn } \\
\text { Cr nr Nevada City }\end{array}$ & $8 / 22 / 2000$ & $15: 10$ & 1 of 2 & $<0.001$ & 0.000 & 1.9 & 0.1 & 0.5 & 0.0 & 0.19 & 0.09 & 2.2 & 0.0 & 0.017 & 0.008 \\
\hline BY114 & $\begin{array}{l}\text { SF Greenhorn Cr } \\
0.7 \text { mi ab Greenhorn } \\
\text { Cr nr Nevada City }\end{array}$ & $8 / 22 / 2000$ & $15: 10$ & $2 \mathrm{of} 2$ & $<0.001$ & 0.001 & 1.8 & 0.1 & 0.5 & 0.0 & 0.14 & 0.06 & 2.1 & 0.0 & $<0.01$ & 0.01 \\
\hline BY122 & $\begin{array}{l}\text { Starr Mine tunnel } \\
\text { inflow nr Grass } \\
\text { Valley }\end{array}$ & 4/23/1999 & $15: 00$ & 1 of 2 & 0.0015 & 0.0003 & 4.6 & 0.0 & 39 & 5 & 0.049 & 0.006 & 1.5 & 0.0 & 0.10 & 0.00 \\
\hline BY122 & $\begin{array}{l}\text { Starr Mine tunnel } \\
\text { inflow nr Grass } \\
\text { Valley }\end{array}$ & 4/23/1999 & $15: 00$ & $2 o f 2$ & 0.0013 & 0.0001 & 4.7 & 0.2 & 38 & 4 & 0.053 & 0.035 & 1.7 & 0.3 & 0.097 & 0.009 \\
\hline BY123 & $\begin{array}{l}\text { Starr Mine tunnel } \\
\text { midway nr Grass } \\
\text { Valley }\end{array}$ & 4/23/1999 & $13: 40$ & 1 of 2 & $<0.0001$ & 0.0001 & 9.1 & 0.2 & 11 & 1 & 0.22 & 0.02 & 2.3 & 0.0 & 0.0016 & 0.0000 \\
\hline BY123 & $\begin{array}{l}\text { Starr Mine tunnel } \\
\text { midway nr Grass } \\
\text { Valley }\end{array}$ & 4/23/1999 & $13: 40$ & $2 \mathrm{of} 2$ & $<0.0001$ & 0.0001 & 8.8 & 0.0 & 12 & 1 & 0.21 & 0.01 & 2.2 & 0.0 & 0.0015 & 0.0005 \\
\hline BY123 & $\begin{array}{l}\text { Starr Mine tunnel } \\
\text { midway nr Grass } \\
\text { Valley }\end{array}$ & 12/15/1999 & $10: 45$ & 1 of 2 & 0.0001 & 0.0001 & 9.6 & 0.1 & 72 & 4 & 0.27 & 0.07 & 3.4 & 0.1 & 0.0023 & 0.0004 \\
\hline BY123 & $\begin{array}{l}\text { Starr Mine tunnel } \\
\text { midway nr Grass } \\
\text { Valley }\end{array}$ & 12/15/1999 & $10: 45$ & $2 \mathrm{of} 2$ & $<0.0001$ & 0.0002 & 11 & 0 & 74 & 1 & 0.23 & 0.03 & 2.4 & 0.1 & 0.0028 & 0.0007 \\
\hline
\end{tabular}


Table 4B. Concentrations of trace metals and selected major elements in filtered water samples, Greenhorn Creek drainage, Nevada County, California—Continued.

[Station name abbreviations: ab, above; bl, below; Cr, creek; Cyn, Canyon; mi, miles; N, north; nr, near; Rd, Road; S, south; SF, South Fork. Number in parentheses is the data parameter code, a five-digit code used in the U.S. Geological Survey computerized data system. $\mu \mathrm{g} / \mathrm{L}$, microgram per liter (equivalent to part per billion); mg/L, milligram per liter (at low concentration, equivalent to part per million); s.d., standard deviation; <, less than; - , not determined]

\begin{tabular}{|c|c|c|c|c|c|c|c|c|c|c|c|c|c|c|c|c|}
\hline \multirow[t]{2}{*}{$\begin{array}{l}\text { Station } \\
\text { map ID }\end{array}$} & \multirow[t]{2}{*}{ Station name } & \multirow[t]{2}{*}{ Date } & \multirow[t]{2}{*}{ Time } & \multirow[t]{2}{*}{$\begin{array}{l}\text { Rep- } \\
\text { licate }\end{array}$} & \multicolumn{2}{|c|}{$\begin{array}{c}\text { Lutetium (Lu), } \\
\text { filtered } \\
(\mu \mathrm{g} / \mathrm{L}) \\
(62844)\end{array}$} & \multicolumn{2}{|c|}{$\begin{array}{c}\text { Magnesium (Mg), } \\
\text { filtered } \\
(\mathrm{mg} / \mathrm{L}) \\
(00925)\end{array}$} & \multicolumn{2}{|c|}{$\begin{array}{c}\text { Manganese (Mn), } \\
\text { filtered } \\
(\mu \mathrm{g} / \mathrm{L}) \\
(01056)\end{array}$} & \multicolumn{2}{|c|}{$\begin{array}{c}\text { Molybdenum (Mo), } \\
\text { filtered } \\
(\mu \mathrm{g} / \mathrm{L}) \\
(01060)\end{array}$} & \multicolumn{2}{|c|}{$\begin{array}{c}\text { Sodium (Na), } \\
\text { filtered } \\
(\mathrm{mg} / \mathrm{L}) \\
(00930)\end{array}$} & \multicolumn{2}{|c|}{$\begin{array}{c}\text { Neodymium (Nd), } \\
\text { filtered } \\
(\mu \mathrm{g} / \mathrm{L}) \\
(50579)\end{array}$} \\
\hline & & & & & value & s.d. & value & s.d. & value & s.d. & value & s.d. & value & s.d. & value & s.d. \\
\hline BY124 & $\begin{array}{l}\text { Starr Mine tunnel outlet } \\
\text { nr Grass Valley }\end{array}$ & $8 / 23 / 2000$ & $17: 30$ & 1of 2 & $<0.001$ & 0.000 & 8 & 2 & 0.2 & 0.0 & 0.44 & 0.07 & 2.8 & 0.1 & $<0.01$ & 0.00 \\
\hline BY124 & $\begin{array}{l}\text { Starr Mine tunnel outlet } \\
\text { nr Grass Valley }\end{array}$ & $8 / 23 / 2000$ & $17: 30$ & $2 o f 2$ & $<0.001$ & 0.001 & 10 & 1 & 0.2 & 0.0 & 0.42 & 0.03 & 2.7 & 0.1 & $<0.01$ & 0.00 \\
\hline BY129 & $\begin{array}{l}\text { Tom and Jerry Mine } \\
\text { drain at tunnel inlet } \\
\text { nr Nevada City }\end{array}$ & $6 / 28 / 2000$ & $12: 00$ & 1 of 2 & $<0.0008$ & 0.0005 & 1.3 & 0.3 & $<0.3$ & 0.1 & $<0.06$ & 0.05 & 1.1 & 0.1 & 0.030 & 0.002 \\
\hline BY129 & $\begin{array}{l}\text { Tom and Jerry Mine } \\
\text { drain at tunnel inlet } \\
\text { nr Nevada City }\end{array}$ & $6 / 28 / 2000$ & $12: 00$ & $2 o f 2$ & $<0.0008$ & 0.0005 & 1.5 & 0.5 & $<0.3$ & 0.1 & 0.08 & 0.10 & 1.1 & 0.1 & 0.031 & 0.006 \\
\hline BY130 & $\begin{array}{l}\text { Tom and Jerry Mine } \\
\text { drain at tunnel outlet } \\
\text { nr Nevada City }\end{array}$ & $6 / 28 / 2000$ & $11: 30$ & 1 of 2 & $<0.0008$ & 0.0003 & 5.4 & 1.8 & 90 & 4 & 0.10 & 0.07 & 1.7 & 0.1 & $<0.004$ & 0.005 \\
\hline BY130 & $\begin{array}{l}\text { Tom and Jerry Mine } \\
\text { drain at tunnel outlet } \\
\text { nr Nevada City }\end{array}$ & $6 / 28 / 2000$ & $11: 30$ & $2 o f 2$ & $<0.0008$ & 0.0007 & 6.3 & 0.6 & 91 & 1 & 0.07 & 0.03 & 1.7 & 0.1 & 0.007 & 0.001 \\
\hline BY131 & $\begin{array}{l}\text { Tom and Jerry Mine } \\
\text { drainage pond nr } \\
\text { Nevada City }\end{array}$ & $8 / 23 / 2000$ & $10: 45$ & 1 of 2 & 0.001 & 0.001 & 2.1 & 0.1 & 3.6 & 0.1 & $<0.04$ & 0.02 & 2.3 & 0.1 & 0.043 & 0.007 \\
\hline BY131 & $\begin{array}{l}\text { Tom and Jerry Mine } \\
\text { drainage pond nr } \\
\text { Nevada City }\end{array}$ & $8 / 23 / 2000$ & $10: 45$ & $2 o f 2$ & $<0.001$ & 0.001 & 1.9 & 0.3 & 3.9 & 0.1 & 0.06 & 0.04 & 2.2 & 0.1 & 0.042 & 0.002 \\
\hline \multicolumn{17}{|c|}{ Disturbed samples } \\
\hline BY123 & $\begin{array}{l}\text { Starr Mine tunnel } \\
\text { midway nr Grass } \\
\text { Valley }\end{array}$ & $12 / 15 / 1999$ & $11: 30$ & 1 of 2 & 0.0001 & 0.0000 & 9.7 & 0.5 & 0.29 & 0.02 & 0.36 & 0.00 & 2.5 & 0.3 & 0.0017 & 0.0003 \\
\hline BY123 & $\begin{array}{l}\text { Starr Mine tunnel } \\
\text { midway nr Grass } \\
\text { Valley }\end{array}$ & $12 / 15 / 1999$ & $11: 30$ & $2 o f 2$ & 0.0002 & 0.0001 & 12 & 0 & 0.28 & 0.02 & 0.37 & 0.00 & 2.7 & 0.4 & 0.0011 & 0.0005 \\
\hline BY129 & $\begin{array}{l}\text { Tom and Jerry Mine } \\
\text { drain at tunnel inlet } \\
\text { nr Nevada City }\end{array}$ & $6 / 28 / 2000$ & $12: 30$ & 1 of 2 & $<0.0008$ & 0.0003 & 5.5 & 1.2 & 0.4 & 0.1 & $<0.06$ & 0.01 & 1.5 & 0.0 & 0.036 & 0.004 \\
\hline BY129 & $\begin{array}{l}\text { Tom and Jerry Mine } \\
\text { drain at tunnel inlet } \\
\text { nr Nevada City }\end{array}$ & $6 / 28 / 2000$ & $12: 30$ & $2 o f 2$ & $<0.0008$ & 0.0001 & 5.7 & 0.1 & 0.5 & 0.1 & 0.08 & 0.02 & 1.4 & 0.1 & 0.041 & 0.004 \\
\hline
\end{tabular}


Table 4B. Concentrations of trace metals and selected major elements in filtered water samples, Greenhorn Creek drainage, Nevada County, California—Continued.

[Station name abbreviations: ab, above; bl, below; Cr, creek; Cyn, Canyon; mi, miles; N, north; nr, near; Rd, Road; S, south; SF, South Fork. Number in parentheses is the data parameter code, a five-digit code used in the U.S. Geological Survey computerized data system. $\mu \mathrm{g} / \mathrm{L}$, microgram per liter (equivalent to part per billion); $\mathrm{mg} / \mathrm{L}$, milligram per liter (at low concentration, equivalent to part per million); s.d., standard deviation; <, less than; - , not determined]

\begin{tabular}{|c|c|c|c|c|c|c|c|c|c|c|c|c|c|c|}
\hline \multirow[t]{2}{*}{$\begin{array}{l}\text { Station } \\
\text { map ID }\end{array}$} & \multirow[t]{2}{*}{ Station name } & \multirow[t]{2}{*}{ Date } & \multirow[t]{2}{*}{ Time } & \multirow[t]{2}{*}{$\begin{array}{l}\text { Rep- } \\
\text { licate }\end{array}$} & \multicolumn{2}{|c|}{$\begin{array}{l}\text { Nickel (Ni), } \\
\text { filtered } \\
(\mu \mathrm{g} / \mathrm{L}) \\
(01065) \\
\end{array}$} & \multicolumn{2}{|c|}{$\begin{array}{c}\text { Lead }(\mathrm{Pb}) \\
\text { filtered } \\
(\mu \mathrm{g} / \mathrm{L}) \\
(01049)\end{array}$} & \multicolumn{2}{|c|}{$\begin{array}{c}\text { Praseodymium }(\mathrm{Pr}), \\
\text { filtered } \\
(\mu \mathrm{g} / \mathrm{L}) \\
(\mathbf{5 0 5 8 2}) \\
\end{array}$} & \multicolumn{2}{|c|}{$\begin{array}{c}\text { Rubidium (Rb), } \\
\text { filtered } \\
(\mu \mathrm{g} / \mathrm{L}) \\
(\mathbf{0 1 1 3 4}) \\
\end{array}$} & \multicolumn{2}{|c|}{$\begin{array}{c}\text { Rhenium (Re), } \\
\text { filtered } \\
(\mu \mathrm{g} / \mathrm{L}) \\
(\mathbf{5 0 5 8 3 )} \\
\end{array}$} \\
\hline & & & & & value & s.d. & value & s.d. & value & s.d. & value & s.d. & value & s.d. \\
\hline \multicolumn{15}{|c|}{ Environmental samples } \\
\hline BY20 & $\begin{array}{l}\text { Boston Mine tunnel } \\
\text { outlet nr Grass } \\
\text { Valley }\end{array}$ & 6/18/1999 & $14: 30$ & 1 of 2 & 6.0 & 0.6 & 0.009 & 0.002 & 0.0026 & 0.0004 & 1.9 & 0.1 & 0.0030 & 0.0002 \\
\hline BY20 & $\begin{array}{l}\text { Boston Mine tunnel } \\
\text { outlet nr Grass } \\
\text { Valley }\end{array}$ & $6 / 18 / 1999$ & $14: 30$ & $2 \mathrm{of} 2$ & 6.1 & 0.4 & 0.008 & 0.004 & 0.0024 & 0.0005 & 1.9 & 0.1 & 0.0031 & 0.0002 \\
\hline BY20 & $\begin{array}{l}\text { Boston Mine tunnel } \\
\text { outlet nr Grass } \\
\text { Valley }\end{array}$ & $12 / 14 / 1999$ & $12: 00$ & 1 of 2 & 3.0 & 0.0 & 0.016 & 0.010 & 0.0006 & 0.0001 & 2.1 & 0.0 & 0.0004 & 0.0000 \\
\hline BY20 & $\begin{array}{l}\text { Boston Mine tunnel } \\
\text { outlet nr Grass } \\
\text { Valley }\end{array}$ & $12 / 14 / 1999$ & $12: 00$ & $2 \mathrm{of} 2$ & 2.9 & 0.0 & 0.017 & 0.001 & 0.0007 & 0.0000 & 2.0 & 0.0 & 0.0011 & 0.0000 \\
\hline BY20 & $\begin{array}{l}\text { Boston Mine tunnel } \\
\text { outlet nr Grass } \\
\text { Valley }\end{array}$ & $5 / 24 / 2000$ & $16: 00$ & 1 of 2 & 4.9 & 0.0 & 0.02 & 0.02 & 0.0037 & 0.0007 & 1.7 & 0.1 & $<0.002$ & 0.000 \\
\hline BY20 & $\begin{array}{l}\text { Boston Mine tunnel } \\
\text { outlet nr Grass } \\
\text { Valley }\end{array}$ & $5 / 24 / 2000$ & $16: 00$ & $2 \mathrm{of} 2$ & 5.0 & 0.1 & 0.01 & 0.01 & 0.0042 & 0.0002 & 1.8 & 0.0 & $<0.002$ & 0.000 \\
\hline BY21 & $\begin{array}{l}\text { Boston Mine wetlands } \\
\text { pond nr Grass Valley }\end{array}$ & 12/14/1999 & $10: 30$ & 1 of 2 & 0.61 & 0.04 & 0.009 & 0.005 & 0.0011 & 0.0002 & 2.5 & 0.0 & 0.0079 & 0.0001 \\
\hline BY21 & $\begin{array}{l}\text { Boston Mine wetlands } \\
\text { pond nr Grass Valley }\end{array}$ & 12/14/1999 & $10: 30$ & $2 \mathrm{of} 2$ & 0.59 & 0.00 & 0.017 & 0.009 & 0.0009 & 0.0002 & 2.6 & 0.0 & 0.0067 & 0.0003 \\
\hline BY21 & $\begin{array}{l}\text { Boston Mine wetlands } \\
\text { pond nr Grass Valley }\end{array}$ & $5 / 24 / 2000$ & $16: 30$ & 1 of 2 & 2.5 & 0.1 & 0.06 & 0.03 & 0.0029 & 0.0005 & 2.3 & 0.0 & $<0.002$ & 0.001 \\
\hline BY21 & $\begin{array}{l}\text { Boston Mine wetlands } \\
\text { pond nr Grass Valley }\end{array}$ & $5 / 24 / 2000$ & $16: 30$ & $2 \mathrm{of} 2$ & 2.3 & 0.1 & 0.03 & 0.01 & 0.0028 & 0.0004 & 2.1 & 0.0 & 0.002 & 0.000 \\
\hline BY58 & $\begin{array}{l}\text { Buckeye Flat Mine } \\
\text { main drain } 0.45 \mathrm{mi} \\
\text { ab Greenhorn } \mathrm{Cr}\end{array}$ & $8 / 21 / 2000$ & 11:00 & 1 of 2 & 93 & 0 & 0.19 & 0.01 & 2.9 & 0.0 & 7.1 & 0.1 & $<0.003$ & 0.001 \\
\hline BY58 & $\begin{array}{l}\text { Buckeye Flat Mine } \\
\text { main drain } 0.45 \mathrm{mi} \\
\text { ab Greenhorn } \mathrm{Cr}\end{array}$ & $8 / 21 / 2000$ & 11:00 & $2 \mathrm{of} 2$ & 94 & 0 & 0.19 & 0.01 & 3.1 & 0.0 & 7.2 & 0.3 & 0.003 & 0.000 \\
\hline BY23 & $\begin{array}{c}\text { Buckeye Flat Mine N } \\
\text { drain to Greenhorn } \\
\text { Cr, Grass Valley }\end{array}$ & 6/18/1999 & $11: 30$ & $2 \mathrm{of} 2$ & 230 & 10 & 0.62 & 0.01 & 5.8 & 0.1 & 5.3 & 0.2 & 0.0008 & 0.0001 \\
\hline
\end{tabular}


Table 4B. Concentrations of trace metals and selected major elements in filtered water samples, Greenhorn Creek drainage, Nevada County, California—Continued.

[Station name abbreviations: ab, above; bl, below; Cr, creek; Cyn, Canyon; mi, miles; N, north; nr, near; Rd, Road; S, south; SF, South Fork. Number in parentheses is the data parameter code, a five-digit code used in the U.S. Geological Survey computerized data system. $\mu \mathrm{g} / \mathrm{L}$, microgram per liter (equivalent to part per billion); $\mathrm{mg} / \mathrm{L}$, milligram per liter (at low concentration, equivalent to part per million); s.d., standard deviation; <, less than; —, not determined]

\begin{tabular}{|c|c|c|c|c|c|c|c|c|c|c|c|c|c|c|}
\hline \multirow[t]{2}{*}{$\begin{array}{l}\text { Station } \\
\text { map ID }\end{array}$} & \multirow[t]{2}{*}{ Station name } & \multirow[t]{2}{*}{ Date } & \multirow[t]{2}{*}{ Time } & \multirow[t]{2}{*}{$\begin{array}{l}\text { Rep- } \\
\text { licate }\end{array}$} & \multicolumn{2}{|c|}{$\begin{array}{l}\text { Nickel (Ni), } \\
\text { filtered } \\
(\mu \mathrm{g} / \mathrm{L}) \\
(01065) \\
\end{array}$} & \multicolumn{2}{|c|}{$\begin{array}{c}\text { Lead }(\mathrm{Pb}) \\
\text { filtered } \\
(\mu \mathrm{g} / \mathrm{L}) \\
(01049)\end{array}$} & \multicolumn{2}{|c|}{$\begin{array}{c}\text { Praseodymium (Pr), } \\
\text { filtered } \\
(\mu \mathrm{g} / \mathrm{L}) \\
(\mathbf{5 0 5 8 2}) \\
\end{array}$} & \multicolumn{2}{|c|}{$\begin{array}{c}\text { Rubidium (Rb), } \\
\text { filtered } \\
(\mu \mathrm{g} / \mathrm{L}) \\
(\mathbf{0 1 1 3 4}) \\
\end{array}$} & \multicolumn{2}{|c|}{$\begin{array}{c}\text { Rhenium (Re), } \\
\text { filtered } \\
(\mu \mathrm{g} / \mathrm{L}) \\
(\mathbf{5 0 5 8 3 )} \\
\end{array}$} \\
\hline & & & & & value & s.d. & value & s.d. & value & s.d. & value & s.d. & value & s.d. \\
\hline BY23 & $\begin{array}{c}\text { Buckeye Flat Mine N } \\
\text { drain to Greenhorn } \\
\text { Cr, Grass Valley }\end{array}$ & $12 / 2 / 1999$ & $11: 00$ & $2 \mathrm{of} 2$ & 140 & 0 & 0.53 & 0.02 & 4.1 & 0.2 & 3.7 & 0.0 & 0.0069 & 0.0001 \\
\hline BY23 & $\begin{array}{c}\text { Buckeye Flat Mine N } \\
\text { drain to Greenhorn } \\
\text { Cr, Grass Valley }\end{array}$ & $8 / 22 / 2000$ & $10: 45$ & 1 of 2 & 160 & 10 & 0.59 & 0.01 & 5.7 & 0.1 & 2.0 & 0.1 & $<0.003$ & 0.001 \\
\hline BY23 & $\begin{array}{c}\text { Buckeye Flat Mine N } \\
\text { drain to Greenhorn } \\
\text { Cr, Grass Valley }\end{array}$ & $8 / 22 / 2000$ & $10: 45$ & $2 \mathrm{of} 2$ & 160 & 0 & 0.60 & 0.00 & 5.9 & 0.0 & 2.1 & 0.0 & $<0.003$ & 0.003 \\
\hline BY116 & $\begin{array}{l}\text { Buckeye Flat Mine } \\
\text { pond drain } 0.15 \mathrm{mi} \\
\text { ab SF Greenhorn } \mathrm{Cr}\end{array}$ & $8 / 21 / 2000$ & $14: 10$ & 1 of 2 & 4.1 & 0.2 & 0.06 & 0.00 & 0.011 & 0.001 & 2.1 & 0.0 & $<0.003$ & 0.001 \\
\hline BY116 & $\begin{array}{l}\text { Buckeye Flat Mine } \\
\text { pond drain } 0.15 \mathrm{mi} \\
\text { ab SF Greenhorn } \mathrm{Cr}\end{array}$ & $8 / 21 / 2000$ & $14: 10$ & $2 \mathrm{of} 2$ & 4.3 & 0.3 & 0.08 & 0.01 & 0.010 & 0.001 & 2.2 & 0.0 & $<0.003$ & 0.001 \\
\hline BY24 & $\begin{array}{l}\text { Buckeye Flat Mine } \\
\text { S drain to SF } \\
\text { Greenhorn Cr, Grass } \\
\text { Valley }\end{array}$ & $8 / 26 / 1999$ & $11: 00$ & 1 of 2 & 8.3 & 0.1 & 1.8 & 0.0 & 0.11 & 0.00 & 1.1 & 0.1 & 0.0014 & 0.0001 \\
\hline BY24 & $\begin{array}{l}\text { Buckeye Flat Mine } \\
\text { S drain to SF } \\
\text { Greenhorn Cr, Grass } \\
\text { Valley }\end{array}$ & $8 / 26 / 1999$ & 11:00 & $2 \mathrm{of} 2$ & 8.0 & 0.1 & 1.7 & 0.0 & 0.098 & 0.003 & 1.0 & 0.0 & 0.0014 & 0.0003 \\
\hline BY24 & $\begin{array}{l}\text { Buckeye Flat Mine } \\
\text { S drain to SF } \\
\text { Greenhorn Cr, Grass } \\
\text { Valley }\end{array}$ & $8 / 22 / 2000$ & $12: 10$ & 1 of 2 & 9.7 & 0.0 & 0.16 & 0.00 & 0.080 & 0.004 & 0.70 & 0.01 & $<0.003$ & 0.001 \\
\hline BY24 & $\begin{array}{l}\text { Buckeye Flat Mine } \\
\text { S drain to SF } \\
\text { Greenhorn Cr, Grass } \\
\text { Valley }\end{array}$ & $8 / 22 / 2000$ & $12: 10$ & $2 \mathrm{of} 2$ & 9.7 & 0.4 & 0.16 & 0.01 & 0.081 & 0.002 & 0.72 & 0.02 & $<0.003$ & 0.001 \\
\hline BY25 & $\begin{array}{l}\text { Buckeye Flat Mine } \\
\text { upper drain }\end{array}$ & $4 / 9 / 2001$ & $10: 00$ & $2 \mathrm{of} 2$ & 67 & 3 & 0.31 & 0.02 & 1.1 & 0.0 & 1.4 & 0.1 & $<0.003$ & 0.002 \\
\hline
\end{tabular}


Table 4B. Concentrations of trace metals and selected major elements in filtered water samples, Greenhorn Creek drainage, Nevada County, California—Continued.

[Station name abbreviations: ab, above; bl, below; Cr, creek; Cyn, Canyon; mi, miles; N, north; nr, near; Rd, Road; S, south; SF, South Fork. Number in parentheses is the data parameter code, a five-digit code used in the U.S. Geological Survey computerized data system. $\mu \mathrm{g} / \mathrm{L}$, microgram per liter (equivalent to part per billion); mg/L, milligram per liter (at low concentration, equivalent to part per million); s.d., standard deviation; <, less than; - , not determined]

\begin{tabular}{|c|c|c|c|c|c|c|c|c|c|c|c|c|c|c|}
\hline \multirow[t]{2}{*}{$\begin{array}{l}\text { Station } \\
\text { map ID }\end{array}$} & \multirow[t]{2}{*}{ Station name } & \multirow[t]{2}{*}{ Date } & \multirow[t]{2}{*}{ Time } & \multirow[t]{2}{*}{$\begin{array}{l}\text { Rep- } \\
\text { licate }\end{array}$} & \multicolumn{2}{|c|}{$\begin{array}{c}\text { Nickel (Ni), } \\
\text { filtered } \\
(\mu \mathrm{g} / \mathrm{L}) \\
(01065) \\
\end{array}$} & \multicolumn{2}{|c|}{$\begin{array}{c}\text { Lead }(\mathrm{Pb}) \\
\text { filtered } \\
(\mu \mathrm{g} / \mathrm{L}) \\
(01049)\end{array}$} & \multicolumn{2}{|c|}{$\begin{array}{c}\text { Praseodymium }(\mathrm{Pr}), \\
\text { filtered } \\
(\mu \mathrm{g} / \mathrm{L}) \\
(\mathbf{5 0 5 8 2}) \\
\end{array}$} & \multicolumn{2}{|c|}{$\begin{array}{c}\text { Rubidium (Rb), } \\
\text { filtered } \\
(\mu \mathrm{g} / \mathrm{L}) \\
(\mathbf{0 1 1 3 4})\end{array}$} & \multicolumn{2}{|c|}{$\begin{array}{c}\text { Rhenium }(\mathrm{Re}), \\
\text { filtered } \\
(\mu \mathrm{g} / \mathrm{L}) \\
(\mathbf{5 0 5 8 3 )} \\
\end{array}$} \\
\hline & & & & & value & s.d. & value & s.d. & value & s.d. & value & s.d. & value & s.d. \\
\hline BY52 & $\begin{array}{l}\text { Greenhorn Cr } 0.3 \mathrm{mi} \\
\text { ab Buckeye Ford nr } \\
\text { Nevada City }\end{array}$ & $1 / 25 / 2000$ & $10: 45$ & 1 of 2 & 5.1 & 0.0 & 0.03 & 0.01 & 0.092 & 0.001 & 1.0 & 0.1 & 0.003 & 0.001 \\
\hline BY52 & $\begin{array}{l}\text { Greenhorn Cr } 0.3 \mathrm{mi} \\
\text { ab Buckeye Ford nr } \\
\text { Nevada City }\end{array}$ & $1 / 25 / 2000$ & $10: 45$ & $2 \mathrm{of} 2$ & 4.9 & 0.2 & 0.02 & 0.01 & 0.096 & 0.001 & 1.0 & 0.1 & $<0.002$ & 0.002 \\
\hline BY59 & $\begin{array}{l}\text { Greenhorn Cr at You } \\
\text { Bet Rd nr Nevada } \\
\text { City }\end{array}$ & $7 / 24 / 2001$ & $11: 00$ & 1 of 2 & 1.9 & 0.1 & 0.028 & 0.012 & 0.0024 & 0.0000 & 1.4 & 0.0 & 0.0060 & 0.0001 \\
\hline BY59 & $\begin{array}{l}\text { Greenhorn Cr at You } \\
\text { Bet Rd nr Nevada } \\
\text { City }\end{array}$ & $7 / 24 / 2001$ & $11: 00$ & $2 \mathrm{of} 2$ & 2.0 & 0.1 & 0.027 & 0.028 & 0.0023 & 0.0002 & 1.5 & 0.0 & 0.0061 & 0.0009 \\
\hline BY59 & $\begin{array}{l}\text { Greenhorn Cr at You } \\
\text { Bet Rd nr Nevada } \\
\text { City }\end{array}$ & $8 / 29 / 2001$ & $15: 00$ & 1 of 2 & 0.89 & 0.01 & $<0.007$ & 0.004 & 0.0026 & 0.0001 & 1.4 & 0.0 & 0.0042 & 0.0004 \\
\hline BY59 & $\begin{array}{l}\text { Greenhorn } \mathrm{Cr} \text { at You } \\
\text { Bet Rd nr Nevada } \\
\text { City }\end{array}$ & $8 / 29 / 2001$ & $15: 00$ & $2 \mathrm{of} 2$ & 0.88 & 0.02 & $<0.007$ & 0.001 & 0.0026 & 0.0001 & 1.5 & 0.0 & 0.0047 & 0.0000 \\
\hline BY59 & $\begin{array}{l}\text { Greenhorn } \mathrm{Cr} \text { at You } \\
\text { Bet Rd nr Nevada } \\
\text { City }\end{array}$ & $9 / 14 / 2001$ & $13: 00$ & 1 of 2 & 1.3 & 0.0 & 0.016 & 0.006 & 0.0018 & 0.0002 & 1.5 & 0.0 & 0.0053 & 0.0002 \\
\hline BY59 & $\begin{array}{l}\text { Greenhorn } \mathrm{Cr} \text { at You } \\
\text { Bet Rd nr Nevada } \\
\text { City }\end{array}$ & $9 / 14 / 2001$ & $13: 00$ & $2 \mathrm{of} 2$ & 1.4 & 0.0 & 0.021 & 0.005 & 0.0020 & 0.0002 & 1.5 & 0.0 & 0.0061 & 0.0001 \\
\hline BY59 & $\begin{array}{l}\text { Greenhorn Cr at You } \\
\text { Bet Rd nr Nevada } \\
\text { City }\end{array}$ & $10 / 15 / 2001$ & $12: 00$ & 1 of 2 & 1.1 & 0.0 & 0.010 & 0.005 & 0.0013 & 0.0003 & 1.2 & 0.0 & 0.0057 & 0.0005 \\
\hline BY59 & $\begin{array}{l}\text { Greenhorn Cr at You } \\
\text { Bet Rd nr Nevada } \\
\text { City }\end{array}$ & $10 / 15 / 2001$ & $12: 00$ & $2 \mathrm{of} 2$ & 1.2 & 0.0 & $<0.004$ & 0.000 & 0.0012 & 0.0000 & 1.2 & 0.0 & 0.0055 & 0.0001 \\
\hline BY59 & $\begin{array}{l}\text { Greenhorn } \mathrm{Cr} \text { at You } \\
\text { Bet Rd nr Nevada } \\
\text { City }\end{array}$ & $11 / 20 / 2001$ & $13: 20$ & 1 of 2 & 3.4 & 0.1 & 0.020 & 0.003 & 0.012 & 0.001 & 1.7 & 0.0 & 0.0066 & 0.0002 \\
\hline BY59 & $\begin{array}{l}\text { Greenhorn Cr at You } \\
\text { Bet Rd nr Nevada } \\
\text { City }\end{array}$ & $12 / 10 / 2001$ & $12: 20$ & 1 of 2 & 5.4 & 0.1 & 0.017 & 0.000 & 0.040 & 0.000 & 1.3 & 0.0 & 0.0045 & 0.0001 \\
\hline
\end{tabular}


Table 4B. Concentrations of trace metals and selected major elements in filtered water samples, Greenhorn Creek drainage, Nevada County, California—Continued.

[Station name abbreviations: ab, above; bl, below; Cr, creek; Cyn, Canyon; mi, miles; N, north; nr, near; Rd, Road; S, south; SF, South Fork. Number in parentheses is the data parameter code, a five-digit code used in the U.S. Geological Survey computerized data system. $\mu \mathrm{g} / \mathrm{L}$, microgram per liter (equivalent to part per billion); mg/L, milligram per liter (at low concentration, equivalent to part per million); s.d., standard deviation; <, less than; - , not determined]

\begin{tabular}{|c|c|c|c|c|c|c|c|c|c|c|c|c|c|c|}
\hline \multirow[t]{2}{*}{$\begin{array}{l}\text { Station } \\
\text { map ID }\end{array}$} & \multirow[t]{2}{*}{ Station name } & \multirow[t]{2}{*}{ Date } & \multirow[t]{2}{*}{ Time } & \multirow[t]{2}{*}{$\begin{array}{l}\text { Rep- } \\
\text { licate }\end{array}$} & \multicolumn{2}{|c|}{$\begin{array}{c}\text { Nickel }(\mathrm{Ni}), \\
\text { filtered } \\
(\mu \mathrm{g} / \mathrm{L}) \\
(01065)\end{array}$} & \multicolumn{2}{|c|}{$\begin{array}{c}\text { Lead }(\mathrm{Pb}), \\
\text { filtered } \\
(\mu \mathrm{g} / \mathrm{L}) \\
(01049) \\
\end{array}$} & \multicolumn{2}{|c|}{$\begin{array}{c}\text { Praseodymium }(\mathrm{Pr}), \\
\text { filtered } \\
(\mu \mathrm{g} / \mathrm{L}) \\
(\mathbf{5 0 5 8 2}) \\
\end{array}$} & \multicolumn{2}{|c|}{$\begin{array}{c}\text { Rubidium (Rb), } \\
\text { filtered } \\
(\mu \mathrm{g} / \mathrm{L}) \\
(\mathbf{0 1 1 3 4}) \\
\end{array}$} & \multicolumn{2}{|c|}{$\begin{array}{c}\text { Rhenium (Re), } \\
\text { filtered } \\
(\mu \mathrm{g} / \mathrm{L}) \\
(\mathbf{5 0 5 8 3 )} \\
\end{array}$} \\
\hline & & & & & value & s.d. & value & s.d. & value & s.d. & value & s.d. & value & s.d. \\
\hline BY59 & $\begin{array}{l}\text { Greenhorn Cr at You } \\
\text { Bet Rd nr Nevada } \\
\text { City }\end{array}$ & $12 / 21 / 2001$ & $13: 00$ & $1 \mathrm{of} 2$ & 4.3 & 0.0 & 0.018 & 0.001 & 0.079 & 0.000 & 1.1 & 0.0 & 0.0022 & 0.0002 \\
\hline BY59 & $\begin{array}{l}\text { Greenhorn Cr at You } \\
\text { Bet Rd nr Nevada } \\
\text { City }\end{array}$ & $12 / 21 / 2001$ & $13: 00$ & $2 \mathrm{of} 2$ & 4.2 & 0.0 & 0.026 & 0.002 & 0.076 & 0.001 & 1.1 & 0.0 & 0.0025 & 0.0003 \\
\hline BY51 & $\begin{array}{l}\text { Greenhorn Cr nr } \\
\text { Headwaters nr Scotts } \\
\text { Flat Reservoir }\end{array}$ & $1 / 25 / 2000$ & $9: 30$ & $1 \mathrm{of} 2$ & 2.3 & 0.5 & 0.06 & 0.05 & 0.059 & 0.003 & 0.56 & 0.04 & $<0.002$ & 0.001 \\
\hline BY51 & $\begin{array}{l}\text { Greenhorn Cr nr } \\
\text { Headwaters nr Scotts } \\
\text { Flat Reservoir }\end{array}$ & $1 / 25 / 2000$ & $9: 30$ & $2 \mathrm{of} 2$ & 2.0 & 0.1 & 0.01 & 0.00 & 0.062 & 0.002 & 0.47 & 0.03 & $<0.002$ & 0.002 \\
\hline BY75 & $\begin{array}{l}\text { Missouri Cyn Cr } 1.2 \mathrm{mi} \\
\text { ab Greenhorn Cr nr } \\
\text { Chicago Park }\end{array}$ & $12 / 14 / 1999$ & $14: 00$ & $1 \mathrm{of} 2$ & 30 & 0 & 0.006 & 0.003 & 0.068 & 0.000 & 4.0 & 0.0 & 0.0041 & 0.0002 \\
\hline BY75 & $\begin{array}{l}\text { Missouri Cyn Cr } 1.2 \mathrm{mi} \\
\text { ab Greenhorn Cr nr } \\
\text { Chicago Park }\end{array}$ & $12 / 14 / 1999$ & $14: 00$ & 2 of 2 & 31 & 1 & 0.020 & 0.021 & 0.068 & 0.001 & 4.1 & 0.1 & 0.0051 & 0.0004 \\
\hline BY86 & $\begin{array}{l}\text { Poore Mine creek ab } \\
\text { tunnel nr Grass } \\
\text { Valley }\end{array}$ & 3/31/1999 & $17: 40$ & $1 \mathrm{of} 2$ & 1.4 & 0.2 & 0.029 & 0.003 & 0.018 & 0.001 & 0.76 & 0.03 & $<0.0004$ & 0.0001 \\
\hline BY86 & $\begin{array}{l}\text { Poore Mine creek ab } \\
\text { tunnel nr Grass } \\
\text { Valley }\end{array}$ & 3/31/1999 & $17: 40$ & $2 \mathrm{of} 2$ & 1.3 & 0.0 & 0.015 & 0.006 & 0.017 & 0.001 & 0.78 & 0.03 & $<0.0004$ & 0.0005 \\
\hline BY87 & $\begin{array}{l}\text { Poore Mine creek bl } \\
\text { tunnel nr Grass } \\
\text { Valley }\end{array}$ & 3/31/1999 & $13: 30$ & $1 \mathrm{of} 2$ & 1.2 & 0.1 & 0.023 & 0.004 & 0.018 & 0.002 & 0.89 & 0.02 & 0.0007 & 0.0006 \\
\hline BY87 & $\begin{array}{l}\text { Poore Mine creek bl } \\
\text { tunnel nr Grass } \\
\text { Valley }\end{array}$ & 3/31/1999 & $13: 30$ & $2 \mathrm{of} 2$ & 1.4 & 0.1 & 0.031 & 0.007 & 0.016 & 0.002 & 0.84 & 0.02 & $<0.0004$ & 0.0001 \\
\hline BY88 & $\begin{array}{l}\text { Poore Mine ground } \\
\text { sluice nr Grass } \\
\text { Valley }\end{array}$ & 4/1/1999 & $15: 40$ & $2 \mathrm{of} 2$ & 11 & 1 & 0.016 & 0.008 & 0.022 & 0.001 & 7.4 & 0.0 & 0.0070 & 0.0009 \\
\hline
\end{tabular}


Table 4B. Concentrations of trace metals and selected major elements in filtered water samples, Greenhorn Creek drainage, Nevada County, California—Continued.

[Station name abbreviations: ab, above; bl, below; Cr, creek; Cyn, Canyon; mi, miles; N, north; nr, near; Rd, Road; S, south; SF, South Fork. Number in parentheses is the data parameter code, a five-digit code used in the U.S. Geological Survey computerized data system. $\mu \mathrm{g} / \mathrm{L}$, microgram per liter (equivalent to part per billion); mg/L, milligram per liter (at low concentration, equivalent to part per million); s.d., standard deviation; <, less than; - , not determined]

\begin{tabular}{|c|c|c|c|c|c|c|c|c|c|c|c|c|c|c|}
\hline \multirow[t]{2}{*}{$\begin{array}{l}\text { Station } \\
\text { map ID }\end{array}$} & \multirow[t]{2}{*}{ Station name } & \multirow[t]{2}{*}{ Date } & \multirow[t]{2}{*}{ Time } & \multirow[t]{2}{*}{$\begin{array}{l}\text { Rep- } \\
\text { licate }\end{array}$} & \multicolumn{2}{|c|}{$\begin{array}{c}\text { Nickel (Ni), } \\
\text { filtered } \\
(\mu \mathrm{g} / \mathrm{L}) \\
(01065)\end{array}$} & \multicolumn{2}{|c|}{$\begin{array}{c}\text { Lead }(\mathrm{Pb}) \\
\text { filtered } \\
(\mu \mathrm{g} / \mathrm{L}) \\
(01049)\end{array}$} & \multicolumn{2}{|c|}{$\begin{array}{c}\text { Praseodymium }(\mathrm{Pr}), \\
\text { filtered } \\
(\mu \mathrm{g} / \mathrm{L}) \\
(\mathbf{5 0 5 8 2})\end{array}$} & \multicolumn{2}{|c|}{$\begin{array}{c}\text { Rubidium (Rb), } \\
\text { filtered } \\
(\mu \mathrm{g} / \mathrm{L}) \\
(\mathbf{0 1 1 3 4})\end{array}$} & \multicolumn{2}{|c|}{$\begin{array}{c}\text { Rhenium (Re), } \\
\text { filtered } \\
(\mu \mathrm{g} / \mathrm{L}) \\
(\mathbf{5 0 5 8 3 )}\end{array}$} \\
\hline & & & & & value & s.d. & value & s.d. & value & s.d. & value & s.d. & value & s.d. \\
\hline BY89 & $\begin{array}{c}\text { Poore Mine pit lake } \\
\text { nr Grass Valley }\end{array}$ & $4 / 1 / 1999$ & $16: 50$ & 1 of 2 & 3.8 & 0.2 & 0.052 & 0.003 & 0.0076 & 0.0005 & 2.1 & 0.1 & 0.0011 & 0.0000 \\
\hline BY89 & $\begin{array}{l}\text { Poore Mine pit lake } \\
\text { nr Grass Valley }\end{array}$ & 4/1/1999 & $16: 50$ & $2 \mathrm{of} 2$ & 3.5 & 0.2 & 0.072 & 0.003 & 0.0066 & 0.0008 & 2.0 & 0.1 & 0.0007 & 0.0005 \\
\hline BY89 & $\begin{array}{l}\text { Poore Mine pit lake } \\
\text { nr Grass Valley }\end{array}$ & $5 / 23 / 2000$ & $10: 45$ & $1 \mathrm{of} 2$ & 60 & 0 & 0.03 & 0.01 & 0.034 & 0.003 & 7.6 & 0.0 & $<0.002$ & 0.000 \\
\hline BY89 & $\begin{array}{l}\text { Poore Mine pit lake } \\
\text { nr Grass Valley }\end{array}$ & $5 / 23 / 2000$ & $10: 45$ & $2 \mathrm{of} 2$ & 61 & 1 & 0.04 & 0.00 & 0.036 & 0.000 & 7.5 & 0.1 & $<0.002$ & 0.000 \\
\hline BY90 & $\begin{array}{l}\text { Poore Mine seep } \\
\text { ab ground sluice } \\
\text { nr Grass Valley }\end{array}$ & 4/1/1999 & $16: 00$ & $1 \mathrm{of} 2$ & 9.4 & 0.4 & 0.007 & 0.002 & 0.0069 & 0.0006 & 6.6 & 0.1 & 0.0021 & 0.0006 \\
\hline BY90 & $\begin{array}{l}\text { Poore Mine seep } \\
\text { ab ground sluice } \\
\text { nr Grass Valley }\end{array}$ & 4/1/1999 & $16: 00$ & $2 \mathrm{of} 2$ & 9.3 & 0.8 & 0.018 & 0.008 & 0.0068 & 0.0007 & 6.5 & 0.3 & 0.0052 & 0.0000 \\
\hline BY91 & $\begin{array}{l}\text { Poore Mine tunnel } \\
\text { effluent nr Grass } \\
\text { Valley }\end{array}$ & 3/31/1999 & $15: 20$ & $2 \mathrm{of} 2$ & 5.9 & 0.8 & 0.019 & 0.011 & 0.0031 & 0.0001 & 5.3 & 0.3 & 0.0044 & 0.0000 \\
\hline BY91 & $\begin{array}{l}\text { Poore Mine tunnel } \\
\text { effluent nr Grass } \\
\text { Valley }\end{array}$ & $12 / 15 / 1999$ & $13: 00$ & $1 \mathrm{of} 2$ & 1.8 & 0.0 & 0.011 & 0.005 & 0.0009 & 0.0002 & 5.7 & 0.2 & 0.0062 & 0.0012 \\
\hline BY91 & $\begin{array}{l}\text { Poore Mine tunnel } \\
\text { effluent nr Grass } \\
\text { Valley }\end{array}$ & $12 / 15 / 1999$ & $13: 00$ & $2 \mathrm{of} 2$ & 1.7 & 0.1 & 0.033 & 0.023 & 0.0010 & 0.0002 & 5.7 & 0.0 & 0.0042 & 0.0003 \\
\hline BY91 & $\begin{array}{l}\text { Poore Mine tunnel } \\
\text { effluent nr Grass } \\
\text { Valley }\end{array}$ & $5 / 23 / 2000$ & $12: 00$ & $1 \mathrm{of} 2$ & 5.6 & 0.0 & 0.01 & 0.01 & 0.0009 & 0.0003 & 5.6 & 0.0 & 0.005 & 0.001 \\
\hline BY91 & $\begin{array}{l}\text { Poore Mine tunnel } \\
\text { effluent nr Grass } \\
\text { Valley }\end{array}$ & $5 / 23 / 2000$ & $12: 00$ & $2 \mathrm{of} 2$ & 5.9 & 0.2 & 0.05 & 0.01 & 0.0014 & 0.0002 & 5.5 & 0.2 & $<0.002$ & 0.001 \\
\hline BY105 & $\begin{array}{l}\text { Sailor Flat Mine main } \\
\text { drain Gulch 01nr } \\
\text { Quaker Hill }\end{array}$ & 7/6/1999 & $16: 00$ & $1 \mathrm{of} 2$ & 33 & 1 & 0.017 & 0.004 & 0.19 & 0.01 & 4.8 & 0.2 & 0.0022 & 0.0001 \\
\hline BY105 & $\begin{array}{l}\text { Sailor Flat Mine main } \\
\text { drain Gulch 01nr } \\
\text { Quaker Hill }\end{array}$ & 7/6/1999 & $16: 00$ & $2 \mathrm{of} 2$ & 33 & 3 & 0.026 & 0.000 & 0.19 & 0.02 & 4.6 & 0.0 & 0.0019 & 0.0005 \\
\hline
\end{tabular}


Table 4B. Concentrations of trace metals and selected major elements in filtered water samples, Greenhorn Creek drainage, Nevada County, California—Continued.

[Station name abbreviations: ab, above; bl, below; Cr, creek; Cyn, Canyon; mi, miles; N, north; nr, near; Rd, Road; S, south; SF, South Fork. Number in parentheses is the data parameter code, a five-digit code used in the U.S. Geological Survey computerized data system. $\mu \mathrm{g} / \mathrm{L}$, microgram per liter (equivalent to part per billion); $\mathrm{mg} / \mathrm{L}$, milligram per liter (at low concentration, equivalen to part per million); s.d., standard deviation; <, less than; - , not determined]

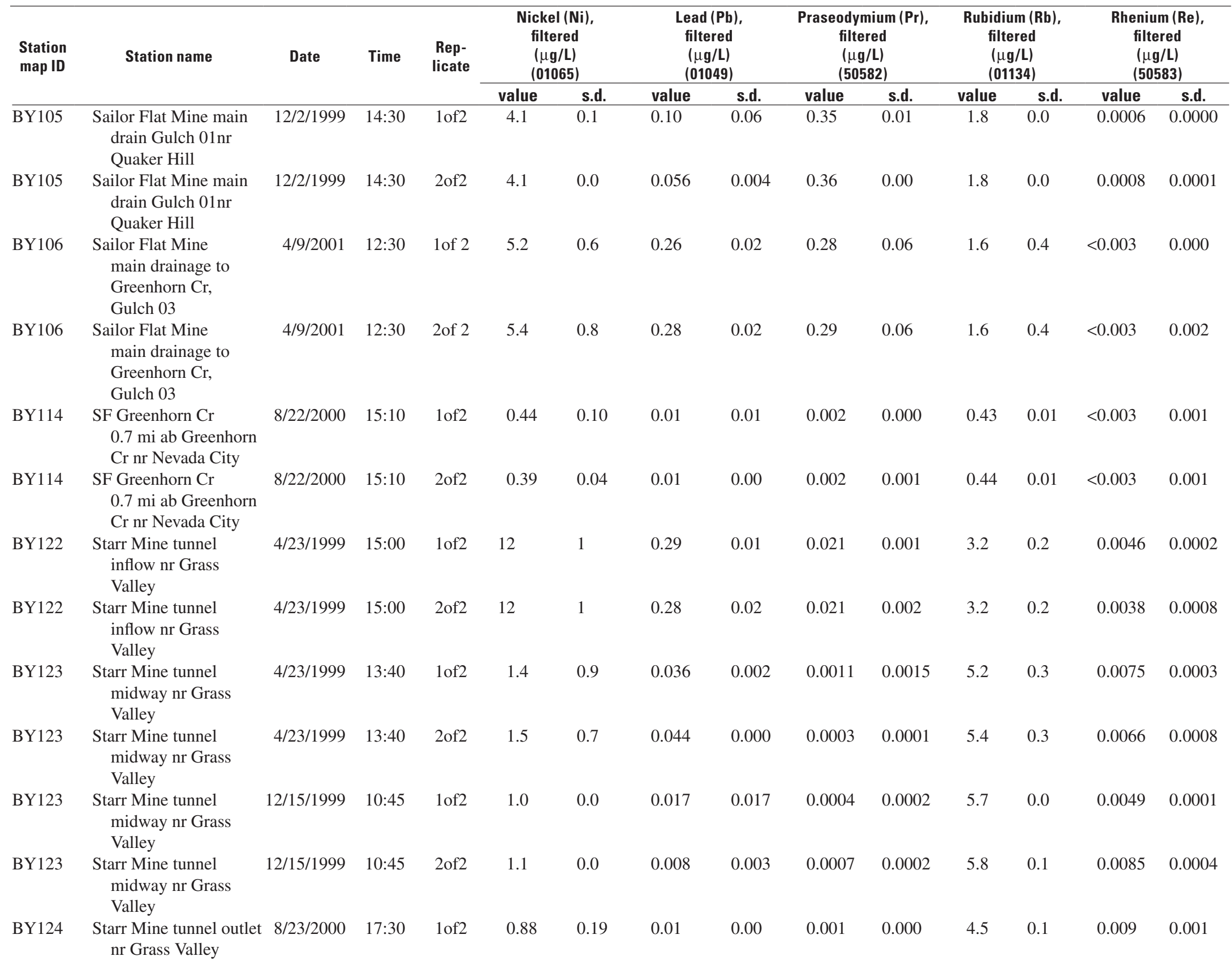


Table 4B. Concentrations of trace metals and selected major elements in filtered water samples, Greenhorn Creek drainage, Nevada County, California—Continued.

[Station name abbreviations: ab, above; bl, below; Cr, creek; Cyn, Canyon; mi, miles; N, north; nr, near; Rd, Road; S, south; SF, South Fork. Number in parentheses is the data parameter code, a five-digit code used in the U.S. Geological Survey computerized data system. $\mu \mathrm{g} / \mathrm{L}$, microgram per liter (equivalent to part per billion); mg/L, milligram per liter (at low concentration, equivalent to part per million); s.d., standard deviation; <, less than; - , not determined]

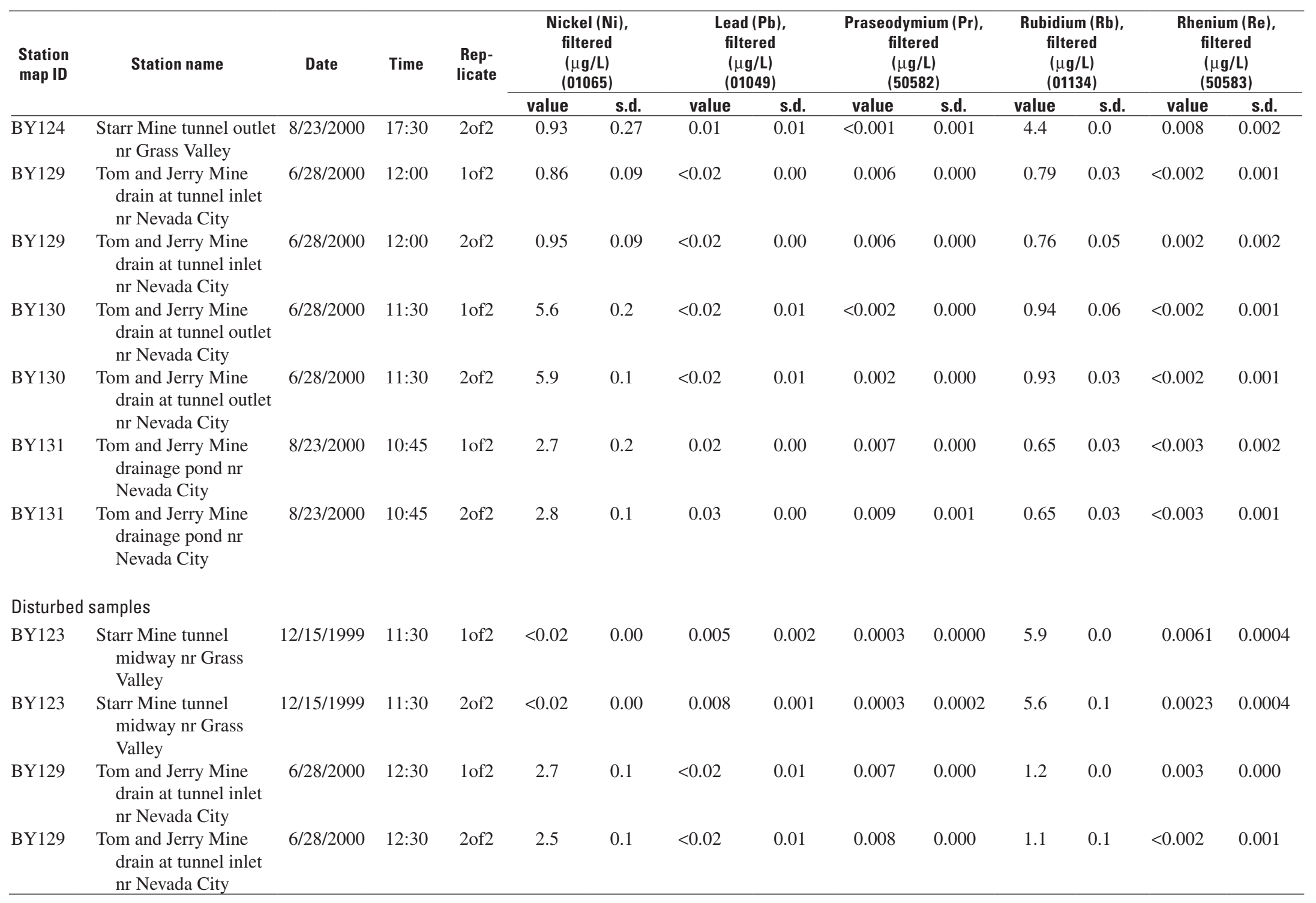


Table 4B. Concentrations of trace metals and selected major elements in filtered water samples, Greenhorn Creek drainage, Nevada County, California—Continued.

[Station name abbreviations: ab, above; bl, below; Cr, creek; Cyn, Canyon; mi, miles; N, north; nr, near; Rd, Road; S, south; SF, South Fork. Number in parentheses is the data parameter code, a five-digit code used in the U.S. Geological Survey computerized data system. $\mu \mathrm{g} / \mathrm{L}$, microgram per liter (equivalent to part per billion); mg/L, milligram per liter (at low concentration, equivalent to part per million); s.d., standard deviation; <, less than; - , not determined]

\begin{tabular}{|c|c|c|c|c|c|c|c|c|c|c|c|c|c|c|}
\hline \multirow[t]{2}{*}{$\begin{array}{l}\text { Station } \\
\text { map ID }\end{array}$} & \multirow[t]{2}{*}{ Station name } & \multirow[t]{2}{*}{ Date } & \multirow[t]{2}{*}{ Time } & \multirow[t]{2}{*}{$\begin{array}{l}\text { Rep- } \\
\text { licate }\end{array}$} & \multicolumn{2}{|c|}{$\begin{array}{c}\text { Sulfur (S), } \\
\text { filtered } \\
\text { (mg/L) } \\
(\mathbf{6 3 7 1 9 )}\end{array}$} & \multicolumn{2}{|c|}{$\begin{array}{c}\text { Antimony (Sb), } \\
\text { filtered } \\
(\mu \mathrm{g} / \mathrm{L}) \\
(01095) \\
\end{array}$} & \multicolumn{2}{|c|}{$\begin{array}{c}\text { Selenium (Se), } \\
\text { filtered } \\
(\mu \mathrm{g} / \mathrm{L}) \\
(01145) \\
\end{array}$} & \multicolumn{2}{|c|}{$\begin{array}{c}\text { Silica (Si), } \\
\text { filtered } \\
(\mathrm{mg} / \mathrm{L}) \\
(00955) \\
\end{array}$} & \multicolumn{2}{|c|}{$\begin{array}{c}\text { Samarium }(\mathrm{Sm}), \\
\text { filtered } \\
(\mu \mathrm{g} / \mathrm{L}) \\
(\mathbf{8 2 3 2 3}) \\
\end{array}$} \\
\hline & & & & & value & s.d. & value & s.d. & value & s.d. & value & s.d. & value & s.d. \\
\hline \multicolumn{15}{|c|}{ Environmental samples } \\
\hline BY20 & $\begin{array}{l}\text { Boston Mine tunnel } \\
\text { outlet nr Grass } \\
\text { Valley }\end{array}$ & 6/18/1999 & $14: 30$ & $1 \mathrm{of} 2$ & - & & 0.036 & 0.003 & 0.12 & 0.07 & 15 & 1 & 0.0033 & 0.0003 \\
\hline BY20 & $\begin{array}{l}\text { Boston Mine tunnel } \\
\text { outlet nr Grass } \\
\text { Valley }\end{array}$ & $6 / 18 / 1999$ & $14: 30$ & $2 \mathrm{of} 2$ & - & & 0.036 & 0.004 & 0.098 & 0.043 & 15 & 0 & 0.0034 & 0.0009 \\
\hline BY20 & $\begin{array}{l}\text { Boston Mine tunnel } \\
\text { outlet nr Grass } \\
\text { Valley }\end{array}$ & $12 / 14 / 1999$ & $12: 00$ & 1 of 2 & - & & 0.031 & 0.003 & 0.22 & 0.06 & 14 & 2 & 0.0011 & 0.0001 \\
\hline BY20 & $\begin{array}{l}\text { Boston Mine tunnel } \\
\text { outlet nr Grass } \\
\text { Valley }\end{array}$ & $12 / 14 / 1999$ & $12: 00$ & $2 \mathrm{of} 2$ & - & & 0.033 & 0.000 & 0.24 & 0.03 & 14 & 2 & 0.0012 & 0.0001 \\
\hline BY20 & $\begin{array}{l}\text { Boston Mine tunnel } \\
\text { outlet nr Grass } \\
\text { Valley }\end{array}$ & $5 / 24 / 2000$ & $16: 00$ & 1 of 2 & 4.3 & 1.0 & 0.075 & 0.037 & $<0.3$ & 0.5 & 15 & 3 & 0.003 & 0.001 \\
\hline BY20 & $\begin{array}{l}\text { Boston Mine tunnel } \\
\text { outlet nr Grass } \\
\text { Valley }\end{array}$ & $5 / 24 / 2000$ & $16: 00$ & $2 \mathrm{of} 2$ & 4.6 & 0.6 & 0.064 & 0.018 & $<0.3$ & 0.3 & 15 & 2 & 0.004 & 0.001 \\
\hline BY21 & $\begin{array}{l}\text { Boston Mine wetlands } \\
\text { pond nr Grass Valley }\end{array}$ & $12 / 14 / 1999$ & $10: 30$ & $1 \mathrm{of} 2$ & - & & 0.042 & 0.002 & $<0.06$ & 0.05 & 4.9 & 0.9 & 0.0012 & 0.0001 \\
\hline BY21 & $\begin{array}{l}\text { Boston Mine wetlands } \\
\text { pond nr Grass Valley }\end{array}$ & $12 / 14 / 1999$ & $10: 30$ & $2 \mathrm{of} 2$ & - & & 0.054 & 0.002 & $<0.06$ & 0.03 & 4.8 & 0.4 & 0.0009 & 0.0007 \\
\hline BY21 & $\begin{array}{l}\text { Boston Mine wetlands } \\
\text { pond nr Grass Valley }\end{array}$ & $5 / 24 / 2000$ & $16: 30$ & $1 \mathrm{of} 2$ & 3.2 & 0.0 & 0.067 & 0.005 & $<0.3$ & 0.2 & 13 & 1 & $<0.003$ & 0.002 \\
\hline BY21 & $\begin{array}{l}\text { Boston Mine wetlands } \\
\text { pond nr Grass Valley }\end{array}$ & $5 / 24 / 2000$ & $16: 30$ & $2 \mathrm{of} 2$ & 2.9 & 0.5 & 0.080 & 0.037 & $<0.3$ & 0.4 & 11 & 0 & $<0.003$ & 0.002 \\
\hline BY58 & $\begin{array}{l}\text { Buckeye Flat Mine } \\
\text { main drain } 0.45 \mathrm{mi} \\
\text { ab Greenhorn } \mathrm{Cr}\end{array}$ & $8 / 21 / 2000$ & $11: 00$ & $1 \mathrm{of} 2$ & 40 & 1 & $<0.005$ & 0.007 & $<0.8$ & 0.5 & 26 & 1 & 2.5 & 0.0 \\
\hline BY58 & $\begin{array}{l}\text { Buckeye Flat Mine } \\
\text { main drain } 0.45 \mathrm{mi} \\
\text { ab Greenhorn } \mathrm{Cr}\end{array}$ & $8 / 21 / 2000$ & 11:00 & $2 \mathrm{of} 2$ & 42 & 1 & 0.005 & 0.004 & $<0.8$ & 0.5 & 29 & 2 & 2.6 & 0.1 \\
\hline BY23 & $\begin{array}{c}\text { Buckeye Flat Mine N } \\
\text { drain to Greenhorn } \\
\text { Cr, Grass Valley }\end{array}$ & $6 / 18 / 1999$ & $11: 30$ & $2 \mathrm{of} 2$ & - & & 0.013 & 0.001 & 1.4 & 0.1 & 37 & 0 & 5.2 & 0.0 \\
\hline
\end{tabular}


Table 4B. Concentrations of trace metals and selected major elements in filtered water samples, Greenhorn Creek drainage, Nevada County, California—Continued.

[Station name abbreviations: ab, above; bl, below; Cr, creek; Cyn, Canyon; mi, miles; N, north; nr, near; Rd, Road; S, south; SF, South Fork. Number in parentheses is the data parameter code, a five-digit code used in the U.S. Geological Survey computerized data system. $\mu \mathrm{g} / \mathrm{L}$, microgram per liter (equivalent to part per billion); mg/L, milligram per liter (at low concentration, equivalent to part per million); s.d., standard deviation; <, less than; - , not determined]

\begin{tabular}{|c|c|c|c|c|c|c|c|c|c|c|c|c|c|c|}
\hline \multirow[t]{2}{*}{$\begin{array}{l}\text { Station } \\
\text { map ID }\end{array}$} & \multirow[t]{2}{*}{ Station name } & \multirow[t]{2}{*}{ Date } & \multirow[t]{2}{*}{ Time } & \multirow[t]{2}{*}{$\begin{array}{l}\text { Rep- } \\
\text { licate }\end{array}$} & \multicolumn{2}{|c|}{$\begin{array}{c}\text { Sulfur (S), } \\
\text { filtered } \\
(\mathrm{mg} / \mathrm{L}) \\
(63719)\end{array}$} & \multicolumn{2}{|c|}{$\begin{array}{c}\text { Antimony (Sb), } \\
\text { filtered } \\
(\mu \mathrm{g} / \mathrm{L}) \\
(\mathbf{0 1 0 9 5 )}\end{array}$} & \multicolumn{2}{|c|}{$\begin{array}{c}\text { Selenium (Se), } \\
\text { filtered } \\
(\mu \mathrm{g} / \mathrm{L}) \\
(01145) \\
\end{array}$} & \multicolumn{2}{|c|}{$\begin{array}{c}\text { Silica (Si), } \\
\text { filtered } \\
(\mathrm{mg} / \mathrm{L}) \\
(00955) \\
\end{array}$} & \multicolumn{2}{|c|}{$\begin{array}{c}\text { Samarium }(\mathrm{Sm}), \\
\text { filtered } \\
(\mu \mathrm{g} / \mathrm{L}) \\
(\mathbf{8 2 3 2 3}) \\
\end{array}$} \\
\hline & & & & & value & s.d. & value & s.d. & value & s.d. & value & s.d. & value & s.d. \\
\hline BY23 & $\begin{array}{c}\text { Buckeye Flat Mine N } \\
\text { drain to Greenhorn } \\
\text { Cr, Grass Valley }\end{array}$ & $12 / 2 / 1999$ & $11: 00$ & 1 of 2 & - & & 0.012 & 0.007 & 0.52 & 0.05 & 34 & 3 & 3.6 & 0.0 \\
\hline BY23 & $\begin{array}{l}\text { Buckeye Flat Mine N } \\
\text { drain to Greenhorn } \\
\text { Cr, Grass Valley }\end{array}$ & $12 / 2 / 1999$ & 11:00 & $2 \mathrm{of} 2$ & - & & 0.009 & 0.000 & 0.56 & 0.01 & 33 & 0 & 3.5 & 0.2 \\
\hline BY23 & $\begin{array}{c}\text { Buckeye Flat Mine N } \\
\text { drain to Greenhorn } \\
\text { Cr, Grass Valley }\end{array}$ & $8 / 22 / 2000$ & $10: 45$ & 1 of 2 & 57 & 3 & 0.015 & 0.007 & $<0.8$ & 0.4 & 40 & 1 & 5.1 & 0.0 \\
\hline BY23 & $\begin{array}{c}\text { Buckeye Flat Mine N } \\
\text { drain to Greenhorn } \\
\text { Cr, Grass Valley }\end{array}$ & $8 / 22 / 2000$ & $10: 45$ & $2 \mathrm{of} 2$ & 52 & 0 & 0.016 & 0.006 & $<0.8$ & 0.4 & 41 & 2 & 4.8 & 0.1 \\
\hline BY116 & $\begin{array}{l}\text { Buckeye Flat Mine } \\
\text { pond drain } 0.15 \mathrm{mi} \\
\text { ab SF Greenhorn } \mathrm{Cr}\end{array}$ & $8 / 21 / 2000$ & $14: 10$ & 1 of 2 & 2.3 & 0.8 & 0.008 & 0.001 & $<0.8$ & 0.8 & 4.8 & 1.3 & 0.013 & 0.004 \\
\hline BY116 & $\begin{array}{l}\text { Buckeye Flat Mine } \\
\text { pond drain } 0.15 \mathrm{mi} \\
\text { ab SF Greenhorn } \mathrm{Cr}\end{array}$ & $8 / 21 / 2000$ & $14: 10$ & $2 \mathrm{of} 2$ & 2.4 & 0.5 & 0.013 & 0.007 & $<0.8$ & 0.7 & 5.2 & 0.8 & 0.013 & 0.005 \\
\hline BY24 & $\begin{array}{l}\text { Buckeye Flat Mine } \\
\text { S drain to SF } \\
\text { Greenhorn Cr, Grass } \\
\text { Valley }\end{array}$ & $8 / 26 / 1999$ & $11: 00$ & 1 of 2 & 2.0 & 0.2 & 0.29 & 0.00 & $<0.1$ & 0.0 & 6.2 & 0.1 & 0.090 & 0.002 \\
\hline BY24 & $\begin{array}{l}\text { Buckeye Flat Mine } \\
\text { S drain to SF } \\
\text { Greenhorn Cr, Grass } \\
\text { Valley }\end{array}$ & $8 / 26 / 1999$ & $11: 00$ & $2 \mathrm{of} 2$ & 2.0 & 0.4 & 0.29 & 0.00 & $<0.1$ & 0.0 & 6.8 & 1.0 & 0.087 & 0.004 \\
\hline BY24 & $\begin{array}{l}\text { Buckeye Flat Mine } \\
\text { S drain to SF } \\
\text { Greenhorn Cr, Grass } \\
\text { Valley }\end{array}$ & $8 / 22 / 2000$ & $12: 10$ & 1 of 2 & 1.7 & 0.3 & 0.009 & 0.004 & $<0.8$ & 0.2 & 6.7 & 1.2 & 0.059 & 0.003 \\
\hline BY24 & $\begin{array}{l}\text { Buckeye Flat Mine } \\
\text { S drain to SF } \\
\text { Greenhorn Cr, Grass } \\
\text { Valley }\end{array}$ & $8 / 22 / 2000$ & $12: 10$ & $2 \mathrm{of} 2$ & 1.8 & 0.6 & 0.006 & 0.000 & $<0.8$ & 0.7 & 7.3 & 2.1 & 0.072 & 0.006 \\
\hline BY25 & $\begin{array}{l}\text { Buckeye Flat Mine } \\
\text { upper drain }\end{array}$ & 4/9/2001 & $10: 00$ & $2 \mathrm{of} 2$ & 15 & 1 & $<0.04$ & 0.00 & $<0.9$ & 0.4 & 15 & 1 & 0.79 & 0.01 \\
\hline
\end{tabular}


Table 4B. Concentrations of trace metals and selected major elements in filtered water samples, Greenhorn Creek drainage, Nevada County, California—Continued.

[Station name abbreviations: ab, above; bl, below; Cr, creek; Cyn, Canyon; mi, miles; N, north; nr, near; Rd, Road; S, south; SF, South Fork. Number in parentheses is the data parameter code, a five-digit code used in the U.S. Geological Survey computerized data system. $\mu \mathrm{g} / \mathrm{L}$, microgram per liter (equivalent to part per billion); mg/L, milligram per liter (at low concentration, equivalent to part per million); s.d., standard deviation; <, less than; —, not determined]

\begin{tabular}{|c|c|c|c|c|c|c|c|c|c|c|c|c|c|c|}
\hline \multirow[t]{2}{*}{$\begin{array}{l}\text { Station } \\
\text { map ID }\end{array}$} & \multirow[t]{2}{*}{ Station name } & \multirow[t]{2}{*}{ Date } & \multirow[t]{2}{*}{ Time } & \multirow[t]{2}{*}{$\begin{array}{l}\text { Rep- } \\
\text { licate }\end{array}$} & \multicolumn{2}{|c|}{$\begin{array}{c}\text { Sulfur (S), } \\
\text { filtered } \\
(\mathrm{mg} / \mathrm{L}) \\
(63719) \\
\end{array}$} & \multicolumn{2}{|c|}{$\begin{array}{c}\text { Antimony (Sb), } \\
\text { filtered } \\
(\mu \mathrm{g} / \mathrm{L}) \\
(01095) \\
\end{array}$} & \multicolumn{2}{|c|}{$\begin{array}{c}\text { Selenium (Se), } \\
\text { filtered } \\
(\mu \mathrm{g} / \mathrm{L}) \\
(01145) \\
\end{array}$} & \multicolumn{2}{|c|}{$\begin{array}{c}\text { Silica (Si), } \\
\text { filtered } \\
(\mathrm{mg} / \mathrm{L}) \\
(00955) \\
\end{array}$} & \multicolumn{2}{|c|}{$\begin{array}{c}\text { Samarium }(\mathrm{Sm}), \\
\text { filtered } \\
(\mu \mathrm{g} / \mathrm{L}) \\
(\mathbf{8 2 3 2 3}) \\
\end{array}$} \\
\hline & & & & & value & s.d. & value & s.d. & value & s.d. & value & s.d. & value & s.d. \\
\hline BY52 & $\begin{array}{l}\text { Greenhorn Cr } 0.3 \mathrm{mi} \\
\text { ab Buckeye Ford nr } \\
\text { Nevada City }\end{array}$ & $1 / 25 / 2000$ & $10: 45$ & 1 of 2 & 1.9 & 0.1 & 0.031 & 0.003 & $<0.8$ & 0.5 & 10 & 1 & 0.098 & 0.002 \\
\hline BY52 & $\begin{array}{l}\text { Greenhorn Cr } 0.3 \mathrm{mi} \\
\text { ab Buckeye Ford nr } \\
\text { Nevada City }\end{array}$ & $1 / 25 / 2000$ & $10: 45$ & 2 of 2 & 1.9 & 0.1 & 0.039 & 0.013 & $<0.8$ & 0.8 & 9.6 & 0.6 & 0.091 & 0.003 \\
\hline BY59 & $\begin{array}{l}\text { Greenhorn Cr at You } \\
\text { Bet Rd nr Nevada } \\
\text { City }\end{array}$ & $7 / 24 / 2001$ & $11: 00$ & 1 of 2 & 6.4 & 0.1 & 0.089 & 0.015 & 0.23 & 0.07 & 13 & 0 & 0.0037 & 0.0001 \\
\hline BY59 & $\begin{array}{l}\text { Greenhorn } \mathrm{Cr} \text { at You } \\
\text { Bet Rd nr Nevada } \\
\text { City }\end{array}$ & $7 / 24 / 2001$ & $11: 00$ & 2 of 2 & 6.4 & 0.0 & 0.093 & 0.019 & 0.14 & 0.03 & 13 & 0 & 0.0034 & 0.0001 \\
\hline BY59 & $\begin{array}{l}\text { Greenhorn } \mathrm{Cr} \text { at You } \\
\text { Bet Rd nr Nevada } \\
\text { City }\end{array}$ & $8 / 29 / 2001$ & $15: 00$ & 1 of 2 & 4.9 & 0.1 & 0.082 & 0.001 & 0.11 & 0.06 & 13 & 0 & 0.0026 & 0.0011 \\
\hline BY59 & $\begin{array}{l}\text { Greenhorn Cr at You } \\
\text { Bet Rd nr Nevada } \\
\text { City }\end{array}$ & $8 / 29 / 2001$ & $15: 00$ & 2 of 2 & 4.9 & 0.1 & 0.083 & 0.001 & 0.10 & 0.02 & 13 & 0 & 0.0021 & 0.0007 \\
\hline BY59 & $\begin{array}{l}\text { Greenhorn Cr at You } \\
\text { Bet Rd nr Nevada } \\
\text { City }\end{array}$ & $9 / 14 / 2001$ & $13: 00$ & 1 of 2 & 5.8 & 0.0 & 0.13 & 0.00 & 0.12 & 0.03 & 12 & 0 & 0.0022 & 0.0005 \\
\hline BY59 & $\begin{array}{l}\text { Greenhorn } \mathrm{Cr} \text { at You } \\
\text { Bet Rd nr Nevada } \\
\text { City }\end{array}$ & $9 / 14 / 2001$ & $13: 00$ & 2 of 2 & 5.9 & 0.0 & 0.14 & 0.00 & 0.14 & 0.04 & 12 & 0 & 0.0036 & 0.0004 \\
\hline BY59 & $\begin{array}{l}\text { Greenhorn } \mathrm{Cr} \text { at You } \\
\text { Bet Rd nr Nevada } \\
\text { City }\end{array}$ & $10 / 15 / 2001$ & $12: 00$ & 1 of 2 & 5.6 & 0.0 & 0.12 & 0.00 & 0.12 & 0.04 & 12 & 0 & 0.0017 & 0.0006 \\
\hline BY59 & $\begin{array}{l}\text { Greenhorn Cr at You } \\
\text { Bet Rd nr Nevada } \\
\text { City }\end{array}$ & $10 / 15 / 2001$ & $12: 00$ & 2 of 2 & 5.5 & 0.0 & 0.11 & 0.00 & 0.15 & 0.03 & 12 & 0 & 0.0018 & 0.0003 \\
\hline BY59 & $\begin{array}{l}\text { Greenhorn Cr at You } \\
\text { Bet Rd nr Nevada } \\
\text { City }\end{array}$ & $11 / 20 / 2001$ & $13: 20$ & 1 of 2 & 7.0 & 0.0 & 0.12 & 0.01 & $<0.2$ & 0.1 & 12 & 0 & 0.013 & 0.001 \\
\hline BY59 & $\begin{array}{l}\text { Greenhorn } \mathrm{Cr} \text { at You } \\
\text { Bet Rd nr Nevada } \\
\text { City }\end{array}$ & $12 / 10 / 2001$ & $12: 20$ & 1 of 2 & 5.9 & 0.0 & 0.16 & 0.00 & 0.20 & 0.02 & 12 & 0 & 0.038 & 0.005 \\
\hline
\end{tabular}


Table 4B. Concentrations of trace metals and selected major elements in filtered water samples, Greenhorn Creek drainage, Nevada County, California—Continued.

[Station name abbreviations: ab, above; bl, below; Cr, creek; Cyn, Canyon; mi, miles; N, north; nr, near; Rd, Road; S, south; SF, South Fork. Number in parentheses is the data parameter code, a five-digit code used in the U.S. Geological Survey computerized data system. $\mu \mathrm{g} / \mathrm{L}$, microgram per liter (equivalent to part per billion); mg/L, milligram per liter (at low concentration, equivalent to part per million); s.d., standard deviation; <, less than; - , not determined]

\begin{tabular}{|c|c|c|c|c|c|c|c|c|c|c|c|c|c|c|}
\hline \multirow[t]{2}{*}{$\begin{array}{l}\text { Station } \\
\text { map ID }\end{array}$} & \multirow[t]{2}{*}{ Station name } & \multirow[t]{2}{*}{ Date } & \multirow[t]{2}{*}{ Time } & \multirow[t]{2}{*}{$\begin{array}{l}\text { Rep- } \\
\text { licate }\end{array}$} & \multicolumn{2}{|c|}{$\begin{array}{c}\text { Sulfur (S), } \\
\text { filtered } \\
(\mathrm{mg} / \mathrm{L}) \\
(63719) \\
\end{array}$} & \multicolumn{2}{|c|}{$\begin{array}{c}\text { Antimony (Sb), } \\
\text { filtered } \\
(\mu \mathrm{g} / \mathrm{L}) \\
(01095) \\
\end{array}$} & \multicolumn{2}{|c|}{$\begin{array}{c}\text { Selenium (Se), } \\
\text { filtered } \\
(\mu \mathrm{g} / \mathrm{L}) \\
(01145) \\
\end{array}$} & \multicolumn{2}{|c|}{$\begin{array}{c}\text { Silica }(\mathrm{Si}), \\
\text { filtered } \\
(\mathrm{mg} / \mathrm{L}) \\
(00955) \\
\end{array}$} & \multicolumn{2}{|c|}{$\begin{array}{c}\text { Samarium }(\mathrm{Sm}), \\
\text { filtered } \\
(\mu \mathrm{g} / \mathrm{L}) \\
(\mathbf{8 2 3 2 3 )}\end{array}$} \\
\hline & & & & & value & s.d. & value & s.d. & value & s.d. & value & s.d. & value & s.d. \\
\hline BY59 & $\begin{array}{l}\text { Greenhorn Cr at You } \\
\text { Bet Rd nr Nevada } \\
\text { City }\end{array}$ & $12 / 21 / 2001$ & $13: 00$ & 1 of 2 & 4.0 & 0.0 & 0.14 & 0.00 & 0.16 & 0.02 & 12 & 0 & 0.087 & 0.000 \\
\hline BY59 & $\begin{array}{l}\text { Greenhorn Cr at You } \\
\text { Bet Rd nr Nevada } \\
\text { City }\end{array}$ & $12 / 21 / 2001$ & $13: 00$ & $2 \mathrm{of} 2$ & 4.0 & 0.0 & 0.14 & 0.01 & 0.12 & 0.05 & 11 & 0 & 0.076 & 0.001 \\
\hline BY51 & $\begin{array}{l}\text { Greenhorn Cr nr } \\
\text { Headwaters nr Scotts } \\
\text { Flat Reservoir }\end{array}$ & $1 / 25 / 2000$ & $9: 30$ & 1 of 2 & 0.14 & 0.02 & 0.021 & 0.006 & $<0.8$ & 0.1 & 7.3 & 0.5 & 0.049 & 0.001 \\
\hline BY51 & $\begin{array}{l}\text { Greenhorn Cr nr } \\
\text { Headwaters nr Scotts } \\
\text { Flat Reservoir }\end{array}$ & $1 / 25 / 2000$ & $9: 30$ & $2 \mathrm{of} 2$ & 0.19 & 0.02 & 0.021 & 0.008 & $<0.8$ & 0.0 & 7.5 & 0.2 & 0.064 & 0.005 \\
\hline BY75 & $\begin{array}{l}\text { Missouri Cyn Cr } 1.2 \mathrm{mi} \\
\text { ab Greenhorn Cr nr } \\
\text { Chicago Park }\end{array}$ & $12 / 14 / 1999$ & $14: 00$ & 1 of 2 & - & & 0.069 & 0.005 & 0.15 & 0.08 & 22 & 3 & 0.055 & 0.004 \\
\hline BY75 & $\begin{array}{l}\text { Missouri Cyn Cr } 1.2 \mathrm{mi} \\
\text { ab Greenhorn Cr nr } \\
\text { Chicago Park }\end{array}$ & $12 / 14 / 1999$ & $14: 00$ & $2 o f 2$ & - & & 0.067 & 0.001 & 0.16 & 0.02 & 28 & 4 & 0.053 & 0.000 \\
\hline BY86 & $\begin{array}{l}\text { Poore Mine creek ab } \\
\text { tunnel nr Grass } \\
\text { Valley }\end{array}$ & 3/31/1999 & $17: 40$ & 1 of 2 & - & & 0.014 & 0.001 & $<0.4$ & 0.2 & 18 & 1 & 0.015 & 0.002 \\
\hline BY86 & $\begin{array}{l}\text { Poore Mine creek ab } \\
\text { tunnel nr Grass } \\
\text { Valley }\end{array}$ & 3/31/1999 & $17: 40$ & $2 \mathrm{of} 2$ & - & & 0.012 & 0.002 & $<0.4$ & 0.3 & 17 & 0 & 0.017 & 0.004 \\
\hline BY87 & $\begin{array}{l}\text { Poore Mine creek bl } \\
\text { tunnel nr Grass } \\
\text { Valley }\end{array}$ & 3/31/1999 & $13: 30$ & 1 of 2 & - & & 0.021 & 0.001 & $<0.4$ & 0.0 & 18 & 0 & 0.015 & 0.000 \\
\hline BY87 & $\begin{array}{l}\text { Poore Mine creek bl } \\
\text { tunnel nr Grass } \\
\text { Valley }\end{array}$ & $3 / 31 / 1999$ & $13: 30$ & $2 \mathrm{of} 2$ & - & & 0.018 & 0.001 & $<0.4$ & 0.1 & 17 & 0 & 0.014 & 0.002 \\
\hline BY88 & $\begin{array}{l}\text { Poore Mine ground } \\
\text { sluice nr Grass } \\
\text { Valley }\end{array}$ & 4/1/1999 & $15: 40$ & $2 o f 2$ & - & & 0.023 & 0.005 & 0.12 & 0.17 & 28 & 1 & 0.025 & 0.002 \\
\hline
\end{tabular}


Table 4B. Concentrations of trace metals and selected major elements in filtered water samples, Greenhorn Creek drainage, Nevada County, California—Continued.

[Station name abbreviations: ab, above; bl, below; Cr, creek; Cyn, Canyon; mi, miles; N, north; nr, near; Rd, Road; S, south; SF, South Fork. Number in parentheses is the data parameter code, a five-digit code used in the U.S. Geological Survey computerized data system. $\mu \mathrm{g} / \mathrm{L}$, microgram per liter (equivalent to part per billion); $\mathrm{mg} / \mathrm{L}$, milligram per liter (at low concentration, equivalent to part per million); s.d., standard deviation; <, less than; - , not determined]

\begin{tabular}{|c|c|c|c|c|c|c|c|c|c|c|c|c|c|c|}
\hline \multirow[t]{2}{*}{$\begin{array}{l}\text { Station } \\
\text { map ID }\end{array}$} & \multirow[t]{2}{*}{ Station name } & \multirow[t]{2}{*}{ Date } & \multirow[t]{2}{*}{ Time } & \multirow[t]{2}{*}{$\begin{array}{l}\text { Rep- } \\
\text { licate }\end{array}$} & \multicolumn{2}{|c|}{$\begin{array}{c}\text { Sulfur (S), } \\
\text { filtered } \\
(\mathrm{mg} / \mathrm{L}) \\
(63719)\end{array}$} & \multicolumn{2}{|c|}{$\begin{array}{c}\text { Antimony (Sb), } \\
\text { filtered } \\
(\mu \mathrm{g} / \mathrm{L}) \\
(01095) \\
\end{array}$} & \multicolumn{2}{|c|}{$\begin{array}{c}\text { Selenium }(\mathrm{Se}), \\
\text { filtered } \\
(\mu \mathrm{g} / \mathrm{L}) \\
(01145) \\
\end{array}$} & \multicolumn{2}{|c|}{$\begin{array}{c}\text { Silica (Si), } \\
\text { filtered } \\
(\mathrm{mg} / \mathrm{L}) \\
(00955)\end{array}$} & \multicolumn{2}{|c|}{$\begin{array}{c}\text { Samarium }(\mathrm{Sm}), \\
\text { filtered } \\
(\mu \mathrm{g} / \mathrm{L}) \\
(\mathbf{8 2 3 2 3 )} \\
\end{array}$} \\
\hline & & & & & value & s.d. & value & s.d. & value & s.d. & value & s.d. & value & s.d. \\
\hline BY89 & $\begin{array}{l}\text { Poore Mine pit lake } \\
\text { nr Grass Valley }\end{array}$ & 4/1/1999 & $16: 50$ & $2 \mathrm{of} 2$ & - & & 0.13 & 0.00 & 0.089 & 0.011 & 14 & 0 & 0.0090 & 0.0013 \\
\hline BY89 & $\begin{array}{c}\text { Poore Mine pit lake } \\
\text { nr Grass Valley }\end{array}$ & $5 / 23 / 2000$ & $10: 45$ & 1 of 2 & 26 & 0 & 0.036 & 0.004 & $<0.3$ & 0.1 & 22 & 1 & 0.030 & 0.002 \\
\hline BY89 & $\begin{array}{l}\text { Poore Mine pit lake } \\
\text { nr Grass Valley }\end{array}$ & $5 / 23 / 2000$ & $10: 45$ & $2 \mathrm{of} 2$ & 28 & 1 & 0.033 & 0.001 & $<0.3$ & 0.2 & 23 & 1 & 0.030 & 0.001 \\
\hline BY90 & $\begin{array}{l}\text { Poore Mine seep } \\
\text { ab ground sluice } \\
\text { nr Grass Valley }\end{array}$ & 4/1/1999 & $16: 00$ & $1 \mathrm{of} 2$ & - & & 0.022 & 0.002 & 0.15 & 0.09 & 27 & 0 & 0.0074 & 0.0008 \\
\hline BY90 & $\begin{array}{l}\text { Poore Mine seep } \\
\text { ab ground sluice } \\
\text { nr Grass Valley }\end{array}$ & 4/1/1999 & $16: 00$ & $2 \mathrm{of} 2$ & - & & 0.023 & 0.002 & 0.16 & 0.02 & 28 & 0 & 0.0081 & 0.0006 \\
\hline BY91 & $\begin{array}{l}\text { Poore Mine tunnel } \\
\text { effluent nr Grass } \\
\text { Valley }\end{array}$ & 3/31/1999 & $15: 20$ & $2 \mathrm{of} 2$ & - & & 0.079 & 0.004 & 0.35 & 0.02 & 30 & 1 & 0.0027 & 0.0008 \\
\hline BY91 & $\begin{array}{l}\text { Poore Mine tunnel } \\
\text { effluent nr Grass } \\
\text { Valley }\end{array}$ & $12 / 15 / 1999$ & $13: 00$ & 1 of 2 & - & & 0.070 & 0.005 & 0.41 & 0.07 & 36 & 2 & 0.0008 & 0.0004 \\
\hline BY91 & $\begin{array}{l}\text { Poore Mine tunnel } \\
\text { effluent nr Grass } \\
\text { Valley }\end{array}$ & $12 / 15 / 1999$ & $13: 00$ & $2 \mathrm{of} 2$ & - & & 0.30 & 0.32 & 0.42 & 0.03 & 37 & 2 & 0.0011 & 0.0005 \\
\hline BY91 & $\begin{array}{l}\text { Poore Mine tunnel } \\
\text { effluent nr Grass } \\
\text { Valley }\end{array}$ & $5 / 23 / 2000$ & $12: 00$ & 1 of 2 & 18 & 1 & 0.071 & 0.001 & $<0.3$ & 0.2 & 38 & 1 & $<0.003$ & 0.003 \\
\hline BY91 & $\begin{array}{l}\text { Poore Mine tunnel } \\
\text { effluent nr Grass } \\
\text { Valley }\end{array}$ & $5 / 23 / 2000$ & $12: 00$ & $2 \mathrm{of} 2$ & 16 & 0 & 0.064 & 0.007 & $<0.8$ & 0.8 & 36 & 1 & $<0.004$ & 0.002 \\
\hline BY105 & $\begin{array}{l}\text { Sailor Flat Mine main } \\
\text { drain Gulch 01nr } \\
\text { Quaker Hill }\end{array}$ & 7/6/1999 & $16: 00$ & $1 \mathrm{of} 2$ & - & & 0.060 & 0.002 & 0.093 & 0.036 & 28 & 0 & 0.23 & 0.00 \\
\hline BY105 & $\begin{array}{l}\text { Sailor Flat Mine main } \\
\text { drain Gulch } 01 \mathrm{nr}\end{array}$ & 7/6/1999 & $16: 00$ & $2 \mathrm{of} 2$ & - & & 0.057 & 0.003 & $<0.4$ & 0.0 & 24 & 0 & 0.21 & 0.00 \\
\hline
\end{tabular}


Table 4B. Concentrations of trace metals and selected major elements in filtered water samples, Greenhorn Creek drainage, Nevada County, California—Continued.

[Station name abbreviations: ab, above; bl, below; Cr, creek; Cyn, Canyon; mi, miles; N, north; nr, near; Rd, Road; S, south; SF, South Fork. Number in parentheses is the data parameter code, a five-digit code used in the U.S. Geological Survey computerized data system. $\mu \mathrm{g} / \mathrm{L}$, microgram per liter (equivalent to part per billion); mg/L, milligram per liter (at low concentration, equivalent to part per million); s.d., standard deviation; <, less than; - , not determined]

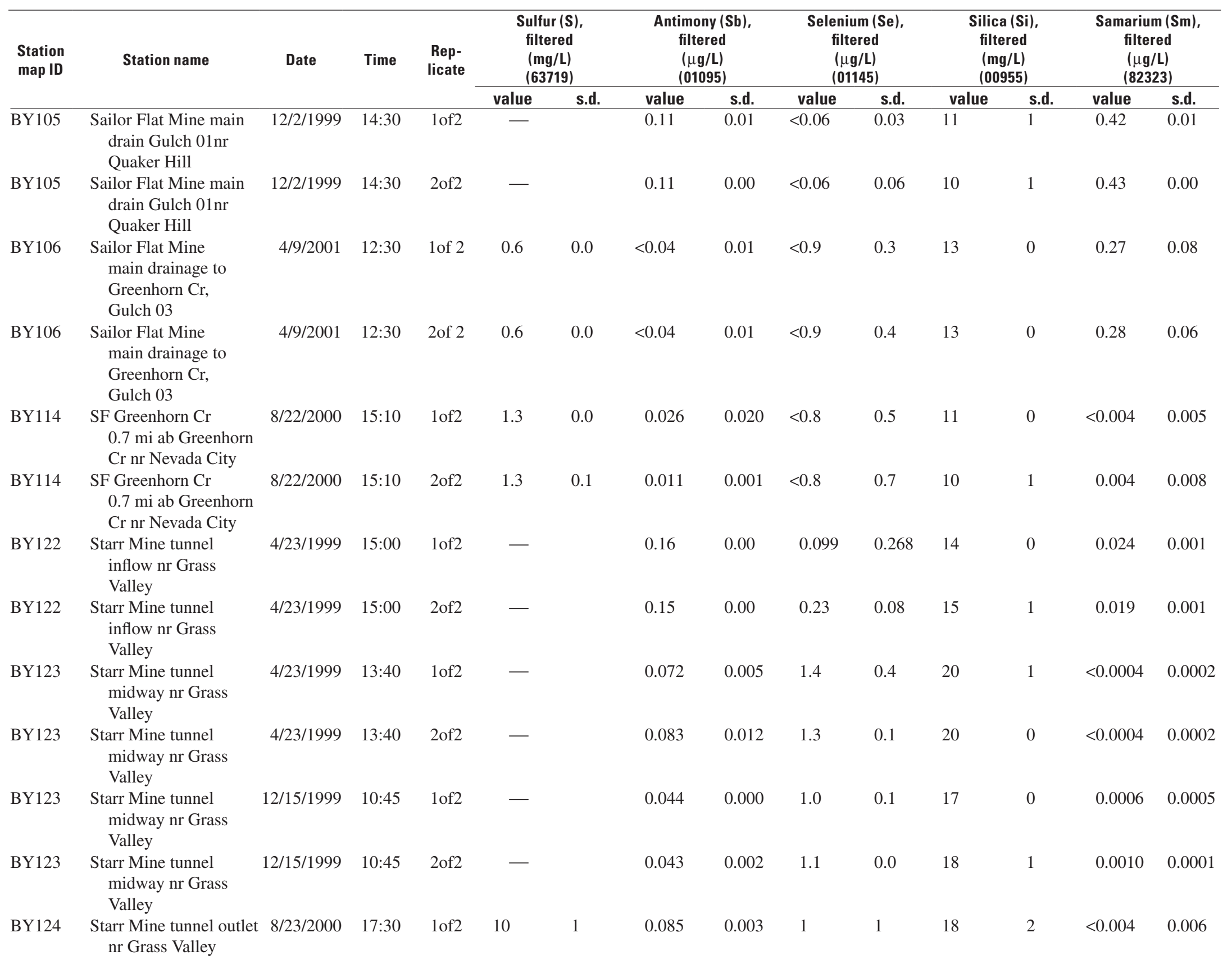


Table 4B. Concentrations of trace metals and selected major elements in filtered water samples, Greenhorn Creek drainage, Nevada County, California—Continued.

[Station name abbreviations: ab, above; bl, below; Cr, creek; Cyn, Canyon; mi, miles; N, north; nr, near; Rd, Road; S, south; SF, South Fork. Number in parentheses is the data parameter code, a five-digit code used in the U.S. Geological Survey computerized data system. $\mu \mathrm{g} / \mathrm{L}$, microgram per liter (equivalent to part per billion); mg/L, milligram per liter (at low concentration, equivalent to part per million); s.d., standard deviation; <, less than; - , not determined]

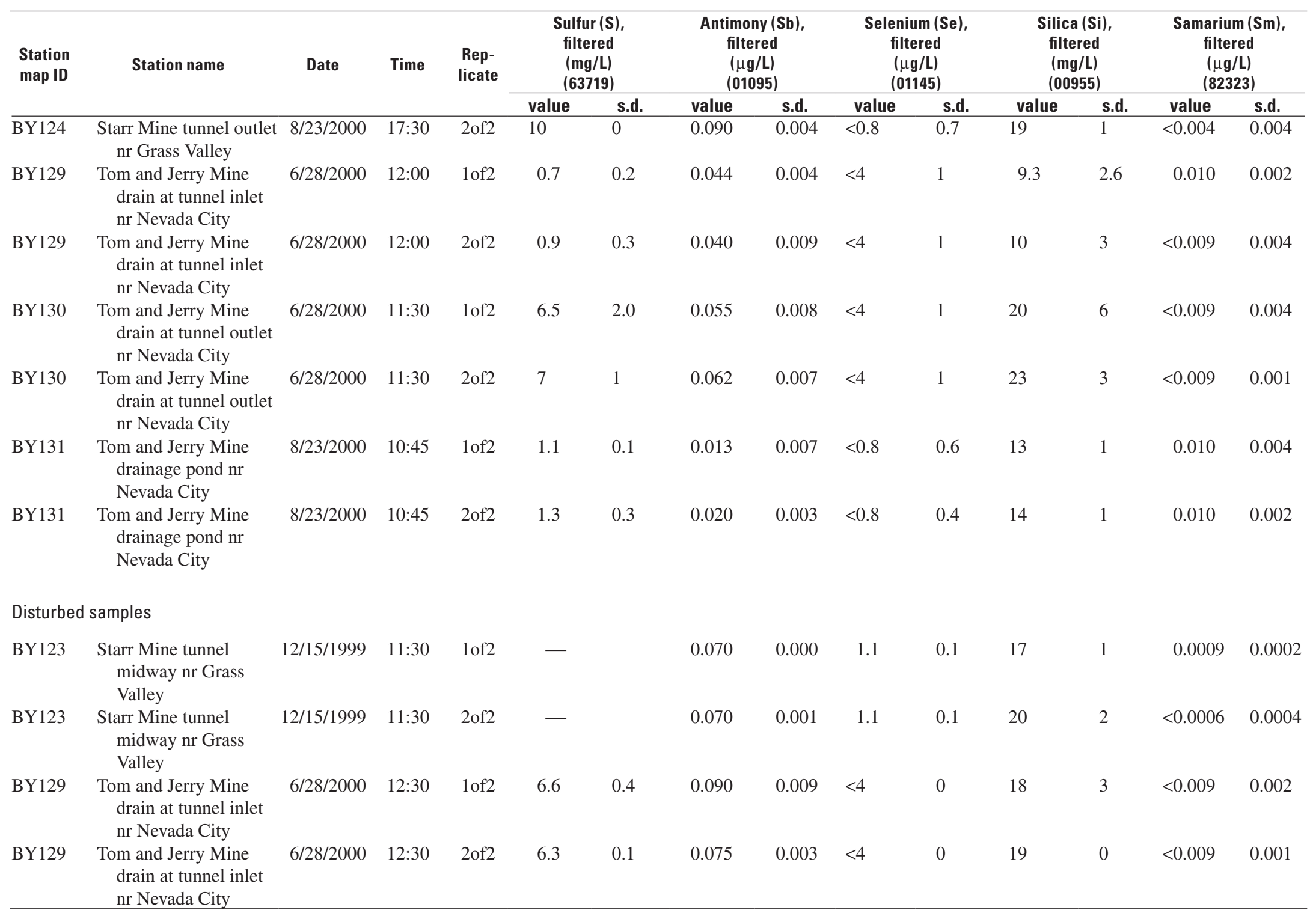


Table 4B. Concentrations of trace metals and selected major elements in filtered water samples, Greenhorn Creek drainage, Nevada County, California—Continued.

[Station name abbreviations: ab, above; bl, below; Cr, creek; Cyn, Canyon; mi, miles; N, north; nr, near; Rd, Road; S, south; SF, South Fork. Number in parentheses is the data parameter code, a five-digit code used in the U.S. Geological Survey computerized data system. $\mu \mathrm{g} / \mathrm{L}$, microgram per liter (equivalent to part per billion); $\mathrm{mg} / \mathrm{L}$, milligram per liter (at low concentration, equivalent to part per million); s.d., standard deviation; <, less than; - , not determined]

\begin{tabular}{|c|c|c|c|c|c|c|c|c|c|c|c|c|}
\hline \multirow[t]{2}{*}{$\begin{array}{l}\text { Station } \\
\text { map ID }\end{array}$} & \multirow[t]{2}{*}{ Station name } & \multirow[t]{2}{*}{ Date } & \multirow[t]{2}{*}{ Time } & \multirow[t]{2}{*}{$\begin{array}{l}\text { Rep- } \\
\text { licate }\end{array}$} & \multicolumn{2}{|c|}{$\begin{array}{c}\text { Strontium (Sr), } \\
\text { filtered } \\
(\mu \mathrm{g} / \mathrm{L}) \\
(\mathbf{0 1 0 8 0}) \\
\end{array}$} & \multicolumn{2}{|c|}{$\begin{array}{c}\text { Terbium (Tb), } \\
\text { filtered } \\
(\mu \mathrm{g} / \mathrm{L}) \\
(\mathbf{5 0 5 8 6 )} \\
\end{array}$} & \multicolumn{2}{|c|}{$\begin{array}{c}\text { Tellurium (Te), } \\
\text { filtered } \\
(\mu \mathrm{g} / \mathrm{L}) \\
(\mathbf{5 0 5 8 5 )} \\
\end{array}$} & \multicolumn{2}{|c|}{$\begin{array}{c}\text { Thorium (Th), } \\
\text { filtered } \\
(\mu \mathrm{g} / \mathrm{L}) \\
(82365)\end{array}$} \\
\hline & & & & & value & s.d. & value & s.d. & value & s.d. & value & s.d. \\
\hline \multicolumn{13}{|c|}{ Environmental samples } \\
\hline BY20 & $\begin{array}{l}\text { Boston Mine tunnel } \\
\text { outlet nr Grass } \\
\text { Valley }\end{array}$ & $6 / 18 / 1999$ & $14: 30$ & 1 of 2 & 91 & 4 & 0.0005 & 0.0001 & $<0.004$ & 0.002 & 0.0006 & 0.0006 \\
\hline BY20 & $\begin{array}{l}\text { Boston Mine tunnel } \\
\text { outlet nr Grass } \\
\text { Valley }\end{array}$ & 6/18/1999 & $14: 30$ & $2 \mathrm{of} 2$ & 91 & 5 & 0.0005 & 0.0002 & $<0.004$ & 0.002 & 0.0003 & 0.0000 \\
\hline BY20 & $\begin{array}{l}\text { Boston Mine tunnel } \\
\text { outlet nr Grass } \\
\text { Valley }\end{array}$ & 12/14/1999 & $12: 00$ & 1 of 2 & 120 & 0 & $<0.0001$ & 0.0001 & $<0.003$ & 0.001 & $<0.001$ & 0.000 \\
\hline BY20 & $\begin{array}{l}\text { Boston Mine tunnel } \\
\text { outlet nr Grass } \\
\text { Valley }\end{array}$ & 12/14/1999 & $12: 00$ & $2 \mathrm{of} 2$ & 120 & 0 & $<0.0001$ & 0.0000 & $<0.003$ & 0.001 & $<0.001$ & 0.000 \\
\hline BY20 & $\begin{array}{l}\text { Boston Mine tunnel } \\
\text { outlet nr Grass } \\
\text { Valley }\end{array}$ & $5 / 24 / 2000$ & $16: 00$ & 1 of 2 & 82 & 2 & 0.0011 & 0.0001 & $<0.02$ & 0.01 & 0.0008 & 0.0002 \\
\hline BY20 & $\begin{array}{l}\text { Boston Mine tunnel } \\
\text { outlet nr Grass } \\
\text { Valley }\end{array}$ & $5 / 24 / 2000$ & $16: 00$ & $2 \mathrm{of} 2$ & 82 & 2 & 0.0008 & 0.0002 & $<0.02$ & 0.02 & 0.0007 & 0.0002 \\
\hline BY21 & $\begin{array}{l}\text { Boston Mine wetlands } \\
\text { pond nr Grass Valley }\end{array}$ & $12 / 14 / 1999$ & $10: 30$ & 1 of 2 & 120 & 0 & 0.0001 & 0.0000 & $<0.003$ & 0.003 & $<0.001$ & 0.001 \\
\hline BY21 & $\begin{array}{l}\text { Boston Mine wetlands } \\
\text { pond nr Grass Valley }\end{array}$ & $12 / 14 / 1999$ & $10: 30$ & $2 \mathrm{of} 2$ & 120 & 0 & $<0.0001$ & 0.0000 & $<0.003$ & 0.002 & $<0.001$ & 0.000 \\
\hline BY21 & $\begin{array}{l}\text { Boston Mine wetlands } \\
\text { pond nr Grass Valley }\end{array}$ & $5 / 24 / 2000$ & $16: 30$ & 1 of 2 & 73 & 1 & $<0.0007$ & 0.0001 & $<0.02$ & 0.02 & 0.0012 & 0.0005 \\
\hline BY21 & $\begin{array}{l}\text { Boston Mine wetlands } \\
\text { pond nr Grass Valley }\end{array}$ & $5 / 24 / 2000$ & $16: 30$ & $2 \mathrm{of} 2$ & 71 & 0 & $<0.0007$ & 0.0000 & $<0.02$ & 0.01 & 0.0011 & 0.0006 \\
\hline BY58 & $\begin{array}{l}\text { Buckeye Flat Mine } \\
\text { main drain } 0.45 \mathrm{mi} \\
\text { ab Greenhorn } \mathrm{Cr}\end{array}$ & $8 / 21 / 2000$ & $11: 00$ & 1 of 2 & 81 & 2 & 0.44 & 0.01 & $<0.08$ & 0.03 & 0.0045 & 0.0023 \\
\hline BY58 & $\begin{array}{l}\text { Buckeye Flat Mine } \\
\text { main drain } 0.45 \mathrm{mi} \\
\text { ab Greenhorn } \mathrm{Cr}\end{array}$ & $8 / 21 / 2000$ & 11:00 & $2 \mathrm{of} 2$ & 82 & 3 & 0.44 & 0.02 & $<0.08$ & 0.05 & 0.0054 & 0.0005 \\
\hline BY23 & $\begin{array}{c}\text { Buckeye Flat Mine N } \\
\text { drain to Greenhorn } \\
\text { Cr, Grass Valley }\end{array}$ & $6 / 18 / 1999$ & $11: 30$ & 1 of 2 & 150 & 10 & 0.91 & 0.00 & $<0.004$ & 0.005 & 0.011 & 0.000 \\
\hline BY23 & $\begin{array}{l}\text { Buckeye Flat Mine N } \\
\text { drain to Greenhorn } \\
\text { Cr, Grass Valley }\end{array}$ & $6 / 18 / 1999$ & $11: 30$ & $2 \mathrm{of} 2$ & 150 & 0 & 0.93 & 0.00 & 0.005 & 0.001 & 0.012 & 0.000 \\
\hline
\end{tabular}


Table 4B. Concentrations of trace metals and selected major elements in filtered water samples, Greenhorn Creek drainage, Nevada County, California—Continued.

[Station name abbreviations: ab, above; bl, below; Cr, creek; Cyn, Canyon; mi, miles; N, north; nr, near; Rd, Road; S, south; SF, South Fork. Number in parentheses is the data parameter code, a five-digit code used in the U.S. Geological Survey computerized data system. $\mu \mathrm{g} / \mathrm{L}$, microgram per liter (equivalent to part per billion); mg/L, milligram per liter (at low concentration, equivalent to part per million); s.d., standard deviation; <, less than; - , not determined]

\begin{tabular}{|c|c|c|c|c|c|c|c|c|c|c|c|c|}
\hline \multirow[t]{2}{*}{$\begin{array}{l}\text { Station } \\
\text { map ID }\end{array}$} & \multirow[t]{2}{*}{ Station name } & \multirow[t]{2}{*}{ Date } & \multirow[t]{2}{*}{ Time } & \multirow[t]{2}{*}{$\begin{array}{l}\text { Rep- } \\
\text { licate }\end{array}$} & \multicolumn{2}{|c|}{$\begin{array}{l}\text { Strontium }(\mathrm{Sr}), \\
\text { filtered } \\
(\mu \mathrm{g} / \mathrm{L}) \\
(\mathbf{0 1 0 8 0}) \\
\end{array}$} & \multicolumn{2}{|c|}{$\begin{array}{c}\text { Terbium (Tb), } \\
\text { filtered } \\
(\mu \mathrm{g} / \mathrm{L}) \\
(\mathbf{5 0 5 8 6 )} \\
\end{array}$} & \multicolumn{2}{|c|}{$\begin{array}{c}\text { Tellurium (Te), } \\
\text { filtered } \\
(\mu \mathrm{g} / \mathrm{L}) \\
(\mathbf{5 0 5 8 5 )} \\
\end{array}$} & \multicolumn{2}{|c|}{$\begin{array}{c}\text { Thorium (Th), } \\
\text { filtered } \\
(\mu \mathrm{g} / \mathrm{L}) \\
(\mathbf{8 2 3 6 5 )} \\
\end{array}$} \\
\hline & & & & & value & s.d. & value & s.d. & value & s.d. & value & s.d. \\
\hline BY23 & $\begin{array}{c}\text { Buckeye Flat Mine N } \\
\text { drain to Greenhorn } \\
\text { Cr, Grass Valley }\end{array}$ & $12 / 2 / 1999$ & $11: 00$ & 1of 2 & 89 & 1 & 0.62 & 0.01 & $<0.003$ & 0.001 & 0.021 & 0.001 \\
\hline BY23 & $\begin{array}{l}\text { Buckeye Flat Mine N } \\
\text { drain to Greenhorn } \\
\text { Cr, Grass Valley }\end{array}$ & 12/2/1999 & 11:00 & $2 \mathrm{of} 2$ & 87 & 0 & 0.61 & 0.03 & $<0.003$ & 0.003 & 0.024 & 0.003 \\
\hline BY23 & $\begin{array}{c}\text { Buckeye Flat Mine N } \\
\text { drain to Greenhorn } \\
\text { Cr, Grass Valley }\end{array}$ & $8 / 22 / 2000$ & $10: 45$ & 1 of 2 & 130 & 0 & 0.84 & 0.01 & $<0.08$ & 0.03 & 0.020 & 0.001 \\
\hline BY23 & $\begin{array}{c}\text { Buckeye Flat Mine N } \\
\text { drain to Greenhorn } \\
\text { Cr, Grass Valley }\end{array}$ & $8 / 22 / 2000$ & $10: 45$ & $2 \mathrm{of} 2$ & 130 & 0 & 0.82 & 0.05 & $<0.08$ & 0.05 & 0.020 & 0.001 \\
\hline BY116 & $\begin{array}{l}\text { Buckeye Flat Mine } \\
\text { pond drain } 0.15 \mathrm{mi} \\
\text { ab SF Greenhorn } \mathrm{Cr}\end{array}$ & $8 / 21 / 2000$ & $14: 10$ & 1 of 2 & 15 & 1 & 0.002 & 0.001 & $<0.08$ & 0.01 & 0.0014 & 0.0007 \\
\hline BY116 & $\begin{array}{l}\text { Buckeye Flat Mine } \\
\text { pond drain } 0.15 \mathrm{mi} \\
\text { ab SF Greenhorn } \mathrm{Cr}\end{array}$ & $8 / 21 / 2000$ & $14: 10$ & $2 \mathrm{of} 2$ & 15 & 1 & 0.002 & 0.001 & $<0.08$ & 0.05 & 0.0015 & 0.0007 \\
\hline BY24 & $\begin{array}{l}\text { Buckeye Flat Mine } \\
\text { S drain to SF } \\
\text { Greenhorn Cr, Grass } \\
\text { Valley }\end{array}$ & $8 / 26 / 1999$ & 11:00 & 1 of 2 & 5.5 & 0.4 & 0.016 & 0.000 & 0.04 & 0.00 & 0.0031 & 0.0006 \\
\hline BY24 & $\begin{array}{l}\text { Buckeye Flat Mine } \\
\text { S drain to SF } \\
\text { Greenhorn Cr, Grass } \\
\text { Valley }\end{array}$ & $8 / 26 / 1999$ & 11:00 & $2 \mathrm{of} 2$ & 5.3 & 0.1 & 0.015 & 0.001 & 0.04 & 0.00 & 0.0028 & 0.0003 \\
\hline BY24 & $\begin{array}{l}\text { Buckeye Flat Mine } \\
\text { S drain to SF } \\
\text { Greenhorn Cr, Grass } \\
\text { Valley }\end{array}$ & $8 / 22 / 2000$ & $12: 10$ & 1 of 2 & 5.8 & 0.2 & 0.013 & 0.002 & $<0.08$ & 0.03 & 0.0020 & 0.0006 \\
\hline BY24 & $\begin{array}{l}\text { Buckeye Flat Mine } \\
\text { S drain to SF } \\
\text { Greenhorn Cr, Grass } \\
\text { Valley }\end{array}$ & $8 / 22 / 2000$ & $12: 10$ & $2 \mathrm{of} 2$ & 5.9 & 0.3 & 0.012 & 0.002 & $<0.08$ & 0.04 & 0.0022 & 0.0008 \\
\hline BY25 & $\begin{array}{l}\text { Buckeye Flat Mine } \\
\text { upper drain }\end{array}$ & 4/9/2001 & $10: 00$ & 1 of 2 & 32 & 6 & 0.14 & 0.03 & $<0.08$ & 0.02 & 0.007 & 0.003 \\
\hline BY25 & $\begin{array}{l}\text { Buckeye Flat Mine } \\
\text { upper drain }\end{array}$ & 4/9/2001 & $10: 00$ & $2 \mathrm{of} 2$ & 32 & 2 & 0.14 & 0.00 & $<0.08$ & 0.04 & 0.007 & 0.004 \\
\hline
\end{tabular}


Table 4B. Concentrations of trace metals and selected major elements in filtered water samples, Greenhorn Creek drainage, Nevada County, California—Continued.

[Station name abbreviations: ab, above; bl, below; Cr, creek; Cyn, Canyon; mi, miles; N, north; nr, near; Rd, Road; S, south; SF, South Fork. Number in parentheses is the data parameter code, a five-digit code used in the U.S. Geological Survey computerized data system. $\mu \mathrm{g} / \mathrm{L}$, microgram per liter (equivalent to part per billion); $\mathrm{mg} / \mathrm{L}$, milligram per liter (at low concentration, equivalent to part per million); s.d., standard deviation; <, less than; —, not determined]

\begin{tabular}{|c|c|c|c|c|c|c|c|c|c|c|c|c|}
\hline \multirow[t]{2}{*}{$\begin{array}{l}\text { Station } \\
\text { map ID }\end{array}$} & \multirow[t]{2}{*}{ Station name } & \multirow[t]{2}{*}{ Date } & \multirow[t]{2}{*}{ Time } & \multirow[t]{2}{*}{$\begin{array}{l}\text { Rep- } \\
\text { licate }\end{array}$} & \multicolumn{2}{|c|}{$\begin{array}{c}\text { Strontium }(\mathrm{Sr}), \\
\text { filtered } \\
(\mu \mathrm{g} / \mathrm{L}) \\
(\mathbf{0 1 0 8 0}) \\
\end{array}$} & \multicolumn{2}{|c|}{$\begin{array}{c}\text { Terbium (Tb), } \\
\text { filtered } \\
(\mu \mathrm{g} / \mathrm{L}) \\
(\mathbf{5 0 5 8 6 )}\end{array}$} & \multicolumn{2}{|c|}{$\begin{array}{c}\text { Tellurium (Te), } \\
\text { filtered } \\
(\mu \mathrm{g} / \mathrm{L}) \\
(50585) \\
\end{array}$} & \multicolumn{2}{|c|}{$\begin{array}{c}\text { Thorium (Th), } \\
\text { filtered } \\
(\mu \mathrm{g} / \mathrm{L}) \\
(\mathbf{8 2 3 6 5 )} \\
\end{array}$} \\
\hline & & & & & value & s.d. & value & s.d. & value & s.d. & value & s.d. \\
\hline BY52 & $\begin{array}{l}\text { Greenhorn Cr } 0.3 \mathrm{mi} \\
\text { ab Buckeye Ford nr } \\
\text { Nevada City }\end{array}$ & $1 / 25 / 2000$ & $10: 45$ & 1 of 2 & 23 & 2 & 0.013 & 0.002 & 0.08 & 0.11 & 0.012 & 0.001 \\
\hline BY52 & $\begin{array}{l}\text { Greenhorn Cr } 0.3 \mathrm{mi} \\
\text { ab Buckeye Ford nr } \\
\text { Nevada City }\end{array}$ & $1 / 25 / 2000$ & $10: 45$ & $2 \mathrm{of} 2$ & 22 & 1 & 0.015 & 0.000 & $<0.06$ & 0.02 & 0.013 & 0.001 \\
\hline BY59 & $\begin{array}{l}\text { Greenhorn Cr at You } \\
\text { Bet Rd nr Nevada } \\
\text { City }\end{array}$ & $7 / 24 / 2001$ & 11:00 & 1 of 2 & 44 & 1 & 0.0004 & 0.0001 & $<0.002$ & 0.001 & 0.008 & 0.007 \\
\hline BY59 & $\begin{array}{l}\text { Greenhorn Cr at You } \\
\text { Bet Rd nr Nevada } \\
\text { City }\end{array}$ & $7 / 24 / 2001$ & 11:00 & $2 \mathrm{of} 2$ & 44 & 1 & 0.0004 & 0.0000 & $<0.002$ & 0.001 & $<0.001$ & 0.000 \\
\hline BY59 & $\begin{array}{l}\text { Greenhorn } \mathrm{Cr} \text { at You } \\
\text { Bet Rd nr Nevada } \\
\text { City }\end{array}$ & $8 / 29 / 2001$ & $15: 00$ & 1 of 2 & 46 & 0 & 0.0006 & 0.0001 & $<0.002$ & 0.005 & $<0.001$ & 0.000 \\
\hline BY59 & $\begin{array}{l}\text { Greenhorn Cr at You } \\
\text { Bet Rd nr Nevada } \\
\text { City }\end{array}$ & $8 / 29 / 2001$ & $15: 00$ & $2 \mathrm{of} 2$ & 47 & 0 & 0.0004 & 0.0001 & $<0.002$ & 0.001 & $<0.001$ & 0.000 \\
\hline BY59 & $\begin{array}{l}\text { Greenhorn Cr at You } \\
\text { Bet Rd nr Nevada } \\
\text { City }\end{array}$ & 9/14/2001 & $13: 00$ & 1 of 2 & 46 & 1 & 0.0003 & 0.0001 & $<0.005$ & 0.003 & 0.001 & 0.001 \\
\hline BY59 & $\begin{array}{l}\text { Greenhorn Cr at You } \\
\text { Bet Rd nr Nevada } \\
\text { City }\end{array}$ & $9 / 14 / 2001$ & 13:00 & $2 \mathrm{of} 2$ & 47 & 0 & 0.0003 & 0.0001 & $<0.005$ & 0.003 & 0.002 & 0.001 \\
\hline BY59 & $\begin{array}{l}\text { Greenhorn Cr at You } \\
\text { Bet Rd nr Nevada } \\
\text { City }\end{array}$ & $10 / 15 / 2001$ & $12: 00$ & 1 of 2 & 46 & 0 & 0.0003 & 0.0001 & $<0.005$ & 0.004 & 0.004 & 0.005 \\
\hline BY59 & $\begin{array}{l}\text { Greenhorn Cr at You } \\
\text { Bet Rd nr Nevada } \\
\text { City }\end{array}$ & $10 / 15 / 2001$ & $12: 00$ & $2 \mathrm{of} 2$ & 46 & 0 & 0.0003 & 0.0000 & $<0.005$ & 0.002 & 0.001 & 0.001 \\
\hline BY59 & $\begin{array}{l}\text { Greenhorn Cr at You } \\
\text { Bet Rd nr Nevada } \\
\text { City }\end{array}$ & $11 / 20 / 2001$ & $13: 20$ & 1 of 2 & 52 & 0 & 0.0020 & 0.0001 & $<0.005$ & 0.002 & 0.003 & 0.003 \\
\hline BY59 & $\begin{array}{l}\text { Greenhorn Cr at You } \\
\text { Bet Rd nr Nevada } \\
\text { City }\end{array}$ & $11 / 20 / 2001$ & $13: 20$ & $2 \mathrm{of} 2$ & 53 & 1 & 0.0021 & 0.0001 & $<0.005$ & 0.003 & 0.002 & 0.002 \\
\hline BY59 & $\begin{array}{l}\text { Greenhorn Cr at You } \\
\text { Bet Rd nr Nevada } \\
\text { City }\end{array}$ & $12 / 10 / 2001$ & $12: 20$ & 1 of 2 & 51 & 0 & 0.0051 & 0.0001 & $<0.007$ & 0.002 & 0.003 & 0.001 \\
\hline
\end{tabular}


Table 4B. Concentrations of trace metals and selected major elements in filtered water samples, Greenhorn Creek drainage, Nevada County, California—Continued.

[Station name abbreviations: ab, above; bl, below; Cr, creek; Cyn, Canyon; mi, miles; N, north; nr, near; Rd, Road; S, south; SF, South Fork. Number in parentheses is the data parameter code, a five-digit code used in the U.S. Geological Survey computerized data system. $\mu \mathrm{g} / \mathrm{L}$, microgram per liter (equivalent to part per billion); $\mathrm{mg} / \mathrm{L}$, milligram per liter (at low concentration, equivalent to part per million); s.d., standard deviation; <, less than; - , not determined]

\begin{tabular}{|c|c|c|c|c|c|c|c|c|c|c|c|c|}
\hline \multirow[t]{2}{*}{$\begin{array}{l}\text { Station } \\
\text { map ID }\end{array}$} & \multirow[t]{2}{*}{ Station name } & \multirow[t]{2}{*}{ Date } & \multirow[t]{2}{*}{ Time } & \multirow[t]{2}{*}{$\begin{array}{l}\text { Rep- } \\
\text { licate }\end{array}$} & \multicolumn{2}{|c|}{$\begin{array}{c}\text { Strontium }(\mathrm{Sr}), \\
\text { filtered } \\
(\mu \mathrm{g} / \mathrm{L}) \\
(\mathbf{0 1 0 8 0})\end{array}$} & \multicolumn{2}{|c|}{$\begin{array}{c}\text { Terbium (Tb), } \\
\text { filtered } \\
(\mu \mathrm{g} / \mathrm{L}) \\
(\mathbf{5 0 5 8 6 )}\end{array}$} & \multicolumn{2}{|c|}{$\begin{array}{c}\text { Tellurium (Te), } \\
\text { filtered } \\
(\mu \mathrm{g} / \mathrm{L}) \\
(50585) \\
\end{array}$} & \multicolumn{2}{|c|}{$\begin{array}{c}\text { Thorium (Th), } \\
\text { filtered } \\
(\mu \mathrm{g} / \mathrm{L}) \\
(82365)\end{array}$} \\
\hline & & & & & value & s.d. & value & s.d. & value & s.d. & value & s.d. \\
\hline BY59 & $\begin{array}{l}\text { Greenhorn Cr at You } \\
\text { Bet Rd nr Nevada } \\
\text { City }\end{array}$ & $12 / 10 / 2001$ & $12: 20$ & $2 \mathrm{of} 2$ & 51 & 1 & 0.0058 & 0.0000 & $<0.007$ & 0.002 & 0.002 & 0.001 \\
\hline BY59 & $\begin{array}{l}\text { Greenhorn Cr at You } \\
\text { Bet Rd nr Nevada } \\
\text { City }\end{array}$ & $12 / 21 / 2001$ & $13: 00$ & 1 of 2 & 37 & 0 & 0.011 & 0.000 & $<0.008$ & 0.001 & 0.0043 & 0.0021 \\
\hline BY59 & $\begin{array}{l}\text { Greenhorn Cr at You } \\
\text { Bet Rd nr Nevada } \\
\text { City }\end{array}$ & $12 / 21 / 2001$ & $13: 00$ & $2 \mathrm{of} 2$ & 36 & 1 & 0.011 & 0.000 & $<0.008$ & 0.000 & 0.0043 & 0.0025 \\
\hline BY51 & $\begin{array}{l}\text { Greenhorn Cr nr } \\
\text { Headwaters nr Scotts } \\
\text { Flat Reservoir }\end{array}$ & $1 / 25 / 2000$ & $9: 30$ & 1 of 2 & 18 & 1 & 0.0061 & 0.0000 & $<0.06$ & 0.04 & 0.011 & 0.002 \\
\hline BY51 & $\begin{array}{l}\text { Greenhorn Cr nr } \\
\text { Headwaters nr Scotts } \\
\text { Flat Reservoir }\end{array}$ & $1 / 25 / 2000$ & $9: 30$ & $2 \mathrm{of} 2$ & 17 & 2 & 0.0081 & 0.0017 & $<0.06$ & 0.02 & 0.010 & 0.001 \\
\hline BY75 & $\begin{array}{l}\text { Missouri Cyn Cr } 1.2 \mathrm{mi} \\
\text { ab Greenhorn Cr nr } \\
\text { Chicago Park }\end{array}$ & $12 / 14 / 1999$ & $14: 00$ & 1 of 2 & 100 & 0 & 0.0097 & 0.0001 & $<0.003$ & 0.002 & 0.002 & 0.001 \\
\hline BY75 & $\begin{array}{l}\text { Missouri Cyn Cr } 1.2 \mathrm{mi} \\
\text { ab Greenhorn Cr nr } \\
\text { Chicago Park }\end{array}$ & $12 / 14 / 1999$ & $14: 00$ & $2 \mathrm{of} 2$ & 100 & 0 & 0.0092 & 0.0000 & $<0.003$ & 0.005 & 0.002 & 0.000 \\
\hline BY86 & $\begin{array}{l}\text { Poore Mine creek ab } \\
\text { tunnel nr Grass } \\
\text { Valley }\end{array}$ & 3/31/1999 & $17: 40$ & 1 of 2 & 41 & 1 & 0.0023 & 0.0005 & $<0.007$ & 0.006 & 0.0005 & 0.0001 \\
\hline BY86 & $\begin{array}{l}\text { Poore Mine creek ab } \\
\text { tunnel nr Grass } \\
\text { Valley }\end{array}$ & 3/31/1999 & $17: 40$ & $2 \mathrm{of} 2$ & 41 & 1 & 0.0020 & 0.0001 & $<0.007$ & 0.008 & 0.0005 & 0.0002 \\
\hline BY87 & $\begin{array}{l}\text { Poore Mine creek bl } \\
\text { tunnel nr Grass } \\
\text { Valley }\end{array}$ & 3/31/1999 & $13: 30$ & 1 of 2 & 42 & 1 & 0.0027 & 0.0003 & $<0.007$ & 0.004 & 0.0004 & 0.0001 \\
\hline BY87 & $\begin{array}{l}\text { Poore Mine creek bl } \\
\text { tunnel nr Grass } \\
\text { Valley }\end{array}$ & $3 / 31 / 1999$ & $13: 30$ & $2 \mathrm{of} 2$ & 40 & 0 & 0.0023 & 0.0004 & $<0.007$ & 0.002 & $<0.0004$ & 0.0001 \\
\hline BY88 & $\begin{array}{l}\text { Poore Mine ground } \\
\text { sluice nr Grass } \\
\text { Valley }\end{array}$ & 4/1/1999 & $15: 40$ & 1 of 2 & 48 & 2 & 0.0046 & 0.0006 & $<0.007$ & 0.002 & 0.0010 & 0.0003 \\
\hline BY88 & $\begin{array}{l}\text { Poore Mine ground } \\
\text { sluice nr Grass } \\
\text { Valley }\end{array}$ & 4/1/1999 & $15: 40$ & $2 \mathrm{of} 2$ & 48 & 1 & 0.0043 & 0.0003 & $<0.004$ & 0.002 & 0.0006 & 0.0003 \\
\hline
\end{tabular}


Table 4B. Concentrations of trace metals and selected major elements in filtered water samples, Greenhorn Creek drainage, Nevada County, California—Continued.

[Station name abbreviations: ab, above; bl, below; Cr, creek; Cyn, Canyon; mi, miles; N, north; nr, near; Rd, Road; S, south; SF, South Fork. Number in parentheses is the data parameter code, a five-digit code used in the U.S. Geological Survey computerized data system. $\mu \mathrm{g} / \mathrm{L}$, microgram per liter (equivalent to part per billion); mg/L, milligram per liter (at low concentration, equivalent to part per million); s.d., standard deviation; <, less than; - , not determined]

\begin{tabular}{|c|c|c|c|c|c|c|c|c|c|c|c|c|}
\hline \multirow[t]{2}{*}{$\begin{array}{l}\text { Station } \\
\text { map ID }\end{array}$} & \multirow[t]{2}{*}{ Station name } & \multirow[t]{2}{*}{ Date } & \multirow[t]{2}{*}{ Time } & \multirow[t]{2}{*}{$\begin{array}{l}\text { Rep- } \\
\text { licate }\end{array}$} & \multicolumn{2}{|c|}{$\begin{array}{c}\text { Strontium }(\mathrm{Sr}), \\
\text { filtered } \\
(\mu \mathrm{g} / \mathrm{L}) \\
(\mathbf{0 1 0 8 0})\end{array}$} & \multicolumn{2}{|c|}{$\begin{array}{c}\text { Terbium (Tb), } \\
\text { filtered } \\
(\mu \mathrm{g} / \mathrm{L}) \\
(\mathbf{5 0 5 8 6 )}\end{array}$} & \multicolumn{2}{|c|}{$\begin{array}{c}\text { Tellurium (Te), } \\
\text { filtered } \\
(\mu \mathrm{g} / \mathrm{L}) \\
(\mathbf{5 0 5 8 5 )}\end{array}$} & \multicolumn{2}{|c|}{$\begin{array}{c}\text { Thorium (Th), } \\
\text { filtered } \\
(\mu \mathrm{g} / \mathrm{L}) \\
(\mathbf{8 2 3 6 5 )}\end{array}$} \\
\hline & & & & & value & s.d. & value & s.d. & value & s.d. & value & s.d. \\
\hline BY89 & $\begin{array}{c}\text { Poore Mine pit lake } \\
\text { nr Grass Valley }\end{array}$ & 4/1/1999 & $16: 50$ & 1 of 2 & 25 & 1 & 0.0012 & 0.0002 & 0.007 & 0.004 & 0.0009 & 0.0000 \\
\hline BY89 & $\begin{array}{l}\text { Poore Mine pit lake } \\
\text { nr Grass Valley }\end{array}$ & 4/1/1999 & $16: 50$ & $2 \mathrm{of} 2$ & 24 & 1 & 0.0006 & 0.0007 & $<0.004$ & 0.002 & 0.0004 & 0.0005 \\
\hline BY89 & $\begin{array}{l}\text { Poore Mine pit lake } \\
\text { nr Grass Valley }\end{array}$ & $5 / 23 / 2000$ & $10: 45$ & 1 of 2 & 120 & 0 & 0.0067 & 0.0001 & $<0.02$ & 0.01 & 0.0008 & 0.0012 \\
\hline BY89 & $\begin{array}{l}\text { Poore Mine pit lake } \\
\text { nr Grass Valley }\end{array}$ & $5 / 23 / 2000$ & $10: 45$ & $2 \mathrm{of} 2$ & 120 & 0 & 0.0057 & 0.0003 & $<0.02$ & 0.02 & 0.0011 & 0.0007 \\
\hline BY90 & $\begin{array}{l}\text { Poore Mine seep } \\
\text { ab ground sluice } \\
\text { nr Grass Valley }\end{array}$ & 4/1/1999 & $16: 00$ & 1 of 2 & 44 & 1 & 0.0016 & 0.0003 & $<0.004$ & 0.004 & 0.0004 & 0.0001 \\
\hline BY90 & $\begin{array}{l}\text { Poore Mine seep } \\
\text { ab ground sluice } \\
\text { nr Grass Valley }\end{array}$ & 4/1/1999 & $16: 00$ & $2 \mathrm{of} 2$ & 44 & 1 & 0.0013 & 0.0002 & $<0.004$ & 0.000 & 0.0008 & 0.0003 \\
\hline BY91 & $\begin{array}{l}\text { Poore Mine tunnel } \\
\text { effluent nr Grass } \\
\text { Valley }\end{array}$ & $3 / 31 / 1999$ & $15: 20$ & 1 of 2 & 87 & 0 & 0.0004 & 0.0000 & $<0.007$ & 0.002 & 0.0012 & 0.0009 \\
\hline BY91 & $\begin{array}{l}\text { Poore Mine tunnel } \\
\text { effluent nr Grass } \\
\text { Valley }\end{array}$ & 3/31/1999 & $15: 20$ & $2 \mathrm{of} 2$ & 91 & 4 & 0.0005 & 0.0002 & $<0.004$ & 0.001 & 0.0002 & 0.0001 \\
\hline BY91 & $\begin{array}{l}\text { Poore Mine tunnel } \\
\text { effluent nr Grass } \\
\text { Valley }\end{array}$ & $12 / 15 / 1999$ & 13:00 & 1 of 2 & 130 & 10 & $<0.0001$ & 0.0001 & $<0.003$ & 0.003 & $<0.001$ & 0.000 \\
\hline BY91 & $\begin{array}{l}\text { Poore Mine tunnel } \\
\text { effluent nr Grass } \\
\text { Valley }\end{array}$ & $12 / 15 / 1999$ & 13:00 & $2 \mathrm{of} 2$ & 130 & 0 & 0.0001 & 0.0001 & 0.005 & 0.002 & $<0.001$ & 0.000 \\
\hline BY91 & $\begin{array}{l}\text { Poore Mine tunnel } \\
\text { effluent nr Grass } \\
\text { Valley }\end{array}$ & $5 / 23 / 2000$ & $12: 00$ & 1 of 2 & 120 & 0 & $<0.0007$ & 0.0005 & $<0.02$ & 0.01 & $<0.0003$ & 0.0002 \\
\hline BY91 & $\begin{array}{l}\text { Poore Mine tunnel } \\
\text { effluent nr Grass } \\
\text { Valley }\end{array}$ & $5 / 23 / 2000$ & $12: 00$ & $2 \mathrm{of} 2$ & 130 & 0 & $<0.0004$ & 0.0001 & $<0.06$ & 0.01 & $<0.001$ & 0.001 \\
\hline BY105 & $\begin{array}{l}\text { Sailor Flat Mine main } \\
\text { drain Gulch 01nr } \\
\text { Quaker Hill }\end{array}$ & 7/6/1999 & $16: 00$ & 1 of 2 & 57 & 2 & 0.030 & 0.001 & 0.007 & 0.003 & 0.021 & 0.001 \\
\hline BY105 & $\begin{array}{l}\text { Sailor Flat Mine main } \\
\text { drain Gulch 01nr } \\
\text { Quaker Hill }\end{array}$ & 7/6/1999 & $16: 00$ & $2 \mathrm{of} 2$ & 56 & 1 & 0.030 & 0.002 & $<0.007$ & 0.005 & 0.018 & 0.000 \\
\hline
\end{tabular}


Table 4B. Concentrations of trace metals and selected major elements in filtered water samples, Greenhorn Creek drainage, Nevada County, California—Continued.

[Station name abbreviations: ab, above; bl, below; Cr, creek; Cyn, Canyon; mi, miles; N, north; nr, near; Rd, Road; S, south; SF, South Fork. Number in parentheses is the data parameter code, a five-digit code used in the U.S. Geological Survey computerized data system. $\mu \mathrm{g} / \mathrm{L}$, microgram per liter (equivalent to part per billion); $\mathrm{mg} / \mathrm{L}$, milligram per liter (at low concentration, equivalent to part per million); s.d., standard deviation; <, less than; - , not determined]

\begin{tabular}{|c|c|c|c|c|c|c|c|c|c|c|c|c|}
\hline \multirow[t]{2}{*}{$\begin{array}{l}\text { Station } \\
\text { map ID }\end{array}$} & \multirow[t]{2}{*}{ Station name } & \multirow[t]{2}{*}{ Date } & \multirow[t]{2}{*}{ Time } & \multirow[t]{2}{*}{$\begin{array}{l}\text { Rep- } \\
\text { licate }\end{array}$} & \multicolumn{2}{|c|}{$\begin{array}{c}\text { Strontium }(\mathrm{Sr}), \\
\text { filtered } \\
(\mu \mathrm{g} / \mathrm{L}) \\
(\mathbf{0 1 0 8 0})\end{array}$} & \multicolumn{2}{|c|}{$\begin{array}{c}\text { Terbium (Tb), } \\
\text { filtered } \\
(\mu \mathrm{g} / \mathrm{L}) \\
(\mathbf{5 0 5 8 6 )}\end{array}$} & \multicolumn{2}{|c|}{$\begin{array}{c}\text { Tellurium (Te), } \\
\text { filtered } \\
(\mu \mathrm{g} / \mathrm{L}) \\
(50585)\end{array}$} & \multicolumn{2}{|c|}{$\begin{array}{c}\text { Thorium (Th), } \\
\text { filtered } \\
(\mu \mathrm{g} / \mathrm{L}) \\
(\mathbf{8 2 3 6 5 )}\end{array}$} \\
\hline & & & & & value & s.d. & value & s.d. & value & s.d. & value & s.d. \\
\hline BY105 & $\begin{array}{l}\text { Sailor Flat Mine main } \\
\text { drain Gulch 01nr } \\
\text { Quaker Hill }\end{array}$ & $12 / 2 / 1999$ & $14: 30$ & 1 of 2 & 20 & 0 & 0.051 & 0.002 & 0.006 & 0.001 & 0.14 & 0.00 \\
\hline BY105 & $\begin{array}{l}\text { Sailor Flat Mine main } \\
\text { drain Gulch 01nr } \\
\text { Quaker Hill }\end{array}$ & $12 / 2 / 1999$ & $14: 30$ & $2 \mathrm{of} 2$ & 19 & 0 & 0.052 & 0.000 & 0.005 & 0.004 & 0.12 & 0.00 \\
\hline BY106 & $\begin{array}{l}\text { Sailor Flat Mine } \\
\text { main drainage to } \\
\text { Greenhorn Cr, } \\
\text { Gulch } 03\end{array}$ & 4/9/2001 & $12: 30$ & 1 of 2 & 11 & 2 & 0.038 & 0.011 & $<0.08$ & 0.02 & 0.11 & 0.04 \\
\hline BY106 & $\begin{array}{l}\text { Sailor Flat Mine } \\
\text { main drainage to } \\
\text { Greenhorn Cr, } \\
\text { Gulch } 03\end{array}$ & 4/9/2001 & $12: 30$ & 2 of 2 & 11 & 2 & 0.037 & 0.009 & $<0.08$ & 0.01 & 0.14 & 0.00 \\
\hline BY114 & $\begin{array}{l}\text { SF Greenhorn Cr } \\
0.7 \text { mi ab Greenhorn } \\
\text { Cr nr Nevada City }\end{array}$ & $8 / 22 / 2000$ & $15: 10$ & 1 of 2 & 40 & 1 & $<0.001$ & 0.001 & $<0.08$ & 0.04 & $<0.001$ & 0.001 \\
\hline BY114 & $\begin{array}{l}\text { SF Greenhorn Cr } \\
0.7 \text { mi ab Greenhorn } \\
\text { Cr nr Nevada City }\end{array}$ & $8 / 22 / 2000$ & $15: 10$ & $2 \mathrm{of} 2$ & 40 & 1 & $<0.001$ & 0.000 & $<0.08$ & 0.05 & $<0.001$ & 0.001 \\
\hline BY122 & $\begin{array}{l}\text { Starr Mine tunnel } \\
\text { inflow nr Grass } \\
\text { Valley }\end{array}$ & 4/23/1999 & $15: 00$ & 1 of 2 & 58 & 3 & 0.0037 & 0.0002 & $<0.004$ & 0.000 & 0.0005 & 0.0002 \\
\hline BY122 & $\begin{array}{l}\text { Starr Mine tunnel } \\
\text { inflow nr Grass } \\
\text { Valley }\end{array}$ & 4/23/1999 & $15: 00$ & $2 \mathrm{of} 2$ & 59 & 2 & 0.0036 & 0.0007 & 0.004 & 0.002 & 0.0004 & 0.0001 \\
\hline BY123 & $\begin{array}{l}\text { Starr Mine tunnel } \\
\text { midway nr Grass } \\
\text { Valley }\end{array}$ & 4/23/1999 & $13: 40$ & 1 of 2 & 210 & 10 & $<0.0001$ & 0.0000 & $<0.004$ & 0.002 & $<0.0002$ & 0.0002 \\
\hline BY123 & $\begin{array}{l}\text { Starr Mine tunnel } \\
\text { midway nr Grass } \\
\text { Valley }\end{array}$ & 4/23/1999 & $13: 40$ & $2 \mathrm{of} 2$ & 210 & 10 & $<0.0001$ & 0.0000 & $<0.004$ & 0.006 & 0.0003 & 0.0003 \\
\hline BY123 & $\begin{array}{l}\text { Starr Mine tunnel } \\
\text { midway nr Grass } \\
\text { Valley }\end{array}$ & 12/15/1999 & $10: 45$ & 1 of 2 & 250 & 0 & $<0.0001$ & 0.0000 & $<0.003$ & 0.004 & $<0.001$ & 0.000 \\
\hline BY123 & $\begin{array}{l}\text { Starr Mine tunnel } \\
\text { midway nr Grass } \\
\text { Valley }\end{array}$ & $12 / 15 / 1999$ & $10: 45$ & $2 \mathrm{of} 2$ & 250 & 0 & $<0.0001$ & 0.0000 & $<0.003$ & 0.003 & 0.002 & 0.002 \\
\hline BY124 & $\begin{array}{l}\text { Starr Mine tunnel outlet } \\
\text { nr Grass Valley }\end{array}$ & $8 / 23 / 2000$ & $17: 30$ & 1 of 2 & 220 & 0 & $<0.001$ & 0.000 & $<0.08$ & 0.03 & 0.0014 & 0.0008 \\
\hline
\end{tabular}


Table 4B. Concentrations of trace metals and selected major elements in filtered water samples, Greenhorn Creek drainage, Nevada County, California—Continued.

[Station name abbreviations: ab, above; bl, below; Cr, creek; Cyn, Canyon; mi, miles; N, north; nr, near; Rd, Road; S, south; SF, South Fork. Number in parentheses is the data parameter code, a five-digit code used in the U.S. Geological Survey computerized data system. $\mu \mathrm{g} / \mathrm{L}$, microgram per liter (equivalent to part per billion); $\mathrm{mg} / \mathrm{L}$, milligram per liter (at low concentration, equivalent to part per million); s.d., standard deviation; <, less than; —, not determined]

\begin{tabular}{|c|c|c|c|c|c|c|c|c|c|c|c|c|}
\hline \multirow[t]{2}{*}{$\begin{array}{l}\text { Station } \\
\text { map ID }\end{array}$} & \multirow[t]{2}{*}{ Station name } & \multirow[t]{2}{*}{ Date } & \multirow[t]{2}{*}{ Time } & \multirow[t]{2}{*}{$\begin{array}{l}\text { Rep- } \\
\text { licate }\end{array}$} & \multicolumn{2}{|c|}{$\begin{array}{c}\text { Strontium }(\mathrm{Sr}), \\
\text { filtered } \\
(\mu \mathrm{g} / \mathrm{L}) \\
(\mathbf{0 1 0 8 0})\end{array}$} & \multicolumn{2}{|c|}{$\begin{array}{c}\text { Terbium (Tb), } \\
\text { filtered } \\
(\mu \mathrm{g} / \mathrm{L}) \\
(50586)\end{array}$} & \multicolumn{2}{|c|}{$\begin{array}{c}\text { Tellurium (Te), } \\
\text { filtered } \\
(\mu \mathrm{g} / \mathrm{L}) \\
(\mathbf{5 0 5 8 5 )}\end{array}$} & \multicolumn{2}{|c|}{$\begin{array}{c}\text { Thorium (Th), } \\
\text { filtered } \\
(\mu \mathrm{g} / \mathrm{L}) \\
(\mathbf{8 2 3 6 5 )}\end{array}$} \\
\hline & & & & & value & s.d. & value & s.d. & value & s.d. & value & s.d. \\
\hline BY124 & $\begin{array}{l}\text { Starr Mine tunnel outlet } \\
\text { nr Grass Valley }\end{array}$ & $8 / 23 / 2000$ & $17: 30$ & $2 \mathrm{of} 2$ & 220 & 10 & $<0.001$ & 0.000 & $<0.08$ & 0.03 & $<0.001$ & 0.001 \\
\hline BY129 & $\begin{array}{l}\text { Tom and Jerry Mine } \\
\text { drain at tunnel inlet } \\
\text { nr Nevada City }\end{array}$ & $6 / 28 / 2000$ & $12: 00$ & 1 of 2 & 49 & 2 & 0.001 & 0.000 & $<0.06$ & 0.04 & $<0.002$ & 0.000 \\
\hline BY129 & $\begin{array}{l}\text { Tom and Jerry Mine } \\
\text { drain at tunnel inlet } \\
\text { nr Nevada City }\end{array}$ & $6 / 28 / 2000$ & $12: 00$ & $2 \mathrm{of} 2$ & 48 & 2 & 0.0011 & 0.0008 & $<0.06$ & 0.02 & 0.0015 & 0.0013 \\
\hline BY130 & $\begin{array}{l}\text { Tom and Jerry Mine } \\
\text { drain at tunnel outlet } \\
\text { nr Nevada City }\end{array}$ & $6 / 28 / 2000$ & $11: 30$ & 1 of 2 & 120 & 10 & $<0.0008$ & 0.0004 & $<0.06$ & 0.03 & $<0.002$ & 0.001 \\
\hline BY130 & $\begin{array}{l}\text { Tom and Jerry Mine } \\
\text { drain at tunnel outlet } \\
\text { nr Nevada City }\end{array}$ & $6 / 28 / 2000$ & $11: 30$ & $2 \mathrm{of} 2$ & 120 & 10 & $<0.0008$ & 0.0003 & $<0.06$ & 0.06 & 0.0018 & 0.0009 \\
\hline BY131 & $\begin{array}{l}\text { Tom and Jerry Mine } \\
\text { drainage pond nr } \\
\text { Nevada City }\end{array}$ & $8 / 23 / 2000$ & $10: 45$ & 1 of 2 & 44 & 1 & $<0.001$ & 0.000 & $<0.08$ & 0.04 & $<0.001$ & 0.001 \\
\hline BY131 & $\begin{array}{l}\text { Tom and Jerry Mine } \\
\text { drainage pond nr } \\
\text { Nevada City }\end{array}$ & $8 / 23 / 2000$ & $10: 45$ & $2 \mathrm{of} 2$ & 44 & 2 & 0.002 & 0.000 & $<0.08$ & 0.03 & $<0.001$ & 0.001 \\
\hline \multicolumn{13}{|c|}{ Disturbed samples } \\
\hline BY123 & $\begin{array}{l}\text { Starr Mine tunnel } \\
\text { midway nr Grass } \\
\text { Valley }\end{array}$ & $12 / 15 / 1999$ & $11: 30$ & 1 of 2 & 250 & 0 & $<0.0001$ & 0.0000 & $<0.003$ & 0.000 & $<0.001$ & 0.000 \\
\hline BY123 & $\begin{array}{l}\text { Starr Mine tunnel } \\
\text { midway nr Grass } \\
\text { Valley }\end{array}$ & $12 / 15 / 1999$ & $11: 30$ & $2 \mathrm{of} 2$ & 240 & 0 & $<0.0001$ & 0.0000 & $<0.003$ & 0.001 & 0.005 & 0.006 \\
\hline BY129 & $\begin{array}{l}\text { Tom and Jerry Mine } \\
\text { drain at tunnel inlet } \\
\text { nr Nevada City }\end{array}$ & $6 / 28 / 2000$ & $12: 30$ & 1 of 2 & 130 & 0 & 0.0013 & 0.0004 & $<0.06$ & 0.00 & 0.0024 & 0.0015 \\
\hline BY129 & $\begin{array}{l}\text { Tom and Jerry Mine } \\
\text { drain at tunnel inlet } \\
\text { nr Nevada City }\end{array}$ & $6 / 28 / 2000$ & $12: 30$ & $2 \mathrm{of} 2$ & 120 & 10 & $<0.0008$ & 0.0003 & $<0.06$ & 0.05 & 0.0019 & 0.0009 \\
\hline
\end{tabular}


Table 4B. Concentrations of trace metals and selected major elements in filtered water samples, Greenhorn Creek drainage, Nevada County, California—Continued.

[Station name abbreviations: ab, above; bl, below; Cr, creek; Cyn, Canyon; mi, miles; N, north; nr, near; Rd, Road; S, south; SF, South Fork. Number in parentheses is the data parameter code, a five-digit code used in the U.S. Geological Survey computerized data system. $\mu \mathrm{g} / \mathrm{L}$, microgram per liter (equivalent to part per billion); $\mathrm{mg} / \mathrm{L}$, milligram per liter (at low concentration, equivalen to part per million); s.d., standard deviation; <, less than; —, not determined]

\begin{tabular}{|c|c|c|c|c|c|c|c|c|c|c|c|c|c|c|}
\hline \multirow[t]{2}{*}{$\begin{array}{l}\text { Station } \\
\text { map ID }\end{array}$} & \multirow[t]{2}{*}{ Station name } & \multirow[t]{2}{*}{ Date } & \multirow[t]{2}{*}{ Time } & \multirow[t]{2}{*}{$\begin{array}{l}\text { Rep- } \\
\text { licate }\end{array}$} & \multicolumn{2}{|c|}{$\begin{array}{c}\text { Thallium (TI), } \\
\text { filtered } \\
(\mu \mathrm{g} / \mathrm{L}) \\
(01057)\end{array}$} & \multicolumn{2}{|c|}{$\begin{array}{c}\text { Uranium (U), } \\
\text { filtered } \\
(\mu \mathrm{g} / \mathrm{L}) \\
(22703)\end{array}$} & \multicolumn{2}{|c|}{$\begin{array}{c}\text { Vanadium }(V), \\
\text { filtered } \\
(\mu \mathrm{g} / \mathrm{L}) \\
(01085) \\
\end{array}$} & \multicolumn{2}{|c|}{$\begin{array}{c}\text { Yttrium }(\mathrm{Y}) \\
\text { filtered } \\
(\mu \mathrm{g} / \mathrm{L}) \\
(01201) \\
\end{array}$} & \multicolumn{2}{|c|}{$\begin{array}{c}\text { Ytterbium }(Y \mathbf{Y b}), \\
\text { filtered } \\
(\mu \mathrm{g} / \mathrm{L}) \\
(01194) \\
\end{array}$} \\
\hline & & & & & value & s.d. & value & s.d. & value & s.d. & value & s.d. & value & s.d. \\
\hline BY20 & $\begin{array}{l}\text { Boston Mine tunnel } \\
\text { outlet nr Grass } \\
\text { Valley }\end{array}$ & 6/18/1999 & $14: 30$ & $1 \mathrm{of} 2$ & 0.0003 & 0.0000 & 0.0068 & 0.0004 & 0.066 & 0.037 & 0.029 & 0.002 & 0.0022 & 0.0003 \\
\hline BY20 & $\begin{array}{l}\text { Boston Mine tunnel } \\
\text { outlet nr Grass } \\
\text { Valley }\end{array}$ & $6 / 18 / 1999$ & $14: 30$ & $2 \mathrm{of} 2$ & 0.0003 & 0.0000 & 0.0061 & 0.0001 & 0.065 & 0.059 & 0.027 & 0.003 & 0.0021 & 0.0006 \\
\hline BY20 & $\begin{array}{l}\text { Boston Mine tunnel } \\
\text { outlet nr Grass } \\
\text { Valley }\end{array}$ & $12 / 14 / 1999$ & $12: 00$ & 1 of 2 & 0.0002 & 0.0000 & 0.016 & 0.001 & $<1$ & 1 & 0.012 & 0.001 & 0.0006 & 0.0001 \\
\hline BY20 & $\begin{array}{l}\text { Boston Mine tunnel } \\
\text { outlet nr Grass } \\
\text { Valley }\end{array}$ & $12 / 14 / 1999$ & $12: 00$ & $2 \mathrm{of} 2$ & $<0.0001$ & 0.0001 & 0.016 & 0.001 & $<1$ & 1 & 0.012 & 0.000 & 0.0008 & 0.0000 \\
\hline BY20 & $\begin{array}{l}\text { Boston Mine tunnel } \\
\text { outlet nr Grass } \\
\text { Valley }\end{array}$ & $5 / 24 / 2000$ & $16: 00$ & $1 \mathrm{of} 2$ & 0.0015 & 0.0000 & 0.009 & 0.001 & $<0.7$ & 0.3 & 0.032 & 0.003 & 0.003 & 0.001 \\
\hline BY20 & $\begin{array}{l}\text { Boston Mine tunnel } \\
\text { outlet nr Grass } \\
\text { Valley }\end{array}$ & $5 / 24 / 2000$ & $16: 00$ & $2 \mathrm{of} 2$ & $<0.001$ & 0.000 & 0.009 & 0.001 & $<0.7$ & 0.3 & 0.033 & 0.004 & 0.003 & 0.000 \\
\hline BY21 & $\begin{array}{l}\text { Boston Mine wetlands } \\
\text { pond nr Grass Valley }\end{array}$ & $12 / 14 / 1999$ & $10: 30$ & 1 of 2 & $<0.0001$ & 0.0000 & 0.0053 & 0.0008 & $<1$ & 1 & 0.011 & 0.001 & 0.0006 & 0.0001 \\
\hline BY21 & $\begin{array}{l}\text { Boston Mine wetlands } \\
\text { pond nr Grass Valley }\end{array}$ & $12 / 14 / 1999$ & $10: 30$ & $2 \mathrm{of} 2$ & 0.0001 & 0.0000 & 0.0072 & 0.0029 & $<1$ & 1 & 0.010 & 0.000 & 0.0007 & 0.0001 \\
\hline BY21 & $\begin{array}{l}\text { Boston Mine wetlands } \\
\text { pond nr Grass Valley }\end{array}$ & $5 / 24 / 2000$ & $16: 30$ & 1of 2 & $<0.001$ & 0.000 & 0.005 & 0.001 & $<0.7$ & 0.1 & 0.027 & 0.000 & 0.002 & 0.000 \\
\hline BY21 & $\begin{array}{l}\text { Boston Mine wetlands } \\
\text { pond nr Grass Valley }\end{array}$ & $5 / 24 / 2000$ & $16: 30$ & $2 \mathrm{of} 2$ & $<0.001$ & 0.000 & 0.004 & 0.001 & $<0.7$ & 0.1 & 0.025 & 0.001 & 0.003 & 0.003 \\
\hline BY58 & $\begin{array}{l}\text { Buckeye Flat Mine } \\
\text { main drain } 0.45 \mathrm{mi} \\
\text { ab Greenhorn } \mathrm{Cr}\end{array}$ & $8 / 21 / 2000$ & $11: 00$ & $1 \mathrm{of} 2$ & 0.16 & 0.00 & 0.23 & 0.00 & $<0.4$ & 0.1 & 16 & 0 & 1.0 & 0.0 \\
\hline BY58 & $\begin{array}{l}\text { Buckeye Flat Mine } \\
\text { main drain } 0.45 \mathrm{mi} \\
\text { ab Greenhorn } \mathrm{Cr}\end{array}$ & $8 / 21 / 2000$ & 11:00 & $2 \mathrm{of} 2$ & 0.18 & 0.00 & 0.25 & 0.00 & $<0.4$ & 0.1 & 16 & 0 & 1.0 & 0.0 \\
\hline BY23 & $\begin{array}{c}\text { Buckeye Flat Mine N } \\
\text { drain to Greenhorn } \\
\text { Cr, Grass Valley }\end{array}$ & $6 / 18 / 1999$ & $11: 30$ & $2 \mathrm{of} 2$ & 0.36 & 0.00 & 0.39 & 0.01 & 0.17 & 0.04 & 35 & 0 & 2.0 & 0.0 \\
\hline
\end{tabular}


Table 4B. Concentrations of trace metals and selected major elements in filtered water samples, Greenhorn Creek drainage, Nevada County, California—Continued.

[Station name abbreviations: ab, above; bl, below; Cr, creek; Cyn, Canyon; mi, miles; N, north; nr, near; Rd, Road; S, south; SF, South Fork. Number in parentheses is the data parameter code, a five-digit code used in the U.S. Geological Survey computerized data system. $\mu \mathrm{g} / \mathrm{L}$, microgram per liter (equivalent to part per billion); $\mathrm{mg} / \mathrm{L}$, milligram per liter (at low concentration, equivalent to part per million); s.d., standard deviation; <, less than; - , not determined]

\begin{tabular}{|c|c|c|c|c|c|c|c|c|c|c|c|c|c|c|}
\hline \multirow[t]{2}{*}{$\begin{array}{l}\text { Station } \\
\text { map ID }\end{array}$} & \multirow[t]{2}{*}{ Station name } & \multirow[t]{2}{*}{ Date } & \multirow[t]{2}{*}{ Time } & \multirow[t]{2}{*}{$\begin{array}{l}\text { Rep- } \\
\text { licate }\end{array}$} & \multicolumn{2}{|c|}{$\begin{array}{c}\text { Thallium (TI), } \\
\text { filtered } \\
(\mu \mathrm{g} / \mathrm{L}) \\
(01057) \\
\end{array}$} & \multicolumn{2}{|c|}{$\begin{array}{c}\text { Uranium (U), } \\
\text { filtered } \\
(\mu \mathrm{g} / \mathrm{L}) \\
(22703) \\
\end{array}$} & \multicolumn{2}{|c|}{$\begin{array}{c}\text { Vanadium }(V), \\
\text { filtered } \\
(\mu \mathrm{g} / \mathrm{L}) \\
(01085) \\
\end{array}$} & \multicolumn{2}{|c|}{$\begin{array}{c}\text { Yttrium (Y), } \\
\text { filtered } \\
(\mu \mathrm{g} / \mathrm{L}) \\
(01201)\end{array}$} & \multicolumn{2}{|c|}{$\begin{array}{c}\text { Ytterbium (Yb), } \\
\text { filtered } \\
(\mu \mathrm{g} / \mathrm{L}) \\
(01194) \\
\end{array}$} \\
\hline & & & & & value & s.d. & value & s.d. & value & s.d. & value & s.d. & value & s.d. \\
\hline BY23 & $\begin{array}{c}\text { Buckeye Flat Mine N } \\
\text { drain to Greenhorn } \\
\text { Cr, Grass Valley }\end{array}$ & $12 / 2 / 1999$ & $11: 00$ & 1 of 2 & 0.25 & 0.00 & 0.40 & 0.01 & $<1$ & 0 & 23 & 0 & 1.4 & 0.0 \\
\hline BY23 & $\begin{array}{c}\text { Buckeye Flat Mine N } \\
\text { drain to Greenhorn } \\
\text { Cr, Grass Valley }\end{array}$ & $12 / 2 / 1999$ & $11: 00$ & $2 \mathrm{of} 2$ & 0.25 & 0.01 & 0.41 & 0.00 & $<1$ & 0 & 22 & 0 & 1.4 & 0.1 \\
\hline BY23 & $\begin{array}{l}\text { Buckeye Flat Mine N } \\
\text { drain to Greenhorn } \\
\text { Cr, Grass Valley }\end{array}$ & $8 / 22 / 2000$ & $10: 45$ & 1 of 2 & 0.31 & 0.00 & 0.42 & 0.00 & $<0.4$ & 0.1 & 29 & 0 & 1.8 & 0.0 \\
\hline BY23 & $\begin{array}{l}\text { Buckeye Flat Mine N } \\
\text { drain to Greenhorn } \\
\text { Cr, Grass Valley }\end{array}$ & $8 / 22 / 2000$ & $10: 45$ & $2 \mathrm{of} 2$ & 0.32 & 0.00 & 0.42 & 0.00 & $<0.4$ & 0.1 & 29 & 1 & 1.9 & 0.0 \\
\hline BY116 & $\begin{array}{l}\text { Buckeye Flat Mine } \\
\text { pond drain } 0.15 \mathrm{mi} \\
\text { ab SF Greenhorn Cr }\end{array}$ & $8 / 21 / 2000$ & $14: 10$ & 1 of 2 & $<0.001$ & 0.001 & 0.002 & 0.001 & $<0.4$ & 0.1 & 0.053 & 0.003 & 0.005 & 0.003 \\
\hline BY116 & $\begin{array}{l}\text { Buckeye Flat Mine } \\
\text { pond drain } 0.15 \mathrm{mi} \\
\text { ab SF Greenhorn Cr }\end{array}$ & $8 / 21 / 2000$ & $14: 10$ & $2 \mathrm{of} 2$ & 0.0011 & 0.0010 & 0.002 & 0.001 & $<0.4$ & 0.1 & 0.057 & 0.005 & 0.005 & 0.001 \\
\hline BY24 & $\begin{array}{l}\text { Buckeye Flat Mine } \\
\text { S drain to SF } \\
\text { Greenhorn Cr, Grass } \\
\text { Valley }\end{array}$ & $8 / 26 / 1999$ & 11:00 & $1 \mathrm{of} 2$ & 0.0080 & 0.0001 & 0.021 & 0.001 & $<0.1$ & 0.1 & 0.66 & 0.03 & 0.051 & 0.000 \\
\hline BY24 & $\begin{array}{l}\text { Buckeye Flat Mine } \\
\text { S drain to SF } \\
\text { Greenhorn Cr, Grass } \\
\text { Valley }\end{array}$ & $8 / 26 / 1999$ & $11: 00$ & $2 \mathrm{of} 2$ & 0.0082 & 0.0001 & 0.019 & 0.000 & $<0.1$ & 0.1 & 0.62 & 0.01 & 0.048 & 0.000 \\
\hline BY24 & $\begin{array}{l}\text { Buckeye Flat Mine } \\
\text { S drain to SF } \\
\text { Greenhorn Cr, Grass } \\
\text { Valley }\end{array}$ & $8 / 22 / 2000$ & $12: 10$ & 1 of 2 & 0.0059 & 0.0012 & 0.021 & 0.002 & $<0.4$ & 0.1 & 0.52 & 0.01 & 0.033 & 0.001 \\
\hline BY24 & $\begin{array}{l}\text { Buckeye Flat Mine } \\
\text { S drain to SF } \\
\text { Greenhorn Cr, Grass } \\
\text { Valley }\end{array}$ & $8 / 22 / 2000$ & $12: 10$ & $2 \mathrm{of} 2$ & 0.0062 & 0.0012 & 0.022 & 0.002 & $<0.4$ & 0.1 & 0.55 & 0.01 & 0.041 & 0.003 \\
\hline BY25 & $\begin{array}{l}\text { Buckeye Flat Mine } \\
\text { upper drain }\end{array}$ & $4 / 9 / 2001$ & $10: 00$ & $2 \mathrm{of} 2$ & 0.051 & 0.001 & 0.034 & 0.002 & $<0.9$ & 0.2 & 5.2 & 0.3 & 0.30 & 0.03 \\
\hline
\end{tabular}


Table 4B. Concentrations of trace metals and selected major elements in filtered water samples, Greenhorn Creek drainage, Nevada County, California—Continued.

[Station name abbreviations: ab, above; bl, below; Cr, creek; Cyn, Canyon; mi, miles; N, north; nr, near; Rd, Road; S, south; SF, South Fork. Number in parentheses is the data parameter code, a five-digit code used in the U.S. Geological Survey computerized data system. $\mu \mathrm{g} / \mathrm{L}$, microgram per liter (equivalent to part per billion); $\mathrm{mg} / \mathrm{L}$, milligram per liter (at low concentration, equivalen to part per million); s.d., standard deviation; <, less than; - , not determined]

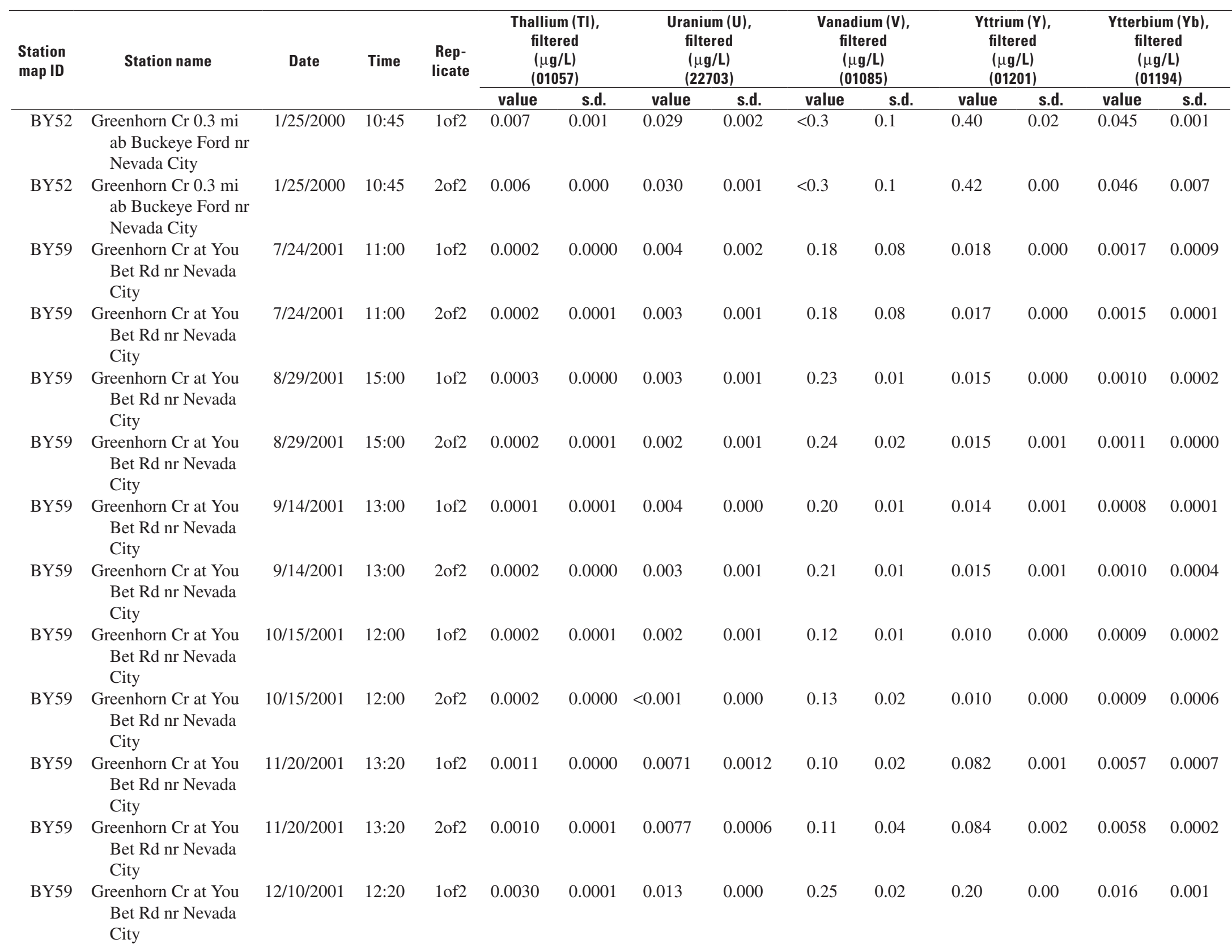


Table 4B. Concentrations of trace metals and selected major elements in filtered water samples, Greenhorn Creek drainage, Nevada County, California—Continued.

[Station name abbreviations: ab, above; bl, below; Cr, creek; Cyn, Canyon; mi, miles; N, north; nr, near; Rd, Road; S, south; SF, South Fork. Number in parentheses is the data parameter code, a five-digit code used in the U.S. Geological Survey computerized data system. $\mu \mathrm{g} / \mathrm{L}$, microgram per liter (equivalent to part per billion); $\mathrm{mg} / \mathrm{L}$, milligram per liter (at low concentration, equivalen to part per million); s.d., standard deviation; <, less than; - , not determined]

\begin{tabular}{|c|c|c|c|c|c|c|c|c|c|c|c|c|c|c|}
\hline \multirow[t]{2}{*}{$\begin{array}{l}\text { Station } \\
\text { map ID }\end{array}$} & \multirow[t]{2}{*}{ Station name } & \multirow[t]{2}{*}{ Date } & \multirow[t]{2}{*}{ Time } & \multirow[t]{2}{*}{$\begin{array}{l}\text { Rep- } \\
\text { licate }\end{array}$} & \multicolumn{2}{|c|}{$\begin{array}{c}\text { Thallium (TI), } \\
\text { filtered } \\
(\mu \mathrm{g} / \mathrm{L}) \\
(01057)\end{array}$} & \multicolumn{2}{|c|}{$\begin{array}{c}\text { Uranium (U), } \\
\text { filtered } \\
(\mu \mathrm{g} / \mathrm{L}) \\
(22703)\end{array}$} & \multicolumn{2}{|c|}{$\begin{array}{c}\text { Vanadium }(V), \\
\text { filtered } \\
(\mu \mathrm{g} / \mathrm{L}) \\
(01085)\end{array}$} & \multicolumn{2}{|c|}{$\begin{array}{c}\text { Yttrium (Y), } \\
\text { filtered } \\
(\mu \mathrm{g} / \mathrm{L}) \\
(01201)\end{array}$} & \multicolumn{2}{|c|}{$\begin{array}{c}\text { Ytterbium (Yb), } \\
\text { filtered } \\
(\mu \mathrm{g} / \mathrm{L}) \\
(01194)\end{array}$} \\
\hline & & & & & value & s.d. & value & s.d. & value & s.d. & value & s.d. & value & s.d. \\
\hline BY59 & $\begin{array}{l}\text { Greenhorn } \mathrm{Cr} \text { at You } \\
\text { Bet Rd nr Nevada } \\
\text { City }\end{array}$ & $12 / 10 / 2001$ & $12: 20$ & 2 of 2 & 0.0029 & 0.0001 & 0.013 & 0.000 & 0.24 & 0.02 & 0.20 & 0.00 & 0.016 & 0.000 \\
\hline BY59 & $\begin{array}{l}\text { Greenhorn Cr at You } \\
\text { Bet Rd nr Nevada } \\
\text { City }\end{array}$ & $12 / 21 / 2001$ & $13: 00$ & 1 of 2 & 0.0062 & 0.0002 & 0.012 & 0.001 & 0.29 & 0.03 & 0.47 & 0.00 & 0.040 & 0.000 \\
\hline BY59 & $\begin{array}{l}\text { Greenhorn } \mathrm{Cr} \text { at You } \\
\text { Bet Rd nr Nevada } \\
\text { City }\end{array}$ & $12 / 21 / 2001$ & $13: 00$ & $2 \mathrm{of} 2$ & 0.0056 & 0.0000 & 0.011 & 0.001 & 0.28 & 0.05 & 0.44 & 0.00 & 0.040 & 0.000 \\
\hline BY51 & $\begin{array}{l}\text { Greenhorn Cr nr } \\
\text { Headwaters nr Scotts } \\
\text { Flat Reservoir }\end{array}$ & $1 / 25 / 2000$ & $9: 30$ & 1 of 2 & 0.004 & 0.002 & 0.006 & 0.000 & $<0.3$ & 0.1 & 0.25 & 0.02 & 0.025 & 0.004 \\
\hline BY51 & $\begin{array}{l}\text { Greenhorn Cr nr } \\
\text { Headwaters nr Scotts } \\
\text { Flat Reservoir }\end{array}$ & $1 / 25 / 2000$ & $9: 30$ & $2 \mathrm{of} 2$ & 0.003 & 0.001 & 0.007 & 0.002 & $<0.3$ & 0.1 & 0.24 & 0.04 & 0.023 & 0.006 \\
\hline BY75 & $\begin{array}{l}\text { Missouri Cyn Cr } 1.2 \mathrm{mi} \\
\text { ab Greenhorn Cr nr } \\
\text { Chicago Park }\end{array}$ & $12 / 14 / 1999$ & $14: 00$ & 1 of 2 & 0.0048 & 0.0000 & 0.013 & 0.001 & $<1$ & 0 & 0.50 & 0.00 & 0.030 & 0.000 \\
\hline BY75 & $\begin{array}{l}\text { Missouri Cyn Cr } 1.2 \mathrm{mi} \\
\text { ab Greenhorn Cr nr } \\
\text { Chicago Park }\end{array}$ & $12 / 14 / 1999$ & $14: 00$ & $2 \mathrm{of} 2$ & 0.0051 & 0.0002 & 0.013 & 0.000 & $<1$ & 1 & 0.49 & 0.02 & 0.029 & 0.003 \\
\hline BY86 & $\begin{array}{l}\text { Poore Mine creek ab } \\
\text { tunnel nr Grass } \\
\text { Valley }\end{array}$ & 3/31/1999 & $17: 40$ & 1 of 2 & 0.0010 & 0.0000 & 0.0038 & 0.0003 & 0.30 & 0.02 & 0.083 & 0.002 & 0.0076 & 0.0006 \\
\hline BY86 & $\begin{array}{l}\text { Poore Mine creek ab } \\
\text { tunnel nr Grass } \\
\text { Valley }\end{array}$ & 3/31/1999 & $17: 40$ & $2 \mathrm{of} 2$ & 0.0010 & 0.0001 & 0.0042 & 0.0001 & 0.28 & 0.00 & 0.082 & 0.001 & 0.0080 & 0.0012 \\
\hline BY87 & $\begin{array}{l}\text { Poore Mine creek bl } \\
\text { tunnel nr Grass } \\
\text { Valley }\end{array}$ & $3 / 31 / 1999$ & $13: 30$ & 1 of 2 & 0.0013 & 0.0000 & 0.0040 & 0.0009 & 0.47 & 0.16 & 0.10 & 0.00 & 0.0080 & 0.0001 \\
\hline BY87 & $\begin{array}{l}\text { Poore Mine creek bl } \\
\text { tunnel nr Grass } \\
\text { Valley }\end{array}$ & $3 / 31 / 1999$ & $13: 30$ & $2 \mathrm{of} 2$ & 0.0010 & 0.0001 & 0.0040 & 0.0007 & 0.35 & 0.03 & 0.097 & 0.002 & 0.0078 & 0.0009 \\
\hline BY88 & $\begin{array}{l}\text { Poore Mine ground } \\
\text { sluice nr Grass } \\
\text { Valley }\end{array}$ & 4/1/1999 & $15: 40$ & $2 \mathrm{of} 2$ & 0.0033 & 0.0008 & 0.0072 & 0.0003 & $<0.06$ & 0.05 & 0.23 & 0.01 & 0.017 & 0.000 \\
\hline
\end{tabular}


Table 4B. Concentrations of trace metals and selected major elements in filtered water samples, Greenhorn Creek drainage, Nevada County, California—Continued.

[Station name abbreviations: ab, above; bl, below; Cr, creek; Cyn, Canyon; mi, miles; N, north; nr, near; Rd, Road; S, south; SF, South Fork. Number in parentheses is the data parameter code, a five-digit code used in the U.S. Geological Survey computerized data system. $\mu \mathrm{g} / \mathrm{L}$, microgram per liter (equivalent to part per billion); mg/L, milligram per liter (at low concentration, equivalent to part per million); s.d., standard deviation; <, less than; - , not determined]

\begin{tabular}{|c|c|c|c|c|c|c|c|c|c|c|c|c|c|c|}
\hline \multirow[t]{2}{*}{$\begin{array}{l}\text { Station } \\
\text { map ID }\end{array}$} & \multirow[t]{2}{*}{ Station name } & \multirow[t]{2}{*}{ Date } & \multirow[t]{2}{*}{ Time } & \multirow[t]{2}{*}{$\begin{array}{l}\text { Rep- } \\
\text { licate }\end{array}$} & \multicolumn{2}{|c|}{$\begin{array}{c}\text { Thallium (TI), } \\
\text { filtered } \\
(\mu \mathrm{g} / \mathrm{L}) \\
(01057) \\
\end{array}$} & \multicolumn{2}{|c|}{$\begin{array}{c}\text { Uranium (U), } \\
\text { filtered } \\
(\mu \mathrm{g} / \mathrm{L}) \\
(22703)\end{array}$} & \multicolumn{2}{|c|}{$\begin{array}{c}\text { Vanadium }(V), \\
\text { filtered } \\
(\mu \mathrm{g} / \mathrm{L}) \\
(01085) \\
\end{array}$} & \multicolumn{2}{|c|}{$\begin{array}{c}\text { Yttrium }(\mathrm{Y}), \\
\text { filtered } \\
(\mu \mathrm{g} / \mathrm{L}) \\
(01201)\end{array}$} & \multicolumn{2}{|c|}{$\begin{array}{c}\text { Ytterbium }(\mathrm{Yb}), \\
\text { filtered } \\
(\mu \mathrm{g} / \mathrm{L}) \\
(01194) \\
\end{array}$} \\
\hline & & & & & value & s.d. & value & s.d. & value & s.d. & value & s.d. & value & s.d. \\
\hline BY89 & $\begin{array}{l}\text { Poore Mine pit lake } \\
\text { nr Grass Valley }\end{array}$ & 4/1/1999 & $16: 50$ & $2 \mathrm{of} 2$ & 0.0007 & 0.0001 & 0.0038 & 0.0006 & $<0.06$ & 0.08 & 0.037 & 0.001 & 0.0042 & 0.0012 \\
\hline BY89 & $\begin{array}{c}\text { Poore Mine pit lake } \\
\text { nr Grass Valley }\end{array}$ & $5 / 23 / 2000$ & $10: 45$ & $1 \mathrm{of} 2$ & 0.0041 & 0.0001 & 0.016 & 0.001 & $<0.7$ & 0.3 & 0.32 & 0.00 & 0.018 & 0.000 \\
\hline BY89 & $\begin{array}{l}\text { Poore Mine pit lake } \\
\text { nr Grass Valley }\end{array}$ & $5 / 23 / 2000$ & $10: 45$ & $2 \mathrm{of} 2$ & 0.0030 & 0.0010 & 0.017 & 0.001 & $<0.7$ & 0.2 & 0.33 & 0.00 & 0.016 & 0.003 \\
\hline BY90 & $\begin{array}{l}\text { Poore Mine seep } \\
\text { ab ground sluice } \\
\text { nr Grass Valley }\end{array}$ & 4/1/1999 & $16: 00$ & $1 \mathrm{of} 2$ & 0.0010 & 0.0001 & 0.0023 & 0.0011 & $<0.06$ & 0.03 & 0.078 & 0.004 & 0.0069 & 0.0001 \\
\hline BY90 & $\begin{array}{l}\text { Poore Mine seep } \\
\text { ab ground sluice } \\
\text { nr Grass Valley }\end{array}$ & 4/1/1999 & $16: 00$ & $2 \mathrm{of} 2$ & 0.0010 & 0.0001 & 0.0023 & 0.0010 & $<0.06$ & 0.04 & 0.077 & 0.000 & 0.0070 & 0.0007 \\
\hline BY91 & $\begin{array}{l}\text { Poore Mine tunnel } \\
\text { effluent nr Grass } \\
\text { Valley }\end{array}$ & 3/31/1999 & $15: 20$ & $2 \mathrm{of} 2$ & 0.0002 & 0.0002 & 0.036 & 0.000 & 0.13 & 0.03 & 0.029 & 0.003 & 0.0006 & 0.0008 \\
\hline BY91 & $\begin{array}{l}\text { Poore Mine tunnel } \\
\text { effluent nr Grass } \\
\text { Valley }\end{array}$ & $12 / 15 / 1999$ & $13: 00$ & 1 of 2 & $<0.0001$ & 0.0000 & 0.058 & 0.005 & $<1$ & 1 & 0.008 & 0.000 & 0.0002 & 0.0001 \\
\hline BY91 & $\begin{array}{l}\text { Poore Mine tunnel } \\
\text { effluent nr Grass } \\
\text { Valley }\end{array}$ & $12 / 15 / 1999$ & $13: 00$ & $2 \mathrm{of} 2$ & 0.0001 & 0.0001 & 0.060 & 0.001 & $<1$ & 1 & 0.009 & 0.000 & 0.0006 & 0.0003 \\
\hline BY91 & $\begin{array}{l}\text { Poore Mine tunnel } \\
\text { effluent nr Grass } \\
\text { Valley }\end{array}$ & $5 / 23 / 2000$ & $12: 00$ & 1of 2 & $<0.001$ & 0.000 & 0.041 & 0.004 & $<0.7$ & 0.4 & 0.011 & 0.001 & $<0.002$ & 0.001 \\
\hline BY91 & $\begin{array}{l}\text { Poore Mine tunnel } \\
\text { effluent nr Grass } \\
\text { Valley }\end{array}$ & $5 / 23 / 2000$ & $12: 00$ & $2 o f 2$ & $<0.001$ & 0.001 & 0.045 & 0.004 & $<0.3$ & 0.0 & 0.010 & 0.002 & $<0.004$ & 0.002 \\
\hline BY105 & $\begin{array}{l}\text { Sailor Flat Mine main } \\
\text { drain Gulch 01nr } \\
\text { Quaker Hill }\end{array}$ & 7/6/1999 & $16: 00$ & 1 of 2 & 0.017 & 0.000 & 0.068 & 0.002 & 0.24 & 0.11 & 0.98 & 0.00 & 0.12 & 0.00 \\
\hline BY105 & $\begin{array}{l}\text { Sailor Flat Mine main } \\
\text { drain Gulch 01nr } \\
\text { Quaker Hill }\end{array}$ & 7/6/1999 & $16: 00$ & $2 \mathrm{of} 2$ & 0.017 & 0.000 & 0.061 & 0.003 & 0.18 & 0.03 & 0.94 & 0.02 & 0.12 & 0.01 \\
\hline
\end{tabular}


Table 4B. Concentrations of trace metals and selected major elements in filtered water samples, Greenhorn Creek drainage, Nevada County, California—Continued.

[Station name abbreviations: ab, above; bl, below; Cr, creek; Cyn, Canyon; mi, miles; N, north; nr, near; Rd, Road; S, south; SF, South Fork. Number in parentheses is the data parameter code, a five-digit code used in the U.S. Geological Survey computerized data system. $\mu \mathrm{g} / \mathrm{L}$, microgram per liter (equivalent to part per billion); $\mathrm{mg} / \mathrm{L}$, milligram per liter (at low concentration, equivalen to part per million); s.d., standard deviation; <, less than; - , not determined]

\begin{tabular}{|c|c|c|c|c|c|c|c|c|c|c|c|c|c|c|}
\hline \multirow[t]{2}{*}{$\begin{array}{l}\text { Station } \\
\text { map ID }\end{array}$} & \multirow[t]{2}{*}{ Station name } & \multirow[t]{2}{*}{ Date } & \multirow[t]{2}{*}{ Time } & \multirow[t]{2}{*}{$\begin{array}{l}\text { Rep- } \\
\text { licate }\end{array}$} & \multicolumn{2}{|c|}{$\begin{array}{c}\text { Thallium (TI), } \\
\text { filtered } \\
(\mu \mathrm{g} / \mathrm{L}) \\
(\mathbf{0 1 0 5 7 )} \\
\end{array}$} & \multicolumn{2}{|c|}{$\begin{array}{c}\text { Uranium (U), } \\
\text { filtered } \\
(\mu \mathrm{g} / \mathrm{L}) \\
(22703)\end{array}$} & \multicolumn{2}{|c|}{$\begin{array}{c}\text { Vanadium }(\mathrm{V}), \\
\text { filtered } \\
(\mu \mathrm{g} / \mathrm{L}) \\
(\mathbf{0 1 0 8 5}) \\
\end{array}$} & \multicolumn{2}{|c|}{$\begin{array}{c}\text { Yttrium (Y), } \\
\text { filtered } \\
(\mu \mathrm{g} / \mathrm{L}) \\
(01201)\end{array}$} & \multicolumn{2}{|c|}{$\begin{array}{c}\text { Ytterbium (Yb), } \\
\text { filtered } \\
(\mu \mathrm{g} / \mathrm{L}) \\
(01194)\end{array}$} \\
\hline & & & & & value & s.d. & value & s.d. & value & s.d. & value & s.d. & value & s.d. \\
\hline BY105 & $\begin{array}{l}\text { Sailor Flat Mine main } \\
\text { drain Gulch 01nr } \\
\text { Quaker Hill }\end{array}$ & $12 / 2 / 1999$ & $14: 30$ & 1 of 2 & 0.025 & 0.000 & 0.30 & 0.00 & $<1$ & 0 & 1.2 & 0.0 & 0.19 & 0.00 \\
\hline BY105 & $\begin{array}{l}\text { Sailor Flat Mine main } \\
\text { drain Gulch 01nr } \\
\text { Quaker Hill }\end{array}$ & $12 / 2 / 1999$ & $14: 30$ & $2 \mathrm{of} 2$ & 0.026 & 0.000 & 0.30 & 0.00 & $<1$ & 1 & 1.2 & 0.0 & 0.20 & 0.00 \\
\hline BY106 & $\begin{array}{l}\text { Sailor Flat Mine } \\
\text { main drainage to } \\
\text { Greenhorn Cr, } \\
\text { Gulch } 03\end{array}$ & 4/9/2001 & $12: 30$ & 1of 2 & 0.019 & 0.003 & 0.33 & 0.07 & $<0.9$ & 0.2 & 1.1 & 0.2 & 0.14 & 0.04 \\
\hline BY106 & $\begin{array}{l}\text { Sailor Flat Mine } \\
\text { main drainage to } \\
\text { Greenhorn Cr, } \\
\text { Gulch } 03\end{array}$ & 4/9/2001 & $12: 30$ & 2 of 2 & 0.023 & 0.001 & 0.33 & 0.07 & $<0.9$ & 0.4 & 1.1 & 0.2 & 0.14 & 0.01 \\
\hline BY114 & $\begin{array}{l}\text { SF Greenhorn } \mathrm{Cr} \\
0.7 \quad \text { i ab Greenhorn } \\
\text { Cr nr Nevada City }\end{array}$ & $8 / 22 / 2000$ & $15: 10$ & $2 \mathrm{of} 2$ & $<0.001$ & 0.001 & 0.007 & 0.001 & $<0.4$ & 0.1 & 0.013 & 0.001 & $<0.003$ & 0.003 \\
\hline BY122 & $\begin{array}{l}\text { Starr Mine tunnel } \\
\text { inflow nr Grass } \\
\text { Valley }\end{array}$ & 4/23/1999 & $15: 00$ & 1 of 2 & 0.0015 & 0.0000 & 0.0044 & 0.0003 & 0.11 & 0.02 & 0.14 & 0.00 & 0.0086 & 0.0004 \\
\hline BY122 & $\begin{array}{l}\text { Starr Mine tunnel } \\
\text { inflow nr Grass } \\
\text { Valley }\end{array}$ & 4/23/1999 & $15: 00$ & $2 \mathrm{of} 2$ & 0.0015 & 0.0002 & 0.0051 & 0.0003 & 0.12 & 0.00 & 0.14 & 0.00 & 0.0085 & 0.0014 \\
\hline BY123 & $\begin{array}{l}\text { Starr Mine tunnel } \\
\text { midway nr Grass } \\
\text { Valley }\end{array}$ & 4/23/1999 & $13: 40$ & 1 of 2 & $<0.0001$ & 0.0000 & 0.036 & 0.001 & 0.074 & 0.007 & 0.010 & 0.005 & $<0.0003$ & 0.0001 \\
\hline BY123 & $\begin{array}{l}\text { Starr Mine tunnel } \\
\text { midway nr Grass } \\
\text { Valley }\end{array}$ & $4 / 23 / 1999$ & $13: 40$ & $2 \mathrm{of} 2$ & $<0.0001$ & 0.0000 & 0.037 & 0.000 & $<0.06$ & 0.01 & 0.0076 & 0.0003 & $<0.0003$ & 0.0001 \\
\hline BY123 & $\begin{array}{l}\text { Starr Mine tunnel } \\
\text { midway nr Grass } \\
\text { Valley }\end{array}$ & 12/15/1999 & $10: 45$ & 1 of 2 & 0.0001 & 0.0001 & 0.044 & 0.001 & $<1$ & 1 & 0.011 & 0.000 & 0.0003 & 0.0000 \\
\hline BY123 & $\begin{array}{l}\text { Starr Mine tunnel } \\
\text { midway nr Grass } \\
\text { Valley }\end{array}$ & 12/15/1999 & $10: 45$ & 2of2 & $<0.0001$ & 0.0001 & 0.044 & 0.001 & $<1$ & 0 & 0.013 & 0.001 & 0.0003 & 0.0000 \\
\hline BY124 & $\begin{array}{l}\text { Starr Mine tunnel outlet } \\
\text { nr Grass Valley }\end{array}$ & $8 / 23 / 2000$ & $17: 30$ & 1 of 2 & $<0.001$ & 0.000 & 0.13 & 0.00 & 1.0 & 0.2 & 0.012 & 0.001 & $<0.003$ & 0.000 \\
\hline
\end{tabular}


Table 4B. Concentrations of trace metals and selected major elements in filtered water samples, Greenhorn Creek drainage, Nevada County, California—Continued.

[Station name abbreviations: ab, above; bl, below; Cr, creek; Cyn, Canyon; mi, miles; N, north; nr, near; Rd, Road; S, south; SF, South Fork. Number in parentheses is the data parameter code, a five-digit code used in the U.S. Geological Survey computerized data system. $\mu \mathrm{g} / \mathrm{L}$, microgram per liter (equivalent to part per billion); mg/L, milligram per liter (at low concentration, equivalent to part per million); s.d., standard deviation; <, less than; - , not determined]

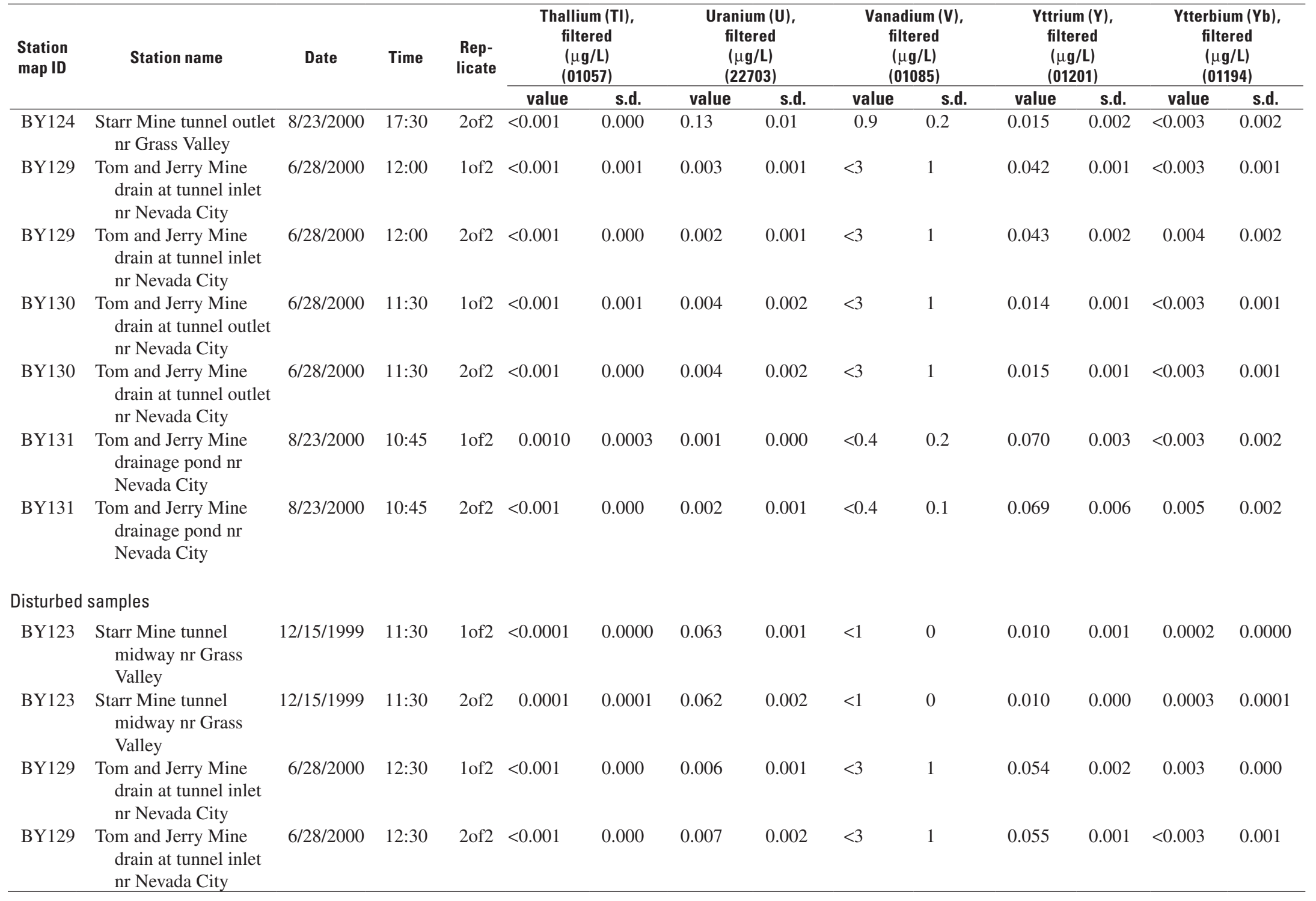


Table 4B. Concentrations of trace metals and selected major elements in filtered water samples, Greenhorn

[Station name abbreviations: ab, above; bl, below; Cr, creek; Cyn, Canyon; mi, miles; N, north; nr, near; Rd, Road; S, south; SF, South Fork. Number in parentheses is the data parameter code, a five-digit code used in the U.S. Geological Survey computerized data system. $\mu \mathrm{g} / \mathrm{L}$, microgram per liter (equivalent to part per billion); $\mathrm{mg} / \mathrm{L}$, milligram per liter (at low concentration, equivalent to part per million); s.d., standard deviation; <, less than; - , not determined]

\begin{tabular}{|c|c|c|c|c|c|c|c|c|}
\hline \multirow[t]{2}{*}{$\begin{array}{l}\text { Station } \\
\text { map ID }\end{array}$} & \multirow[t]{2}{*}{ Station name } & \multirow[t]{2}{*}{ Date } & \multirow[t]{2}{*}{ Time } & \multirow[t]{2}{*}{$\begin{array}{l}\text { Rep- } \\
\text { licate }\end{array}$} & \multicolumn{2}{|c|}{$\begin{array}{c}\text { Zinc }(Z n), \\
\text { filtered } \\
(\mu \mathrm{g} / \mathrm{L}) \\
(01090)\end{array}$} & \multicolumn{2}{|c|}{$\begin{array}{c}\text { Zirconium (Zr), } \\
\text { filtered } \\
(\mu \mathrm{g} / \mathrm{L}) \\
(01160)\end{array}$} \\
\hline & & & & & value & s.d. & value & s.d. \\
\hline \multicolumn{9}{|c|}{ Environmental samples } \\
\hline BY20 & $\begin{array}{l}\text { Boston Mine tunnel } \\
\text { outlet nr Grass } \\
\text { Valley }\end{array}$ & 6/18/1999 & $14: 30$ & 1 of 2 & 1.6 & 0.0 & 0.0016 & 0.0002 \\
\hline BY20 & $\begin{array}{l}\text { Boston Mine tunnel } \\
\text { outlet nr Grass } \\
\text { Valley }\end{array}$ & $6 / 18 / 1999$ & $14: 30$ & $2 \mathrm{of} 2$ & 1.4 & 0.1 & 0.0016 & 0.0002 \\
\hline BY20 & $\begin{array}{l}\text { Boston Mine tunnel } \\
\text { outlet nr Grass } \\
\text { Valley }\end{array}$ & $12 / 14 / 1999$ & $12: 00$ & 1 of 2 & 2.4 & 0.3 & 0.0019 & 0.0011 \\
\hline BY20 & $\begin{array}{l}\text { Boston Mine tunnel } \\
\text { outlet nr Grass } \\
\text { Valley }\end{array}$ & $12 / 14 / 1999$ & $12: 00$ & 2 of 2 & 1.9 & 0.2 & 0.0022 & 0.0007 \\
\hline BY20 & $\begin{array}{l}\text { Boston Mine tunnel } \\
\text { outlet nr Grass } \\
\text { Valley }\end{array}$ & $5 / 24 / 2000$ & $16: 00$ & 1 of 2 & 2.9 & 0.2 & 0.005 & 0.002 \\
\hline BY20 & $\begin{array}{l}\text { Boston Mine tunnel } \\
\text { outlet nr Grass } \\
\text { Valley }\end{array}$ & $5 / 24 / 2000$ & $16: 00$ & $2 o f 2$ & 3.0 & 0.0 & $<0.004$ & 0.002 \\
\hline BY21 & $\begin{array}{l}\text { Boston Mine wetlands } \\
\text { pond nr Grass Valley }\end{array}$ & $12 / 14 / 1999$ & $10: 30$ & 1 of 2 & 2.3 & 0.3 & 0.0019 & 0.0001 \\
\hline BY21 & $\begin{array}{l}\text { Boston Mine wetlands } \\
\text { pond nr Grass Valley }\end{array}$ & $12 / 14 / 1999$ & $10: 30$ & $2 o f 2$ & 2.4 & 0.1 & 0.0020 & 0.0000 \\
\hline BY21 & $\begin{array}{l}\text { Boston Mine wetlands } \\
\text { pond nr Grass Valley }\end{array}$ & $5 / 24 / 2000$ & $16: 30$ & 1 of 2 & 6.2 & 0.3 & 0.007 & 0.001 \\
\hline BY21 & $\begin{array}{l}\text { Boston Mine wetlands } \\
\text { pond nr Grass Valley }\end{array}$ & $5 / 24 / 2000$ & $16: 30$ & $2 \mathrm{of} 2$ & 2.4 & 0.2 & 0.007 & 0.003 \\
\hline BY58 & $\begin{array}{l}\text { Buckeye Flat Mine } \\
\text { main drain } 0.45 \mathrm{mi} \\
\text { ab Greenhorn } \mathrm{Cr}\end{array}$ & $8 / 21 / 2000$ & $11: 00$ & 1 of 2 & 200 & 0 & $<0.01$ & 0.01 \\
\hline BY58 & $\begin{array}{l}\text { Buckeye Flat Mine } \\
\text { main drain } 0.45 \mathrm{mi} \\
\text { ab Greenhorn } \mathrm{Cr}\end{array}$ & $8 / 21 / 2000$ & $11: 00$ & $2 o f 2$ & 200 & 10 & 0.014 & 0.007 \\
\hline BY23 & $\begin{array}{c}\text { Buckeye Flat Mine N } \\
\text { drain to Greenhorn } \\
\text { Cr, Grass Valley }\end{array}$ & $6 / 18 / 1999$ & $11: 30$ & 1 of 2 & 560 & 0 & 0.011 & 0.003 \\
\hline
\end{tabular}


Table 4B. Concentrations of trace metals and selected major elements in filtered water samples, Greenhorn Creek drainage, Nevada County, California_-Continued.

[Station name abbreviations: ab, above; bl, below; Cr, creek; Cyn, Canyon; mi, miles; N, north; nr, near; Rd, Road; S, south; SF, South Fork. Number in parentheses is the data parameter code, a five-digit code used in the U.S. Geological Survey computerized data system. $\mu \mathrm{g} / \mathrm{L}$, microgram per liter (equivalent to part per billion); $\mathrm{mg} / \mathrm{L}$, milligram per liter (at low concentration, equivalent to part per million); s.d., standard deviation; <, less than; - , not determined]

\begin{tabular}{|c|c|c|c|c|c|c|c|c|}
\hline \multirow[t]{2}{*}{$\begin{array}{l}\text { Station } \\
\text { map ID }\end{array}$} & \multirow[t]{2}{*}{ Station name } & \multirow[t]{2}{*}{ Date } & \multirow[t]{2}{*}{ Time } & \multirow[t]{2}{*}{$\begin{array}{l}\text { Rep- } \\
\text { licate }\end{array}$} & \multicolumn{2}{|c|}{$\begin{array}{c}\text { Zinc }(Z n), \\
\text { filtered } \\
(\mu \mathrm{g} / \mathrm{L}) \\
(01090)\end{array}$} & \multicolumn{2}{|c|}{$\begin{array}{c}\text { Zirconium (Zr), } \\
\text { filtered } \\
(\mu \mathrm{g} / \mathrm{L}) \\
(01160) \\
\end{array}$} \\
\hline & & & & & value & s.d. & value & s.d. \\
\hline BY23 & $\begin{array}{c}\text { Buckeye Flat Mine N } \\
\text { drain to Greenhorn } \\
\text { Cr, Grass Valley }\end{array}$ & $6 / 18 / 1999$ & $11: 30$ & $2 \mathrm{of} 2$ & 550 & 10 & 0.0090 & 0.0012 \\
\hline BY23 & $\begin{array}{c}\text { Buckeye Flat Mine N } \\
\text { drain to Greenhorn } \\
\text { Cr, Grass Valley }\end{array}$ & $12 / 2 / 1999$ & 11:00 & 1 of 2 & 300 & 0 & 0.041 & 0.001 \\
\hline BY23 & $\begin{array}{c}\text { Buckeye Flat Mine N } \\
\text { drain to Greenhorn } \\
\text { Cr, Grass Valley }\end{array}$ & $12 / 2 / 1999$ & 11:00 & $2 \mathrm{of} 2$ & 290 & 0 & 0.041 & 0.001 \\
\hline BY23 & $\begin{array}{c}\text { Buckeye Flat Mine N } \\
\text { drain to Greenhorn } \\
\text { Cr, Grass Valley }\end{array}$ & $8 / 22 / 2000$ & $10: 45$ & 1 of 2 & 370 & 0 & 0.026 & 0.007 \\
\hline BY23 & $\begin{array}{c}\text { Buckeye Flat Mine N } \\
\text { drain to Greenhorn } \\
\text { Cr, Grass Valley }\end{array}$ & $8 / 22 / 2000$ & $10: 45$ & $2 \mathrm{of} 2$ & 370 & 0 & 0.021 & 0.001 \\
\hline BY116 & $\begin{array}{l}\text { Buckeye Flat Mine } \\
\text { pond drain } 0.15 \mathrm{mi} \\
\text { ab SF Greenhorn Cr }\end{array}$ & $8 / 21 / 2000$ & $14: 10$ & 1 of 2 & 8.3 & 0.1 & $<0.01$ & 0.01 \\
\hline BY116 & $\begin{array}{l}\text { Buckeye Flat Mine } \\
\text { pond drain } 0.15 \mathrm{mi} \\
\text { ab SF Greenhorn Cr }\end{array}$ & $8 / 21 / 2000$ & $14: 10$ & 2 of 2 & 8.8 & 0.3 & $<0.01$ & 0.00 \\
\hline BY24 & $\begin{array}{l}\text { Buckeye Flat Mine } \\
\text { S drain to SF } \\
\text { Greenhorn Cr, Grass } \\
\text { Valley }\end{array}$ & $8 / 26 / 1999$ & 11:00 & 1 of 2 & 20 & 2 & 0.0083 & 0.0006 \\
\hline BY24 & $\begin{array}{l}\text { Buckeye Flat Mine } \\
\text { S drain to SF } \\
\text { Greenhorn Cr, Grass } \\
\text { Valley }\end{array}$ & $8 / 26 / 1999$ & 11:00 & $2 \mathrm{of} 2$ & 20 & 1 & 0.0055 & 0.0007 \\
\hline BY24 & $\begin{array}{l}\text { Buckeye Flat Mine } \\
\text { S drain to SF } \\
\text { Greenhorn Cr, Grass } \\
\text { Valley }\end{array}$ & $8 / 22 / 2000$ & $12: 10$ & 1 of 2 & 14 & 0 & $<0.01$ & 0.00 \\
\hline BY24 & $\begin{array}{l}\text { Buckeye Flat Mine } \\
\text { S drain to SF } \\
\text { Greenhorn Cr, Grass } \\
\text { Valley }\end{array}$ & $8 / 22 / 2000$ & $12: 10$ & $2 \mathrm{of} 2$ & 14 & 0 & $<0.01$ & 0.00 \\
\hline
\end{tabular}


Table 4B. Concentrations of trace metals and selected major elements in filtered water samples, Greenhorn

[Station name abbreviations: ab, above; bl, below; Cr, creek; Cyn, Canyon; mi, miles; N, north; nr, near; Rd, Road; S, south; SF, South Fork. Number in parentheses is the data parameter code, a five-digit code used in the U.S. Geological Survey computerized data system. $\mu \mathrm{g} / \mathrm{L}$, microgram per liter (equivalent to part per billion); $\mathrm{mg} / \mathrm{L}$, milligram per liter (at low concentration, equivalent to part per million); s.d., standard deviation; <, less than; - , not determined]

\begin{tabular}{|c|c|c|c|c|c|c|c|c|}
\hline \multirow[t]{2}{*}{$\begin{array}{l}\text { Station } \\
\text { map ID }\end{array}$} & \multirow[t]{2}{*}{ Station name } & \multirow[t]{2}{*}{ Date } & \multirow[t]{2}{*}{ Time } & \multirow[t]{2}{*}{$\begin{array}{l}\text { Rep- } \\
\text { licate }\end{array}$} & \multicolumn{2}{|c|}{$\begin{array}{c}\text { Zinc }(Z n), \\
\text { filtered } \\
(\mu \mathrm{g} / \mathrm{L}) \\
(01090)\end{array}$} & \multicolumn{2}{|c|}{$\begin{array}{c}\text { Zirconium }(\mathrm{Zr}), \\
\text { filtered } \\
(\mu \mathrm{g} / \mathrm{L}) \\
(\mathbf{0 1 1 6 0 )}\end{array}$} \\
\hline & & & & & value & s.d. & value & s.d. \\
\hline BY25 & $\begin{array}{l}\text { Buckeye Flat Mine } \\
\text { upper drain }\end{array}$ & $4 / 9 / 2001$ & $10: 00$ & 1 of 2 & 79 & 11 & 0.015 & 0.011 \\
\hline BY25 & $\begin{array}{l}\text { Buckeye Flat Mine } \\
\text { upper drain }\end{array}$ & $4 / 9 / 2001$ & $10: 00$ & 2 of 2 & 76 & 0 & 0.017 & 0.004 \\
\hline BY52 & $\begin{array}{l}\text { Greenhorn Cr } 0.3 \mathrm{mi} \\
\text { ab Buckeye Ford nr } \\
\text { Nevada City }\end{array}$ & $1 / 25 / 2000$ & $10: 45$ & 1 of 2 & 4.2 & 0.5 & 0.044 & 0.004 \\
\hline BY52 & $\begin{array}{l}\text { Greenhorn Cr } 0.3 \mathrm{mi} \\
\text { ab Buckeye Ford nr } \\
\text { Nevada City }\end{array}$ & $1 / 25 / 2000$ & $10: 45$ & $2 \mathrm{of} 2$ & 3.6 & 0.3 & 0.047 & 0.003 \\
\hline BY59 & $\begin{array}{l}\text { Greenhorn Cr at You } \\
\text { Bet Rd nr Nevada } \\
\text { City }\end{array}$ & $7 / 24 / 2001$ & 11:00 & 1 of 2 & 1.5 & 0.1 & 0.0091 & 0.0058 \\
\hline BY59 & $\begin{array}{l}\text { Greenhorn Cr at You } \\
\text { Bet Rd nr Nevada } \\
\text { City }\end{array}$ & $7 / 24 / 2001$ & 11:00 & $2 o f 2$ & 1.7 & 0.0 & 0.0026 & 0.0003 \\
\hline BY59 & $\begin{array}{l}\text { Greenhorn Cr at You } \\
\text { Bet Rd nr Nevada } \\
\text { City }\end{array}$ & $8 / 29 / 2001$ & $15: 00$ & 1 of 2 & 0.67 & 0.08 & 0.0019 & 0.0006 \\
\hline BY59 & $\begin{array}{l}\text { Greenhorn Cr at You } \\
\text { Bet Rd nr Nevada } \\
\text { City }\end{array}$ & $8 / 29 / 2001$ & $15: 00$ & $2 \mathrm{of} 2$ & 0.55 & 0.02 & 0.0035 & 0.0015 \\
\hline BY59 & $\begin{array}{l}\text { Greenhorn Cr at You } \\
\text { Bet Rd nr Nevada } \\
\text { City }\end{array}$ & $9 / 14 / 2001$ & $13: 00$ & 1 of 2 & 0.90 & 0.05 & 0.0022 & 0.0019 \\
\hline BY59 & $\begin{array}{l}\text { Greenhorn Cr at You } \\
\text { Bet Rd nr Nevada } \\
\text { City }\end{array}$ & $9 / 14 / 2001$ & 13:00 & $2 \mathrm{of} 2$ & 1.2 & 0.0 & 0.0036 & 0.0010 \\
\hline BY59 & $\begin{array}{l}\text { Greenhorn Cr at You } \\
\text { Bet Rd nr Nevada } \\
\text { City }\end{array}$ & $10 / 15 / 2001$ & $12: 00$ & 1 of 2 & 1.5 & 0.0 & 0.0031 & 0.0013 \\
\hline BY59 & $\begin{array}{l}\text { Greenhorn Cr at You } \\
\text { Bet Rd nr Nevada } \\
\text { City }\end{array}$ & $10 / 15 / 2001$ & $12: 00$ & $2 o f 2$ & 1.5 & 0.0 & 0.0036 & 0.0016 \\
\hline BY59 & $\begin{array}{l}\text { Greenhorn Cr at You } \\
\text { Bet Rd nr Nevada } \\
\text { City }\end{array}$ & $11 / 20 / 2001$ & $13: 20$ & 1 of 2 & 2.5 & 0.1 & 0.0026 & 0.0006 \\
\hline
\end{tabular}


Table 4B. Concentrations of trace metals and selected major elements in filtered water samples, Greenhorn Creek drainage, Nevada County, California_-Continued.

[Station name abbreviations: ab, above; bl, below; Cr, creek; Cyn, Canyon; mi, miles; N, north; nr, near; Rd, Road; S, south; SF, South Fork. Number in parentheses is the data parameter code, a five-digit code used in the U.S. Geological Survey computerized data system. $\mu \mathrm{g} / \mathrm{L}$, microgram per liter (equivalent to part per billion); $\mathrm{mg} / \mathrm{L}$, milligram per liter (at low concentration, equivalent to part per million); s.d., standard deviation; <, less than; - , not determined]

\begin{tabular}{|c|c|c|c|c|c|c|c|c|}
\hline \multirow[t]{2}{*}{$\begin{array}{l}\text { Station } \\
\text { map ID }\end{array}$} & \multirow[t]{2}{*}{ Station name } & \multirow[t]{2}{*}{ Date } & \multirow[t]{2}{*}{ Time } & \multirow[t]{2}{*}{$\begin{array}{l}\text { Rep- } \\
\text { licate }\end{array}$} & \multicolumn{2}{|c|}{$\begin{array}{c}\text { Zinc }(\mathrm{Zn}), \\
\text { filtered } \\
(\mu \mathrm{g} / \mathrm{L}) \\
(01090)\end{array}$} & \multicolumn{2}{|c|}{$\begin{array}{c}\text { Zirconium }(\mathrm{Zr}), \\
\text { filtered } \\
(\mu \mathrm{g} / \mathrm{L}) \\
(\mathbf{0 1 1 6 0 )}\end{array}$} \\
\hline & & & & & value & s.d. & value & s.d. \\
\hline BY59 & $\begin{array}{l}\text { Greenhorn Cr at You } \\
\text { Bet Rd nr Nevada } \\
\text { City }\end{array}$ & $11 / 20 / 2001$ & $13: 20$ & 2 of 2 & 2.2 & 0.1 & 0.0035 & 0.0010 \\
\hline BY59 & $\begin{array}{l}\text { Greenhorn Cr at You } \\
\text { Bet Rd nr Nevada } \\
\text { City }\end{array}$ & $12 / 10 / 2001$ & $12: 20$ & 1 of 2 & 2.8 & 0.0 & 0.017 & 0.001 \\
\hline BY59 & $\begin{array}{l}\text { Greenhorn } \mathrm{Cr} \text { at You } \\
\text { Bet Rd nr Nevada } \\
\text { City }\end{array}$ & $12 / 10 / 2001$ & $12: 20$ & 2 of 2 & 3.0 & 0.1 & 0.016 & 0.006 \\
\hline BY59 & $\begin{array}{l}\text { Greenhorn } \mathrm{Cr} \text { at You } \\
\text { Bet Rd nr Nevada } \\
\text { City }\end{array}$ & $12 / 21 / 2001$ & 13:00 & 1 of 2 & 1.3 & 0.0 & 0.032 & 0.005 \\
\hline BY59 & $\begin{array}{l}\text { Greenhorn Cr at You } \\
\text { Bet Rd nr Nevada } \\
\text { City }\end{array}$ & $12 / 21 / 2001$ & 13:00 & $2 \mathrm{of} 2$ & 1.4 & 0.1 & 0.025 & 0.003 \\
\hline BY51 & $\begin{array}{l}\text { Greenhorn Cr nr } \\
\text { Headwaters nr Scotts } \\
\text { Flat Reservoir }\end{array}$ & $1 / 25 / 2000$ & $9: 30$ & 1 of 2 & 4.2 & 2.9 & 0.041 & 0.005 \\
\hline BY51 & $\begin{array}{l}\text { Greenhorn Cr nr } \\
\text { Headwaters nr Scotts } \\
\text { Flat Reservoir }\end{array}$ & $1 / 25 / 2000$ & $9: 30$ & $2 \mathrm{of} 2$ & 1.3 & 0.1 & 0.035 & 0.004 \\
\hline BY75 & $\begin{array}{l}\text { Missouri Cyn Cr } 1.2 \mathrm{mi} \\
\text { ab Greenhorn Cr nr } \\
\text { Chicago Park }\end{array}$ & $12 / 14 / 1999$ & $14: 00$ & 1 of 2 & 33 & 1 & 0.0065 & 0.0008 \\
\hline BY75 & $\begin{array}{l}\text { Missouri Cyn Cr } 1.2 \mathrm{mi} \\
\text { ab Greenhorn Cr nr } \\
\text { Chicago Park }\end{array}$ & $12 / 14 / 1999$ & $14: 00$ & $2 o f 2$ & 32 & 1 & 0.0077 & 0.0009 \\
\hline BY86 & $\begin{array}{l}\text { Poore Mine creek ab } \\
\text { tunnel nr Grass } \\
\text { Valley }\end{array}$ & $3 / 31 / 1999$ & $17: 40$ & 1 of 2 & 2.1 & 0.0 & 0.010 & 0.001 \\
\hline BY86 & $\begin{array}{l}\text { Poore Mine creek ab } \\
\text { tunnel nr Grass } \\
\text { Valley }\end{array}$ & $3 / 31 / 1999$ & $17: 40$ & 2 of 2 & 1.5 & 0.1 & 0.0093 & 0.0016 \\
\hline
\end{tabular}


Table 4B. Concentrations of trace metals and selected major elements in filtered water samples, Greenhorn

[Station name abbreviations: ab, above; bl, below; Cr, creek; Cyn, Canyon; mi, miles; N, north; nr, near; Rd, Road; S, south; SF, South Fork. Number in parentheses is the data parameter code, a five-digit code used in the U.S. Geological Survey computerized data system. $\mu \mathrm{g} / \mathrm{L}$, microgram per liter (equivalent to part per billion); $\mathrm{mg} / \mathrm{L}$, milligram per liter (at low concentration, equivalent to part per million); s.d., standard deviation; <, less than; - , not determined]

\begin{tabular}{|c|c|c|c|c|c|c|c|c|}
\hline \multirow[t]{2}{*}{$\begin{array}{l}\text { Station } \\
\text { map ID }\end{array}$} & \multirow[t]{2}{*}{ Station name } & \multirow[t]{2}{*}{ Date } & \multirow[t]{2}{*}{ Time } & \multirow[t]{2}{*}{$\begin{array}{l}\text { Rep- } \\
\text { licate }\end{array}$} & \multicolumn{2}{|c|}{$\begin{array}{c}\text { Zinc }(Z n), \\
\text { filtered } \\
(\mu \mathrm{g} / \mathrm{L}) \\
(01090)\end{array}$} & \multicolumn{2}{|c|}{$\begin{array}{c}\text { Zirconium (Zr), } \\
\text { filtered } \\
(\mu \mathrm{g} / \mathrm{L}) \\
(\mathbf{0 1 1 6 0 )} \\
\end{array}$} \\
\hline & & & & & value & s.d. & value & s.d. \\
\hline BY87 & $\begin{array}{l}\text { Poore Mine creek bl } \\
\text { tunnel nr Grass } \\
\text { Valley }\end{array}$ & $3 / 31 / 1999$ & $13: 30$ & 1 of 2 & 1.4 & 0.1 & 0.010 & 0.003 \\
\hline BY87 & $\begin{array}{l}\text { Poore Mine creek bl } \\
\text { tunnel nr Grass } \\
\text { Valley }\end{array}$ & 3/31/1999 & $13: 30$ & $2 o f 2$ & 1.8 & 0.1 & 0.010 & 0.001 \\
\hline BY88 & $\begin{array}{l}\text { Poore Mine ground } \\
\text { sluice nr Grass } \\
\text { Valley }\end{array}$ & 4/1/1999 & $15: 40$ & 1 of 2 & 9.2 & 0.2 & 0.0026 & 0.0006 \\
\hline BY88 & $\begin{array}{l}\text { Poore Mine ground } \\
\text { sluice nr Grass } \\
\text { Valley }\end{array}$ & 4/1/1999 & $15: 40$ & $2 o f 2$ & 9.7 & 0.6 & 0.0057 & 0.0128 \\
\hline BY89 & $\begin{array}{c}\text { Poore Mine pit lake } \\
\text { nr Grass Valley }\end{array}$ & 4/1/1999 & $16: 50$ & 1 of 2 & 11 & 0 & 0.0019 & 0.0011 \\
\hline BY89 & $\begin{array}{c}\text { Poore Mine pit lake } \\
\text { nr Grass Valley }\end{array}$ & 4/1/1999 & $16: 50$ & 2 of 2 & 10 & 0 & 0.0016 & 0.0001 \\
\hline BY89 & $\begin{array}{l}\text { Poore Mine pit lake } \\
\text { nr Grass Valley }\end{array}$ & $5 / 23 / 2000$ & $10: 45$ & 1 of 2 & 85 & 0 & 0.005 & 0.002 \\
\hline BY89 & $\begin{array}{l}\text { Poore Mine pit lake } \\
\text { nr Grass Valley }\end{array}$ & $5 / 23 / 2000$ & $10: 45$ & 2 of 2 & 85 & 4 & 0.004 & 0.001 \\
\hline BY90 & $\begin{array}{l}\text { Poore Mine seep } \\
\text { ab ground sluice } \\
\text { nr Grass Valley }\end{array}$ & 4/1/1999 & $16: 00$ & 1 of 2 & 8.5 & 0.3 & $<0.0006$ & 0.0027 \\
\hline BY90 & $\begin{array}{l}\text { Poore Mine seep } \\
\text { ab ground sluice } \\
\text { nr Grass Valley }\end{array}$ & 4/1/1999 & $16: 00$ & $2 \mathrm{of} 2$ & 7.9 & 0.0 & 0.0039 & 0.0016 \\
\hline BY91 & $\begin{array}{l}\text { Poore Mine tunnel } \\
\text { effluent nr Grass } \\
\text { Valley }\end{array}$ & 3/31/1999 & $15: 20$ & 1 of 2 & 6.5 & 0.0 & 0.035 & 0.001 \\
\hline BY91 & $\begin{array}{l}\text { Poore Mine tunnel } \\
\text { effluent nr Grass } \\
\text { Valley }\end{array}$ & 3/31/1999 & $15: 20$ & $2 o f 2$ & 6.6 & 0.3 & 0.0048 & 0.0002 \\
\hline
\end{tabular}


Table 4B. Concentrations of trace metals and selected major elements in filtered water samples, Greenhorn Creek drainage, Nevada County, California_-Continued.

[Station name abbreviations: ab, above; bl, below; Cr, creek; Cyn, Canyon; mi, miles; N, north; nr, near; Rd, Road; S, south; SF, South Fork. Number in parentheses is the data parameter code, a five-digit code used in the U.S. Geological Survey computerized data system. $\mu \mathrm{g} / \mathrm{L}$, microgram per liter (equivalent to part per billion); $\mathrm{mg} / \mathrm{L}$, milligram per liter (at low concentration, equivalent to part per million); s.d., standard deviation; <, less than; - , not determined]

\begin{tabular}{|c|c|c|c|c|c|c|c|c|}
\hline \multirow[t]{2}{*}{$\begin{array}{l}\text { Station } \\
\text { map ID }\end{array}$} & \multirow[t]{2}{*}{ Station name } & \multirow[t]{2}{*}{ Date } & \multirow[t]{2}{*}{ Time } & \multirow[t]{2}{*}{$\begin{array}{l}\text { Rep- } \\
\text { licate }\end{array}$} & \multicolumn{2}{|c|}{$\begin{array}{c}\text { Zinc }(Z \mathbf{n}) \text {, } \\
\text { filtered } \\
(\mu \mathrm{g} / \mathrm{L}) \\
(01090)\end{array}$} & \multicolumn{2}{|c|}{$\begin{array}{c}\text { Zirconium (Zr), } \\
\text { filtered } \\
(\mu \mathrm{g} / \mathrm{L}) \\
(\mathbf{0 1 1 6 0 )}\end{array}$} \\
\hline & & & & & value & s.d. & value & s.d. \\
\hline BY91 & $\begin{array}{l}\text { Poore Mine tunnel } \\
\text { effluent nr Grass } \\
\text { Valley }\end{array}$ & $12 / 15 / 1999$ & 13:00 & 1 of 2 & 2.9 & 0.0 & 0.0013 & 0.0004 \\
\hline BY91 & $\begin{array}{l}\text { Poore Mine tunnel } \\
\text { effluent nr Grass } \\
\text { Valley }\end{array}$ & $12 / 15 / 1999$ & $13: 00$ & $2 \mathrm{of} 2$ & 3.1 & 0.4 & 0.0025 & 0.0004 \\
\hline BY91 & $\begin{array}{l}\text { Poore Mine tunnel } \\
\text { effluent nr Grass } \\
\text { Valley }\end{array}$ & $5 / 23 / 2000$ & $12: 00$ & 1 of 2 & 7.6 & 0.2 & 0.005 & 0.004 \\
\hline BY91 & $\begin{array}{l}\text { Poore Mine tunnel } \\
\text { effluent nr Grass } \\
\text { Valley }\end{array}$ & $5 / 23 / 2000$ & $12: 00$ & $2 \mathrm{of} 2$ & 7.5 & 0.0 & $<0.004$ & 0.002 \\
\hline BY105 & $\begin{array}{l}\text { Sailor Flat Mine main } \\
\text { drain Gulch 01nr } \\
\text { Quaker Hill }\end{array}$ & 7/6/1999 & $16: 00$ & 1 of 2 & 27 & 0 & 0.11 & 0.00 \\
\hline BY105 & $\begin{array}{l}\text { Sailor Flat Mine main } \\
\text { drain Gulch 01nr } \\
\text { Quaker Hill }\end{array}$ & 7/6/1999 & $16: 00$ & $2 \mathrm{of} 2$ & 25 & 2 & 0.12 & 0.00 \\
\hline BY105 & $\begin{array}{l}\text { Sailor Flat Mine main } \\
\text { drain Gulch 01nr } \\
\text { Quaker Hill }\end{array}$ & $12 / 2 / 1999$ & $14: 30$ & 1 of 2 & 8.0 & 1.0 & 0.29 & 0.00 \\
\hline BY105 & $\begin{array}{l}\text { Sailor Flat Mine main } \\
\text { drain Gulch 01nr } \\
\text { Quaker Hill }\end{array}$ & $12 / 2 / 1999$ & $14: 30$ & $2 \mathrm{of} 2$ & 7.4 & 0.3 & 0.28 & 0.01 \\
\hline BY106 & $\begin{array}{l}\text { Sailor Flat Mine } \\
\text { main drainage to } \\
\text { Greenhorn Cr, } \\
\text { Gulch } 03\end{array}$ & $4 / 9 / 2001$ & $12: 30$ & 1 of 2 & 4.4 & 0.3 & 0.31 & 0.08 \\
\hline BY106 & $\begin{array}{l}\text { Sailor Flat Mine } \\
\text { main drainage to } \\
\text { Greenhorn Cr, } \\
\text { Gulch } 03\end{array}$ & $4 / 9 / 2001$ & $12: 30$ & 2 of 2 & 4.7 & 0.5 & 0.32 & 0.09 \\
\hline
\end{tabular}


Table 4B. Concentrations of trace metals and selected major elements in filtered water samples, Greenhorn

[Station name abbreviations: ab, above; bl, below; Cr, creek; Cyn, Canyon; mi, miles; N, north; nr, near; Rd, Road; S, south; SF, South Fork. Number in parentheses is the data parameter code, a five-digit code used in the U.S. Geological Survey computerized data system. $\mu \mathrm{g} / \mathrm{L}$, microgram per liter (equivalent to part per billion); $\mathrm{mg} / \mathrm{L}$, milligram per liter (at low concentration, equivalent to part per million); s.d., standard deviation; <, less than; - , not determined]

\begin{tabular}{|c|c|c|c|c|c|c|c|c|}
\hline \multirow[t]{2}{*}{$\begin{array}{l}\text { Station } \\
\text { map ID }\end{array}$} & \multirow[t]{2}{*}{ Station name } & \multirow[t]{2}{*}{ Date } & \multirow[t]{2}{*}{ Time } & \multirow[t]{2}{*}{$\begin{array}{l}\text { Rep- } \\
\text { licate }\end{array}$} & \multicolumn{2}{|c|}{$\begin{array}{c}\text { Zinc }(\mathrm{Zn}), \\
\text { filtered } \\
(\mu \mathrm{g} / \mathrm{L}) \\
(01090)\end{array}$} & \multicolumn{2}{|c|}{$\begin{array}{c}\text { Zirconium }(\mathrm{Zr}), \\
\text { filtered } \\
(\mu \mathrm{g} / \mathrm{L}) \\
(\mathbf{0 1 1 6 0 )}\end{array}$} \\
\hline & & & & & value & s.d. & value & s.d. \\
\hline BY114 & $\begin{array}{l}\text { SF Greenhorn Cr } 0.7 \text { i } \\
\text { ab Greenhorn Cr nr } \\
\text { Nevada City }\end{array}$ & $8 / 22 / 2000$ & $15: 10$ & 1 of 2 & $<0.2$ & 0.1 & $<0.01$ & 0.01 \\
\hline BY114 & $\begin{array}{l}\text { SF Greenhorn Cr } \\
0.7 \text { mi ab Greenhorn } \\
\text { Cr nr Nevada City }\end{array}$ & $8 / 22 / 2000$ & $15: 10$ & $2 \mathrm{of} 2$ & $<0.2$ & 0.1 & 0.065 & 0.067 \\
\hline BY122 & $\begin{array}{l}\text { Starr Mine tunnel } \\
\text { inflow nr Grass } \\
\text { Valley }\end{array}$ & 4/23/1999 & $15: 00$ & 1 of 2 & 16 & 0 & 0.0020 & 0.0001 \\
\hline BY122 & $\begin{array}{l}\text { Starr Mine tunnel } \\
\text { inflow nr Grass } \\
\text { Valley }\end{array}$ & 4/23/1999 & $15: 00$ & $2 \mathrm{of} 2$ & 17 & 1 & 0.0029 & 0.0005 \\
\hline BY123 & $\begin{array}{l}\text { Starr Mine tunnel } \\
\text { midway nr Grass } \\
\text { Valley }\end{array}$ & 4/23/1999 & $13: 40$ & 1 of 2 & 0.53 & 0.08 & 0.0028 & 0.0006 \\
\hline BY123 & $\begin{array}{l}\text { Starr Mine tunnel } \\
\text { midway nr Grass } \\
\text { Valley }\end{array}$ & 4/23/1999 & $13: 40$ & $2 \mathrm{of} 2$ & 0.59 & 0.02 & 0.0043 & 0.0004 \\
\hline BY123 & $\begin{array}{l}\text { Starr Mine tunnel } \\
\text { midway nr Grass } \\
\text { Valley }\end{array}$ & $12 / 15 / 1999$ & $10: 45$ & 1 of 2 & 2.1 & 0.3 & 0.0027 & 0.0001 \\
\hline BY123 & $\begin{array}{l}\text { Starr Mine tunnel } \\
\text { midway nr Grass } \\
\text { Valley }\end{array}$ & $12 / 15 / 1999$ & $10: 45$ & $2 \mathrm{of} 2$ & 1.7 & 0.3 & 0.0030 & 0.0008 \\
\hline BY124 & $\begin{array}{l}\text { Starr Mine tunnel outlet } \\
\text { nr Grass Valley }\end{array}$ & $8 / 23 / 2000$ & $17: 30$ & 1 of 2 & 0.4 & 0.1 & $<0.01$ & 0.01 \\
\hline BY124 & $\begin{array}{l}\text { Starr Mine tunnel outlet } \\
\text { nr Grass Valley }\end{array}$ & $8 / 23 / 2000$ & $17: 30$ & $2 \mathrm{of} 2$ & 0.4 & 0.1 & $<0.01$ & 0.00 \\
\hline BY129 & $\begin{array}{l}\text { Tom and Jerry Mine } \\
\text { drain at tunnel inlet } \\
\text { nr Nevada City }\end{array}$ & $6 / 28 / 2000$ & $12: 00$ & 1 of 2 & 0.5 & 0.2 & 0.010 & 0.008 \\
\hline BY129 & $\begin{array}{l}\text { Tom and Jerry Mine } \\
\text { drain at tunnel inlet } \\
\text { nr Nevada City }\end{array}$ & $6 / 28 / 2000$ & $12: 00$ & $2 \mathrm{of} 2$ & 0.4 & 0.1 & $<0.006$ & 0.003 \\
\hline
\end{tabular}


Table 4B. Concentrations of trace metals and selected major elements in filtered water samples, Greenhorn Creek drainage, Nevada County, California_-Continued.

[Station name abbreviations: ab, above; bl, below; Cr, creek; Cyn, Canyon; mi, miles; N, north; nr, near; Rd, Road; S, south; SF, South Fork. Number in parentheses is the data parameter code, a five-digit code used in the U.S. Geological Survey computerized data system. $\mu \mathrm{g} / \mathrm{L}$, microgram per liter (equivalent to part per billion); $\mathrm{mg} / \mathrm{L}$, milligram per liter (at low concentration, equivalent to part per million); s.d., standard deviation; <, less than; - , not determined]

\begin{tabular}{|c|c|c|c|c|c|c|c|c|}
\hline \multirow[t]{2}{*}{$\begin{array}{l}\text { Station } \\
\text { map ID }\end{array}$} & \multirow[t]{2}{*}{ Station name } & \multirow[t]{2}{*}{ Date } & \multirow[t]{2}{*}{ Time } & \multirow[t]{2}{*}{$\begin{array}{l}\text { Rep- } \\
\text { licate }\end{array}$} & \multicolumn{2}{|c|}{$\begin{array}{c}\text { Zinc }(Z n), \\
\text { filtered } \\
(\mu \mathrm{g} / \mathrm{L}) \\
(01090)\end{array}$} & \multicolumn{2}{|c|}{$\begin{array}{c}\text { Zirconium }(\mathrm{Zr}), \\
\text { filtered } \\
(\mu \mathrm{g} / \mathrm{L}) \\
(\mathbf{0 1 1 6 0 )}\end{array}$} \\
\hline & & & & & value & s.d. & value & s.d. \\
\hline BY130 & $\begin{array}{l}\text { Tom and Jerry Mine } \\
\text { drain at tunnel outlet } \\
\text { nr Nevada City }\end{array}$ & $6 / 28 / 2000$ & $11: 30$ & 1 of 2 & 1.1 & 0.3 & $<0.006$ & 0.004 \\
\hline BY130 & $\begin{array}{l}\text { Tom and Jerry Mine } \\
\text { drain at tunnel outlet } \\
\text { nr Nevada City }\end{array}$ & $6 / 28 / 2000$ & $11: 30$ & $2 o f 2$ & 1.1 & 0.3 & $<0.006$ & 0.000 \\
\hline BY131 & $\begin{array}{l}\text { Tom and Jerry Mine } \\
\text { drainage pond nr } \\
\text { Nevada City }\end{array}$ & $8 / 23 / 2000$ & $10: 45$ & 1 of 2 & 1.6 & 0.1 & $<0.01$ & 0.00 \\
\hline BY131 & $\begin{array}{l}\text { Tom and Jerry Mine } \\
\text { drainage pond nr } \\
\text { Nevada City }\end{array}$ & $8 / 23 / 2000$ & $10: 45$ & $2 \mathrm{of} 2$ & 1.5 & 0.1 & $<0.01$ & 0.00 \\
\hline \multicolumn{9}{|c|}{ Disburbed samples } \\
\hline BY123 & $\begin{array}{l}\text { Starr Mine tunnel } \\
\text { midway nr Grass } \\
\text { Valley }\end{array}$ & $12 / 15 / 1999$ & $11: 30$ & 1 of 2 & 3.2 & 0.1 & 0.0022 & 0.0001 \\
\hline BY123 & $\begin{array}{l}\text { Starr Mine tunnel } \\
\text { midway nr Grass } \\
\text { Valley }\end{array}$ & $12 / 15 / 1999$ & $11: 30$ & $2 o f 2$ & 1.5 & 0.2 & 0.0026 & 0.0016 \\
\hline BY129 & $\begin{array}{l}\text { Tom and Jerry Mine } \\
\text { drain at tunnel inlet } \\
\text { nr Nevada City }\end{array}$ & $6 / 28 / 2000$ & $12: 30$ & 1 of 2 & 1.1 & 0.3 & 0.032 & 0.030 \\
\hline BY129 & $\begin{array}{l}\text { Tom and Jerry Mine } \\
\text { drain at tunnel inlet } \\
\text { nr Nevada City }\end{array}$ & $6 / 28 / 2000$ & $12: 30$ & 2 of 2 & 0.5 & 0.1 & 0.008 & 0.007 \\
\hline
\end{tabular}




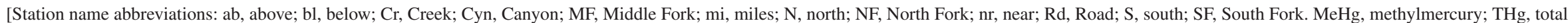
mercury. n, number of organisms; g, gram; $\mu \mathrm{g} / \mathrm{g}$, microgram per gram; - , not determined]

\begin{tabular}{|c|c|c|c|c|c|c|c|c|c|c|c|c|}
\hline $\begin{array}{l}\text { Station } \\
\text { map ID }\end{array}$ & Station name & $\begin{array}{l}\text { Collection } \\
\text { date }\end{array}$ & $\begin{array}{l}\text { Common } \\
\text { name }\end{array}$ & Order & Family & $\begin{array}{c}\text { Sample } \\
\text { size } \\
\text { (n) }\end{array}$ & $\begin{array}{l}\text { Live } \\
\text { weight } \\
\text { (g) }\end{array}$ & $\begin{array}{l}\text { Moisture } \\
\text { (percent) }\end{array}$ & $\begin{array}{c}\text { Total } \\
\text { mercury } \\
\text { ( } \mu \mathrm{g} / \mathrm{g} \text { wet })\end{array}$ & $\begin{array}{l}\text { Methyl- } \\
\text { mercury } \\
\text { ( } \mu \mathrm{g} / \mathrm{g} \text { wet) }\end{array}$ & $\begin{array}{c}\mathrm{MeHg} / \mathrm{THg} \\
\text { (percent) }\end{array}$ & Age \\
\hline BY20 & $\begin{array}{l}\text { Boston Mine tunnel outlet nr Grass } \\
\text { Valley }\end{array}$ & $8 / 31 / 99$ & Banana slug & Gastropoda & Arionidae & 3 & 50.87 & 84.2 & - & 0.033 & - & Undetermined \\
\hline BY20 & $\begin{array}{l}\text { Boston Mine tunnel outlet nr Grass } \\
\text { Valley }\end{array}$ & $8 / 21 / 00$ & Banana slug & Gastropoda & Arionidae & 3 & 40.82 & 81.8 & - & 0.0092 & - & Undetermined \\
\hline BY20 & $\begin{array}{l}\text { Boston Mine tunnel outlet nr Grass } \\
\quad \text { Valley }\end{array}$ & $9 / 13 / 01$ & Banana slug & Gastropoda & Arionidae & 3 & 61.19 & 82.9 & - & 0.0081 & - & Undetermined \\
\hline BY58 & $\begin{array}{l}\text { Buckeye Flat Mine main drain } 0.45 \\
\text { mi ab Greenhorn } \mathrm{Cr}\end{array}$ & $8 / 22 / 00$ & Banana slug & Gastropoda & Arionidae & 3 & 50.45 & 81.5 & - & 0.0060 & - & Undetermined \\
\hline BY58 & $\begin{array}{l}\text { Buckeye Flat Mine main drain } 0.45 \\
\text { mi ab Greenhorn } \mathrm{Cr}\end{array}$ & $9 / 13 / 01$ & Banana slug & Gastropoda & Arionidae & 3 & 46.64 & 84.5 & - & 0.0070 & - & Undetermined \\
\hline BY23 & $\begin{array}{l}\text { Buckeye Flat Mine N drain to } \\
\text { Greenhorn Cr Grass Valley }\end{array}$ & $8 / 22 / 00$ & Banana slug & Gastropoda & Arionidae & 3 & 43.67 & 88.7 & - & 0.0056 & - & Undetermined \\
\hline BY116 & $\begin{array}{l}\text { Buckeye Flat Mine pond drain } 0.15 \\
\text { mi ab SF Greenhorn } \mathrm{Cr}\end{array}$ & $8 / 22 / 00$ & Banana slug & Gastropoda & Arionidae & 3 & 33.60 & 89.5 & - & 0.014 & - & Undetermined \\
\hline BY116 & $\begin{array}{l}\text { Buckeye Flat Mine pond drain } 0.15 \\
\text { mi ab SF Greenhorn } \mathrm{Cr}\end{array}$ & $9 / 12 / 01$ & Banana slug & Gastropoda & Arionidae & 3 & 25.34 & 84.6 & - & 0.048 & - & Undetermined \\
\hline BY25 & Buckeye Flat Mine upper drain & $10 / 19 / 01$ & Banana slug & Gastropoda & Arionidae & 3 & 47.97 & 80.9 & 0.039 & 0.013 & 33 & Undetermined \\
\hline BY146 & $\begin{array}{l}\text { Missouri Cyn } 1.6 \mathrm{mi} \text { ab Greenhorn Cr } \\
\text { nr Chicago Park }\end{array}$ & $9 / 25 / 00$ & Banana slug & Gastropoda & Arionidae & 3 & 35.94 & 87.6 & - & 0.024 & - & Undetermined \\
\hline BY75 & $\begin{array}{l}\text { Missouri Cyn Cr } 1.2 \text { mi ab Greenhorn } \\
\text { Cr nr Chicago Park }\end{array}$ & $8 / 31 / 99$ & Banana slug & Gastropoda & Arionidae & 2 & 36.87 & 82.2 & - & 0.0014 & - & Undetermined \\
\hline BY75 & $\begin{array}{l}\text { Missouri Cyn Cr } 1.2 \text { mi ab Greenhorn } \\
\text { Cr nr Chicago Park }\end{array}$ & $8 / 21 / 00$ & Banana slug & Gastropoda & Arionidae & 3 & 105.94 & 79.5 & - & 0.0031 & - & Undetermined \\
\hline BY75 & $\begin{array}{l}\text { Missouri Cyn Cr } 1.2 \text { mi ab Greenhorn } \\
\text { Cr nr Chicago Park }\end{array}$ & $9 / 15 / 01$ & Banana slug & Gastropoda & Arionidae & 3 & 39.53 & 80.3 & - & 0.0035 & - & Undetermined \\
\hline BY147 & NF MF Missouri Cyn nr Chicago Park & $9 / 25 / 00$ & Banana slug & Gastropoda & Arionidae & 3 & 43.67 & 87.6 & - & 0.036 & - & Undetermined \\
\hline BY147 & NF MF Missouri Cyn nr Chicago Park & $9 / 15 / 01$ & Banana slug & Gastropoda & Arionidae & 3 & 26.56 & 82.9 & - & 0.020 & - & Undetermined \\
\hline BY86 & $\begin{array}{l}\text { Poore Mine Creek ab tunnel nr Grass } \\
\text { Valley }\end{array}$ & $9 / 26 / 00$ & Banana slug & Gastropoda & Arionidae & 3 & 28.83 & 84.1 & - & 0.006 & - & Undetermined \\
\hline BY90 & $\begin{array}{l}\text { Poore Mine Seep above ground sluice } \\
\text { nr Grass Valley }\end{array}$ & $9 / 26 / 00$ & Banana slug & Gastropoda & Arionidae & 3 & 50.19 & 84.2 & - & 0.017 & - & Undetermined \\
\hline BY91 & $\begin{array}{l}\text { Poore Mine tunnel effluent nr Grass } \\
\text { Valley }\end{array}$ & $8 / 13 / 99$ & Banana slug & Gastropoda & Arionidae & 3 & 53.86 & 83.6 & - & 0.0010 & - & Undetermined \\
\hline BY91 & $\begin{array}{l}\text { Poore Mine tunnel effluent nr Grass } \\
\text { Valley }\end{array}$ & $8 / 21 / 00$ & Banana slug & Gastropoda & Arionidae & 3 & 50.97 & 85.6 & - & 0.0072 & - & Undetermined \\
\hline BY91 & $\begin{array}{l}\text { Poore Mine tunnel effluent nr Grass } \\
\text { Valley }\end{array}$ & $10 / 17 / 01$ & Banana slug & Gastropoda & Arionidae & 3 & 50.35 & 85.5 & - & 0.010 & - & Undetermined \\
\hline BY114 & $\begin{array}{l}\text { SF Greenhorn Cr } 0.7 \text { mi ab Greenhorn } \\
\text { Cr nr Nevada City }\end{array}$ & $8 / 22 / 00$ & Banana slug & Gastropoda & Arionidae & 3 & 70.67 & 86.8 & - & 0.0043 & - & Undetermined \\
\hline BY113 & SF Greenhorn Cr $0.8 \mathrm{mi}$ ab Greenhorn & $9 / 13 / 01$ & Banana slug & Gastropoda & Arionidae & 3 & 38.46 & 89.8 & 0.087 & 0.0076 & 9 & Undetermined \\
\hline
\end{tabular}
Cr nr Nevada City 
Table 11A. Mercury and methylmercury concentration data for invertebrates, Greenhorn Creek drainage, Nevada County, California-Continued.

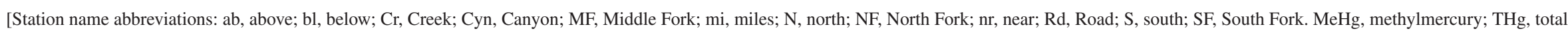
mercury. $\mathrm{n}$, number of organisms; g, gram; $\mu \mathrm{g} / \mathrm{g}$, microgram per gram; - , not determined]

\begin{tabular}{|c|c|c|c|c|c|c|c|c|c|c|c|c|}
\hline $\begin{array}{l}\text { Station } \\
\text { map ID }\end{array}$ & Station name & $\begin{array}{l}\text { Collection } \\
\text { date }\end{array}$ & $\begin{array}{c}\text { Common } \\
\text { name }\end{array}$ & Order & Family & $\begin{array}{c}\text { Sample } \\
\text { size } \\
\text { (n) }\end{array}$ & $\begin{array}{c}\text { Live } \\
\text { weight } \\
\text { (g) }\end{array}$ & $\begin{array}{l}\text { Moisture } \\
\text { (percent) }\end{array}$ & $\begin{array}{c}\text { Total } \\
\text { mercury } \\
(\mu \mathrm{g} / \mathrm{g} \text { wet })\end{array}$ & $\begin{array}{c}\text { Methyl- } \\
\text { mercury } \\
\text { ( } \mu \mathrm{g} / \mathrm{g} \text { wet) }\end{array}$ & $\begin{array}{c}\mathrm{MeHg} / \mathrm{THg} \\
\text { (percent) }\end{array}$ & Age \\
\hline BY124 & $\begin{array}{l}\text { Starr Mine tunnel outlet nr Grass } \\
\text { Valley }\end{array}$ & $8 / 21 / 00$ & Banana slug & Gastropoda & Arionidae & 2 & 52.97 & 81.5 & - & 0.0052 & - & Undetermined \\
\hline BY130 & $\begin{array}{l}\text { Tom Jerry Mine drain at tunnel outlet } \\
\text { nr Nevada City }\end{array}$ & $8 / 23 / 00$ & Banana slug & Gastropoda & Arionidae & 3 & 41.66 & 87.9 & - & 0.015 & - & Undetermined \\
\hline BY130 & $\begin{array}{l}\text { Tom Jerry Mine drain at tunnel outlet } \\
\text { nr Nevada City }\end{array}$ & $8 / 23 / 00$ & Banana slug & Gastropoda & Arionidae & 3 & 34.86 & 85.9 & - & 0.0036 & - & Undetermined \\
\hline BY130 & $\begin{array}{l}\text { Tom Jerry Mine drain at tunnel outlet } \\
\text { nr Nevada City }\end{array}$ & $9 / 13 / 01$ & Banana slug & Gastropoda & Arionidae & 3 & 40.90 & 82.0 & - & 0.0076 & 一 & Undetermined \\
\hline BY130 & $\begin{array}{l}\text { Tom Jerry Mine drain at tunnel outlet } \\
\text { nr Nevada City }\end{array}$ & $9 / 13 / 01$ & Banana slug & Gastropoda & Arionidae & 3 & 43.56 & 88.2 & - & 0.017 & 一 & Undetermined \\
\hline BY180 & Greenhorn Cr .2 mi bl The Narrows & $10 / 17 / 01$ & Dobsonfly & Megaloptera & Corydalidae & 1 & 0.73 & 63.4 & 0.10 & 0.067 & 64 & Immature \\
\hline BY57 & $\begin{array}{l}\text { Greenhorn Cr } 0.3 \text { mi bl Sailor Flat } \\
\text { West drain nr Nevada City }\end{array}$ & $9 / 29 / 99$ & Dobsonfly & Megaloptera & Corydalidae & 4 & 2.84 & 75.6 & - & 0.027 & 一 & Immature \\
\hline BY57 & $\begin{array}{l}\text { Greenhorn Cr } 0.3 \text { mi bl Sailor Flat } \\
\text { West drain nr Nevada City }\end{array}$ & 9/29/99 & Dobsonfly & Megaloptera & Corydalidae & 3 & 1.40 & 76.6 & - & 0.033 & - & Immature \\
\hline BY75 & $\begin{array}{l}\text { Missouri Cyn Cr } 1.2 \text { mi ab Greenhorn } \\
\text { Cr nr Chicago Park }\end{array}$ & $8 / 31 / 99$ & Dobsonfly & Megaloptera & Corydalidae & 1 & 1.43 & 70.5 & - & 0.18 & - & Immature \\
\hline BY75 & $\begin{array}{l}\text { Missouri Cyn Cr } 1.2 \text { mi ab Greenhorn } \\
\text { Cr nr Chicago Park }\end{array}$ & $8 / 21 / 00$ & Dobsonfly & Megaloptera & Corydalidae & 1 & 4.13 & 67.2 & - & 0.20 & - & Immature \\
\hline BY75 & $\begin{array}{l}\text { Missouri Cyn Cr } 1.2 \text { mi ab Greenhorn } \\
\text { Cr nr Chicago Park }\end{array}$ & $8 / 21 / 00$ & Dobsonfly & Megaloptera & Corydalidae & 1 & 3.95 & 70.0 & - & 0.19 & - & Immature \\
\hline BY75 & $\begin{array}{l}\text { Missouri Cyn Cr } 1.2 \text { mi ab Greenhorn } \\
\text { Cr nr Chicago Park }\end{array}$ & $9 / 15 / 01$ & Dobsonfly & Megaloptera & Corydalidae & 1 & 4.98 & 68.0 & 0.14 & 0.078 & 56 & Immature \\
\hline BY147 & NF MF Missouri Cyn nr Chicago Park & $9 / 25 / 00$ & Dobsonfly & Megaloptera & Corydalidae & 3 & 0.84 & 82.6 & - & 0.39 & - & Immature \\
\hline BY91 & $\begin{array}{l}\text { Poore Mine tunnel effluent nr Grass } \\
\text { Valley }\end{array}$ & $10 / 17 / 01$ & Dobsonfly & Megaloptera & Corydalidae & 1 & 2.27 & 76.9 & 0.060 & 0.039 & 65 & Immature \\
\hline BY114 & $\begin{array}{l}\text { SF Greenhorn Cr } 0.7 \text { mi ab Greenhorn } \\
\text { Cr nr Nevada City }\end{array}$ & $8 / 22 / 00$ & Dobsonfly & Megaloptera & Corydalidae & 3 & 1.65 & 75.3 & - & 0.068 & 一 & Immature \\
\hline BY113 & $\begin{array}{l}\text { SF Greenhorn Cr } 0.8 \text { mi ab Greenhorn } \\
\text { Cr nr Nevada City }\end{array}$ & $8 / 22 / 00$ & Dobsonfly & Megaloptera & Corydalidae & 3 & 2.05 & 75.9 & - & 0.048 & 一 & Immature \\
\hline BY113 & $\begin{array}{l}\text { SF Greenhorn Cr } 0.8 \text { mi ab Greenhorn } \\
\text { Cr nr Nevada City }\end{array}$ & $9 / 13 / 01$ & Dobsonfly & Megaloptera & Corydalidae & 3 & 3.96 & 70.4 & 0.11 & 0.030 & 27 & Immature \\
\hline BY115 & $\begin{array}{l}\text { SF Greenhorn Cr bl Boston Mine nr } \\
\text { Nevada City }\end{array}$ & $8 / 21 / 00$ & Dobsonfly & Megaloptera & Corydalidae & 1 & 0.60 & 77.8 & - & 0.21 & - & Immature \\
\hline BY130 & $\begin{array}{l}\text { Tom Jerry Mine drain at tunnel outlet } \\
\text { nr Nevada City }\end{array}$ & $8 / 23 / 00$ & Dobsonfly & Megaloptera & Corydalidae & 1 & 1.44 & 73.4 & 一 & 0.047 & - & Immature \\
\hline BY20 & $\begin{array}{l}\text { Boston Mine tunnel outlet nr Grass } \\
\text { Valley }\end{array}$ & $8 / 21 / 00$ & Dragonfly & Odonata & Cordulegastridae & 1 & 1.04 & 78.6 & - & 0.89 & - & Immature \\
\hline BY21 & Boston Mine wetlands pond nr Grass & $8 / 12 / 99$ & Dragonfly & Odonata & Aeshnidae & 3 & 3.84 & 76.7 & 0.071 & 0.070 & 99 & Immature \\
\hline
\end{tabular}


Table 11A. Mercury and methylmercury concentration data for invertebrates, Greenhorn Creek drainage, Nevada County, California-Continued.

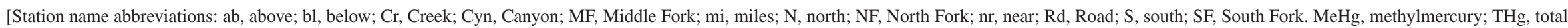
mercury. $\mathrm{n}$, number of organisms; g, gram; $\mu \mathrm{g} / \mathrm{g}$, microgram per gram; - , not determined]

\begin{tabular}{|c|c|c|c|c|c|c|c|c|c|c|c|c|}
\hline $\begin{array}{l}\text { Station } \\
\text { map ID }\end{array}$ & Station name & $\begin{array}{l}\text { Collection } \\
\text { date }\end{array}$ & $\begin{array}{l}\text { Common } \\
\text { name }\end{array}$ & Order & Family & $\begin{array}{c}\text { Sample } \\
\text { size } \\
\text { (n) }\end{array}$ & $\begin{array}{l}\text { Live } \\
\text { weight } \\
\text { (g) }\end{array}$ & $\begin{array}{l}\text { Moisture } \\
\text { (percent) }\end{array}$ & $\begin{array}{c}\text { Total } \\
\text { mercury } \\
(\mu \mathrm{g} / \mathrm{g} \text { wet })\end{array}$ & $\begin{array}{l}\text { Methyl- } \\
\text { mercury } \\
\text { ( } \mu \mathrm{g} / \mathrm{g} \text { wet) }\end{array}$ & $\begin{array}{c}\mathrm{MeHg} / \mathrm{THg} \\
\text { (percent) }\end{array}$ & Age \\
\hline BY21 & $\begin{array}{l}\text { Boston Mine wetlands pond nr Grass } \\
\text { Valley }\end{array}$ & $8 / 21 / 00$ & Dragonfly & Odonata & Aeshnidae & 8 & 2.08 & 82.6 & - & 0.032 & - & Immature \\
\hline BY21 & $\begin{array}{l}\text { Boston Mine wetlands pond nr Grass } \\
\text { Valley }\end{array}$ & $9 / 13 / 01$ & Dragonfly & Odonata & Libellulidae & 8 & 3.78 & 82.5 & 0.016 & 0.014 & 89 & Immature \\
\hline BY21 & $\begin{array}{l}\text { Boston Mine wetlands pond nr Grass } \\
\text { Valley }\end{array}$ & $9 / 13 / 01$ & Dragonfly & Odonata & Aeshnidae & 7 & 2.45 & 83.6 & 0.036 & 0.034 & 95 & Immature \\
\hline BY21 & $\begin{array}{l}\text { Boston Mine wetlands pond nr Grass } \\
\text { Valley }\end{array}$ & $9 / 13 / 01$ & Dragonfly & Odonata & Aeshnidae & 10 & 2.42 & 82.1 & 0.027 & 0.026 & 97 & Immature \\
\hline BY21 & $\begin{array}{l}\text { Boston Mine wetlands pond nr Grass } \\
\text { Valley }\end{array}$ & $9 / 13 / 01$ & Dragonfly & Odonata & Aeshnidae & 7 & 2.15 & 82.0 & 0.028 & 0.029 & 102 & Immature \\
\hline BY116 & $\begin{array}{l}\text { Buckeye Flat Mine pond drain } 0.15 \\
\text { mi ab SF Greenhorn Cr }\end{array}$ & $8 / 22 / 00$ & Dragonfly & Odonata & Cordulegastridae & 3 & 2.62 & 83.6 & 0.10 & 0.092 & 90 & Immature \\
\hline BY116 & $\begin{array}{l}\text { Buckeye Flat Mine pond drain } 0.15 \\
\text { mi ab SF Greenhorn } \mathrm{Cr}\end{array}$ & $9 / 12 / 01$ & Dragonfly & Odonata & Aeshnidae & 2 & 1.18 & 83.5 & 0.065 & 0.047 & 72 & Immature \\
\hline BY116 & $\begin{array}{l}\text { Buckeye Flat Mine pond drain } 0.15 \\
\text { mi ab SF Greenhorn } \mathrm{Cr}\end{array}$ & $9 / 12 / 01$ & Dragonfly & Odonata & Gomphidae & 5 & 0.96 & 81.8 & 0.12 & 0.10 & 83 & Immature \\
\hline BY116 & $\begin{array}{l}\text { Buckeye Flat Mine pond drain } 0.15 \\
\text { mi ab SF Greenhorn } \mathrm{Cr}\end{array}$ & $9 / 12 / 01$ & Dragonfly & Odonata & Aeshnidae & 4 & 1.12 & 81.6 & 0.063 & 0.063 & 100 & Immature \\
\hline BY24 & $\begin{array}{l}\text { Buckeye Flat Mine S drain to SF } \\
\text { Greenhorn Cr Grass Valley }\end{array}$ & 8/26/99 & Dragonfly & Odonata & Aeshnidae & 4 & 2.86 & 79.3 & - & 1.6 & - & Immature \\
\hline BY24 & $\begin{array}{l}\text { Buckeye Flat Mine S drain to SF } \\
\text { Greenhorn Cr Grass Valley }\end{array}$ & $8 / 22 / 00$ & Dragonfly & Odonata & Aeshnidae & 3 & 0.99 & 83.2 & - & 0.32 & - & Immature \\
\hline BY24 & $\begin{array}{l}\text { Buckeye Flat Mine S drain to SF } \\
\text { Greenhorn Cr Grass Valley }\end{array}$ & $9 / 12 / 01$ & Dragonfly & Odonata & Aeshnidae & 4 & 1.95 & 78.4 & 0.087 & 0.079 & 91 & Immature \\
\hline BY24 & $\begin{array}{l}\text { Buckeye Flat Mine S drain to SF } \\
\text { Greenhorn Cr Grass Valley }\end{array}$ & $9 / 12 / 01$ & Dragonfly & Odonata & Aeshnidae & 10 & 0.76 & 83.6 & 0.11 & 0.10 & 92 & Immature \\
\hline BY144 & $\begin{array}{l}\text { Coon Hollow Cr drain tunnel outlet nr } \\
\text { Dutch Flat }\end{array}$ & $9 / 26 / 00$ & Dragonfly & Odonata & Aeshnidae & 6 & 0.92 & 83.5 & - & 0.085 & - & Immature \\
\hline BY144 & $\begin{array}{l}\text { Coon Hollow Cr drain tunnel outlet nr } \\
\text { Dutch Flat }\end{array}$ & $9 / 26 / 00$ & Dragonfly & Odonata & Aeshnidae & 6 & 1.20 & 85.1 & - & 0.031 & - & Immature \\
\hline BY180 & Greenhorn $\mathrm{Cr} .2 \mathrm{mi}$ bl The Narrows & $10 / 17 / 01$ & Dragonfly & Odonata & Cordulegastridae & 1 & 1.21 & 82.2 & 0.058 & 0.056 & 97 & Immature \\
\hline BY180 & Greenhorn $\mathrm{Cr} .2 \mathrm{mi}$ bl The Narrows & $10 / 17 / 01$ & Dragonfly & Odonata & Aeshnidae & 4 & 1.40 & 84.6 & 0.10 & 0.10 & 108 & Immature \\
\hline BY56 & $\begin{array}{l}\text { Greenhorn Cr bl SF Greenhorn Cr nr } \\
\text { Nevada City }\end{array}$ & $9 / 14 / 01$ & Dragonfly & Odonata & Cordulegastridae & 1 & 1.27 & 86.4 & 0.021 & 0.014 & 65 & Immature \\
\hline BY75 & $\begin{array}{l}\text { Missouri Cyn Cr } 1.2 \text { mi ab Greenhorn } \\
\text { Cr nr Chicago Park }\end{array}$ & $8 / 31 / 99$ & Dragonfly & Odonata & Cordulegastridae & 7 & 2.97 & 81.6 & - & 0.12 & - & Immature \\
\hline BY75 & $\begin{array}{l}\text { Missouri Cyn Cr } 1.2 \text { mi ab Greenhorn } \\
\text { Cr nr Chicago Park }\end{array}$ & $8 / 21 / 00$ & Dragonfly & Odonata & Cordulegastridae & 6 & 3.99 & 85.1 & - & 0.084 & - & Immature \\
\hline BY75 & $\begin{array}{l}\text { Missouri Cyn Cr } 1.2 \text { mi ab Greenhorn } \\
\text { Cr nr Chicago Park }\end{array}$ & $9 / 15 / 01$ & Dragonfly & Odonata & Gomphidae & 14 & 3.29 & 79.0 & 0.12 & 0.10 & 81 & Immature \\
\hline BY75 & $\begin{array}{l}\text { Missouri Cyn Cr } 1.2 \mathrm{mi} \text { ab Greenhorn } \\
\text { Cr nr Chicago Park }\end{array}$ & $9 / 15 / 01$ & Dragonfly & Odonata & Cordulegastridae & 5 & 5.15 & 85.1 & 0.074 & 0.074 & 100 & Immature \\
\hline
\end{tabular}
Cr nr Chicago Park 
Table 11A. Mercury and methylmercury concentration data for invertebrates, Greenhorn Creek drainage, Nevada County, California-Continued.

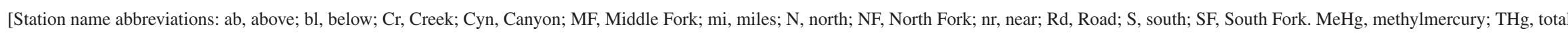
mercury. $\mathrm{n}$, number of organisms; g, gram; $\mu \mathrm{g} / \mathrm{g}$, microgram per gram; - , not determined]

\begin{tabular}{|c|c|c|c|c|c|c|c|c|c|c|c|c|}
\hline $\begin{array}{l}\text { Station } \\
\text { map ID }\end{array}$ & Station name & $\begin{array}{l}\text { Collection } \\
\text { date }\end{array}$ & $\begin{array}{l}\text { Common } \\
\text { name }\end{array}$ & Order & Family & $\begin{array}{c}\text { Sample } \\
\text { size } \\
\text { (n) }\end{array}$ & $\begin{array}{l}\text { Live } \\
\text { weight } \\
\text { (g) }\end{array}$ & $\begin{array}{l}\text { Moisture } \\
\text { (percent) }\end{array}$ & $\begin{array}{c}\text { Total } \\
\text { mercury } \\
\text { ( } \mu \mathrm{g} / \mathrm{g} \text { wet })\end{array}$ & $\begin{array}{c}\text { Methyl- } \\
\text { mercury } \\
\text { ( } \mu \mathrm{g} / \mathrm{g} \text { wet) }\end{array}$ & $\begin{array}{c}\mathrm{MeHg} / \mathrm{THg} \\
\text { (percent) }\end{array}$ & Age \\
\hline BY147 & NF MF Missouri Cyn nr Chicago Park & $9 / 25 / 00$ & Dragonfly & Odonata & Cordulegastridae & 1 & 0.92 & 82.6 & - & 0.36 & - & Immature \\
\hline BY147 & NF MF Missouri Cyn nr Chicago Park & $9 / 15 / 01$ & Dragonfly & Odonata & Gomphidae & 12 & 2.88 & 80.7 & 0.37 & 0.34 & 92 & Immature \\
\hline BY147 & NF MF Missouri Cyn nr Chicago Park & $9 / 15 / 01$ & Dragonfly & Odonata & Aeshnidae & 3 & 1.69 & 82.0 & 0.32 & 0.29 & 93 & Immature \\
\hline BY147 & NF MF Missouri Cyn nr Chicago Park & $9 / 15 / 01$ & Dragonfly & Odonata & Cordulegastridae & 2 & 2.42 & 83.7 & 0.28 & 0.26 & 94 & Immature \\
\hline BY86 & $\begin{array}{l}\text { Poore Mine Creek ab tunnel nr Grass } \\
\text { Valleyley }\end{array}$ & $9 / 26 / 00$ & Dragonfly & Odonata & Gomphidae & 5 & 1.28 & 78.5 & - & 0.049 & - & Immature \\
\hline BY91 & $\begin{array}{l}\text { Poore Mine tunnel effluent nr Grass } \\
\text { Valleyley }\end{array}$ & 8/13/99 & Dragonfly & Odonata & Cordulegastridae & 9 & 1.27 & 81.4 & - & 0.022 & - & Immature \\
\hline BY91 & $\begin{array}{l}\text { Poore Mine tunnel effluent nr Grass } \\
\text { Valleyley }\end{array}$ & $8 / 21 / 00$ & Dragonfly & Odonata & Cordulegastridae & 2 & 1.86 & 82.6 & - & 0.037 & - & Immature \\
\hline BY91 & $\begin{array}{l}\text { Poore Mine tunnel effluent nr Grass } \\
\text { Valley }\end{array}$ & $10 / 17 / 01$ & Dragonfly & Odonata & Cordulegastridae & 5 & 4.63 & 81.4 & 0.036 & 0.027 & 75 & Immature \\
\hline BY115 & $\begin{array}{l}\text { SF Greenhorn Cr bl Boston Mine nr } \\
\text { Nevada City }\end{array}$ & $9 / 13 / 01$ & Dragonfly & Odonata & Cordulegastridae & 1 & 0.92 & 72.8 & 0.35 & 0.33 & 95 & Immature \\
\hline BY124 & $\begin{array}{l}\text { Starr Mine tunnel outlet nr Grass } \\
\text { Valley }\end{array}$ & $8 / 21 / 00$ & Dragonfly & Odonata & Cordulegastridae & 3 & 2.10 & 85.0 & - & 0.042 & - & Immature \\
\hline BY148 & Starr Pit pond nr Grass Valley & $8 / 12 / 99$ & Dragonfly & Odonata & Aeshnidae & 2 & 1.83 & 86.4 & - & 0.011 & - & Immature \\
\hline BY148 & Starr Pit pond nr Grass Valley & $8 / 21 / 00$ & Dragonfly & Odonata & Aeshnidae & 6 & 4.32 & 79.1 & - & 0.015 & - & Immature \\
\hline BY148 & Starr Pit pond nr Grass Valley & $8 / 21 / 00$ & Dragonfly & Odonata & Aeshnidae & 16 & 1.72 & 82.8 & - & 0.015 & - & Immature \\
\hline BY148 & Starr Pit pond nr Grass Valley & $8 / 21 / 00$ & Dragonfly & Odonata & Libellulidae & 5 & 2.40 & 85.5 & - & 0.013 & - & Immature \\
\hline BY130 & $\begin{array}{l}\text { Tom Jerry Mine drain at tunnel outlet } \\
\text { nr Nevada City }\end{array}$ & $8 / 23 / 00$ & Dragonfly & Odonata & Cordulegastridae & 4 & 1.76 & 82.2 & - & 0.18 & - & Immature \\
\hline BY130 & $\begin{array}{l}\text { Tom Jerry Mine drain at tunnel outlet } \\
\text { nr Nevada City }\end{array}$ & 9/13/01 & Dragonfly & Odonata & Cordulegastridae & 3 & 1.84 & 83.8 & 0.29 & 0.31 & 107 & Immature \\
\hline BY130 & $\begin{array}{l}\text { Tom Jerry Mine drain at tunnel outlet } \\
\text { nr Nevada City }\end{array}$ & 9/13/01 & Dragonfly & Odonata & Cordulegastridae & 5 & 0.87 & 84.2 & 0.34 & 0.41 & 120 & Immature \\
\hline BY130 & $\begin{array}{l}\text { Tom Jerry Mine drain at tunnel outlet } \\
\text { nr Nevada City }\end{array}$ & $9 / 13 / 01$ & Dragonfly & Odonata & Cordulegastridae & 3 & 2.33 & 84.3 & 0.033 & 0.036 & 108 & Immature \\
\hline BY131 & $\begin{array}{l}\text { Tom Jerry Mine drainage pond nr } \\
\text { Nevada City }\end{array}$ & $9 / 13 / 01$ & Dragonfly & Odonata & Cordulegastridae & 4 & 2.62 & 83.0 & 0.016 & 0.014 & 90 & Immature \\
\hline BY131 & $\begin{array}{l}\text { Tom Jerry Mine drainage pond nr } \\
\text { Nevada City }\end{array}$ & $9 / 13 / 01$ & Dragonfly & Odonata & Cordulegastridae & 3 & 2.49 & 78.2 & 0.019 & 0.018 & 97 & Immature \\
\hline BY131 & $\begin{array}{l}\text { Tom Jerry Mine drainage pond nr } \\
\text { Nevada City }\end{array}$ & $9 / 13 / 01$ & Dragonfly & Odonata & Aeshnidae & 5 & 1.47 & 82.4 & 0.021 & 0.022 & 106 & Immature \\
\hline BY131 & $\begin{array}{l}\text { Tom Jerry Mine drainage pond nr } \\
\text { Nevada City }\end{array}$ & $9 / 13 / 01$ & Dragonfly & Odonata & Cordulegastridae & 3 & 3.03 & 87.1 & 0.019 & 0.022 & 115 & Immature \\
\hline BY75 & $\begin{array}{l}\text { Missouri Cyn Cr } 1.2 \text { mi ab Greenhorn } \\
\text { Cr nr Chicago Park }\end{array}$ & $8 / 31 / 99$ & $\begin{array}{l}\text { Giant Water } \\
\text { Bug }\end{array}$ & Hemiptera & Belostomatidae & 1 & 3.27 & 66.4 & 0.33 & 0.34 & 102 & Adult \\
\hline BY75 & $\begin{array}{l}\text { Missouri Cyn Cr } 1.2 \text { mi ab Greenhorn } \\
\text { Cr nr Chicago Park }\end{array}$ & $8 / 21 / 00$ & $\begin{array}{l}\text { Giant Water } \\
\text { Bug }\end{array}$ & Hemiptera & Belostomatidae & 1 & 3.95 & 63.9 & - & 0.47 & - & Adult \\
\hline
\end{tabular}


Table 11A. Mercury and methylmercury concentration data for invertebrates, Greenhorn Creek drainage, Nevada County, California-Continued.

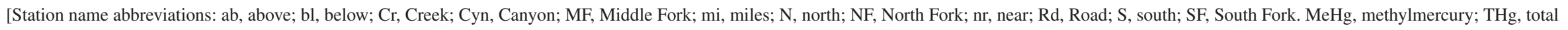
mercury. $\mathrm{n}$, number of organisms; g, gram; $\mu \mathrm{g} / \mathrm{g}$, microgram per gram; - , not determined]

\begin{tabular}{|c|c|c|c|c|c|c|c|c|c|c|c|c|}
\hline $\begin{array}{l}\text { Station } \\
\text { map ID }\end{array}$ & Station name & $\begin{array}{l}\text { Collection } \\
\text { date }\end{array}$ & $\begin{array}{l}\text { Common } \\
\text { name }\end{array}$ & Order & Family & $\begin{array}{c}\text { Sample } \\
\text { size } \\
\text { (n) }\end{array}$ & $\begin{array}{l}\text { Live } \\
\text { weight } \\
\text { (g) }\end{array}$ & $\begin{array}{l}\text { Moisture } \\
\text { (percent) }\end{array}$ & $\begin{array}{c}\text { Total } \\
\text { mercury } \\
\text { ( } \mu \mathrm{g} / \mathrm{g} \text { wet })\end{array}$ & $\begin{array}{l}\text { Methyl- } \\
\text { mercury } \\
\text { ( } \mathrm{gg} / \mathrm{g} \text { wet) }\end{array}$ & $\begin{array}{l}\text { MeHg/THg } \\
\text { (percent) }\end{array}$ & Age \\
\hline BY20 & $\begin{array}{l}\text { Boston Mine tunnel outlet nr Grass } \\
\text { Valley }\end{array}$ & 8/12/99 & $\begin{array}{l}\text { Predaceous } \\
\text { diving } \\
\text { beetle }\end{array}$ & Coleoptera & Dytiscidae & 10 & 1.01 & 49.9 & - & 0.31 & - & Adult \\
\hline BY20 & $\begin{array}{l}\text { Boston Mine tunnel outlet nr Grass } \\
\text { Valley }\end{array}$ & $8 / 12 / 99$ & $\begin{array}{l}\text { Predaceous } \\
\text { diving } \\
\text { beetle }\end{array}$ & Coleoptera & Dytiscidae & 10 & 1.02 & 48.9 & - & 0.22 & - & Adult \\
\hline BY20 & $\begin{array}{l}\text { Boston Mine tunnel outlet nr Grass } \\
\text { Valley }\end{array}$ & $8 / 21 / 00$ & $\begin{array}{l}\text { Predaceous } \\
\text { diving } \\
\text { beetle }\end{array}$ & Coleoptera & Dytiscidae & 22 & 2.14 & 57.3 & - & 0.50 & - & Adult \\
\hline BY20 & $\begin{array}{l}\text { Boston Mine tunnel outlet nr Grass } \\
\text { Valley }\end{array}$ & 9/13/01 & $\begin{array}{l}\text { Predaceous } \\
\text { diving } \\
\text { beetle }\end{array}$ & Coleoptera & Dytiscidae & 7 & 0.49 & 53.6 & 0.61 & 0.30 & 49 & Adult \\
\hline BY21 & $\begin{array}{l}\text { Boston Mine wetlands pond nr Grass } \\
\text { Valley }\end{array}$ & $8 / 12 / 99$ & $\begin{array}{l}\text { Predaceous } \\
\text { diving } \\
\text { beetle }\end{array}$ & Coleoptera & Dytiscidae & 5 & 1.10 & 58.8 & - & 0.31 & - & Adult \\
\hline BY21 & $\begin{array}{l}\text { Boston Mine wetlands pond nr Grass } \\
\text { Valley }\end{array}$ & 9/13/01 & $\begin{array}{l}\text { Predaceous } \\
\text { diving } \\
\text { beetle }\end{array}$ & Coleoptera & Dytiscidae & 20 & 1.30 & 61.5 & 0.049 & 0.031 & 63 & Adult \\
\hline BY22 & $\begin{array}{l}\text { Buckeye Flat Mine drain } 0.1 \mathrm{mi} \text { ab } \\
\text { Greenhorn } \mathrm{Cr}\end{array}$ & 9/29/99 & $\begin{array}{l}\text { Predaceous } \\
\text { diving } \\
\text { beetle }\end{array}$ & Coleoptera & Dytiscidae & 20 & 1.77 & 55.1 & 0.036 & 0.030 & 82 & Adult \\
\hline BY58 & $\begin{array}{l}\text { Buckeye Flat Mine main drain } 0.45 \\
\text { mi ab Greenhorn } \mathrm{Cr}\end{array}$ & 8/22/00 & $\begin{array}{l}\text { Predaceous } \\
\text { diving } \\
\text { beetle }\end{array}$ & Coleoptera & Dytiscidae & 40 & 1.47 & 48.0 & - & 0.11 & - & Adult \\
\hline BY58 & $\begin{array}{l}\text { Buckeye Flat Mine main drain } 0.45 \\
\text { mi ab Greenhorn } \mathrm{Cr}\end{array}$ & 8/22/00 & $\begin{array}{l}\text { Predaceous } \\
\text { diving } \\
\text { beetle }\end{array}$ & Coleoptera & Dytiscidae & 12 & 0.79 & 47.2 & - & 0.060 & - & Adult \\
\hline BY58 & $\begin{array}{l}\text { Buckeye Flat Mine main drain } 0.45 \\
\text { mi ab Greenhorn } \mathrm{Cr}\end{array}$ & $9 / 13 / 01$ & $\begin{array}{l}\text { Predaceous } \\
\text { diving } \\
\text { beetle }\end{array}$ & Coleoptera & Dytiscidae & 15 & 0.96 & 51.6 & 0.080 & 0.029 & 36 & Adult \\
\hline BY58 & $\begin{array}{l}\text { Buckeye Flat Mine main drain } 0.45 \\
\text { mi ab Greenhorn } \mathrm{Cr}\end{array}$ & 9/13/01 & $\begin{array}{l}\text { Predaceous } \\
\text { diving } \\
\text { beetle }\end{array}$ & Coleoptera & Dytiscidae & 30 & 1.14 & 52.5 & 0.082 & 0.038 & 47 & Adult \\
\hline BY116 & $\begin{array}{l}\text { Buckeye Flat Mine pond drain } 0.15 \\
\text { mi ab SF Greenhorn Cr }\end{array}$ & $8 / 22 / 00$ & $\begin{array}{l}\text { Predaceous } \\
\text { diving } \\
\text { beetle }\end{array}$ & Coleoptera & Dytiscidae & 26 & 1.75 & 55.2 & - & 0.12 & - & Adult \\
\hline BY116 & $\begin{array}{l}\text { Buckeye Flat Mine pond drain } 0.15 \\
\text { mi ab SF Greenhorn Cr }\end{array}$ & 9/12/01 & $\begin{array}{l}\text { Predaceous } \\
\text { diving } \\
\text { beetle }\end{array}$ & Coleoptera & Dytiscidae & 9 & 0.70 & 53.3 & 0.089 & 0.050 & 57 & Adult \\
\hline BY24 & $\begin{array}{l}\text { Buckeye Flat Mine S drain to SF } \\
\text { Greenhorn Cr Grass Valley }\end{array}$ & $8 / 22 / 00$ & $\begin{array}{l}\text { Predaceous } \\
\text { diving } \\
\text { beetle }\end{array}$ & Coleoptera & Dytiscidae & 40 & 1.52 & 50.8 & 0.18 & 0.17 & 94 & Adult \\
\hline
\end{tabular}


Table 11A. Mercury and methylmercury concentration data for invertebrates, Greenhorn Creek drainage, Nevada County, California-Continued.

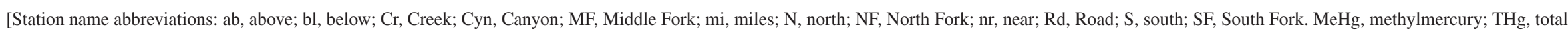
mercury. $\mathrm{n}$, number of organisms; g, gram; $\mu \mathrm{g} / \mathrm{g}$, microgram per gram; - , not determined]

\begin{tabular}{|c|c|c|c|c|c|c|c|c|c|c|c|c|}
\hline $\begin{array}{l}\text { Station } \\
\text { map ID }\end{array}$ & Station name & $\begin{array}{l}\text { Collection } \\
\text { date }\end{array}$ & $\begin{array}{l}\text { Common } \\
\text { name }\end{array}$ & Order & Family & $\begin{array}{c}\text { Sample } \\
\text { size } \\
\text { (n) }\end{array}$ & $\begin{array}{c}\text { Live } \\
\text { weight } \\
\text { (g) }\end{array}$ & $\begin{array}{l}\text { Moisture } \\
\text { (percent) }\end{array}$ & $\begin{array}{c}\text { Total } \\
\text { mercury } \\
(\mu \mathrm{g} / \mathrm{g} \text { wet })\end{array}$ & $\begin{array}{c}\text { Methyl- } \\
\text { mercury } \\
\text { ( } \mathrm{g} / \mathrm{g} \text { wet) }\end{array}$ & $\begin{array}{l}\mathrm{MeHg} / \mathrm{THg} \\
\text { (percent) }\end{array}$ & Age \\
\hline BY24 & $\begin{array}{l}\text { Buckeye Flat Mine S drain to SF } \\
\text { Greenhorn Cr Grass Valley }\end{array}$ & $9 / 12 / 01$ & $\begin{array}{l}\text { Predaceous } \\
\text { diving } \\
\text { beetle }\end{array}$ & Coleoptera & Dytiscidae & 11 & 0.76 & 50.6 & 0.11 & 0.078 & 68 & Adult \\
\hline BY24 & $\begin{array}{l}\text { Buckeye Flat Mine S drain to SF } \\
\text { Greenhorn Cr Grass Valley }\end{array}$ & $9 / 12 / 01$ & $\begin{array}{l}\text { Predaceous } \\
\text { diving } \\
\text { beetle }\end{array}$ & Coleoptera & Dytiscidae & 29 & 0.96 & 50.1 & 0.15 & 0.12 & 78 & Adult \\
\hline BY25 & Buckeye Flat Mine upper drain & 10/19/01 & $\begin{array}{l}\text { Predaceous } \\
\text { diving } \\
\text { beetle }\end{array}$ & Coleoptera & Dytiscidae & 10 & 0.75 & 55.0 & 0.13 & 0.078 & 61 & Adult \\
\hline BY144 & $\begin{array}{l}\text { Coon Hollow Cr drain tunnel outlet nr } \\
\text { Dutch Flat }\end{array}$ & $9 / 26 / 00$ & $\begin{array}{l}\text { Predaceous } \\
\text { diving } \\
\text { beetle }\end{array}$ & Coleoptera & Dytiscidae & 23 & 1.54 & 53.6 & - & 0.11 & - & Adult \\
\hline BY146 & $\begin{array}{l}\text { Missouri Cyn } 1.6 \mathrm{mi} \text { ab Greenhorn Cr } \\
\text { nr Chicago Park }\end{array}$ & $9 / 25 / 00$ & $\begin{array}{l}\text { Predaceous } \\
\text { diving } \\
\text { beetle }\end{array}$ & Coleoptera & Dytiscidae & 20 & 1.34 & 58.4 & - & 0.11 & - & Adult \\
\hline BY145 & $\begin{array}{l}\text { Missouri Cyn Cr Tributary nr Chicago } \\
\text { Park }\end{array}$ & $9 / 25 / 00$ & $\begin{array}{l}\text { Predaceous } \\
\text { diving } \\
\text { beetle }\end{array}$ & Coleoptera & Dytiscidae & 20 & 1.45 & 55.0 & - & 0.10 & - & Adult \\
\hline BY147 & NF MF Missouri Cyn nr Chicago Park & $9 / 25 / 00$ & $\begin{array}{l}\text { Predaceous } \\
\text { diving } \\
\text { beetle }\end{array}$ & Coleoptera & Dytiscidae & 20 & 1.32 & 55.2 & - & 0.21 & - & Adult \\
\hline BY147 & NF MF Missouri Cyn nr Chicago Park & $9 / 25 / 00$ & $\begin{array}{l}\text { Predaceous } \\
\text { diving } \\
\text { beetle }\end{array}$ & Coleoptera & Dytiscidae & 20 & 1.31 & 55.0 & - & 0.14 & - & Adult \\
\hline BY147 & NF MF Missouri Cyn nr Chicago Park & $9 / 15 / 01$ & $\begin{array}{l}\text { Predaceous } \\
\text { diving } \\
\text { beetle }\end{array}$ & Coleoptera & Dytiscidae & 25 & 1.80 & 56.0 & 0.23 & 0.17 & 73 & Adult \\
\hline BY90 & $\begin{array}{l}\text { Poore Mine Seep above ground sluice } \\
\text { nr Grass Valley }\end{array}$ & $9 / 26 / 00$ & $\begin{array}{l}\text { Predaceous } \\
\text { diving } \\
\text { beetle }\end{array}$ & Coleoptera & Dytiscidae & 20 & 1.45 & 54.5 & - & 0.034 & - & Adult \\
\hline BY105 & $\begin{array}{l}\text { Sailor Flat Mine main drain Gulch } 01 \\
\text { nr Quaker Hill }\end{array}$ & $8 / 24 / 99$ & $\begin{array}{l}\text { Predaceous } \\
\text { diving } \\
\text { beetle }\end{array}$ & Coleoptera & Dytiscidae & 25 & 1.76 & 51.7 & 0.039 & 0.032 & 83 & Adult \\
\hline BY148 & Starr Pit pond nr Grass Valley & $8 / 21 / 00$ & $\begin{array}{l}\text { Predaceous } \\
\text { diving } \\
\text { beetle }\end{array}$ & Coleoptera & Dytiscidae & 11 & 2.29 & 62.6 & - & 0.23 & - & Adult \\
\hline BY130 & $\begin{array}{l}\text { Tom Jerry Mine drain at tunnel outlet } \\
\text { nr Nevada City }\end{array}$ & $8 / 23 / 00$ & $\begin{array}{l}\text { Predaceous } \\
\text { diving } \\
\text { beetle }\end{array}$ & Coleoptera & Dytiscidae & 20 & 1.88 & 53.5 & - & 0.18 & - & Adult \\
\hline BY130 & $\begin{array}{l}\text { Tom Jerry Mine drain at tunnel outlet } \\
\text { nr Nevada City }\end{array}$ & $8 / 23 / 00$ & $\begin{array}{l}\text { Predaceous } \\
\text { diving } \\
\text { beetle }\end{array}$ & Coleoptera & Dytiscidae & 9 & 0.63 & 55.5 & - & 0.041 & - & Adult \\
\hline
\end{tabular}


Table 11A. Mercury and methylmercury concentration data for invertebrates, Greenhorn Creek drainage, Nevada County, California-Continued.

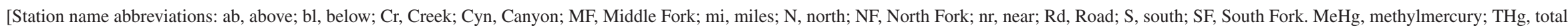
mercury. $\mathrm{n}$, number of organisms; g, gram; $\mu \mathrm{g} / \mathrm{g}$, microgram per gram; - , not determined]

\begin{tabular}{|c|c|c|c|c|c|c|c|c|c|c|c|c|}
\hline $\begin{array}{l}\text { Station } \\
\text { map ID }\end{array}$ & Station name & $\begin{array}{l}\text { Collection } \\
\text { date }\end{array}$ & $\begin{array}{l}\text { Common } \\
\text { name }\end{array}$ & Order & Family & $\begin{array}{c}\text { Sample } \\
\text { size } \\
\text { (n) }\end{array}$ & $\begin{array}{c}\text { Live } \\
\text { weight } \\
\text { (g) }\end{array}$ & $\begin{array}{l}\text { Moisture } \\
\text { (percent) }\end{array}$ & $\begin{array}{c}\text { Total } \\
\text { mercury } \\
\text { ( } \mu \mathrm{g} / \mathrm{g} \text { wet })\end{array}$ & $\begin{array}{l}\text { Methyl- } \\
\text { mercury } \\
\text { ( } \mu \mathrm{g} / \mathrm{g} \text { wet) }\end{array}$ & $\begin{array}{l}\mathrm{MeHg} / \mathrm{THg} \\
\text { (percent) }\end{array}$ & Age \\
\hline BY130 & $\begin{array}{l}\text { Tom Jerry Mine drain at tunnel outlet } \\
\text { nr Nevada City }\end{array}$ & $9 / 13 / 01$ & $\begin{array}{l}\text { Predaceous } \\
\text { diving } \\
\text { beetle }\end{array}$ & Coleoptera & Dytiscidae & 13 & 1.16 & 57.6 & 0.18 & 0.11 & 61 & Adult \\
\hline BY130 & $\begin{array}{l}\text { Tom Jerry Mine drain at tunnel outlet } \\
\text { nr Nevada City }\end{array}$ & 9/13/01 & $\begin{array}{l}\text { Predaceous } \\
\text { diving } \\
\text { beetle }\end{array}$ & Coleoptera & Dytiscidae & 15 & 1.01 & 49.9 & 0.12 & 0.083 & 69 & Adult \\
\hline BY131 & $\begin{array}{l}\text { Tom Jerry Mine drainage pond nr } \\
\text { Nevada City }\end{array}$ & $9 / 13 / 01$ & $\begin{array}{l}\text { Predaceous } \\
\text { diving } \\
\text { beetle }\end{array}$ & Coleoptera & Dytiscidae & 30 & 1.07 & 50.9 & 0.070 & 0.055 & 78 & Adult \\
\hline BY57 & $\begin{array}{l}\text { Greenhorn Cr } 0.3 \text { mi bl Sailor Flat } \\
\text { West drain nr Nevada City }\end{array}$ & 9/29/99 & $\begin{array}{l}\text { Predaceous } \\
\text { stonefly }\end{array}$ & Plecoptera & Perlidae & 10 & 2.01 & 74.3 & - & 0.026 & - & Immature \\
\hline BY57 & $\begin{array}{l}\text { Greenhorn Cr } 0.3 \text { mi bl Sailor Flat } \\
\text { West drain nr Nevada City }\end{array}$ & 9/29/99 & $\begin{array}{l}\text { Predaceous } \\
\text { stonefly }\end{array}$ & Plecoptera & Perlidae & 10 & 2.36 & 72.4 & - & 0.045 & - & Immature \\
\hline BY53 & $\begin{array}{l}\text { Greenhorn } \mathrm{Cr} \text { ab SF Greenhorn } \mathrm{Cr} \mathrm{nr} \\
\text { Nevada City }\end{array}$ & $9 / 14 / 01$ & $\begin{array}{l}\text { Predaceous } \\
\text { stonefly }\end{array}$ & Plecoptera & Perlidae & 25 & 0.95 & 79.8 & 0.13 & 0.084 & 65 & Immature \\
\hline BY56 & $\begin{array}{l}\text { Greenhorn Cr bl SF Greenhorn Cr nr } \\
\text { Nevada City }\end{array}$ & $9 / 14 / 01$ & $\begin{array}{l}\text { Predaceous } \\
\text { stonefly }\end{array}$ & Plecoptera & Perlidae & 11 & 0.90 & 78.0 & 0.15 & 0.094 & 64 & Immature \\
\hline BY59 & $\begin{array}{l}\text { Greenhorn Crk at You Bet Rd nr } \\
\text { Nevada City }\end{array}$ & $8 / 31 / 99$ & $\begin{array}{l}\text { Predaceous } \\
\text { stonefly }\end{array}$ & Plecoptera & Perlidae & 13 & 1.21 & 73.0 & - & 0.047 & - & Immature \\
\hline BY75 & $\begin{array}{l}\text { Missouri Cyn Cr } 1.2 \text { mi ab Greenhorn } \\
\text { Cr nr Chicago Park }\end{array}$ & $8 / 21 / 00$ & $\begin{array}{l}\text { Predaceous } \\
\text { stonefly }\end{array}$ & Plecoptera & Perlidae & 10 & 0.44 & 75.1 & - & 0.14 & - & Immature \\
\hline BY75 & $\begin{array}{l}\text { Missouri Cyn Cr } 1.2 \mathrm{mi} \text { ab Greenhorn } \\
\text { Cr nr Chicago Park }\end{array}$ & $9 / 15 / 01$ & $\begin{array}{l}\text { Predaceous } \\
\text { stonefly }\end{array}$ & Plecoptera & Perlidae & 16 & 0.39 & 72.7 & 0.23 & 0.19 & 84 & Immature \\
\hline BY91 & $\begin{array}{l}\text { Poore Mine tunnel effluent nr Grass } \\
\text { Valley }\end{array}$ & 8/13/99 & $\begin{array}{l}\text { Predaceous } \\
\text { stonefly }\end{array}$ & Plecoptera & Perlidae & 7 & 1.08 & 71.4 & - & 0.044 & - & Immature \\
\hline BY91 & $\begin{array}{l}\text { Poore Mine tunnel effluent nr Grass } \\
\text { Valley }\end{array}$ & $10 / 17 / 01$ & $\begin{array}{l}\text { Predaceous } \\
\text { stonefly }\end{array}$ & Plecoptera & Perlidae & 6 & 0.52 & 70.1 & 0.043 & 0.036 & 84 & Immature \\
\hline BY114 & $\begin{array}{l}\text { SF Greenhorn Cr } 0.7 \text { mi ab Greenhorn } \\
\text { Cr nr Nevada City }\end{array}$ & $8 / 22 / 00$ & $\begin{array}{l}\text { Predaceous } \\
\text { stonefly }\end{array}$ & Plecoptera & Perlidae & 10 & 2.52 & 71.7 & - & 0.14 & - & Immature \\
\hline BY114 & $\begin{array}{l}\text { SF Greenhorn Cr } 0.7 \text { mi ab Greenhorn } \\
\text { Cr nr Nevada City }\end{array}$ & 9/13/01 & $\begin{array}{l}\text { Predaceous } \\
\text { stonefly }\end{array}$ & Plecoptera & Perlidae & 6 & 1.31 & 70.4 & 0.23 & 0.17 & 73 & Immature \\
\hline BY114 & $\begin{array}{l}\text { SF Greenhorn Cr } 0.7 \text { mi ab Greenhorn } \\
\text { Cr nr Nevada City }\end{array}$ & 9/13/01 & $\begin{array}{l}\text { Predaceous } \\
\text { stonefly }\end{array}$ & Plecoptera & Perlidae & 12 & 1.05 & 75.0 & 0.15 & 0.12 & 81 & Immature \\
\hline BY113 & $\begin{array}{l}\text { SF Greenhorn Cr } 0.8 \text { mi ab Greenhorn } \\
\text { Cr nr Nevada City }\end{array}$ & $8 / 22 / 00$ & $\begin{array}{l}\text { Predaceous } \\
\text { stonefly }\end{array}$ & Plecoptera & Perlidae & 10 & 2.28 & 75.2 & - & 0.040 & - & Immature \\
\hline BY113 & $\begin{array}{l}\text { SF Greenhorn Cr } 0.8 \text { mi ab Greenhorn } \\
\text { Cr nr Nevada City }\end{array}$ & 9/13/01 & $\begin{array}{l}\text { Predaceous } \\
\text { stonefly }\end{array}$ & Plecoptera & Perlidae & 12 & 1.43 & 77.0 & 0.11 & 0.059 & 55 & Immature \\
\hline BY113 & $\begin{array}{l}\text { SF Greenhorn Cr } 0.8 \text { mi ab Greenhorn } \\
\text { Cr nr Nevada City }\end{array}$ & 9/13/01 & $\begin{array}{l}\text { Predaceous } \\
\text { stonefly }\end{array}$ & Plecoptera & Perlidae & 5 & 1.07 & 76.5 & 0.11 & 0.081 & 76 & Immature \\
\hline BY115 & $\begin{array}{l}\text { SF Greenhorn Cr bl Boston Mine nr } \\
\text { Nevada City }\end{array}$ & 9/28/99 & $\begin{array}{l}\text { Predaceous } \\
\text { stonefly }\end{array}$ & Plecoptera & Perlidae & 14 & 1.61 & 73.1 & - & 0.20 & - & Immature \\
\hline BY115 & $\begin{array}{l}\text { SF Greenhorn Cr bl Boston Mine nr } \\
\text { Nevada City }\end{array}$ & $8 / 21 / 00$ & $\begin{array}{l}\text { Predaceous } \\
\text { stonefly }\end{array}$ & Plecoptera & Perlidae & 11 & 1.27 & 72.5 & 0.40 & 0.31 & 79 & Immature \\
\hline
\end{tabular}


Table 11A. Mercury and methylmercury concentration data for invertebrates, Greenhorn Creek drainage, Nevada County, California-Continued.

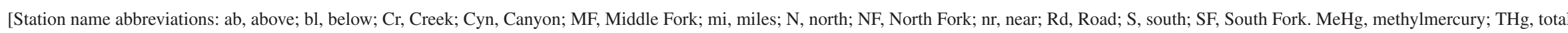
mercury. $\mathrm{n}$, number of organisms; g, gram; $\mu \mathrm{g} / \mathrm{g}$, microgram per gram; - , not determined]

\begin{tabular}{|c|c|c|c|c|c|c|c|c|c|c|c|}
\hline $\begin{array}{l}\text { Station } \\
\text { map ID }\end{array}$ & Station name & $\begin{array}{l}\text { Collection } \\
\text { date }\end{array}$ & $\begin{array}{c}\text { Common } \\
\text { name }\end{array}$ & Family & $\begin{array}{c}\text { Sample } \\
\text { size } \\
\text { (n) }\end{array}$ & $\begin{array}{c}\text { Live } \\
\text { weight } \\
\text { (g) }\end{array}$ & $\begin{array}{l}\text { Moisture } \\
\text { (percent) }\end{array}$ & $\begin{array}{c}\text { Total } \\
\text { mercury } \\
\text { ( } \mu \mathrm{g} / \mathrm{g} \text { wet) }\end{array}$ & $\begin{array}{c}\text { Methyl- } \\
\text { mercury } \\
\text { ( } \mu \mathrm{g} / \mathrm{g} \text { wet) }\end{array}$ & $\begin{array}{c}\mathrm{MeHg} / \mathrm{THg} \\
\text { (percent) }\end{array}$ & Age \\
\hline$\overline{B Y 115}$ & $\begin{array}{l}\text { SF Greenhorn Cr bl Boston Mine nr } \\
\text { Nevada City }\end{array}$ & $9 / 13 / 01$ & $\begin{array}{l}\text { Predaceous Plecoptera } \\
\text { stonefly }\end{array}$ & Perlidae & 10 & 0.53 & 78.6 & 0.47 & 0.52 & 109 & Immature \\
\hline BY20 & $\begin{array}{l}\text { Boston Mine tunnel outlet nr Grass } \\
\text { Valley }\end{array}$ & $8 / 12 / 99$ & Water strider Hemiptera & Gerridae & 26 & 1.36 & 61.3 & - & 0.26 & - & Adult \\
\hline BY20 & $\begin{array}{l}\text { Boston Mine tunnel outlet nr Grass } \\
\quad \text { Valley }\end{array}$ & $8 / 21 / 00$ & Water strider Hemiptera & Gerridae & 14 & 0.81 & 58.3 & - & 0.22 & - & Adult \\
\hline BY20 & $\begin{array}{l}\text { Boston Mine tunnel outlet nr Grass } \\
\text { Valley }\end{array}$ & $9 / 13 / 01$ & Water strider Hemiptera & Gerridae & 13 & 0.73 & 56.9 & 0.35 & 0.24 & 68 & Adult \\
\hline BY21 & $\begin{array}{l}\text { Boston Mine wetlands pond nr Grass } \\
\text { Valley }\end{array}$ & $8 / 12 / 99$ & Water strider Hemiptera & Gerridae & 50 & 1.13 & 62.3 & - & 0.061 & - & Adult \\
\hline BY21 & $\begin{array}{l}\text { Boston Mine wetlands pond nr Grass } \\
\text { Valley }\end{array}$ & $8 / 21 / 00$ & Water strider Hemiptera & Gerridae & 24 & 0.58 & 58.7 & - & 0.13 & - & Adult \\
\hline BY22 & $\begin{array}{l}\text { Buckeye Flat Mine drain } 0.1 \mathrm{mi} \mathrm{ab} \\
\text { Greenhorn } \mathrm{Cr}\end{array}$ & 9/29/99 & Water strider Hemiptera & Gerridae & 20 & 1.39 & 55.9 & - & 0.090 & - & Adult \\
\hline BY58 & $\begin{array}{l}\text { Buckeye Flat Mine main drain } 0.45 \\
\text { mi ab Greenhorn } \mathrm{Cr}\end{array}$ & $8 / 22 / 00$ & Water strider Hemiptera & Gerridae & 34 & 1.78 & 65.3 & - & 0.068 & - & Adult \\
\hline BY58 & $\begin{array}{l}\text { Buckeye Flat Mine main drain } 0.45 \\
\text { mi ab Greenhorn } \mathrm{Cr}\end{array}$ & $9 / 13 / 01$ & Water strider Hemiptera & Gerridae & 25 & 1.64 & 60.5 & 0.12 & 0.13 & 106 & Adult \\
\hline BY23 & $\begin{array}{l}\text { Buckeye Flat Mine N drain to } \\
\text { Greenhorn Cr Grass Valley }\end{array}$ & $8 / 22 / 00$ & Water strider Hemiptera & Gerridae & 25 & 1.61 & 68.6 & - & 0.093 & - & Adult \\
\hline BY23 & $\begin{array}{l}\text { Buckeye Flat Mine N drain to } \\
\text { Greenhorn Cr Grass Valley }\end{array}$ & $8 / 22 / 00$ & Water strider Hemiptera & Gerridae & 25 & 1.62 & 66.0 & - & 0.087 & 一 & Adult \\
\hline BY116 & $\begin{array}{l}\text { Buckeye Flat Mine pond drain } 0.15 \\
\text { mi ab SF Greenhorn } \mathrm{Cr}\end{array}$ & $8 / 22 / 00$ & Water strider Hemiptera & Gerridae & 32 & 2.02 & 62.8 & 0.14 & 0.16 & 111 & Adult \\
\hline BY116 & $\begin{array}{l}\text { Buckeye Flat Mine pond drain } 0.15 \\
\text { mi ab SF Greenhorn } \mathrm{Cr}\end{array}$ & $9 / 12 / 01$ & Water strider Hemiptera & Gerridae & 25 & 1.79 & 58.9 & 0.18 & 0.11 & 59 & Adult \\
\hline BY116 & $\begin{array}{l}\text { Buckeye Flat Mine pond drain } 0.15 \\
\text { mi ab SF Greenhorn } \mathrm{Cr}\end{array}$ & $9 / 12 / 01$ & Water strider Hemiptera & Gerridae & 25 & 1.77 & 61.8 & 0.15 & 0.12 & 80 & Adult \\
\hline BY24 & $\begin{array}{l}\text { Buckeye Flat Mine S drain to SF } \\
\text { Greenhorn Cr Grass Valley }\end{array}$ & $8 / 26 / 99$ & Water strider Hemiptera & Gerridae & 32 & 1.93 & 59.7 & 0.52 & 0.55 & 107 & Adult \\
\hline BY24 & $\begin{array}{l}\text { Buckeye Flat Mine S drain to SF } \\
\text { Greenhorn C Grass Valley }\end{array}$ & $8 / 22 / 00$ & Water strider Hemiptera & Gerridae & 25 & 1.65 & 60.6 & - & 0.16 & - & Adult \\
\hline BY24 & $\begin{array}{l}\text { Buckeye Flat Mine S drain to SF } \\
\text { Greenhorn Cr Grass Valley }\end{array}$ & $9 / 12 / 01$ & Water strider Hemiptera & Gerridae & 25 & 1.56 & 60.2 & 0.19 & 0.12 & 64 & Adult \\
\hline BY24 & $\begin{array}{l}\text { Buckeye Flat Mine S drain to SF } \\
\text { Greenhorn Cr Grass Valley }\end{array}$ & $9 / 12 / 01$ & Water strider Hemiptera & Gerridae & 25 & 1.53 & 63.1 & 0.15 & 0.11 & 73 & Adult \\
\hline BY25 & Buckeye Flat Mine upper drain & $10 / 19 / 01$ & Water strider Hemiptera & Gerridae & 25 & 1.62 & 58.1 & 0.13 & 0.081 & 61 & Adult \\
\hline BY144 & $\begin{array}{l}\text { Coon Hollow Cr drain tunnel outlet nr } \\
\text { Dutch Flat }\end{array}$ & $9 / 26 / 00$ & Water strider Hemiptera & Gerridae & 30 & 1.84 & 57.2 & - & 0.15 & - & Adult \\
\hline BY144 & $\begin{array}{l}\text { Coon Hollow Cr drain tunnel outlet nr } \\
\text { Dutch Flat }\end{array}$ & $9 / 26 / 00$ & Water strider Hemiptera & Gerridae & 30 & 1.80 & 58.6 & - & 0.14 & 一 & Adult \\
\hline
\end{tabular}


Table 11A. Mercury and methylmercury concentration data for invertebrates, Greenhorn Creek drainage, Nevada County, California-Continued.

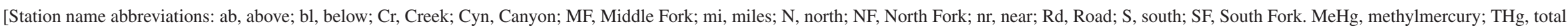
mercury. $\mathrm{n}$, number of organisms; g, gram; $\mu \mathrm{g} / \mathrm{g}$, microgram per gram; - , not determined]

\begin{tabular}{|c|c|c|c|c|c|c|c|c|c|c|c|}
\hline $\begin{array}{l}\text { Station } \\
\text { map ID }\end{array}$ & Station name & $\begin{array}{l}\text { Collection } \\
\text { date }\end{array}$ & $\begin{array}{l}\text { Common } \\
\text { name }\end{array}$ & Family & $\begin{array}{c}\text { Sample } \\
\text { size } \\
\text { (n) }\end{array}$ & $\begin{array}{c}\text { Live } \\
\text { weight } \\
\text { (g) }\end{array}$ & $\begin{array}{l}\text { Moisture } \\
\text { (percent) }\end{array}$ & $\begin{array}{c}\text { Total } \\
\text { mercury } \\
\text { ( } \mu \mathrm{g} / \mathrm{g} \text { wet) }\end{array}$ & $\begin{array}{c}\text { Methyl- } \\
\text { mercury } \\
\text { ( } \mu \mathrm{g} / \mathrm{g} \text { wet) }\end{array}$ & $\begin{array}{l}\mathrm{MeHg} / \mathrm{THg} \\
\text { (percent) }\end{array}$ & Age \\
\hline BY55 & $\begin{array}{l}\text { Greenhorn Cr } 0.1 \text { mi bl Sailor Flat } \\
\text { West drain nr Nevada City }\end{array}$ & $9 / 29 / 99$ & Water strider Hemiptera & Gerridae & 20 & 1.08 & 56.0 & - & 0.076 & - & Adult \\
\hline BY180 & Greenhorn Cr $0.2 \mathrm{mi}$ bl The Narrows & $10 / 17 / 01$ & Water strider Hemiptera & Gerridae & 25 & 1.39 & 59.0 & 0.10 & 0.091 & 90 & Adult \\
\hline BY57 & $\begin{array}{l}\text { Greenhorn Cr } 0.3 \text { mi bl Sailor Flat } \\
\text { West drain nr Nevada City }\end{array}$ & 9/29/99 & Water strider Hemiptera & Gerridae & 24 & 1.31 & 59.0 & - & 0.080 & - & Adult \\
\hline BY59 & $\begin{array}{l}\text { Greenhorn Cr at You Bet Rd nr } \\
\text { Nevada City }\end{array}$ & $8 / 31 / 99$ & Water strider Hemiptera & Gerridae & 23 & 1.54 & 53.2 & - & 0.11 & - & Adult \\
\hline BY60 & $\begin{array}{l}\text { Greenhorn Creek bl Buckeye drain nr } \\
\text { Nevada City }\end{array}$ & 9/29/99 & Water strider Hemiptera & Gerridae & 25 & 1.53 & 61.2 & - & 0.089 & - & Adult \\
\hline BY146 & $\begin{array}{l}\text { Missouri Cyn } 1.6 \mathrm{mi} \text { ab Greenhorn } \mathrm{Cr} \\
\text { nr Chicago Park }\end{array}$ & $9 / 25 / 00$ & Water strider Hemiptera & Gerridae & 30 & 1.85 & 56.6 & - & 0.20 & - & Adult \\
\hline BY75 & $\begin{array}{l}\text { Missouri Cyn Cr } 1.2 \text { mi ab Greenhorn } \\
\text { Cr nr Chicago Park }\end{array}$ & $8 / 31 / 99$ & Water strider Hemiptera & Gerridae & 21 & 1.13 & 61.8 & - & 0.10 & - & Adult \\
\hline BY75 & $\begin{array}{l}\text { Missouri Cyn Cr } 1.2 \text { mi ab Greenhorn } \\
\text { Cr nr Chicago Park }\end{array}$ & $8 / 21 / 00$ & Water strider Hemiptera & Gerridae & 22 & 1.25 & 65.8 & - & 0.30 & - & Adult \\
\hline BY75 & $\begin{array}{l}\text { Missouri Cyn Cr } 1.2 \text { mi ab Greenhorn } \\
\text { Cr nr Chicago Park }\end{array}$ & $8 / 21 / 00$ & Water strider Hemiptera & Gerridae & 25 & 1.47 & 64.5 & - & 0.12 & - & Adult \\
\hline BY75 & $\begin{array}{l}\text { Missouri Cyn Cr } 1.2 \text { mi ab Greenhorn } \\
\text { Cr nr Chicago Park }\end{array}$ & $9 / 15 / 01$ & Water strider Hemiptera & Gerridae & 25 & 1.50 & 62.4 & 0.10 & 0.069 & 72 & Adult \\
\hline BY145 & $\begin{array}{l}\text { Missouri Cyn Cr Tributary nr Chicago } \\
\text { Park }\end{array}$ & $9 / 25 / 00$ & Water strider Hemiptera & Gerridae & 30 & 1.86 & 57.1 & - & 0.34 & - & Adult \\
\hline BY147 & NF MF Missouri Cyn nr Chicago Park & $9 / 25 / 00$ & Water strider Hemiptera & Gerridae & 30 & 1.78 & 55.9 & - & 0.27 & - & Adult \\
\hline BY147 & NF MF Missouri Cyn nr Chicago Park & $9 / 15 / 01$ & Water strider Hemiptera & Gerridae & 25 & 1.65 & 60.6 & 0.30 & 0.17 & 58 & Adult \\
\hline BY86 & $\begin{array}{l}\text { Poore Mine Creek ab tunnel nr Grass } \\
\text { Valley }\end{array}$ & $9 / 26 / 00$ & Water strider Hemiptera & Gerridae & 25 & 1.68 & 53.8 & - & 0.11 & - & Adult \\
\hline BY86 & $\begin{array}{l}\text { Poore Mine Creek ab tunnel nr Grass } \\
\text { Valley }\end{array}$ & $9 / 26 / 00$ & Water strider Hemiptera & Gerridae & 25 & 1.52 & 53.8 & - & 0.11 & - & Adult \\
\hline BY90 & $\begin{array}{l}\text { Poore Mine Seep above ground sluice } \\
\text { nr Grass Valley }\end{array}$ & $9 / 26 / 00$ & Water strider Hemiptera & Gerridae & 20 & 1.16 & 57.6 & - & 0.13 & 一 & Adult \\
\hline BY91 & $\begin{array}{l}\text { Poore Mine tunnel effluent nr Grass } \\
\text { Valley }\end{array}$ & $8 / 21 / 00$ & Water strider Hemiptera & Gerridae & 22 & 1.21 & 66.2 & - & 0.16 & - & Adult \\
\hline BY91 & $\begin{array}{l}\text { Poore Mine tunnel effluent nr Grass } \\
\text { Valley }\end{array}$ & $10 / 17 / 01$ & Water strider Hemiptera & Gerridae & 25 & 1.50 & 59.6 & 0.13 & 0.12 & 92 & Adult \\
\hline BY105 & $\begin{array}{l}\text { Sailor Flat Mine main drain Gulch } 01 \\
\text { nr Quaker Hill }\end{array}$ & $8 / 24 / 99$ & Water strider Hemiptera & Gerridae & 22 & 1.41 & 53.8 & - & 0.089 & - & Adult \\
\hline BY105 & $\begin{array}{l}\text { Sailor Flat Mine main drain Gulch } 01 \\
\text { nr Quaker Hill }\end{array}$ & $8 / 24 / 99$ & Water strider Hemiptera & Gerridae & 21 & 1.31 & 56.8 & - & 0.075 & - & Adult \\
\hline BY114 & $\begin{array}{l}\text { SF Greenhorn Cr } 0.7 \text { mi ab Greenhorn } \\
\text { Cr nr Nevada City }\end{array}$ & $8 / 22 / 00$ & Water strider Hemiptera & Gerridae & 36 & 2.28 & 58.0 & - & 0.16 & - & Adult \\
\hline BY114 & $\begin{array}{l}\text { SF Greenhorn Cr } 0.7 \text { mi ab Greenhorn } \\
\text { Cr nr Nevada City }\end{array}$ & $9 / 13 / 01$ & Water strider Hemiptera & Gerridae & 25 & 1.52 & 62.4 & 0.25 & 0.17 & 69 & Adult \\
\hline
\end{tabular}


Table 11A. Mercury and methylmercury concentration data for invertebrates, Greenhorn Creek drainage, Nevada County, California-Continued

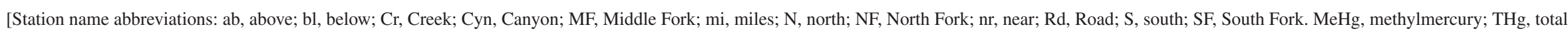
mercury. $\mathrm{n}$, number of organisms; g, gram; $\mu \mathrm{g} / \mathrm{g}$, microgram per gram; —, not determined]

\begin{tabular}{|c|c|c|c|c|c|c|c|c|c|c|c|}
\hline $\begin{array}{l}\text { Station } \\
\text { map ID }\end{array}$ & Station name & $\begin{array}{l}\text { Collection } \\
\text { date }\end{array}$ & $\begin{array}{c}\text { Common } \\
\text { name }\end{array}$ & Family & $\begin{array}{c}\text { Sample } \\
\text { size } \\
\text { (n) }\end{array}$ & $\begin{array}{c}\text { Live } \\
\text { weight } \\
\text { (g) }\end{array}$ & $\begin{array}{l}\text { Moisture } \\
\text { (percent) }\end{array}$ & $\begin{array}{c}\text { Total } \\
\text { mercury } \\
(\mu \mathrm{g} / \mathrm{g} \text { wet })\end{array}$ & $\begin{array}{c}\text { Methyl- } \\
\text { mercury } \\
\text { ( } \mu \mathrm{g} / \mathrm{g} \text { wet) }\end{array}$ & $\begin{array}{c}\mathrm{MeHg} / \mathrm{THg} \\
\text { (percent) }\end{array}$ & Age \\
\hline BY114 & $\begin{array}{l}\text { SF Greenhorn Cr } 0.7 \text { mi ab Greenhorn } \\
\text { Cr nr Nevada City }\end{array}$ & $9 / 13 / 01$ & Water strider Hemiptera & Gerridae & 25 & 1.53 & 59.7 & 0.25 & 0.21 & 84 & Adult \\
\hline BY113 & $\begin{array}{l}\text { SF Greenhorn Cr } 0.8 \text { mi ab Greenhorn } \\
\text { Cr nr Nevada City }\end{array}$ & $8 / 22 / 00$ & Water strider Hemiptera & Gerridae & 21 & 1.33 & 57.6 & - & 0.074 & - & Adult \\
\hline BY113 & $\begin{array}{l}\text { SF Greenhorn Cr } 0.8 \text { mi ab Greenhorn } \\
\text { Cr nr Nevada City }\end{array}$ & $9 / 13 / 01$ & Water strider Hemiptera & Gerridae & 25 & 1.42 & 57.4 & 0.10 & 0.065 & 64 & Adult \\
\hline BY115 & $\begin{array}{l}\text { SF Greenhorn Cr bl Boston Mine nr } \\
\text { Nevada City }\end{array}$ & 9/28/99 & Water strider Hemiptera & Gerridae & 20 & 1.22 & 57.1 & - & 0.18 & - & Adult \\
\hline BY115 & $\begin{array}{l}\text { SF Greenhorn Cr bl Boston Mine nr } \\
\text { Nevada City }\end{array}$ & $8 / 21 / 00$ & Water strider Hemiptera & Gerridae & 28 & 1.57 & 62.6 & - & 0.22 & - & Adult \\
\hline BY115 & $\begin{array}{l}\text { SF Greenhorn Cr bl Boston Mine nr } \\
\text { Nevada City }\end{array}$ & $9 / 13 / 01$ & Water strider Hemiptera & Gerridae & 25 & 1.29 & 63.5 & 0.20 & 0.21 & 107 & Adult \\
\hline BY124 & $\begin{array}{l}\text { Starr Mine tunnel outlet nr Grass } \\
\text { Valley }\end{array}$ & $8 / 21 / 00$ & Water strider Hemiptera & Gerridae & 9 & 0.48 & 57.5 & - & 0.12 & - & Adult \\
\hline BY148 & Starr Pit pond nr Grass Valley & $8 / 12 / 99$ & Water strider Hemiptera & Gerridae & 15 & 0.58 & 51.7 & - & 0.10 & - & Adult \\
\hline BY148 & Starr Pit pond nr Grass Valley & $8 / 21 / 00$ & Water strider Hemiptera & Gerridae & 35 & 0.98 & 63.9 & - & 0.10 & - & Adult \\
\hline BY130 & $\begin{array}{l}\text { Tom and Jerry Mine drain at tunnel } \\
\text { outlet nr Nevada City }\end{array}$ & $8 / 23 / 00$ & Water strider Hemiptera & Gerridae & 22 & 1.22 & 64.3 & - & 0.13 & - & Adult \\
\hline BY130 & $\begin{array}{l}\text { Tom and Jerry Mine drain at tunnel } \\
\text { outlet nr Nevada City }\end{array}$ & $8 / 23 / 00$ & Water strider Hemiptera & Gerridae & 30 & 1.86 & 65.8 & - & 0.073 & - & Adult \\
\hline BY130 & $\begin{array}{l}\text { Tom and Jerry Mine drain at tunnel } \\
\text { outlet nr Nevada City }\end{array}$ & $9 / 13 / 01$ & Water strider Hemiptera & Gerridae & 17 & 1.13 & 53.8 & 0.25 & 0.31 & 127 & Adult \\
\hline BY130 & $\begin{array}{l}\text { Tom and Jerry Mine drain at tunnel } \\
\text { outlet nr Nevada City }\end{array}$ & $9 / 13 / 01$ & Water strider Hemiptera & Gerridae & 25 & 1.64 & 57.2 & 0.090 & 0.064 & 71 & Adult \\
\hline BY131 & $\begin{array}{l}\text { Tom and Jerry Mine drainage pond nr } \\
\text { Nevada City }\end{array}$ & $9 / 13 / 01$ & Water strider Hemiptera & Gerridae & 16 & 1.03 & 54.5 & 0.066 & 0.066 & 99 & Adult \\
\hline
\end{tabular}


[Station name abbreviations: nr, near. MeHg, methylmercury; THg, total mercury. n, number of organisms; g, gram; $\mu \mathrm{g} / \mathrm{g}$, microgram per gram; - , not determined]

\begin{tabular}{|c|c|c|c|c|c|c|c|c|c|c|c|c|}
\hline $\begin{array}{l}\text { Station } \\
\text { map ID }\end{array}$ & Station name & $\begin{array}{l}\text { Collection } \\
\text { date }\end{array}$ & Common name & Order & Family & $\begin{array}{c}\text { Sample } \\
\text { size } \\
(\mathrm{n})\end{array}$ & $\begin{array}{c}\text { Live } \\
\text { weight } \\
\text { (g) }\end{array}$ & $\begin{array}{l}\text { Moisture } \\
\text { (percent) }\end{array}$ & $\begin{array}{c}\text { Total } \\
\text { mercury } \\
\text { ( } \mu \mathrm{g} / \mathrm{g} \text { wet })\end{array}$ & $\begin{array}{c}\text { Methyl- } \\
\text { mercury } \\
\text { ( } \mu \mathrm{g} / \mathrm{g} \text { wet })\end{array}$ & $\begin{array}{c}\mathrm{MeHg} / \mathrm{THg} \\
\text { (percent) }\end{array}$ & Age \\
\hline BY199 & Bear River at Highway $20 \mathrm{nr}$ Emigrant Gap & $9 / 21 / 00$ & Dobsonfly & Megaloptera & Corydalidae & 1 & 1.52 & 74.9 & 0.035 & 0.038 & 109 & Immature \\
\hline BY199 & Bear River at Highway $20 \mathrm{nr}$ Emigrant Gap & $9 / 15 / 01$ & Dobsonfly & Megaloptera & Corydalidae & 4 & 3.48 & 75.7 & 0.040 & 0.029 & 73 & Immature \\
\hline BY199 & Bear River at Highway $20 \mathrm{nr}$ Emigrant Gap & $9 / 15 / 01$ & Dobsonfly & Megaloptera & Corydalidae & 4 & 0.61 & 79.6 & 0.041 & 0.04 & 98 & Immature \\
\hline BY199 & Bear River at Highway $20 \mathrm{nr}$ Emigrant Gap & $8 / 23 / 02$ & Dobsonfly & Megaloptera & Corydalidae & 2 & 3.03 & 71.2 & 0.05 & 0.048 & 96 & - \\
\hline BY199 & Bear River at Highway $20 \mathrm{nr}$ Emigrant Gap & $9 / 15 / 01$ & Dragonfly & Odonata & Aeshnidae & 7 & 3.89 & 81.9 & 0.022 & 0.014 & 64 & Immature \\
\hline BY199 & Bear River at Highway $20 \mathrm{nr}$ Emigrant Gap & $8 / 23 / 02$ & Dragonfly & Odonata & Aeshnidae & 8 & 3.63 & 79.9 & 0.024 & 0.026 & 108 & Immature \\
\hline BY199 & Bear River at Highway $20 \mathrm{nr}$ Emigrant Gap & $9 / 15 / 01$ & $\begin{array}{l}\text { Giant water } \\
\text { bug }\end{array}$ & Hemiptera & $\begin{array}{l}\text { Belosto- } \\
\text { matidae }\end{array}$ & 1 & 4.47 & 72.6 & 0.022 & 0.015 & 68 & Adult \\
\hline BY199 & Bear River at Highway $20 \mathrm{nr}$ Emigrant Gap & $9 / 15 / 01$ & $\begin{array}{l}\text { Predaceous } \\
\quad \text { diving beetle }\end{array}$ & Coleoptera & Dytiscidae & 24 & 1.07 & 54.8 & 0.061 & 0.041 & 67 & Adult \\
\hline BY199 & Bear River at Highway $20 \mathrm{nr}$ Emigrant Gap & $9 / 15 / 01$ & $\begin{array}{l}\text { Predaceous } \\
\quad \text { diving beetle }\end{array}$ & Coleoptera & Dytiscidae & 7 & 0.59 & 57.6 & 0.14 & 0.15 & 107 & Adult \\
\hline BY199 & Bear River at Highway $20 \mathrm{nr}$ Emigrant Gap & $8 / 23 / 02$ & $\begin{array}{l}\text { Predaceous } \\
\quad \text { diving beetle }\end{array}$ & Coleoptera & Dytiscidae & 11 & 0.75 & 56.7 & 0.14 & 0.14 & 100 & Adult \\
\hline BY199 & Bear River at Highway $20 \mathrm{nr}$ Emigrant Gap & $10 / 1 / 99$ & $\begin{array}{l}\text { Predaceous } \\
\text { stonefly }\end{array}$ & Plecoptera & Perlidae & 14 & 1.94 & 69.2 & 0.037 & 0.039 & 105 & Immature \\
\hline BY199 & Bear River at Highway $20 \mathrm{nr}$ Emigrant Gap & $9 / 21 / 00$ & $\begin{array}{l}\text { Predaceous } \\
\text { stonefly }\end{array}$ & Plecoptera & Perlidae & 5 & 1.2 & 68.9 & - & 0.067 & - & Immature \\
\hline BY199 & Bear River at Highway $20 \mathrm{nr}$ Emigrant Gap & $9 / 15 / 01$ & $\begin{array}{l}\text { Predaceous } \\
\text { stonefly }\end{array}$ & Plecoptera & Perlidae & 12 & 2.5 & 69.3 & 0.076 & 0.060 & 79 & Immature \\
\hline BY199 & Bear River at Highway $20 \mathrm{nr}$ Emigrant Gap & $9 / 15 / 01$ & $\begin{array}{l}\text { Predaceous } \\
\text { stonefly }\end{array}$ & Plecoptera & Perlidae & 12 & 1.7 & 72.2 & 0.056 & 0.058 & 104 & Immature \\
\hline BY199 & Bear River at Highway $20 \mathrm{nr}$ Emigrant Gap & $8 / 23 / 02$ & $\begin{array}{l}\text { Predaceous } \\
\text { stonefly }\end{array}$ & Plecoptera & Perlidae & 12 & 1.5 & 68.8 & 0.052 & 0.044 & 85 & Immature \\
\hline BY199 & Bear River at Highway $20 \mathrm{nr}$ Emigrant Gap & $10 / 1 / 99$ & Water strider & Hemiptera & Gerridae & 21 & 1.1 & 57.2 & - & 0.027 & - & Adult \\
\hline BY199 & Bear River at Highway $20 \mathrm{nr}$ Emigrant Gap & $9 / 21 / 00$ & Water strider & Hemiptera & Gerridae & 26 & 1.3 & 76.1 & 0.028 & 0.027 & 96 & Adult \\
\hline BY199 & Bear River at Highway $20 \mathrm{nr}$ Emigrant Gap & $9 / 15 / 01$ & Water strider & Hemiptera & Gerridae & 25 & 1.3 & 64.7 & 0.070 & 0.050 & 71 & Adult \\
\hline BY199 & Bear River at Highway $20 \mathrm{nr}$ Emigrant Gap & $8 / 23 / 02$ & Water strider & Hemiptera & Gerridae & 25 & 1.4 & 63.0 & 0.045 & 0.041 & 91 & Adult \\
\hline
\end{tabular}




\section{Appendix A. Map of Tunnel Locations and Photographs of Field Sites, Greenhorn Creek Drainage, Nevada County, California}

(Note: Additional descriptions of photographs in figures A2 through A26 are in table C3.) 


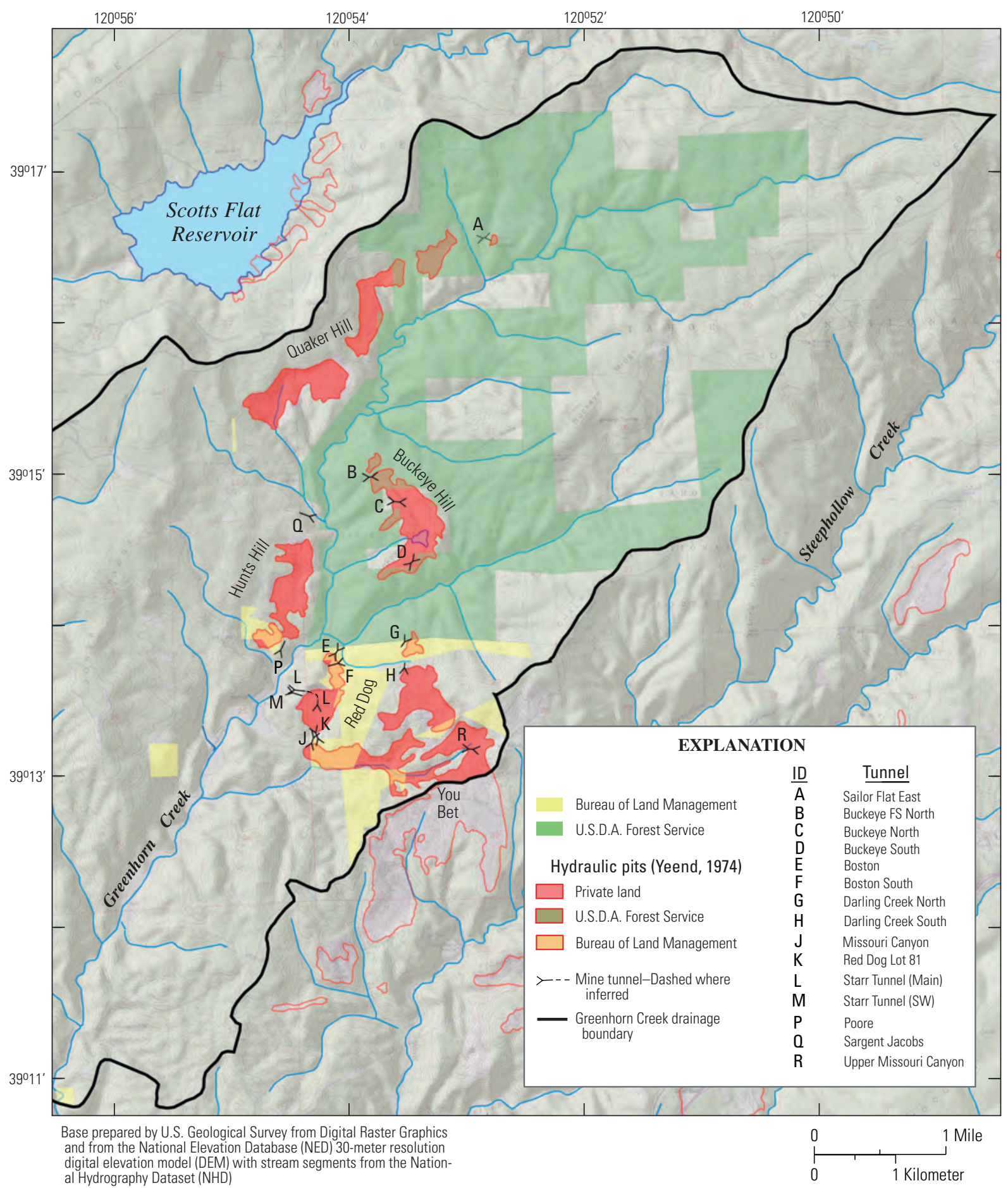

Figure A1. Locations of mine tunnels, hydraulic pits, and lands managed by federal land management agencies, Greenhorn Creek drainage, Nevada County, California. Boundaries of public lands provided by the Bureau of Land Management and the U.S. Department of Agriculture-Forest Service. Tunnel locations accurate to within approximately 300 feet. 


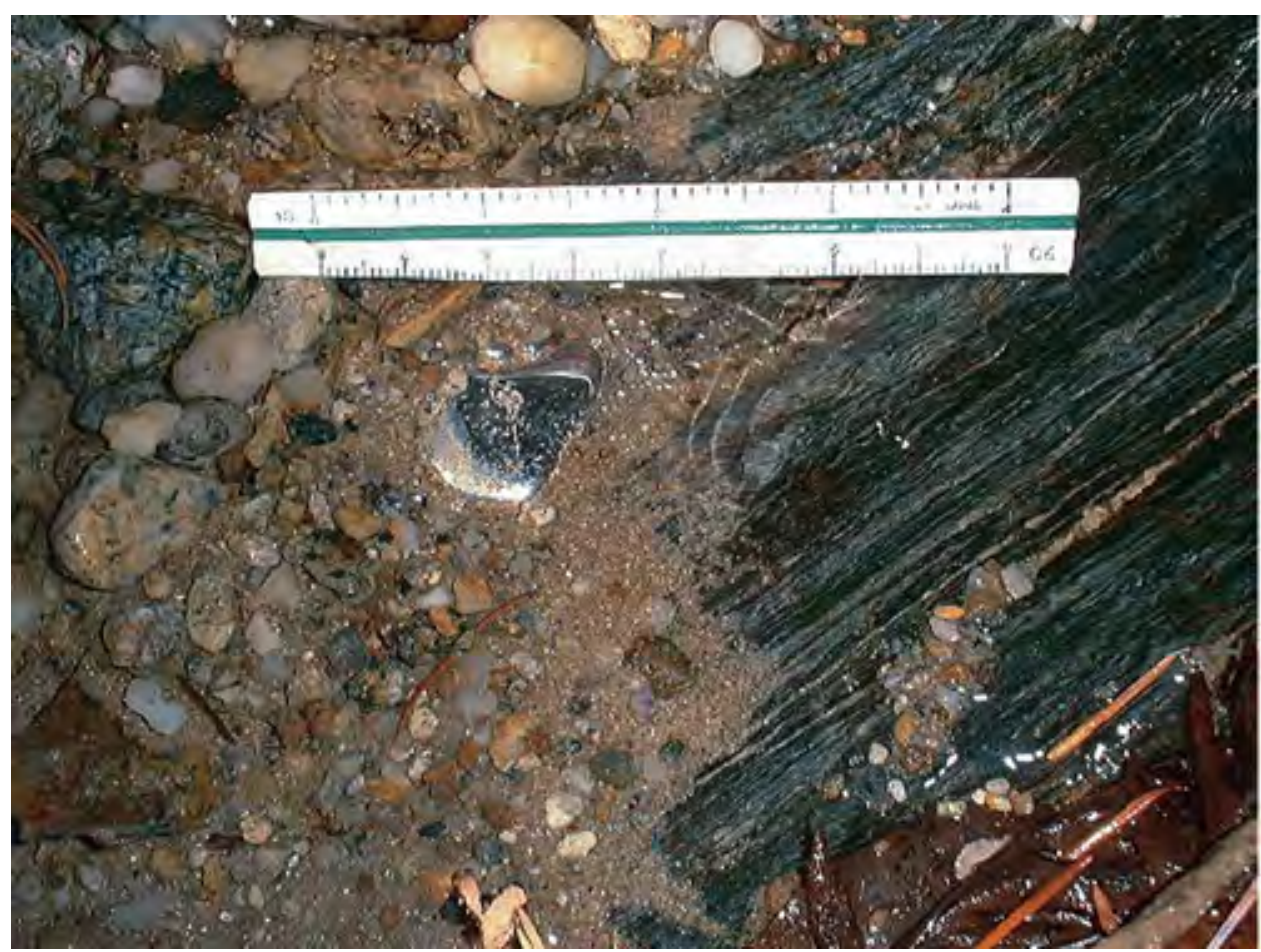

Figure A2. Main drainage gulch at Sailor Flat (station BY105) with visible mercury bead about one inch in diameter, Greenhorn Creek drainage, Nevada County, California.

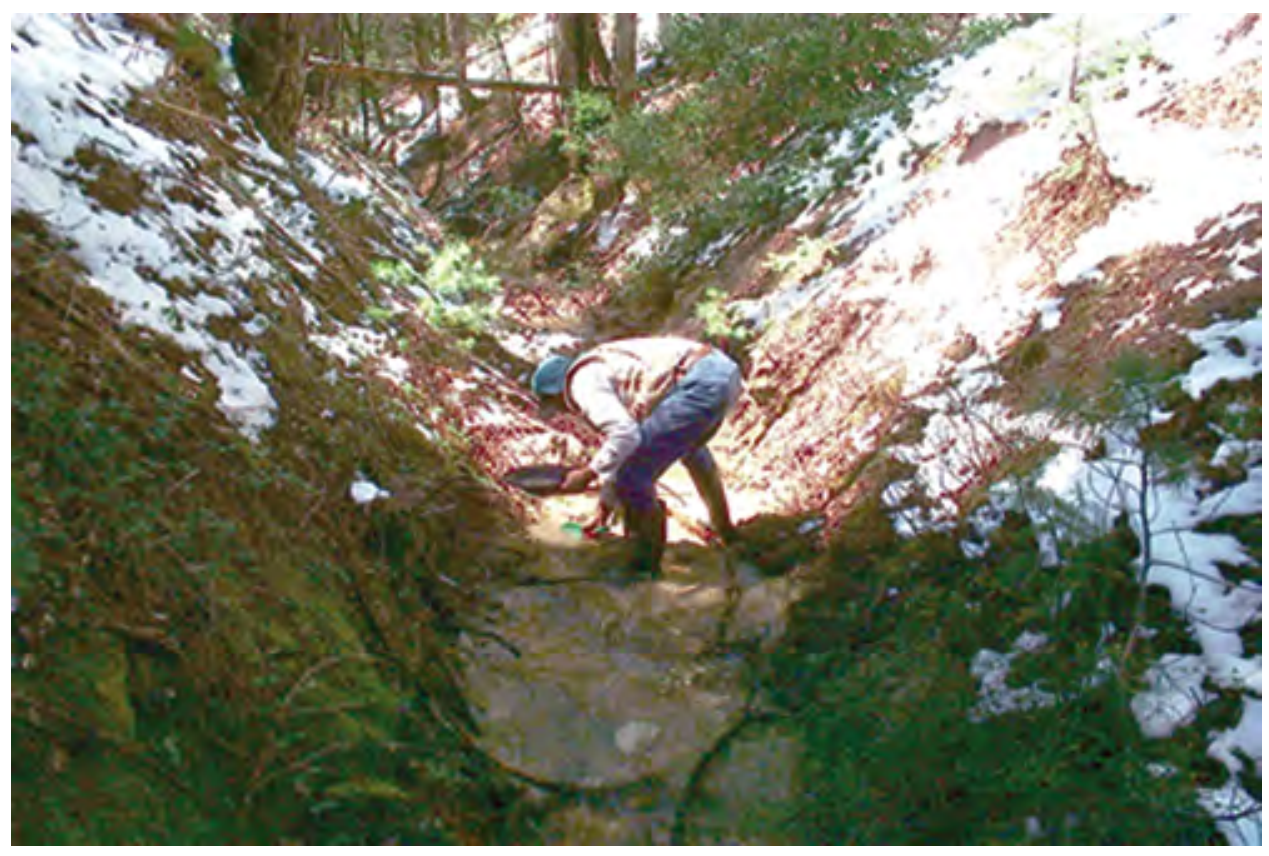

Figure A3. Sailor Flat main drainage to Greenhorn Creek (station BY106), Nevada County, California. 


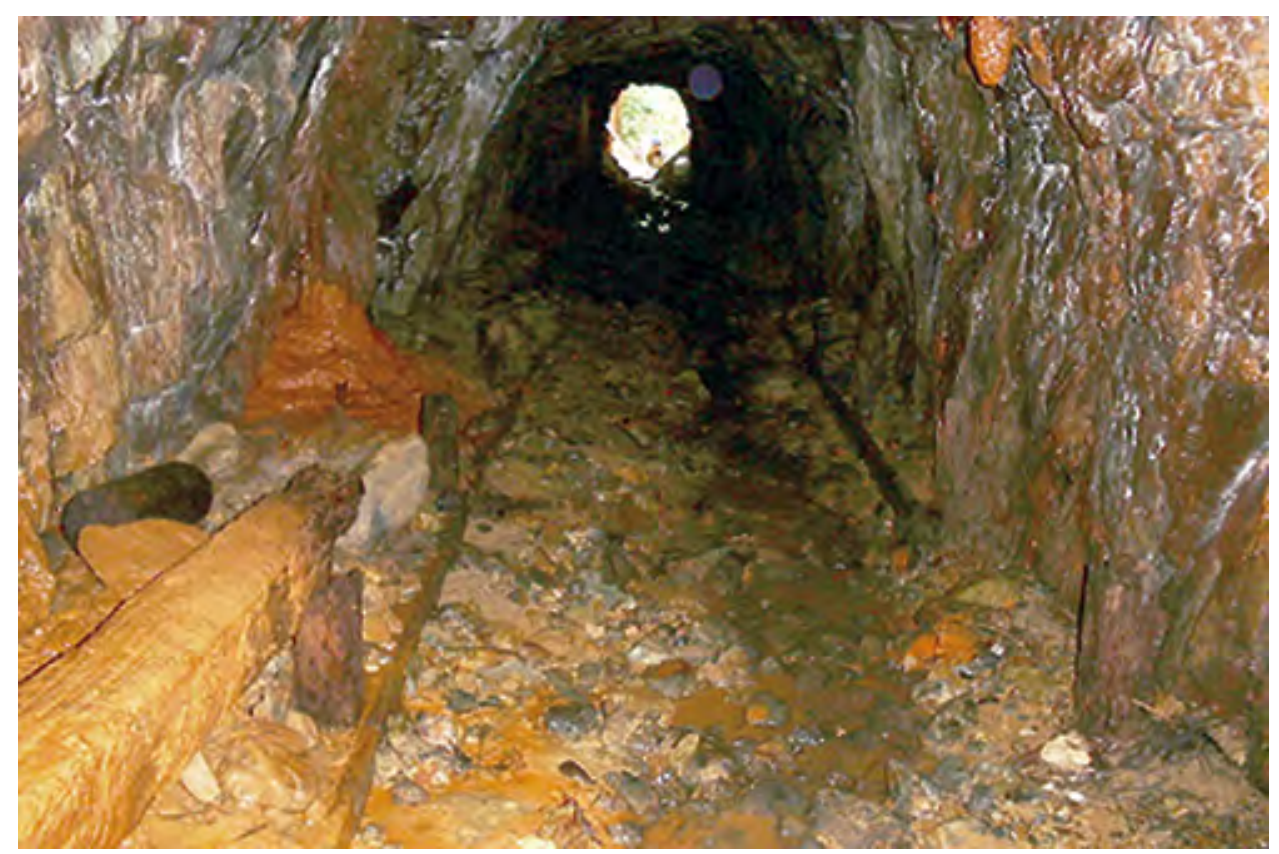

Figure A4. Tom and Jerry Mine tunnel outlet (station BY130), Greenhorn Creek drainage, Nevada County, California.

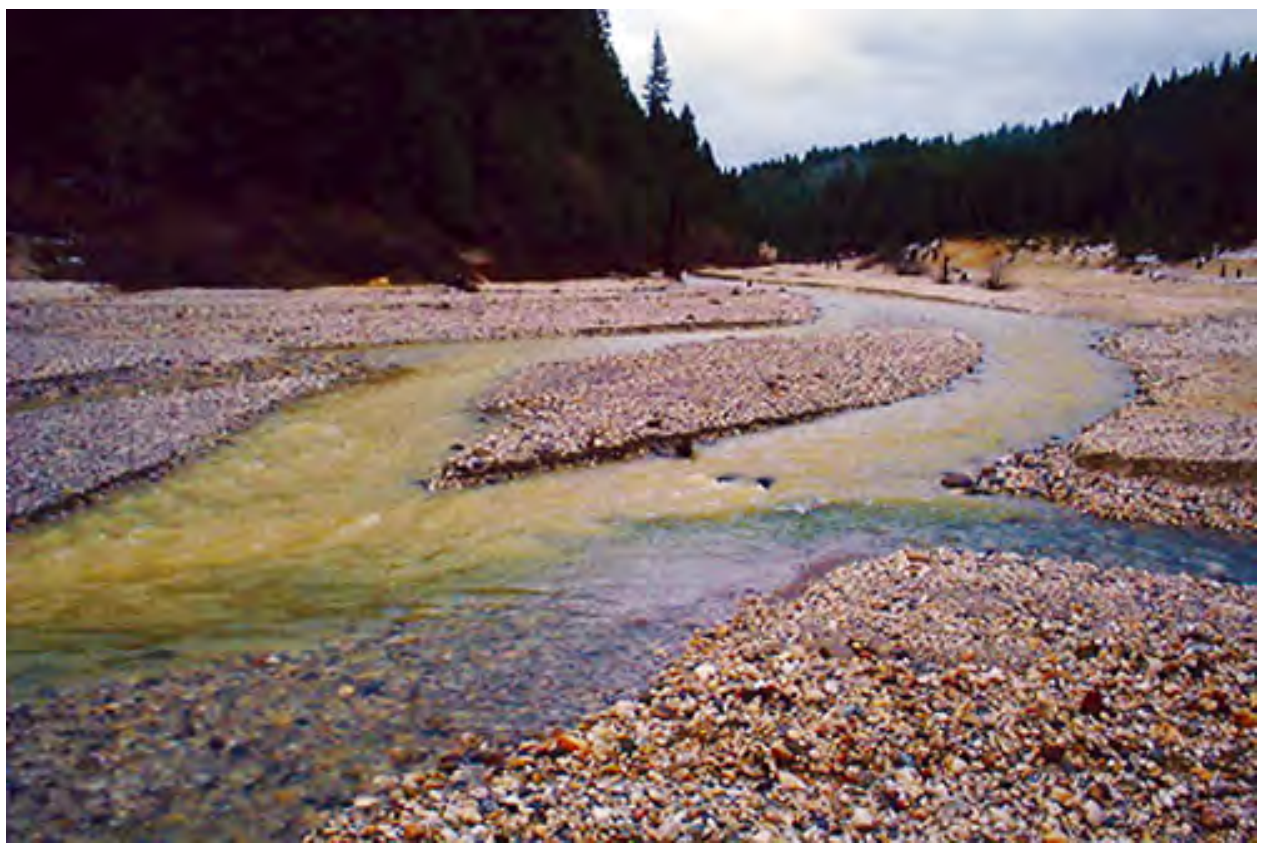

Figure A5. Buckeye main drain entering Greenhorn Creek (station BY58), Nevada County, California. 


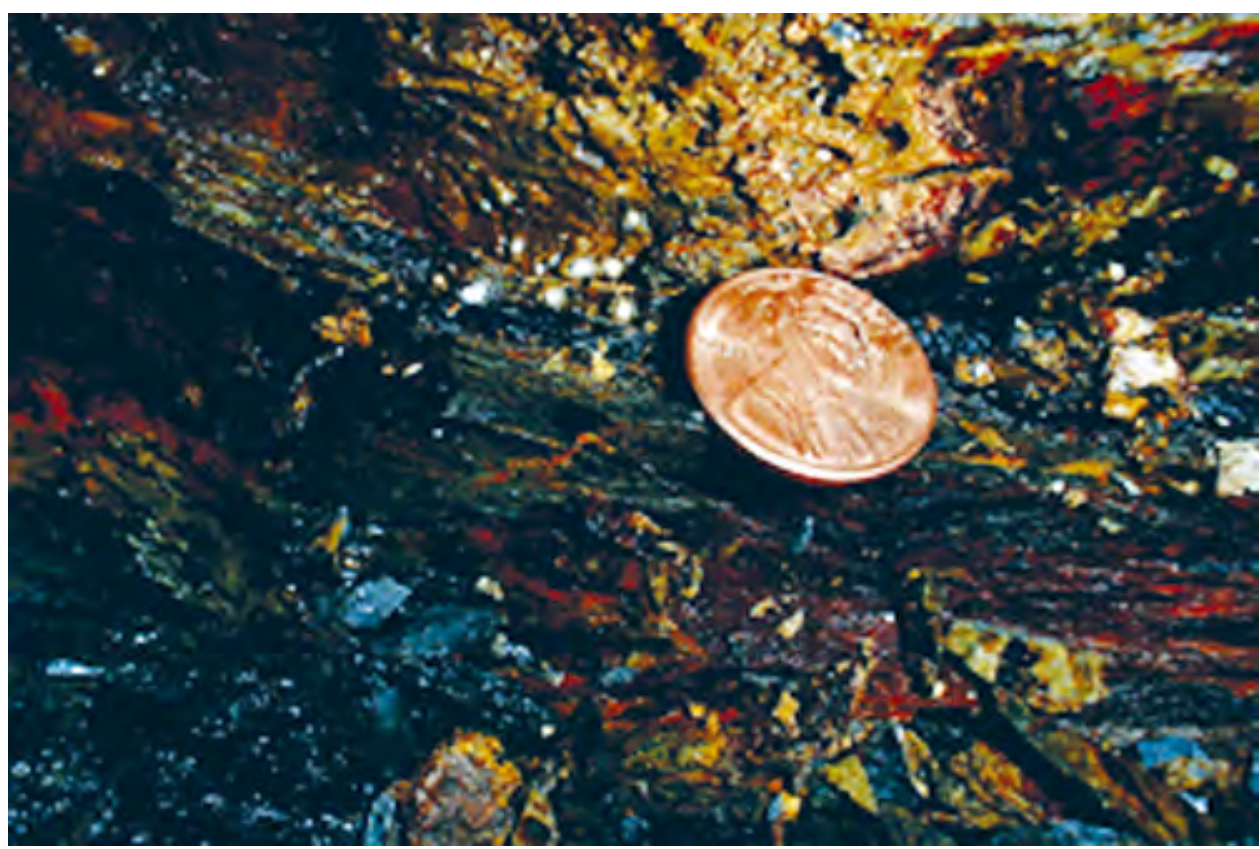

Figure A6. mercury beads on bedrock at Boston Mine tunnel outlet (station BY20), Greenhorn Creek drainage, Nevada County, California.

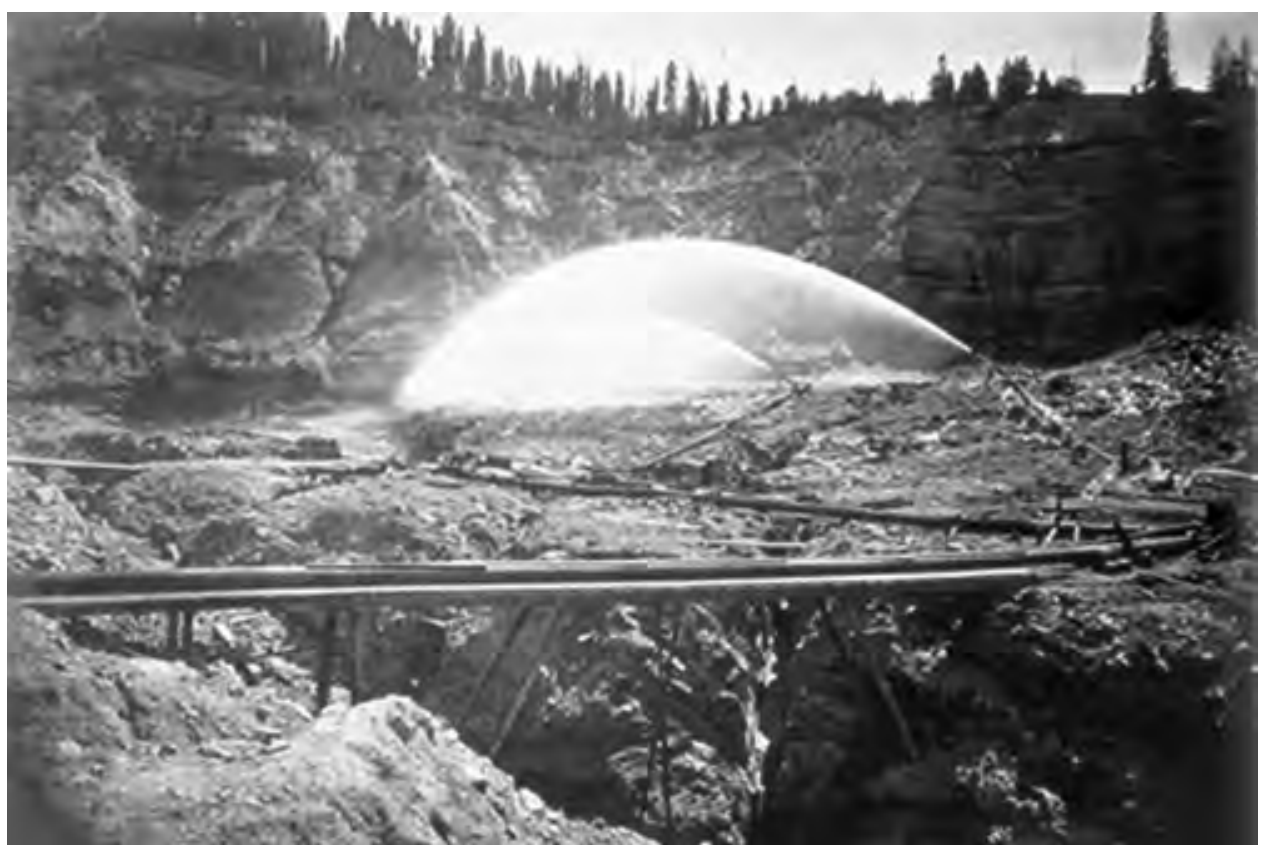

Figure A7. Historical photograph of Boston Mine (station BY21), Greenhorn Creek drainage, Nevada County, California. Photograph courtesy of the Bancroft Library, University of California, Berkeley. 


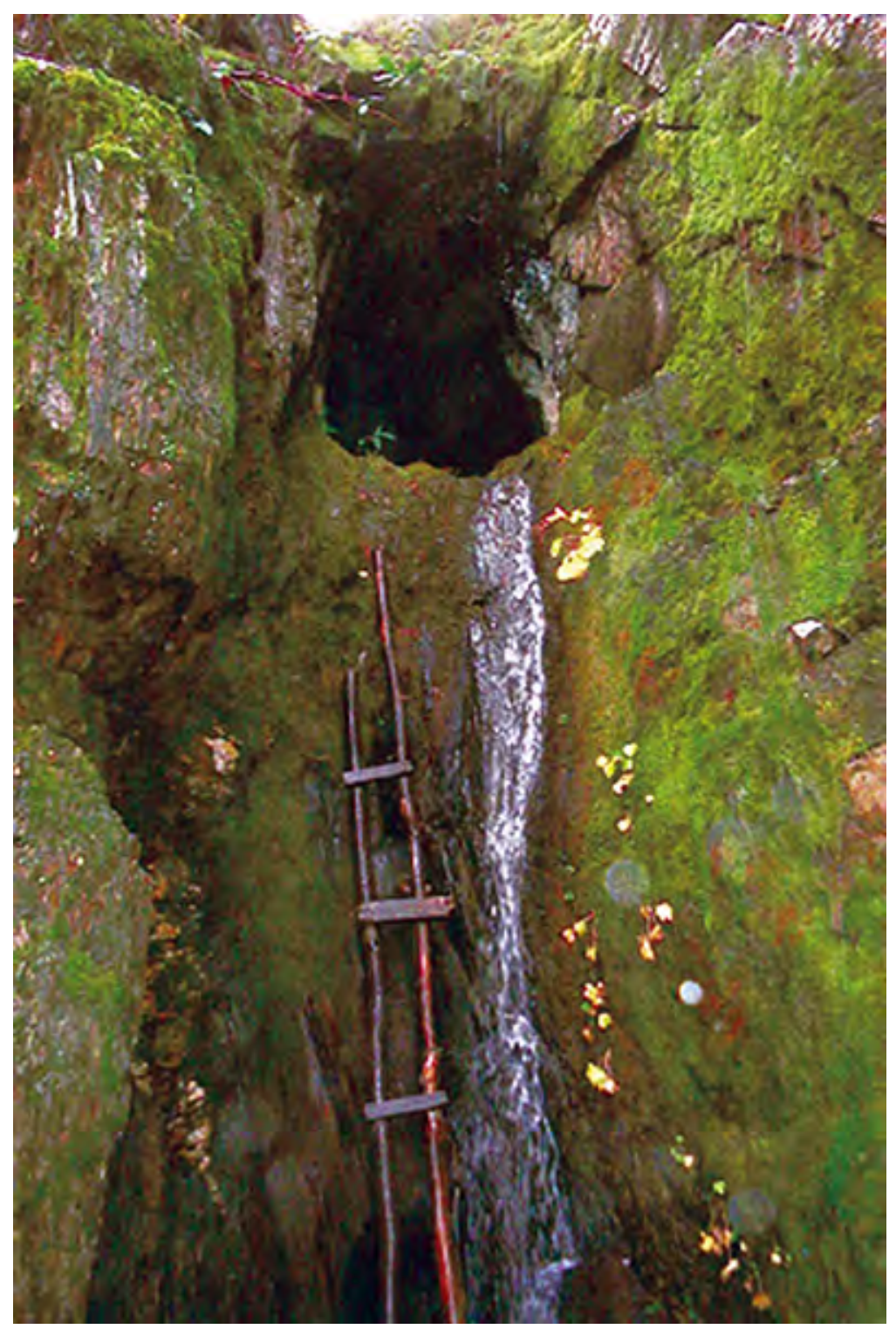

Figure A8. Dscharge from Boston Mine tunnel outlet (station BY20), Greenhorn Creek drainage, Nevada County, California. 


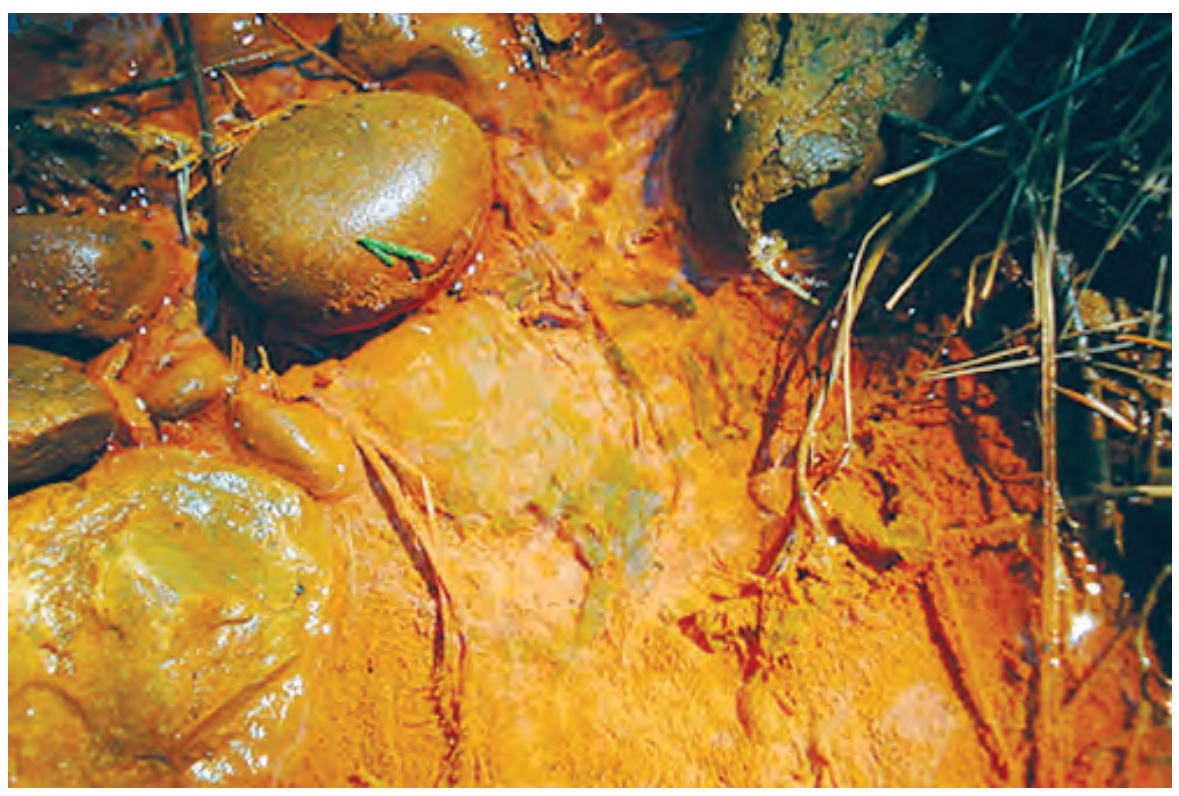

Figure A9. Iron precipitate in ground sluice at Poore Mine (station BY88), Greenhorn Creek drainage, Nevada County, California.

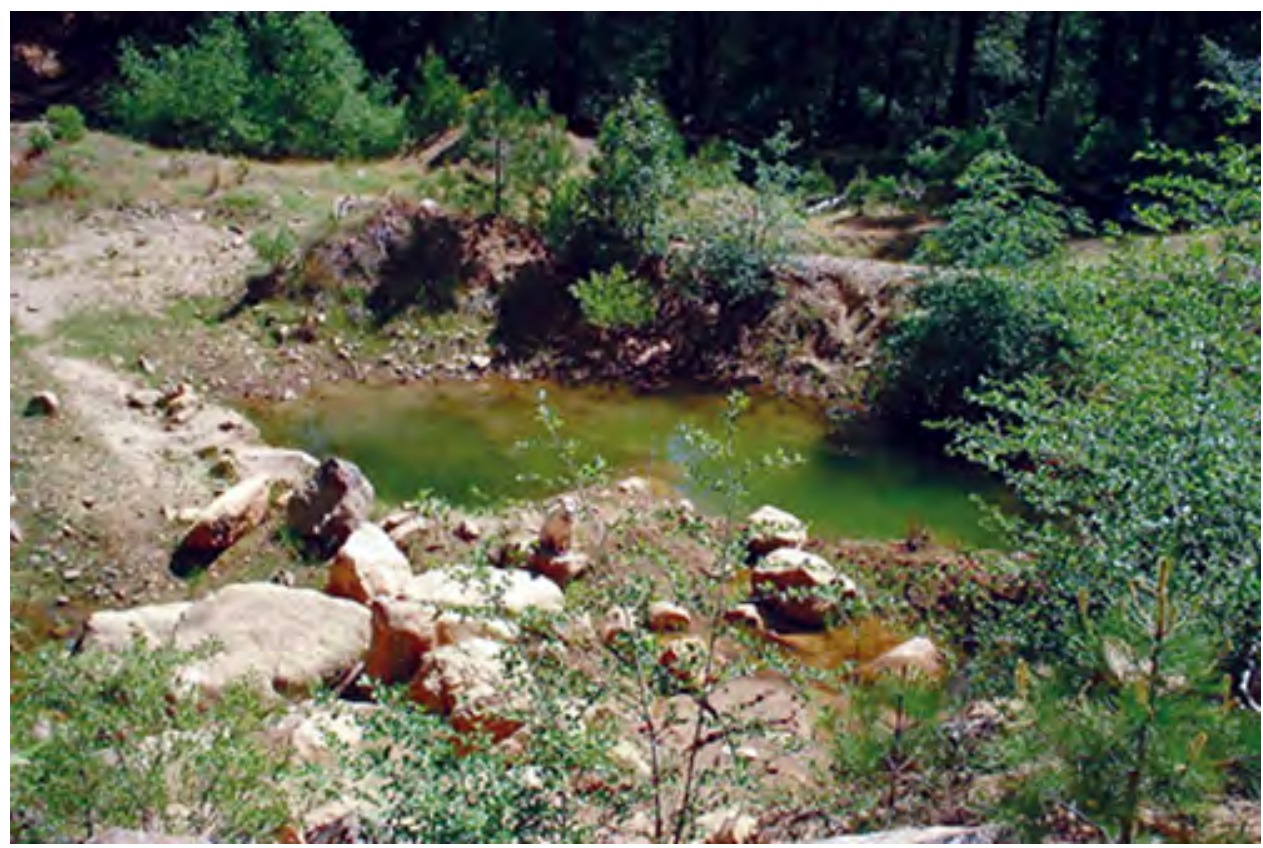

Figure A10. Poore Mine pit lake (station BY89), Greenhorn Creek drainage, Nevada County, California. 


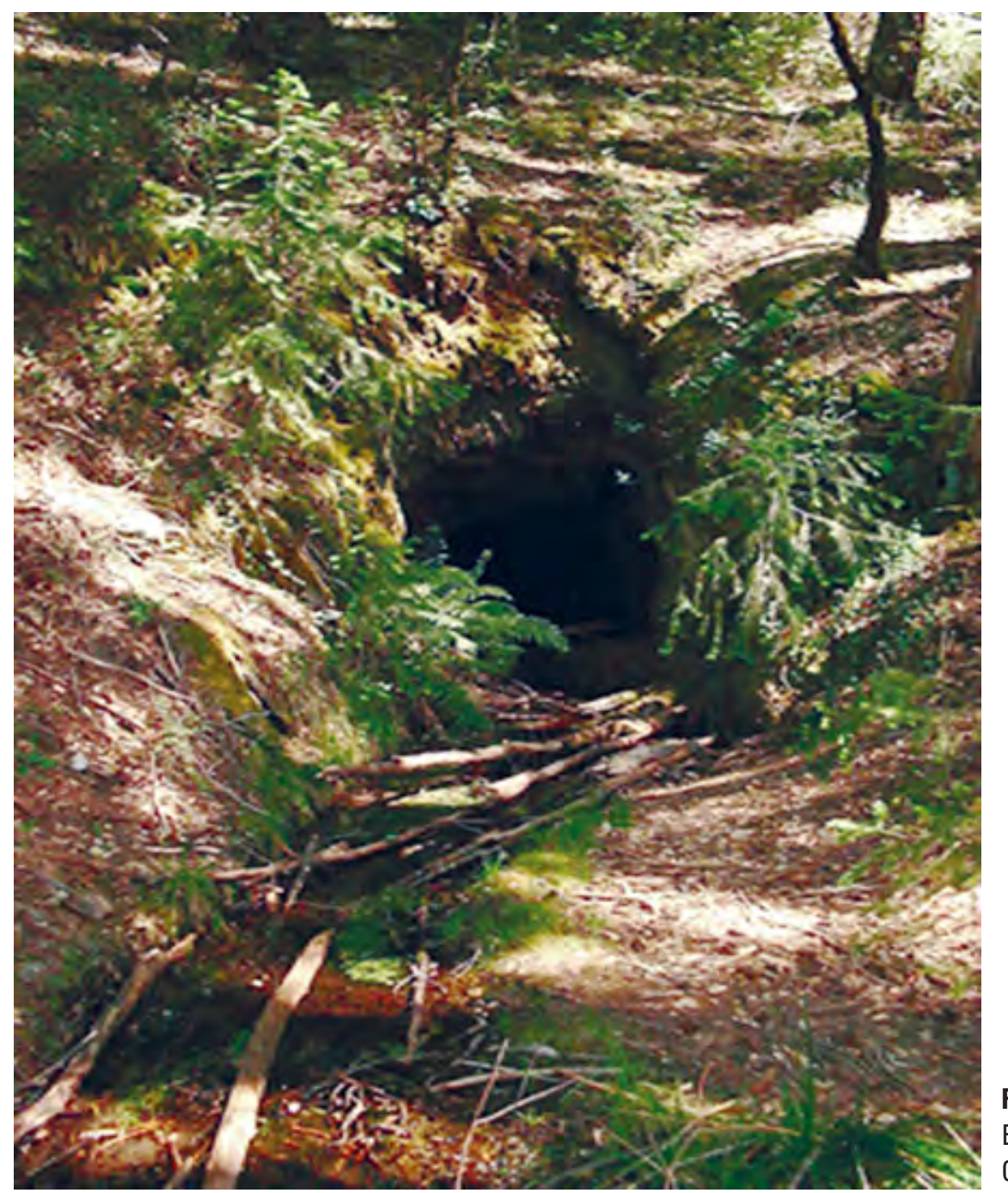

Figure A11. Poore Mine tunnel outlet (station BY91), Greenhorn Creek drainage, Nevada County, California.

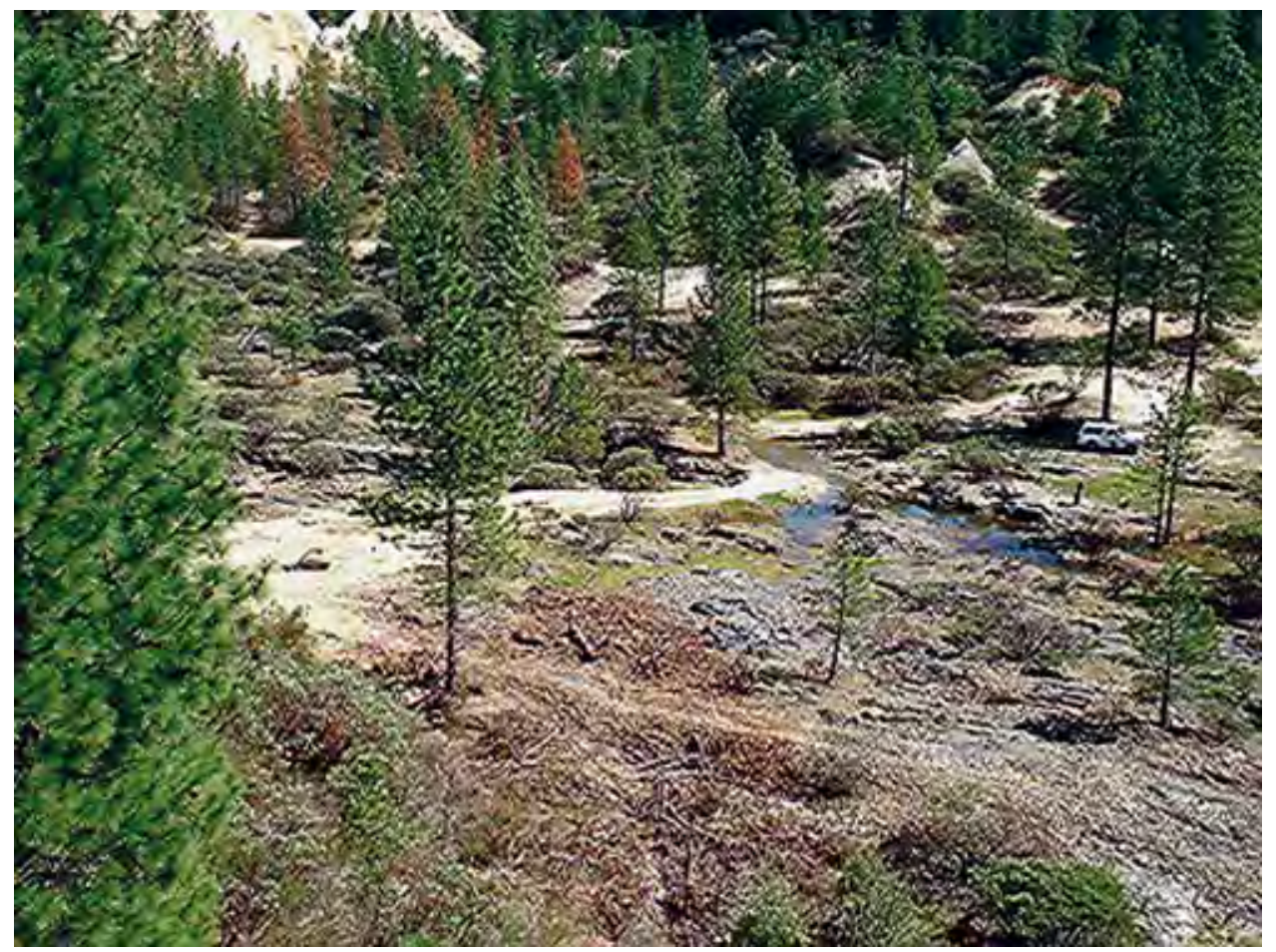

Figure A12. Starr Mine tunnel inlet area (station BY122), Greenhorn Creek drainage, Nevada County, California. 


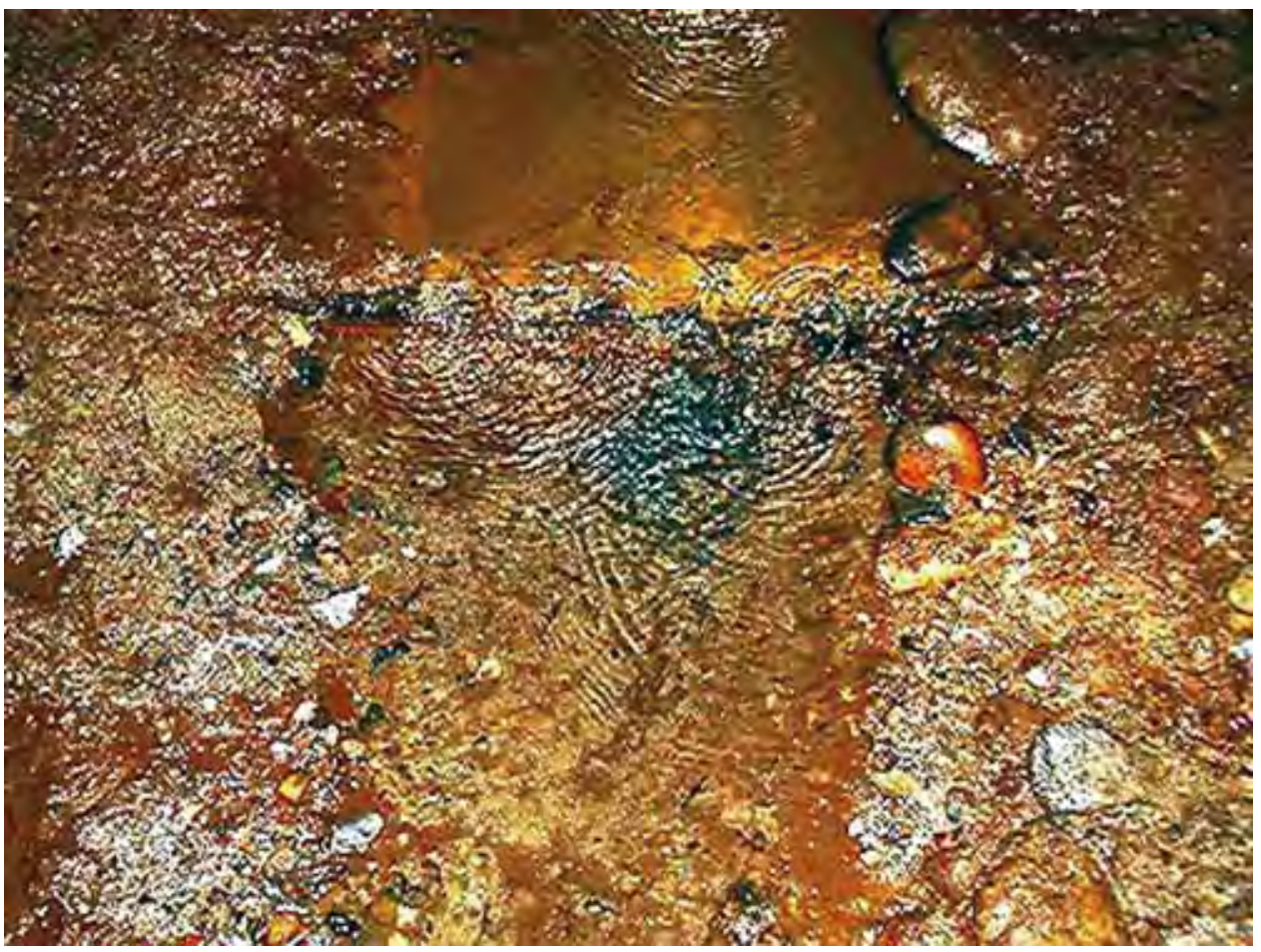

Figure A13. Midway station in Starr Mine tunnel (station BY123), Greenhorn Creek drainage, Nevada County, California.

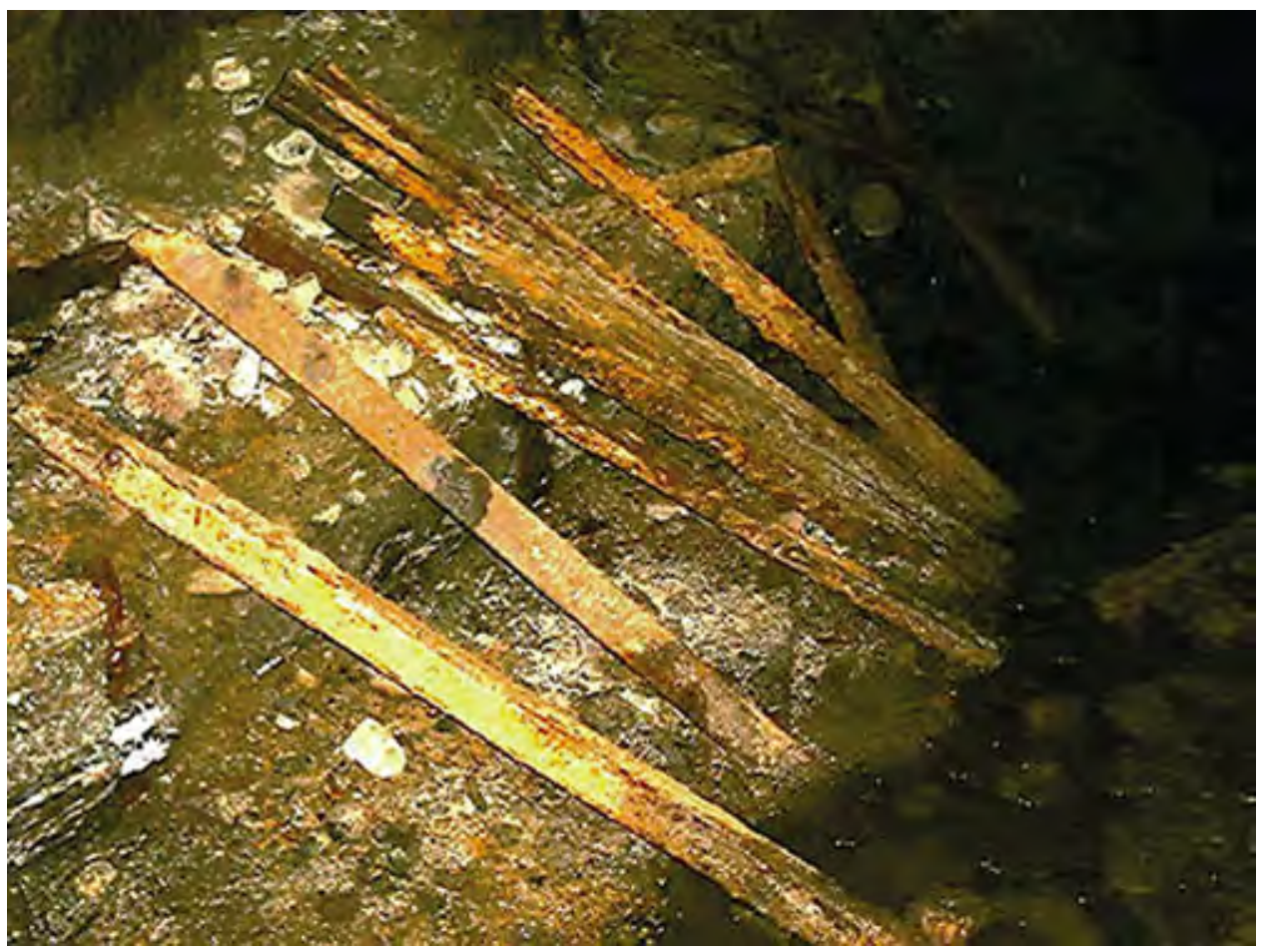

Figure A14. Starr Mine tunnel, near outlet (station BY124), Greenhorn Creek drainage, Nevada County, California. 


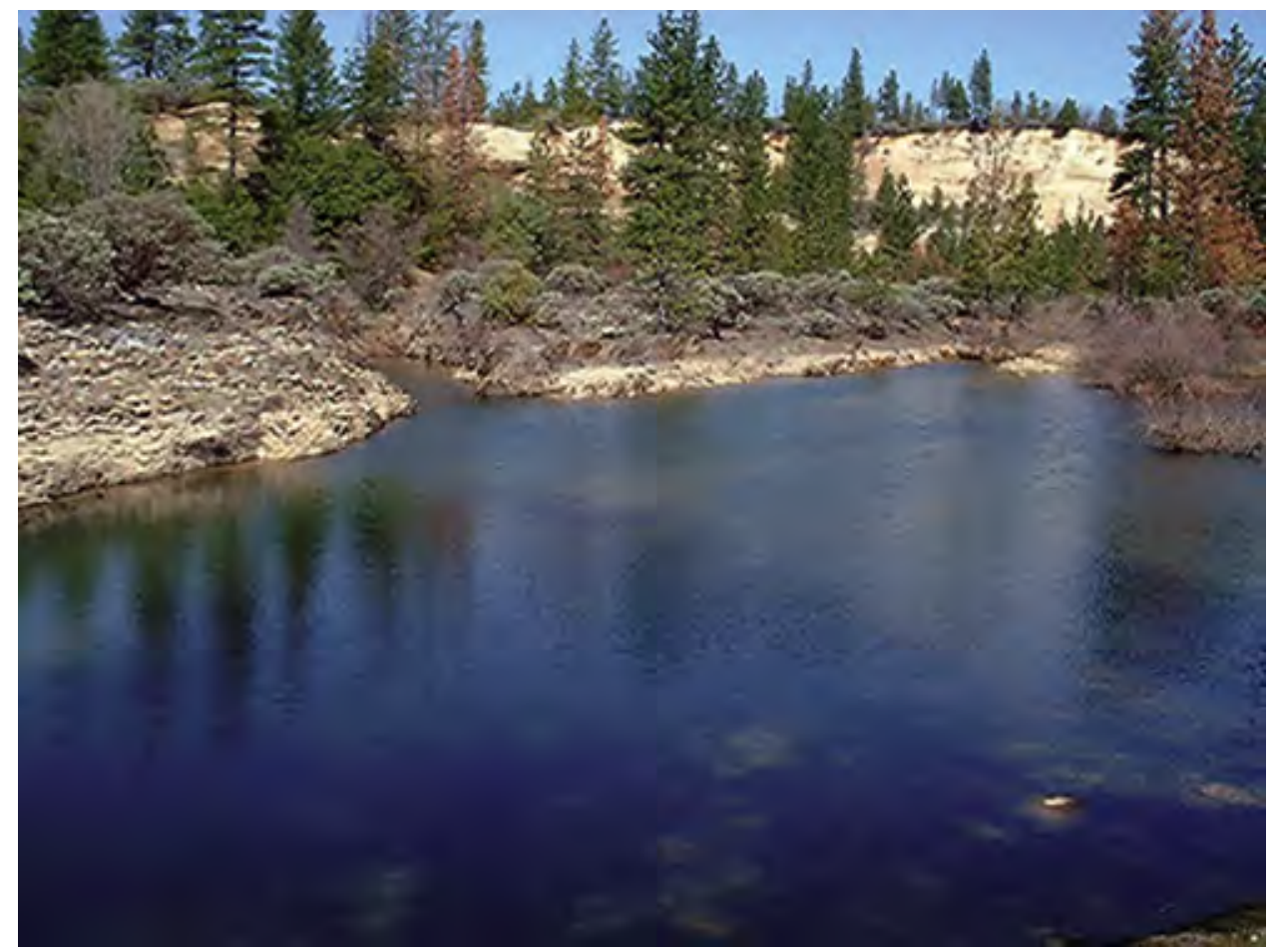

Figure A15. Starr Mine pit lake (station BY148), Greenhorn Creek drainage, Nevada County, California.

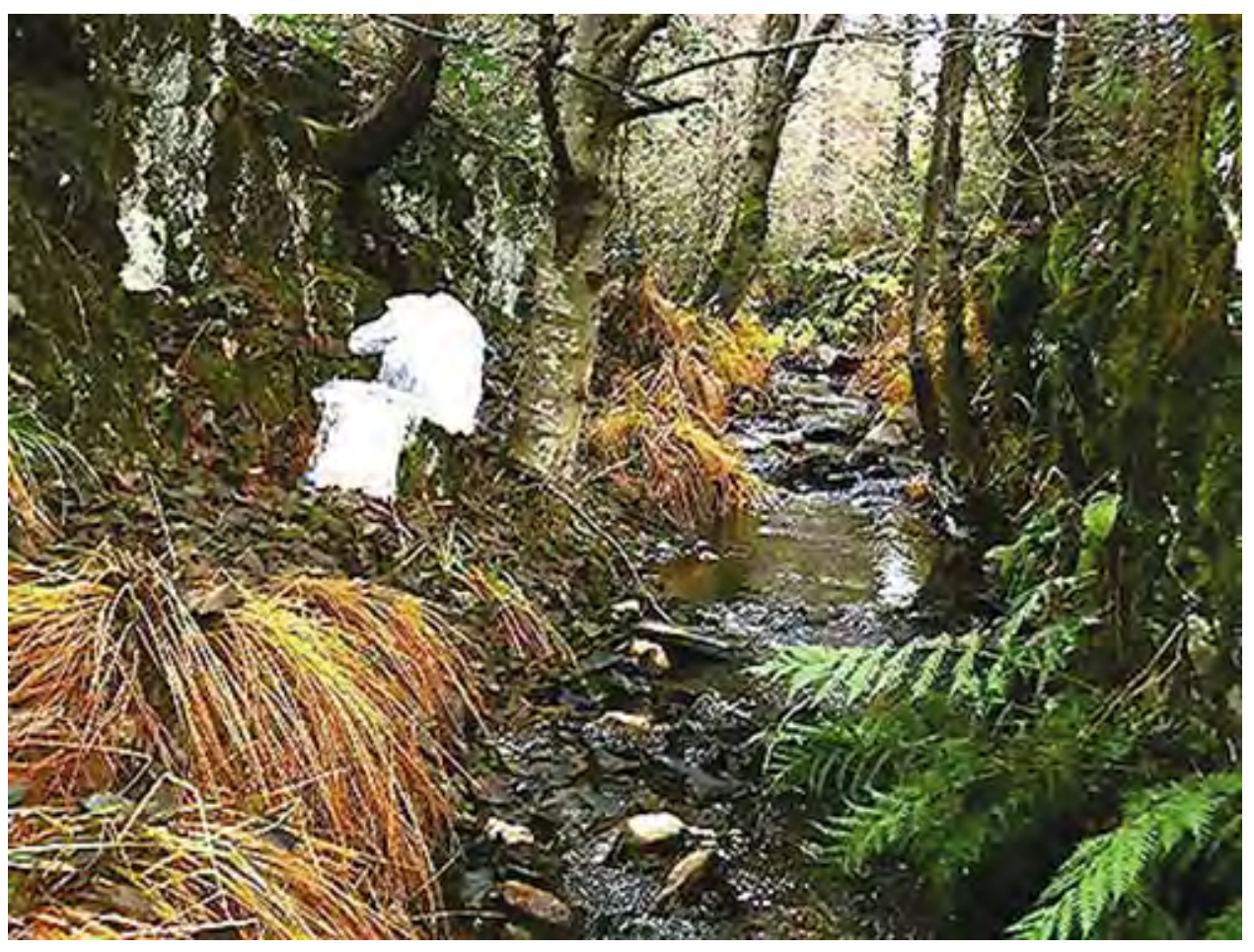

Figure A16. Missouri Canyon ground sluice (station BY146), Greenhorn Creek drainage, Nevada County, California. 


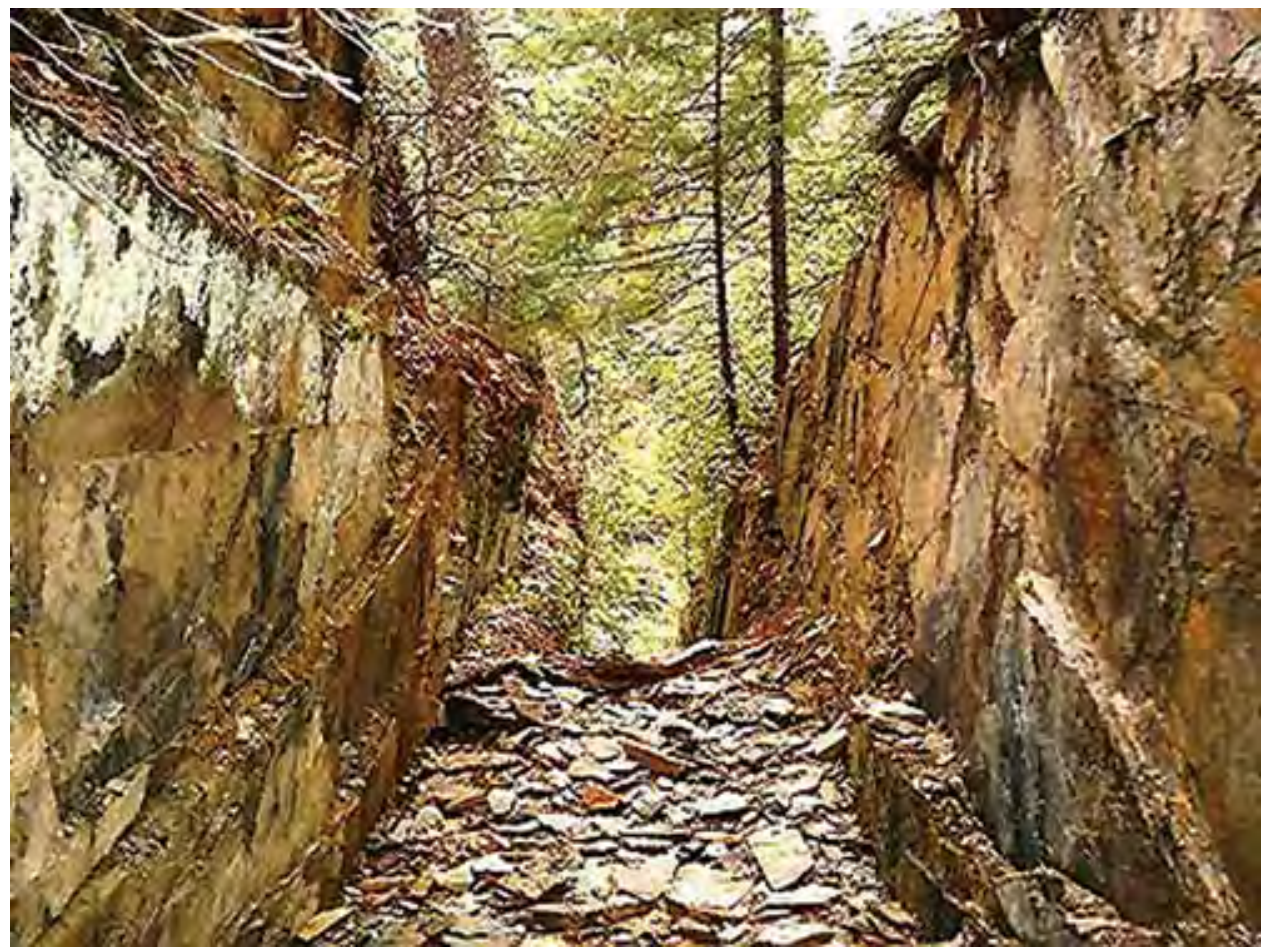

Figure A17. Missouri Canyon ground sluice above creek (station BY75), Greenhorn Creek drainage, Nevada County, California.

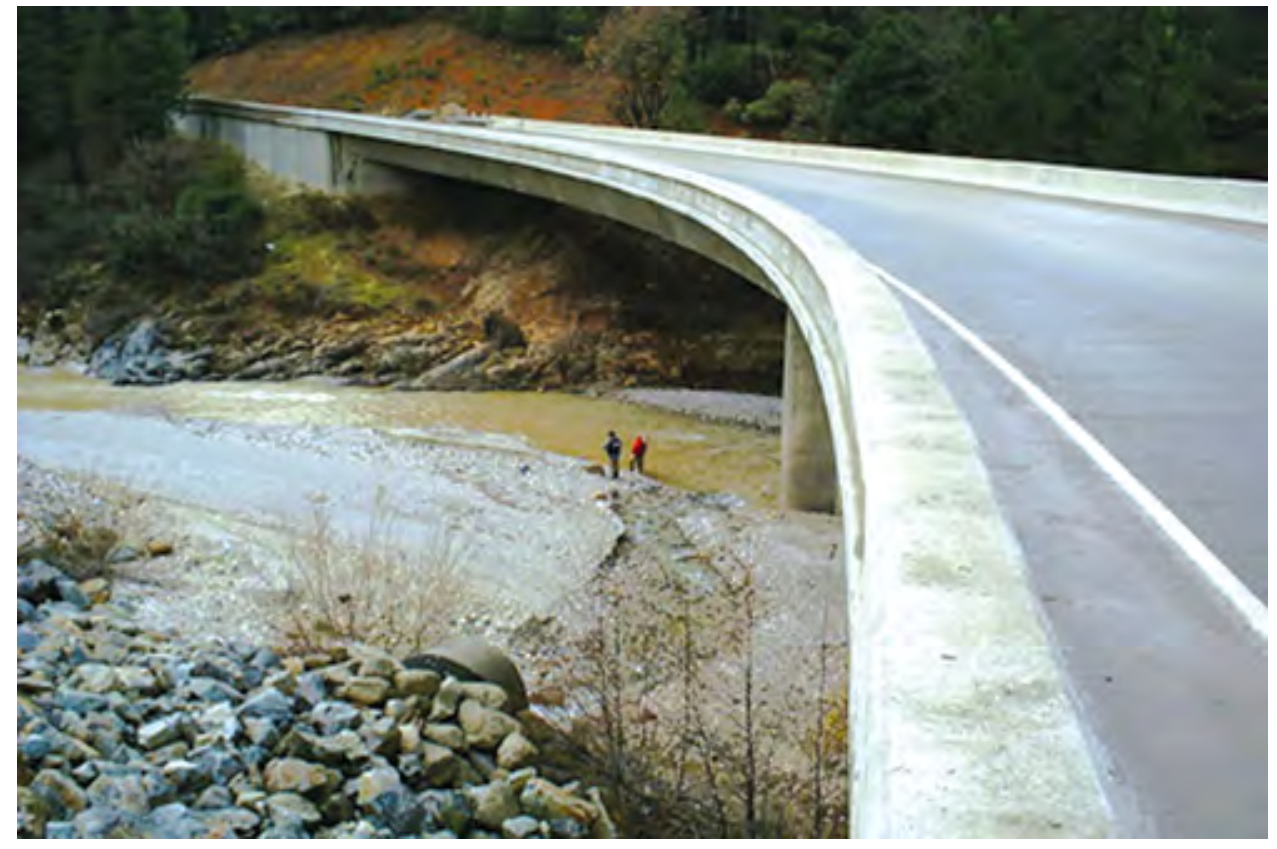

Figure A18. Greenhorn Creek at You Bet Road (station BY59), Nevada County, California. 


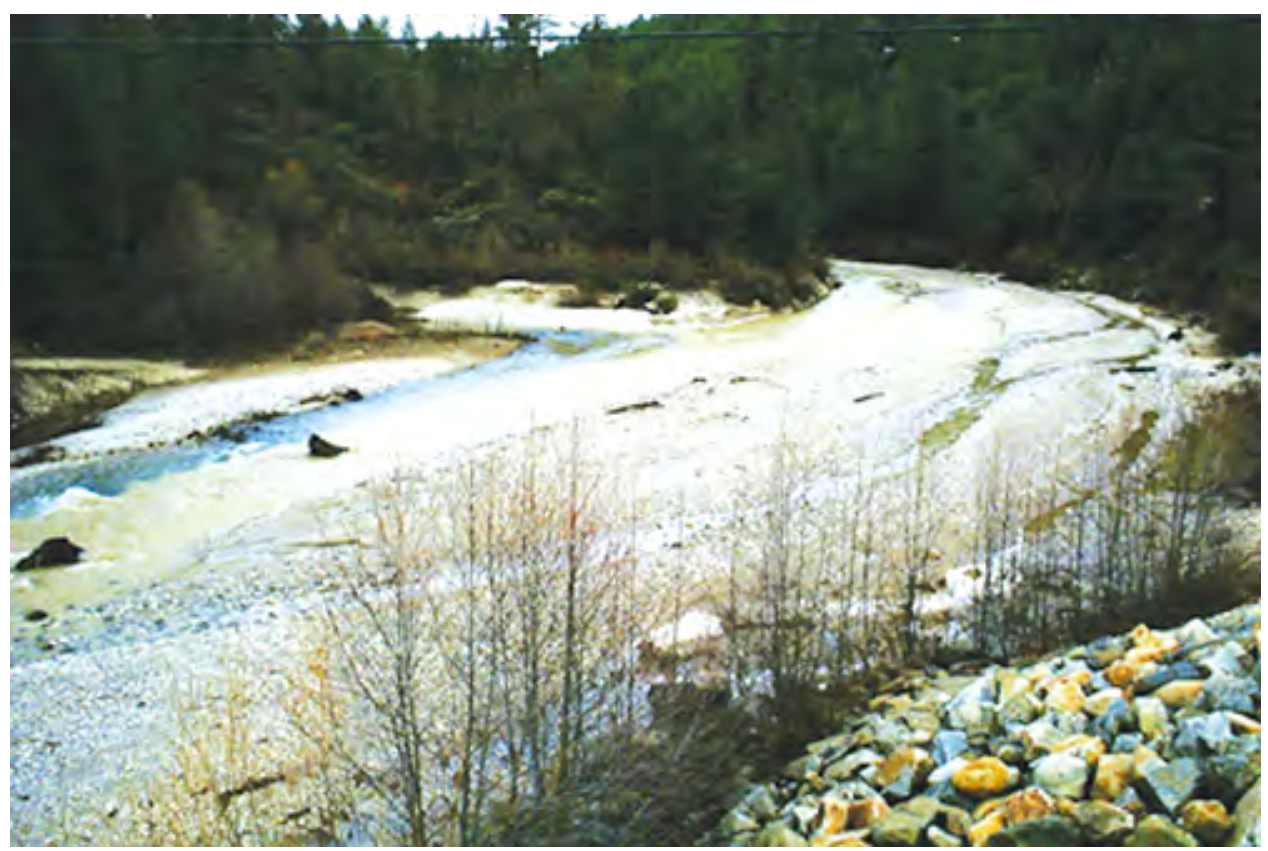

Figure A19. Confluence of Little Greenhorn Creek with Greenhorn Creek upstream of You Bet Road (station BY59), Nevada County, California.

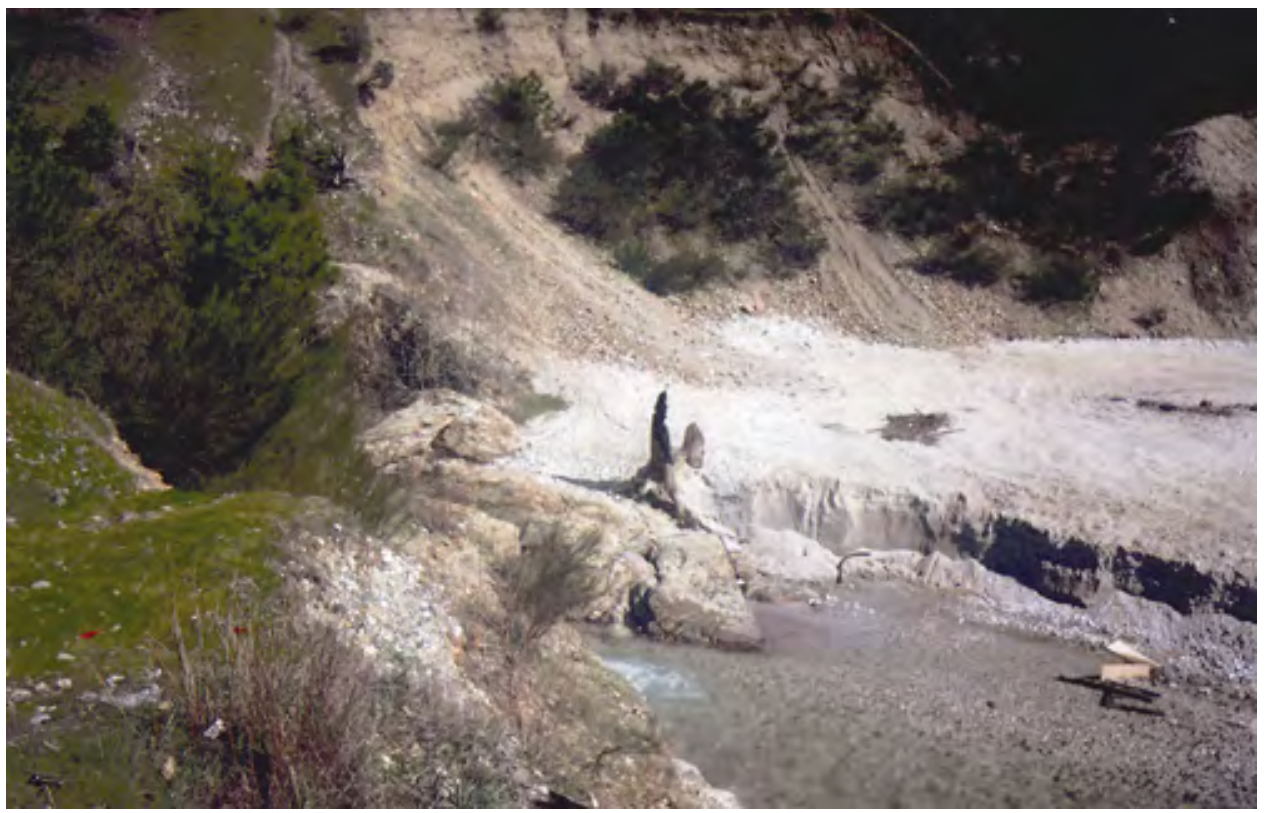

Figure A20. Streambank showing flood incision of gravels, Greenhorn Creek at the Narrows (station BY180), Nevada County, California. 


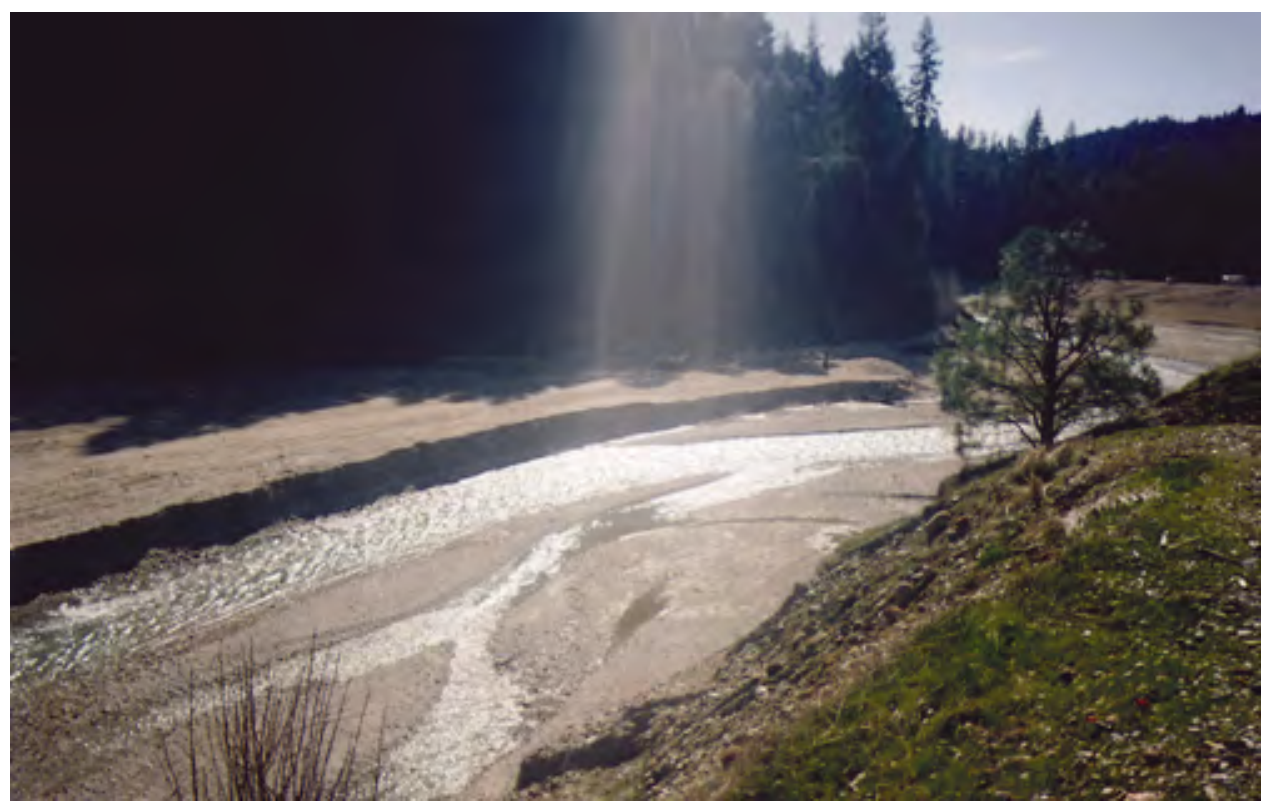

Figure A21. Greenhorn Creek at the Narrows, looking downstream (station BY180), Nevada County, California.

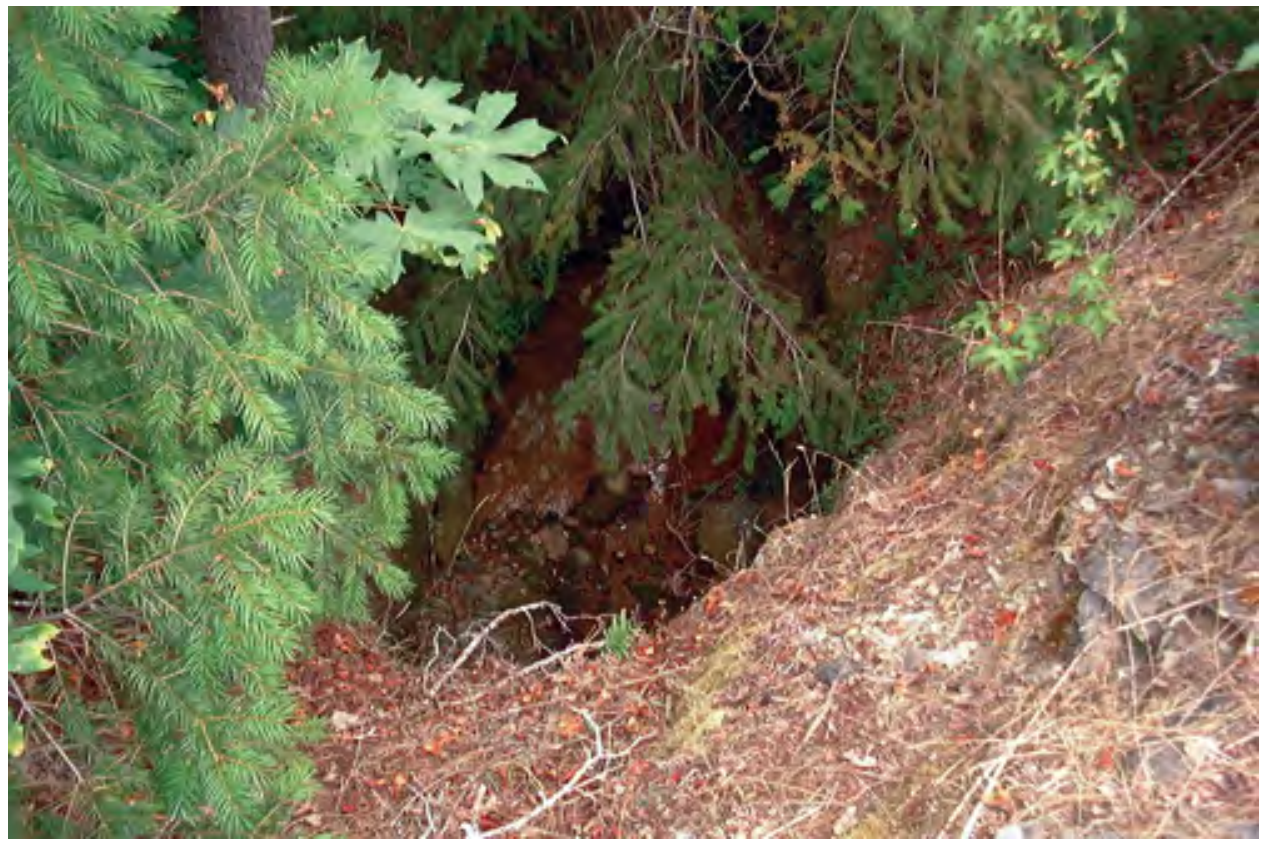

Figure A22. Hunts Hill drainage tunnel outlet, near Buckeye Ford in Greenhorn Creek, Nevada County, California. 


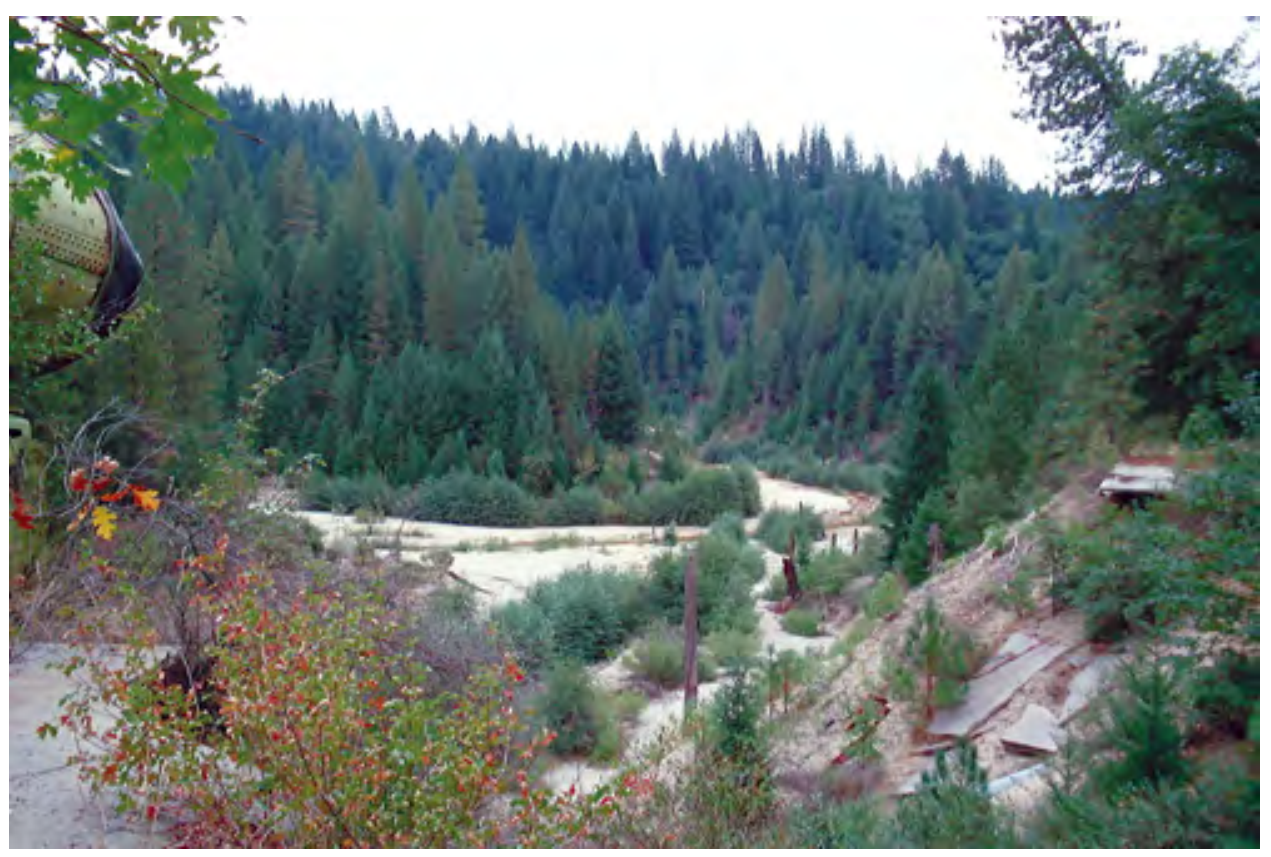

Figure A23. Confluence of Gas Canyon with Greenhorn Creek (station BY52), Nevada County, California.

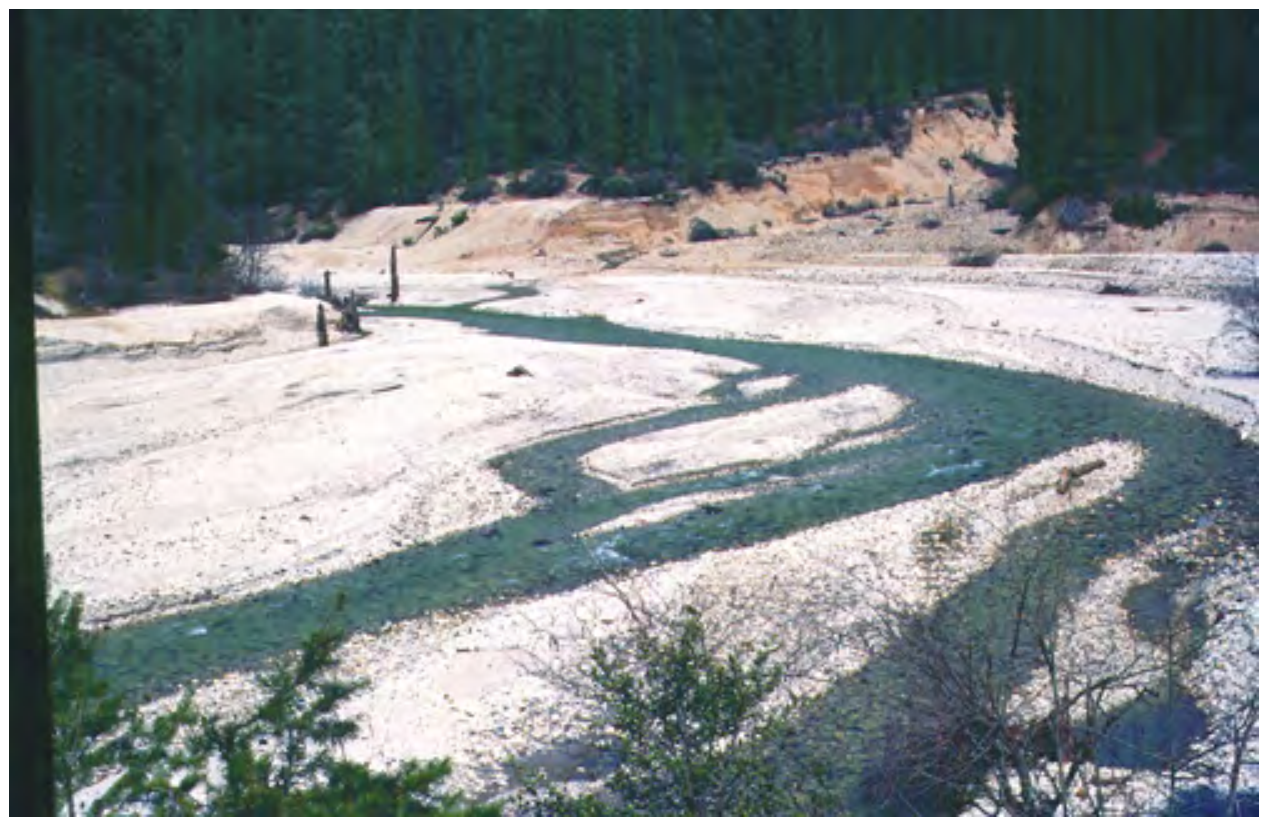

Figure A24. Greenhorn Creek at Buckeye Drain, looking upstream (station BY60), Nevada County, California. 


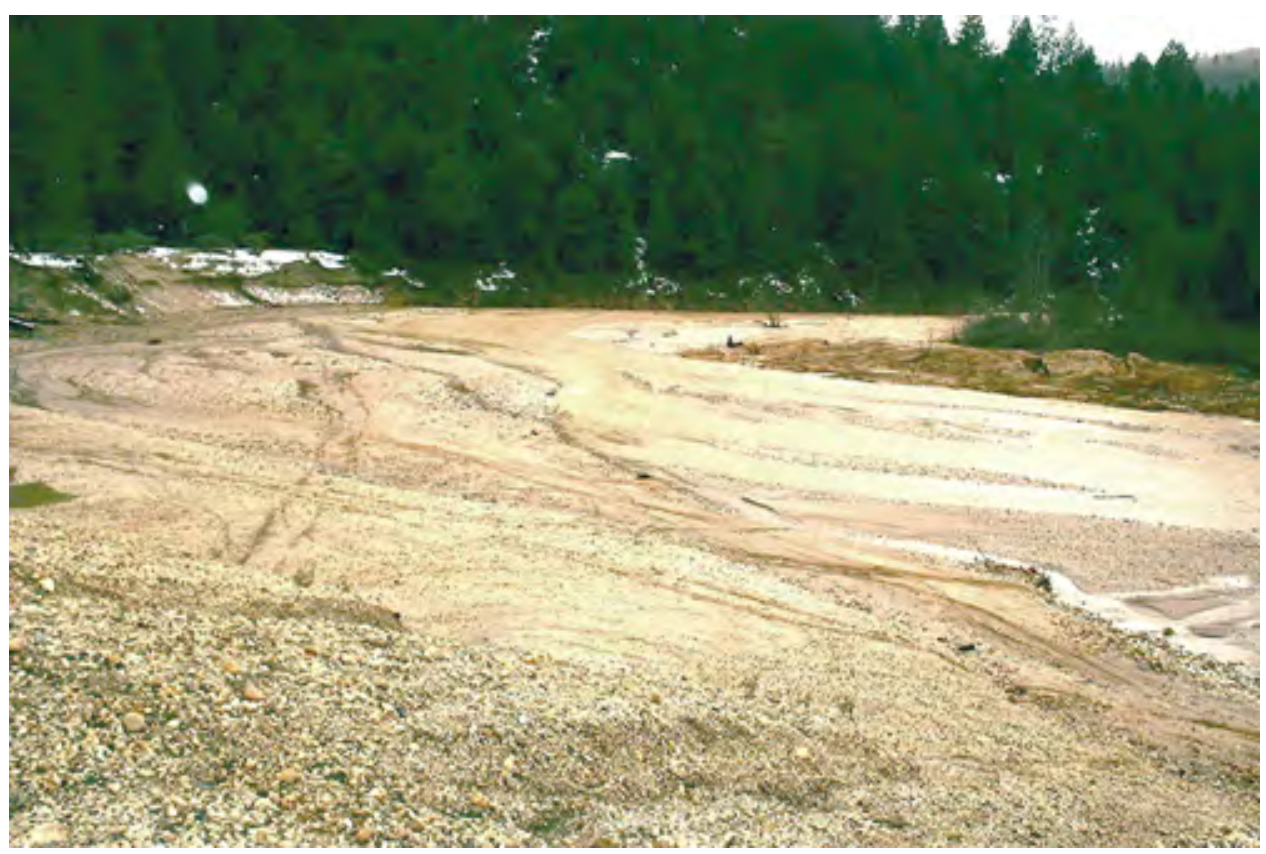

Figure A25. Greenhorn Creek at Red Dog Road crossing, Nevada County, California.

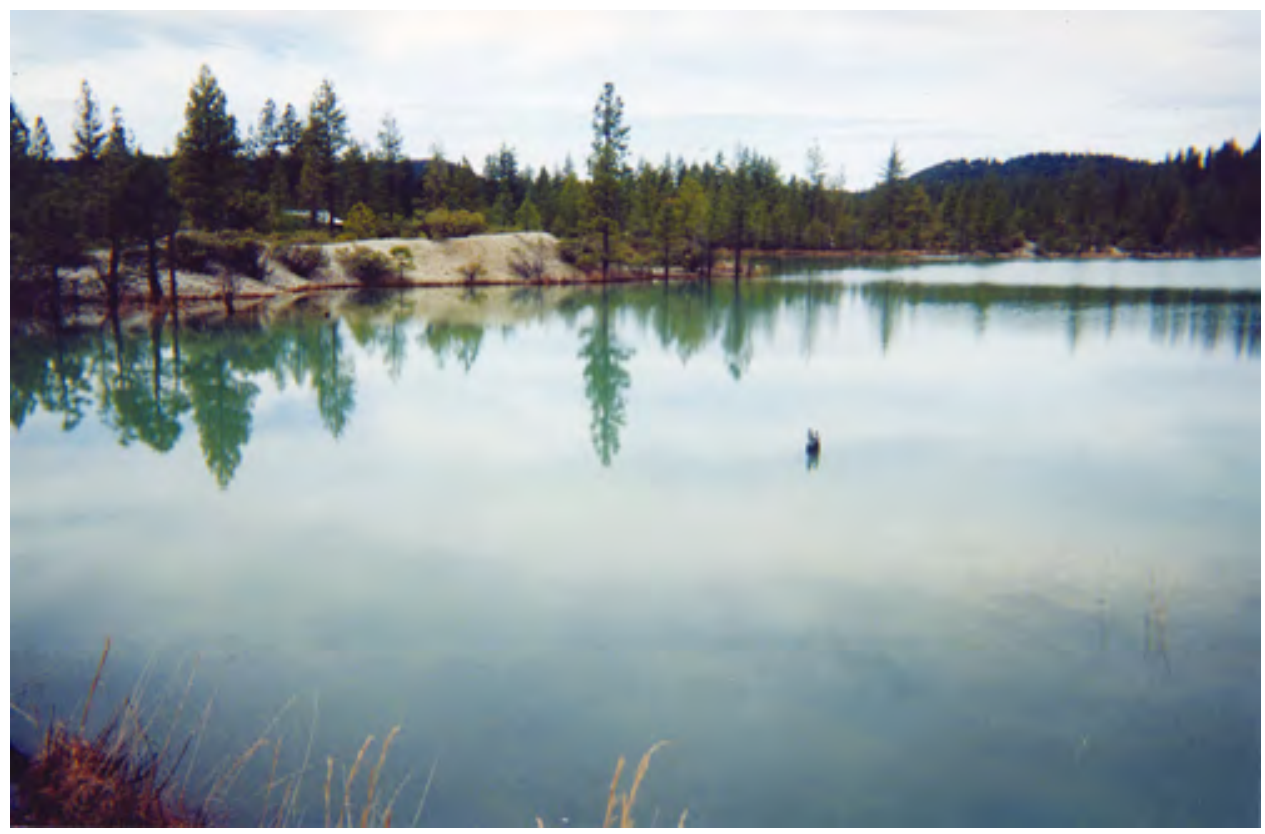

Figure A26. Pit lake at Buckeye Flat Mine, Greenhorn Creek drainage, Nevada County, California. 
INTENTIONALLY BLANK PAGE 
Appendix B. Quality Assurance and Quality Control Plots for Chemical Analyses of Water Samples from the Greenhorn Creek Drainage, Nevada County, California 


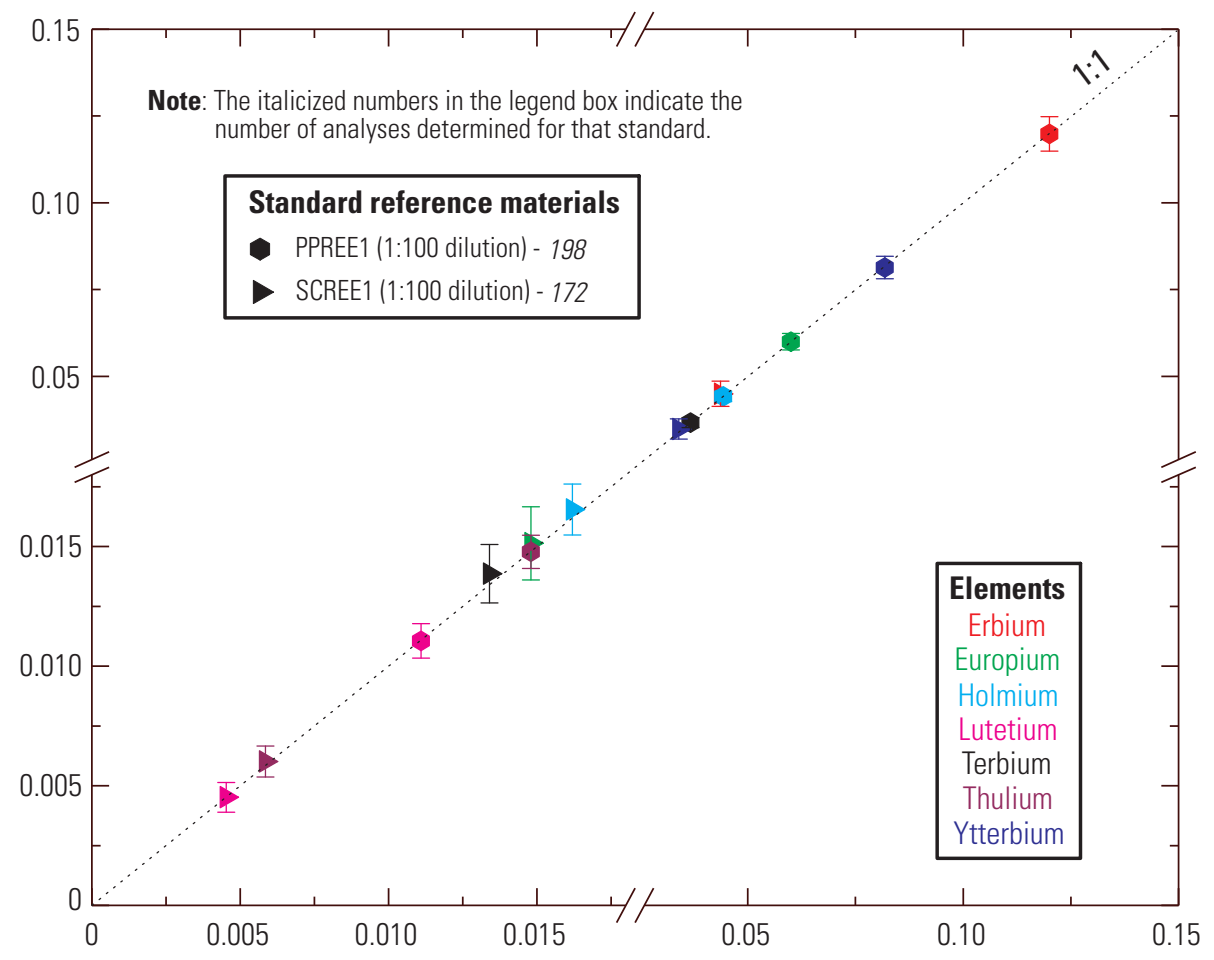

Reported value, in micrograms per liter

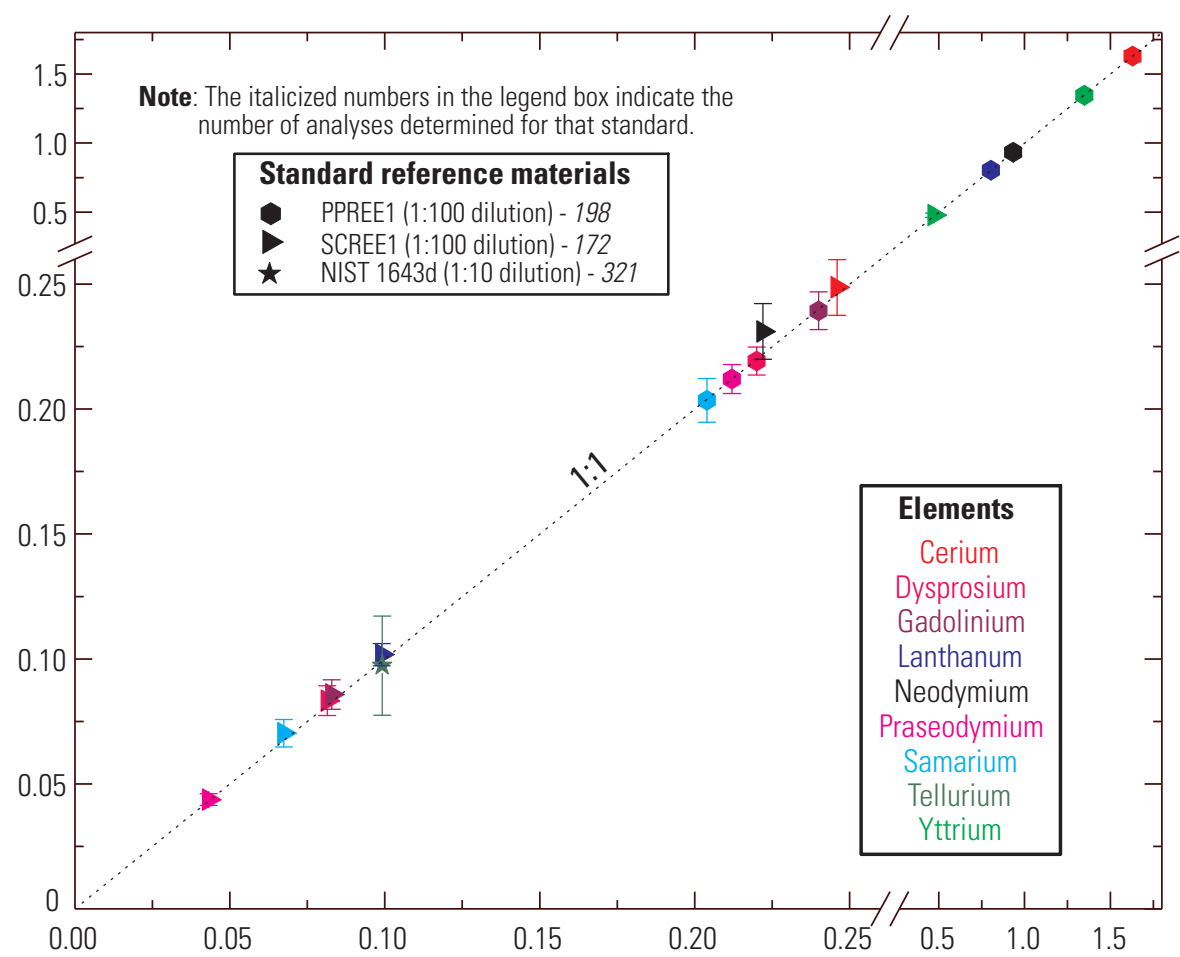

Reported value, in micrograms per liter
Figure B1. Correlation plot of observed values versus reported values for erbium, europium, holmium, lutetium, terbium, thulium, and ytterbium determined in standard reference materials.
Figure B2. Correlation plot of observed values versus reported values for cerium, dysprosium, gadolinium, lanthanum, neodymium, praseodymium, samarium, tellurium, and yttrium determined in standard reference materials. 

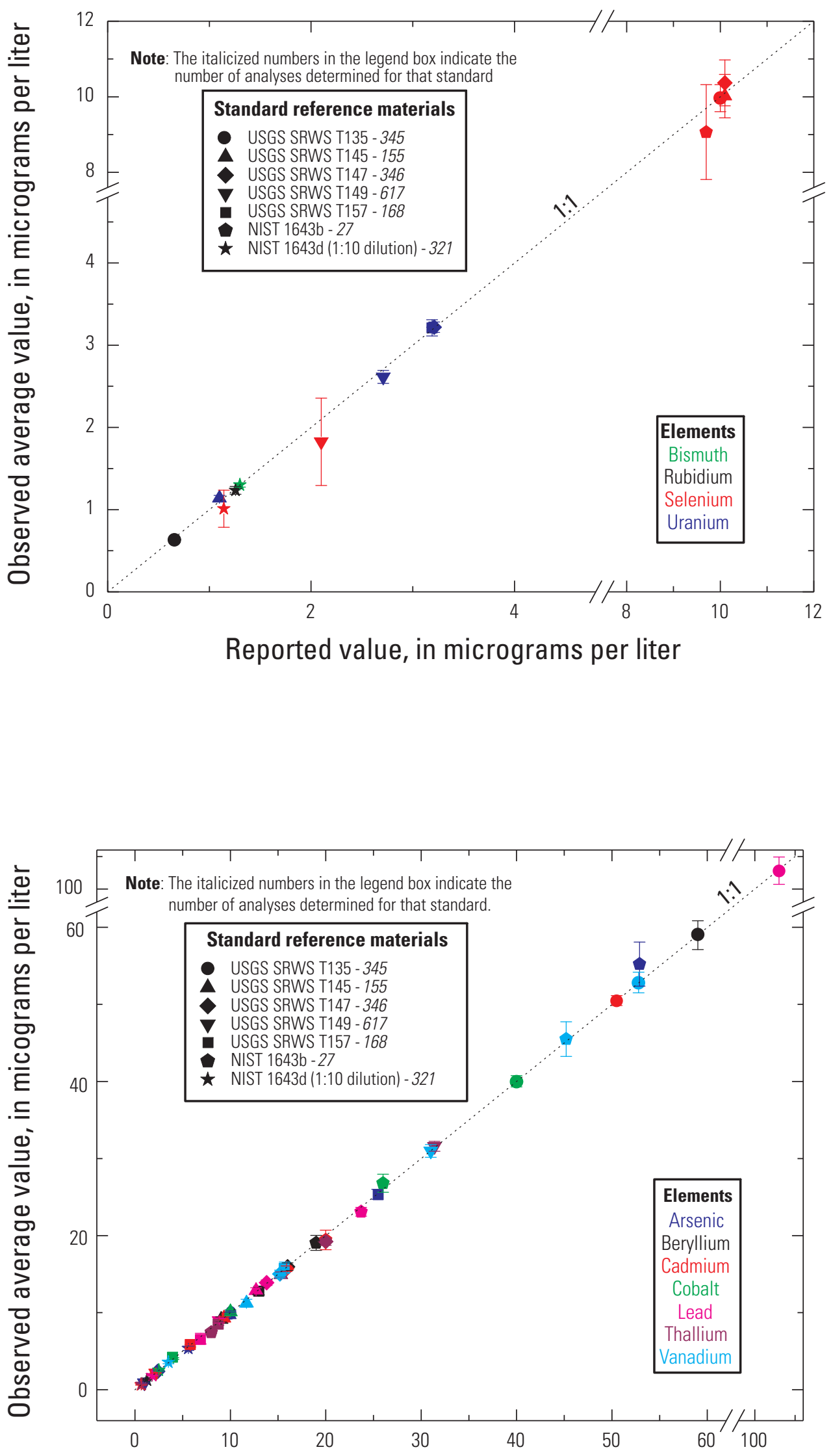

Reported value, in micrograms per liter
Figure B3. Correlation plot of observed values versus reported values for bismuth, rubidium, selenium, and uranium determined in standard reference materials.
Figure B4. Correlation plot of observed values versus reported values for arsenic, beryllium, cadmium, cobalt, lead, thallium, and vanadium determined in standard reference materials. 

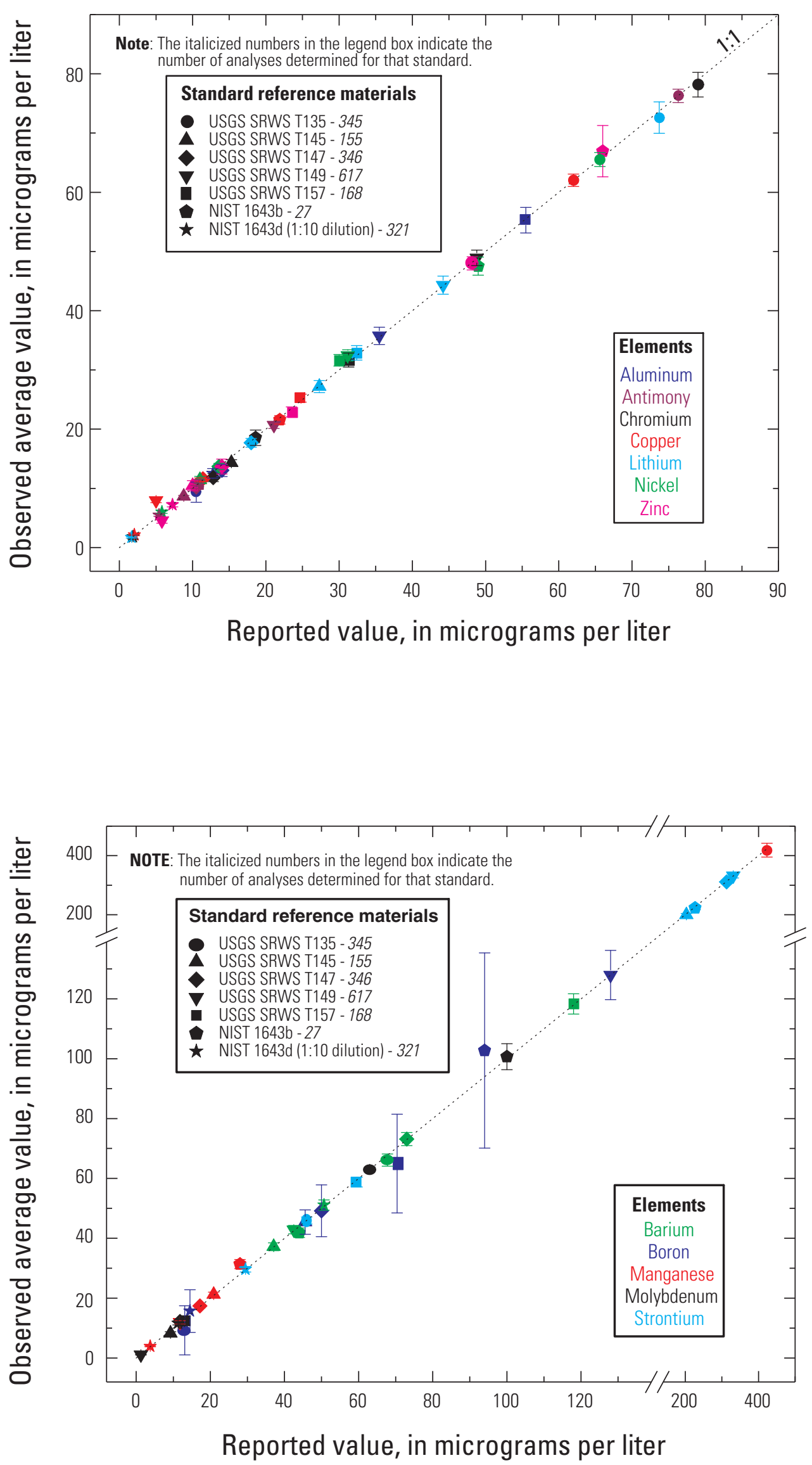

Figure B5. Correlation plot of observed values versus reported values for aluminum, antimony, chromium, copper, lithium, nickel, and zinc determined in standard reference materials.
Figure B6. Correlation plot of observed values versus reported values for barium, boron, manganese, molybdenum, and strontium determined in standard reference materials. 


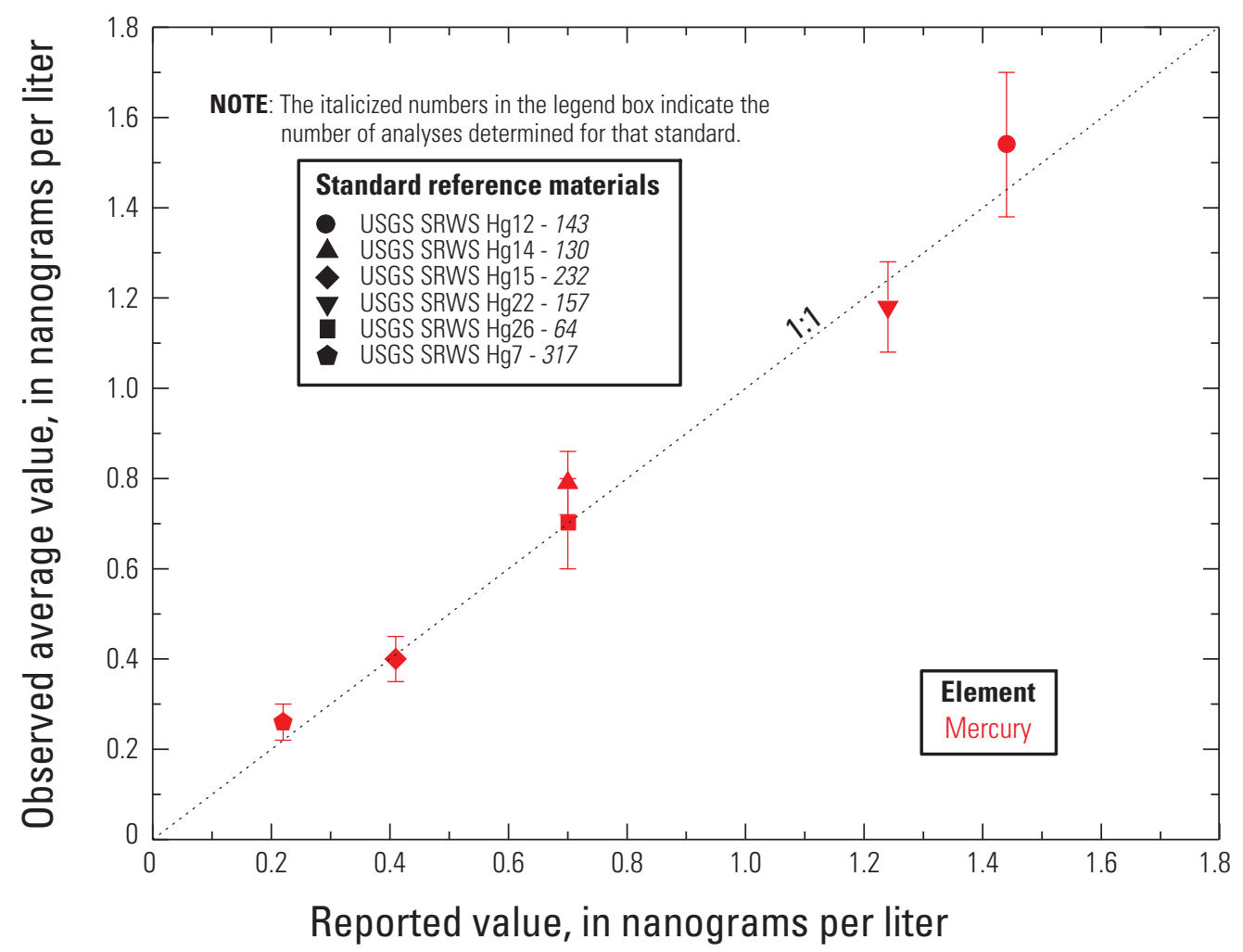

Figure B7. Correlation plot of observed values versus reported values for mercury determined in standard reference materials. 


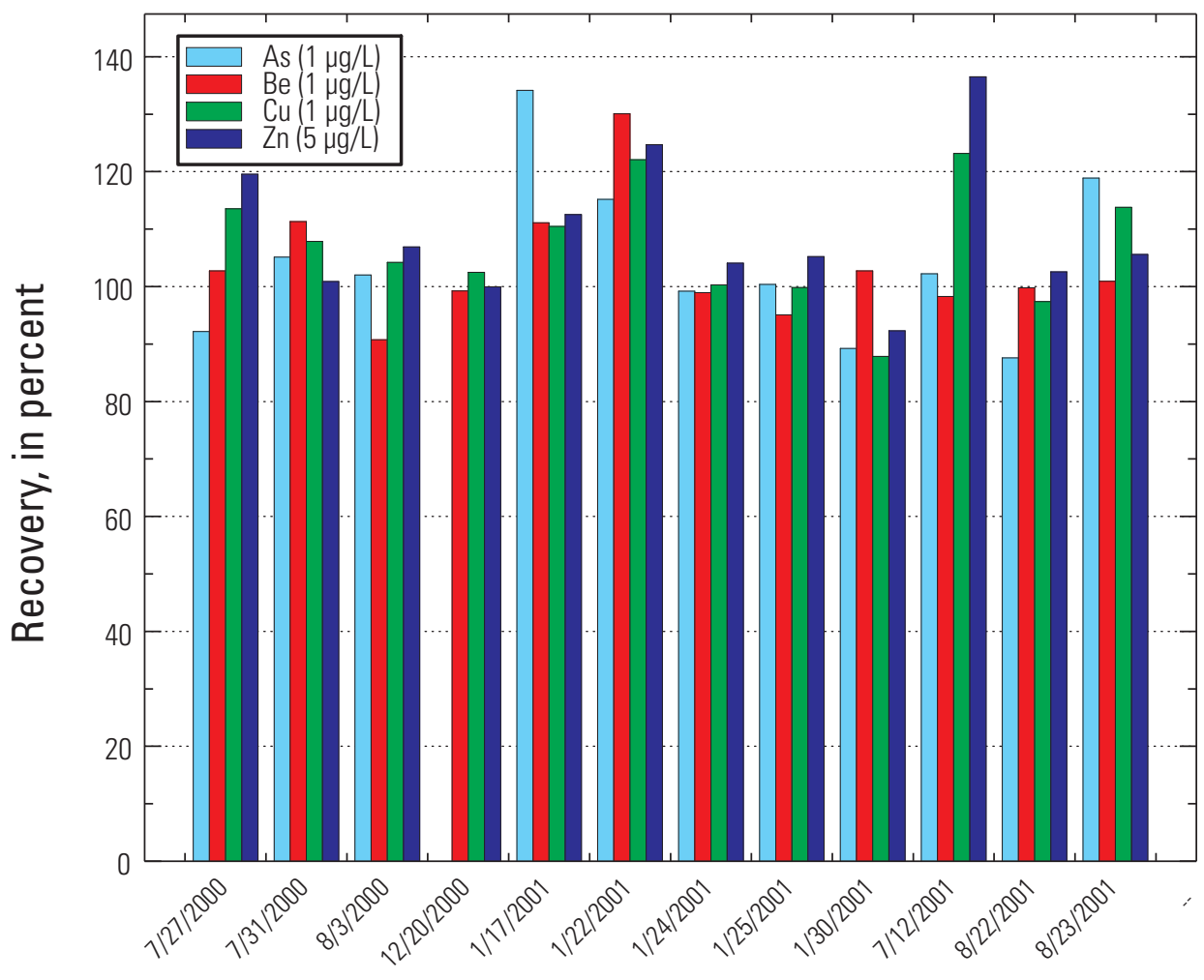

Analysis date

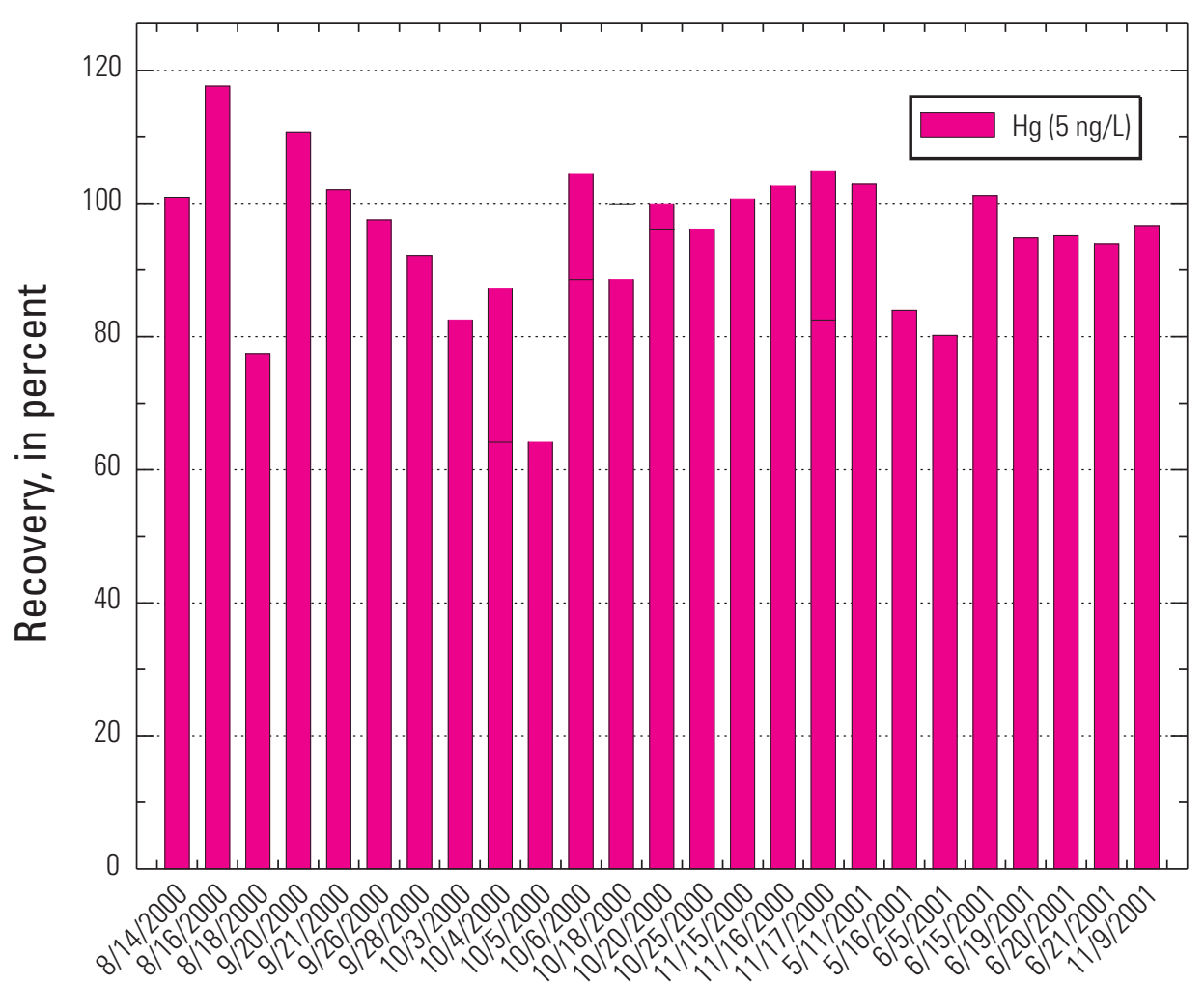

Analysis date
Figure B8. Bar graph plotting recovery (in percent) for arsenic (As), beryllium (Be), copper (Cu), and zinc $(\mathrm{Zn})$ spiked in blanks for selected analysis dates. $\mu \mathrm{g} / \mathrm{L}$, microgram per liter.
Figure B9. Bar graph plotting recovery (in percent) for mercury $(\mathrm{Hg}$ ) spiked in blanks for selected analysis dates. $\mathrm{ng} / \mathrm{L}$, nanogram per liter. 


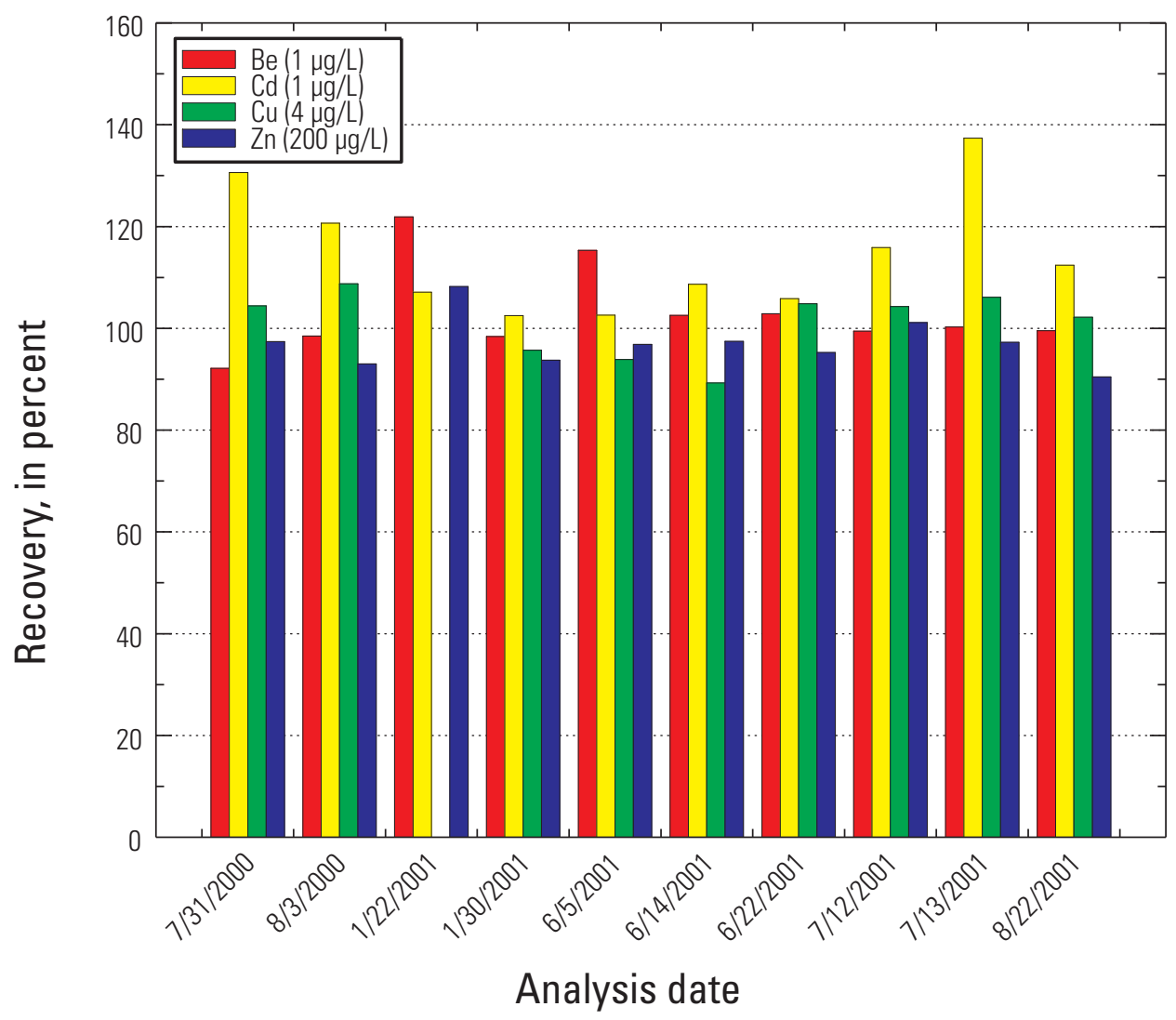

Figure B10. Graph plotting recovery (in percent) for beryllium (Be), cadmium (Cd), copper (Cu), and zinc $(\mathrm{Zn})$ spiked in samples for selected analysis dates. $\mu \mathrm{g} / \mathrm{L}$, microgram per liter. 


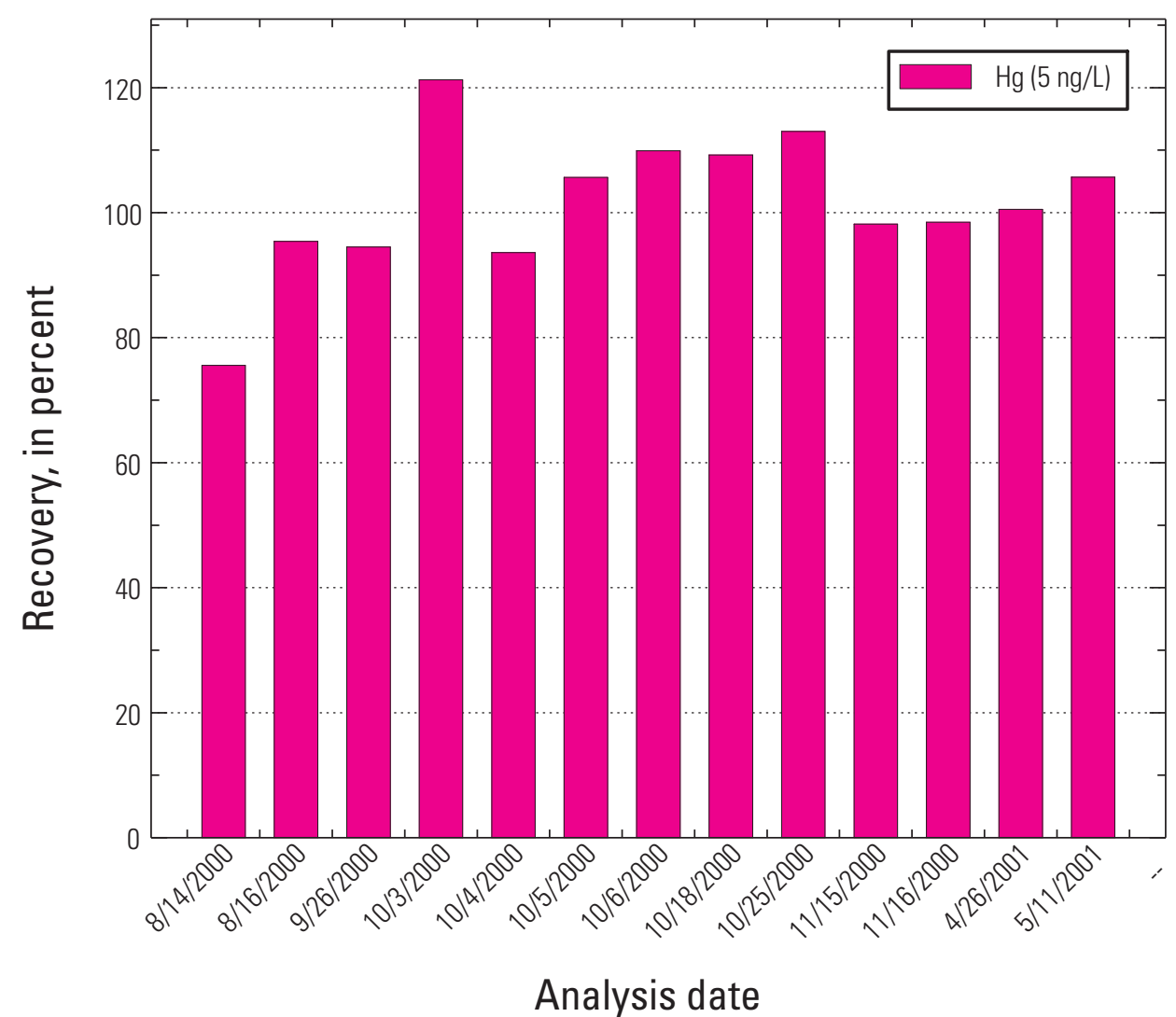

Figure B11. Plotting recovery (in percent) for mercury spiked in samples for selected analysis dates. $\mathrm{ng} / \mathrm{L}$, nanogram per liter. 

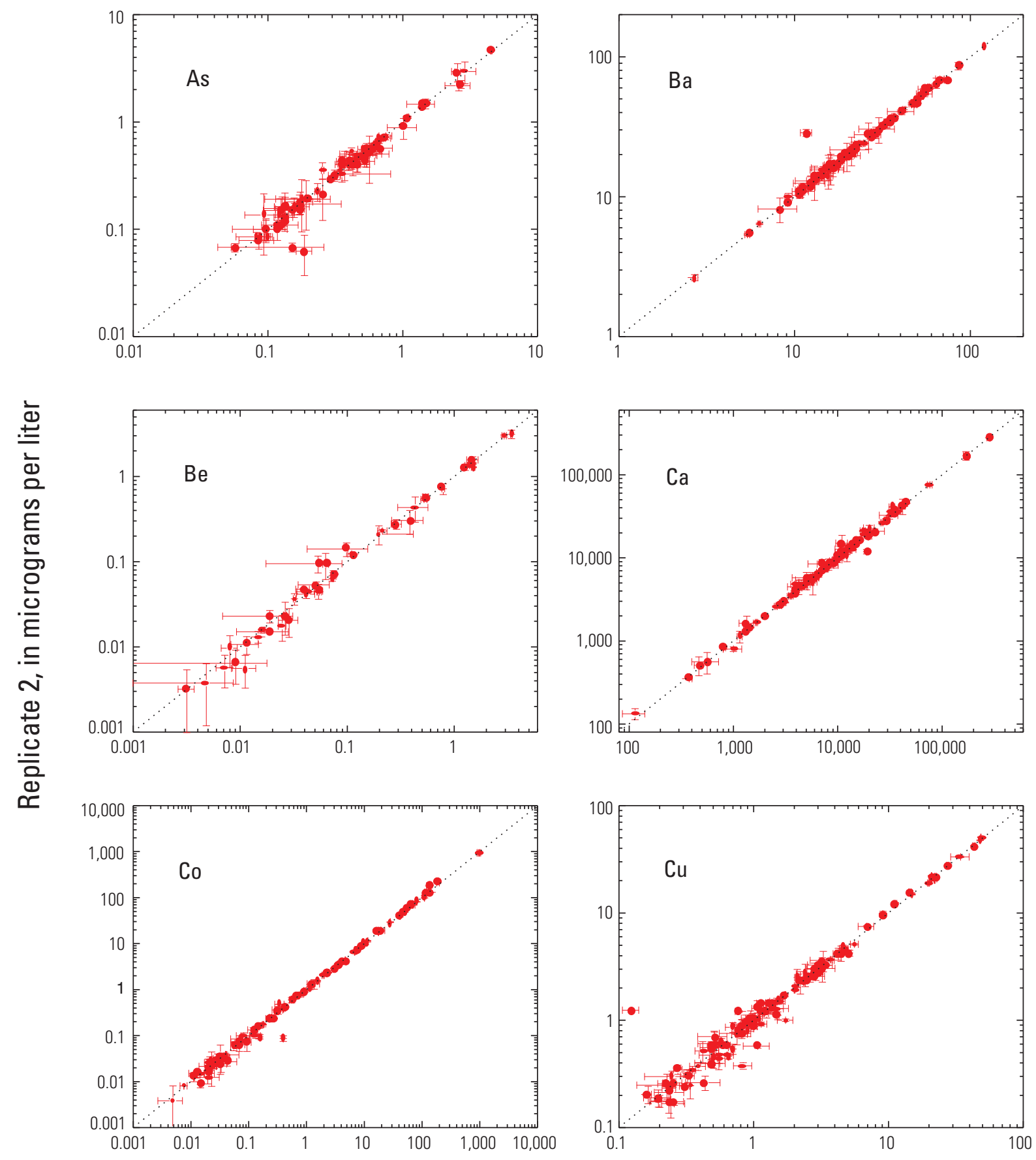

Replicate 1, in micrograms per liter

Figure B12. Field replicate analyses of six elements: arsenic (As), barium (Ba), beryllium (Be), calcium (Ca), cobalt (Co), and copper (Cu). [Each replicate sample analyzed in triplicate; mean value shown with standard deviation.] 

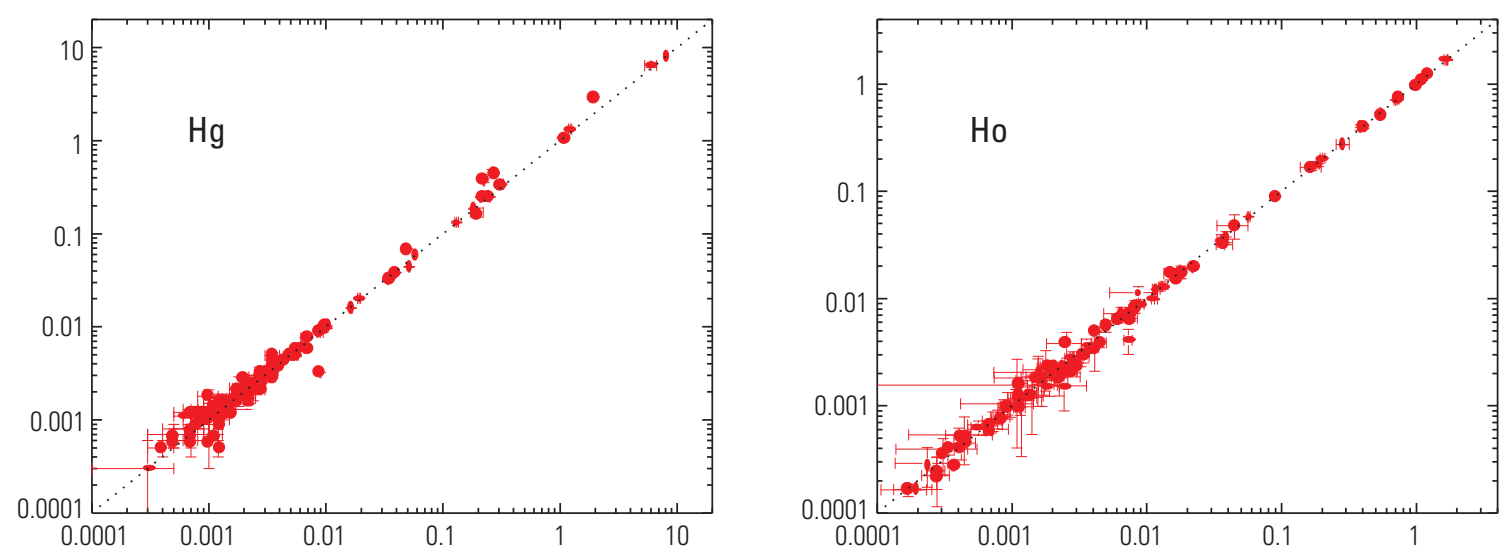

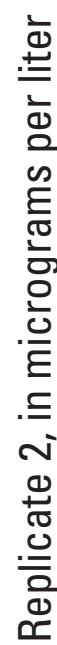
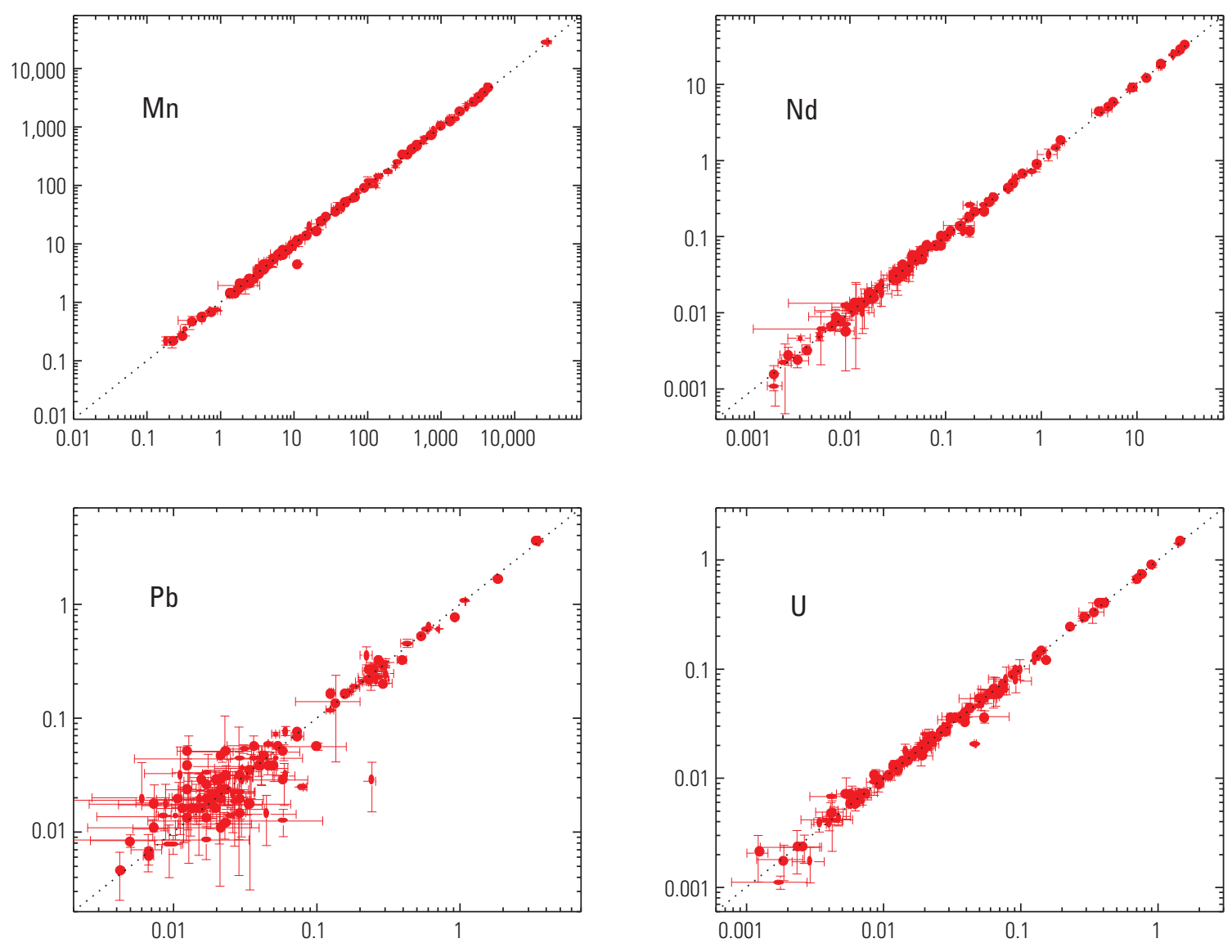

Replicate 1, in micrograms per liter

Figure B13. Correlation plots of field replicate analyses of six elements: mercury (Hg), holmium (Ho), manganese (Mn), neodymium $(\mathrm{Nd})$, lead $(\mathrm{Pb})$, and uranium $(\mathrm{U})$. [Each replicate sample analyzed in triplicate; mean value shown with standard deviation.] 

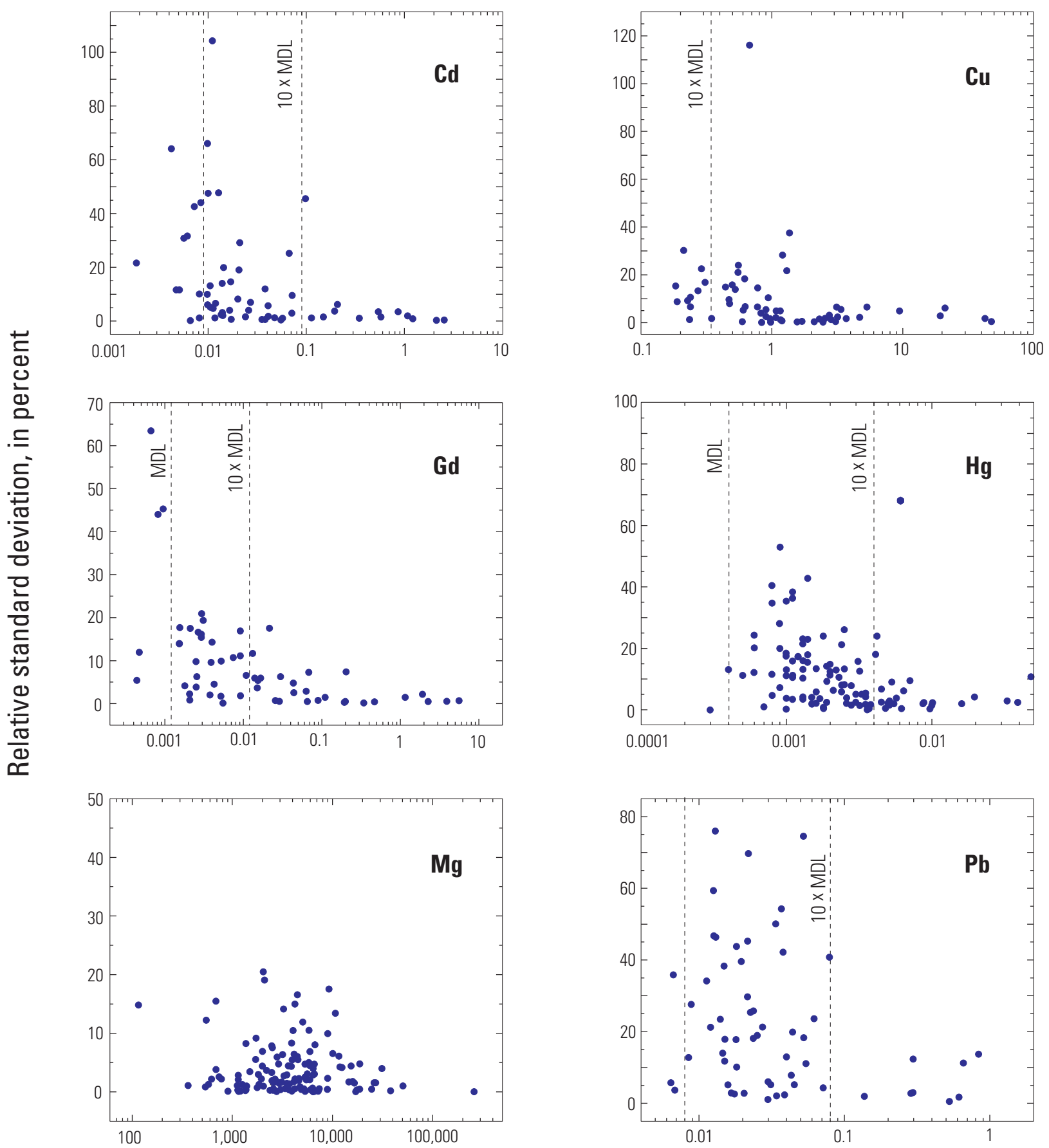

Concentration, in micrograms per liter

Figure B14. Percent relative standard deviation versus concentration for six elements: cadmium (Cd), copper (Cu), gadolinium $(\mathrm{Gd})$, mercury $(\mathrm{Hg})$, magnesium $(\mathrm{Mg})$, and lead $(\mathrm{Pb})$. MDL, method detection limit. 


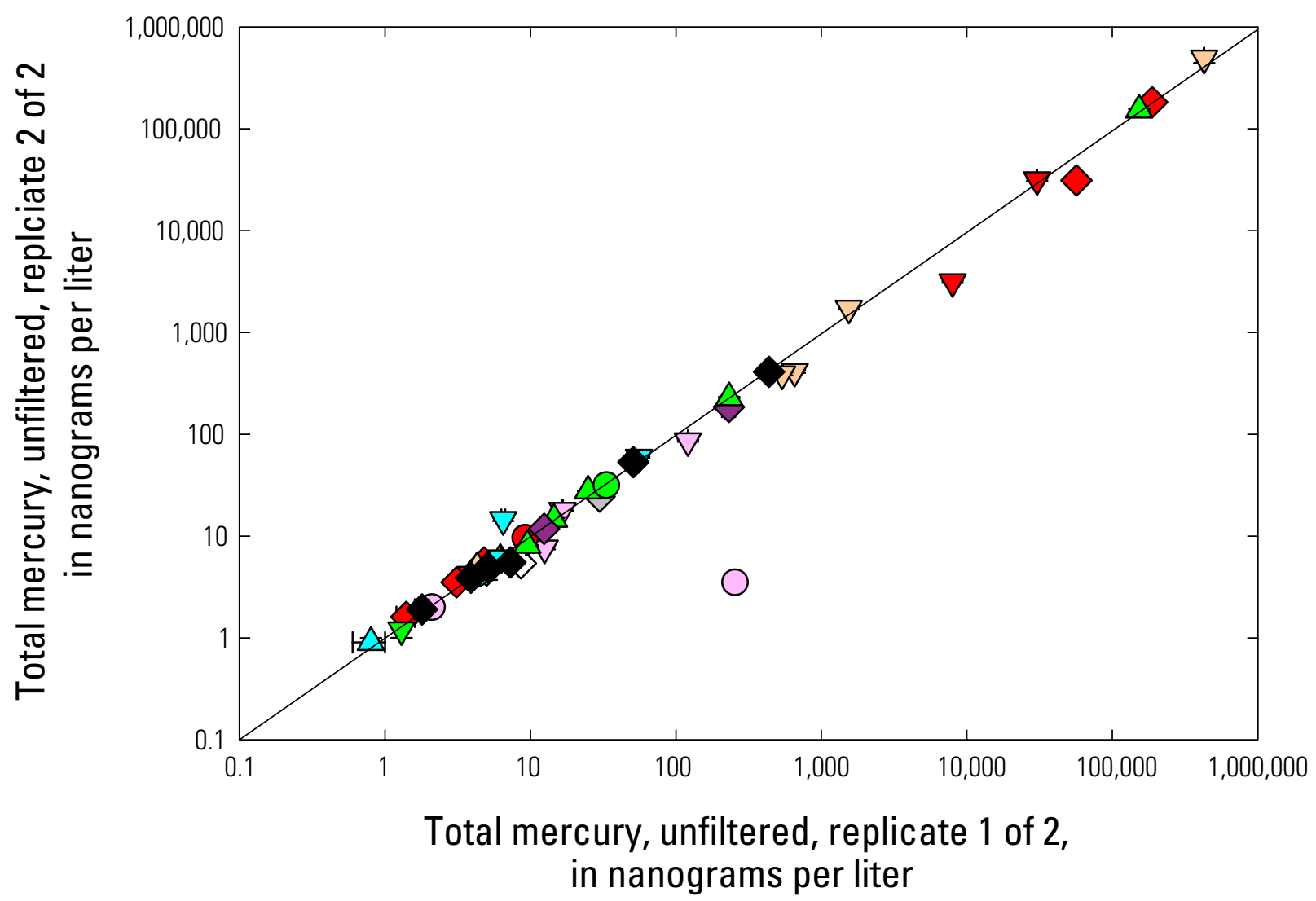

EXPLANATION

\begin{tabular}{lll}
\multicolumn{1}{c}{ Station group } & \multicolumn{2}{c}{ Type of sampling station } \\
$\square$ Boston & $\square$ Missouri Canyon & $\triangle$ Ground sluice \\
Buckeye & $\bigcirc$ Poore & $\bigcirc$ Pit lake/pond/wetland \\
$\square$ Greenhorn, main stem & SF Greenhorn & $\diamond$ Stream \\
$\square$ Headwaters & $\square$ Sailor Flat & $\nabla$ Tunnel \\
Integrator & $\square$ Starr &
\end{tabular}

Figure B15. Total mercury concentrations in unfiltered water showing relation of replicate 1 of 2 and replicate 2 of 2. [Each replicate analyzed in triplicate; mean value shown with standard deviation.] 


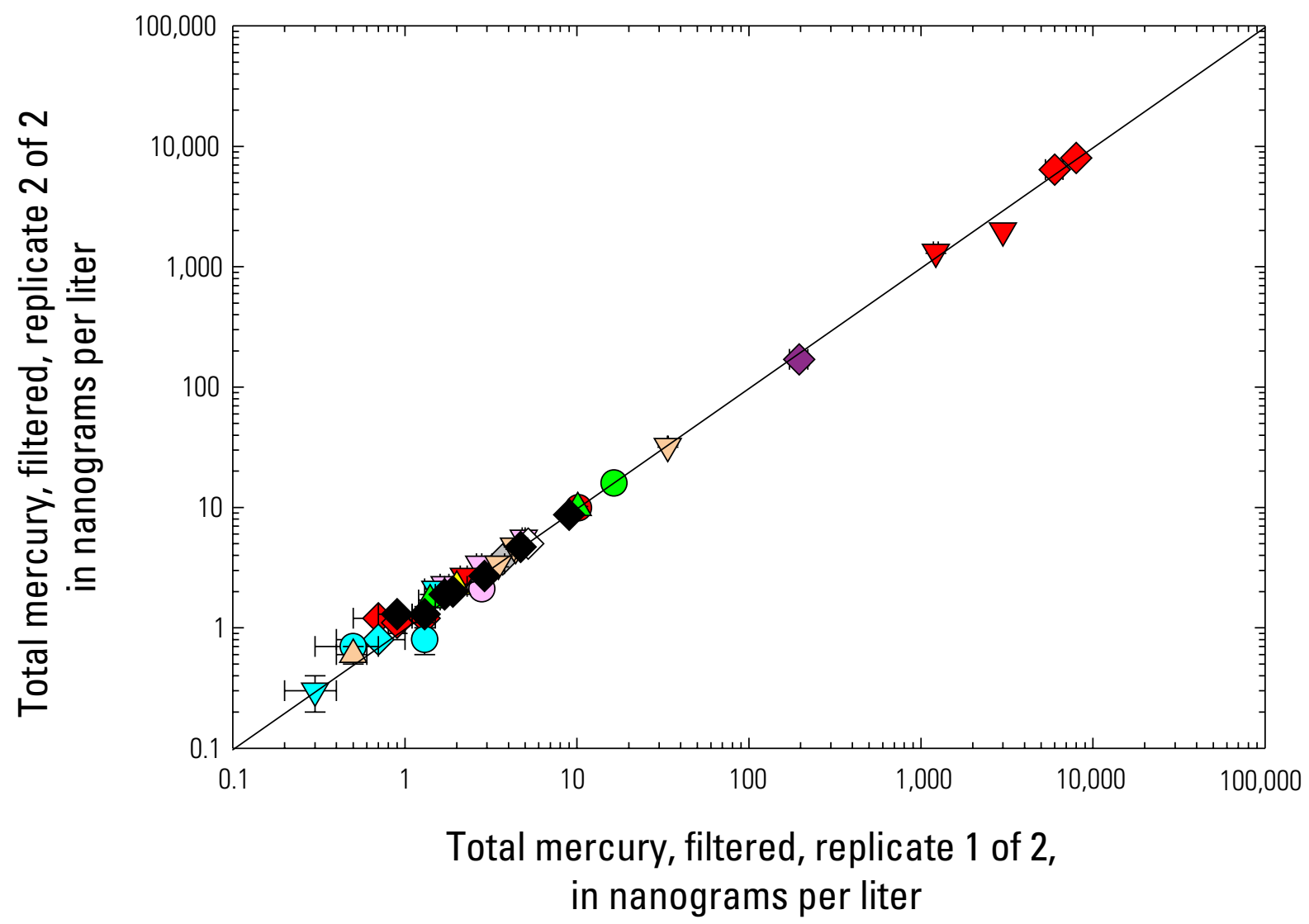

EXPLANATION

\begin{tabular}{llll}
\multicolumn{1}{c}{ Station group } & \multicolumn{2}{c}{ Type of sampling station } \\
$\square$ Boston & $\square$ Missouri Canyon & $\triangle$ Ground sluice \\
Buckeye & & $\bigcirc$ Pit lake/pond/wetland \\
$\square$ Greenhorn, main stem & $\square$ SF Greenhorn & $\triangle$ Stream \\
$\square$ Headwaters & $\square$ Sailor Flat & $\nabla$ Tunnel \\
Integrator & $\square$ Starr &
\end{tabular}

Figure B16. Total mercury concentrations in filtered water showing relation of replicate 1 of 2 and replicate 2 of 2. [Each replicate analyzed in triplicate; mean value shown with standard deviation.] 


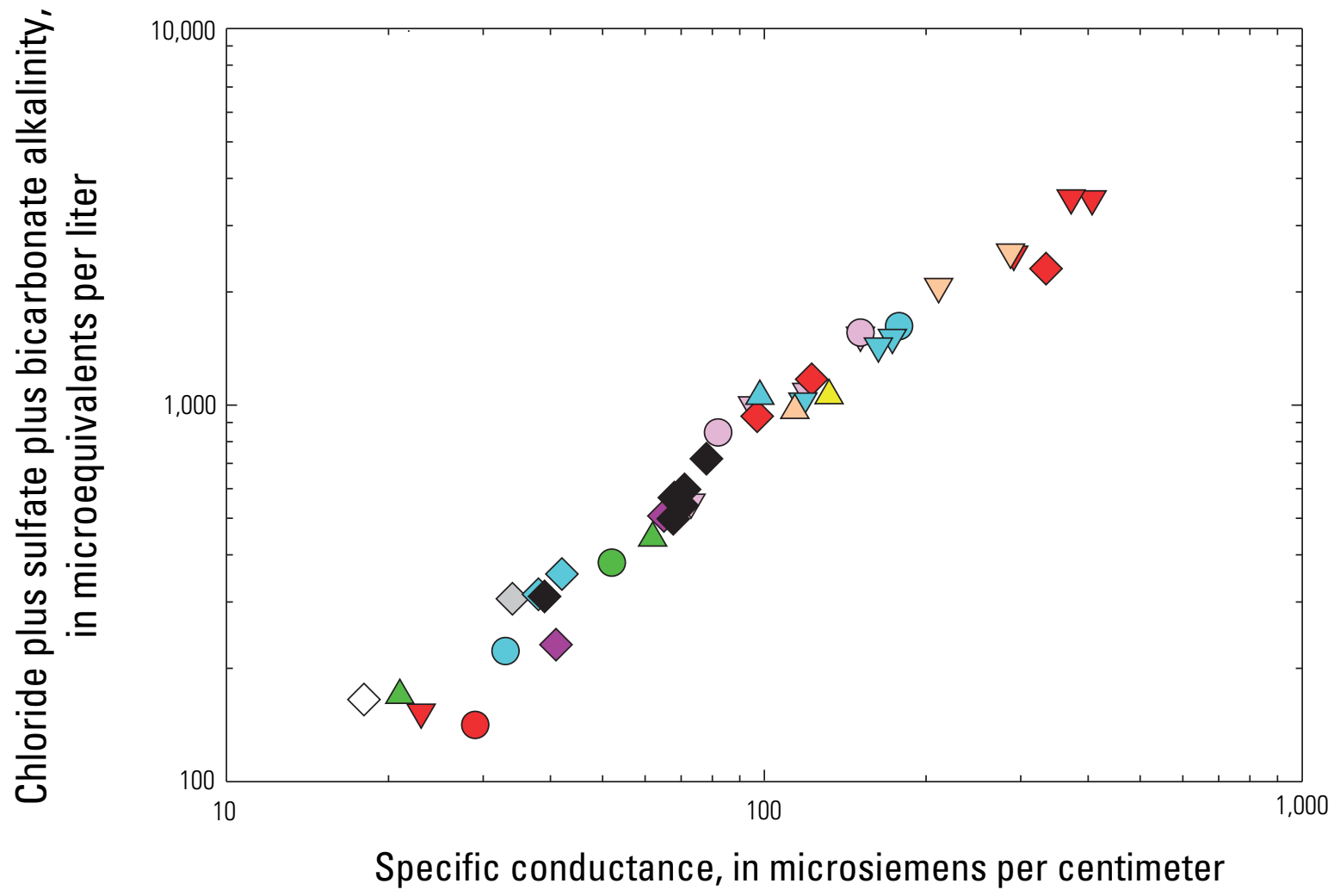

\section{EXPLANATION}

\begin{tabular}{lll}
\multicolumn{1}{c}{ Station group } & \multicolumn{2}{c}{ Type of sampling station } \\
$\square$ Boston & $\square$ Integrator & $\triangle$ Ground sluice \\
Buckeye & $\square$ Poore & $\bigcirc$ Pit lake/pond/wetland \\
$\square$ Greenhorn-Main stem & $\square$ SF Greenhorn & $\searrow$ Stream \\
$\square$ Missouri Canyon & $\square$ Sailor Flat & $\nabla$ Tunnel \\
$\square$ Headwaters & $\square$ Starr &
\end{tabular}

Figure B17. Relation of specific conductance with sum of microequivalent concentrations of chloride, sulfate, and bicarbonate alkalinity. 


\section{Appendix C. Locations and Characteristics of Sampling Stations and Tunnels, Greenhorn Creek Drainage, Nevada County, California}




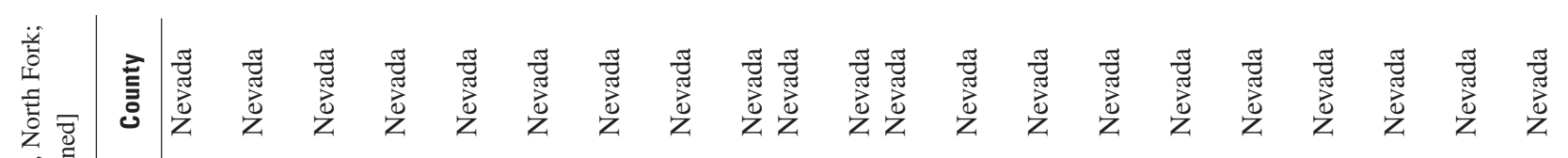

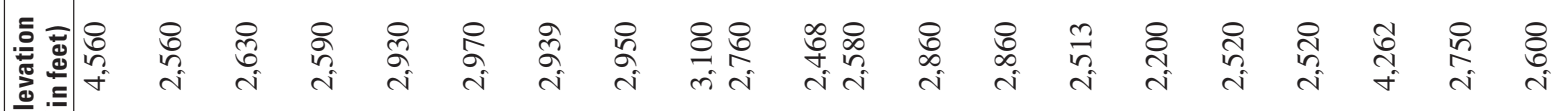

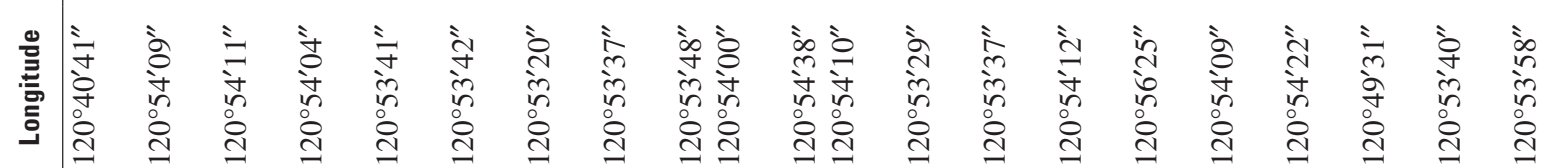

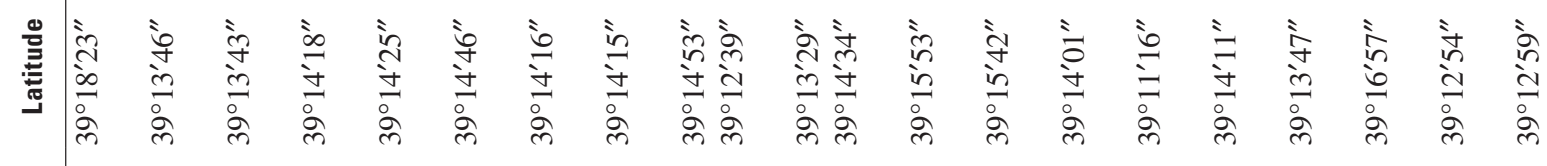

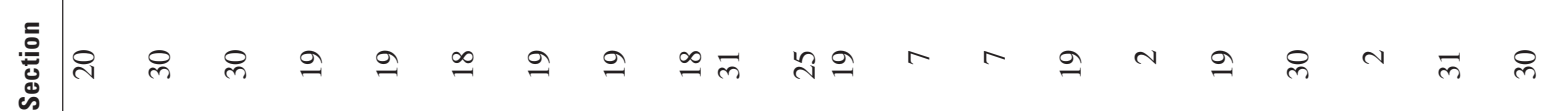
急

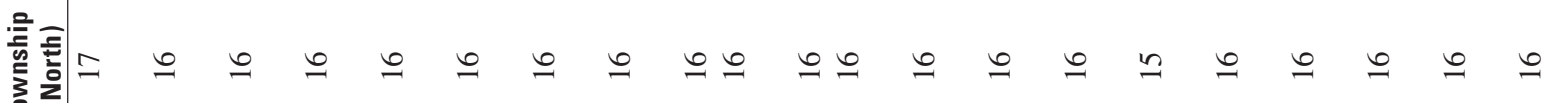

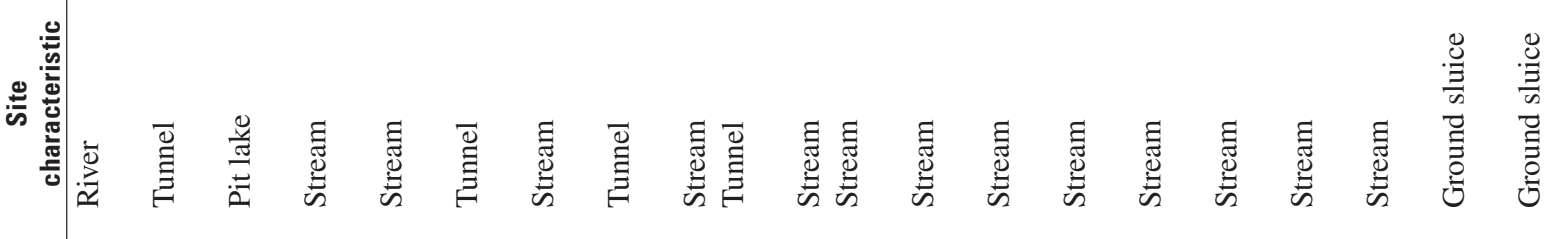

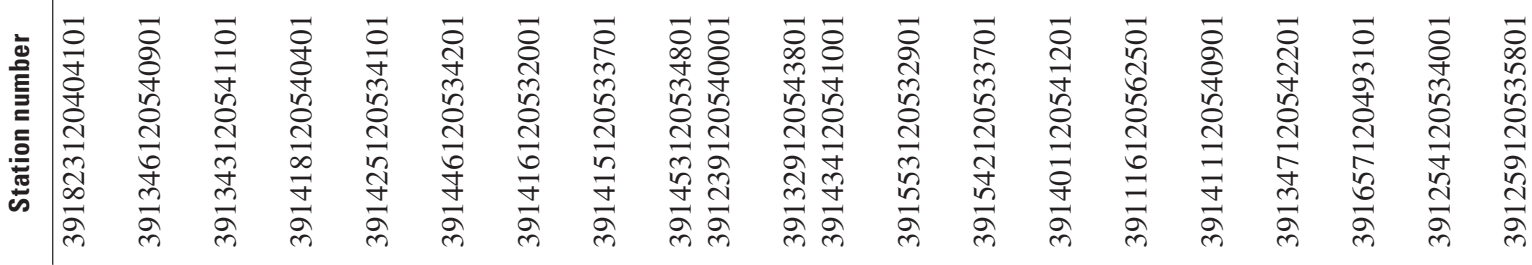

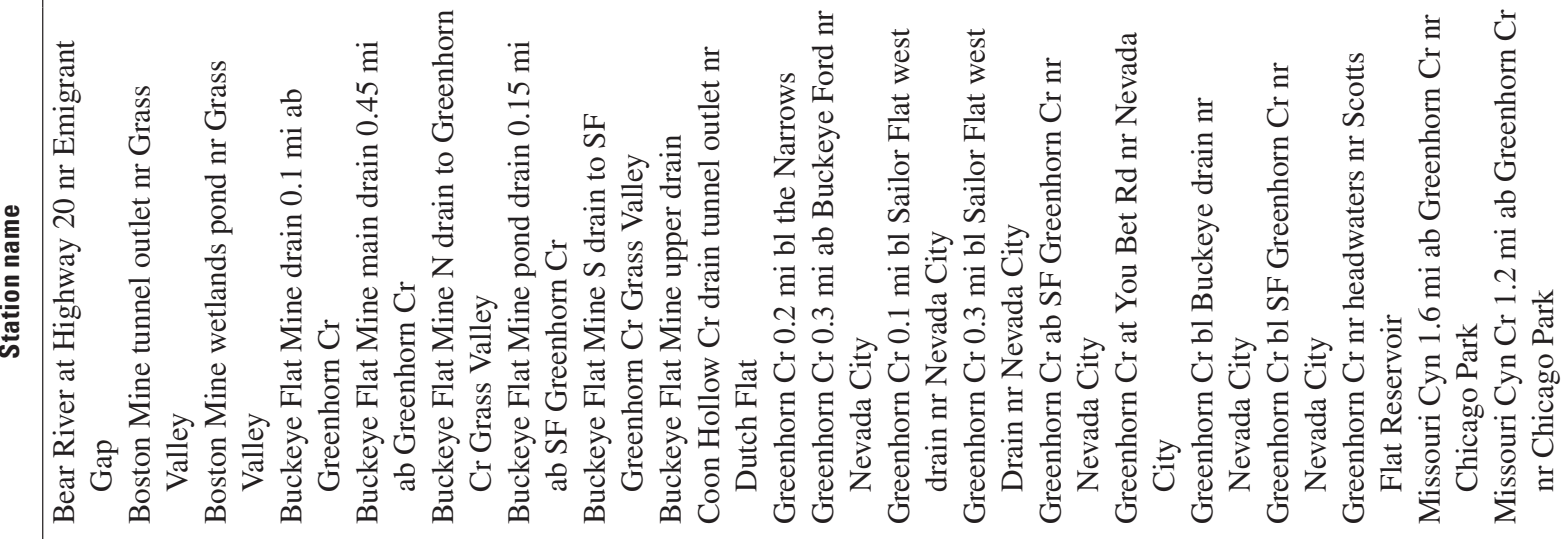

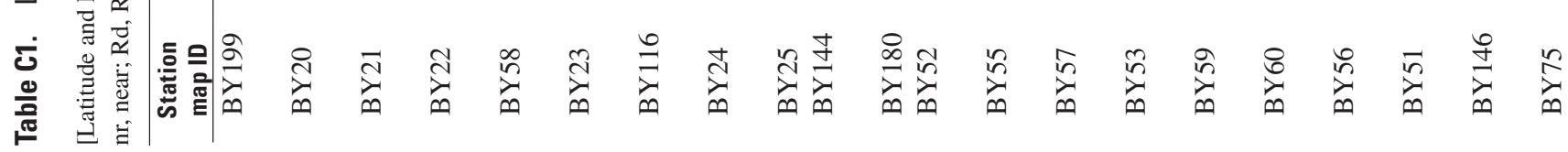




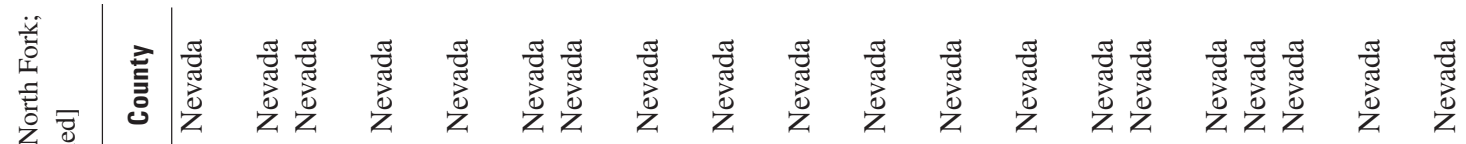

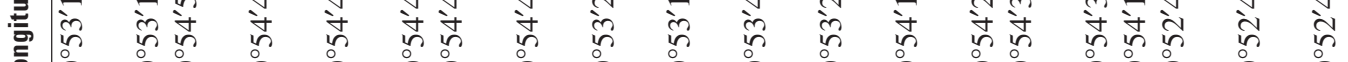
ప

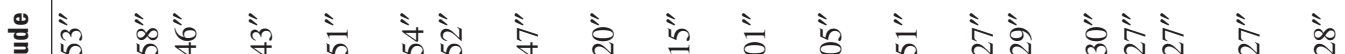

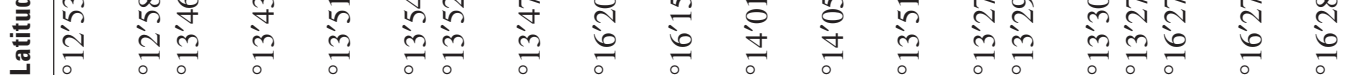

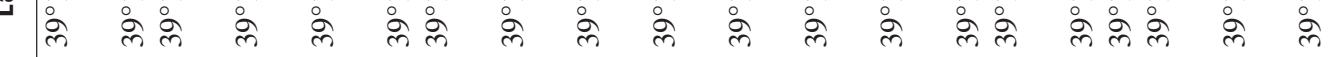
递 $\bar{m}$ 旁 密을

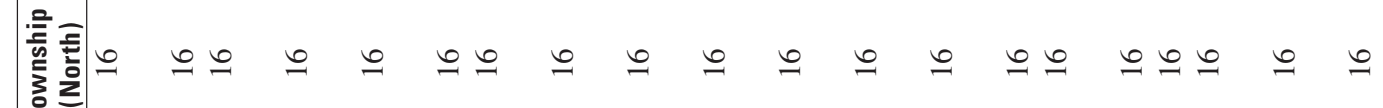
政 

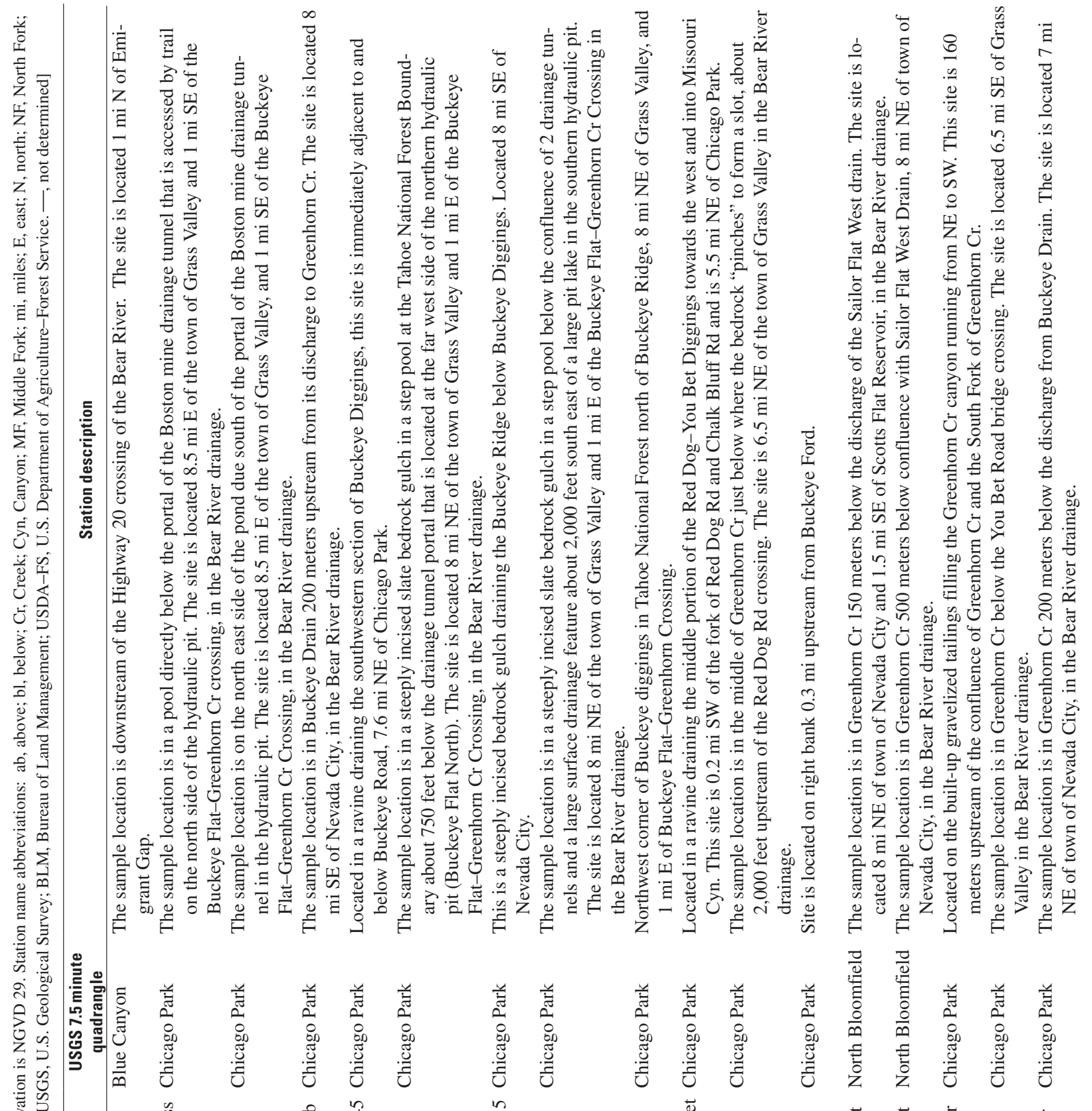


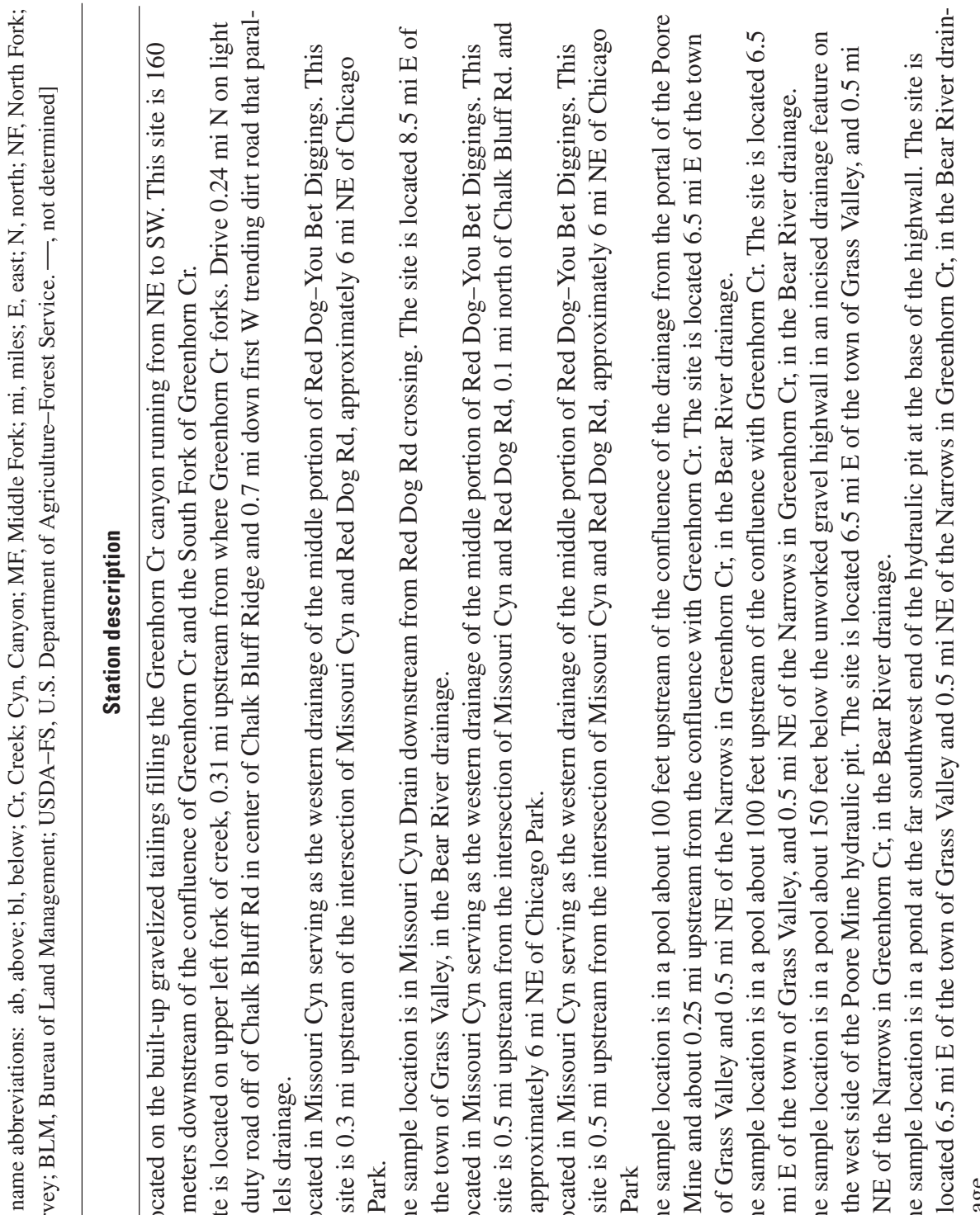

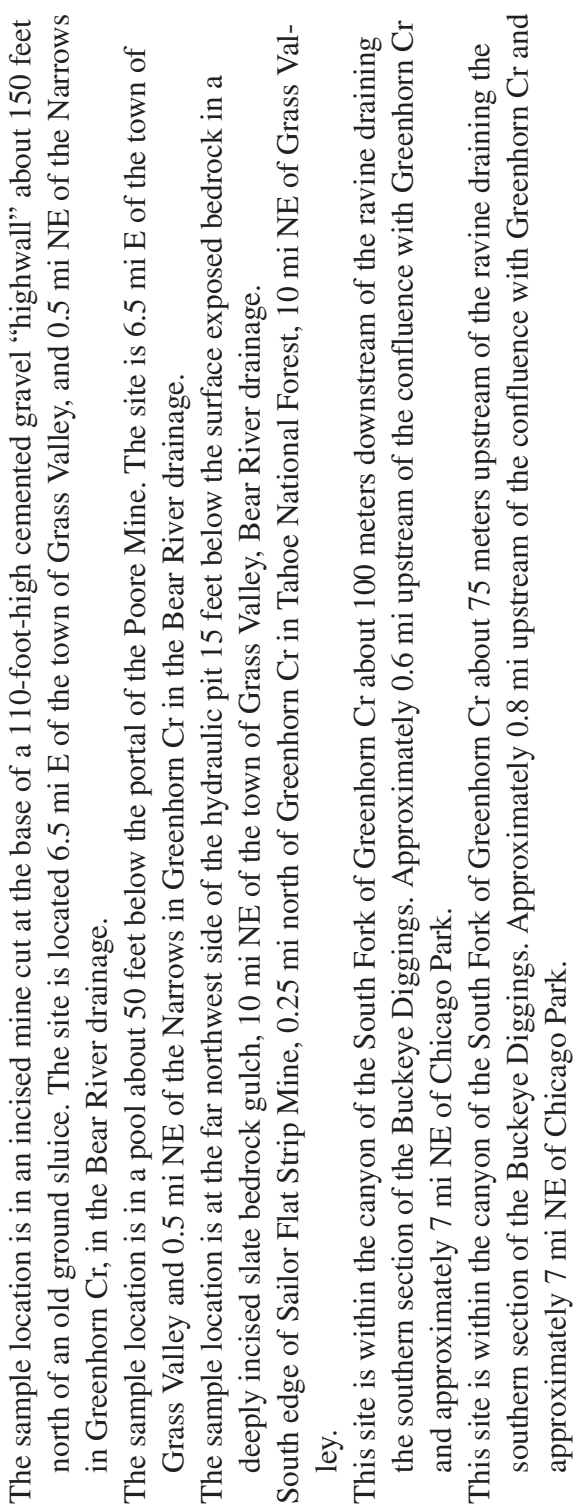

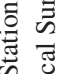

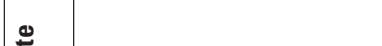

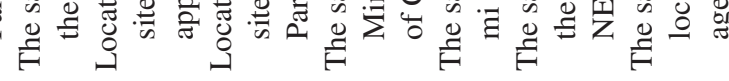

ते

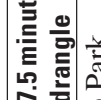

흘

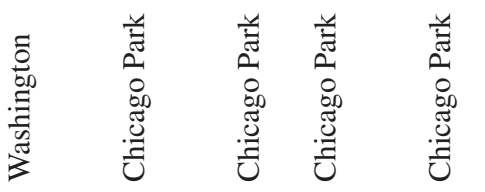

范

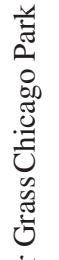

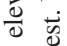

कु

चु चै

U

$\stackrel{\hat{\imath}}{3}$

艺

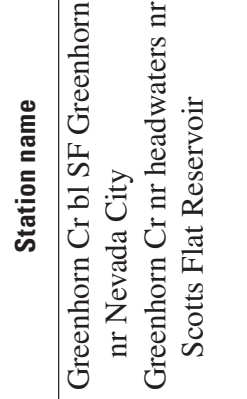

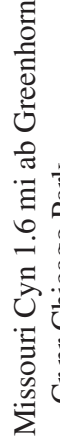

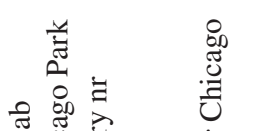

节苞密

吾

离 क्ष

党

造 in

范

तุ

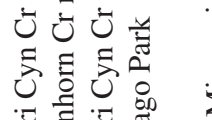

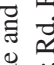

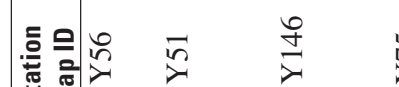

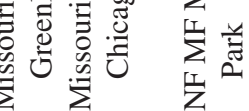

咅

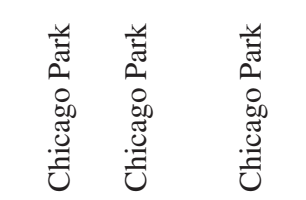

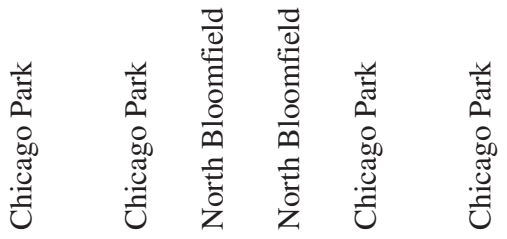

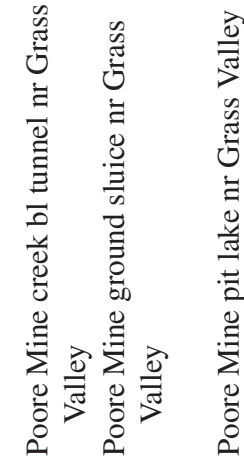

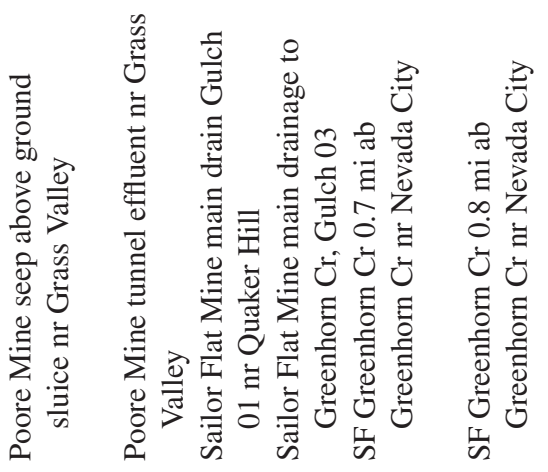

(1)

के

里守

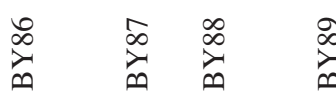

$\infty$

ஓ 


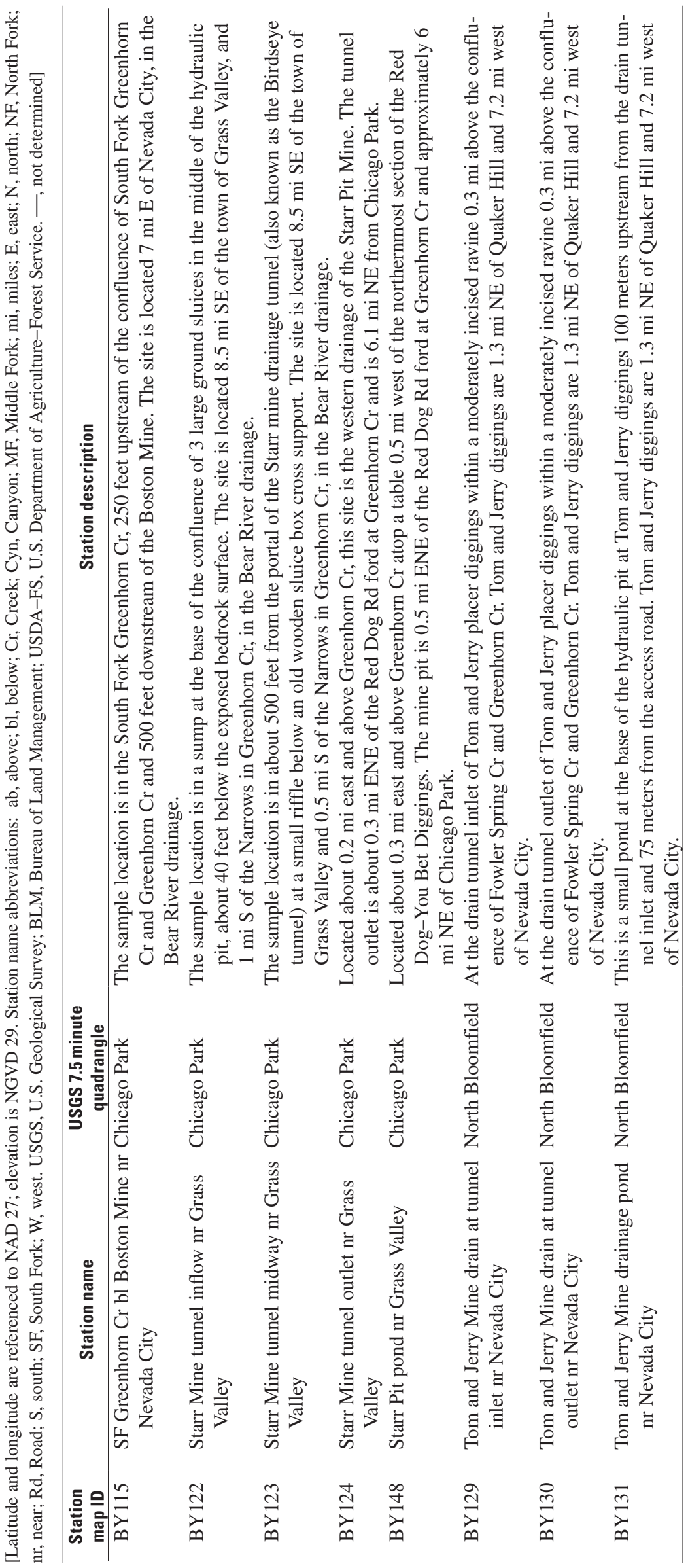




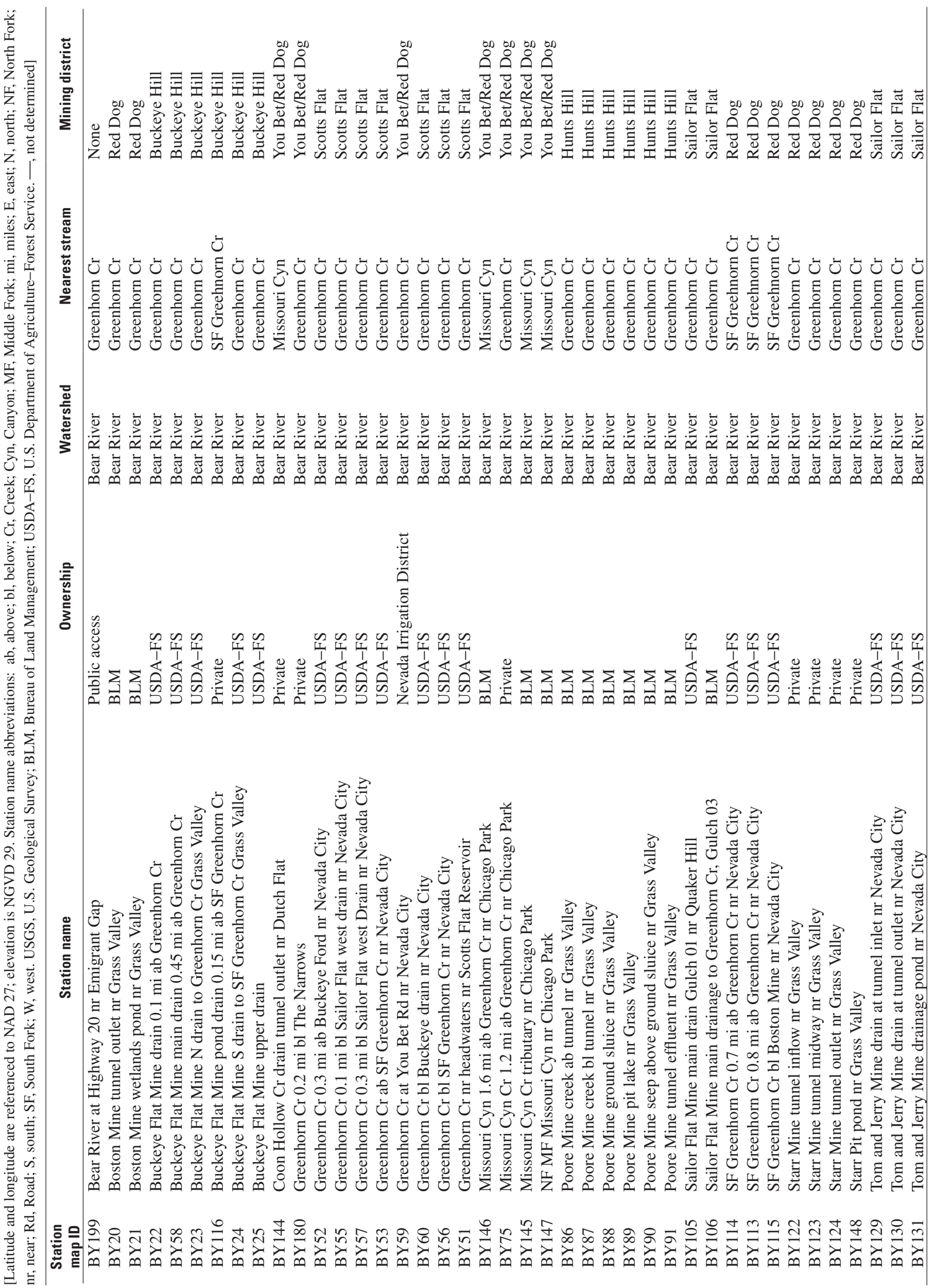




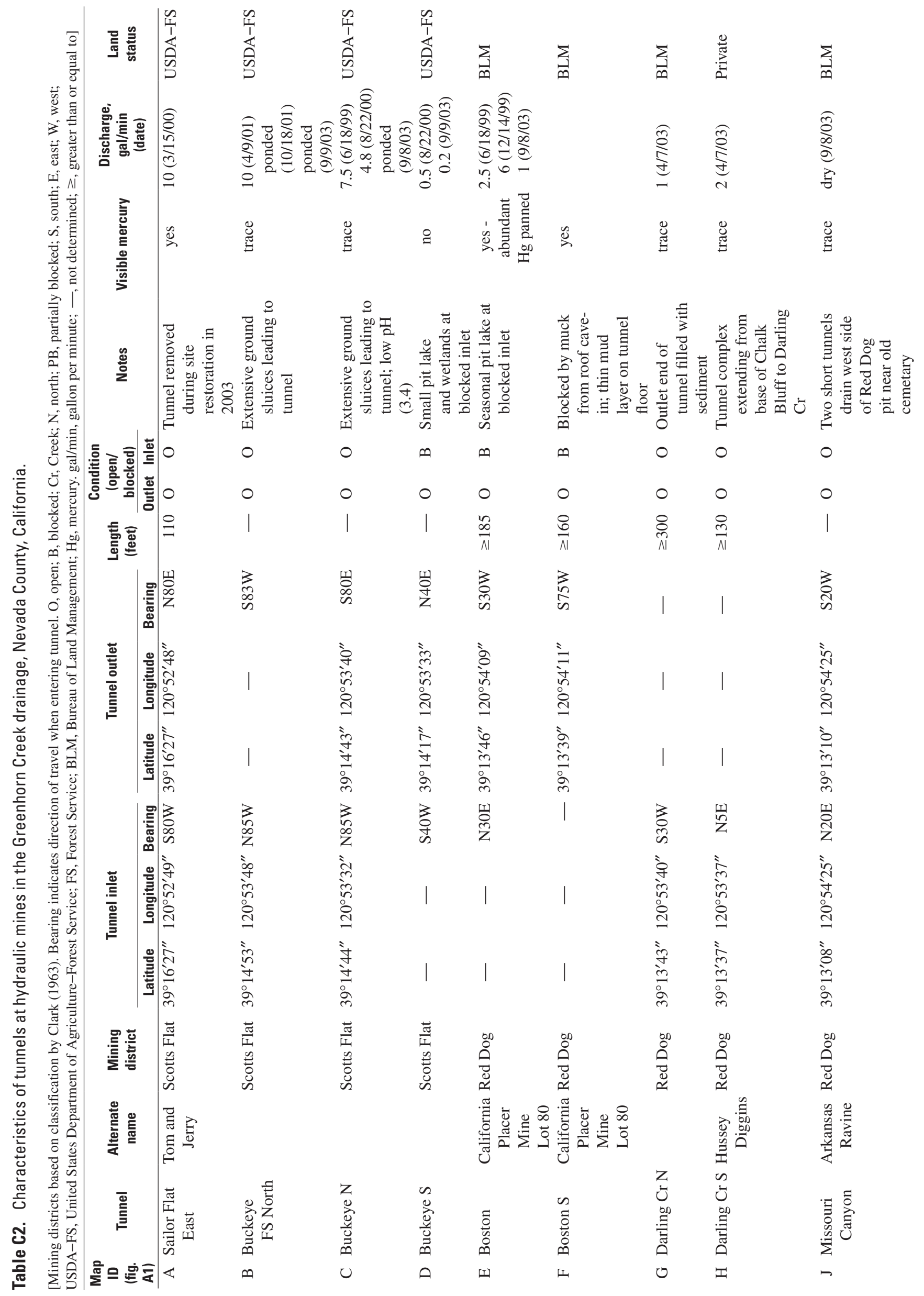




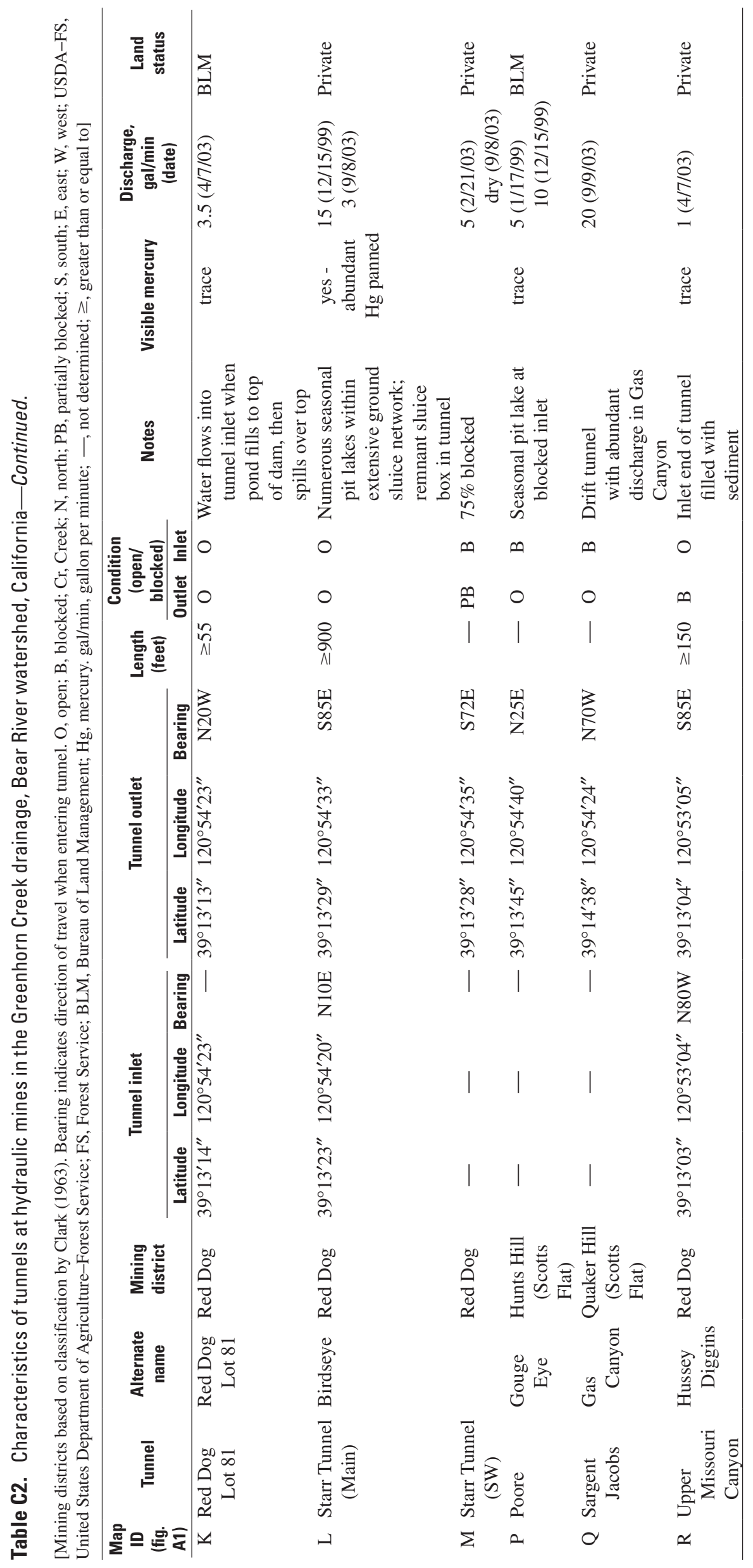


Table C3. Descriptions of photographs of sampling stations, Greenhorn Creek drainage, Nevada County, California.

[Station name abbreviations: ab, above; bl, below; Cr, Creek; Cyn, Canyon; mi, mile; nr, near; Rd, Road; ***, not USGS station. SWRCB, State Water Resources Control Board]

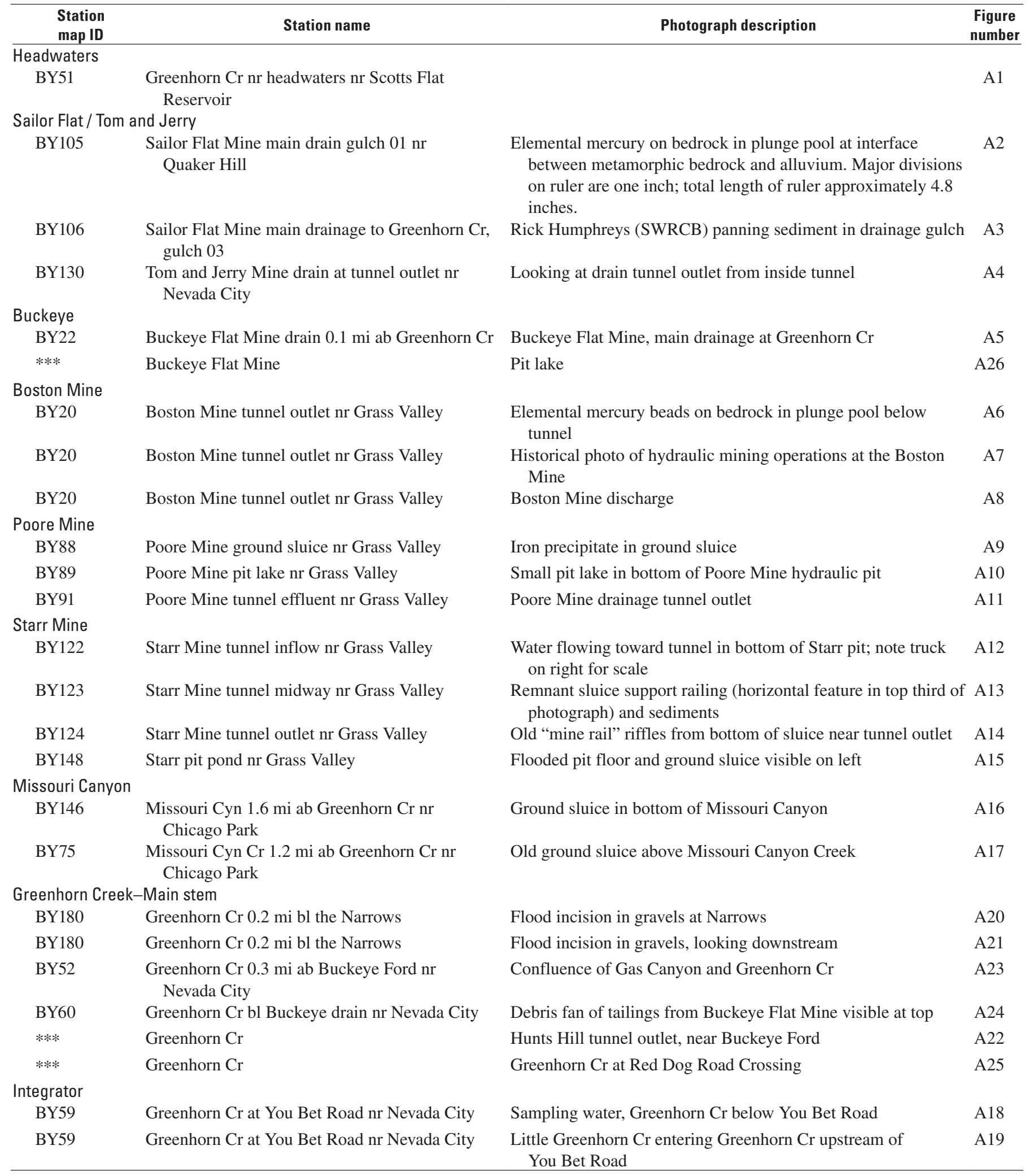


Appendix D. Quality Assurance and Quality Control Information for Chemical Analyses of Samples from the Greenhorn Creek Drainage, Nevada County, California 
Table D1. Trace elements analyzed by inductively coupled plasmamass spectrometry, and corresponding median detection limits.

[Elements defined in front matter and in Tables 4A and 4B. n, number of analyses during 1999-2001. $\mu \mathrm{g} / \mathrm{L}$, microgram per liter]

\begin{tabular}{|c|c|c|}
\hline Element & $\begin{array}{c}\text { Dectection } \\
\quad \operatorname{limit} \\
(\mu \mathrm{g} / \mathrm{L})\end{array}$ & $\mathrm{n}$ \\
\hline $\mathrm{Al}$ & 0.2 & 419 \\
\hline As & 0.05 & 415 \\
\hline B & 11 & 420 \\
\hline $\mathrm{Ba}$ & 0.02 & 446 \\
\hline $\mathrm{Be}$ & 0.03 & 459 \\
\hline $\mathrm{Bi}$ & 0.003 & 447 \\
\hline $\mathrm{Cd}$ & 0.009 & 430 \\
\hline $\mathrm{Ce}$ & 0.0005 & 449 \\
\hline Co & 0.007 & 447 \\
\hline $\mathrm{Cr}$ & 0.3 & 432 \\
\hline Cs & 0.01 & 442 \\
\hline $\mathrm{Cu}$ & 0.03 & 445 \\
\hline Dy & 0.001 & 472 \\
\hline $\mathrm{Er}$ & 0.0009 & 467 \\
\hline $\mathrm{Eu}$ & 0.0005 & 476 \\
\hline $\mathrm{Gd}$ & 0.001 & 463 \\
\hline Но & 0.0002 & 471 \\
\hline $\mathrm{La}$ & 0.0007 & 452 \\
\hline $\mathrm{Li}$ & 0.07 & 421 \\
\hline $\mathrm{Lu}$ & 0.0002 & 466 \\
\hline $\mathrm{Mn}$ & 0.06 & 436 \\
\hline Mo & 0.03 & 442 \\
\hline $\mathrm{Nd}$ & 0.001 & 464 \\
\hline $\mathrm{Ni}$ & 0.03 & 443 \\
\hline $\mathrm{Pb}$ & 0.008 & 399 \\
\hline $\operatorname{Pr}$ & 0.0004 & 472 \\
\hline $\mathrm{Rb}$ & 0.003 & 449 \\
\hline $\mathrm{Re}$ & 0.001 & 475 \\
\hline $\mathrm{Sb}$ & 0.004 & 450 \\
\hline $\mathrm{Se}$ & 0.4 & 423 \\
\hline $\mathrm{Sm}$ & 0.002 & 474 \\
\hline $\mathrm{Sr}$ & 0.1 & 434 \\
\hline $\mathrm{Tb}$ & 0.0003 & 469 \\
\hline $\mathrm{Te}$ & 0.02 & 462 \\
\hline Th & 0.0006 & 436 \\
\hline $\mathrm{Tl}$ & 0.005 & 439 \\
\hline $\mathrm{Tm}$ & 0.0003 & 460 \\
\hline $\mathrm{U}$ & 0.001 & 453 \\
\hline V & 0.1 & 414 \\
\hline $\mathrm{Y}$ & 0.0008 & 451 \\
\hline $\mathrm{Yb}$ & 0.0007 & 472 \\
\hline $\mathrm{Zn}$ & 0.2 & 425 \\
\hline $\mathrm{Zr}$ & 0.003 & 444 \\
\hline
\end{tabular}


Table D2. Selected trace elements and regression correlation coefficients $\left(R^{2}\right)$ for correlation plots of observed versus reported values of analyses of standard reference materials.

\begin{tabular}{cl}
\hline Element & \multicolumn{1}{|c}{$\mathbf{R}^{2}$} \\
\hline $\mathrm{Al}$ & 0.9995 \\
$\mathrm{As}$ & 0.9996 \\
$\mathrm{~B}$ & 0.9903 \\
$\mathrm{Ba}$ & 0.9986 \\
$\mathrm{Be}$ & 1.000 \\
$\mathrm{Cd}$ & 0.9999 \\
$\mathrm{Co}$ & 0.9996 \\
$\mathrm{Cr}$ & 0.9995 \\
$\mathrm{Cu}$ & 0.9974 \\
$\mathrm{Li}$ & 0.9997 \\
$\mathrm{Mn}$ & 0.9881 \\
$\mathrm{Mo}$ & 0.9998 \\
$\mathrm{Ni}$ & 0.9980 \\
$\mathrm{~Pb}$ & 0.9999 \\
$\mathrm{Sb}$ & 1.000 \\
$\mathrm{Se}$ & 0.9955 \\
$\mathrm{Sr}$ & 0.9998 \\
$\mathrm{Tl}$ & 0.9989 \\
$\mathrm{U}$ & 0.9965 \\
$\mathrm{~V}$ & 0.9998 \\
$\mathrm{Zn}$ & 0.9994 \\
\hline
\end{tabular}


Table D3. Data for total mercury in blanks.

[value, mean of triplicate analyses; MilliQ is water deionization unit manufactured by Millipore, Inc.; s.d., standard deviation of triplicate analyses; ng/L, nanogram per liter; <, less than]

\begin{tabular}{|c|c|c|c|c|c|}
\hline \multirow[b]{2}{*}{ Blank type } & \multirow[b]{2}{*}{ Replicate } & \multirow[b]{2}{*}{ Date } & \multirow[b]{2}{*}{ Time } & \multicolumn{2}{|c|}{ Total mercury } \\
\hline & & & & $\begin{array}{l}\text { value } \\
\text { (ng/L) }\end{array}$ & $\begin{array}{c}\text { s.d. } \\
\text { (ng/L) }\end{array}$ \\
\hline Source Blank - Deionized Water - MilliQ & 1 of 2 & $11 / 30 / 99$ & 11:00 & 3.3 & 0.2 \\
\hline Source Blank - Deionized Water - MilliQ & 2 of 2 & $11 / 30 / 99$ & 11:00 & 2.1 & 0.4 \\
\hline Equipment Blank - Teflon-lined Churn & 1 of 2 & 12/1/99 & $10: 30$ & 3.8 & 0.0 \\
\hline Equipment Blank - Teflon-lined Churn & 2 of 2 & $12 / 1 / 99$ & $10: 30$ & $<0.6$ & 0.3 \\
\hline Equipment Blank - Teflon-lined Churn & 1 of 1 & $1 / 26 / 00$ & $5: 00$ & 0.9 & 0.2 \\
\hline Equipment Blank - Churn \#5 & 1 of 1 & $1 / 26 / 00$ & 5:00 & 1.2 & 0.1 \\
\hline Equipment Blank - Jerrican BY01 & 1 of 1 & $1 / 26 / 00$ & 5:00 & 0.5 & 0.4 \\
\hline Equipment Blank - Jerrican BY02 & 1 of 1 & $1 / 26 / 00$ & 5:00 & $<0.6$ & 0.0 \\
\hline Equipment Blank - Jerrican BY03 & 1 of 1 & $1 / 26 / 00$ & 5:00 & $<0.6$ & 0.1 \\
\hline Equipment Blank - MilliQ Holding Bottle & 1 of 1 & $1 / 26 / 00$ & 5:00 & 1.6 & 0.1 \\
\hline Equipment Blank - Holding Bottle \#4 & 1 of 1 & $1 / 26 / 00$ & $5: 00$ & 0.8 & 0.3 \\
\hline Process Blank - Churn 2 FB-C2 & 1 of 1 & $2 / 27 / 00$ & $10: 45$ & $<0.4$ & 0.2 \\
\hline Process Blank - Churn 2 FB-C2 & 1 of 1 & $2 / 28 / 00$ & $11: 30$ & $<0.4$ & 0.1 \\
\hline
\end{tabular}


Table D4. Data for methylmercury in blanks.

[ng/L, nanogram per liter; <, less than; - , not determined)

\begin{tabular}{lccc}
\hline \multicolumn{1}{c}{ Type of blank } & Date & Time & \begin{tabular}{c} 
Methylmercury \\
\cline { 3 - 4 }
\end{tabular} \\
\hline Equipment Blank - Churn \# HT-1 & & $\begin{array}{c}\text { Unfiltered } \\
\text { (ng/L) }\end{array}$ & $\begin{array}{c}\text { Filtered } \\
\text { (ng/L) }\end{array}$ \\
Equipment Blank - Holding Bottle \#4 & $6 / 17 / 1999$ & - & $<0.04$ \\
Equipment Blank - Holding Bottle \#3 & $6 / 17 / 1999$ & $14: 30$ & $<0.04$ \\
Process Blank - Holding Bottle \#2 & $8 / 21 / 2000$ & $20: 30$ & $<0.04$ \\
Process Blank - Holding Bottle \#4 & $8 / 21 / 2000$ & $18: 45$ & $<0.04$ \\
Process Blank & $8 / 21 / 2000$ & $18: 45$ & $<0.04$ \\
\hline
\end{tabular}




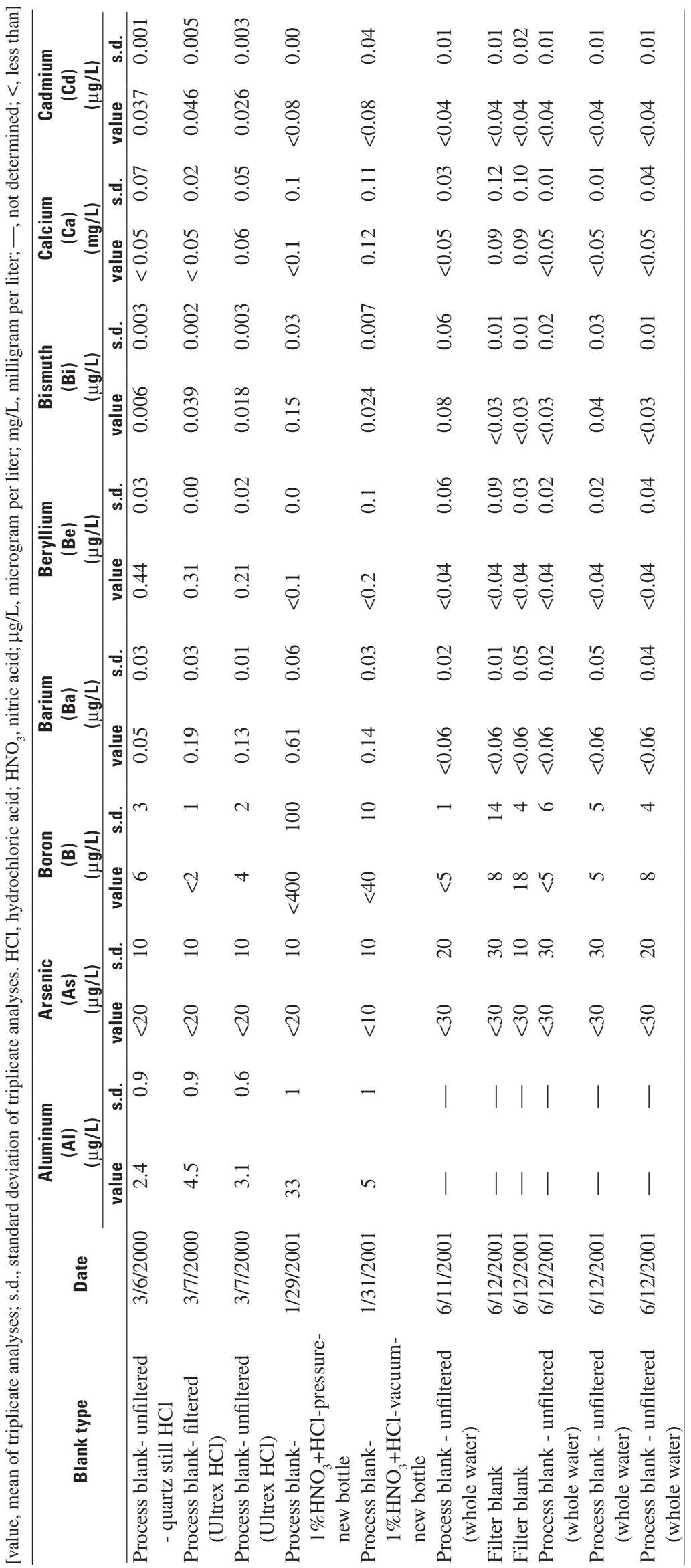




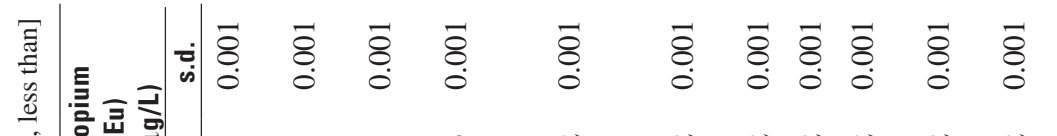

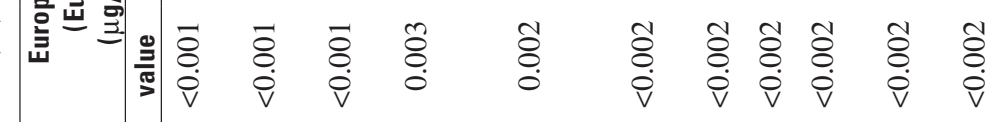

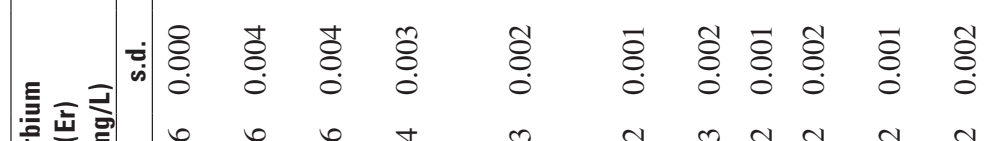

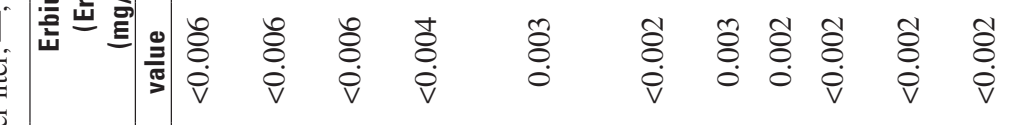

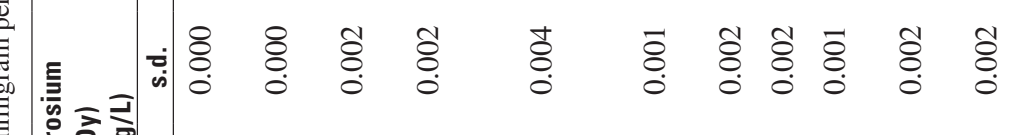

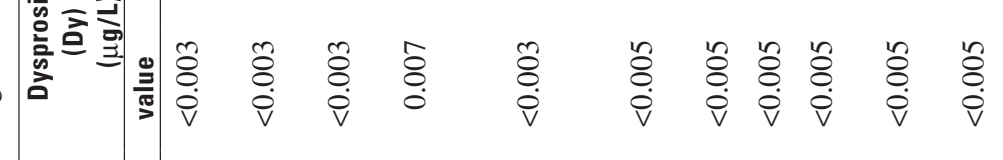

$$
\begin{aligned}
& \text { 苟 }
\end{aligned}
$$

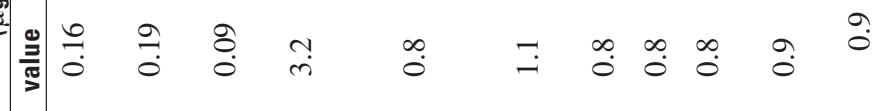

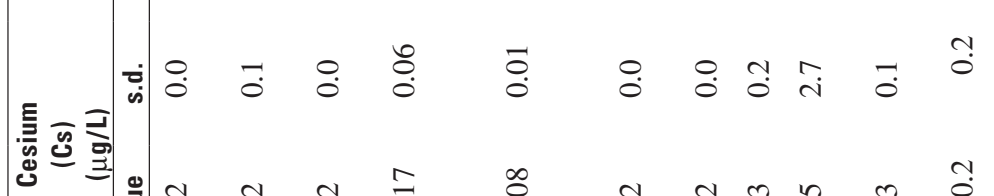

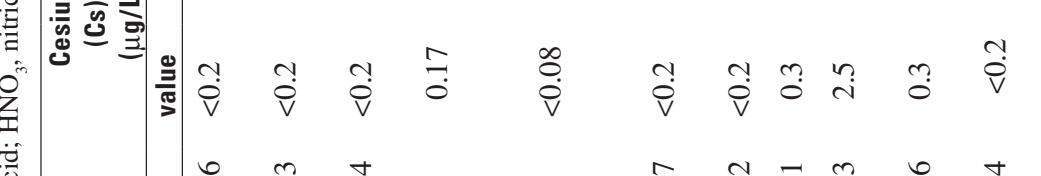

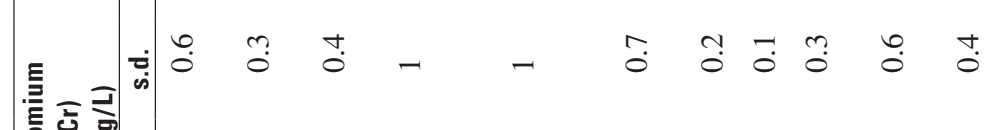

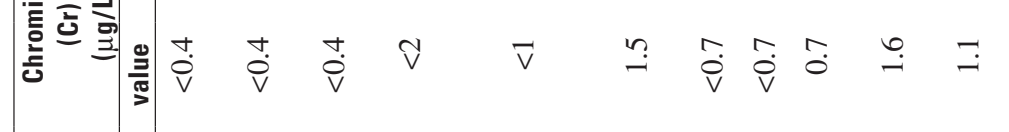

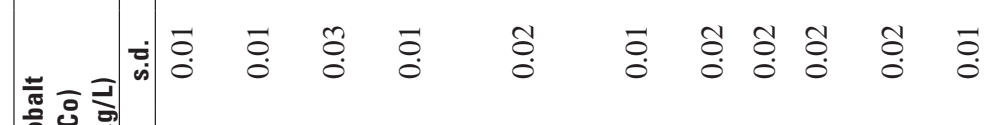

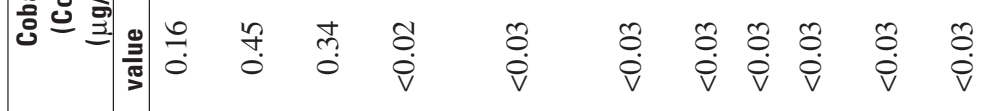

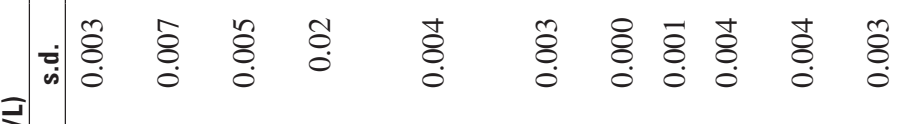

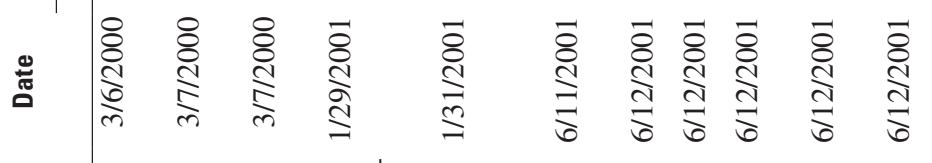

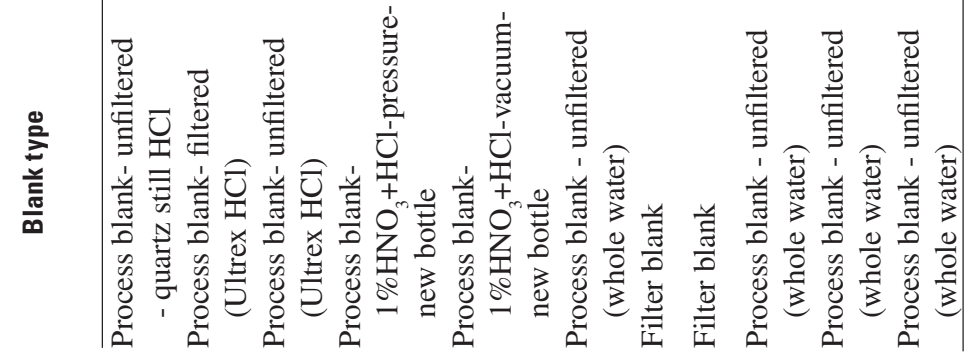




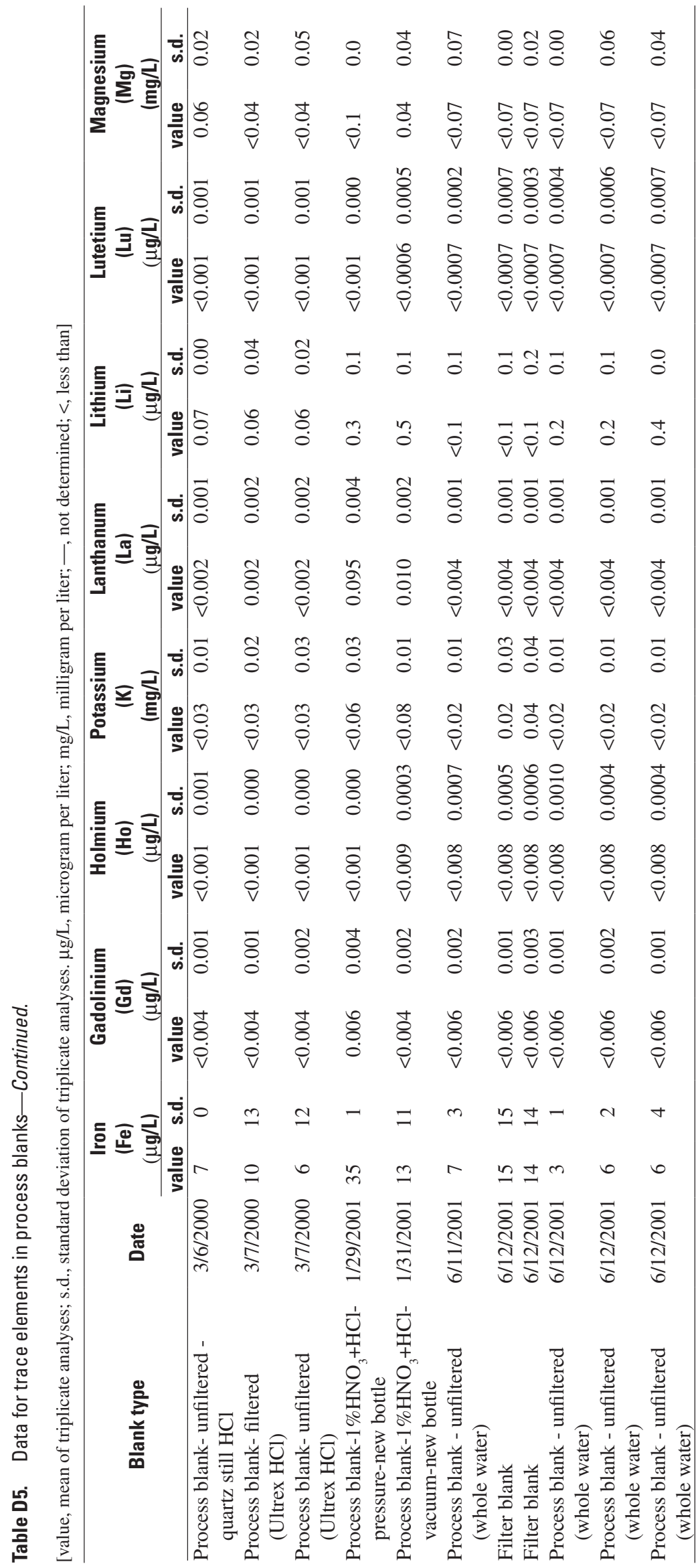




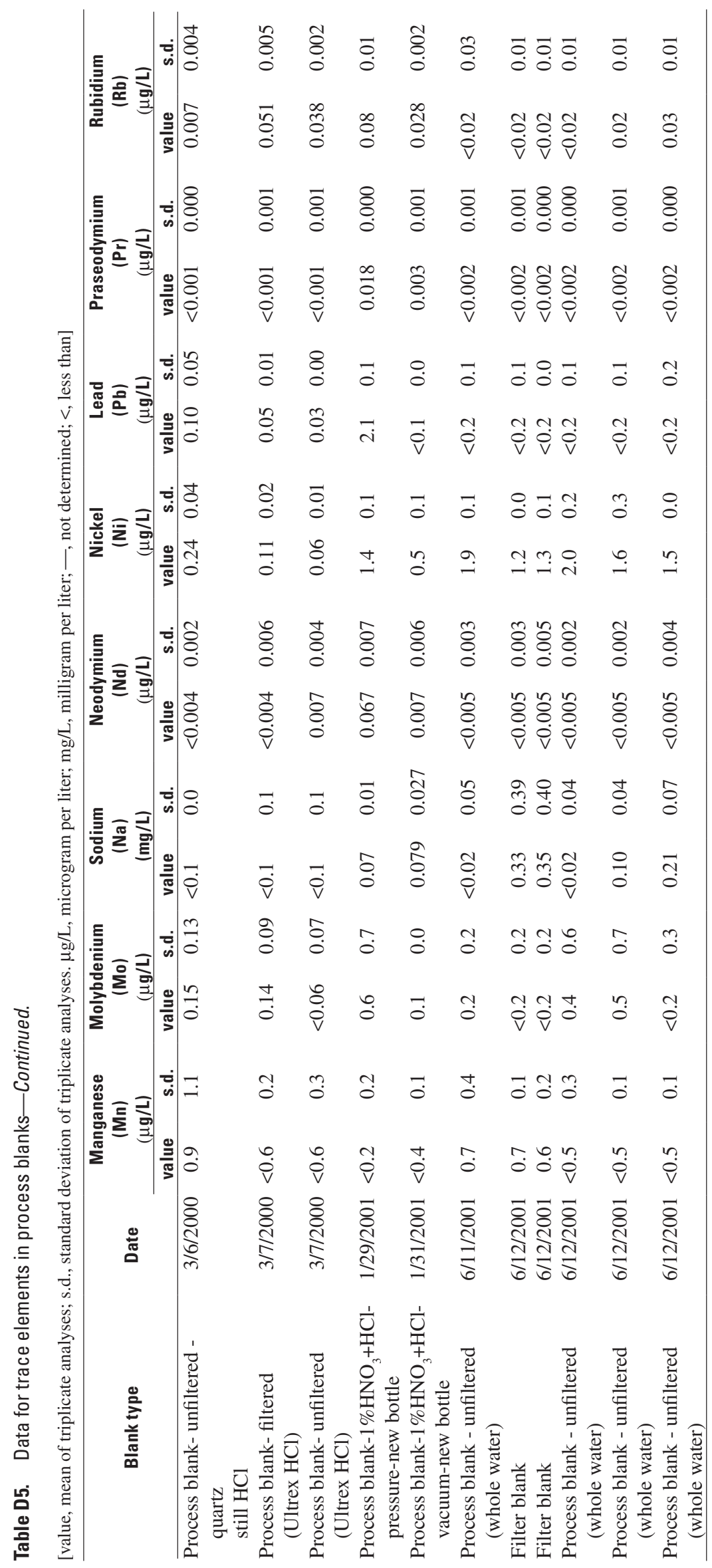




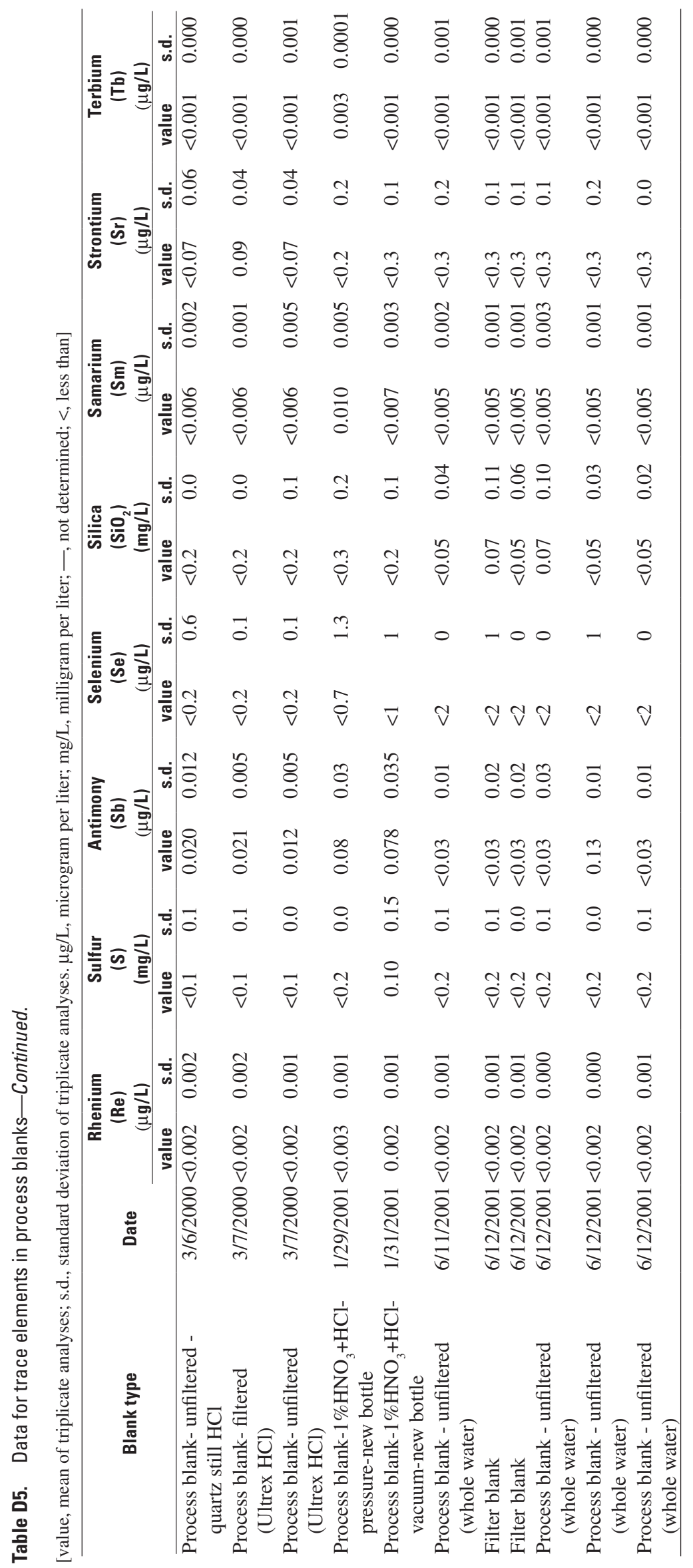




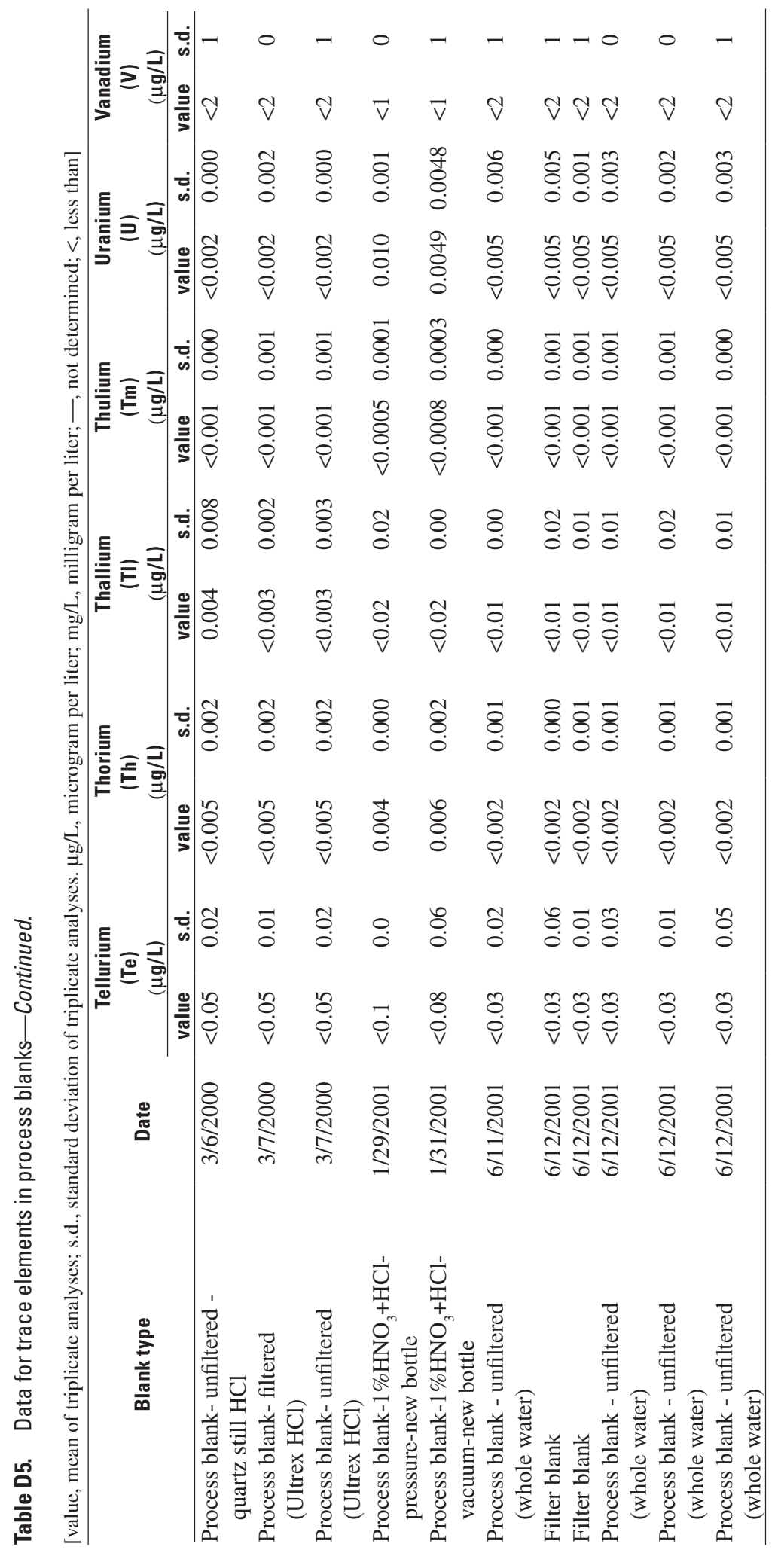




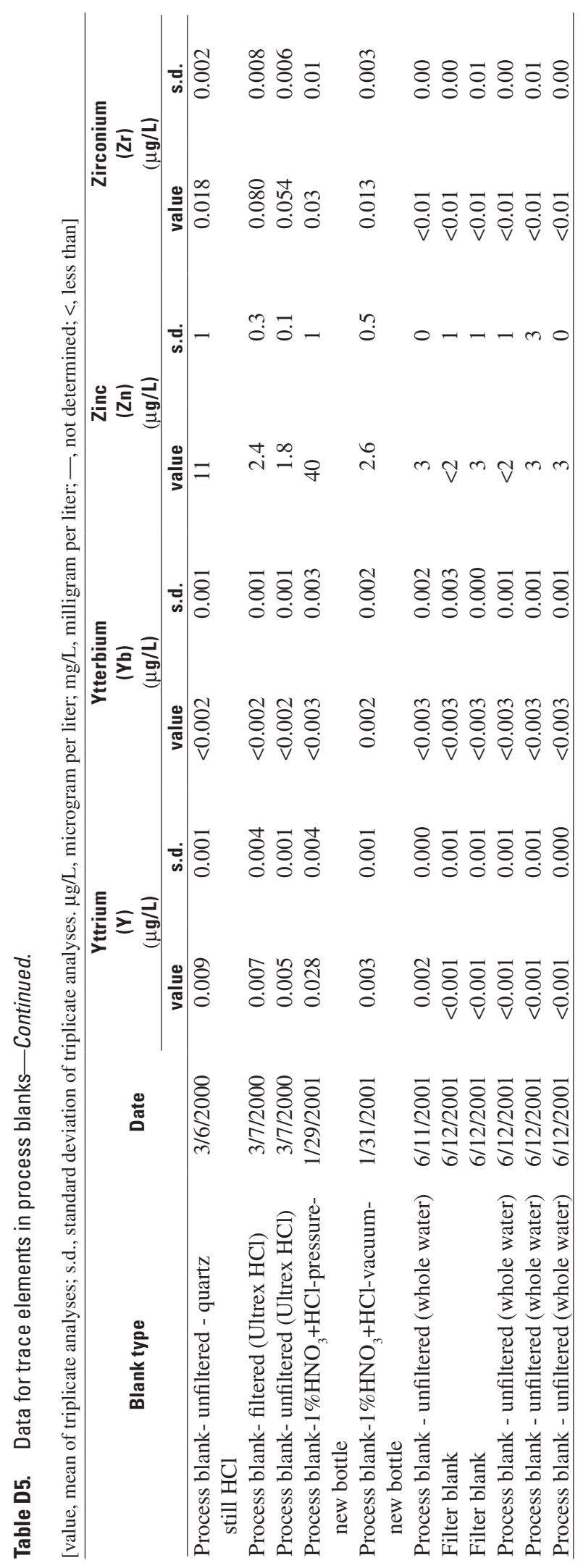




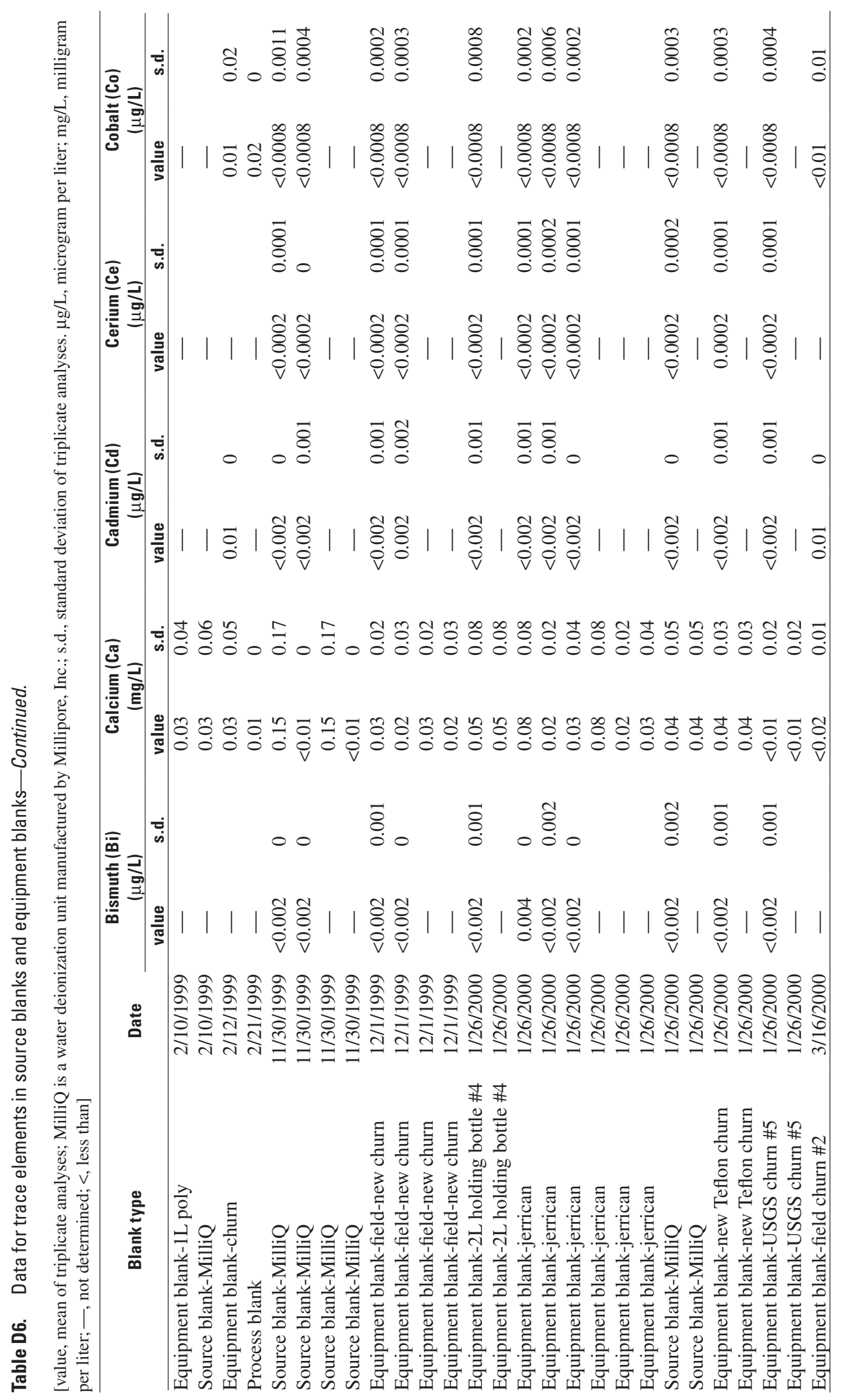




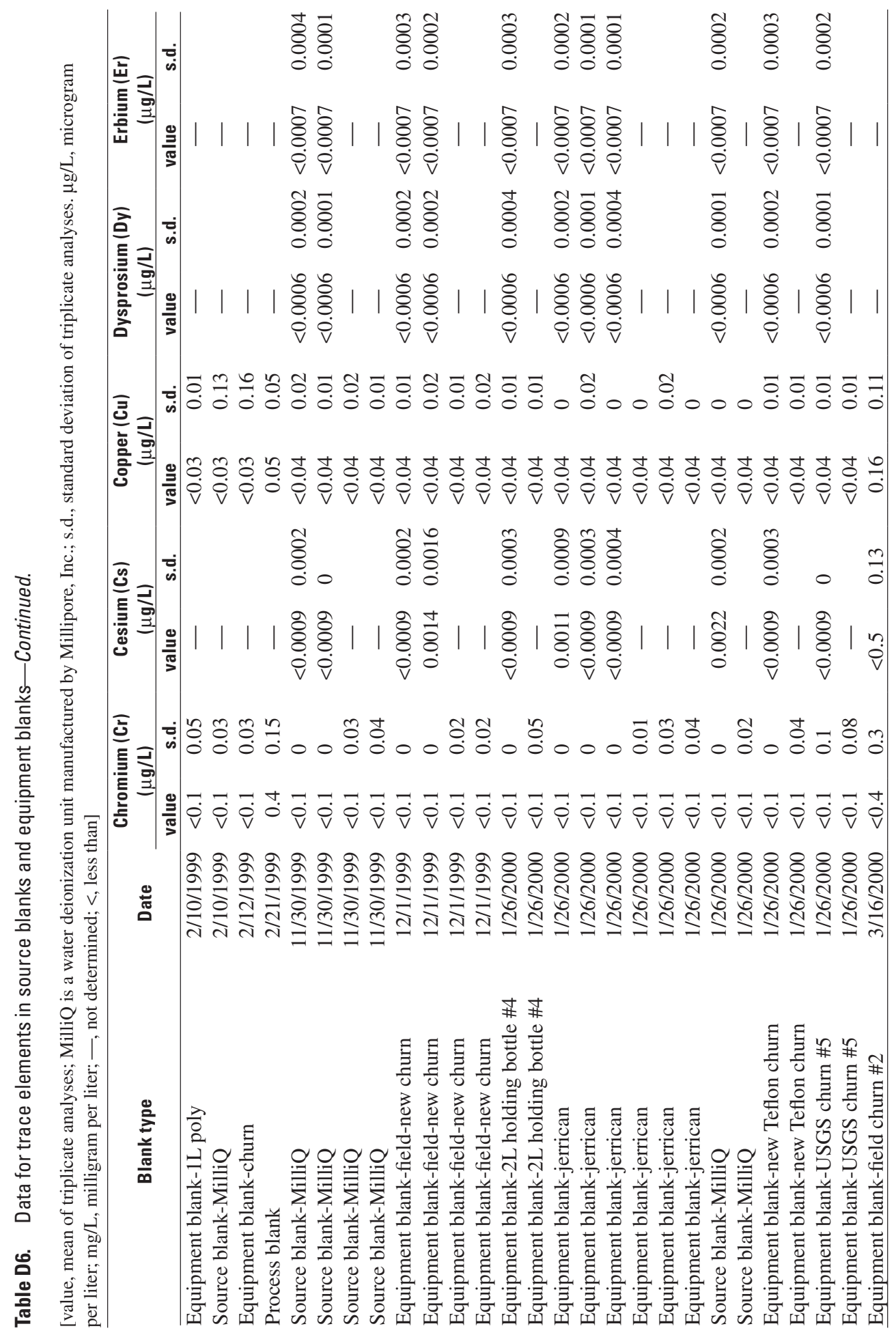




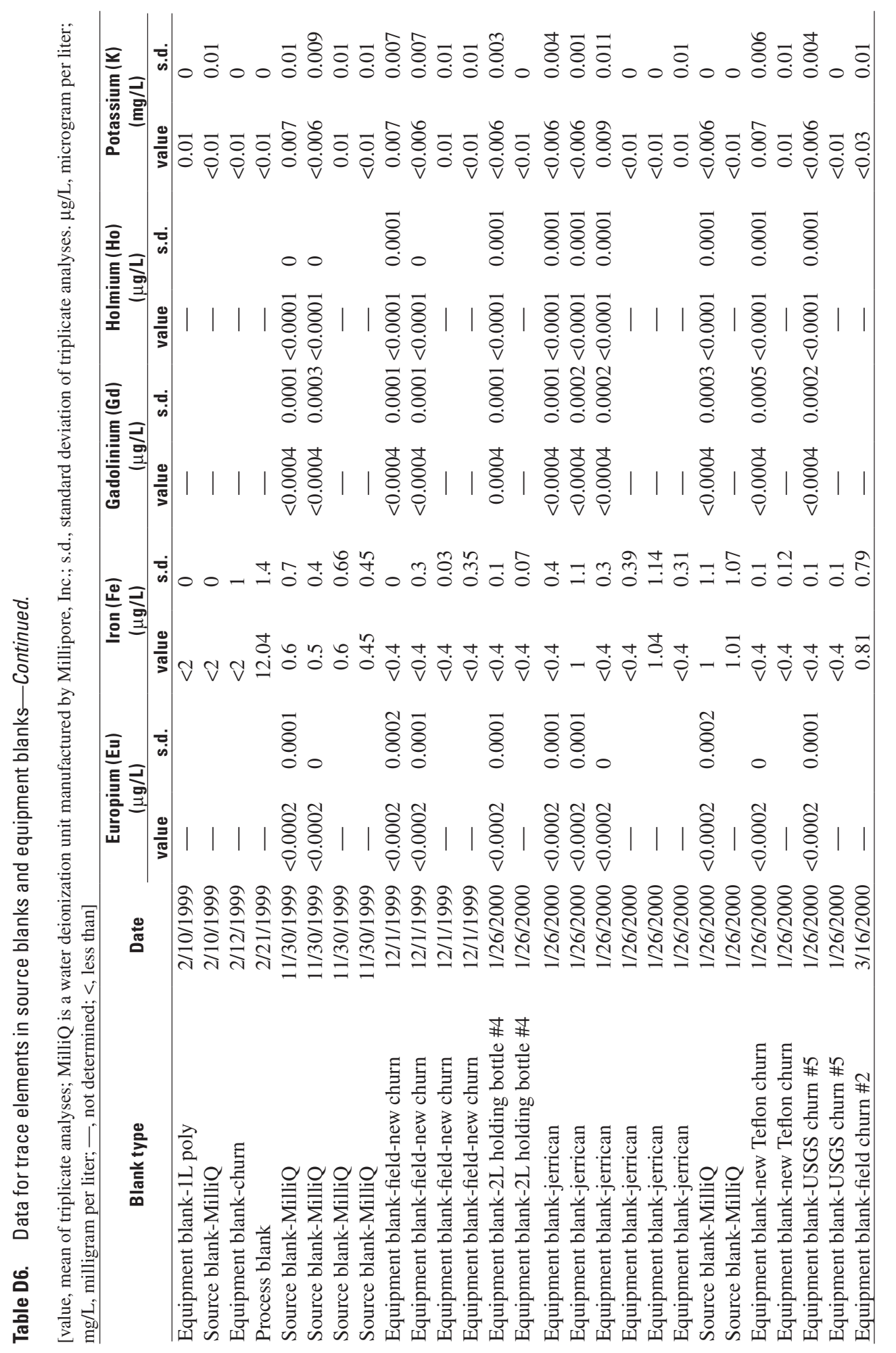




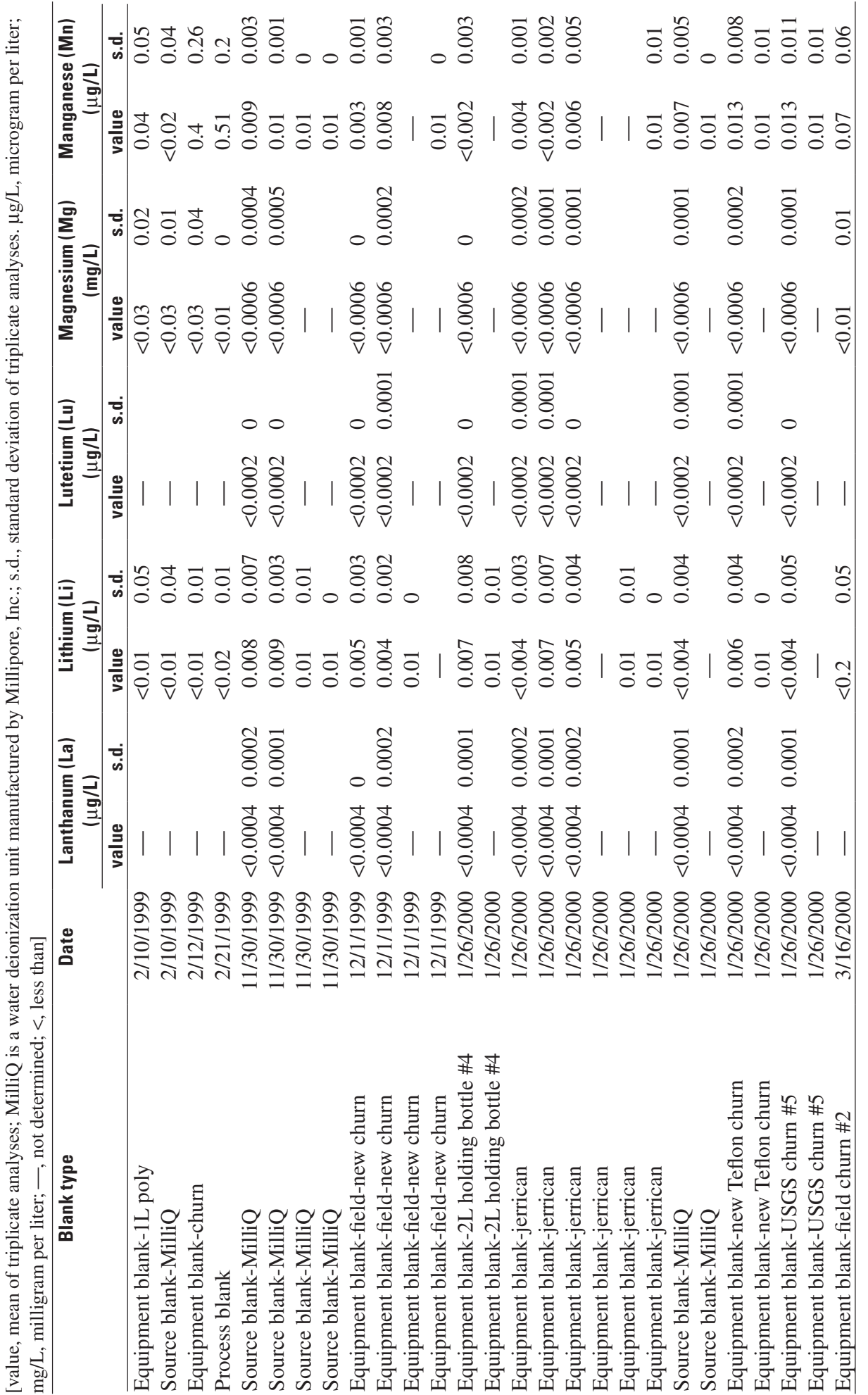




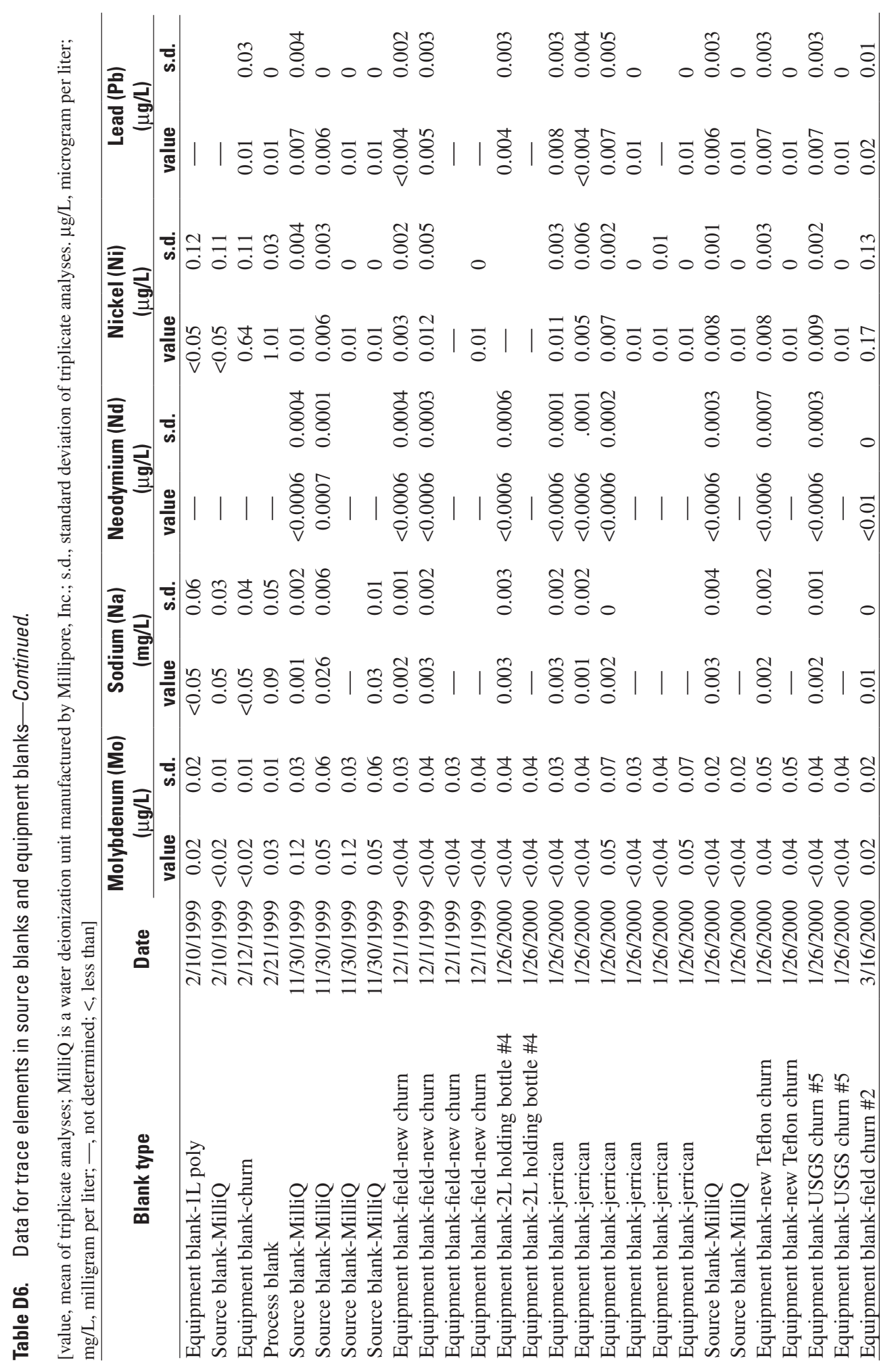




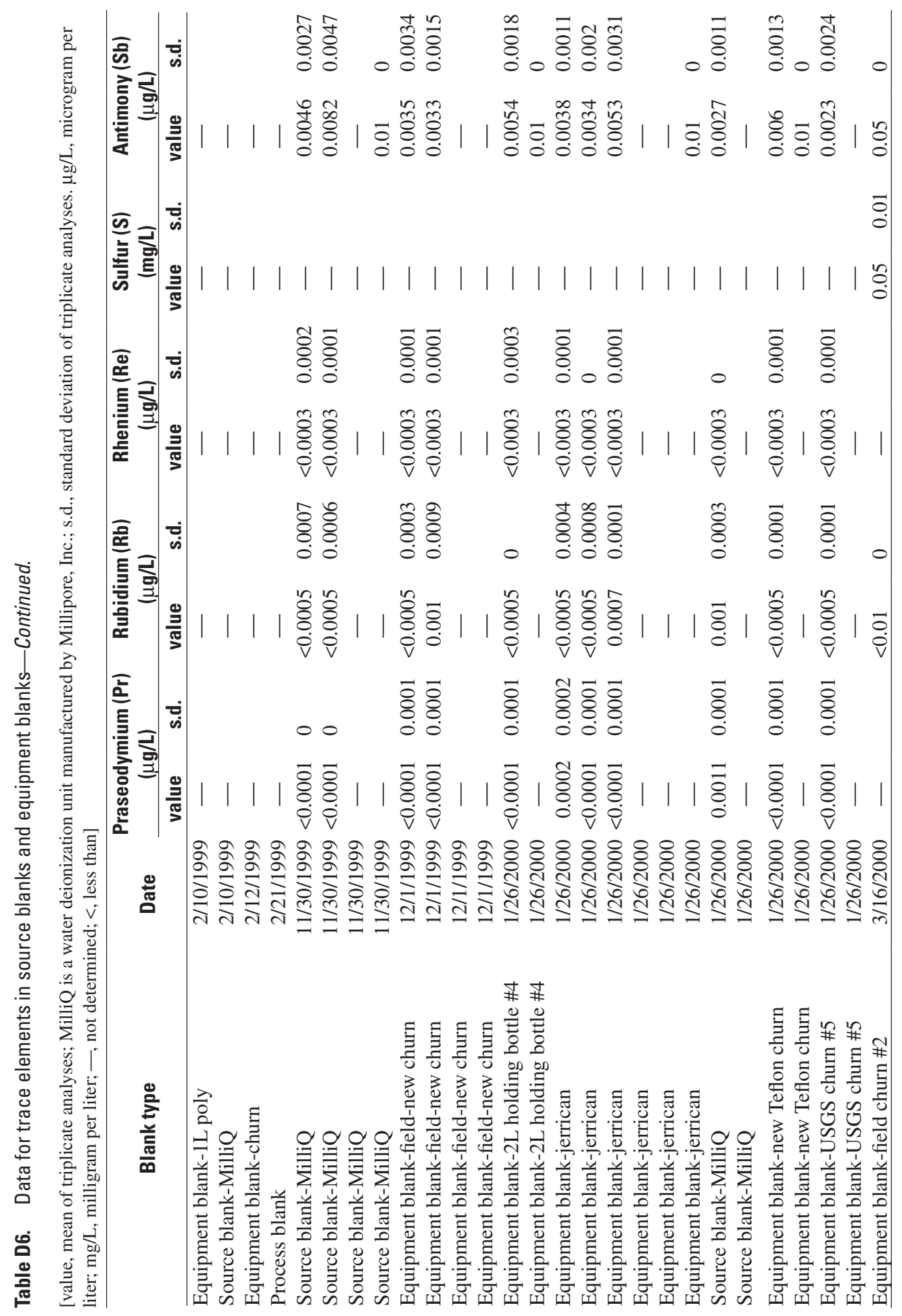




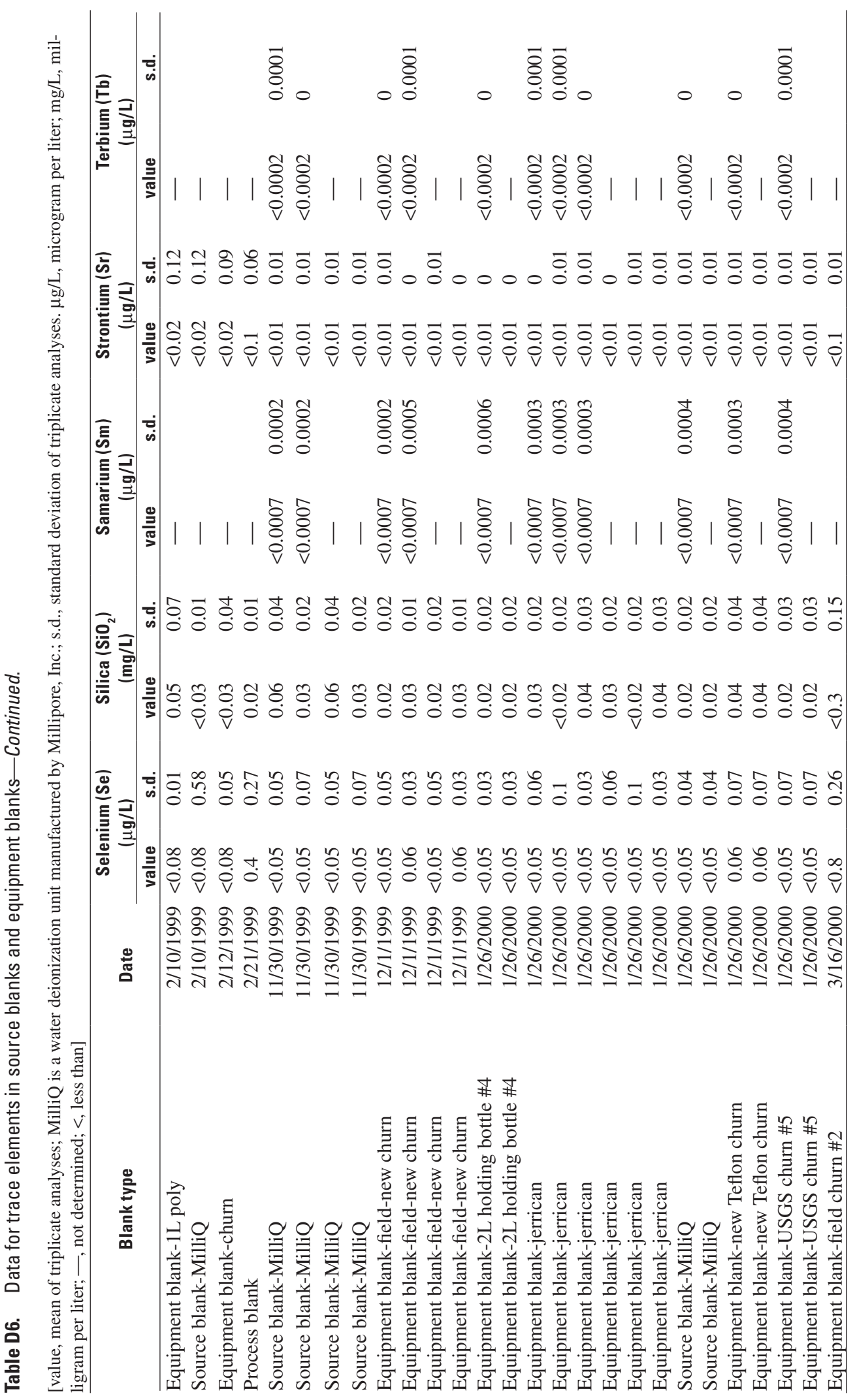




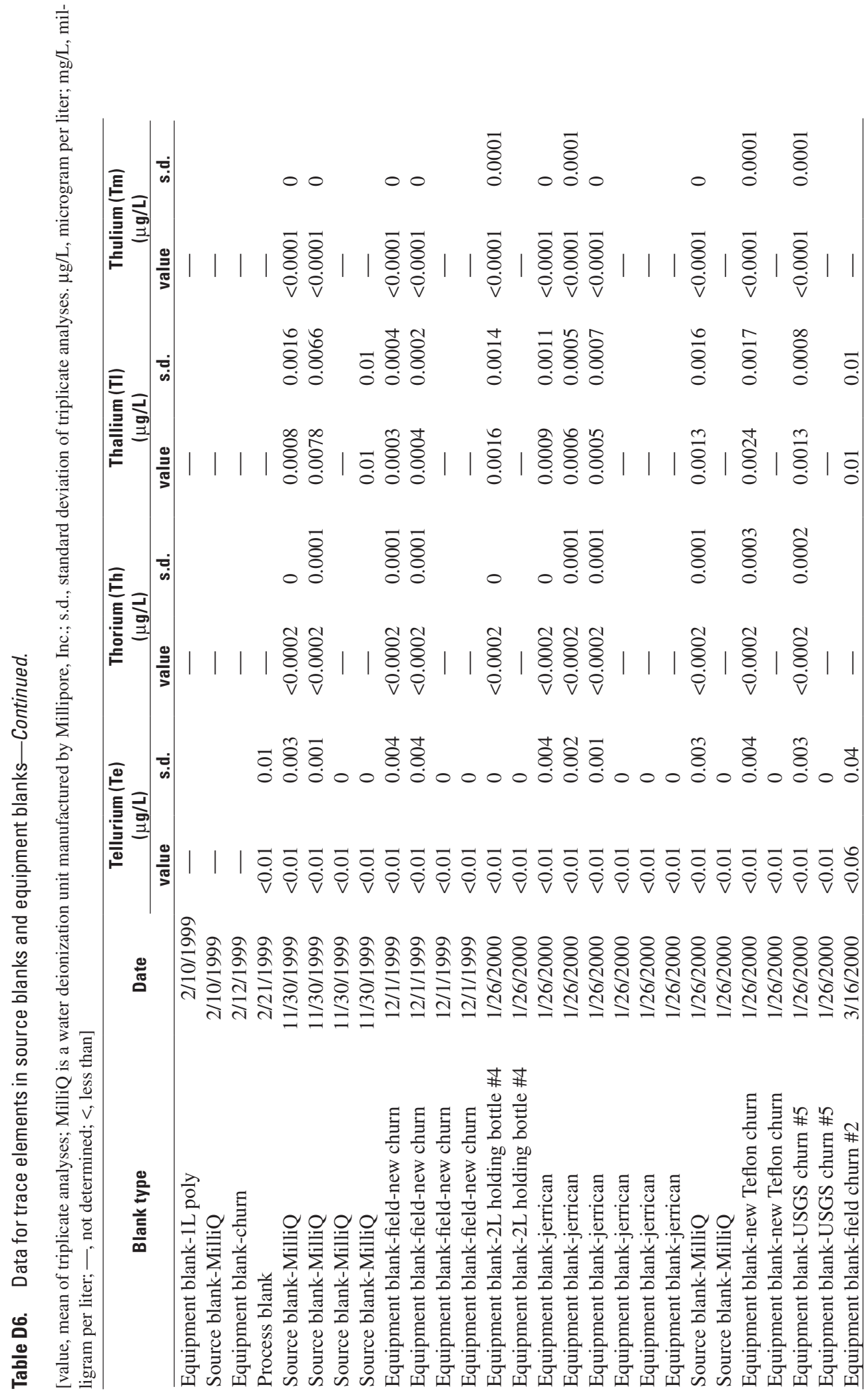




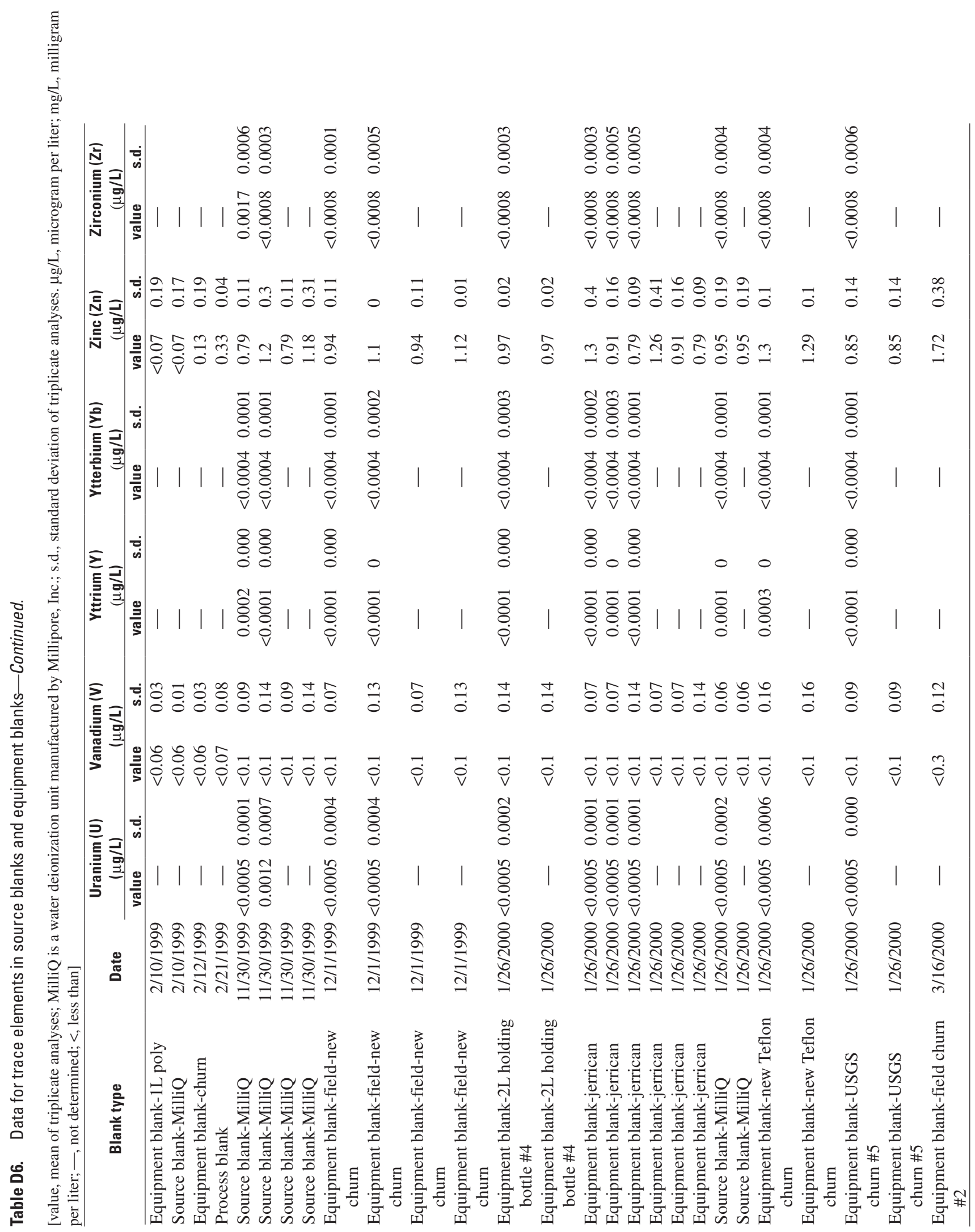




\section{Glossary}

amalgamation The process by which a metal is united in an alloy with mercury. Metals known to amalgamate include gold, silver, copper, and tin.

bench deposit Gravel deposits located anywhere from tens to several hundreds of feet above a stream channel or flood plain.

bioaccumulation The process by which a chemical constituent derived from the environment builds up in the tissues of an organism.

cemented gravels Gravels and sand particles bound together in a sedimentary formation by geological materials usually containing calcium, silica, or iron.

detritivore An animal that consumes decomposing organic particles (detritus), deriving nutrition primarily from microbes on the particles.

elemental mercury The pure form of mercury, the only element to be stable as a liquid at room temperature; also known as quicksilver.

equal width increment (EWI) method A method for collecting an integrated crosssectional sample of a flowing river or stream. The sample is obtained by collecting a volume proportional to the amount of flow at each of several equally spaced verticals in a cross section.

"hot spot" An area characterized by an anomalously high concentration of one or more contaminants of interest. In this study, mercury and methylmercury concentrations in water, sediment, and biota were considered in defining "hot spots."

hydraulic mining A method of mining in which a bank of gold-bearing earth or gravel is washed away by a powerful jet of water and carried into sluices, where the gold drops out because of its higher density. mercury load The amount (mass) of mercury transported by a river or stream passing a specific location during a given time; typical units for mercury load are grams per day and kilograms per year.

methylmercury (MeHg) An organic form of mercury (formula $\mathrm{CH}_{3} \mathrm{Hg}^{+}$) that is readily bioaccumulated. It is more toxic to humans and other biota than native (elemental) mercury.

placer gold Gold grains or flakes in an unconsolidated sediment deposit. Also known as alluvial gold.

predatory insect An insect that feeds on another species of insect (or, rarely, on a member of the same species)

remediation Remedying or mitigating the effects of pollution of lands or waters, or restoring land or waters to their former state.

retort (1) (verb) to distill or decompose by heat; (2) (noun) vessel in which gold-mercury amalgam is heated to drive off the mercury as vapor. The mercury vapor is cooled in a condenser pipe and is recovered as a liquid. A similar process was used to extract mercury from cinnabar (mercury sulfide) ores and concentrates.

RPD Relative Percentage Difference Quantity computed for the evaluation of precision (or variability) of laboratory analytical data using randomly submitted split samples.

$\mathrm{RPD}=\frac{\text { difference between reported values }}{\text { average reported value }} \times 100$

RSD Relative Standard Deviation A quantity computed for the evaluation of precision (or variability) of data. Relative standard deviation is the standard deviation of a series of measurements divided by the average of those measurements times 100:

$$
\mathrm{RSD}=\frac{\text { standard deviation }}{\text { average reported value }} \times 100
$$


sluice (1) (verb) To mine an alluvial deposit using hydraulic mining methods (2) (noun) A long, trough-like box set on a gentle slope of about 1:20, through which placer gravel is carried by a stream of water. The sand, gravel, and finer sediments are carried away while most of the gold and other heavy minerals are caught in riffles. Amalgamation (reaction with mercury) is commonly practiced by putting native (elemental) mercury between riffles.

stamp mill An apparatus, and the building containing it, in which rock is crushed by descending pestles (stamps), operated by water power or steam power. Amalgamation (reaction with mercury) was commonly combined with crushing to recover gold and (or) silver. tenor The percentage or average content of metal or precious metal in an ore. As commonly used, it is synonymous with an approximate concentration.

Tertiary channel deposit Ancient gravel deposit, commonly auriferous (gold-bearing), composed of stream alluvium of Tertiary age (1.8 to 65 million years before present). Tertiary channel deposits are abundant in the Sierra Nevada gold belt of California where many have been covered by extensive volcanic eruptions and subsequently elevated by mountain uplifts. They are now deeply-buried channels, high above the present stream beds.

total mercury (THg) The sum of all forms or species of mercury in a sample of water, sediment, or biota. 
可.

政

을 돟

롤 동 꼭

옹

꾸음

름윽

옹

우욤

果

궁

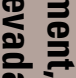

을

卷

言蛋

흘 옹

붕

छ

익

올

co

오을

营

言

추 흘

里

ㅎ. 를

음

을 के

金 흠

응 믕

을 몰.

กิ 